UNITED STATES DEPARTMENT OF THE INTERIOR

Harold L. Ickes, Secretary

GEOLOGICAL SURVEY

W. C. Mendenhall, Director

Water-Supply Paper 844

\title{
FLOODS OF MARCH 1938 \\ IN SOUTHERN CALIFORNIA
}

BY

$\because \%$

(3.

$\therefore$

HAROLD C. TROXELL AND QFHERs

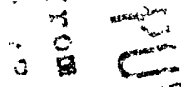

Prepared in cooperation with the

FEDERAL EMERGENCY ADMINISTRATION OF PEBLICWORKS

AND WORKS PROGRESS ADMINISTIִATPQNC?

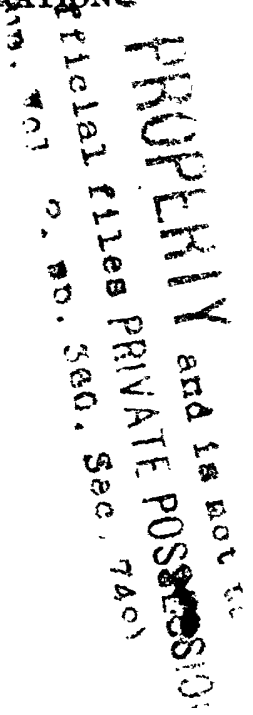

UNITED STATES

GOVERNMENT PRINTING OFFICE

WASHINGTON : 1042

For ale by the Superintendent of Documents, Washington, D. C. - - - - - - Price \$1.25 

Introduction

Administration and personnel . . . . . . . . . . . . . . . 2

Acknowledgments . . . . . . . . . . . . . . . . . . . . . . 3

General features of the storm and flood . . . . . . . . . . . .

Collection of rainfall and runoff records ............... 14

Heteorologic and hydrologic conditions . . . . . . . . . . . . 15

Preclpitation records ........................ . 15

Preparation of isohyetal maps . . . . . . . . . . . . . . . . 46

Local rainfall characteristics . . . . . . . . . . . . . . . 48

San Dimas Experimental Forest . . . . . . . . . . . . . . . 54

Chronologic distribution . . . . . . . . . . . . . . . 58

Rainfall intensities . . . . . . . . . . . . . . . . 61

Comparison of rates of intensity with rates in previous storms . 91

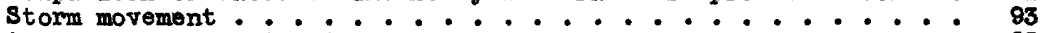

Antecedent precipitation . . . . . . . . . . . . 95

Snow ... . . . . . . . . . . . . . . . . . . . . . . . 96

Basic discharge records : : : : : : : : : : : : : : : 98

Santa Ana River Basin .... . . . . . . . . . . . . . 105

Santiago Creok at Santiago Reservoir, near vilia Park : : 105

San Gabriel River Basin . . . . . . . . . . . . . . 106

San Gabriel River at flood-control reservoir No. i, near

Azusa . . . . . . . . . . . . . . . 106

San Gabriel River at Morris Reservoir, near Azusa . . . . 108

West Fork of San Gabriel River at flood-control reservoir

No. 2 , near Camp Rincon . . : . . . . . . . . . 109

Sawplt Creek at flood-control reservolr near Monrovia . . 111

San Dimas Creek at flood-control reservolr near San Dimas - 112

Fern Canyon Watershed No. 2, near San Dimas ......... 114

Ferm Canyon Watershed No. 3, near San Dimas : . . . . : 114

Wolfskill Creek near San Dimas................ 115

West Fork of San Dimas Creok near San Dimas . . . . . . . 116

Dalton Creek at flood-control reservoir near Glendora . . . 117

Iive 0ak Creek at flood-control reservoir near La Verne : 118

Thompson Creek at flood-control reservoir near Claremont . : 120

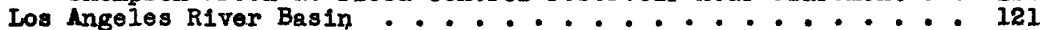

Pacolma Creek at flood-control reservoir near San Fermando: 121

Tujunga Creek at flood-control reservolr near Sunland . . . 123

Santa Anita Creek at flood-control reservoir near sierra

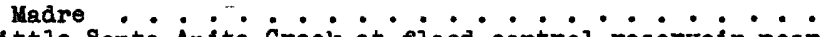

Little Santa Anita Creek at flood-control reservoir near

Sierra Madre........................ 126

Eaton Creek at flood-control reservoir near Pasadena . . . 127

Calleguas Creek Basin . . . . . . . . . . . . . . . . . 129

Honda Barranca at Beryliwood Road, near Somis . . . . . 129

Honda Barranca at Perkins Road, near Somis . : . : . : . 129

Laximum inflow to reservolirg . . . . . . 131

Studies of besic discharge records in relation to bas in characteristics and rainfall

Basin characteristićs

Runoff characteristics . . . . . . . . . . . . . . . . . 140

First phase, February 27 and 28 : . . . . . . . . : 141

Second phase, March 1 ................... 148

Thira phase, March 2 : : : : : : 148

Fourth phase, $6 \mathrm{p}$.

Relation between rates of precipitation and rates of runoff : - I49

Lag between precipitation and runoff............. 168

Infiltration . : . : : : : : : : . : 166

Determination of fiood discharge : : : : : : : : : : : : : 168

Computation of peak discharge . . . . . . . . . . . . . . 168

Slope-area determinations . . . . . . . . . . . 169

Flow over weirs ......................... 170

Flow through contracted openings: . . . . . . . . . . 172

Bxtension of rating curves... . . . . . . . . . . 178

Discharge in unstable channels.............. 173

Computation of continuous records of discharge . . . . . . . . 174

Dexivation of discharge for periods of missing records . . : 176

Consiatency of determinations of flood discharge........ 188 
Page

Explanation of data . . . . . . . . . . . . . 184

Tia Juana River Basin . . . . . . . . . . . . 186

Cottonmood Creek at Morena Dam . . . . . . . . . . . 186

Cottonmood Creek near Dulzura . . . . . . . . . . . 186

Cottonmood Creok above Tecate Creek, near Dulzura . . . . 187

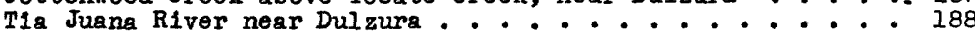

Tia Juana River near Nestor . . . . . . . . . . . . 189

Campo Creek near Campo ..................... 190

otay River Basín . . . . . . . . . . . . . . . . . . . 191

Otay River at Savage Dam . : . : . . . . . . . . . . 191

Sweetwater River Basin................ . . . . . 192

Sweetwater River at Sweotwater Dam: . . . . . . . . . . 192

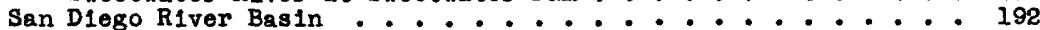

San Diego River at El Capitan Dam, near Lakeside...... 192

San Diego River near Santeo . . . . . . . . 193

Boulder Creek at Cuyamaca Reservoir, near Julian . . . . 194

San Vicente Creek at Foster . . : . . . . . . . 195

San Dieguito River Basin . . . . . . . . . . . . . . . . . 196

Santa Ysabel Creek near Mesa Grande : : . : . : . : 196

San Dieguito River at Lake Hodges . : . : : . . : : 197

San Iuls Rey River Basin . . . . . . . . . . . . . . . . . 197

San Iuis Rey River near Bonsail : : : : . : . . . 197

San Luis Rey River at Oceanside . . . . . . . . . . . . . 198

Santa Margarita River Basin ................ . . 199

Temecula Creek at Nigger Canyon, near Temecula . . . . . . 199

Temecula Creek at Raliroad Canyon, near Temecula . : : : 200

Santa Margarita River near Fall Brook . . . . . . . . . 200

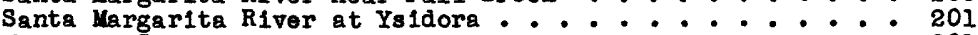

Murrieta Creek at Temecula ................. . 201

San Juan Creek Basin . . . . : : . . . . . . . . . : . 202

San Juan Creek near San Juan Capistrano . . . . . . . . 202

Trabuco Creek near San Juan Caplstrano . . . . . . . . . . 202

Aliso Creek Basin . . . . . : . : . : 203

Aliso Creek at El Toro:. . . . . . . . . . . . . . 203

Santa Ana River Basín . . . . . . . . . . . . . . . 204

Santa Ana River near Mentone... . . . . . . . . . 204

Santa Ana River near Prado... . . . . . . . . . . . 205

Santa Ana River at Santa Ana............... 206

Mill Creek near Craftonville... . . . . . . . . . . 207

Plunge Creek near East Highlands . . . . . . . . . . . 208

San Timoteo Creek near Redlands . . . . . . . . . . . 209

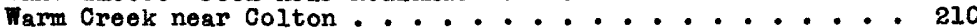

Strawberry Creok near Arrowhead Springs . . . . . . . . . 211

Waterman Canyon Creek near Arrowhead Springs . . . . . . . 212

City Creek near Highland. . . . . . . . . . . . . . 213

Devil Canyon Creek near San Bernardino........... . 214

Lytle Creek near Fontana . . . . . . . . . . . . . . 215

Iytle Creok (east channel) at San Bernardino . . . . . . . 215

Iytle Creek (west channel) at Colton ............ 216

Cajon Creek near Keenbrook .................. 217

Lone Pine Creok near Keenbrook .................... 218

Day Creek near Etlwanda .. . . . . . . . . . . . . . 219

Cucamonga Creek near Upland : : . . . . . . 219

San Jacinto River néar San Jacinto . . . . . . . . . . . 220

San Jacinto River at Railroad Canjon Reservoir, near

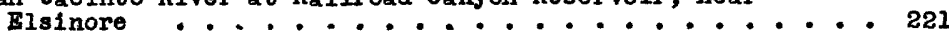

San Jacinto River neap Elsinore . . . . . . . . . . . . 221

Els Inore Lake at Elsinore ................. . . 222

Temescal Creek near Corona . . . . . . . . . . . . . . . 223

Chino Creek near Prado : : : : : : : : : : : 223

San Antonio Creek near claremont . . . . . . : . . . 224

Santiago Creek near Villa Park. . . . . . . . . . . . . 225

Santiago Creek at Santa Ana . . . . . . . . . . . . 226

Irvine Ranch dralnage canal near Tustin . . . . . . . . 227

San Gabriol River Bas in . . . . . . . . . . . . . . . 228

Bast Fork of San Gabriel River near Camp Bonita . . . . 288

San Gabriel River at Foothill Boulevard, near Azusa . . . . 229

San Gabriel River at Plco.................." 230

San Gabriel River at Spring street, near Los Alamitos: : 231

Fest Fork of San Gabriel River at Camp Rincon . . . . : . 232

Rogera Creek near Azusa .. . . . . . . . . . . . . . . . 232

Pish Creek near Duarte... . . . . . . . . . . . . 233

Sawpit Creek near Monrovia... . . . . . . . . . . . . . 234

San Dimas Creek near San Dimas . . . . . . . . . . . . 234

Dalton Creek near Glendora ................. . 235

Iittle Dalton Creek near alendora : . : . . : . : . 236 
Records of stage and discharge at stream-gaging stations--Continued

San Gabriel River Basin--Continued

Walnut Creok near Baldwin Park . . . . . . . . . . . 237

San Jose Creek near Whittier . . . . . . . . . . . . . 238

Cojote Creok near Artes1a . . . . . . . . . . . . . . 239

Brea Creok at Fullerton . . . . . . . . . . . . . . . 240

Carbon Crook at 0linda... . . . . . . . . . . . . . . 241

Los Angeles River Basin . . . . . . . . . . . . . . . . . 242

Los Angeles River near Van Nuys . . . . . . . . . . . . 242

Los Angeles RIver near UnIversal city : : : : : : . : : . : 243

Los Angeles River at Los Angeles . . : : : : : : : : : 244

Ios Angeles River near Domey . . . . . . . . . . . . . . 245

Los Angeles River at Long Beach . . . . . . . . . . . . . 246

Browns Creek at Chatsworth . . . . . . . . . . . . . . . 246

Pacolma Creek near San Fermando . . . . . . . . . . . . . 247

Pacolma Croek at Mission Acres........... . . . . . 247

Tujunga Creek near Eolby Ranch . . . . . . . . . . . . 248

Tu junga Creok near Sunland .... . . . . . . . . . . . . 249

Tujunga Creok near San Fernando . . . . . . . . . . . . . 249

Little Tujunga Creek near San Ferriando . . . . . . . . . . 250

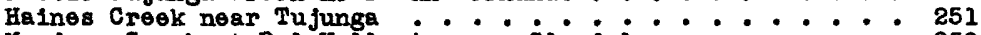

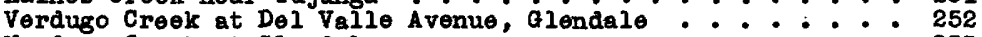

Verdugo Creek at alendale . . . . . . . . . . . . . . 253

Arrojo Seco near Pasadena . . . . . . . . . . . . . . 254

Santa An1ta Creok near Slerra Madro . . . . . . . . . . 255

Little Santa Anita Creek near Sierra Madre... . . . . . 255

Baton Creek near Pasadena . . . . . . . . . . . . . . 256

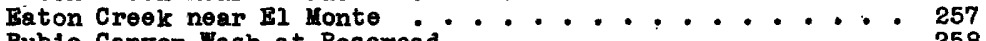

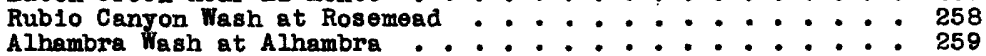

Rio Hondo near BI Monte

Rio Hondo near Montebel10 . . . . . . . . . . . . . . . 261

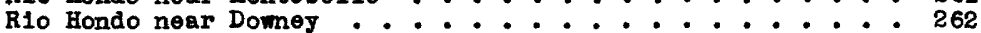

Ballona Creok Bas in ..... . . . . . . . . . . . . . . . 263

Centinela Creok near Culver City. . . . . . . . . . . . . 263

Ballona Creok near Culver Clty : . : . . . . . . . . . 264

Benedict Canyon storm drain at Culver city . . . . . . . 265

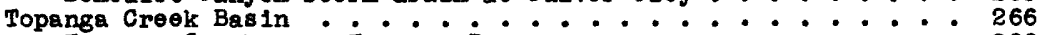

Topenga Creek near Topanga Beach . . . . . . . . . . . . 266

Malibu Creek Basin . . . . . . . . . 267

Mal1bu Crook at Crater Camp, near Calabasas : . . . . . 267

Santa Clara River Basin . . : . . . . . . . . 268

Santa Clara Rivor noar Saugus : : . : : : : : . : . : 268

Plru Creek near Piru . . . . . . . . . . . . . . . . 269

Sespe Creok near Filimore . . . . . . . . . . . . . . . 270

Santa Paula Creok near Santa Paula . . . . . . . . . . . . 271

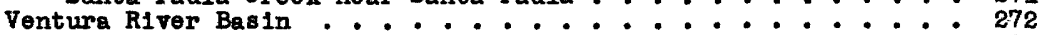

Matilija Crook at Matilija.................. 272

Ventura River near Ventura . . . . . . . . . . . . . 273

Cojote Creok near ventura .... . . . . . . . . . . . . . 274

Santa Inez River Basin . . . : : : : : : 275

Santa Inez River at Juncal Reservolr, near Montecito. . . . 275

Santa Ynez RIver near Santa Barbara . . . . . . . . . . 276

Santa Ynez River below Gibraltar Dam, noar Santa Barbara - 277

Santa Ynez River near Santa Inez . . . . . . . . . . . . 278

Santa Ynez River near Lompoc . . . : : : : : : : . : . . : 279

Santa Marla Rivor Basin

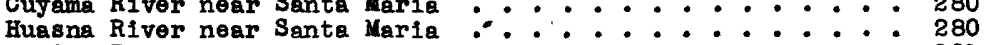

Salton Sea Basin

Palm Canjon Creek near Palm Springs . . . . . . . . . . . 281

Mojeve River Basin ....... . . . . . . . . . . . . . . . . 282

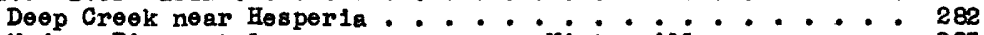

Ho jave River at lower narrows, near victorvilie . . . . . 283

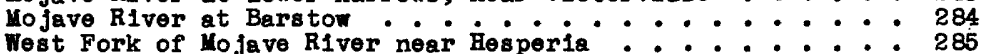

Sumary of flood discharges . . . . . . . . . : . . . . . 286

Physlographic and geologic features affecting flood runoff, by

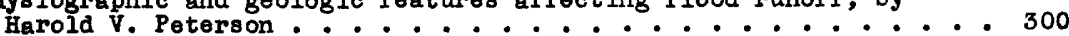

Introduction . . . . . . . . . . . . . . . . . . . . 300

San Dlego area :

Kountaln highiands: . . . . . . . . . . . . . . . $30 s$

Coastal belt......................... 304

River valieys . . . . . . . . . . . . . . . . . . 304

Fffect of geology on flood runoff : . : . . . . . . . . . 305

San Bernardino mounta in area. . . . . . . . . . . . . 307

Geology of the San Bernardino Range ............ 308 
Physlographic and geologic features affecting flood runoff--Continued

San Bernardino mountain area--Cont inued

Effect of the geology on the runoff .......... 310

Mo jave River............................. 311

Santa Ana River ......................... 312

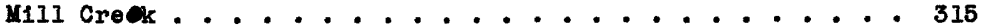

The frontal streams : . . . . . . . . . 317

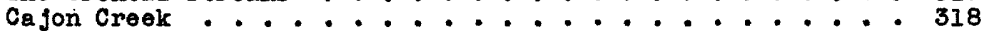

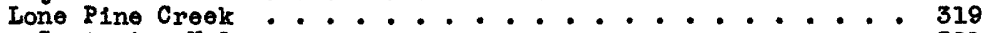

Opper Santa Ana Valley ................. 321

San Gabriel mountain area ................ 322

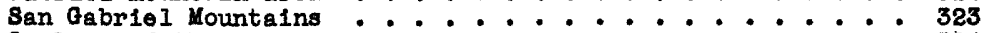

Geology of the mountain area.............. 324

Faulting. . . . . . . . . . . . . 325

Effect of geology on flood runoff ............... 326

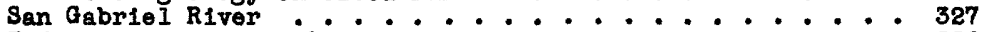

Tujunga and Pacoima Creoks ............... 330

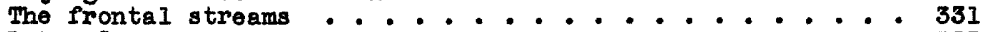

Iytle Creok ................... 335

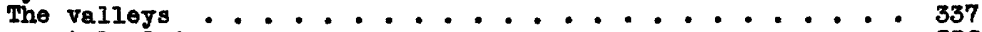

Tho coastal plain $: \ldots$

Santa Clara, Ventura, Santa Ynez, and Santa Maria drainage

basins ......................... 340

Relation of rainfali and runoff to goology ........ 341

Santa Clara River ................. 342

Plru Creok . . . . . . . . . . . . . 344

Sespe Creek : : : : : : : : : : 344

Other parts of Santa clara drainago basin : : : : 346

Ventura River .................. 346

Santa Ynez R1ver . . . . . . . . . . . . . 348

Santa Karia River ................ . . 350

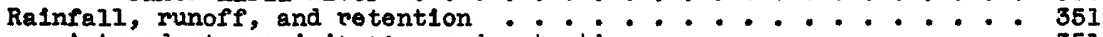

Antecodent precipitation and retention ........... 351

San Diego, San Dleguito, San Luis Rey, and Santa Margarita River

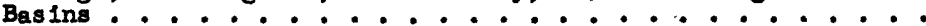

Santa Ana, San Gabriel, and Los Angeles River Basins : $: \therefore 364$

Santa Clara and Ventura River Basins............ 368

Santa Ynez and Santa Maria River Basins :........ 368

Artificlal storage ................... 369

Debris . . . . . . . . . . . . . . 372

Interception of debris by reservolrs and debris basins . . . . . 372

Movement of debris in stream channels on the valley floor. . 377

Flood damage . . . . . . . . . . . . . . . . . . . . . 381

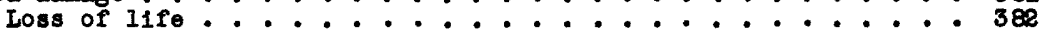

Flood-damage survey ..................... 388

History of floods in southern California................ 385

Index . . . . . . . . . . . . . . . . . . . . 385

\section{ILLUSTRATIONS}

Page

Plate 1. Mouth of Santa Ana River, March 3, 1938 ....... . 14

2. Overflor at mouth of Santa Ana River, shoing area between Santa Ana River and Huntington Beach submerged on March 3,1938

3. A, B, Bebris deposits in Colton .

4. Break in levee of Santa Ana River, March 3,1938 : : : : 14

5. Overflowed land along Santiago Creek . . . . . . . . 14

6. A. B, Debris deposits in overfloved citrus grove . . . . . 14

7. Sceñes on Los Angeles River near Los Angeles: A, Wash-out on rallroed right-of-way; $B$, Damage to channel ..... 14

8. San Fernando Valiey, March 2,1938 . . . . . : : 14

9. Art1ficial channels across débris cones, March 2, 1938 : A, Outlet channel from Dunsmuir debris basin; $\underline{B}$, Outlet channel from Eagle-coss debris basin.... . . .

10. Property damaged by flood: A, Destruction by meandering stream; B, Debris depositez̃ by meandering stream.?

Isohyetal map of southern part of southerm california showing total precipitation, in inches, Fobruary 27 to

March 4, 1938 ...... ... ....... In pocket 
- Plate 12. Isohyetal map of central part of southern California, shoring total precipitation, in inches, February 27

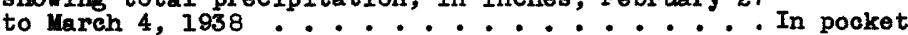

13. Isohyetal map of northiestern part of southern California, showing total precipitation, in inches, February 27 to Karch 4, 1938 . . . . . . . . In pocket

14. Precipitation in part of southern California for indicated intervals of the maximum 24-hour period, $10 \mathrm{p} . \mathrm{m}$. March 1 to 10 p.m. March 2, 1938 . . . . . . . . 94

15. Ferm Canyon Watershods Nos. 1,2 , and 3 ........ 102

16. A, Iast Itiwanda Creok near Etiwanda; B, Slope-area reach on Lone Pine Creek near Keenbrook . . . . . . . 174

17. Reconstructed gaging stations replacing structures destroyed during the floods of March 1938: A, San Gabriel River near Azusa; B, Cucamonga Creék near Upland . . . . . . . . . . . . . . . . .

18. Reconstructed gaging stations replacing structures destroyed during the floods of March 1938: A, San Antonio Creek near Claremont; $\underline{B}$, Deep Creok near

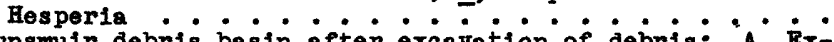

19. Dunsmuir debris basin after excavation of debris: $A$, Excavated basin and Inflow structure; $B$, Outflow structure.

20. Dunsmuir debris basin full of debris, March 11, 1938: A, View upstream showing debris deposits up to altitude of inflow structures; $\mathrm{B}$, Vlew downstream ......

21. Santa Ana River below lower santa Ana Canyon, March $\dot{3}$, 1938 . . . 394

22. Santa Ana River at mouth of Chino Creek, March 3, $1938^{\circ} 394$

23. Santa Ana River near Norco, March 3, $1938 . .$. ... 394

24. Break in levee of Santa Ana River, March 3, 1938 . . . 394

25. Overflow afea along Santa Ana River, March 3, $1938^{\circ}$. . 394

26. Flooded citrus and walnut groves along Santa Ana River, March 3, 1938 . 394

Figure 1. Map of California showing area covered by this report..

2. Map of area covered by this report showing principal drainage systems ....................

3. Profiles of Santa Ana River and its main tributaries -

4. Profiles of San Gabriel River and its main tributaries:

5. Relation between altituae and precipitation in the vicinity of Mount Islip and schematic cross sections showing relative position of rain gages............

6. Cross sections showing altitude and precipitation in Ventura County and in Los Angeles and San Gabriel River Basins .....................

7. Cross sections showing altitude and precipitation in besins of Santa Ana and San Iuis Rey Rivers and

8. Tsohyetal map and cross sections shoing aititude and precipitation in San Dimas Inperimental Forest ... .

9. Mass curves of accumulated precipitation at representative stations, February 27 to March 3, 1938......

10. Hourly precipitation at representative stations, February 27 to March 3, 1938 . . . .

11. Hours of precipitation and percentage of total, in excess of indicated rates, for groups of stations $A$ to $B$ in San Gabriel and San Bernardino Mountains and foothill areas .........................

12. Average hourly precipitation for groups of stations is to

13. Mass curves of accumulated precipitation for groups of stations A to E....................

14. Time of occurrence of maximin periods of precipitation for groups of stations $A$ to $\mathbf{B}$..............

15. Rates of precipitation for groups of stations $\mathbf{A}$ to $\mathbf{B}$. .

16. Graphs of discharge into various reservolrs in southerm Califormia, March 1938 . . . . . . . . . . .

17. Naximum rates of runoff for periods of 1 minute to 16 hours in certain areas in San Gabriel and Los Angeles River and Calleguas Creok Besins ...........

18. Ratio of maximum discharge for indicated period to maximum 24-hour discharge in areas above certain dams in Santa Ana, San Gabriel, and Los Angeles River Basins

19. Tujunga Creek Basin, showing segments used in deterinining naximam concentration of area. 
Figure 20. Distribution of drainage area above flood-control dams Page in San Gabriel and Ios Angeles River Basins .... 138

21. Hourly precipitation and munoff, February 27 to March 5 , for West Fork of San Gabriel River at floodcontrol dam No. 2 ................... 14

22. Hourly precipitation and runoff, February 27 to Maroh 5, for Itve Oak Creek t flood-control dam . . . . . I43

23. Accumiated precipitation, accumulated runof, and retention, February 27 to March 5 , for basin of liost Fork of San Gabriel River at fload-control dax Ho. 2 . 144

24. Accumilated precipitation, accumblated munof, and retention, Pobruary 27 to March 5, for Live Oak Creek

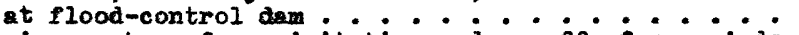

25. Laximum rates of precipitation and munoff, for periods of 5 minutes to 24 hours, in basins of liest Pork of San Gabriel River and IIve Oak Creek .........

26. Laximum rates of precipitation and runoff, for periods of 5 minutes to 24 hours, in San Dimss Creok Basin and Fern Canyon Watersheds Wos. 2 and 3 ..........

27. Relation between rates of precipitation and rates of runoff during maximum periods of 24 hours and less for typical areas in San Gabriel and Los Angeles River Basins . . . . . . . . . . . . . . .

28. Relation between maximum rates of precipitation and maximum rates of munoff during indicated perlods of time for lowest rates of runoff . . . . . . . . 159

29. Relation between maximum rates of precipitation and maximam rates of runoff during indicated periods of time for intermediate rates of runoff . . .... I60

30. Relation between maximum rates of precipitetion and maximum rates of rumoff during indicated period of time for highest rates of runoff ${ }^{2}$. picipitation

31. Time of occurrence of maximum periods of precipitation
and munoff for besins of West Fork of San Gabriel River and Little Santa Anita Creek and Ferm Cenjon Watershed No. 2 ................. .

32. Sketch map, water-surface profiles, and cross sections of the slope-area reach of Ione Pine Creek near

33. Cross sections t cablewaj on East Fork of san Gabriei River near Camp Bonita, February and March 1938 tes of precipitation and munoff, for periods of 5 minutes to 24 hours, for C1ty Creek neax Highland

35. Time of occurrence of indicated maximum precipitation periods as observed at Devil Canyon and Del Rosa and probable time of occurrence of runoff of City Creek, with discharge hydrograph of City Creek near Highland, March 1-4... . . . . . . . . . . . . .

36. Retention curves for certain stations in Santa ina, San Gabriel, and Los Angeles River Basins ........

37. Precipitation, runoff, and gross and net retention on Strawberry Creok during rainy seasons of 1926-27 and

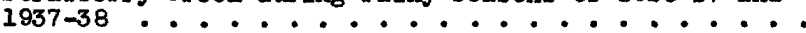

38. Precipitation, runoff, and gross and net retention on
Santa Anita Creek during rainy seasons of 1926-27 and
$1937-38$

38. Precipitation, runoff, and gross and net retention on
Santa Anita Creek during rainy seasons of 1926-27 and
$1937-38$

38. Precipitation, runoff, and gross and net retention on
Santa Anita Creek during rainy seasons of 1926-27 and
$1937-38$

39. Relation between everage siope of drainage bas in and deposition of debris in reservolrs and debris besins of the Los Angeles County Flood Control District...

40. Altitude of point of zero flow at stream-gaging station on Santa Ana River near Prado, 1919-38........ 
Table 1. Daily precipitation, in inches, at standard gages for the perlod February 27 to March 4, 1938 ..........

2. Daily precipitation, in inches, at recording gages for

3. Summary of information regarding distribution of standard rain-gage records for storm of February 27 to March 4, 1938 , in southern California............

4. Percentage of total storm precipitation on indicated days at 10 representative recording stations in Los Angeles and adjacent counties for the period February 27 to March 3, 1938 . . . . . . . . . . . . . . .

5. Precipitation, in inches, for period ending at indicated time, February and March 1938 . . . . . . . . .

6. Duration and amount of precipitation, in excess of ind1cated rates, February 27 to March 4, 1938 ........

7. Maximum rates of precipitation, in inches per hour, for shorter perlods within the storm perlod February 27 to March 4, 1938 . . . . . . . . . . . . . . . .

8. Location, altitude, and storm precipitation for certain groups of stations in Santa Ana, San Gabriel, and Los Angeles River Basins ... . . . . . . . . . . . .

9. Maximum precipitation during indicated periods for period of record at selected stations . . . . . . . . . .

10. Maximum deily precipitation during March 1938 at selected stations compared with maximum previously recorded . . .

11. Precipitation and departure from normal, in inches, at typical stations, for perfods from July 1, 1937 to February 5, 1938 ....................

12. Maximum rates of discharge for indicated periods and excess of peak discharge (duration 1 minute) over those rates in San Dimas and Calleguas Creek Basins, March 2 , 1938 . . . . . . . . . . . . .

13. Ratio of maximum discharg for indicated periods to maximum 24-hour discharge for certain areas in Santa Ana, San Gabriel, and Los Angeles River Basins .....

14. Characteristics of distribution of area in drainage
basins above certain flood-control dams in San Gabriel
and Los Angeles River Basins.

14. Characteristics of distribution of area in drainage
basins above certain flood-control dams in San Gabriel
and Los Angeles River Basins.

14. Characteristics of distribution of area in drainage
basins above certain flood-control dams in San Gabriel
and Los Angeles River Basins.

15. Topographic characteristics of certain drainage basins above point of measurement in Santa Ana, San Gabriel, and Los Angeles River Basins .............

16. Runoff characteristics during fiood of February-March 1938 for certain areas in San Gabriel and Los Angeles

17. Maximum rates of rainfail and runoff, in inches per hour for given periods of time, for certain areas in Santa Ana, San Gabriel, and Los Angeles River Basins....

18. Duration, in hours, and rates of munoff, in inches per hour, for given rates of precipitation at typical stations in the San Gabriel and Los Angeles River Basins.

19. Ratios of maximum rates of precipitation and runoff for given periods to those for maximum 24-hour period for certain areas in San Gabriel River Basin........

20. Time of occurrence of center of mass of precipitation, center of mass of runoff, and lag between times of occurrence, at typical stations in San Gabriel and Los

21. Precipitation and runoff for given periods on $1 / 40$-acre

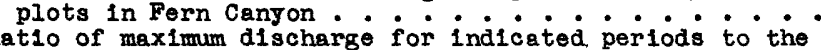

22. Rat10 of maximum discharge for indicated periods to the
maximum 24-hour discharge for City and San Dimas Creeks and for the average for 14 areas in southern California.

23. Sumtary of flood discharges . . . . . . . . . . .

24. Average depth of precipitation, in inches, required to produce runoff on certain streams in the San Diego

25. Net retention in straberry and Santa Anita creck Basins for the rainy seasons of 1925-26, 1926-27, and 1937-38.

26. Rainfall, munoff, and retention for the period February 27 to March 10, 1938 , for selected streams in southern California .....................

27. Storage and flow at reservoirs in santa Ana, san Gabriel, and Los Angeles River Basins during floods of February and March 1938 .............. 
Table 28. Deposition of debris in reservolrs and debris basins of the Los Angeles County Flood Control Distriet..... 374 29. Loss in capacity of reservolrs of the Los Angeles County Flood Control District............. 375

30. Scour and fill in channel of the Santa Ana River due to flood of March 1938 ................. . 379

31. Loss of life in southern california due to storm of

February 27 to March 3, 1938 . . . . . . 382

32. Damage in southern californie caused by storm of February 27 to March 4, 1888 .................. 384 
FLOQDS OF MRCH 1938 IN SOUTHERM CATIFORMII

Harold C. Troxell and others

\section{ABSTRACT}

During the winter of 1937-38 California was visited by two disastrous floods, one in December 1937, in the northern part of the state, 1 and the other in March 1938; in the southern part of the State. The present volume is a report on the flood in the southern part of the State.

A series of heary rainstorms in the coastal area, extending from San Diego on the south to San Iuis Oblspo on the north and inland to parts of the Mojave Deser.t, produced extreme floods. These floods, which appear to have been the greatest within the last 70 years, caused the loss of 87 lives and damage estimated at $\$ 78,602,000$.

The storm seems to have centered in the San Bernardino and San Gabriel Mountain areas tributary to the Los Angeles, San Gabriel, Santa Ana, and Mojave River Basins. These mountain areas are among the highest in southern Callfornia, ranging in altitude from about 1,000 to 11,485 feet above sea level. Their average precipitation for the period February 27 to March 4 was about 22.5 inches, and the greatest precipitation reeorded was 32.20 inches at Kelly's Kamp, in the San Gabriel Mountalns between Ontario and Cucamonga Peaks, at an altitude of 8,300 feet.

The typical drainage areas within this mountain region are small, short, rough, and steep, the average land slope ranging from about 35 to 65 percent. In much of the region, considerably more than haif of the average rainfall of 22.5 inches was absorbed in the so11 mantle and underlying rock and held in storage at the end of the storm period, notwithstanding the many factors conducive to rapid surface munoff.

The rates of rainfall during the storm perlod of March 1938 were not particularly high as compared with the rates in other storm periods in the same region. Only for periods as long as 24 hours do the maximum rates of rainfall appear to equal or exceed those of earlier storms. The maxi-. mum discharge coming t the culmination of these maximum 24-hour periods, produced in some areas a runoff of more than 1,000 second-feet per squaro mile. This report indicates that these high rates of flood runoff occurred at a time when antecedent rainfall had been such as to fill most of the space avallable for subsurface storage.

The relations between rates of rainfall and rates of runoff for the 24-hour and shorter periods of maximum rainfall as obtained for a number of areas controlled by reservoirs are included in this report. Many features incidental to these relations have also been indicated.

In a region marked by the sharp topographic and geologic contrasts that exist in seuthern California many factors affect rates of flood runoff. Considered from a broad viewpoint the area consists of a series of mountain ranges and intervening vallegs, varying in size and altitude, that werge into the coastal plain. The mountains represent great upthrust or upfolded blocks of the earth's crust, and the valleys are depressed segments filled with debris eroded from the surrounding mountains. The surface conditions affeeting rates of munoff throughout the mountain area range from steep slopes of mch-fractured rock overlain by a thin soll antio, interspersed with outcrops of practically impervious unweathered rock, to almost level surfaces covered by a porous soll mantle and underlain by thick depositis of highly permeable alluvium.

The region is geologically young, with erosion proceeding at an active rate within the mountain area. During the flood of March 1938 the streams moved down their moantain channels a great quantity of debris. much of which had accumulated since the time of previous major floods. Heasured on an areal basis the debris load in parts of the region exceeded 70 acre-feet per square mile. This movement of debris from the. stroam channels had the effect of reducing the storage capacity of many of the mountain reservolrs as much as 78 percent.

1 Doalashan, H. D., and Briggs, R. C., Floods of December 1937 in northerm Califomia; U. S. Geol. Survey Water-Supply Paper 843, 497 pp., 1940. 


\section{INTRODUCTION}

During the period February 27 to March 4, 1938, southern California was visited by a series of heavy rainstorms, centered along the coastal slopes of the San Gabriel and San Bernardino Mountains, that produced flood discharge far in excess of any previously recorded. Although the rates of rainfall for short periods were not unusually high, the maximum rates for a 24-hour period at many of the mountain stations exceeded any within the period of record. According to reports of the Corps of Eng1neers, United States Army, the resulting floods claimed the lives of 87 persons and caused damage amounting to $\$ 78,602,000$.

More Information has been ava1lable concerning these floods than for any of the previous floods in this region because of the concern that had developed among the residents in matters pertaining to flood control and conservation of water. Records were obtalned from approximately 730 preclpitation stations, of which about 70 were equipped with recording gages. The flood runoff, or the maximum discharge, has been determined at about 160 places in southern California. Difficulties involved in obtaining satisfactory records of runoff in the mountain area have made it necessary to compute a part of these records on the basis of the relation of munoff to rainfall. The basic factors in this relationship were determined mainly from the records obtained during the flood period at a group of flood-control reservoirs in Los Angeles County. Much information on the movement of debris down the chennels of steep mountain streams was obtained at each of these reservolrs as well as at a group of debris basins, and some of it is included in this report.

In order to complle and make avallable to the general public data obtained during this flood period, the President of the United States on May 23, 1933, approved the allotment of the sum of $\$ 3,779$ by the Works Progress Administration to the Geological Survey, Un1ted States Department of the Interior. In June 1938, the Public Works Administration, acting In accordance with the National Industrial Recoverg Act of 1938 , 2llotted to the Geological Survey the sum of $\$ 21,000$ to assist in the preparation of the present report, which embodies these data.

\section{ADMINISTRATION AND PERSOMEL}

The field and office work incident to the preparation of this report were performed by the water-resources branch of the Geological survey under the general administrative direction first, of $\mathrm{N}$. C. Grover, chief 
hydraulic engineer unt1l his retirement on January 31, 1939; then of $C . G$. Paulsen, acting chief hydraulic engineer until October 17, 1939; and finally of G. I. Parker, chief hydraulic englneer since October 17, 1939. Mr. Paulsen has adminlstered the rork also as chief of the division of surface water:

The actual field work and collection and tabulation of the basic information on stages and discharges and the many other tasks in the preparation of the report were done by the personnel of the Los Angeles suboffice of the San Francisco district office of the division of surface water, under the direction of H. D. McGlashan, district engineer.

The activities in the Los Angeles office have been carried on under the supervision of F. C. Ebert, senior hydraulic engineer in charge, assisted by Harold C. Troxell. Mr. Troxell has been largely responsible for the special hydraulic and hydrologic studies that have contributed to the preparation of the report. The mamuscript was reviewed by 1. . G. Hoyt, consulting engineer, of the conservation branch of the Geological Survey. The finel assembling and arranging of the report was carried on under the direction of R. W. Davenport, chief of the division of water utilization. Many of the members of the Geologlcal Survey staff have participated in the collection and preparation of the information embodied in the report, especially George E. Carroll, R. Stanley Lord, and Komneth R. Melin, all of the Los Angeles office. H. V. Peterson, also of the Los Angeles office, prepared the section entitled "Physlographic and geologic features affecting flood munoff". Much of the tabulation and typing necessary for the report was done as project 365-03-3-34 of the Works Progress Administration, under the direction of Herbert $C$. Legg, administrator for southern California, and Edwin B. Scheuer, coordinator of research, surveys, and statistical projects. In carrying on this work the permanent fleld and office staffs were assisted by temporary employees appointed by the Secretary of the Interior under the provisions of the National Industrial Recovery Act.

\section{ACKROWLEDOMENTS}

The Corps of Engineers, United States Army, through Maj. Theodore Wyman, Jr., district engineer, Los Angeles, Calif, made available, for use in this report, information collected by that organization on the floods of March 1938, data on historic floods, flood damage, flood runoff, and precipitation, and photographs. The Los Angeles County Flood Control District, through H. E. Hedger, chief engineer, furnished most of the 
precipitation records for that county and detalled records of operation of the reservolrs and of silt doposition in the reservolrs and debris basins. The Orange County Flood Control District, through M. N. Thompson, chief engineer, furmished precipitation and runoff data for the Santa Ana River. Basin. The United States Weather Bureau and the Division of Water Resources of the State of California, Department of Public Works, furnished data on precipitation. The United States Forest Service furnished data on precipitation and runoff collected in the San Dimas Experimental Forest.

Much information was obtained from other sources, including individuals, corporations, and city officials, ackowledgments of which are given as far as practicable, at appropriate places in the report.

\section{GENERAL FEATURES OF THE STORM AND FLOOD}

The rains that caused the severe floods of March 1938 in southern California began with general light fall on February 27 and during the early hours of kebruary' 28. The later hours of February 28 were marked by senerally intense and continuous precipitation. On March 1 there was a lull, followed on March 2 by the heaviest rains of the storm and on March 3 by light and intermittent rain, which continued in some places through March 4. Although the storm is generally referred to as that of Fobruary 27 to March 4, it had coased over most of the area on March 3.

The heavy rains covered the Pacific coast area for about $250 \mathrm{~m} 1108$ north of the boundary with Mexico and extended inland 50 to 100 miles. (Soe fig. 1.)

The storm appears to have centered in the San Gabriel and $S a n$ Bernardino Mountain areas tributary to the Santa Ana, San Gabriel, and Los Angeles Rivers. Many of the rainfall records indicated from 20 to 30 inches of rain during the period February 27 to March 4. The heaviest rainfall for this period, 32.20 inches, was observed at Kelly's Kamp, in the San Gabriel Mountains between Ontario and Cucamonga Peaks, at an altitude of 8,300 feot. The rainfall decreased sharply to the north and south of this area, the recording stations at San Diego and San Luis Oblspo showing 4.26 inches and 3.67 inches, respectively. The rainfall decreased sharply also toward the Mojave Desert area; at Table Mountain (altitude, 7,500 feet); about 12 miles porthwest of Kelly's Kamp, the rainfall was 10.40 Inches, and at victorville (altitude, 2,713 feot), about 30 miles northeast of Kelly's Kamp, it was 3.16 inches. These records were not typical of the headwaters of the Mojave River, for there 


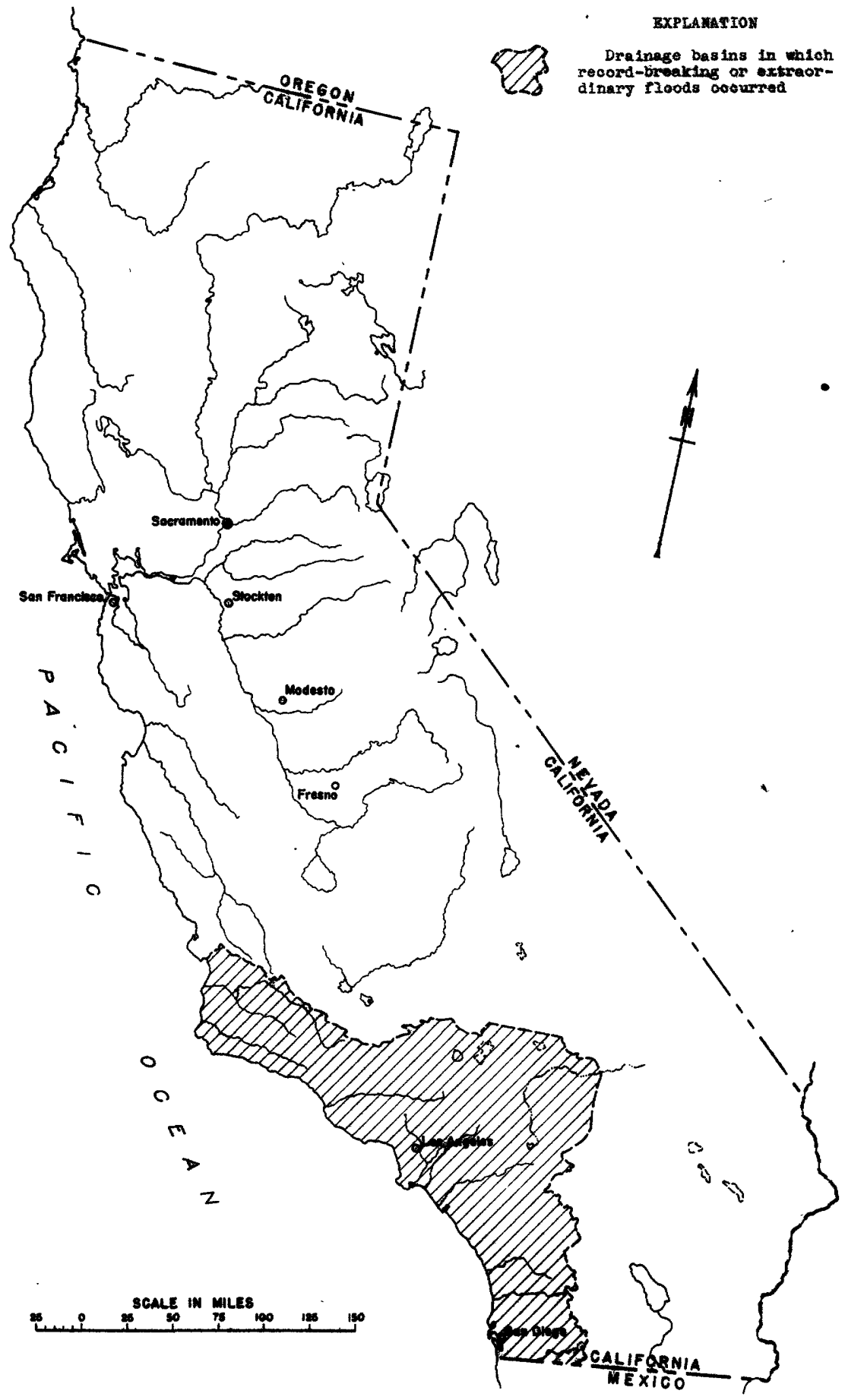

Figure 1.- Map of California showing area covered by this report. 
was a rainfall of 30.49 inches at Lake Arrowhead and 28.58 inches at Sawpit Canyon.

Daingerfield 2 gives the following account of this storm and the antecedent weather conditions:

The season of 1937-38 (beginning July 1, 1937) has been marked by wide contrasts in rainfall over southern California. Los Angeles was rainless from May 31 to October 2, 1937, inclusive, a period of 125 days, followed by 67 days, or unt11 December 8, 1937, with only two light showers, totaling 0,03 inch. The combined period of 192 days had only 0.03 inch of precipitation. This long dry period is second only to that of 1927, when no measurable rainfall occurred at Los Angeles for the 197day period from April 13 to October 26, although traces were recorded each month. The recent drought was rather definitely ended by the rain of December $9-12,1937$, and precipitation was amplo and approximately normal during the remainder of December and through January, 1938.

The closing days of January, however, were marked by intense disturbences over the Gulf of Alaska and adjacent North Pacific waters, with strong southeasterly trends near the Pacific coast of North America, which has been absent or deficlent in the earlier depressions of the season. This change in storm development and movement ushered in a period of almost continuous and frequently heavy rainfall over this area of California during the first half of February, during which period the soll became well saturated with molsture.

The tendency to form disturbances near and off the Pacific coast featured the late January, February, and early March perlod. A storm which developed northeast of Hawail moved slowly eastward (followed by a well-developed $\mathrm{Paciflc} \mathrm{hlgh}$ ) and moved inland near the Bay region on February 9. This was an exceptionally violent disturbance, attended by precipitation throughout California, heavy over the northerm part of the State, and high winds and gales over most cosstal districts and inland to and bejond Sacramento.

A serles of North Pacific and Gulf of Alaska depressions prevalled from February 26 to March 4, 1938, extending their maximum sphere of influence hundreds of miles farther southeastward than normally over the Pacific coast area. On February 27, a rather shallow disturbance moved slowly in over the southern California coest, attended by a general light rain on the 27 th and intermittently through the 28 th. During the night of February 28-March 1, heavy rain occurred as the result of the passage of a cold front, which moved in from the northwest. No general rain occurred after this frontal passage unt1l March 2, with the arrival of a more intense disturbance. This disturbance likewise developed northeast of the Hawalian Islands, and while the center moved to the Oregon coast, the region of greatest intensity was over southern $C_{a}$ if fornia. Warmfront action and a pronounced orographic effect produced the greatest intensities and amounts of precipitation of the entire storm period, which were directly the cause of the great flood.

The origin of the storm is briefly described in a report of the Corps

of Engineers, United States Army, 3 as follows:

The storm, or rather series of storms, which caused the flood of Narch, 1938, orlginated in Siberle, circled southward over the Paciflc Ocean to Midway Island, swinging eastward near Hawa11, and thence to the California coast. In their long, encircling course over the Pacific Ocean, a great amount of molsture was absorbed. Reaching southern Cal1fornla, the air masses swept in at high speed at almost right angles to the main mountain ranges. As the molst air was thrust upward by these obstructions, it encountered the colder upper air. The rapid condensation resulting caused excessively heavy rainfall.

2 Dalngerfleld, L. H., Southern Califormia rain and flood, February 27 to Karch 4, 1938: Monthiy Weather Rev., vol.66, no. 5, p. 139, 1938.

3 Santa Ane River, Callf., flood control, Hydrology of flood of March 1938, p. 2, U. S. EngIneer Office, Los Angeles, August 1938 (processed). 
Discussions of the more intricate features of the synoptic analysis of this storm have been prepared by Plerse, of the United States Weather Bureau; ${ }^{4}$ Burke, of the Los Angeles County Flood Control District; 5 and. the staff of the Los Angeles district office, Corps of Bngineers, United States Amg. 6

The intensity of rainfall for relatively short periods, although high In men of the storm-affected area, was generally less than hed been previously recorded, and only in the maximum rainfall for a 24-hour period did this storm exceed most previous storms. In the mountein area, however, the maximum 24-hour precipltation for 1938 apparently has not been exceeded during the past 30 jears. The storm of March 1938 had many of the characteristics of the storm of December 31, 1933, to January 1, 1934, sometimes called the "New Year's storm of 1934", which was notable for the large amount of flood damage in the La Cañada Valleg.7

In the storm of March 1938, the maximum rate of rainfall for a 5minute period was 4.80 inches an hour, as recorded by a rain gage in the Rossmoyne fire area in the Verdugo Mountains near Glendale. The maximum rate for a 1 -hour period was 1.99 inches at Clear Creek, in the San Gabriel Mountains.

The main streams affected by this storm were the San Diego, San Luls Rey, Santa Margarita, Santa Ana, San Gabriel, Los Angeles, Santa Clara, Ventura, Santa Inez, and Santa Marla Rivers. (See flg. 2.) The basins of the Santa Ana, $S_{a n}$ Gabriel, and Los Angeles Rivers and parts of that of the Sante Clara River contributed the greatest flood runoff. Profiles of the Santa Ana and San Gabriel Rivers and thelr main tributaries are shown in figures 3 and 4.

The flood munoff from the storm of March 1938 was espectally heavy In the larger streams in the mountains and in the main streams crossing the valley floor. It is belleved that the peak discharges over a considerable part of the area exceeded any since those of the floods of 1861-62, which are generally accepted as the greatest previousiy known in the region. Of the more recent floods for which there are records of discharge, those of 1914 and 1916 rank as having the highest peaks prior to 1938.

4 Plerce, C. H., Synoptic analysis of the southern California flood of March 2, 1938: Monthly Weather Rev., vol., 66, no. 5, p. 135, 1938.

5 Burke, M. F., Flood of March 2, 1938, Los Angeles County Flood Control District, May 20, 1938 (processed).

6 Los Angeles County drainage a rea, Calif., Report on engineering aspects, flood of March 1938, U. S. Englneer Office, Los Angeles, August 1938 (processed).

7 Troxell, H. C., and Peterson, J. Q., Flood in La Cañada Valley, California, January 1, 1934: U. S. Geol. Survey Water-Supply Paper 796m, pp. $53-98,1937$. 


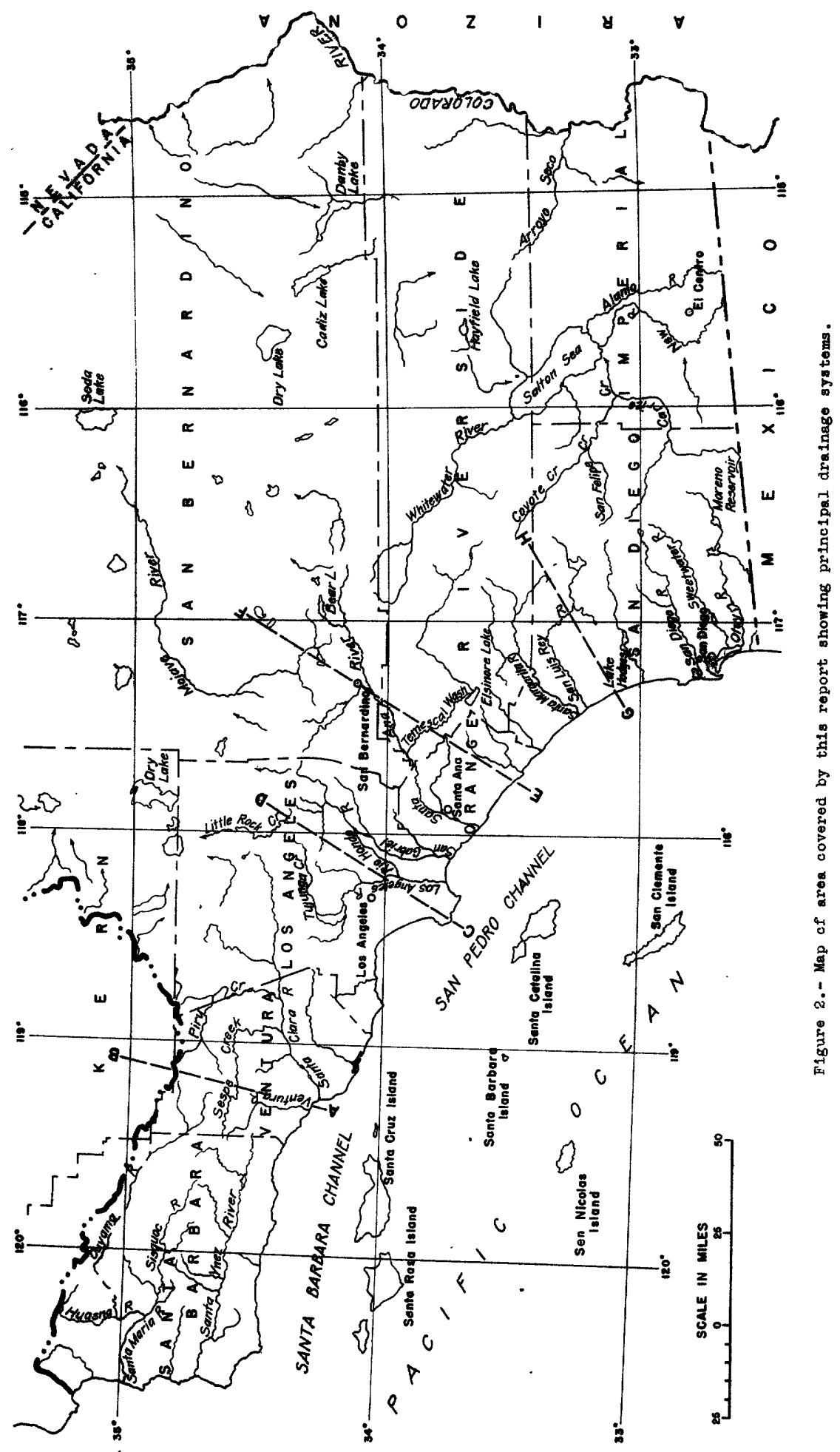




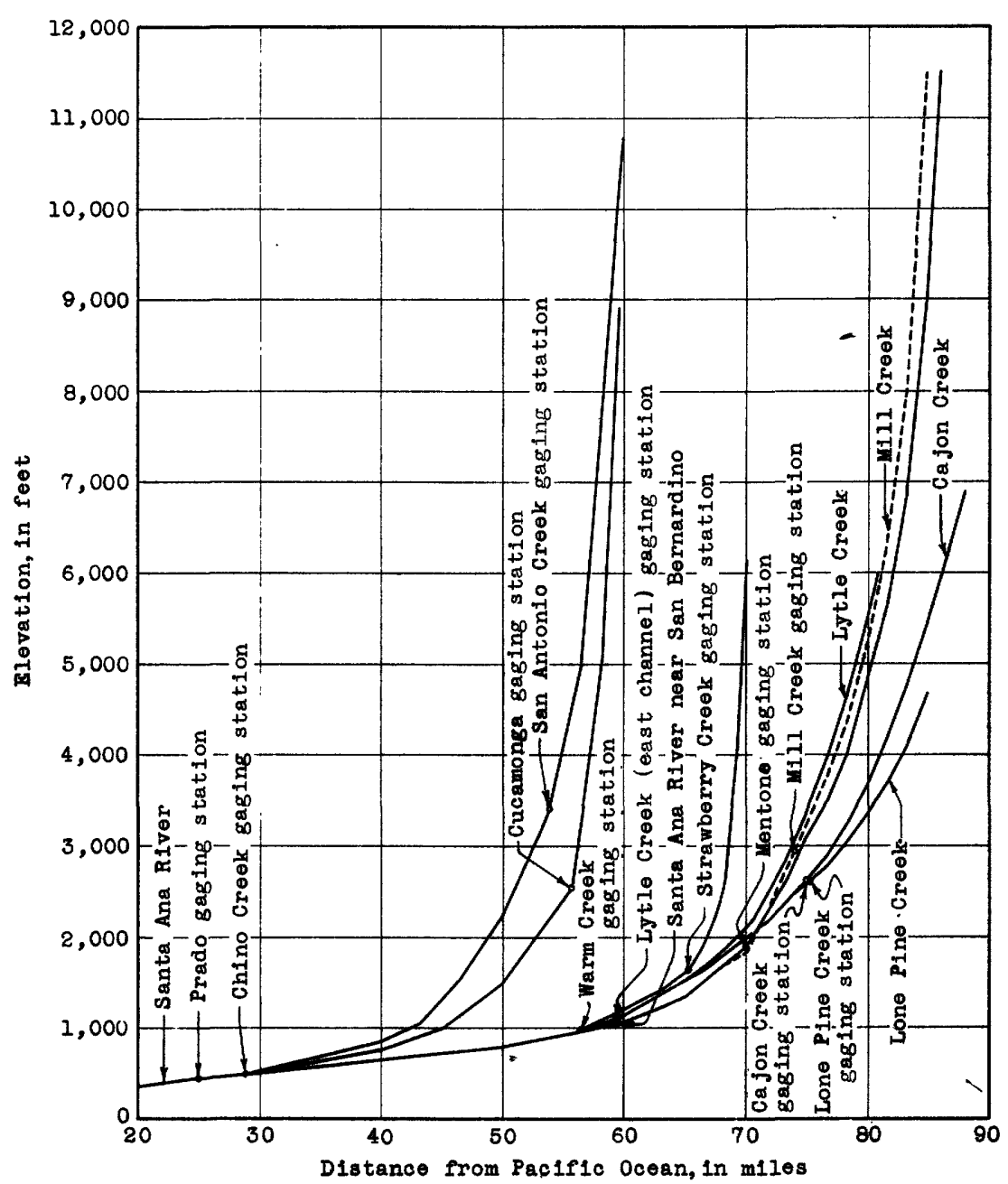

F1gure 3.- Profiles of Santa Ana River and 1ts main tributaries. 


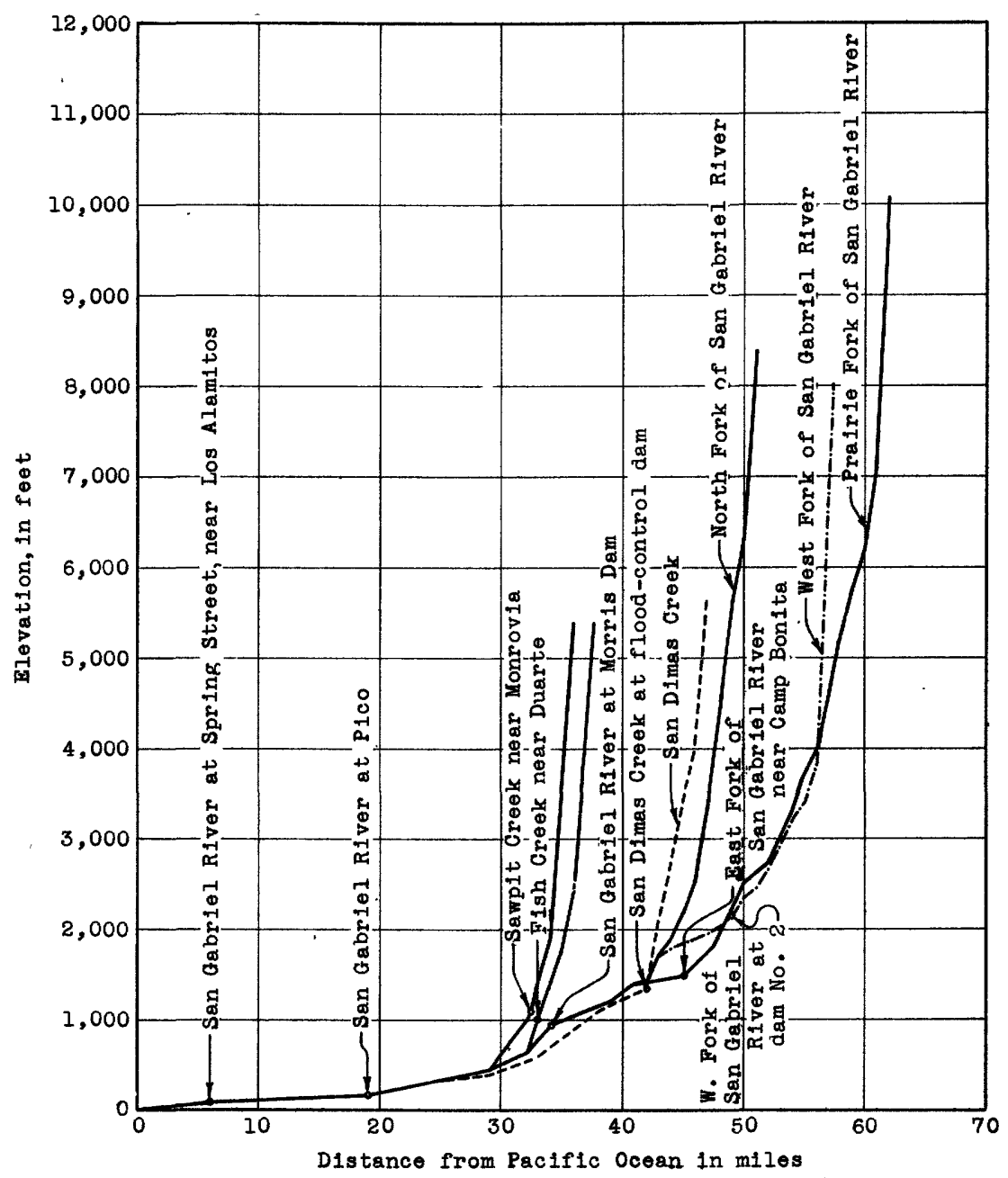

Figure 4.- Profiles of San Gabriel River and its main tributaries. 
The Santa Ana, San Gabriel, and Las Angeles River systems drain the most highly developed areas of southern $C_{a}$ lfornia: practically all the arable land from the mountains on the north to the ocean on the south is occupled by farms, orchards, and towns. When the floods were at their highest, generally during the afternoon of March 2, water and debris poured from the steep mountain canjons and spread over mach of the land bordering the streams. Areas near the mountains were covered with debris and extensive tracts along the lower courses of the streams were inundated. (See pls. 1 and 2.)

During the period February 27 to March 3 the total direct flood runoff from the mountain areas, in mean depth in inches, ranged from less than 1 inch to more than 13 inches. Rates of maximum discharge ranged from 200 to 600 second-feet per square mile and were estimated to be as high as 1,000 second-feet per square mile in some places. The streams flowing in the steep, narrow canyons moved enormous quantities of debris and greatly disturbed the material in the bottom of the canyons. The damage in the canjons, however, where the population is sparse, was small in comparison with that on the outer slopes and floors of the valleys.

The area centering at Colton, where Warm and Iytle Creeks join the Santa Ana River, was extensively overflowed (see pl. 3); and parts of the cities of San Bermardino and Riverside were also submerged. Above the city of Santa Ana, where breaks occurred in the levees (see pl. 4), large areas were inundated and some of the floodwater entered an old river channel and damaged many homes and orchards. (See pls. 5 and 6.) This spreading of the floodwaters above the city of Santa Ana reduced the peak discharge at that place to about half the peak at the Orange-Riverside County line, about $18 \mathrm{miles}$ upstream.

At the stream-measurement station on Santa Ana River near Mentone, a peak discharge of 52,300 second-feet occurred during the flood of March 1938, as compared with a peak discharge of 29,100 second-feet on January $27,1916$.

On the San Gabriel River the flood was the greatest of record, notwithstanding the large storage capacity of San Cabriel No. 1 and Morris Reservolrs, on the main stream, and additional regulation on tributaries. Much of the floodwater of the San Gabriel River flowed into the Rlo Hondo soon after leaving the mountains and thence into the Los Angeles River near Downey. Comparatively little damage was done along the lower reaches of the San Gabriel except in an area near the ocean and along coyote Creek. 
At San Gabriel River flood-control dam No. 1, on the San Gabriel River about $3 \frac{t}{2}$ miles above the Geological Survey's stream-measurement station near Azusa, the partly regulated peak discharge on March 2, 1938, was about 90,000 second-feet as compared with an unregulated peak discharge of 40,000 second-feet at the gaging station near Azusa on January 18, 1916.

On the Los Angeles River the flood of March 1938 exceeded all previous floods for which records are available. Pacoima and Tujunga Creeks, Arroyo Seco, and other tributuries reached unusually high stages. The highly developed areas along the Los Angeles River and 1ts tributaries in the city of Los Angeles sustained the greatest damage. (See pl. 7.) Iarge tracts in San Fernando Valley were inundated, and bridges, highways, and rallroads were severely damaged. (See pl. 8.) The flow from Rio Hondo added considerable water to the Los Angeles River below Los Angeles and increased the peak flow at the city of Long Beach, although comparatively little damage was done to that city. At a point a mile upstream from the Main Street Bridge in Los Angeles, above the Arroyo Seco, the discharge of the Los Angeles River was about 67,000 second-feet, as compared to an estimated discharge of about 31,100 second-feet at the Main Street Bridge during the flood of January and February 1914.8 During the storm of March 1938 the flood-control reservolrs on Pacolme and Tujunga Creeks and Arroyo Seco were fllled to capacity and undoubtedly contributed somewhat to the regulation of the discharge.

In the Santa Clara and Ventura Riyer Basins the flood runoff exceeded the maximum of record in many localities. The flood on Santa.Clara River did considerable damage to the Southern Pacific Ra1lroad in Soledad CanJon and to cltrus groves and several highway bridges in the Santa Paula area. The maximum discharge of the Santa Clara River at Santa Paula on March 2, 1938, was about 120,000 second-feet. The maximum discharge of the Ventura River near Ventura was 39,200 second-feet, as compared with the previously recorded maximum of 23,000 second-feet on December 31 , 1933.

The maximum discharge of the Santa Ynez River near Lompoc was about 50,100 second-feet on March 3, 1938, as compared with 41,800 second-feet on January 25, 1914.

B From reports of the Board of Engineers, Flood Control, Los Angeles, p. 248, July 27, 1915 . 
In the Mojave River and Rock Creok Besins record-breaking floods resulted from the heary raine that fell on the north slopes of the San Cabriel and San Bernardino Mountains. As these streans flow onto the Mojave Desert, in the Great Basin, littlo damage was done except to highways and the railroad in the vicinity of Victorville, Barstow, and Yermo.

In the remainder of the area covered by the storm, the maximux discharges of the streams during the flood of March 1938 were not of for proportions and heve been exceeded several times during the pertod of record.

Flood stages are most keenly felt by occupants of a flooded area when the safety of their lives or homes is menaced. The manner in which man has challenged nature on some of the streams of southern California is conspicuous. Streans that formerly moandered freely over debris cones and valley floors are now restricted to narrow channels (seo pl.9), fixed by the over-encroaching developwents of rapidly increasing popu1ation. It seoms probable that wuch of the flood runoff from the mountains that forwerly was absorbed by the very porous gravel cones, bullt to a thickess of many hundreds of feet by flood debris, now moves rap1dy, through concrete storm drains, across the debris cones and the naturally absorptive velley floors. The older river channels that carry the runoff to the ocean are now texed beyond their capacities by concentrated flows. For these ressons, it 18 no doubt true that storm rainfalls of no greater intensity are considerably more disastrous, in many areas, under present conditions than under former conditions. The corerIng of absorptive oones and valley floors with houses and streots, the construction and other surfic1al changes in mountain areas, and the changes in land cover due to $f$ ires and other causes all change, within a comparatively short time, the runoff characteristics of a drainage basin, especially in an area as young geologically as southern Cal1fornia. The evaluation of these infiuences on the runoff from the raroh storm seems beyond the limits of conjecture.

During the floods of March 1938 houses, both large and small, were deatrojed (soe pl. 10), and highways, bridges, and many othor public improvements wore washed awey or damaged. Citrus groves along the Santa Ana River were overflowed, and largo losses resulted. Hore than 290,000 acres of land was inundated in Los Angeles, San Bernardino, Orango, Riverside, and Ventura Counties. Owing to the rapidity with wich the flood waters collected in the river channels there was not suffieient time to waxn many of the residents in the threatened aress. As a result, 87 ifres wero 
lost, mainly along the Santa Ana and Los Angeles Rivers, and many poople were driven from their homes. The property damage caused in southern Califormia by the floods is estimated by the Corps of Engineers, $U$. $s$. Army, at about $\$ 78,602,000$.

\section{COLLECTION OF RAIMFALI AND RUNOFF RECORDS}

As the floods of March 1938 in southern Californla were extraordinary In respect to loss of Iffe and property damage, s pecial effort has been made to collect and compile in this report all avaliable information relating to meteorologic conditions that mag have had a bearing on their magnitude and other characteristics. Ilkewise, an attempt has been made to present all available stream-flow records during the flood period, including estimates of peak discharges. Concerning the adequacy of the information obtained, the Corps of Engineers 9 summarizes the difficulties encountered by the various fact-finding agencies as follows:

Notwithstanding the great amount of hydrological data collected, results have been disappointing in many respeots, principalis due to the lack of direct measurements of discharge during the peak period. The streams in this area produce floods of exceedingly short duration. The rapldiy changing stages do not allow sufficient time for the collection of necessary data. Transportation is disrupted to a great extent. Waterstage recorders, In many cases, were damaged or destroyed before the peak arrived. Bridges from which discharge measurements were made during the lower stages were swept away. Current-meters were damaged or lost because of the debris and high velocity of flow. Surveys to determine fill or scour were, in many cases, rendered valueless because of the lack of data concerning the conditions of the stream bed before the flood. Information was missing in some cases as to just where the channels were at the time of the peak.

At many river-measurement stations maintained by the Geological Survey, it has been necessary to flll in incomplete records by a comparison. of observations in a given basin with observations in adjacent basins; by surveys and studies of cross sections and slopes of stream channels; and by the conversion of rainfall into runoff by means of such relationships as could be determined in basins for which continuous records are ava11able. Throughout this report an endeavor has been made to indicate olearly the souroe of the data and the methods used in deriving such results as are not based on actual observations or arrived at by standard methods. The publication, so far as practioable, of all the base data obtained will enable engineers to judge as to the reasonableness of the assumptions and to make independent studies of the records. Flood runoff data $a$ re included in this report for all localities indicated on plates 11, 12, and 13 .

9 Los Angeles County drainage area, Callf., Report on engineering aspects, flood of March 1938, U. S. Engineer Office, August 1938 (processed). 


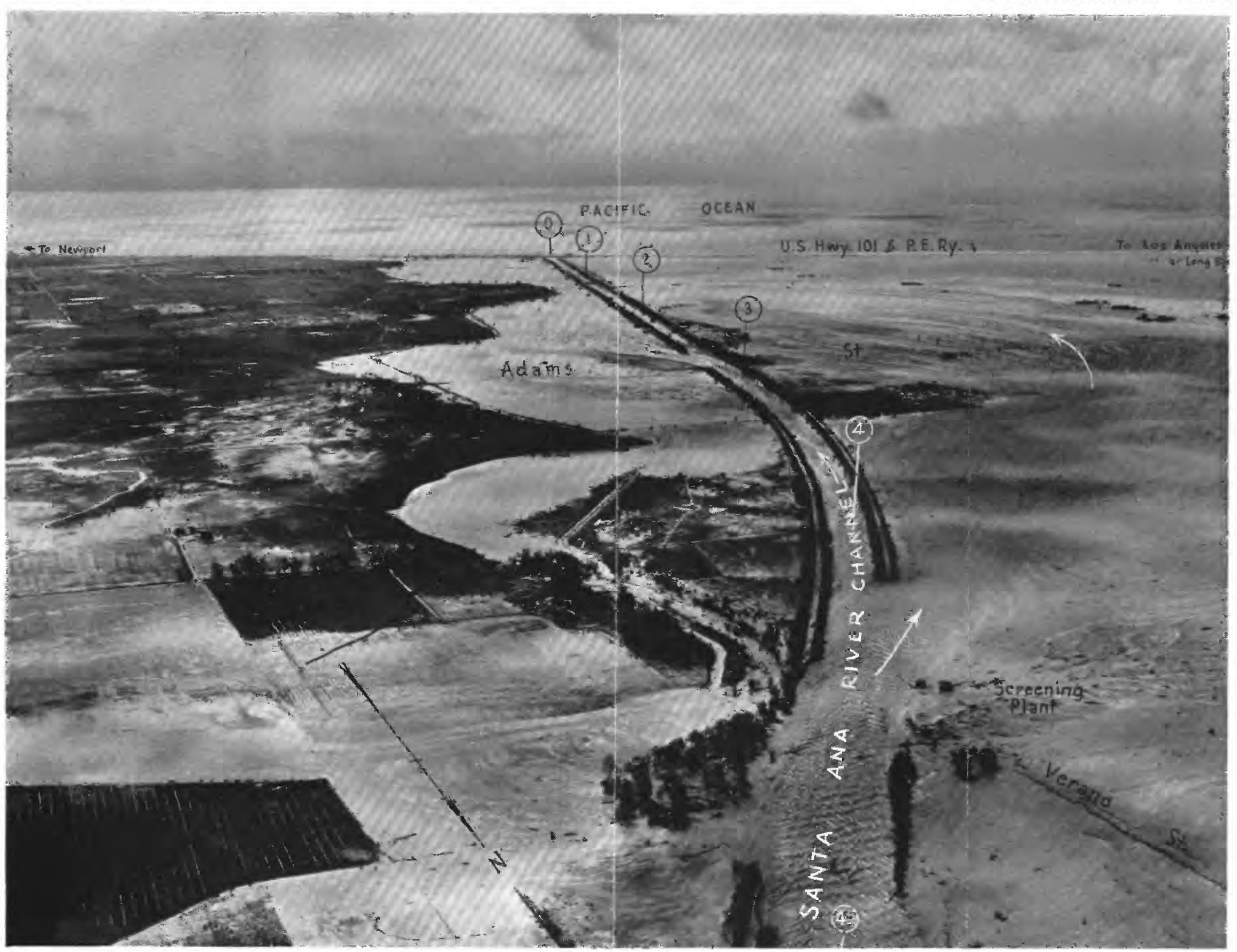

MOUTH OF SANTA ANA RIVER, MARCH 3, 1938.

Figures along channel represent distance in miles from Pacific Ocean. Courtesy of Fairchild Aerial Surveys, Inc. 


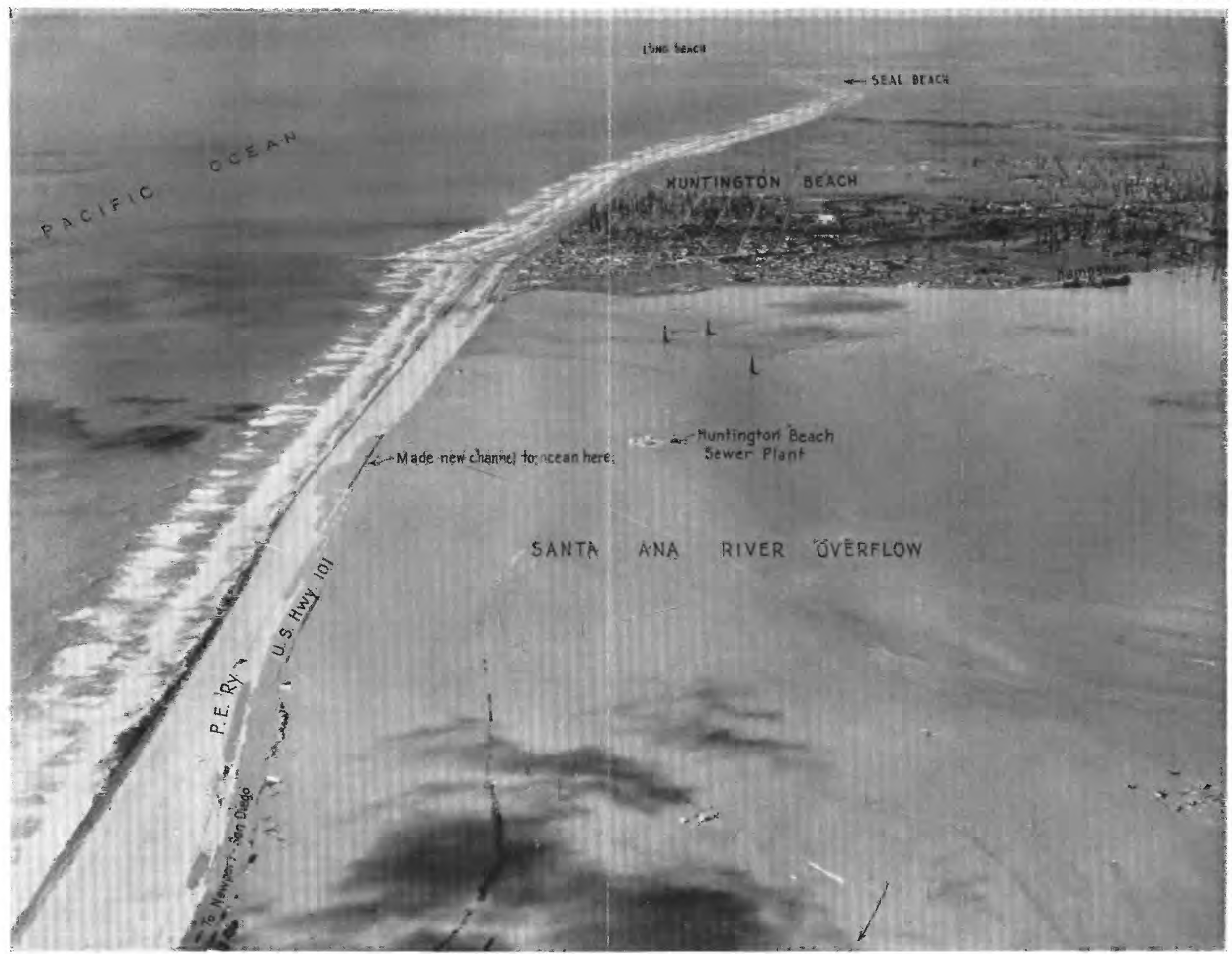

OVERFLOW AT MOUTH OF SANTA A\A RIVER, SHOWING AREA BETWEEN SANTA ANA RIVER AND HUNTINGTON BEACH SUBMERGED ON MARCH 3, 1938. Courtesy of Fairchild Aerial Surveys, Inc. 



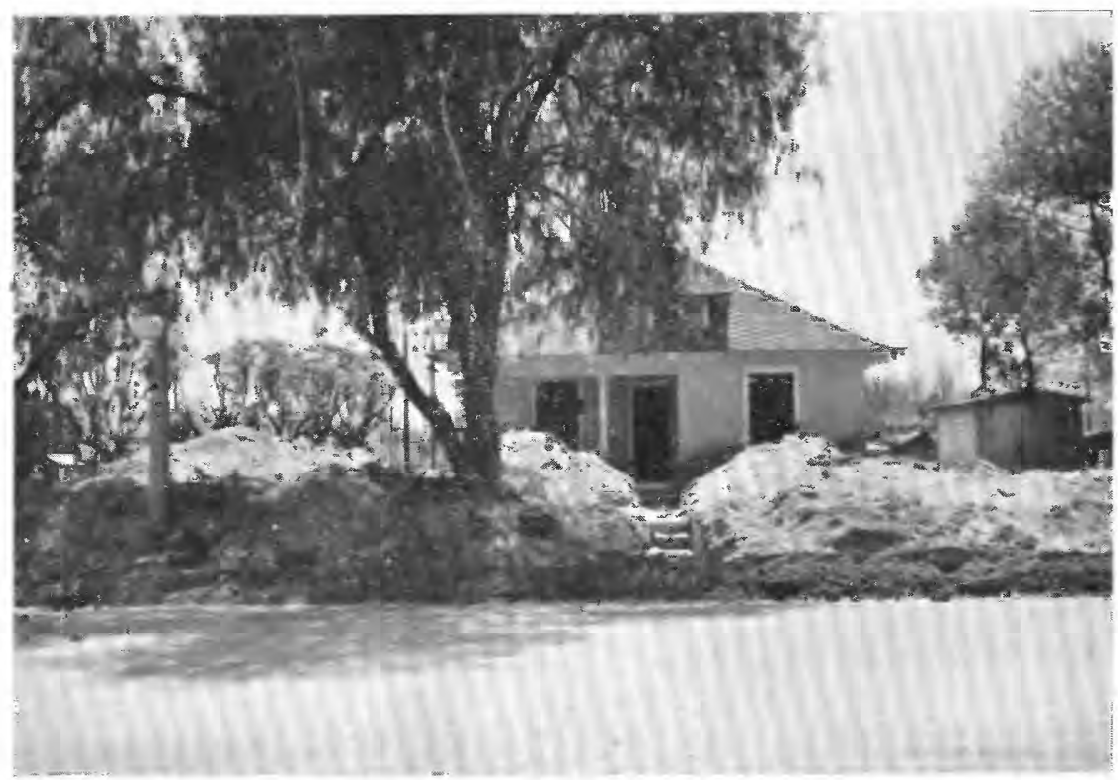

A.

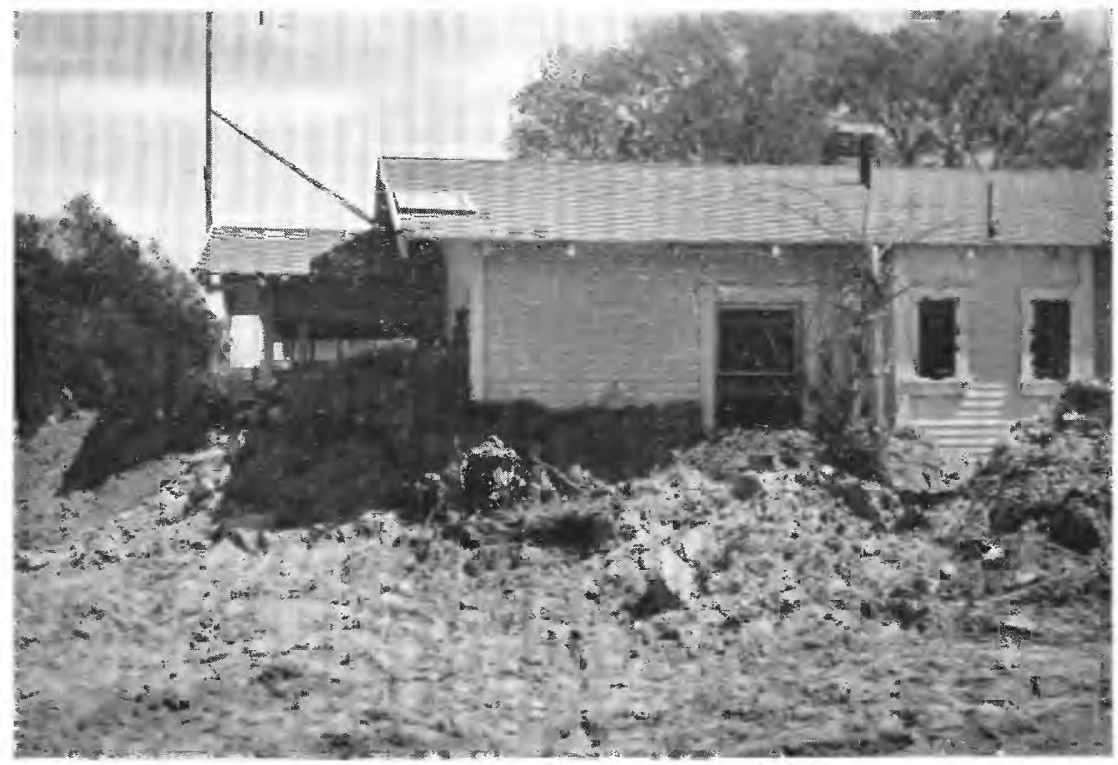

B.

DEBRIS DEPOSITS IN COLTON.

Damage caused by flood runoff of Lytle Creek. 



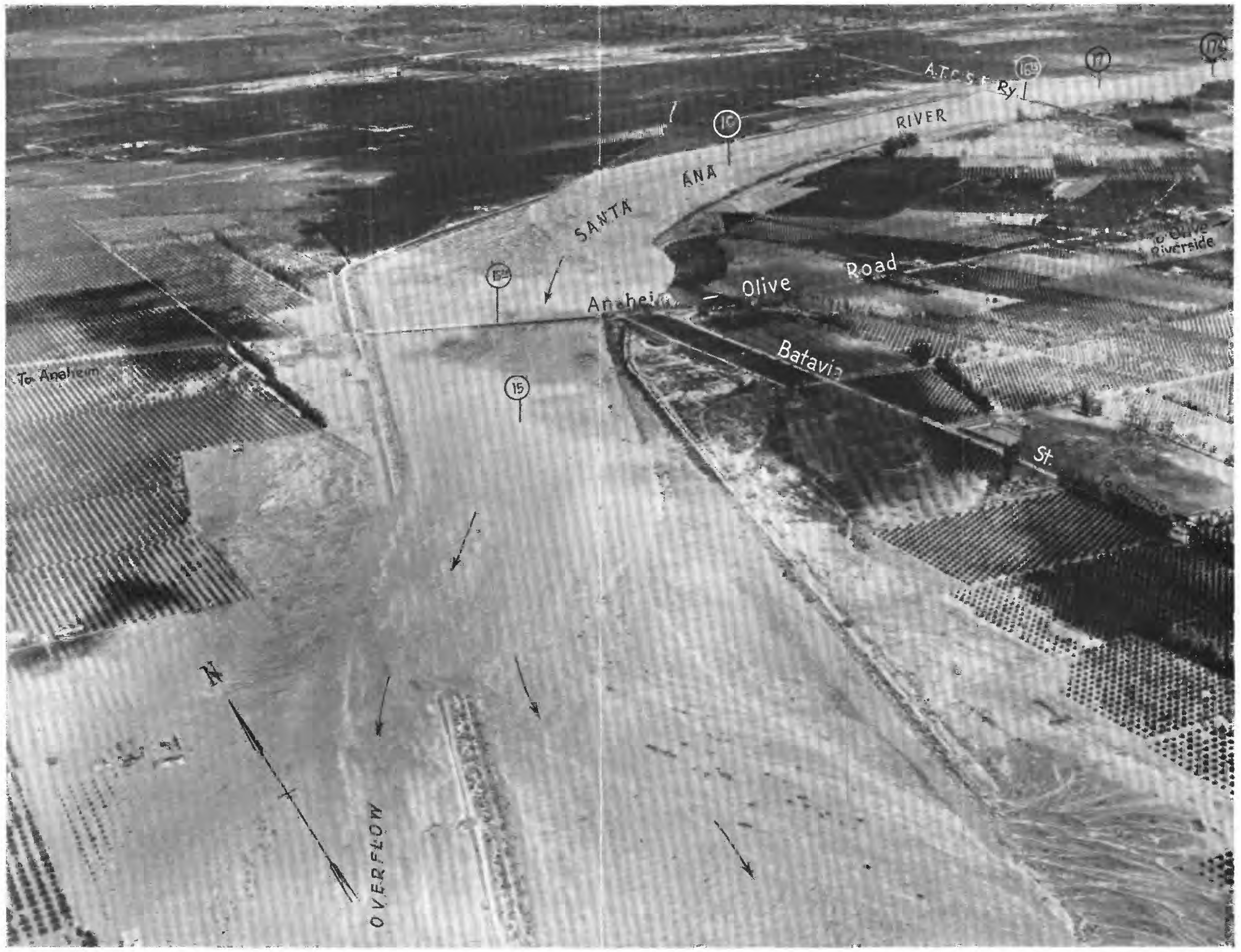

BREAK IN LEVEE OF SANTA AN'A RIVER, MARCH 3, 1938.

Figures along channel represent distance in miles from Pacific Ocean. Courtesy of Fairchild Aerial Surveys, Inc. 


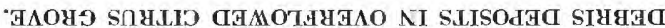
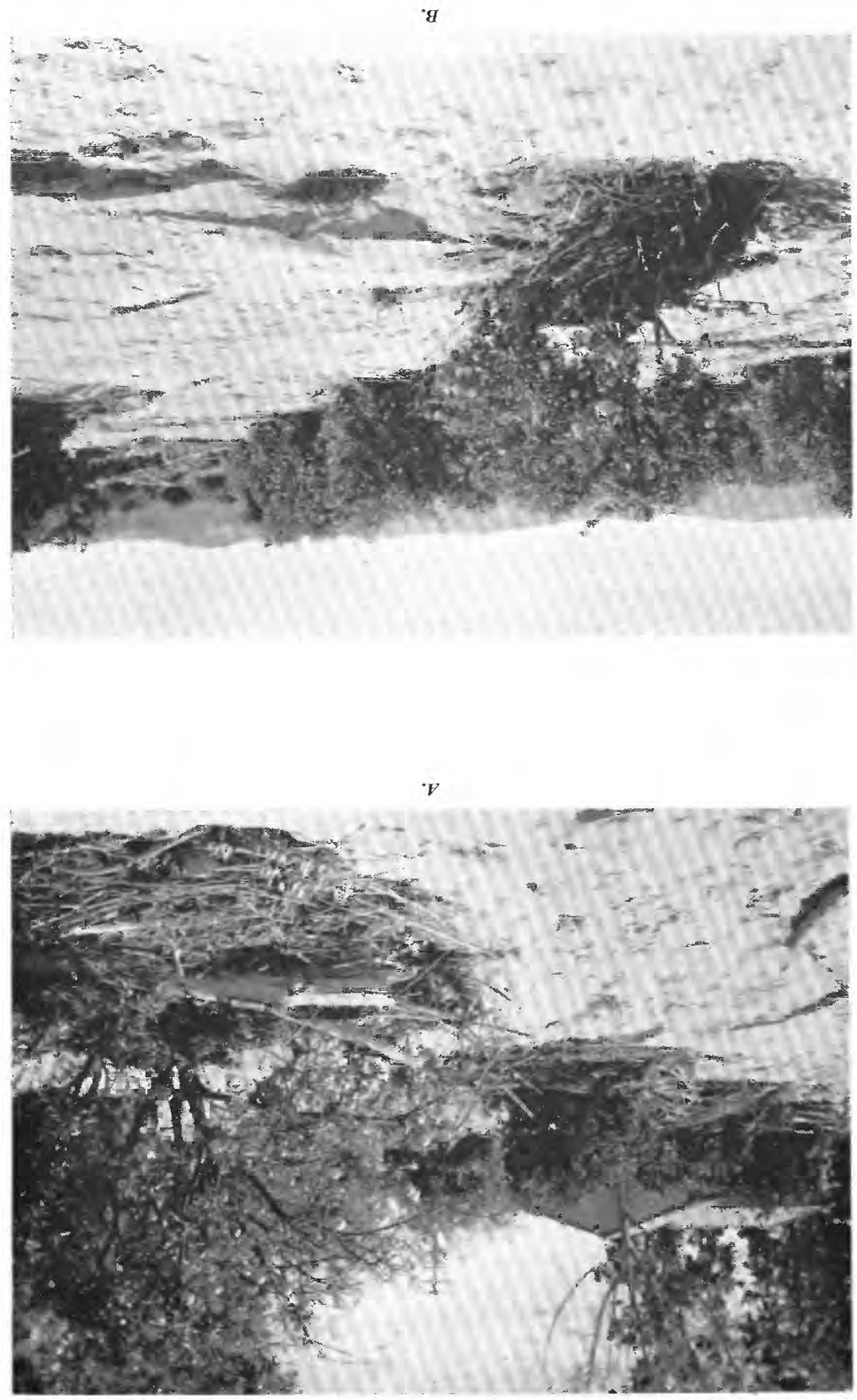


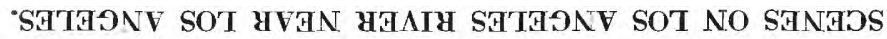
"THNNVHO OL 'SOVLVA ' $\boldsymbol{g}$

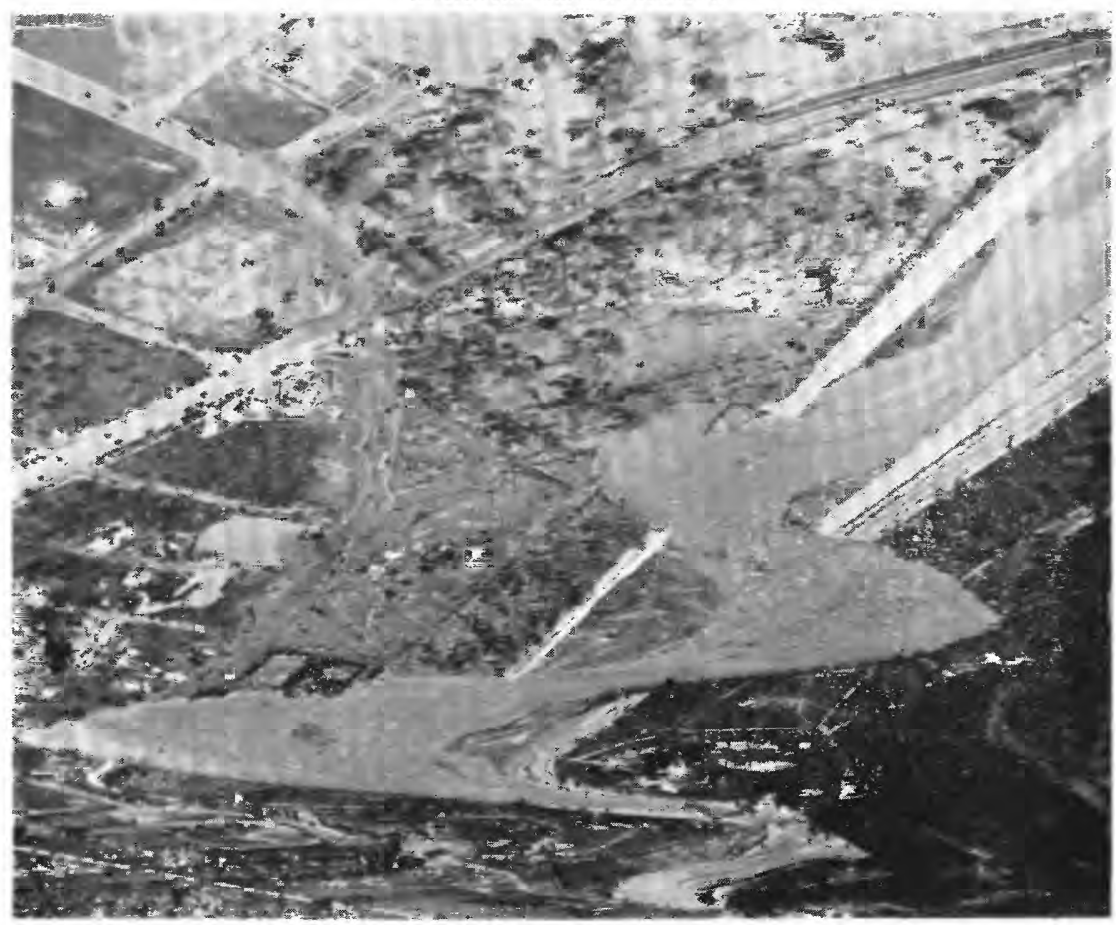

XVA-HO-LHAI GVOQTIVY NO JOO'HSVA 'V

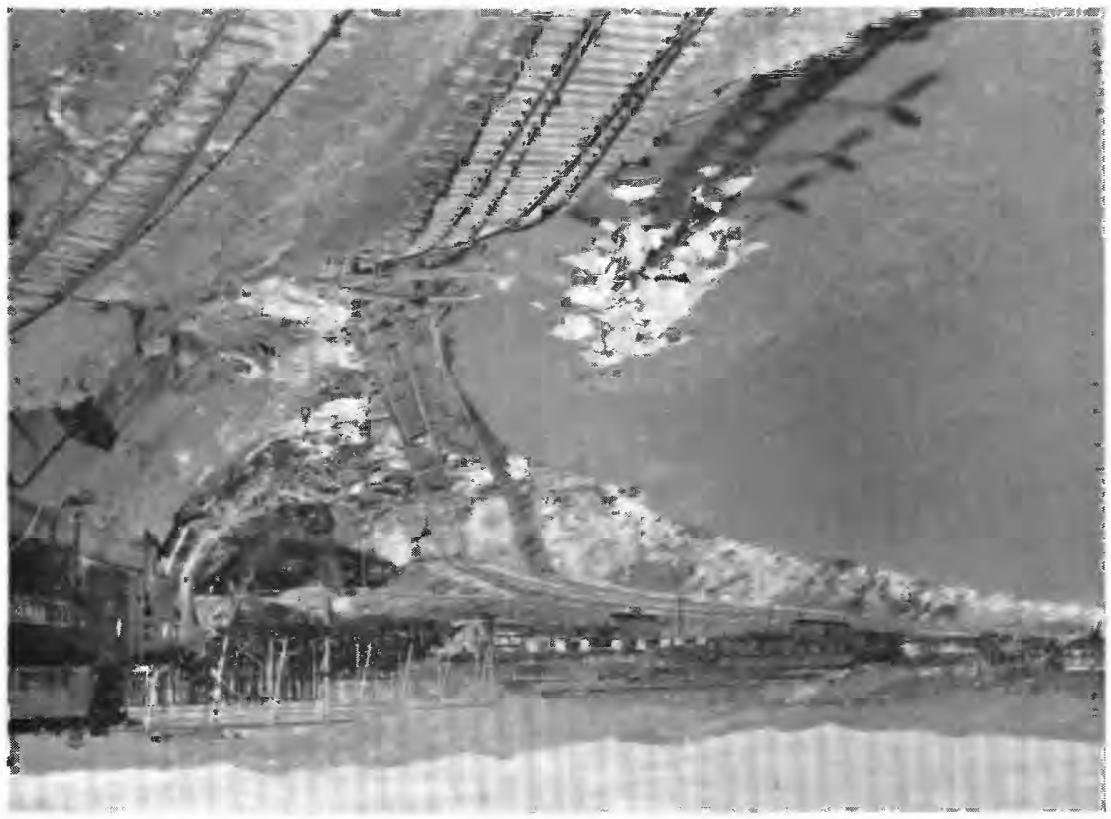




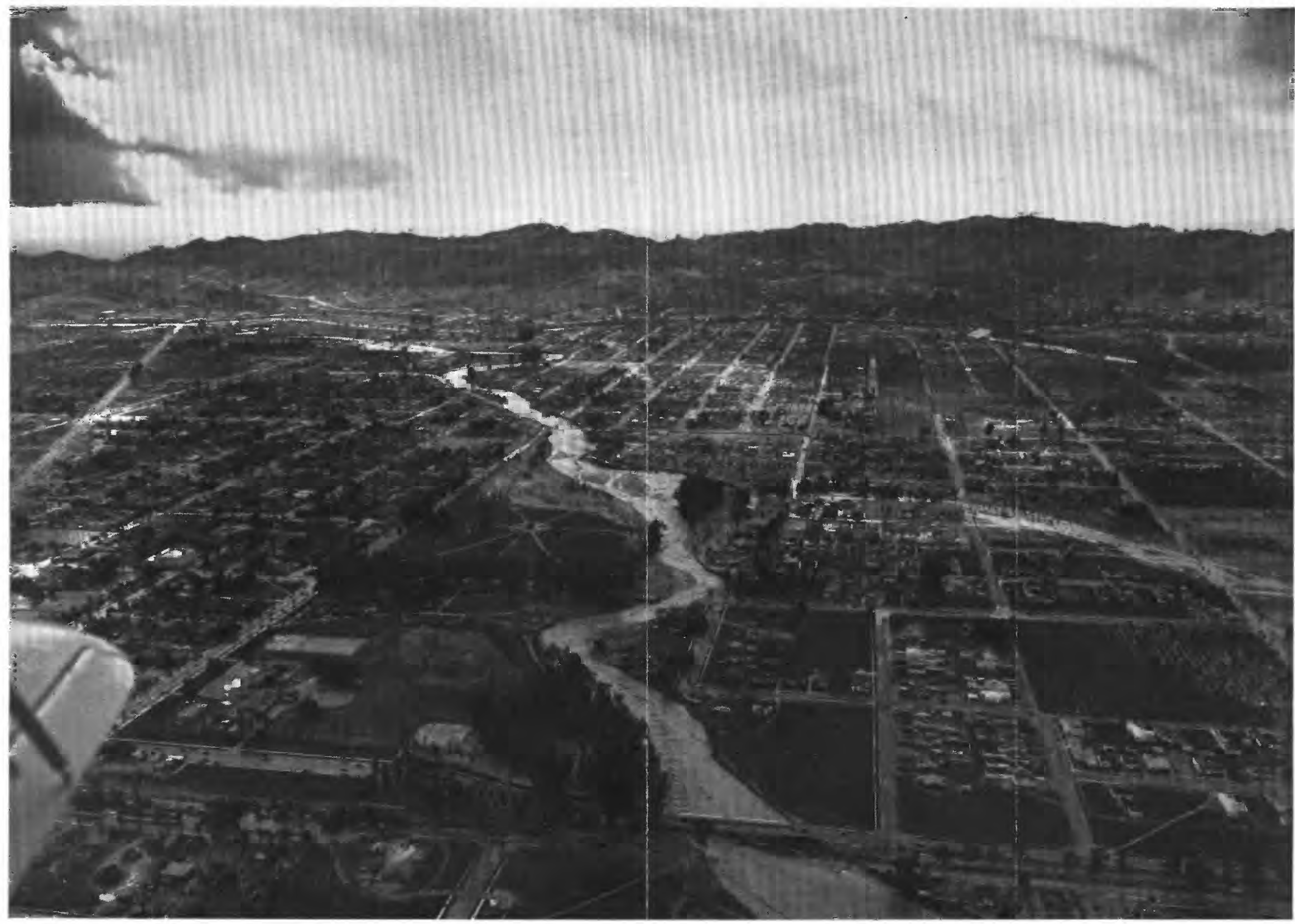

SAN FERNANDO VALLEY, MARCH 2, 1938.

Note small, meandering chanrel in right center. Courtesy of 115th Photo Section, California National Guard. 


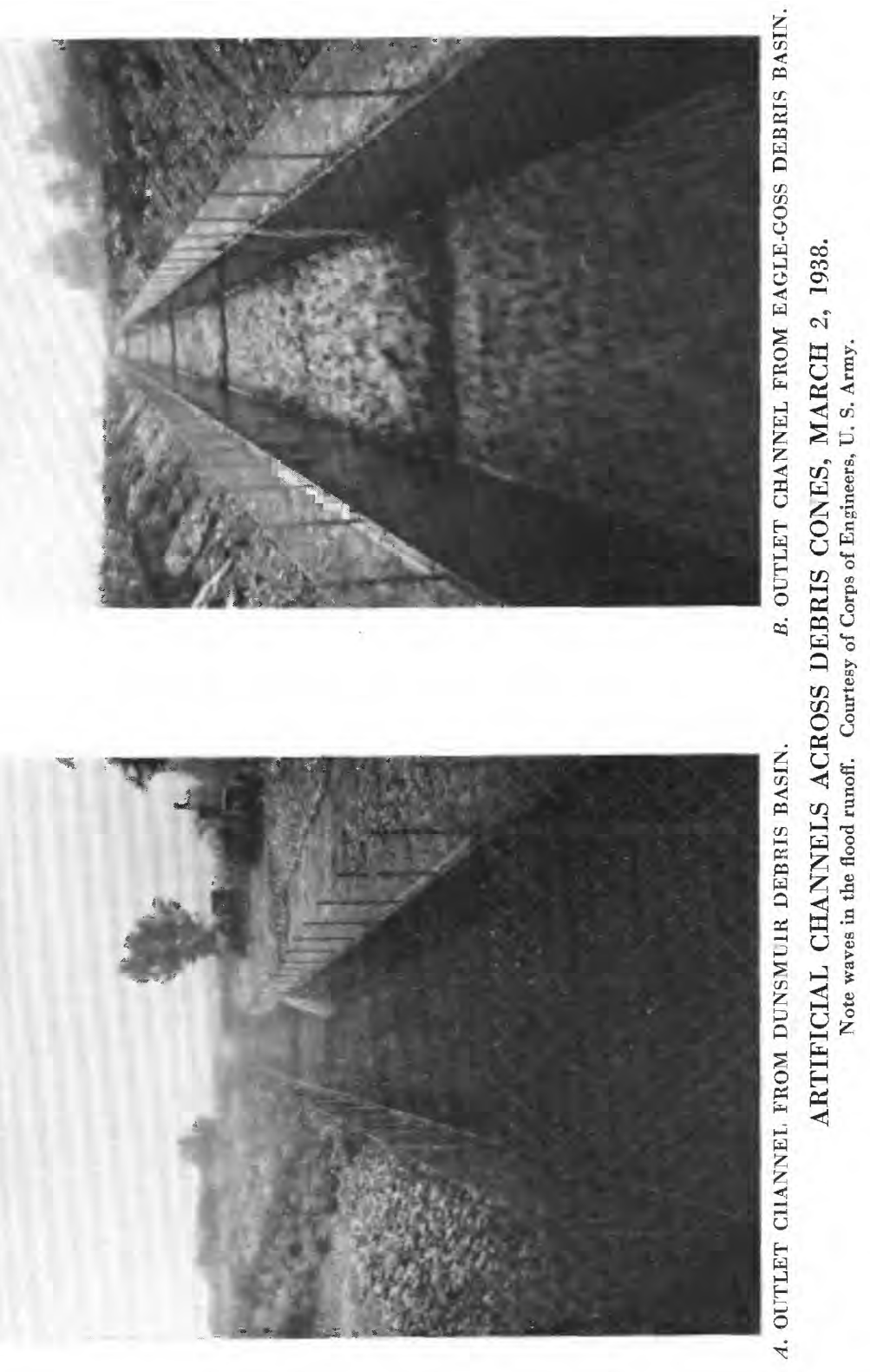




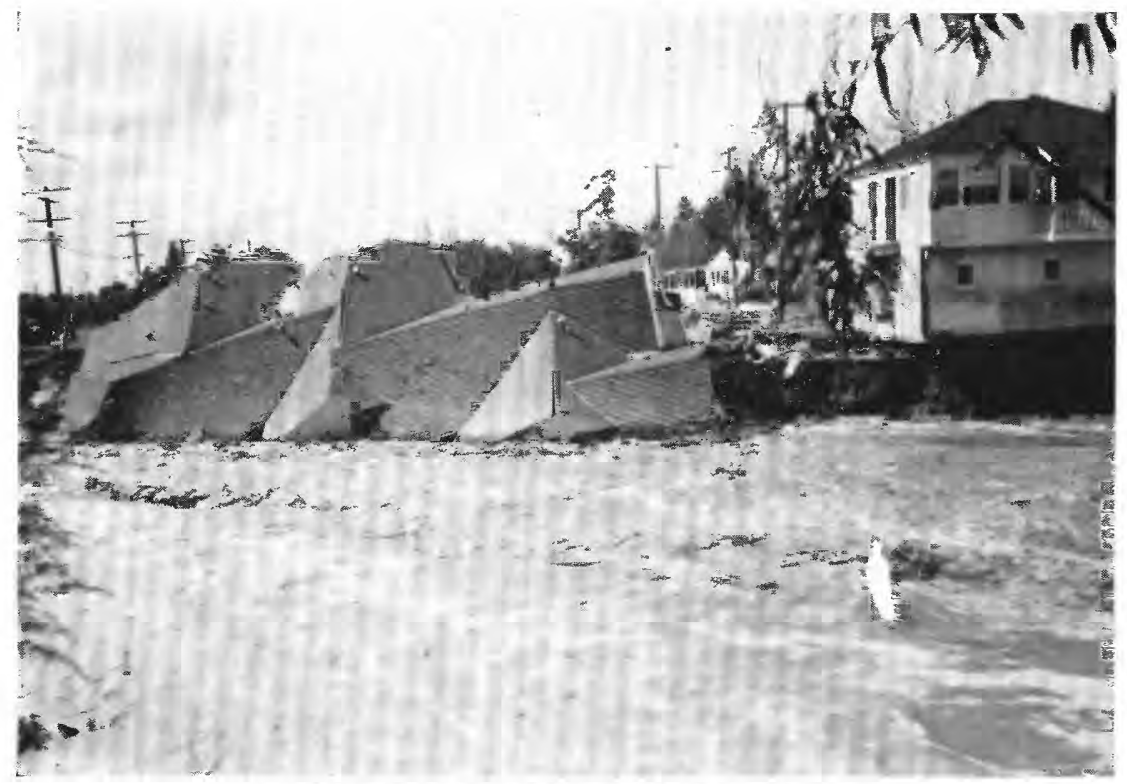

A. DESTRUCTION BY MEANDERING STREAM.

Note composition of stream banks.

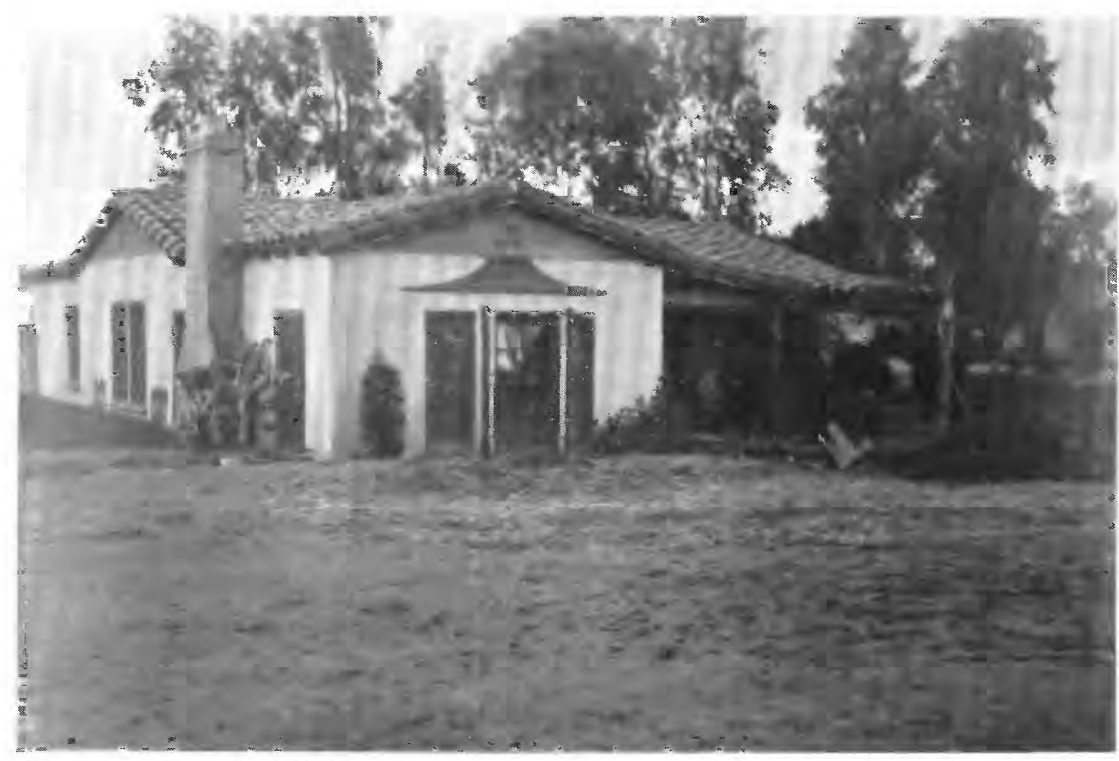

B. DEBRIS DEPOSITED BY MEANDERING STREAM.

Note that deposit is as high as fence at front of house but much lower at rear.

PROPERTY DAMAGED BY FLOOD.

Courtesy of Ralph Willis. 
METEOROLOGIC AND HYDROLOGIC CONDITIONS

\section{Precipitation records}

In the arid and semiarid climate of southern California rainfall is a domant factor in the life of the people, and the collection of data on precipitation is, therefore, of great importance and public interest. Many governmental agencies furnish data on rainfalf to supplement those collected by the United states Weather Bureau. Among these ageneies are the Forest Service and Soll Conservation Service of the United States Department of Agriculture; Corps of Bngineers, United States Arry; Californie State Division of Water Resources; Los Angeles County Flood Control District; Orang' County Flood Control District; Ventura County Engineer; and Los Angeles City Water Department. In addition to the establishment of their own stations, these agencies have aided in furnishing gages to Individuals for operation in sparsely populated areas where no precipitation records were ava1lable. Many rain gages have also been operated by private individuals and by cities and tows for their own information. Some of these records may be subject to error in interpretation, owing to faulty location of gages or topographic influence, but generally information thus collected makes a substantial contribution to the knowledge of precipitation in the area. As a result of this widespread activity and interest, southern Califormia has a more complete coverage of precipitation stations than most other sections of the United states.

The floods of March 1938 were the direct result of rain which fell during the storm perlod February 27 to March 4. The dally precipitation data for this period are presented in table 1 , which embodies the records at 733 standard rain-gage stations in the region covered by this report in which direct observations were made of the accumulated catch. The stations are arranged alphabetically in 22 groups corresponding to areas defined by drainage basins or other topographic features. For each station are given the lecation, altitude, dally rainfall, total rainfall for the storm period, name of observer or source of the record, and a note concerming time of observation if other than in the afternoon. The latitude and longltude of each station are recorded to the nearest minute, or to the nearest second if the close spacing of the gages in an area makes this desirable or if already so determined. Altitudes were obtained from topographic maps of the Geological Survey and are accurate within 10 to 100 feet, depending on the contour interval of the maps. Small amounts of rainfall observed at some stations on February 26 and Mareh 5 were ccmbined with amounts recorded on February 27 and March 4, respectively in order to complete the record for the entire storm. 


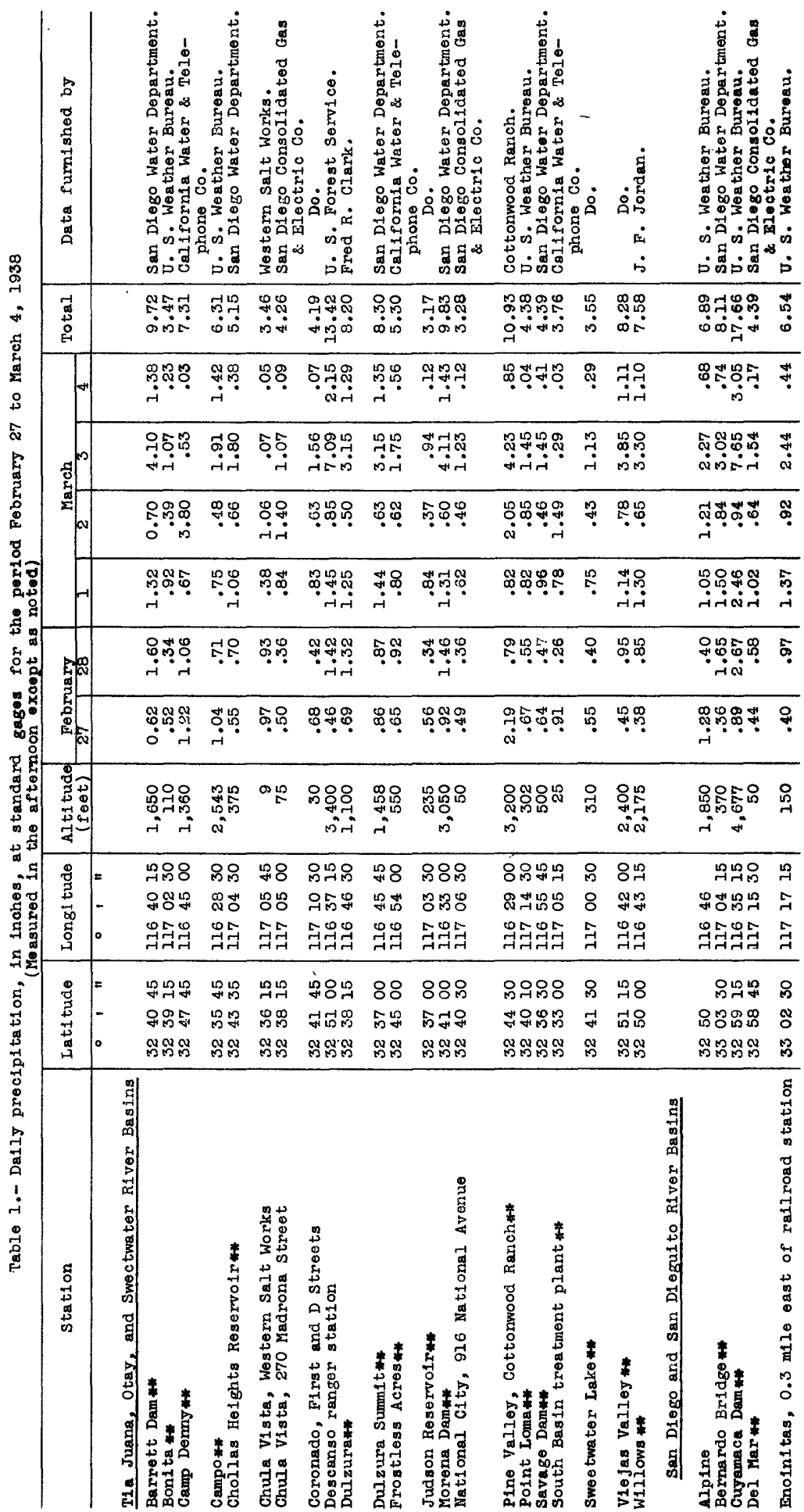




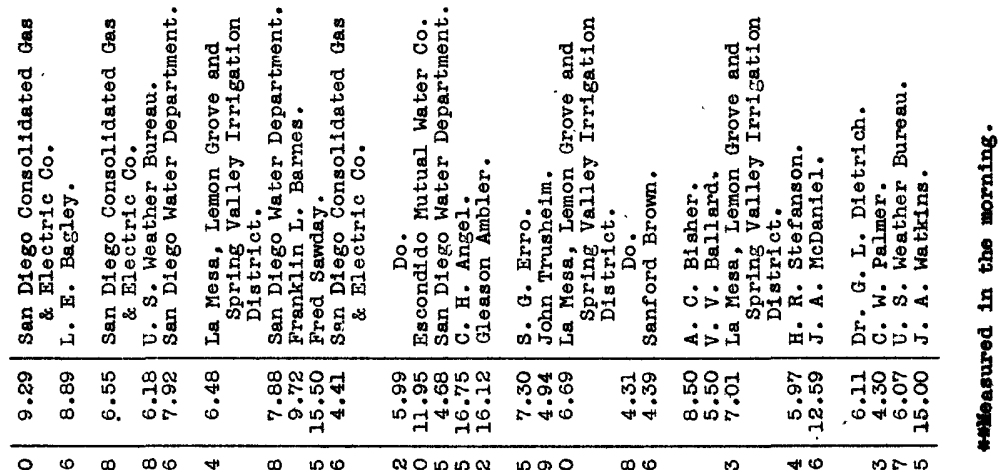

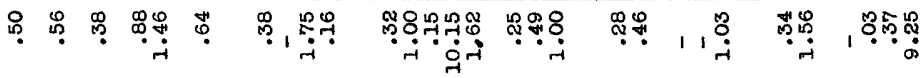

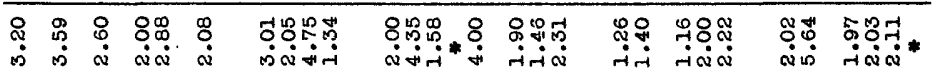

\begin{tabular}{|c|}
\hline \\
\hline 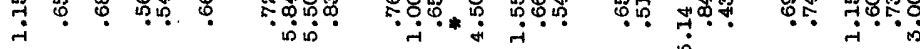 \\
\hline
\end{tabular}

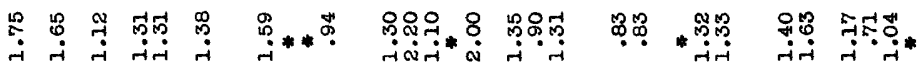

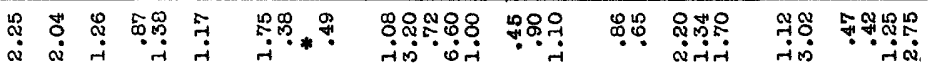

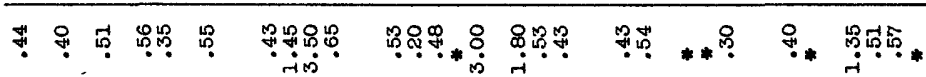

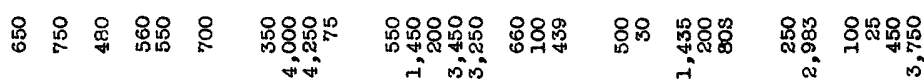

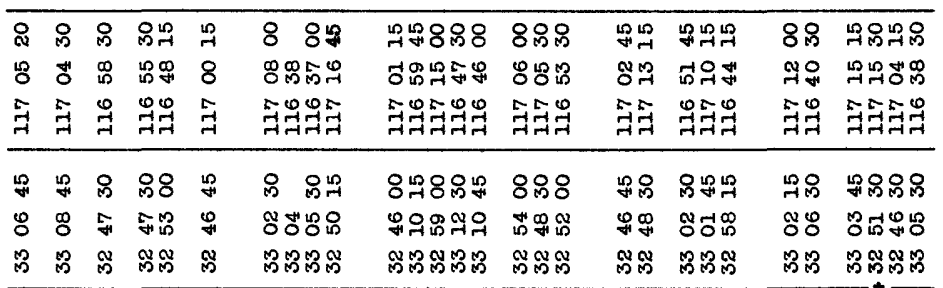

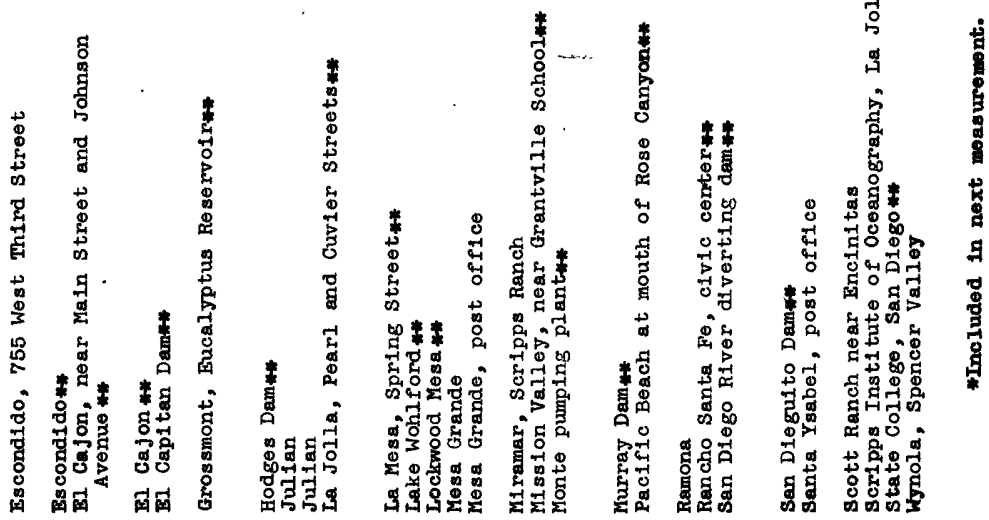




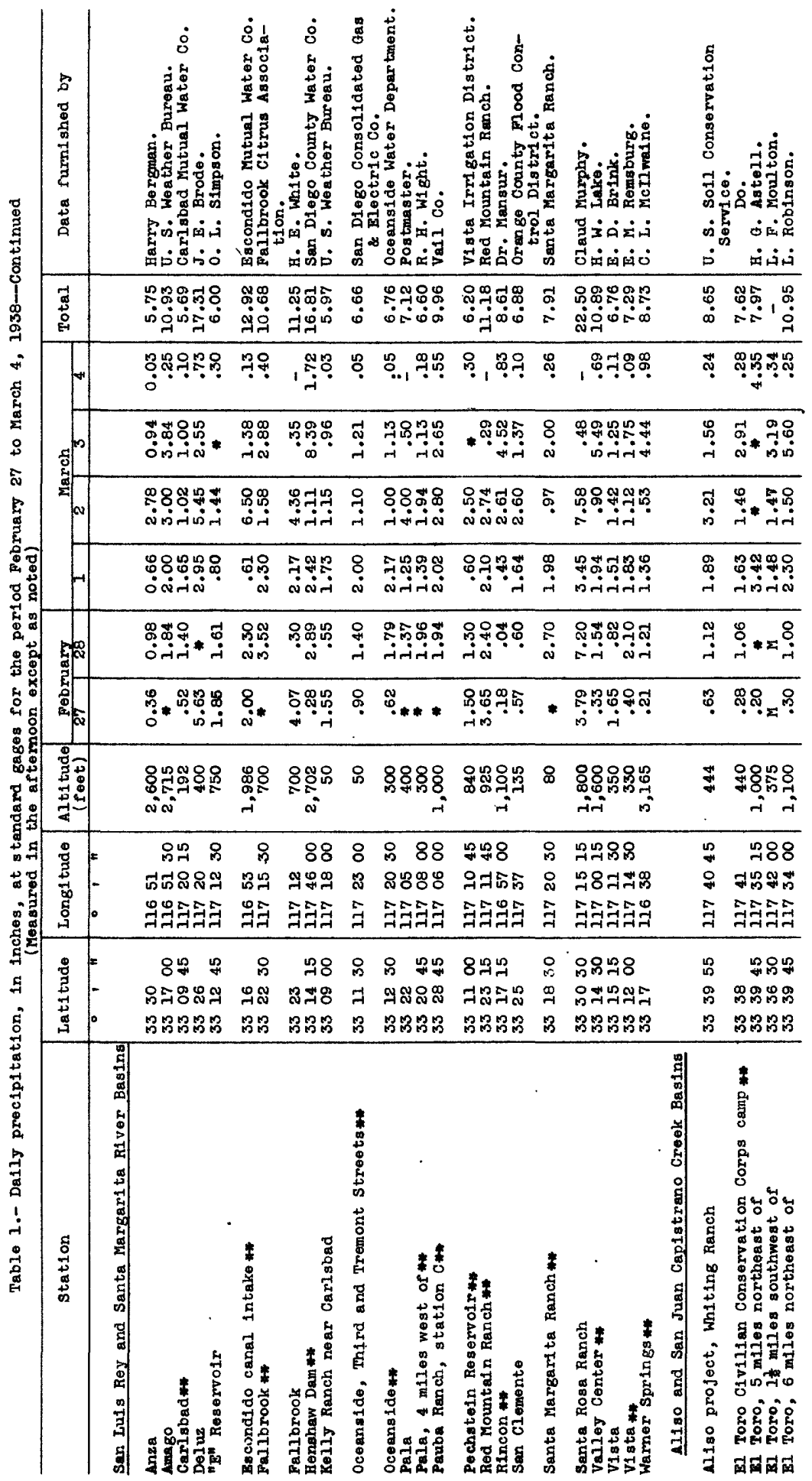




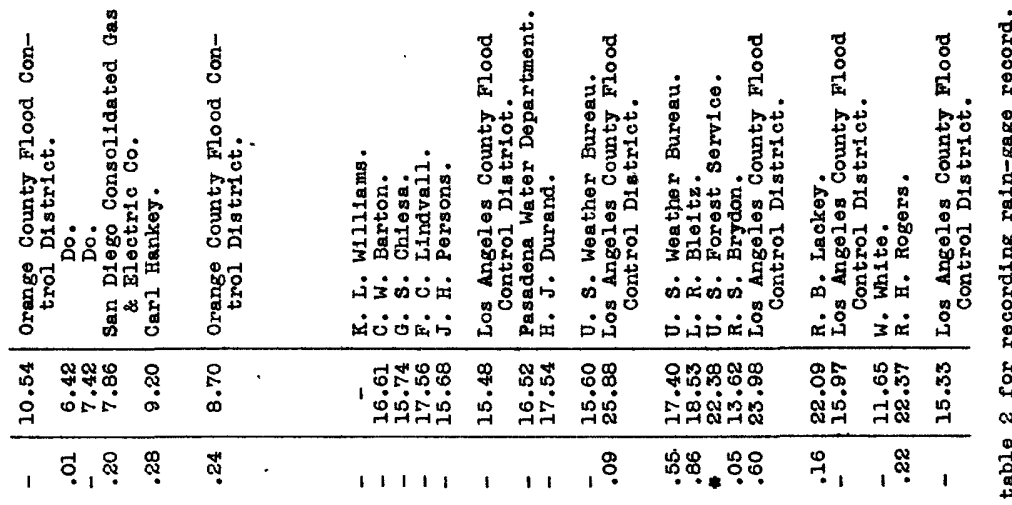

कृ

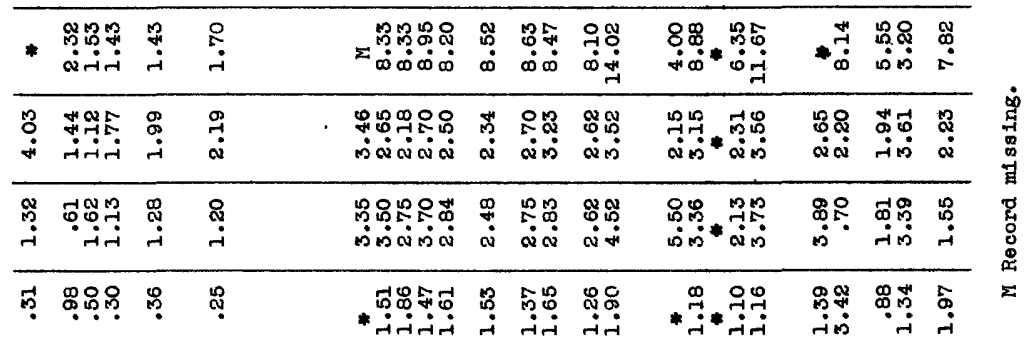

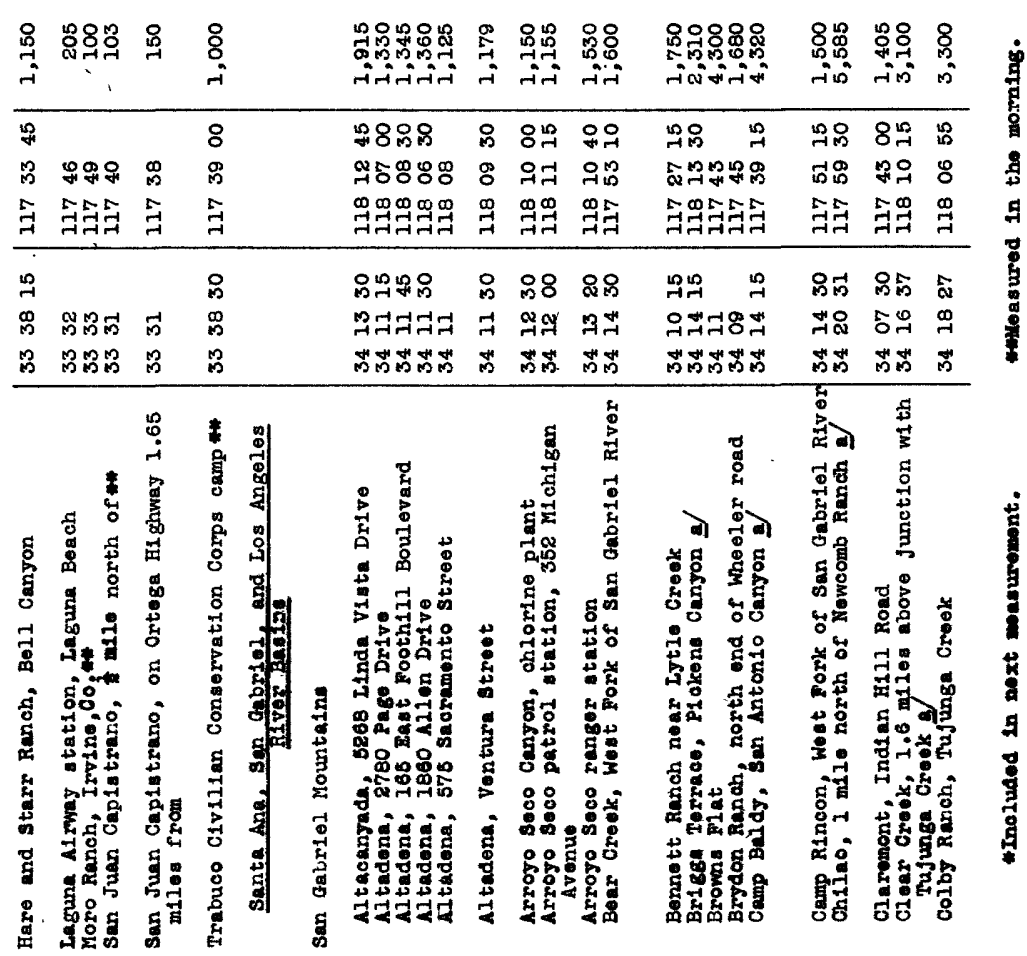




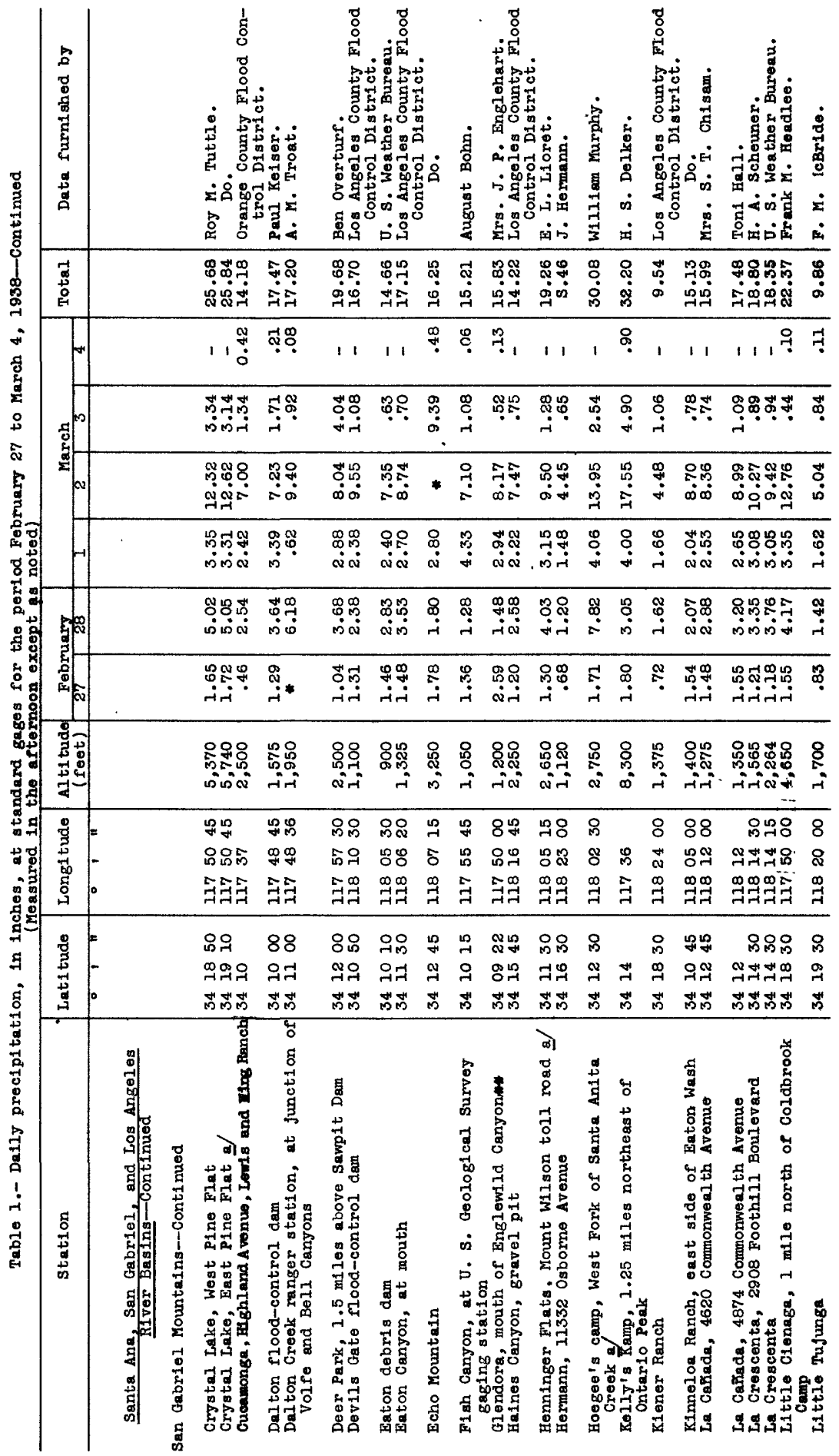




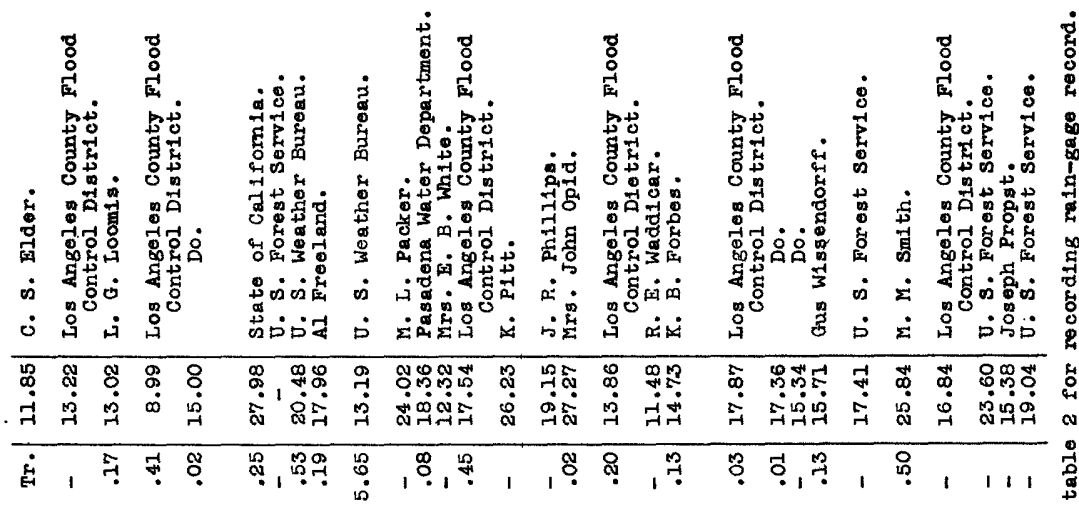

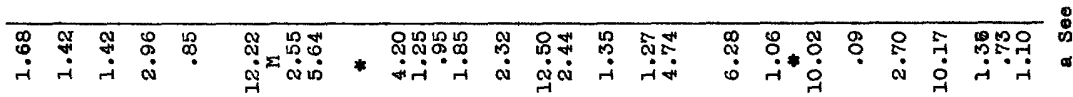

包

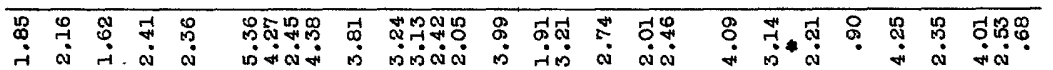

कौ

今.

员 品

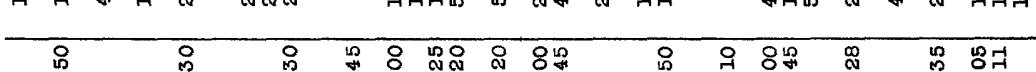

Ұ Ұ

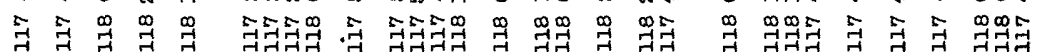

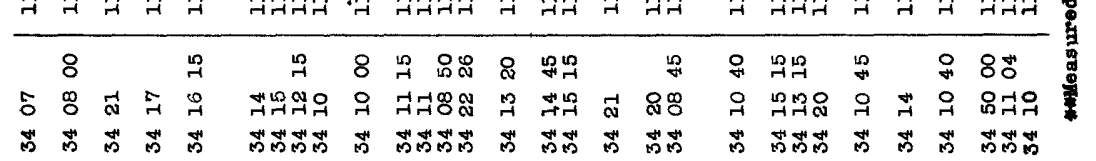

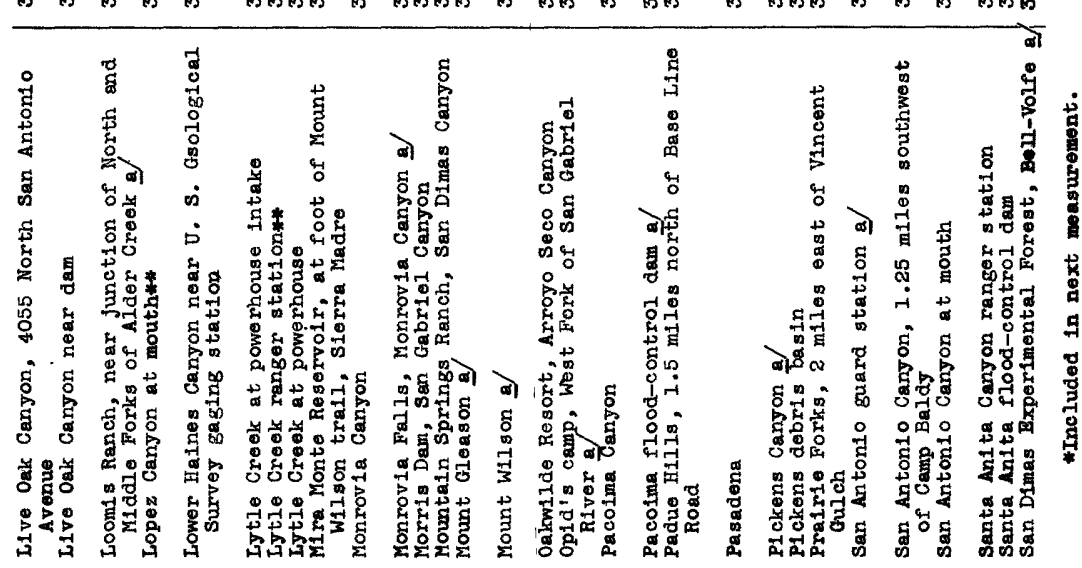




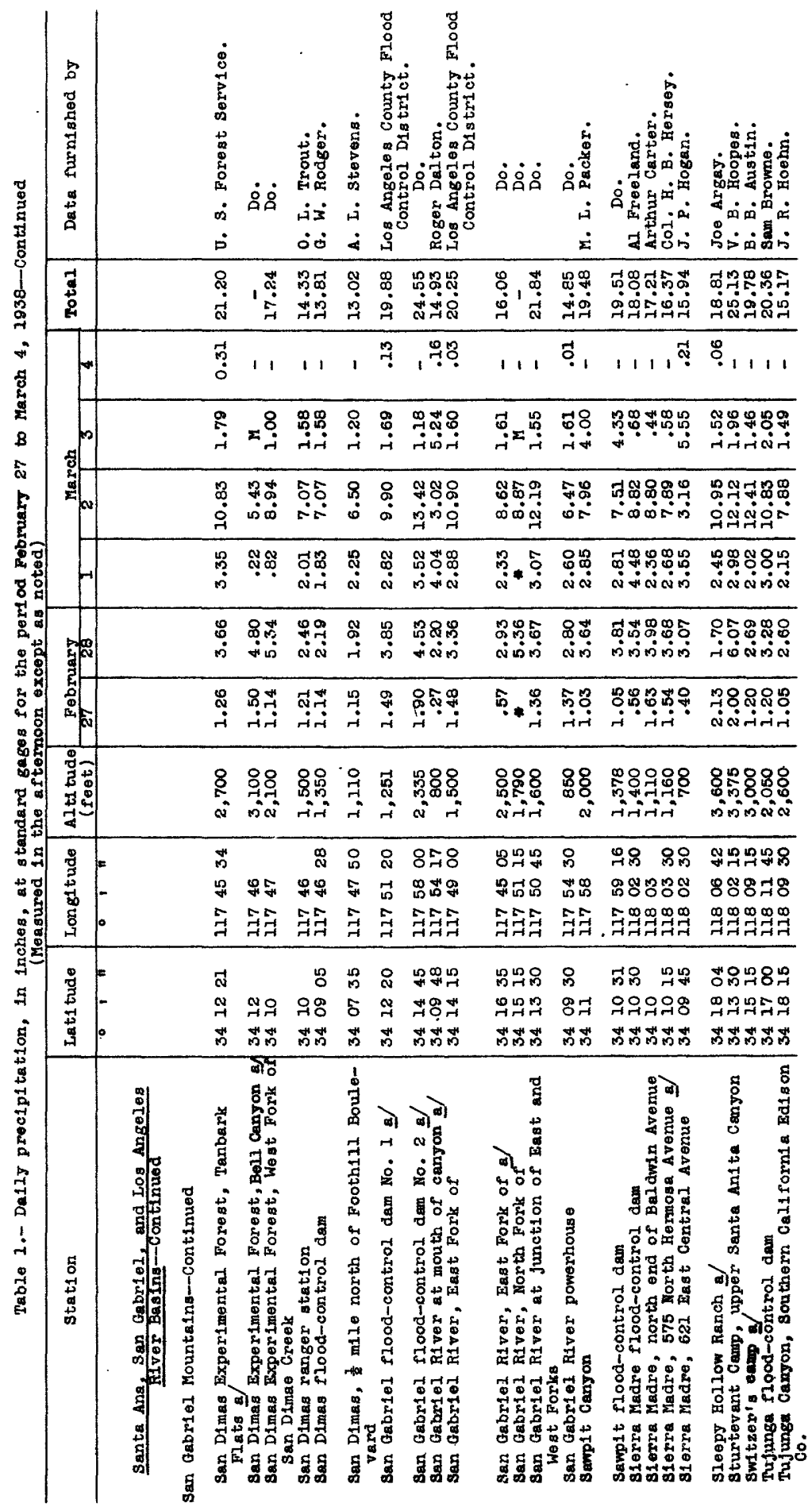




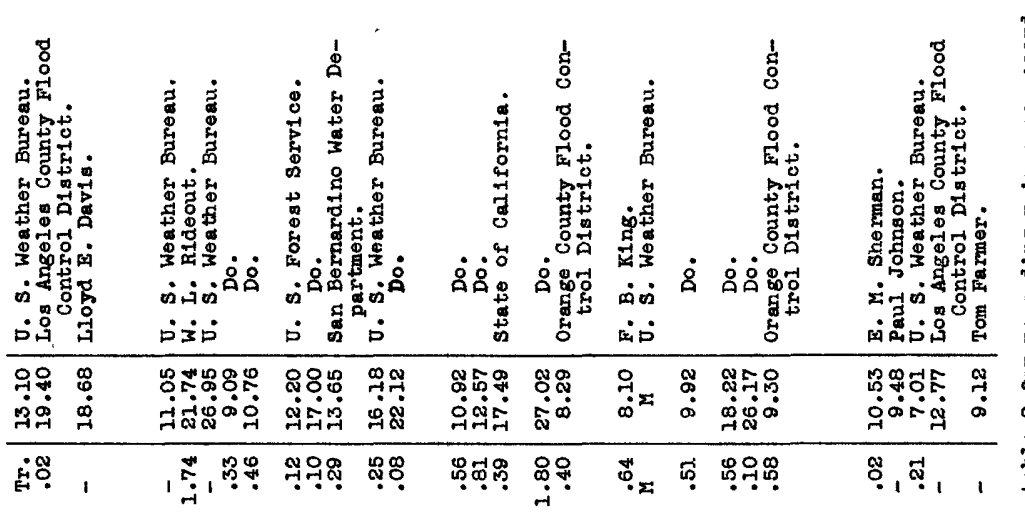

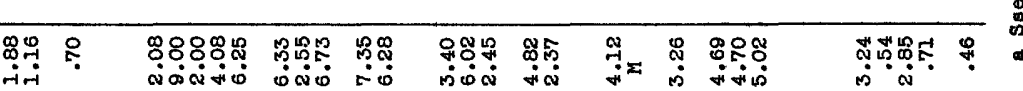

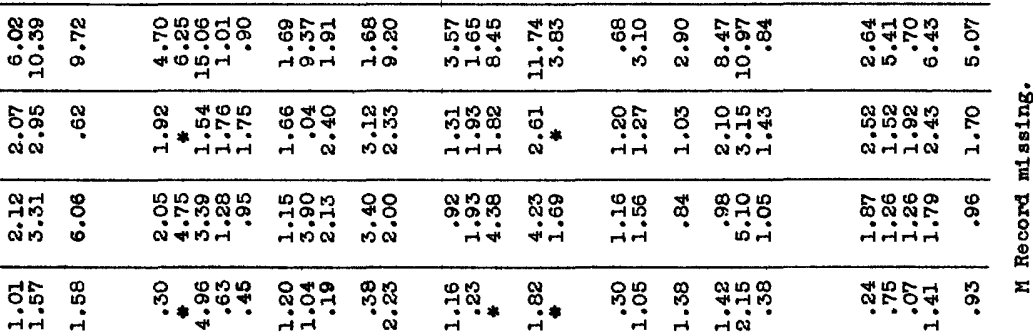

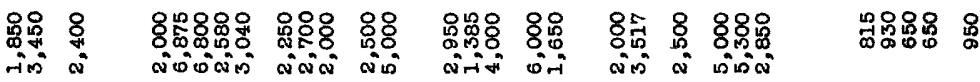

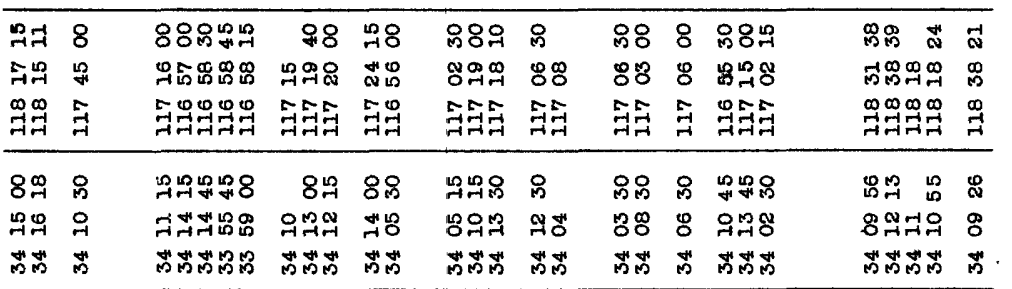

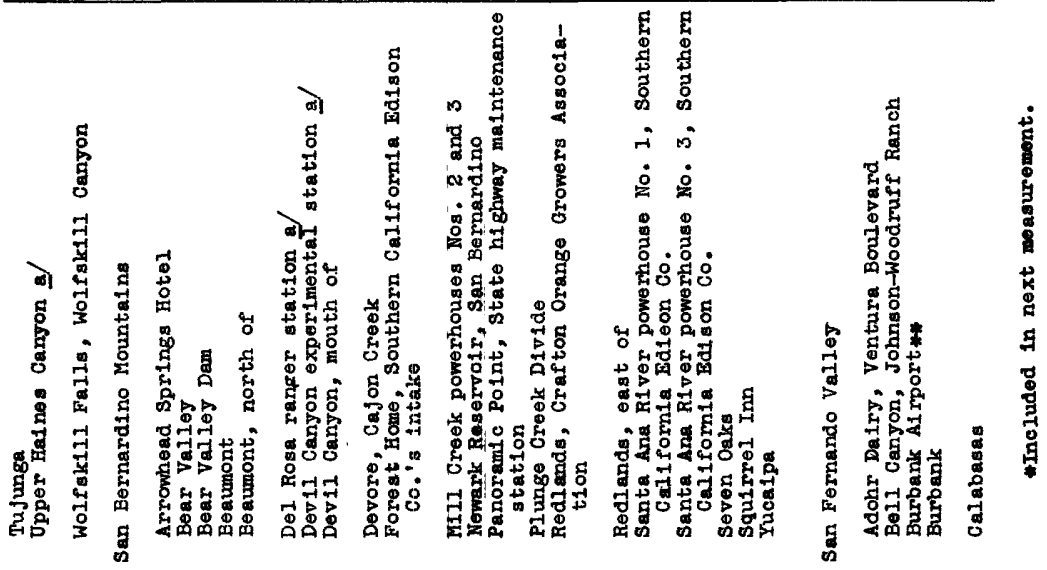




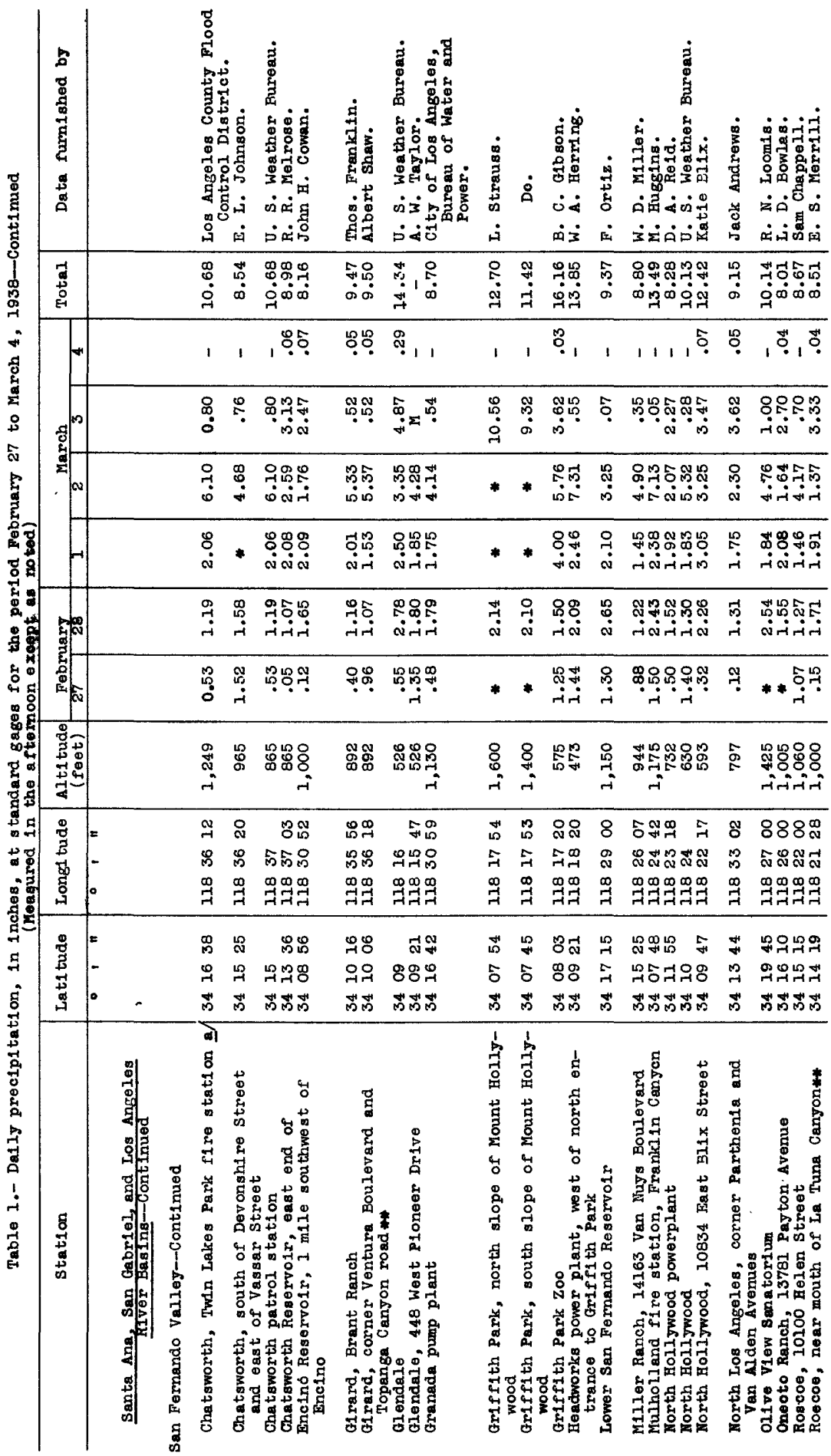




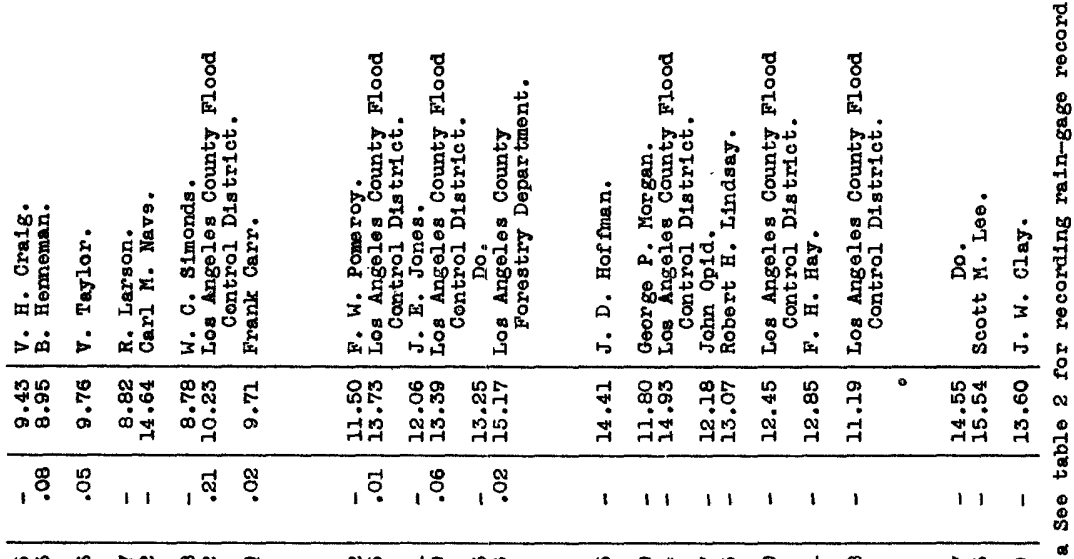

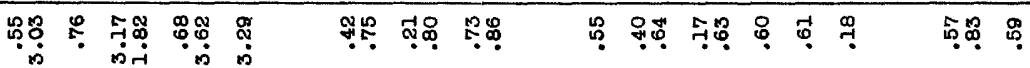

\begin{tabular}{|c|c|c|c|c|c|c|c|c|c|c|c|c|c|c|c|}
\hline 我芯 & 今. & 노요 & 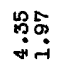 & $\underset{\alpha}{\ddot{\alpha}}$ & 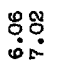 & 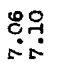 & 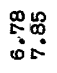 & $\stackrel{2}{\circ}$ & ثै: & 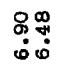 & $\overrightarrow{0}$ & $\stackrel{\hat{\alpha}}{\grave{0}}$ & $\begin{array}{l}\vec{\phi} \\
\stackrel{\overrightarrow{0}}{\circ}\end{array}$ & : & $\stackrel{-1}{-}$ \\
\hline 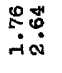 & & 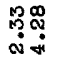 & 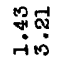 & $\stackrel{\sharp}{\sharp ! ~}$ & 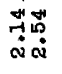 & 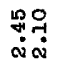 & 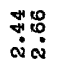 & $\stackrel{\widehat{T}}{\stackrel{4}{a}}$ & 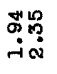 & 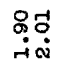 & $\underset{\sim}{\stackrel{\leftrightarrow}{*}}$ & $\stackrel{9}{a}$ & $\begin{array}{l}\stackrel{\infty}{\infty} \\
\stackrel{-}{+} \\
\end{array}$ & 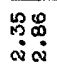 & 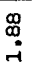 \\
\hline 㱐 & $\stackrel{\sharp}{\sharp}$ & 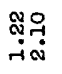 & ڤึ: & مُ & 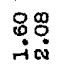 & 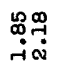 & 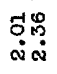 & 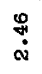 & 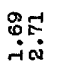 & 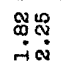 & $\stackrel{\overrightarrow{1}}{\ddot{a}}$ & $\begin{array}{l}m \\
\stackrel{m}{a} \\
\dot{\alpha}\end{array}$ & $\begin{array}{l}\overrightarrow{0} \\
\stackrel{-}{+}\end{array}$ & $\begin{array}{l}\text { P: } \\
\dot{\sim}: \\
\dot{\alpha}\end{array}$ & ס् \\
\hline$=$ & 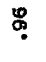 & 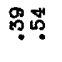 & & 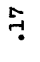 & 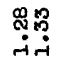 & \$્ન & $H_{1}$ & -1 & تُّ" & 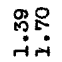 & $\begin{array}{l}\stackrel{8}{0} \\
\stackrel{\sim}{+}\end{array}$ & مُ & 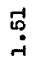 & تِّ & $*$ \\
\hline
\end{tabular}

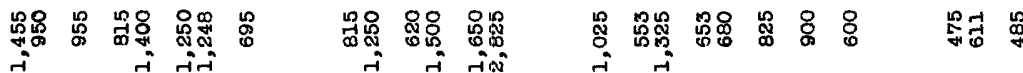

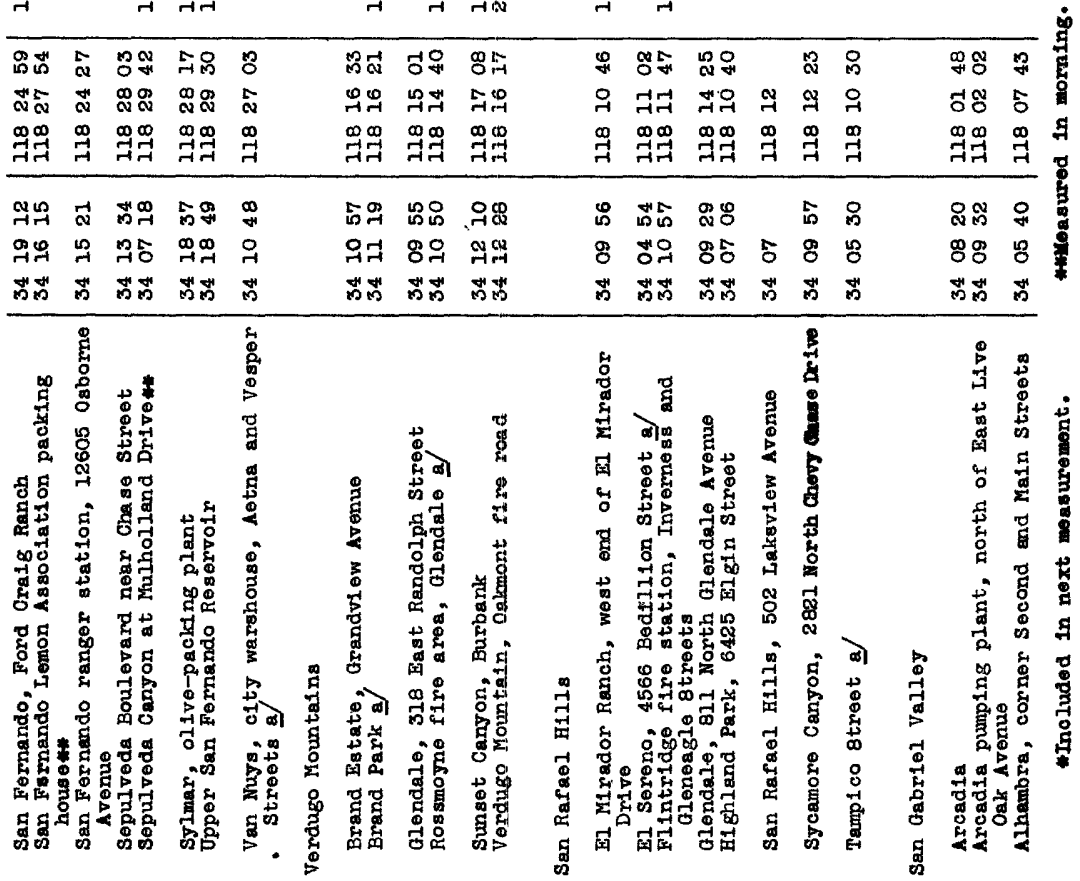




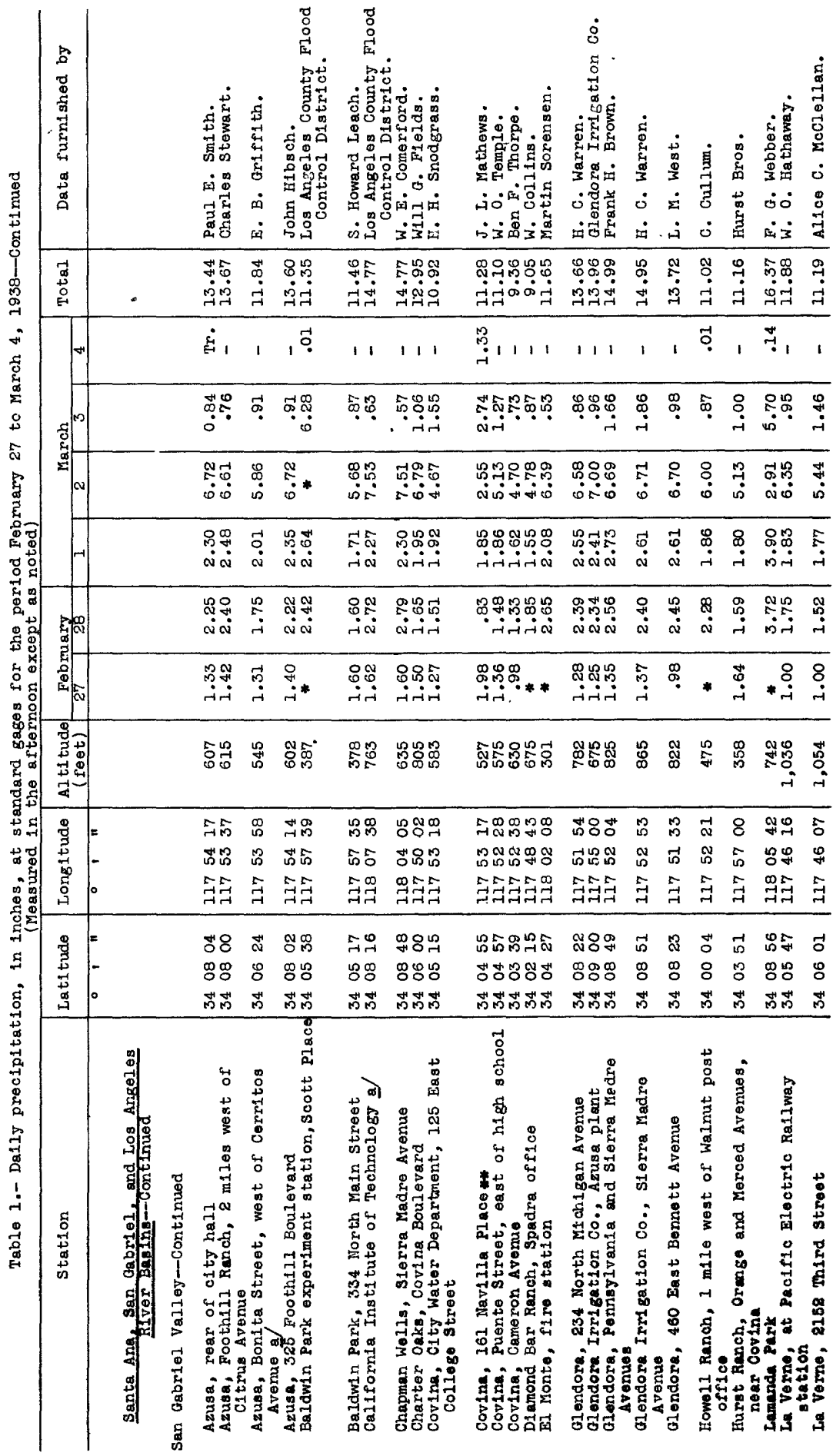




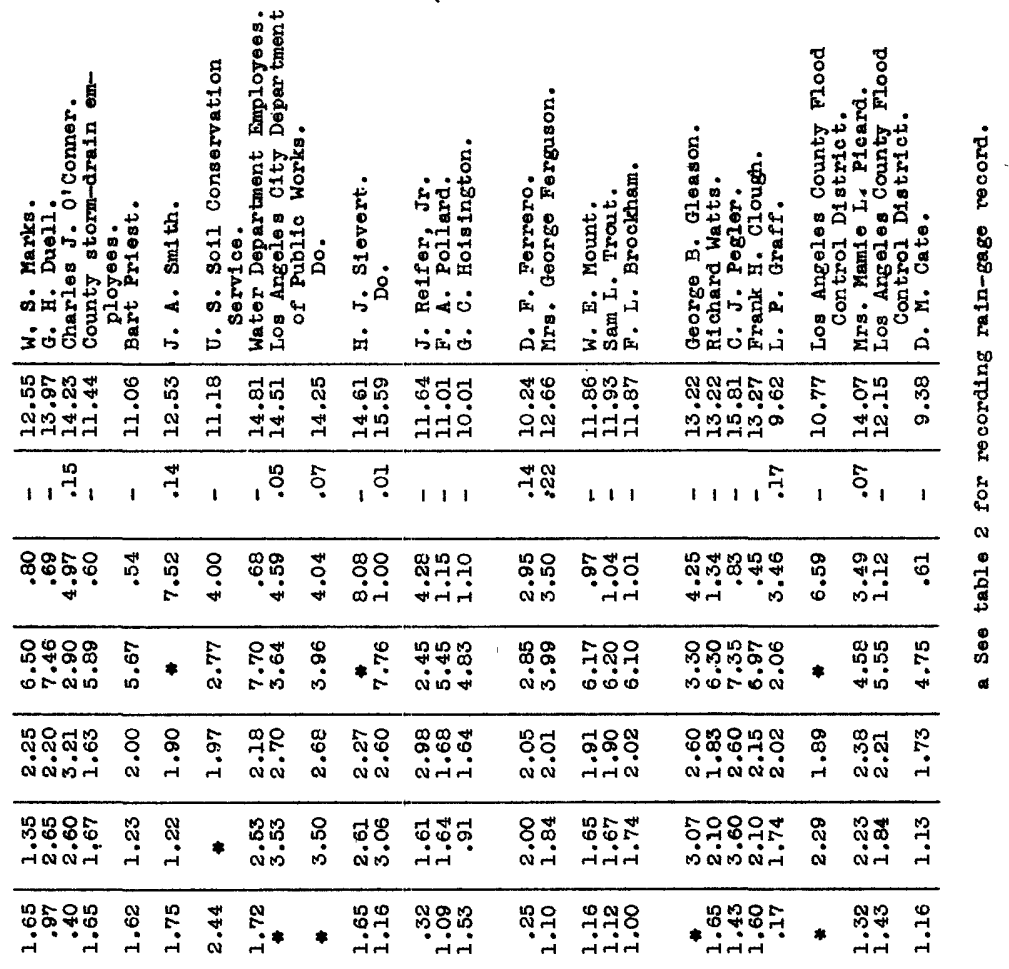

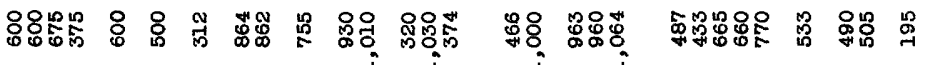

\begin{tabular}{|c|c|c|c|c|c|c|c|c|c|c|c|c|}
\hline 58 & 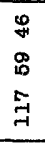 & 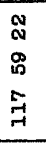 & $\begin{array}{l}m \\
\infty \\
0 \\
0 \\
\\
-1\end{array}$ & $\begin{array}{l}\infty \\
0 \\
\infty \\
\infty \\
-\pi \\
-7 \\
\end{array}$ & $\begin{array}{l}\mathscr{0} \\
0 \\
\infty \\
-1 \\
-1\end{array}$ & $\begin{array}{l}8 \infty \\
80 \\
\infty \\
-1 \\
\rightarrow=\end{array}$ & 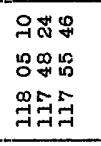 & 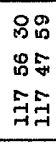 & 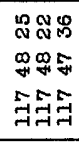 & 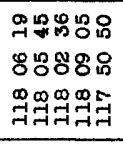 & -1 & 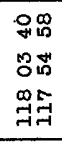 \\
\hline & & \& & $\stackrel{n}{-1}$ & ํㅗำ & p & 20 & 8 & 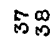 & & & & ผูก \\
\hline & 8 & 8 & 5 & : & 5 & 영우 & ำเ & ำ & 88 & $8 \%$ & & 58 \\
\hline (n) & ले & In & "ే & ถึं & దే & 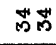 & 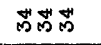 & ஜึ心 & 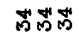 & फีฒ & m & लें \\
\hline . & 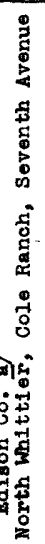 & 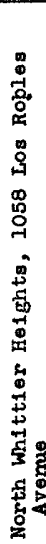 & 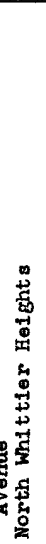 & $\begin{array}{r} \\
0 \\
0\end{array}$ & تี & 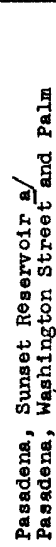 & 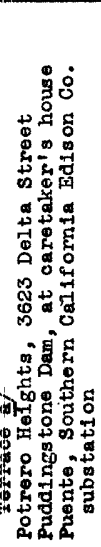 & 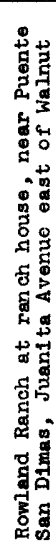 & 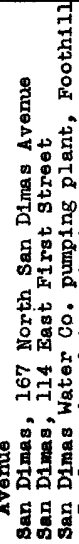 & 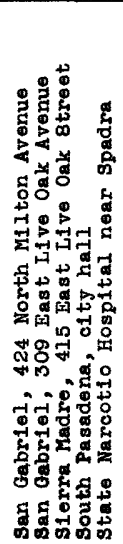 & 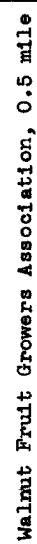 & 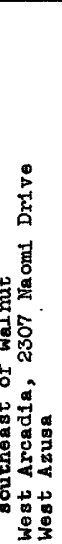 \\
\hline
\end{tabular}




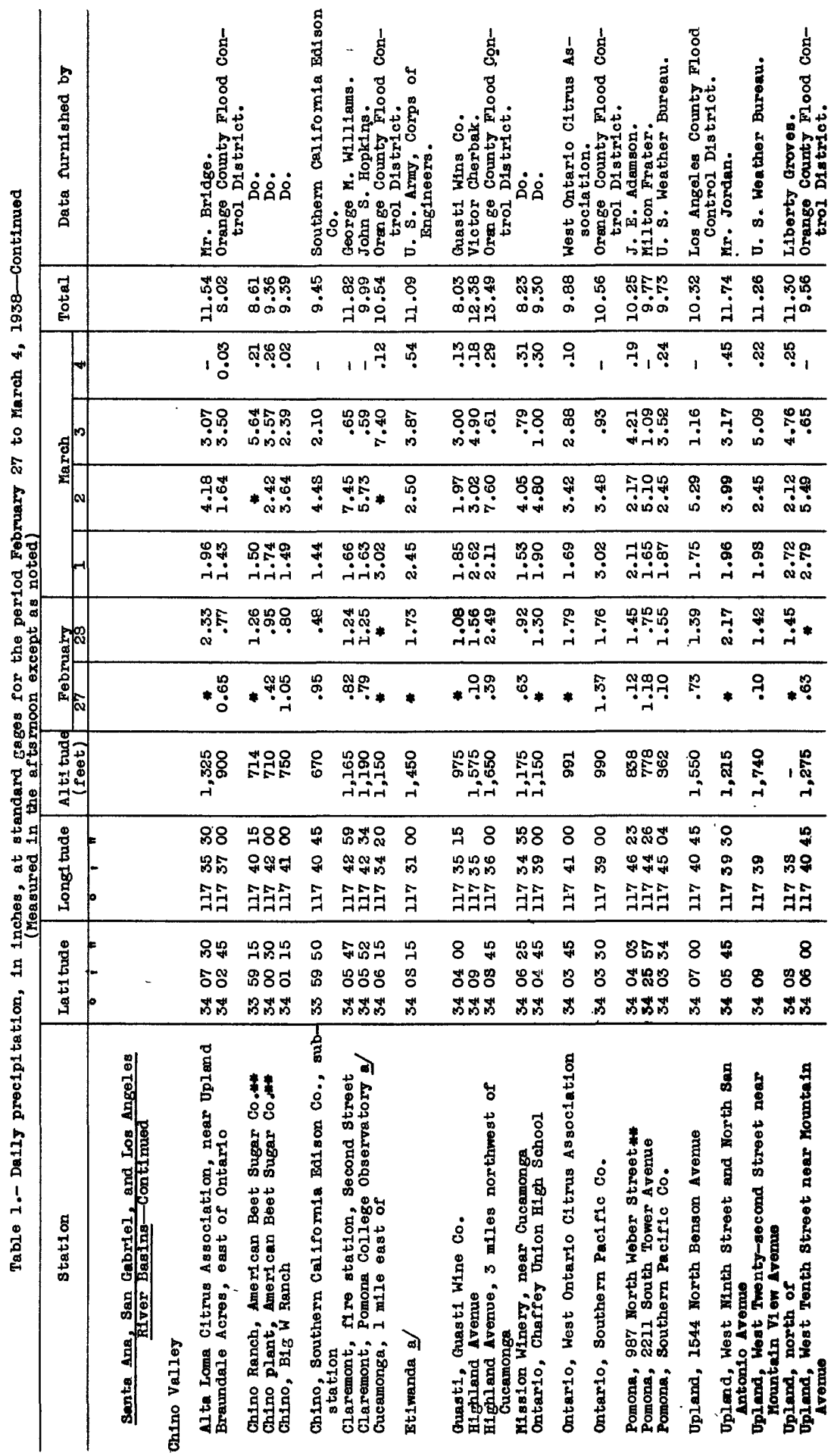




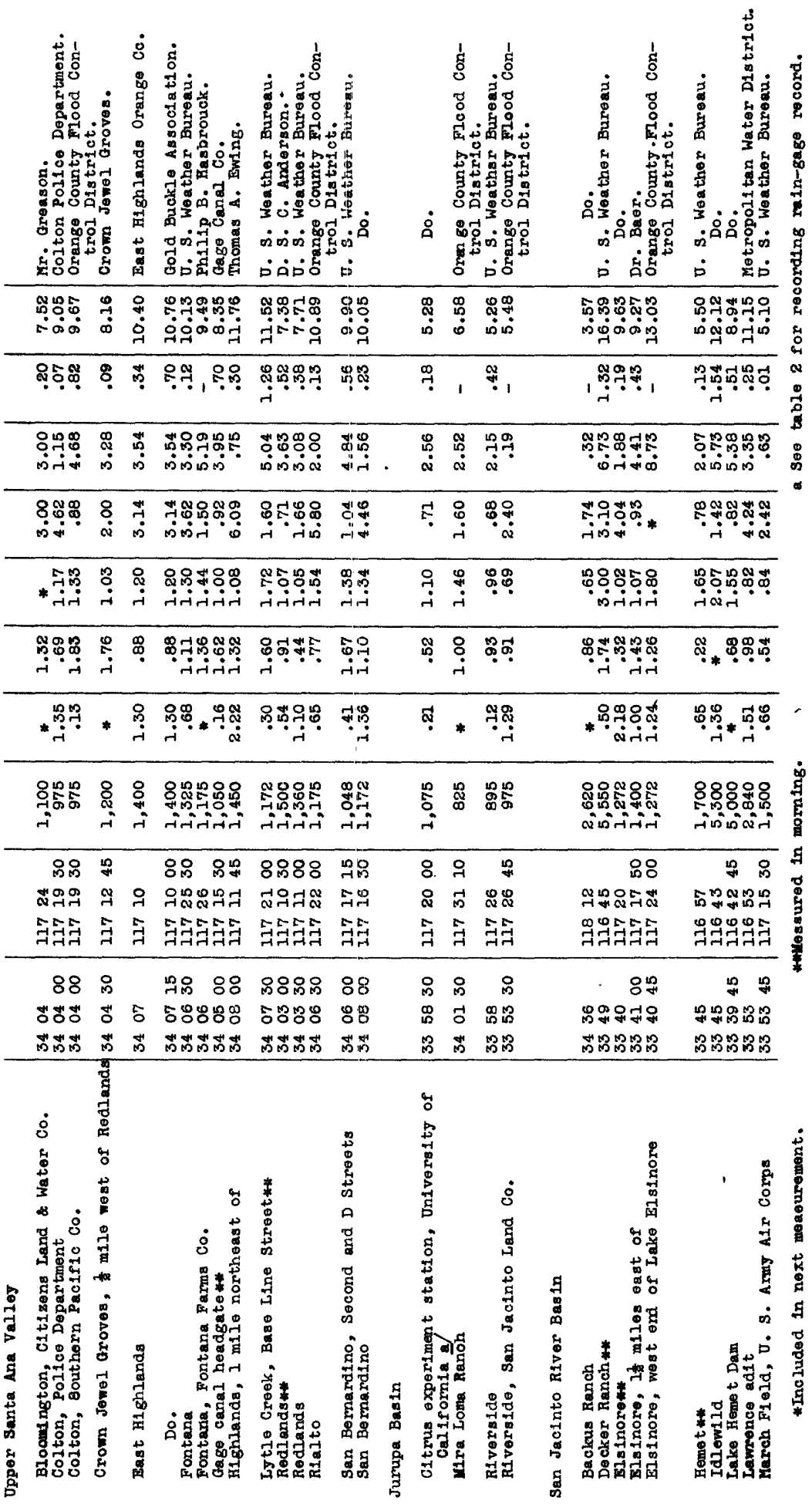




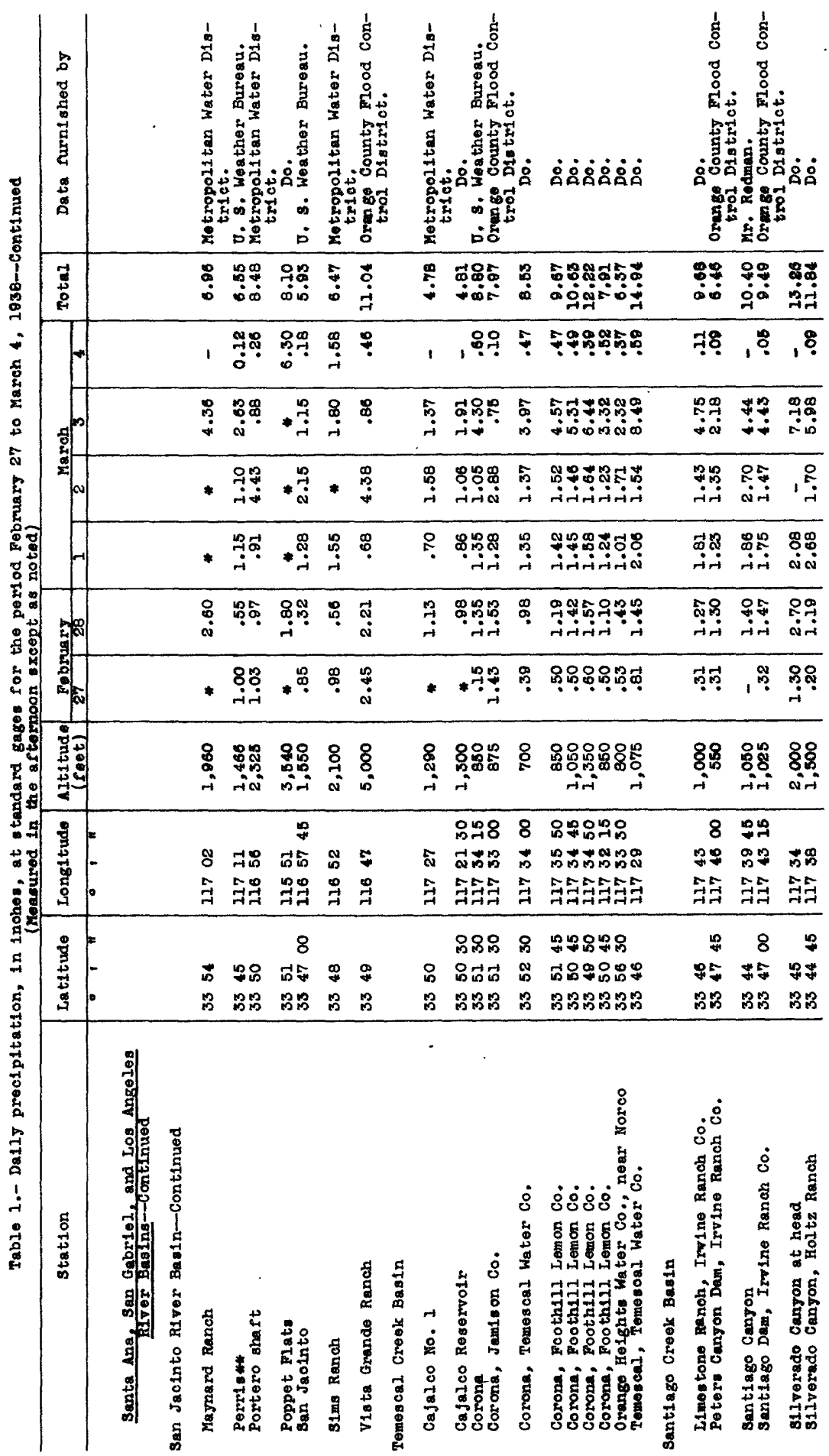




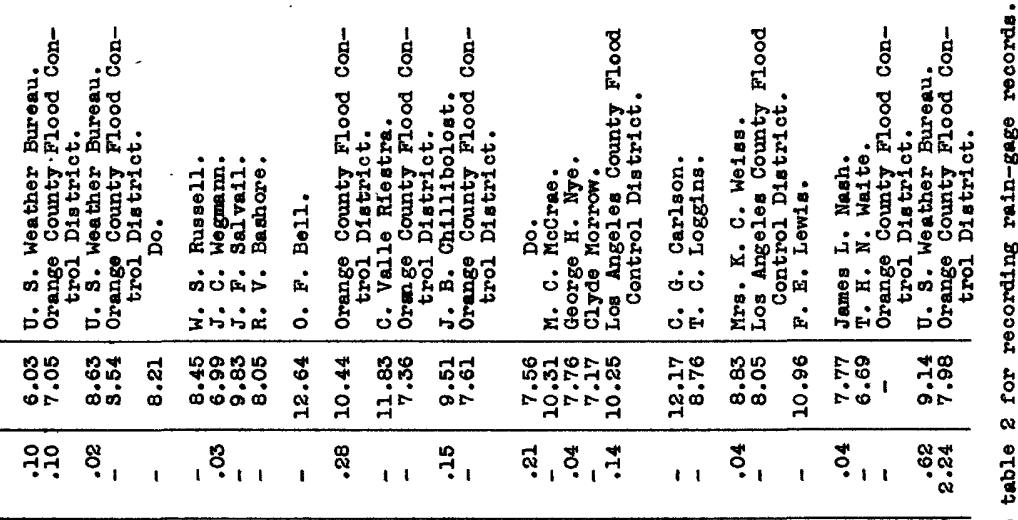

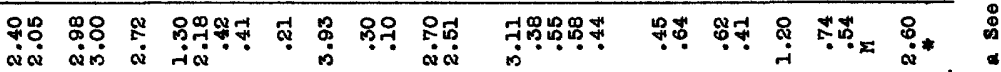

\begin{tabular}{|c|c|c|c|c|c|c|c|c|c|c|c|c|}
\hline त्ष & 7ై & $\underset{\alpha}{\alpha}$ & 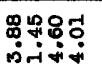 & 芯 & $\stackrel{m}{a}$ & 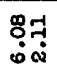 & 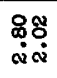 & 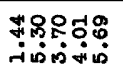 & 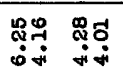 & מִ & 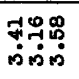 & 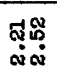 \\
\hline 号品 & 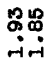 & $\stackrel{\leftrightarrow}{:}$ & 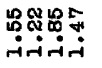 & 8 & $\stackrel{4}{9}$ & 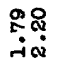 & : & 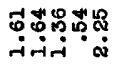 & 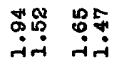 & تص & 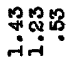 & 营 \\
\hline ה: & (ิ) & $\stackrel{\circ}{:}$ & ख़ी & $\begin{array}{l}\stackrel{2}{\alpha} \\
\text { a }\end{array}$ & $\underset{\sim}{\sim}$ & 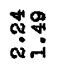 & 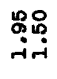 & 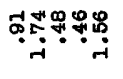 & & $\stackrel{+}{\sharp}$ & דี. & $\stackrel{\mathscr{p}_{\oplus}}{*}$ \\
\hline קִ & & & 180 & 莒. & & צु० & ద్తు & 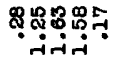 & 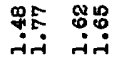 & 䍙 & 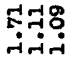 & $\stackrel{5}{~+. * ~}$ \\
\hline
\end{tabular}

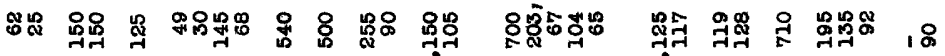

\begin{tabular}{|c|c|c|c|c|c|c|c|c|c|c|c|c|c|}
\hline 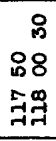 & 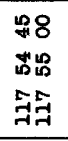 & $\begin{array}{l}\stackrel{0}{0} \\
\text { స }\end{array}$ & 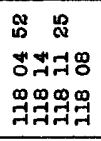 & $\begin{array}{l}\mathscr{P} \\
\& \\
\& \\
9 \\
7 \\
\end{array}$ & 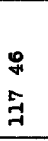 & 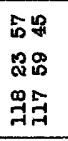 & 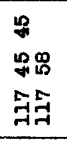 & & 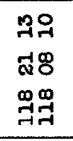 & 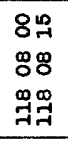 & $\begin{array}{l}8 \\
8 \\
8 \\
5 \\
7\end{array}$ & 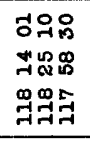 & 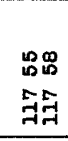 \\
\hline $\begin{array}{r}8 \\
232\end{array}$ & 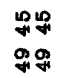 & i⿱口 & 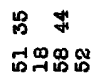 & & i̊ & & $\begin{array}{l}: 8 \\
\text { 용 }\end{array}$ & & $\begin{array}{l}6 \% \\
680 \\
8 \%\end{array}$ & $\begin{array}{l}\text { 品品 } \\
\text { 号5 }\end{array}$ & $\begin{array}{l}8 \\
8 \\
0\end{array}$ & & ถึก๊ \\
\hline ค月 & $B P$ & 品 & 品品品品 & मे & $B$ & ถีค月 & 盟品 & 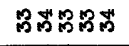 & 荋盟 & 量品 & 8 & 路品 & B \\
\hline
\end{tabular}

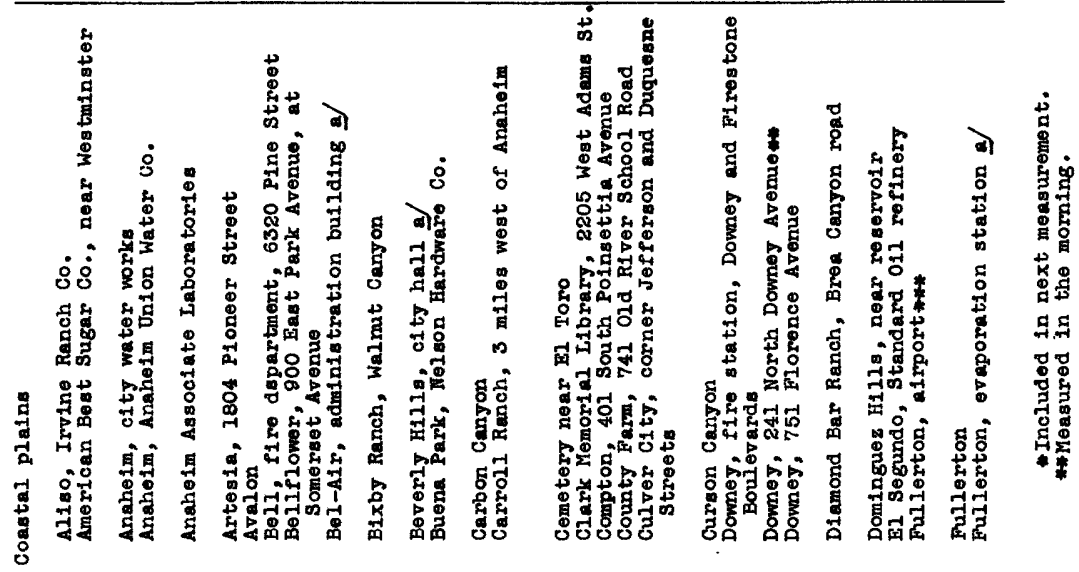




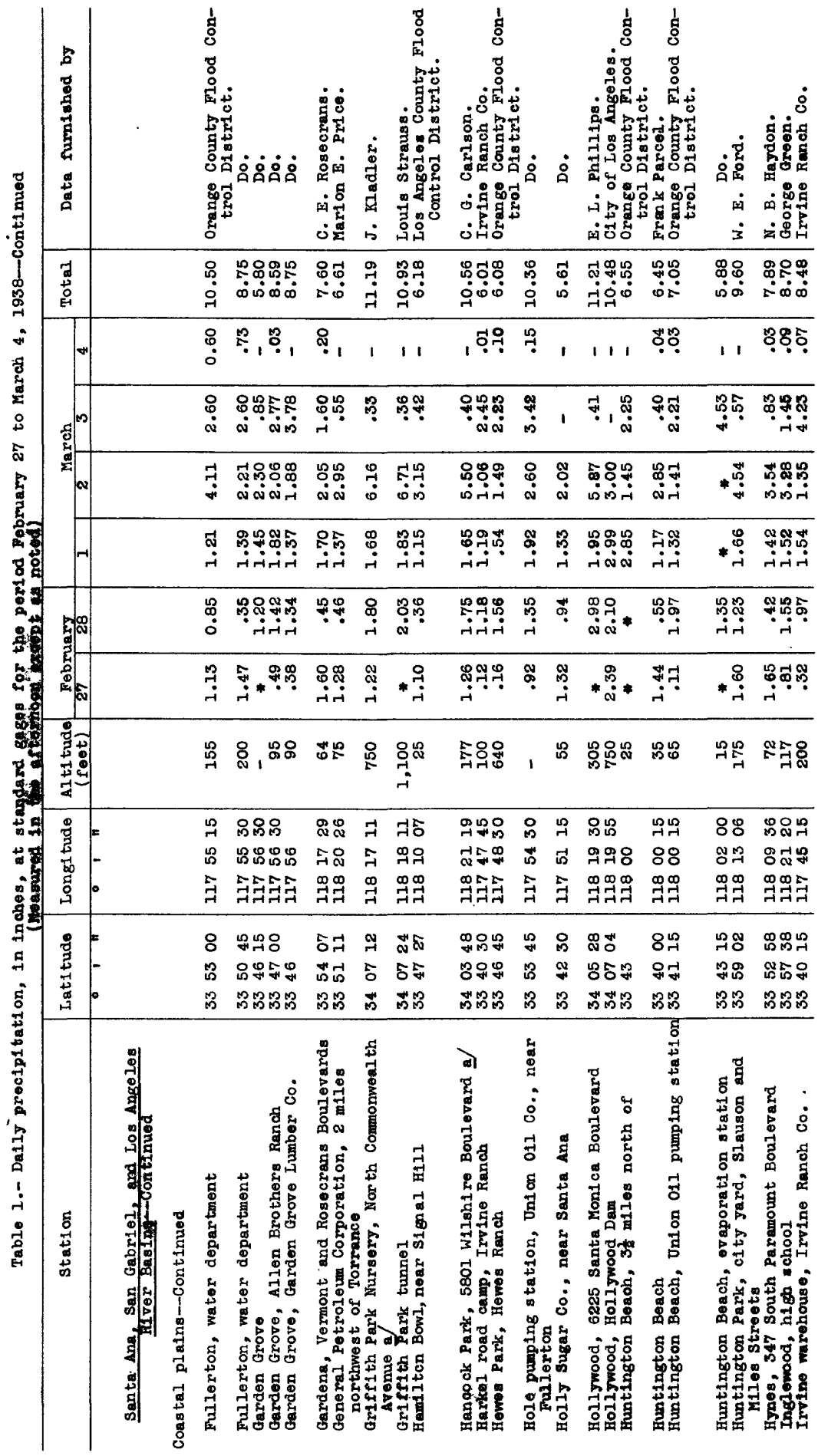




\begin{tabular}{|c|c|c|c|c|c|c|c|c|}
\hline 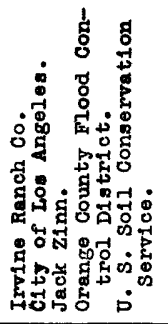 & $\dot{\circ} \dot{\circ}$ & 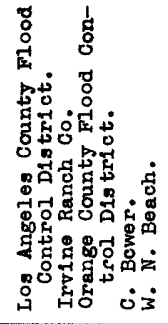 & 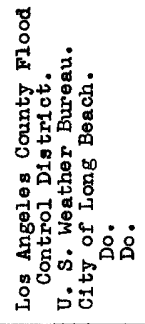 & 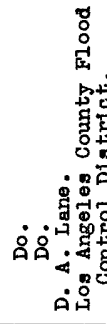 & & 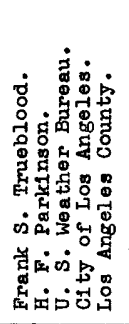 & 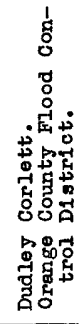 & 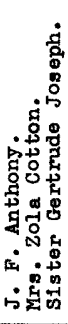 \\
\hline 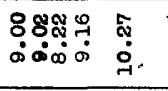 & 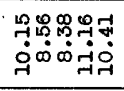 & 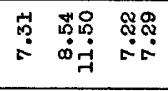 & 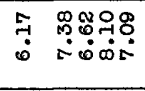 & 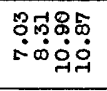 & $\underset{0}{\stackrel{0}{0}}$ & 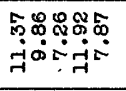 & 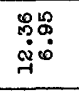 & $\%$ \\
\hline 10 & ฟีન્ & 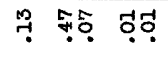 & , †ำ & 8 & 1 & 7 & & \\
\hline
\end{tabular}

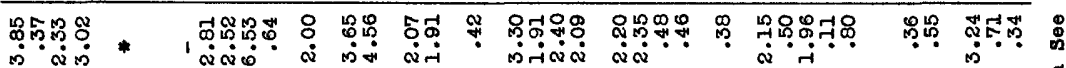

\begin{tabular}{|c|c|c|c|c|c|c|c|c|c|c|}
\hline 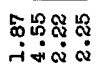 & $\stackrel{8}{\mathscr{0}}$ & 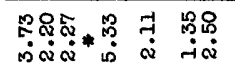 & सुन्य & $\stackrel{\mathscr{m}}{\stackrel{2}{:}}$ & 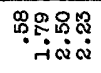 & 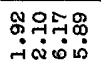 & $\stackrel{8}{\circ}$ & 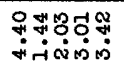 & 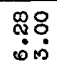 & 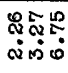 \\
\hline 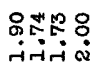 & $\stackrel{9}{9}$ & 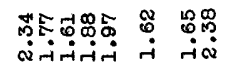 & 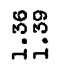 & $\underset{i}{i}$ & 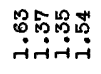 & 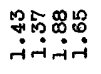 & స్ & 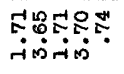 & 요 & 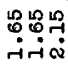 \\
\hline 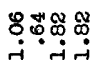 & 品 & 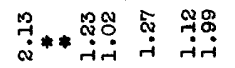 & Фุ. & ஜึ. & 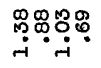 & 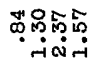 & i: & 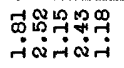 & 萬 & 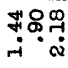 \\
\hline & & 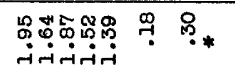 & : & $\underset{i}{\stackrel{9}{i}}$ & ชัะธำ & 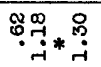 & 7 & 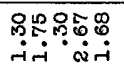 & №ㅇ & 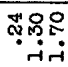 \\
\hline
\end{tabular}

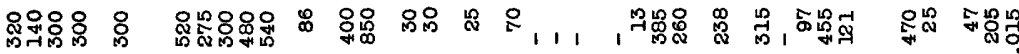

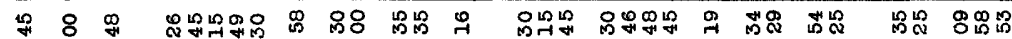

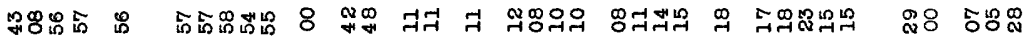

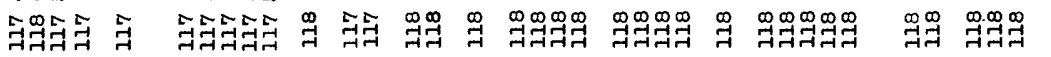

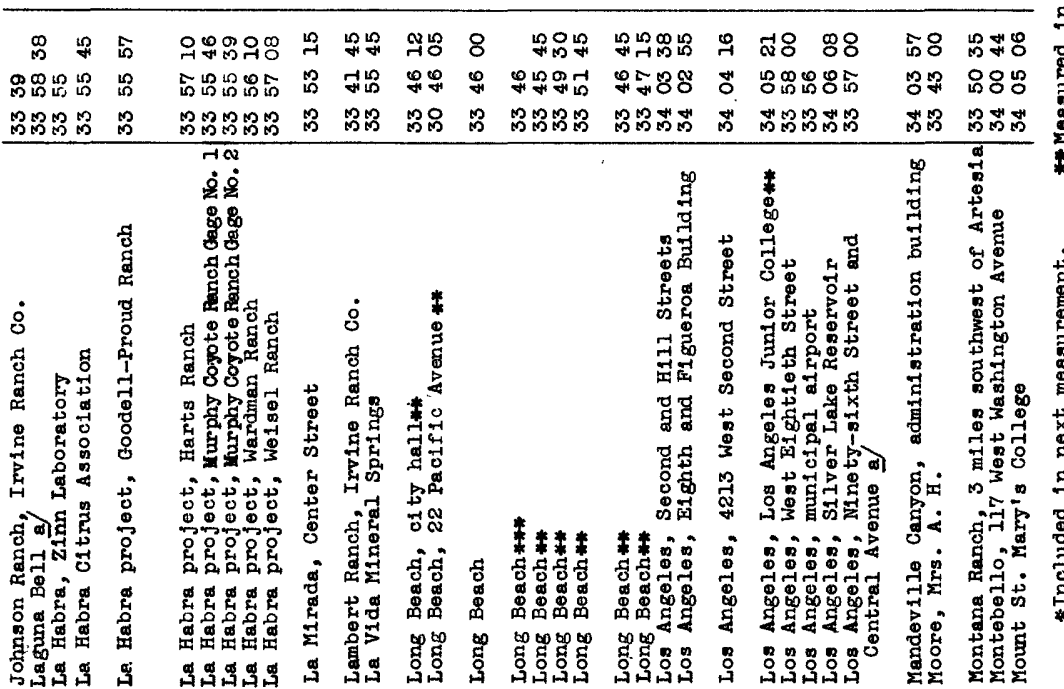




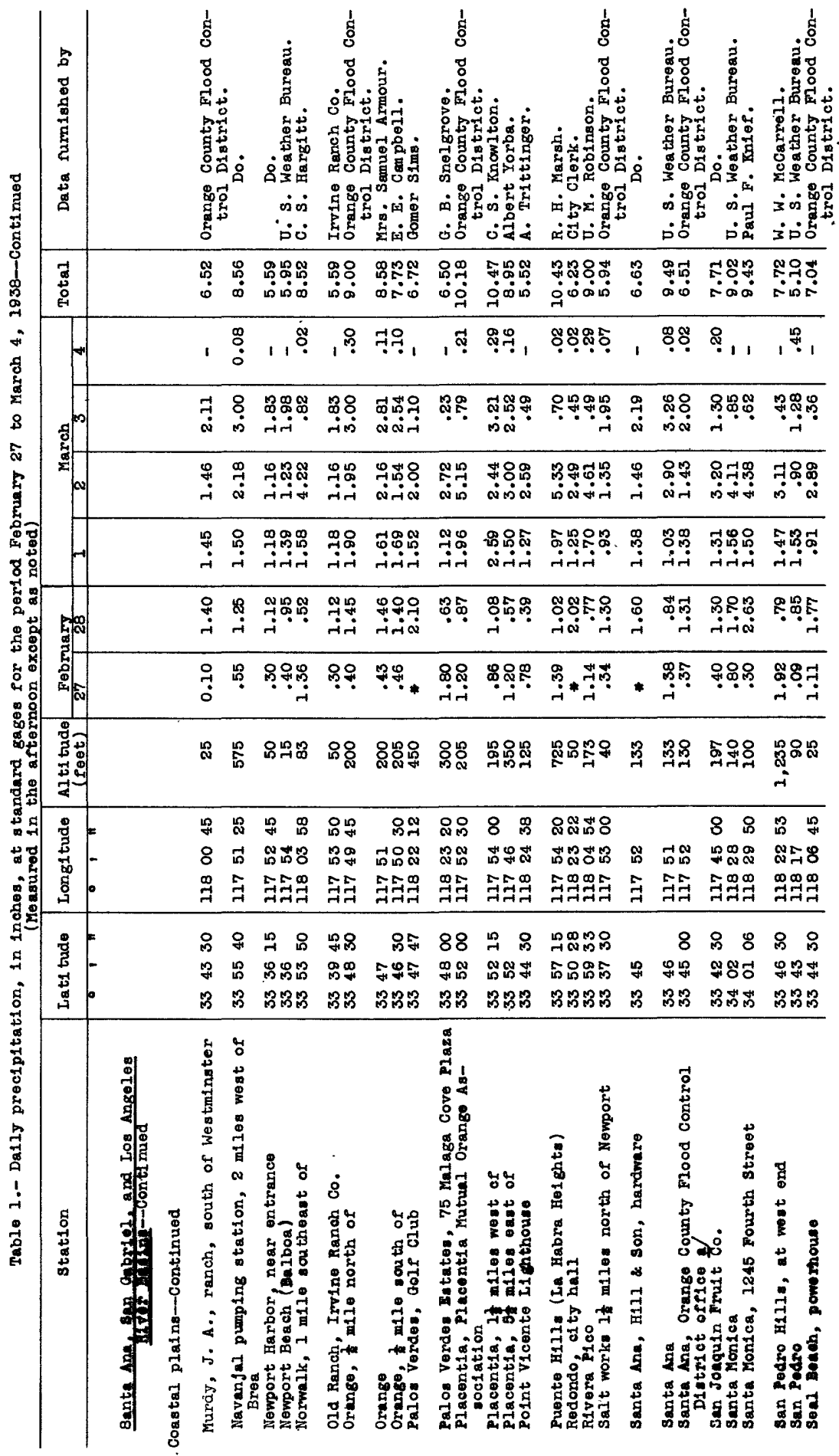




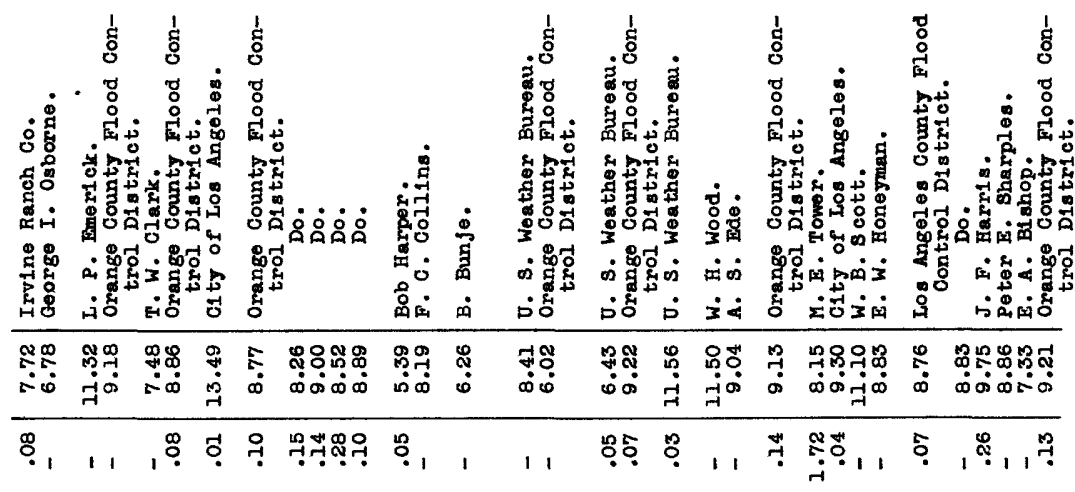

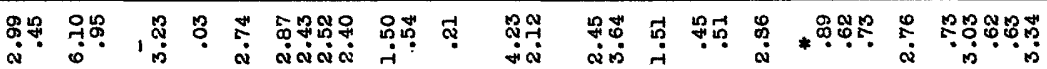

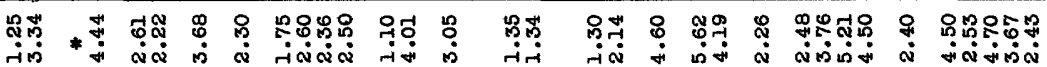

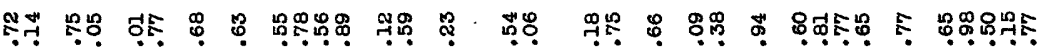

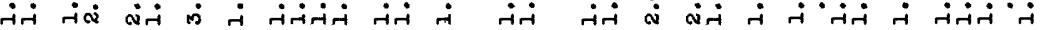

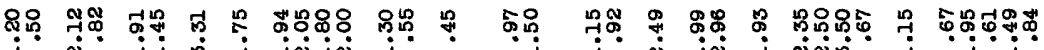

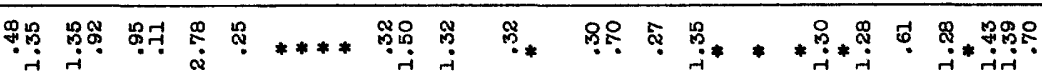

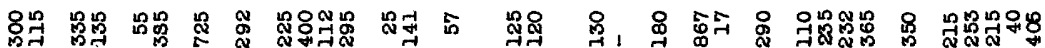

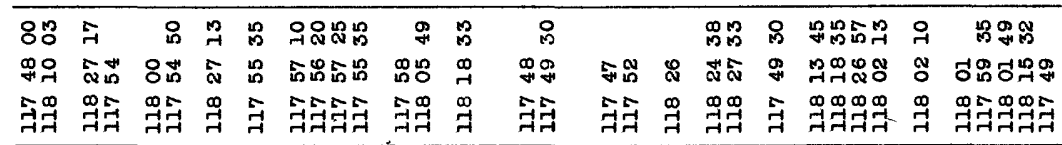

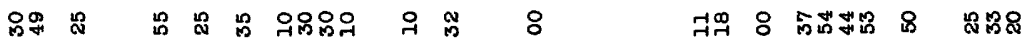

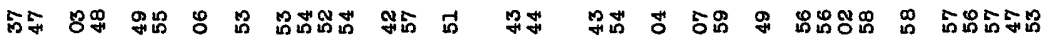

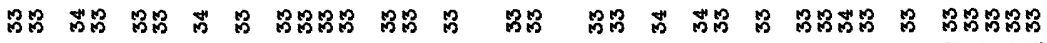

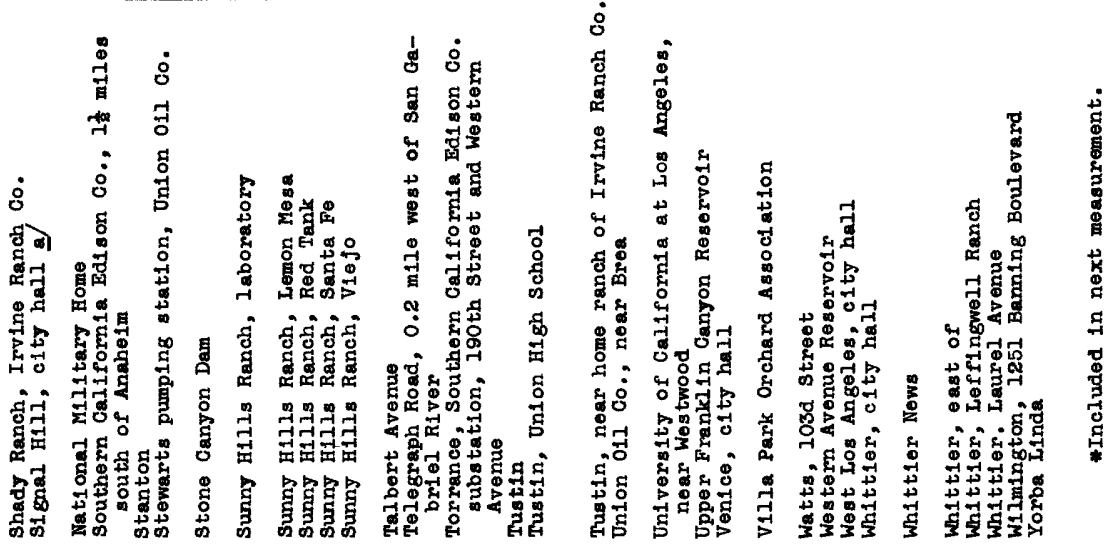




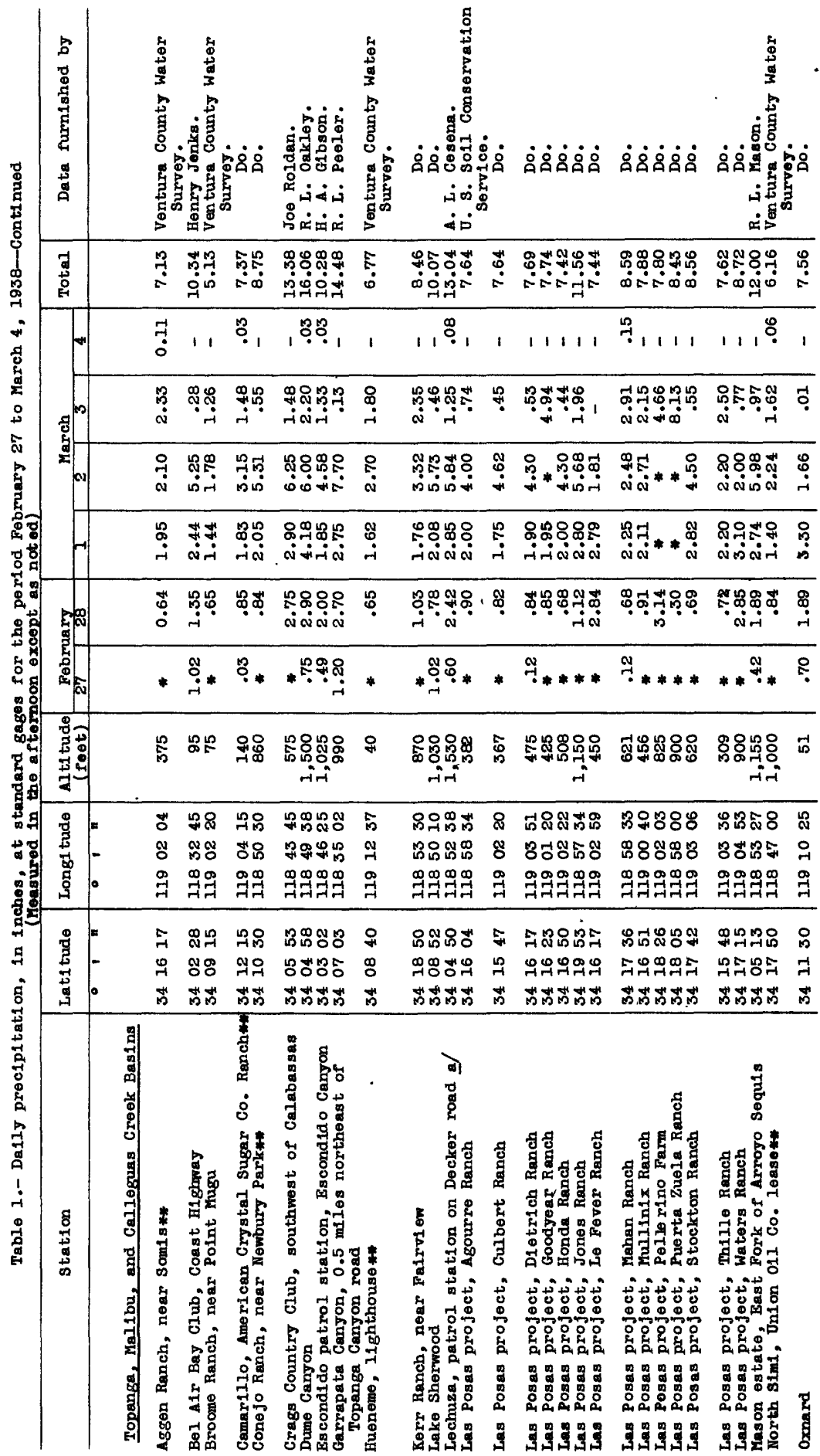




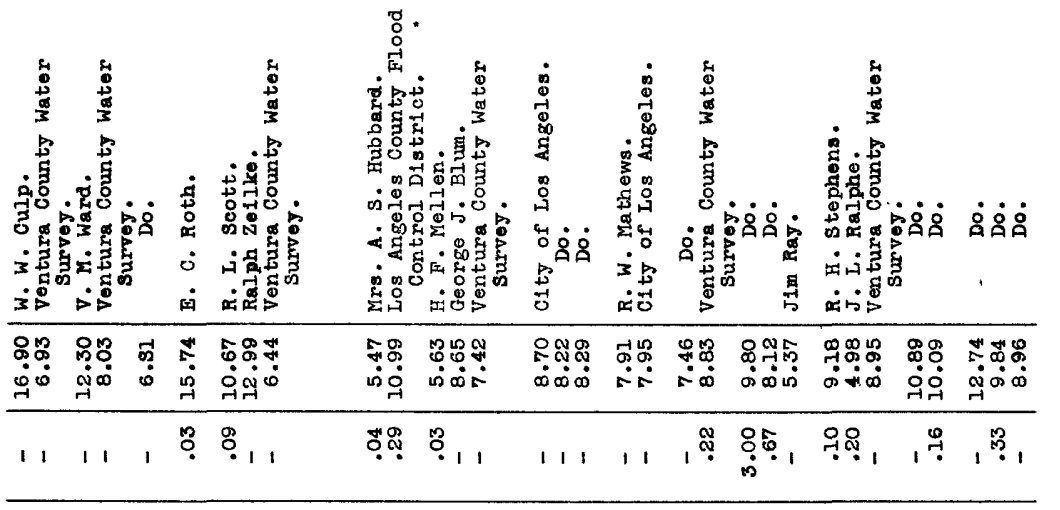

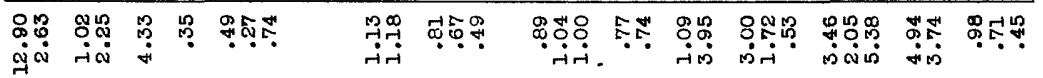

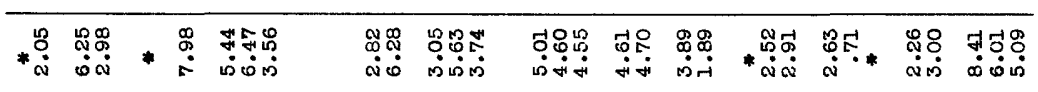

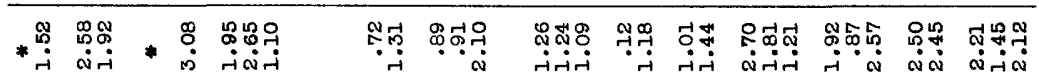

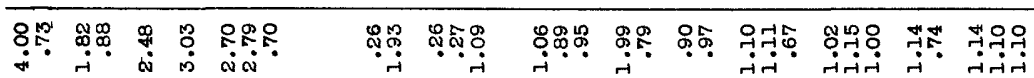

* *

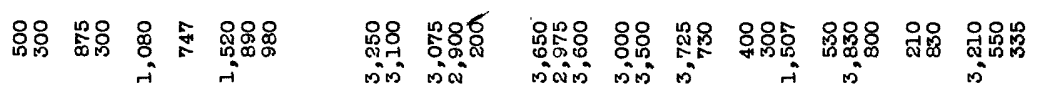

\begin{tabular}{|c|c|c|c|c|c|c|c|c|c|c|c|c|c|}
\hline ஸ゙' & 㤐品 & $\stackrel{n}{\sim}$ & $\infty$ & 8요 & 앴 & ณீณ8 & 뭉요 & q & 경ㅇ & 녹ㅇㅇ & 웅요 & 경 & 옹요 \\
\hline 电吕 & Fis & 2 & 品 & ஜ̊హి & D્A & น马잉 & నిన్స & $\pi \mathbb{N}$ & 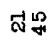 & क्नम & ถึีต & ㅋำ & 국옹 \\
\hline 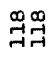 & 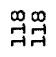 & $\underset{\sim}{\stackrel{\infty}{-1}}$ & $\underset{\sim}{\rightrightarrows}$ & 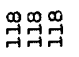 & $\begin{array}{l}\infty \\
\rightarrow \\
\rightarrow \\
\vec{H}\end{array}$ & 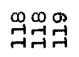 & $\begin{array}{l}\infty \\
\exists \rightarrow-\infty \\
\exists \rightarrow-7\end{array}$ & $\begin{array}{l}\infty \infty \\
\rightarrow \\
\vec{F}\end{array}$ & $\begin{array}{l}\infty \infty \\
\underset{7}{-} \rightarrow\end{array}$ & 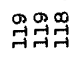 & 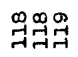 & $\begin{array}{l}\stackrel{9}{ت} \\
\overrightarrow{7}\end{array}$ & 象 \\
\hline B욱 & ฉ & $\stackrel{n}{4}$ & $\varnothing$ & $\mathfrak{\infty}$ & 녹오 & 덩연 & ஃ০ & g & 암욱 & 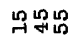 & 8 옹 & 윰용 & 뭄요 \\
\hline பี & 8 & 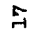 & 용 & 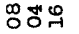 & ตెన & ฉূ心 & 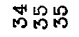 & 品品 & 周 & న్రిల్ల & సె & $8 \%$ & 吕怘 \\
\hline 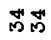 & が心 & m゙ & B̈ & 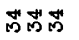 & 代 & ヴガが & 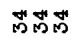 & ભే心ే & ભ゙ฒี & 芯柋心゙ & 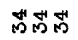 & 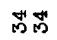 & 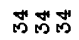 \\
\hline
\end{tabular}

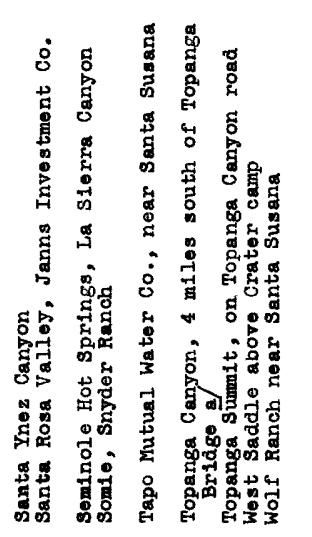




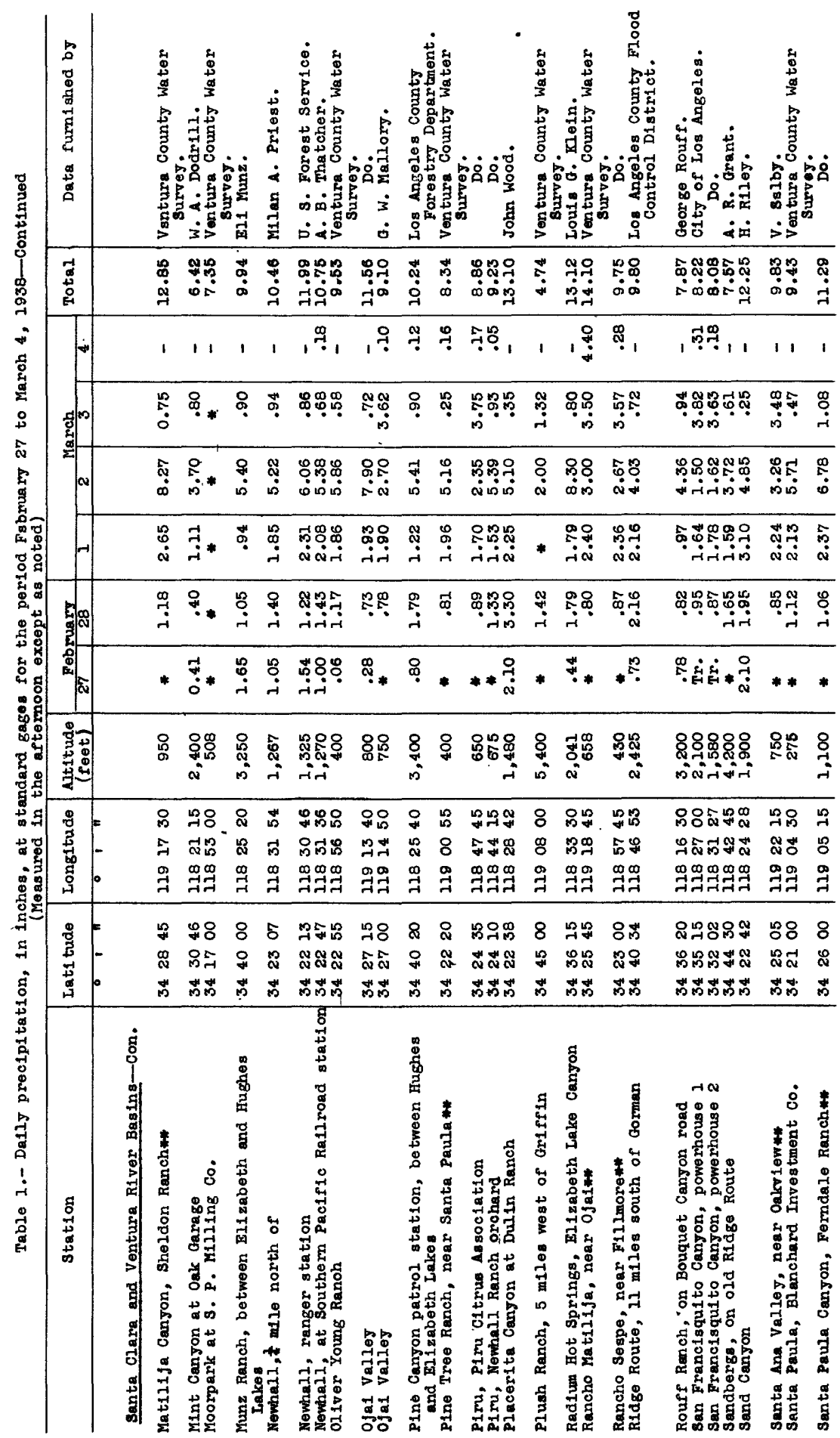




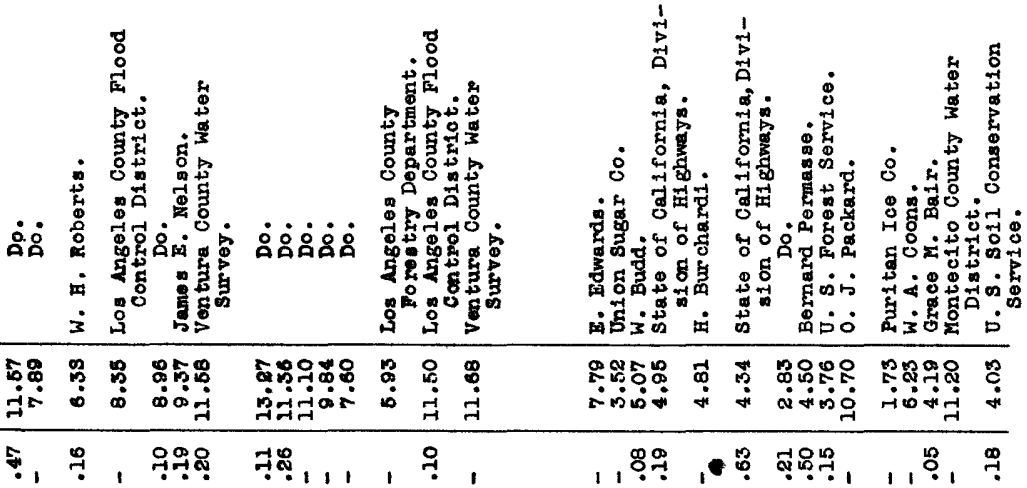

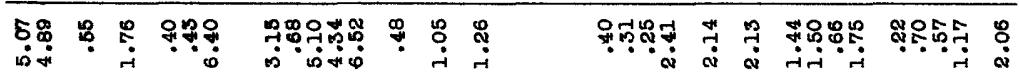

\begin{tabular}{|c|c|c|}
\hline 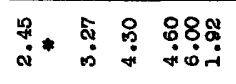 & 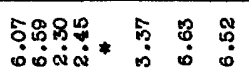 & 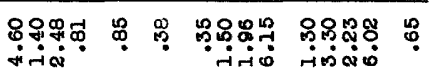 \\
\hline 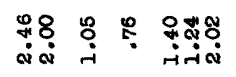 & 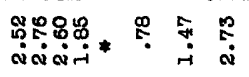 & * \\
\hline 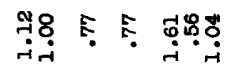 & 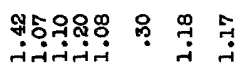 & 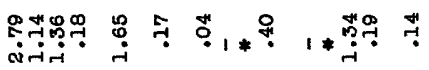 \\
\hline : & **** & 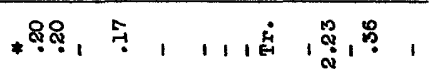 \\
\hline
\end{tabular}

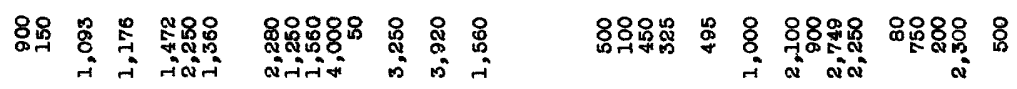

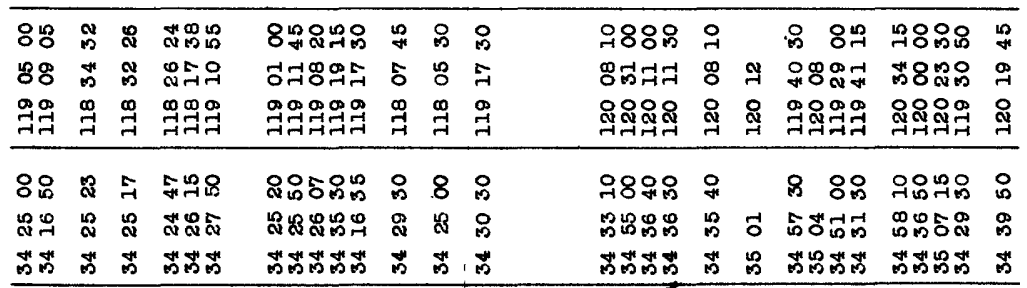

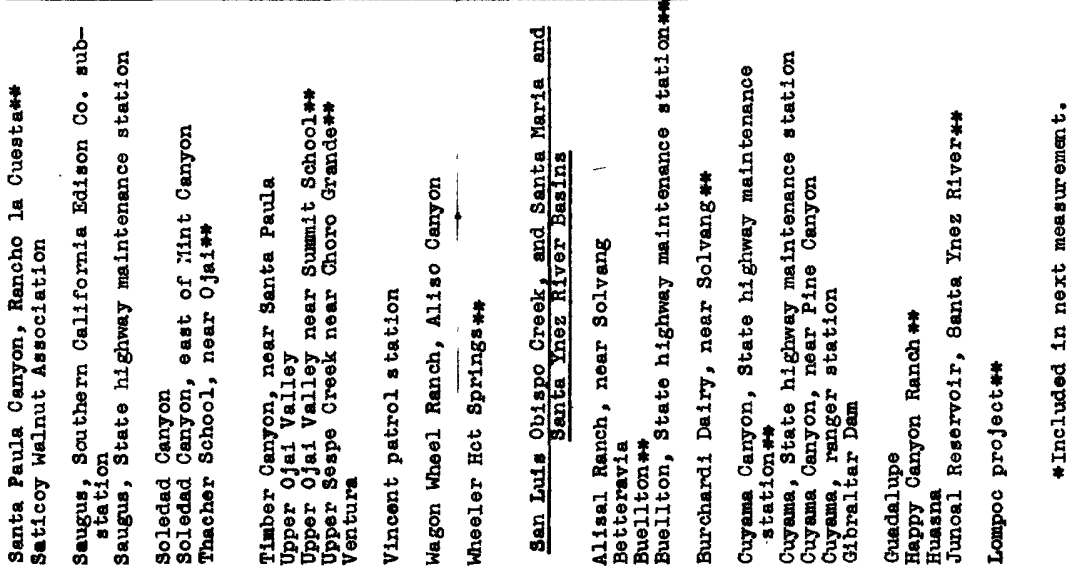




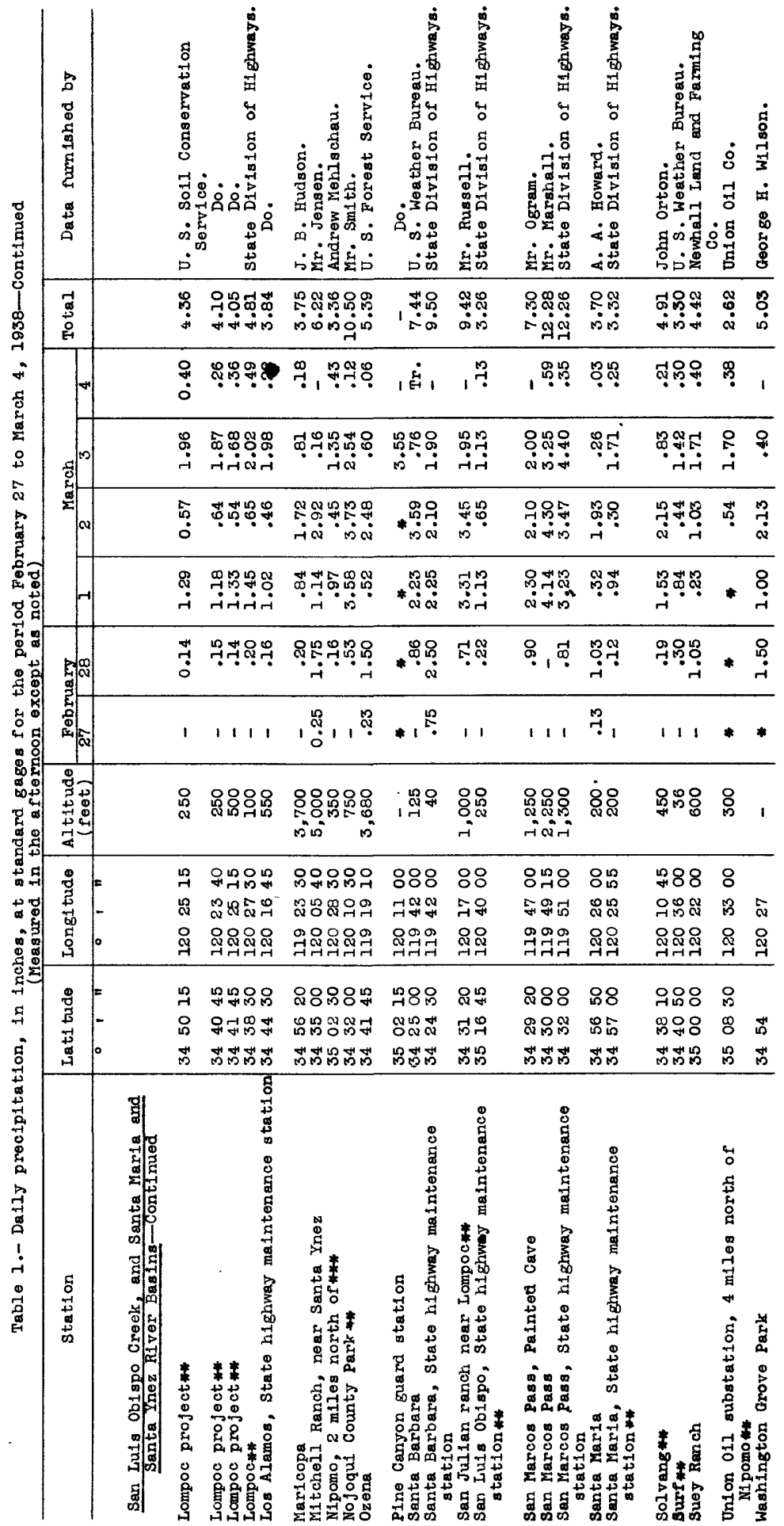




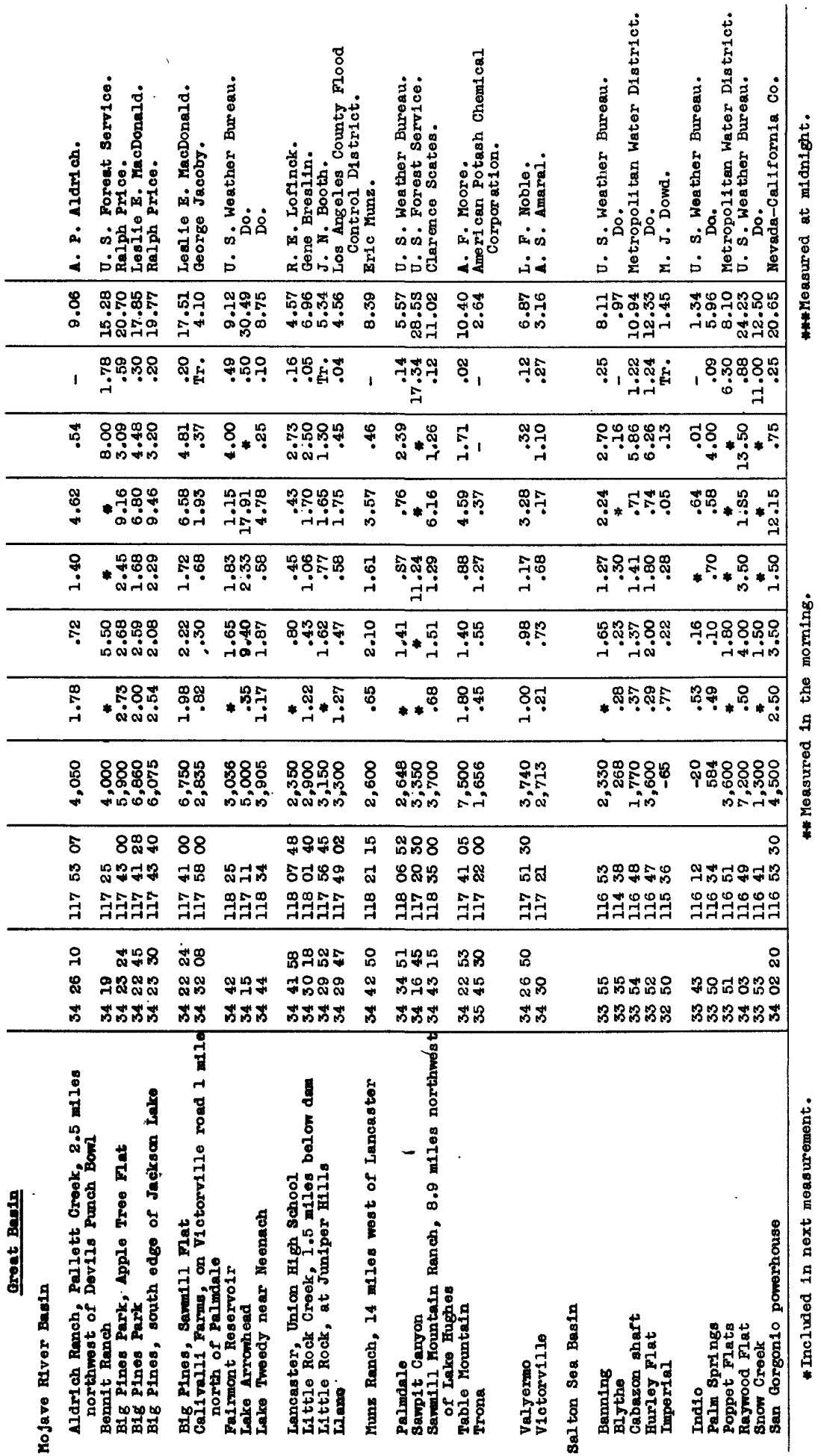




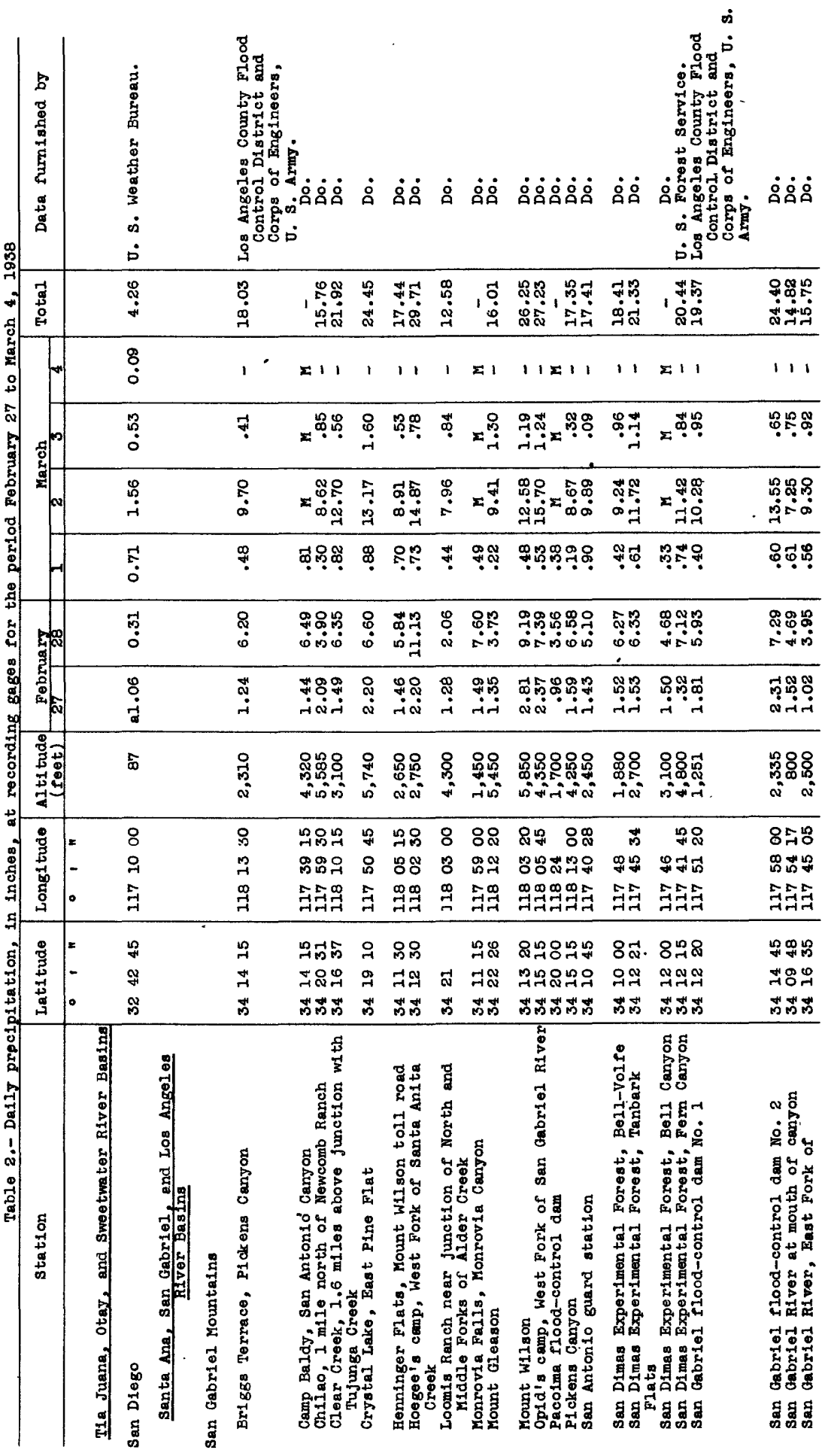




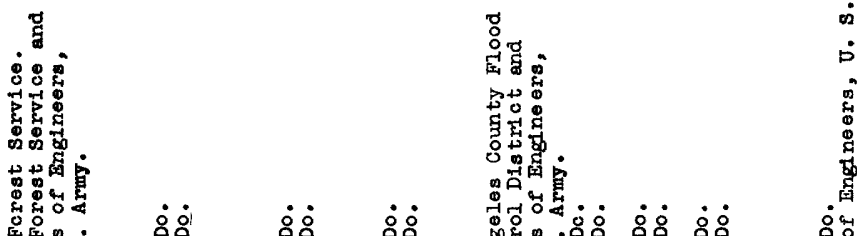

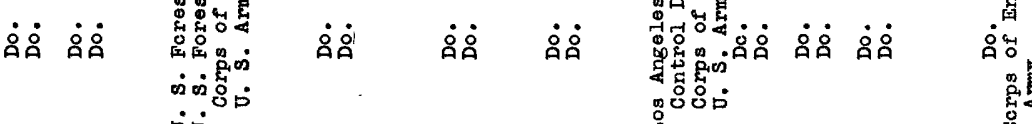
में

\begin{tabular}{|c|c|c|c|c|c|c|c|c|c|c|}
\hline 离 & 11 & 㫄 & 10 & 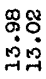 & 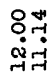 & م. & 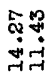 & 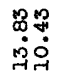 & 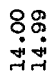 & 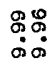 \\
\hline
\end{tabular}

$\sum 1 \quad 1 \pm 1,1$

ֻุำ

\begin{tabular}{|c|c|c|c|c|c|c|c|c|c|}
\hline 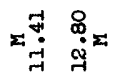 & $\stackrel{\vec{D}}{\dot{0}^{2}}$ & $=\hat{\widehat{a}}$ & क्ष: & 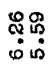 & $\begin{array}{l}\stackrel{0}{\circ} \\
\dot{\text { in }}\end{array}$ & 式: & 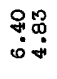 & 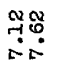 & 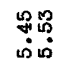 \\
\hline :̊ & 욤요 & 무류. & \& & 苫品 & 4 & ?? & ซึt & 요 & ?ִ \\
\hline
\end{tabular}

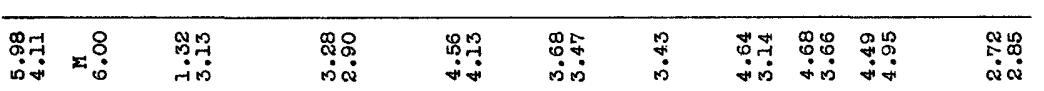

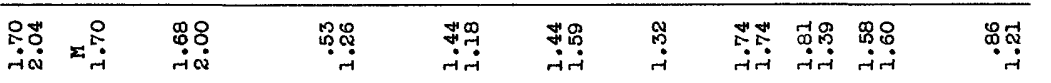

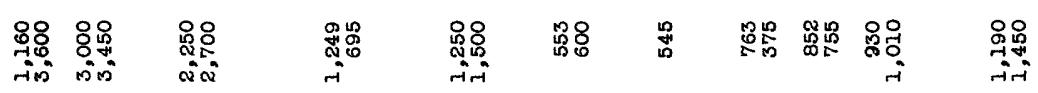

\begin{tabular}{|c|c|c|c|c|c|c|c|c|c|c|}
\hline 요 & $\stackrel{\mathscr{P}}{\rightarrow-1}$ & 악 & $\approx ్)^{\infty}$ & 떵ㅇ & 요요 & ஜึ & מ্: & કが & సૈ & カ่'8 \\
\hline 8: & ஓே円 & : & ஜัฒ & ஸ્ન & 거어 & 吕 & 55 & ஜํ & ஜே & Fin \\
\hline 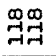 & 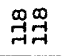 & 氠 & $\begin{array}{l}\infty \\
-\pi \\
-\pi \\
-7\end{array}$ & $\underset{\text { 番 }}{\infty}$ & 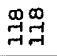 & 곡 & 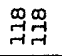 & 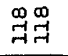 & 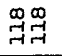 & 氙点 \\
\hline セேరా & 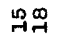 & 8 & $\stackrel{\infty}{\infty} \underset{\sim}{\infty}$ & 억요 & 교 & $\stackrel{\text { 心 }}{ }$ & 엉 & ㅇำ & พ3\% & : \\
\hline 요 & מำ & 옴 & 욱요 & Fi유 & రુઠீٌ & $\because$ & ๑๐ั & จิธิ์ & 옹어 & ํํㅇ \\
\hline मేळే & मี & लేळ̈ & जేలే & ガં & लै लै & $\vec{m}$ & लिख & ヴガ & 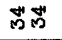 & ஸ゙'ガ \\
\hline
\end{tabular}
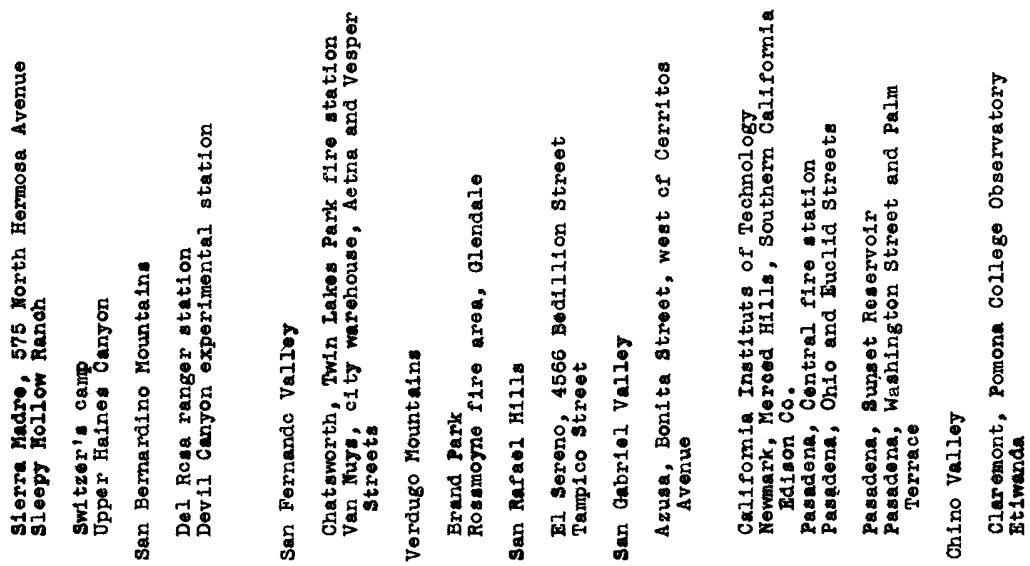


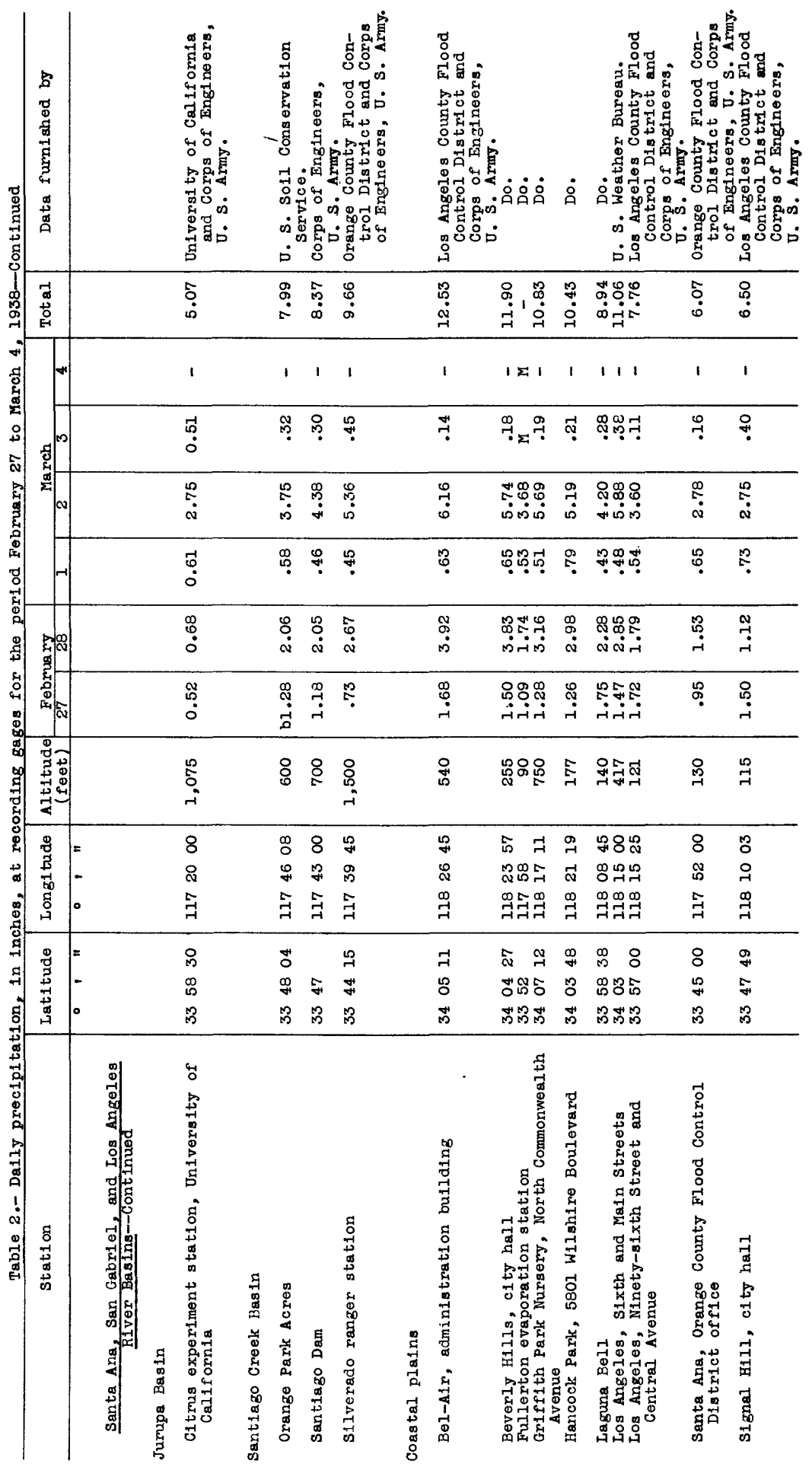




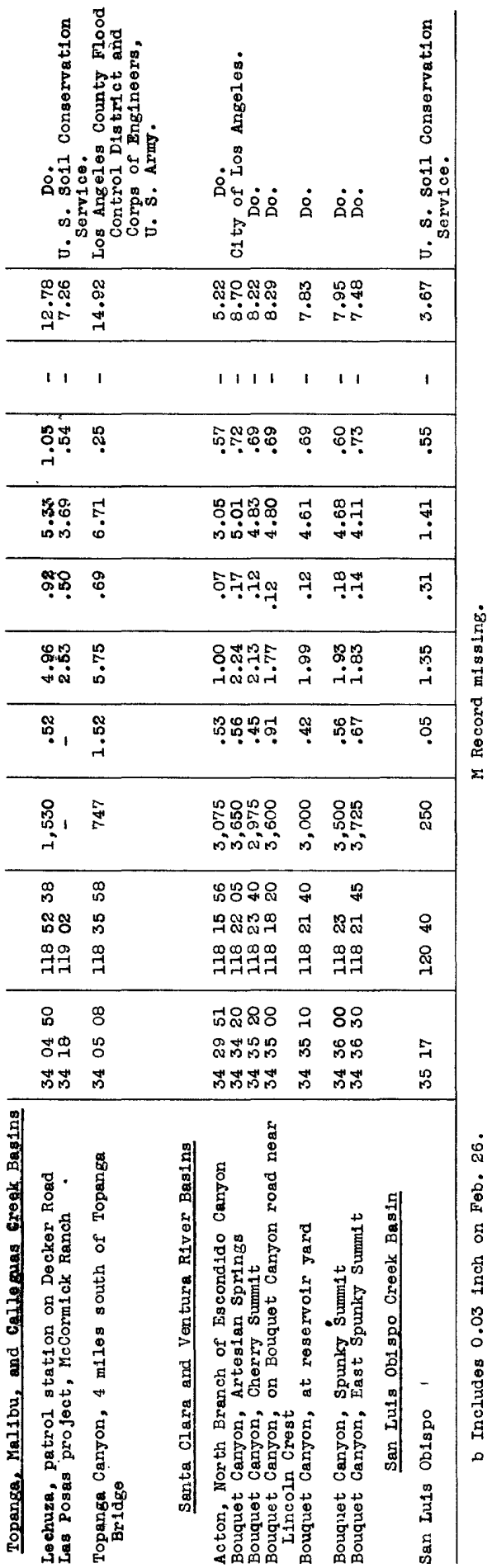


The amounts of precipitation as shown for the individual days are not strictly comparable, as the observations at the various stations were not always made simultaneously. Host of the amounts given in table 1 represent the rainfall during the 24-hour period preceding the time of the daily observation. Rainfall occurring during the dajlight hours, however, may be recorded under the date of occurrence if observations were made in the late aftermoon, or under the date of the following day if readings were made in the early morning.

Recording rain gages are maintained near many of the standard rain gages for which data are given in table 1. The two gages are usually operated for the purpose of comparison, and frequently there is considerable difference between the catch in the two. It should be emphasized that only the records for the standard-can rain gages are presented in table 1. All recording rain-gage records are presented in table 2 in a form similar to that used in table 1. The stations in table 1 for which recording-gage data are given in table 2 are indicated by a footnote reference. As the amounts given in table 2 represent rainfall occurring from midnight to midnight, the arrangement of the records is capable of presenting several features of the storm not discernible from readings made at different times during the day at the standard gages. By reference to several precipitation stations having gages of both types (see tables 1 and 2), the difference resulting from elther unequal catch or different times of reading will be readily apparent.

\section{Preparation of isohyetal maps}

Although most of the precipitation fell in two or more rather distinct storms, both storms occurred within the period February 27 to March 4, which has been treated as one storm period in the preparation of the isohjetal map. The isohjetal map for the entire storm period was developed from the records of the standard rain gages as given in table 1. The location of each rain gage and the total storm rainfall were plotted on topographic sheets. For areas where there were a large number of station records and changes in topographic aspect were pronounced, maps on a scale of 1 mile to the inch were used; such areas included the coastal slopes of the San Gabriel, San Bernardino, Santa Ana, Verdugo, and Santa Monica Mountains, and the San Rafael Hills. For the remaining areas, where there were either fewer precipitation records or greater uniformity in topographic aspect, as in the valley areas, the information was plotted on topographic maps having a scale of 4 miles to the inch. The final 
1sohyetal map, on a scale of 4 miles to the Inch, 1 seproduced as plates 11-13 in this report. In locating the position of the isohyetals, full use has been made of such relations as could be determined between altitude, topographic aspect, and storm precipitation. In his recent account of the storm, Daingerfield 10 states:

The San Gabriel and San Bernardino mountains, with their east-west trend, lying directly across the pass of the molsture-laden winds, performed the functions of fixed and permenent "fronts", mechanically accomplishing the chilling of tropical maritime air and precipitating its mois ture.

Under the conditions described, fairly definite relations between altitude and storm rainfall can reasonably be expected. Figure 5 presents an example of these relations. On the upper half of the figure, the storm rainfall for both the coastal and desert slopes of Mount Islip and vicinity was plotted. against altitude, and graphs were drawn to show the relation between altitude and rainfall. The relative position of the rain gages with respect to topography is indicated on the lower half of this figure. Also included on the graphs, for the purpose of comparison, are records of a few gages located in the Santa Ana and San Bernardino Mountains. These grapha indicate that the storm rainfall on the coastal side of Hount Islip varied at a rate of about 3 inches per 1,000 feet of change in altitude and on the desert side at a rate of about 6 inches per 1,000 feet, although as discussed in the following section, "Local storm characteristics", the altitude-rainfall relations were not so well defined in all perts of the area as in the part represented by figure 5 .

In arawing the isohjetals, an effort was made to obtain the altituderainfall relations throughout the storm area, but there are many purely local conditions that seem to obscure any such relation. This is especially true in the mountains, where for nearly every storm many small areas show consistently higher or lower rainfall than the surrounding areas. The Los Angeles County Flood Control District has observed many such areas within the county and has mainteined standard or recording rain gages in them for a number of years. In some parts of the mountains, therefore, there is perhaps a tendency to have a disproportionate number of gages indicating special conditions as compared with the number indicating normal conditions. As rain gages in the mountains must generally be located at or near places of human habitation, it is often difficult to arrange for the collection of records in desirable but remote places. 

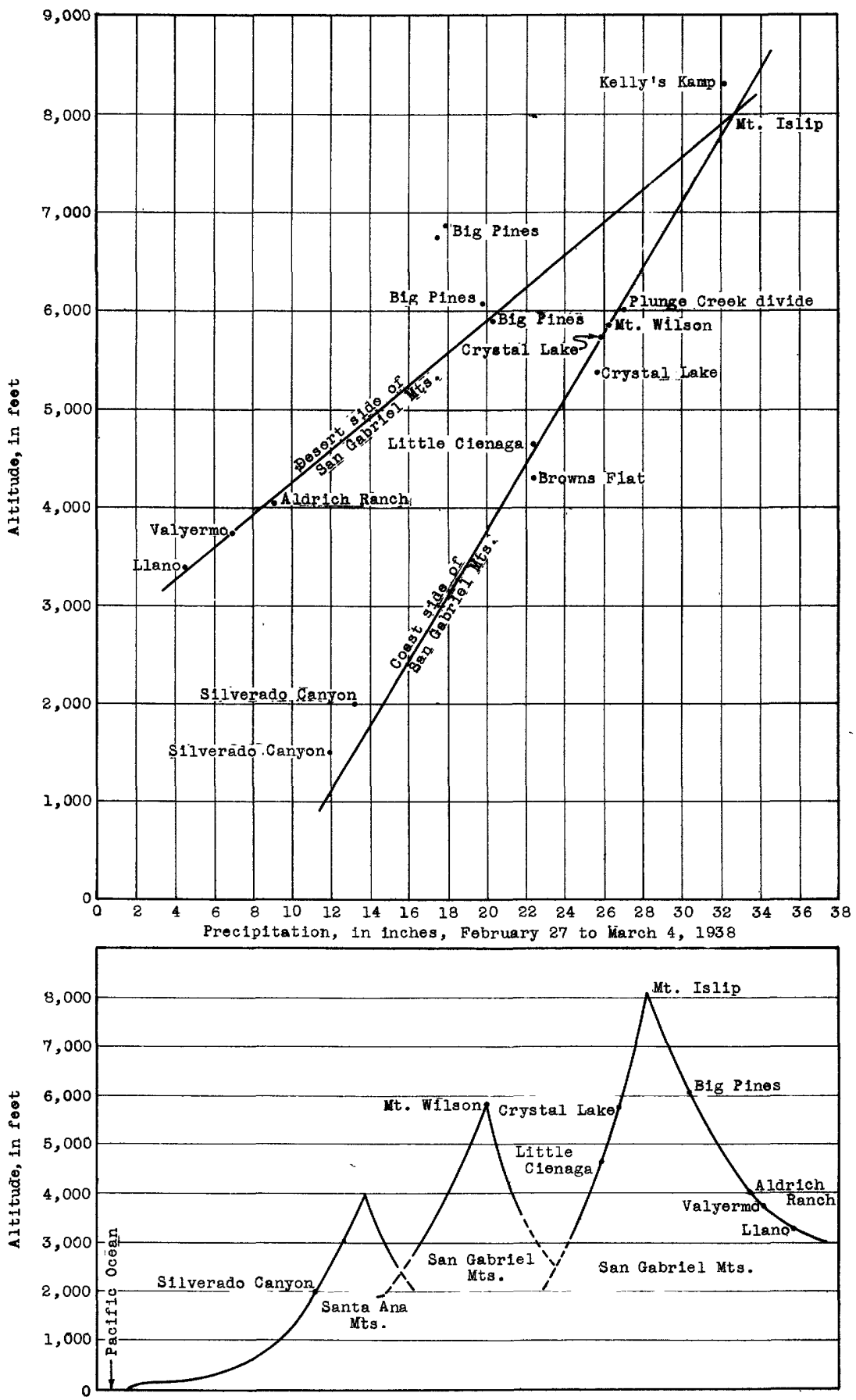

Figure 5.- Relation between altitude and precipitation in the vicinity of Mount Islip and schematic cross sections showing relative position of rain gages. 
For most drainage areas in southern California, each rainfall observation for the storm period was plotted against altitude, and the relations were developed in a similar manner to those indicated on figure 5 . In areas that falled to show definite relations, a series of parallel curves indicating a change in rainfall of 3 inches per 1,000 feet of difference in altitude were passed through each observation. With such an altitude-rainfali relation established, the construction of the isohyetal map was somewhat simplifled. Starting with the recorded precipitation at the various stations and using the indicated altitude-rainfall relation, points for flxing the position of each isohyetal were located at right angles to the land contours above and below each rain gage. The 1sohyetals were, in the main, drawn parallel to ground-surface contours, with gradual or abrupt transitions according to the influence of topographic features.

In those basins where there were few precipitation stations, especlally in the headwaters of the Ventura, Santa Clara, and Cuyama Rivers, the location of the 1sohyetals may be subject to considerable error. Ilkewise, the 1sohyetal map for much of the desert region, such as the Deep Oreek and West Fork of Mojave River Basins, may not represent actual conditions. In parts of the area, the isohyetals have been shown as dashed lines to indicate that their position is uncertain.

Table 3 shows for each of 22 drainage areas listed in table 1 the number of rain gages; the approximate area in square miles; the range in altitude of the rain gages and of the drainage area; the average precipitation over the area during the storm period February 27 to March 4 obtained by planimetering the area between each 1sohyetal; and a statement of opinion as to the probable accuracy of the isohyetals according to the classifications well defined, fairly well defined, and poorly defined.

\section{Local rainfall characteristics}

Analysis of the precipltation records used in the preparation of the 1sohyetal map shows that certain areas have rainfall characteristics that appear to be related to local altitude and topography. Some of these areas and their individual characteristics are described as follows:

Some of the heaviest rainfall occurred just over the 5,000- to 6,000foot divide of the south frontel ridge that parallels the main range of the San Gabriel Mountains. The upward movement of the molst tropical air mass, caused by the blocking of 1ts passage eastward by these steep costal slopes, apparently continued after the mass passed the divide and 


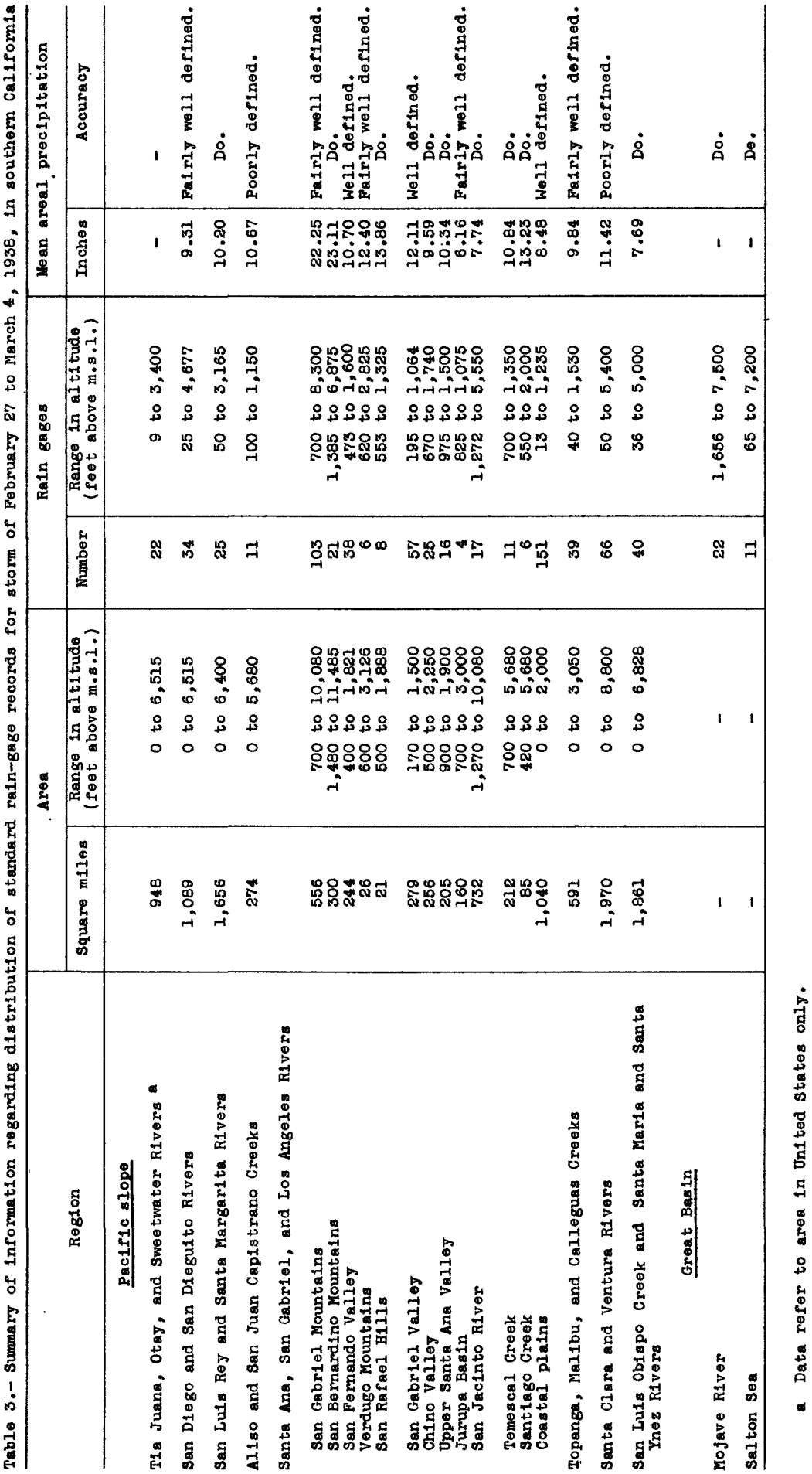


resulted in the condensation and excessive precipitation of its moisture on the northern slopes of these ridges. The very heavy precipitation at such stations as Opid's camp, Arrowhead Lake, and Hoegee's camp may be explained in part by such topographic influences.

The effect of local conditions and of differences in exposure of the gages is 1llustrated by rainfall records in the vicinity of Julian, where three gages within a radus of 3 miles and with a difference in altitude of less than 500 feet showed a variation in rainfall of 5.8 inches. So wide a divergence suggests that the isohyetals may only roughly represent actual conditions.

An example of the effect of mountain ranges on rainfall is indicated by the records from the rain gages in the San Luis Rey River Basin. Two gages at Mesa Grande, at an altitude of more than 3,000 feet and on the coastal slope of the range, indicated a rainfall in excess of 16 inches. The gage at Henshaw Dam, in the San Luls Rey River gap between the ranges, showed similar precipitation. At Warner springs (altitude, 3,165 feet), less then 10 miles east of the coastal range, there was only 8.73 inches of rainfall.

The low precipitation in the area around Riverside and in parts of the San Jacinto River Basin may be ascribed to the influence of the Santa Ana Mountains on the incoming maritime air mass, an influence similar to that indicated for the mountains near Warner Springs.

The abrupt changes in topography and altitude in the San Gabriel Mountains, whose runoff is tributary to the Santa Ang, San Gabriel, and Los Angeles Rivers, introduce great difficulty in the development of isohyetals. Rainfall data for this area, however, are more complete than for any other mountainous section of southern California.

The altitude-rainfall relations for the vicinity of Mount Islip are show on figure 5. Simflar conditions were assumed to apply in all places where the individual observations checked this curve reasonably

- we1l. For example, the total rainfall on the divide at the head of Pickens Canyon, at an altitude of 4,250 feet, was 17.36 inches, but $2 \frac{1}{2}$ miles north, at Tujunga flood-control dam, at an altitude of 2,050 feet - or 2,200 feet lower - the total was 20.36 inches. The rainfall recorded at the Tujunga flood-control dam is considerably greater than the amount normally to be expected for this altitude. (see fig. 5.) This unusually heary rainfall way result from the effect of the steep mountain canyon on the upward movement of the warm, moisture-laden maritime air masses, which apparently continue their upward course for some distance over the 
ridge. The accompanying chilling process would thus produce excessive precipitation for a short distance beyond. Likewise, at Opld's camp, (altitude, 4,350 feet), 3 miles northwest of Mount wilson, the rainfall was 27.27 inches, as compared with 26.23 inches at Mount Wilson (altitude, 5,850 feet). Actually there was a zone of heavy rainfall north of the frontal ridge and paralleling the mountain range that extends for a distance of about 20 miles from Tujunga flood-control dam to the junction of the East and West Forks of the San Gabriel River.

With the exception of the rainfall of 32.20 inches at Kelly's Kamp (altitude, 8,300 feet), the heaviest precipitation in the southerm Cal1formia area was 30.08 inches at Hoegee's camp (altitude, 2,750 feet), which is located on the east side of a north-south mountain spur formed by Mount Wilson and Mount Harvard in the West Fork of Santa Anita Creek. Inasmuch as the tropical air mass seemed to move eastward, this short spur extending across the path of the storm may have produced a condition similar to that already described.

Similar characteristics were observed for this eame group of stations during the 2-day storm period of December 31, 1933, to January 1 , 1934, known locally as the "New Year's storm of 1934", when 19.20 inches of rain fell at Hoegee's camp. This exceeds by 1.10 inches the rainfall for the maximum 2-day period in the storm of February 27 to March 4, 1938. At opid's camp, the rainfall of 17.21 inches during the "New Year's storm of $1934^{\prime \prime}$ was 0.12 inch less than that during the maximum 2-day period for the 1938 storm. A similar comparison of these two storms at the Clear Creek station and at Tujunga flood-control dam shows an excess of only about 3 inches for the 1938 storm. At Mount wilson, the rainfall of 14.69 inches for the $1934 \mathrm{storm}$ was but 1.13 inches 1 ess than that for the maximum 2-day period during the 1938 storm.

A group of rain gages, including those at Mount Glesson (altitude, 5,450 feet), Chilao (altitude, 5,585 feet), and Loomis Ranch (altitude, 4,300 feet), are located about 10 miles directly north of this area of extremely heavy rainfall, to the west of Mount Islip and somewhat nearer the ocean. They indicate that considerably less rain fell in this area than in areas of comparable altitude on the frontal range. Mount aleason had a rainfall of 17.54 inches at an altitude of 5,450 feet, as compared with 25.0 Inches for that altitude as indicated by the Mount Islip altitude-rainfall relations. The greater rainfall at Mount Islip may be explained, in part, by its greater height and by the fact that it is approached on the coastal side by two straight north-south canyons, the 
canjons of Bear Creek and North Fork of San Gabriel River, which are more than 7 miles long. The prevalling winds are mainly from the south and are belleved to have caused free movement of the molsture-laden air mass up these canyons, with heaviest rainfall at the head of the canyons as a result of the more rapid upward movement of air currents there. Loomis Ranch showed the least rainfall of the stations in this group, a fact which may be attributed to its lower altitude. During the 1934 storm, also, Loomis Ranch had a lower rainfall than the nearby stations.

Each station in these several groups showed characteristics during the 1938 storm similar to those it showed in the 1934 storm, indicating that as a result of local topographic conditions certain localities have a tendency toward predominantly lighter or heavier rainfall as considered in relation to each other. There are undoubtediy other localities throughout the southern Califormia mountains that possess similar characteristics but because of insufficient rainfall records have not as yet been identified.

In the San Bermardino Mountains, also, are found local conditions that are likely to affect the rainfall of surrounding areas. The rainfall shown by the Plunge Creek gage (altitude, 6,000 feet) is almost identical in amount with that to be expected from the normal altituderainfall relation indicated on flgure 5. Many of the San Bernardino Mountain stations, however, show considerably less rainfall than that indicated on figure 5 for their respective altitudes.

As Indicated in the analysis of the observations from stations similarly situated in the San Gabriel Mountains, records from stations affected by local conditions are representative of relatively small areas that may recelve comparatively light or heavy rainfall with a considerable degree of regularity. Because of the scarcity of gages elsewhere, such observations are of necessity assumed to be representative of many more areas than those to which they actually apply.

Isohyetals in Bear Valley and parts of Cajon Pass are not so rellable as In the remainder of the San Bermardino area, mainly becauee of lack of precipitation data.

Rainfall at the base of the Verdugo Mountains, between San Fernando and La Cañada Valleys, also showed considerable varlation, caused probably by the local topography. A poorly defined altitude-rainfall relation indicated that the rainfall varied from 8.5 inches for the $1,000-$ foot altitude, in the western part, to 13 inches for the same altitude in the eastern part. An isohyetal map of the New Year's storm of 1934, 
prepared by the Los Angeles County Flood Control District, shows characteristics of distribution very similar to those of the 1938 storm.

The effect of local topography is strikingly lllustrated in figures 6 and 7 which show four topographic cross sections of the southern Cal1fornia coastal plain, with the recorded rainfall for the 1938 storm plotted directly above them. One cross section is of an area in Ventura County, and the others of areas that cross the basins of Los Angeles, San Gabriel, Santa Ana, San Luis Rey Rivers and Temecula Creek. In none of the areas represented by the sections shown does the highest rainfall appear to have occurred at the point of highest altitude.

\section{San Dimas Experimental Forest}

The San Dimas Experimental Forest, located in the Sierra Madre Mountains northeast of Glendora, covers 17,000 acres and includes the entire drainage basins of San Dimas and Dalton Canyons above the flood-control dams maintained by the Los Angeles County Flood Control District. The investigation being conducted by the United States Forest Service in this area involves a determination of the relation of the chaparral vegetation to the yield of usable water from mountain drainage basins and of the $\theta x-$ tent to which this vegetation reduces flood runoff and erosion. Exact measurements of precipitation and runoff are necessary for the solution of these important problems. The entire area has been divided into two major basins, which, in turn, are subdivided into 10 component areas of 1 to 14 square miles each.

Rainfall is measured at the ond of each storm at about 300 gages spaced half a mile apart along a system of trails built on the contours at altitudes of $2,100,3,100,4,100$, and 5,100 feet. In addition, records of rainfall intensity are obtained from 15 recording rain gages distributed throughout the area in situations as nearly equal as possible both as to altitude and area.

Through the apoperation of Dr. E. I. Kotok, Director of the California Forest and Range Experimental Station, J. D. Sinclair, silviculturist in charge of the San Dimas Experimental Forest, and members of their staffs, an isohyetal map for the storm perlod February 27 to March 4 in the San Dimas Experimental Forest was drawn. (See fig. 8.) On this map is a random cross-section line through the area, showing rainfall and altitude. The extent to which local influences may obscure the relation betweon altitude and rainfall is clearly ovident from this map. An altitude-rainfall relation developed under these conditions is limited in 


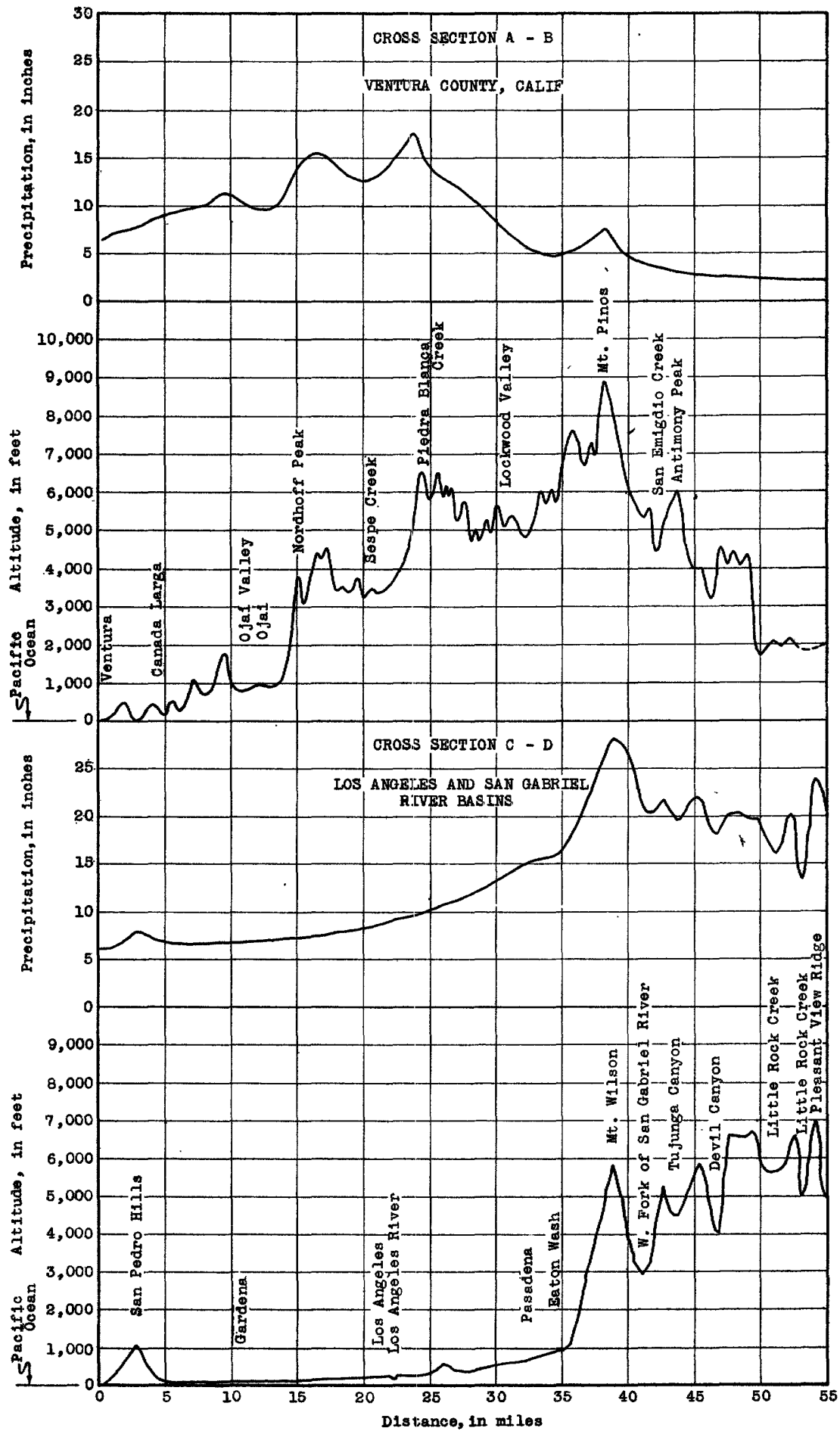

Figure 6.- Cross sections showing altitude and preclpitatiox in Ventura County and in Los Angeles and San Gabriel River Basins. 

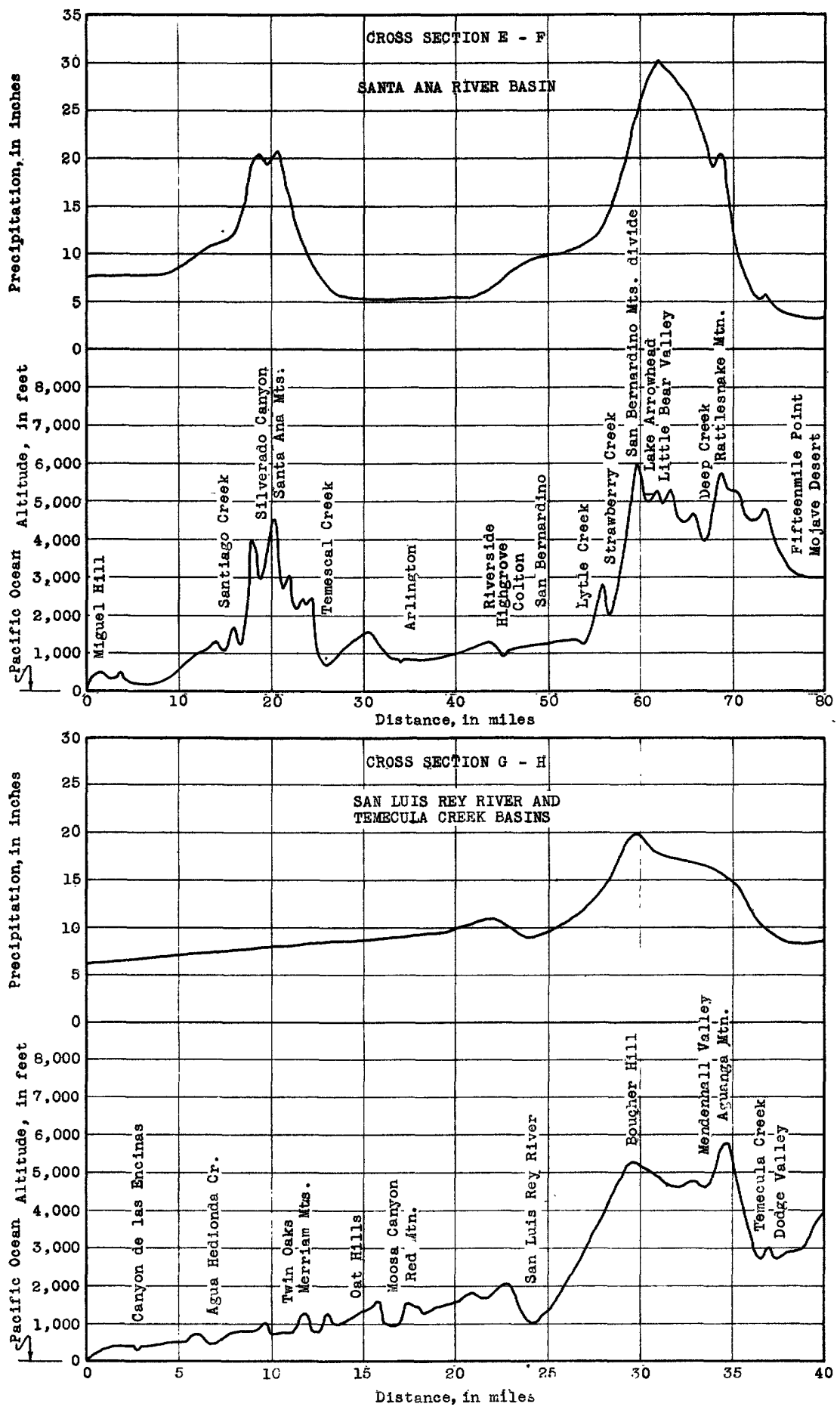

Figure 7.- Cross sections showing altitude and precipitation in basins of Santa Ana and San Luls Rey Ripers and Temecula Creek. 

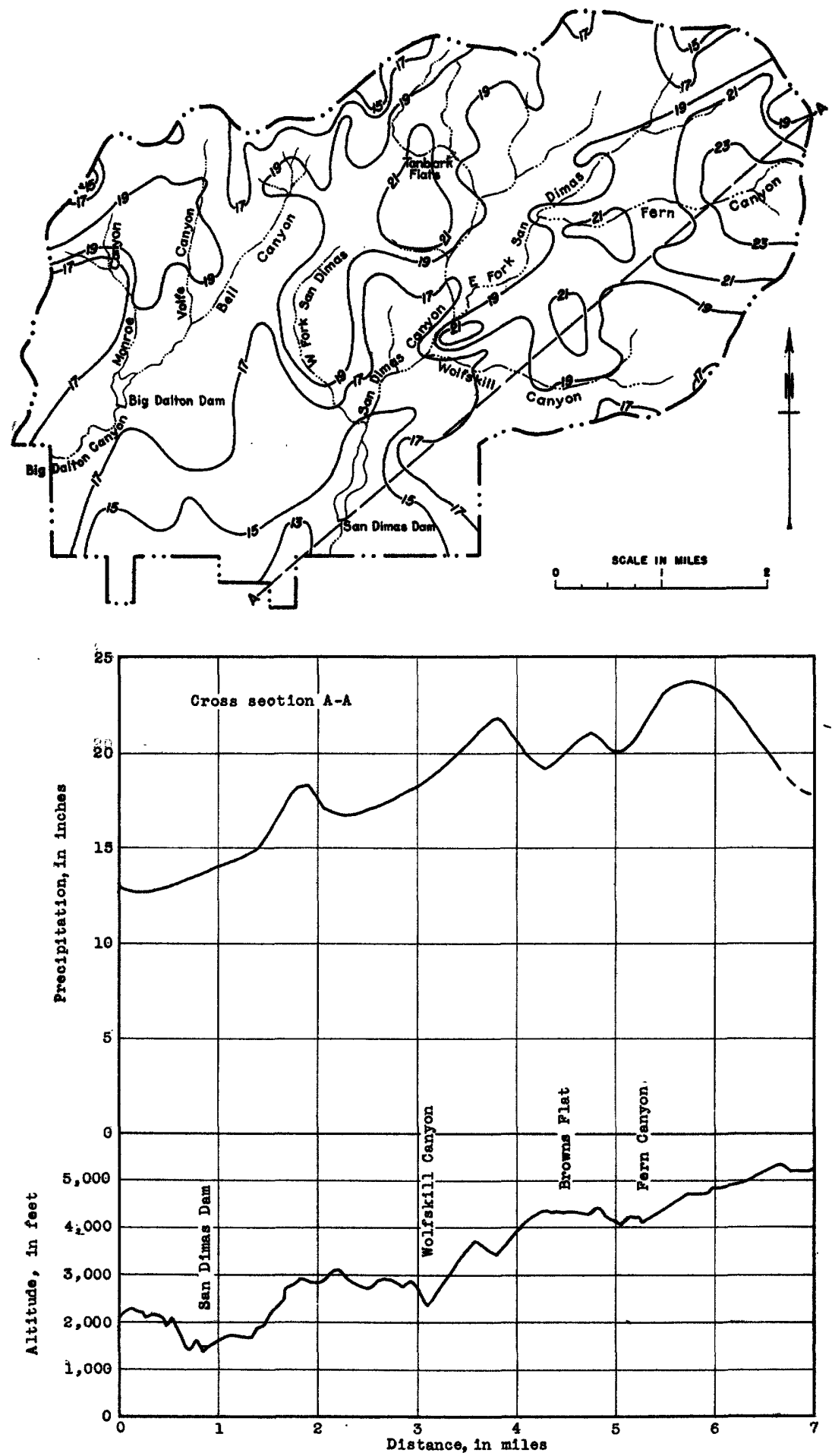

Figure 8.- Isohyetel map and cross sections showing altitude and precipitation in San Dimas Experimental Forest. 
Its application, and isohyetals based strictly on such a relationship, especially in areas where the data are meager, should be used with caution.

\section{Chronologic distribution}

The precipitation of the storm of February 27 to March 4, 1938, was marked by four phases. The flrst phase occurred on February 27 and 28. From the morning of February 27 to the early hours of the morning of February 28 the rain was light and intermittent. During the remainder of February 28 the precipitation was generally continuous and intense. Many of the precipitation stations, especially those near the coast, reported meximum intensities of short duration during this phase. In the second phase, on March 1, no appreciable precipitation occurred from 1 a.m. to 10 or 11 p.m. This lull was followed by the third phase, on March 2 , when the rainfall was extremely heavy. At stations in the headwater areas of many streams, especially the Los Angeles, San Gabriel, and Santa Ana Rivers, the maximum 24-hour rainfall was the greatest on record. Some stations recorded maximum rates during the morning of March 2 and others recorded them during the afternoon. The data obtained during the storm were not so complete for areas north and south of these basins, but available observations indicate that the storm there was less severe than in the intermediate areas. Following the intense rain of March 2 , the storm entered 1ts fourth and closing phase on March 3, when light and intermittent rains continued generally unt1l late afternoon. During the period March 4-10 there was either no rainfall at any station or too little to be significant.

The four phases of the storm are readily visualized by noting the mass curves of accumulated rainfall at several representative stations, as shown in figure 9. (Mass curves of accumulated precipitation for typlcal groups of stations are shown in fig. 13.) The periods of maximum intensity are also clearly indicated in figure 10, which shows the hourly precipitation at representative stations. The general eastward movement of the center of the storm is indicated by comparing the mass curves for the Topanga and Sleepy Hollow stations in figure 9. During the period of intense precipitation on February 28 the Topanga station showed considerably more accumulated rainfall than the Sleepy Hollow station, 32 miles to the northeast. This excess continued until shortly after noon on March 2, when the precipitation in the Topanga area began to slacken, but that In the Sleepy Hollow area did not slacken unt1l several hours later. The 


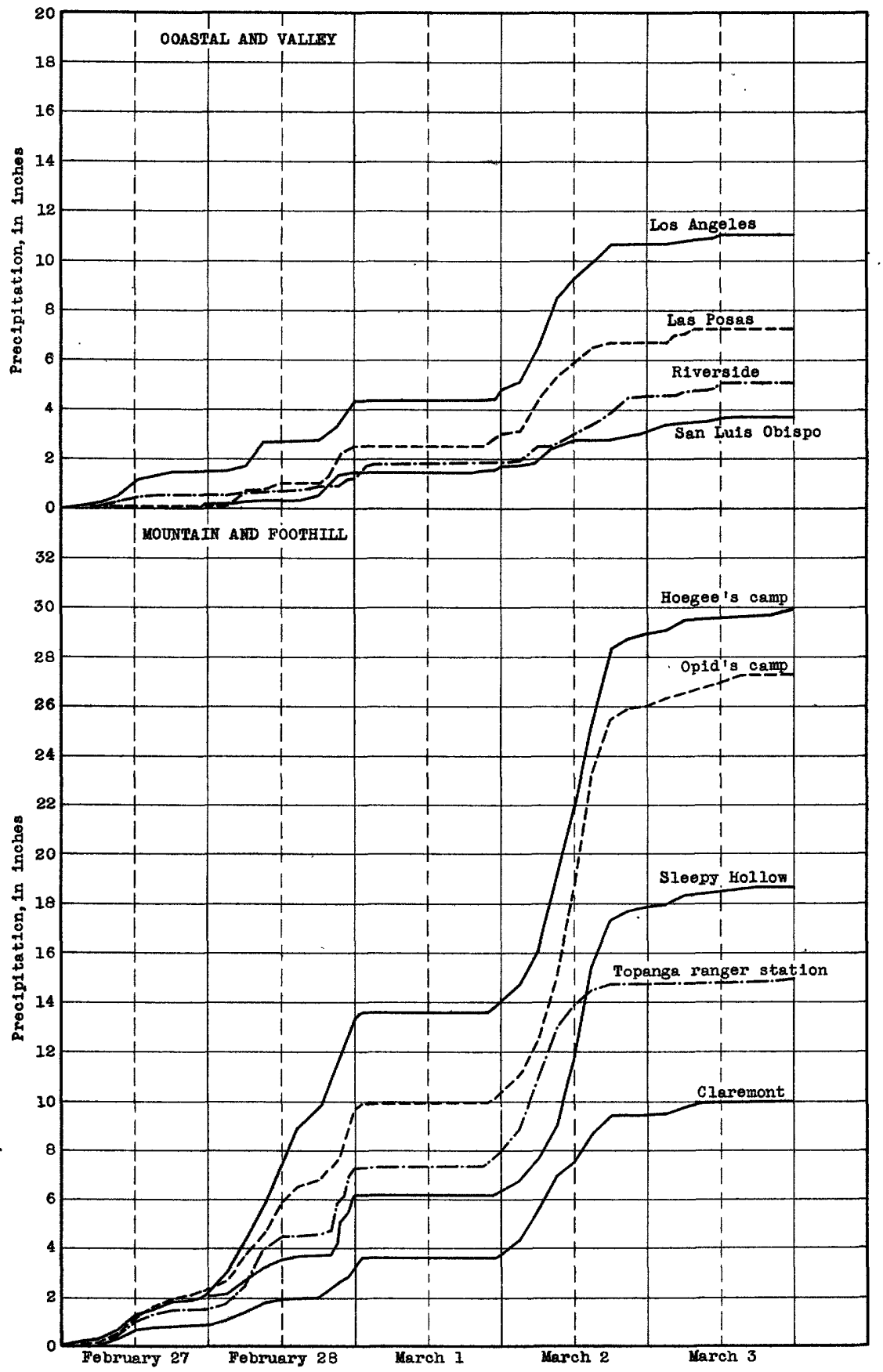

Figure 9.- Mass curves of accumulated precipitation at representative stations, February 27 to March 3, 1938. 


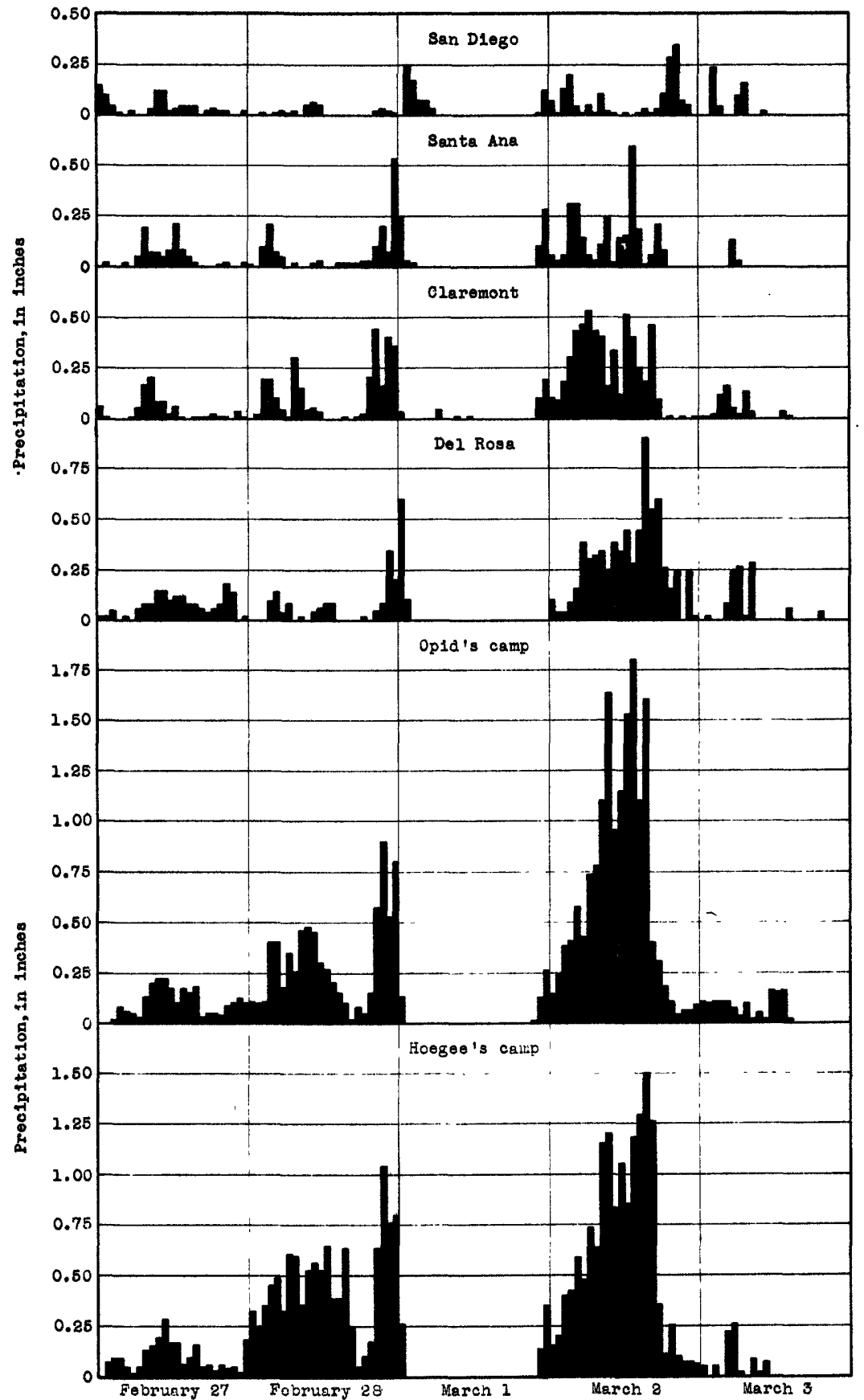

Figure 10.- Hourly precipitation at representative stations, February 27 to March 3 , 1938. 
extremely heavy 12-hour rainfall, especially in the mountains, is graphically indicated by the mass curves of precipitation at Hoegee's camp and Opld's camp. The mass curve for the San Luls Oblspo station indicates that this station was outside the area of heavy precipitation. Table 4 , which gives the percentage of the total storm rainfall occurring at representative stations during each day of the storm, presents also data on the periods of rainfall and indicates the variations in the storm characteristics of the affected region. The average of 58 records from recordIng gages in the Los Angeles, San Gabriel, and Santa Ana areas, Included in this table, shows that about 52 percent of the totel rainfall fell on March 2, which was the day of maximum rainfall during the storm.

Table 4.- Percentage of total storm precipitation on indicated days at 10 representative recording stations in Los Angeles and adjacent counties for the period February 27 to March 3, 1938

\begin{tabular}{|c|c|c|c|c|c|c|}
\hline \multirow[t]{2}{*}{ Station } & \multicolumn{2}{|c|}{ Februery } & \multicolumn{3}{|c|}{ March } & \multirow{2}{*}{$\begin{array}{c}\text { Total } \\
\text { (inches) }\end{array}$} \\
\hline & & 28 & 1 & 2 & 3 & \\
\hline oegee's camp, West Fork of Santa & 7.4 & 37.5 & 2.4 & 50.1 & 2.6 & 29.71 \\
\hline $\begin{array}{l}\text { Opld's camp, West Fark of San Gam } \\
\text { briel Rlver }\end{array}$ & 8.7 & 27.1 & 2.0 & 57.6 & 4.6 & 27.23 \\
\hline $\begin{array}{l}\text { Sleepy Hollow Ranch } \\
\text { Topanga Canyon, } 4 \text { miles south of }\end{array}$ & $\begin{array}{l}11.0 \\
10.2\end{array}$ & $\begin{array}{l}22.0 \\
38.5\end{array}$ & $\begin{array}{l}1.4 \\
4.6\end{array}$ & $\begin{array}{l}61.3 \\
45.0\end{array}$ & $\begin{array}{l}4.3 \\
1.7\end{array}$ & $\begin{array}{l}18.62 \\
14.92\end{array}$ \\
\hline $\begin{array}{l}\text { Topanga Bridge } \\
\text { Del Rosa ranger station } \\
\text { Los Angeles, Sixth and Main } \\
\text { Streets }\end{array}$ & $\begin{array}{l}14.5 \\
13.3\end{array}$ & $\begin{array}{l}11.5 \\
25.8\end{array}$ & $\begin{array}{l}6.0 \\
4.3\end{array}$ & $\begin{array}{l}59.3 \\
53.2\end{array}$ & $\begin{array}{l}8.7 \\
3.4\end{array}$ & 11 \\
\hline $\begin{array}{l}\text { Claremont, Pomona College Obser- } \\
\text { vatory }\end{array}$ & 8.6 & 27.2 & 3.8 & 54.5 & 5.9 & 9.99 \\
\hline Santa Ana, Orange County Flood & 15.7 & 25.2 & 10.7 & 45.8 & 2.6 & 6.07 \\
\hline $\begin{array}{l}\text { San Diego } \\
\text { San Luis Obispo }\end{array}$ & $\begin{array}{r}21.3 \\
1.4\end{array}$ & $\begin{array}{r}7.8 \\
36.8\end{array}$ & $\begin{array}{r}18.0 \\
8.4\end{array}$ & $\begin{array}{l}39.5 \\
38.4\end{array}$ & $\begin{array}{l}13 \cdot 4 \\
15 \cdot 0\end{array}$ & $\begin{array}{l}3.95 \\
3.67\end{array}$ \\
\hline Average of 63 recording gages & 9.8 & 29.7 & 3.6 & 52.0 & 4.9 & \\
\hline
\end{tabular}

\section{Rainfall intensities}

Among the first recording rain gages to be established outside the Ios Angeles metropolitan area were those located in the San Gabriel Mountains. Prior to 1916 the United States Forest Service, the United States Weather Bureau, and the County of Los Angeles, entered Into cooperetive agreement that provided for the establishment and operation of certain recording rainfall stations in the mountain areas. These stations were located at Mount Wilson, Sister Elsie Peak, Haines Canyon (upper station), and Alder Creek (Loomis Ranch). The gages et Haines Canyon and Sister Elsie Peak which were some distance from human habitation, were inspected weekly by employees of the United States Forest Service. This duty was later assumed by the Geological Survey and more recently by the Los Angeles County Flood Control District. 
The hydrologic division of the Los Angeles County Flood Control District was created in 1927, and Walter J. Wood, the present chief hydraulic engineer, and Finley B. Laverty and F. H. Hays, former chief hydraulic engineers, were largely responsible for establishing the large number of recording rain-gage stations now in operation in Los Angeles County. As a result of the combined activities of the various agencles previously. referred to, more than 60 recording rain gages were in operation during the storm of February 27 to March 4, 1938, in an area covering about 7,000 square miles and including both mountains and valleys.

In order to facilitate a more detalled analysis of the rainfall records, the hourly records of rainfall at the automatic recording gages are assembled in table 5. The stations are the same as those for which the daily precipitation is given in table 2 .

Tables 6 and 7 present data deduced from the original records on the intensity of the precipitation during the storm perlod February 27 to March 4, 1938, and 1ts duration at different rates of intensity. Table 6 shows the number of hours in the storm perlod during which the intensity exceeded the given rates, which range from 0.2 of an inch to 1.4 inches per hour, and also the total amount of preclpitation that fell at rates In excess of the indicated rates. Table 7 shows the maximum rates of precipitation, in inches per hour, for specified periods, ranging from 5 minutes to 120 hours, within the storm period. In table 7 the specified periods are continuous, but in table 6 they may be elther continuous or discontinuous. The basic data for tables 6 and 7 were furnished, in the mein, by the Corps of Englneers, United States Army, and the Los Angeles County Flood Control District.

In some localities, especially on the coastal plain, the maximum rates of precipitation for the short perlods occurred on February 28 , or outside the maximum 24-hour perlod of the storm, which corresponded in general to the calendar day March 2. Generally, at the stations given in table 5, each maximum period of 3 hours or longer occurred within the next longer maximum period.

The recording-gage records in the foothill and mountain areas have been analyzed, and five groups, $A$ to $E$, Inclusive, have been selected that seem to be more or less distinct in the total amount of rainfall, the relation between duration and average intensity, and the time of occurrence. 


\begin{tabular}{|c|c|c|c|c|c|}
\hline 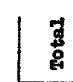 & 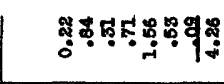 & & å? & 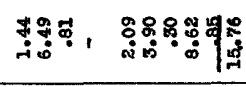 & Fi \\
\hline न & ํㅜㅇ웅휴: , , & & ํㅜㅇ & 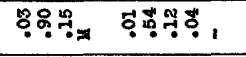 & , \\
\hline$\Rightarrow$ & 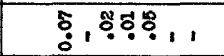 & & 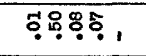 & 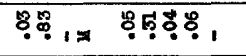 & ,윰윤 \\
\hline 요 & : & & 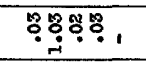 & 产只 1 & $\div 8, \div$, \\
\hline a & 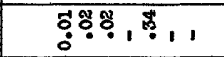 & & 高草, & 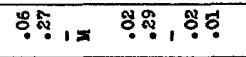 & 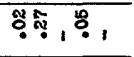 \\
\hline$\infty$ & 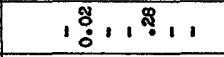 & & 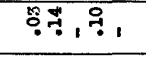 & ธุ๋ & 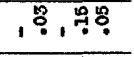 \\
\hline- & $10,1,1$ & & $. \stackrel{8}{10}, 1$ & 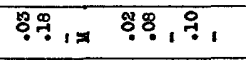 & 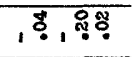 \\
\hline$\circ$ & 1\% & & 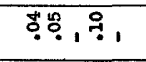 & \%ั\% & ๆำ ำ \\
\hline$\infty$ & $\ldots \ldots$ & & \%ั: & : & 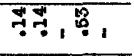 \\
\hline+ & , & & 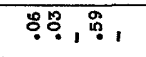 & 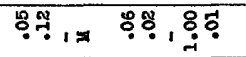 & 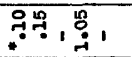 \\
\hline$\infty$ & , 1. & & 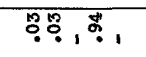 & ๆ๐ , & :ㅇำ \\
\hline$n^{2}$ & 1, 1, 1 & & 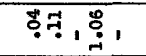 & 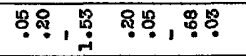 & 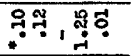 \\
\hline$\omega$ & 10,1 & & 우 & 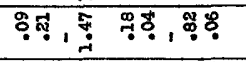 & ㅇำ \\
\hline 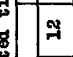 & 10,111 & & $\because \div, ?$ & ஜ우 & 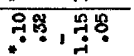 \\
\hline 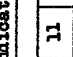 & 17\%유. & & 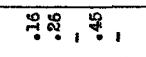 & 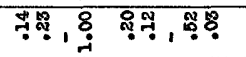 & 유 \\
\hline 요 & 1 & & $\because 5,5$ & ๙ุ. & 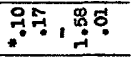 \\
\hline$a$ & 18, , & & ๆำ , & 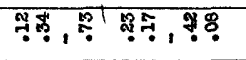 & 유, \\
\hline$\infty$ & , , & & 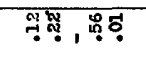 & ตัด : & 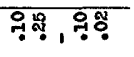 \\
\hline$\sim$ & 1, 1: & & 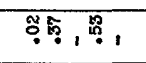 & 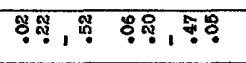 & 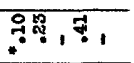 \\
\hline$\infty$ & ' & & , ๆ. & 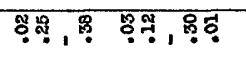 & 윰ำ \\
\hline$\infty$ & 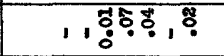 & & 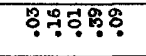 & 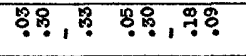 & 유, \\
\hline+ & 1. & & 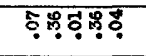 & 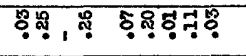 & , ๆ. \\
\hline$\infty$ & 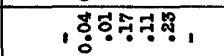 & & 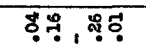 & 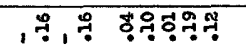 & 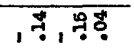 \\
\hline$\infty$ & 1 & & 1웅 & 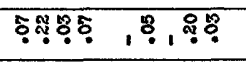 & , \\
\hline$\rightarrow$ & $10,1,1$ & & , ๑ำ: & 훙ํㅠ융 & , ถุำ \\
\hline 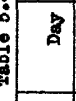 & 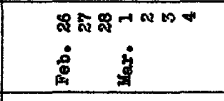 & & 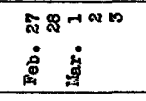 & 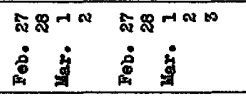 & 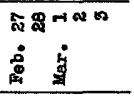 \\
\hline 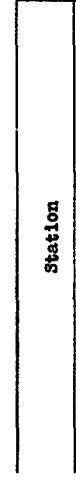 & 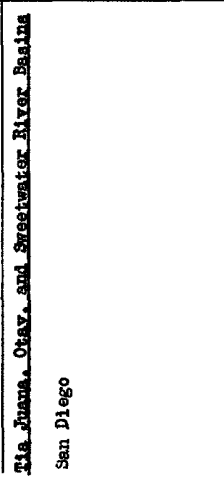 & 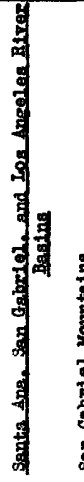 & 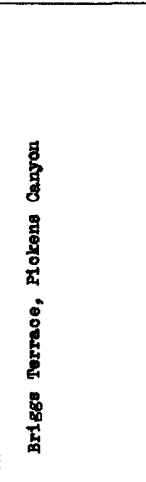 & 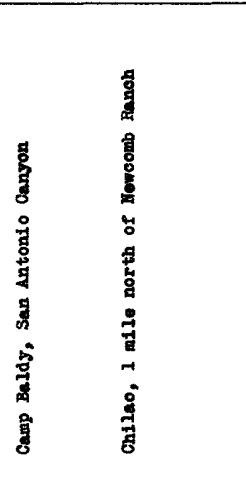 & 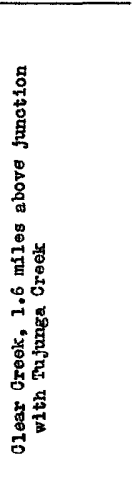 \\
\hline
\end{tabular}




\begin{tabular}{|c|c|c|c|c|c|}
\hline & $\therefore 9$ & 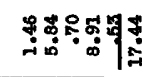 & 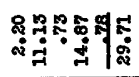 & $\operatorname{Fin}=1$ & ?? \\
\hline$\approx$ & ตํํำ? & 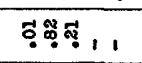 & 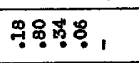 & 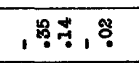 & 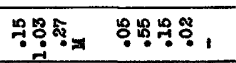 \\
\hline $\mid \vec{F}$ & की & 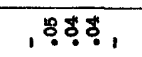 & 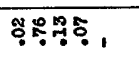 & 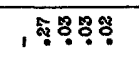 & , \\
\hline \% & 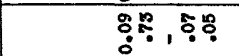 & 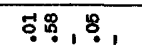 & 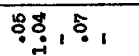 & 1958 & 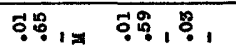 \\
\hline a & 萬器, & , & 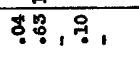 & 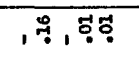 & 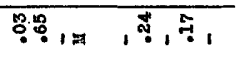 \\
\hline$\infty$ & 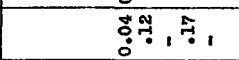 & 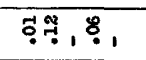 & 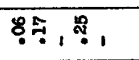 & . & ชั \\
\hline 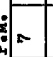 & ดั & है। & 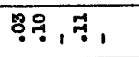 & 1 , , & , \\
\hline- & 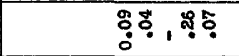 & 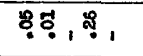 & 菓, & 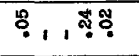 & 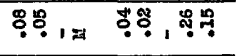 \\
\hline of & $77_{0}^{7}, 15$ & 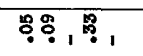 & ) & 䔽, , & 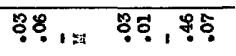 \\
\hline+ & 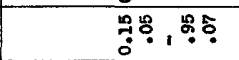 & 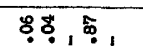 & 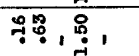 & 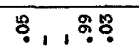 & 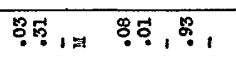 \\
\hline$\infty$ & 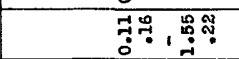 & 菖骂, 范, & 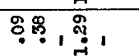 & 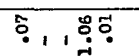 & 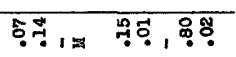 \\
\hline$\infty$ & 경운, & :ั\% & 禺, & ๆ , , & 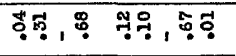 \\
\hline$\rightarrow$ & 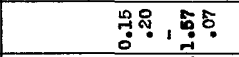 & 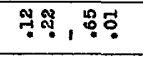 & 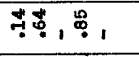 & ๆุ , , & 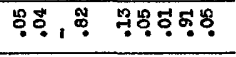 \\
\hline$\approx$ & 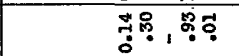 & 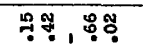 & 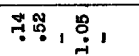 & 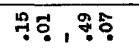 & 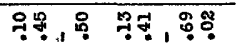 \\
\hline 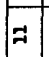 & 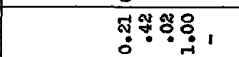 & 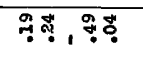 & 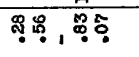 & । & 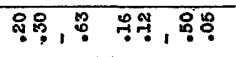 \\
\hline i & 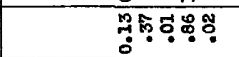 & 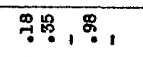 & 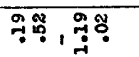 & 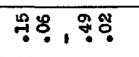 & 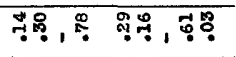 \\
\hline 0 & 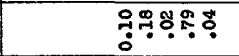 & 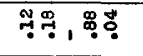 & 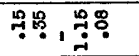 & 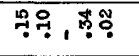 & 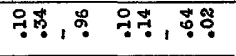 \\
\hline$\infty$ & דับ & 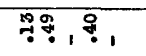 & , & 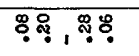 & 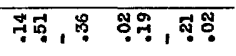 \\
\hline$\sim$ & 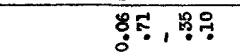 & 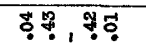 & 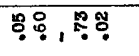 & ํํำ , ูำ & 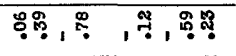 \\
\hline$\circ$ & . & , & 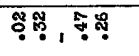 & 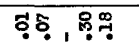 & 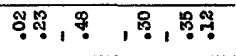 \\
\hline 5 & 苟 & 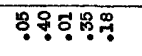 & 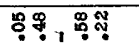 & 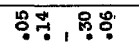 & 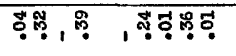 \\
\hline$*$ & 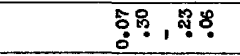 & 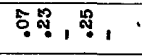 & 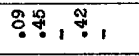 & 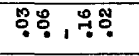 & 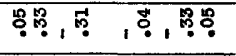 \\
\hline$\approx$ & ๑ั: & 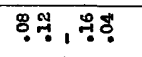 & 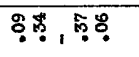 & ) & ه̆ \\
\hline N & จุด & 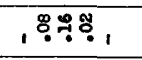 & 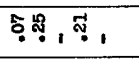 & $\ldots, 5$ & 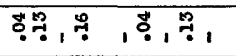 \\
\hline$H$ & 18:30\% & , & 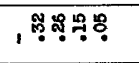 & , 1 & 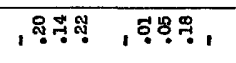 \\
\hline 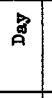 & 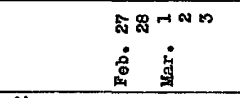 & 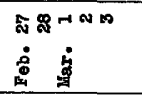 & 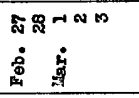 & 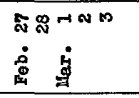 & 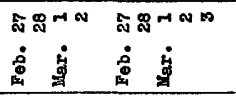 \\
\hline & 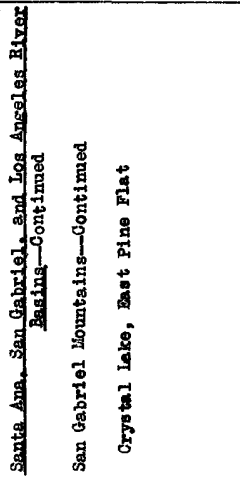 & 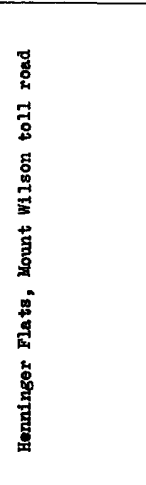 & 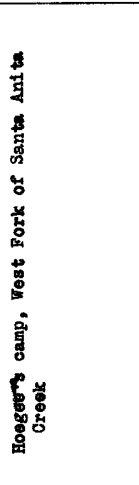 & 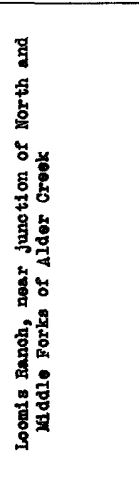 & 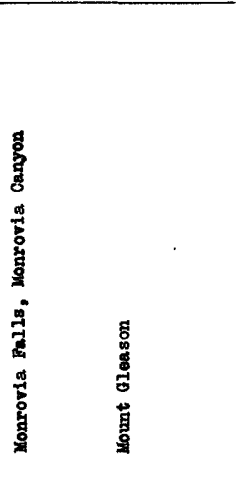 \\
\hline
\end{tabular}




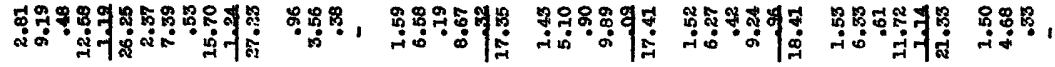

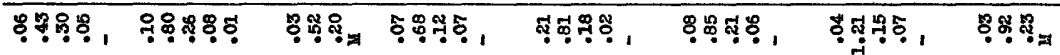

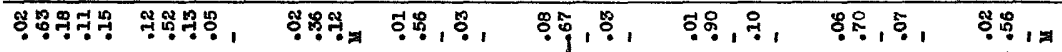

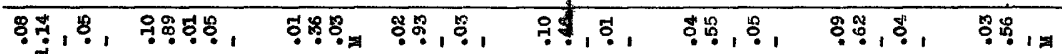

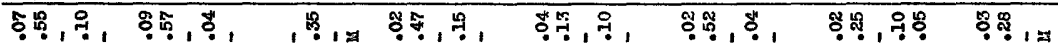

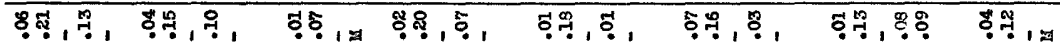

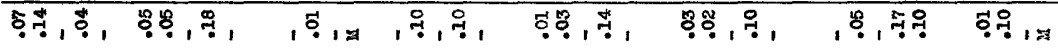

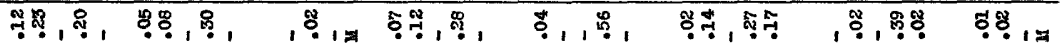

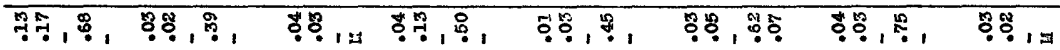

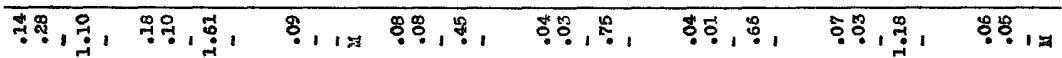

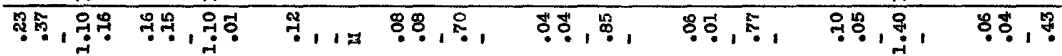

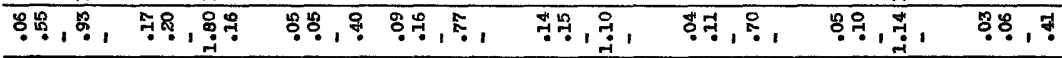

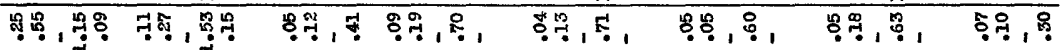

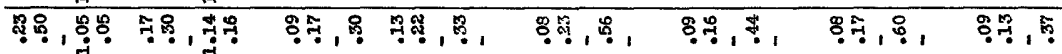

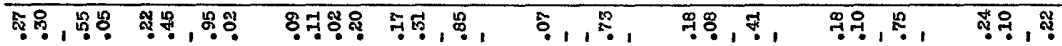

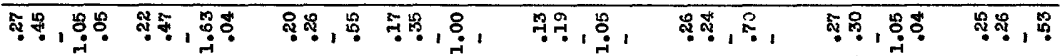

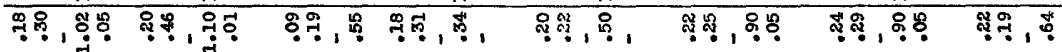
ศัด,

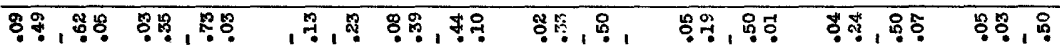

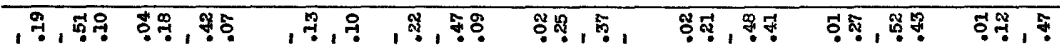

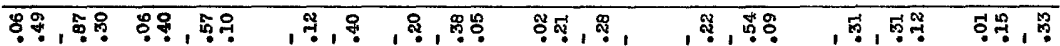

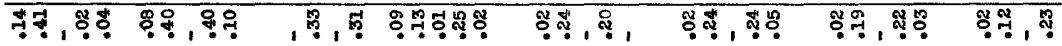

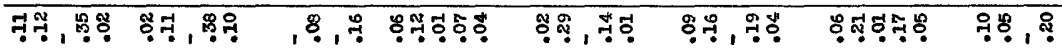

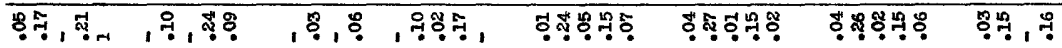

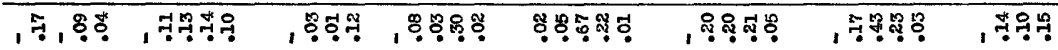

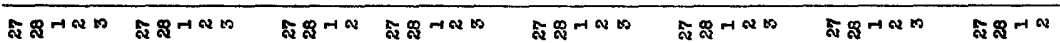

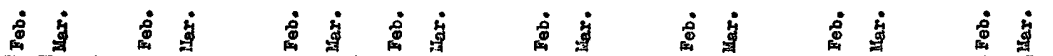

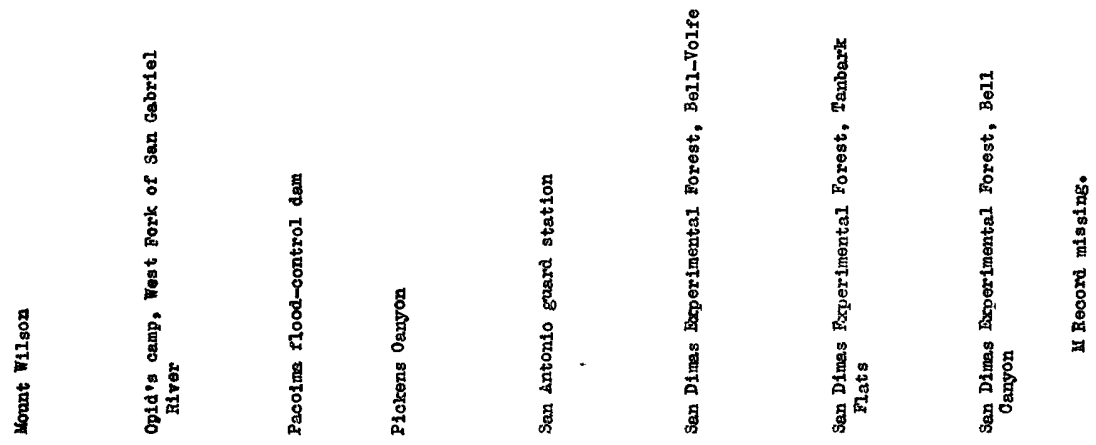




\begin{tabular}{|c|c|c|c|c|c|c|c|}
\hline$\left|\begin{array}{l}1 \\
0 \\
0 \\
0\end{array}\right|$ & & $\therefore-i$ & تص & 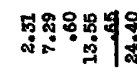 & 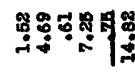 & 象象: & ن \\
\hline$\approx$ & & 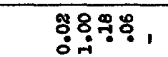 & 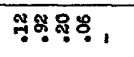 & 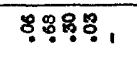 & ชุธ్ & 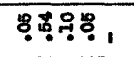 & $8: 8 \%$ \\
\hline I & & , & 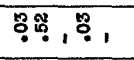 & "ั. & 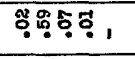 & 草: & 60. \\
\hline 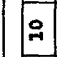 & & 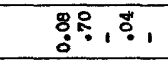 & : : : & ஜ̊̊, & : & 8: & 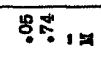 \\
\hline a & & 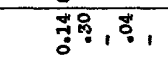 & :ำ, & కั & 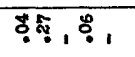 & 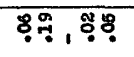 & $5 ! 11$ \\
\hline$\infty$ & & 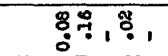 & 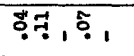 & 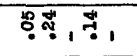 & 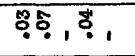 & 80,1 & 19 \\
\hline 2 & & 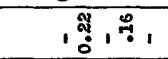 & ธัด. ริ & ఫึ? & 5\%, & ธุด. & 95 \\
\hline 0 & & 1,19 & 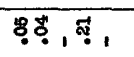 & 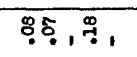 & \%ั, , গำ & 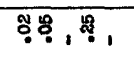 & 5.11 \\
\hline$\infty$ & & , & :0\%, & $\stackrel{80}{0}$, & 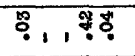 & $\stackrel{0}{\circ}, 1 \%$ & $50.1=$ \\
\hline+ & & , đু & 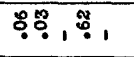 & नुठ । & 0 & न1, & $8 \% 17$ \\
\hline$\infty$ & & 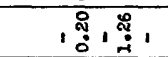 & รั? , & 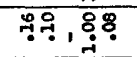 & 薃, & $8 \%, 8 \%$ & 17. \\
\hline$\approx$ & & , 染, & 军 & $\because \frac{9}{9}, 1$ & 8,19 & \%ั: : & $8 \% 1.1$ \\
\hline-1 & & 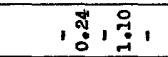 & 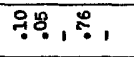 & 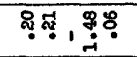 & : & $8 \%, \$$ & 85.8 \\
\hline$\nexists$ & & , & 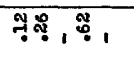 & 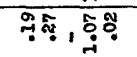 & ศึ? & ธุด , & नี่ \\
\hline$\Rightarrow$ & & , & 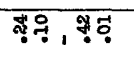 & ชุำ & งุ๋ & 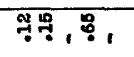 & สุ? ใ? \\
\hline 0 & & 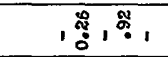 & 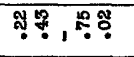 & 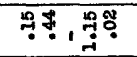 & 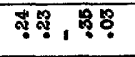 & เ งั , : & สุด \\
\hline$\infty$ & & . & 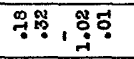 & 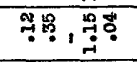 & 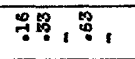 & $, \stackrel{n}{7}, \stackrel{0}{0}$ & ๆ? \% \\
\hline$\infty$ & & , 品, 范, & 象, & $\because 9$, & ๆ. & 骂品, 9 & 궁 \\
\hline a & & , 哭, & 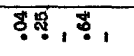 & !? & कें? & 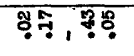 & 吅象象: \\
\hline$\infty$ & & , 范, 寻 & 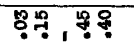 & 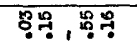 & 융뭉, & 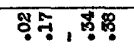 & . \\
\hline$\infty$ & & 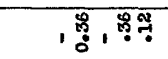 & 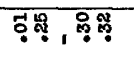 & : & 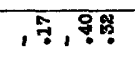 & ศ़ำ , สุตุ & 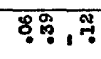 \\
\hline+ & & , צִ & 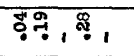 & 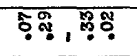 & ठั & 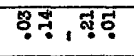 & 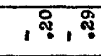 \\
\hline$\infty$ & & 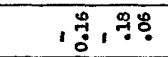 & : & 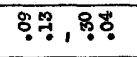 & 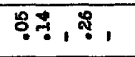 & 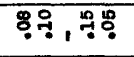 & คำด \\
\hline$\approx$ & & 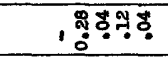 & ํํำ, 중 & \%ั & \%ั & :55:8\% & 귝둥ํㅇ \\
\hline-1 & & 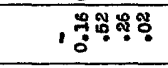 & , วับุำ & , ซิสุร , & , ดัดูร & , ดัดั & 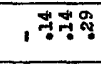 \\
\hline . & & 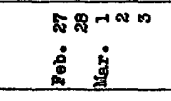 & 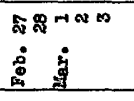 & 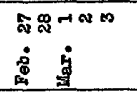 & 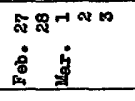 & 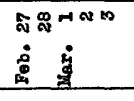 & 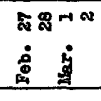 \\
\hline 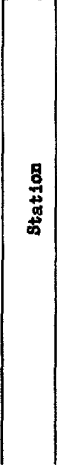 & 㝵 & 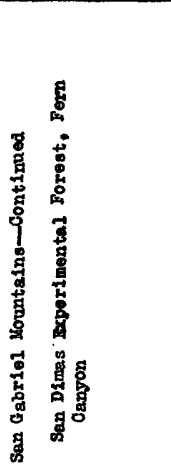 & 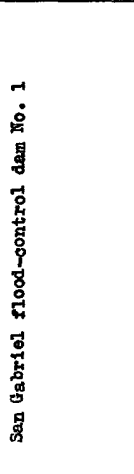 & 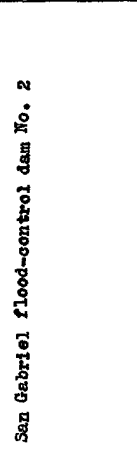 & 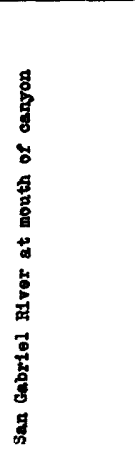 & 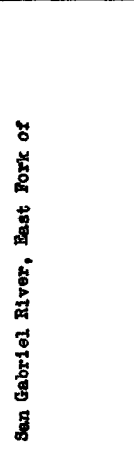 & 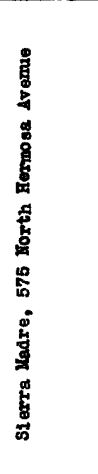 \\
\hline
\end{tabular}




\begin{tabular}{|c|c|c|c|c|c|c|}
\hline 熟 & & 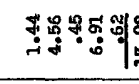 & 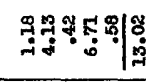 & 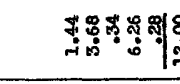 & 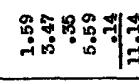 & 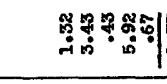 \\
\hline$\approx$ & & 웅영윰뭉, & 1 & 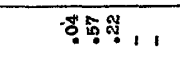 & 1 & 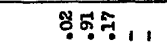 \\
\hline$F$ & & ) & 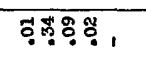 & , & , I & 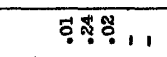 \\
\hline 9 & & 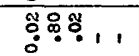 & , & 带骂, , & 草等, 1 & 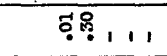 \\
\hline$\sigma_{0}$ & & 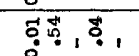 & वุo & 范, 1, & , & , ו \\
\hline$\infty$ & & , & 19.9 & , & भे & , 1, \\
\hline s & & 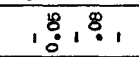 & , ו & 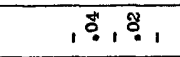 & 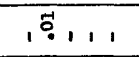 & ธุดัด ดั, \\
\hline$\circ$ & & 年, & 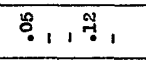 & . & , 50, & $\overline{0}, 1,5$ \\
\hline هـ & & 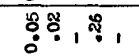 & । & ום & 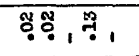 & 1, 1 \\
\hline 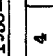 & & 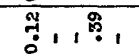 & 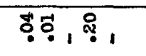 & 苟, , & 萡, , & , 骂, \\
\hline$\infty$ & & (5)⿱宀⿻三丨口 & , & 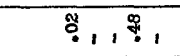 & ธัด, & $\frac{1}{9}, 1,1$ \\
\hline$\approx$ & & 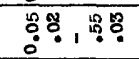 & דั & 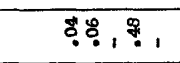 & 象草， & 器, , \\
\hline- & & : & $87.5 \%$ & 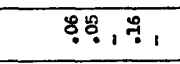 & नุธ, ब, & 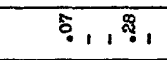 \\
\hline$\approx$ & & नु० & 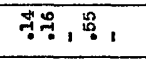 & $7 \%$ & 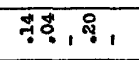 & 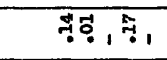 \\
\hline$F$ & & 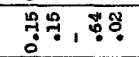 & รุำดัด & $\$$ & ו וִ & 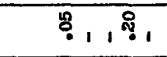 \\
\hline \% & & 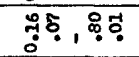 & 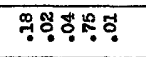 & \$ุ? & ब़ุุ & 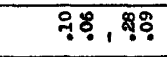 \\
\hline o & & 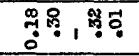 & 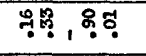 & 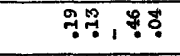 & มุะั , : & $7 \%, 58$ \\
\hline$\infty$ & & ตุำ & 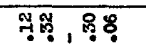 & สุด & 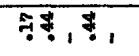 & 겸 \\
\hline - & & 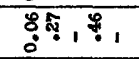 & 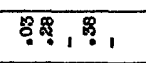 & 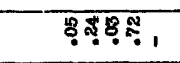 & 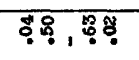 & 49: \\
\hline - & & 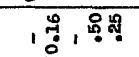 & 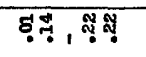 & 98,89 & ตัด , 2ุำ & नุ. \\
\hline هـ & & , & 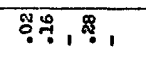 & 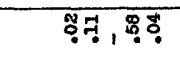 & 98. & †๐, \\
\hline+ & & 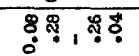 & 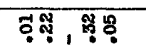 & ธุด & 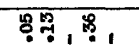 & 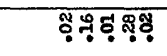 \\
\hline s & & 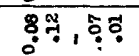 & 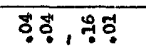 & $8 \%, 8$ & 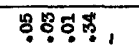 & क्ष: \\
\hline ه & & 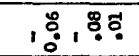 & $\therefore$, & 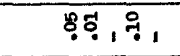 & 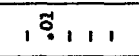 & 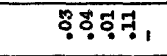 \\
\hline - & & . & ( & , ธุดำด & . & 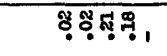 \\
\hline 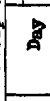 & & 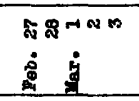 & 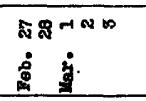 & 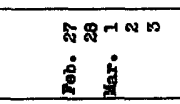 & 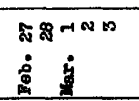 & 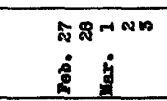 \\
\hline & 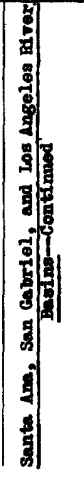 & 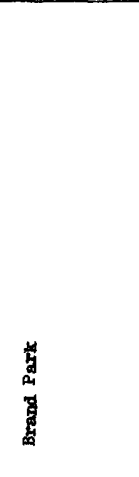 & 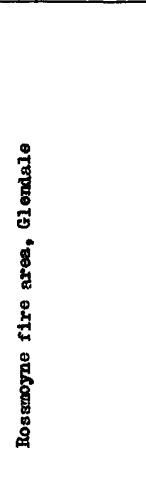 & 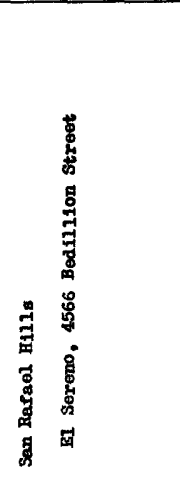 & $\begin{array}{l}\mathbf{\$} \\
\stackrel{5}{5} \\
: \\
: \\
:\end{array}$ & 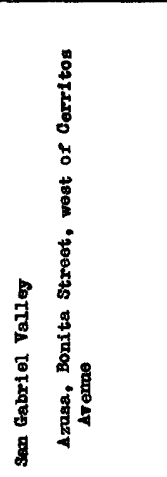 \\
\hline
\end{tabular}




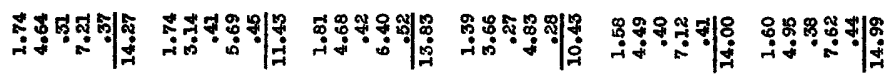

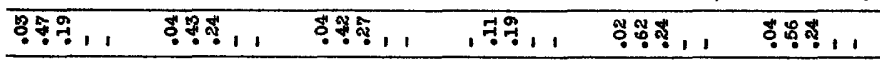

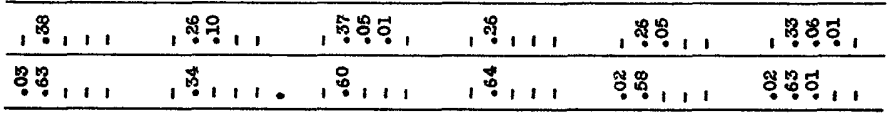

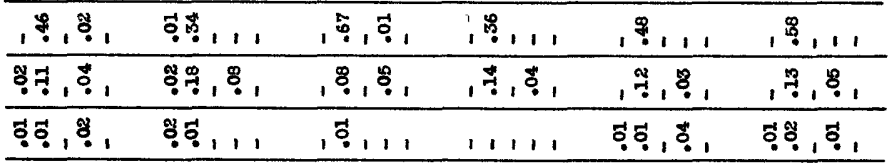

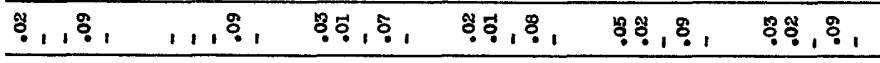

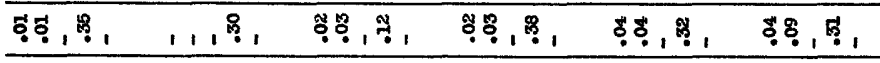

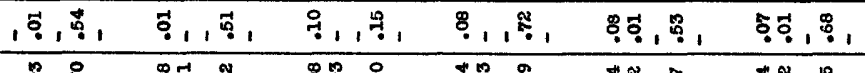

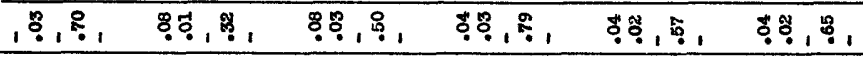

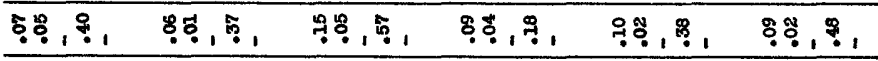

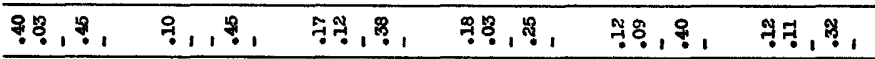

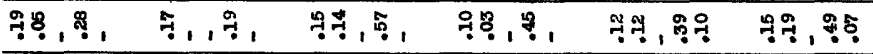

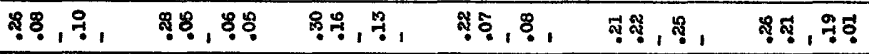

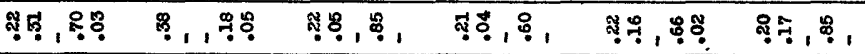

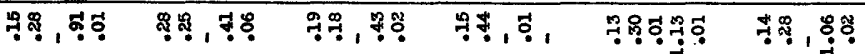

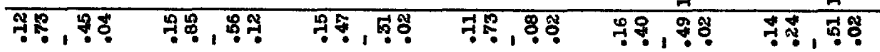

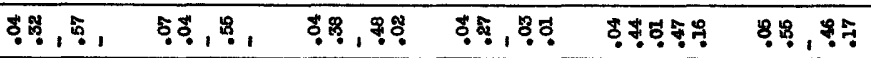

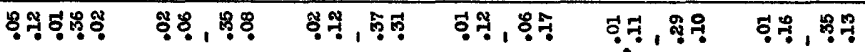

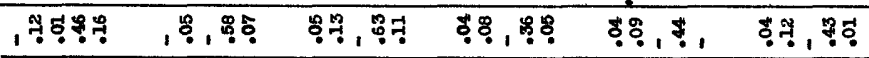

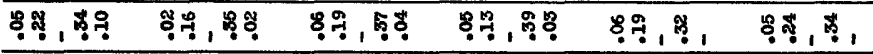

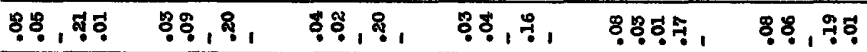

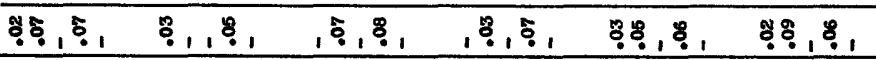
1ำด ,

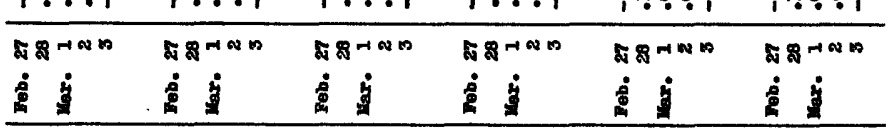

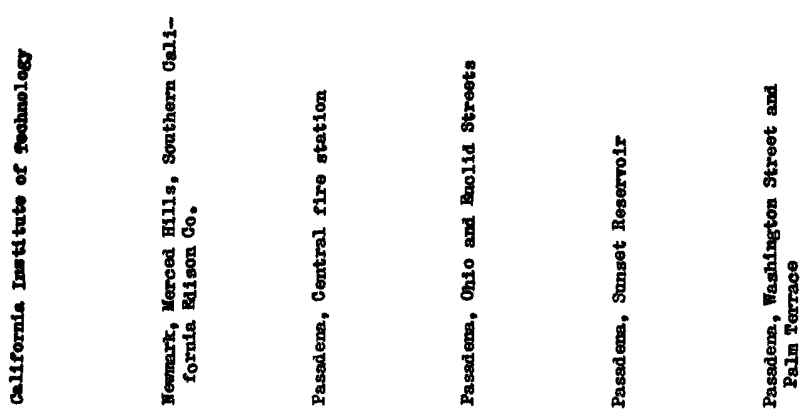




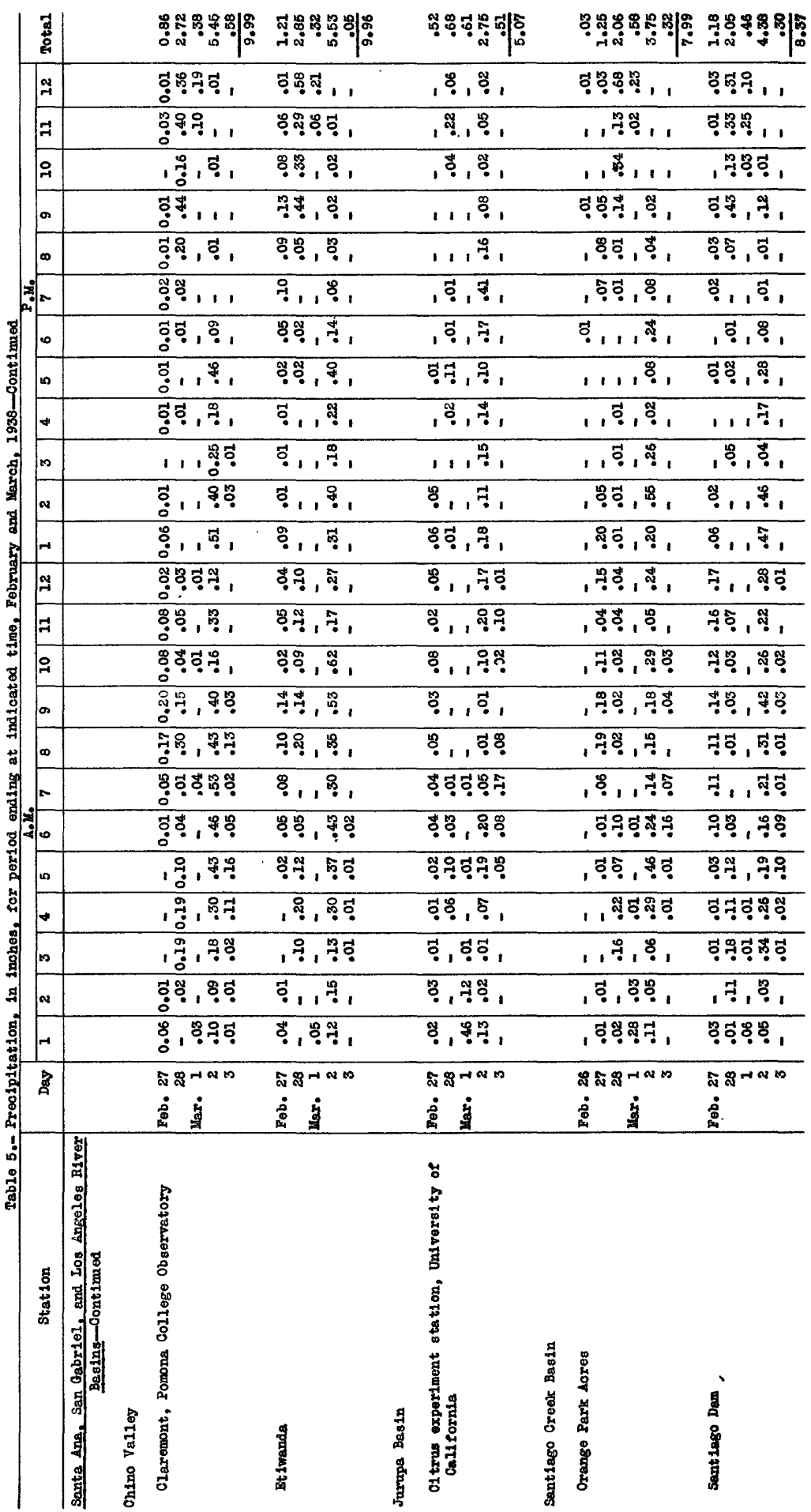




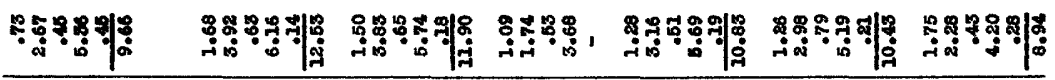

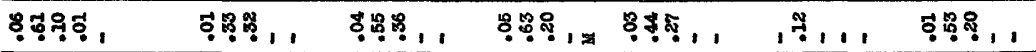

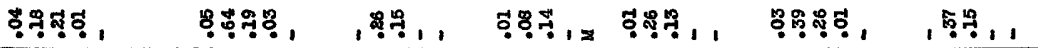

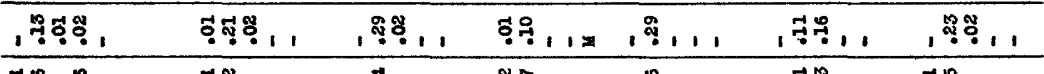

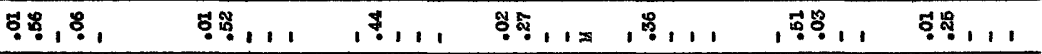

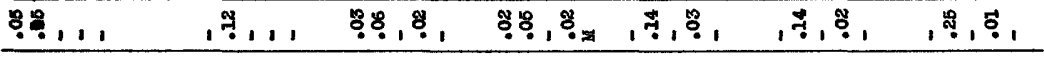

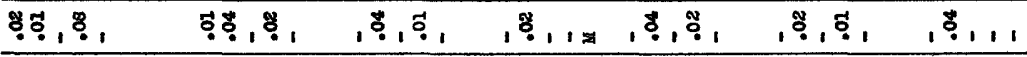

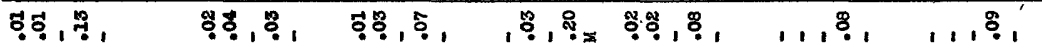

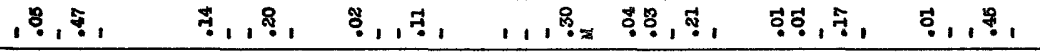

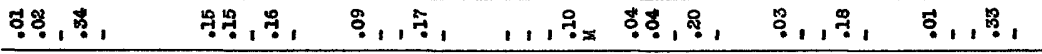

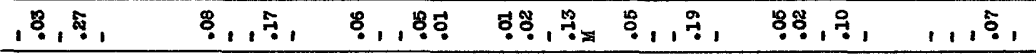

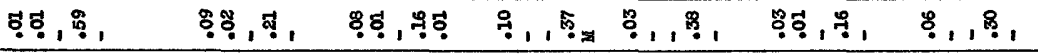

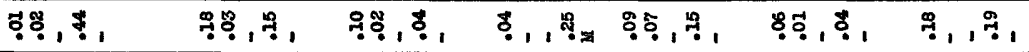

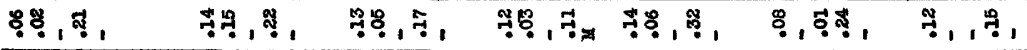

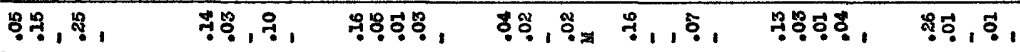

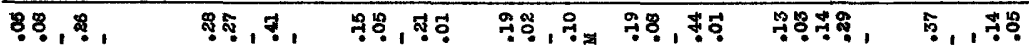

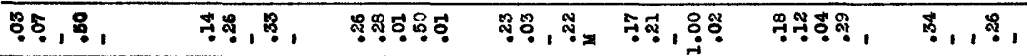

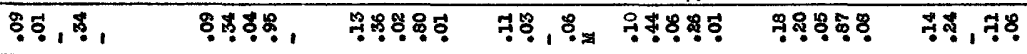

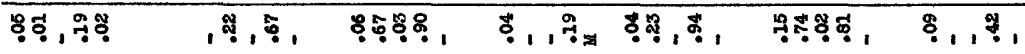

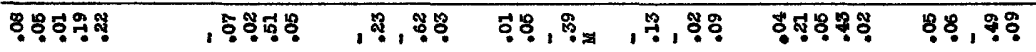

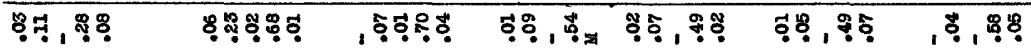

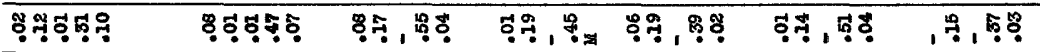

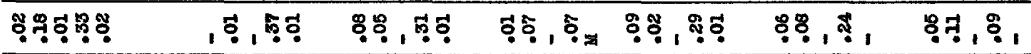

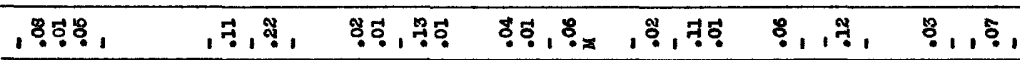

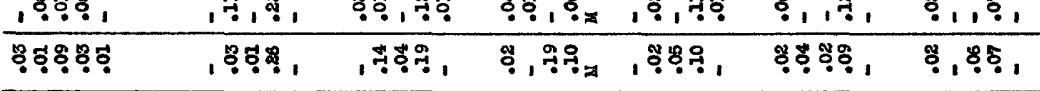

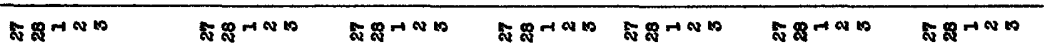

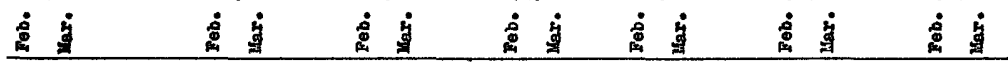

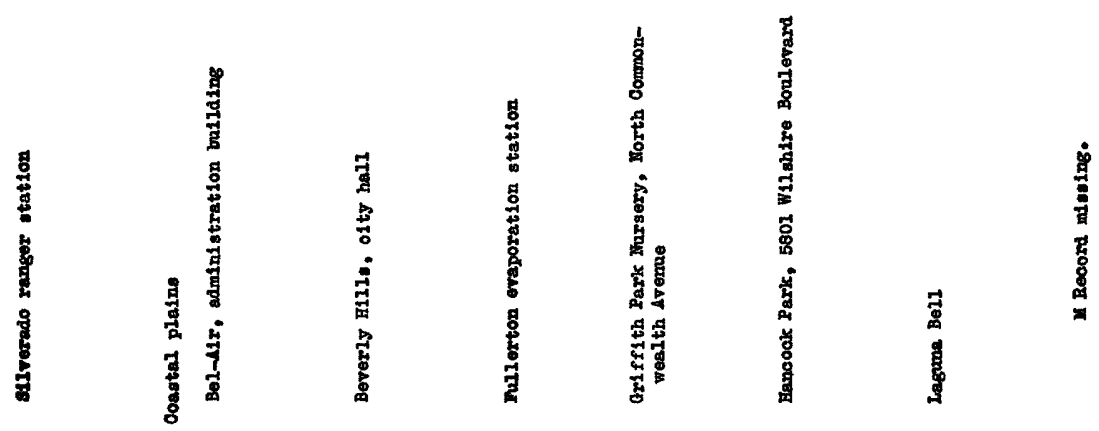




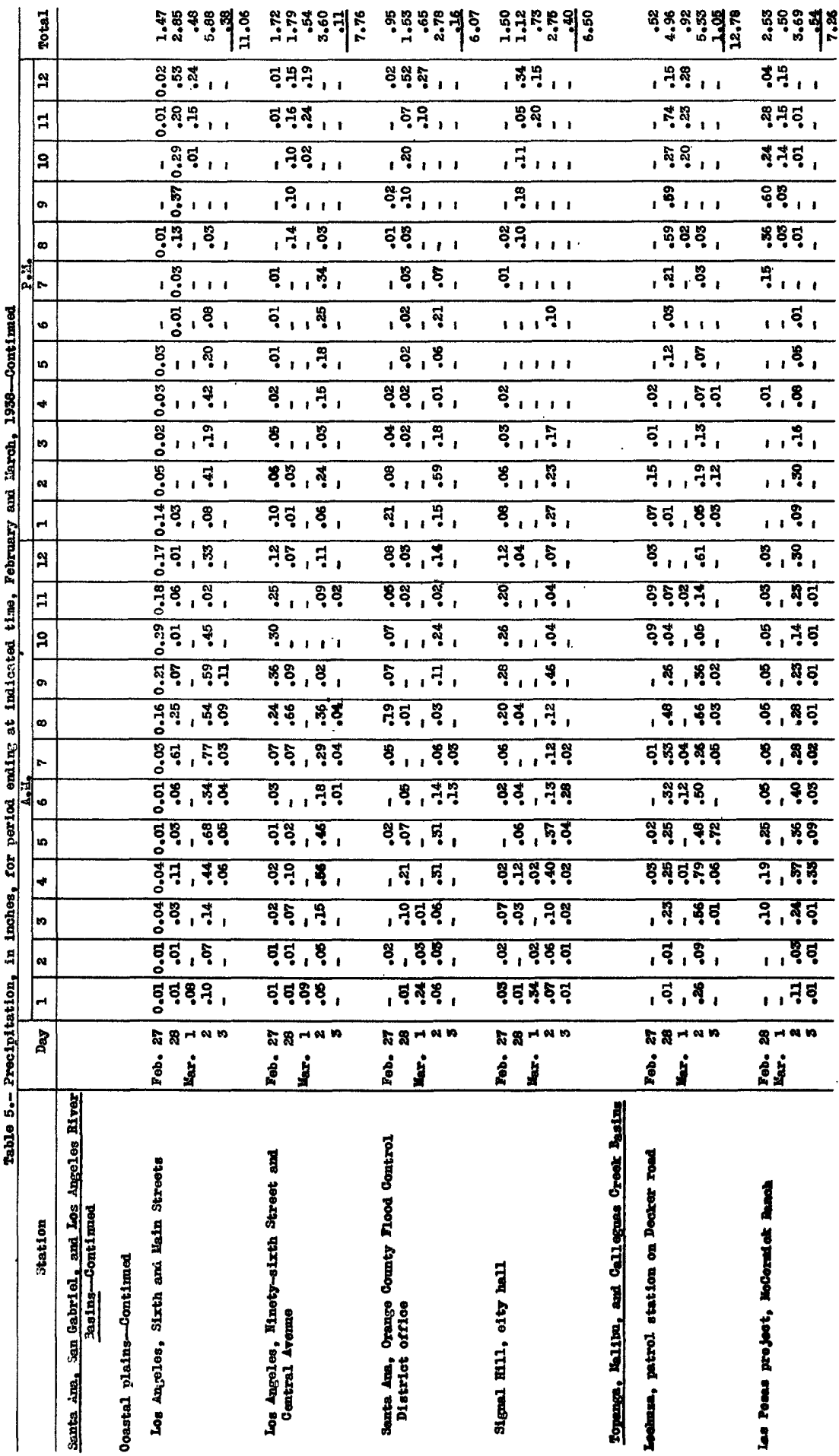




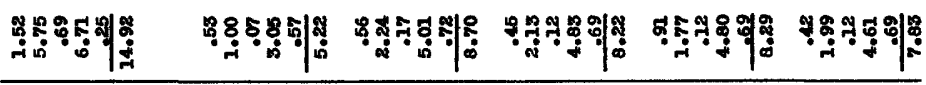

\begin{tabular}{|c|c|c|c|c|c|}
\hline 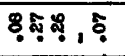 & 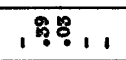 & ?. & 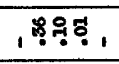 & 1. & $\cdots, 1$ \\
\hline , & 411 & 85. & ta. & $8 \%$ & $8 \%$ \\
\hline 8.8 & \& 11 & 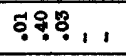 & 萬它, I & ด़ & 9 \\
\hline ו & 18.8 & , I & 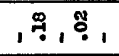 & 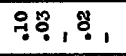 & , \\
\hline , वै, & $1, \%$ & , & , 骂, 范, & $5 \div, 8,1$ & $\vdots_{1}$ \\
\hline . & , , , & 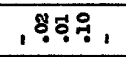 & $8, \%$, & ธุด. & 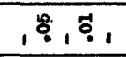 \\
\hline. & , , & 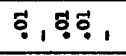 & $11, \stackrel{0}{1}$ & ă & $1, \%$ \\
\hline : & 1,7 & 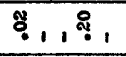 & 8,1, & 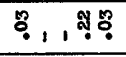 & : \\
\hline 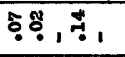 & $8,1,5$ & 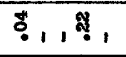 & 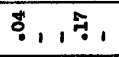 & $89.9 \%$ & 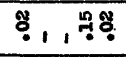 \\
\hline ד, & ，， ， & । & 莦, , & \%ุำ & 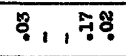 \\
\hline , , & 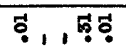 & 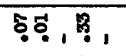 & ر & 8 & । \\
\hline 3ั. & 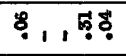 & $\stackrel{8}{8}, \ldots$ & 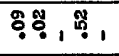 & $77_{1}, \%$ & $8,1,1$ \\
\hline , & 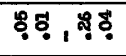 & । & 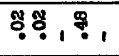 & 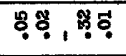 & । \\
\hline 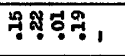 & $\overbrace{1}$ & 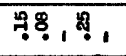 & 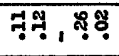 & 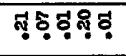 & 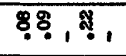 \\
\hline ฺุด & 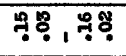 & 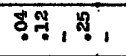 & $5 \%$ & 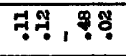 & | \\
\hline 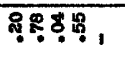 & ธุด & , & , & 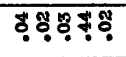 & , \\
\hline 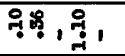 & $8 \%$ & ， & 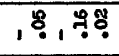 & 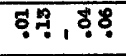 & $10 \%$ \\
\hline 菓, & 1,18 & 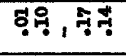 & 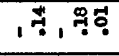 & 18.90 & $, 8,18 \%$ \\
\hline 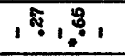 & $, 8,97$ & $1 \%$ & 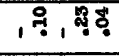 & , & $, 8,19$, \\
\hline 5075 & $18 \%$ & 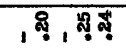 & ดัด & 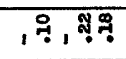 &, 1 \\
\hline 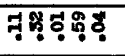 & ๑ & 18,48 & ชุ สุส & 18.74 & 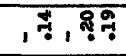 \\
\hline $5 \div, \frac{7}{9}, 1$ & , & 19.98 & $18,7 \%$ & 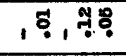 & $18,79 \%$ \\
\hline 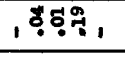 & 1,85 & 1,1980 & $1,8 \%$ & , & 1050 \\
\hline , 8ํㅜㅁำ, & $1,180 \%$ & 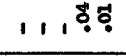 & , 1, 1, & , & 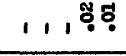 \\
\hline & $8 \%$ & : & 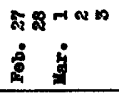 & 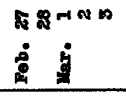 & \\
\hline
\end{tabular}




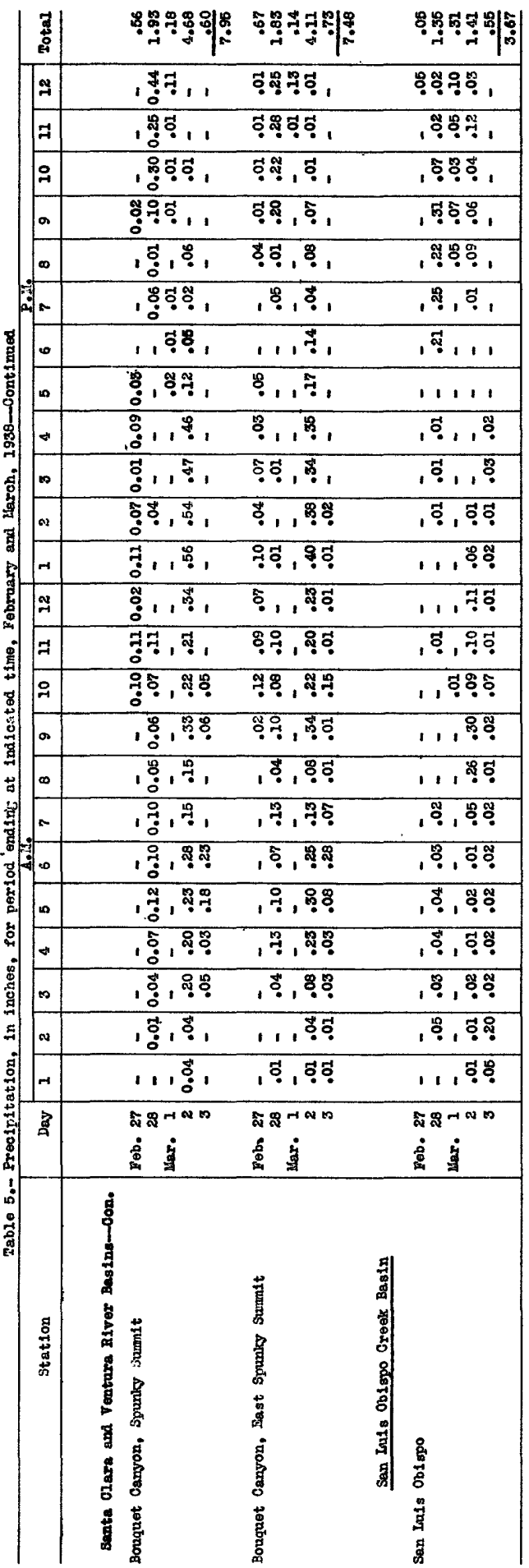




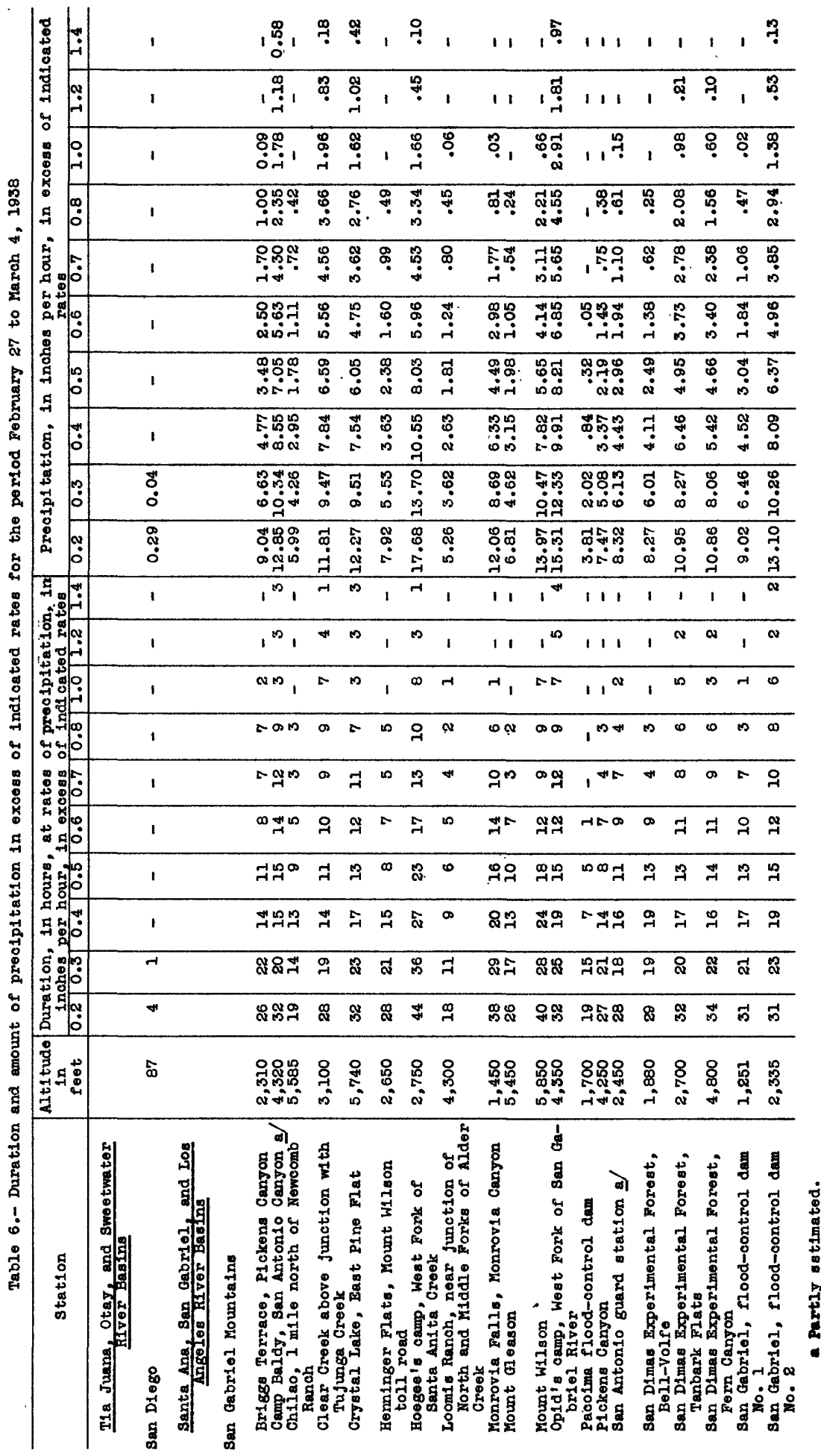




\begin{tabular}{|c|c|c|c|c|c|c|c|c|c|c|c|c|}
\hline 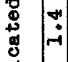 & & 11111 & 11 & 1 & & 11 & & 11 & 1 & 111 & 11 & 11 \\
\hline 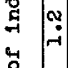 & & $111 \stackrel{\text { \$્ڤ }}{0}$ & 11 & 1 & & 11 & & 11 & 1 & 111 & 11 & 11 \\
\hline & & 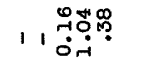 & i: & 1 & & 11 & & 11 & I & 111 & 11 & $\stackrel{0}{?}$ \\
\hline \begin{tabular}{l|l}
0 & $\infty$ \\
& 0 \\
\end{tabular} & & 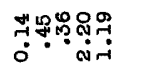 & 움웅 & 1 & & 11 & & 11 & & 궁ำ & $\stackrel{0}{0}, 1$ & 菖骂 \\
\hline वै. & & 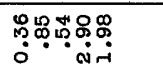 & ஷฺุ & 1 & & 옴ํำ & & 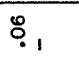 & $\stackrel{4}{9}$ & 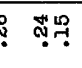 & $\stackrel{0}{7}$ & क़? \\
\hline 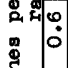 & & 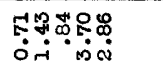 & 品吕 & 1 & & ผุ้ & & 10\% & 웅 & 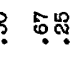 & 品早? & 可产 \\
\hline \begin{tabular}{l|l|}
$\Xi$ & 0 \\
$\Xi$ & 0
\end{tabular} & & 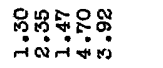 & 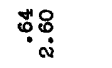 & $\stackrel{9}{\oplus}$ & & 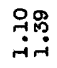 & & ?ִיף & \& & ?. & 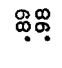 & 占 \\
\hline : & & 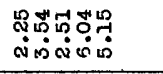 & 承吕 & :ै & & 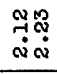 & & : & 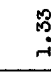 & 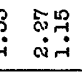 & 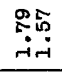 & 若 \\
\hline 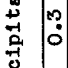 & & 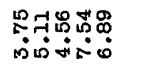 & 幽号 & 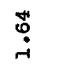 & & 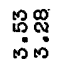 & & 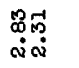 & $\stackrel{m}{m}$ & 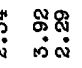 & 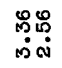 & 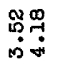 \\
\hline 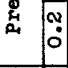 & & 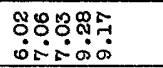 & 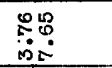 & $\begin{array}{l}\circ \\
\text { in }\end{array}$ & & 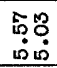 & & 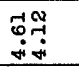 & $\stackrel{\infty}{\leftrightarrow}$ & 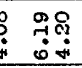 & 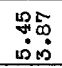 & 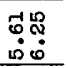 \\
\hline \$ & & 11111 & 11 & 1 & & 11 & & 11 & $\mathrm{I}$ & $1 \quad 11$ & 11 & 11 \\
\hline 年 & & $1,1^{N H}$ & 11 & 1 & & 11 & & 11 & 1 & $1 \quad 11$ & 11 & 11 \\
\hline 象 영웅 & & $11^{-1000}$ & $1^{-1}$ & 1 & & 11 & & 11 & 1 & 111 & 11 & A- \\
\hline 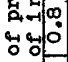 & & WMATL & HD & 1 & & 11 & & 11 & - & A th & $r_{1}$ & $4 \infty$ \\
\hline 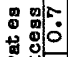 & & $m$ m & กเ & 1 & & NM & & ${ }^{\infty} 1$ & $\infty$ & ar & HA & $r \infty$ \\
\hline $\begin{array}{llll}4 & 0 \\
+ & 0 & 0\end{array}$ & & $D=-\infty O a$ & $\tan$ & 1 & & が & & $\mathrm{BN}$ & m & nल & の & $\infty 0$ \\
\hline 点 & & 엄억 & $A$ & $m$ & & $\infty \infty$ & & Don & + & N & an & $\infty 0$ \\
\hline s : & & 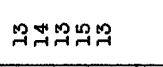 & $0_{H}^{H}$ & $\omega$ & & $\pi^{\circ}$ & & 표 & 禹 & $7^{\infty}$ & $3^{2}$ & नM \\
\hline 过 & & テテニญฺ & న్సి & 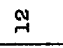 & & ת? & & 교 & $\stackrel{m}{-2}$ & 踏 & $8 \pi$ & $\stackrel{్}{\oplus}$ \\
\hline : & & ๙ననస్心 & สజ & $\stackrel{n}{\rightarrow}$ & & జజ & & त्व & - & $\stackrel{9}{\infty}$ & జ్ & $\stackrel{\alpha}{\infty}$ \\
\hline 荧 & & 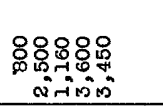 & 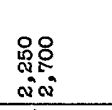 & 总 & & $\begin{array}{l}\text { 员: } \\
\text { ât } \\
\text { Ait- }\end{array}$ & & 点吕 & 尔 & 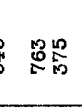 & 窝品 & $\begin{array}{r}\circ 0 \\
\text { 윰 } \\
i \\
\end{array}$ \\
\hline 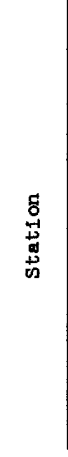 & 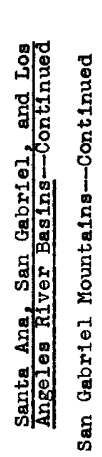 & 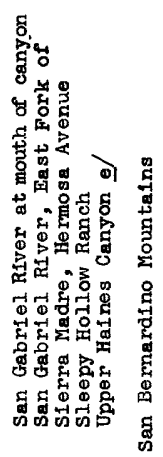 & 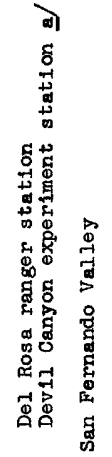 & 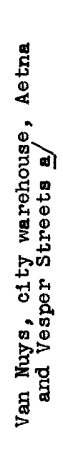 & 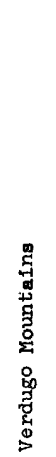 & 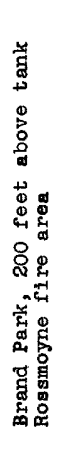 & 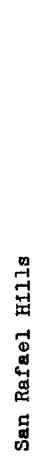 & 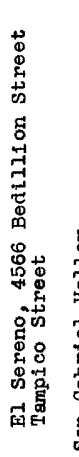 & 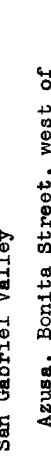 & 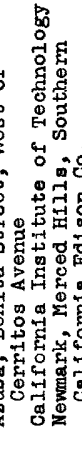 & 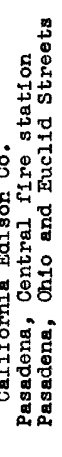 & 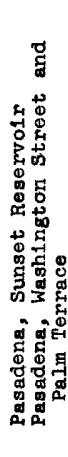 \\
\hline
\end{tabular}




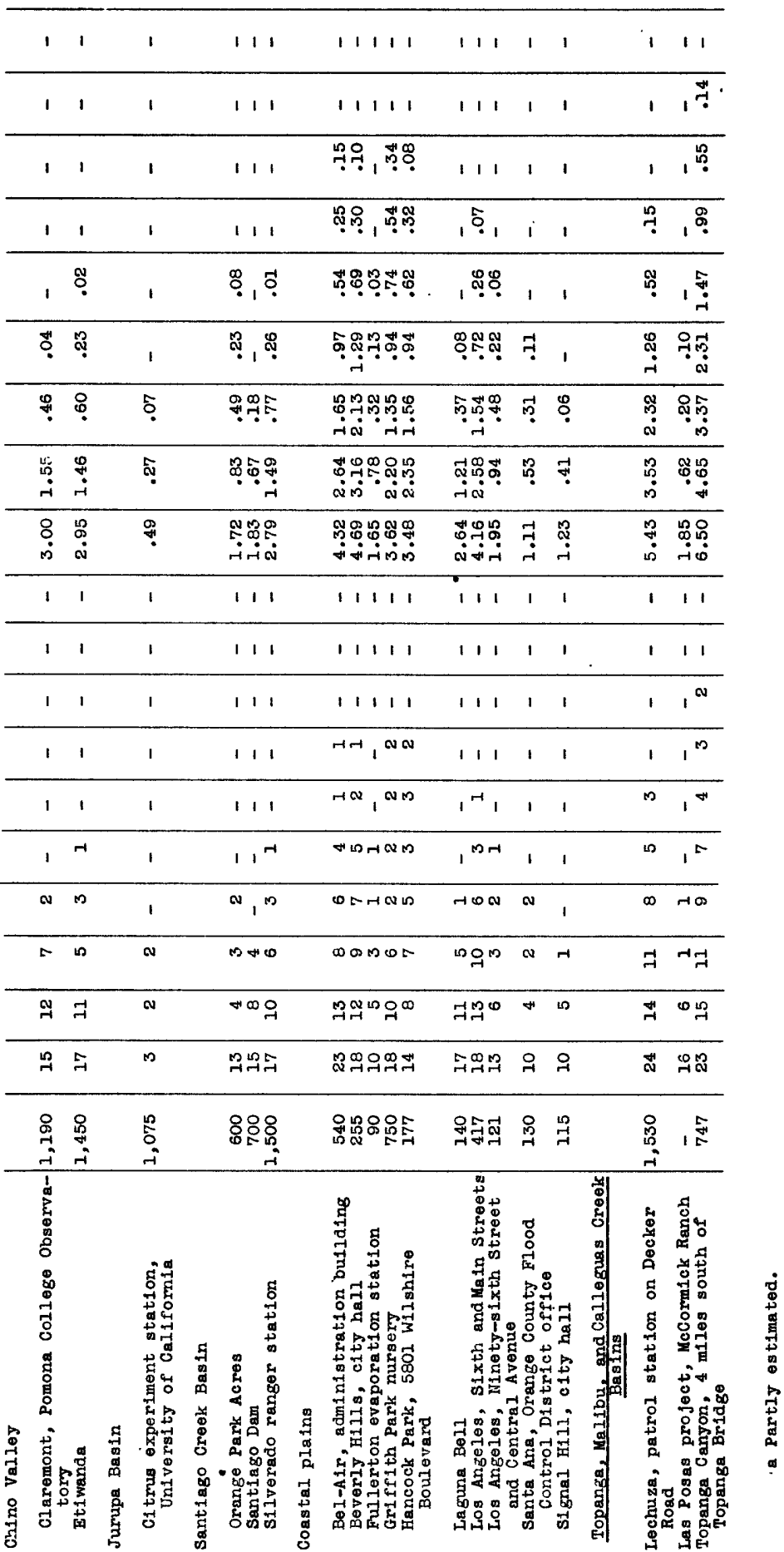




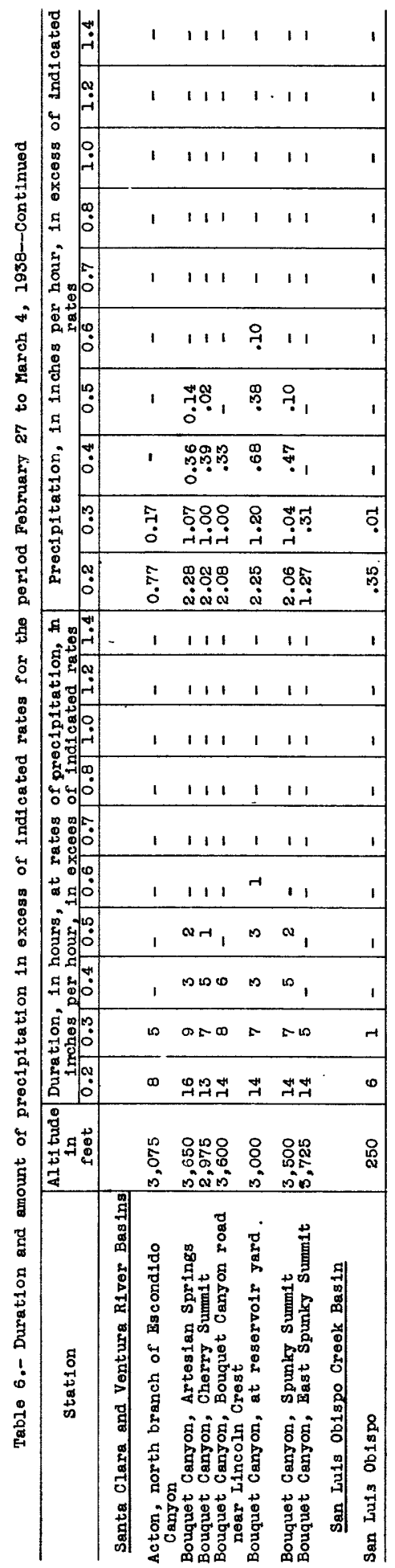




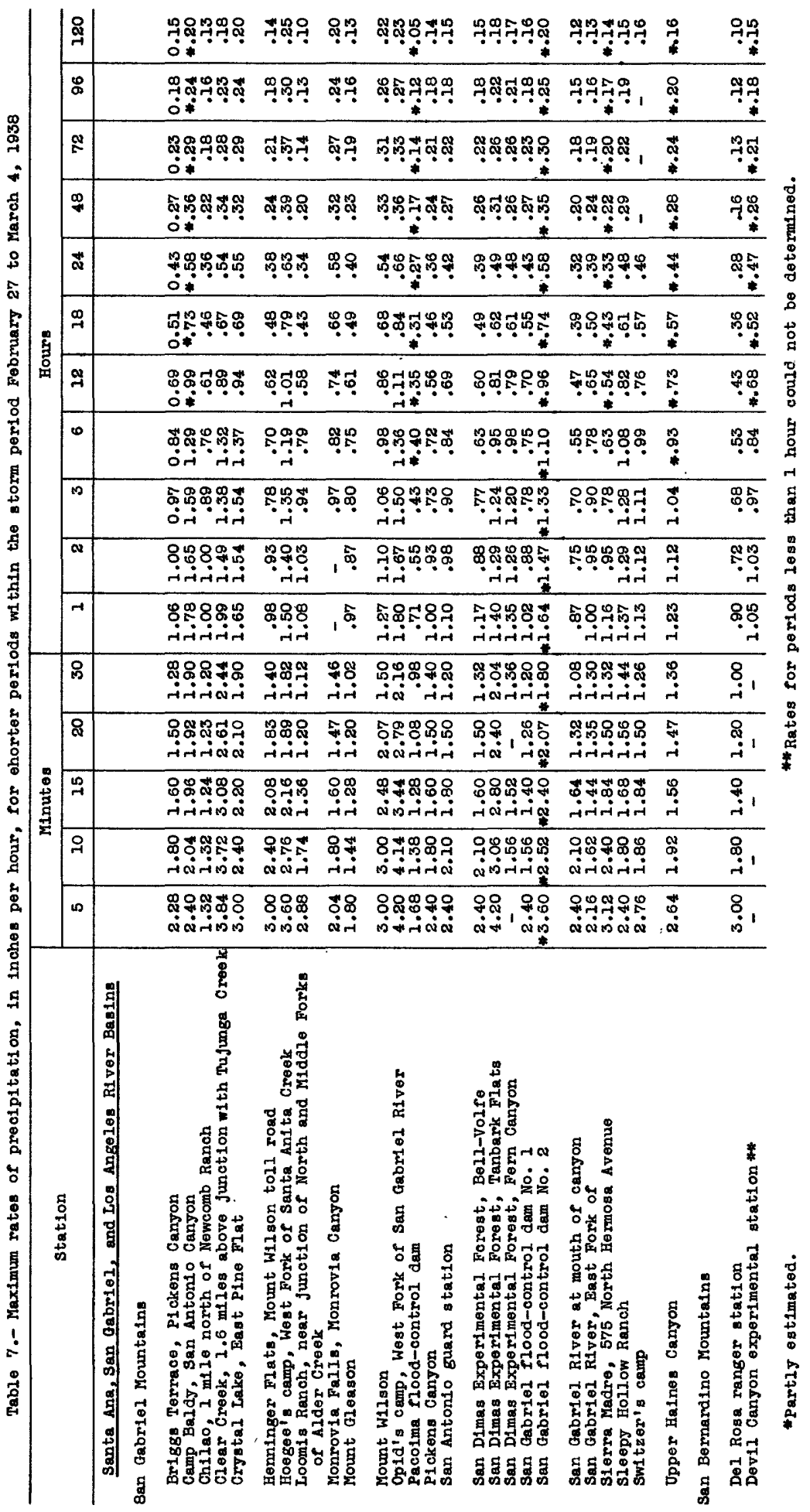




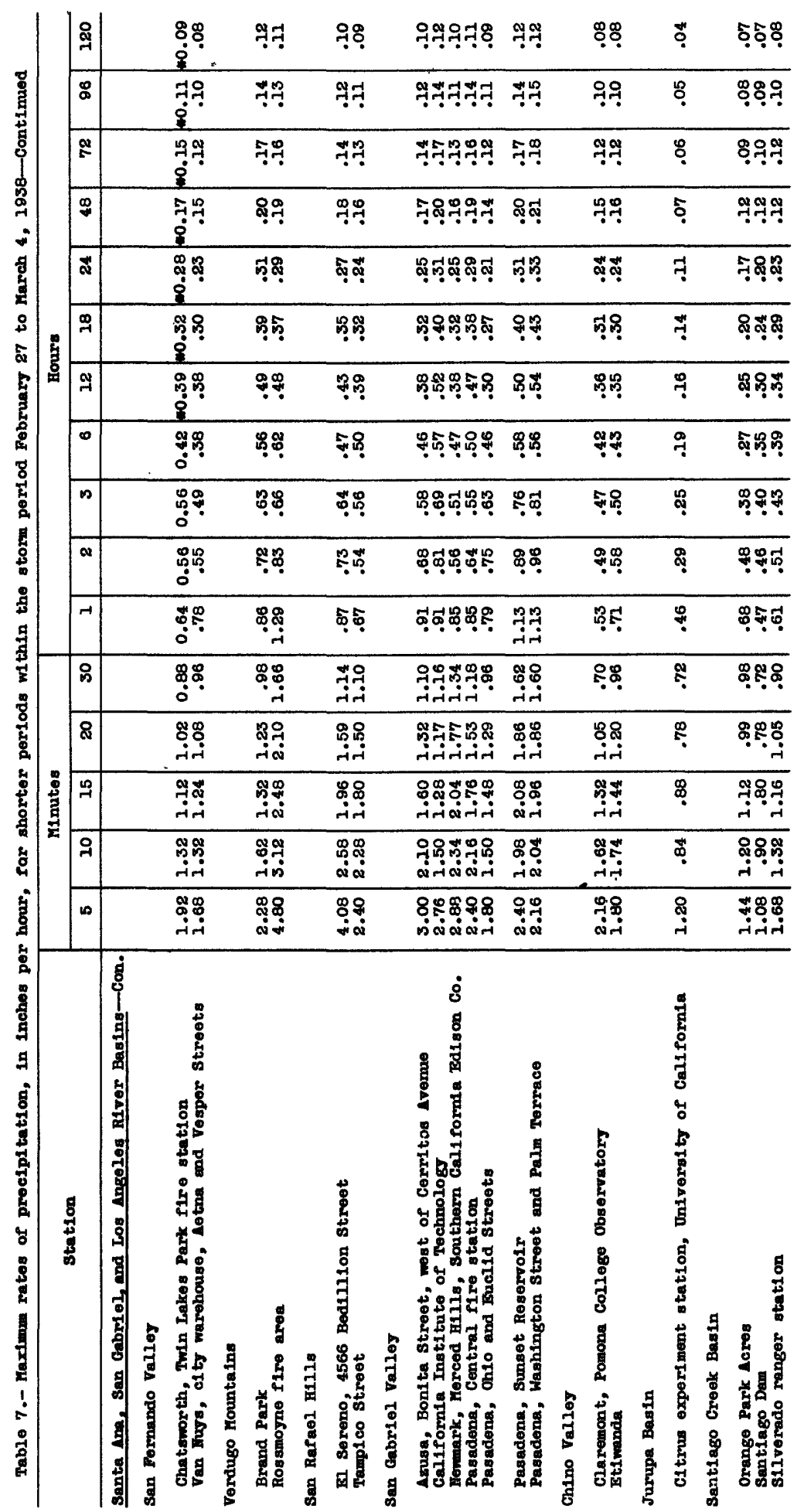


ชำ

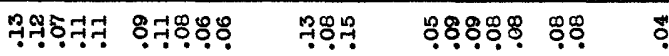

\begin{tabular}{|c|}
\hline 0 \\
\hline
\end{tabular}

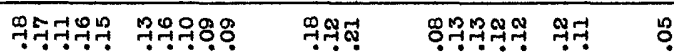

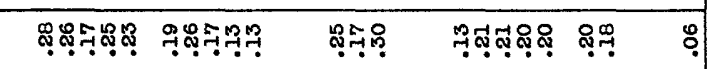

\begin{tabular}{|c|c|c|}
\hline ดีด & 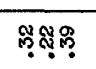 & 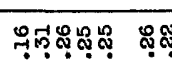 \\
\hline 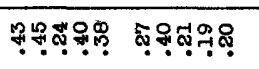 & 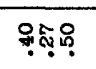 & 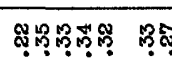 \\
\hline
\end{tabular}

\begin{tabular}{|c|c|c|c|}
\hline 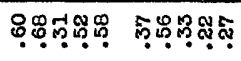 & พุำำ? & 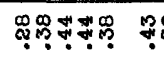 & $\dddot{?}$ \\
\hline 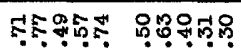 & G99\%? & 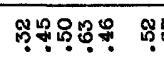 & $\stackrel{\leftrightarrow}{\leftrightarrow}$ \\
\hline 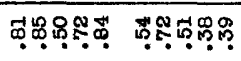 & 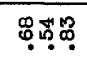 & : & ष \\
\hline 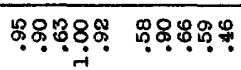 & 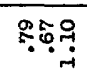 & 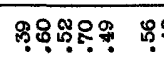 & : \\
\hline 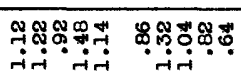 & 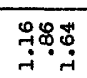 & $\vec{a}_{1} \ldots 1,1$ & ?ִ! \\
\hline 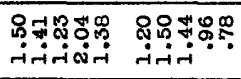 & 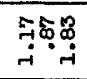 & 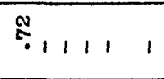 & ִִ \\
\hline 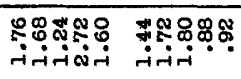 & 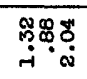 & $\mathscr{\Phi}_{1}$ & 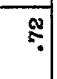 \\
\hline 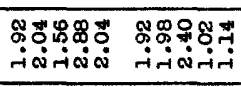 & 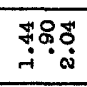 & $\stackrel{8}{8} 111$ & 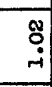 \\
\hline 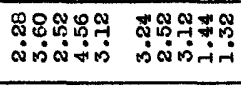 & 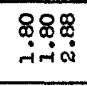 & & $\stackrel{8}{8}$ \\
\hline
\end{tabular}

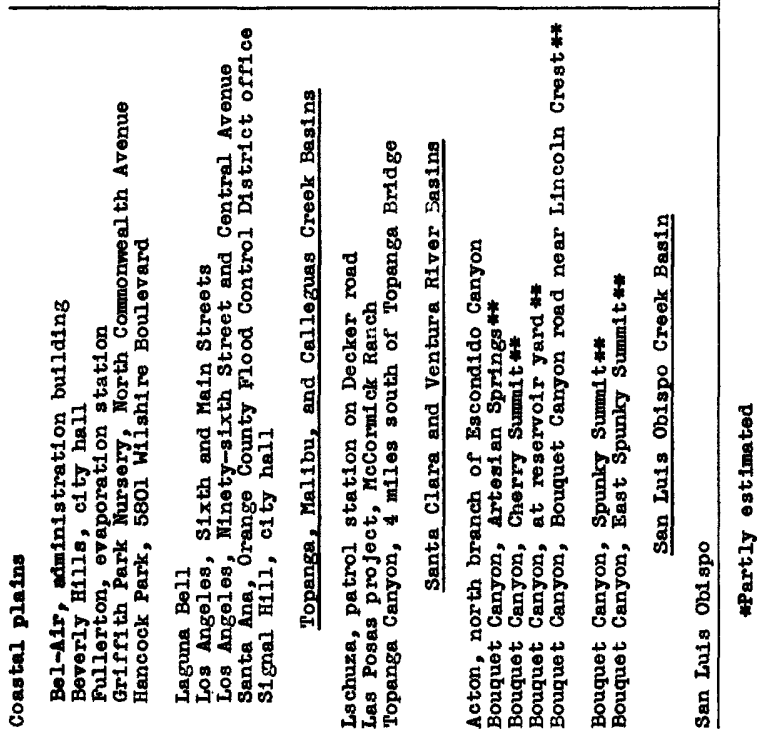


Table 8 lists the stations in the severgl groups and gives for each the latitude and longltude, altitude, and recorded rainfall for the total storm perlod and for March 2 , the day of heaviest rainfall. General descriptions of the different groups follow.

Group A. Stations at relatively high altitudes on the slopes of the second mountain range to the north and east of the zone of heaviest rainfal1.

Group B. Stations near or just beyond the first divide and in the zone of heaviest rainfall in the San Gabriel and San Bermardino Mountains.

Group C. Stations on the coastal side of the first divide in a zone of heavy though somewhat less average precipitation than observed at the stations of group B.

Group D. Stations on the coastal side of the first divide at a somewhat lower average altitude and in a zone of somewhat less average precipitation than the stations of group $C$.

Group E. Stations situated along the toe of the San Gabriel Mountains, in a zone of less rainfall than the other groups.

Table 8.- Location, altitude, and storm precipitation, for certain groups of stations in Santa Ana, San Gabriel, and Los Angeles River Basins

\begin{tabular}{|c|c|c|c|c|c|}
\hline Station and group & Latitude & Long 1 & $\begin{array}{l}\text { Altitude } \\
\text { (feet) }\end{array}$ & $\frac{\text { Prec }}{\text { Mar. }}$ & $T$ \\
\hline Group A & & & & & \\
\hline $\begin{array}{l}\text { Chilao, } 1 \text { mile north of } \\
\text { Nemcomb Ranch } \\
\text { Loomis Ranch near junction } \\
\text { of North and Niddle Forks } \\
\text { of Alder Creek }\end{array}$ & $\left|\begin{array}{lll}34 & 20 & 31 \\
34 & 21\end{array}\right|$ & $\begin{array}{lll}117 & 59 & 30 \\
118 & 03\end{array}$ & $\begin{array}{l}5,585 \\
4,300\end{array}$ & $\begin{array}{l}8.62 \\
7.96\end{array}$ & $\begin{array}{l}15.7 \\
12.5\end{array}$ \\
\hline $\begin{array}{l}\text { Mount Gleas on } \\
\text { East Fork of San Gabriel } \\
\text { River } \\
\text { Group B }\end{array}$ & $\begin{array}{lll}34 & 22 & 26 \\
34 & 16 & 35\end{array}$ & $\begin{array}{lll}118 & 12 & 20 \\
117 & 45 & 05\end{array}$ & $\begin{array}{l}5,450 \\
2,500\end{array}$ & $\begin{array}{l}9.41 \\
9.30\end{array}$ & $\begin{array}{l}16.01 \\
15.75\end{array}$ \\
\hline $\begin{array}{l}\text { amp Baldy, San Antonio } \\
\text { Canyon }\end{array}$ & 341415 & $11739 \quad 15$ & 4,320 & 210.77 & 019.5 \\
\hline $\begin{array}{l}\text { Crystal Lake, Fast Pine Flat } \\
\text { Hoegee's camp, West Fork of } \\
\text { Santa Anlta Creek }\end{array}$ & $\begin{array}{lll}34 & 19 & 10 \\
34 & 12 & 30\end{array} \mid$ & $\begin{array}{lll}117 & 50 & 45 \\
118 & 02 & 30\end{array}$ & $\begin{array}{l}5,740 \\
2,750\end{array}$ & $\begin{array}{l}13.17 \\
14.87\end{array}$ & $\begin{array}{l}24 . \\
29 .\end{array}$ \\
\hline $\begin{array}{c}\text { Opid's camp, West Fork of } \\
\text { San Gabriel River } \\
\text { Group C }\end{array}$ & $\begin{array}{lll}34 & 15 & 15\end{array}$ & $\begin{array}{lll}118 & 05 & 45\end{array}$ & 4,350 & 15.70 & 27.22 \\
\hline $\begin{array}{l}\text { Devil Canyon experimental } \\
\text { station }\end{array}$ & $\begin{array}{lll}34 & 13 & 00\end{array}$ & $117 \quad 19 \quad 40$ & 2,700 & a9.37 & 015.30 \\
\hline $\begin{array}{l}\text { Monrovia Falls, Monrovia } \\
\text { Canjon }\end{array}$ & 341115 & 1175900 & 1,450 & a7.32 & al6 \\
\hline $\begin{array}{l}\text { Mount Wilson } \\
\text { San Antonio guard station } \\
\text { San Dimas Experimental For- } \\
\text { est, Tanbark Flats } \\
\text { GroupD }\end{array}$ & $\left|\begin{array}{lll}34 & 13 & 20 \\
34 & 10 & 45 \\
34 & 12 & 21\end{array}\right|$ & $\begin{array}{lll}118 & 03 & 20 \\
117 & 40 & 28 \\
117 & 45 & 34\end{array}$ & $\begin{array}{l}5,850 \\
2,450 \\
2,700\end{array}$ & $\begin{array}{r}12.58 \\
9.89 \\
11.72\end{array}$ & \\
\hline $\begin{array}{l}\text { Henninger Flats, Mount Wil- } \\
\text { son toli road } \\
\text { Pickens Canyon } \\
\text { San Gabriel River at mouth } \\
\text { of canyon }\end{array}$ & $\left|\begin{array}{lll}34 & 11 & 30 \\
34 & 15 & 15 \\
34 & 09 & 48\end{array}\right|$ & $\begin{array}{lll}118 & 05 & 15 \\
118 & 13 & 00 \\
117 & 54 & 17\end{array}$ & $\begin{array}{r}2,650 \\
4,250 \\
800\end{array}$ & $\begin{array}{l}8.91 \\
8.67 \\
7.25\end{array}$ & $\begin{array}{l}17.44 \\
17.35 \\
14.82\end{array}$ \\
\hline
\end{tabular}

a Incomplete record. 
Table 8.- Location, altitude, and storm precipitation, for certain groups of stations in Santa Ana, San Gabriel, and Los Angeles River Basins--Con.

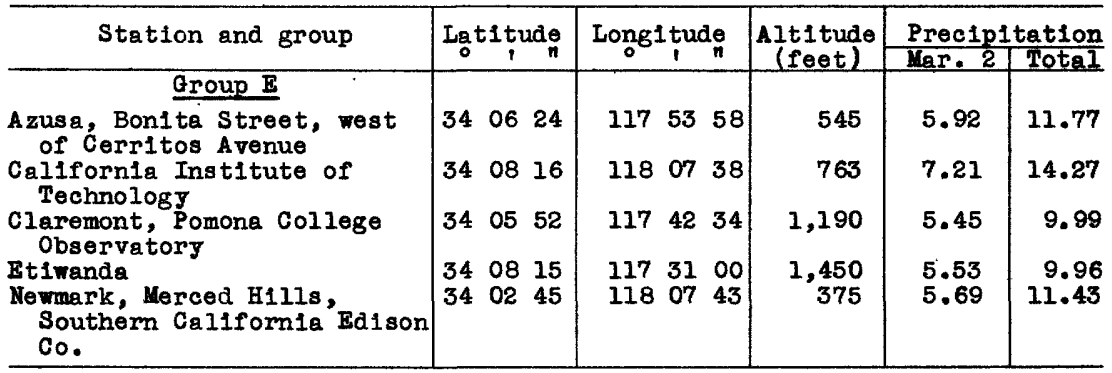

The rainfall characteristics at the stations in these groups are believed to be representative of those of much of the area in which the greater part of the flood runoff originated. The date collected at these stations have therefore been analyzed in detall. For the three stations at which the recording rain-gage record is incomplete, the missing part was computed from additional readings made by observers and from mass curves similar to those in figure 9.

Figure 11, besed on data in table 6 , shows graphically for each group of stations the average number of hours of rainfall at rates in excess of those indicated, and also the percentage of the total precipitation that fell at rates in excess of those indicated. The graphs show the increase In the intensity of rainfall from the group E stations, along the toe of the mountains, to the group B stations, in the belt of heaviest precipitation near or just beyond the first divide, and a decrease from the group B stations to the group A stations, which are located farther north and some of which are high on the slopes of the secondary divide.

Figure 12 gives, for each group of stations, the average hourly rainfall throughout the storm period based on the recording-gage data, and figure 13 gives, for each group, the accumulated precipitation in the form of mass curves, determined from the data given in figure 12.

All stations show two outstanding periods of intense precipitation, separated by an interval of about 20 hours on March 1, when little or no rain fell at any station. In general, little flood munof occurred durIng the first major storm period, and the maximum flood runoff in all the arees was associated with the precipitation during the second major storm perlod, the third phase of the storm, which occurred March 2 . It was the rain that fell on this day that was mainly responsible for the intense flood runoff, and the storm characteristics of this third phase have therefore been further analyzed. 

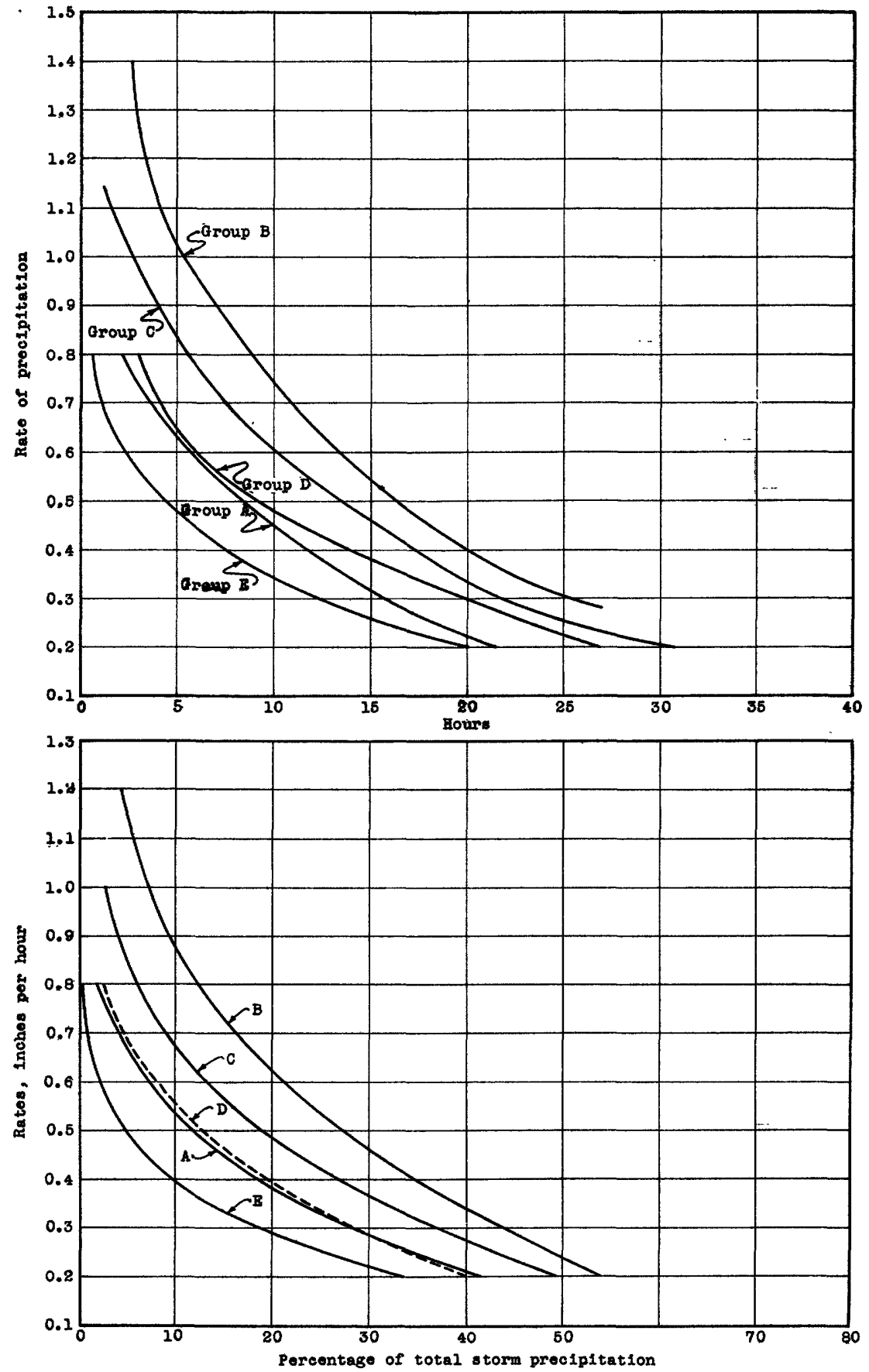

Figure 11.- Hours of preclpitation and percentage of total, in excess of Indicated rates, for groups of stations $A$ to $E$ in San Gabriel and San Bernardino Mountalns and footh111 areas. 


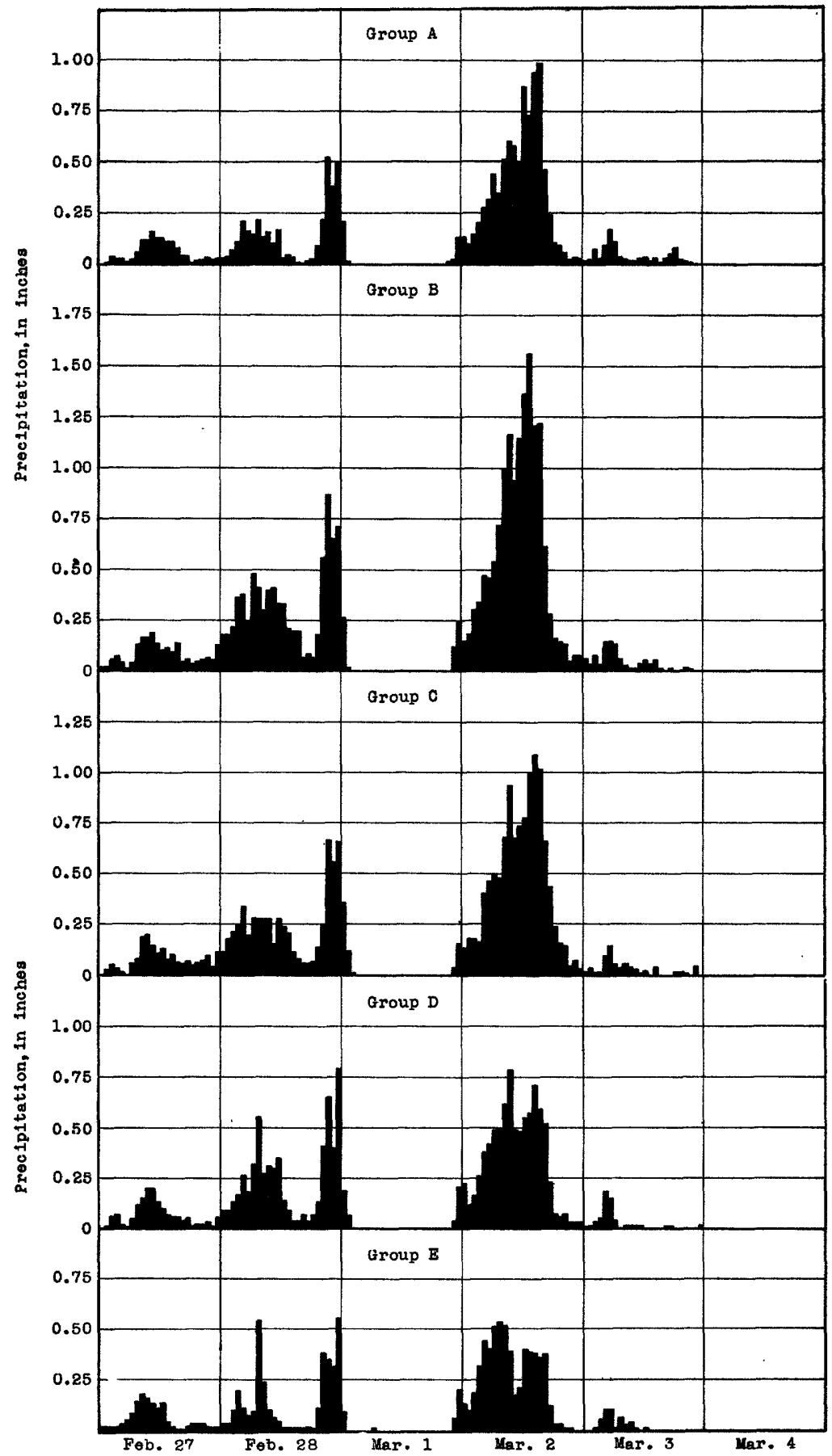

Figure 12.- Average hourly precipitation for groups of stations $A$ to $E$. 


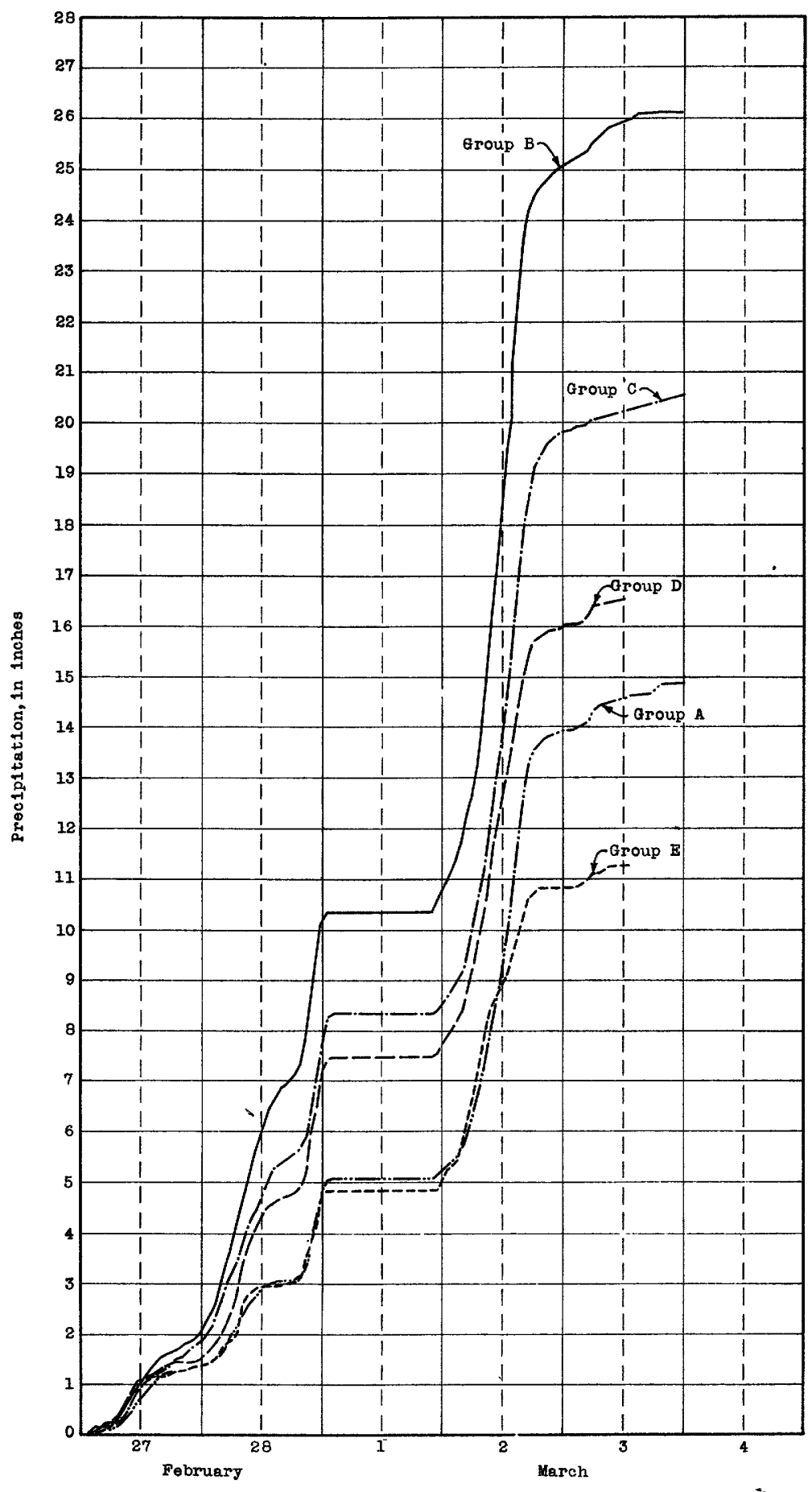

Figure 13.- Mass curves of accumalated precipitation for groups cf stations A to $\mathbb{B}$. 


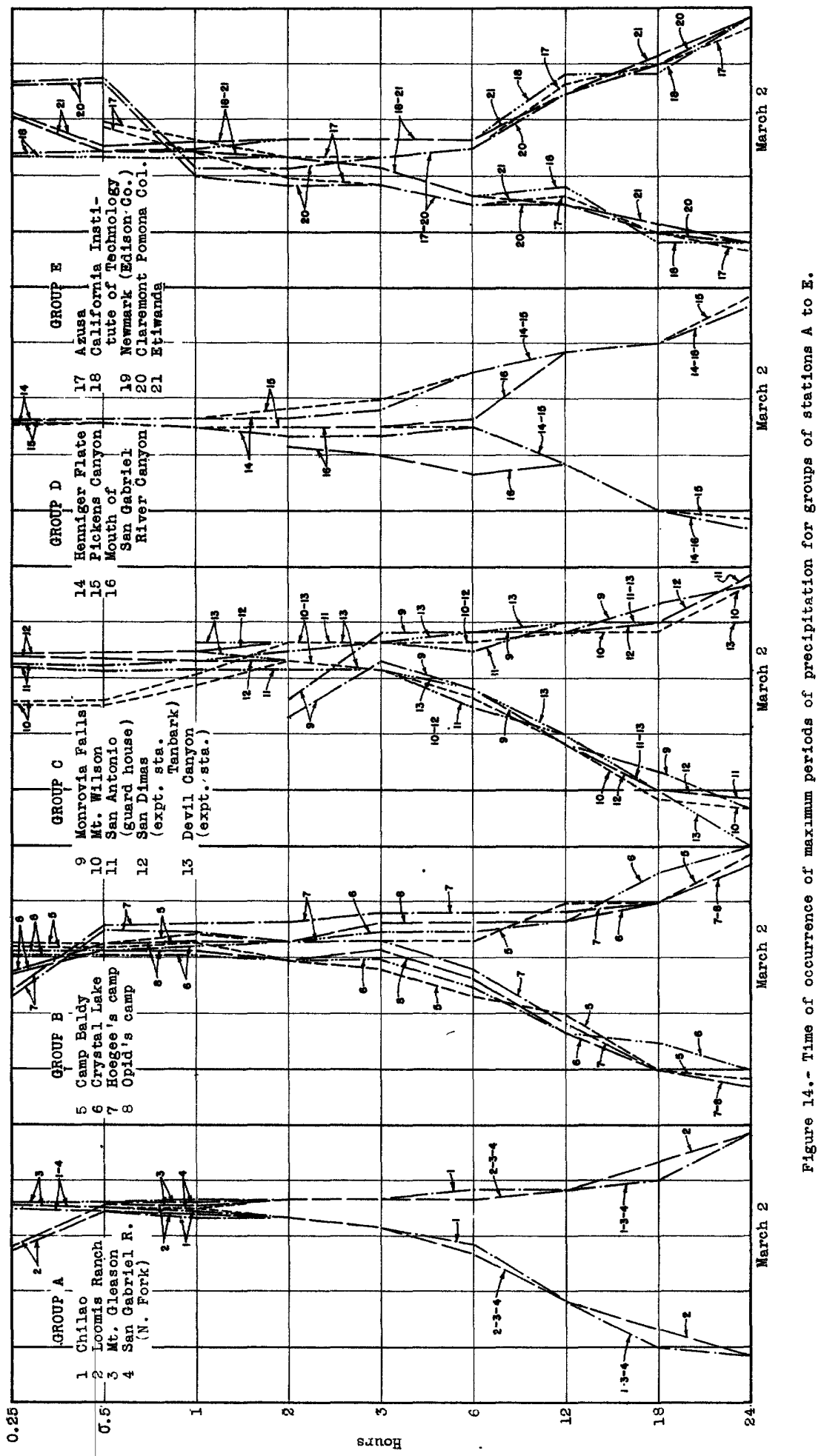




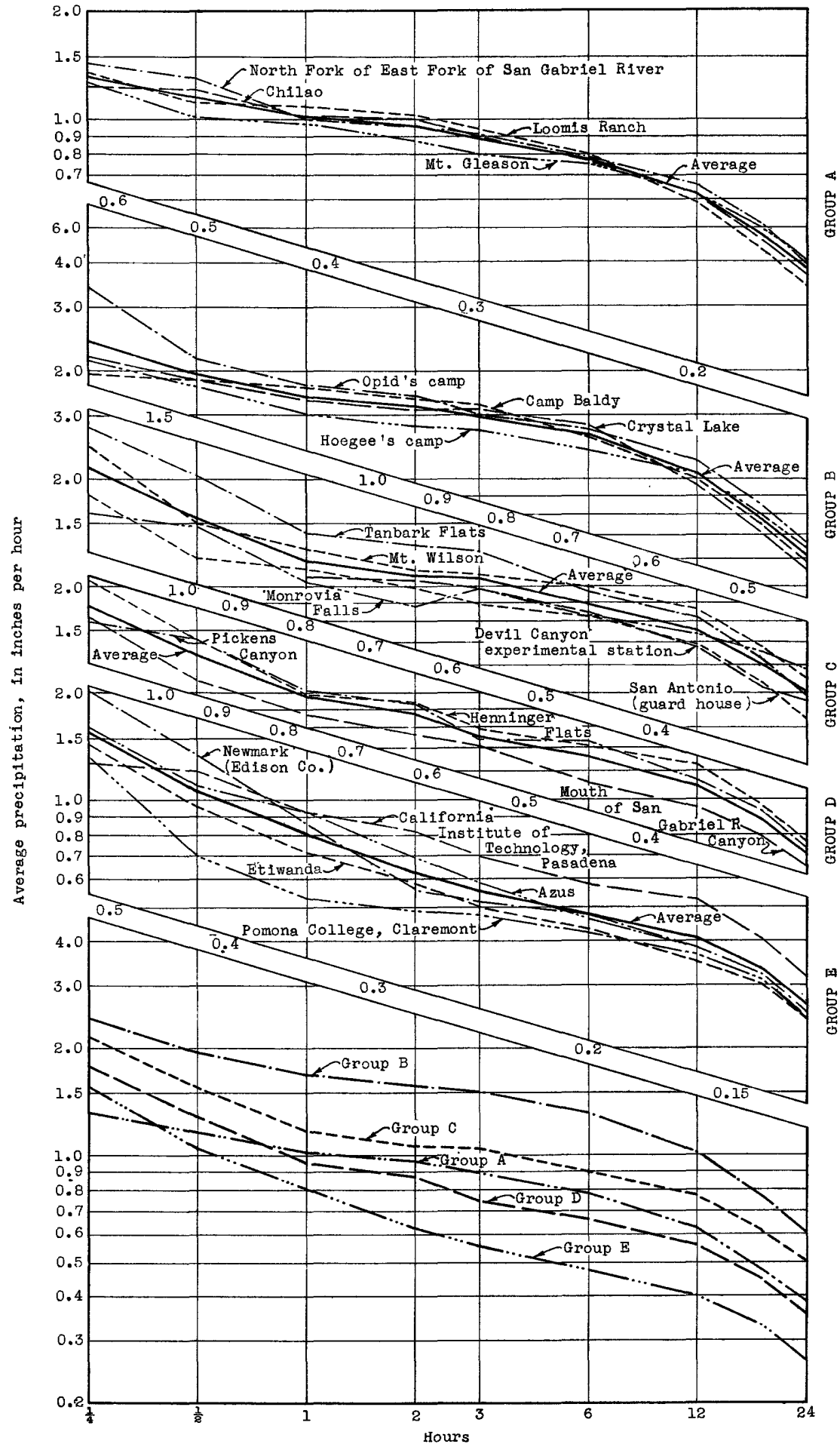

Figure 15.- Rates of precipitation for groups of stations $A$ to $E$. 
Figure 14 gives the time of occurrence of the maximum rate of rainfall at the stations of groups $A$ to $E$, during the second major storm period, for consecutive periods of 15 minutes to 24 hours. The rates of precipitation for periods of less than 15 minutes often reflected purely local storm conditions not characteristic of any large area. It may be observed that, with the exception of group $E$, each maximum period usually occurred within the succeeding longer maximum period. Generally, if a shorter maximum period occurred outside the succeeding longer maximum period, the rate during the shorter maximum period was only slightly higher than the rate for corresponding periods within the longer maximum period.

Figure 15 shows the observed rates of precipitation at each station in groups $A$ to $E$ for periods ranging from 15 minutes to 24 hours on March 2. These rates are presented logarithmically, because on this type of projection, the tendency for lines to be parallel indicates similar characteristics of rainfall distribution. Considering the scattered locations of these stations, the close agreement for groups $A$ and $B$, in the San Gabriel Mountains, is significant.

In the San Bernardino Mountains there were no recording rain gages in the area characterized by the group $B$ type of rainfall. However, the record of the 8-inch standard rain gage at Lake Arrowhead (altitude; 5,000 feet) showed characteristics of precipitation similar to group $B$. On the basis of readings made at approximately 2-hour intervals, this record indicated a rate of 1.55 inches per hour for the maximum 2-hour period, 1.15 inches per hour for the maximum 6-hour period, 0.98 inch per hour for the maximam 8-hour period, and 0.88 inch per hour for the maximum 10-hour period. The station at Big Bear Lake Dam, situated to the east of this station, might also be considered in group $B$, as the record from its standard gage showed 1.06 inches per hour for the maximum 5-hour period and 1.00 inch per hour for the maximum 10-hour period.

At the bottom of figure 15 the average graph for each of these groups of stations is given. The precipitation represented by groups $A$ and $B$ is characterized by a relatively greater prevalence of rainfall at higher rates than for the other groups. The rate for the maximum 15minute period was 3.47 times that for the maximum 24-hour period in group $A$ and 4.08 times that in group B. The rates for the maximum 1-hour, 2-hour, 3-hour, and 6-hour periods were 2.68, 2.51, 2.29, and 2.02 times, respectively, that for the maximum 24-hour period in group A. The 
corresponding ratios for group B were $2.84,2.63,2.45$, and 2.17 . It was the precipitation of this type that produced the major part of the runoff on such streams as the San Gabriel River and San Antonio and Cucamonga Creeks.

The average graphs for groups $C$ and $D$ show storm characteristics similar to each other but different from those found for groups $A$ and $B$. For perlods shorter than 2 hours, the average curves for groups C and D curve upward more sharply than for groups $A$ and $B$. The rate for the maximum 15-minute period for group $C$ was 4.32 times the maximum 24-hour rate; the corresponding ratio of the maximu 15-mimute and 24-hour periods for group D was 5.00. For perlods of 1 hour, 2 hours, 3 hours, and 6 hours, the graph for group C indicated precipitation rates $2.34,2.10,2.06$, and 1.78 times the maximum 24-hour rate; for group D, the corresponding ratios were $2.76,2.44,2.12$, and 1.85 . On small streams this type of precipitation would tend to produce sharp peak discharges.

The average curve for group $E$ on figure 15 is characterized by even shorter perlods of the heavier rainfall; the maximum rate for a 15-mimute period was 5.96 times that for a 24 -hour period. For periods of 1 hour, 2 hours, 3 hours, and 6 hours, the ratios were $3.07,2.38,2.11$, and 1.81 , respectively. The resulting runoff would tend to have even sharper peaks than any of the other groups. In many of the smaller canyons at the lower altitudes in the vicinity of Pasadena, Monrovia, and Ia Cañada, the characteristics of flood flow were determined from this type of rainfall.

Rainfall stations located on the coastal plain and in the Verdugo, Santa Monica, and Santa Ana Mountains all showed characteristics very similar to those indicated by group $\mathrm{E}$.

The five groups of stations indicate factors that might tend to produce a heavier and more continuous flow for the higher altitudes than for the lower altitudes.

The data on intensity for the part of the storm period when the rainfall was heaviest have been correlated with observed rates of runoff at the reservolrs maintained by the Los Angeles Flood Control District and other agencies, and from the relations thus obteined many missing records have been fllled in, especially for the perlod of major flow at a considerable number of river-measurement stations where, for reasons elsewhere explained, observations of stage and discharge were interrupted or lost during the flood perlod. 


\section{Comparison of rates of intens 1ty with rates in previous storms}

The records from several typleal recording rain-gage stations cover a period sufficiently long to make possible a comparison between intensities of rainfall during the storm of March 1938 and several previous porlods of heavy rains. Burke's report 11 conteins a table listing the rainfall for selected periods of time during the March 1938 storm and maxima for previous storms at nine stations in Los Angeles County. This interesting table, somewhat modifled, is included in this report as table 9. It indicates that, except at Hoegee's camp, Clear Creekstation, and Camp Baldy, the rates of raintall for perlods of 30 minutes or less during the 1938 storm were lower than have previously been recorded during the relatively short time that the gages have been in operation. According to Burke the maximum rate of precipitation for a 5-minute period recorded during the 1938 storm at Opid's camp (4.20 inches an hour) has been exceeded four times during the 12 years of record. The maximum 5-minute rate of 2.52 inches an hour observed at Los Angeles during this storm has been exceeded 17 times during the 38 years of record. For longer periods up to 24 hours, the rates of rainfall observed during the $1938 \mathrm{storm} a \mathrm{p}-$ proach the previous maximum rates, and at three of the nine locations exceed them. At Opld's camp the rate of 1.80 inches for the maximum 1-hour perlod recorded on March 2, 1938, has been exceeded only once in the 12jear perlod of record, and the rate for the maximum 24-hour period was the highest ever observed. At Los Angeles, the rate of 0.90 Inch for the maximum 1-hour period during this storm has been exceeded only four times in 38 years, and the rate for the maximum 24-hour period has been exceeded only once.

Table 9 further shows that at the Los Angeles station of the United States Weather Bureau during the 38 years of record prior to March 2 , 1938, the maximum 24-hour rate of precipitation occurred during the New Year's storm of 1934. In addition, at each of the other valley-floor and footh1ll stations--Van Nuys, Sierra Madre, Claremont, and Hancock Park-the maximum 24-hour rate occurred during the same storm. It was only in the more mountainous sections of the San Gabriel Mountains, at Opld's Camp, Hoegee's camp, Clear Creek, and Camp Baldy, that the maximum 24hour rate during the 1938 storm exceeded that for the 1934 storm and all other storms in the comparatively short periods of record at these stations. 


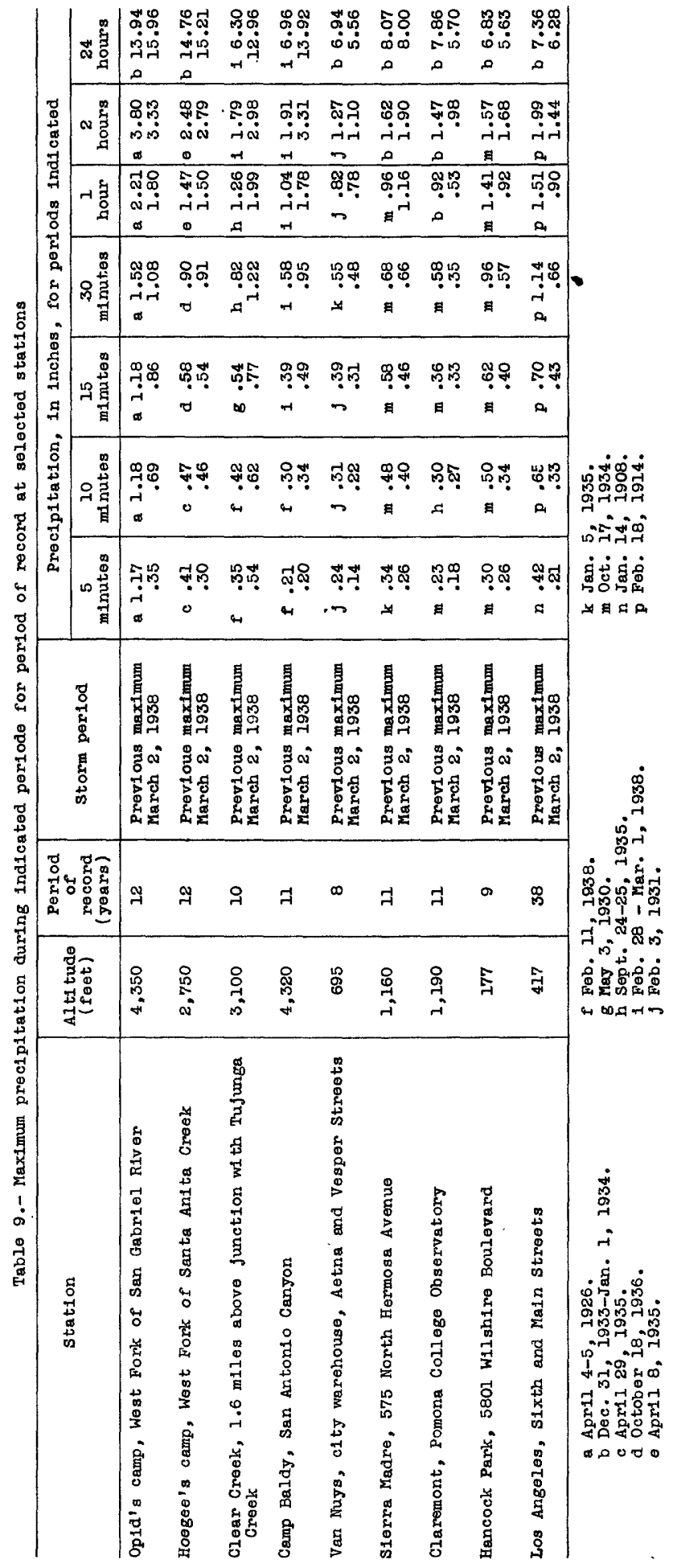


During the 38 years of record at Los Angeles the heaviest rainfall for perlods ranging from 10 minutes to 2 hours occurred on February 18, 1914, and the rates for 10-minute, 15-minute, 30-minute, 1-hour, and 2hour perlods were $2.24,1.84,1.73,1.67$, and 1.38 times as high, respectively, as for the 1938 storm. Thus, the March 1938 rainfall at Ios Angeles did not at any time exceed in intensity rate, that previously recorded.

Records of short-period rainfall intensities during previous floods are not avallable for the San Bernardino Mountains, and a comparison is therefore impossible. However, a comparison of the observations taken in these mountains during the 1938 storm with those taken in the San Gabriel Mountains indicates that conditions in the two ranges were in all probability similar. The following comparison is quoted from the record of storms since 1884 as complied by the Bear Valley Mutual Water Company:

In 1891,32 inches of rain fell in 48 hours at Bear Valley Dam, as compared with 21.75 inches in 1916 and 18 inches in March 1938, for a similar perlod. The maximum 24-hour precipitation of 15.06 inches during the March 1938 storm was exceeded only by the February 22-23, 1891, storm.

In table 10, there are 11 sted, for 25 selected stations at which records are avallable for perlods ranging from 15 to 44 years, the maximum da1ly precipitation recorded prior to March 1938, the maximum for March 1938, and the number of times the latter record has been exceeded. The maximum dally rainfall for the March 1938 storm has been exceeded at 17 of the stations and, on an average, twice at each station. Many of these dally records were not obtained from recording rain gages and so are not strictly comparable with those so obtalned. They seem to ind1cate, however, that in much of the lower part of the region, the maximum dally rainfall during the 1938 storm was not especially extreme. At the two stations of the group having the highest altitudes--Mount Wilson, 5,850 feet, and Camp Baldy, San Antonio Canyon, 4,320 feet--the maximum dally precipltation for the $1938 \mathrm{storm}$ has apparently not been exceeded during perlods of record of 30 and 17 Jears, respectively.

\section{Storm movement}

From the rainfall-runoff studies of the storm of March 1938, it appears that the flood discharge was closely related to the precipitation that fell during the second major perlod of the storm, the 24-hour period from about $10 \mathrm{p} . \mathrm{m}$. March I to $10 \mathrm{p.m}$. March 2. For the area of heavlest precipitation, where there was a fairly complete coverage by recording rain gages, 1sohyetal maps (pl. 14) have been prepared indicating the 
Table 10.- Maximum daily precipitation during March 1938 at selected stations compared with maximum previously recorded.

\begin{tabular}{|c|c|c|c|c|c|}
\hline \multirow[b]{2}{*}{ Station } & \multirow[b]{2}{*}{ Altitude } & \multirow{2}{*}{$\begin{array}{l}\text { Period } \\
\text { of } \\
\text { record } \\
\text { (years) }\end{array}$} & \multicolumn{2}{|c|}{$\begin{array}{l}\text { Maximum daily } \\
\text { precipitation }\end{array}$} & \multirow{2}{*}{$\begin{array}{c}\text { Times } \\
\text { March } \\
1938 \\
\text { maximum } \\
\text { has been } \\
\text { exceeded }\end{array}$} \\
\hline & & & $\begin{array}{l}\text { Prior to } \\
\text { March } \\
1938 \\
\text { (inches) }\end{array}$ & \begin{tabular}{|c|} 
March \\
2 or 3 \\
1938 \\
(inches)
\end{tabular} & \\
\hline $\begin{array}{l}\text { Azusa, Bonita Street, } \\
\text { west of Cerritos Avenue }\end{array}$ & 545 & 44 & 7.18 & 5.86 & 4 \\
\hline $\begin{array}{l}\text { Claremont, Pomona College } \\
\text { Observatory }\end{array}$ & 1,190 & 41 & 7.82 & 5.73 & 2 \\
\hline $\begin{array}{l}\text { Newhall, at Southern } \mathrm{Pa}- \\
\text { cific Co. railroad sta- } \\
\text { tion }\end{array}$ & 1,270 & 39 & 6.02 & 5.38 & 3 \\
\hline $\begin{array}{l}\text { Sierra Madre, north end } \\
\text { of Baldwin A venue }\end{array}$ & 1,110 & 39 & 7.84 & 8.80 & 0 \\
\hline $\begin{array}{l}\text { San Dimas, } 167 \text { North San } \\
\text { Dimas Avenue }\end{array}$ & 963 & 38 & 6.98 & 6.17 & 2 \\
\hline $\begin{array}{l}\text { Acton, fust east of sum- } \\
\text { mit, Mint Canyon Road }\end{array}$ & 3,250 & 35 & 3.75 & 2.82 & 6 \\
\hline $\begin{array}{l}\text { Mount Wilson } \\
\text { Girard, Brant Ranch } \\
\text { Arroyo Seco Canyon, } \\
\text { chlorine plant }\end{array}$ & $\begin{array}{r}5,850 \\
892 \\
1,150\end{array}$ & $\begin{array}{l}30 \\
24 \\
22\end{array}$ & $\begin{array}{l}11.0 \\
4.55 \\
9.40\end{array}$ & $\begin{array}{r}11.83 \\
5.33 \\
8.63\end{array}$ & $\begin{array}{l}0 \\
0 \\
1\end{array}$ \\
\hline $\begin{array}{l}\text { Azusa, Foothill Ranch, } 2 \\
\text { miles west of Citrus } \\
\text { Avenue }\end{array}$ & 615 & 22 & 9.53 & 6.61 & 2 \\
\hline $\begin{array}{l}\text { Claremont, Indian H111 } \\
\text { Road }\end{array}$ & 1,405 & 20 & 7.77 & 5.55 & 3 \\
\hline $\begin{array}{l}\text { Santa Anita Canyon ranger } \\
\text { station }\end{array}$ & 1,950 & 20 & 8.70 & 10.64 & 0 \\
\hline $\begin{array}{l}\text { San Dimas, } \\
\text { Street }\end{array}$ & 960 & 20 & 5.86 & 6.20 & 0 \\
\hline $\begin{array}{l}\text { Montana Ranch, } 3 \text { miles } \\
\text { southwest of Artesia }\end{array}$ & 47 & 19 & 4.00 & 3.24 & 2 \\
\hline $\begin{array}{l}\text { Sylmar, ollve-packing } \\
\text { plant }\end{array}$ & 1,250 & 19 & 5.26 & 4.35 & 1 \\
\hline $\begin{array}{l}\text { Camp Baldy, San Antonio } \\
\text { Canyon }\end{array}$ & 4,320 & 17 & 10.65 & 11.67 & 0 \\
\hline $\begin{array}{l}\text { Diamond Bar Ranch, Spadra } \\
\text { Offlce }\end{array}$ & 675 & 17 & 6.62 & 4.78 & 1 \\
\hline $\begin{array}{l}\text { Glendale, } 811 \text { North Glen- } \\
\text { dale Avenue }\end{array}$ & 653 & 17 & 7.15 & 6.90 & 1 \\
\hline $\begin{array}{l}\text { Pomona, } 987 \text { North Weber } \\
\text { Street }\end{array}$ & 838 & 16 & 5.60 & 4.21 & 3 \\
\hline $\begin{array}{l}\text { Altadena, } 575 \text { Sacramento } \\
\text { Street }\end{array}$ & 1,125 & 16 & 6.91 & 8.20 & 0 \\
\hline $\begin{array}{l}\text { Covina, Puente street, } \\
\text { east of high school }\end{array}$ & 575 & 16 & 8.30 & 5.13 & 1 \\
\hline $\begin{array}{l}\text { San Fernando Lemon Asso- } \\
\text { clation packing house }\end{array}$ & 950 & 15 & 7.55 & 3.03 & 3 \\
\hline $\begin{array}{l}\text { Kinneloa Ranch, east side } \\
\text { of Eaton Wash }\end{array}$ & 1,400 & 15 & 9.20 & 8.70 & 1 \\
\hline $\begin{array}{l}\text { Iamanda Park } \\
\text { Altadena, Ventura Streot }\end{array}$ & $\begin{array}{r}742 \\
1,179 \\
\end{array}$ & $\begin{array}{r}15 \\
15 \\
\end{array}$ & $\begin{array}{r}11.42 \\
5.87 \\
\end{array}$ & $\begin{array}{l}5.70 \\
8.52 \\
\end{array}$ & $\begin{array}{l}1 \\
0\end{array}$ \\
\hline
\end{tabular}

Note.- Data based on report of Los Angeles County Flood Control District.

amount of rain that fell during each 2-hour period from 10 p.m. Karch 1 to 2 a.m. March 2 and each 1 -hour perlod from 2 a.m. to 10 p.m. March 2. In parts of the mountain areas and in San Jacinto River Basin, the isohyetals have been developed from observations at standard gages, the total rainfall being alstributed on the basis of records at the nearest recoraing gage. 


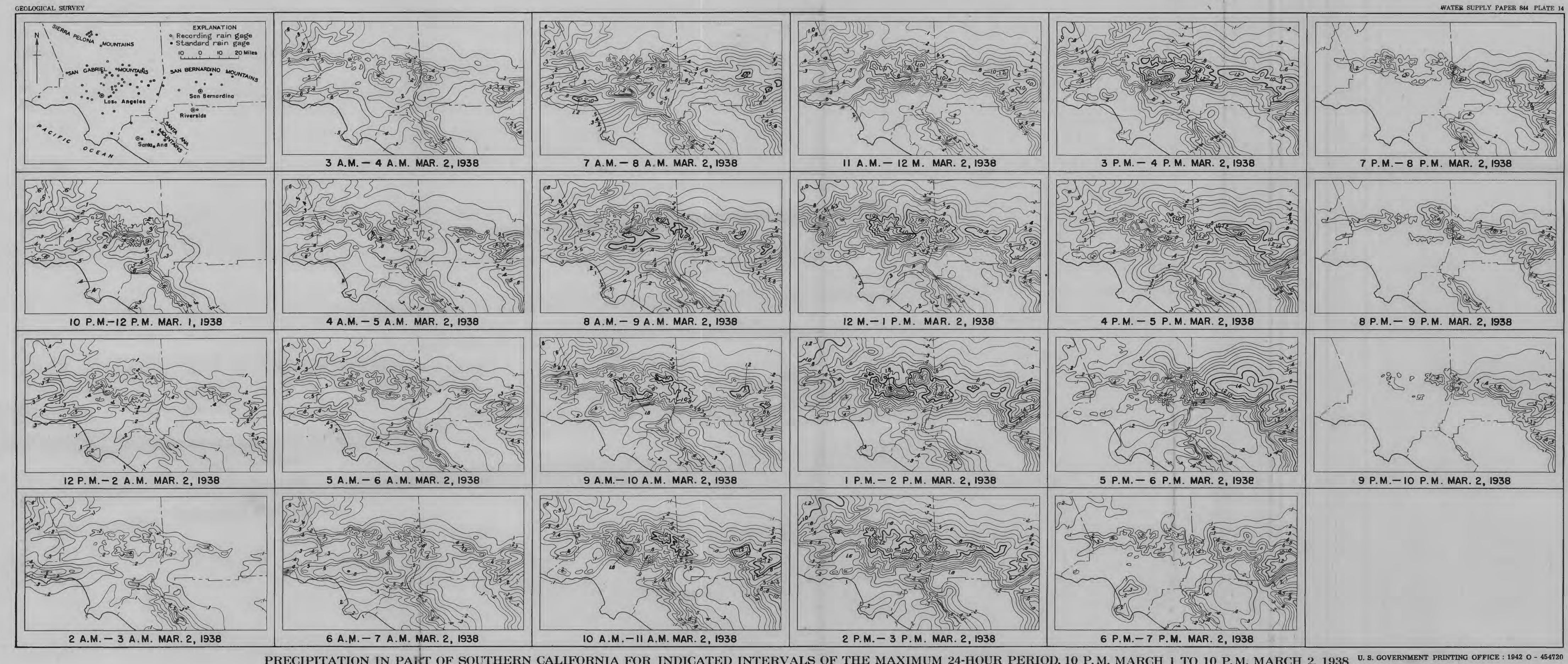

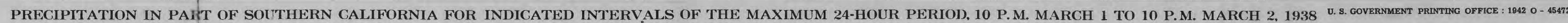


The general eastward movement of the storm and the tendency for the heaviest rainfall to occur in the mountains is clearly indicated by the series of rainfall maps shown on plate 14. With the exception of a period from about 7 to 9 a.m. March 2 , when rain fell at a rate of 1 inch an hour on the coastal plain, the area over which a rate was 1 inch or more an hour lies chiefly in the San Gabriel and San Bernardino Mountains. From about noon until 2 p.m. March 2 rain was falling at the highest intensity and over the largest area in the San Gabriel and San Bernardino Mountains. Following this period there was a general eastwara movement of the storm, and by $7 \mathrm{p} . \mathrm{m}$. the rainfall rate had dropped to 0.1 inch an hour in the central part of the area, and it was only in the extreme eastern part that the rate exceeded 1 inch an hour. By 9 p.m. the rate at all stations was reduced to less than 1 inch an hour.

\section{Antecodent procipitation}

In southern California the greater part of the annual precipitation occurs during a comparatively short rainy season that is followed by a long dry season, during which there is a gradual draining out of the ground water and a loss of moisture through evaporation and transpiration. As the rainy season progresses the absorptive capacity of the ground tends gradually to be fully utilized, and the magnitude of the runoff following any storm therefore depends to a large extent on the time of occurrence of the storm within the reiny season and the amount of antecedent precipitation. Table 11 gives, for each of eight typical stations, the total precipitation and departure from normal for the periods July 1 , 1937, to January 31, 1938, and February 1-25, 1938, and the total departure from normal for the period July 1, 1937, to February 25, 1938. The selected stations are distributed over the area from San Diego on the south to San Iuis Obispo on the north. The stations in the midale part of the table represent the area of heaviest rainfall during the storm of Fobruary 27 to March 4, 1938. The remaining stations are' situated around the margin of the storm area.

From table 11 it is evident that the seasonal precipitation prior to February 1, 1938, was below normal except at Pattiway and Santa Maria. On the other hand, the precipitation for the first 25 days in February was. so greatly above normal as to more than make up for the deficiency prior to February 1. Squirrel Inn and Mount Wilson, located near one of the storm centers, had excesses of 9.67 and 8.32 Inches above normal, respectively, during the first 25 days in February, and similar trends of less 
Table 11.- Precipitation and departure from normal, in inches, at typical stations, for periods from July 1, 1937, to Feb. 25, 1938

\begin{tabular}{|c|c|c|c|c|c|}
\hline \multirow[t]{2}{*}{ Station } & \multicolumn{2}{|c|}{$\begin{array}{l}\text { Precipitation } \\
\text { July } 1,1937 \\
\text { to } \\
\text { Jan. } 31,1938\end{array}$} & \multicolumn{2}{|c|}{$\begin{array}{l}\text { Precipitation } \\
\text { Feb. I-25, } 1938\end{array}$} & \multirow[t]{2}{*}{$\begin{array}{l}\text { Departure } \\
\text { from normal } \\
\text { July 1, } 1937 \\
\text { to } \\
\text { Feb. 25, } 1938\end{array}$} \\
\hline & Total & Departure & Total & Departure & \\
\hline $\begin{array}{l}\text { San Diego } \\
\text { Cuyamaca } \\
\text { Santa Ana } \\
\text { Riverside } \\
\text { San Bernardino }\end{array}$ & $\begin{array}{r}2.13 \\
10.16 \\
4.30 \\
3.01 \\
5.58\end{array}$ & $\begin{array}{l}-3.25 \\
-8.35 \\
-2.40 \\
-2.52 \\
-2.11\end{array}$ & $\begin{array}{l}2.11 \\
8.39 \\
4.95 \\
1.83 \\
4.02\end{array}$ & $\begin{array}{r}+0.30 \\
+1.77 \\
+2.56 \\
-.08 \\
+1.31\end{array}$ & $\begin{array}{r}-2.95 \\
-6.58 \\
+.16 \\
-2.60 \\
-.80\end{array}$ \\
\hline $\begin{array}{l}\text { Squirrel Inn } \\
\text { Mount Wilson } \\
\text { San Fernando } \\
\text { Los Angeles } \\
\text { Oxnard }\end{array}$ & $\begin{array}{r}14.84 \\
11.67 \\
5.41 \\
5.18 \\
5.93\end{array}$ & $\begin{array}{l}-4.06 \\
-1.92 \\
-2.09 \\
-2.63 \\
-.86\end{array}$ & $\begin{array}{r}17.03 \\
14.02 \\
6.82 \\
5.49 \\
3.06\end{array}$ & $\begin{array}{l}+9.67 \\
+8.32 \\
+3.75 \\
+2.75 \\
+1.10\end{array}$ & $\begin{array}{r}+5.61 \\
+6.40 \\
+1.66 \\
+.12 \\
+.24\end{array}$ \\
\hline $\begin{array}{l}\text { Ofai } \\
\text { Pattiway } \\
\text { Santa Barbara } \\
\text { Santa Maria } \\
\text { San Luis Obispo }\end{array}$ & $\begin{array}{r}7.40 \\
4.77 \\
6.55 \\
8.03 \\
11.01\end{array}$ & $\begin{array}{r}-2.79 \\
+.27 \\
-3.09 \\
+.88 \\
-.32\end{array}$ & $\begin{array}{r}9.60 \\
2.45 \\
7.34 \\
6.23 \\
11.68\end{array}$ & $\begin{array}{r}+5.89 \\
+.42 \\
+3.88 \\
+3.77 \\
+7.97\end{array}$ & $\begin{array}{r}+3.10 \\
+.69 \\
+.79 \\
+4.65 \\
+7.65\end{array}$ \\
\hline
\end{tabular}

magnitude are shown for all stations except Riverside.

The effect of this generaliy heavy precipitation prior to the March storm was to increase the soll moisture, to lessen the capacity for storage afforded by surface detention, and in much of the mountain area to replenish, in part at least, the ground-water storage. These conditions tended to increase the direct runoff resulting from the storm of February 27 to March 4 to an amount above what it would have been if there had been less rain in the period just prior to the storm.

The influence of antecedent precipitation is discussed further in the section entitled "Rainfall, runoff, and retention".

\section{$\underline{\text { Snow }}$}

Records of snowfall prior to and during the storm of March 1938 are few and fragmentary. At Bear Valley Dam (altitude, 6,800 feet), on the headwaters of Bear Creek, a tributary of the Santa Ana River in the San Bernardino Mountains, the following observations were made by the president and general manager of the Bear Valley Mutual Water Co.: 12

In this 1938 storm, we estimate that the average depth of snow at the beginning of the storm on the watershed was 18 inches. With 9 inches added at the close of the storm, the appearance of the 'snow cover in the valley was very much the same at the end as at the beginning. At no time did the snow lose its structure or slush down. The rain seemed to pass right through it and come runing out under the snow. Temperatures early In the storm of March 2 were $36^{\circ}$ to $38^{\circ}$, but about $4 \mathrm{a} \cdot \mathrm{m}$. on March 3 the temperature dropped to $28^{\circ}$, the precipitation changed to sleet and snow, and the runoff decreased immediately. 
At the Big Pines station of the Los Angeles County Flood Control District (altitude, 6,860 feet), on the desert side of the San Gabriel Mountains, in the Great Basin, 19 inches of snow was reported on the ground on February 27, 7 Inches fell during the storm, and 23 inches remained on March 4. Table Mountain station (alt1tude, 7,500 feet), a short distance from B1g Pines station, recorded 32 inches of snow on the ground on February 27, a fall of 8 inches during the storm, and 27 inches remaining March 4. At a few other stations the records are not so complete, owing to infrequent observations.

At Kelly's Kamp (altitude, 8,300 feet), in the San Antonio Creek Basin, 72 inches of snow was reported on the ground on February 28 and 60 Inches on March 9, but of the 32 inches of storm precipitation, only a trace fell in the form of snow. Mount Gleason station (altitude; 5,450 feet), on the headwaters of Pacolma Creek, recorded 3 inches, of snow on the ground on February 13, 2 inches on February 28, and none on March 15. At Opld's camp (altitude, 4,350 feet), in the basin of West Fork of San Gabriel River, in the San Gabriel Mountains, there was no snow on the ground on February 28, 1 inch on March 3 and half an inch on March 4. These stations represent most of the areas in southern California in which snow is an important factor in seasonal runoff.

These fragmentary observations in the San Bermardino and San Gabriel Mountaing indicate the probable absence of snow at the beginning of the storm period at an altitude of about 5,000 feet or less. Above 6,000 feet there was a snow cover of considerable depth, with a maximum of more than 70 inches in some aress at an altitude of 8,000 feet and above. It appears that at the higher altitudes some snow accumulated during the storm, and that at the lower altitudes there was no material depletion in snow cover. At stations located at an altitude of more than 6,000 feet, some of the precipitation during the later part of the storm occurred as sleet and snow. In general, the snowmelt is belleved to have contributed but little to the flood runoff, and in the headwater areas the flood runoff may have been less than it would otherwise have been because some of the precipitation occurred as snow, which remained on the ground after the storm. Roughly, 30 percent of the area of the basins above the stream-measurement stations in the San Gabriel and San Bernardino Mountains is above 6,000 feet, and 5 percent is above 8,000 feet.

In Ban Diego, Ventura, and Santa Barbara Counties the snowfall is usually light and seldom remains long on the ground. The snowfall in these counties from February 27 to March 4, 1938, was negligible. 


\section{BASIC DISCHARGE RECORDS}

During a major flood it is extremely difflcult, if not impossible, to obtain continuous accurate records of discharge of the streams in southern California. In determining discharge at a stream-measurement station, current-meter measurements are made to establish a rating curve that will show the discharge for any given stage at a given time. Ordinarily the rating curve can be defined by measurements for the usual range of stage, but its definition is more difficult for unusually high stages, and increasingly difficult for flood stages so high as to be of rare occurrence, when conditions for obtaining measurements are most unfavorable.

Complete definition of the rating curve to the highest peak can be obtained only by an adequate number of timely measurements and a record of channel conditions during the flood. In southern California, this ideal is difficult to attain owing to the fact that it is practicaliy impossible to make current-meter measurements during the higher flood stages. The streams have flashy floods with excessively high velocities and usually move large quantities of debris and suspended material. Their flood crests frequently occur almost simultaneously throughout the area, and often during the night. The fleld engineer is further handicapped during flood perlods by washed-out or obstructed routes of travel and at times by damage to bridges or cableways from which discharge measurements are made.

For many streams in both the mountain and valley areas, the continual shifting of their channels owing to scour, f1ll, or bank cutting make It impossible to develop a stage-discharge relation even if it is possible to obtain a large number of measurements. On the Los Angeles River at Los Angeles, 44 current-meter measurements made in the period February 28 to March 5 showed that no stage-discharge relation could be defined. For this reason many of the gage-helght records in the main flood area were of little value as an indication of the magnitude of the flood runoff.

There were, however, within the flood area a number of flood-control reservolrs from the records of whose contents it was possible to compute falrly satisfactory records of muoff for the flood period. Similar records were avaliable for certain reservolrs that are maintained for purposes other than flood control. For a, small number of other streams, also, the records of discharge through the flood rise are belleved to be 
rellable with respect to rates of flow for short periods. The aforementioned records are presented herein as "basic discharge records" and are analyzed in relation to rainfall rates and other pertinent factors in order to develop relations which are of general interest and which may be used in interpreting the often meager data obtainable at streammeasurement stations.

In measuring the outflow from these reservoirs, the ratings for the valves and spillways are based mainly on current-meter measurements, a.lthough a few are based on model ratings. The operation of the release valves in the dams made it necessary to compute the inflow and outflow from the reservoir for intervals of time between readings of the valve openings. This opening and closing of the valves necessarily affects the record of stage in the reservoir and makes difflcult the sccurate determination of inflow data for very short intervals of time. At some reservoirs the operation of these valves resulted in so much drawdown at the water-stage recorder that the record was not a correct index of inflow. For such periods observations made on staff gages, which are not affected by drawdown, were used as a true index of reservolr stages. During the periods of greatest discinarge the staff gages were read frequently.

Shortly after the floods the flood-control reservolrs were resurreyed by the Califormia Institute of Technology, the Los Angeles'County Flood Control District, and the United States Soll Conservation Service, and new capacity tables were prepared. In determining the inflow to the reservolrs the older capacity tables were used prior to the time of the maximum discharge and the new capacity tables subsequently.

The estimated storage, which is added to or subtracted from the spillway discharge and talve release in order to determine the inflow, was based on the area of the water surface at the time of observation, the rate of change in area,. and the rate of change in the altitude of the water surfaces. The surveys made after the storm period indicate that the area of the water surface for each gage helght had been decreased somewhat by debris deposition, with the higher gage heights showing the least change in area. The shift of capacity tables at the time of the maximum discharge has the tendency to increase the computed inflow on the rising stages during the periods of greatest runoff and to decrease to a much slighter degree, however, the computed inflow on the friling stages. 
Most of these reservolrs were practically full prior to the periods of maximum discharge, so there was little fluctuation in the area and altitude of their water surfaces during these perlods. The rather small capacity of the reservoirs found in these steep, narrow canyons tends to reduce further the influence of change in storage on the inflow computations. It is under these conditions that the change in storage factors would have the minimum influence on the inflow computations.

The computed inflow to these reservolrs includes not only the water but the debris that was carried with it and deposited in the reservoirs. As incoming debris entered the upper end of a reservoir, it caused the displacement of water at a rate and in a volume equivalent to its own. The section on debris (pp. 372-376) discusses the deposition of debris in the reservoirs. Theoretically, debris movement varies with the fifth or sixth power of the velocity. Since the highest velocity is associated with the greatest discharge, it might be assumed that the greatest inflow of debris would also be associated with the greatest discharge. Avail$a b l e$ information indicates that streams tend to carry more debris on $\mathrm{ris}-$ ing than on falling stages. For this reason it may be assumed that most of the inflow of debris occurred during the greater discharges.

Table 28 (p. 374) shows, in acre-feet per square mile of drainage area, the debris deposited in each of 13 reservoirs, as determined from surveys before and after the flood, and the inflow to each for the maximum 24 hours. In the aggregate, the debris inflow is computed to be between 8 and 9 percent of the maximum 24-hour inflow, with the percentages for individual reservolrs ranging generally from 5 to 15. Probably the debris was associated mostly with the rising side of the flood hydrograph and with perhaps less than half of the volume of flow in the maximum 24 hours, thus apparentiy increasing the content of debris considerably above the percentages indicated. The significance of this complicating factor in relation to the computed inflow and the correlation of rainfall and runoff rates must be kept in mind.

The complicating factor of debris enters into the measurement of the flood flow of all the mountain streams of southern California, as is pointed out in other places in this report. The reservoir records permitted a more reliable determination of the flow including the debris than was possible at any of the gaging stations. The records are especlaliy valuable in furnishing information as to the volume of debris 
borne by the flood waters of natural streams, this being a subject on which reliable data are very meager.

The inflow and outflow for the flood-control reservolrs during the main part of the storm perlod and the peak discharges have been computed by the Geological Survey from data furnished by the Los Angeles County Flood Control District, but these data were not complete at the time this report was prepared. Owing to differences in interpretation of the basic data, some of the results differ by relatively small amounts from those reported by the flood-control District.13 The records for Morris Reservoir were computed and furnished by the City of Pasadena Water Department. The records for Santiago Creek Reservoir were computed and furnished by the Corps of Englneers and the Orange County Flood Control District.

Records were furnished by the United States Forest Service for two basins in Fern Canyon, designated Watersheds Nos. 2 and 3 , having drainage areas of 0.063 and 0.084 square mile, respectively. No runoff records were obtained from the Watershed No. 1 because of damage to the measuring equipment. These watersheds are small, contiguous basins lying along the eastern divide of the San Dimas Creek drainage area, within the San Dimas Experimental Forest. (See pl. 15.) They have an average altitude of about 5,000 feet and an average slope of about 65 percent. The soil mantie varies in depth from less than 1 foot to more than 2 feet, with an average of about 1 foot. At the time of the flood of March 1938, each basin had a fairly heavy and uniform chaparral cover. At the lower end of each basin is a concrete dam, founded on bedrock, above which is a concrete-ilned reservoir with a capacity of 10,000 cubic feet, bullt to trap the erosional debris. The runoff of these basins was measured by a modifled 3-foot Parshall flume and a $90^{\circ} \mathrm{V}$-notch weir.

The United States Forest Service has also made available basic records on Wolfskill Creek and West Fork of San Dimas Creek, in the San Dimas Creek Basin. In addition, the United States Soll Conservation Service maintained several broadcrested-weir gaging stations on Honda Barranca in the Los Posas area, for which records are presented.

In general there are given for each reservoir a description of the station, a table of mean daily and monthly inflow to the reservolr

13 Los Angeles County Flood Control District, Blennial report on hydrologic data, seasons 1936-37 and 1937-38, June $30,1939$. 
throughout the 3-month period February to April 1938, a table of hourly or bihourly inflow for the period February 27 to March 10, 1938, and corresponding tables of outflow. The presentation of the data has followed a uniform plan as far as practicable.

The station description gives, in the first paragraph, the location of the reservolr and the altitude of the stream bed at the dam. A second paragraph gives characteristics of the drainage area upstream from the dam. Each drainage area as measured on the most recent topographic maps, and its average altitude was determined by planimeter. It was then divided into small areas, ranging from 1,000 feet to 1 mile square each, and the average slope of each was estimated. The average of these est1mates was taken as the average slope for the entire area. The average slope of the main stream channel was obtained by dividing the difference between the maximum and minimum altitudes of the channel by the length of the channel as scaled from the topographic maps. The next paragraph, relating to the gage-height record, gives the method of determining the stage during the flood. In a fourth paragraph is given the method of computing the discharge. This is followed by a paragraph giving the maximum discharge and the time of its occurrence. The method of estimating the maximum discharge is discussed later. The sixth and last paragraph, under the heading "Remarks", begins with a statement concerning the reliability of the record; it then gives the altitude of the spillway, the area of the reservolr at the spillway, the capacity of the reservoir before and after the floods, a statement concerning storage or diversion upstream from the reseryoir, and other miscellaneous information. Similar descriptions for stations other than those at reservoirs were also furnished.

Following each description, if the records are avallable, is a table showing the mean daily inflow in second-feet, and the monthly inflow in acre-feet for the period February 1, 1938, to April 30, 1938. This covers the actual flobd perlod and sufficient time before and after it to establish the relations of flood discharges to the prevalent discharges and to give a general perspective of the March flood. The table of inflow at indicated times was obtained from inflow hydrographs developed on the basis of the gage-height record from the water-stage recorder on the reservoir, supplemented by staff readings and records of valve operation. The table of outflow from each reservolr is presented immediately following the table of inflow. 
要

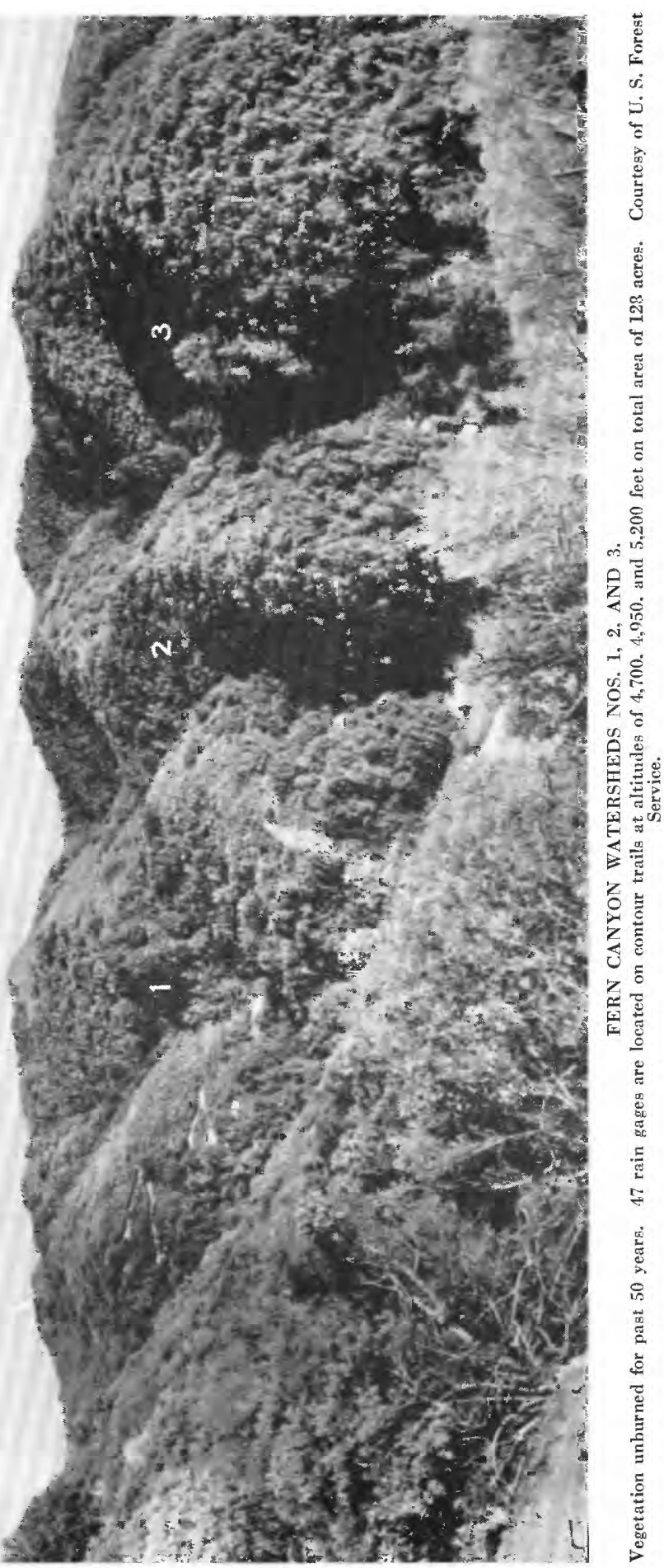



The outflow from the reservolrs was governed mainly by the opening and closing of valves, resulting in a steplike hydrograph. Dally discharges were computed from this steplike hydrograph, but in computing the outflow at indicated times, a smoothed hydrograph was used; the total discharge for any hour is approximately the same computed from elther hydrograph.

Hydrographs of inflow into five of the flood-control reservoirs, namely, those on Tujunga Creok, West Fork of San Gabriel River, Santa Anita Creok, Eaton Creok, and IIve Oak Creok, are show on figure 16. The drainage areas of these streams represent a wide variety of basin characteristics, with that of Eaton Creek having the steepest slope and that of Ilve Oak Creek the gentlest. The West Fork of San Gabriel River had the greatest runoff to the square mile, however, and Ilve Oak Creok the least.

$4547200-42-8$ 


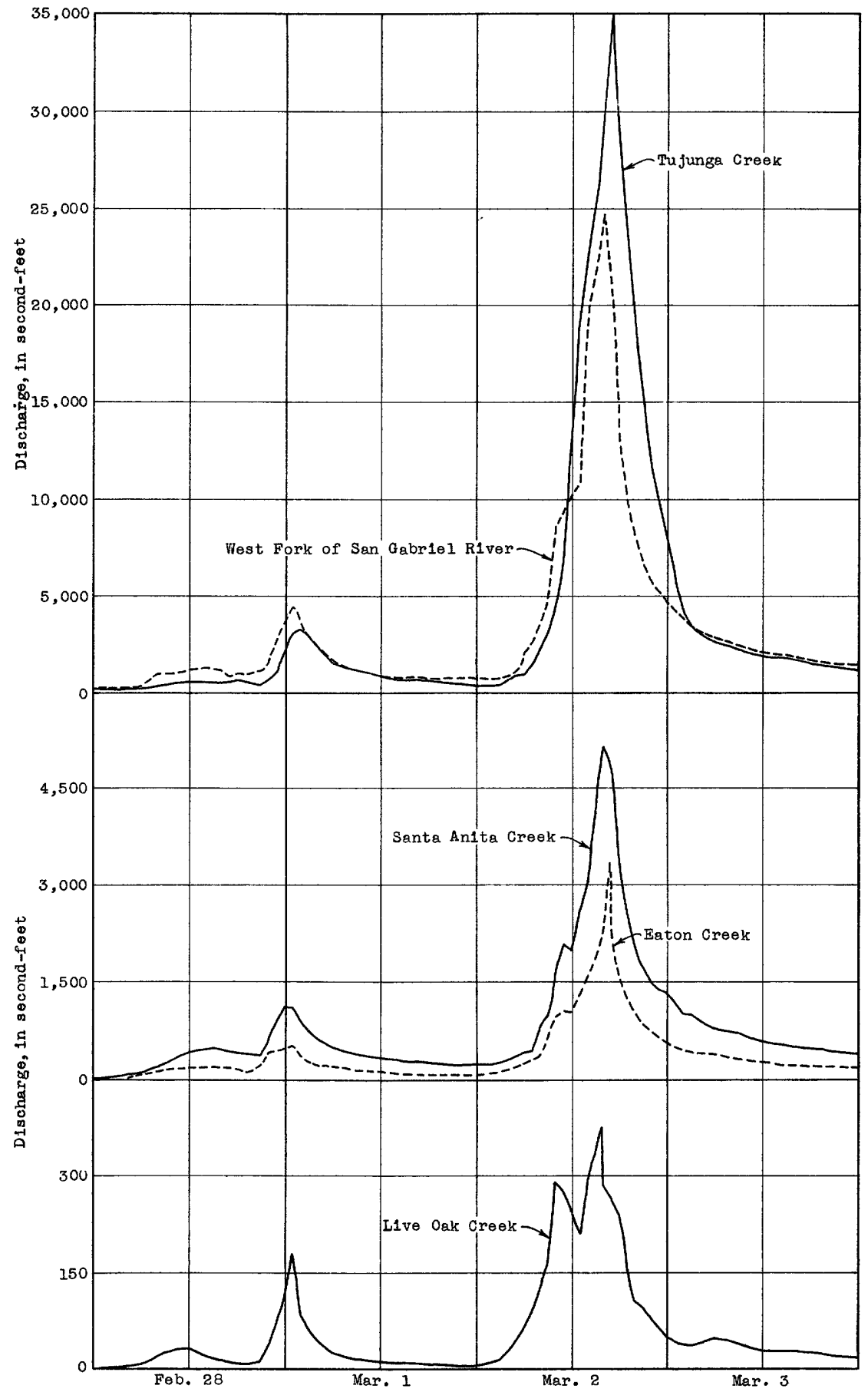

Figure 16.- Graphs of discharge Into various reservo1rs in southern Callforn1a, March 1938. 


\section{Santa Ana River Basin}

Santiago Creok at Santiago Reservolr, near Villa Park, Calif.

Iocation. - Water-stage recorder, 1at. $33^{\circ} 47^{100^{n}}$, Iong. $117^{\circ} 43^{\prime} 35^{n}$, In Lomas de Sant1ago grant, 2 miles east of Orange County Park, and 5 miles east of V1lla Park, Orange

county. Altitude of stream bed, 680 feet.

Drainage area.-Area, 62.5 square miles. Average altitude, 2,120 feet. Maximum alt1tude, 5,680 feet. Average slope, 45 percent. Iength of main stream channel, 13.0 miles. Average slope of maln stream channel, 7.3 percent.

Gage-height record. - Water-stage recorder graph.

Discharge record.- Computed from record of stage, stage-capac1ty tables, and sp111way rating curve.

Maxlma.- 1938: Inflow, 9,850 second-feet 5 p.m. Mar. 2.

Outflow, 5,670 second-feet $9: 30$ p.m. Mar. 2.

Remarks.- Records good. Cepac1ty at sp11iway leve1, 25,000 acre-feet. Reservolr operated by Irvine Co. and Serrano and Carpenter Irrigation Districts. Records furnished by Corps of Engineers, U. S. Army, and Orange County Flood Control District through M. N. Thompson, chief engineer.

Mean da1ly Inflow, in second-fe日t, 1938

\begin{tabular}{|c|c|c|c|c|c|c|}
\hline $\begin{array}{l}\text { Feb. } \\
\text { Mar. }\end{array}$ & $\begin{array}{r}28 \\
1 \\
2 \\
3 \\
4 \\
5\end{array}$ & $\begin{array}{l}\ldots \ldots \\
\ldots \ldots \\
\ldots \ldots \\
\ldots \ldots\end{array}$ & $\begin{array}{r}243 \\
586 \\
4,200 \\
2,430 \\
1,150 \\
803\end{array}$ & Mar. & $\begin{array}{r}6 \\
7 \\
8 \\
9 \\
10 \\
11\end{array}$ & $\begin{array}{l}\ldots \ldots \\
\ldots \ldots \\
\ldots \ldots \\
\ldots \ldots \\
\ldots \ldots\end{array}$ \\
\hline
\end{tabular}

Inflow Feb. 28 to Mar. 11, in acre-feet..25,180

Inflow, in second-feet, at indicated time, 1938

\begin{tabular}{|c|c|c|c|c|c|c|c|c|c|c|c|}
\hline $\mathrm{Hr}$. & Feb. $2 B$ & Mar. 1 & Mar. 2 & Mar. 3 & Mar. 4 & Mar. 5 & Mar. 6 & Mar. 7 & Mar. 8 & Mar. 9 & Mar. 10 \\
\hline $\begin{array}{r}1 \\
2 \\
3 \\
4 \\
5 \\
6 \\
7 \\
8 \\
9 \\
10 \\
11 \\
N \\
1 \\
2 \\
3 \\
4 \\
5 \\
6 \\
7 \\
8 \\
9 \\
10 \\
11 \\
M\end{array}$ & $\begin{array}{r}90 \\
92 \\
92 \\
92 \\
104 \\
150 \\
203 \\
\\
189 \\
184 \\
\\
190 \\
211 \\
249 \\
370 \\
660 \\
1,050 \\
1,420\end{array}$ & $\begin{array}{r}1,600 \\
1,700 \\
1,410 \\
1,000 \\
867 \\
760 \\
655 \\
570 \\
550 \\
512 \\
405 \\
342 \\
\\
321 \\
313 \\
308 \\
299 \\
270 \\
241 \\
224 \\
211 \\
211 \\
220 \\
232 \\
248\end{array}$ & $\begin{array}{r}328 \\
430 \\
500 \\
675 \\
920 \\
1,040 \\
1,280 \\
1,750 \\
2,700 \\
3,060 \\
3,600 \\
4,130 \\
5,300 \\
6,950 \\
7,800 \\
8,650 \\
9,850 \\
9,100 \\
7,800 \\
6,900 \\
5,950 \\
5,270 \\
4,700 \\
4,170\end{array}$ & $\begin{array}{l}3,800 \\
3,400 \\
3,130 \\
3,040 \\
2,980 \\
2,900 \\
2,840 \\
2,800 \\
2,690 \\
2,600 \\
2,470 \\
2,330 \\
2,220 \\
2,120 \\
2,060 \\
2,000 \\
1,930 \\
1,870 \\
1,800 \\
1,710 \\
1,650 \\
1,600 \\
1,550 \\
1,500\end{array}$ & $\begin{array}{r}1,410 \\
1,350 \\
1,300 \\
1,240 \\
1,190 \\
1,130 \\
1,080 \\
1,040 \\
1,010 \\
980 \\
940 \\
910\end{array}$ & 790 & 660 & 580 & 555 & $\begin{array}{l}530 \\
530\end{array}$ & 505 \\
\hline
\end{tabular}

Mean da1ly outflow, in second-feet, 1938

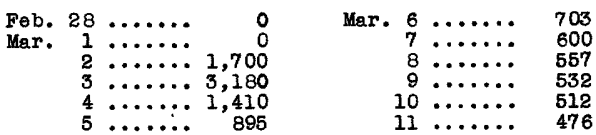

Outflow Feb. 28 to Mar. 11, In acre-feet. 20,960 
Outflow, In second-feet, at Indicated time, 1938

\begin{tabular}{|c|c|c|c|c|c|c|c|c|c|c|c|}
\hline Eir. & Feb. 28 & Mar. 1 & Mar. 2 & Mar. 3 & Mar. 4 & Mar. 5 & Mer. 6 & $\operatorname{Mar} .7$ & Mar. 8 & Mar. 9 & Max. 10 \\
\hline $\begin{array}{r}1 \\
2 \\
3 \\
4 \\
5 \\
6 \\
7 \\
8 \\
9 \\
10 \\
11 \\
5 \\
1 \\
2 \\
3 \\
4 \\
5 \\
6 \\
7 \\
8 \\
9 \\
10 \\
11 \\
4 \\
\end{array}$ & 0 & 0 & $\begin{array}{r}0 \\
0 \\
330 \\
1,070 \\
2,030 \\
3,160 \\
4,270 \\
5,010 \\
5,480 \\
5,630 \\
5,630 \\
5,520 \\
5,310\end{array}$ & $\begin{array}{l}5,060 \\
4,770 \\
4,460 \\
4,180 \\
3,950 \\
3,790 \\
3,650 \\
3,520 \\
3,390 \\
3,270 \\
3,140 \\
3,020 \\
2,900 \\
2,780 \\
2,670 \\
2,570 \\
2,480 \\
2,390 \\
2,310 \\
2,220 \\
2,120 \\
2,040 \\
1,980 \\
1,920\end{array}$ & $\begin{array}{l}1,850 \\
1,800 \\
1,740 \\
1,700 \\
1,650 \\
1,600 \\
1,540 \\
1,510 \\
1,470 \\
1,440 \\
1,410 \\
1,380 \\
1,340 \\
1,300 \\
1,260 \\
1,240 \\
1,210 \\
1,190 \\
1,170 \\
1,150 \\
1,120 \\
1,100 \\
1,080 \\
1,060\end{array}$ & $\begin{array}{r}1,030 \\
990 \\
960 \\
940 \\
910 \\
890 \\
860 \\
840 \\
810 \\
810 \\
790 \\
770\end{array}$ & $\begin{array}{l}760 \\
745 \\
740 \\
728 \\
710 \\
705 \\
685 \\
680 \\
670 \\
660 \\
646 \\
635 \\
\end{array}$ & $\begin{array}{l}580 \\
574 \\
562 \\
\end{array}$ & $\begin{array}{l}560 \\
550 \\
542\end{array}$ & $\begin{array}{l}530 \\
530 \\
530\end{array}$ & $\begin{array}{l}520 \\
512\end{array}$ \\
\hline
\end{tabular}

Supplemental record.- Mar. 2, 9830 p.m. 5,670 sec.-ft.

\section{San Gabriol River Basin}

Ban Gabriel River at flood-control reservolr Ho. 1, near Azusa, Calif.

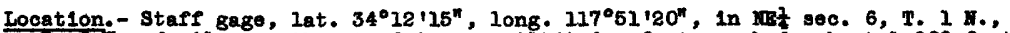
R. 9 ., 6 miles northeast of Azusa. Alt ltude of stream bed, about 1,200 feet.

Drainage area. - Area, 202 square miles. Average altitude, 4,500 feet. Iaximum alt1tude, I0,080 feet. Average slope, 49 percent. Length of main stream channel, 24,5 miles. Average slope of main stream channs $1,6.9$ percent.

Qage-helght record.- Staff gage read da1ly at 8 a.m., more frequently during stcrm period, and at about 4-minute intervals $3-7$ p.m. Har. 2 .

Discharge record. - Computed from record of stage, sp1liway rating table, valve rating curves, valve-operation record, and changes in storage based on record of stage and stage-capac1ty tables; stage-capacity table based on survey of 1937 used to time of peak stage of Har. 2 (7830 p.m.), table based on survey of october 1938 used thereafter.

Maxima.- 1938: Inflow, 90,000 second-feet 4 p.m. Mar. 2.

Outflow, 56,700 second-feet $7: 30$ p.m. Mar. 2.

Remarks. - Records good. Alt1tude of sp111way, 1,453 feet. Area at sp111way level, 523 acres (Oct. 1938). Capac1ty at splilway level befcre storm of Mar. $2,53,334$ acre-feet; after stom, 47, 191 acre-feet. Partial regulation at flood-control dam Ho. 2 cn West Fork of San Gabrlel River, 10 miles upstream. Outelct goes directlJ Into Lorris Reservelr. Basic data Fob. 27 to Mar. II and entire rocord for rest of Pebruary, Narch, and April furnished by Ios Angeles County Flood Control D1strict, through H. B. Hedger, chief engineer.

Mean daily inflow, in second-feet, 1938

\begin{tabular}{|c|c|c|c|c|c|c|c|c|c|c|c|}
\hline Day & Feb. & Mar. & Apr. & Day & Feb. & Mar. & Apr. & Day & Feb. & Mar. & Apr. \\
\hline $\begin{array}{r}1 \\
2 \\
3 \\
4 \\
5 \\
6 \\
7 \\
8 \\
9 \\
10\end{array}$ & $\begin{array}{l}215 \\
129 \\
510 \\
749 \\
420 \\
286 \\
228 \\
206 \\
291 \\
367\end{array}$ & $\begin{array}{r}5,290 \\
31,100 \\
15,700 \\
7,790 \\
4,570 \\
3,730 \\
3,390 \\
3,100 \\
3,480 \\
2,080\end{array}$ & $\begin{array}{l}525 \\
763 \\
699 \\
688 \\
773 \\
747 \\
700 \\
632 \\
648 \\
692\end{array}$ & $\begin{array}{l}11 \\
12 \\
13 \\
14 \\
15 \\
16 \\
17 \\
18 \\
19 \\
20\end{array}$ & $\begin{array}{l}963 \\
903 \\
554 \\
448 \\
378 \\
329 \\
300 \\
294 \\
312 \\
269\end{array}$ & $\begin{array}{l}1,220 \\
4,190 \\
3,400 \\
2,550 \\
2,040 \\
1,470 \\
1,270 \\
1,220 \\
1,170 \\
1,100\end{array}$ & $\begin{array}{l}667 \\
719 \\
595 \\
554 \\
516 \\
488 \\
474 \\
475 \\
496 \\
506\end{array}$ & $\begin{array}{l}21 \\
22 \\
23 \\
24 \\
25 \\
26 \\
27 \\
28 \\
29 \\
30 \\
31\end{array}$ & $\begin{array}{r}253 \\
249 \\
236 \\
239 \\
240 \\
233 \\
419 \\
2,850\end{array}$ & $\begin{array}{r}1,260 \\
1,330 \\
1,280 \\
1,210 \\
1,190 \\
1,110 \\
1,070 \\
1,050 \\
940 \\
854 \\
801\end{array}$ & $\begin{array}{l}488 \\
476 \\
488 \\
505 \\
511 \\
436 \\
398 \\
390 \\
405 \\
387\end{array}$ \\
\hline 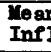 & & & & & & & & & $\begin{array}{r}460 \\
25,530\end{array}$ & $\begin{array}{r}3,610 \\
222,100\end{array}$ & $\begin{array}{r}561 \\
33,390 \\
\end{array}$ \\
\hline
\end{tabular}


Inflow, in seoond-feet, at indlcated time, 1938

\begin{tabular}{|c|c|c|c|c|c|c|}
\hline Hour & Pebmuary 27 & Pebruary 28 & March 1 & March 2 & March 3 & Harch 4 \\
\hline $\begin{array}{r}1 \\
2 \\
3 \\
4 \\
5 \\
6 \\
7 \\
8 \\
9 \\
10 \\
11 \\
11\end{array}$ & $\begin{array}{l}242 \\
238 \\
236 \\
233 \\
232 \\
246 \\
262 \\
280 \\
295 \\
310 \\
330 \\
352\end{array}$ & $\begin{array}{r}833 \\
855 \\
865 \\
892 \\
945 \\
1,070 \\
1,530 \\
2,160 \\
2,510 \\
2,900 \\
3,200 \\
3,360\end{array}$ & $\begin{array}{r}10,600 \\
9,530 \\
8,960 \\
8,570 \\
8,320 \\
8,000 \\
6,800 \\
5,320 \\
4,930 \\
4,700 \\
4,420 \\
4,120\end{array}$ & $\begin{array}{r}3,070 \\
3,160 \\
3,300 \\
3,200 \\
3,120 \\
3,170 \\
7,600 \\
9,200 \\
10,700 \\
15,500 \\
20,200 \\
27,600\end{array}$ & $\begin{array}{l}26,900 \\
24,000 \\
19,200 \\
18,800 \\
20,700 \\
19,900 \\
18,800 \\
17,700 \\
16,600 \\
15,900 \\
15,100 \\
14,500\end{array}$ & $\begin{array}{l}9,760 \\
9,300 \\
8,800 \\
8,540 \\
8,320 \\
8,000\end{array}$ \\
\hline \multirow[t]{2}{*}{$\begin{array}{r}1 \\
2 \\
3 \\
4 \\
5 \\
6 \\
7 \\
8 \\
9 \\
10 \\
11 \\
12 \\
\end{array}$} & $\begin{array}{l}375 \\
405 \\
443 \\
489 \\
535 \\
575 \\
612 \\
647 \\
685 \\
730 \\
775 \\
805\end{array}$ & $\begin{array}{l}3,540 \\
3,800 \\
3,940 \\
3,680 \\
3,490 \\
3,350 \\
3,300 \\
3,380 \\
3,450 \\
3,700 \\
6,800 \\
9,100\end{array}$ & $\begin{array}{l}3,910 \\
3,780 \\
3,600 \\
3,360 \\
2,890 \\
3,040 \\
3,460 \\
3,300 \\
3,140 \\
3,060 \\
3,000 \\
3,010\end{array}$ & $\begin{array}{l}39,000 \\
57,300 \\
69,500 \\
90,000 \\
81,000 \\
72,000 \\
59,500 \\
48,300 \\
40,800 \\
35,800 \\
31,300 \\
29,000\end{array}$ & $\begin{array}{l}13,700 \\
13,100 \\
12,800 \\
12,500 \\
12,000 \\
11,700 \\
11,400 \\
11,100 \\
10,800 \\
10,600 \\
10,300 \\
10,000\end{array}$ & $\begin{array}{l}7,730 \\
7,550 \\
6,900 \\
5,680 \\
5,390 \\
5,200\end{array}$ \\
\hline & March 5 & March 6 & March 7 & March 8 & March 9 & March 10 \\
\hline $\begin{array}{r}2 \\
4 \\
6 \\
8 \\
10 \\
1 \\
2 \\
4 \\
6 \\
8 \\
10 \\
11 \\
\end{array}$ & $\begin{array}{l}5,020 \\
4,900 \\
4,810 \\
4,700 \\
4,610 \\
4,480 \\
4,320 \\
4,200 \\
4,310 \\
4,380 \\
4,360 \\
4,300\end{array}$ & $\begin{array}{l}4,270 \\
4,220 \\
4,150 \\
4,090 \\
3,570 \\
2,840 \\
2,810 \\
3,700 \\
3,760 \\
3,740 \\
3,690 \\
3,630\end{array}$ & $\begin{array}{l}3,610 \\
3,590 \\
3,550 \\
3,500 \\
3,400 \\
3,350 \\
3,320 \\
3,290 \\
3,260 \\
3,220 \\
3,210 \\
3,200\end{array}$ & $\begin{array}{l}3,180 \\
3,170 \\
3,160 \\
3,120 \\
2,710 \\
2,110 \\
2,420 \\
2,630 \\
3,260 \\
3,900 \\
3,980 \\
3,950\end{array}$ & $\begin{array}{l}3,950 \\
3,980 \\
4,000 \\
4,010 \\
3,990 \\
3,920 \\
3,330 \\
2,790 \\
2,200 \\
2,700 \\
3,250 \\
3,290\end{array}$ & $\begin{array}{l}3,280 \\
3,250 \\
3,220 \\
3,190 \\
2,370 \\
1,600 \\
1,280 \\
1,220 \\
1,100 \\
1,080 \\
1,140 \\
1,210\end{array}$ \\
\hline
\end{tabular}

Mean da1ly outflow, in second-feet, 1938

\begin{tabular}{|c|c|c|c|c|c|c|c|c|c|c|c|}
\hline Day & Feb. & Mar. & Apr. & Day & $\mathrm{Feb}$. & Nar. & Apr. & Day & Feb. & Mar. & Apr. \\
\hline $\begin{array}{r}1 \\
2 \\
3 \\
4 \\
5 \\
6 \\
7 \\
8 \\
9 \\
10\end{array}$ & $\begin{array}{l}133 \\
177 \\
181 \\
147 \\
162 \\
191 \\
177 \\
204 \\
199 \\
239\end{array}$ & $\begin{array}{r}0 \\
11,800 \\
16,700 \\
8,080 \\
4,640 \\
3,790 \\
3,420 \\
3,100 \\
3,540 \\
2,300\end{array}$ & $\begin{array}{l}1,150 \\
2,820 \\
2,770 \\
2,700 \\
2,640 \\
2,560 \\
2,490 \\
2,400 \\
2,300 \\
2,190\end{array}$ & $\begin{array}{l}11 \\
12 \\
13 \\
14 \\
15 \\
16 \\
17 \\
18 \\
19 \\
20\end{array}$ & $\begin{array}{l}285 \\
309 \\
183 \\
137 \\
288 \\
321 \\
304 \\
282 \\
538 \\
524\end{array}$ & $\begin{array}{l}1,410 \\
3,790 \\
3,490 \\
2,590 \\
2,140 \\
1,520 \\
1,350 \\
1,330 \\
1,330 \\
1,320\end{array}$ & $\begin{array}{r}1,420 \\
499 \\
501 \\
504 \\
504 \\
504 \\
504 \\
503 \\
503 \\
503\end{array}$ & $\begin{array}{l}21 \\
22 \\
23 \\
24 \\
25 \\
26 \\
27 \\
28 \\
29 \\
30 \\
31\end{array}$ & $\begin{array}{l}528 \\
532 \\
507 \\
504 \\
502 \\
478 \\
489 \\
291\end{array}$ & $\begin{array}{r}1,140 \\
1,380 \\
1,380 \\
1,380 \\
1,380 \\
1,370 \\
1,370 \\
1,370 \\
1,360 \\
1,360 \\
932\end{array}$ & $\begin{array}{r}503 \\
502 \\
501 \\
501 \\
167 \\
1 \\
1 \\
1 \\
1 \\
1 \\
1\end{array}$ \\
\hline & & & & & & & & & $\begin{array}{r}315 \\
7,480 \\
\end{array}$ & $\begin{array}{r}2,970 \\
182,600 \\
\end{array}$ & $\begin{array}{r}1,070 \\
63,760 \\
\end{array}$ \\
\hline
\end{tabular}

Outflow, in second-foet, at indlcated time, 1938

\begin{tabular}{|c|c|c|c|c|c|c|}
\hline Hour & February 27 & February 28 & Larch I & March 2 & March 3 & Harch 4 \\
\hline $\begin{array}{r}1 \\
2 \\
3 \\
4 \\
5 \\
6 \\
7 \\
8 \\
9 \\
10 \\
11 \\
11 \\
\\
1 \\
2 \\
3 \\
4 \\
5 \\
6 \\
7 \\
8 \\
9 \\
10 \\
11 \\
11 \\
\end{array}$ & $\begin{array}{l}490 \\
490 \\
490 \\
490 \\
489 \\
488 \\
487 \\
486 \\
487 \\
488 \\
490 \\
492\end{array}$ & $\begin{array}{r}494 \\
496 \\
498 \\
500 \\
510 \\
520 \\
527 \\
0 \\
0 \\
0 \\
0 \\
0 \\
0 \\
\end{array}$ & $\begin{array}{l}0 \\
0 \\
0 \\
0 \\
0 \\
0 \\
0 \\
0 \\
0 \\
0 \\
0 \\
0\end{array}$ & $\begin{array}{r}0 \\
0 \\
0 \\
0 \\
0 \\
0 \\
0 \\
0 \\
0 \\
0 \\
0 \\
36,700 \\
56,400 \\
54,400 \\
47,000 \\
40,600 \\
35,400 \\
31,600 \\
\end{array}$ & $\begin{array}{l}29,200 \\
26,600 \\
24,400 \\
22,800 \\
21,500 \\
20,500 \\
19,700 \\
18,800 \\
17,700 \\
16,800 \\
15,900 \\
15,200 \\
14,500 \\
13,800 \\
13,300 \\
13,000 \\
12,600 \\
12,100 \\
11,800 \\
11,500 \\
11,200 \\
11,000 \\
10,700 \\
10,300\end{array}$ & $\begin{array}{r}10,200 \\
10,000 \\
9,800 \\
9,600 \\
9,300 \\
9,100 \\
8,900 \\
8,700 \\
8,600 \\
8,500 \\
8,350 \\
8,200 \\
8,000 \\
7,940 \\
7,860 \\
7,740 \\
7,600 \\
7,400 \\
6,830 \\
6,180 \\
5,970 \\
5,700 \\
5,500 \\
5,400\end{array}$ \\
\hline
\end{tabular}


Outflor, in second-feet, at indioated t1me, 1938--Cont imied

\begin{tabular}{|c|c|c|c|c|c|c|}
\hline Hour & March 5 & March 6 & March 7 & March 8 & March 9 & March 10 \\
\hline 2 & 5,180 & 4,310 & 3,620 & 3,190 & 3,960 & 3,250 \\
4 & 5,000 & 4,270 & 3,600 & 3,160 & 3,980 & 3,250 \\
6 & 4,850 & 4,220 & 3,550 & 3,160 & 3,980 & 3,250 \\
8 & 4,780 & 4,190 & 3,520 & 3,180 & 3,980 & 3,220 \\
10 & 4,680 & 3,960 & 3,440 & 3,020 & 3,980 & 3,050 \\
II & 4,580 & 3,320 & 3,400 & 2,500 & 3,980 & 2,320 \\
2 & 4,420 & 2,960 & 3,360 & 2,470 & 3,820 & 1,820 \\
4 & 4,300 & 3,200 & 3,320 & 3,100 & 3,250 & 1,540 \\
6 & 4,270 & 3,640 & 3,280 & 2,920 & 2,860 & 1,340 \\
8 & 4,360 & 3,720 & 3,230 & 3,430 & 2,540 & 1,220 \\
10 & 4,360 & 3,760 & 3,210 & 3,820 & 2,930 & 1,180 \\
M & 4,360 & 3,680 & 3,200 & 3,940 & 3,150 & 1,160 \\
\hline
\end{tabular}

Supplemental record.- Mar. 2, 7:30 p.m., 56,700 sec.-ft.

San Gabriel River at Morris Reservoir, near Azusa, Calif.

Lccation. - Water-stage recorder, lat. $34^{\circ} 10^{\prime} 25^{\prime \prime}$, long. $117^{\circ} 52^{\prime} 50^{\prime \prime}$, in Net sec. 13 , T. 1 N., R. 10 N., 3t miles northeast of Azusa. Altitude of stream bed, about 950 feet.

Drainage area.- Area, 211 square miles. Average altitude, 4,480 feet. Maximum altitude, 10,080 feet. Average slope, 49 percent. Length of main stream channel, 27.5 miles. Áverage slope of main stream channel, 6.3 percent.

Gage-helght record. - Water-stage recorder graph and frequent staff-gage readings.

D1scharge record.- Inflow computed from outflow, records of evaporation and diversion, and changes in storage, based on record of stage and stage-capacity tables. Outflow computed from record of stage, splllway rating curve, vaive rating curve, and gate and valve operation record.

Maxime.- 1938: Inflow, 66,200 second-feet 7:25 p.m. Mar. 2 .

1894-1937: Discharge, 40,000 second-feet Jan. 18, 1916.

Remarks.- Records good. Altitude of spillway, 1,152 feet; top of gates, 1,170 feet. Area at spillway level, 414 acres. Capacity before storm of Mar. 2, at spillway level, 31,700 acre-feet and at top of gates, 38,690 acre-feet; capacity after storm, 30,180 and 37,170 acre-feet, respectively. Inflow partially regulated by floodcontrol reservoir No. 1,3 miles upstream, and flocd-control reservoir Ho. 2, 13 miles upstream on Fest Fork of San Gabriel River. Records of inflow and records of outflow for Feb. 22 to Apr. 30 furnished by Oity of Pasadena Water Department.

Mean daily inflow, in second-feet, 1938

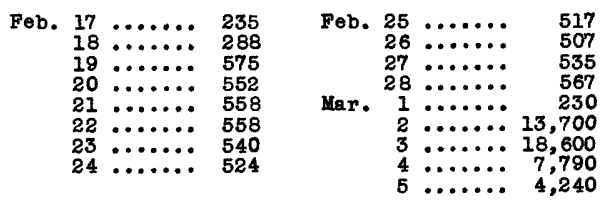

Inflow Feb. 17 to Mar. 5 in acre-feet...100,200

Inflow, in second-feet, at indicated time, 1938

\begin{tabular}{|c|c|c|c|c|}
\hline Hour & March 2 & March 3 & March 4 & March 5 \\
\hline $\begin{array}{r}1 \\
2 \\
3 \\
4 \\
5 \\
6 \\
7 \\
8 \\
9 \\
10 \\
11 \\
N\end{array}$ & $\begin{array}{r}200 \\
200 \\
240 \\
280 \\
350 \\
500 \\
720 \\
1,000 \\
1,350 \\
1,470 \\
1,540 \\
1,700\end{array}$ & $\begin{array}{l}33,600 \\
29,700 \\
27,600 \\
24,800 \\
21,600 \\
21,000 \\
20,100 \\
18,400 \\
17,200 \\
16,500 \\
16,300 \\
16,500\end{array}$ & $\begin{array}{r}10,500 \\
10,000 \\
9,660 \\
9,240 \\
9,030 \\
8,860 \\
8,660 \\
8,500 \\
8,260 \\
8,050 \\
7,900 \\
7,750\end{array}$ & $\begin{array}{l}4,770 \\
4,580 \\
4,500 \\
4,330 \\
4,240 \\
4,120\end{array}$ \\
\hline $\begin{array}{r}1 \\
2 \\
3 \\
4 \\
5 \\
6 \\
7 \\
8 \\
9 \\
10 \\
11 \\
M \\
\end{array}$ & $\begin{array}{r}2,000 \\
2,580 \\
3,000 \\
3,400 \\
4,100 \\
34,500 \\
61,000 \\
57,800 \\
51,400 \\
44,000 \\
38,300 \\
35,300\end{array}$ & $\begin{array}{l}16,400 \\
16,100 \\
15,800 \\
15,400 \\
14,900 \\
14,400 \\
13,900 \\
13,300 \\
13,000 \\
12,500 \\
12,000 \\
11,200\end{array}$ & $\begin{array}{l}7,600 \\
7,500 \\
7,320 \\
7,200 \\
7,060 \\
6,960 \\
6,400 \\
5,850 \\
5,640 \\
5,400 \\
5,180 \\
5,020\end{array}$ & $\begin{array}{l}4,040 \\
3,980 \\
3,940 \\
3,910 \\
3,880 \\
3,880\end{array}$ \\
\hline
\end{tabular}

Supplemental record.- Mar. 2, 7:25 p.m., 66,200 sec.-ft. 
Mean dally outflow, in second-feet, 1938

\begin{tabular}{|c|c|c|c|c|c|c|c|c|c|c|c|}
\hline Day & Feb. & Mar. & Apr. & Day & $\mathrm{Feb}$. & Mar. & Apr. & Day & Feb. & Mar. & Apr. \\
\hline $\begin{array}{r}1 \\
2 \\
3 \\
4 \\
5 \\
6 \\
7 \\
8 \\
9 \\
10\end{array}$ & $\begin{array}{l}3.8 \\
3.3 \\
5.5 \\
7 \\
6 \\
5.5 \\
4.2 \\
25 \\
72 \\
85\end{array}$ & $\begin{array}{r}205 \\
12,730 \\
21,660 \\
8,620 \\
4,520 \\
3,900 \\
3,840 \\
4,460 \\
4,940 \\
2,670\end{array}$ & $\begin{array}{r}860 \\
1,330 \\
1,280 \\
1,640 \\
2,410 \\
2,620 \\
2,500 \\
2,320 \\
2,290 \\
2,010\end{array}$ & $\begin{array}{l}11 \\
12 \\
13 \\
14 \\
15 \\
16 \\
17 \\
18 \\
19 \\
20\end{array}$ & $\begin{array}{l}89 \\
92 \\
89 \\
89 \\
88 \\
88 \\
88 \\
88 \\
89 \\
92\end{array}$ & $\begin{array}{l}1,620 \\
1,950 \\
2,690 \\
2,700 \\
2,380 \\
1,920 \\
1,140 \\
1,760 \\
1,750 \\
1,420\end{array}$ & $\begin{array}{r}1,190 \\
462 \\
418 \\
414 \\
414 \\
383 \\
392 \\
395 \\
397 \\
400\end{array}$ & $\begin{array}{l}21 \\
22 \\
23 \\
24 \\
25 \\
26 \\
27 \\
28 \\
29 \\
30 \\
31\end{array}$ & $\begin{array}{r}94 \\
98 \\
99 \\
170 \\
341 \\
308 \\
180 \\
86\end{array}$ & $\begin{array}{r}700 \\
580 \\
1,030 \\
1,360 \\
1,300 \\
1,300 \\
1,210 \\
1,310 \\
1,310 \\
1,310 \\
790 \\
\end{array}$ & $\begin{array}{c}403 \\
406 \\
409 \\
343 \\
96 \\
13 \\
12 \\
9.5 \\
9 \\
8.5\end{array}$ \\
\hline \multicolumn{9}{|c|}{ 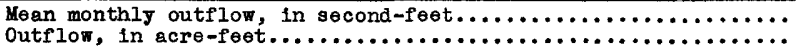 } & $\begin{array}{r}88.8 \\
4,930\end{array}$ & $\begin{array}{r}3,196 \\
196,500\end{array}$ & $\begin{array}{r}861 \\
51,240\end{array}$ \\
\hline
\end{tabular}

Outflow, in second-feet, at inalcated time, 1938

\begin{tabular}{|c|c|c|c|c|}
\hline Hour & March 2 & March 3 & March 4 & March 5 \\
\hline $\begin{array}{r}1 \\
2 \\
3 \\
4 \\
5 \\
6 \\
7 \\
8 \\
9 \\
10 \\
11 \\
\text { N }\end{array}$ & $\begin{array}{r}45 \\
60 \\
60 \\
60 \\
75 \\
105 \\
165 \\
255 \\
390 \\
615 \\
735 \\
855\end{array}$ & $\begin{array}{l}32,200 \\
28,700 \\
28,500 \\
27,300 \\
20,200 \\
20,800 \\
20,500 \\
19,500 \\
16,200 \\
16,500 \\
17,500 \\
21,300\end{array}$ & $\begin{array}{r}13,800 \\
12,100 \\
11,000 \\
10,300 \\
9,850 \\
9,500 \\
9,200 \\
8,960 \\
8,700 \\
8,500 \\
8,300 \\
8,100\end{array}$ & $\begin{array}{l}5,250 \\
5,000 \\
4,830 \\
4,630 \\
4,500 \\
4,400\end{array}$ \\
\hline $\begin{array}{r}1 \\
2 \\
3 \\
4 \\
5 \\
6 \\
7 \\
8 \\
9 \\
10 \\
11 \\
M \\
\end{array}$ & $\begin{array}{r}1,020 \\
1,290 \\
1,600 \\
5,230 \\
13,500 \\
20,200 \\
50,400 \\
55,500 \\
51,800 \\
46,200 \\
39,600 \\
33,500 \\
\end{array}$ & $\begin{array}{l}20,500 \\
21,000 \\
20,500 \\
21,200 \\
21,500 \\
19,500 \\
18,300 \\
19,400 \\
19,400 \\
19,300 \\
18,700 \\
16,700\end{array}$ & $\begin{array}{l}8,000 \\
7,800 \\
7,600 \\
7,500 \\
7,400 \\
7,300 \\
7,050 \\
6,700 \\
6,350 \\
6,100 \\
5,900 \\
5,650\end{array}$ & $\begin{array}{l}4,360 \\
4,250 \\
4,160 \\
4,060 \\
4,020 \\
4,000\end{array}$ \\
\hline
\end{tabular}

Supplemental records.- Mar. 2, 7:05 p.m., 65,700 sec.-ft.; $7: 12$ p.m., 65,400 sec.-ft.; $7: 20$ p.m., 51,800 sec.-ft.; 8:25 p.m., 56,300 sec. $-f t$.

West Fork of San Gabriel River at flood-control reservoir No. 2, near Camp Rincon, Calif.

Locat1on.- Staff gage, lat. $34^{\circ} 14^{\prime} 45^{\prime \prime}$, long. $117^{\circ} 57^{\prime} 50^{\prime \prime}$, near center sec. 19, T. 2 N., R. 10 W., 6 miles west of Camp Rincon. Altitude of stream bed, 2,140 fe日t.

Drainage area.- Area, 40.4 square miles. Average altitude, 4,290 feet. Maximur altitude, 8,038 feet. Average slope, 47 percent. Length of main stream channel, 8.5 miles. Average slope of main stream channel, 13 percent.

Gage-he1ght record.- Staff gage read dally at 8 a.m.; more frequently during storm period.

Dlscharge record.- Computed from splilway rating curve, valve rating curves, valve operation record, record of stage, discharge measurements, and changes in storage based on record of atage and stage-capacity tables; stage-capacity table dated Dctober 1935 used to time of peak stage of Mar. $2(4: 30 \mathrm{p.m.}$, and table corrected by silt survey made after storm used thereafter.

Maxima.- 1938: Inflow, 26,900 second-fe日t 4:15 p.m. Mar. 2. Outflow, 24,200 second-feet $4: 30$ p.m. Mar. 2 .

Remarks.- Records good. Altitude of spillway, 2,385 feet. Area at splllway level, 143 acres. Capacity at splliway level before storm of Mar. 2, 12, 298 acre-feet; after storm, 10,787 acre-feet. Basic data Feb. 27 to Mar. 11 and entire record Feb. $1-26$ and Mar. 12 to Apr. 30 furnished by Los Angeles County Flood Control District through H. E. Hedger, chlef englneer. 
Mean dally inflow, in second-feet, 1938

\begin{tabular}{|c|c|c|c|c|c|c|c|c|c|c|c|}
\hline Day & Fob. & Mar. & Apr. & Day & Feb. & Mar. & Apr. & Day & Feb. & Mar. & Apr. \\
\hline $\begin{array}{r}1 \\
2 \\
3 \\
4 \\
5 \\
6 \\
7 \\
8 \\
9 \\
10\end{array}$ & $\begin{array}{r}93 \\
39 \\
246 \\
305 \\
132 \\
70 \\
54 \\
46 \\
107 \\
109\end{array}$ & $\begin{array}{r}1,430 \\
8,100 \\
2,420 \\
995 \\
613 \\
428 \\
313 \\
350 \\
276 \\
294\end{array}$ & $\begin{array}{r}107 \\
101 \\
100 \\
99 \\
95 \\
94 \\
79 \\
79 \\
77 \\
75\end{array}$ & $\begin{array}{l}11 \\
12 \\
13 \\
14 \\
15 \\
16 \\
17 \\
18 \\
19 \\
20\end{array}$ & $\begin{array}{r}549 \\
288 \\
162 \\
113 \\
91 \\
71 \\
61 \\
75 \\
73 \\
62\end{array}$ & $\begin{array}{l}246 \\
853 \\
539 \\
469 \\
388 \\
321 \\
276 \\
264 \\
238 \\
227\end{array}$ & $\begin{array}{l}70 \\
71 \\
72 \\
69 \\
65 \\
60 \\
61 \\
56 \\
56 \\
52\end{array}$ & $\begin{array}{l}21 \\
22 \\
23 \\
24 \\
25 \\
26 \\
27 \\
28 \\
29 \\
30 \\
31\end{array}$ & $\begin{array}{r}53 \\
48 \\
47 \\
42 \\
41 \\
42 \\
140 \\
1,030\end{array}$ & $\begin{array}{l}203 \\
197 \\
190 \\
185 \\
178 \\
155 \\
155 \\
163 \\
145 \\
126 \\
111\end{array}$ & $\begin{array}{l}52 \\
48 \\
48 \\
54 \\
61 \\
56 \\
45 \\
45 \\
51 \\
57\end{array}$ \\
\hline \multicolumn{9}{|c|}{ 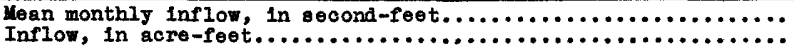 } & $\begin{array}{r}150 \\
8,310 \\
\end{array}$ & $\begin{array}{r}673 \\
41,350 \\
\end{array}$ & $\begin{array}{r}68.5 \\
4,080 \\
\end{array}$ \\
\hline
\end{tabular}

Inflow, in second-fe日t, at Indicated time, 1938

\begin{tabular}{|c|c|c|c|c|c|c|c|c|c|c|}
\hline Hr. & Feb. 27 & Fob. 28 & Mar. 1 & Mar. 2 & Mar. 3 & Mar. 4 & Mar. 5 & Mar. 6 & Mar. 7 & Mar. 8 \\
\hline $\begin{array}{r}1 \\
2 \\
3 \\
4 \\
5 \\
6 \\
7 \\
8 \\
9 \\
10 \\
11 \\
11\end{array}$ & $\begin{array}{l}45 \\
45 \\
45 \\
46 \\
46 \\
46 \\
46 \\
46 \\
55 \\
65 \\
77 \\
95\end{array}$ & $\begin{array}{r}248 \\
244 \\
242 \\
267 \\
320 \\
425 \\
625 \\
960 \\
1,060 \\
1,130 \\
1,170 \\
1,200\end{array}$ & $\begin{array}{r}4,600 \\
3,350 \\
2,720 \\
2,300 \\
1,860 \\
1,680 \\
1,540 \\
1,400 \\
1,200 \\
1,040 \\
915 \\
880\end{array}$ & $\begin{array}{r}705 \\
735 \\
780 \\
850 \\
1,180 \\
1,940 \\
2,580 \\
3,600 \\
5,100 \\
8,600 \\
9,300 \\
10,200\end{array}$ & $\begin{array}{l}4,350 \\
3,860 \\
3,430 \\
3,240 \\
3,080 \\
2,870 \\
2,700 \\
2,550 \\
2,400 \\
2,270 \\
2,170 \\
2,100\end{array}$ & $\begin{array}{r}1,290 \\
1,250 \\
1,180 \\
1,120 \\
1,040 \\
975\end{array}$ & 675 & 480 & 311 & $\begin{array}{l}288 \\
273 \\
287 \\
308 \\
375 \\
392\end{array}$ \\
\hline $\begin{array}{r}1 \\
2 \\
3 \\
4 \\
5 \\
6 \\
7 \\
8 \\
9 \\
10 \\
11 \\
1 \\
\end{array}$ & $\begin{array}{l}123 \\
162 \\
204 \\
230 \\
246 \\
260 \\
284 \\
269 \\
263 \\
259 \\
255 \\
250 \\
\end{array}$ & $\begin{array}{r}1,220 \\
1,300 \\
1,580 \\
1,160 \\
890 \\
950 \\
985 \\
1,040 \\
1,180 \\
1,940 \\
2,850 \\
3,450 \\
\end{array}$ & $\begin{array}{l}860 \\
843 \\
830 \\
820 \\
806 \\
798 \\
787 \\
775 \\
763 \\
735 \\
695 \\
680 \\
\end{array}$ & $\begin{array}{r}10,800 \\
20,000 \\
21,800 \\
24,700 \\
20,300 \\
13,000 \\
10,000 \\
8,050 \\
6,500 \\
5,700 \\
5,200 \\
4,730\end{array}$ & $\begin{array}{l}2,010 \\
1,930 \\
1,850 \\
1,790 \\
1,700 \\
1,650 \\
1,590 \\
1,510 \\
1,460 \\
1,410 \\
1,370 \\
1,330 \\
\end{array}$ & $\begin{array}{l}905 \\
850 \\
805 \\
765 \\
733 \\
720 \\
\end{array}$ & 490 & $\begin{array}{r}350 \\
333 \\
\end{array}$ & 295 & $\begin{array}{l}410 \\
435 \\
455 \\
360 \\
320 \\
300 \\
\end{array}$ \\
\hline
\end{tabular}

Supplemental record.- Mar. 2, 4:15 p.m., 26,900 sec.-ft.

Mean dafly cutflow, in second-fert, 1938

\begin{tabular}{|c|c|c|c|c|c|c|c|c|c|c|c|}
\hline Day & Feb. & Mar. & Apr. & Day & Feb. & Mar. & Apr. & Day & Feb. & Mar. & Apr. \\
\hline $\begin{array}{r}1 \\
8 \\
3 \\
4 \\
5 \\
6 \\
7 \\
8 \\
9 \\
10\end{array}$ & $\begin{array}{l}7.5 \\
9 \\
12 \\
26 \\
30 \\
28 \\
26 \\
25 \\
23 \\
25\end{array}$ & $\begin{array}{r}181 \\
6,810 \\
2,980 \\
1,655 \\
741 \\
733 \\
860 \\
934 \\
1,290 \\
533\end{array}$ & $\begin{array}{r}97 \\
96 \\
96 \\
95 \\
95 \\
94 \\
74 \\
56 \\
24 \\
7\end{array}$ & $\begin{array}{l}11 \\
12 \\
13 \\
14 \\
15 \\
16 \\
17 \\
18 \\
19 \\
20\end{array}$ & $\begin{array}{l}30 \\
38 \\
34 \\
34 \\
34 \\
33 \\
30 \\
28 \\
26 \\
24\end{array}$ & $\begin{array}{r}123 \\
540 \\
332 \\
283 \\
192 \\
14 \\
15 \\
84 \\
129 \\
155\end{array}$ & $\begin{array}{l}6 \\
6 \\
6 \\
6.5 \\
7 \\
7 \\
7 \\
7 \\
7 \\
7\end{array}$ & $\begin{array}{l}21 \\
28 \\
23 \\
24 \\
25 \\
26 \\
27 \\
28 \\
29 \\
30 \\
31 \\
\end{array}$ & $\begin{array}{l}23 \\
22 \\
22 \\
21 \\
20 \\
20 \\
21 \\
31\end{array}$ & $\begin{array}{r}225 \\
286 \\
288 \\
286 \\
282 \\
266 \\
268 \\
272 \\
177 \\
135 \\
93 \\
\end{array}$ & $\begin{array}{l}7.5 \\
8 \\
7.5 \\
8 \\
8.5 \\
9 \\
9 \\
9 \\
9 \\
9\end{array}$ \\
\hline \multicolumn{9}{|c|}{$\begin{array}{l}\text { Mean monthly outflow, in second-feet. } \ldots \ldots \ldots \ldots \ldots \ldots \ldots \ldots \ldots \ldots \ldots \ldots \ldots \ldots \\
\text { Outflcw, in acre-feet. } \ldots \ldots \ldots \ldots \ldots \ldots \ldots \ldots \ldots \ldots \ldots \ldots \ldots \ldots \ldots \ldots \ldots \ldots \ldots\end{array}$} & $\begin{array}{r}25.1 \\
1,390 \\
\end{array}$ & $\begin{array}{r}683 \\
41,970 \\
\end{array}$ & $\begin{array}{r}29.5 \\
1,760 \\
\end{array}$ \\
\hline
\end{tabular}

Outflow, in second-feet, at indicated time, 1938

\begin{tabular}{|c|c|c|c|c|c|c|}
\hline Hour & February 27 & Fobruary 28 & March 1 & March 2 & Harch 3 & Saroh 4 \\
\hline $\begin{array}{r}1 \\
2 \\
3 \\
4 \\
5 \\
6 \\
7 \\
8 \\
9 \\
10 \\
11 \\
1 \\
\\
1 \\
2 \\
3 \\
4 \\
5 \\
6 \\
7 \\
8 \\
9 \\
10 \\
11 \\
1 \\
\end{array}$ & $\begin{array}{l}20 \\
20 \\
20 \\
20 \\
21 \\
21 \\
21 \\
21 \\
21 \\
21 \\
21 \\
22\end{array}$ & $\begin{array}{l}22 \\
22 \\
22 \\
23 \\
26 \\
29 \\
33 \\
37 \\
39 \\
42 \\
45 \\
52\end{array}$ & $\begin{array}{r}65 \\
75 \\
82 \\
87 \\
90 \\
92 \\
\\
94 \\
95 \\
236 \\
377 \\
378 \\
380 \\
381 \\
382\end{array}$ & $\begin{array}{r}386 \\
387 \\
392 \\
397 \\
403 \\
411 \\
423 \\
4,150 \\
6,430 \\
6,840 \\
14,500 \\
19,600 \\
22,800 \\
23,400 \\
16,700 \\
12,300 \\
9,530 \\
7,730 \\
6,430 \\
6,710 \\
7,550\end{array}$ & $\begin{array}{l}7,050 \\
5,050 \\
3,730 \\
3,460 \\
3,270 \\
3,170 \\
3,060 \\
2,860 \\
2,710 \\
2,610 \\
2,420 \\
2,380 \\
2,320 \\
2,280 \\
2,240 \\
2,230 \\
2,230 \\
2,210 \\
2,200 \\
2,190\end{array}$ & $\begin{array}{r}2,170 \\
2,150 \\
2,140 \\
2,120 \\
2,100 \\
2,070 \\
2,050 \\
2,020 \\
654 \\
654 \\
657 \\
658 \\
659\end{array}$ \\
\hline
\end{tabular}


Outflow, in second-feet, et Indicated time, 1938--Continued

\begin{tabular}{|c|c|c|c|c|c|c|}
\hline Hour & March 5 & March 6 & March 7 & March 8 & March 9 & March 10 \\
\hline $\begin{array}{r}2 \\
4 \\
6 \\
8 \\
10 \\
1 \\
2 \\
4 \\
6 \\
8 \\
10 \\
1\end{array}$ & $\begin{array}{l}659 \\
660 \\
660 \\
660 \\
927 \\
924 \\
921\end{array}$ & $\begin{array}{r}915 \\
905 \\
25 \\
25 \\
911 \\
910 \\
903 \\
895\end{array}$ & $\begin{array}{l}883 \\
871 \\
861 \\
861 \\
850 \\
840 \\
832\end{array}$ & $\begin{array}{r}821 \\
813 \\
18 \\
18 \\
1,770 \\
1,720 \\
1,670\end{array}$ & $\begin{array}{r}1,610 \\
1,510 \\
1,420 \\
646 \\
641 \\
321 \\
1,420 \\
1,390 \\
\end{array}$ & $\begin{array}{r}1,330 \\
1,290 \\
260 \\
7 \\
7 \\
7 \\
337 \\
335 \\
\end{array}$ \\
\hline
\end{tabular}

Supplemental record.- Mar. 2, 4:30 p.m., 24,200 sec.-ft.

Sawpit Creẹ at flood-contrcl reservolr near Monrovia, Callf.

Iocation. - Water-stage recorder, lat. $34^{\circ} 10^{\prime} 30^{n}$, long. $117^{\circ} 59^{\prime} 00^{n}$, near center sec. 13 , 1. IH., R. 11 W., 2 miles nerth of Monrovia. Altitude of stream bed, 1,228 feet.

Drainege area.- Area, 3.27 square miles. Average altitude, 2,960 feet. Heximm alt1tude, 5,390 feet. Average slope, 50 percent. Iength of main stream channel, 3.1

miles. Average slope of main stream channel, 25 percent.

Gage-he1ght record.- Water-stage recorder graph and frequent staff-gage readings.

Discharge record.- Computed from spillway rating curve, valve rating curves, valve operation records, record of stage, discharge messurements, and atage-capacity tables: stage-capacity table based on survey of october 1935 used to time of peak stage of Mar. 2 ( 6 p.m.), table corrected by silt survey made after storm used thereafter.

Yaxima.- 1938: Inflow, 1,000 second-feet 4:15 p.m. Mar. 2.

1932-37: Mean hourly inflcw, 240 second-feet $2-3$ a.m. Jan. 1, 1934.

Remarks.- Records fair. Altitude of spillway, 1,360 feet. Area at sp1llway level, 7.9 acres (after stom of Mar. 2). Capacity at spiliway level before atorm, 389 acre-feet; after storm, 320 acre-feet. Basic data Feb. 27 to Mar. 11 and entire record for rest of February, Merch, and April furnished by Los Angeles County Flood Control District, through H. E. Hedger, chlef engineer.

Mean dally inflow, in second-feet, 1938

\begin{tabular}{|c|c|c|c|c|c|c|c|c|c|c|c|}
\hline Day & Feb. & Mar. & Apr. & Dey & Feb. & Mar. & Apr. & Day & Feb. & Mar. & Apr. \\
\hline $\begin{array}{r}1 \\
2 \\
3 \\
4 \\
5 \\
6 \\
7 \\
8 \\
9 \\
10\end{array}$ & $\begin{array}{c}3.4 \\
1.4 \\
22 \\
16 \\
6 \\
3.0 \\
2.1 \\
1.0 \\
4.2 \\
4.6\end{array}$ & $\begin{array}{r}83 \\
371 \\
132 \\
54 \\
35 \\
34 \\
27 \\
17 \\
15 \\
15\end{array}$ & $\begin{array}{l}8 \\
8 \\
7.5 \\
7.5 \\
7.5 \\
7 \\
6.5 \\
6 \\
6 \\
6\end{array}$ & $\begin{array}{l}11 \\
12 \\
13 \\
14 \\
15 \\
16 \\
17 \\
18 \\
19 \\
20\end{array}$ & $\begin{array}{l}9.5 \\
9 \\
6 \\
4.8 \\
3.3 \\
3.3 \\
3.2 \\
3.1 \\
3.0 \\
2.4\end{array}$ & $\begin{array}{l}14 \\
21 \\
17 \\
15 \\
14 \\
14 \\
13 \\
13 \\
12 \\
11\end{array}$ & $\begin{array}{l}5.5 \\
5.5 \\
5.5 \\
5.5 \\
5.5 \\
5 \\
5 \\
5 \\
5 \\
4.9\end{array}$ & $\begin{array}{l}21 \\
22 \\
23 \\
24 \\
25 \\
26 \\
27 \\
28 \\
29 \\
30 \\
31 \\
\end{array}$ & $\begin{array}{l}1.5 \\
1.2 \\
1.4 \\
1.5 \\
1.4 \\
1.0 \\
1.3 \\
44\end{array}$ & $\begin{array}{l}11 \\
10 \\
9.5 \\
9 \\
8.5 \\
8.5 \\
8.5 \\
8.5 \\
8.5 \\
8.5 \\
8.5 \\
\end{array}$ & $\begin{array}{l}4.8 \\
4.8 \\
4.8 \\
5 \\
5 \\
4.4 \\
3.9 \\
3.4 \\
3.4 \\
3.3\end{array}$ \\
\hline \multicolumn{9}{|c|}{ 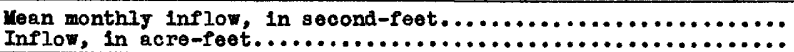 } & $\begin{array}{r}5.88 \\
326\end{array}$ & $\begin{array}{r}33.1 \\
2,040\end{array}$ & $\begin{array}{r}5.51 \\
328\end{array}$ \\
\hline
\end{tabular}

Inflow, in second-feet, at indicated time, 1938

\begin{tabular}{|c|c|c|c|c|c|c|c|c|c|c|c|c|}
\hline Hr. & Feb.27 & Feb.28 & Mar.1 & Mar.2 & Mar. 3 & Mar. 4 & $\operatorname{Mar} .5$ & Mar. 6 & Mar. 7 & Mar. 8 & Mar.9 & Mâr.10 \\
\hline \begin{tabular}{|c|}
1 \\
2 \\
3 \\
4 \\
5 \\
6 \\
7 \\
8 \\
9 \\
10 \\
71 \\
1
\end{tabular} & $\begin{array}{r}0.9 \\
.9 \\
.9 \\
.9 \\
.9 \\
.9 \\
1.0 \\
1.4 \\
2.7 \\
2.1 \\
1.6 \\
1.3\end{array}$ & $\begin{array}{l}2.1 \\
3.2 \\
5.5 \\
13 \\
22 \\
31 \\
42 \\
60 \\
73 \\
68 \\
63 \\
58\end{array}$ & $\begin{array}{r}245 \\
183 \\
151 \\
128 \\
110 \\
97 \\
87 \\
81 \\
75 \\
68 \\
62 \\
59\end{array}$ & $\begin{array}{r}49^{\circ} \\
51 \\
52 \\
58 \\
76 \\
99 \\
156 \\
180 \\
260 \\
314 \\
320 \\
455\end{array}$ & $\begin{array}{l}240 \\
220 \\
205 \\
196 \\
185 \\
174 \\
163 \\
150 \\
134 \\
129 \\
122 \\
115\end{array}$ & $\begin{array}{l}68 \\
64 \\
60 \\
57 \\
54 \\
52\end{array}$ & 36 & 36 & 30 & 17 & 16 & 14 \\
\hline $\begin{array}{r}1 \\
2 \\
3 \\
4 \\
5 \\
6 \\
7 \\
8 \\
9 \\
10 \\
11 \\
4\end{array}$ & $\begin{array}{l}.8 \\
1.0 \\
1.3 \\
1.4 \\
1.4 \\
1.4 \\
1.4 \\
1.4 \\
1.4 \\
1.4 \\
1.4 \\
1.6\end{array}$ & $\begin{array}{r}54 \\
39 \\
31 \\
25 \\
23 \\
22 \\
21 \\
19 \\
32 \\
120 \\
156 \\
170 \\
\end{array}$ & $\begin{array}{l}57 \\
54 \\
50 \\
47 \\
46 \\
44 \\
43 \\
42 \\
40 \\
41 \\
44 \\
47 \\
\end{array}$ & $\begin{array}{l}515 \\
615 \\
800 \\
925 \\
815 \\
680 \\
620 \\
575 \\
470 \\
352 \\
300 \\
265 \\
\end{array}$ & $\begin{array}{r}106 \\
97 \\
93 \\
90 \\
87 \\
84 \\
83 \\
82 \\
81 \\
80 \\
79 \\
79 \\
\end{array}$ & $\begin{array}{l}50 \\
48 \\
46 \\
44 \\
42 \\
40\end{array}$ & 32 & 32 & 20 & 16 & 15 & $\begin{array}{l}13 \\
13 \\
20\end{array}$ \\
\hline
\end{tabular}

Supplemental record.- Mar. 2, $5: 15$ p.m., 1,000 sec.-ft. 
Mean daily outflow, in second-feet, 1938

\begin{tabular}{|c|c|c|c|c|c|c|c|c|c|c|c|}
\hline Day & Feb. & Mar. & Apr. & Day & $F \in b$. & Mar. & Apr. & Day & Feb. & Mar. & Apr. \\
\hline $\begin{array}{r}1 \\
2 \\
3 \\
4 \\
5 \\
6 \\
7 \\
8 \\
9 \\
10\end{array}$ & $\begin{array}{l}0 \\
0 \\
2.9 \\
16 \\
8.5 \\
7 \\
4.5 \\
8 \\
9.5 \\
0\end{array}$ & $\begin{array}{r}63 \\
231 \\
149 \\
75 \\
71 \\
68 \\
59 \\
27 \\
16 \\
15\end{array}$ & $\begin{array}{l}9 \\
7.5 \\
7.5 \\
8 \\
7 \\
7 \\
6.5 \\
6 \\
5.5 \\
5.5\end{array}$ & $\begin{array}{l}11 \\
12 \\
13 \\
14 \\
15 \\
16 \\
17 \\
18 \\
19 \\
20\end{array}$ & $\begin{array}{l}7 \\
9.5 \\
9.5 \\
7.5 \\
4.9 \\
4.4 \\
4.1 \\
1.7 \\
0 \\
0\end{array}$ & $\begin{array}{l}13 \\
20 \\
17 \\
15 \\
15 \\
14 \\
13 \\
12 \\
11 \\
11\end{array}$ & $\begin{array}{l}6 \\
5.5 \\
5.5 \\
5.5 \\
5.5 \\
5.5 \\
5 \\
5 \\
5 \\
4.9\end{array}$ & $\begin{array}{l}21 \\
22 \\
23 \\
24 \\
25 \\
26 \\
27 \\
28 \\
29 \\
30 \\
31\end{array}$ & $\begin{array}{l}0 \\
0 \\
0 \\
0 \\
0 \\
0 \\
5.5 \\
16\end{array}$ & $\begin{array}{l}10 \\
10 \\
13 \\
9 \\
8 \\
8.5 \\
8.5 \\
8.5 \\
9 \\
8.5 \\
8 \\
\end{array}$ & $\begin{array}{l}4.8 \\
4.8 \\
4.8 \\
5 \\
5 \\
4.6 \\
3.9 \\
3.2 \\
3.4 \\
3.2\end{array}$ \\
\hline \multicolumn{9}{|c|}{ 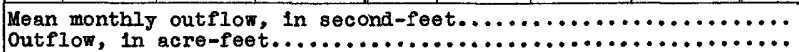 } & $\begin{array}{r}4.52 \\
251\end{array}$ & $\begin{array}{r}32.8 \\
2,020\end{array}$ & $\begin{array}{r}5.52 \\
328 \\
\end{array}$ \\
\hline
\end{tabular}

Outflor, in second-feet, at indicated time, 1938

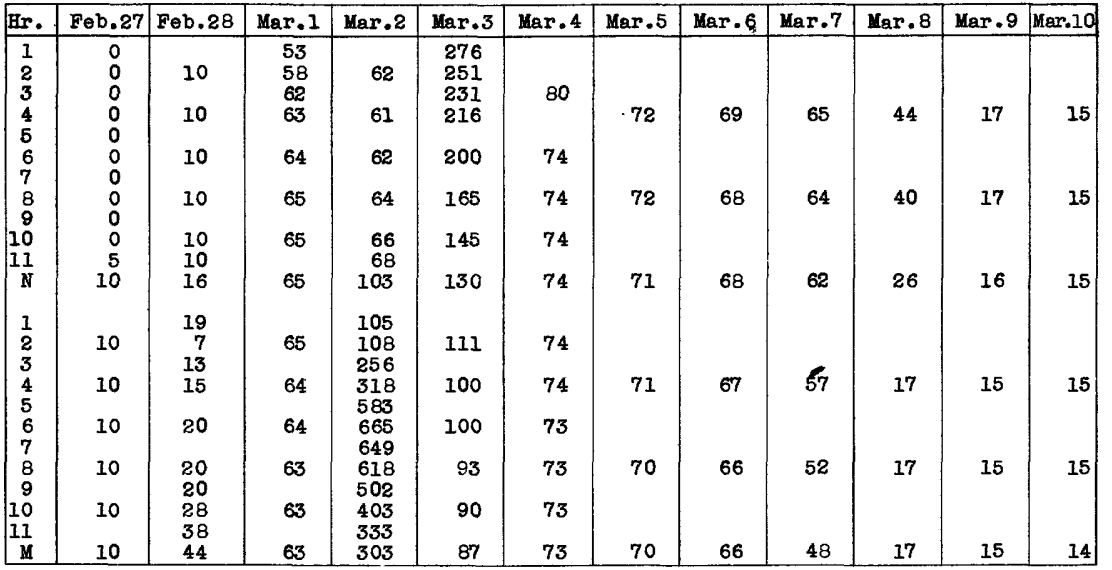

San Dimas Creek at flood-control reservoir near San Dimas, Calif.

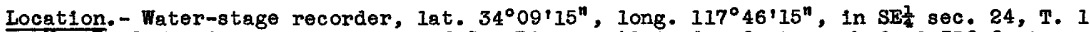
K., R. 9 W., 4 miles northeast of San Dimas. Altitude of stream bed, 1,350 feet.

Drainage area.- Area, 16.5 square miles. Average altitude, 3,130 feet. Maximum altitude, 5,558 feet. Average slope, 42 percent. Length of main stream channel, 6 miles. Average slope of main stream channel, 13 percent.

Gage-height record. - Water-atage recorder graph and staff-gage readings.

Discharge record.- Computed from spillway rating curve, valve rating curves, valve operation record, record of stage, and stage-capacity tables; stage-capecity table based on survey of December 1935 used to time of peak stage on Mar. $2(5: 15$ p.m.), table corrected by silt survey made after storm used thereafter.

Maxima.- 1938: Inflow, 4,600 second-feet $5: 10$ p.m. Mar. 2.

1932-37: Mean hourly inflow, 422 second-feet $3-4$ a.m. Jan. 1, 1934.

Remarks.- Records fair. Altitude of sp1llway, 1,462 feet. Area at sp1llway level, 36 acres (after storm of Mar. 2). Capacity of spillway level before storm, 1,399 acrefeet; after storm, 1,188 acre-feet. Basic data Feb. 27 to Mar. 2 and entire record for rest of February, March, and April furnished by Los Angeles County Flood Control District, through H. E. Hedger, chlef engineer.

Mean daily inflow, in second-feet, 1938

\begin{tabular}{|c|c|c|c|c|c|c|c|c|c|c|c|}
\hline Day & Feb. & Mar. & Apr. & Day & $F \in b$. & Mar. & Apr. & Day & Feb. & Mar. & Apr. \\
\hline $\begin{array}{r}1 \\
2 \\
3 \\
4 \\
5 \\
6 \\
7 \\
8 \\
9 \\
10\end{array}$ & $\begin{array}{l}13 \\
5.5 \\
32 \\
30 \\
13 \\
8 \\
5.5 \\
5.5 \\
15 \\
13\end{array}$ & $\begin{array}{r}331 \\
1,580 \\
461 \\
191 \\
135 \\
95 \\
77 \\
76 \\
61 \\
54\end{array}$ & $\begin{array}{l}31 \\
30 \\
28 \\
28 \\
26 \\
25 \\
24 \\
23 \\
22 \\
21\end{array}$ & $\begin{array}{l}11 \\
12 \\
13 \\
14 \\
15 \\
16 \\
17 \\
18 \\
19 \\
20\end{array}$ & $\begin{array}{c}42 \\
34 \\
18 \\
15 \\
10 \\
9 \\
8 \\
8 \\
7.5 \\
5.5\end{array}$ & $\begin{array}{r}50 \\
121 \\
116 \\
97 \\
83 \\
72 \\
66 \\
58 \\
59 \\
55\end{array}$ & $\begin{array}{l}21 \\
22 \\
20 \\
17 \\
15 \\
16 \\
15 \\
14 \\
15 \\
16\end{array}$ & $\begin{array}{l}21 \\
22 \\
23 \\
24 \\
25 \\
26 \\
27 \\
28 \\
29 \\
30 \\
31 \\
\end{array}$ & $\begin{array}{c}6 \\
6 \\
4.9 \\
3.7 \\
3.7 \\
5 \\
11 \\
144\end{array}$ & $\begin{array}{l}51 \\
48 \\
44 \\
43 \\
42 \\
38 \\
37 \\
36 \\
36 \\
33 \\
32 \\
\end{array}$ & $\begin{array}{l}15 \\
15 \\
14 \\
17 \\
18 \\
15 \\
15 \\
15 \\
15 \\
15\end{array}$ \\
\hline \multicolumn{12}{|c|}{ 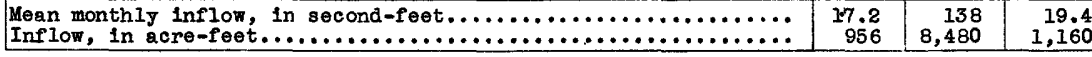 } \\
\hline
\end{tabular}


Inflow, in second-feet, at indleated t1me, 1938

\begin{tabular}{|c|c|c|c|c|c|c|c|c|c|c|c|c|}
\hline $\mathrm{Hr}$. & Feb.27 & Feb.28 & $\operatorname{Mar} .1$ & Mar.2 & Mar.3 & Mar. 4 & Kar.5 & Mar. 6 & $\operatorname{Mar} .7$ & $\operatorname{Mar} .8$ & $\operatorname{Mar} .9$ & $\tan 70$ \\
\hline $\begin{array}{l}1 \\
2\end{array}$ & 5 & 20 & $\begin{array}{l}1,420 \\
1,270\end{array}$ & $\begin{array}{r}97 \\
108\end{array}$ & 800 & 247 & 154 & & & & & \\
\hline 4 & 5 & 34 & 545 & 139 & 705 & 219 & 156 & 102 & 82 & 74 & 63 & 58 \\
\hline 6 & 6 & 58 & $\begin{array}{l}400 \\
360\end{array}$ & 219 & 600 & 210 & 157 & & & & & \\
\hline $\begin{array}{l}7 \\
8\end{array}$ & 13 & 93 & $\begin{array}{l}300 \\
265\end{array}$ & $\begin{array}{l}343 \\
500\end{array}$ & 530 & 200 & 156 & 97 & 79 & 78 & 62 & 55 \\
\hline 10 & 16 & 170 & 218 & 1,220 & 441 & 192 & 150 & & & & & \\
\hline N & 14 & 171 & 183 & 1,730 & 363 & 183 & 138 & 93 & 77 & 80 & 61 & 53 \\
\hline $\begin{array}{l}1 \\
2\end{array}$ & 13 & $\begin{array}{l}181 \\
170\end{array}$ & $\begin{array}{l}167 \\
150\end{array}$ & $\begin{array}{l}2,090 \\
3,200\end{array}$ & 330 & 177 & 120 & & & & & \\
\hline 4 & 14 & 160 & $\begin{array}{l}140 \\
130\end{array}$ & $\begin{array}{l}4,100 \\
4,010\end{array}$ & 314 & 170 & 116 & 92 & 74 & 80 & 60 & 52 \\
\hline $\begin{array}{l}5 \\
6\end{array}$ & 14 & 149 & 111 & 3,850 & 298 & 165 & 114 & & & & & \\
\hline $\begin{array}{l}7 \\
8\end{array}$ & 12 & $\begin{array}{l}132 \\
139\end{array}$ & $\begin{array}{r}104 \\
98\end{array}$ & $\begin{array}{l}2,600 \\
2,300\end{array}$ & 293 & 161 & 113 & 88 & 74 & 73 & 59 & 50 \\
\hline $\begin{array}{r}9 \\
10\end{array}$ & 11 & $\begin{array}{l}159 \\
230\end{array}$ & $\begin{array}{l}92 \\
87\end{array}$ & $\begin{array}{l}1,900 \\
1,280\end{array}$ & 284 & 157 & 110 & & & & & \\
\hline 11 & 14 & $\begin{array}{l}410 \\
790\end{array}$ & $\begin{array}{l}86 \\
88\end{array}$ & $\begin{array}{r}1,010 \\
870\end{array}$ & 270 & 154 & 108 & 84 & 72 & 66 & 60 & 50 \\
\hline
\end{tabular}

Supplemental record.- Mar. 2, 5:10 p.m., 4,600 sec.-ft.

Mean dally cutflow, in second-feet, 1938

\begin{tabular}{|r|c|r|r||r|r|r|r||r|r|r|r|}
\hline Day & Feb. & Mar. & Apr. & Day & Feb. & Mar. & Apr. & Day & Feb. & Mar. & Apr. \\
\hline 1 & 0 & 235 & 30 & 11 & 0 & 38 & 23 & 21 & 0 & 53 & 2.1 \\
2 & 0 & 1,290 & 26 & 12 & 6 & 76 & 23 & 22 & 0 & 53 & 2.1 \\
3 & 0 & 720 & 26 & 13 & 14 & 109 & 7.5 & 23 & 0 & 47 & 2.1 \\
4 & 0 & 319 & 26 & 14 & 15 & 128 & 0 & 24 & 0 & 44 & 1.6 \\
5 & 0 & 193 & 25 & 15 & 14 & 109 & 0 & 25 & 0 & 44 & 0 \\
6 & 0 & 106 & 25 & 16 & 10 & 68 & 0 & 26 & 0 & 43 & 0 \\
7 & 0 & 96 & 24 & 17 & 11 & 61 & 0 & 27 & 5 & 37 & 3.0 \\
8 & 0 & 70 & 24 & 18 & 6.5 & 32 & 0 & 28 & 74 & 32 & 9 \\
9 & 0 & 68 & 24 & 19 & 0 & 80 & $\ldots .8$ & 29 & & 32 & 12 \\
10 & 0 & 47 & 23 & 20 & 0 & 64 & 2.1 & 30 & & 32 & .12 \\
\hline
\end{tabular}

Outflow, in second-feet at indicated t1me, 1938

\begin{tabular}{|c|c|c|c|c|c|c|c|c|c|c|c|c|}
\hline Hr. & Feb.27 & Feb.28 & Mar.1 & Mer.2 & Mar.3 & $\operatorname{Mar} .4$ & Mar.5 & $\operatorname{Mar} .6$ & Mar .7 & Har. 8 & Mar.9 & Mar.10 \\
\hline $\begin{array}{l}1 \\
2\end{array}$ & & 18 & $\begin{array}{l}113 \\
115\end{array}$ & 250 & $\begin{array}{r}995 \\
1,090\end{array}$ & 328 & & & & & & \\
\hline $\begin{array}{l}3 \\
4\end{array}$ & & 18 & $\begin{array}{l}120 \\
255\end{array}$ & 248 & $\begin{array}{r}1,120 \\
960\end{array}$ & 325 & 300 & 105 & 103 & 69 & 69 & 68 \\
\hline $\begin{array}{l}5 \\
6\end{array}$ & & 19 & 255 & 248 & 780 & 325 & & & & & & \\
\hline $\begin{array}{l}7 \\
8\end{array}$ & & 19 & 255 & 248 & 720 & 325 & 295 & 105 & 103 & 69 & 69 & $5 \overline{2}$ \\
\hline $\begin{array}{r}9 \\
10\end{array}$ & & $\begin{array}{r}19 \\
110\end{array}$ & 255 & 250 & 717 & 323 & & & & & - & \\
\hline 11 & 0 & 110 & 255 & $\begin{array}{l}458 \\
785\end{array}$ & 710 & 320 & 287 & 105 & 103 & 69 & 69 & 36 \\
\hline 1 & & & & 798 & & & & & & & & \\
\hline 2 & 0 & 110 & 255 & $\begin{array}{r}830 \\
2.370\end{array}$ & 702 & 318 & 0 & & & & & \\
\hline 4 & 0 & 110 & 255 & 3,700 & 695 & 316 & 0 & 105 & 102 & 70 & 69 & 36 \\
\hline $\begin{array}{l}5 \\
6 \\
7\end{array}$ & 18 & 110 & 255 & 4,200 & 685 & 314 & 145 & & 102 & & & \\
\hline 8 & 18 & 110 & 252 & 2,630 & 675 & 311 & 145 & 105 & 69 & 70 & 69 & $\bar{z} 6$ \\
\hline $\begin{array}{r}9 \\
10\end{array}$ & 18 & 110 & 250 & $\begin{array}{l}2,350 \\
1,560\end{array}$ & $\begin{array}{l}330 \\
330\end{array}$ & 308 & 125 & & & & & \\
\hline$\stackrel{11}{M}$ & 18 & 111 & 250 & $\begin{array}{l}1,240 \\
1,090\end{array}$ & 328 & 305 & 105 & 104 & 69 & 70 & 69 & 36 \\
\hline
\end{tabular}

Supplemental record. - Mar. 2, 5:15 p.m., 4,370 sec.-ft. 
Fern Canjon Watershed No. 2, near San Dimas, Callf.

Location. - Water-stage recorders and flume, lat. $34^{\circ} 11^{\prime} 55^{\prime \prime}$, Iong. $.17^{\circ} 42^{\prime} 08^{n}$, in sec. 3, 1. 1 N., R. 8 i.., In San Dimas Experimental Foreet, about 8 miles northeast of San Dimas. Altitude, about 4,500 foet.

Dralnage area.- Area, 0.063 square mile. Average altitude, 5,000 foet. Maximum alt1tude, 5,400 feet. Average slope, 65 percent. Length of maln stream channel, 0.41 mile. A verage slope of main stream channel, 42 percent.

Gage-he1ght record. - Water-stage recorder graphs.

Discharge record.- The punoff is measured by a modifled 3-foot Parghald flume and represents the inflow into a small concrete-lined reservolr of $10,000-c u b 1 c-f o o t$ capacity. A $90^{\circ} \mathrm{V}$-notch weir is used in confunction with flume for low flows.

Ratings of flume and weir used throughout the period.

Yaximam. - 1938: D1scharge, 28.8 second-feet 4 p.m. Mar. 2.

Remerks.- Records furnished by U. S. Forest Service.

Mean da12y discharge, In second-feet, 2938
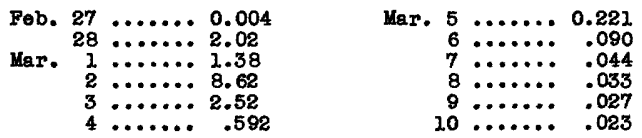

Runoff Feb. 27 to Mar. 10 , in acre-feet.... 30.9

Discharge, in second-feet, at indicated time, 1938

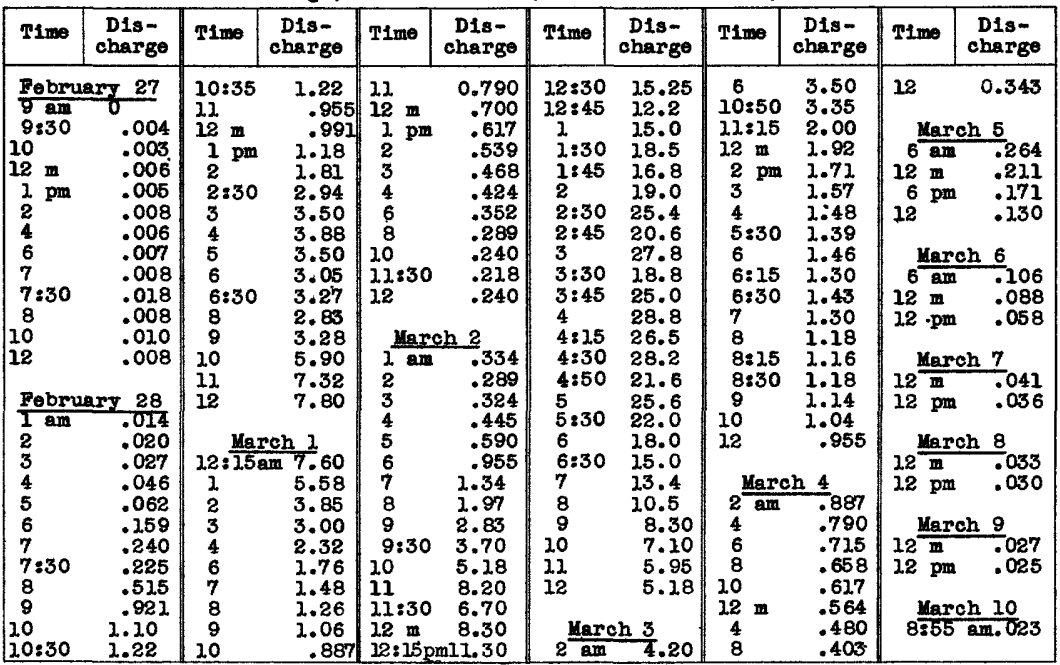

Fern Canyon Watershed No. 3, near San Dimas, Callf.

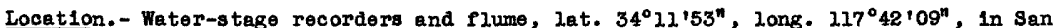
Dimas Experimental Forest, about 8 miles northoast of San Dimas. Altitude, about 4,500 feet.

Drainage area.- Area, 0.084 squars mile. Average alt1tude, 5,000 feet. Maximum alt1tude, 5,400 feet. Average slope, 65 percent. Length of mein stream chamel, 0.52 mile. Average slope of main stream channel, 33 percent.

Gage-height record.- Water-stage recorder graphs.

Discharge record.- The runoff is measured by a modifled 3-foot Parshall flume and represents the inflow into a smell concrete-lined reservoir of $10,000-0 u b 1 c-f o o t$ capacity. A $90^{\circ} \mathrm{V}$-notch weir is used in conjunction with flume for low flows. Ratings of flume and weir used throughout the period.

Maximum.- 1938: Discharge, 19.0 second-feet 4 and 4:15 p.m. Mar. 2 .

Remarks.- Records fumished by U. S. Forest Service. 
D1scharge, in second-feet, at Inaicated time, 1938

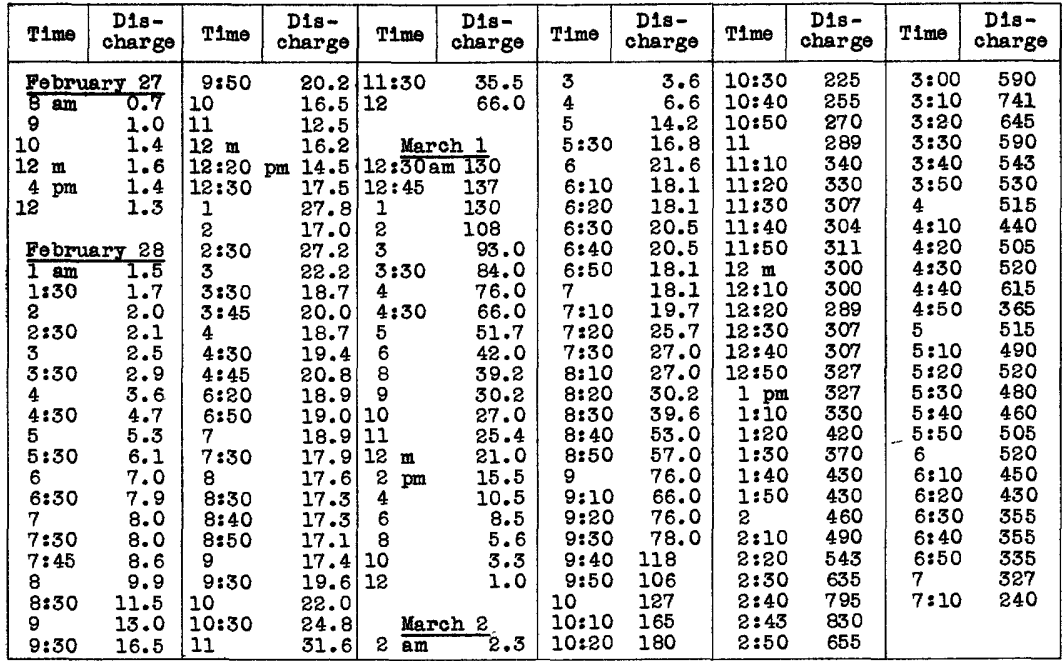

West Fork of San Dimas Creok near San Dimas, Callf.

Location. - Water-stage recorders and flumes, lat. $34^{\circ} 10^{\prime} 10^{\prime \prime}$, long. $117^{\circ} 46^{\prime} 15^{\prime \prime}$, In SEtSist, soc. 13, T. I N., R. 9 W., in San Dimas Experlmental Forest, about 4.5 miles northeast of San Dimas. Altitude, about 1,600 feet.

Drainage area.- Area, 1.66 square miles. Average alt1tude, 2,360 feet. Maximum alt1tude, 3,100 feet. Average s lope, 47 percent. Length of main stream channel, 2.5 m1les. Average s lope of main stream channel, 11.4 percent.

Gage-helght record. - Water-stage recorder graph.

D1scharge record. - The runoff is measured by a 10-foot modifled Parshall flume and a $2-$ foot San Dimas flume for lower flcws. Rating of flumes used throughout the period.

Max1mum.- 1938: D1scharge, 664 second-fe日t 4:05 p.m. Mar. 2.

Remarks.- Records furnished by 0 . S. Forest Service.

Mean da1ly discharge, in second-feet, 1938

$$
\begin{aligned}
& \text { Feb. } \begin{array}{llll}
27 & \ldots \ldots & 1.1 \\
28 & \ldots & \ldots & 16.3
\end{array} \\
& \text { Mar. } 1 \ldots . . .41 .4
\end{aligned}
$$

\begin{tabular}{|c|c|c|c|c|c|c|c|c|c|c|c|}
\hline TIme & $\begin{array}{c}\text { D1s- } \\
\text { charge }\end{array}$ & Ime & $\begin{array}{c}\text { D1s- } \\
\text { charge }\end{array}$ & T1me & $\begin{array}{l}\text { D18- } \\
\text { charge }\end{array}$ & TIme & $\begin{array}{c}\text { Dis- } \\
\text { charge }\end{array}$ & Time & $\begin{array}{c}\text { Dis- } \\
\text { charge }\end{array}$ & Time & $\begin{array}{l}\text { D1s- } \\
\text { harge }\end{array}$ \\
\hline $\begin{array}{l}\frac{\text { Febmu }}{7 \mathrm{am}} \\
9 \\
9: 30 \\
10 \\
11 \\
12 \\
1 \mathrm{pm} \\
2 \\
3 \\
4 \\
5 \\
6 \\
7 \\
8 \\
12 \\
\\
\text { Febru } \\
1: \mathrm{am} \\
1: 20 \\
1: 40 \\
2 \\
2: 20 \\
2: 40 \\
3 \\
3: 30 \\
4 \\
4: 30 \\
5\end{array}$ & $\begin{array}{r}\text { ory } 27 \\
0.5 \\
.8 \\
1.4 \\
1.9 \\
2.4 \\
2.3 \\
1.8 \\
1.6 \\
1.4 \\
1.4 \\
1.4 \\
1.3 \\
1.2 \\
1.2 \\
1.0\end{array}$ & $\begin{array}{c}5: 30 \\
6 \\
7 \\
8 \\
12 \mathrm{~m} \\
1 \\
2 \\
3 \\
4 \\
5 \\
6 \\
7 \\
7: 30 \\
8 \\
8: 30 \\
9 \\
9: 30 \\
10 \\
\\
12: 15 \\
12: 30 \\
1 \\
1: 30 \\
2 \\
2: 10 \\
2: 20 \\
2: 40 \\
3\end{array}$ & $\begin{array}{r}7.3 \\
7.8 \\
9.4 \\
13.1 \\
24.0 \\
20.7 \\
20.4 \\
19.0 \\
16.9 \\
15.4 \\
15.0 \\
14.5 \\
14.1 \\
13.8 \\
14.2 \\
16.1 \\
21.2 \\
31.5\end{array}$ & $\begin{array}{l}3: 20 \\
4 \\
5 \\
6: 20 \\
7 \\
8: 40 \\
9 \\
10 \\
11 \\
12 \mathrm{~m} \\
12 \mathrm{pm} \\
2 \\
4 \\
5 \\
7 \\
9 \\
10 \\
12 \\
\\
4 \text { Marc } \\
4 \\
5 \\
5: 30 \\
6 \\
6: 30 \\
7 \\
7: 20 \\
7: 40 \\
8\end{array}$ & $\begin{array}{l}78.5 \\
63.8 \\
49.0 \\
38.9 \\
41.9 \\
45.9 \\
44.8 \\
34.0 \\
29.5 \\
26.5 \\
23.4 \\
21.0 \\
20.2 \\
18.1 \\
17.8 \\
16.2 \\
14.2 \\
14.0\end{array}$ & $\begin{array}{c}9: 40 \\
9: 50 \\
10 \\
10: 10 \\
10: 20 \\
10: 25 \\
10: 35 \\
10: 40 \\
10: 50 \\
11: 05 \\
11: 05 \\
11: 10 \\
11: 20 \\
11: 40 \\
12 \mathrm{~m} \\
12: 10 \mathrm{pm} \\
12: 20 \\
12: 30 \\
12: 40 \\
12: 50 \\
1 \\
1: 10\end{array}$ & $\begin{array}{c}68.5 \\
78.5 \\
101 \\
112 \\
118 \\
124 \\
142 \\
152 \\
164 \\
184 \\
214 \\
236 \\
248 \\
261 \\
259 \\
248 \\
229 \\
195 \\
174 \\
164 \\
160 \\
166 \\
1677 \\
195 \\
206 \\
219 \\
232 \\
244 \\
259\end{array}$ & $\begin{array}{l}3: 10 \\
3: 15 \\
3: 20 \\
3: 30 \\
3: 45 \\
3: 50 \\
3: 55 \\
4 \\
4: 05 \\
4: 10 \\
4: 15 \\
4: 20\end{array}$ & $\begin{array}{l}425 \\
460 \\
500 \\
530 \\
580 \\
610 \\
585 \\
558 \\
585 \\
595 \\
568 \\
615 \\
650 \\
664 \\
630 \\
530 \\
475\end{array}$ & $\begin{aligned} 4: 25 \\
4: 35 \\
4: 45 \\
4: 50 \\
4: 55 \\
5 \\
5: 10 \\
5: 20 \\
5: 30 \\
5: 40 \\
5: 45 \\
5: 50 \\
5: 55 \\
6 \\
6: 10 \\
6: 20 \\
6: 30 \\
6: 40 \\
6: 50 \\
7 \\
7: 10 \\
7: 30 \\
8 \\
8: 30 \\
9 \\
9: 30 \\
10\end{aligned}$ & $\begin{array}{l}355 \\
378 \\
330 \\
390 \\
450 \\
470 \\
492 \\
415 \\
350 \\
300 \\
254 \\
289 \\
322 \\
292 \\
251 \\
232 \\
221 \\
215 \\
206 \\
180 \\
164 \\
157 \\
145 \\
130 \\
112 \\
104 \\
101\end{array}$ \\
\hline
\end{tabular}

D1scharge, in second-feet, at indicated time, 1938 
Dalton Creok at flood-control reserrolr near Glendora, Galif.

Location.- Water-s tage recorder, lat. $34^{\circ} 10^{\prime} 10^{\prime \prime}$, long. $117^{\circ} 48^{\prime} 30^{\prime \prime}$, near center sec. 15, 1. IN., R. 9 W., $3 \frac{3}{8}$ miles northeast of Glendora. Altitude of stream bed, 1,565 feot.

Drainage area.- Area, 4.49 square miles. Average altitude, 2,590 feot. Maximum altitude, 3,6ट 6 feot. Average slope, 40 percent. Length of ma1n stream channel, 3.5 miles. Average slope of in stream channel, 11 percent.

Gage-height record. - Water-stage recorder graph and frequent staff-gage readings.

D1scharge record.- Computed from valve rating curves, valve operation records, record of stage, discharge measurements, and stage-capacity tables; stage-capacity table based on survey of November 1934 used to t ime of peak stage cf Mar. '2 (8:30 p.m.), table corrected by silt survey made after storm used thereafter.

Maxima.- 1938: 'Inflow, 1,350 second-feet 3:15 p.m. Mar. 2 . Outflow, 739 second-feet 6 p.m. Mar. 2 .

1929-37: Mean hourly inflow, 227 second-fe日t $3-4$ a.m. Jan. 1, 1934.

Remarks, - Records good. Altitude of spillway, 1,706 feet. Ares at spillway level, 23 acres (after storn of Mar. 2). Capacity at spillway level before storm, 040 acre feet; after storm, 974 acre-feet. Baslc data Feb. 27 to Mar. 10 and complete record for rest of February, March, and April furnished by Los Angeles County Flood Control District, through'H. $\mathrm{B}$. Hedger, chief engineer.

Mean daily inflow, in second - feet, 1938

\begin{tabular}{|r|l|l|l||r|l|l|l|l||r|r|r|r|}
\hline Day & Feb. & Mar. & Apr. & Day & Fob. & Mar. & Apr. & Day & Fob. & Mar. & Apr. \\
\hline 1 & 1.6 & 101 & 5 & 11 & 8.5 & 15 & 4.5 & 21 & 1.6 & 9 & 3.3 \\
2 & .6 & 416 & 6 & 12 & 8 & 39 & 4.7 & 22 & 1.6 & 8.5 & 3.4 \\
3 & 7 & 147 & 5 & 13 & 5.5 & 38 & 4.7 & 23 & .8 & 7 & 3.4 \\
4 & 5 & 67 & 5 & 14 & 3.4 & 33 & 3.9 & 24 & 1.1 & 8.5 & 3.4 \\
5 & 1.9 & 45 & 5 & 15 & 2.7 & 27 & 4 & 25 & 1.2 & 9.5 & 3.5 \\
6 & 1.6 & 37 & 4.5 & 16 & 2 & 20 & 4 & 26 & .8 & 9.5 & 3.5 \\
7 & 1.8 & 31 & 4.5 & 17 & 2.1 & 18 & 4 & 27 & 2.8 & 9.5 & 3.5 \\
8 & 1.3 & 31 & 3.7 & 18 & 2 & 14 & 3.4 & 28 & 50 & 10 & 2.6 \\
9 & 3.2 & 22 & 3.9 & 19 & 2.7 & 11 & 3.3 & 29 & & 8 & 3.6 \\
10 & 2.5 & 17 & 4.5 & 20 & 2 & 11 & 3.3 & 30 & & 7 & 3.6 \\
\end{tabular}

Inflow, in second-feet, at indicated time, 1938

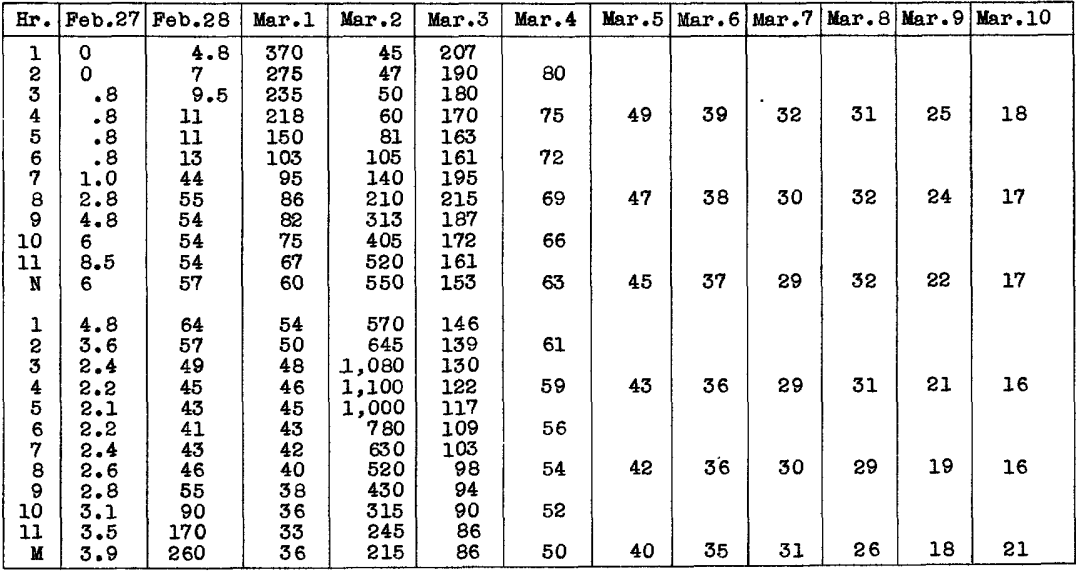

Supplemental record.- Mar. $2,3: 15$ p.m., 1,350 sec.-ft.

Hean dally outflow, in second-feet, 1938

\begin{tabular}{|c|c|c|c|c|c|c|c|c|c|c|c|}
\hline Day & Feb. & Mar. & $\mathrm{Apr}$. & Day & $\mathrm{F} \oplus \mathrm{b}$. & Mar. & Apr. & Day & Feb. & Mar. & Apr. \\
\hline $\begin{array}{r}1 \\
2 \\
3 \\
4 \\
5 \\
6 \\
7 \\
8 \\
9 \\
10\end{array}$ & $\begin{array}{l}0 \\
0 \\
0 \\
0 \\
0 \\
0 \\
0 \\
0 \\
0 \\
0\end{array}$ & $\begin{array}{r}10 \\
179 \\
117 \\
173 \\
96 \\
94 \\
60 \\
48 \\
33 \\
37\end{array}$ & $\begin{array}{l}4.5 \\
4.5 \\
4.5 \\
4.5 \\
4.5 \\
4.5 \\
4.5 \\
4.5 \\
2.5 \\
0\end{array}$ & $\begin{array}{l}11 \\
12 \\
13 \\
14 \\
15 \\
16 \\
17 \\
18 \\
19 \\
20\end{array}$ & $\begin{array}{l}0 \\
0 \\
0 \\
0 \\
0 \\
0 \\
0 \\
0 \\
0 \\
0\end{array}$ & $\begin{array}{l}14 \\
16 \\
16 \\
16 \\
18 \\
25 \\
25 \\
21 \\
12 \\
10\end{array}$ & $\begin{array}{l}0 \\
0 . \\
0 \\
0 \\
0 \\
0 \\
0 \\
0 \\
0 \\
0\end{array}$ & $\begin{array}{l}21 \\
22 \\
23 \\
24 \\
25 \\
26 \\
27 \\
28 \\
29 \\
30 \\
31\end{array}$ & $\begin{array}{l}0 \\
0 \\
0 \\
0 \\
0 \\
0 \\
0 \\
0\end{array}$ & $\begin{array}{l}10 \\
10 \\
10 \\
6.5 \\
5 \\
5 \\
9 \\
12 \\
8.5 \\
7 \\
6\end{array}$ & $\begin{array}{l}0 \\
0 \\
0 \\
0 \\
0 \\
0 \\
0 \\
0 \\
0 \\
0\end{array}$ \\
\hline \multicolumn{9}{|c|}{ 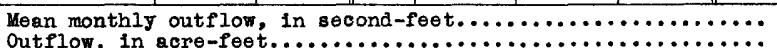 } & $\begin{array}{l}0 \\
0\end{array}$ & $\begin{array}{r}35.8 \\
2,200\end{array}$ & $\begin{array}{r}1.28 \\
76\end{array}$ \\
\hline
\end{tabular}


Outflow, in second-reet, at indicated time, 1938

\begin{tabular}{|c|c|c|c|c|c|c|c|c|c|c|c|c|}
\hline $\mathrm{Hr}$. & Feb.27 & $\mathrm{Feb} .28$ & Mar. 1 & $\operatorname{Mar} \cdot 2$ & Mar.3 & $\operatorname{Lax} \cdot 4$ & $\operatorname{Mar} .5$ & $\operatorname{Mar} .6$ & Har.7 & Mar. 8 & $\operatorname{Lax} .9$ & Har. 10 \\
\hline $\begin{array}{r}1 \\
2 \\
3 \\
4 \\
5 \\
6 \\
7 \\
8 \\
9 \\
10 \\
11 \\
11 \\
\\
1 \\
2 \\
3 \\
4 \\
5 \\
6 \\
7 \\
8 \\
9 \\
10 \\
11 \\
4\end{array}$ & $\begin{array}{l}0 \\
0 \\
0 \\
0 \\
0 \\
0\end{array}$ & $\begin{array}{l}0 \\
0 \\
0 \\
0 \\
0 \\
0\end{array}$ & $\begin{array}{r}0 \\
0 \\
0 \\
0 \\
0 \\
0 \\
0 \\
\\
0 \\
0 \\
15 \\
29 \\
29 \\
29 \\
29 \\
29\end{array}$ & $\begin{array}{r}29 \\
29 \\
29 \\
30 \\
30 \\
\\
31 \\
32 \\
81 \\
131 \\
134 \\
456 \\
739 \\
460 \\
462 \\
462 \\
328 \\
327 \\
231\end{array}$ & $\begin{array}{r}138 \\
138 \\
138 \\
138 \\
83 \\
83 \\
84 \\
84 \\
84 \\
84 \\
84 \\
84 \\
\\
84 \\
112 \\
123 \\
106 \\
123 \\
140 \\
140 \\
140 \\
140 \\
140 \\
244 \\
346\end{array}$ & $\begin{array}{r}344 \\
342 \\
340 \\
337 \\
335 \\
333 \\
330 \\
164 \\
0 \\
0 \\
83 \\
166 \\
149 \\
132 \\
66 \\
66 \\
33 \\
66 \\
66 \\
66 \\
66 \\
66 \\
66 \\
66\end{array}$ & $\begin{array}{r}66 \\
0 \\
66 \\
112 \\
111\end{array}$ & $\begin{array}{r}105 \\
83 \\
62\end{array}$ & 60 & $\begin{array}{l}59 \\
59 \\
30\end{array}$ & $\begin{array}{l}29 \\
43 \\
43\end{array}$ & $\begin{array}{l}42 \\
42 \\
21 \\
17 \\
17\end{array}$ \\
\hline
\end{tabular}

Supplemental records.- Mar. 2, 4:01 p.m., 449 sec.-ft.; 5:01 p.m., 732 se0.-ft.; and $6: 01$ p.m., 458 sec.-it.

LIve Oak Creek at flood-control reservolr near La Verne, Callf.

Location. - Water-stage recorder, 1at. $34^{\circ} 07^{\prime} 45^{\prime \prime}$, long. $117^{\circ} 44^{\prime} 45^{n}$, in $\mathrm{mit}$ sec. 32 , I. IN., R. 8 W., 2t miles northeast of La Verne. Altitude of' stream bed, 1,430 feet.

Drainage area.- Area, 2.30 square miles. Average alt1tude, 2,220 feet. Maximm alt1tide, 3,694 feet. Average slope, 36 percent. Iength of main atream channel, 2.75 miles. Average slope of main stream channe1, 16 percent.

Gage-he1ght record.- Water-stage recorder graph;stalf-gage observations da11y at 8 a.m. and more irequentiy during storm periods.

Discharge record.- Computed from valve rating curve, valve operation record, record of stage, and stage-capacity tables; atage-capacity table based on survey of larch 1936 used to time of peak stage of lias. 2 (6:20 p.m.), table based on survey of May 1938 used thereafter.

Maxima.- 1938: Inflow, 373 second-feet 3:45 p.m. Mar. 2 .

Outflow, 200 second-feet about 5:30 p.m. Mar. 2

1936-37: Kean Inflow, 139 second-feet between 6 and 6:30 p.m. Feb. 6, 1937.

Remerks.- Records good for Harch and fair for February and April. Altitude of spillway, 1,497 feet. Area at sp111way level, 11.6 acrss (Nay 1938). Capac1ty at splilway level before storm of Mar. 2, 242 acre-feet, after storm, 229 acre-feet. Basic data Feb. 27 to Mar. 12 and entire record Feb. 1-26 and Mar. 13 to Apr. 30 furmished by Los Angeles County Flood Control Distriot, through H. B. Hedger, chier engineer.

Mean da1ly inflow, in second-feet, 1938

\begin{tabular}{|c|c|c|c|c|c|c|c|c|c|c|c|}
\hline Day & Feb. & Mar. & Apr. & Day & Feb. & Mar. & Apr. & Day & Feb. & Mar. & Apr. \\
\hline $\begin{array}{r}1 \\
2 \\
3 \\
4 \\
5 \\
6 \\
7 \\
8 \\
9 \\
10\end{array}$ & $\begin{array}{l}0.2 \\
0 \\
1.5 \\
1.2 \\
.5 \\
.1 \\
.1 \\
.1 \\
.9 \\
.6\end{array}$ & $\begin{array}{r}28 \\
148 \\
35 \\
13 \\
10 \\
6 \\
4.4 \\
3.1 \\
3.0 \\
3.3\end{array}$ & $\begin{array}{r}0.8 \\
.7 \\
.7 \\
.7 \\
.7 \\
.5 \\
.5 \\
.5 \\
.4 \\
.4\end{array}$ & $\begin{array}{l}11 \\
12 \\
13 \\
14 \\
15 \\
16 \\
17 \\
18 \\
19 \\
20\end{array}$ & $\begin{array}{r}4.9 \\
3.3 \\
1.2 \\
.5 \\
.5 \\
.2 \\
.2 \\
.1 \\
.1 \\
.1\end{array}$ & $\begin{array}{l}3.2 \\
13 \\
11 \\
6 \\
4.9 \\
4.1 \\
3.4 \\
2.8 \\
2.3 \\
2.1\end{array}$ & $\begin{array}{r}0.4 \\
.4 \\
.5 \\
.5 \\
.5 \\
.4 \\
.4 \\
.4 \\
.4 \\
.4\end{array}$ & $\begin{array}{l}21 \\
22 \\
23 \\
24 \\
25 \\
26 \\
27 \\
28 \\
29 \\
30 \\
31\end{array}$ & $\begin{array}{c}0 \\
0 \\
0 \\
0 \\
0 \\
0 \\
19^{* 4}\end{array}$ & $\begin{array}{l}1.9 \\
1.7 \\
1.5 \\
1.3 \\
1.2 \\
1.1 \\
1.1 \\
1.2 \\
1 \\
1.1 \\
.9\end{array}$ & $\begin{array}{r}0.4 \\
.4 \\
.3 \\
.6 \\
1.2 \\
.8 \\
.6 \\
.5 \\
.5 \\
.4\end{array}$ \\
\hline \multicolumn{12}{|c|}{ 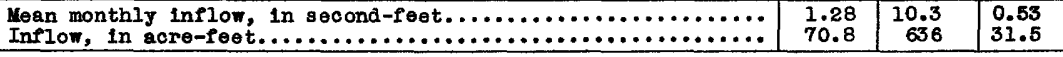 } \\
\hline
\end{tabular}


Inflow, in second-feet, at indicated time, 1938

\begin{tabular}{|c|c|c|c|c|c|}
\hline Hour & February 27 & February 28 & Karch 1 & March 2 & March 3 \\
\hline $\begin{array}{r}1 \\
2 \\
3 \\
4 \\
5 \\
6 \\
7 \\
8 \\
9 \\
10 \\
11 \\
\mathbf{H}\end{array}$ & $\begin{array}{l}0 \\
0 \\
0 \\
0 \\
0 \\
0 \\
0 \\
1.0 \\
1.3 \\
1.2 \\
.9 \\
.7\end{array}$ & $\begin{array}{r}0.1 \\
.1 \\
1.0 \\
1.4 \\
2.5 \\
7.5 \\
8.5 \\
11 \\
25 \\
31 \\
37 \\
34\end{array}$ & $\begin{array}{r}177 \\
85 \\
62 \\
41 \\
31 \\
27 \\
23 \\
19 \\
16 \\
14 \\
13 \\
12\end{array}$ & $\begin{array}{r}13 \\
15 \\
16 \\
17 \\
29 \\
64 \\
110 \\
130 \\
168 \\
290 \\
278 \\
250\end{array}$ & $\begin{array}{r}46 \\
42 \\
41 \\
39 \\
40 \\
53 \\
56 \\
49 \\
43 \\
38 \\
34 \\
31\end{array}$ \\
\hline $\begin{array}{r}1 \\
2 \\
3 \\
4 \\
5 \\
6 \\
7 \\
8 \\
9 \\
10 \\
11 \\
11\end{array}$ & $\begin{array}{l}.6 \\
.5 \\
.5 \\
.4 \\
.4 \\
.3 \\
.3 \\
.3 \\
.3 \\
.3 \\
.2 \\
.2\end{array}$ & $\begin{array}{r}25 \\
19 \\
16 \\
14 \\
12 \\
10 \\
9 \\
11 \\
14 \\
40 \\
72 \\
115\end{array}$ & $\begin{array}{l}11 \\
10 \\
10 \\
9.5 \\
9 \\
8 \\
7 \\
6 \\
5 \\
3.2 \\
2.6 \\
6\end{array}$ & $\begin{array}{r}214 \\
290 \\
335 \\
285 \\
269 \\
241 \\
158 \\
103 \\
92 \\
84 \\
71 \\
54\end{array}$ & $\begin{array}{l}30 \\
29 \\
29 \\
28 \\
27 \\
26 \\
25 \\
23 \\
21 \\
20 \\
19 \\
18\end{array}$ \\
\hline
\end{tabular}

Supplemental record.- Mar. 2, 3:45 p.m., 373 sec.-ft.

Mean daily outflow, in second-feet, 1938

\begin{tabular}{|c|c|c|c|c|c|c|c|c|c|c|c|}
\hline Day & Feb. & Mar. & Apr. & Day & Feb. & Mar. & Apr. & Day & Feb. & Nar. & Apr. \\
\hline $\begin{array}{r}1 \\
2 \\
3 \\
4 \\
5 \\
6 \\
7 \\
8 \\
9 \\
10\end{array}$ & $\begin{array}{l}0 \\
0 \\
0 \\
0 \\
0 \\
0 \\
0 \\
0 \\
0 \\
0\end{array}$ & $\begin{array}{c}26 \\
88 \\
55 \\
22 \\
19 \\
17 \\
15 \\
13 \\
6.5 \\
3.2\end{array}$ & $\begin{array}{l}0 \\
0 \\
0 \\
0 \\
0 \\
0 \\
0 \\
0 \\
0 \\
0\end{array}$ & $\begin{array}{l}11 \\
12 \\
13 \\
14 \\
15 \\
16 \\
17 \\
18 \\
19 \\
20\end{array}$ & $\begin{array}{l}0 \\
0 \\
0 \\
0 \\
0 \\
0 \\
0 \\
0 \\
0 \\
0\end{array}$ & $\begin{array}{l}3.2 \\
7.5 \\
7.5 \\
5.5 \\
5.5 \\
5.5 \\
5.5 \\
5.0 \\
2.8 \\
3.3\end{array}$ & $\begin{array}{l}0 \\
0 \\
0 \\
0 \\
0 \\
0 \\
0 \\
0 \\
0 \\
0\end{array}$ & $\begin{array}{l}21 \\
22 \\
23 \\
24 \\
25 \\
26 \\
27 \\
28 \\
29 \\
30 \\
31\end{array}$ & $\begin{array}{l}0 \\
0 \\
0 \\
0 \\
0 \\
0 \\
0 \\
.7\end{array}$ & $\begin{array}{l}3.2 \\
3.1 \\
2.6 \\
2.6 \\
0.8 \\
0 \\
0 \\
0 \\
0 \\
0 \\
0\end{array}$ & $\begin{array}{l}0 \\
0 \\
0 \\
0 \\
0 \\
0 \\
0 \\
0 \\
0 \\
0\end{array}$ \\
\hline \multicolumn{9}{|c|}{ 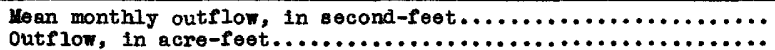 } & $\begin{array}{r}0.025 \\
1.39\end{array}$ & $\begin{array}{r}10.4 \\
639\end{array}$ & $\begin{array}{l}0 \\
0\end{array}$ \\
\hline
\end{tabular}

Outriow, in second-feet, at indicated time, 1938

\begin{tabular}{|c|c|c|c|c|c|c|c|c|c|c|c|c|}
\hline Hr. & Feb.27 & Feb.28 & Mar. 1 & $\operatorname{Mar}, 2$ & Mar.3 & Mar. 4 & Mar. 5 & Mar. 6 & Nar .7 & Mar. 8 & Mar. 9 & Mar.10 \\
\hline $\begin{array}{r}1 \\
2 \\
3 \\
4 \\
5 \\
6 \\
7 \\
8 \\
9 \\
10 \\
11 \\
1 \\
1 \\
1 \\
2 \\
3 \\
4 \\
5 \\
6 \\
7 \\
8 \\
9 \\
10 \\
11 \\
4\end{array}$ & 0 & 0 & $\begin{array}{l}40 \\
40 \\
40 \\
40 \\
40 \\
40 \\
28 \\
15 \\
15 \\
\\
15 \\
15 \\
15 \\
15 \\
15 \\
15 \\
25\end{array}$ & $\begin{array}{r}25 \\
25 \\
25 \\
25 \\
25 \\
50 \\
75 \\
75 \\
75 \\
88 \\
150 \\
162 \\
195 \\
195 \\
175 \\
150 \\
113 \\
100 \\
88 \\
75\end{array}$ & $\begin{array}{l}75 \\
75 \\
75 \\
75 \\
74 \\
74 \\
\\
74 \\
25 \\
25 \\
25 \\
25 \\
25 \\
25\end{array}$ & $\begin{array}{l}25 \\
25 \\
25 \\
25 \\
25 \\
19 \\
19 \\
19 \\
19 \\
19 \\
19 \\
19 \\
19\end{array}$ & $\begin{array}{l}19 \\
18 \\
18\end{array}$ & 17 & $\begin{array}{l}15 \\
15\end{array}$ & $\begin{array}{l}14 \\
13\end{array}$ & $\begin{array}{l}12 \\
4.5 \\
3.2 \\
3.2 \\
3.2 \\
3.2\end{array}$ & 3.2 \\
\hline
\end{tabular}

Supplementai record.- Mar. 2, 5:30 p.m., 200 sec.-ft.

$4547200-42-9$ 
Thompson Creek at flood-control reservoir near Claremont, Calif.

Location.- Staff gage, lat. $34^{\circ} 08^{\prime} 30^{\prime \prime}$, long. $11^{\circ} 42^{\prime} 30^{\prime \prime}$, In swt sec. 27. T. 1 X., R 8 W., 3 miles north of claremont. Aititude of stream bed, 1,582 feet.

Drainage area.- Area, 3.91 square miles. Average altitude, 2,670 feet. Maximum altitude, 4,700 feet. Average slope, 36 percent. Length of main stream channel, 2.5 miles. Average slope of main stream channel, 24 percent.

Gage-helght record.- Staff gage read at irregular intervals except during storm period, when several observations were made dally.

Discharge record. - Computed from valve rating curves, valve operation record, record of stage, observations of depth of flow in release flume, and stage-capacity table; stage-capacity table based on survey of November 1932 used throughout storm period.

Maxima.- 1938: Inflow, 669 second-feet 2:30 p.m. Mar. 2.

Outflow, 133 second-feet 12:30 p.m. Mar. 3

1932-37: Mean hourly inflow, 91 second-feet between 8 and 9 a.m. Feb. 9, 1932.

Remarks.- Records good Feb. 28 to Mar. 6; major portion of inflow for perlods Feb. 127 and Mar. 7 to Apr. 30 diverted above dam to spreading grounds. Altitude of spillway, 1, 640 feet. Area at spiliway level, 35 acres. Capacity at splilway level before storm of Mar. 2, 812 acre-feet; after storm, 786 acre-feet. Basic data Feb. 28 to Mar. 6 and entire record Feb. 1-27 and Mar. 7 to Apr. 30 furmished by Ios Angeles County Flood Control District, through H. E. Hedger, chief engineer.

Mean daily inflow, in second-feet, 1938

\begin{tabular}{|c|c|c|c|c|c|c|c|c|c|c|c|}
\hline Day & $\mathrm{Feb}$. & Mar. & Apr. & Day & Feb. & Mar. & Apr. & Day & Feb. & Mar. & Apr. \\
\hline $\begin{array}{r}1 \\
2 \\
3 \\
4 \\
5 \\
6 \\
7 \\
8 \\
9 \\
10\end{array}$ & $\begin{array}{l}0 \\
0 \\
0 \\
0 \\
0 \\
0 \\
0 \\
0 \\
0 \\
.1\end{array}$ & $\begin{array}{r}42 \\
259 \\
101 \\
44 \\
25 \\
16 \\
9 \\
0 \\
0 \\
0\end{array}$ & $\begin{array}{l}0 \\
0 \\
0 \\
0 \\
0 \\
0 \\
0 \\
0 \\
0 \\
0\end{array}$ & $\begin{array}{l}11 \\
12 \\
13 \\
14 \\
15 \\
16 \\
17 \\
18 \\
19 \\
20\end{array}$ & $\begin{array}{l}1.0 \\
5.2 \\
4.8 \\
0 \\
0 \\
0.2 \\
0 \\
0 \\
0 \\
0\end{array}$ & $\begin{array}{l}3.4 \\
14 \\
11 \\
5.5 \\
1.8 \\
0 \\
0 \\
0 \\
0 \\
0\end{array}$ & $\begin{array}{l}0 \\
0 \\
0 \\
0 \\
0 \\
0 \\
0 \\
0 \\
0 \\
0\end{array}$ & $\begin{array}{l}21 \\
22 \\
23 \\
24 \\
25 \\
26 \\
27 \\
28 \\
29 \\
30 \\
31\end{array}$ & $\begin{array}{r}0 \\
0 \\
0 \\
0 \\
0 \\
0 \\
0 \\
24\end{array}$ & $\begin{array}{l}0 \\
0 \\
0 \\
0 \\
0 \\
0 \\
0 \\
0 \\
0 \\
0 \\
0\end{array}$ & $\begin{array}{l}0 \\
0 \\
0 \\
0 \\
0 \\
0 \\
0 \\
0 \\
0 \\
0\end{array}$ \\
\hline \multicolumn{9}{|c|}{ 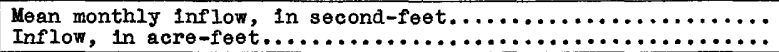 } & $\begin{array}{r}1.26 \\
70 \\
\end{array}$ & $\begin{array}{r}17 \cdot 2 \\
1,050\end{array}$ & $\begin{array}{l}0 \\
0\end{array}$ \\
\hline
\end{tabular}

Inflow, in second-feet, at indicated time, 1938

\begin{tabular}{|c|c|c|c|c|c|c|c|c|c|}
\hline Hour & $F \oplus b .27$ & $F e b .28$ & Mar. 1 & Mar . 2 & Mar. 3 & Mar. 4 & Mar. 5 & Mar. 6 & Mar. 7 \\
\hline $\begin{array}{r}1 \\
2 \\
3 \\
4 \\
5 \\
6 \\
7 \\
8 \\
9 \\
10 \\
11 \\
\mathrm{~N}\end{array}$ & $\begin{array}{l}0 \\
0 \\
0 \\
0 \\
0 \\
0 \\
0 \\
0 \\
0 \\
0 \\
0 \\
0\end{array}$ & $\begin{array}{l}0.2 \\
.2 \\
.2 \\
\\
30.3 \\
39 \\
27 \\
23\end{array}$ & $\begin{array}{r}170 \\
155 \\
117 \\
75 \\
51 \\
43 \\
40 \\
37 \\
35 \\
32 \\
29 \\
27\end{array}$ & $\begin{array}{r}13 \\
15 \\
17 \\
24 \\
34 \\
55 \\
98 \\
130 \\
233 \\
375 \\
430 \\
398\end{array}$ & $\begin{array}{r}126 \\
119 \\
120 \\
153 \\
142 \\
132 \\
126 \\
120 \\
115 \\
108 \\
102 \\
96\end{array}$ & $\begin{array}{l}62 \\
56 \\
50 \\
46 \\
43 \\
39\end{array}$ & $\begin{array}{l}29 \\
26 \\
23\end{array}$ & 16 & 9 \\
\hline $\begin{array}{r}1 \\
2 \\
3 \\
4 \\
5 \\
6 \\
7 \\
8 \\
9 \\
10 \\
11 \\
M\end{array}$ & $\begin{array}{l}0 \\
0 \\
0 \\
0 \\
0 \\
0 \\
0 \\
0 \\
0 \\
0 \\
0 \\
0\end{array}$ & $\begin{array}{l}22 \\
21 \\
20 \\
20 \\
19 \\
18 \\
18 \\
18 \\
19 \\
23 \\
35 \\
66\end{array}$ & $\begin{array}{l}25 \\
22 \\
18 \\
16 \\
15 \\
14 \\
14 \\
13 \\
12 \\
12 \\
11 \\
12\end{array}$ & $\begin{array}{l}380 \\
460 \\
535 \\
508 \\
540 \\
530 \\
405 \\
292 \\
236 \\
212 \\
173 \\
136\end{array}$ & $\begin{array}{l}92 \\
88 \\
84 \\
80 \\
77 \\
75 \\
74 \\
73 \\
71 \\
70 \\
69 \\
67\end{array}$ & $\begin{array}{l}37 \\
36 \\
35 \\
34 \\
34 \\
33\end{array}$ & $\begin{array}{l}22 \\
21 \\
19\end{array}$ & & \\
\hline
\end{tabular}

Supplemental record.- Mar. 2, 2:30 p.m., 669 sec.-ft.

Mean daily outflow, in second-feet, 1938

\begin{tabular}{|c|c|c|c|c|c|c|c|c|c|c|c|}
\hline Day & Feb. & Mar. & Apr. & Day & $F \in b$. & Mar. & Apr. & Day & $F e b$. & Mar. & Apr. \\
\hline $\begin{array}{r}1 \\
2 \\
3 \\
4 \\
5 \\
6 \\
7 \\
8 \\
9 \\
10\end{array}$ & $\begin{array}{l}0 \\
0 \\
0 \\
0 \\
0 \\
0 \\
0 \\
0 \\
0 \\
0\end{array}$ & $\begin{array}{r}0 \\
16 \\
101 \\
87 \\
39 \\
25 \\
17 \\
12 \\
20 \\
23\end{array}$ & $\begin{array}{l}1.7 \\
1.7 \\
1.7 \\
1.7 \\
1.6 \\
1.6 \\
1.6 \\
1.6 \\
1.6 \\
1.6\end{array}$ & $\begin{array}{l}11 \\
12 \\
13 \\
14 \\
15 \\
16 \\
17 \\
18 \\
19 \\
20\end{array}$ & $\begin{array}{l}0 \\
0 \\
0 \\
0 \\
0 \\
0 \\
0 \\
0 \\
0 \\
0\end{array}$ & $\begin{array}{l}25 \\
.5 \\
3.1 \\
10 \\
10 \\
11 \\
13 \\
13 \\
12 \\
12\end{array}$ & $\begin{array}{l}1.3 \\
1.3 \\
1.3 \\
1.3 \\
1.3 \\
1.3 \\
1.3 \\
1.2 \\
1.2 \\
1.2\end{array}$ & $\begin{array}{l}21 \\
22 \\
23 \\
24 \\
25 \\
26 \\
27 \\
28 \\
29 \\
30 \\
31\end{array}$ & $\begin{array}{l}0 \\
.1 \\
: 1 \\
: 1 \\
: 1 \\
: \frac{1}{1} \\
0.1 \\
0^{-1}\end{array}$ & $\begin{array}{l}5 \\
1.2 \\
1.2 \\
1.7 \\
1.8 \\
1.7 \\
2.2 \\
2.3 \\
2.1 \\
2.0 \\
2.1\end{array}$ & $\begin{array}{r}1.0 \\
1.0 \\
1.0 \\
1.0 \\
1.0 \\
1.0 \\
.9 \\
.9 \\
.9 \\
.9\end{array}$ \\
\hline Mea & ath & & & & & & & & $\begin{array}{r}0.02 \\
1.2\end{array}$ & $\begin{array}{r}15.3 \\
938\end{array}$ & $\begin{array}{l}1.29 \\
76.8\end{array}$ \\
\hline
\end{tabular}


Outflow, In second-feet, at indicated time, 1938

\begin{tabular}{|c|c|c|c|c|c|}
\hline Hour & March 2 & March 3 & March 4 & March 5 & March 6 \\
\hline $\begin{array}{r}1 \\
2 \\
3 \\
4 \\
5 \\
6 \\
7 \\
8 \\
9 \\
9 \\
10 \\
11 \\
\mathbb{N} \\
1 \\
1 \\
2 \\
3 \\
4 \\
5 \\
6 \\
7 \\
8 \\
9 \\
10 \\
11 \\
\mathbb{M}\end{array}$ & $\begin{array}{r}0 \\
0 \\
39 \\
52 \\
59 \\
63 \\
67 \\
68 \\
70\end{array}$ & $\begin{array}{r}70 \\
72 \\
74 \\
76 \\
77 \\
78 \\
79 \\
79 \\
80 \\
80 \\
80 \\
81 \\
132 \\
131 \\
130 \\
129 \\
128 \\
128 \\
127 \\
125 \\
123 \\
121 \\
120 \\
119\end{array}$ & $\begin{array}{c}117 \\
114 \\
111 \\
107 \\
104 \\
101 \\
98 \\
96 \\
50 \\
49 \\
49 \\
47 \\
45\end{array}$ & $\begin{array}{l}32 \\
28\end{array}$ & $\begin{array}{l}24 \\
26 \\
32\end{array}$ \\
\hline
\end{tabular}

Supplemental record.- Mar. 3, $12: 30$ p.m., 133 sec.-ft.

\section{Los Angeles River Basin}

Pacolma Creek at flood-control reservoir near San Fernando, Cal1f.

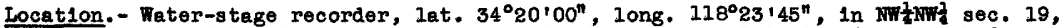
T. 3 N., R. 14 M., 4 miles northesist of San Fermando. Altitude of stream bed, 1,650 soet.

Drainage area.- Area, 27.8 square miles. Averege altitude, 3,890 foet. Maximum eltitude, 6,500 feet. Average slope, 41 percent. Length of main stream channel, 15.6 m1les. Average slope of maln stroem channel, 5.2 percent.

Gage-helght record. - Water-stage recorder graph; staff-gage readings dally at 8 a.m. and more frequently during storm perlod.

Discharge record.- Computed from spillway rating curve, valve rating curves, valve operation record, record of stage and stage-capacity tables; stage-capacity table based on a urvey of January 1936 used to time of peak stage of Mar. 3 (1 a.m.);

table corrected by s11t survey made after storm used thereafter.

Maxima.- 1938: Inflow, 8,450 second-feet 3:50 p.m. Mar. 2 . outflow, 2,440 second-feet 12 p.m. Mar. 2 to 2 .m. Mar. 3 .

1914, 1927-37: 5,400 second-feet Februkry 1914

Remarks.- Records good. Alt1tude of sp1llway, 1,950 feet. Area at spillway level, 63 acres (after storm of Mar. 2). Capaclty at splilway level before storm, 5,592 acrefoot; after storm, 5,004 core-foet. Bas1c data Fob. 27 to Nar. 11 and entíre rocord Fob, 1-26 and Mer. 12 to Apr. 30 furnished by Los Angeles County Flood Control D1s-

trict, through H. F. Hedger, chlef eng ineer.

Mean dally inflow, in second-feet, 1938

\begin{tabular}{|r|r|r|r||r|r|r|r||r|r|r|r|}
\hline Day & Fob. & Mar. & Apr. & Day & Fob. & Mar. & Apr. & Day & Fob. & Mar. & Apr. \\
\hline 1 & 14 & $\mathbf{3 5 5}$ & 60 & 11 & 198 & 130 & 36 & 21 & 27 & 132 & 28 \\
2 & 14 & 2,430 & 55 & 12 & 146 & 310 & 34 & 22 & 25 & 119 & 27 \\
3 & 100 & 1,520 & 53 & 13 & 84 & 445 & 36 & 23 & 24 & 106 & 26 \\
4 & 113 & 579 & 47 & 14 & 61 & 364 & 35 & 24 & 21 & 96 & 31 \\
5 & 55 & 367 & 45 & 15 & 49 & 304 & 34 & 25 & 21 & 87 & 39 \\
6 & 32 & 279 & 44 & 16 & 42 & 254 & 33 & 26 & 21 & 78 & 28 \\
7 & 24 & 225 & 42 & 17 & 38 & 215 & 32 & 27 & 26 & 74 & 24 \\
8 & 20 & 193 & 39 & 18 & 35 & 183 & 31 & 28 & 116 & 71 & 23 \\
9 & 24 & 156 & 39 & 19 & 35 & 159 & 29 & 29 & & 66 & 27 \\
10 & 30 & 140 & 37 & 20 & 30 & 146 & 28 & 30 & & 62 & 28 \\
\end{tabular}


Inflow, in second-feet, at Indleated time, 1938

\begin{tabular}{|c|c|c|c|c|c|c|c|c|c|c|c|c|}
\hline Hr. & $\mathrm{Feb} .27$ & Feb.28 & Mar.1 & Mar.2 & Mar.3 & $\operatorname{Mar} .4$ & Mar. 5 & Hax.6 & Har.7 & Mar. 8 & Mar. 9 & Lar. 10 \\
\hline $\begin{array}{r}1 \\
2 \\
3 \\
4 \\
5 \\
6 \\
7 \\
8 \\
9 \\
10 \\
11 \\
\text { II }\end{array}$ & $\begin{array}{l}14 \\
14 \\
14 \\
14 \\
15 \\
15 \\
16 \\
17 \\
18 \\
20 \\
24 \\
26\end{array}$ & $\begin{array}{r}37 \\
37 \\
37 \\
42 \\
54 \\
51 \\
47 \\
54 \\
82 \\
95 \\
106 \\
119\end{array}$ & $\begin{array}{l}435 \\
590 \\
690 \\
685 \\
500 \\
390 \\
375 \\
364 \\
350 \\
336 \\
371 \\
306\end{array}$ & $\begin{array}{r}261 \\
276 \\
300 \\
343 \\
375 \\
407 \\
495 \\
630 \\
815 \\
1,060 \\
1,190 \\
1,700\end{array}$ & $\begin{array}{l}2,480 \\
2,370 \\
2,220 \\
2,100 \\
2,000 \\
1,880 \\
1,800 \\
1,690 \\
1,600 \\
1,540 \\
1,500 \\
1,450\end{array}$ & $\begin{array}{l}805 \\
780 \\
748 \\
718 \\
695 \\
665 \\
636 \\
620 \\
608 \\
597 \\
570 \\
537\end{array}$ & 377 & 293 & 230 & 200 & 156 & 142 \\
\hline $\begin{array}{r}1 \\
2 \\
3 \\
4 \\
5 \\
6 \\
7 \\
8 \\
9 \\
10 \\
11 \\
1\end{array}$ & $\begin{array}{l}27 \\
28 \\
29 \\
33 \\
36 \\
38 \\
40 \\
39 \\
39 \\
38 \\
38 \\
37\end{array}$ & $\begin{array}{l}141 \\
140 \\
135 \\
131 \\
129 \\
128 \\
130 \\
136 \\
169 \\
270 \\
300 \\
412\end{array}$ & $\begin{array}{l}289 \\
275 \\
262 \\
253 \\
250 \\
249 \\
248 \\
247 \\
246 \\
244 \\
242 \\
247\end{array}$ & $\begin{array}{l}3,120 \\
3,840 \\
5,950 \\
7,850 \\
5,730 \\
5,000 \\
4,700 \\
3,860 \\
3,320 \\
2,950 \\
2,710 \\
2,550\end{array}$ & $\begin{array}{r}1,380 \\
1,320 \\
1,260 \\
1,180 \\
1,140 \\
1,070 \\
1,030 \\
1,000 \\
960 \\
920 \\
890 \\
860\end{array}$ & $\begin{array}{l}528 \\
520 \\
516 \\
508 \\
480 \\
474 \\
469 \\
460 \\
454 \\
448 \\
440 \\
436\end{array}$ & 329 & 259 & 203 & 178 & 148 & $\begin{array}{c}138 \\
135\end{array}$ \\
\hline
\end{tabular}

Supplemental record.- Max. $2,3: 50$ p.m., 8,450 sec.-ft.

Hean dally outflcw, In second-feet, 1938

\begin{tabular}{|r|c|r|r||r|r|r|r||r|r|r|r|}
\hline Day & Feb. & Mar. & Apr. & Day & Feb. & Mar. & Apr. & Day & Feb. & Mar. & Apr. \\
\hline 1 & 0 & 56 & 50 & 11 & 0 & 297 & 74 & 21 & 33 & 265 & 27 \\
2 & 0 & 618 & 50 & 12 & 18 & 243 & 87 & 22 & 33 & 268 & 25 \\
3 & 0 & 1,740 & 50 & 13 & 30 & 296 & 83 & 23 & 33 & 249 & 25 \\
4 & 0 & 676 & 77 & 14 & 19 & 301 & 86 & 24 & 33 & 144 & 25 \\
5 & 0 & 416 & 108 & 15 & 8 & 240 & 62 & 25 & 28 & 106 & 25 \\
6 & 0 & 330 & 106 & 16 & 0 & 304 & 47 & 26 & 22 & 120 & 17 \\
7 & 0 & 293 & 104 & 17 & 26 & 302 & 46 & 27 & 8 & 91 & 22 \\
8 & 0 & 316 & 100 & 18 & 32 & 297 & 46 & 28 & 8 & 76 & 22 \\
9 & 0 & 310 & 97 & 19 & 22 & 292 & 35 & 29 & & 61 & 22 \\
10 & 0 & 304 & 95 & 20 & 33 & 286 & 30 & 30 & & 57 & 9.5 \\
\end{tabular}

Outflow, in second-feet, at indicated t1me, 1938

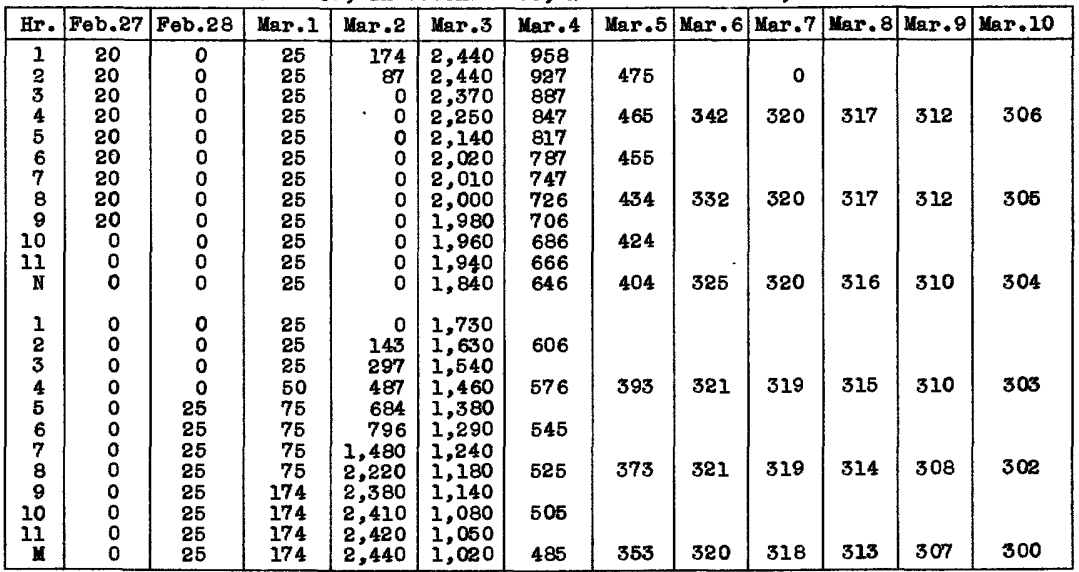


Tujunga Creek at flood-control reservolr near Sunland, Galif.

Location. - Water-atage recorder, lat. $34^{\circ} 17^{\prime} 30^{\prime \prime}$, long. $118^{\circ} 11^{\prime} 1^{\prime \prime}$, in NEt sec. 1 , T. 2 N., R. 13 W., $10 \mathrm{mlles}$ northesst of Sunland. Altitude of stream bed, 2,104 feet.

Drainage area.- Area, 81.4 aquare miles. Average altitude, 4,500 feet. Maximum alt1tude, 7,078 feet. Average slope, 40 percent. Length of main stream channel, 12 miles. Average slope of main stream channel, 7.8 percent.

Gage-height record.- Water-atage recorder graph except 2 - 6 p.m. Mar. 2; staff-gage readings daily at $8 \mathrm{a} . \mathrm{m}$. and more frequently curing atorm period; maximum stage determined from floodmarks.

Discharge record. - Computed from record of atage, spiliway rating curve, valve rating curves, valve operation record, discharge measurements, and a tage-capacity tables; stage-capacity table of December 1935 used to time of peak stage (5:20 p.m. Mar. 2), table corrected by silt survey made after storm used the reafter.

Haxima.- 1938: Inflow, 35,000 second-feet 5 p.m., Mar. 2.

1931-37: Mean hourly inflow, 2,430 second-feet 3 to 4 a.m., Jan. 1, 1934.

Remarks.- Recorâs fair. Spillway discharge affected by debris Mar. 5-6. Altitude of spiliway, 2,290 feet. Area at spiliway level, 74.4 acres (after gtorm of Mar. 2 ). Storage capacity before atorm, 6,240 acre-feet; after storm, 4,734 acre-feet. Basic data Feb. 27 to Mar. 11 and entire record for remainder of February, March, and

April furnished by Los Angeles County Flood Control District, through H. E. Hedger, chtef engineer.

Mean daily inflow, in second-feet, 1938

\begin{tabular}{|c|c|c|c|c|c|c|c|c|c|c|c|}
\hline Day & Feb. & Mar. & Apr. & Day & Feb. & Har. & Apr. & Day & Feb. & Mar. & Apr. \\
\hline $\begin{array}{r}1 \\
2 \\
3 \\
4 \\
5 \\
6 \\
7 \\
8 \\
9 \\
10\end{array}$ & $\begin{array}{r}66 \\
39 \\
118 \\
150 \\
66 \\
34 \\
25 \\
25 \\
59 \\
91\end{array}$ & $\begin{array}{r}1,240 \\
11,600 \\
2,330 \\
867 \\
521 \\
415 \\
327 \\
324 \\
247 \\
236\end{array}$ & $\begin{array}{r}112 \\
117 \\
115 \\
108 \\
102 \\
82 \\
82 \\
84 \\
82 \\
82\end{array}$ & $\begin{array}{l}11 \\
12 \\
13 \\
14 \\
15 \\
16 \\
17 \\
18 \\
18 \\
20\end{array}$ & $\begin{array}{r}532 \\
228 \\
112 \\
81 \\
65 \\
51 \\
39 \\
37 \\
55 \\
43\end{array}$ & $\begin{array}{l}204 \\
825 \\
558 \\
405 \\
347 \\
301 \\
259 \\
272 \\
242 \\
205\end{array}$ & $\begin{array}{l}84 \\
85 \\
91 \\
81 \\
80 \\
78 \\
73 \\
72 \\
67 \\
66\end{array}$ & $\begin{array}{l}21 \\
22 \\
23 \\
24 \\
25 \\
26 \\
27 \\
28 \\
29 \\
30 \\
31\end{array}$ & $\begin{array}{r}38 \\
38 \\
35 \\
32 \\
35 \\
35 \\
106 \\
501\end{array}$ & $\begin{array}{l}199 \\
188 \\
184 \\
171 \\
166 \\
143 \\
133 \\
142 \\
135 \\
126 \\
117\end{array}$ & $\begin{array}{l}62 \\
60 \\
62 \\
72 \\
69 \\
62 \\
59 \\
57 \\
64 \\
61\end{array}$ \\
\hline \multicolumn{12}{|c|}{ 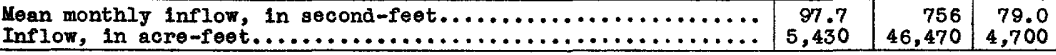 } \\
\hline
\end{tabular}

Inflow, in second-feet, at indicated time, 1938

\begin{tabular}{|c|c|c|c|c|c|c|c|c|c|c|c|c|}
\hline $\mathbf{E r}$. & Feb.27 & Feb.28 & Mar. 1 & Uar.2 & Mar.3 & Mar. 4 & Mar. 5 & Mar . 6 & Mar.7 & Mar. 8 & Mar. 9 & Har.10 \\
\hline $\begin{array}{r}1 \\
2 \\
3 \\
4 \\
5 \\
6 \\
7 \\
8 \\
9 \\
10 \\
11 \\
11\end{array}$ & $\begin{array}{l}15 \\
24 \\
33 \\
40 \\
40 \\
38 \\
35 \\
32 \\
30 \\
36 \\
48 \\
57\end{array}$ & $\begin{array}{l}160 \\
179 \\
188 \\
196 \\
210 \\
240 \\
280 \\
318 \\
490 \\
580 \\
553 \\
580\end{array}$ & $\begin{array}{r}3,140 \\
3,330 \\
3,060 \\
2,310 \\
1,750 \\
1,430 \\
1,300 \\
1,220 \\
1,160 \\
1,020 \\
925 \\
882\end{array}$ & $\begin{array}{r}420 \\
420 \\
437 \\
655 \\
805 \\
1,000 \\
1,450 \\
2,200 \\
3,280 \\
4,300 \\
7,400 \\
13,000\end{array}$ & $\begin{array}{l}5,750 \\
4,250 \\
3,420 \\
3,000 \\
2,700 \\
2,600 \\
2,530 \\
2,400 \\
2,220 \\
2,150 \\
2,070 \\
1,890\end{array}$ & $\begin{array}{r}1,090 \\
1,030 \\
970 \\
920 \\
875 \\
857\end{array}$ & 574 & 428 & 327 & 324 & 247 & 236 \\
\hline $\begin{array}{r}1 \\
2 \\
3 \\
4 \\
5 \\
6 \\
7 \\
8 \\
9 \\
10 \\
11 \\
M\end{array}$ & $\begin{array}{r}66 \\
82 \\
102 \\
131 \\
173 \\
229 \\
275 \\
253 \\
244 \\
238 \\
221 \\
200\end{array}$ & $\begin{array}{r}600 \\
600 \\
585 \\
595 \\
620 \\
640 \\
515 \\
470 \\
440 \\
720 \\
1,100 \\
2,130\end{array}$ & $\begin{array}{l}820 \\
775 \\
715 \\
680 \\
650 \\
620 \\
590 \\
547 \\
520 \\
492 \\
469 \\
448\end{array}$ & $\begin{array}{r}18,700 \\
23,100 \\
25,000 \\
30,000 \\
35,000 \\
28,500 \\
23,200 \\
18,300 \\
14,900 \\
11,600 \\
9,550 \\
7,860\end{array}$ & $\begin{array}{l}1,880 \\
1,780 \\
1,700 \\
1,610 \\
1,550 \\
1,470 \\
1,400 \\
1,350 \\
1,290 \\
1,230 \\
1,190 \\
1,150\end{array}$ & $\begin{array}{l}815 \\
773 \\
746 \\
723 \\
700 \\
675\end{array}$ & 451 & 392 & & & & . \\
\hline
\end{tabular}

Mean dally outflow, in second-feet, 1938

\begin{tabular}{|c|c|c|c|c|c|c|c|c|c|c|c|}
\hline Day & Fob. & Mar. & Apr. & Day & Feb. & Mar. & Apr. & Day & Feb. & Mar. & Apr. \\
\hline $\begin{array}{r}1 \\
2 \\
3 \\
4 \\
5 \\
6 \\
7 \\
8 \\
9 \\
10\end{array}$ & $\begin{array}{l}13 \\
21 \\
7 \\
2.5 \\
40 \\
45 \\
45 \\
45 \\
0 \\
0\end{array}$ & $\begin{array}{r}627 \\
10,900 \\
2,530 \\
893 \\
524 \\
405 \\
818 \\
775 \\
717 \\
342\end{array}$ & $\begin{array}{r}100 \\
109 \\
111 \\
108 \\
137 \\
155 \\
155 \\
104 \\
68 \\
69\end{array}$ & $\begin{array}{l}11 \\
12 \\
13 \\
14 \\
15 \\
16 \\
17 \\
18 \\
19 \\
20\end{array}$ & $\begin{array}{l}13 \\
55 \\
76 \\
76 \\
57 \\
26 \\
50 \\
31 \\
38 \\
57\end{array}$ & $\begin{array}{l}196 \\
557 \\
619 \\
383 \\
320 \\
433 \\
429 \\
283 \\
248 \\
213\end{array}$ & $\begin{array}{l}71 \\
72 \\
73 \\
74 \\
50 \\
18 \\
18 \\
8 \\
2.7 \\
0\end{array}$ & $\begin{array}{l}21 \\
22 \\
23 \\
24 \\
25 \\
26 \\
27 \\
28 \\
29 \\
30 \\
31\end{array}$ & $\begin{array}{l}57 \\
57 \\
57 \\
57 \\
57 \\
57 \\
17 \\
60\end{array}$ & $\begin{array}{r}225 \\
191 \\
169 \\
195 \\
67 \\
170 \\
171 \\
144 \\
97 \\
99 \\
97\end{array}$ & $\begin{array}{r}0 \\
0 \\
0 \\
0 \\
0 \\
0 \\
0 \\
0 \\
0 \\
31\end{array}$ \\
\hline 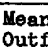 & 11 & & & & & & & & $\begin{array}{r}39.9 \\
2,210\end{array}$ & $\begin{array}{r}769 \\
47,280\end{array}$ & $\begin{array}{r}51.1 \\
3,040\end{array}$ \\
\hline
\end{tabular}


Outflow, in second-feet, at indicated t1me, 1938

\begin{tabular}{|c|c|c|c|c|c|c|c|c|c|c|c|c|}
\hline Fr. & Feb.27 & Feb.28 & Mar.1 & Mar.2 & Mar. 3 & Mar. 4 & Mar. 5 & $-\operatorname{Mar} \cdot 6$ & Mar.7 & Mar. 8 & Mar. $\theta$ & Mar.10 \\
\hline $\begin{array}{r}1 \\
2 \\
3 \\
4 \\
5 \\
6 \\
7 \\
8 \\
9 \\
10 \\
11 \\
1 \\
\\
1 \\
2 \\
3 \\
4 \\
5 \\
6 \\
7 \\
8 \\
9 \\
10 \\
11 \\
x\end{array}$ & $\begin{array}{l}52 \\
52 \\
52 \\
52 \\
52 \\
52 \\
52 \\
26 \\
0 \\
0 \\
0 \\
0\end{array}$ & $\begin{array}{r}0 \\
-0 \\
0 \\
0 \\
0 \\
0 \\
0 \\
80 \\
160 \\
160 \\
160 \\
160 \\
160\end{array}$ & $\begin{array}{l}518 \\
518 \\
518 \\
518 \\
560 \\
584 \\
571 \\
571 \\
530 \\
530 \\
520 \\
507 \\
507 \\
507 \\
480 \\
456 \\
430 \\
712 \\
994 \\
990 \\
990 \\
985 \\
985 \\
980\end{array}$ & $\begin{array}{r}980 \\
975 \\
975 \\
970 \\
970 \\
970 \\
970 \\
970 \\
1,120 \\
1,130 \\
1,560 \\
8,090 \\
\\
15,300 \\
21,400 \\
23,200 \\
28,200 \\
32,200 \\
31,400 \\
25,100 \\
20,300 \\
16,400 \\
13,100 \\
10,600 \\
8,330\end{array}$ & $\begin{array}{l}6,620 \\
5,200 \\
3,970 \\
3,150 \\
2,800 \\
2,630 \\
2,590 \\
2,520 \\
2,410 \\
2,220 \\
2,130 \\
2,060 \\
1,960 \\
1,870 \\
1,780 \\
1,690 \\
1,600 \\
1,520 \\
1,480 \\
1,410 \\
1,360 \\
1,310 \\
1,260 \\
1,210\end{array}$ & $\begin{array}{r}1,170 \\
1,130 \\
1,090 \\
1,050 \\
1,020 \\
985 \\
960 \\
930 \\
910 \\
895 \\
882 \\
875 \\
\\
865 \\
845 \\
820 \\
800 \\
775 \\
757 \\
742 \\
730 \\
718 \\
703 \\
690 \\
680\end{array}$ & $\begin{array}{l}650 \\
625 \\
600 \\
585 \\
570 \\
515 \\
470 \\
445 \\
431 \\
420 \\
413 \\
408\end{array}$ & 410 & $\begin{array}{l}375 \\
365 \\
360 \\
360 \\
370 \\
380\end{array}$ & $\begin{array}{r}1,040 \\
1,020 \\
640 \\
190 \\
190\end{array}$ & $\begin{array}{l}940 \\
930 \\
905\end{array}$ & $\begin{array}{r}295 \\
205 \\
205 \\
205 \\
205 \\
0 \\
200\end{array}$ \\
\hline
\end{tabular}

Supplemental record.- Mar. 2, 5:20 p.m., 32,500 sec.-ft.

Sante Anlta Creok at flood-control reservolr near slerra Madre, Callf.

Location.- Water-stage recorder, lat. $34^{\circ} 11^{\prime} 10^{\prime \prime}$, long. $11^{\circ} \mathrm{OI} 10^{\prime \prime}$, near center of sec.

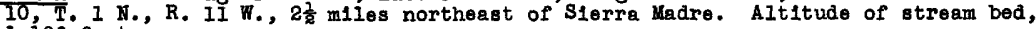
1,100 feot.

Drainage area.- Area, 10.8 square miles. Average altitude, 3,660 feet. Naximum alt1tude, 5, 886 feet. Average slope, 47 percent. Iength of main stream channel, 4.2 miles. Average slope of main stream channel, 20 percent.

Gage-helght record.- Water-stage recorder graph to about 2 p.m. Mar. 2 . Frequent staff-gage readings during storm period; one reading daily at 8 a.m. at other times.

Discharge record. - Computed from record of stage, spillway discharge curves, valve rating ourves, valve operation record, discharge measurements, and stage-capac1ty tables; stage-capacity table based on survey of February 1936 used to time of peak stage of Mar. $2(4: 20$ p.m.), table based on surveg of July 1938 used thereafter.

Maxima.- 1938: Inflow, 5,490 second-feet 4:10 p.m. Mar. 2 .

1932-37: Mean daily inflow, 323 second-feet Jan. $i, i 934$.

Remarks.- Records good except those for Mar. 3 to Apr. 30, which ape fair. Area at lower splliway level, 10.8 acres (July 1938). Oapacity at lower splllway level before storm of Mar. 2, 1,010 acre-feet, efter storm, 683 acre-feet. Alt1tude of sp11lwey, 1,316 feet. Besic date Feb. 27 to Mar. 11 and entire record for rest of February, March, and April furnished by Los Angeles County Flood Control District, through H. E. Hedger, chlef engineer.

Mean de11y inflow, in second-feet, 1938

\begin{tabular}{|c|c|c|c|c|c|c|c|c|c|c|c|}
\hline $\mathrm{Day}$ & Fob. & Mar. & Apr. & Dey & $F \in b$. & Mar. & Apr. & Day & $\mathrm{Feb}$. & Mar. & Apr. \\
\hline $\begin{array}{r}1 \\
2 \\
3 \\
4 \\
5 \\
6 \\
7 \\
8 \\
9 \\
10\end{array}$ & $\begin{array}{l}18 \\
7.5 \\
74 \\
74 \\
29 \\
17 \\
14 \\
11 \\
21 \\
19\end{array}$ & $\begin{array}{r}423 \\
1,780 \\
666 \\
285 \\
185 \\
143 \\
123 \\
107 \\
90 \\
86\end{array}$ & $\begin{array}{l}36 \\
34 \\
32 \\
30 \\
28 \\
27 \\
27 \\
27 \\
27 \\
26\end{array}$ & $\begin{array}{l}11 \\
12 \\
13 \\
14 \\
15 \\
16 \\
17 \\
18 \\
19 \\
20\end{array}$ & $\begin{array}{l}64 \\
50 \\
32 \\
25 \\
19 \\
16 \\
15 \\
16 \\
17 \\
13\end{array}$ & $\begin{array}{r}77 \\
146 \\
129 \\
108 \\
93 \\
85 \\
78 \\
74 \\
69 \\
67\end{array}$ & $\begin{array}{l}26 \\
26 \\
26 \\
26 \\
26 \\
26 \\
25 \\
24 \\
24 \\
24\end{array}$ & $\begin{array}{l}21 \\
22 \\
23 \\
24 \\
25 \\
26 \\
27 \\
28 \\
29 \\
30 \\
31\end{array}$ & $\begin{array}{c}11 \\
11 \\
10 \\
10 \\
9 \\
8.5 \\
15 \\
332\end{array}$ & $\begin{array}{l}62 \\
58 \\
55 \\
51 \\
49 \\
47 \\
45 \\
43 \\
42 \\
40 \\
38\end{array}$ & $\begin{array}{l}24 \\
23 \\
23 \\
23 \\
23 \\
22 \\
21 \\
21 \\
20 \\
20\end{array}$ \\
\hline \multicolumn{12}{|c|}{ 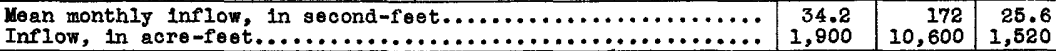 } \\
\hline
\end{tabular}


Inflow, in second-feet, at indicated time, 1938

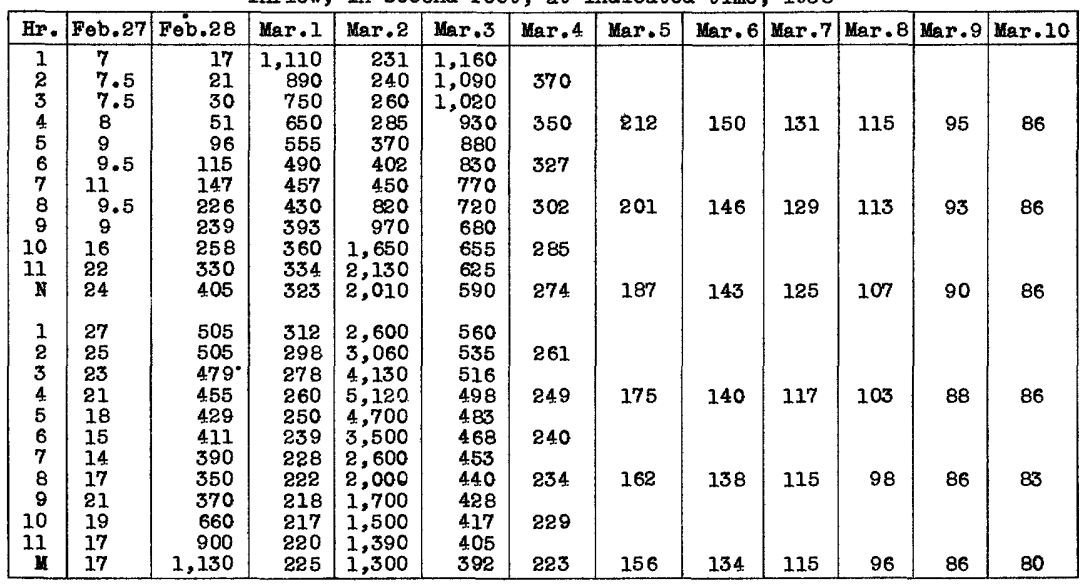

Supplemental record.- Mar. 2, 4:10 p.m., 5,490 sec.-ft.

Mean da1ly outflow, in second-feet, 1938

\begin{tabular}{|c|c|c|c|c|c|c|c|c|c|c|c|}
\hline Day & Feb. & Mar. & Apr. & Day & Fob. & Mar. & Apr. & Day & Feb. & Mar. & Apr. \\
\hline $\begin{array}{r}1 \\
2 \\
3 \\
4 \\
5 \\
6 \\
7 \\
8 \\
9 \\
10\end{array}$ & $\begin{array}{c}8 \\
12 \\
4.7 \\
43 \\
46 \\
28 \\
29 \\
38 \\
14 \\
0\end{array}$ & $\begin{array}{r}559 \\
1,520 \\
692 \\
292 \\
188 \\
146 \\
124 \\
107 \\
90 \\
86\end{array}$ & $\begin{array}{l}37 \\
37 \\
36 \\
34 \\
34 \\
34 \\
35 \\
35 \\
36 \\
37\end{array}$ & $\begin{array}{l}11 \\
12 \\
13 \\
14 \\
15 \\
16 \\
17 \\
18 \\
19 \\
20\end{array}$ & $\begin{array}{c}19 \\
56 \\
55 \\
43 \\
32 \\
23 \\
17 \\
12 \\
8.5 \\
12\end{array}$ & $\begin{array}{r}77 \\
144 \\
130 \\
109 \\
93 \\
85 \\
79 \\
74 \\
70 \\
67\end{array}$ & $\begin{array}{l}21 \\
.3 \\
1.1 \\
19 \\
26 \\
26 \\
25 \\
24 \\
24 \\
24\end{array}$ & $\begin{array}{l}21 \\
22 \\
23 \\
24 \\
25 \\
26 \\
27 \\
28 \\
29 \\
30 \\
31 \\
\end{array}$ & $\begin{array}{r}11 \\
11 \\
11 \\
11 \\
11 \\
11 \\
13 \\
104\end{array}$ & $\begin{array}{l}62 \\
59 \\
55 \\
51 \\
57 \\
42 \\
46 \\
42 \\
40 \\
42 \\
39 \\
\end{array}$ & $\begin{array}{l}24 \\
24 \\
23 \\
22 \\
23 \\
22 \\
21 \\
21 \\
20 \\
21\end{array}$ \\
\hline \multicolumn{9}{|c|}{ 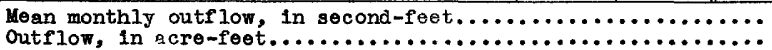 } & $\begin{array}{r}24.4 \\
1,360 \\
\end{array}$ & 10,450 & $\begin{array}{r}26 \\
1,520 \\
\end{array}$ \\
\hline
\end{tabular}

Outflow, in second-feet, at indicated time, 1938

\begin{tabular}{|c|c|c|c|c|c|c|c|c|c|c|c|c|}
\hline Elr. & Feb.27 & Feb.28 & Mar.1 & Mar . 2 & Mar 3 & Mar. 4 & Mar. 5 & Mar. 6 & Mar.7 & Mar. 8 & Mar.9 & Mar. 10 \\
\hline $\begin{array}{r}1 \\
2 \\
3 \\
4 \\
5 \\
6 \\
7 \\
8 \\
9 \\
10 \\
11 \\
N \\
1 \\
1 \\
2 \\
3 \\
4 \\
5 \\
6 \\
7 \\
8 \\
9 \\
10 \\
11 \\
\text { H }\end{array}$ & $\begin{array}{r}0 \\
25 \\
-25 \\
25 \\
25 \\
25 \\
25\end{array}$ & $\begin{array}{r}25 \\
25 \\
25 \\
\\
25 \\
25 \\
50 \\
75 \\
75 \\
\\
75 \\
75 \\
75 \\
75 \\
138 \\
200 \\
200 \\
200 \\
200 \\
467 \\
564\end{array}$ & $\begin{array}{l}573 \\
580 \\
584 \\
583 \\
580 \\
575 \\
568 \\
560 \\
550 \\
541 \\
530 \\
518 \\
506\end{array}$ & $\begin{array}{r}494 \\
484 \\
477 \\
367 \\
256 \\
263 \\
402 \\
548 \\
570 \\
\\
983 \\
2,450 \\
3,850 \\
5,060 \\
4,960 \\
3,320 \\
2,790 \\
1,950 \\
1,760 \\
1,610 \\
1,520 \\
1,350\end{array}$ & $\begin{array}{r}1,230 \\
1,130 \\
1,060 \\
980 \\
930 \\
870 \\
807 \\
745 \\
705 \\
665 \\
645 . \\
605 \\
\\
590 \\
550 \\
530 \\
510 \\
495 \\
480 \\
467 \\
453 \\
440 \\
4.25 \\
411 \\
398\end{array}$ & $\begin{array}{l}383 \\
370 \\
359 \\
346 \\
336 \\
325 \\
318 \\
310 \\
302 \\
297 \\
290 \\
280 \\
269 \\
260 \\
260 \\
260 \\
258 \\
254 \\
250 \\
246 \\
241 \\
236 \\
231 \\
226\end{array}$ & 160 & 144 & 115 & 105 & 93 & 86 \\
\hline
\end{tabular}

Supplemental record.- Mer. 2, 4:20 p.m., 5,260 sec.-ft. 
Little Santa Anita Creek at flood-control reservolr near Sierra Madre, Calif.

Location.- Water-stage recorder, lat. $34^{\circ} 10^{\prime} 40^{n}$, long. $118^{\circ} 02^{\prime} 40^{n}$, near center of sec.

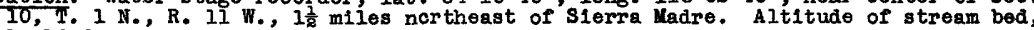
1,110 feet.

Drainage area.- Area, 2.39 square miles. Average altitude, 3,170 feet. Maximum alt1tude, 5,433 feet. Average slope, 50 percent. Length of maln stream channel, 3.0 miles. Average slope of maln stream channel, 27 percent.

Gage-he1ght record.- Water-stage recorder graph.

Discharge record. - Outflow obtalned from record of water-stage recorder located 270 feet below the dam (cperated by Los Angeles County Flood Control District). Inflow " Feb. 1 - 27 and Mar. 4 to Apr. 30, taken as equal to measured outflow; inflow Feb. 27 to Mar. 3 computed from measured outflow and changes in storage, based on record of stage and stage-capacity tables. Stage capacity table based on survey of April 1932 used to 2 p.m. Mar. 2 , and table based on survey made after storm used after 12 p.m. Mar. 2 ; capacity loss distributed during period 2 - 12 p.m. Mar. 2 .

Maxima.- 1938: Inflow, 633 second-feet 4:10 p.m. Mar. 2 . Outflow, 630 second-feet $4: 10$ p.m. Mar. 2 .

Remarks.- Records falr. Alt1tude of splllway, 1,172 feet. Area at sp1llway level, 1.04 acres (Apr1l 1938). Capacity at spillway level before storm of Mar. 2, 47.4 acre-feet, capacity after storm, 8.5 acre-feet. Basio data Feb. 28 to Mar. 3 and entire record $\mathrm{Feb} .1-27$ and Mar. 5 to Apr. 30 furnished by Los Angeles county Flood Control District, through $\mathrm{H}$. F. Hedger, chlef engineer.

\begin{tabular}{|r|l|r|l||r|r|r|r||r|r|r|r|}
\hline Day & Feb. & Mar. & A pr. & Day & Feb. & Mar. & A pr. & Day & Feb. & Mar. & Apr. \\
\hline 1 & 0.9 & 67 & 5.5 & 11 & 6.5 & 19 & 4.7 & 21 & 0.7 & 27 & 3.7 \\
2 & 0 & 214 & 5 & 12 & 5.5 & 38 & 5 & 22 & .5 & 16 & 3.0 \\
3 & 6.5 & 118 & 5 & 13 & 3.3 & 35 & 5.5 & 23 & .4 & 8.5 & 2.2 \\
4 & 8.5 & 48 & 6 & 14 & 2.8 & 20 & 6 & 24 & .3 & 8 & 3.3 \\
5 & 3.9 & 38 & 6 & 15 & 2.0 & 30 & 6 & 25 & .2 & 11 & 3.3 \\
6 & 1.8 & 32 & 5.5 & 16 & 1.6 & 14 & 6 & 26 & .2 & 11 & 3.3 \\
7 & 1.1 & 28 & 5 & 17 & 1.2 & 12 & 5 & 27 & 1.5 & 7.5 & 2.8 \\
8 & .8 & 16 & 4.7 & 18 & 1.1 & 13 & 2.8 & 28 & 47 & 7 & 2.5 \\
9 & 2.4 & 12 & 5 & 19 & .9 & 19 & 1.6 & 29 & & 7.5 & 2.8 \\
10 & 1.8 & 16 & 5 & 20 & .7 & 24 & 2.2 & 30 & & 4.7 & 2.3 \\
\end{tabular}

Inflow, in second-feet, at indicated time, 1938

\begin{tabular}{|c|c|c|c|c|c|}
\hline Hour & February 27 & February 28 & Maroh I & March 2 & March 3 \\
\hline $\begin{array}{r}1 \\
2 \\
3 \\
4 \\
5 \\
6 \\
7 \\
8 \\
9 \\
10 \\
11 \\
\text { N }\end{array}$ & $\begin{array}{r}0.2 \\
.4 \\
.4 \\
.6 \\
1.7 \\
3.6\end{array}$ & $\begin{array}{c}3.3 \\
7 \\
15 \\
23 \\
36 \\
19 \\
24 \\
38 \\
53\end{array}$ & $\begin{array}{r}133 \\
132 \\
123 \\
105 \\
92 \\
85 \\
80 \\
75 \\
72 \\
66 \\
62 \\
57\end{array}$ & $\begin{array}{r}40 \\
45 \\
49 \\
56 \\
65 \\
92 \\
76 \\
80 \\
143 \\
220 \\
236 \\
212\end{array}$ & $\begin{array}{l}180 \\
164 \\
151 \\
138 \\
126 \\
112\end{array}$ \\
\hline $\begin{array}{r}1 \\
2 \\
3 \\
4 \\
5 \\
6 \\
7 \\
8 \\
9 \\
10 \\
11 \\
\mathbf{M}\end{array}$ & $\begin{array}{l}2.3 \\
2.0 \\
2.2 \\
2.2 \\
1.8 \\
1.8\end{array}$ & $\begin{array}{r}60 \\
73 \\
92 \\
76 \\
65 \\
58 \\
54 \\
58 \\
77 \\
103 \\
125 \\
134\end{array}$ & $\begin{array}{l}53 \\
48 \\
44 \\
41 \\
39 \\
36 \\
34 \\
33 \\
33 \\
34 \\
35 \\
38\end{array}$ & $\begin{array}{l}252 \\
370 \\
465 \\
560 \\
403 \\
363 \\
318 \\
272 \\
247 \\
230 \\
214 \\
198\end{array}$ & $\begin{array}{r}100 \\
86 \\
79 \\
76 \\
73 \\
70\end{array}$ \\
\hline
\end{tabular}

Supplemental record.- Mar. 2, 4:10 p.m., 633 sec.-ft. 
Mean daily outflow, in second-feet, 1938

\begin{tabular}{|c|c|c|c|c|c|c|c|c|c|c|c|}
\hline Day & Feb. & Mar. & Apr. & Day & Feb. & Mar. & Apr. & Day & Feb. & Mar. & Apr. \\
\hline $\begin{array}{r}1 \\
2 \\
3 \\
4 \\
5 \\
6 \\
7 \\
8 \\
9 \\
10\end{array}$ & $\begin{array}{l}0.9 \\
0 \\
6.5 \\
8.5 \\
3.9 \\
1.8 \\
1.1 \\
.8 \\
2.4 \\
1.8\end{array}$ & $\begin{array}{r}64 \\
192 \\
118 \\
48 \\
38 \\
32 \\
28 \\
16 \\
12 \\
16\end{array}$ & $\begin{array}{l}5.5 \\
5 \\
5 \\
6 \\
6 \\
5.5 \\
5 \\
4.7 \\
5 \\
5\end{array}$ & $\begin{array}{l}11 \\
12 \\
13 \\
14 \\
15 \\
16 \\
17 \\
18 \\
19 \\
20\end{array}$ & $\begin{array}{r}6.5 \\
5.5 \\
3.3 \\
2.8 \\
2.0 \\
1.6 \\
1.2 \\
1.1 \\
.9 \\
.7\end{array}$ & $\begin{array}{l}19 \\
38 \\
35 \\
20 \\
30 \\
14 \\
12 \\
13 \\
19 \\
24\end{array}$ & $\begin{array}{l}4.7 \\
5 \\
5.5 \\
6 \\
6 \\
6 \\
5 \\
2.8 \\
1.6 \\
2.2\end{array}$ & $\begin{array}{l}21 \\
22 \\
23 \\
24 \\
25 \\
26 \\
27 \\
28 \\
29 \\
30 \\
31\end{array}$ & $\begin{array}{r}0.7 \\
.5 \\
.4 \\
.3 \\
.2 \\
.2 \\
1.5\end{array}$ & $\begin{array}{l}27 \\
16 \\
8.5 \\
8 \\
11 \\
11 \\
7.5 \\
7 \\
7.5 \\
4.7 \\
4.7\end{array}$ & $\begin{array}{l}3.7 \\
3.0 \\
2.2 \\
3.3 \\
3.3 \\
3.3 \\
2.8 \\
2.5 \\
2.8 \\
3.3\end{array}$ \\
\hline \multicolumn{9}{|c|}{ 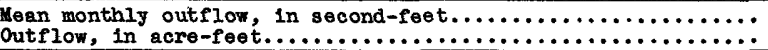 } & $\begin{array}{r}3.65 \\
203\end{array}$ & $\begin{array}{r}29.2 \\
1,800\end{array}$ & $\begin{array}{r}4.26 \\
253\end{array}$ \\
\hline
\end{tabular}

Outflow, in second-feet, at indicated time, 1938

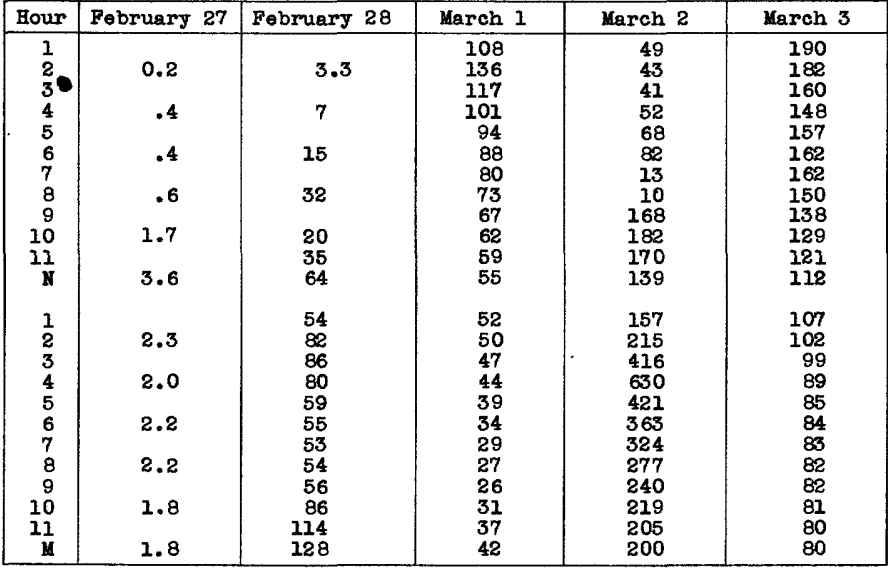

Supplemental record.- Mar. 2, 4:10 p.m., 630 sec.-ft.

Eaton Creek at flood-control reservoir near Pasadena, Calif.

Location.- Water-stage recorder, 1at. $34^{\circ} 10^{\prime} 05^{n}$, Iong. $118^{\circ} 05^{\prime} 30^{\prime \prime}$, in Santa Anita grant, 2 miles northeast of Pasadena, Los Angeles County. Alt1tude of stream bed, 840 feet.

Drainage area.- Area, 9.48 equare miles. Average altitude, 3,100 feet. Maximum altitude, 6,156 feet. Average slope, 52 percent. Iength of main stream channel, 6 miles. Average slope of main stream channel, 17 percent.

Gage-helght record.- Water-stage recorder; staff-gage observations made daily at 8 a.m. and more frequently during storm period.

Discharge record.- Computed from spillway rating curve, valve rating curve, valve operation record, record of stage, and stage-capacity tables; stage-capacity table based on survey of November 1937 used to time of peak stage of Mar. $2(4: 45 \mathrm{p} . \mathrm{m} .)^{\prime}$, and table corrected by silt survey made after storm used thereafter. Inflow Feb. 1 - 26 and Mar. 28 to Apr. 30 taken from records of station Baton Creak near Pasadena", 1 mile upstream. Small outflows measured by weir.

Maxima.- 1938: Inflow, 3,340 second-feet 4:40 p.m. Mar. 2. Outflow, 2,800 second-feet $4: 45$ p.m. Mar. 2 .

Remarks. - Records good except those for Feb. 1 - 26 and Mar. 28 to Apr. 30, which are falr. Altitude of spillway, 888 feet. Area at spillway level, 39 acres (after storm of Mar. 2). Capacity at spillway level before storm of Uar. 2, 940 acre-

feet, after storm, 698 acre-feet. The city of Pasadena Water Department diverts water from Eaton Creek during periods of low flow. Basic data Feb. 27 to Mar. 27 furnished by Los Angeles County Flood Control District, through H. E. Hedger, chlef engineer. 
Mean da1ly inflow, in second-feet, 1938

\begin{tabular}{|c|c|c|c|c|c|c|c|c|c|c|c|}
\hline Day & Feb. & Mar. & Apr. & Day & Feb. & Mar. & Apr. & Day & $\mathrm{Feb}$. & Mar. & Apr. \\
\hline $\begin{array}{r}1 \\
2 \\
3 \\
4 \\
5 \\
6 \\
7 \\
8 \\
9 \\
10\end{array}$ & $\begin{array}{l}9 \\
1.9 \\
36 \\
46 \\
20 \\
4.6 \\
0 \\
0 \\
8.8 \\
6.3\end{array}$ & $\begin{array}{r}161 \\
882 \\
300 \\
126 \\
82 \\
64 \\
57 \\
52 \\
42 \\
39\end{array}$ & $\begin{array}{c}18 \\
13 \\
9 \\
11 \\
12 \\
11 \\
11 \\
10 \\
8 \\
8.5\end{array}$ & $\begin{array}{l}11 \\
12 \\
13 \\
14 \\
15 \\
16 \\
17 \\
18 \\
19 \\
20\end{array}$ & $\begin{array}{c}45 \\
40 \\
20 \\
11 \\
7.5 \\
6 \\
12 \\
5 \\
5 \\
3.0\end{array}$ & $\begin{array}{l}38 \\
74 \\
55 \\
44 \\
32 \\
31 \\
33 \\
26 \\
25 \\
27\end{array}$ & $\begin{array}{l}9 \\
14 \\
12 \\
10 \\
8.5 \\
8 \\
7 \\
6 \\
6 \\
7.5\end{array}$ & $\begin{array}{l}21 \\
22 \\
23 \\
24 \\
25 \\
26 \\
27 \\
28 \\
29 \\
30 \\
31 .\end{array}$ & $\begin{array}{c}2.0 \\
2.0 \\
2.0 \\
2.0 \\
2.0 \\
4.0 \\
8 \\
155\end{array}$ & $\begin{array}{r}26 \\
24 \\
22 \\
24 \\
22 \\
-19 \\
20 \\
23 \\
22 \\
18 \\
17 \\
\end{array}$ & $\begin{array}{l}8 \\
7 \\
6 \\
5.5 \\
5 \\
4.5 \\
4.5 \\
4.5 \\
3.5 \\
4.5\end{array}$ \\
\hline \multicolumn{9}{|c|}{ 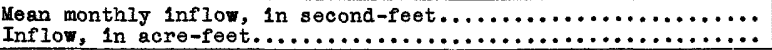 } & $\begin{array}{r}16.6 \\
921 \\
\end{array}$ & $\begin{array}{r}78.2 \\
.4,810 \\
\end{array}$ & $\begin{array}{r}8.42 \\
501 \\
\end{array}$ \\
\hline
\end{tabular}

Inflow, in seconä-fe日t, at indicated time, 1938

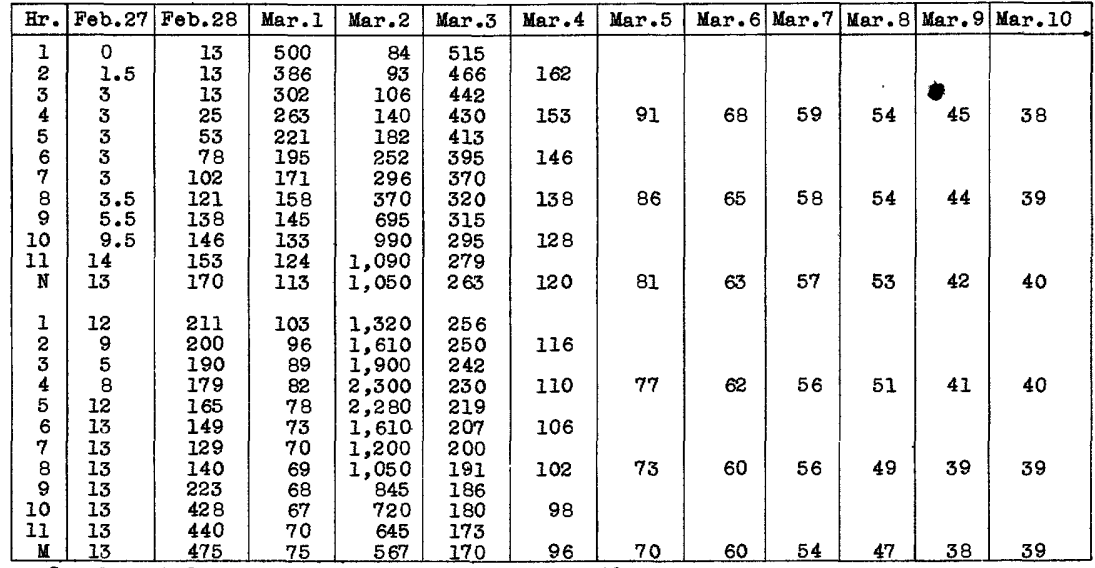

Supplemental record.- Mar. 2, 4:40 p.m., 3,340 sec.-ft.

Mean dally outflow, in second-feet, 1938

\begin{tabular}{|c|c|c|c|c|c|c|c|c|c|c|c|}
\hline Day & Feb. & Mar. & Apr. & Day & Feb. & Mar. & Apr. & Day & $\mathrm{Feb}$. & Mar. & Apr. \\
\hline $\begin{array}{r}1 \\
2 \\
3 \\
4 \\
5 \\
6 \\
7 \\
8 \\
9 \\
10\end{array}$ & $\begin{array}{l}0 \\
0 \\
0 \\
0 \\
0 \\
0 \\
0 \\
0 \\
0 \\
0\end{array}$ & $\begin{array}{r}191 \\
694 \\
295 \\
144 \\
129 \\
128 \\
87 \\
58 \\
61 \\
60\end{array}$ & $\begin{array}{l}0 \\
0 \\
5 \\
4.1 \\
2.2 \\
2.2 \\
11 \\
23 \\
23 \\
23\end{array}$ & $\begin{array}{l}11 \\
12 \\
13 \\
14 \\
15 \\
16 \\
17 \\
18 \\
19 \\
20\end{array}$ & $\begin{array}{l}0 \\
0 \\
0 \\
0 \\
0 \\
0 \\
0 \\
0 \\
0 \\
0\end{array}$ & $\begin{array}{l}16 \\
34 \\
32 \\
15 \\
49 \\
55 \\
35 \\
45 \\
45 \\
30\end{array}$ & $\begin{array}{c}9.5 \\
4.3 \\
19 \\
16 \\
15 \\
5.5 \\
4.3 \\
4.0 \\
3.5 \\
2.4\end{array}$ & $\begin{array}{l}21 \\
22 \\
23 \\
24 \\
25 \\
26 \\
27 \\
28 \\
29 \\
30 \\
31\end{array}$ & $\begin{array}{l}0 \\
0 \\
0 \\
0 \\
0 \\
0 \\
0 \\
6\end{array}$ & $\begin{array}{l}21 \\
21 \\
21 \\
18 \\
17 \\
16 \\
17 \\
31 \\
49 \\
0 \\
0\end{array}$ & $\begin{array}{l}2.9 \\
4.0 \\
3.5 \\
2.3 \\
2.3 \\
.9 \\
0 \\
0 \\
0 \\
0\end{array}$ \\
\hline \multicolumn{9}{|c|}{ 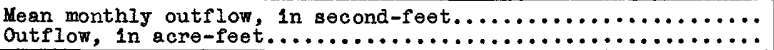 } & $\begin{array}{r}214 \\
11.9\end{array}$ & $\begin{array}{r}77.8 \\
4.780\end{array}$ & $\begin{array}{r}6.43 \\
383\end{array}$ \\
\hline
\end{tabular}

Outflow, in second-feet, at indicated time, 1938

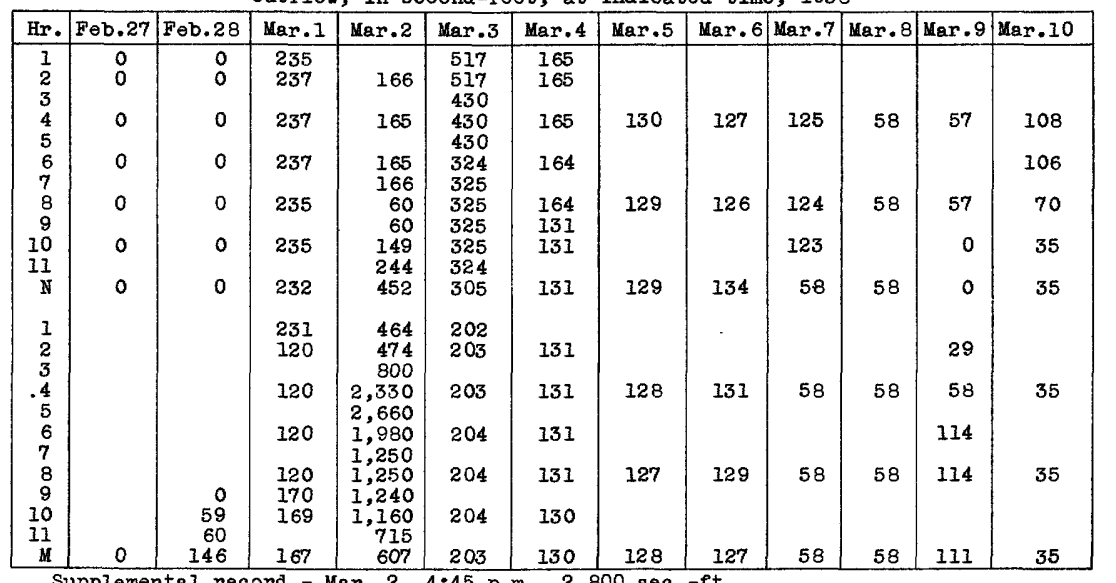




\section{Galloguas Crook Basin}

Honda Barranca at Berylwood Road, near Somis, Calif.

Locstion.- Water-stage recorder and weir control, lat. $34^{\circ} 17^{\prime} 22^{n}$, long. $119^{\circ} 02^{1} 32^{n}$, in

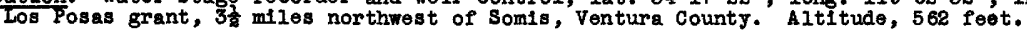

Drainage ares.- Area, 1.35 square miles. Average slope, 35 percent.

Gage-helght record. - Water-stage recorder graph.

Discharge record.- Stage-discharge relation determined from broad-crested weir rating. Maximum.- 1938: Discharge, 256 second-feet 9:05 p.m. Feb. 28.

Remarks.- Records good. Records furnished by U. S. Soll Conservation Service.

Mean dally discharge, in second-feet, 1938

$$
\begin{aligned}
& \text { Feb. } 28 \text {....... } 13 \\
& \text { Mar. }{ }_{2}^{1} \ldots \ldots \ldots 2^{24}{ }^{6} \\
& 3 \ldots \ldots . .24
\end{aligned}
$$

\begin{tabular}{|c|c|c|c|c|}
\hline Hour & February 28 & March 1 & March 2 & March 3 \\
\hline $\begin{array}{r}1 \\
2 \\
3 \\
4 \\
5 \\
6 \\
7 \\
8 \\
9 \\
10 \\
11 \\
11\end{array}$ & $\begin{array}{c}0 \\
0 \\
.2 \\
.9 \\
14.5 \\
9 \\
5 \\
3.1 \\
1.9 \\
1.1 \\
.7 \\
.4\end{array}$ & $\begin{array}{l}2.4 \\
.6 \\
.2 \\
0 \\
0 \\
0 \\
0 \\
0 \\
0 \\
0 \\
0.1\end{array}$ & $\begin{array}{l}15 \\
14 \\
23 \\
70 \\
35 \\
45 \\
25 \\
15 \\
30 \\
26 \\
35 \\
45\end{array}$ & $\begin{array}{c}13 \\
13 \\
13 \\
40 \\
14 \\
14 \\
14 \\
14 \\
19 \\
13 \\
1.5 \\
.6\end{array}$ \\
\hline $\begin{array}{r}1 \\
2 \\
3 \\
4 \\
5 \\
6 \\
7 \\
8 \\
9 \\
10 \\
11 \\
\mathbf{M}\end{array}$ & $\begin{array}{l}.2 \\
0 \\
0 \\
0 \\
0 \\
2.4 \\
2.1 \\
23 \\
200 \\
16 \\
40 \\
7.6\end{array}$ & $\begin{array}{r}.1 \\
.1 \\
.2 \\
.2 \\
.2 \\
.3 \\
.3 \\
.4 \\
.4 \\
.7 \\
2.2 \\
6.4\end{array}$ & $\begin{array}{l}18 \\
64 \\
19 \\
13 \\
13 \\
13 \\
13 \\
13 \\
13 \\
13 \\
13 \\
13\end{array}$ & $\begin{array}{l}.3 \\
.2 \\
.1 \\
0 \\
0 \\
0 \\
0 \\
0 \\
0 \\
0 \\
0 \\
0\end{array}$ \\
\hline
\end{tabular}

Runoff Feb. 28 to Mar. 3 , in acre-feet ...88

Discharge, in second-feet, at inalcated time, 1938

Supplemental record.-Feb. 28, 9:05 p.m., 256 sec.-ft.

Honda Barranca at Perkins Road, near Somis, Calif.

Location.- Water-stage recorder and concrete weir control, lat. $34^{\circ} 16^{\prime} 05^{\prime \prime}$, long. $119^{\circ}$ $0^{\prime} 5^{n}$, In Los Poses grant, at Perkins road, 3 miles west af Somis, Ventura County. Alt1 tude, 349 feet.

Draingge area.- Area, 2.86 square miles. Average slope, 27 percont.

Gage-height record.- Water-stage recorder graph.

Discharge record.- Stage-d1scharge relation determined from broad-crested we1r rating. Maximum.- 1938: Discharge, 486 second-feet 9:20 p.m. Feb. 28.

Remarks.- Records good. Records furnished by U. S. Soll Conservation Service.

Mean dafly discharge, in second-feet, 1938

$$
\begin{aligned}
& \text { Feb. } 28 \text {...... } 26 \\
& \text { Mar. } 1 \ldots \ldots \text {. } 1.0 \\
& \begin{array}{l}
2 \\
3
\end{array} \ldots \ldots \ldots .119
\end{aligned}
$$

Runoff Feb.28 to Har.3, in acre-feet .... 328 
Discharge, in second-reet, at indicated time, 1838

\begin{tabular}{|c|c|c|c|c|}
\hline Hour & Febmuary 28 & March 1 & March 2 & March 3 \\
\hline $\begin{array}{r}1 \\
2 \\
3 \\
4 \\
5 \\
6 \\
7 \\
8 \\
9 \\
10 \\
11 \\
1\end{array}$ & $\begin{array}{c}0 \\
0 \\
0 \\
0 \\
0 \\
60 \\
5 \\
.7 \\
0 \\
0 \\
0 \\
0 \\
0\end{array}$ & $\begin{array}{l}0 \\
0 \\
0 \\
0 \\
0 \\
0 \\
0 \\
0 \\
0 \\
0 \\
0 \\
0\end{array}$ & $\begin{array}{l}50 \\
50 \\
37 \\
160 \\
230 \\
350 \\
210 \\
110 \\
241 \\
163 \\
210 \\
330\end{array}$ & $\begin{array}{r}17 \\
22 \\
23 \\
250 \\
50 \\
24 \\
9 \\
36 \\
30 \\
0 \\
0 \\
0\end{array}$ \\
\hline $\begin{array}{r}1 \\
2 \\
3 \\
4 \\
5 \\
6 \\
7 \\
8 \\
9 \\
10 \\
11 \\
\mathbf{4}\end{array}$ & $\begin{array}{r}0 \\
0 \\
0 \\
0 \\
0 \\
0 \\
0 \\
40 \\
172 \\
100 \\
237 \\
4\end{array}$ & $\begin{array}{l}0 \\
0 \\
0 \\
0 \\
0 \\
0 \\
0 \\
0 \\
0 \\
0 \\
5.5 \\
\mathbf{3 7}^{5}\end{array}$ & $\begin{array}{c}110 \\
190 \\
210 \\
57 \\
51 \\
34 \\
8 \\
4.9 \\
7.0 \\
7.0 \\
7.5 \\
11\end{array}$ & $\begin{array}{l}0 \\
0 \\
0 \\
0 \\
0 \\
0 \\
0 \\
0 \\
0 \\
0 \\
0 \\
0\end{array}$ \\
\hline
\end{tabular}

Supplemental record.- Feb. 28, $9: 20$ p.m., 486 sec.-ft. 


\section{MAXIMUM INFLOW TO RESERVOIRS}

As previousiy explained, for several reservoirs the records of stage and outflow were used in conjunction with the capacity tables to compute rates of inflow. For those reservoirs for which the differences in contents over periods of time constitute a material factor in the determination of Inflow, the results will tend to be more rellable for long than for short perlods. The differences in contents for very short periods of time may be subject to considerable inaccuracy or even be indeterminable, and therefore the method is not well suited to the determination of rates of inflow at any instant or for any very short period.

During the period of maximum discharge the inflow to reservoirs was not conputed for any interval of less than 15 minutes. Therefore, further study was made in order to estimate the maximum or peak rates of inflow as given in the preceding tables.

Records of instantaneous rates of discharge throughout the flood rise were determined at five stations, which are listed in table 12. These records are included, also, in the basic discharge records presented in the next preceding section. Table 12 gives, for each of these stations, the peak discharge rate (inches per hour for 1 minute), the maximum 5-, 10-, 15-, and 30-minute and 1-hour rates, and the percentage of excess of the peak rate above the maximum 5-, 10-, 15-, and 30-minute and 1-hour rates. The percentages of excess, as given in table 12 , appear to have a tendency to increase as the discharge rates decrease.

Table 12,- Maximan rates of discharge for indlcated psriods and excess of peak discharge (duration 1 minute) over those rates in San Dimas and Calleguas Creez Basins, Narch 2 , 1938

\begin{tabular}{|c|c|c|c|c|c|c|c|c|c|c|}
\hline \multirow[t]{2}{*}{ Stream } & \multirow[t]{2}{*}{$\begin{array}{l}\text { Drain- } \\
\text { age } \\
\text { area } \\
\text { (sq. } \\
\text { mi.) }\end{array}$} & \multicolumn{6}{|c|}{$\begin{array}{l}\text { Maximum disoharge } \\
\text { (Inches per hour) }\end{array}$} & \multicolumn{3}{|c|}{$\begin{array}{l}\text { Excess of peak } \\
\text { (1 minute) over } \\
\text { maximum discharge } \\
\text { (percent) }\end{array}$} \\
\hline & & 1 & $\begin{array}{c}5 \\
\text { min. }\end{array}$ & $\begin{array}{l}10 \\
\text { min. }\end{array}$ & $\begin{array}{l}15 \\
\text { min. }\end{array}$ & $\begin{array}{c}30 \\
\text { min. }\end{array}$ & $\stackrel{1}{\text { hour }}$ & $\begin{array}{l}15 \\
\min .\end{array}$ & $\begin{array}{c}30 \\
\text { min. }\end{array}$ & $\stackrel{1}{\text { hour }}$ \\
\hline \multicolumn{11}{|l|}{ San Dimas Creok } \\
\hline $\begin{array}{l}\text { Wolfskill Creek near } \\
\text { Sen Dimas }\end{array}$ & 2.78 & 0.463 & 0.451 & 0.439 & 0.424 & 0.390 & 0.379 & 9.2 & 18.7 & 22.2 \\
\hline $\begin{array}{l}\text { West Fork of San } \\
\text { Dimas Creek, near } \\
\text { San Dimas }\end{array}$ & 1.66 & .620 & .615 & .609 & .602 & .574 & .559 & 3.0 & 8.0 & 10.9 \\
\hline $\begin{array}{l}\text { Fern Canyon Water- } \\
\text { shed Mo. } 3\end{array}$ & .084 & .350 & .346 & .340 & .334 & .324 & . 314 & 4.8 & $8.0^{\circ}$ & 11.5 \\
\hline $\begin{array}{l}\text { Ferm Canyon Water- } \\
\text { shed Iro. } 2\end{array}$ & .063 & .710 & .703 & .698 & .691 & .671 & .651 & 2.7 & 5.8 & 9.1 \\
\hline Calleguas Creaic & & & & & & & & & & \\
\hline $\begin{array}{l}\text { Honda Barranca at } \\
\text { Perkins Road, noar } \\
\text { Somis }\end{array}$ & 2.86 & .214 & .211 & .206 & .203 & .190 & .166 & 5.4 & 12.6 & 28.9 \\
\hline
\end{tabular}


In figure 17, the discharge rates for each of the stations are plotted against corresponding intervala of time, using logarithmic scales. For this purpose, the peak discharge is assumed to correspond to a time interval of 1 minute. The plottings have been extended to longer intervals than are glven in table 12. Discharge rates have also been plotted on the flgure for some of the reservolrs for which the maximum average rates of Inflow are belleved to be reliably determined for the shorter intervals of time.

In general, the ratios of the maximum mean discharge, for progressively shorter intervals of time, to the maximum 24-hour discharge have been found to increase with enough regularity to suggest that the trend of these ratios could be utilized in estimating the peak inflow to the reservolrs. The foregoing rather fragmentary observations as to the relation between peak rates of flow and the average rates over selected perlods of time have been useful in estimating the peak inflow.

With the exception of those for the Ferm Canyon stations, none of the records in table 12 cover conditions in the zones of heaviest rainfall and thus may not reflect in all particulars the conditions of flow that prevalled with munoff from higher rainfall intensities. There is the possibility that none of the records in table 12 are necessarily applicable to the behavior of flow in some of the steep channels in which higher and relatively sharper peaks may have occurred. As pointed out in the preceding section, the peak discharge was made up of debris in an unknown but perhaps substant1al amount.

Table 13 shows, for each of 14 dralnage basins for which basic discharge records have been presented, Ita area, the peak discharge (est1mated for most of the streams) and maximum 24-hour discharge in secondfeet per square $\mathrm{m} 1 \mathrm{l} \theta$, and the ratios of the peak discharge and maximum mean diacharge for Indicated periods to the maximum 24-hour discharge.

Figure 18 shows these ratios graphically for several of the stations represented in table 13 and also the average ratios for the 14 stations plotted against the corresponding intervals of time.

These ratios reflect generally and in a composite way the factors affecting the concentration of flow in individual streams, such as amount and rate of precipitation, direction of storm travel, size and shape of drainage basin, slope of stream channel and general slope of basin, infiltration rate and retention capacity of basin, and vegetative cover. It is to be inferred that the greater the afference in these factors in the 


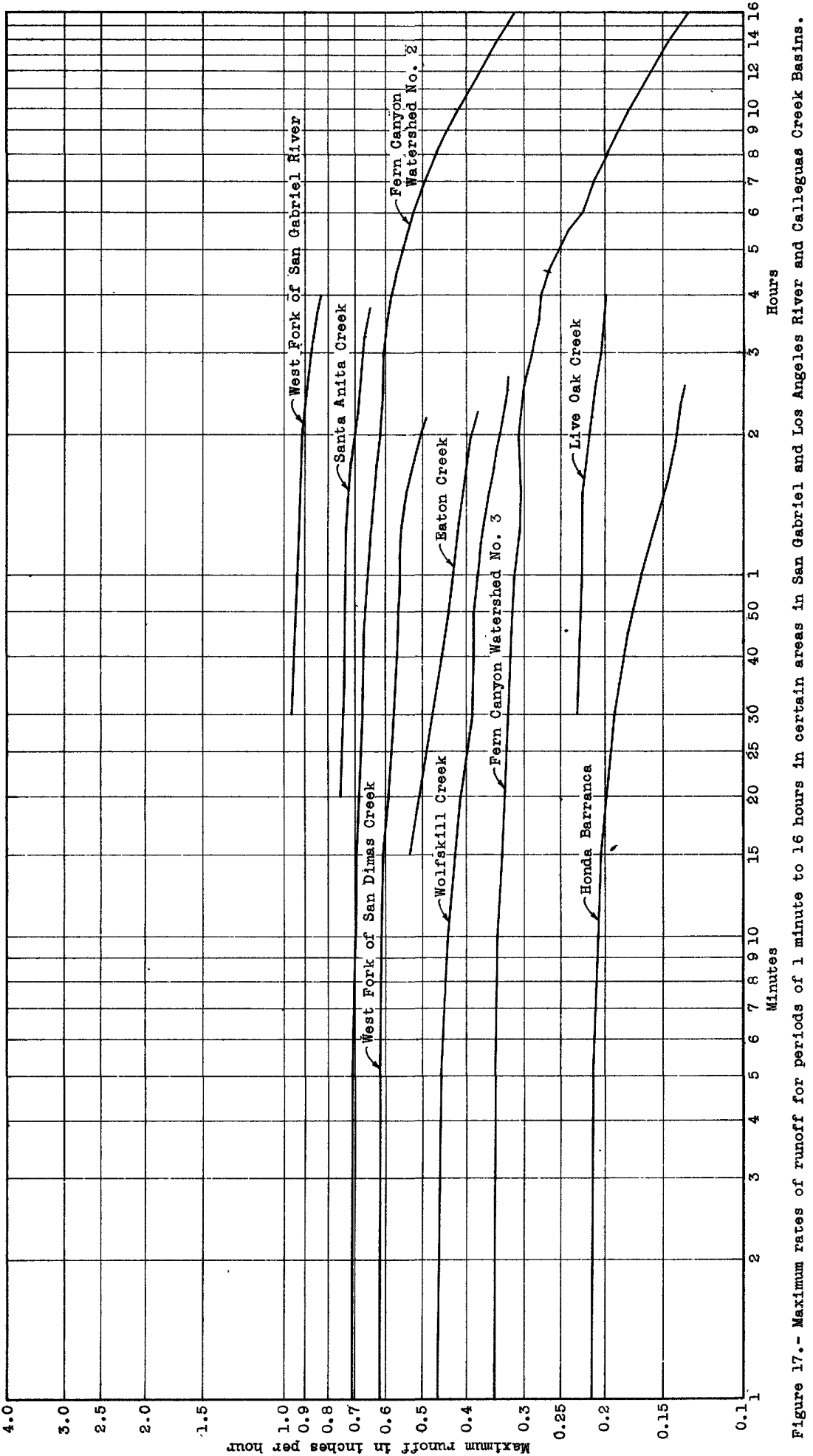




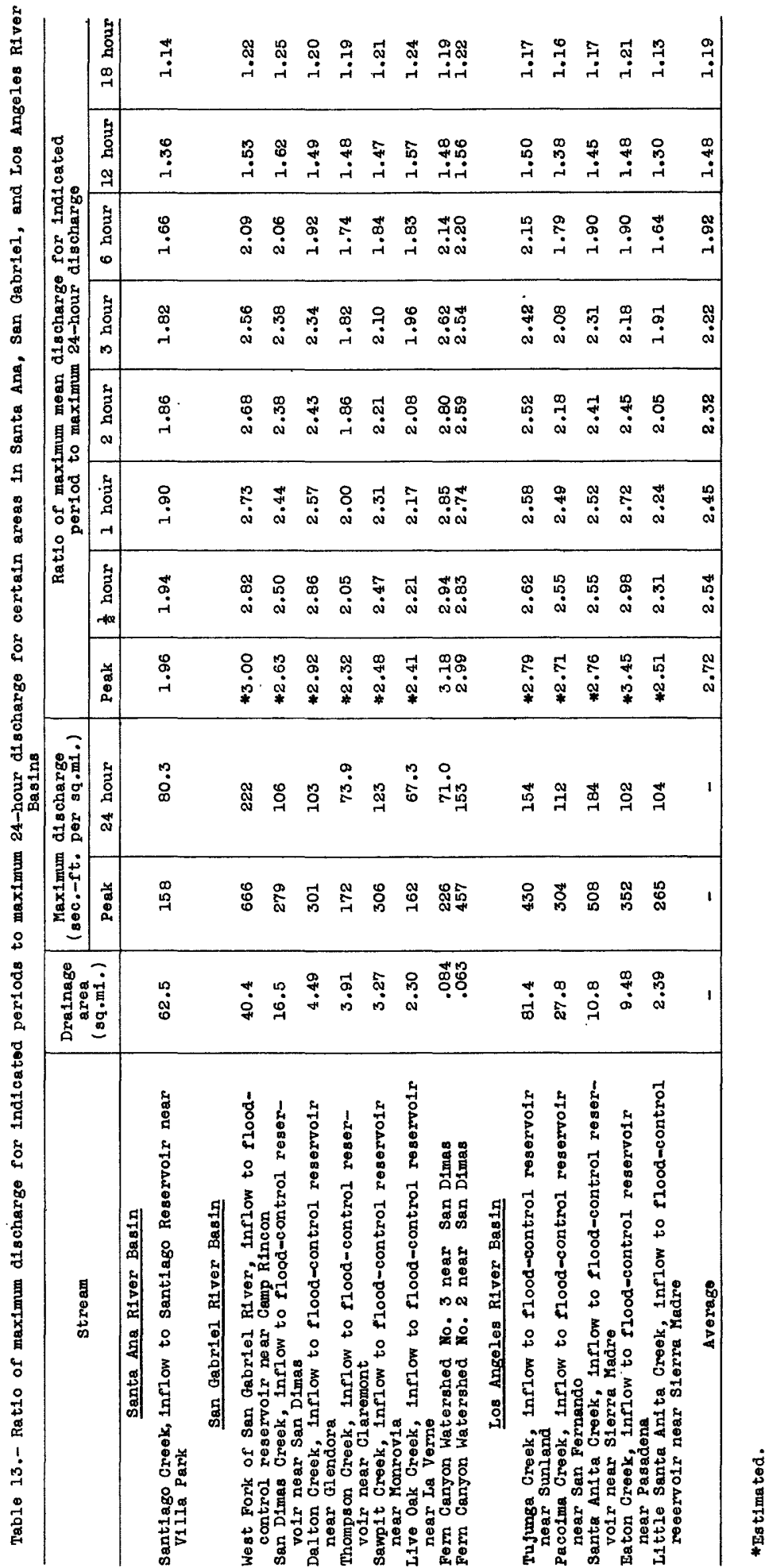




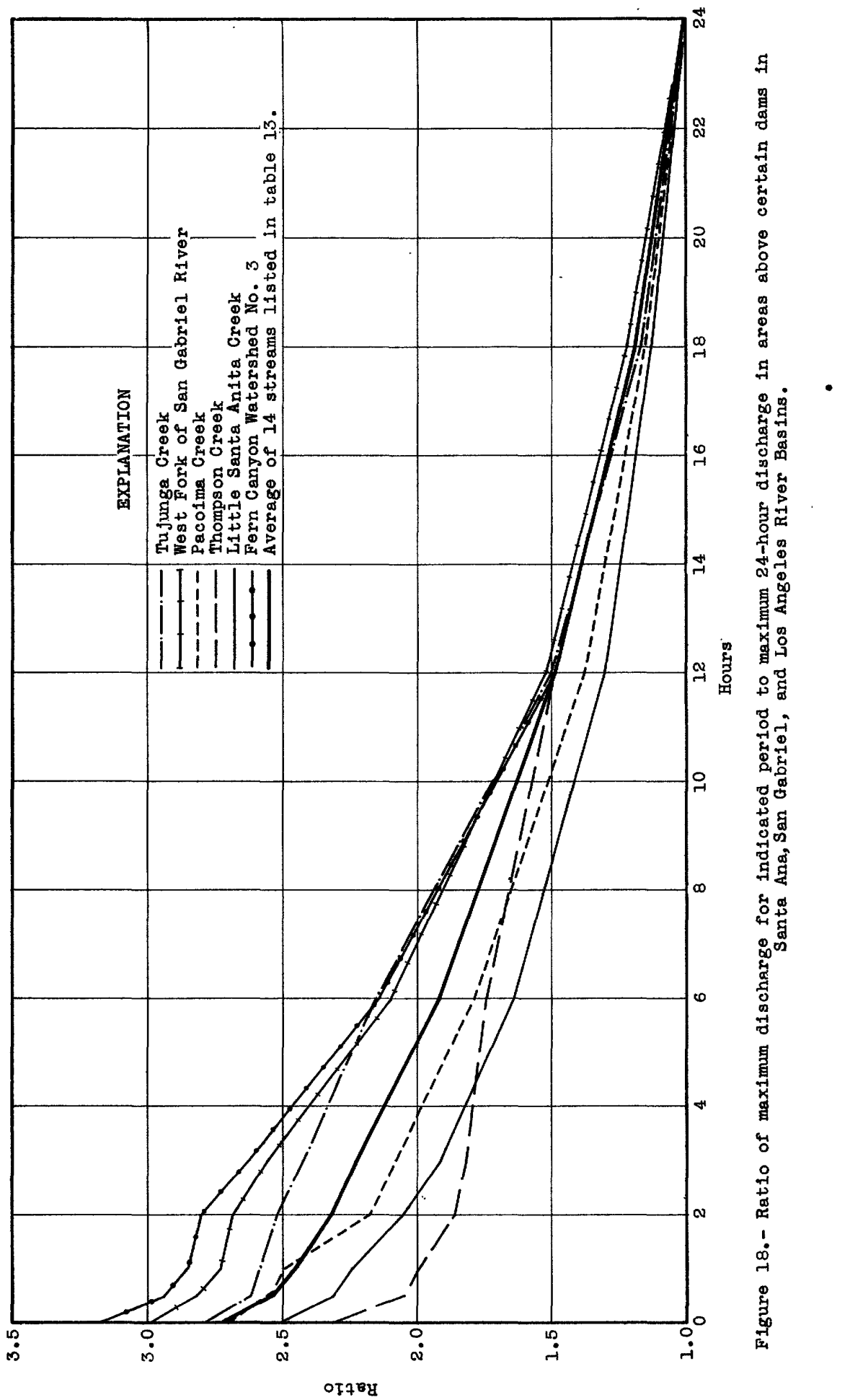


several basins, the wider the variation in the magnitude of the ratios during the floods.

Although the estimates of maximum or peak inflow to the reservoirs is obtained by somewhat indirect methods into which individual judgment enters, it is believed that the margin of probable error is relatively small and that, in general, the results are acceptable for determinations of extremely high flood flows and materially more accurate than the results obtained for the floods of March 1938 at other places in this region.

\section{STUDIES OF BASIC DISCHARGE RECORDS IN RELATION TO BASIN CHARACTERISTICS}

\section{AND RAINFAII}

For the region most severely affected by the storms and floods of March 1938, many rainfall records have been presented and discussed in earlier sections of this report. Also, avallable basic discharge records believed to be reliable, have been presented in the section immediately preceding. For most of the stream basins covered by these records there are given, in tables to be introduced hereafter, pertinent data regarding the area, channel slopes, form relations, and other characteristics that may be examined with regard to their influence on the concentration of flood runoff. (See tables 14 and 15.)

The studies of the hydrologic features relate, first, to volumes of rainfall and flood runoff expressed in depth in inches over these areas, and second, to rates of rainfall and runoff. Since the flood proper was largely associated with the heavy rain that fell on March 2, the analyses of rates of rainfall and runoff have been confined to the rates for the maximum 24-hour period. The discussion of the precipitation and runoff on an areal basis relates to the entire storm and flood period.

The analyses of the runoff data on an areal basis are primarily of interest in problems relating to the storage of water at or near the source either in reservolrs or by means of land-management or cultural practices whereby the natural retention capacity of the basins can be maintained. The analyses showing the extent to which the runoff was concentrated with respect to time, the relations between the maximum rates of rainfall and runoff, and the time interval or "lag" between the occurrence of rain and its appearance as stream flow are largely of interest in problems relating to the control and disposal of water after it has reached the stream channels. They are essential to the designing of spillways, bridge openings, bypasses, flood channels, and other engineering structures and flood-control devices. 


\section{Basin characteristios}

The word "besin" is used at many places in this report to mean only the area upstream from the gaging station at which the runoff is measured. Therefore, under this usage, in the consideration of runoff 1 means the entire basin of a given stream only if the gaging station discussed is near the mouth of the stream.

By means of suitable maps certain drainage basins hereinafter listed were divided areally into segments or groups of segments, the upper limit of the first segment being 1 mile upstream from the reference station (Lowermost point in the basin), and the upper limit of each succeeding segment or group being 1 mile upstream from that of the segment or group preceding 1t, or, when it is reached, the watershed line of the basin. The division of a drainage area into segments is 1llustrated in figure 19 , which represents Tujunga Creek above the flood-control dam.

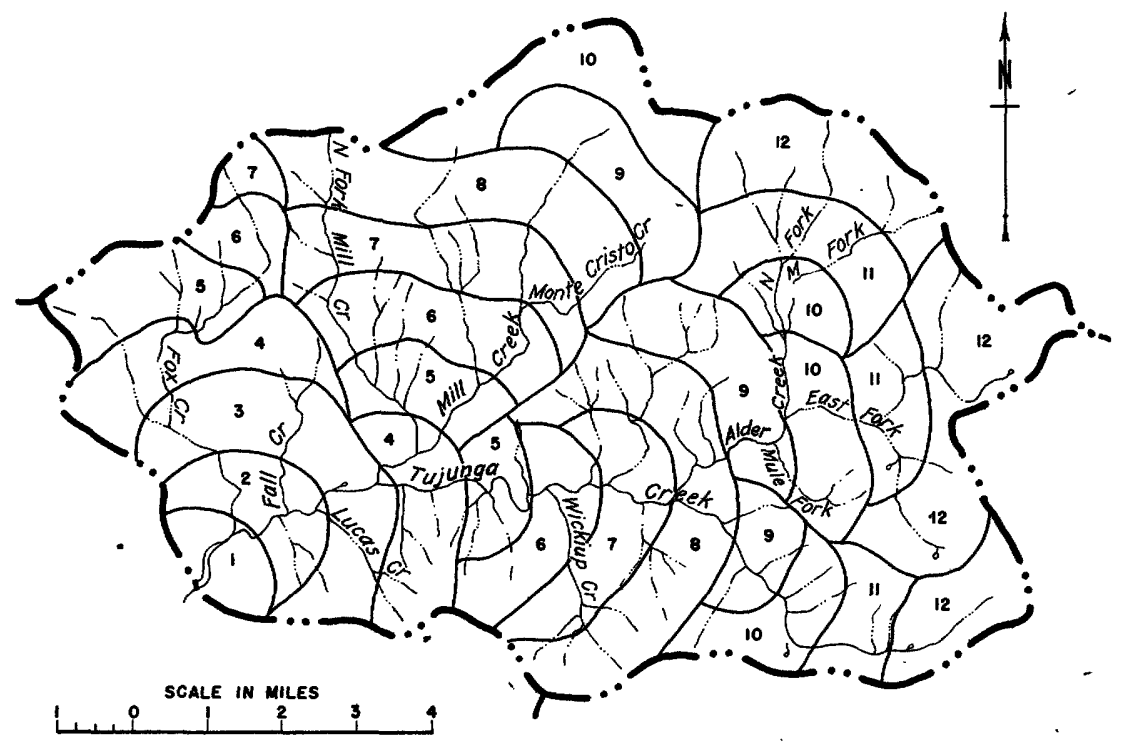

Figure 19.- Tujunge Creek Basin, showing segments used in determining maximum concentration of area.

Figure 20 shows the distribution of the segments of the drainage basins in accordance with this method of subdivision. The influence that the shape of the basin may have had on the concentration of runoff may be considered by studying this figure. For example, Pacoima Creok Basin, except for the segment 9 to $10 \mathrm{mlles}$ from the dam, has no segment or group of segments as shown equal in area to 10 percent of the total drainage basin. The basin of West Fork of San Gabrlel River, which produced the greatest muff to the square mile measured at the flood-control dams durIng the flood of March 1938, has no group of segments upstream from the dam with an area as great as 20 percent of the entire drainage basin. 


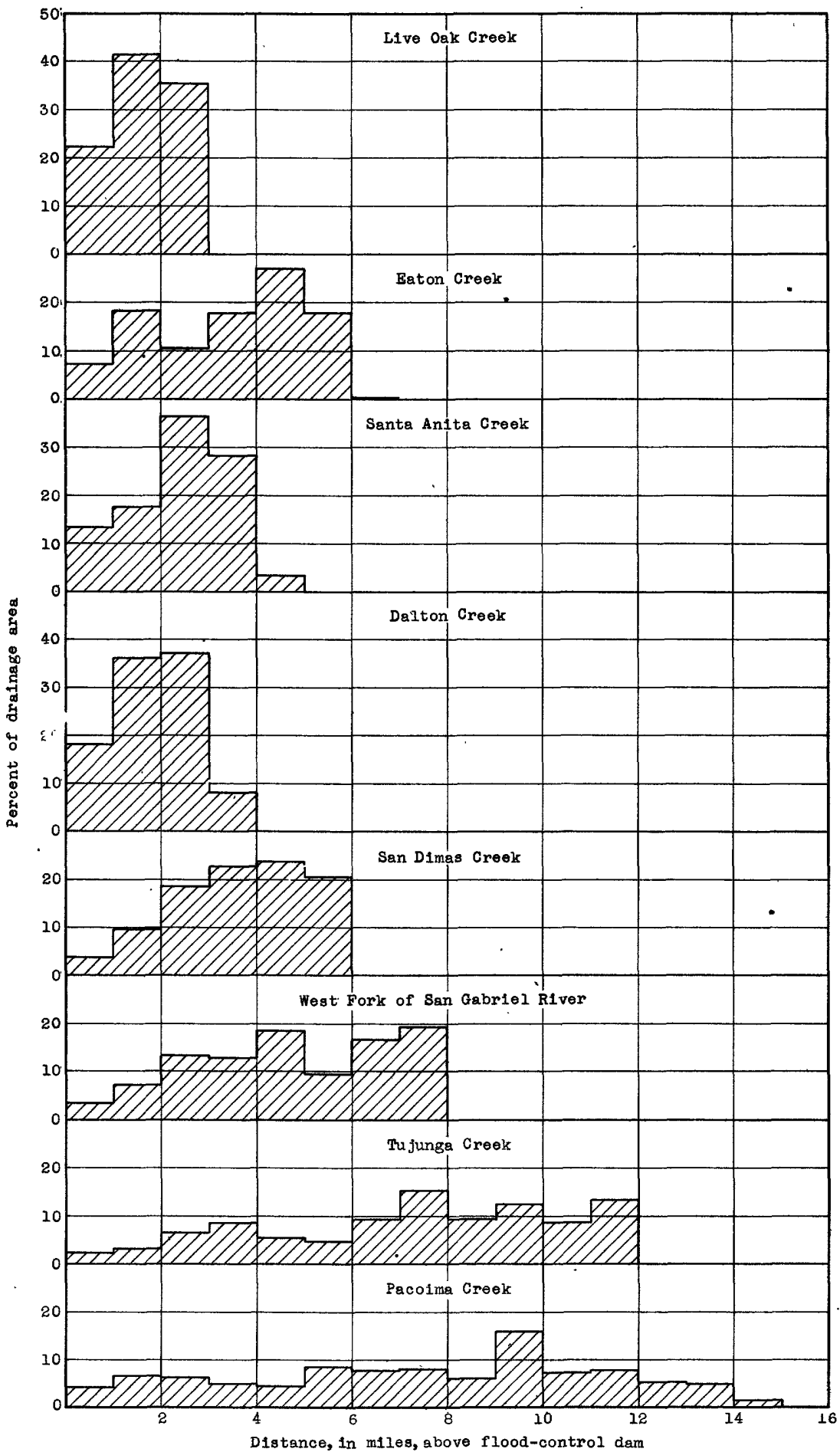

Figure 20.- Distribution of drainage area above flood-control dams in San Gabriel and Los Angeles River Basins. 
However, the group of segments 6 to 8 miles upstream from the flood-control dam represents about 36 percent of the total drainage basin. Dalton Creek Basin shows a concentration of 37.9 percent of 1 ts area, or 1.7 square miles, within 2 to 3 miles of the dam. Table 14 shows the segment or group of segments, classifled on the basis above described, that contains the maximum concentration of drainage area. For example, for Tujunga Creek the group of segments with the largest area (12.5 square miles) is 7 to 8 miles upstream from the flood-control dam measured along natural water courses. The average distance from the dam of all the segments in the entire drainage basin is shown in the last column to be $7.24 \mathrm{miles}$.

Table 14.- Characteristics of distribution of area in arainage basins above certain flood-control dams in San Gabriel and Los Angeles River Basins

\begin{tabular}{|c|c|c|c|c|}
\hline \multirow[b]{2}{*}{ Stream } & \multirow[b]{2}{*}{$\begin{array}{l}\text { Dralnage } \\
\text { area } \\
\text { (square } \\
\text { miles) }\end{array}$} & \multirow{2}{*}{$\begin{array}{l}\text { Maximum } \\
\text { concentration } \\
\text { of area in } \\
\text { segment } \\
\text { l mile wide } \\
\text { (square miles) }\end{array}$} & $\begin{array}{r}\text { Distance, } \\
\text { from }\end{array}$ & in miles, \\
\hline & & & $\begin{array}{l}\text { To maximum } \\
\text { concentra- } \\
\text { tion of } \\
\text { area }\end{array}$ & $\begin{array}{l}\text { To center } \\
\text { of total } \\
\text { dra inage } \\
\text { area }\end{array}$ \\
\hline San Gabriel River Basin & & & & \\
\hline $\begin{array}{l}\text { West Fork of San Gabriel } \\
\text { River } \\
\text { San Dimas Creek } \\
\text { Dalton Creek } \\
\text { Thompson Creek } \\
\text { Sawpit Creek } \\
\text { Live Oak Creek }\end{array}$ & $\begin{array}{l}40.4 \\
16.5 \\
4.49 \\
3.91 \\
3.27 \\
2.30\end{array}$ & $\begin{array}{l}7.9 \\
4.0 \\
1.7 \\
1.8 \\
1.3 \\
1.0\end{array}$ & $\begin{array}{l}7-8 \\
4-5 \\
2-3 \\
2-3 \\
2-3 \\
1-2\end{array}$ & $\begin{array}{l}4.92 \\
3.65 \\
1.86 \\
2.27 \\
1.78 \\
1.63\end{array}$ \\
\hline Los Angeles River Basin & & & & \\
\hline $\begin{array}{l}\text { Tujunga Creek } \\
\text { Pacolma Creek } \\
\text { Santa Anita Creek } \\
\text { Eaton Creek } \\
\text { Iittle Santa Anita Creek }\end{array}$ & $\begin{array}{l}81 \cdot 4 \\
27.8 \\
10.8 \\
9.48 \\
2.39\end{array}$ & $\begin{array}{r}12.5 \\
4.4 \\
3.8 \\
2.6 \\
1.1\end{array}$ & $\begin{array}{l}7-8 \\
9-10 \\
2-3 \\
4-5 \\
1-2\end{array}$ & $\begin{array}{l}7.24 \\
7.45 \\
2.41 \\
3.44 \\
1.41\end{array}$ \\
\hline
\end{tabular}

The topographic characteristics of the drainage basins studied herein are shown in table 15. The areas of these basins range from 0.063 square mile, for Fern Canyon Watershed No. 2, to 81.4 square m1les, for Tujunga Creek Basin. The land slopes of all the basins are steep; the two Fern Canyon Watersheds have the greatest slope--65 percent-and Iive oak and Thompson Creek Basins have the smallest slope--36 percent. The average altitudes in these basins range between 2,000 and 5,000 feet. Bxcluding the sma11 Fern Creek Watersheds, Tujunga Creek Basin has the highest average altitude.

The size of the contributing area, the slope of the land areas and stream channels, and the shape of the basin, Inssmuch as they determine distances and time of travel of the flood waters, undoubtedly influence the period of concentration of the flood runoff. Beief study of the data given in tables 15 and 17 (see pp. 140 and 150) suggests that the concentration characteristics of the several basins listed may be related directly or 
Table 15.- Topographic charaoteristics of certain drainage basins above point of measurement in Santa Ana, San Gabriel, and Los Angeles River Basins

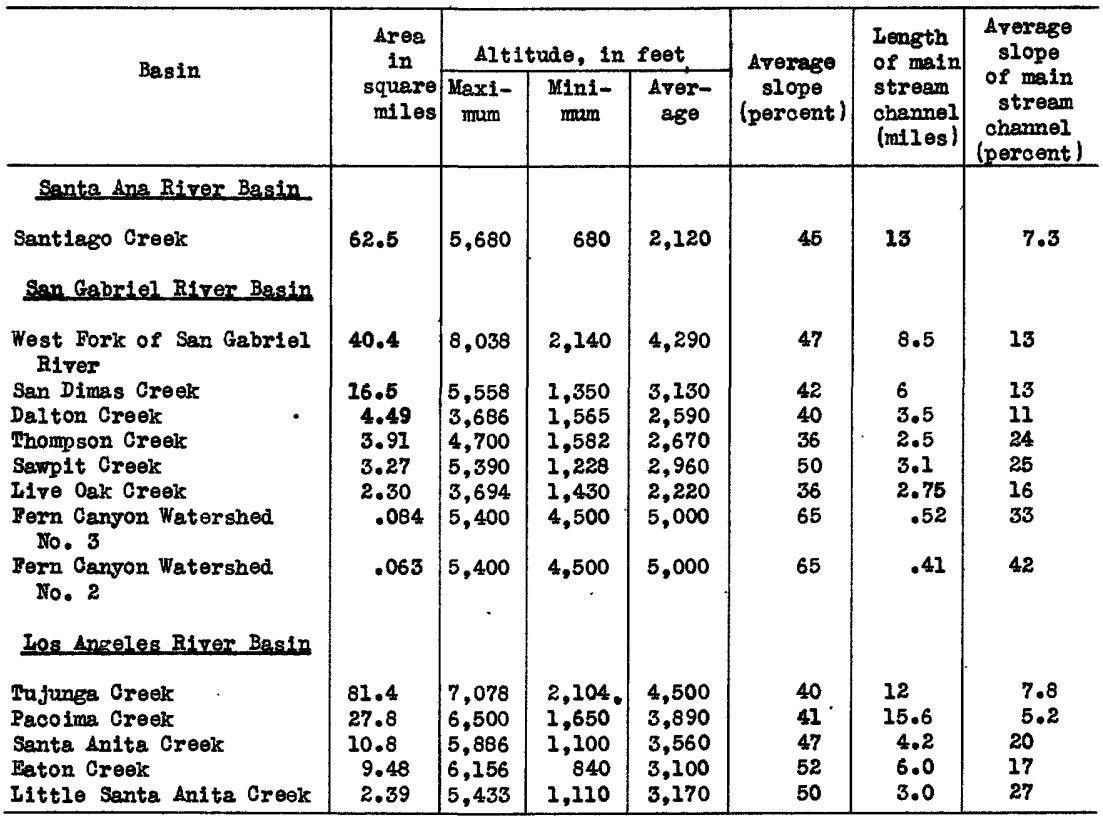

Indirectly to the mean land slope. Other influences can perhaps be detected, and the basic data are here recorded for examination by other investigators. Nevertheless, for the floods of March 1938 the rainfall characteristics seem to have greatly influenced, and indeed to have dominated, the manoff characteristios.

\section{Runoff characteristics}

There were four distinct pheses in the munoff resulting from the storm of February 27 to March 4, corresponding in many respects to the four phases of the storm as previously described. The f1rst phase is associated with the rain that fell on February 27 and 28 . On February 27 there was generally very little stream flow, but beginning about noon February 28, the flow gradually increased and reached a minor peak near or shortly after midnight, following the higher rates of rainfall near the end of the 2-day per1od. The second runoff phase lasted from about 1 a.m. to midnight March 1 when there was an almost complete cessation of rain and a gradual decrease in stream flow. The third runoff phase began during the early morning of March 2 and reached a climax generally between 2 and 5 p.m. on the same day, when most streams reached the1r maximum rates of discharge for the flood. Although the rain did not cease 
unt1l later, the discharge rates were generaliy lower after the early evening of Jarch 2. The fourth runoff phase lasted from the time of the peak on March 2 through March 10.

For basins for which continuous observations of runof are avallable throughout the flood, analyses have been made of hourly records of rainfall, munof, and retention, or the difference between the accumulated rainfall and the runoff. These analyses have been used as a check on the accuracy of derivations of discharge made to complete records at streamflow measurement stations. They are presented herein for use by engineers and hydrologists interested in flood runoff, especially under the physiographic and climatic conditions existing in southern california. Graphs of hourly rainfall and runoff, in mean depth in inches, in figures 21 and 22 , and graphs of the accumulated precipitation, runoff, and retention, in figures 23 and 24 , are presented for West Fork of San Gabriel River and Live Oak Creek Basins, the two drainage areas showing the widest range in these factors, for the period February 27 to March 5. In figures 23 and 24 the periods covered by the runoff phases are indicated. The method of determining the rainfall in the basing is described on page 151 .

First phase, February 27 and 28

Table 16 shows the amount, in inches, of accumulated rain that fell over the 13 basins during the first storm, before the accumulated runoff beginning at midnight on February 26 equaled $0.10 \mathrm{inch}$, and the accumulated rainfall and runoff and the retention, or difference between the rainfall and runoff, at the time of the first peak, which occurred generally elther shortly before or shortly after midnight February 28. With the exception of the basin of the West Fork of San Gabriel River there was an accumulated retention in each of the 13 basins of between 3 and 4.8 inches before there was an accumulated runoff of 0.10 inch; the average retention for the 13 basins was about 3.9 inches. This conaltion was reached toward noon on February 28, 24 hours or more after the begiming of the storm. Although the accumlated rain up to the time of the first peak averaged about 8.5 inches, the total runoff averaged only 0.7 inch, indicating an average retention at that time of about 7.8 inches. The duration of rainfall in the first storm at rates in excess of 0.02 inch an hour was about 30 hours, and on this time bas 1s there was an average increase in retention in the basins of about 0.26 inch an hour. 


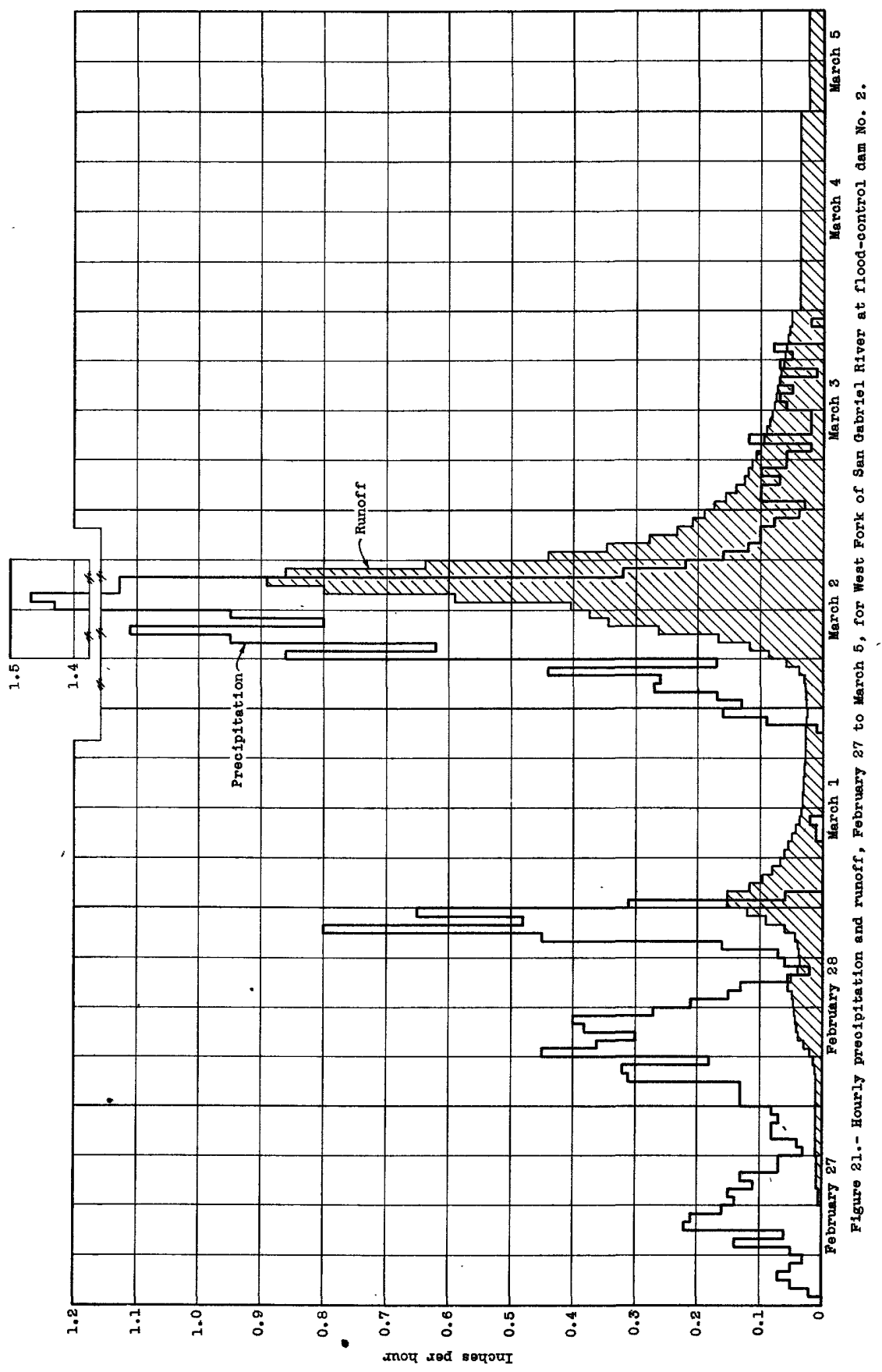




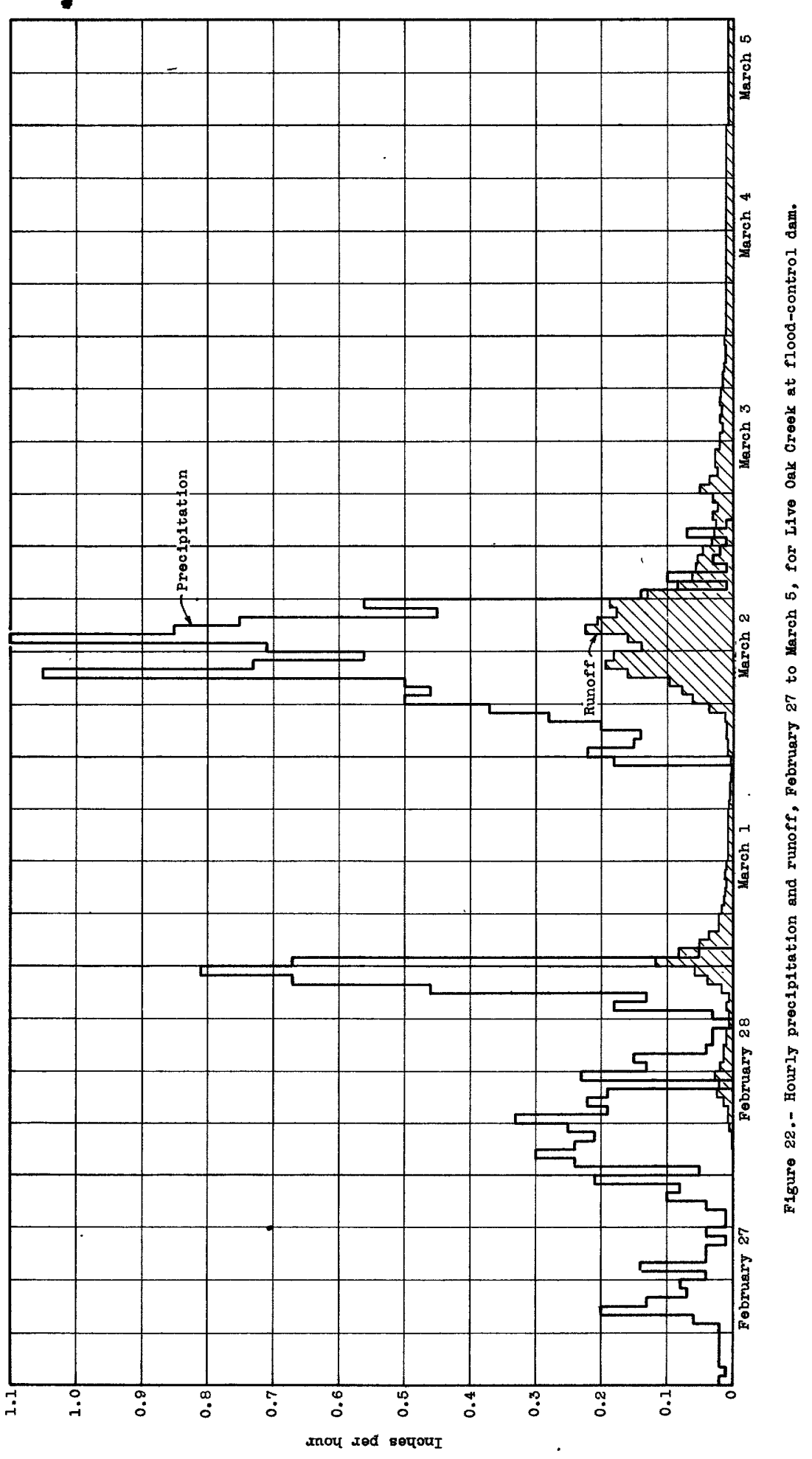




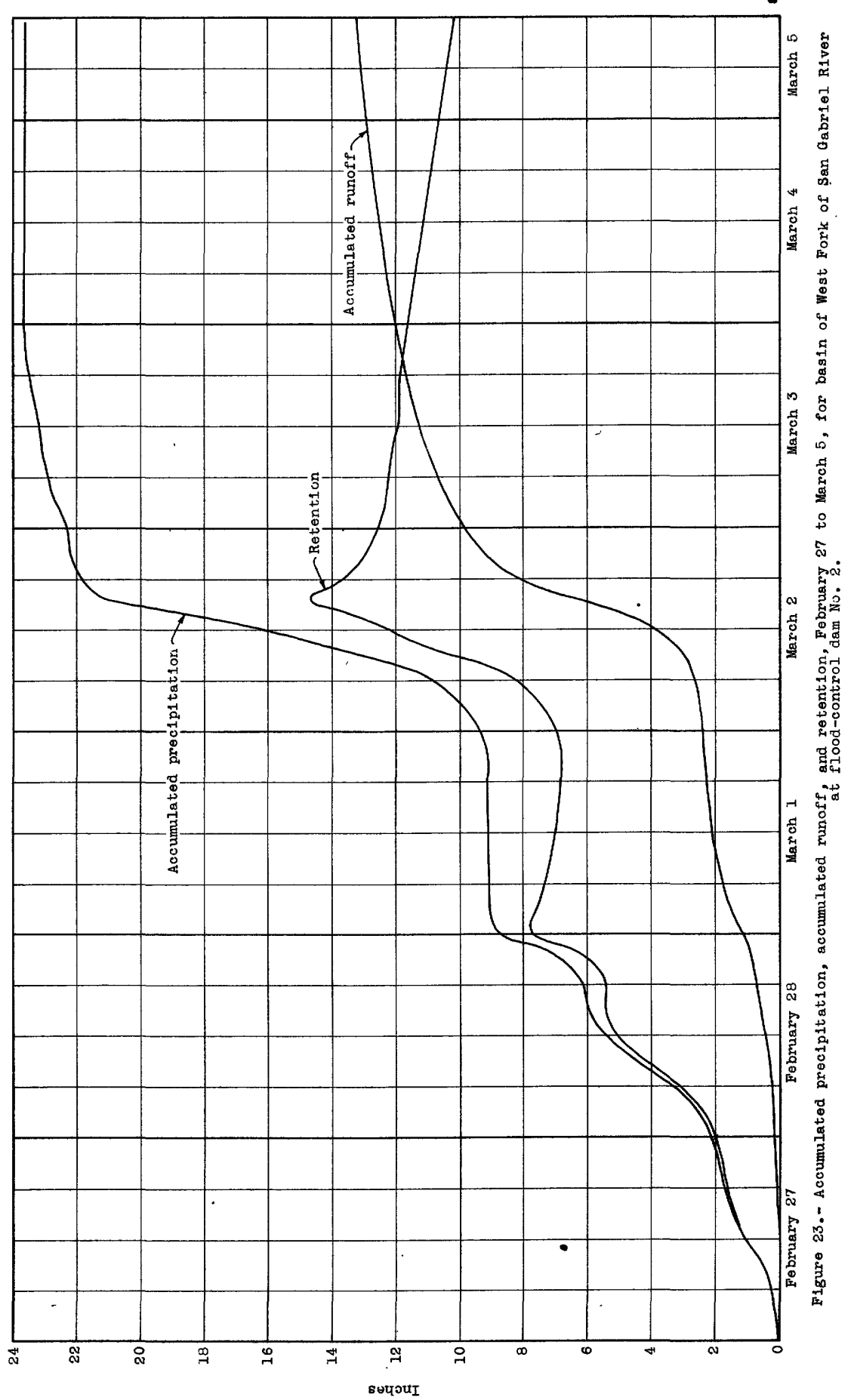




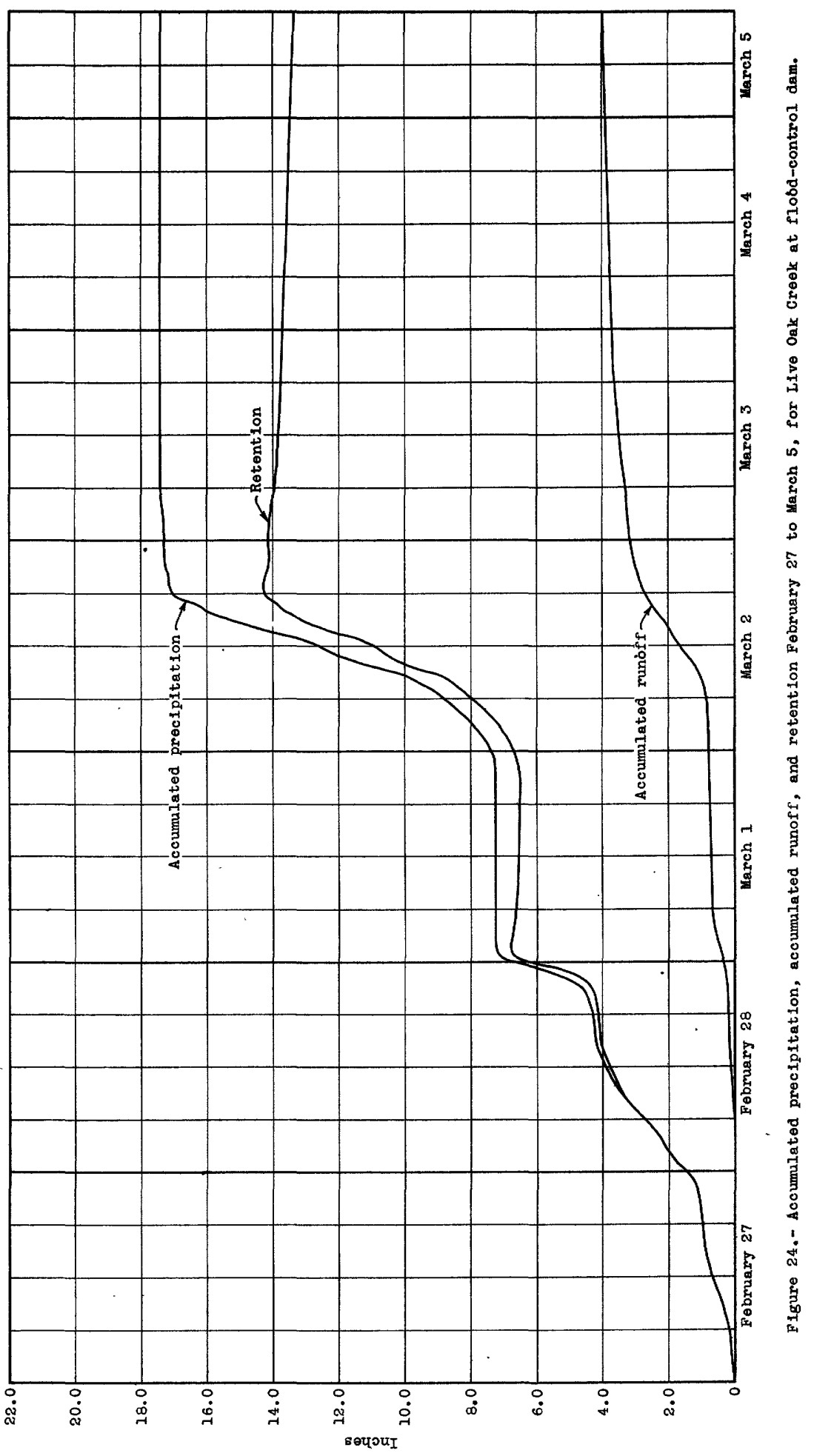




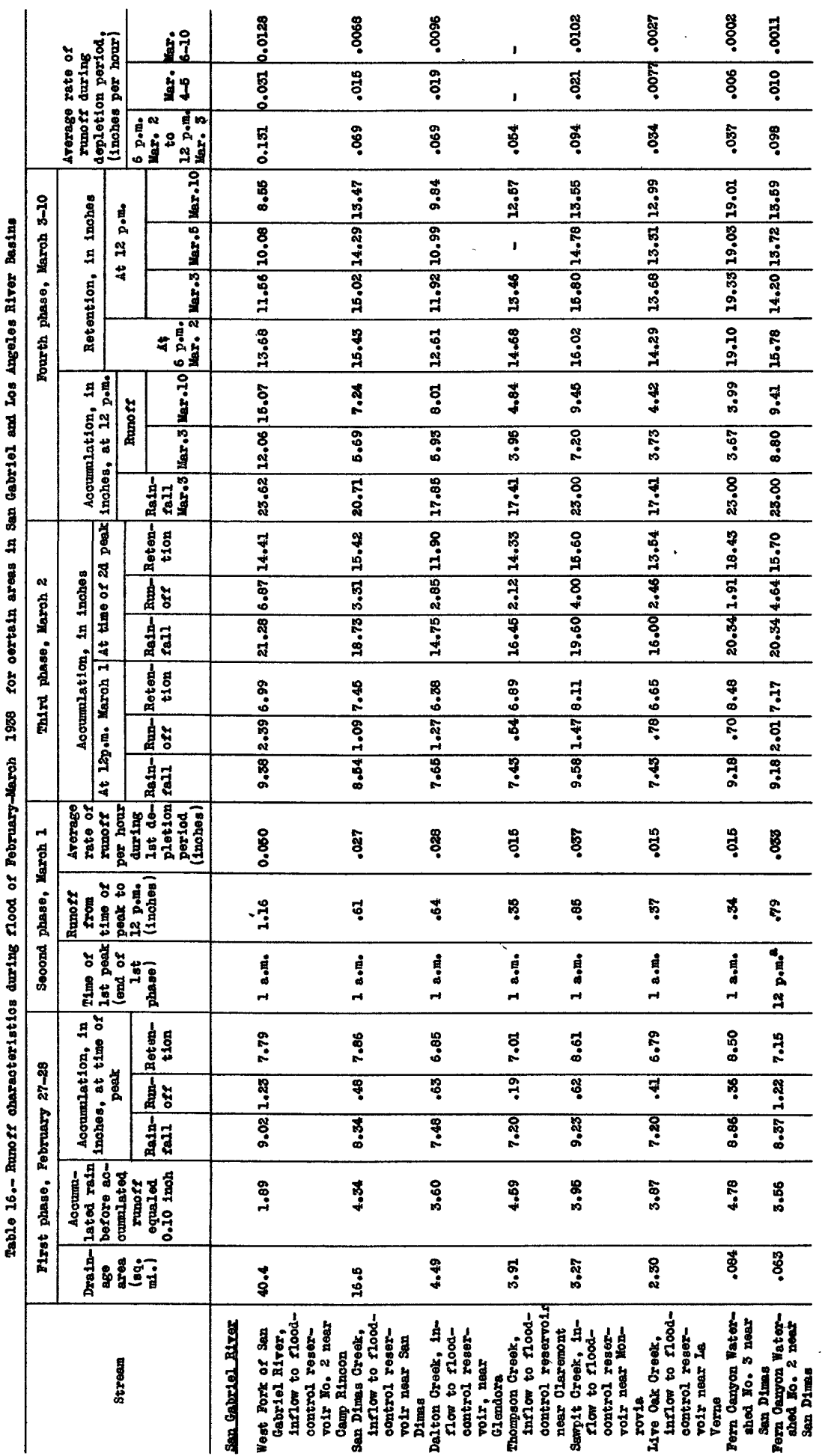




\begin{tabular}{|c|c|c|c|c|}
\hline 8 & ; & $\stackrel{0}{\circ}$ & \%ั & $\ddot{\square}$ \\
\hline$\stackrel{8}{9}$ & ğ & $\begin{array}{l} \\
9\end{array}$ & $\tilde{a}$ & $\frac{8}{8}$ \\
\hline \%ั & 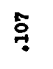 & 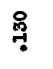 & $\tilde{8}$ & : \\
\hline $\begin{array}{l}\vec{a} \\
\end{array}$ & 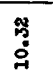 & 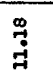 & $\stackrel{4}{9}$ & 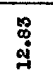 \\
\hline 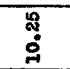 & 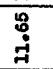 & 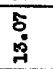 & 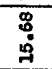 & 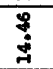 \\
\hline 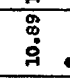 & 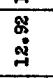 & $\frac{1}{8}$ & 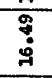 & 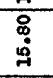 \\
\hline 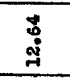 & 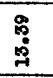 & $\stackrel{8}{\circ}$ & 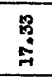 & 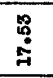 \\
\hline 吕 & 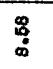 & 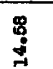 & 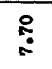 & $\begin{array}{l}5 \\
\end{array}$ \\
\hline 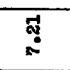 & 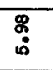 & 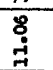 & 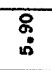 & $\stackrel{8}{8}$ \\
\hline $\begin{array}{l}\stackrel{\circ}{\dot{m}} \\
\end{array}$ & 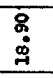 & 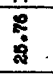 & 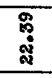 & 芯 \\
\hline 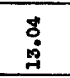 & 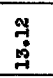 & 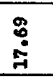 & $\stackrel{7}{8}$ & 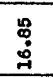 \\
\hline $\begin{array}{ll} \\
\\
0 \\
0\end{array}$ & $\begin{array}{r} \\
\\
8 \\
\end{array}$ & 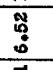 & 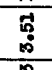 & 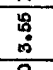 \\
\hline 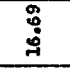 & 芯 & d. & 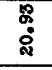 & 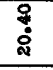 \\
\hline 惫 & $\stackrel{8}{3}$ & 莒 & హ్ & $\stackrel{\dot{\infty}}{\infty}$ \\
\hline 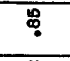 & $\%$ & 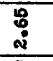 & $\sqrt[4]{4}$ & $\stackrel{9}{i}$ \\
\hline 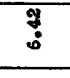 & $\begin{array}{ll}8 \\
\\
\end{array}$ & 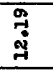 & 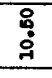 & 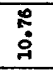 \\
\hline : & ఫి & $\stackrel{7}{a}$ & g̊ & $\frac{7}{9}$ \\
\hline ? & ? & g̊ & ฉั & $\underset{\sim}{\not}$ \\
\hline 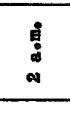 & $\begin{array}{ll}0 \\
\vdots \\
0\end{array}$ & \begin{tabular}{|l} 
\\
\\
9 \\
\end{tabular} & $\stackrel{5}{9}$ & 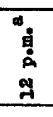 \\
\hline$\stackrel{5}{0}$ & $\stackrel{5}{\square}$ & $\stackrel{9}{\stackrel{9}{2}}$ & $\begin{array}{l}0 \\
0 \\
\end{array}$ & $\stackrel{8}{2}$ \\
\hline \% & $\$$ & 㫄 & ?ִ & ?ִים \\
\hline$\stackrel{8}{0}$ & $\begin{array}{l}0 \\
\end{array}$ & 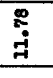 & $\begin{array}{l}0 \\
\stackrel{0}{\circ}\end{array}$ & 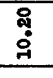 \\
\hline$\stackrel{g}{\sharp}$ & $\$_{\dot{H}}$ & \%ัم & $\stackrel{9}{3}$ & มू \\
\hline$\stackrel{\dot{D}}{\mathbf{D}}$ & $\stackrel{\leftrightarrow}{*}$ & $\stackrel{8}{\circ}$ & $\stackrel{3}{3}$ & : \\
\hline & & & & 98 \\
\hline
\end{tabular}




\section{Second phase, March 1}

Table 16 shows the total retention in each basin at the time of the first peak, near or shortly after midnight February 28, the total runoff from the time of the peak until midnight March 1, and the average rate of runoff per hour. Since there was little rain on March 1 , the latter rop resents essentialiy the rate of depletion in the water that was retained in the basin as ground or channel storage at the end of the first storm. The contribution to the runoff from groundwater storage due to antecedent rainfall was relatively inappreclable. This araining out on March I, Immediately following the first peak, averaged about 0.03 inch an hour over the several basins, or about a tenth of the average rate at which the retention accumulated.

Third phase, March 2

Rain of the second storm period started to fall at about $10 \mathrm{p} . \mathrm{m}$. March $I$ and continued unt1I about midnight March 2, with the greatest intensity occurring near noon on March 2 and with fairly little intensity during the late afternoon and evening. All streams rose rapidy and reached maximum peaks generally between 2 and 5 p.m.

Table 16 shows the accumulated rainfall, the munfe, and the retention in inches at midnight on March I, about the beginning of the second storm, and the accumulated rainfali, runoff, and retention at the peak of the flood. In the period of about 16 hours between midnight of March 1 and the peak on March 2, there was an average accumulation of about 10.0 Inches of rain, 2.3 inches of runoff, and 7.7 inches of retention, the increase in the retention belng at an average rate of about 0.48 inch an hour, or 85 percent in excess of the average rate of increase during the longer but less intense first storm. The average retention at the time of the peak was 15 inches.

Fourth phase, 6 p.m. March 2 to midnight March 10

Generally by about 6 p.m. March 2 all streams had started to recede and there was a gradual decrease in flow unt1I March II, when adaltional rain increased the flow. Table 16 shows the total accumulated rainfall. at 12 p.m. March 3, which includes essentially all the rain associated with the floods and a small amount that fell after the major peak stages on March 3 and that may have influenced the rate of recession; the tota. accumulated runoff through $12 \mathrm{p.m}$. March 3 , which probably includes 
essentlaliy all water in channel and surface storage at the time of the peak; and the accumilated runoff at $12 \mathrm{p.m}$. March 10. Since there was relatively little rain from March 4 to 10 , the difference in accumulated runoff at the two indicated times represents essentlally a draining out of ground water. The retention as shown in table 16 at a stated hour on each of four days, namely, $6 \mathrm{p.m}$. on March 2 and midnight on March 3, 5, and 10, was not corrected for the evaporation and transpiration losses which may have occurred during the time intervals involved or for the rainfali from March 4 to 10. The results approximate the addition to basin storage because of the March storm. Table 16 shows also the average rates of runoff during three parts of the depletion period, namely, the 30-hour perlod from 6 p.m. March 2 to midnight March 3, the 2-day perlod of March 4 and 5, and the 5-day perlod of March 6 to 10 . It may be noted that there was a marked decrease in the average rates of depletion in the three periods.

\section{Relation between rates of precipitation and rates of runoff}

The greatest rise of the floods of March 1938 occurred on March 2 and resulted from the rains that fell on the same day. It has been shown previousiy ( $f 1 g .14$ ) that during the maximum 24 hours of rainfall, for periods of maximum rates of precipitation in progressively decreasing intervals of time, each succeeding perlod generally fell within the next longer perlod. Flood rlses as shown on discharge hydrographs tend generally to have the same characteristic, and this is particularly true for the floods of March 1938, as w131 be illustrated hereafter. This tendency suggested a study of the relation of the maximum rates of precipitation to the maximum rates of runoff for corresponding intervals of time for aralnage basins for which there are basic discharge records. Table 17 is a compliation of maximum rates of rainfali and runoff for 14 drainage basins for selected intervals of time ranging from 15 minutes to 24 hours each.

The average precipltation in the drainage basing was determined from the 1sohyetal map of the area for the total storm perlod February 27 to March 4. It was then distributed as to time on the basis of the records at a selected recording gage or gages. The rates of precipitation at the recording gages are summarized in table 2 . 


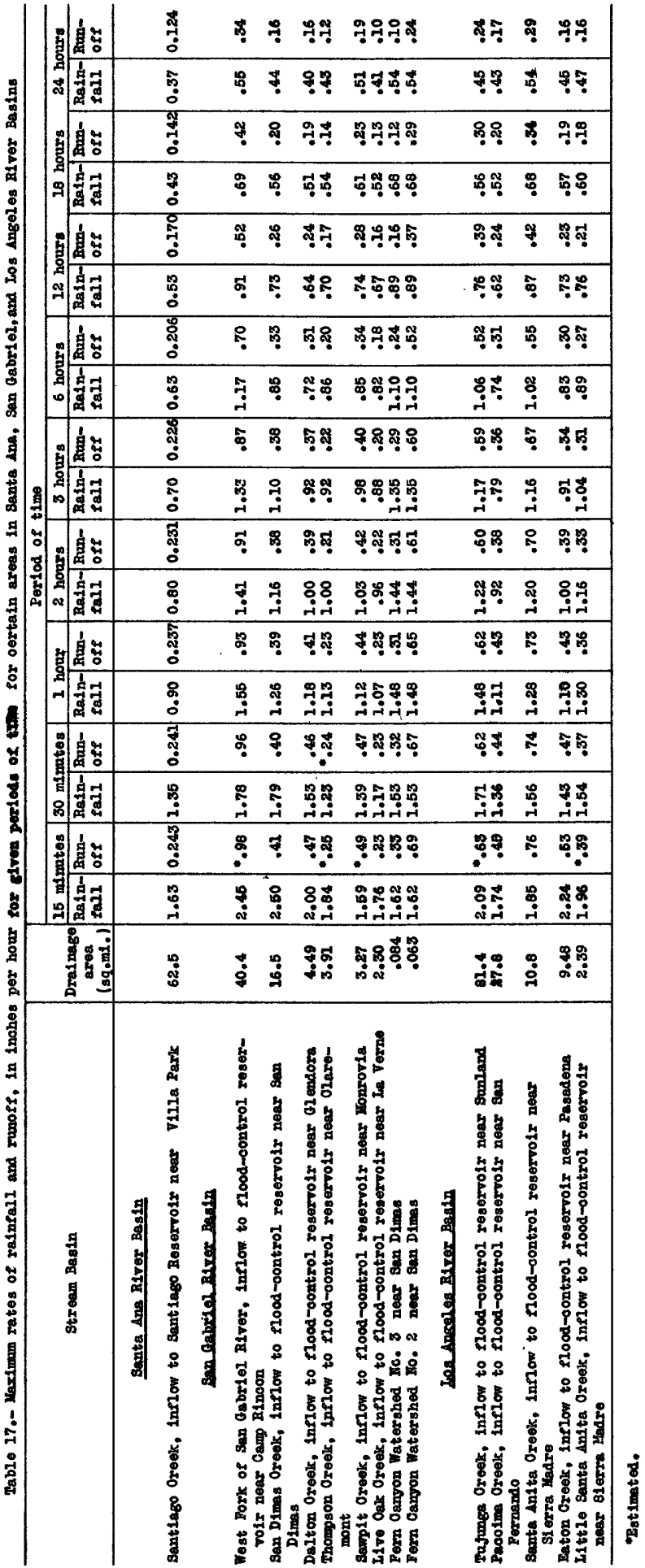


The assumption that the average rates of precipitation over a drainage basin w111 vary as do the rates at a nearby recording gage or else those obtained by averaging the rates for two or more nearby gages has certain inherent features that would seem to make it more rellable for the longer than for the shorter intervals for which rates are determined. The precipitation appears to have been extraordinarily uniform over the entire area, and the drainage basins are small in relation to the veloc1ty of the storm; consequently, the rates for the basins may be accepted as quite reliable for the longer intervals of time. obviously, however, there may be a question as to whether the uniformity of precipitation for short intervals of time, such as 1 hour or less, is such that the average rates over a basin w11l be closely related, e1ther as to intensity or as to time of occurrence, to the rates obtained at one or more recording stations in the basin or within a few miles of 1 t.

The runoff records for the West Fork of San Gabriel River at floodcontrol dam No. 2 and for IIve Oak Creek, as presented in the basic discharge records, are typlcal of the extremes of flood discharge as determined from the reservolr records.

In figure 25 the rates of precipitation and runoff for both the West Fork of San Gabriel River and Live Oak Creek Basins are plotted to logarithmic scales against the durations of these respective rates. For this plotting, the data in table 19 have been supplemented to obtain additional points in these basins, and rates of precipitation have been computed to the maximum 5-minute interval.

The major part of the drainage of the West Fork of San Gabriel River was within the zone of heaviest rainfall during the storm of March 1938 , and characteristics of its rainfall are included among those show for the group B stations on figures 11 to 15. From the 1sohyetal map (pl. 17) the average rainfall on this drainage area of 40.4 square miles was found to be 23.62 inches for the period of the storm. The distribution of the rainfall was based on the records at Opld's camp, Crystal Lake, and San Gabriel flood-control dam No. 2 .

IIve. Oak Creok Basin, on the coastal side of the first divide, had somewhat less rainfall than the basin of the West Fork of San Gabriel River and averaged 17.41 inches over the 2.30 square miles. The rainfall intensities for this basin were distributed on the basis of the automatic record at the mouth of San Antonio Canyon, about 4 miles to the northeast, at an altitude a little above the average for Live 0ak Creek Basin. The 
record from the San Antonio Canyon gage seems to put that station in group C (f1gs. 11 to 15 ).

Similarly, figure 26 shows the maximum rates of precipitation and runoff for periods of 5 minutes to 24 hours for San Dimas Creok and Fern Canjon Watersheds Nos. 2 and 3 , which are within the basin of San Dimas Creek. This basin has an area of 16.5 square miles, and in 1 are 174 standard and 10 recording rain gages maintained by the United States Forest Service. The average precipitation over the basin during the storm was 20.71 inches, which was distributed on the basis of the recording rain gage at Tanbark Flats. The record at Tanbark Flats (alt1tude, 2,700 feet) puts this station in group $C$.

The rainfall record in Fern Canyon (alt1tude, 4,800 feet), also in the drainage basin of San Dimas Creek, seems to conform to the records of the group B stations. The drainage basin of San Dimas Creok has a mean altitude of about 3,130 feet, w1th maximum and minimum altitudes of about 5,600 and 1,400 feet, respectively. Evidently much of the rainfall in the basin would have followed more closely the Fern Canyon type of distribution than that shown by the Tanbark Flats station. The altitude of the latter station is nearer the mean altitude of the drainage area and so may represent the distribution of precipitation rates over the entire basin more accurately than the Ferm Canyon station.

The rainfall over Fern Canyon Watersheds Nos. 2 and 3 was pract1cally Identical as determined by observations at 93 rain gages within these two drainage areas. The rainfall was analyzed on the basis of the Fern Canyon recording gage record.

It may be observed in the graphs for the flve basins show in figures 25 and 26 that under the method of plotting used, for time intervals of 1 hour or more the precipitation and runoff graphs for the respective basins are approximately parallel.

Graphs showing the runoff rates for Fern Canyon Watersheds Nos. 2 and 3 Indicate marked differences in the characteristics of these two drainage basins. These differences seem to result in part from the differences in their geology. That part of the mountains in which the Fern Canyon Watersheds are situated is underlain by a metamorphic-igneous bedrock complex, the San Gabriel formation of Miller. 14 Schists and

14 Millẹr, W. J., Geology of westerm Sen Gabriel Mountains: Univ. Calif. at Los Angeles, Pubs. In Math. and Phys. Sciences, vol. 1, no. 1, pp. 50-51, 1934 . 


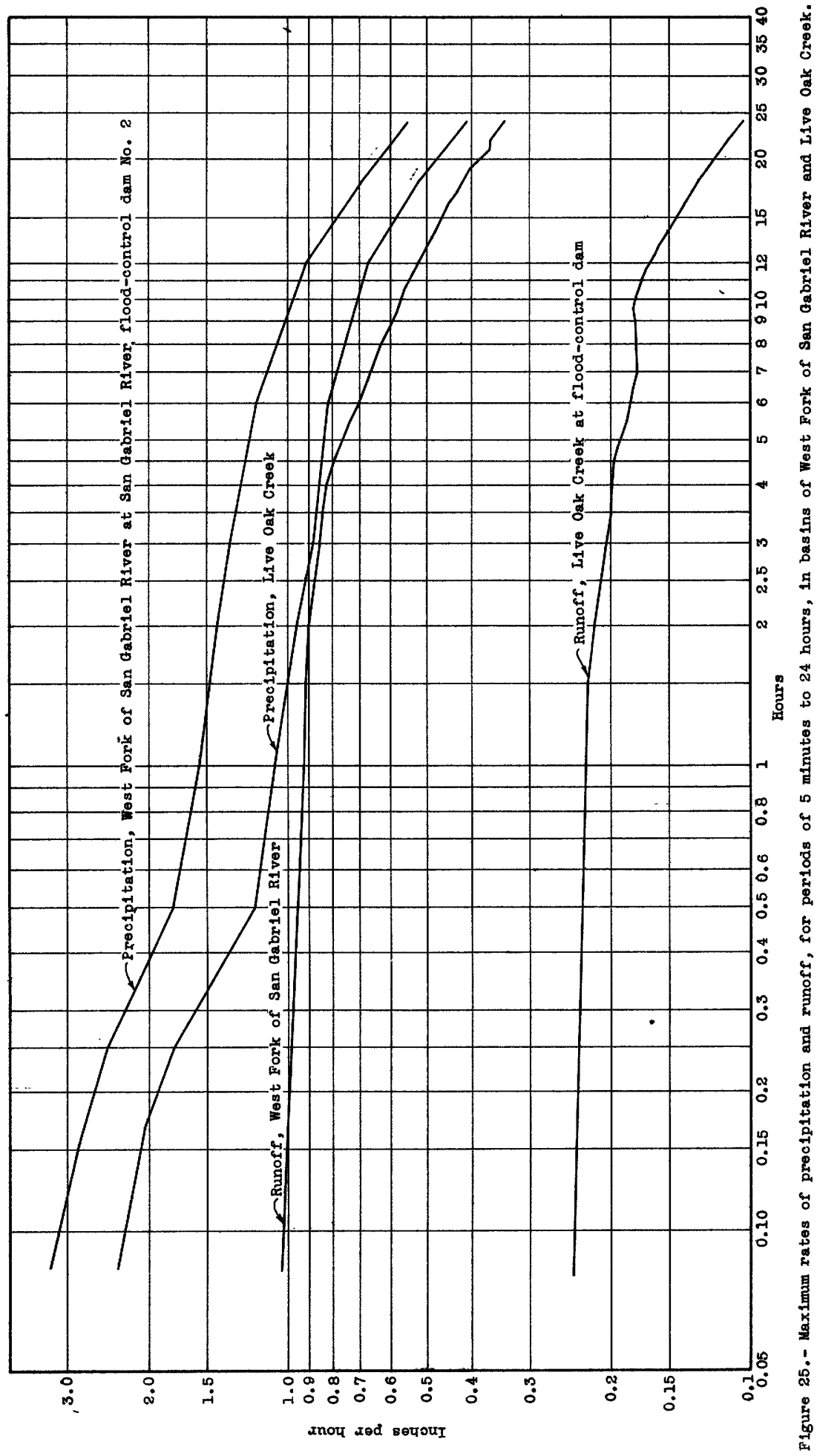




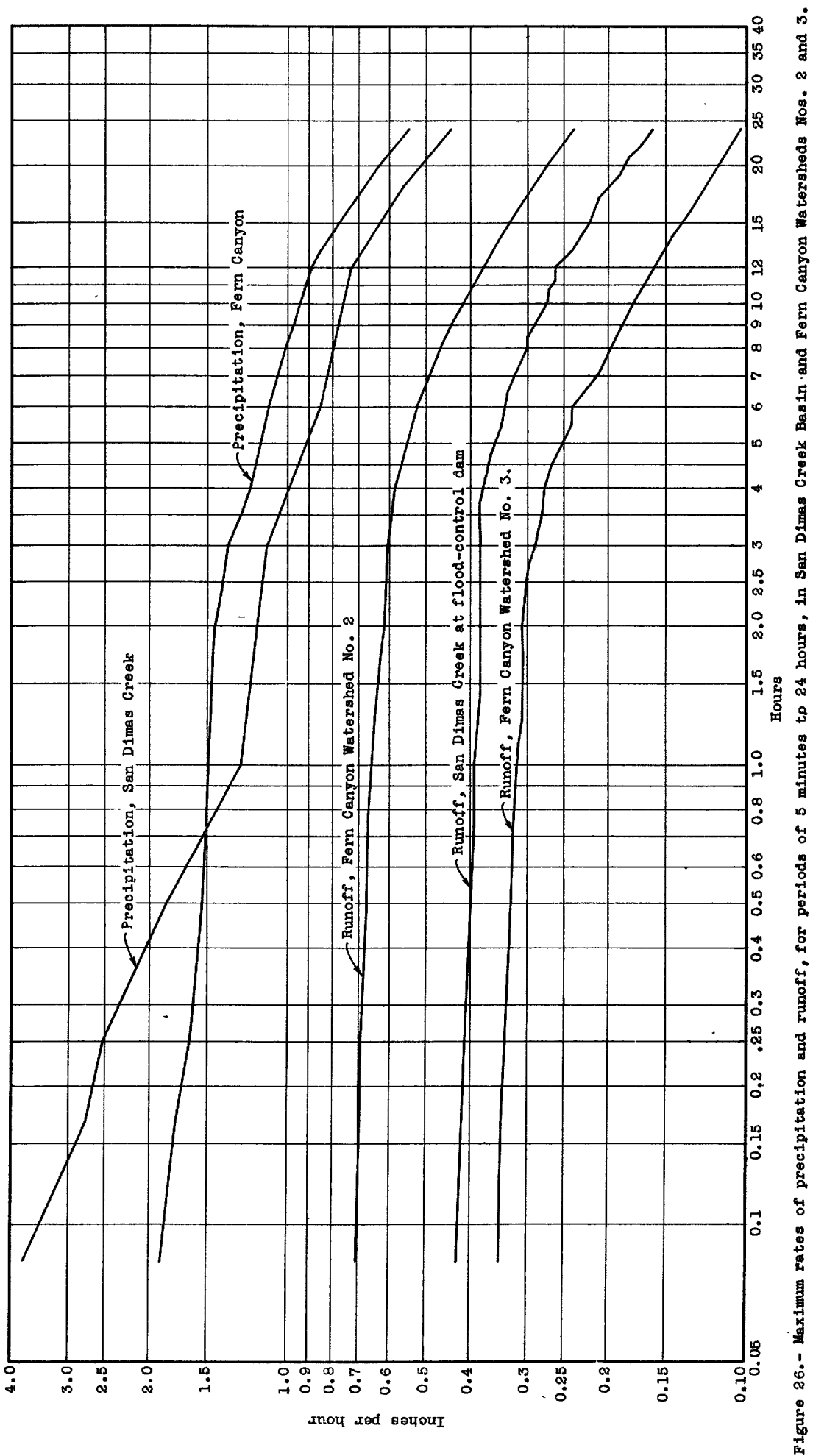


granitic gneisses predominate and are intruded by many pegmatite and ap11te dikes. The dikes, though locally crosscutting, generally follow schistosity planes in the metamorphic country rock for long distances. As a result of these conditions, the bedrock is characterized by a very prominent, steoply inclined planer structure of consistent orientation throughout the area.

In a report on a geologic examination 15 of the area after the 1938 flood it is indicated that watershed No. 3, although larger than No. 2 , has always had less runoff, both for seasons and for Individual floods. In watershed No. 3 the planar structure of the bedrock lies normal to the drainage axis and dips falrly ste日ply downstream. This permits a more rapid percolation of water than in watershed No. 2 , which is so orlented that the schistosity planes of the same rock types intersect its arainage axis at an acute angle, with correspondingly less opportunity for percolation and more opportunity for runoff to reach the stream channel.

The relation between maximum rates of precipitation and runoff for intervals of 24 hours and less is shown on figure 27 for five representative basins, namely, Live Oak, San Dimas, and Santa Anita Creok Basins, West Fork of San Gabriel River Basin, and Fern Canjon Watershed No. 2. The data used in the plotting are those show in table 19, supplemented by other data for better definition of the relation. The number noted against a plotted point indicates the length of the interval in hours appliceble thereto.

The decided break in some of these curves for intervals longer than about 12 hours is due to the falling off of the rates of rainfall for these longer intervals as compared to the rates of runoff, the runoff reflecting in this way the influence of ground-water seepage in the basin. This relation indicates that the rates of runoff corresponding to a fixed rate of rainfall have a tendency to increase as the durations of such rates increase. This tendency is demonstrated by tablo 18, in which are complled data taken from figure 27. For example, for an average rate of precipitation of 1.0 Inch an hour, lasting for 1.5 hours, on Live Oak Creok, the resulting average runoff was 0.22 inch an hour, and for the same average precipitation lasting for 4 hours on San'Dimas Creok, the resulting runoff was $0.38 \mathrm{inch}$ an hour. The corresponding figures increase progressively to 9.7 hours and 0.59 Inch on the West Fork of San Gabrlel

15 Storey, H. C., The Ferm watersheds, characteristic runoff differences: Mem. Rept. California Forest and Range Exp. Sta., San Dimas Exp. Forest, Sept. 19, 1939. 


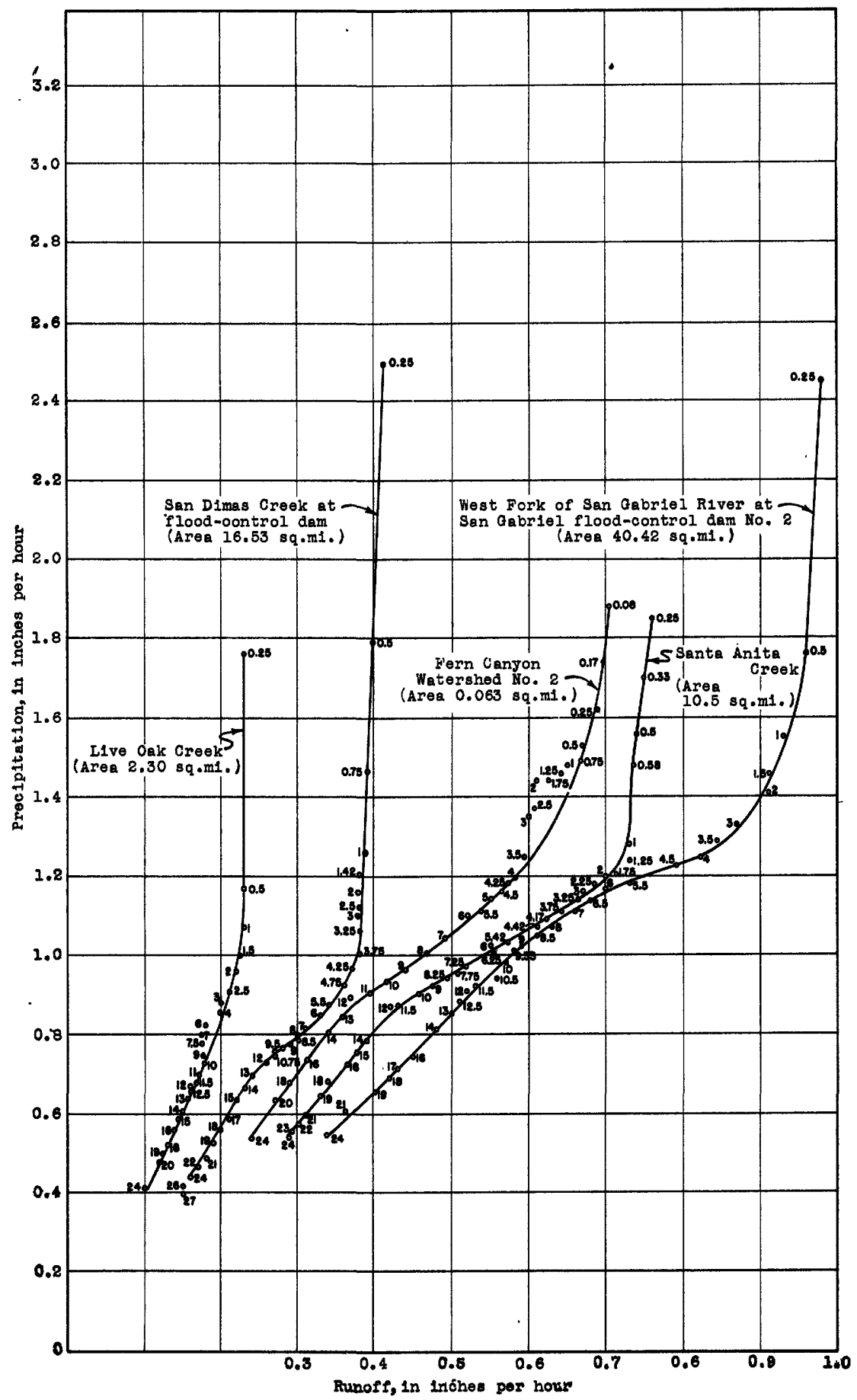

F1gure 27.- Relation between rates of precipitation and rates of runoff during maximum periode of 24 hours and less for typlcel areas in San Gabriel and Los Angelos River Basins. 


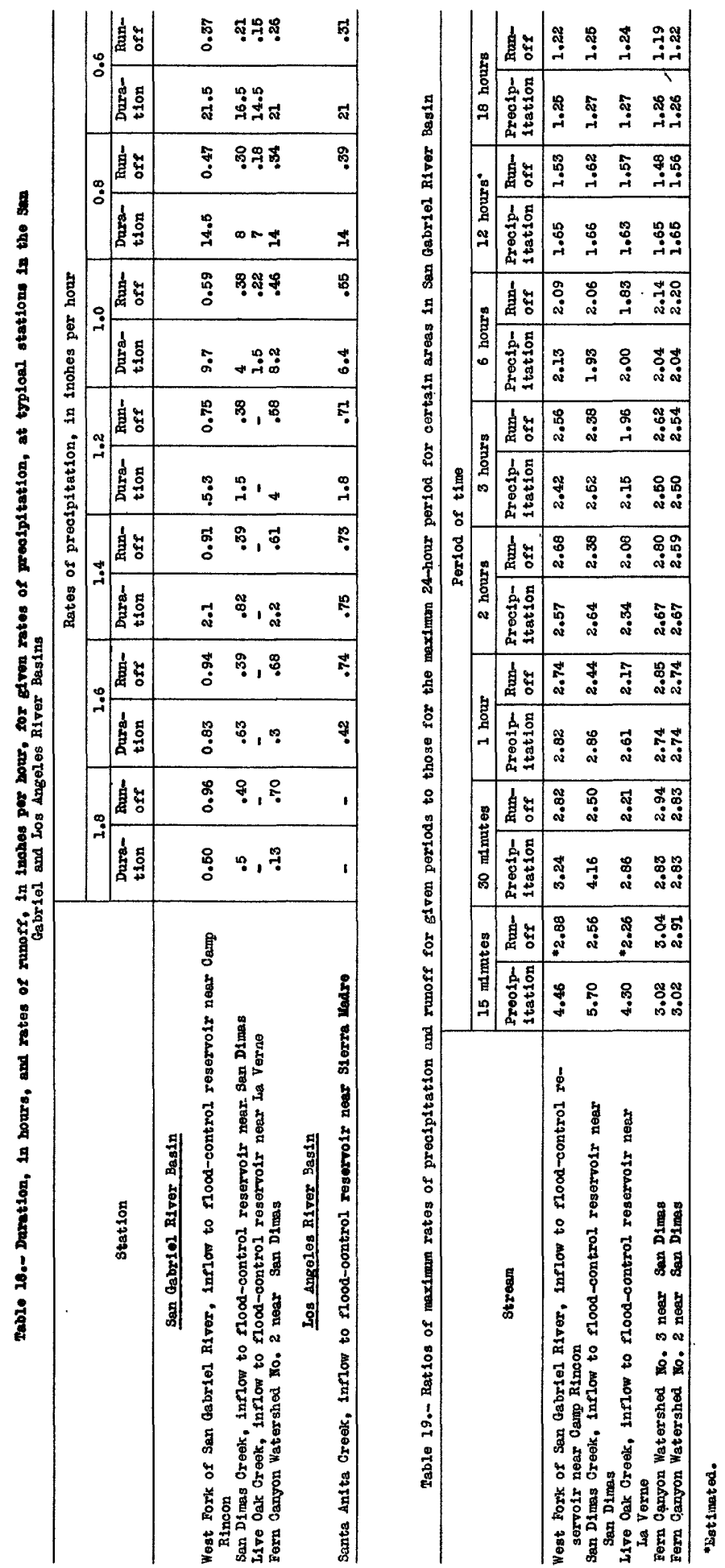


River. A like tendency is shown for parts of the curves representing rates for 18 to 24 hours. A study of the rates of rainfall and runoff for 24 hours, as given in the last two columns of table 17, discloses a rough relation betweon such rates, which may be used in estimating runoff from drainage basins for which only rainfall records were ava1lable.

For further 1llustration of the relation between precipitation and runoff the data of table 19 have been plotted in three groups, as in figures 28 to 30 , In general accordance with the magnitude of the rates of rumoff prevalent in the basins.

These graphs 1liustrate further the similarity in the intensityduration relations for precipltation and runoff. The tendency of these curves to conform to straight lines drawn from the origin through the plotted points marks characteristics of the data that tended to produce parallelism in the logarithmic plotting of the relation as previously noted.

Table 19 shows, for the drainage basins analyzed in figures 25 and 26, the ratios to the maximum rates of precipitation and runoff for a 24hour period of the maximum rates for selected shorter periods. It may be noted that, for the 11sted basins, the runoff ratio for the 1-hour perlod varies from the precipitation ratio by +4 to -17 percent. The tablo $11-$ lustrates the similarity in the intensity-duration relation of precipitation and runoff for the several basins, such similarity being fundamentally the same as that referred to in the preceding paragraph as tending to produce parallel lines under logarithmic plotting.

The foregoing graphic and tabular analyses indicate that for perlods of time shorter than 1 hour the rainfall rates seem to increase more sharply than the runoff rates. For examplo, tablo 19 shows that on San Dimes Creok, for the l-hour and half-hour periods, respectively, the preclpitation ratio changed from 2.86 to 4.16 , whereas the runoff ratio changed from 2.44 to 2.50. This difference 1s explainable in part by the fact that surface detention and channel storage in the stream system tend to modulate the reflection of high rates of rainfall for short intervals into high rates of runoff for corresponding intervals. It is important In these analyses not to overlook the fact that the computed alscharge, particularly the highest alscharge, includes debris, which is a complicating factor of unknown but probably substantial amount. 


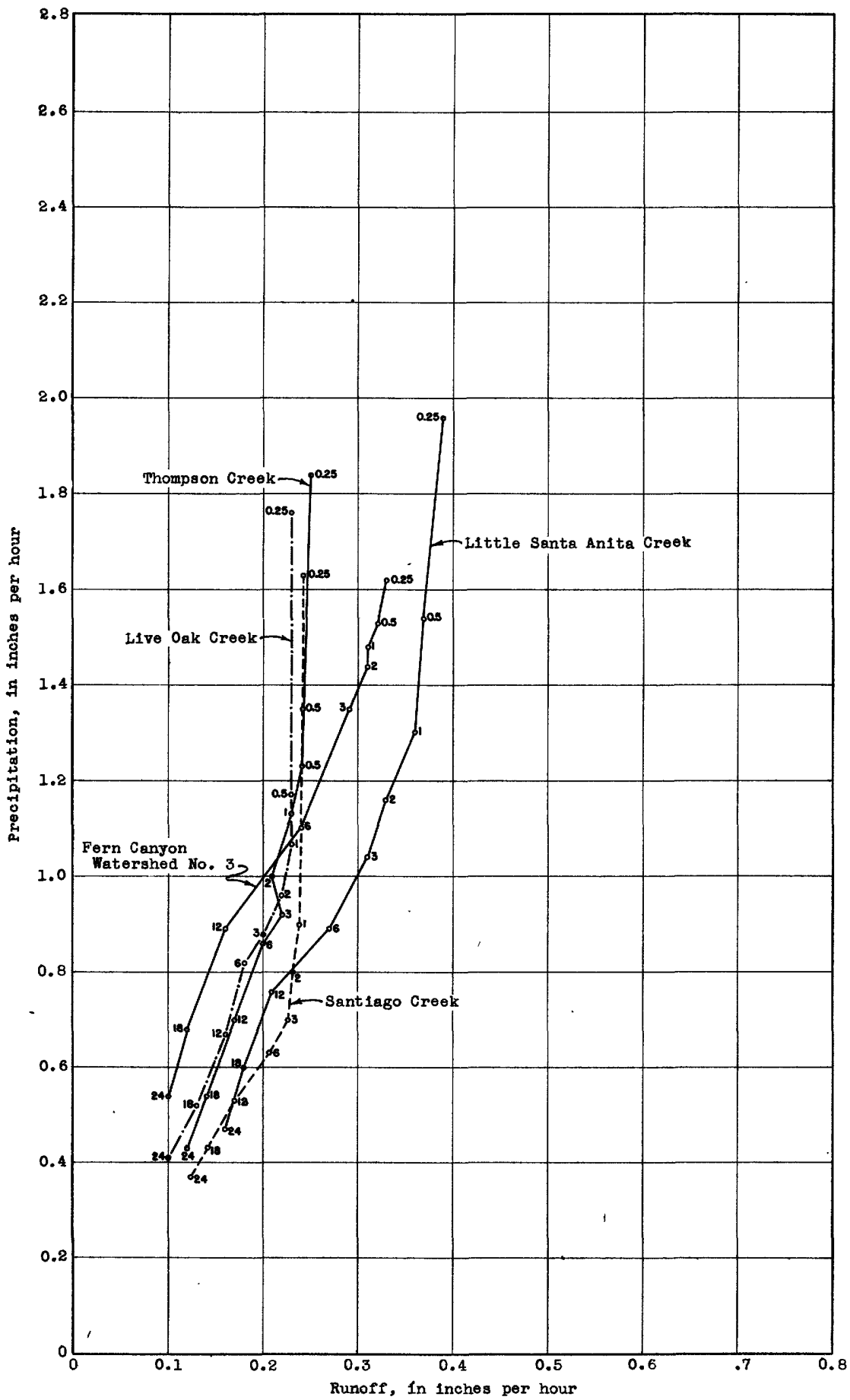

Figure 28. - Relation between maximum rates of precipitation and maximum rates of runoff during indicated periods of time for loweat rates of runoff. 


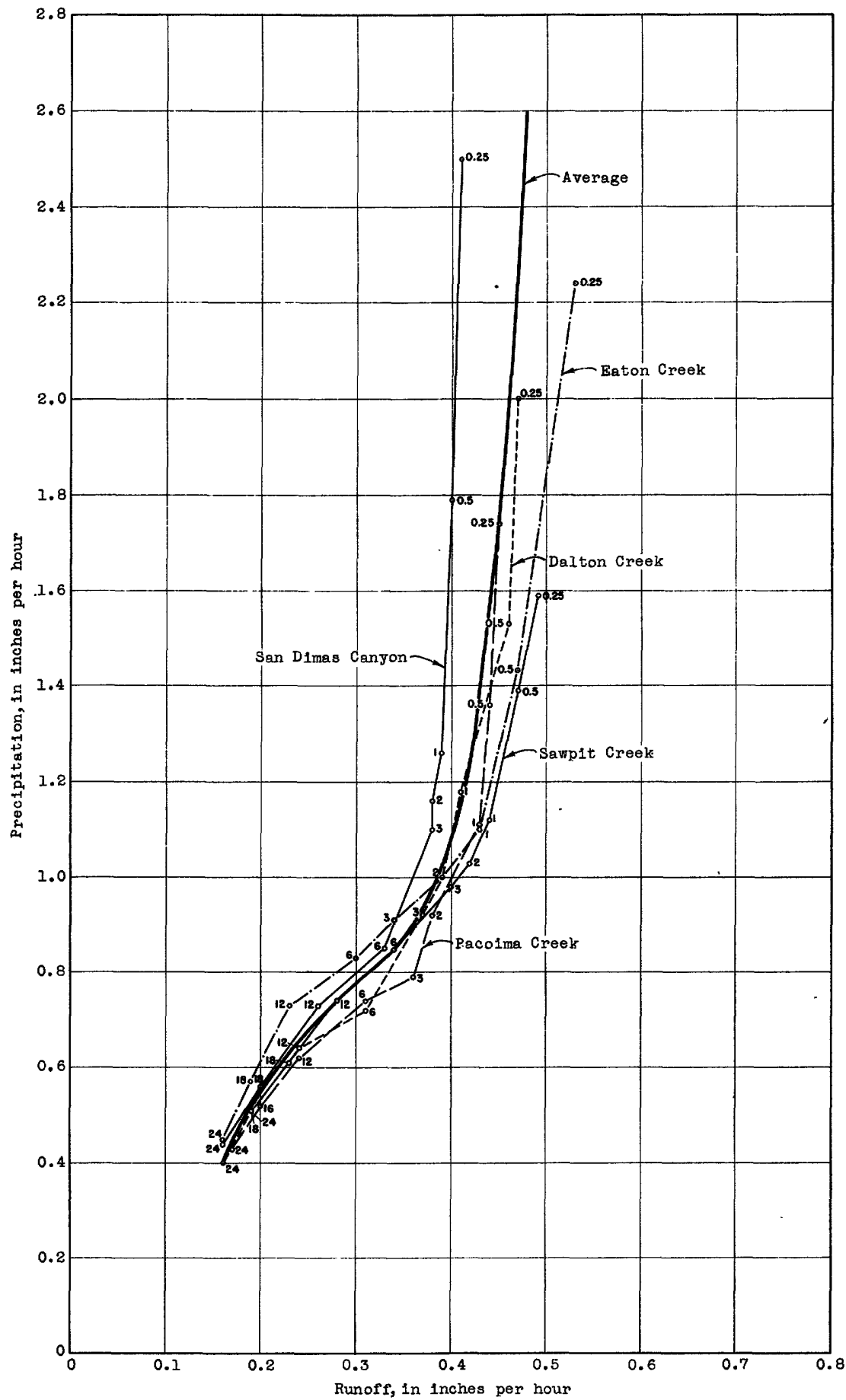

Figure 29.- Relation between maximum rates of precipitation and maximum rates of runoff during indicated periods of time for intermediate rates of runoff. 


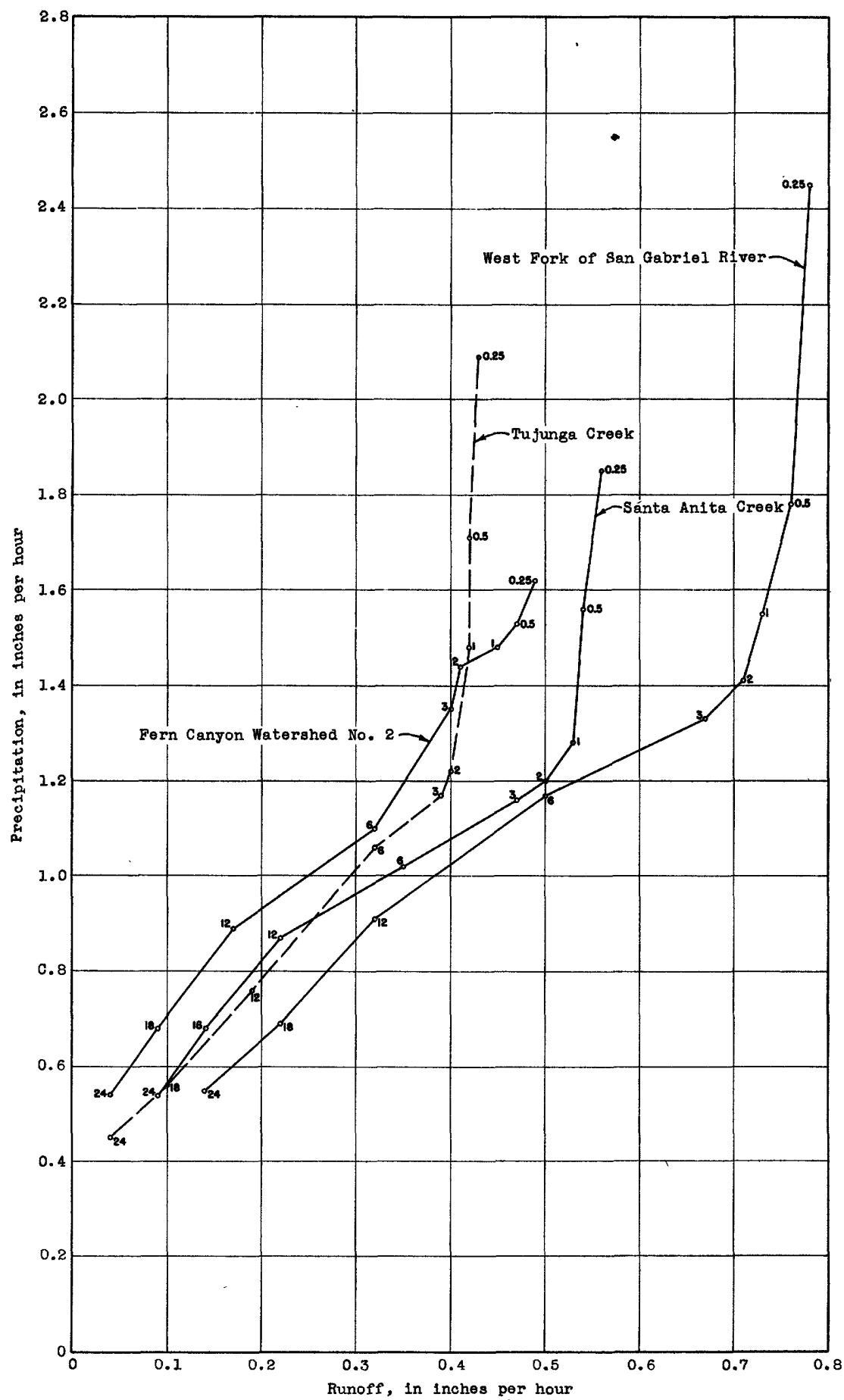

Figure 30.- Relation between maximum rates of precipitation and maximum rates of runoff during indicated perlod of time for highest rates of munoff. 
The difference may be due also, in part, to the fact that the rainfall rates over a given basin are estimated on the basis of the average rates at one, or at the most, only a few recording rain gages, located in or near that basin, "which method of procedure, as has previously been noted, may tend to make the computed rainfall rates less rellable for intervals of 1 hour or less.

Lag between precipitation and runoff

The systematic relations found between rates of precipitation and rates of runoff suggest a comparison, between different drainage areas, of the times of their occurrence similar to the comperison, furnished by figure 14, of the times of occurrence of maximum rates of precipitation. Figure 31 furnishes a comparison of the times of occurrence of maximum rates of precipitation and runoff in West Fork of San Gabriel River and Little Santa Antta Creek Basins and Ferm Canjon Watershed No. 2.

The graphs representing runoff rates define periods of time which without exception are subsequent to those for the corresponding rainfall rates. The interval between the times corresponding to the centers of mass of the rainfall and munoff occurring in comparable perlods, such as 1 hour or 2 hours, is denoted "lag". This term, as commonly used, is the time-difference in phase between salient features of rainfall and associated munoff. Table 20 shows, for the above-mentioned three basins and for Santa Anita Creek Basin, the time of occurrence of the center of mass of precipitation, of the center of mass of runoff, and of the lag as determined therefrom for selected periods ranging from 5 minutes to 24 hours each.

In Fern Canyon Watershed No. 2, the lag in time of occurrence between the rainfall and the assoclated runoff appears to be generally from I굴 to 3 hours for perlods of 12 hours and less, which seems to be rather excessive for a dralnage area of only 0.063 square mile with a maximum distance of water travel of about $0.4 \mathrm{mile}$ from the divide to the gaging station. This apparently excessive lag tends to support the conclision that much of the runoff moved through subterranean channels, with the result that the flow was slower than the flow in surface channels and may have been subjected to the influence of ground-water storage. The ind 1cated lag may have been due in part to the peculiarities of rainfall distribution. The rainfall involved in this comparison continued until about 6 p.m. March 2, when 1t stopped almost completelf as indicated by the mass curves on figures 9 and 13 . 


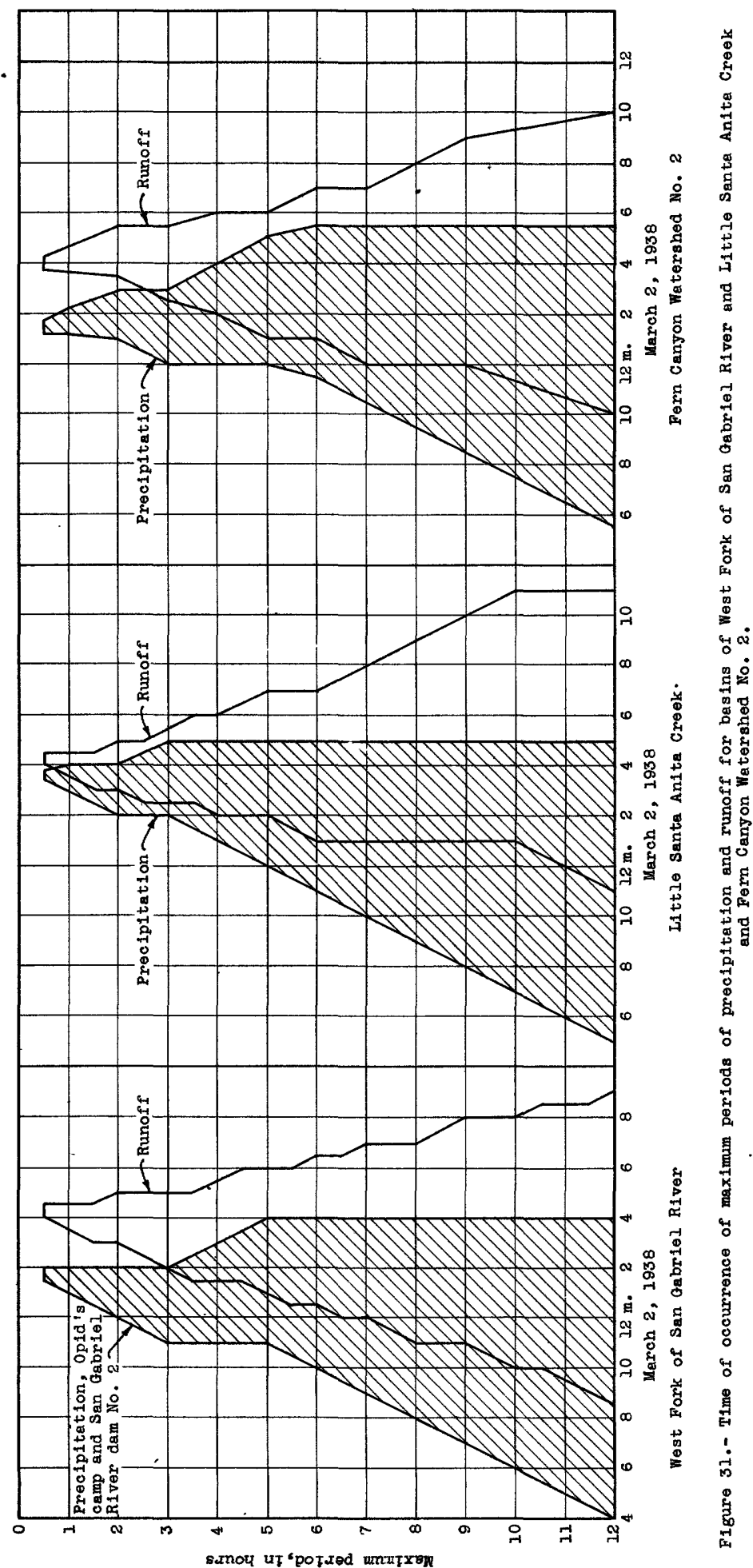




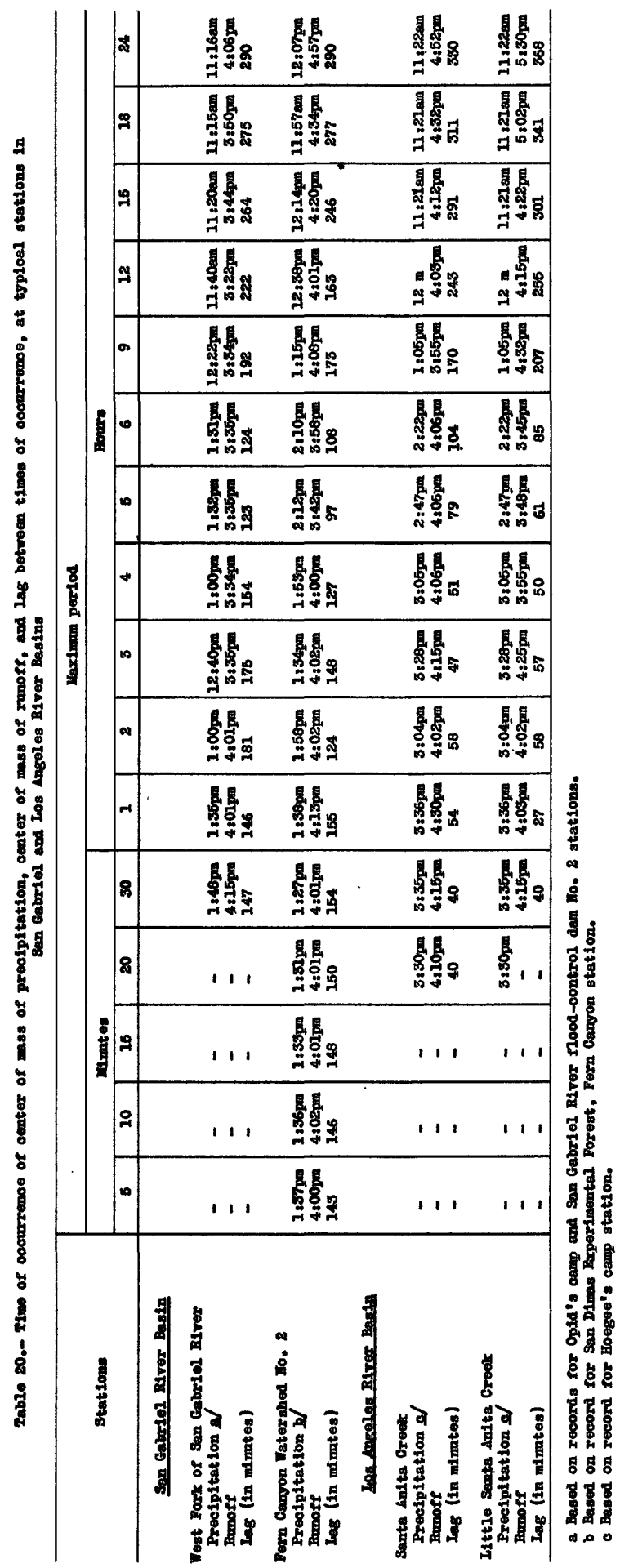


Between $3: 30$ and 4 p.m. March 2 , there was a sharp shower, direct surface runoff from which may have synchronized with the outflow from ground water from antecedent precipitation discharging into the channel and so have produced the maximum discharge at the gaging station.

The lag on the West Fork of San Gabriel River appears to vary between 2 and 3 hours for periods of 6 hours and less. For longer periods than the maximum 6 hours, the lag increases progressively to about 5 hours for the maximum 24-hour period, which probably indicates a greater influence of basin storage on the discharge.

Table 14 gives the travel distance from the center of the drainage area of the West Fork of San Gabriel River to the point of measurement as 4.92 miles. If $1 t$ is assumed that this represents the average travel distance for each particle of water in runoff, and that the lag represents the average travel time, for the maximum period of 30 minutes, this travel time would have been 147 minutes, which represents a mean velocity of 2.95 feet a second. Mean velocities of similar magnitude may be determined for other maximum periods. Such velocities seem rather low for the average drainage basin slope of 47 percent. It is believed that these apparently low rates of travel may be due to the passage of many of the particles of water through the underlying rock formations, and to the slower passage of others as surface runoff down minute channels on the mountain slopes before joining the larger tributaries.

It is probable that the peak discharge of the West Fork of San Gabriel River was caused in part by a heavy, short shower just before 4 p.m., the direct surface runoff from which combined with a large contribution of ground-water seepage. It seems likely that this sharp shower would have influenced the lag less for the longer periods.

On Santa Anita Creek, for which the center of the drainage area is. about 2.41 miles from the gaging station, the lag for the maximum $30-$ minute period was 40 minutes. On assumptions similar to those previously made, the time of travel of the particles from the time of their precipitation to the time they passed the point of measurement would indicate a velocity of 5.3 feet per second. On Ifttle Santa Anita Creek the lag of 40 minutes for the maximum 30-minute period would indicate a velocity of 3.1 feet per second, which is comparable with the velocity indicated for the West Fork of San Gabriel River. In both the Santa Anita and Ittle Santa An1ta Creok Basins, the maximum runoff was caused by the sharp shower which occurred between 3 and 4 p.m., and produced the maximum rates of rainfall for the storm period (see group B, Hoegee's camp, fig. 15). 
The lag between rainfall and munoff is a manifestation of the retardIng effect of the topographic and geologic characteristics of the basin-- . such as slope of the streams, roughness of channels, and vegetation covermon the time required for rainfall to become runoff. Observations seem to indicate that the greater the lag the less close will be the relation between rates of rainfall and munoff for the shorter periods of time, and the less responsive in increase will the stream flow be to the rainfall. Moreover, as previously pointed out, the lag as determined may embody conditions with respect to the distribution of rainfall and the concentration of runoff, which were peculiar to the storm and floods of March 1938.

\section{Infiltration}

It is shown elsewhere in this report (see tables 16 and 26) that the quantities of water retained in surface and ground storage during the floods of March 1938 in southern Cal1fornia were exceptionally large. The high rates of retention are attributed in large part to the high rates of infiltration that have been found to prevail during flood periods throughout much of the mountain area in Los Angeles and San Bernardino Counties. Some interesting information concerning the characteristics of Infiltration is available from data collected in the course of experiments by the United States Forest Service in the San Dimas Experimental Forest. Measurements of precipitation and direct surface runoff during the storm of March 1938 on experimental plots, each a fortieth of an acre in area, Indicate extremely low rates of direct surface runoff or, conversely, extremely high rates of infiltration. The records of precipitation and runoff from three of a group of nine adjacent plots of the Ferm Canyon series, showing greatest, medium, and lowest runoff, are shown in table 21.

The plots are at an altitude of about 5,000 feet and have a slope of about 60 percent, which makes them comparable with areas at the headwaters of most of the streams in the San Gabriel and San Bernardino Mountains that are subject to excessive floods. They are comparable, also, In geology, slope, and vegetative cover, with many of the headwater areas in other basins. The soil mantle on the plots is about 1 foot deep and overlies badly fractured bedrock. They have a fairly heavy and uniform growth of chaparral. Variations in soil mantle and the underlying rock structure, in addition to general tendencies toward inherent plot variability, are undoubtedly the cause of the main differences in the observed 
Table 21.- Precipitation and runoff for given periods on $1 / 40$-acre plots in Fern Canyon

\begin{tabular}{|c|c|c|c|c|}
\hline \multirow{2}{*}{ Period } & \multirow{2}{*}{$\begin{array}{l}\text { Precipitation } \\
\text { in inches } \\
\text { per hour }\end{array}$} & \multicolumn{3}{|c|}{ Maximum runoff in inches per hour } \\
\hline & & Plot 349 & Plot 341 & Plot 345 \\
\hline $\begin{array}{l}10 \text { minutes } \\
15 \\
30 \\
1 \text { hour } \\
2 \\
3 \\
4 \\
6 \\
9 \\
12\end{array}$ & $\begin{array}{r}1.56 \\
1.52 \\
1.36 \\
1.35 \\
1.26 \\
1.20 \\
1.12 \\
.98 \\
.91 \\
.79\end{array}$ & $\begin{array}{r}0.0186 \\
.0186 \\
.0176 \\
.0162 \\
.0159 \\
.0143 \\
.0132 \\
.0107 \\
.0099 \\
.0083\end{array}$ & $\begin{array}{l}0.0198 \\
.0176 \\
.0121 \\
.0101 \\
.0109 \\
.0099 \\
.0090 \\
.0085 \\
.0070 \\
.0056\end{array}$ & $\begin{array}{l}0.0086 \\
.0065 \\
.0055 \\
.0050 \\
.0048 \\
.0047 \\
.0043 \\
.0036 \\
.0032 \\
.0027\end{array}$ \\
\hline $\begin{array}{l}\text { Total, In Inches, } \\
\text { Feb. } 27 \text { to Har. } 3\end{array}$ & 20.44 & .17 & .08 & .06 \\
\hline
\end{tabular}

munoff rates. For a total rainfall of 20.44 inches during the storm, these three plóts showed a direct surface runoff of only $0.17,0.08$, and 0.06 inch each, respectively. With an average rate of rainfall of 1.52 inches an hour for a 15-minute period, the direct surface rumoff amounted to less than 0.02 Inch an hour. Observations made on plots at Tanbark Flats showed characteristics very similar to those of the plots at Fern Canyon.

Ferm Canyon Watersheds Nos. 2 and 3 (see pl. 15) are less than half a mile from the Fern Canyon plots. Of these watersheds, No. 2 showed a maximum runoff of 0.69 inch an hour for a $15-\operatorname{minute}$ period, and No. 3 showed 0.33 inch an hour, maxima that are considerably higher than those for the plots.

Observations at the plots indicate a surface munoff of less than 0.2 inch, but a considerably higher rate of infiltration than watersheds Nos. 2 and 3, which, with a storm rainfall of 23 inches, produced a runoff of about 9 and 4 inches, respectively. (See table 16.) It appears that a considerable part of the precipitation that was absorbed by the soil mantle, as shom by the plot experiments, passed on through the underlyIng much-fractured rock and thence into the streams and that its movement was rather rapid. This procedure is, more pronounced for Watershed No. 2 than Watershed No. 3. The geologic conditions which mag account, in part, for this difference in rate of movement are discussed on page 334. The rate was undoubtediy influenced by the steepness of the land surface, the depth of the soll mentle, the nature and extent of rock outcrops, and the amount of water already in storage within the formations.

The apparently rapid flow of a large part of the infiltrated water to the stream channel seems to introduce difficulty in the derivation of infiltration capacitios which are of practical utility. 
The method most commonly employed by the Geological Survey in the determination of stream discharge consists of the determination, first, of a stage-discharge relation or rating by means of current-meter measurements of discharge at various stages from low water to high water and the application of this rating to the records of stage. If the stagedischarge relation for a station has been determined throughout the range of flow, it is evident that if the stage at any particular time is known and normal flow conditions prevall, the rate of discharge past the station at that time may be ascertained by application of the rating. It is very difficult to determine the discharge during abnormal flood stages in most localities, and the accuracy of the result arrived at usually depends on surveys, analyses, and computations, by various more or less indirect methods, for extending the stage-discharge relation beyond the range covered by current-meter measurements.

The difficulties which make it practically impossible to obtain direct measurements of discharge or to define stage-discharge relations during flood periods at most stream-measurement stations in southern California have been discussed in the section on basic discharge records. Records of discharge during the floods of March 1938 have of necessity been determined by a combination of the methods that have been developed by the Geological Survey in connection with stream gaging and studies of other floods. Particularly helpful in this connection have been the checks upon estimates of discharge afforded by the relations between rainfall and runoff as developed from a study of the basic discharge records and described in the next preceding section of this report.

\section{Computation of peak discharge}

Peak discharges were evaluated in a variety of ways suited to the conditions at the site and the kind of basic information that was available or could be found. Wherever possible, the results as determined by one method were verifled by checking them with results as determined by other methods.

The discharges were determined by slope-area surveys and related methods based fundamentally on the application of the Chezy formula. They were also determined from data concerning flow over weirs and through contracted openings, by the extension of rating curves with the ald of various techniques, by analyses of reservoir records as previously described, and by various methods involving comparison of basic factors. 
Peak discharges computed from a survey of pertinent conditions made subsequent to the peak are subject to errors introduced by unstable channels. Valley-floor streams usually have beds composed of sand and light gravel. At many places in these streams there was considerable scour during the higher stages, and the scour was probably greatest at the time of the peak discharge. On these streams the bed was prodded with a round iron rod and the rod forced by hand to a comparatively firm bottom that was considered to have been the bottom of the section at the time of the peak discharge.

Streams in the mountain areas have steep slopes and consequently high velocities. Their beds are gsnerelly composed of loose boulders and gravel, which are moved in large quantities because of the high velocities. These swift streams move from side to side in the canyons, depositIng or removing debris first on one side of the channel and then on the other. Cross-sectional areas determined after the floods have subsided may or may not be the same as at the time of the peak discharge. Debris moving downstream may have raised the water surface locally unt1l it was higher for smaller discharges than for the peak discharge. Turbulence may have caused splash or run-up marks on the banks higher than the controlling high-water levels. Because it is necessary to apply indirect methods of determination for such streams attempts were made to select reaches where these unfavorable influences would be least complicating.

Field data were obtainsd for certain streams in the San Bernardino and San Gabriel Mountains at places where channel conditions were extremely unfavorable, and it was found desirable to discard peak discharges computed from the date obtained at some of these places. A view showing typical channel conditions on these streams is presented in plate 16,A.

\section{Slope-area determinations}

Peak discharges were determined at many river-measurement stations and on miscellaneous streams by means of slope-area studies. Discharges were computed from measurements of the cross-sectional areas and slope by the use of formulas which have had extsnsive practical application. The formula generally used was the Manning formula, usually written as:

$$
V=\frac{1.486}{n} R^{2 / 3} s^{\frac{1}{2}}
$$

in which $V=$ mean velocity in feet per second, $n=$ coefficient of roughness, $R=$ hydraulic radius in feet, and $S=$ friction slope. 
The selection of values of $n$ has been guided by the results of the Survey's experience in the use of the slope-area method, and the values chosen were selected in the light of the few data avallable for streams in southern California.

The friction slope was generally computed from the hydraulic gradient of the peak stage as determined from high-water marks, on the basis of the difference in weighted velocity heads at the end sections. 15 a For streams whose velocity at the downstream section was less than at the upstream section, It was assumed that there was a 50 percent recovery of the theoretical kinetic energy head. Cross sections were divided into parts to provide for variation in the hydraulic radil and coefficient of roughness.

As an example of the application of the slope-area method, the basic data used in determining the peak discharge of Lone Pine Creek near Keenbrook are shown in figure 32. A view of the reach is shown in plate 16,B.

On some streams reaches of channel were survejed in which the changes In channel shape and slope made them unsulted to normal methods of analysis. The peak discharge was computed singly for each cross section on the basis of the Manning formula or the varied-flow equation given by Rouse. 16

\section{Flow over welrs}

The basic formula for the computation of flow over weirs is commonly expressed as $Q=\mathrm{CLH}^{\mathrm{n}}$, in which $Q=$ discharge in second-feet, $C=\operatorname{coeff1-}$ clent for the weir, $L=$ effective length of crest in feet, $H=$ head in feet on the crest measured far enough above the weir to avold surface draw-down, and $n=$ the exponent of $H$. The exponent $\underline{n}$ may be assumed to be 1.5, and under this assumption the coefficlent $c$ will ordinarily vary with the head and with differences in slope of crest. The velocity of approach in the channel above the weir affects the discharge over the weir and practically increases the head on the crest by an amount equivalent to the corresponding velocity head.

I5a Johnson, Hollister, The New York State flood of July 1935: U. S. Geol. Survey Water-Suppiy Paper 773-E, pp. 253-254, 1936. 16 Rouse, Hunter, Fluid mechanics for hydraulic engineers, p. 290, McGraw-Hi1l Book Co., 1938 . 


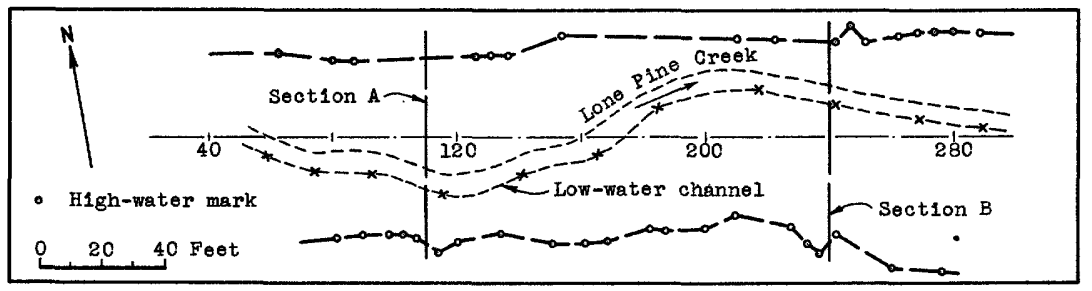

Sketch map
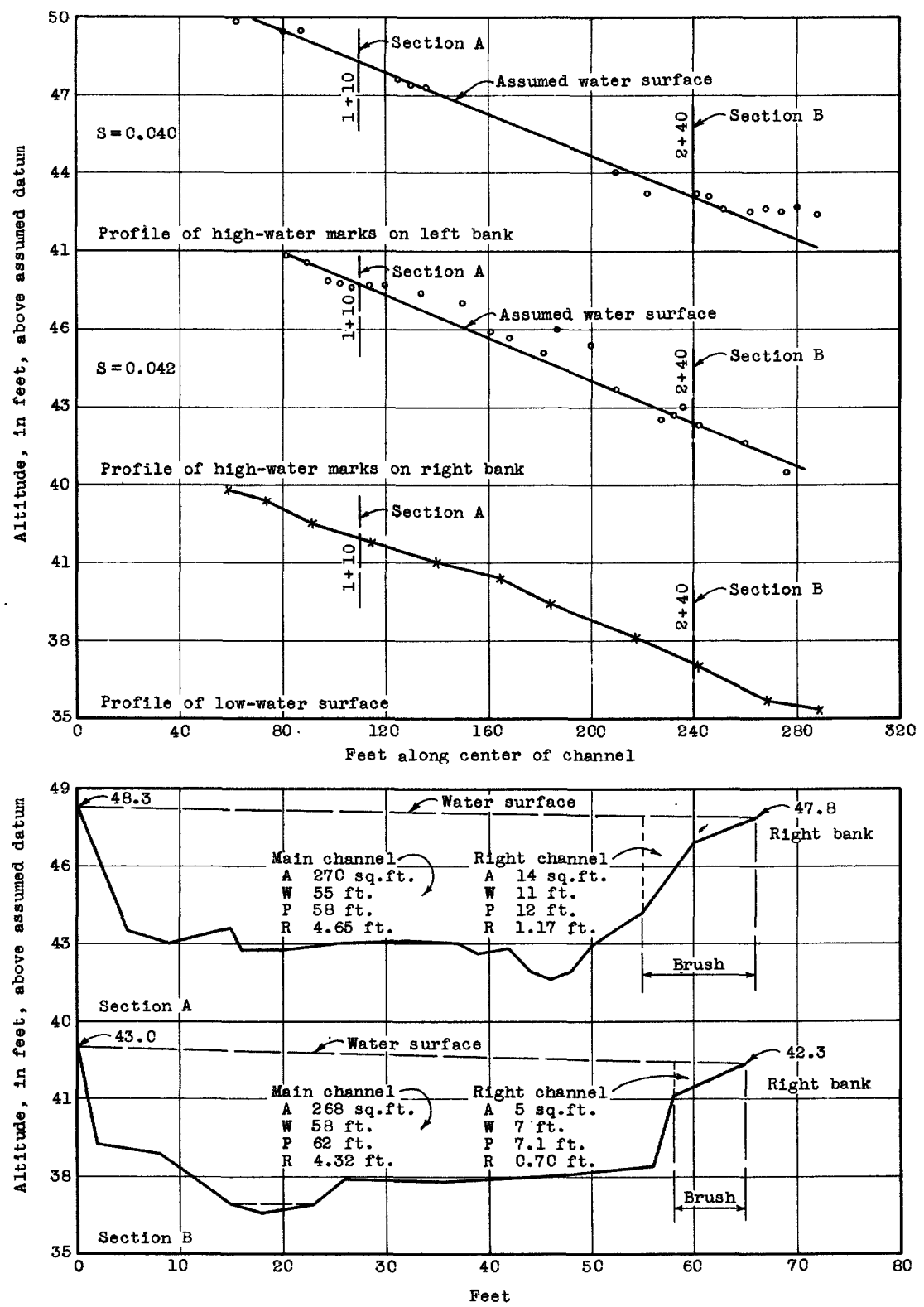

Figure 32.- Sketch map, water-surface profiles, and cross sections of the slope-area reach of Lone Pine Creek near Keenbrook. 
The basic formula was modified to make it conform to conditions peculiar to the places of application. For example, if the crest of a weir was submerged, this submergence was considered in computing the discharge over the weir. The exponent $n$ was generally taken as 1.5 , and values of C were seiected from data summarized in Water-Supply Paper 200, "Weir experiments, coefficlents, and formulas," or from those contained in hanäbooks.

Flow through contracted openings

The peak discharge was computed at some sections where the stream channel was constricted by bridge abutments or by natural rock formations. At these sections, the area of the cross section of the opening was much less than that of a cross section of the channel above, resulting in an Increase in velocity through the contracted section. This increase in velocity could be produced only by converting head into velocity, and the head so used caused a sharp drop in the water surface through the opening. The velocity was computed by the formula, $V=c \sqrt{2 g h}$, in which $V=v e l o c-$ Ity in feet per second, $c=$ coefficient of section, $g=$ gravity, and $h=$ head at most contracted section (surface drop through opening plus velocIty of approach).

The values selected for $C$ have been based mostly on experiments by Yarne11. 17

\section{Extension of rating curves}

The rating curve showing the relations of stage to discharge may, under favorable circumstances, be extended to stages and discharges higher than the range defined by current-meter measurements. The most favorable conditions for the accurate extension of a rating curve are furmished by well-defined rapids or riffles below the gaging station at all stages, and a uniform increase of channel cross section as the stage increases without abrupt changes in area or the adition of overflow channels.

Many of the gaging stations within the area affected by the March floods were of necessity located where both the benks and the bed of the natural stream channel were composed of easily erosible sand or light gravel, so that there was not a permanent relation between stage and dis$\operatorname{charg} \theta$.

17 Yarnell, David I., P1le trestles as channel obstructions; U. S. Dept. Agr1., Tech. Bull. 429, July 1934; Bridge plers as channel obstructions, U. S. Dept. Agri., Tech. Bull. 442, November 1934. 
There were some channels, however, in which the process of scour and f1ll was less active. At these stations it is belleved the rating curves can be extended with a falr degree of accuracy by assuming that nature, by scour and fill within the channel, maintains a rather definite relation between slope and elements of the cross section. This relation is obtained by plotting, to logarithmic scales, the mean depth against the mean velocity for each current-meter measurement. The rating curve may be extended to the peak stage by computing the mean depth from the cross section believed to have existed at this stage. The extension, of course, involves evaluation of the friction factor and slope through the range of stage. This method is further discussed in the succeeding section on "Discharge in unstable channels."

Discharge in unstable channels

For streams having unstable channels the relation between stage and discharge is generally variable, and the determination of discharge may involve the introduction into the process of one or more additional factors and the development of multiple rating curves. Conditions conducive to instability are prevalent in the alluvial channels of the streams of southern California, particularly during floods. It has been found useful on these streams to plot logarithmically mean depth against mean velocity for each discharge measurement.

It can be assumed that this relationship may be expressed as $v=C d^{n}$, in which $V=$ velocity in feet per second, $C=$ a constant, $d=$ mean depth In feet, and $n=$ the exponent of $d$. In channels in which the width is great compared to the depth, the hydraulic radius of the Manning formula should approach in value the mean depth, and the exponent of the mean depth should approach the value of the exponent of the hydraulic radius. The results of a current-meter measurement at the control section will define the constant $\mathrm{C}$, which represents the $\left(\frac{1.486 \mathrm{~s} \frac{2}{2}}{\mathrm{n}}\right)$ factor of the Manning formula. Assuming that this constant is applicable for a wide range of stage, then it is possible to develop a rating curve for this control section that was applicable at the time the measurement was made.

Additional current-meter measurements made at the same site may show a different constant, indicating a change in slope or friction factor. Using each new measurement and its resulting constant, additional rating curves can be developed that embody cross section, slope, and friction factor at the time the measurement was made. 
In the application of this method to determine flood discharges, advantage should be taken of all pertinent information regarding the conditions of flow at the higher stages and of any determinations of discharge by other indirect methods as previously discussed.

\section{Computation of continuous records of discharge}

The most accurate continuous records of flow during the flood were based on volumetric determinations of discharge into reservoirs. The computations were made from records of stage in the reservolrs, taking Into consideration, also, the information avallable concerning storage capacity and discharge through spillways and other outlet structures. The records of inflow to these reservolrs and of flow at certain other stations are included in a preceding section of this report, "Basic discharge records," in which the methods of determining them and their accuracy are discussed. The records in that section have been utilized in the analysis of basic relationships between rainfall and runoff.

Continuous records through the flood perlod were also avallable for a group of stations, principally on the valley floor, operated by the Corps of Engineers, United States Army, and the Los Angeles County Flood Control District. At these stations current-meter measurements of discharge were made at frequent intervals. For example, during the period February 28 to March 4, 1938, 18 measurements were made on Ballona Creek near Culver City and 44 on Los Angeles River at Los Angeles. Even at stations where frequent measurements were made, however, parts of the records are questionable because of unstable channel conditions.

At a large number of the gaging stations the records for the flood period were seriously deficlent. At 26 stations the water-stage recorders and accompenying equipment installed by the Geological Survey were completely destroyed. Many water-stage recorders installed by the Los Angeles and Orange County Flood Control Districts were also destroyed. At these gaging stations all records made during and shortly prior to the major flood period were lost.

Several of the gage-house structures were battered and overtopped by the flood, whlch stopped the recorder clocks and caused the loss of subsequent records. The wells of many stations were filled with silt, making them inoperative during parts of the flood perlod. The scouring and fillIng of the stream beds at many stations made it impossible or very difficult to establish any relation between gage height and discharge. This is 1llustrated by flgure 33, which shows actual cross sections of channel 


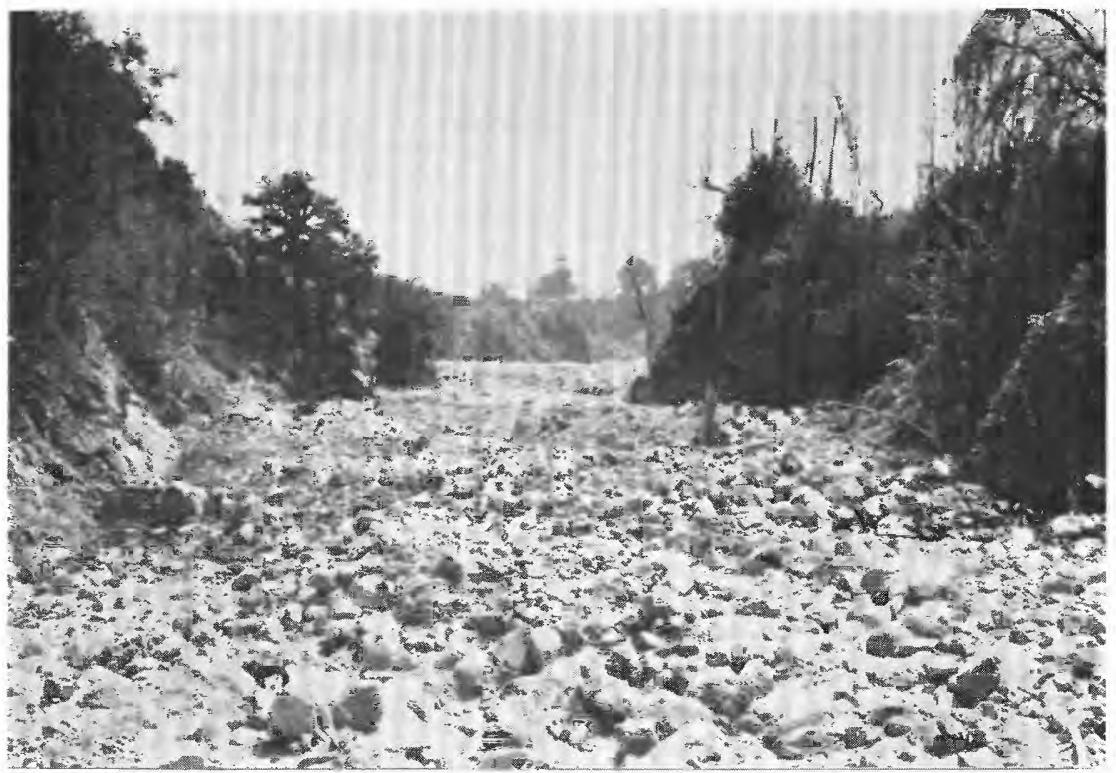

A. EAST ETIWANDA CREEK NEAR ETIII ANDA.

Looking downstream at reach below falls and near mouth of canyon: a typical mountain stream channel.

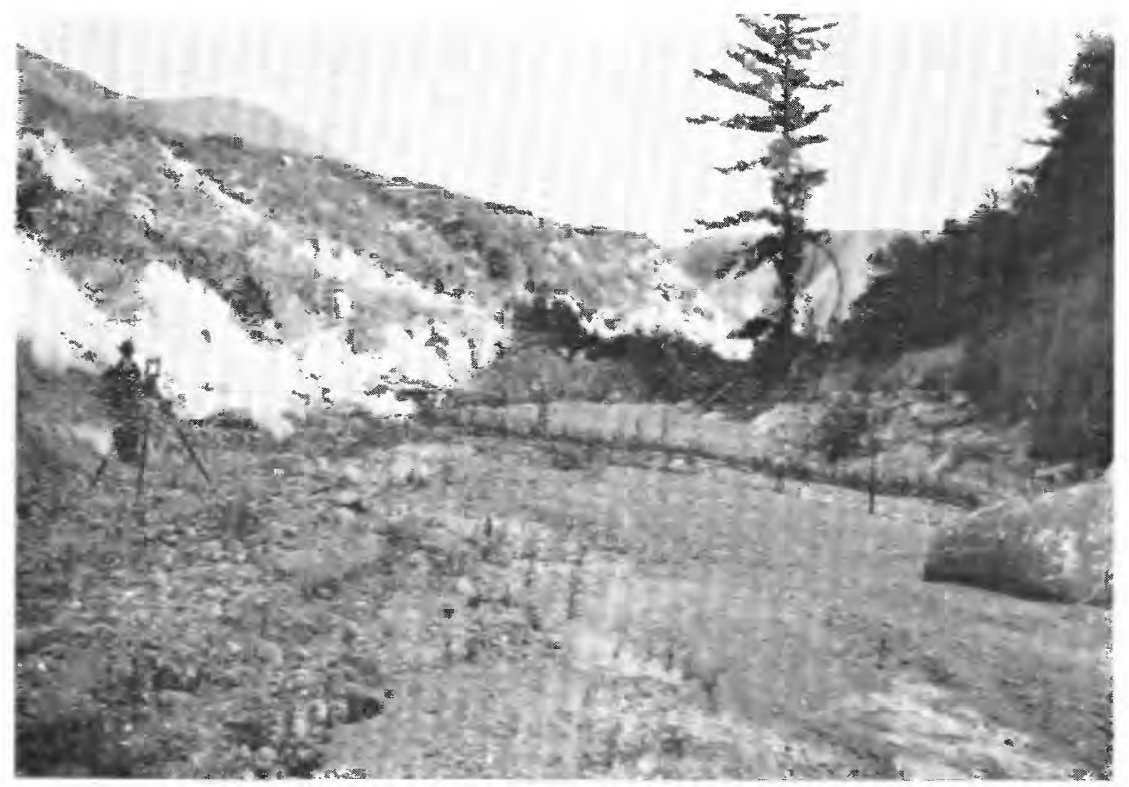

B. SLOPE-AREA REACH ON LONE PINE CREEK NEAR KEENBROOK.

Looking downstream. 



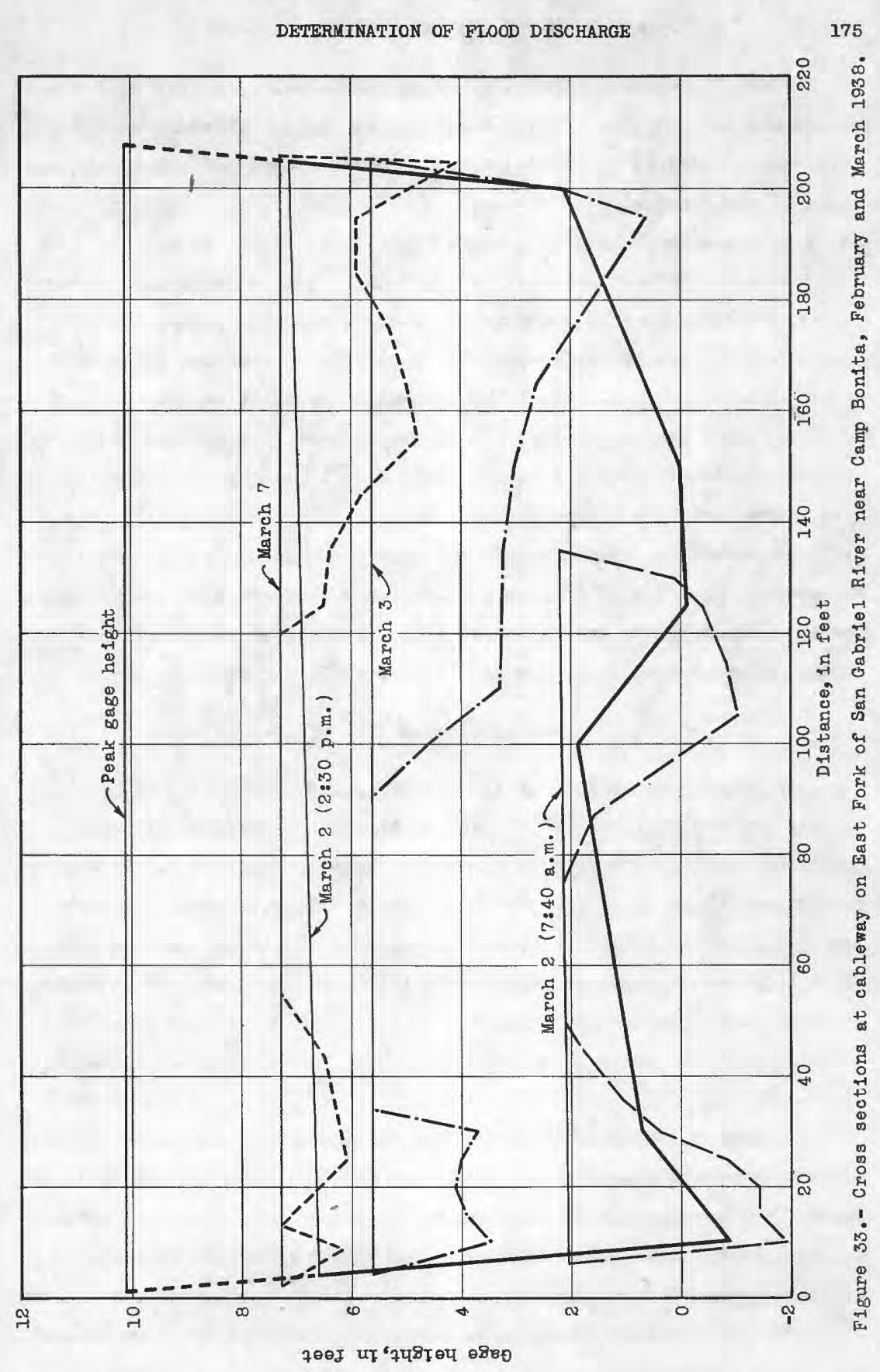


of the East Fork of San Gabriel River at the cableway at Camp Bonita as determined several times during the flood period. At several rivermeasurement stations artificial masonry controls were badly battered and rendered ineffective. The destruction of highways also interfered serious Iy in the work of obtaining needed date at gaging stations.

Every possible effort has been made to fill in missing records made during the flood so that wherever practicable complete records of discharge volumes may be available. All available information has been ut1lized to make these substituted records as reliable as possible. Many of the missing records were filled in and checked by comparing hydrographs for the stations to which they appertain with hydrographs for stations of like characteristics. The relations developed in the analysis of rainfall and munoff for the basic discharge records have also been utilized extensively in estimating missing records. A discussion of the procedure and an example of the derivation of flow for a gaging station with a period of missing record is discussed in the following section.

Derivation of discharge for periods of missing records

The general procedure for determining the discharge for periods of missing or unsatisfactory record was to prepare hydrographs based on all avallable data, including such discharges as were complled from fragmentary observations of stage, current-meter measurements, and slope-area and other similar determinations of discharge. These hydrographs were plotted on semilogarithmic paper, with the discharge expressed in inches of runoff over the area or in second-feet per square mile in order that the data might be compared readily with those for adjacent or comparable areas.

In general, the records at the various gaging stations were complete and fairly satisfactory to the morning of March 2, although some had already been lost through the destruction of the recording-gage structures. Commonly, therefore, the problem resolved itself into a determination of the discharge during the period of the main flood, on March 2 , and during the period of recession until such time as observations could be resumed.

In many instances helpful information regarding the flow was obtained by translating the rates of rainfall for a given drainage basin, based on recording rain-gage records within or-near the basin for the maximum 24 hours, into rates of discharge by the application of relations determined from the basic discharge records. The method used in completing the missing record for City Creek near Highland is presented as a 
typical example of the procedure. The avallable information consisted of a gage-helght record continuing unt1l about noon March 2 , and the results of a slope-area determination of the peak discharge.

The isohyetal map indicates that the average precipltation over City Creok Basin (drainage area, 19.8 squere m1les) during the entire storm period was about 19 inches. This rainfall was distributed through the period on the bas is of continuous records obtained from the recording rain gage at Del Rosa (altitude, 2,250 feet), located near the mouth of Strawberry Creok, about 5 miles southwest of the center of City Creek Basin. On this basis the precipitation for the basin was 11.2 inches for the maximum 24-hour period, or at a rate of 0.47 inch an hour; 8.5 inches for the maximum 12-hour period, or at a rate of 0.71 inch an hour; and proportionately less for shorter periods down to the maximum 15-minute period, for which the rate was estimated as 2.3 inches an hour. These rainfall rates are plotted on flgure 34.

The relation between rates of rainfall and runoff for C1ty Creok Basin are assumed to be very similar to those for the basins of Eaton, Dalton, Sawpit, Pacolma, and San Dimas Creoks as shown in figure 29. For this group, the maximum 24-hour rainfall ranged from 9.6 to 12.2 inches, the average being 11.0 inches.

On figure 29 is shown also an average curve of these relations for the basins above named, which was assumed to apply also to City Creok. By applying the assumed rates of rainfall to this average curve the corresponding rates of runoff were determined and used to define the runoff curve shown on flgure 34. This figure is comparable with figures 25 and 26.

The slope-area determination for Clty Creek near HIghland Indicates a peak discharge of 6,900 second-feet which is equivalent to 348 secondfeet to the square mile, or a rate of runoff of 0.54 Inch an hour. This hourly rate is plotted on flgure 34 as representing a discharge that lasted for 5 minutes. By this point and the other points representing rates of runoff, obtained as above described, a line is defined that is assumed to show the rates of runoff for the Clty Creok station for perlods of time ranging from 5 minutes to 24 hours.

On figure 35 are plotted the times of occurrence of the various maximum rainfall periods as observed for the recording rain gages at Del Rosa and Dovil Canyon. 


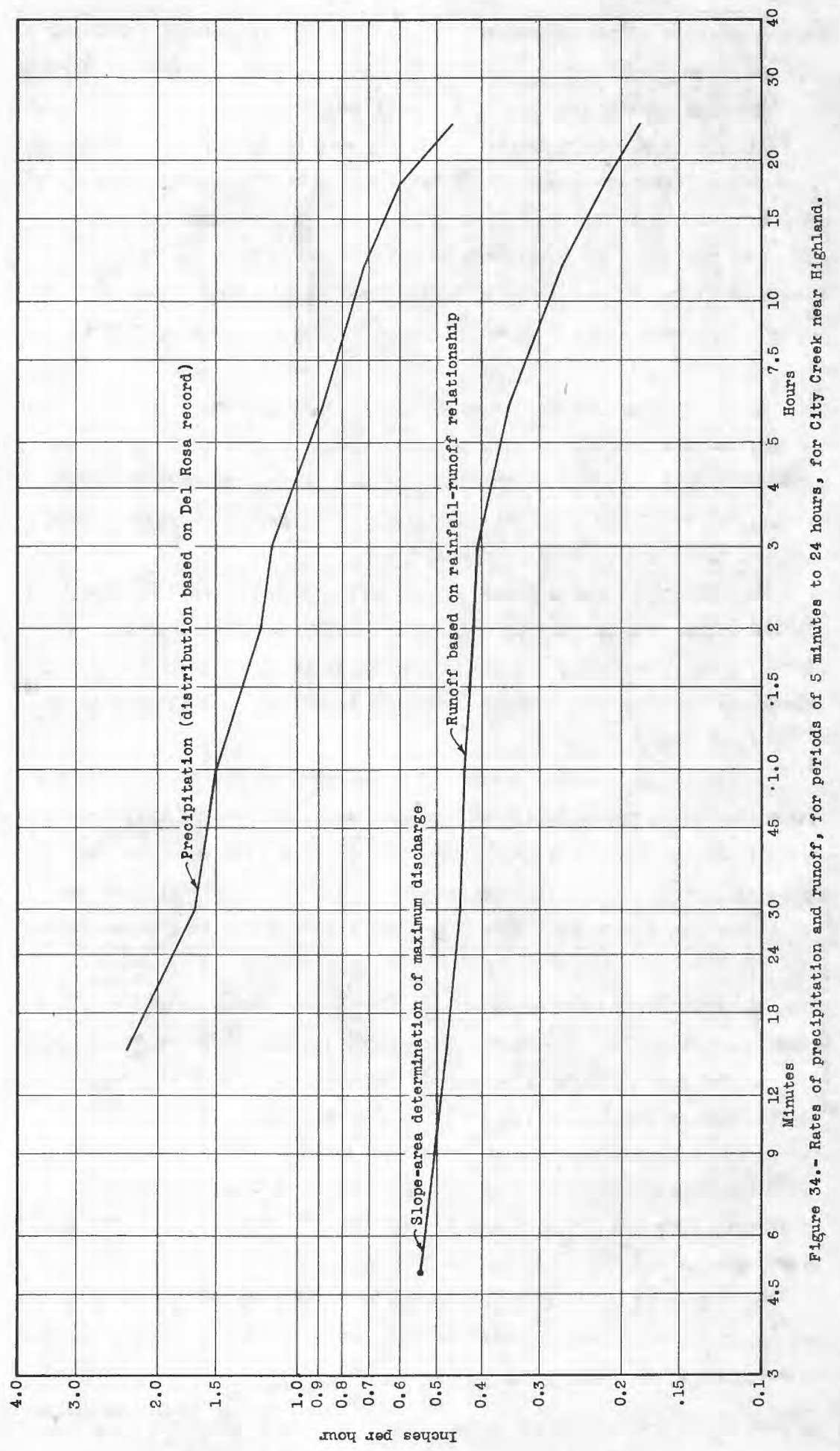



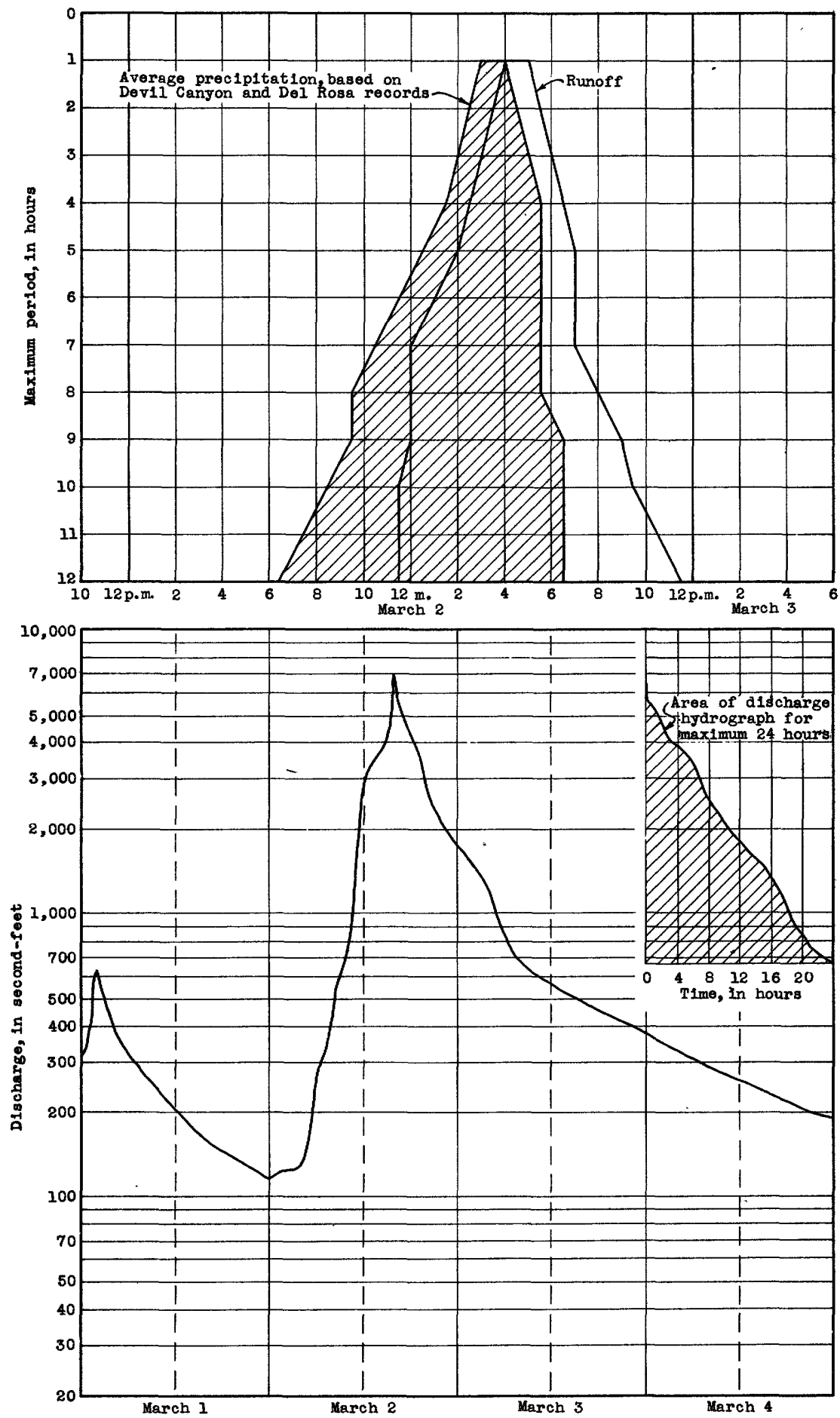

Figure 35.- Time of occurrence of indicated maximum precipitation perlods as obeerved at Devil Ganjon and Del Roea, and probable time of occurrence of runoff of Clty Creok,

- with discharge hydrograph of City Creek near H1ghland, March 1-4. 
On the basis of the general knowledge of the behavior of streams in the region, the lag between rainfall and runoff on City creek for the maximum 1-hour period was assumed to be about an hour. The beginnings of the meximum perlods of more than 6 hours were within the period of the observed record. The lag for the 12 -hour period is assumed to be 5 hours and is comparable with the data shown on figure 31.

On figure 36 is show, also, the estimated hydrograph of C1ty Creek for the perlod March 1-4. The record of discharge from midnight of Febmary 28 to noon of March 2 was obtained from the gage-height record and a fairly well defined stage-discharge relation.

In the upper right hand part of the lower part of figure 35 is a curve that shows the rates of discharge for the maximum 24-hour period, ranged in the order of magnitude as derived from the data obtained from the runoff curve show on figure 34. With this curve showing the rates of discharge and the curve in the upper part of figure 35 indicating the times of occurrence of maximum average rates for specified intervals, the hydrograph was completed through the maximum 24-hour period.

Table 22 fumishes a comparison, between C1ty Creek and San Dimas Creek on the basis of the ratios of the maximum discharge and the maximum average discharge for selected perlods to the discharge and average discharge for the maximum 24-hour perlod as well as the ratio of the maximum average discharge of each of the 14 areas given in table 13 to the average discharge for the maximum 24-hour period in that area. The values derived for City Creek seem reasonable on the basis of this comparison. The slight differences in storm pattern and topography between the basins of City and San Dimas Creeks may account for the higher ratio on C1ty Creek of the maximum discharge to discharge for the maximum 24-hour period.

Table 22.- Ratio of maximum discharge for indicated periods to the maximum 24-hour discharge for C1ty and San Dimas Creeks and for the average of 14 areas in southern California

\begin{tabular}{|c|c|c|c|c|c|c|c|}
\hline S.tream & $\begin{array}{l}\text { Drain- } \\
\text { age } \\
\text { area }\end{array}$ & Peak & $\begin{array}{c}1 \\
\text { hour }\end{array}$ & $\begin{array}{c}3 \\
\text { hours }\end{array}$ & $\begin{array}{c}6 \\
\text { hours }\end{array}$ & $\begin{array}{l}12 \\
\text { hours }\end{array}$ & $\begin{array}{l}18 \\
\text { hours }\end{array}$ \\
\hline Santa Ana River Bas In & & & & & & & \\
\hline $\begin{array}{l}\text { City Creek } \\
\text { San Gabriel River Basin }\end{array}$ & 19.8 & 3.11 & 2.50 & 2.33 & 2.01 & 1.52 & 1.25 \\
\hline San Dimas Creek & 16.5 & 2.63 & $2 \cdot 44$ & 2.38 & 2.06 & 1.62 & 1.25 \\
\hline Average of 14 areas ${ }^{2}$ & - & 2.72 & 2.45 & 2.22 & 1.92 & 1.48 & 1.19 \\
\hline
\end{tabular}

a From table 13. 
The discharge of C1ty Creek for the period March 4-1l was estimated on the basis of a general study of recession characteristics as described below and a current-meter measurement on March 11 .

On figure 36 are drawn retention curves for several of the stations listed in table 17. The characteristics of these curves show on logarithmic profection seem to conform to a theory that the discharges following flood peaks, if so plotted, approximate a linear relation to the amounts of water retained as ground storage.

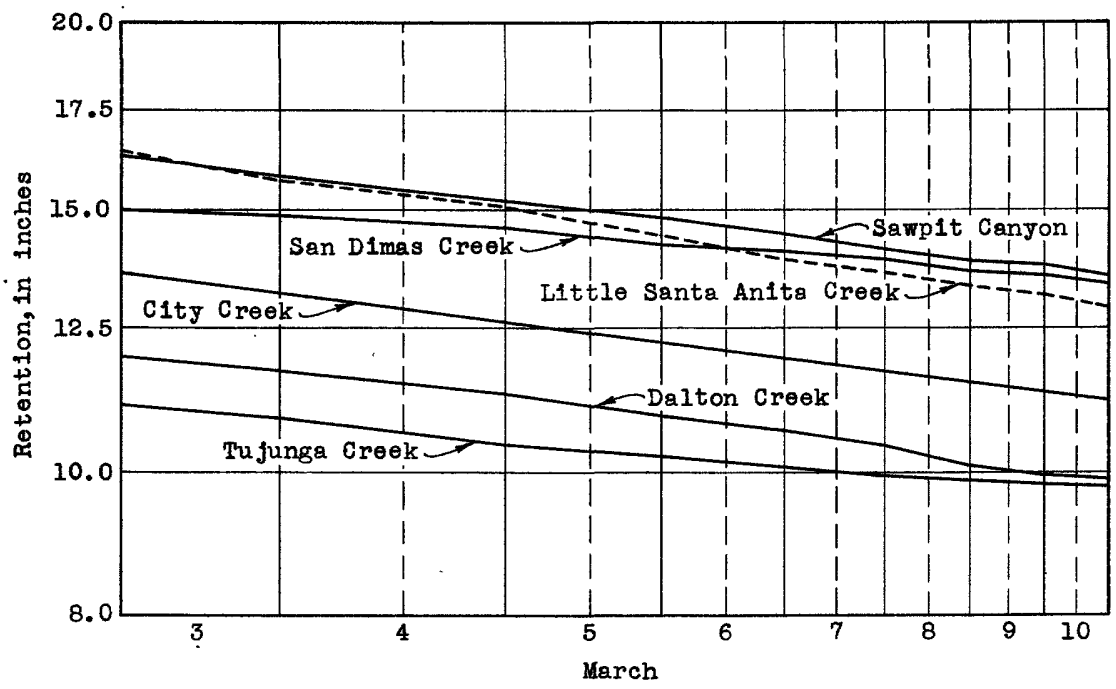

Figure 36.- Retention curves for certain stations in Santa Ana, San Gabriel, and Los Angeles River Basins.

Beginning with the retention in City Creek Basin at noon on March 3, computed from the hydrograph just developed, a straight line was drawn approximately parallel to the other lines, and the runoff for each day was then determined from the drop in this retention curve. These amounts of runoff appear reasonable and wẹre checked by the current-meter measurement on March 11. The signiffcance of retention is discussed at some length in a later section of this report.

The determination of the record by the foregoing procedure necessar$11 \mathrm{y}$ involves assumptions with respect to the factors ontering into it, such as the rainfall rates that occurred on City Creek, the time of their occurrence, the selection of a suitable relationship between rainfall rates and runoff, and the estimate of the lag between rainfall and runoff in City Creek Basin. Also affecting the computed record are the possible complicating influences due to basing the fundamental rainfall-runoff records on discharge that includes more or less debris flow. The 
procedure Involves to a large degree the application of the experience and knowledge of the authors, gained in their long-time observation of the runoff characteristics of streams in southern Califormip.

From the records of discharge throughout the flood period as thus deduced, the mean dally dischatge for each of the missing days may be readily computed and complete record thus be provided for publication.

The foregoing method and modifications of it for adaptation to varying circumstances, as well as other devices, have been used to fill in the discharge record for days for which this record is missing. The description accompanying the discharge tables gives information regarding the methods used in completing the records. High accuracy cannot be claimed for the results, but they are belleved to be trustworthy enough to be used in studying the general characteristics of flood runoff in the region.

\section{Consistency of determinations of flood discharge}

Comparison of the intensity-duration relation for the runoff of city Creek Basin near Highland, as shown in figure 34, with the intensityduration relation for the runoff of other drainage areas, as shown in figures 25 and 26 and elsewhere, seems to demonstrate that the maximum runoff rates of City Creok Basin as indicated by the rainfali-munoff study and also by the slope-area determination tend to be mutualiy confirmatory. Similar confirmation was obtained at several other places.

At the following gaging stations substantial differences were found between estimates of discharge obtained by rainfall-runoff studies and those obtained by slope-area and other methods: Dev1l Canjon Creek near San Bernardino, Waterman Canyon Creek near Arrowhead Springs, Cucamonga Creek near Jpland, San Antonio Creek near Claremont, and Strawberry Creek near Arrowhead Springs. These differences reflect the difficulties inherent in the determination of peak discharges under the existing conditions as well as the part played by personal judgment in the interpretation of the data. The estimates of discharge as published in this report are intermediate between those obtained by rainfali-runoff studies and those obtalned by slope-area or other methods. An evaluation of the maximum discharge of these streams has been made on the basis of the composite judgment of engineers of the Geological Survey.

There was a wide discrepancy between the estimates of discharge of the Santa Ana River near Prado as obtained by different methods and different engineers. These estlmates ranged from less than 80,000 to more 


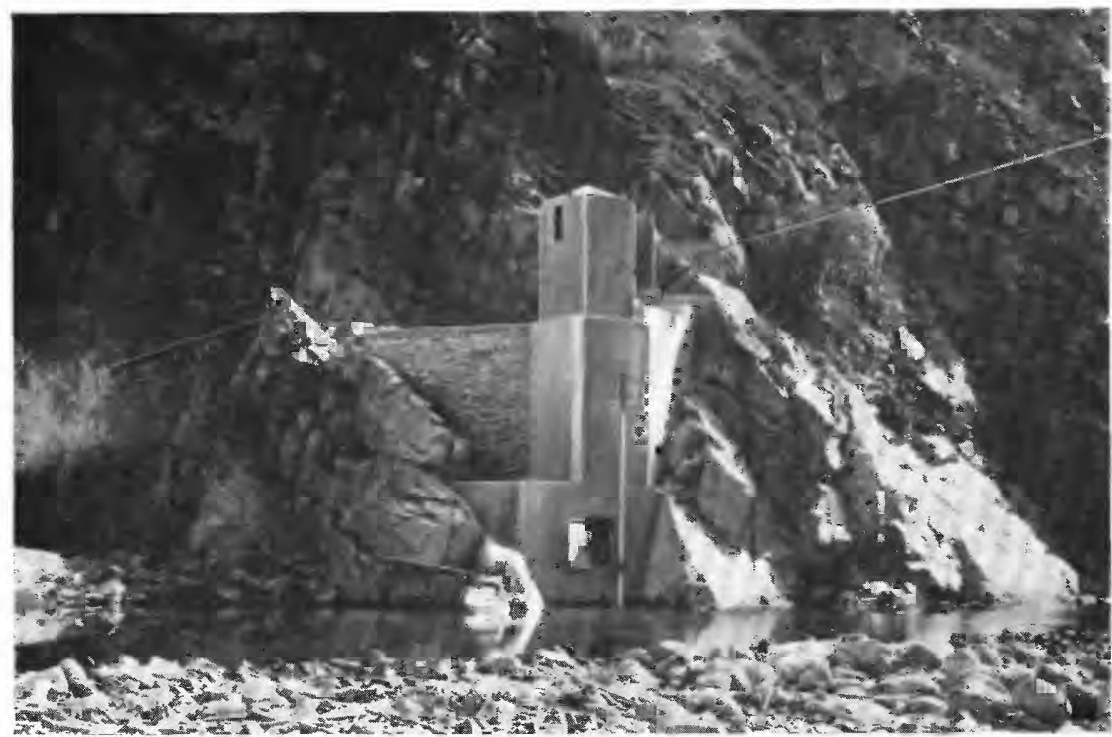

A. SAN GABRIEL RIVER NEAR AZUSA.

In lower end of San Gabriel Canyon. Slope of channel about 1 percent.

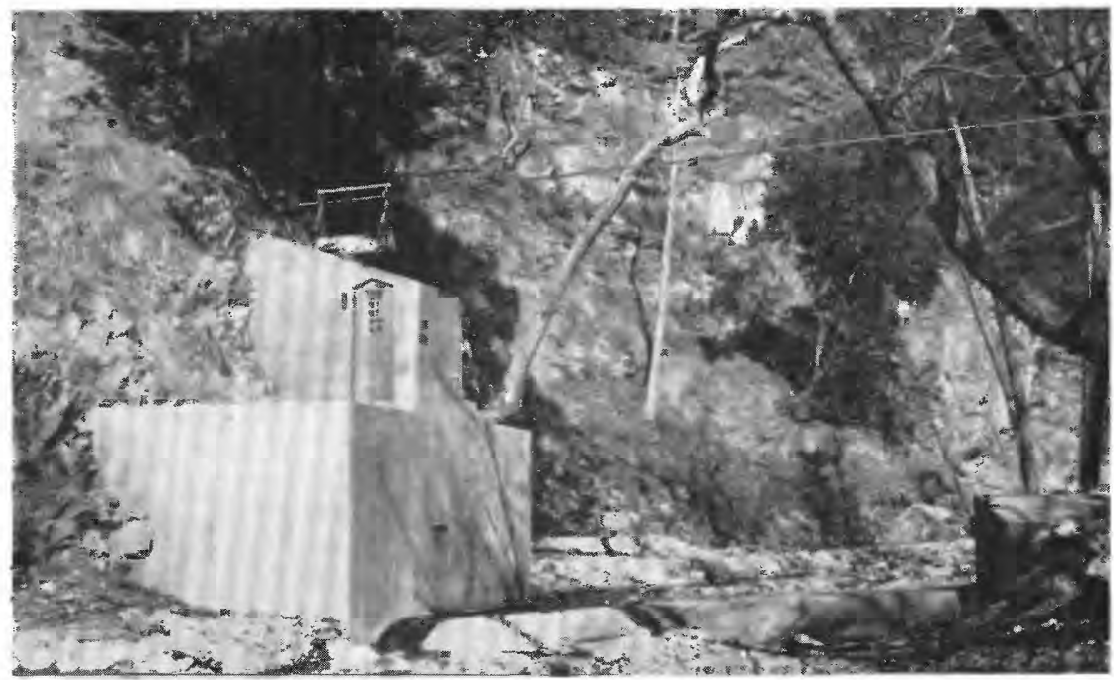

B. CUCAMONGA CREEK NEAR UPLAND.

Shelter is designed to withstand rocks falling from the canyon walls. Slope of channel about 4 percent.

\section{RECONSTRUGTED GAGING STATIONS REPLACING STRUCTURES DESTROYED DURING THE FLOODS OF MARCH 1938.}




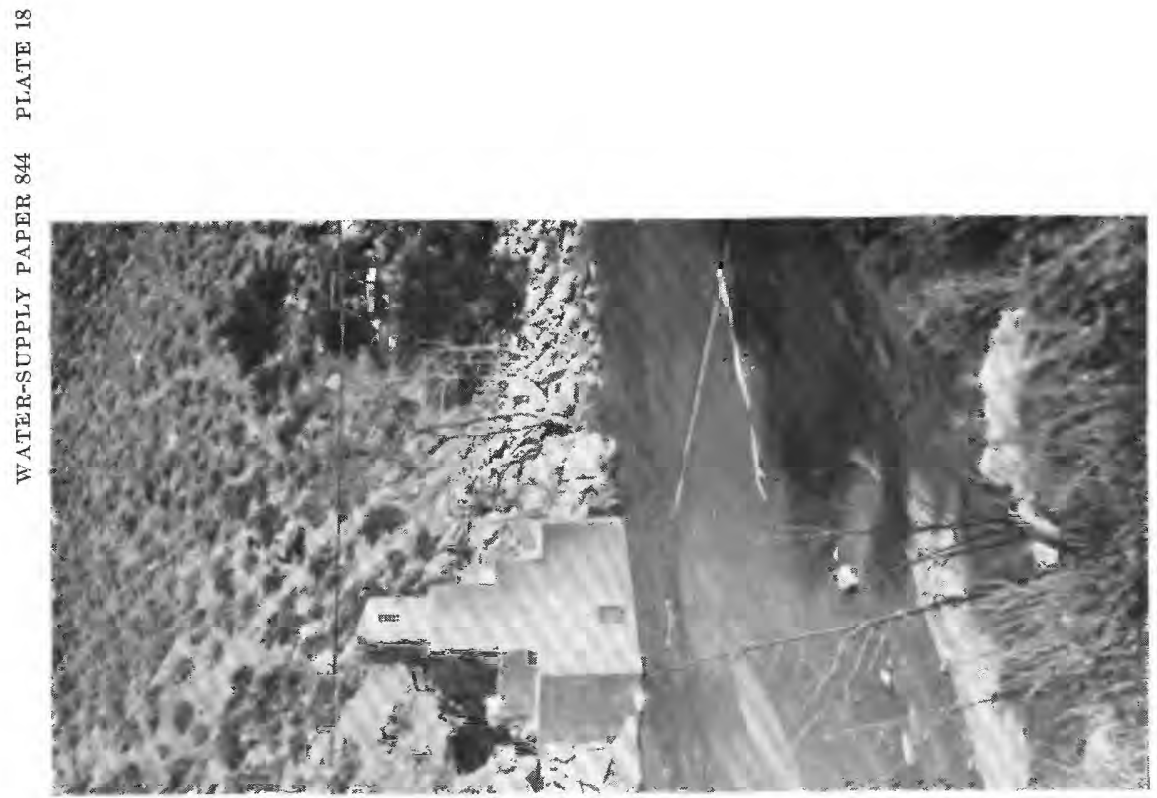

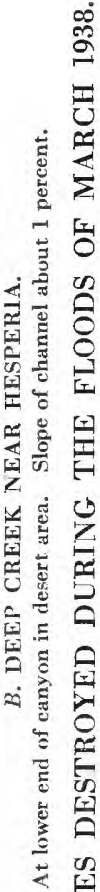

$\stackrel{\infty}{\circ}$

:

$\Sigma$

5

$\cong$

둥

它

स是

$\simeq$

붕

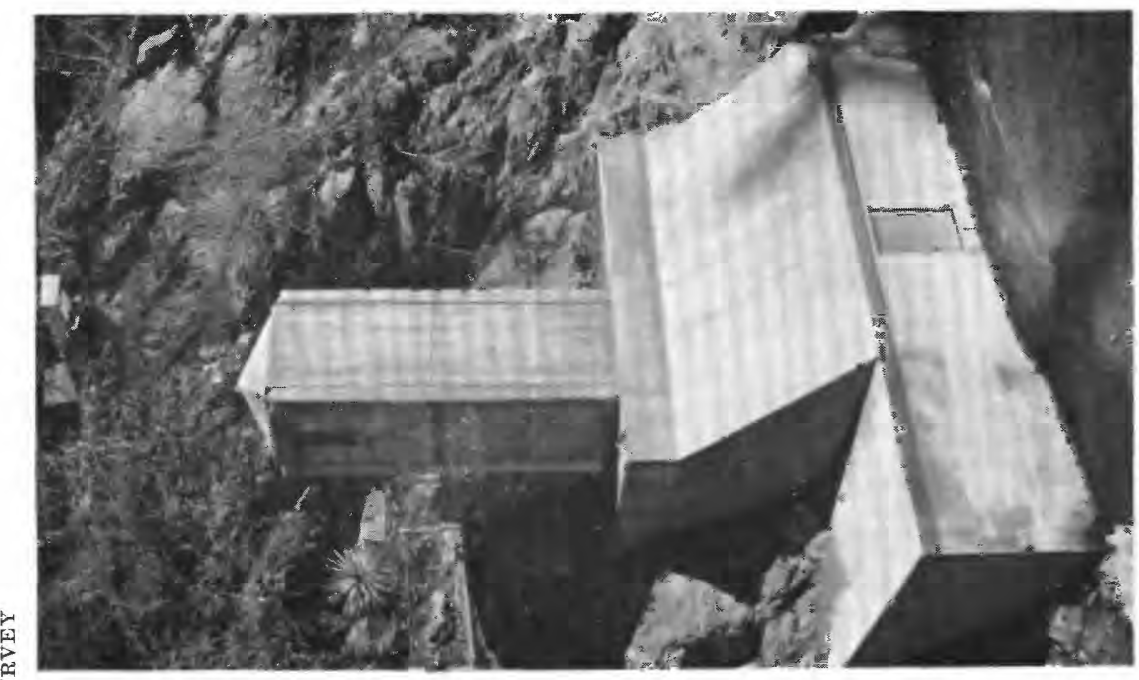

它

ह

의 는

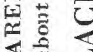

डै है

$\approx$ 드

तथ

$z \div$

뇡

क

¿ ह

ह

o \&

ส

<

藏芯

$-\overbrace{0}^{\circ}$

w 
than 140,000 second-feet. The difficulties at this gaging station included that of the uncertainty as to the cross-sectional area of the chamel, that of the definition of the slope of the water surface at the time of the maximum discharge, and other complications typical of the shifting channels found in the unstable alluvial material of flood plains on the valley floor. The maximum discharge adopted for publication in this report is 100,000 second-feet. Obviously this estimate is subject to considerable error, and wherever it is used appropriate allowance should be made for its possible inaccuracy.

Great effort was made to collect accurate and complete fleld data on practically all the larger streams in the area covered by the flood in order to better determine their maximum discharges. The surveys were made by engineers of the Geological Survey chosen e1ther because they had long been familiar with the streams in this region or because of their long experience in studying floods in other sections of the United states. A large amount of time was spent, both in the field and office, in attempting to coordinate and reconclie results that were discordant because of the extraordinarily unfavorable conditions prevalling along these stream channels. A record of all the information collected, however, and the results of the computations are on f1le at the Los Angeles office of the Survey and are avallable to anyone who may wish to make further study of them.

\section{RECORDS OF STAGE AND DISCHARGE AT STREAM-GAGINC STATIONS}

One of the principal purposes of this report is to record the ava11able information regarding the discharge of streams in southern California during the floods of March 1938 in more deta1l than appears in WaterSupply Papers 860 and 861, the reports on th1s region for 1937-38 pubIlshed by the Geologieal Survey as parts 10 and 11 of 1 ts annual series on surface water supply of the United States. This information is useful In comparative studies of the characteristics of floods and in planning flood protection and control by means of reservolrs, levees, and channel improvements. The records should also be helpful in the planning of future bridge and highway construction as well as in the design and layout of hydraulic and other structures that may have a relation to the channels of the streams or their flood plains.

The records published in this report are for streams on which floods occurred in March 1938 or which are adjacent to the margins of the storm area and therefore serve to define the extent of the floods. In this 
section are presented the avallable records for most of the stream-gaging atations in the flooded region. The records for a few stations have already been presented as bas1c discharge records. The latter were analyzed to bring out details that might lead to a better interpretation of the often meager data avallable at most stations.

\section{Explanation of data}

The data systematically collected at stream-gaging stations consist of records of stage, measurements of discharge, and other technioal information useful in determining the daily flow. The records of stage are generally obtained by water-stage recorders, whlch give a continuous graphlc record of the fluctuations. Measurements of discharge are usually made with a current-meter according to methods outlined in standard textbooks on the measurement of stream discharge. Typical stream-gaging stat1ons, equipped with water-stage recorder and measuring cable and car, are shown in plates 17 and 18.

Because of the unusually destructive character of the floods of March 1938, as already outlined, many of the regular stream-gaging stations were destroyed or so badly damaged that no record of stage was obtained. Furthermore, it was practically impossible during the flood period to obtain current-meter measurements in either the mountain or the valley-floor areas. Other difficulties in the determination of discharge have been discussed in the preceding section and at other places in this report.

In general the data presented for each stream-gaging station comprise a description of the station, a table of dally and monthly discharge throughout the 3-month period February to April 1938, and a table of stage and discharge at hourly or other indicated intervals, if these are avallable, generally from February 27 to March 4. The presentation of the data has followed a uniform plan as far as practicable.

The description of the station contains, in the first paragraph, information relating to the location of the gage and the approximate altitude of the stream channel at the gage. The second paragraph gives certain characteristics of the drainage area. The third paragraph, which relates to the gage-helght record, gives a statement as to the type of record and the period for which a gage-helght record was avallable. The fourth paragraph, ent1tled "Discharge record," contains information concerning the method used to compute the discharge. If a gage-height record was avallable and the stage-discharge relation could be defined, 
Information is given concerning the range to which the rating ourve is defined by ourrent-meter measurements and the method used to extend the rating curve to the peak of the flood. The flfth paragraph, headed "Maxima," Ilsts the discharge, time, day, and gage helght, if avallable, of the peak flow during the March flood. Similar Information 1s Included for the largest flood that occurred during the period of record preceding Karch 1938. The sixth and final paragraph, under the heading "Remarks," furnishes data concerning storage or diversions upstream from the station and other miscellaneous information.

The table following each station description shows the mean daily discharge in second-feet, meen monthly discharge in second-feet, and monthly runoff in acre-feet, generally for the period Fobmary 1 to April 30, 1938. This covers the flood period and sufficient time before and after to show the relation of flood discharges to the prevalent discharges and to give a general perspective of the March floods.

The table of gage helghts and discharges at indicated times was designed to present the detalls of the rise and recession of the flood. It is accompanied by footnotes giving supplemental records of discharge if these are needed to define more accurately the subsidiary peaks or valleys In the hydrograph that would otherwise not be disclosed in the houriy or bihourly listing. For those stations for which there is little fluctuation in the hydrograph, mean values are sometimes used, as indicated by braces showing the period for which the mean is given. The stages at indicated times were obtalned from the water-stage recorders insofar as such records were avallable. The discharges at indicated times were generally obtained from the gage helghts by application of the rating table, usually allowing for correctlons due to shifting-control.

The records are arranged in accordance with the regular plan used by the Geological Survey in its annual series of reports on the surface water supply of the United States. The only exception is that in the annual series, records for the part of the Great Basin that is in California (part 10) are published separately from those for the remainder of cal1fornia (part 11), whereas in this report the Great Basin records follow Immediately after those for the south Pacific slope basing.

Reference should be made to the water-supply papers of the Goological Survey for other records of flow of many of the streams discussed in this report.

The records in this report are based on all the information avaliable at the time of its compllation. Any revisions found necessary will be published in subsequent water-supply papers. 


\section{Tia Juana River Basin}

Cottonwood Creek at Morena Dam, Cal1f.

Iocation. - Iat. $32^{\circ} 41^{\prime} 00^{\prime \prime}$, Iong. $116^{\circ} 32^{\prime} 55^{\prime \prime}$, in SW sec. 14 T. 17 S., R. 4 E., at Morena Dam, 1-3/4 miles above junction with Hauser Creek. Altitude of stream bed, 2,882 feet.

Drainage area.- 120 square miles.

Gage-height record.- Gage read dally at 7 a.m.

Remarks.- Records of discharge computed from discharge over spillway, change in con-

tents, leakage, evaporation, and direct rainfall on surface of reservoir. Altitude of top of splilway gates, 3,045 feet. Storage capacity, 67,211 acre-feet. Records furnished by City of San Diego Water Department.

Gage height, in feet, and contents, in acre-feet, 1938

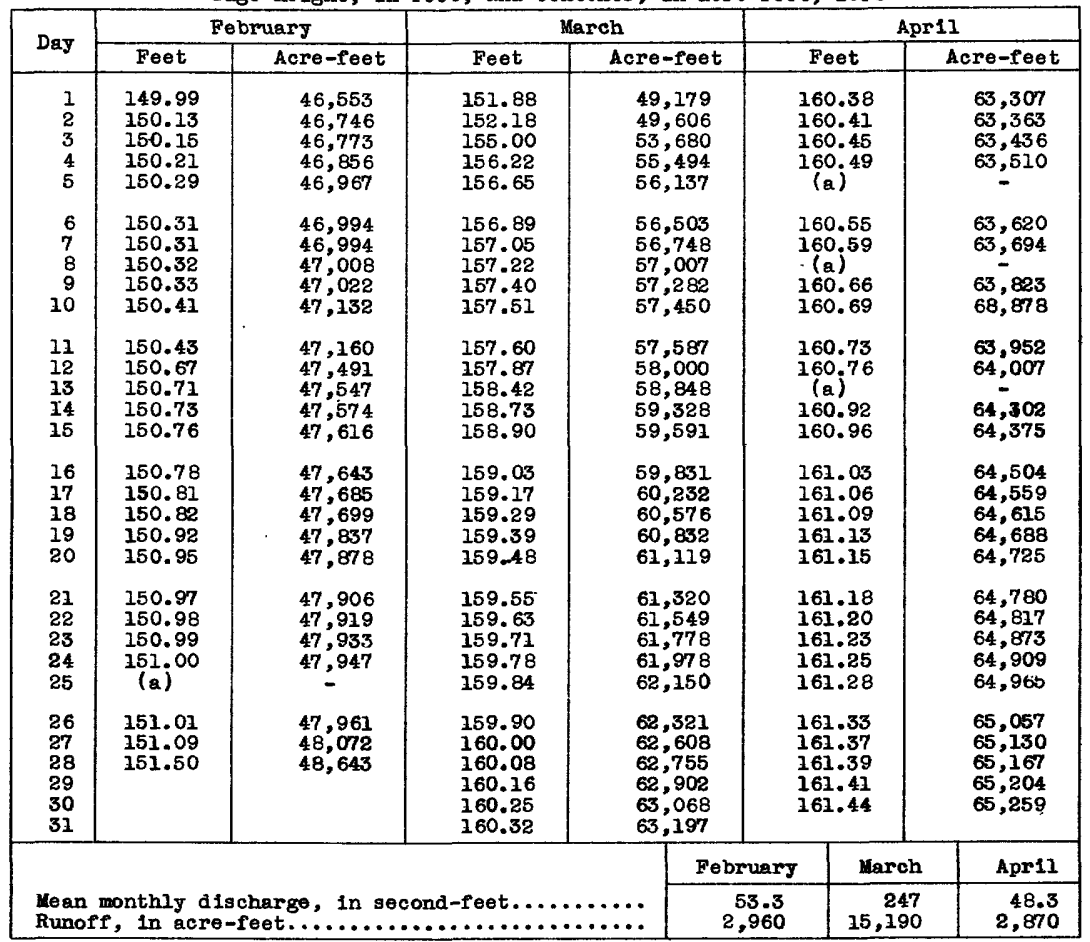

a Unable to read gage owing to heavy wind.

Gottonwood Creek near Dulzura, Calif.

Location.- Iat. $32^{\circ} 40^{1} 45^{n}$, long. $116^{\circ} 40^{\prime} 20^{n}$, in Mth sec. 22, T. 17 S., R. 3 E., at Barrett Dam, about 1 milo aomatream from junetion ith Pine Valley Creek, and about 17 miles northeast of Dulzura. Altitude of gtream bed, 1,446 feet.

Drainage area.- 250 square miles.

Gage-height record.- Gage read daily at 7 a.m.

Remarks.- Records of discharge represent flow into Barrett Resorvoir, computed from discharge over spillway, change in contents, leakage, evaporation, and direct rainfall on surface of reservoir. Altitude of top of epiliway gatee, 1,615 feet. Storage capacity, 42,796 acre-feet. Additional storage obtained by placing sandbags on spillway. Records furnished by City of San Diego. 
Gage height, in feet, and contents, in acre-feet, 1938

\begin{tabular}{|c|c|c|c|c|c|c|c|c|c|}
\hline \multirow{2}{*}{ Day } & \multicolumn{2}{|c|}{ February } & \multicolumn{3}{|c|}{ March } & \multicolumn{4}{|c|}{ Apr 11} \\
\hline & Feet & Acre-feet & Feet & \multicolumn{2}{|c|}{ Acre-feet } & \multicolumn{2}{|c|}{ Feet } & & Acre-feet \\
\hline $\begin{array}{l}1 \\
2 \\
3 \\
4 \\
5\end{array}$ & $\begin{array}{l}160.02 \\
160.14 \\
160.21 \\
160.28 \\
160.35\end{array}$ & $\begin{array}{l}35,501 \\
35,595 \\
35,651 \\
35,706 \\
35,761\end{array}$ & $\begin{array}{l}162.56 \\
163.20 \\
169.20 \\
169.59 \\
169.32\end{array}$ & \multicolumn{2}{|c|}{$\begin{array}{l}37,524 \\
38,043 \\
43,073 \\
43,411 \\
43,177\end{array}$} & \multicolumn{2}{|c|}{$\begin{array}{l}168.93 \\
168.94 \\
168.92 \\
168.91 \\
168.90\end{array}$} & & $\begin{array}{l}42,839 \\
42,848 \\
42,831 \\
42,822 \\
42,814\end{array}$ \\
\hline $\begin{array}{r}6 \\
7 \\
8 \\
9 \\
10\end{array}$ & $\begin{array}{l}160.39 \\
160.44 \\
160.45 \\
160.50 \\
160.59\end{array}$ & $\begin{array}{l}35,793 \\
35,832 \\
35,840 \\
35,879 \\
35,950\end{array}$ & $\begin{array}{l}169.19 \\
169.09 \\
169.05 \\
169.05 \\
169.01\end{array}$ & \multicolumn{2}{|c|}{$\begin{array}{l}43,064 \\
42,977 \\
42,943 \\
42,943 \\
42,908\end{array}$} & \multicolumn{2}{|c|}{$\begin{array}{l}168.93 \\
168.94 \\
168.95 \\
168.91 \\
168.90\end{array}$} & & $\begin{array}{l}42,839 \\
42,848 \\
42,856 \\
42,822 \\
42,814\end{array}$ \\
\hline $\begin{array}{l}11 \\
12 \\
13 \\
14 \\
15\end{array}$ & $\begin{array}{l}160.63 \\
160.81 \\
160.90 \\
161.00 \\
161.03\end{array}$ & $\begin{array}{l}35,982 \\
36,124 \\
36,195 \\
36,274 \\
36,298\end{array}$ & $\begin{array}{l}169.00 \\
169.10 \\
169.38 \\
169.29 \\
169.17\end{array}$ & \multicolumn{2}{|c|}{$\begin{array}{l}42,899 \\
42,986 \\
43,229 \\
43,151 \\
43,047\end{array}$} & \multicolumn{2}{|c|}{$\begin{array}{l}168.93 \\
168.94 \\
168.98 \\
169.10 \\
169.19\end{array}$} & & $\begin{array}{l}42,839 \\
42,848 \\
42,882 \\
42,986 \\
43,064\end{array}$ \\
\hline $\begin{array}{l}16 \\
17 \\
18 \\
19 \\
20\end{array}$ & $\begin{array}{l}161.08 \\
161.11 \\
161.14 \\
161.25 \\
161.30\end{array}$ & $\begin{array}{l}36,338 \\
36,362 \\
36,386 \\
36,473 \\
36,513\end{array}$ & $\begin{array}{l}169.10 \\
169.07 \\
169.05 \\
169.01 \\
169.00\end{array}$ & \multicolumn{2}{|c|}{$\begin{array}{l}42,986 \\
42,960 \\
42,943 \\
42,908 \\
42,899\end{array}$} & \multicolumn{2}{|c|}{$\begin{array}{l}169.25 \\
169.31 \\
169.38 \\
169.45 \\
169.50\end{array}$} & & $\begin{array}{r}43,116 \\
43,168 \\
43,229 \\
43,289 \\
43,333\end{array}$ \\
\hline $\begin{array}{l}21 \\
22 \\
23 \\
24 \\
25\end{array}$ & $\begin{array}{l}161 \cdot 31 \\
161 \cdot 34 \\
161 \cdot 37 \\
161 \cdot 39 \\
161 \cdot 41\end{array}$ & $\begin{array}{l}36,521 \\
36,545 \\
36,569 \\
36,585 \\
36,601\end{array}$ & $\begin{array}{l}168.99 \\
168.98 \\
168.96 \\
168.94 \\
168.93\end{array}$ & \multicolumn{2}{|c|}{$\begin{array}{l}42,891 \\
42,882 \\
42,865 \\
42,848 \\
42,839\end{array}$} & \multicolumn{2}{|c|}{$\begin{array}{l}169.55 \\
169.58 \\
169.60 \\
169.61 \\
169.66\end{array}$} & & $\begin{array}{l}43,376 \\
43,402 \\
43,419 \\
43,428 \\
43,471\end{array}$ \\
\hline $\begin{array}{l}26 \\
27 \\
28 \\
29 \\
30 \\
31\end{array}$ & $\begin{array}{l}161.43 \\
161.50 \\
162.00\end{array}$ & $\begin{array}{l}36,617 \\
36,673 \\
37,072\end{array}$ & $\begin{array}{l}168.95 \\
168.97 \\
168.94 \\
169.95 \\
168.94 \\
168.95\end{array}$ & \multicolumn{2}{|c|}{$\begin{array}{l}42,856 \\
42,874 \\
42,848 \\
42,856 \\
42,848 \\
42,856\end{array}$} & \multicolumn{2}{|c|}{$\begin{array}{l}169.70 \\
169.73 \\
169.75 \\
169.78 \\
169.79\end{array}$} & & $\begin{array}{l}43,506 \\
43,532 \\
43,549 \\
43,575 \\
43,584\end{array}$ \\
\hline \multicolumn{7}{|c|}{ Fobruary } & \multicolumn{2}{|c|}{ March } & Aprel \\
\hline $\begin{array}{l}\text { Mean } \\
\text { Runoff }\end{array}$ & 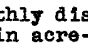 & in & & & \multicolumn{2}{|c|}{$\begin{array}{r}37.1 \\
2,060\end{array}$} & \multicolumn{2}{|c|}{$\begin{array}{r}212 \\
13,040\end{array}$} & $\begin{array}{r}93.4 \\
5,560\end{array}$ \\
\hline
\end{tabular}

Cottonwood Creek above Tecate Creek, near Dulzura, Calif.

Location.- Water-stage recorder, lat. $32^{\circ} 34^{\prime} 10^{\prime \prime}$, long. $116^{\circ} 45^{\prime} 40^{n}$, in sec. 27, T. 18 S., k. 2 k., half a mile upstream from confluence with Tecate Creek and $5 \frac{1}{5} \mathrm{mlles} s o u$ th of Dulzura. Altitude, about 580 feet.

Drainage area. - 316 square miles.

Gage-height record.- Water-s tage recorder graph.

Discharge record.- Stage-discharge relation defined by current-meter measurements beIow 540 second-feet; extended to peak stage on the basis of mean depth-mean velocity relation.

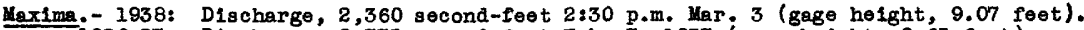
1936-37: D1scharge, 2,775 second-feet Feb. 7, 1937 (gage height, 9.65 feet).

Remarks.- Records good. Regulation at Barrett and Morens Reservolrs, 10 and 18 miles, respectively, above station.

Mean daily discharge, in second-feet, 1938

\begin{tabular}{|c|c|c|c|c|c|c|c|c|c|c|c|}
\hline Day & Feb. & Mar. & Apr. & Day & Feb. & Mer. & Apr. & Day & Feb. & Mar. & Apr. \\
\hline $\begin{array}{r}1 \\
2 \\
3 \\
4 \\
5 \\
6 \\
7 \\
8 \\
9 \\
10\end{array}$ & $\begin{array}{l}3.5 \\
5 \\
2.5 \\
3.9 \\
3.5 \\
2.3 \\
2.1 \\
1.9 \\
2.5 \\
5\end{array}$ & $\begin{array}{r}120 \\
281 \\
1,460 \\
1,040 \\
631 \\
427 \\
326 \\
298 \\
283 \\
238\end{array}$ & $\begin{array}{l}92 \\
82 \\
78 \\
79 \\
75 \\
67 \\
63 \\
59 \\
51 \\
42\end{array}$ & $\begin{array}{l}11 \\
12 \\
13 \\
14 \\
15 \\
16 \\
17 \\
18 \\
19 \\
20\end{array}$ & $\begin{array}{l}6 \\
20 \\
10 \\
7 \\
6 \\
5.5 \\
4.8 \\
3.9 \\
6 \\
5\end{array}$ & $\begin{array}{l}219 \\
497 \\
606 \\
517 \\
397 \\
328 \\
292 \\
259 \\
219 \\
192\end{array}$ & $\begin{array}{l}41 \\
37 \\
41 \\
37 \\
25 \\
19 \\
13 \\
10 . \\
8 \\
7.5\end{array}$ & $\begin{array}{l}21 \\
22 \\
23 \\
24 \\
25 \\
26 \\
27 \\
28 \\
29 \\
30 \\
31\end{array}$ & $\begin{array}{r}3.5 \\
3.2 \\
3.0 \\
2.8 \\
2.5 \\
2.3 \\
22 \\
47\end{array}$ & $\begin{array}{r}167 \\
144 \\
135 \\
128 \\
121 \\
121 \\
121 \\
116 \\
114 \\
106 \\
96 \\
\end{array}$ & $\begin{array}{l}7.5 \\
6.5 \\
6.5 \\
6 \\
7 \\
7.5 \\
6 \\
5 \\
4.2 \\
6\end{array}$ \\
\hline \multicolumn{12}{|c|}{ 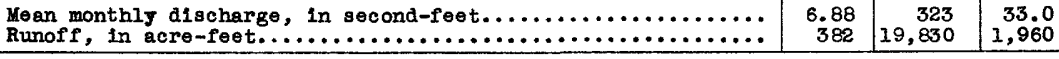 } \\
\hline
\end{tabular}


Gage holght, in foot, and difcharge, in socond-foet, at indioated time, 1938

\begin{tabular}{|c|c|c|c|c|c|c|c|c|c|c|c|c|}
\hline \multirow{2}{*}{\begin{tabular}{|l|}
\multicolumn{6}{|c}{} \\
\\
\end{tabular}} & Foot & Soo.ft. & Foot & Soo.ft. & eot & seo.ft. & Feet & seo.ft. & Feet & seo.ft. & Poot & 8eo.ft. \\
\hline & \multicolumn{2}{|c|}{ Fobmuary 27} & \multicolumn{2}{|c|}{ Fobmary 28} & \multicolumn{2}{|c|}{ March 1} & \multicolumn{2}{|c|}{ Maroh 2} & \multicolumn{2}{|c|}{ Maroh 3} & \multicolumn{2}{|c|}{ Maroh 4} \\
\hline $\begin{array}{l}1 \\
2 \\
3 \\
4 \\
5 \\
6\end{array}$ & 2.18 & 7.8 & $\begin{array}{l}3.20 \\
3.08 \\
2.96 \\
2.87 \\
2.80 \\
2.74\end{array}$ & $\begin{array}{r}101 \\
85 \\
71 \\
61 \\
54 \\
48\end{array}$ & $\begin{array}{l}2.78 \\
2.80 \\
2.85 \\
2.93 \\
3.35 \\
3.35\end{array}$ & $\begin{array}{r}58 \\
54 \\
58 \\
67 \\
184 \\
124\end{array}$ & $\begin{array}{l}2.98 \\
2.98 \\
2.99 \\
3.08 \\
3.04 \\
3.07\end{array}$ & $\begin{array}{l}73 \\
73 \\
74 \\
79 \\
80 \\
84\end{array}$ & $\begin{array}{l}8.00 \\
8.18 \\
7.75 \\
7.20 \\
6.70 \\
6.35\end{array}$ & $\begin{array}{l}1,800 \\
1,900 \\
1,700 \\
1,480 \\
1,170 \\
1,000\end{array}$ & $\begin{array}{l}6.65 \\
6.60 \\
6.60 \\
6.60 \\
6.60 \\
6.60\end{array}$ & $\begin{array}{l}1,150 \\
1,130 \\
1,130 \\
1,130 \\
1,130 \\
1,130\end{array}$ \\
\hline \begin{tabular}{r|}
7 \\
8 \\
9 \\
10 \\
11 \\
8
\end{tabular} & & & $\begin{array}{l}2.69 \\
2.65 \\
2.68 \\
2.69 \\
2.57 \\
2.57\end{array}$ & $\begin{array}{l}43 \\
40 \\
37 \\
35 \\
34 \\
34\end{array}$ & $\begin{array}{l}3.38 \\
3.43 \\
3.60 \\
3.73 \\
3.68 \\
3.67\end{array}$ & $\begin{array}{l}128 \\
136 \\
165 \\
180 \\
180 \\
178\end{array}$ & $\begin{array}{l}3.09 \\
3.13 \\
3.30 \\
3.42 \\
3.48 \\
3.54\end{array}$ & $\begin{array}{r}86 \\
92 \\
116 \\
135 \\
144 \\
154\end{array}$ & $\begin{array}{l}10 \\
.20 \\
18 \\
10 \\
10 \\
.85 \\
.40\end{array}$ & $\begin{array}{r}890 \\
936 \\
928 \\
890 \\
956 \\
1,820\end{array}$ & $\begin{array}{l}6.60 \\
6.60 \\
6.60 \\
6.60 \\
6.60 \\
6.55\end{array}$ & $\begin{array}{l}1,130 \\
1,130 \\
1,130 \\
1,130 \\
1,130 \\
1,100\end{array}$ \\
\hline $\begin{array}{l}1 \\
8 \\
3 \\
4 \\
5 \\
6\end{array}$ & 2.28 & 15 & $\begin{array}{l}2.57 \\
2.56 \\
2.57 \\
2.60 \\
2.68 \\
2.62\end{array}$ & $\begin{array}{l}34 \\
33 \\
34 \\
36 \\
37 \\
37\end{array}$ & $\begin{array}{l}3.74 \\
3.67 \\
3.67 \\
3.48 \\
3.38 \\
3.30\end{array}$ & $\begin{array}{l}192 \\
178 \\
160 \\
144 \\
128 \\
116\end{array}$ & $\begin{array}{l}3.60 \\
3.75 \\
4.00 \\
4.17 \\
4.26 \\
4.87\end{array}$ & $\begin{array}{l}185 \\
184 \\
247 \\
286^{\circ} \\
307 \\
310\end{array}$ & $\begin{array}{l}7.60 \\
8.80 \\
9.00 \\
8.70 \\
8.45 \\
7.85\end{array}$ & $\begin{array}{l}1, \\
2, \\
2, \\
2, \\
2, \\
1,\end{array}$ & $\begin{array}{l}6.50 \\
6.45 \\
6.40 \\
6.30 \\
6.25 \\
6.20\end{array}$ & $\begin{array}{r}1,080 \\
1,060 \\
1,080 \\
980 \\
956 \\
986\end{array}$ \\
\hline $\begin{array}{r}7 \\
8 \\
9 \\
10 \\
1\end{array}$ & 2.50 & $\begin{array}{l}44 \\
92\end{array}$ & $\begin{array}{l}2.60 \\
2.59 \\
2.59 \\
2.62 \\
2.69 \\
2.73\end{array}$ & $\begin{array}{l}36 \\
38 \\
35 \\
37 \\
43 \\
47\end{array}$ & $\begin{array}{l}3.23 \\
3.18 \\
3.12 \\
3.07 \\
3.02\end{array}$ & $\begin{array}{r}106 \\
93 \\
90 \\
84 \\
78 \\
73\end{array}$ & $\begin{array}{l}4.30 \\
4.60 \\
5.00 \\
6.10 \\
6.50 \\
7.75\end{array}$ & $\begin{array}{r}318 \\
403 \\
524 \\
865 \\
1,160 \\
1,880\end{array}$ & $\begin{array}{l}7.15 \\
7.00 \\
6.85 \\
6.85 \\
6.75 \\
6.70\end{array}$ & $\begin{array}{l}1,400 \\
1,320 \\
1,300 \\
1,240 \\
1,200 \\
1170\end{array}$ & $\begin{array}{l}6.16 \\
6.10 \\
6.05 \\
6.00 \\
5.95 \\
8.90\end{array}$ & $\begin{array}{l}886 \\
-806 \\
\end{array}$ \\
\hline
\end{tabular}

Supplementel record. - Mar. 3, 8ะ30 o.m., 8.07 foet., 2,360 000.-ft.; Mar. 5, 6 a.m., $6.66 \mathrm{ft} ., 710000 .-\mathrm{ft} . ; 6 \mathrm{p.m.}, 5.24 \mathrm{ft} ., 562000 .-\mathrm{ft}$.

T1. Juana R1ver near Dulzusa, Calle.

Locat1on. - Water-atage rocorder, lat. $32^{\circ} 33^{\prime} 60^{\prime \prime}$, long. $116^{\circ} 46^{\prime} 28^{n}$, in 800.33 , T. 18 S., R, $2 \mathrm{~g} .$, half a milo downstream from confluence of Cottonwood and Tecate Creeke, and 6 i miles south of Dulzusa. Altitude, about 650 foet.

Drainege area.- 478 oquare m1les, of wh10h 62 square miles it in Mex100.

Gage-he1zht rooord. - Water-stage recorder graph.

D1eoharge reoord. - Stuge-d1soharge relation defined by ourrent-meter moaturemente below 659 eoond-ioet; extended to peak stage on bue1s of mean depth-mean veloo1ty relation.

Haxima.- 1938: D1eoherge, 3,520 seo ond-feet 11 a.m. Har. 3 (gage he1eht, 6.77 feet). 1936-37: D10oharge, 4,700 second-feet Fob. 7, 1037, (gage ho1ght, 6.60 feet).

Remarks.- Recorkis good. Flow oubjeot to rogulation by storage in Worene und Barrett ireervo1re, operuted by elty of San Diego.

Mean de11s diecharge, in second-feet, 1938

\begin{tabular}{|c|c|c|c|c|c|c|c|c|c|c|c|}
\hline Day & Pob. & Mar. & Apr. & Day & Fob. & Mar. & Apr. & Dey & Beb. & Mer. & Apr. \\
\hline $\begin{array}{r}1 \\
2 \\
3 \\
4 \\
6 \\
6 \\
7 \\
8 \\
9 \\
10\end{array}$ & $\begin{array}{l}9 \\
13 \\
10 \\
11 \\
14 \\
11 \\
8.6 \\
7 \\
7.6\end{array}$ & $\begin{array}{r}283 \\
510 \\
2,650 \\
1,180 \\
800 \\
580 \\
428 \\
384 \\
367 \\
314\end{array}$ & $\begin{array}{l}123 \\
113 \\
109 \\
107 \\
104 \\
96 \\
88 \\
80 \\
69 \\
69\end{array}$ & $\begin{array}{l}11 \\
12 \\
15 \\
14 \\
15 \\
16 \\
17 \\
18 \\
18 \\
20\end{array}$ & $\begin{array}{l}13 \\
67 \\
39 \\
28 \\
21 \\
18 \\
16 \\
14 \\
16 \\
16\end{array}$ & $\begin{array}{l}286 \\
682 \\
738 \\
658 \\
499 \\
406 \\
354 \\
314 \\
277 \\
247\end{array}$ & $\begin{array}{l}59 \\
58 \\
65 \\
68 \\
50 \\
42 \\
37 \\
31 \\
27 \\
24\end{array}$ & $\begin{array}{l}21 \\
22 \\
25 \\
24 \\
28 \\
26 \\
27 \\
28 \\
29 \\
30 \\
31\end{array}$ & $\begin{array}{c}11 \\
10 \\
8.5 \\
8.6 \\
6.5 \\
5.5 \\
40 \\
124\end{array}$ & $\begin{array}{l}287 \\
205 \\
100 \\
179 \\
164 \\
164 \\
167 \\
186 \\
184 \\
146 \\
131\end{array}$ & $\begin{array}{l}23 \\
22 \\
22 \\
21 \\
23 \\
26 \\
28\end{array}$ \\
\hline \multicolumn{12}{|c|}{ 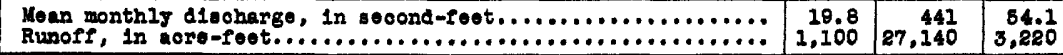 } \\
\hline
\end{tabular}


Gage height, in feet, and diseharge, in second-feet at indiested time, 1938

\begin{tabular}{|c|c|c|c|c|c|c|c|c|c|c|c|c|}
\hline \multirow{2}{*}{ 量 } & Poet & Soc.ft. & Foet & Soc.ft. & Poet & Sec.ft. & Fost & sec.ft. & Foet & Sec.ft. & Foot & Sec.ft. \\
\hline & \multicolumn{2}{|c|}{ Febmuary 27} & \multicolumn{2}{|c|}{ Febmuary 28} & \multicolumn{2}{|c|}{ March 1} & \multicolumn{2}{|c|}{ March 2} & \multicolumn{2}{|c|}{ March 3} & \multicolumn{2}{|c|}{ March 4} \\
\hline $\begin{array}{l}1 \\
2 \\
3 \\
4 \\
5 \\
6\end{array}$ & 1.56 & $>14$ & $\begin{array}{l}2.18 \\
2.12\end{array}$ & 141 & $\begin{array}{l}2.07 \\
2.10 \\
2.17 \\
2.19 \\
2.29 \\
2.32\end{array}$ & $\begin{array}{l}124 \\
134 \\
160 \\
168 \\
212 \\
226\end{array}$ & $\begin{array}{l}2.33 \\
2.34 \\
2.33 \\
2.34 \\
2.36 \\
2.36\end{array}$ & $\begin{array}{l}230 \\
235 \\
230 \\
235 \\
247 \\
247\end{array}$ & $\begin{array}{l}4.80 \\
4.97 \\
5.32 \\
5.55 \\
5.45 \\
5.40\end{array}$ & $\begin{array}{l}2,230 \\
2,440 \\
2,800 \\
3,120 \\
3,020 \\
2,980\end{array}$ & & \\
\hline $\begin{array}{r}7 \\
8 \\
9 \\
10 \\
11 \\
11\end{array}$ & 1.73 & 34 & 2.05 & 98 & $\begin{array}{l}2.40 \\
2.42 \\
2.47 \\
2.57 \\
2.66 \\
2.64\end{array}$ & $\begin{array}{l}271 \\
283 \\
314 \\
381 \\
446 \\
430\end{array}$ & $\begin{array}{l}2.37 \\
2.41 \\
2.45 \\
2.50 \\
2.57 \\
2.55\end{array}$ & $\begin{array}{l}253 \\
277 \\
302 \\
334 \\
381 \\
367\end{array}$ & $\begin{array}{l}5.46 \\
5.53 \\
5.50 \\
5.65 \\
5.77 \\
5.68\end{array}$ & $\begin{array}{l}3,080 \\
3,180 \\
3,140 \\
3,350 \\
3,520 \\
3,390\end{array}$ & & \\
\hline $\begin{array}{l}1 \\
2 \\
3 \\
4 \\
5 \\
6\end{array}$ & 1.81 & 50 & 2.05 & 98 & $\begin{array}{l}2.64 \\
2.63 \\
2.61 \\
2.58 \\
2.55 \\
2.49\end{array}$ & $\begin{array}{l}430 \\
423 \\
409 \\
388 \\
367 \\
328\end{array}$ & $\begin{array}{l}2.56 \\
2.60 \\
2.73 \\
2.80 \\
2.87 \\
2.91\end{array}$ & $\begin{array}{l}374 \\
402 \\
502 \\
564 \\
627 \\
663\end{array}$ & $\begin{array}{l}5.27 \\
5.32 \\
4.85 \\
4.90 \\
5.15 \\
4.83\end{array}$ & $\begin{array}{l}2,830 \\
2,900 \\
2,400 \\
2,460 \\
2,680 \\
2,380\end{array}$ & & \\
\hline $\begin{array}{r}7 \\
8 \\
9 \\
10 \\
11 \\
\end{array}$ & 1.93 & 80 & $\begin{array}{l}2.02 \\
2.07\end{array}$ & $\begin{array}{r}88 \\
104 \\
\end{array}$ & $\begin{array}{l}2.46 \\
2.43 \\
2.39 \\
2.37 \\
2.36 \\
2.34\end{array}$ & $\begin{array}{l}308 \\
289 \\
265 \\
253 \\
247 \\
235\end{array}$ & $\begin{array}{l}2.93 \\
3.03 \\
3.27 \\
3.68 \\
3.88 \\
4.20\end{array}$ & $\begin{array}{r}681 \\
780 \\
890 \\
1,170 \\
1,330 \\
1,620\end{array}$ & $\begin{array}{l}4.62 \\
4.50 \\
4.37 \\
4.33 \\
4.38 \\
4.32\end{array}$ & $\begin{array}{l}2,160 \\
2,040 \\
1,900 \\
1,860 \\
1,910 \\
1,850\end{array}$ & & \\
\hline
\end{tabular}

Supplemental records.- Mar. 3, 2:30 p.m., 2,550. sec.-ft.; 4:15 p.m., 2,540 sec.-ft.; $5: 45$ p.m., 2,540 sec. -ft.

T1a Juana River near Nestor, Calif.

Location. - Water-stage recorder, lat. $32^{\circ} 32^{\prime} 55^{\prime \prime}$, long. $117^{\circ} 05^{\prime} 15^{\prime \prime}$, on line between secs. 3 and 4, T. 19 S., R. 2 W., it miles south of Nestor, and 3 miles upetream from mouth. Alt1tude, 25 feot.

Drainage area.- 1,668 square miles, of which 1,198 square miles is in Mexico.

Gage-he1ght record. - Water-stage recorder graph.

Discharge record.- Stage-discharge relation defined by current-meter measurements below 4,100 second-feet; extended to peak stage on basis of mean depth-mean velocity relation.

Maxima.- 1938: Discharge, 6,760 second-feet 8 a.m. Mar. 3 (gage he1ght, 6.38 feet). 1936-37: Discharge, 17,700 second-feet Fob. 7, 1937 (gage helght, 8.20 feet).

Remarks. - Records good. Flow subject to regulation by storage in Morena and Barrett keservoirs, operated by city of San Diego, and Rodriguez Reservolr, operated by Government of Mexico.

Mean daily discharge, in second-feet, 1938

\begin{tabular}{|c|c|c|c|c|c|c|c|c|c|c|c|}
\hline Day & Feb. & Mar. & Apr. & Day & Feb. & Mar. & Apr. & Day & Feb. & Mar. & Apr. \\
\hline $\begin{array}{r}1 \\
2 \\
3 \\
4 \\
5 \\
6 \\
7 \\
8 \\
9 \\
10\end{array}$ & $\begin{array}{l}0 \\
0 \\
0 \\
0 \\
0 \\
0 \\
0 \\
0 \\
0 \\
0\end{array}$ & $\begin{array}{r}221 \\
439 \\
4,950 \\
3,010 \\
1,640 \\
1,090 \\
680 \\
618 \\
601 \\
412\end{array}$ & $\begin{array}{l}144 \\
130 \\
122 \\
119 \\
116 \\
110 \\
100 \\
88 \\
76 \\
63\end{array}$ & $\begin{array}{l}11 \\
12 \\
13 \\
14 \\
15 \\
16 \\
17 \\
18 \\
19 \\
20\end{array}$ & $\begin{array}{l}0 \\
0 \\
0 \\
0 \\
3.3 \\
3.5 \\
3.7 \\
3.9 \\
3.9 \\
4.4\end{array}$ & $\begin{array}{r}322 \\
816 \\
1,540 \\
1,100 \\
670 \\
496 \\
398 \\
358 \\
322 \\
294\end{array}$ & $\begin{array}{l}59 \\
57 \\
61 \\
67 \\
63 \\
49 \\
42 \\
36 \\
31 \\
28\end{array}$ & $\begin{array}{l}21 \\
22 \\
23 \\
24 \\
25 \\
26 \\
27 \\
28 \\
29 \\
30 \\
31\end{array}$ & $\begin{array}{l}5 \\
4.4 \\
3.5 \\
2.8 \\
1.7 \\
0 \\
14 \\
42\end{array}$ & $\begin{array}{l}272 \\
240 \\
220 \\
205 \\
193 \\
185 \\
189 \\
189 \\
181 \\
169 \\
158\end{array}$ & $\begin{array}{l}26 \\
20 \\
19 \\
18 \\
18 \\
18 \\
20 \\
20 \\
18 \\
17\end{array}$ \\
\hline \multicolumn{9}{|c|}{ 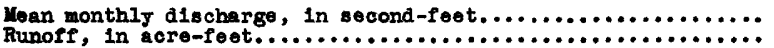 } & $\begin{array}{r}3.43 \\
191\end{array}$ & $\begin{array}{r}715 \\
43,990\end{array}$ & $\begin{array}{r}58.5 \\
3,480 \\
\end{array}$ \\
\hline
\end{tabular}


Gage height, in feet, and discharge, in second-feet, at indicated time, 1938

\begin{tabular}{|c|c|c|c|c|c|c|c|c|}
\hline \multirow{2}{*}{ Hour } & Feet & sec.ft. & Feet & Sec.ft. & Feet & Sec.ft. & Feet & Sec.ft. \\
\hline & \multicolumn{2}{|c|}{ March 1} & \multicolumn{2}{|c|}{ March 2} & \multicolumn{2}{|c|}{ March 3} & \multicolumn{2}{|c|}{ Harch 4} \\
\hline $\begin{array}{l}1 \\
2 \\
3 \\
4 \\
5 \\
6\end{array}$ & $\begin{array}{l}2.93 \\
2.93 \\
2.99 \\
3.01 \\
3.02 \\
3.04\end{array}$ & $\begin{array}{r}85 \\
85 \\
100 \\
105 \\
108 \\
113\end{array}$ & $\begin{array}{l}3.63 \\
3.62 \\
3.61 \\
3.59 \\
3.56 \\
3.54\end{array}$ & $\begin{array}{l}405 \\
398 \\
391 \\
377 \\
358 \\
334\end{array}$ & $\begin{array}{l}5.15 \\
5.37 \\
5.63 \\
5.95 \\
6.05 \\
6.15\end{array}$ & $\begin{array}{r}2,880 \\
3,470 \\
4,200 \\
5,220 \\
5,560 \\
5,920\end{array}$ & & 4,030 \\
\hline $\begin{array}{r}7 \\
8 \\
9 \\
10 \\
11 \\
11\end{array}$ & $\begin{array}{l}3.06 \\
3.08 \\
3.10 \\
3.13 \\
3.16 \\
3.17\end{array}$ & $\begin{array}{l}122 \\
127 \\
134 \\
144 \\
154 \\
158\end{array}$ & $\begin{array}{l}3.50 \\
3.47 \\
3.46 \\
3.43 \\
3.41 \\
3.40\end{array}$ & $\begin{array}{l}328 \\
310 \\
304 \\
288 \\
277 \\
272\end{array}$ & $\begin{array}{l}6.25 \\
6.38 \\
6.30 \\
6.10 \\
5.95 \\
5.90\end{array}$ & $\begin{array}{l}6,300 \\
6,760 \\
6,480 \\
5,810 \\
5,280 \\
5,110\end{array}$ & & 2,890 \\
\hline $\begin{array}{l}1 \\
2 \\
3 \\
4 \\
5 \\
6\end{array}$ & $\begin{array}{l}3.22 \\
3.34 \\
3.45 \\
3.52 \\
3.56 \\
3.58\end{array}$ & $\begin{array}{l}177 \\
230 \\
288 \\
328 \\
352 \\
364\end{array}$ & $\begin{array}{l}3.41 \\
3.42 \\
3.44 \\
3.48 \\
3.53 \\
3.65\end{array}$ & $\begin{array}{l}277 \\
282 \\
294 \\
316 \\
346 \\
426\end{array}$ & $\begin{array}{l}5.95 \\
5.90 \\
5.67 \\
5.50 \\
5.30 \\
5.35\end{array}$ & $\begin{array}{l}5,280 \\
5,110 \\
4,370 \\
3,890 \\
3,330 \\
3,470\end{array}$ & & 3,064 \\
\hline $\begin{array}{r}7 \\
8 \\
9 \\
10 \\
11 \\
M\end{array}$ & $\begin{array}{l}3.58 \\
3.57 \\
3.58 \\
3.61 \\
3.63 \\
3.63\end{array}$ & $\begin{array}{l}370 \\
364 \\
370 \\
391 \\
405 \\
405\end{array}$ & $\begin{array}{l}3.75 \\
3.83 \\
3.90 \\
4.00 \\
4.25 \\
4.55\end{array}$ & $\begin{array}{r}512 \\
583 \\
650 \\
754 \\
1,080 \\
1,560\end{array}$ & $\begin{array}{l}5.55 \\
5.90 \\
6.20 \\
6.17 \\
6.05 \\
5.97\end{array}$ & $\begin{array}{l}4,030 \\
5,110 \\
6,160 \\
6,060 \\
5,640 \\
5,360\end{array}$ & & $\begin{array}{l}2,790 \\
2,420\end{array}$ \\
\hline
\end{tabular}

Campc Creek near Campo, Calif.

Location. - Water-stage recorder, lat. $32^{\circ} 35^{\prime} 20^{\prime \prime}$, long. $116^{\circ} 31^{\prime} 35^{\prime \prime}$, in sec. 24, T. 18 S., R. 4 E., at state highway about $3 \frac{1}{2}$ miles southwest of Campo. Altitude, about 2,180 feet.

Drainage area.- 84 square miles, of which 4 square miles are in Mexicc.

Gage-height record. - Water-stage recorder graph except for pericd 3 p.m. Mar. 4 to $12 \mathrm{~m}$. Mar. 8, gage heights for which were estimated.

Discharge record. - Stage-discharge relation defined by current-meter measurements below 42 second-feet; extended to peak stage on basis of mean depth-mean velocity relation.

Maxima-- 1938: Discharge, 584 sscond-feet 1 a.m. Mar. 3 (gage height, 2.73 feet). 1936-37: Discharge, 1,470 second-feet Feb. 6, ig37 (gage he1ght, 3.80 feet).

Remarks.- Records gocd.

Mean daily discharge, in second-feet, 1938

\begin{tabular}{|c|c|c|c|c|c|c|c|c|c|c|c|}
\hline Day & Feb. & Mar. & Apr. & Day & Feb. & Mar. & Apr. & Day & Feb. & Mar. & Apr. \\
\hline $\begin{array}{r}1 \\
2 \\
3 \\
4 \\
5 \\
6 \\
7 \\
8 \\
9 \\
10\end{array}$ & $\begin{array}{c}11 \\
13 \\
7.5 \\
9 \\
10 \\
6.5 \\
5.5 \\
4.8 \\
6.5 \\
9.5\end{array}$ & $\begin{array}{r}42 \\
89 \\
315 \\
161 \\
41 \\
41 \\
45 \\
41 \\
32 \\
26\end{array}$ & $\begin{array}{l}14 \\
12 \\
12 \\
12 \\
12 \\
11 \\
10 \\
9.5 \\
9.5 \\
9.5\end{array}$ & $\begin{array}{l}11 \\
12 \\
13 \\
14 \\
15 \\
16 \\
17 \\
18 \\
19 \\
20\end{array}$ & $\begin{array}{c}13 \\
24 \\
15 \\
11 \\
9.5 \\
10 \\
9 \\
8 \\
15 \\
11\end{array}$ & $\begin{array}{l}26 \\
60 \\
39 \\
31 \\
87 \\
26 \\
26 \\
24 \\
21 \\
21\end{array}$ & $\begin{array}{l}9.5 \\
10 \\
17 \\
18 \\
14 \\
11 \\
9.5 \\
8.5 \\
9 \\
8.5\end{array}$ & $\begin{array}{l}21 \\
22 \\
23 \\
24 \\
25 \\
26 \\
27 \\
28 \\
29 \\
30 \\
31 \\
\end{array}$ & $\begin{array}{l}8.5 \\
7 \\
7 \\
6.5 \\
6 \\
6 \\
34 \\
34\end{array}$ & $\begin{array}{l}18 \\
18 \\
17 \\
17 \\
16 \\
19 \\
23 \\
18 \\
21 \\
18 \\
15 \\
\end{array}$ & $\begin{array}{c}7 \\
9 \\
10 \\
7.5 \\
9 \\
10 \\
9.5 \\
8.5 \\
8.5 \\
11\end{array}$ \\
\hline \multicolumn{9}{|c|}{ 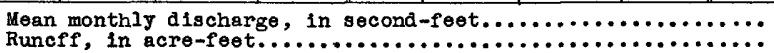 } & $\begin{array}{r}11.4 \\
630\end{array}$ & 2,650 & $\begin{array}{r}10.6 \\
628\end{array}$ \\
\hline
\end{tabular}


Gage neight, in feet, and discharge, in second-feet, at indicated time, 1938

\begin{tabular}{|c|c|c|c|c|c|c|c|c|c|c|c|c|}
\hline \multirow{2}{*}{ 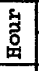 } & Feet & Sec.ft. & Foet & Sec.ft. & Feet & sec.ft. & Feet & Sec.ft. & Feet & Sec.ft. & Feet & Sec.ft. \\
\hline & \multicolumn{2}{|c|}{ February 27} & \multicolumn{2}{|c|}{ February 2s } & \multicolumn{2}{|c|}{ March 1} & \multicolumn{2}{|c|}{ March 2} & \multicolumn{2}{|c|}{ March 3} & \multicolumn{2}{|c|}{ March 4} \\
\hline $\begin{array}{l}1 \\
2 \\
3 \\
4 \\
5 \\
6\end{array}$ & $\begin{array}{r}0.75 \\
.78 \\
.79 \\
.83 \\
.86 \\
.90\end{array}$ & $\begin{array}{l}9.1 \\
10.9 \\
11.4 \\
14 \\
16 \\
19\end{array}$ & $\begin{array}{l}1.13 \\
1.12 \\
1.12 \\
1.11 \\
1.10 \\
1.08\end{array}$ & $\begin{array}{l}41 \\
40 \\
40 \\
39 \\
38 \\
36\end{array}$ & $\begin{array}{l}1.06 \\
1.05 \\
1.06 \\
1.10 \\
1.13 \\
1.18\end{array}$ & $\begin{array}{l}32 \\
31 \\
32 \\
37 \\
40 \\
47\end{array}$ & $\begin{array}{r}0.99 \\
.99 \\
.99 \\
.99 \\
1.00 \\
1.05\end{array}$ & $\begin{array}{l}26 \\
26 \\
26 \\
26 \\
26 \\
31\end{array}$ & $\begin{array}{l}2.73 \\
2.48 \\
2.33 \\
2.17 \\
2.16 \\
2.16\end{array}$ & $\begin{array}{l}584 \\
450 \\
376 \\
309 \\
304 \\
304\end{array}$ & $\begin{array}{l}1.77 \\
1.85 \\
1.87 \\
1.87 \\
1.87 \\
1.89\end{array}$ & $\begin{array}{l}173 \\
197 \\
203 \\
203 \\
203 \\
210\end{array}$ \\
\hline \begin{tabular}{r|}
7 \\
8 \\
9 \\
10 \\
11 \\
11
\end{tabular} & $\begin{array}{l}.06 \\
1.02 \\
1.03 \\
1.03 \\
1.04 \\
1.07\end{array}$ & $\begin{array}{l}24 \\
29 \\
30 \\
30 \\
31 \\
34\end{array}$ & $\begin{array}{l}1.06 \\
1.05 \\
1.03 \\
1.03 \\
1.02 \\
1.02\end{array}$ & $\begin{array}{r}33 \\
32 \\
30 \\
30 \\
29 \\
-\quad 29\end{array}$ & $\begin{array}{l}1.35 \\
1.34 \\
1.33 \\
1.30 \\
1.25 \\
1.20\end{array}$ & $\begin{array}{l}73 \\
71 \\
69 \\
65 \\
57 \\
49\end{array}$ & $\begin{array}{l}1.08 \\
1.08 \\
1.10 \\
1.12 \\
1.15 \\
1.23\end{array}$ & $\begin{array}{l}34 \\
34 \\
37 \\
39 \\
42 \\
54\end{array}$ & $\begin{array}{l}2.18 \\
2.25 \\
2.35 \\
2.48 \\
2.41 \\
2.33\end{array}$ & $\begin{array}{l}313 \\
342 \\
385 \\
450 \\
415 \\
376\end{array}$ & $\begin{array}{l}1.90 \\
1.90 \\
1.90 \\
1.91 \\
1.90 \\
1.89\end{array}$ & $\begin{array}{l}213 \\
213 \\
213 \\
216 \\
213 \\
210\end{array}$ \\
\hline $\begin{array}{l}1 \\
2 \\
3 \\
4 \\
5\end{array}$ & $\begin{array}{l}1.06 \\
1.08 \\
1.10 \\
1.13 \\
1.18 \\
1.30\end{array}$ & $\begin{array}{l}33 \\
36 \\
38 \\
41 \\
48 \\
66\end{array}$ & $\begin{array}{l}1.03 \\
1.03 \\
1.03 \\
1.03 \\
1.06 \\
1.11\end{array}$ & $\begin{array}{l}30 \\
30 \\
30 \\
30 \\
33 \\
39\end{array}$ & $\begin{array}{l}1.18 \\
1.17 \\
1.15 \\
1.12 \\
1.10 \\
1.08\end{array}$ & $\begin{array}{l}47 \\
45 \\
44 \\
39 \\
37 \\
34\end{array}$ & $\begin{array}{l}1.30 \\
1.45 \\
1.40 \\
1.35 \\
1.34 \\
1.36\end{array}$ & $\begin{array}{l}65 \\
91 \\
82 \\
73 \\
71 \\
75\end{array}$ & $\begin{array}{l}2.20 \\
2.10 \\
2.07 \\
2.05 \\
2.07 \\
1.97\end{array}$ & $\begin{array}{l}321 \\
281 \\
269 \\
262 \\
248 \\
234\end{array}$ & $\begin{array}{l}1.87 \\
1.77 \\
1.70 \\
1.60 \\
1.53 \\
1.48\end{array}$ & $\begin{array}{l}203 \\
173 \\
153 \\
128 \\
110 \\
100\end{array}$ \\
\hline \begin{tabular}{r|}
7 \\
8 \\
9 \\
10 \\
11
\end{tabular} & $\begin{array}{l}1.32 \\
1.27 \\
1.23 \\
1.18 \\
1.16 \\
1.15\end{array}$ & $\begin{array}{l}75 \\
61 \\
55 \\
48 \\
45 \\
44 \\
\end{array}$ & $\begin{array}{l}1.10 \\
1.09 \\
1.08 \\
1.07 \\
1.06 \\
1.06\end{array}$ & $\begin{array}{l}38 \\
37 \\
36 \\
34 \\
33 \\
33 \\
\end{array}$ & $\begin{array}{r}1.07 \\
1.05 \\
1.03 \\
1.01 \\
.98 \\
.98\end{array}$ & $\begin{array}{l}33 \\
31 \\
29 \\
27 \\
25 \\
25 \\
\end{array}$ & $\begin{array}{l}1.40 \\
1.50 \\
1.65 \\
2.00 \\
2.45 \\
2.65 \\
\end{array}$ & $\begin{array}{r}82 \\
102 \\
138 \\
244 \\
435 \\
536 \\
\end{array}$ & $\begin{array}{l}1.94 \\
1.91 \\
1.87 \\
1.86 \\
1.76 \\
1.70\end{array}$ & $\begin{array}{l}223 \\
213 \\
200 \\
197 \\
167 \\
151 \\
\end{array}$ & $\begin{array}{l}1.40 \\
1.40 \\
1.39 \\
1.38 \\
1.37 \\
1.37\end{array}$ & $\begin{array}{l}84 \\
84 \\
82 \\
80 \\
78 \\
78 \\
\end{array}$ \\
\hline
\end{tabular}

\section{Otaj River Basin}

Otay River at Savage Dam, Calif.

Location. - Lat. $32^{\circ} 36^{\prime} 40^{\prime \prime}$, long. $116^{\circ} 55^{\prime} 40^{n}$, in Nit sec. 18, T. 18 S., R. I E., at Seqage Dam, at Lower Otay Reservolr. Zero of gage is 347.20 feet abore mean sea. level. Altitude of stream bed, 347 feet.

Drainage area.- Area, 98 square miles : Average altitude, 1,535 feet. Maximum altitude, 3,755 feet. Average slope, 26 percent. Length of main stream channel, 15 miles. Average slope of main stream channel, 4.1 percent.

Gage-height record. - Gage read dally at $7 \mathrm{a.m}$.

Remariks.- Records of discharge represent inflow to Lower Otay Reservoir, computed from discharge over spillway, change in contents, leakage, evaporation, and direct rainfall on surface of reservoir. Altitude of top of spilimay gates, 491 feet. Storage capacity, 56,314 acre-feet. Records furnished by City of San Diego Water Department.

Gage helght, in feet, and contents, in acre-feet, 1938

\begin{tabular}{|c|c|c|c|c|c|c|}
\hline \multirow{2}{*}{ Day } & \multicolumn{2}{|c|}{ Fobruary } & \multicolumn{2}{|c|}{ March } & \multicolumn{2}{|c|}{ April } \\
\hline & Feet & Acrelfeet & Feet & Acre-feet & Feot & Acre-feet \\
\hline $\begin{array}{l}\frac{1}{2} \\
3 \\
4 \\
5\end{array}$ & $\begin{array}{l}139.93 \\
139.95 \\
139.92 \\
139.89 \\
139.91\end{array}$ & $\begin{array}{l}51,944 \\
51,968 \\
51,933 \\
51,897 \\
51,921\end{array}$ & $\begin{array}{l}139.78 \\
140.04 \\
142.98 \\
144.46 \\
144.41\end{array}$ & $\begin{array}{l}\mathbf{5 1}, \mathbf{7 6 8} \\
\mathbf{5 2}, 075 \\
\mathbf{5 5}, 666 \\
\mathbf{5 7}, \mathbf{5 5 0} \\
\mathbf{5 7 , 4 8 6}\end{array}$ & $\begin{array}{l}143.52 \\
143.51 \\
143.49 \\
143.45 \\
143.41\end{array}$ & $\begin{array}{l}56,339 \\
56,327 \\
56,301 \\
56,251 \\
56,200\end{array}$ \\
\hline $\begin{array}{r}6 \\
7 \\
8 \\
9 \\
10\end{array}$ & $\begin{array}{l}139.90 \\
139.86 \\
139.83 \\
139.81 \\
139.78\end{array}$ & $\begin{array}{l}51,909 \\
51,862 \\
51,827 \\
51,803 \\
51,768\end{array}$ & $\begin{array}{l}144.21 \\
143.98 \\
143.92 \\
143.88 \\
143.84\end{array}$ & $\begin{array}{l}57,228 \\
56,922 \\
56,846 \\
56,795 \\
56,745\end{array}$ & $\begin{array}{l}143.40 \\
143.38 \\
143.36 \\
143.28 \\
143.28\end{array}$ & $\begin{array}{l}56,187 \\
56,162 \\
56,137 \\
56,036 \\
56,036\end{array}$ \\
\hline $\begin{array}{l}11 \\
12 \\
13 \\
14 \\
15\end{array}$ & $\begin{array}{l}139.76 \\
139.83 \\
139.83 \\
139.79 \\
139.76\end{array}$ & $\begin{array}{l}51,744 \\
51,827 \\
51,827 \\
51,780 \\
51,744\end{array}$ & $\begin{array}{l}143.81 \\
143.88 \\
144.13 \\
144.18 \\
144.07\end{array}$ & $\begin{array}{l}56,707 \\
56,795 \\
57,125 \\
57,189 \\
57,047\end{array}$ & $\begin{array}{l}143.26 \\
143.26 \\
143.26 \\
143.25 \\
143.23\end{array}$ & $\begin{array}{l}56,010 \\
56,010 \\
56,010 \\
55,998 \\
55,972\end{array}$ \\
\hline $\begin{array}{l}16 \\
17 \\
18 \\
19 \\
20\end{array}$ & $\begin{array}{l}139.74 \\
139.73 \\
139.72 \\
139.68 \\
139.66\end{array}$ & $\begin{array}{l}51,721 \\
51,709 \\
51,697 \\
51,650 \\
51,627\end{array}$ & $\begin{array}{l}144.07 \\
143.92 \\
143.84 \\
143.78 \\
143.76\end{array}$ & $\begin{array}{l}57,047 \\
56,846 \\
56,745 \\
56,669 \\
56,643\end{array}$ & $\begin{array}{l}143.23 \\
143.22 \\
143.21 \\
143.19 \\
143.18\end{array}$ & $\begin{array}{l}\mathbf{5 5}, 972 \\
55,960 \\
55,947 \\
55,922 \\
55,909\end{array}$ \\
\hline $\begin{array}{l}21 \\
22 \\
23 \\
24 \\
25\end{array}$ & $\begin{array}{l}139.63 \\
139.61 \\
139.57 \\
139.55 \\
139.51\end{array}$ & $\begin{array}{l}51,591 \\
51,568 \\
51,521 \\
51,497 \\
51,450\end{array}$ & $\begin{array}{l}143.73 \\
143.70 \\
143.70 \\
143.68 \\
143.64\end{array}$ & $\begin{array}{l}56,601 \\
56,567 \\
56,567 \\
56,542 \\
56,491\end{array}$ & $\begin{array}{l}143.18 \\
143.19 \\
143.19 \\
143.20 \\
143.20\end{array}$ & $\begin{array}{l}55,909 \\
55,922 \\
55,922 \\
55,934 \\
55,934\end{array}$ \\
\hline $\begin{array}{l}26 \\
27 \\
28 \\
29 \\
30 \\
31 \\
\end{array}$ & $\begin{array}{l}139.47 \\
139.46 \\
139.58\end{array}$ & $\begin{array}{l}51,403 \\
51,391 \\
51,532\end{array}$ & $\begin{array}{l}143.62 \\
143.60 \\
143.58 \\
143.56 \\
143.56 \\
143.54\end{array}$ & $\begin{array}{l}56,466 \\
56,441 \\
56,415 \\
56,390 \\
56,390 \\
56,365\end{array}$ & $\begin{array}{l}143.21 \\
143.22 \\
143.22 \\
143.23 \\
143.25\end{array}$ & $\begin{array}{l}55,947 \\
55,960 \\
55,960 \\
55,972 \\
55,998\end{array}$ \\
\hline \multirow{2}{*}{\multicolumn{5}{|c|}{ 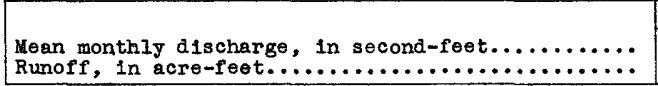 }} & & Apr11 \\
\hline & & & & & 17 & $\begin{array}{r}4.32 \\
257 \\
\end{array}$ \\
\hline
\end{tabular}




\section{Sweetwater River Basin}

Sweotwater RIver at Sweetwater Dam, Callf.

Location. - Lat. $32^{\circ} 41^{\prime} 20^{\prime \prime}$, long. $117^{\circ} 00^{\prime} 35^{\prime \prime}$, at Sweetwater Dam, in Le Nacion grant, 6 milos east of National C1ty, San Diogo County, and about 8 miles upstrean from mouth. Alt1tude of stroam bod, about 150 feot.

Dralnage area.- Area, 181 square milos. Average altitude, 2,277 feot. Maximum alt1tuide, 6,515 foot. Average slope, 29 percont. Length of main stream chennel, 43 wt les. Average slope of main streaim channol, 2.7 percent.

Gage-helght rocord.- Staff gage road da1ly betweon 7 and $8 \mathrm{a.m}$.

Remiks.- Records of discharge represent flow Into swoetwater Reservolr, computed from discharge over splilway, change in centents, leakage, evaporation, and direct rainfall on surface of reservolr. Storage capac1ty, 30,973 acre-feet. Records furnished by Callfornia Water and Telephone Company.

Gage holght, in feet, and contents, in acre-feet, 1938

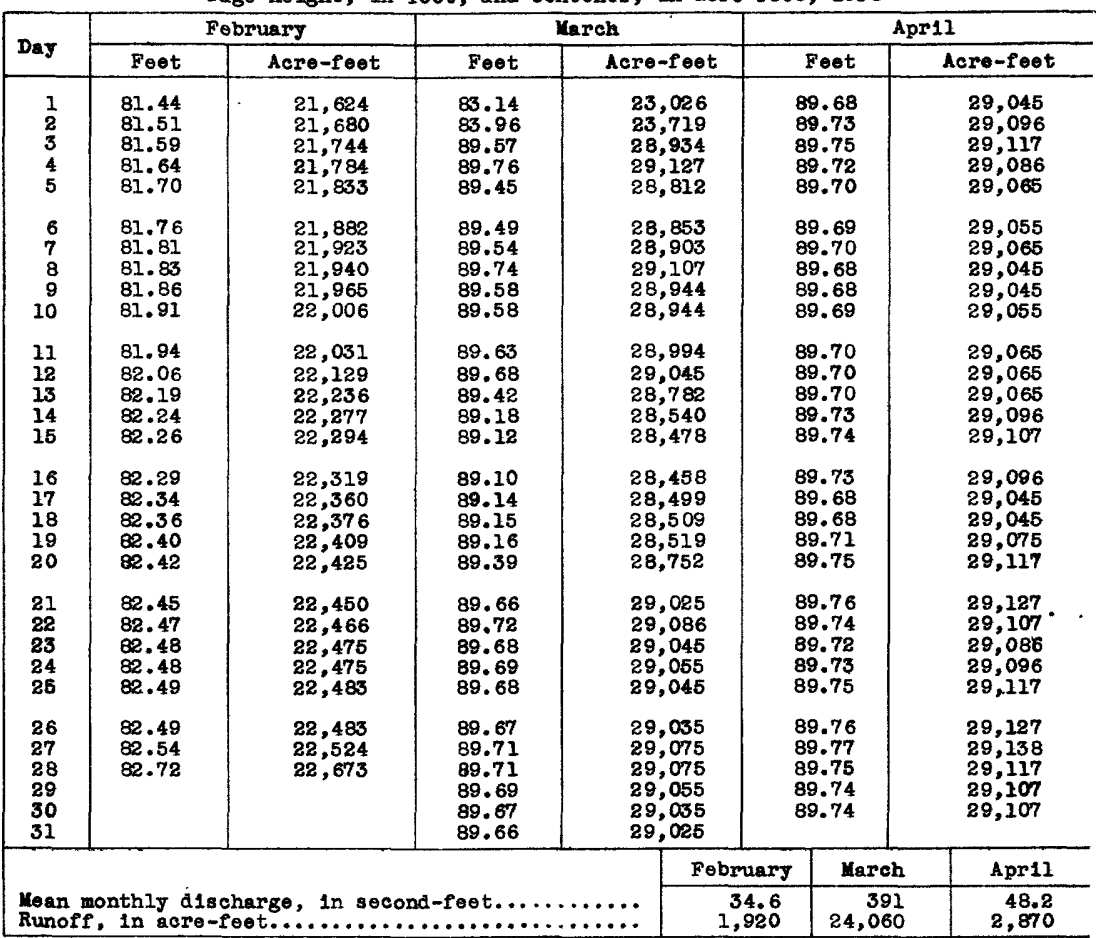

\section{San Diego River Basin}

San Diego River at El Capitan Dam, near Lakeside, Calif.

Location. - Lat. $32^{\circ} 53^{\prime} 00^{n}$, long. $116^{\circ} 48^{\prime} 40^{n}$, In NEt sec. 7, T. 15 S., R. 2 E., t E1 Capltan Dam, I mile downs tream from mouth of Chocolate Creok, and 7 miles east of Lakeside. Altitude of stream bed, 553 foet.

Draingge area.- Area, 190 square miles. Average altitude, 2,905 feet. Maximalt1tude, 6,515 foet. Average slope, 38 percent. Iongth of in stream channel, 14.5 miles. Average slope of main stream, 7.5 percent.

Gage-height record. - Gage read dally at 7 a.m.-

Remapks.- Records of discharge represent flow Into Bl Capitan Reservo1r, computed from discharge over spillway, change in contents, leakage, evaporation, and direct rainfall on surface of reservolr. Alt1tude of splilway, 750 feet. Storage capacity. 116,500 acre-feet. Additional storage obtalned by placing sandbags on spillear. Records furnished by Clty of San Diego Water Department. 
Gage he1ght, in reet, and oontents, in acre-reet, 1938

\begin{tabular}{|c|c|c|c|c|c|c|c|c|}
\hline \multirow[b]{2}{*}{ Day } & \multicolumn{2}{|c|}{ Fobruary } & \multicolumn{3}{|c|}{ March } & \multicolumn{3}{|c|}{ Apr11 } \\
\hline & Feet & Aore-feet & Feet & \multicolumn{2}{|c|}{ Acre-feet } & \multicolumn{2}{|c|}{ Foet } & Acre-foet \\
\hline $\begin{array}{l}1 \\
2 \\
3 \\
4 \\
5\end{array}$ & $\begin{array}{l}177.65 \\
177.75 \\
177.80 \\
177.84 \\
177.98\end{array}$ & $\begin{array}{l}88,377 \\
88,509 \\
88,576 \\
88,629 \\
88,816\end{array}$ & $\begin{array}{l}180.68 \\
181.46 \\
188.15 \\
192.50 \\
194.06\end{array}$ & \multicolumn{2}{|c|}{$\begin{array}{r}92,460 \\
93,530 \\
103,024 \\
109,497 \\
111,877\end{array}$} & \multicolumn{2}{|c|}{$\begin{array}{r}197.77 \\
197.87 \\
197.92 \\
197.99 \\
198.07\end{array}$} & $\begin{array}{l}117,664 \\
117,808 \\
117,908 \\
118,013 \\
118,140\end{array}$ \\
\hline $\begin{array}{r}6 \\
7 \\
8 \\
9 \\
10\end{array}$ & $\begin{array}{l}178.05 \\
178.09 \\
178.11 \\
178.14 \\
178.28\end{array}$ & $\begin{array}{l}88,909 \\
88,962 \\
88,989 \\
89,029 \\
89,135\end{array}$ & $\begin{array}{l}194.95 \\
195.50 \\
196.85 \\
196.36 \\
196.68\end{array}$ & \multicolumn{2}{|c|}{$\begin{array}{l}113,249 \\
114,102 \\
114,647 \\
115,444 \\
115,945\end{array}$} & \multicolumn{2}{|c|}{$\begin{array}{l}198.10 \\
198.08 \\
198.05 \\
198.08 \\
197.99\end{array}$} & $\begin{array}{l}118,188 \\
118,156 \\
118,108 \\
118,061 \\
118,013\end{array}$ \\
\hline $\begin{array}{l}11 \\
12 \\
13 \\
14 \\
16\end{array}$ & $\begin{array}{l}178.27 \\
178.56 \\
178.70 \\
178.79 \\
178.82\end{array}$ & $\begin{array}{l}89,202 \\
89,580 \\
89,778 \\
89,898 \\
89,939\end{array}$ & $\begin{array}{l}196.83 \\
197.23 \\
197.78 \\
197.86 \\
197.59\end{array}$ & \multicolumn{2}{|c|}{$\begin{array}{l}116,338 \\
116,811 \\
117,680 \\
117,807 \\
117,379\end{array}$} & \multicolumn{2}{|c|}{$\begin{array}{l}197.97 \\
197.96 \\
197.96 \\
198.04 \\
198.05\end{array}$} & $\begin{array}{l}117,981 \\
117,966 \\
117,966 \\
118,008 \\
118,108\end{array}$ \\
\hline $\begin{array}{l}16 \\
17 \\
18 \\
19 \\
20\end{array}$ & $\begin{array}{l}178.86 \\
178.90 \\
178.94 \\
179.01 \\
179.06\end{array}$ & $\begin{array}{l}89,993 \\
90,046 \\
90,100 \\
90,194 \\
90,262\end{array}$ & $\begin{array}{l}197 \cdot 48 \\
197.39 \\
187.37 \\
197.33 \\
197.30\end{array}$ & \multicolumn{2}{|c|}{$\begin{array}{l}117,206 \\
117,065 \\
117,052 \\
116,969 \\
116,921\end{array}$} & \multicolumn{2}{|c|}{$\begin{array}{l}198.02 \\
188.01 \\
198.00 \\
198.02 \\
198.07\end{array}$} & $\begin{array}{l}118,061 \\
118,045 \\
118,089 \\
118,061 \\
118,140\end{array}$ \\
\hline $\begin{array}{l}21 \\
22 \\
23 \\
24 \\
25\end{array}$ & $\begin{array}{l}179.10 \\
179.12 \\
179.14 \\
179.16 \\
179.14\end{array}$ & $\begin{array}{l}90,315 \\
90,342 \\
90,369 \\
90,396 \\
90,369\end{array}$ & $\begin{array}{l}197.29 \\
187.28 \\
187.27 \\
197.27 \\
187.26\end{array}$ & \multicolumn{2}{|c|}{$\begin{array}{l}116,906 \\
116,890 \\
116,874 \\
116,874 \\
116,858\end{array}$} & \multicolumn{2}{|c|}{$\begin{array}{l}198.11 \\
108.14 \\
198.17 \\
198.18 \\
198.21\end{array}$} & $\begin{array}{l}118,204 \\
118,851 \\
118,809 \\
118,316 \\
118,363\end{array}$ \\
\hline $\begin{array}{l}26 \\
27 \\
28 \\
29 \\
30 \\
31\end{array}$ & $\begin{array}{l}179.13 \\
179.21 \\
179.84\end{array}$ & $\begin{array}{l}90,356 \\
90,464 \\
91,316\end{array}$ & $\begin{array}{l}197.25 \\
197.26 \\
187.24 \\
197.23 \\
197.40 \\
197.60\end{array}$ & \multicolumn{2}{|c|}{$\begin{array}{l}116,842 \\
116,858 \\
116,827 \\
116,811 \\
117,079 \\
117,395\end{array}$} & \multicolumn{2}{|c|}{$\begin{array}{l}198.29 \\
198.28 \\
198.25 \\
198.24 \\
198.28\end{array}$} & $\begin{array}{l}118,490 \\
118,474 \\
118,426 \\
118,410 \\
118,474\end{array}$ \\
\hline \multirow{2}{*}{\multicolumn{5}{|c|}{ 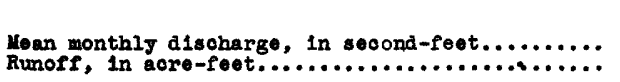 }} & \multicolumn{2}{|c|}{ February } & \multicolumn{2}{|c|}{ Maroh } \\
\hline & & & & & \multicolumn{2}{|c|}{$\begin{array}{r}86.1 \\
4,780\end{array}$} & $\begin{array}{r}687 \\
42,260\end{array}$ & $\begin{array}{r}86.9 \\
5,110\end{array}$ \\
\hline
\end{tabular}

3an Dlego River near Santee, Cal1f.

Location. - Water-stage recorder, lat. 32 $49^{\prime} 20^{\prime \prime}$, 1ong. $117^{\circ} 03^{\prime} 25^{\prime \prime}$, In Misalon Gorge, In IV ulssion San Diege grant, 6 miles west of Santee, San Diego County. Altitude, about 205 feet.

Drainaze areu.- Area, 380 square miles. Average altitude, 2, 070 feet. Maximan altitude, 6,515 feet. Average slope, 38 percent. Length of main stream chemnel, 40 miles. Average lope of maln stream channel, 2.9 percent.

Gage-height record.- Water-stage recorder graph.

Discharge record. - Stage-discharge relation dofined by ourrent-meter measurements below 4,570 second-feet; extended logaritheically to peak atage.

Nexime- - 1838: Discharge, 7,350 second-feet 2 a.m. Kar, 3 (gage he1ght, 7.05 feet). 1912-37: Discharge, 70,200 second-feet Jan. 27 , 1916, by slope-area mothod.

Remarks.- Records good. Flow partlally regulated by Bl Capitan Reservo1r, $26 \mathrm{mlles}$ upstream. C1ty of San Diego afverts water from El Capitan Reaervoir.

Nean dally discharge, in second-feet, 1938

\begin{tabular}{|c|c|c|c|c|c|c|c|c|c|c|c|}
\hline Day & Feb. & Mar. & Apr. & Day & $\mathrm{Feb}$. & Mar. & Apr. & Day & Feb. & Mar. & Apr. \\
\hline $\begin{array}{r}1 \\
2 \\
3 \\
4 \\
5 \\
6 \\
7 \\
8 \\
9 \\
10\end{array}$ & $\begin{array}{r}0.6 \\
.4 \\
.4 \\
.4 \\
.4 \\
.2 \\
.2 \\
.2 \\
.6 \\
.9\end{array}$ & $\begin{array}{r}496 \\
1,700 \\
3,770 \\
925 \\
470 \\
295 \\
200 \\
189 \\
165 \\
100\end{array}$ & $\begin{array}{l}31 \\
24 \\
24 \\
27 \\
31 \\
80 \\
91 \\
88 \\
77 \\
75\end{array}$ & $\begin{array}{l}11 \\
12 \\
13 \\
14 \\
15 \\
16 \\
17 \\
18 \\
19 \\
20\end{array}$ & $\begin{array}{r}1.0 \\
2.1 \\
1.2 \\
.9 \\
.7 \\
.9 \\
.9 \\
.6 \\
1.0 \\
.6\end{array}$ & $\begin{array}{r}87 \\
379 \\
1,250 \\
1,220 \\
745 \\
590 \\
486 \\
400 \\
307 \\
259\end{array}$ & $\begin{array}{l}65 \\
60 \\
68 \\
75 \\
75 \\
75 \\
77 \\
77 \\
26 \\
14\end{array}$ & $\begin{array}{l}21 \\
22 \\
23 \\
24 \\
25 \\
26 \\
27 \\
28 \\
29 \\
30 \\
31\end{array}$ & $\begin{array}{r}0.4 \\
.3 \\
.3 \\
.3 \\
.3 \\
.2 \\
5.5 \\
24\end{array}$ & $\begin{array}{r}230 \\
210 \\
195 \\
174 \\
168 \\
156 \\
153 \\
145 \\
137 \\
65 \\
37\end{array}$ & $\begin{array}{l}18 \\
11 \\
13 \\
13 \\
24 \\
68 \\
65 \\
68 \\
45 \\
54\end{array}$ \\
\hline \multicolumn{12}{|c|}{ 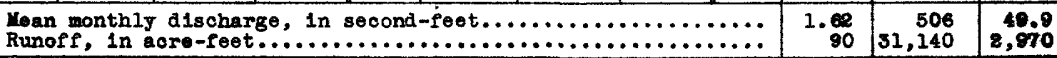 } \\
\hline
\end{tabular}


Gage height, in feet, and discharge, in second-feet, at indicated time, 1938

\begin{tabular}{|c|c|c|c|c|c|c|c|c|c|c|c|c|}
\hline \multirow{2}{*}{ 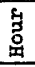 } & Peet & Sec.ft. & Feet & Sec.ft. & Feet & Sec.ft. & Feet & Sec.ft. & Feet & Sec.ft. & Feet & Sec.ft. \\
\hline & \multicolumn{2}{|c|}{ Pebruary 27} & \multicolumn{2}{|c|}{ February 28} & \multicolumn{2}{|c|}{ March 1} & \multicolumn{2}{|c|}{ March 2} & \multicolumn{2}{|c|}{ March 3} & \multicolumn{2}{|c|}{ Harch 4} \\
\hline $\begin{array}{l}1 \\
2 \\
3 \\
4 \\
5 \\
6\end{array}$ & $\begin{array}{r}0.13 \\
.17 \\
.19 \\
.20 \\
.21 \\
.22\end{array}$ & $\begin{array}{l}0.3 \\
.5 \\
.6 \\
.7 \\
.8 \\
.9\end{array}$ & $\begin{array}{r}0.70 \\
.69 \\
.68 \\
.64 \\
.62 \\
.61\end{array}$ & $\begin{array}{l}37 \\
35 \\
34 \\
27 \\
24 \\
23\end{array}$ & $\begin{array}{r}0.60 \\
.64 \\
.75 \\
1.11 \\
2.00 \\
2.40\end{array}$ & $\begin{array}{r}21 \\
27 \\
47 \\
132 \\
480 \\
710\end{array}$ & $\begin{array}{l}1.63 \\
1.63 \\
1.68 \\
1.69 \\
1.75 \\
1.95\end{array}$ & $\begin{array}{l}307 \\
307 \\
328 \\
332 \\
358 \\
455\end{array}$ & $\begin{array}{l}6.95 \\
7.05 \\
6.57 \\
6.00 \\
5.75 \\
5.53\end{array}$ & $\begin{array}{r}7,110 \\
7,350 \\
6,250 \\
5,160 \\
4,710 \\
4,320\end{array}$ & $\begin{array}{l}3.14 \\
3.10 \\
3.03 \\
3.02 \\
3.00 \\
2.92\end{array}$ & $\begin{array}{l}1,230 \\
1,200 \\
1,130 \\
1,120 \\
1,110\end{array}$ \\
\hline $\begin{array}{r}7 \\
5 \\
9 \\
10 \\
11 \\
\mathrm{~N}\end{array}$ & $\begin{array}{l}.23 \\
.27 \\
.30 \\
.31 \\
.32 \\
.34\end{array}$ & $\begin{array}{l}.9 \\
1.3 \\
1.5 \\
1.8 \\
2.1 \\
2.7\end{array}$ & $\begin{array}{l}.60 \\
.60 \\
.59 \\
.58 \\
.58 \\
.58\end{array}$ & $\begin{array}{l}21 \\
21 \\
20 \\
19 \\
19 \\
19\end{array}$ & $\begin{array}{l}2.40 \\
2.31 \\
2.21 \\
2.92 \\
2.80 \\
2.50\end{array}$ & $\begin{array}{r}710 \\
656 \\
596 \\
1,100 \\
1,000 \\
780\end{array}$ & $\begin{array}{l}2.10 \\
2.18 \\
2.30 \\
2.50 \\
3.09 \\
3.01\end{array}$ & $\begin{array}{r}535 \\
579 \\
650 \\
780 \\
1,250 \\
1,180\end{array}$ & & & & $\begin{array}{l}1,030 \\
1,020 \\
1,030 \\
1,060 \\
1,100 \\
1,050\end{array}$ \\
\hline $\begin{array}{l}1 \\
2 \\
3 \\
4 \\
5 \\
6\end{array}$ & $\begin{array}{l}.34 \\
.34 \\
.41 \\
.44 \\
.49 \\
.50\end{array}$ & $\begin{array}{l}2.7 \\
2.7 \\
5 \\
6.7 \\
9.4 \\
10\end{array}$ & $\begin{array}{l}.58 \\
.58 \\
.58 \\
.58 \\
.58 \\
.58\end{array}$ & $\begin{array}{l}19 \\
19 \\
19 \\
19 \\
19 \\
19\end{array}$ & $\begin{array}{l}37 \\
31 \\
25 \\
17 \\
10 \\
02\end{array}$ & $\begin{array}{l}692 \\
656 \\
620 \\
573 \\
535 \\
491\end{array}$ & $\begin{array}{l}95 \\
35 \\
73 \\
68 \\
54 \\
41\end{array}$ & $\begin{array}{l}1,120 \\
1,490 \\
1,900 \\
1,830 \\
1,680 \\
1,550\end{array}$ & $\begin{array}{l}5.68 \\
5.31 \\
4.78 \\
4.32 \\
3.97 \\
3.70\end{array}$ & $\begin{array}{l}70 \\
10 \\
20 \\
80 \\
60 \\
70\end{array}$ & $\begin{array}{l}30 \\
70 \\
63 \\
58 \\
52\end{array}$ & $\begin{array}{l}944 \\
871 \\
822 \\
787 \\
745 \\
731\end{array}$ \\
\hline $\begin{array}{r}7 \\
8 \\
9 \\
10 \\
11 \\
11\end{array}$ & $\begin{array}{l}.50 \\
.49 \\
.49 \\
.50 \\
.60 \\
.72\end{array}$ & $\begin{array}{l}10 \\
9.4 \\
9.4 \\
10 \\
21 \\
41\end{array}$ & $\begin{array}{l}.58 \\
.58 \\
.58 \\
.58 \\
.60\end{array}$ & $\begin{array}{l}19 \\
19 \\
19 \\
19 \\
21 \\
21\end{array}$ & $\begin{array}{l}1.95 \\
1.87 \\
1.79 \\
1.73 \\
1.69\end{array}$ & $\begin{array}{l}455 \\
415 \\
375 \\
349 \\
332 \\
315\end{array}$ & $\begin{array}{l}3.48 \\
4.32 \\
5.70 \\
6.45 \\
6.50\end{array}$ & $\begin{array}{l}1,620 \\
2,560 \\
4,690 \\
6,050 \\
6,110 \\
6,110\end{array}$ & $\begin{array}{l}3.54 \\
3.40 \\
3.34 \\
3.24 \\
3.17 \\
3.10\end{array}$ & $\begin{array}{l}10 \\
70 \\
10 \\
20 \\
60 \\
00\end{array}$ & $\begin{array}{l}2.45 \\
2.41 \\
2.39 \\
2.33 \\
2.31\end{array}$ & $\begin{array}{l}698 \\
674 \\
662 \\
626 \\
614 \\
608\end{array}$ \\
\hline
\end{tabular}

Boulder Creek at Cuyameca Reservolr, near Jullan, Callf.

Iocation.- Lat. $32^{\circ} 59^{\prime} 15^{\prime \prime}$, Iong. $116^{\circ} 35^{\prime} 10^{n}$, in MEt sec. 8, T. 14 S., R. 4 E., at Cuyamaca Reservolr, 7 miles south of Julian. Altitude of stream bed, 4,590 feet.

Drainage area.- Area, 12.0 square miles. Average altitude, 4,976 foet. Maximum altitude, 6,515 feet. Average slope, 20 percent. Iength of main stream channel, 3.5 miles. Average slope of main stream chemel, 7.5 percent.

Gage-helght record.- Gage read da1ly at 7 a.m.

Remarks. - Records of discharge computed from discharge over sp11lway, change in contents, leakage, evaporation, and direct rainfall on surface of reservoir. Altitude of crest of dam, 4,633 feet. Storage capacity, 11,600 acre-feet. Records furnished by Ia Mesa, Lemon Grove, and Spring Valley Irrigation Diatrict.

Gage height, in feet, and contents, in acre-feet, 1938

\begin{tabular}{|c|c|c|c|c|c|c|c|c|}
\hline \multirow{2}{*}{ Day } & \multicolumn{2}{|c|}{ February } & \multicolumn{3}{|c|}{ March } & \multicolumn{3}{|c|}{ Apre11 } \\
\hline & Feet & Acre-feet & Feet & \multicolumn{2}{|c|}{ Acre-feet } & \multicolumn{2}{|c|}{ Feet } & Acre-feet \\
\hline $\begin{array}{l}1 \\
2 \\
3 \\
4 \\
5\end{array}$ & $\begin{array}{l}30.62 \\
30.83 \\
30.83 \\
30.92 \\
31.12\end{array}$ & $\begin{array}{l}7,565 \\
7,738 \\
7,738 \\
7,806 \\
7,978\end{array}$ & $\begin{array}{l}32.83 \\
33.00 \\
35.25 \\
35.79 \\
35.58\end{array}$ & \multicolumn{2}{|c|}{$\begin{array}{r}9,414 \\
9,551 \\
11,457 \\
11,904 \\
11,732\end{array}$} & \multicolumn{2}{|c|}{$\begin{array}{l}35.33 \\
35.33 \\
35.37 \\
35.37 \\
35.37\end{array}$} & $\begin{array}{l}11,525 \\
11,525 \\
11,559 \\
11,559 \\
11,559\end{array}$ \\
\hline $\begin{array}{r}6 \\
7 \\
8 \\
9 \\
10\end{array}$ & $\begin{array}{l}31.12 \\
31.12 \\
31 \cdot 12 \\
31 \cdot 12 \\
31.21\end{array}$ & $\begin{array}{l}7,978 \\
7,978 \\
7,978 \\
7,978 \\
8,047\end{array}$ & $\begin{array}{l}35.33 \\
35.25 \\
35.33 \\
35.33 \\
35.33\end{array}$ & \multicolumn{2}{|c|}{$\begin{array}{l}11,525 \\
11,457 \\
11,525 \\
11,525 \\
11,525\end{array}$} & \multicolumn{2}{|c|}{$\begin{array}{l}35.37 \\
35.37 \\
35.37 \\
35.33 \\
35.33\end{array}$} & $\begin{array}{l}11,559 \\
11,559 \\
11,559 \\
11,525 \\
11,525\end{array}$ \\
\hline $\begin{array}{l}11 \\
12 \\
13 \\
14 \\
15\end{array}$ & $\begin{array}{l}31.21 \\
31.37 \\
31.50 \\
31.50 \\
31.50\end{array}$ & $\begin{array}{l}8,047 \\
8,185 \\
8,288 \\
8,288 \\
8,288\end{array}$ & $\begin{array}{l}35.37 \\
35.75 \\
35.53 \\
35.42 \\
35.33\end{array}$ & \multicolumn{2}{|c|}{$\begin{array}{l}11,491 \\
11,870 \\
11,732 \\
11,595 \\
11,525\end{array}$} & \multicolumn{2}{|c|}{$\begin{array}{l}35.33 \\
35.29 \\
35.37 \\
35.42 \\
35.46\end{array}$} & $\begin{array}{l}11,525 \\
11,491 \\
11,559 \\
11,595 \\
11,629\end{array}$ \\
\hline $\begin{array}{l}16 \\
17 \\
18 \\
19 \\
20\end{array}$ & $\begin{array}{l}31.50 \\
31.50 \\
31.50 \\
31.62 \\
31.67\end{array}$ & $\begin{array}{l}8,288 \\
8,288 \\
8,288 \\
8,391 \\
8,426\end{array}$ & $\begin{array}{l}35.42 \\
35.37 \\
35.42 \\
35.42 \\
35.42\end{array}$ & \multicolumn{2}{|c|}{$\begin{array}{l}11,595 \\
11,559 \\
11,595 \\
11,595 \\
11,595\end{array}$} & \multicolumn{2}{|c|}{$\begin{array}{l}35.46 \\
35.46 \\
35.42 \\
35.42 \\
35.42\end{array}$} & $\begin{array}{r}11,689 \\
11,629 \\
-11,595 \\
11,595 \\
11,595\end{array}$ \\
\hline $\begin{array}{l}21 \\
22 \\
23 \\
24 \\
25\end{array}$ & $\begin{array}{l}31.71 \\
31.71 \\
31.71 \\
31.71 \\
31.71\end{array}$ & $\begin{array}{l}8,460 \\
8,460 \\
8,460 \\
8,460 \\
8,460\end{array}$ & $\begin{array}{l}35.42 \\
35.42 \\
35.49 \\
35.42 \\
35.42\end{array}$ & \multicolumn{2}{|c|}{$\begin{array}{l}11,595 \\
11,595 \\
11,595 \\
11,595 \\
11,595\end{array}$} & \multicolumn{2}{|c|}{$\begin{array}{l}35.42 \\
35.42 \\
35.37 \\
35.37 \\
35.37\end{array}$} & $\begin{array}{l}11,595 \\
11,595 \\
11,542 \\
11,542 \\
11,542\end{array}$ \\
\hline $\begin{array}{l}26 \\
27 \\
28 \\
29 \\
30 \\
31\end{array}$ & $\begin{array}{l}31.71 \\
31.83 \\
32.33\end{array}$ & $\begin{array}{l}8,460 \\
8,564 \\
9,000\end{array}$ & $\begin{array}{l}35.46 \\
35.46 \\
35.46 \\
35.50 \\
35.50 \\
35.42\end{array}$ & \multicolumn{2}{|c|}{$\begin{array}{l}11,629 \\
11,629 \\
11,629 \\
11,663 \\
11,663 \\
11,595\end{array}$} & \multicolumn{2}{|c|}{$\begin{array}{l}35.46 \\
35.46 \\
35.46 \\
35.46 \\
35.50\end{array}$} & $\begin{array}{r}11,629 \\
11,629 \\
11,629 \\
11,629 \\
11,663\end{array}$ \\
\hline \multirow{2}{*}{\multicolumn{5}{|c|}{ 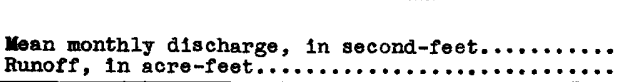 }} & \multicolumn{2}{|c|}{ February } & March & Apr 11 \\
\hline & & & & & \multicolumn{2}{|c|}{$\begin{array}{r}33 \\
1,849 \\
\end{array}$} & $\begin{array}{r}116 \\
7,146 \\
\end{array}$ & $\begin{array}{r}16 \\
940 \\
\end{array}$ \\
\hline
\end{tabular}


San Vicente Creek at Foster, Caiff.

Iocation. - Water-stage recorder, lat. $32^{\circ} 54^{\prime} 40^{\prime \prime}$, long. $116^{\circ} 55^{\prime} 35^{\prime \prime}$, in sec. 31 , T. 14 S., R. 1 E., half a mile north of Foster. Altitude, about 750 feet.

Drainage area.- Area, 75 square miles.. Average altitude, 1,640 feet. Maximum alt1tude, 4,132 feet. Average slope, 36 percent. Iength of main stream channel, 14 miles. Average stope of main stream channel, 4.5 percent.

Gage-he1ght record.- Water-stage recorder graph except Feb. 1-10, 20-26, Apr. 1-18 and $20-30$.

Discharge record.- Stage-discharge relation defined by current-meter measurements below 1,320 second-feet; extended to peak stage on basis of mean depth-mean velocity relation. Discharge for perlods of missing gage-helght record determined from current-meter measurements and comparable hydrographs of adjacent streams.

Maxima.- 1938: Discharge, 5,270 second-feet 11 p.m. Mar. 2 (gage height, 5.59 feet). 1915, 1916, 1936-37: D1scharge, 18,600 second-feet Jan. 27, 1916, by slopearea method.

Remarks.- Records falr.

Mean da1ly discharge, in second-feet, 1938

\begin{tabular}{|c|c|c|c|c|c|c|c|c|c|c|c|}
\hline Day & Feb. & Mar. & Apr. & Day & Feb. & Mar. & Apr. & Day & Feb. & Mar. & Apr. \\
\hline $\begin{array}{r}1 \\
2 \\
3 \\
4 \\
5 \\
6 \\
7 \\
8 \\
9 \\
10\end{array}$ & $\begin{array}{r}0.2 \\
.1 \\
.1 \\
.3 \\
.2 \\
.1 \\
.1 \\
.1 \\
.2 \\
.2\end{array}$ & $\begin{array}{r}433 \\
1,380 \\
1,790 \\
545 \\
272 \\
160 \\
133 \\
154 \\
138 \\
143\end{array}$ & $\begin{array}{l}26 \\
25 \\
24 \\
22 \\
21 \\
20 \\
18 \\
17 \\
17 \\
18\end{array}$ & $\begin{array}{l}11 \\
12 \\
13 \\
14 \\
15 \\
16 \\
17 \\
18 \\
19 \\
20\end{array}$ & $\begin{array}{r}1.0 \\
.2 \\
.2 \\
.2 \\
.2 \\
.2 \\
.1 \\
.1 \\
.2 \\
.2\end{array}$ & $\begin{array}{r}143 \\
346 \\
302 \\
198 \\
143 \\
122 \\
118 \\
100 \\
91 \\
67\end{array}$ & $\begin{array}{l}19 \\
20 \\
23 \\
20 \\
17 \\
16 \\
14 \\
12 \\
11 \\
10\end{array}$ & $\begin{array}{l}21 \\
22 \\
23 \\
24 \\
25 \\
26 \\
27 \\
28 \\
29 \\
30 \\
31\end{array}$ & $\begin{array}{r}0.2 \\
.2 \\
.1 \\
.1 \\
.1 \\
65^{.1} \\
123\end{array}$ & $\begin{array}{l}58 \\
52 \\
49 \\
42 \\
40 \\
34 \\
35 \\
35 \\
38 \\
34 \\
29 \\
\end{array}$ & $\begin{array}{r}10 \\
9 \\
9 \\
10 \\
20 \\
17 \\
14 \\
12 \\
10 \\
10\end{array}$ \\
\hline \multicolumn{9}{|c|}{ 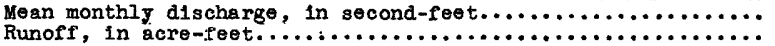 } & $\begin{array}{r}6.89 \\
383 \\
\end{array}$ & $\begin{array}{r}233 \\
14,330 \\
\end{array}$ & $\begin{array}{r}16.4 \\
974 \\
\end{array}$ \\
\hline
\end{tabular}

Gage height, in feet, and discharge, in second-feet, at indicated time, 1938

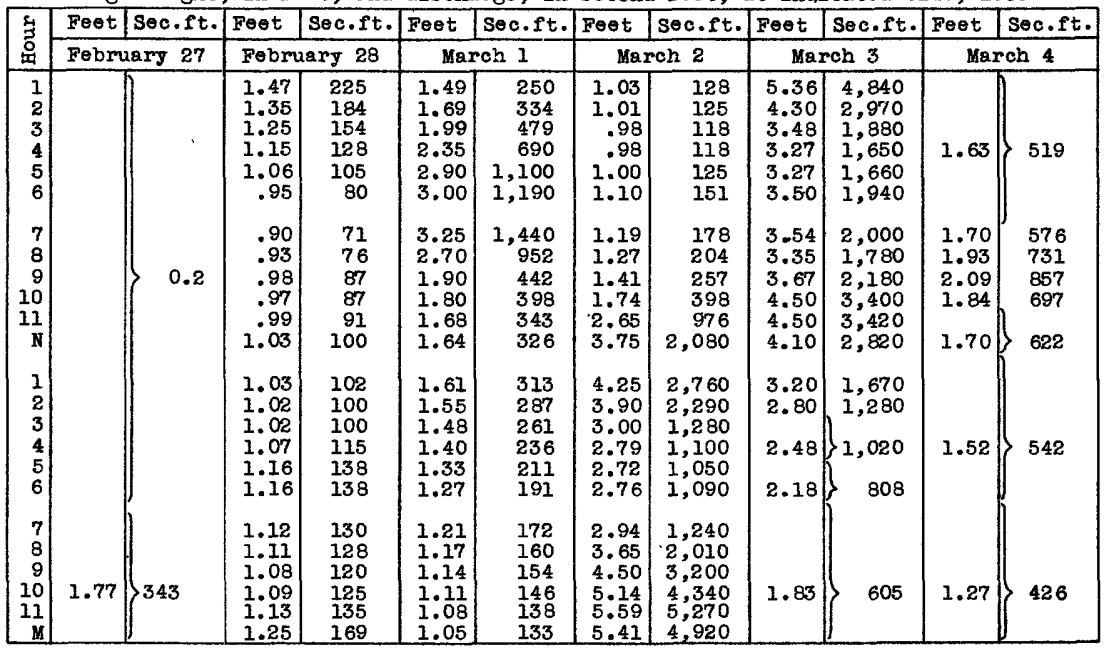




\section{San Diegufto River Besin}

Santa Yaabel Creek near Yeaa Grande, Callf.

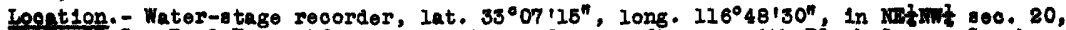

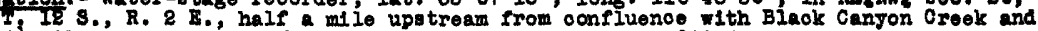
4 miler outhweat of Mera Grande. Alt1tude, about 2,000 feet.

Dratnage area.- Area, 58 gquare mlles. Arerage altitude, 3,360 feet. Meximam alt1tude, 5, 577 feet. Average lope, 40 percent. Iength of main etream ohamme1, 7.6 :* miles. Average lope of main stream ohannel, 8.8 percent.

Gege-ho1ght reoord. - Water-etage reoorder graph, except for perlod 4 p.w. Uar. 3 to 12 m. Vlar. 6, gage helght for which were interpolated.

Disoharge reoord,- Stago-discharge relation derined by ourrent-ater meagurements beION 1,140 geond-feet; extended to peak otage on basis of mean depth-mean veloofty relation.

Lit1m. - 1938: D1woharge, 8,000 vecond-feet 11820 p.m. Yar. 2 (gage helght, 7.30 feet). $1918-28$, 1936-37; Dlecharge, 21,100 gecond-feet Jan. 27, 1916.

Hean dally divcherge, in vecond-feet, 1038

\begin{tabular}{|c|c|c|c|c|c|c|c|c|c|c|c|}
\hline Des & 5ob. & Mar. & Apr. & Day & Peb. & Mne. & Apr. & Dar & Dob. & Mar. & Apr. \\
\hline $\begin{array}{r}1 \\
8 \\
5 \\
4 \\
8 \\
6 \\
7 \\
8 \\
0 \\
10\end{array}$ & $\begin{array}{l}16 \\
14 \\
11 \\
50 \\
26 \\
17 \\
18 \\
13 \\
14 \\
28\end{array}$ & $\begin{array}{r}443 \\
1,750 \\
2,000 \\
769 \\
454 \\
247 \\
158 \\
178 \\
108 \\
76\end{array}$ & $\begin{array}{l}68 \\
68 \\
68 \\
68 \\
86 \\
70 \\
76 \\
78 \\
68 \\
60\end{array}$ & $\begin{array}{l}11 \\
12 \\
13 \\
14 \\
15 \\
16 \\
17 \\
18 \\
19 \\
20\end{array}$ & $\begin{array}{l}88 \\
66 \\
34 \\
26 \\
28 \\
26 \\
82 \\
19 \\
32 \\
20\end{array}$ & $\begin{array}{r}68 \\
709 \\
618 \\
402 \\
288 \\
218 \\
226 \\
162 \\
145 \\
143\end{array}$ & $\begin{array}{l}54 \\
51 \\
76 \\
76 \\
64 \\
46 \\
40 \\
38 \\
34 \\
34\end{array}$ & $\begin{array}{l}21 \\
88 \\
28 \\
24 \\
85 \\
28 \\
87 \\
80 \\
80 \\
80 \\
31\end{array}$ & $\begin{array}{l}18 \\
16 \\
16 \\
16 \\
14 \\
13 \\
168 \\
185\end{array}$ & $\begin{array}{r}128 \\
113 \\
105 \\
94 \\
94 \\
105 \\
108 \\
98 \\
108 \\
98 \\
08\end{array}$ & $\begin{array}{l}34 \\
38 \\
34 \\
54 \\
84 \\
48 \\
38 \\
40 \\
48 \\
49\end{array}$ \\
\hline & & & & & & & & & $\begin{array}{r}35.2 \\
1,960\end{array}$ & $\begin{array}{r}350 \\
20,320\end{array}$ & $\begin{array}{r}63.6 \\
3,200\end{array}$ \\
\hline
\end{tabular}

Gage holght, in feet, and disoharge, In recond-feet, at Indioated time, 1038

\begin{tabular}{|c|c|c|c|c|c|c|c|c|c|c|c|c|}
\hline \multirow{2}{*}{8} & Feet & $800 . \mathrm{ft}_{\mathrm{s}}$ & Fet & seo.ft. & Feet & seo.ft. & Deet & seo.ft. & Feet & $800 . \mathrm{ft}$ & Foet & $800 . \mathrm{ft}$ \\
\hline & Pebx & nasy 27 & \multicolumn{2}{|c|}{ Pobruary 28} & \multicolumn{2}{|c|}{ Maroh 1} & \multicolumn{2}{|c|}{ Maroh 8} & \multicolumn{2}{|c|}{ Maroh 3} & \multicolumn{2}{|c|}{ Waroh 4} \\
\hline $\begin{array}{l}1 \\
8 \\
5 \\
4 \\
5 \\
6\end{array}$ & & & $\begin{array}{l}2.15 \\
2.10 \\
2.05 \\
2.00 \\
2.00 \\
1.98\end{array}$ & $\begin{array}{l}240 \\
205 \\
169 \\
138 \\
138 \\
113\end{array}$ & $\begin{array}{l}2.25 \\
2.30 \\
2.50 \\
3.05 \\
3.35 \\
3.44\end{array}$ & $\begin{array}{r}312 \\
350 \\
496 \\
894 \\
1,160 \\
1,230\end{array}$ & $\begin{array}{l}2.00 \\
2.01 \\
2.08 \\
2.10 \\
2.20 \\
2.30\end{array}$ & $\begin{array}{l}138 \\
143 \\
191 \\
205 \\
278 \\
350\end{array}$ & $\begin{array}{l}5.50 \\
4.50 \\
4.30 \\
4.10 \\
3.95 \\
4.00\end{array}$ & $\begin{array}{l}3,930 \\
2,410 \\
2,150 \\
1,910 \\
1,740 \\
1,800\end{array}$ & & \\
\hline $\begin{array}{r}7 \\
8 \\
9 \\
10 \\
12 \\
11\end{array}$ & 1.45 & 6.6 & $\begin{array}{l}2.00 \\
2.00 \\
2.05 \\
2.08 \\
2.05 \\
2.00\end{array}$ & $\begin{array}{l}138 \\
138 \\
159 \\
169 \\
168 \\
138\end{array}$ & $\begin{array}{l}3.10 \\
2.80 \\
2.70 \\
2.50 \\
2.80 \\
2.40\end{array}$ & $\begin{array}{l}934 \\
706 \\
636 \\
566 \\
496 \\
425\end{array}$ & $\begin{array}{l}2.40 \\
2.60 \\
2.85 \\
3.50 \\
4.50 \\
5.10\end{array}$ & $\begin{array}{r}428 \\
566 \\
741 \\
1,280 \\
2,420 \\
3,300\end{array}$ & $\begin{array}{l}4.10 \\
4.20 \\
5.70 \\
5.75 \\
4.70 \\
4.00\end{array}$ & $\begin{array}{l}1,910 \\
2,030 \\
4,270 \\
4,360 \\
2,680 \\
1,800\end{array}$ & & \\
\hline $\begin{array}{l}2 \\
8 \\
5 \\
4 \\
8 \\
8\end{array}$ & $\begin{array}{l}1.56 \\
1.65 \\
1.85 \\
2.35\end{array}$ & $\begin{array}{r}14 \\
26 \\
72 \\
388\end{array}$ & $\begin{array}{l}2.00 \\
2.10 \\
2.20 \\
2.30 \\
2.20 \\
2.15\end{array}$ & $\begin{array}{l}138 \\
205 \\
276 \\
350 \\
276 \\
240\end{array}$ & $\begin{array}{l}2.30 \\
2.20 \\
2.17 \\
2.14 \\
2.10 \\
2.10\end{array}$ & $\begin{array}{l}350 \\
276 \\
254 \\
235 \\
205 \\
205\end{array}$ & $\begin{array}{l}4.40 \\
4.10 \\
3.80 \\
3.80 \\
3.75 \\
3.85\end{array}$ & $\begin{array}{l}2,290 \\
1,800 \\
1,690 \\
1,580 \\
1,520 \\
1,680\end{array}$ & $\begin{array}{l}3.76 \\
3.88 \\
3.68 \\
3.88 \\
3.83 \\
3.49\end{array}$ & $\begin{array}{l}1,820 \\
1,480 \\
1,390 \\
1,350 \\
1,300 \\
1,240\end{array}$ & & \\
\hline $\begin{array}{r}7 \\
8 \\
9 \\
10 \\
11 \\
1\end{array}$ & $\begin{array}{l}3.20 \\
3.10 \\
2.75 \\
2.35 \\
2.25 \\
2.20\end{array}$ & $\begin{array}{r}1,010 \\
934 \\
671 \\
388 \\
512 \\
278\end{array}$ & $\begin{array}{l}2.10 \\
2.15 \\
2.11 \\
2.15 \\
2.10 \\
2.13\end{array}$ & $\begin{array}{l}205 \\
226 \\
212 \\
240 \\
208 \\
226\end{array}$ & $\begin{array}{l}2.05 \\
2.00 \\
2.00 \\
2.00 \\
2.00 \\
2.00\end{array}$ & $\begin{array}{l}168 \\
138 \\
138 \\
138 \\
138 \\
138\end{array}$ & $\begin{array}{l}4.10 \\
4.70 \\
5.16 \\
5.70 \\
6.85 \\
6.00\end{array}$ & $\begin{array}{l}1,910 \\
2,680 \\
3,380 \\
4,870 \\
6,540 \\
4,840\end{array}$ & $\begin{array}{l}3.45 \\
3.40 \\
3.35 \\
3.31 \\
3.28 \\
3.23\end{array}$ & $\begin{array}{l}1,230 \\
1,180 \\
1,140 \\
1,100 \\
1,080 \\
1,080\end{array}$ & & \\
\hline
\end{tabular}

Supplemental reoord. - Mar. 2, $11: 20$ p.m., $7.30 \mathrm{ft}$, , 8,000 ve0.-ft. 
San Dieguito River at Lake Hodges, Calif.

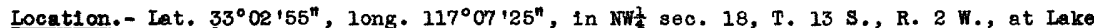
Hodfes Dam, 5t miles southest of Escondido. Altitude of stream bed, 200 feet.

Drainage area.- Area, 303 square miles. Average altitude, 1,690 feot. Maximum altitude, 4,I32 feet. Average slope, 38 percent. Iength of main stream channel, 37

miles. Average slope of main stream channe1, 1.8 percent.

Gege-height recora.- Staff gage read dally at 7 a.m."

Remarks.- Records of discharge represent flow into Lake Hodges, computed from discharge over spillway, change in contents, leakage, evaporation, and direct rainfall on surface of lake. Altitude of spilivay, 315 feet. Storage capac1ty, 37,530 acrefeet. Additional storage obtained by placing sandbags on spillway. Divergions for irrigation in San Pasquel Valley above Lake Hodgos. Record furnishod by City of San Diego Water Department.

Gage height, in feet, and contents, in acre-fe日t, 1938

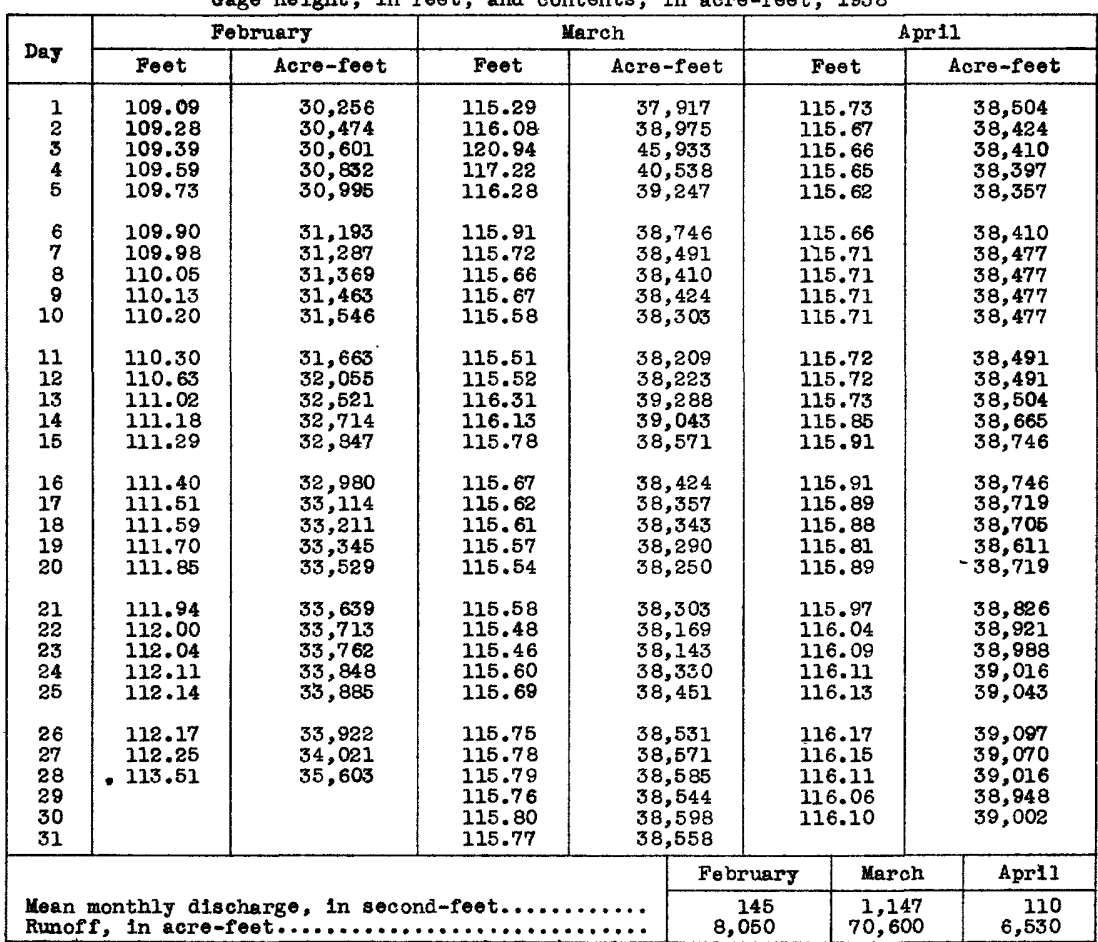

San Luis Rey River Basin

San Luls Rey River nsar. Bonsa11, Cal1f.

Location. - Water-stage recorder, 1at. $33^{\circ} 15^{\prime} 05^{\prime \prime}$, long. $117^{\circ} 14^{\prime} 55^{\prime \prime}$, in $\mathrm{NEt}$ sec. 1, T. IIS., R. 4 W., three-quarters of a mile downstream from highway bridge on FallbrookEscondido road, and 3 miles southwest of Bonsall. Altitude, about 120 feet.

Drainaze area.- Area, 514 square miles. Average altitude, 2,510 feet. Maximum altitude, 6,405 feet. Average slope, 25 percent. Iength of main stream channel, 58 miles. Average slope of main stream channe1, 2.1 percent.

Gage-helght record. - Water-stage recorder graph.

Discharge record.- Stage-discharge relation defined by current-meter measurements beI0 2,360 second-feet; extended to peak stage on basis of mean depth-mean velocity relation.

Maxima.- 1938: Discharge, 18,100 second-feet 1 a.m. Mar. 3 (gage he1ght, 12.60 feet). 1912-18, 1929-37: Discharge, 16,700 second-feet seb. 7, 1937.

Remarks.- Records good. Regulation at lake Henshaw, 41 miles upstream. 
Mean daily discharge, in second-feet, 1938

\begin{tabular}{|c|c|c|c|c|c|c|c|c|c|c|c|}
\hline Day & Feb. & Mar. & Apr. & Day & Feb. & Mar. & Apr. & Day & Feb. & Mar. & Apr. \\
\hline $\begin{array}{r}1 \\
2 \\
3 \\
4 \\
5 \\
6 \\
7 \\
8 \\
9 \\
10\end{array}$ & $\begin{array}{r}58 \\
48 \\
43 \\
81 \\
112 \\
67 \\
51 \\
47 \\
50 \\
51\end{array}$ & $\begin{array}{r}2,060 \\
4,040 \\
9,660 \\
2,180 \\
1,110 \\
757 \\
608 \\
591 \\
475 \\
394\end{array}$ & $\begin{array}{r}130 \\
118 \\
112 \\
106 \\
108 \\
99 \\
87 \\
81 \\
78 \\
76\end{array}$ & $\begin{array}{l}11 \\
12 \\
13 \\
14 \\
15 \\
16 \\
17 \\
18 \\
19 \\
20\end{array}$ & $\begin{array}{r}64 \\
106 \\
78 \\
58 \\
53 \\
56 \\
50 \\
43 \\
53 \\
54\end{array}$ & $\begin{array}{r}345 \\
1,310 \\
1,140 \\
739 \\
505 \\
439 \\
401 \\
341 \\
294 \\
266\end{array}$ & $\begin{array}{r}72 \\
76 \\
-100 \\
102 \\
89 \\
85 \\
81 \\
74 \\
71 \\
71\end{array}$ & $\begin{array}{l}21 \\
22 \\
23 \\
24 \\
25 \\
26 \\
27 \\
28 \\
29 \\
30 \\
31\end{array}$ & $\begin{array}{r}43 \\
42 \\
42 \\
38 \\
36 \\
35 \\
116 \\
\mathrm{a} 504\end{array}$ & $\begin{array}{l}246 \\
229 \\
193 \\
172 \\
178 \\
190 \\
164 \\
159 \\
162 \\
148 \\
136 \\
\end{array}$ & $\begin{array}{l}69 \\
67 \\
64 \\
64 \\
89 \\
81 \\
71 \\
67 \\
67 \\
81\end{array}$ \\
\hline \multicolumn{10}{|c|}{ 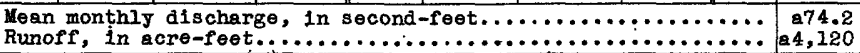 } & $\begin{array}{r}956 \\
58,770\end{array}$ & $\begin{array}{r}84.5 \\
5,030\end{array}$ \\
\hline
\end{tabular}

a Revised; supersedes flgures published in Water-Supply Paper 861, p. 24.

Gage helght, In feet, and discharge, in second-feet, at indicated time, 1938

\begin{tabular}{|c|c|c|c|c|c|c|c|c|c|c|c|c|c|c|}
\hline \multirow{2}{*}{ ริ } & Feet & $\begin{array}{c}\text { Sec.- } \\
\text { ft. }\end{array}$ & Feet & $\begin{array}{c}\text { Sec. } \\
\text { ft. }\end{array}$ & Feet & $\begin{array}{c}\text { sec. } \\
\text { ft. }\end{array}$ & Feet & $\begin{array}{c}\text { sec. } \\
\text { ft. }\end{array}$ & Feet & $\begin{array}{c}\text { Sec. } \\
\text { ft. }\end{array}$ & Feet & $\mid \begin{array}{c}\text { Sec.- } \\
f t .\end{array}$ & Feet & {$\left[\begin{array}{c}\text { Sec. - } \\
\mathrm{ft} .\end{array}\right.$} \\
\hline & \multicolumn{2}{|c|}{$F \in b .27$} & \multicolumn{2}{|c|}{$F \in b .28$} & \multicolumn{2}{|c|}{ Mar. 1} & \multicolumn{2}{|c|}{ Mar. 2} & \multicolumn{2}{|c|}{ Mar. 3} & \multicolumn{2}{|c|}{ Mar. 4} & \multicolumn{2}{|c|}{ Mar. 5} \\
\hline $\begin{array}{l}1 \\
2 \\
3 \\
4 \\
5 \\
6\end{array}$ & $>0.40$ & 45 & $\begin{array}{l}1.57 \\
1.48 \\
1.45 \\
1.42 \\
1.55 \\
1.70\end{array}$ & $\begin{array}{l}365 \\
329 \\
317 \\
305 \\
357 \\
421\end{array}$ & $\begin{array}{l}2.35 \\
2.90 \\
3.50 \\
3.97 \\
4.50 \\
4.34\end{array}$ & $\begin{array}{r}751 \\
1,110 \\
1,580 \\
2,030 \\
2,560 \\
2,400\end{array}$ & $\begin{array}{l}2.75 \\
2.65 \\
2.75 \\
2.75 \\
2.86 \\
3.00\end{array}$ & $\begin{array}{r}994 \\
929 \\
994 \\
994 \\
1,070 \\
1,170\end{array}$ & $\begin{array}{l}12.60 \\
12.25 \\
11.75 \\
11.50 \\
11.45 \\
11.0\end{array}$ & $\begin{array}{l}18,100 \\
17,200 \\
15,900 \\
15,400 \\
15,200 \\
14,000\end{array}$ & $\begin{array}{l}4.95 \\
4.63 \\
4.65 \\
4.52 \\
4.4 \\
4.32\end{array}$ & $\begin{array}{l}3,050 \\
2,660 \\
2,680 \\
2,540 \\
2,420 \\
2,340\end{array}$ & $\begin{array}{l}3.33 \\
3.17 \\
3.2\end{array}$ & $\begin{array}{l}1,390 \\
1,260 \\
1,280\end{array}$ \\
\hline $\begin{array}{r}7 \\
8 \\
9 \\
10 \\
11 \\
\text { N }\end{array}$ & & & $\begin{array}{l}1.78 \\
1.81 \\
1.81 \\
1.80 \\
1.80 \\
1.95\end{array}$ & $\begin{array}{l}457 \\
470 \\
470 \\
466 \\
466 \\
540\end{array}$ & $\begin{array}{l}4.47 \\
5.27 \\
5.31 \\
5.07 \\
4.72 \\
4.34\end{array}$ & $\begin{array}{l}2,530 \\
3,520 \\
3,560 \\
3,250 \\
2,820 \\
2,400\end{array}$ & $\begin{array}{l}3.15 \\
3.50 \\
3.75 \\
4.23 \\
4.48 \\
4.58\end{array}$ & $\begin{array}{l}1,270 \\
1,570 \\
1,790 \\
2,270 \\
2,520 \\
2,620\end{array}$ & $\begin{array}{c}10.5 \\
10.1 \\
9.65 \\
9.25 \\
8.85 \\
8.7\end{array}$ & $\begin{array}{r}12,900 \\
12,000 \\
10,900 \\
10,100 \\
9,340 \\
9,040\end{array}$ & $\begin{array}{l}4.35 \\
4.21 \\
4.37 \\
4.26 \\
4.23 \\
4.35\end{array}$ & $\begin{array}{l}2,370 \\
2,230 \\
2,390 \\
2,280 \\
2,250 \\
2,370\end{array}$ & $\begin{array}{l}3.05 \\
3.05 \\
2.95\end{array}$ & $\left\{\begin{array}{l}1,170 \\
1,170 \\
1,100\end{array}\right.$ \\
\hline $\begin{array}{l}1 \\
2 \\
3 \\
4 \\
5 \\
6\end{array}$ & .62 & 81 & $\begin{array}{l}2.06 \\
2.13 \\
2.09 \\
2.04 \\
2.09 \\
2.06\end{array}$ & $\begin{array}{l}597 \\
635 \\
612 \\
586 \\
612 \\
597\end{array}$ & $\begin{array}{l}4.07 \\
4.17 \\
4.41 \\
4.40 \\
4.17 \\
3.90\end{array}$ & $\begin{array}{l}2,130 \\
2,230 \\
2,470 \\
2,460 \\
2,230 \\
1,960\end{array}$ & $\begin{array}{l}4.88 \\
5.73 \\
6.32 \\
6.48 \\
6.58 \\
6.63\end{array}$ & $\begin{array}{l}2,990 \\
3,990 \\
4,950 \\
5,180 \\
5,330 \\
5,400\end{array}$ & $\begin{array}{l}8.55 \\
8.15 \\
7.8 \\
7.5 \\
7.0 \\
6.6\end{array}$ & $\begin{array}{l}8, \\
7 \\
7 \\
6, \\
5 \\
5\end{array}$ & $\begin{array}{l}28 \\
17 \\
07 \\
93 \\
88 \\
90\end{array}$ & $\begin{array}{l}2,300 \\
2,190 \\
2,090 \\
1,950 \\
1,900 \\
1,920\end{array}$ & $\begin{array}{l}2.8 \\
2.75 \\
2.76\end{array}$ & $\begin{array}{l}993 \\
961 \\
961\end{array}$ \\
\hline $\begin{array}{r}7 \\
8 \\
9 \\
10 \\
11 \\
11\end{array}$ & $\begin{array}{l}1.14 \\
1.50 \\
1.77 \\
1.75\end{array}$ & $\begin{array}{l}156 \\
211 \\
337 \\
452 \\
444\end{array}$ & $\begin{array}{l}2.07 \\
2.06 \\
2.05 \\
2.05 \\
2.07 \\
2.06\end{array}$ & $\begin{array}{l}602 \\
597 \\
590 \\
590 \\
602 \\
597\end{array}$ & $\begin{array}{l}3.60 \\
3.45 \\
3.15 \\
3.00 \\
2.80 \\
2.80\end{array}$ & $\begin{array}{l}1,660 \\
1,550 \\
1,290 \\
1,180 \\
1,040 \\
1,040\end{array}$ & $\begin{array}{r}6.88 \\
7.53 \\
7.88 \\
8.83 \\
10.40 \\
12.00\end{array}$ & $\begin{array}{r}5,780 \\
6,850 \\
7,540 \\
9,340 \\
12,700 \\
16,600\end{array}$ & $\begin{array}{l}6.15 \\
5.85 \\
5.6 \\
5.35 \\
5.15 \\
5.1\end{array}$ & $\begin{array}{l}4,650 \\
4,220 \\
3,880 \\
3,550 \\
3,310 \\
3,250\end{array}$ & $\begin{array}{l}3.68 \\
3.65 \\
3.5 \\
3.5 \\
3.5 \\
3.45\end{array}$ & $\begin{array}{l}1,700 \\
1,670 \\
1,550 \\
1,550 \\
1,550 \\
1,510\end{array}$ & $\begin{array}{l}2.75 \\
2.80 \\
2.75\end{array}$ & $\begin{array}{l}954 \\
987 \\
954\end{array}$ \\
\hline
\end{tabular}

San Luis Rey River at Oceanside, Cal1f.

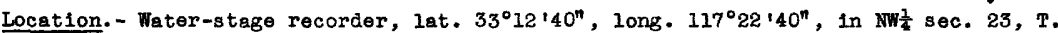
11 S., R. 5 W., half a mile above State highway bridge at Oceanside. Altitude, about 20 feet.

Drainage area.- Area, 557 square miles. Average altitude, 2,334 feet. Maximum altitude, 6,400 feet. Average slope, 33 percent. Length of main stream channel, 62.5 miles. Average slope of main stream channel, 1.9 percent.

Gege-height record.- Water-stage recorder graph.

Discharge record. - Stage-discharge relation defined by ourrent-meter measurements below 2,320 second-feet; extended to peak stage on besis of mean depth-mean velocity relation. Discharge computed by shifting-control method and based in part on comparison with discharge of San Luis Rey River near Bonsall.

Maxima.- 1938: Discharge, 16,500 second-feet 5:30 8.m. Mar. 3 (gage height, 10.77 feet).

1912-14, 1916, 1929-37: Discharge, 95,600 second-feet Jan. 12, 1916.

Remarks.- Records poor, due to shifting control. Storage at Lake Henshaw; numerous diversions above station. 
Hean dally discharge, in second-feet, 1938

\begin{tabular}{|c|c|c|c|c|c|c|c|c|c|c|c|}
\hline Day & Feb. & Mar. & Apr. & Day & Feb. & Mar. & Apr. & Day & Feb. & Mar. & Apr. \\
\hline $\begin{array}{r}1 \\
2 \\
3 \\
4 \\
5 \\
6 \\
7 \\
8 \\
9 \\
10\end{array}$ & $\begin{array}{l}38 \\
44 \\
38 \\
51 \\
85 \\
70 \\
47 \\
40 \\
38 \\
44\end{array}$ & $\begin{array}{r}2,000 \\
2,860 \\
11,300 \\
2,650 \\
1,480 \\
1,060 \\
798 \\
674 \\
568 \\
465\end{array}$ & $\begin{array}{r}122 \\
113 \\
113 \\
110 \\
117 \\
117 \\
98 \\
80 \\
66 \\
64\end{array}$ & $\begin{array}{l}11 \\
12 \\
13 \\
14 \\
15 \\
16 \\
17 \\
18 \\
19 \\
20\end{array}$ & $\begin{array}{l}52 \\
81 \\
88 \\
65 \\
55 \\
54 \\
57 \\
52 \\
55 \\
61\end{array}$ & $\begin{array}{r}390 \\
1,160 \\
1,140 \\
613 \\
462 \\
402 \\
421 \\
421 \\
39 I \\
330\end{array}$ & $\begin{array}{r}66 \\
69 \\
91 \\
110 \\
95 \\
87 \\
77 \\
69 \\
61 \\
53\end{array}$ & $\begin{array}{l}21 \\
22 \\
23 \\
24 \\
25 \\
26 \\
27 \\
28 \\
29 \\
30 \\
31\end{array}$ & $\begin{array}{r}55 \\
46 \\
38 \\
35 \\
34 \\
32 \\
57 \\
347\end{array}$ & $\begin{array}{l}301 \\
262 \\
213 \\
193 \\
166 \\
200 \\
161 \\
151 \\
161 \\
156 \\
137\end{array}$ & $\begin{array}{r}51 \\
51 \\
51 \\
53 \\
84 \\
102 \\
69 \\
53 \\
51 \\
61\end{array}$ \\
\hline \multicolumn{9}{|c|}{ 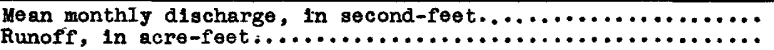 } & 3,490 & $\begin{array}{r}1,022 \\
62,850\end{array}$ & $\begin{array}{r}80.1 \\
4,770\end{array}$ \\
\hline
\end{tabular}

Gage height, in feet, and discharge, in second-feet, at indicated time, I938

\begin{tabular}{|c|c|c|c|c|c|c|c|c|c|c|c|c|}
\hline \multirow{2}{*}{$\begin{array}{l}5 \\
\\
\end{array}$} & Feet & sec.ft. & Feet & Sec.ft. & Feet & sec.ft. & Feet & sec.ft. & Feet & sec.ft. & Feet & Sec.ft. \\
\hline & \multicolumn{2}{|c|}{ February 27} & \multicolumn{2}{|c|}{ February 28} & \multicolumn{2}{|c|}{ March I } & \multicolumn{2}{|c|}{ March 2} & \multicolumn{2}{|c|}{ March 3} & \multicolumn{2}{|c|}{ March 4} \\
\hline $\begin{array}{l}1 \\
2 \\
3 \\
4 \\
5 \\
6\end{array}$ & & & $\begin{array}{l}4.4 I \\
4.48 \\
4.70 \\
4.94 \\
5.01 \\
4.99\end{array}$ & $\begin{array}{l}I I 4 \\
136 \\
210 \\
310 \\
345 \\
340\end{array}$ & $\begin{array}{l}5.27 \\
5.38 \\
5.45 \\
5.47 \\
5.97 \\
6.35\end{array}$ & $\begin{array}{r}517 \\
587 \\
640 \\
656 \\
I, 070 \\
1,500\end{array}$ & $\begin{array}{l}5.74 \\
5.75 \\
5.77 \\
5.77 \\
5.80 \\
5.82\end{array}$ & $\begin{array}{r}I, 250 \\
I, I 60 \\
I, I 40 \\
I, 070 \\
I, 010 \\
938\end{array}$ & $\begin{array}{r}9.10 \\
9.77 \\
10.18 \\
10.40 \\
10.74 \\
10.45\end{array}$ & $\begin{array}{l}11,200 \\
13,100 \\
14,400 \\
15,200 \\
16,400 \\
16,000\end{array}$ & & $\begin{array}{l}4,300 \\
3,800 \\
3,600 \\
3,400 \\
3,300 \\
3,100\end{array}$ \\
\hline $\begin{array}{r}7 \\
8 \\
9 \\
10 \\
11 \\
N\end{array}$ & & & $\begin{array}{l}4.94 \\
4.85 \\
4.82 \\
4.83 \\
4.91 \\
4.97\end{array}$ & $\begin{array}{l}315 \\
274 \\
270 \\
265 \\
305 \\
335\end{array}$ & $\begin{array}{l}6.52 \\
6.55 \\
6.56 \\
6.84 \\
6.93 \\
6.91\end{array}$ & $\begin{array}{l}I, 710 \\
1,750 \\
I, 890 \\
2,460 \\
2,960 \\
3,300\end{array}$ & $\begin{array}{l}5.91 \\
6.04 \\
6.16 \\
6.42 \\
6.57 \\
6.72\end{array}$ & $\begin{array}{l}1,019 \\
1,150 \\
1,280 \\
1,590 \\
1,770 \\
1,980\end{array}$ & $\begin{array}{l}9.95 \\
9.58 \\
8.97 \\
8.65 \\
8.22 \\
7.98\end{array}$ & $\begin{array}{l}15,500 \\
14,900 \\
14,100 \\
13,900 \\
13,300 \\
12,500\end{array}$ & & $\begin{array}{l}2,920 \\
2,800 \\
2,600 \\
2,500 \\
2,430 \\
2,400\end{array}$ \\
\hline $\begin{array}{l}1 \\
2 \\
3 \\
4 \\
5 \\
6\end{array}$ & & & $\begin{array}{l}5.02 \\
5.03 \\
5.04 \\
5.07 \\
5.10 \\
5.19\end{array}$ & $\begin{array}{l}360 \\
365 \\
376 \\
393 \\
409 \\
463\end{array}$ & $\begin{array}{l}6.75 \\
6.55 \\
6.34 \\
6.26 \\
6.36 \\
6.37\end{array}$ & $\begin{array}{l}3,180 \\
2,990 \\
2,610 \\
2,490 \\
2,640 \\
2,650\end{array}$ & $\begin{array}{l}6.83 \\
6.82 \\
6.82 \\
7.17 \\
7.48 \\
7.55\end{array}$ & $\begin{array}{l}2,140 \\
2,120 \\
2,400 \\
3,150 \\
3,960 \\
4,400\end{array}$ & $\begin{array}{l}7.70 \\
7.40 \\
7.10 \\
6.70 \\
6.51 \\
6.26\end{array}$ & $\begin{array}{r}11,900 \\
10,900 \\
10,000 \\
9,800 \\
9,600 \\
8,800\end{array}$ & .76 & 2,320 \\
\hline $\begin{array}{r}7 \\
8 \\
9 \\
10 \\
11 \\
M\end{array}$ & & & $\begin{array}{l}5.20 \\
5.22 \\
5.22 \\
5.26 \\
5.25 \\
5.22\end{array}$ & $\begin{array}{l}475 \\
487 \\
487 \\
511 \\
505 \\
487\end{array}$ & $\begin{array}{l}6.28 \\
6.16 \\
6.00 \\
5.85 \\
5.78 \\
5.77\end{array}$ & $\begin{array}{l}2,520 \\
2,340 \\
2,090 \\
1,880 \\
1,660 \\
1,520\end{array}$ & $\begin{array}{l}7.64 \\
7.70 \\
7.78 \\
8.00 \\
8.30 \\
8.65\end{array}$ & $\begin{array}{l}4,910 \\
5,400 \\
5,960 \\
6,850 \\
8,000 \\
9,450\end{array}$ & $\begin{array}{l}5.94 \\
5.68 \\
5.48 \\
5.31 \\
5.17 \\
5.11\end{array}$ & $\begin{array}{l}8,000 \\
7,190 \\
6,400 \\
5,800 \\
5,200 \\
4,600\end{array}$ & \}$_{4.55}$ & 2,050 \\
\hline
\end{tabular}

Supplemental record.- Mar. 3, 5:30 a.m., I0.77 ft., I6,500 sec.-ft.

\section{Santa Margarita River Basin}

Temecula Creek at Nigger Canyon, near Temecula, Calif.

Location. - Water-stage recorder, Iat. $33^{\circ} 29^{1} 40^{n}$, Iong. $116^{\circ} 59^{\prime} 00^{n}$, in Pauba grant, at upper end of Nigger Canjon, a quarter of a mile downstream from Arrojo Seco, ana 10 miles east of temecula, Riverside County. Altitude, about 1,350 feet.

Drainage area.- Area, 319 square miles. Average altitude, 3,400 feet. Maximum altitude, 6,200 feet. Average slope, 30 percent. Length of main stream channeI, 26.5 miles. Average siope of main stream channeI, 3.4 percent.

Gage-height record.- Water-stage reccrder graph, except Mar. 4 to 3 p.m. Mar. 6.

Discharge record. - Discharge estimated from hydrograph defined by current-meter measurements, gage-helght record, and comparison with adjacent stations.

Maxime.- I938: Discharge, 14,600 second-feet 10 p.m. Mar. 2, (gage he1ght, 11.27 feet). 1923-37: Discharge, I7, 100 second-feet Feb. 16, 1927 .

Remarks.- Records poor, due to extreme shifting of channel at high stages. No diversions above station. Results of some discharge measurements furnished by santa Margarita Ranch.

$4547200-42-14$ 
Mean dally discharge, in second-feet, 1938

\begin{tabular}{|c|c|c|c|c|c|c|c|c|c|c|c|}
\hline Day & Feb. & Mar. & Apr. & Day & Feb. & Mar. & Apr. & Day & Feb. & Mar. & Apr. \\
\hline $\begin{array}{r}1 \\
2 \\
3 \\
4 \\
5 \\
6 \\
7 \\
8 \\
9 \\
10\end{array}$ & $\begin{array}{l}23 \\
22 \\
35 \\
95 \\
50 \\
28 \\
18 \\
17 \\
20 \\
24\end{array}$ & $\begin{array}{r}180 \\
4,100 \\
3,500 \\
1,300 \\
400 \\
140 \\
125 \\
115 \\
95 \\
90\end{array}$ & $\begin{array}{l}42 \\
39 \\
44 \\
34 \\
30 \\
30 \\
30 \\
30 \\
30 \\
36\end{array}$ & $\begin{array}{l}11 \\
12 \\
13 \\
14 \\
15 \\
16 \\
17 \\
18 \\
19 \\
20\end{array}$ & $\begin{array}{l}48 \\
79 \\
69 \\
65 \\
62 \\
55 \\
46 \\
41 \\
43 \\
33\end{array}$ & $\begin{array}{r}95 \\
235 \\
195 \\
180 \\
155 \\
115 \\
115 \\
110 \\
80 \\
60\end{array}$ & $\begin{array}{l}37 \\
38 \\
38 \\
36 \\
33 \\
29 \\
28 \\
26 \\
28 \\
27\end{array}$ & $\begin{array}{l}21 \\
22 \\
23 \\
24 \\
25 \\
26 \\
27 \\
28 \\
29 \\
30 \\
31\end{array}$ & $\begin{array}{l}25 \\
21 \\
16 \\
16 \\
16 \\
16 \\
35 \\
50\end{array}$ & $\begin{array}{l}60 \\
60 \\
65 \\
62 \\
65 \\
68 \\
55 \\
50 \\
55 \\
48 \\
41 \\
\end{array}$ & $\begin{array}{l}27 \\
26 \\
25 \\
23 \\
30 \\
18 \\
17 \\
19 \\
26 \\
30\end{array}$ \\
\hline \multicolumn{9}{|c|}{ 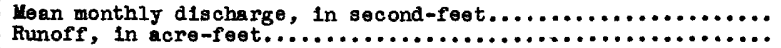 } & $\begin{array}{r}38.1 \\
2,120\end{array}$ & $\begin{array}{r}388 \\
23,830\end{array}$ & $\begin{array}{r}30.2 \\
1,800\end{array}$ \\
\hline
\end{tabular}

Temecula Creek at Railroad Canyon, near Temecula, Calif.

Location. - Water-atage recorder, lat. $33^{\circ} 28^{\prime} 25^{\prime \prime}$, long. $117^{\circ} 08^{\prime} 35^{\prime \prime}$, In Temecula grant, at upper end of Temecula or Rallroad Canyon, an elghth of a mile downtream from Junction with Murrieta Creek, and lt miles south of Temecula, Riverside County. Altitude, about 950 feet.

Drainage area.-Area, 592 square miles. Average altitude, 2,663 fe日t. Maximm altitude, 6,200 feet. Average slope, 26 percent. Length of main stream channel, 41 miles. Average slope of main stream channel, 2.4 percent.

Gage-helght record.- Water-stage recorder graph.

Discharge record. - Stage-discharge relation defined by current-meter measurements below 9,220 second-feet. Discharge estimated from hydrograph based on current-meter measurements and gage-height record, and by couparison with adjacent stations.

Maxima-- 1938: Discharge, 21,700 second-feet (estimated) 10 p.m. Mar. 2 (gege helght, 13.91 feet)

1923-37: D1scharge, about 27,600 second-feet Feb. 16, 1927.

Remarks.- Records poor, due to extreme ahifting of chenneI. Flood flow not affected by diversions. Gage-helght record and results of discharge measurements furnished by Santa Margarita Ranch.

Mean dally disciarge, in second-feet, 1938

\begin{tabular}{|c|c|c|c|c|c|c|c|c|c|c|c|}
\hline Day & Feb. & Mar. & Apr. & Day & Feb. & Mar. & Apr. & Day & Feb. & Mar. & Apr. \\
\hline $\begin{array}{r}1 \\
2 \\
3 \\
4 \\
5 \\
6 \\
7 \\
8 \\
9 \\
10\end{array}$ & $\begin{array}{l}18 \\
11 \\
13 \\
27 \\
18 \\
12 \\
11 \\
11 \\
19 \\
23\end{array}$ & $\begin{array}{r}2,620 \\
11,500 \\
8,000 \\
1,700 \\
950 \\
320 \\
250 \\
350 \\
220 \\
190\end{array}$ & $\begin{array}{l}60 \\
59 \\
58 \\
50 \\
51 \\
50 \\
44 \\
41 \\
46 \\
40\end{array}$ & $\begin{array}{l}11 \\
12 \\
13 \\
14 \\
15 \\
16 \\
17 \\
18 \\
19 \\
20\end{array}$ & $\begin{array}{l}42 \\
46 \\
25 \\
19 \\
15 \\
12 \\
12 \\
13 \\
21 \\
18\end{array}$ & $\begin{array}{r}180 \\
1,810 \\
740 \\
360 \\
290 \\
210 \\
200 \\
160 \\
120 \\
115\end{array}$ & $\begin{array}{l}35 \\
40 \\
61 \\
54 \\
47 \\
31 \\
28 \\
25 \\
25 \\
25\end{array}$ & $\begin{array}{l}21 \\
22 \\
23 \\
24 \\
25 \\
26 \\
27 \\
28 \\
29 \\
30 \\
31\end{array}$ & $\begin{array}{r}16 \\
14 \\
12 \\
12 \\
13 \\
14 \\
130 \\
450\end{array}$ & $\begin{array}{r}100 \\
92 \\
98 \\
90 \\
90 \\
90 \\
82 \\
73 \\
78 \\
70 \\
63\end{array}$ & $\begin{array}{l}32 \\
29 \\
28 \\
27 \\
35 \\
27 \\
21 \\
27 \\
30 \\
44\end{array}$ \\
\hline \multicolumn{12}{|c|}{ 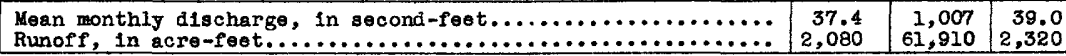 } \\
\hline
\end{tabular}

Santa Margarita River near Fall Brook, Calif.

Location. - Water-atage recorder, lat. $33^{\circ} 24^{\prime} 05^{n}$, long. $117^{\circ} 15^{\prime} 10^{n}$, in sec. 12, T. 9 S., R. 4 H., 2 miles north of Fali Brook. Aititude, about 350 feet.

Drainage area.- Area, 645 square miles. Average altitude, 2,54l feet. Maximum altitude, 6,200 feet. Average slope, 33 percent. Length of main etream channel, 50 miles. Average slope of main atream channel, 2.2 percent.

Gage-helght record. - Water-stage recorder graph.

Discharge record. - Discharge estimated frow hydrograph based on current-metex measurements and gage-helght record, and by comparison with adjacent stations.

Maxima.- 1938: Discharge, 28,000 second-feet (eatimated) 11 p.m. Mar. 2 (gege he1pht, 14.0 feet).

1924-37: Discharge, about 33,100 second-feet Feb. 16, 1927.

Remarks.- Records poor, due to extreme shifting of channel. No diversions during flood period. Resuits of discharge measurements furnished by Santa Margarita Ranch. 
Mean da11y disohnere, in geoond-feet, 1938

\begin{tabular}{|c|c|c|c|c|c|c|c|c|c|c|c|}
\hline Day & Beb. & Har. & Apr. & Day & Fob. & Mar. & Apr. & Day & Fob. & Nar. & Apr. \\
\hline $\begin{array}{r}1 \\
2 \\
3 \\
4 \\
5 \\
6 \\
7 \\
8 \\
9 \\
10\end{array}$ & $\begin{array}{l}30 \\
23 \\
20 \\
48 \\
30 \\
21 \\
20 \\
28 \\
40 \\
43\end{array}$ & $\begin{array}{r}3,890 \\
18,000 \\
10,400 \\
2 ; 000 \\
1,000 \\
700 \\
400 \\
480 \\
360 \\
320\end{array}$ & $\begin{array}{l}92 \\
92 \\
90 \\
80 \\
79 \\
76 \\
68 \\
68 \\
70 \\
64\end{array}$ & $\begin{array}{l}11 \\
12 \\
13 \\
14 \\
16 \\
16 \\
17 \\
18 \\
19 \\
80\end{array}$ & $\begin{array}{l}80 \\
88 \\
48 \\
30 \\
86 \\
28 \\
20 \\
28 \\
30 \\
80\end{array}$ & $\begin{array}{r}300 \\
2,140 \\
980 \\
500 \\
480 \\
365 . \\
300 \\
870 \\
240 \\
200\end{array}$ & $\begin{array}{r}65 \\
66 \\
120 \\
88 \\
70 \\
84 \\
84 \\
46 \\
48 \\
44\end{array}$ & $\begin{array}{l}21 \\
22 \\
83 \\
24 \\
25 \\
26 \\
27 \\
28 \\
89 \\
30 \\
31\end{array}$ & $\begin{array}{r}18 \\
18 \\
18 \\
15 \\
14 \\
13 \\
186 \\
580\end{array}$ & $\begin{array}{l}165 \\
155 \\
150 \\
148 \\
140 \\
138 \\
130 \\
185 \\
185 \\
113 \\
110\end{array}$ & $\begin{array}{r}80 \\
45 \\
40 \\
37 \\
58 \\
48 \\
37 \\
48 \\
80 \\
100\end{array}$ \\
\hline \multicolumn{9}{|c|}{ 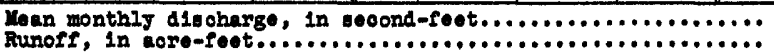 } & $\begin{array}{r}68.7 \\
2.980\end{array}$ & $\begin{array}{r}1,240 \\
76,800\end{array}$ & 3.840 \\
\hline
\end{tabular}

Santa Margarita River at Yaldora, Calif.

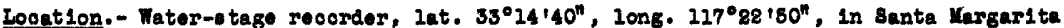
J Lo Flores grant, 1 mile downtream from Yílore, san Diego Counts, and about 2 illes upotream from mouth. Alt1 tude, about 15 feet.

Drainare eres.- Area, 740 cquare miles. Arezage alt1tude, 8,327 feet. Maximum alt1tide, 0,800 feet. Arerage lope, 31 pereent. Iength of min otream ohannel, 65 miles. Average lope of min atreal channel, 1.7 peroent.

Gere-heleht reoond. - Fater-atage reoorder graph.

Dipohayze reoord. - Dieohargo estinated from hydrograph based on ourrent-meter masureinte and gare-helght seoord, and by compario on vith ad jesent tations.

Maxim.- 1938; D1soharge, 31,000 ecoond-feet (eotiunted) 1 a.m. Mar. 3, (gage hoight, 1K.70 feet).

1983-37: D1soharge, 33,600 second-feet (eet1mated) Feb. 16, 1827.

Remarle. - Records poor, due to extreme abifting of ohannel. No diversions during 100d perlod. Gage-helght record and results of disobarge moasurements furnighed by santa Margar1ta Ranch.

Mean da11y discharge, In seoond-feet, 1938

\begin{tabular}{|c|c|c|c|c|c|c|c|c|c|c|c|}
\hline Day & Pob. & Mar. & Apr. & Day & Fob. & Yar. & Apr. & Day & Pob. & Mar. & Apr. \\
\hline $\begin{array}{r}1 \\
8 \\
3 \\
4 \\
8 \\
6 \\
7 \\
8 \\
9 \\
10\end{array}$ & $\begin{array}{l}44 \\
48 \\
35 \\
51 \\
68 \\
45 \\
35 \\
33 \\
58 \\
60\end{array}$ & $\begin{array}{r}6,870 \\
13,000 \\
15,500 \\
3,000 \\
1,600 \\
1,300 \\
840 \\
900 \\
850 \\
390\end{array}$ & $\begin{array}{l}185 \\
117 \\
111 \\
107 \\
104 \\
101 \\
100 \\
98 \\
96 \\
94\end{array}$ & $\begin{array}{l}11 \\
12 \\
13 \\
14 \\
18 \\
16 \\
17 \\
18 \\
18 \\
80\end{array}$ & $\begin{array}{r}80 \\
240 \\
100 \\
80 \\
70 \\
50 \\
43 \\
36 \\
50 \\
65\end{array}$ & $\begin{array}{r}350 \\
2,600 \\
1,600 \\
950 \\
690 \\
550 \\
450 \\
340 \\
330 \\
300\end{array}$ & $\begin{array}{r}98 \\
90 \\
128 \\
138 \\
104 \\
88 \\
83 \\
79 \\
76 \\
74\end{array}$ & $\begin{array}{l}21 \\
82 \\
83 \\
24 \\
28 \\
26 \\
27 \\
88 \\
89 \\
30 \\
31\end{array}$ & $\begin{array}{r}50 \\
40 \\
36 \\
34 \\
30 \\
88 \\
800 \\
975\end{array}$ & $\begin{array}{l}235 \\
280 \\
210 \\
205 \\
190 \\
170 \\
200 \\
205 \\
190 \\
180 \\
130\end{array}$ & $\begin{array}{l}70 \\
60 \\
77 \\
66 \\
80 \\
80 \\
65 \\
85 \\
60 \\
80\end{array}$ \\
\hline & & & & & & & & & $\begin{array}{r}94.8 \\
5,230\end{array}$ & $\begin{array}{r}1,730 \\
106,400\end{array}$ & $\begin{array}{r}89.7 \\
5.340\end{array}$ \\
\hline
\end{tabular}

Mursieta Oreek at Temooule, Calif.

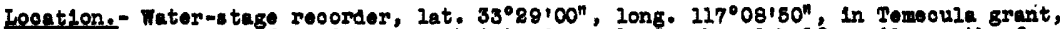
$0.6 \mathrm{mI}$ le upatream from debouohment into Tenecula Creek and half a mile wouth of Temoule, Rivers 1de County. Altitude, about 1,050 feet.

Draince aree.- Area, 280 square m110. Average alt 1tude, 1,710 feet. Maximum alt1tiue, 4,600 feet. Average lope, 23 percent. Iength of mein stream ohunne1, 31 miles. Average lope of maln tream channel, 2.1 peroent.

Gege-he1sht reoord. - Water-atage reoorder graph.

Dilohnse reoord.- Diecharge estimated from hjdrograph based on ourrent-meter neasuremont and Eeg-helght rooord, and by comparison with ad jeoent atatione.

Maxime.- 1938: D1soharge, 16,800 seoond-foet (estimated) 8 p.m. Mar. 2 (gege he1ght, 5.7 feet).

1930-37; Disoharge, 7,800 geoond-feet Fob, 16, 1982.

Remarte.- Reoords poor, due to extreme inteting of ohannel. Gage-holght reoord and teveral disoharge measurements furnished by Santa Margarita Ranoh. 
Mean dally discharge, in second-feet, 1938

\begin{tabular}{|c|c|c|c|c|c|c|c|c|c|c|c|}
\hline Day & Feb. & Mar. & Apr. & Day & Feb. & Mar. & Apr. & Day & Feb. & Mar. & Apr. \\
\hline $\begin{array}{r}1 \\
2 \\
3 \\
4 \\
5 \\
6 \\
7 \\
8 \\
9 \\
10\end{array}$ & $\begin{array}{l}5.0 \\
4.0 \\
2.8 \\
6.0 \\
4.7 \\
2.8 \\
2.4 \\
2.3 \\
7.0 \\
8.0\end{array}$ & $\begin{array}{r}1,870 \\
7,200 \\
3,200 \\
360 \\
120 \\
83 \\
62 \\
85 \\
54 \\
40\end{array}$ & $\begin{array}{r}11 \\
9.0 \\
8.5 \\
8.0 \\
7.5 \\
7.5 \\
6.5 \\
5.5 \\
5.5 \\
5.5\end{array}$ & $\begin{array}{l}11 \\
12 \\
13 \\
14 \\
15 \\
16 \\
17 \\
18 \\
19 \\
20\end{array}$ & $\begin{array}{l}21 \\
24 \\
8.0 \\
4.6 \\
3.9 \\
3.08 \\
3.5 \\
3.5 \\
6.5 \\
4.6\end{array}$ & $\begin{array}{r}33 \\
950 \\
250 \\
105 \\
71 \\
48 \\
45 \\
39 \\
29 \\
25\end{array}$ & $\begin{array}{l}5.0 \\
5.5 \\
7.5 \\
6.5 \\
5.5 \\
5.0 \\
4.9 \\
4.5 \\
4.1 \\
4.0\end{array}$ & $\begin{array}{l}21 \\
22 \\
23 \\
24 \\
25 \\
26 \\
27 \\
28 \\
29 \\
30 \\
31\end{array}$ & $\begin{array}{r}3.5 \\
3.4 \\
3.2 \\
3.2 \\
3.0 \\
3.0 \\
68 \\
320\end{array}$ & $\begin{array}{l}22 \\
20 \\
19 \\
17 \\
17 \\
16 \\
15 \\
13 \\
14 \\
14 \\
13\end{array}$ & $\begin{array}{l}4.1 \\
4.0 \\
3.8 \\
3.8 \\
5.0 \\
4.5 \\
4.1 \\
3.8 \\
3.7 \\
5.0\end{array}$ \\
\hline & & & & & & & & & $\begin{array}{r}10.1 \\
1,060\end{array}$ & $\begin{array}{r}479 \\
29,450\end{array}$ & 5.6 \\
\hline
\end{tabular}

\section{San Juan Creok Basin}

San Juan Cregk near San Juan Caplatwarro, Cal15.

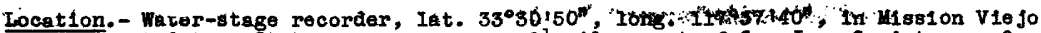
grant, at Ortega State Highway Bridge, $2 \frac{1}{3}$ mlies east of San Juan Cap1strano, Orange County. Altitude, about iso feet.

Drainage area.- Area, 110 square miles. Average altitude, 1,470 feet. Maximum altitude, 4,525 feet. Average slope, 30 percent. Length of matn stream channel, 18 miles. Average slope of main stream channel, 4.6 percent.

Gage-he1ght record. - Water-3tage recorder graph exeept $12 \mathrm{~m}$. Feb. 28 to 2 p.m. Mar. 1 and 10 a.m. lar. 2 to 12 m. Mar. 14.

Discharge record. - Stage-discharge relation defined by current-meter measurements below 436 second-feet; peak discharge determined by slope-area measurement. Reoords from $12 \mathrm{~m}$. Feb. 28 to $2 \mathrm{p.m}$. Mar. 1 and 9 a.m. Mar. 2 to Apr. 30 based on currentmeter measurements, slope-area measurement of peak discharge, and comparison with record of inflow to Santiago Reservoir.

Maxima.- 1938: Discharge, 13,000 seoond-feet about 10 p.m. Mar. 2. 1929-37: Discharge, 8,300 second-feet Feb, 6, 1937.

Remarks.- Reoords poor.

Mean dally discharge, in second-feet, 1938

\begin{tabular}{|c|c|c|c|c|c|c|c|c|c|c|c|}
\hline Day & Feb. & Mar. & Apr. & Day & Feb. & Mar. & Apr. & Dey & Feb. & Mar. & Apr. \\
\hline $\begin{array}{r}1 \\
2 \\
3 \\
4 \\
5 \\
6 \\
7 \\
8 \\
9 \\
20\end{array}$ & $\begin{array}{l}18 \\
10 \\
10 \\
14 \\
9 \\
9 \\
9 \\
8.5 \\
8.5 \\
8.5\end{array}$ & $\begin{array}{r}550 \\
4,100 \\
3,400 \\
1,000 \\
600 \\
410 \\
315 \\
265 \\
265 \\
225\end{array}$ & $\begin{array}{l}77 \\
74 \\
71 \\
68 \\
67 \\
63 \\
61 \\
54 \\
53 \\
52\end{array}$ & $\begin{array}{l}11 \\
12 \\
13 \\
14 \\
15 \\
16 \\
17 \\
18 \\
19 \\
20\end{array}$ & $\begin{array}{l}11 \\
47 \\
1.5 \\
10 \\
8.5 \\
8 \\
7 \\
7.5 \\
7.5 \\
7\end{array}$ & $\begin{array}{l}188 \\
600 \\
540 \\
480 \\
410 \\
330 \\
230 \\
195 \\
183 \\
175\end{array}$ & $\begin{array}{l}51 \\
50 \\
50 \\
50 \\
50 \\
47 \\
44 \\
41 \\
38 \\
39\end{array}$ & $\begin{array}{l}21 \\
22 \\
23 \\
24 \\
25 \\
26 \\
27 \\
28 \\
29 \\
30 \\
31\end{array}$ & $\begin{array}{l}7.5 \\
8 \\
8 \\
8 \\
8 \\
8 \\
10 \\
41\end{array}$ & $\begin{array}{l}170 \\
160 \\
150 \\
139 \\
115 \\
115 \\
112 \\
115 \\
96 \\
85 \\
80\end{array}$ & $\begin{array}{l}41 \\
37 \\
38 \\
39 \\
41 \\
43 \\
44 \\
45 \\
46 \\
46\end{array}$ \\
\hline \multicolumn{9}{|c|}{ 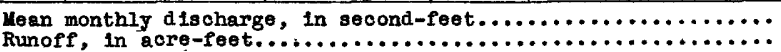 } & $\begin{array}{r}11.8 \\
660\end{array}$ & $\begin{array}{r}509 \\
31,320\end{array}$ & $\begin{array}{r}50.7 \\
3,010\end{array}$ \\
\hline
\end{tabular}

Trabuco Creek near San Juan Capistrano, Calif.

Looation.- Nater-stage recorder, lat. $33^{\circ} 31^{\prime} 30^{n}$, Iong. $117^{\circ} 40^{\prime} 15^{\prime \prime}$, in SWt 800.25 , T.

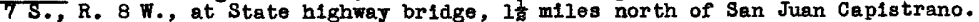
Altitude, 200 feet.

Drainage area.- Area, 36.5 square miles. Average altitude, 1,770 feet. Maximum altitude, 5,680 feet. Average slope, 41 percent. Length of main atream chenne1, 20 miles. Average slope of main stream channel, 5.2 percent.

Gage-he1ght record.- Water-stage recorder greph except Nar. 3-4 owing to silting of we11.

Discharge record. - Stage-discharge relation falriy well defined below 300 second-feet by current-neter measurements, and extended above on bas is of slope-area determ1nations. Discharge for Mar. 3 and 4 was obtalned by comparison with other streams.

Maxima.- 1938: Discharge, 4,400 second-feet Mar. 2. 1930-37: Discharge, 9,240 second-feet Feb. 6, 1937.

Remarks.- Fecords furnished by Orange County Flood Control Distriot, through M. N. Thompson, chief engineer. 
Mean daily discharge, in second-feet, 1938

\begin{tabular}{|c|c|c|c|c|c|c|c|c|c|c|c|}
\hline Day & Fob. & Mar. & Apr. & Day & Peb. & Mar. & Apr. & Day & Feb. & Mar. & Apr. \\
\hline $\begin{array}{r}1 \\
2 \\
3 \\
4 \\
5 \\
6 \\
7 \\
8 \\
9 \\
10\end{array}$ & $\begin{array}{l}0.1 \\
0 \\
0.1 \\
0 \\
0 \\
0 \\
0 \\
0 \\
0 \\
0\end{array}$ & $\begin{array}{r}254 \\
1,480 \\
820 \\
331 \\
245 \\
195 \\
156 \\
113 \\
92 \\
85\end{array}$ & $\begin{array}{l}32 \\
30 \\
29 \\
27 \\
26 \\
24 \\
23 \\
22 \\
21 \\
20\end{array}$ & $\begin{array}{l}11 \\
12 \\
13 \\
14 \\
15 \\
16 \\
17 \\
18 \\
19 \\
20\end{array}$ & $\begin{array}{c}0.1 \\
15 \\
4.0 \\
0 \\
0 \\
0 \\
0 \\
0 \\
0 \\
0\end{array}$ & $\begin{array}{r}79 \\
215 \\
211 \\
182 \\
169 \\
152 \\
138 \\
115 \\
103 \\
92\end{array}$ & $\begin{array}{l}19 \\
18 \\
17 \\
16 \\
15 \\
14 \\
14 \\
13 \\
12 \\
11\end{array}$ & $\begin{array}{l}21 \\
22 \\
23 \\
24 \\
25 \\
26 \\
27 \\
28 \\
29 \\
30 \\
31\end{array}$ & $\begin{array}{r}0 \\
0 \\
0 \\
0 \\
0 \\
0 \\
0 \\
1.5\end{array}$ & $\begin{array}{l}76 \\
64 \\
58 \\
54 \\
51 \\
47 \\
44 \\
40 \\
38 \\
34 \\
33 \\
\end{array}$ & $\begin{array}{l}10 \\
10 \\
10 \\
10 \\
27 \\
19 \\
12 \\
11 \\
12 \\
22\end{array}$ \\
\hline \multicolumn{9}{|c|}{ 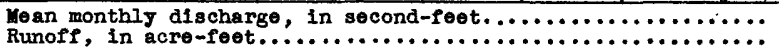 } & $\begin{array}{r}1.23 \\
68\end{array}$ & $\begin{array}{r}186 \\
11,440\end{array}$ & $\begin{array}{r}18.2 \\
1,080\end{array}$ \\
\hline
\end{tabular}

Gage height, in feet, and discharge, in second-reet, at indicated time, 1938

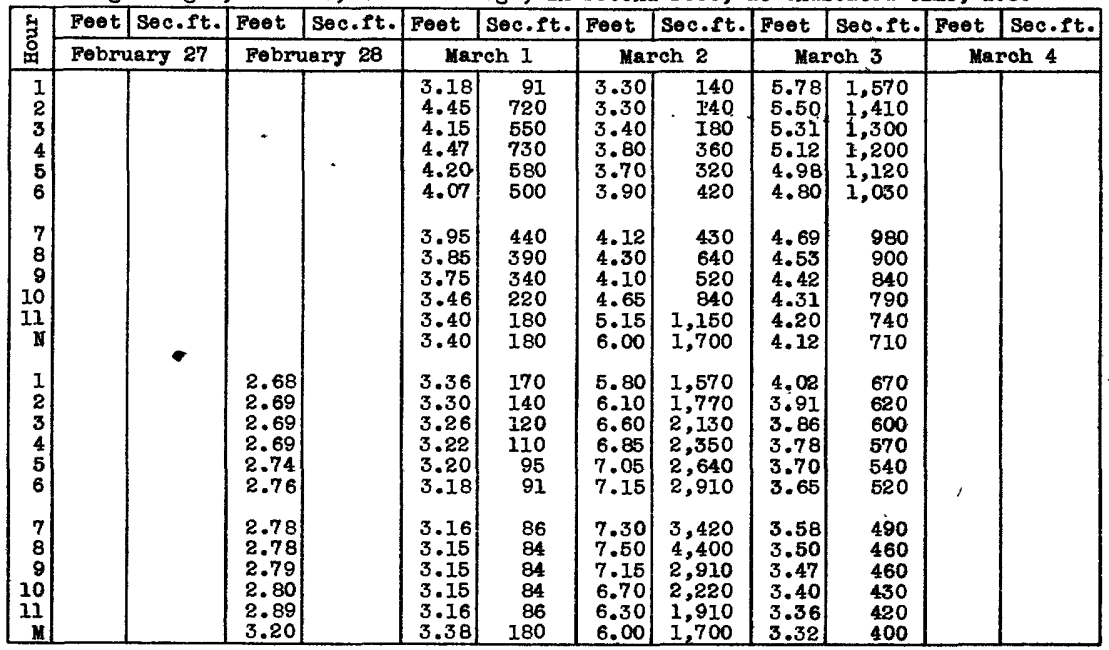

\section{Allso Creok Basin}

Aliso Creok at El Taro, Gallf.

Location. - Water-gtage recorder, lat. $33^{\circ} 37^{\prime} 15^{\prime \prime}$, long. $127^{\circ} 41^{\prime} 20^{n}$, In Canada de Ioa Alisos grant, at Second Street Bridge at Bl Toro, Orange Countj. Altitude, 440 feet.

Drainage area.- Area, 8.5 square miles. Average altitude, 997 feet. Maximum altitude; 2,430 feet. Length of main stream channel, 5 miles. Average slope of main atream channel, 7.5 percent.

Gage-height record. - Water-stage recorder graph.

Discharge record.- Stage-discharge relation defined below 300 second-fe日t by measurements; extended above on basis of slope-area determination.

Maxima.- 1938: Discharge, 1,280 aecond-feet Mar. 2. 1930-37: Discharge, 1,950 second-feet Feb. 6, 1937.

Remarks.- Records furnished by Orange County Flood Control District, through H. N. Thomps on, chief engineer.

Mean dally discharge, in second-feet, 1938 .

\begin{tabular}{|c|c|c|c|c|c|c|c|c|c|c|c|}
\hline Day & Feb. & Mar. & Apr. & Day & Fob. & Mar. & Apr. & Day & Feb. & Mar. & Apr. \\
\hline $\begin{array}{r}1 \\
2 \\
3 \\
4 \\
5 \\
6 \\
7 \\
8 \\
9 \\
10\end{array}$ & $\begin{array}{l}9 \\
0 \\
.1 \\
0 \\
0 \\
0 \\
0 \\
0 \\
8 \\
0\end{array}$ & $\begin{array}{r}104 \\
404 \\
66 \\
15 \\
7 \\
2.5 \\
1.0 \\
17 \\
1.0 \\
.7\end{array}$ & $\begin{array}{l}0 \\
0 \\
0 \\
0 \\
0 \\
0 \\
0 \\
0 \\
0 \\
0\end{array}$ & $\begin{array}{l}11 \\
12 \\
13 \\
14 \\
15 \\
16 \\
17 \\
18 \\
19 \\
20\end{array}$ & $\begin{array}{r}17 \\
7 \\
0 \\
0 \\
0 \\
0 \\
0 \\
0 \\
0 \\
0\end{array}$ & $\begin{array}{c}0.2 \\
46 \\
26 \\
7 \\
5 \\
3.0 \\
3.0 \\
3.0 \\
4.0 \\
5\end{array}$ & $\begin{array}{l}0 \\
0 \\
0 \\
0 \\
0 \\
0 \\
0 \\
0 \\
0 \\
0\end{array}$ & $\begin{array}{l}21 \\
22 \\
23 \\
24 \\
25 \\
26 \\
27 \\
28 \\
29 \\
30 \\
31\end{array}$ & $\begin{array}{l}0 \\
0 \\
0 \\
0 \\
0 \\
0 \\
39^{.1}\end{array}$ & $\begin{array}{l}4.0 \\
1.0 \\
0 \\
0 \\
0 \\
0 \\
0 \\
0 \\
0 \\
0 \\
0\end{array}$ & $\begin{array}{l}0 \\
0 \\
0 \\
0 \\
3.1 \\
0 \\
0 \\
0 \\
0 \\
0\end{array}$ \\
\hline $\begin{array}{l}\text { Mea } \\
\text { Run }\end{array}$ & ir & is & 1 & & & & & $\cdots$ & $\begin{array}{r}2.89 \\
161\end{array}$ & $\begin{array}{r}23.4 \\
1,440\end{array}$ & $\begin{array}{r}0.10 \\
6\end{array}$ \\
\hline
\end{tabular}


Qage holght, in feet, and discharge, in second-feet, at indicated time, 2938

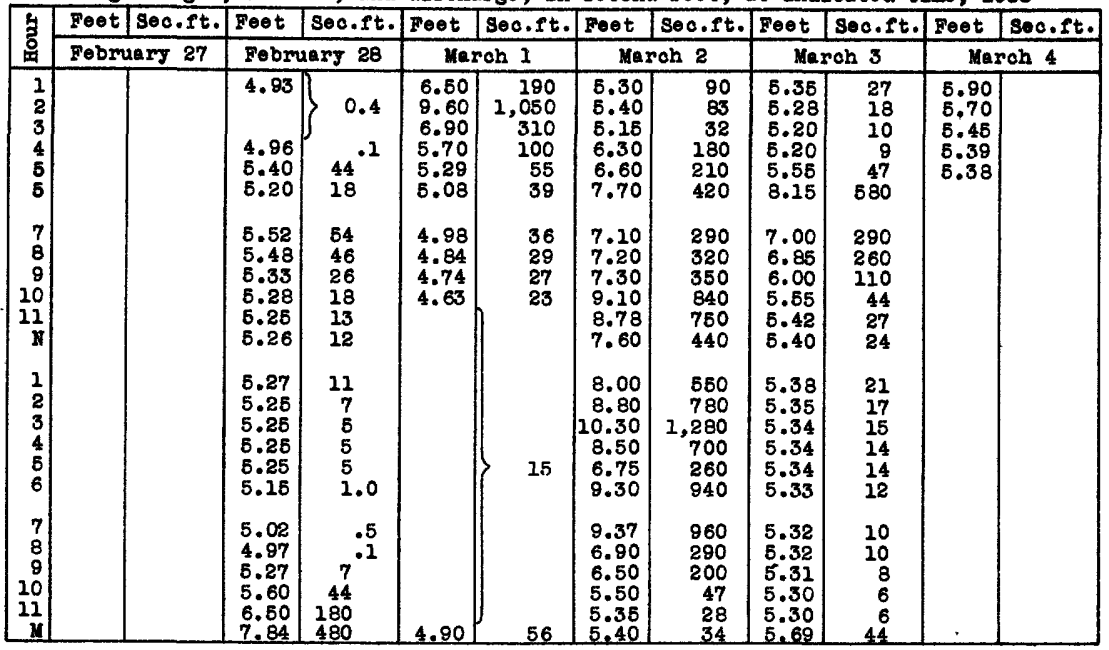

Sante Ana R1ver Basin

Santa Ana River near Nentone, Oal1f.

Locetion. - Water-stage recorder, lat. $34^{\circ} 06^{\prime} 40^{n}$, long. $227^{\circ} 06^{1} 00^{n}$, In swt sec. 4, T. 1 S., K. 2 T., near mouth of canyon, 0.4 mile upgtream from Yontone power plant of Southern Cailfornia Bdison Co., and 3 i miles northeast of Mentone. Altitude, about 1,900 feet.

Drainage aree.- Area, 144 square miles (excluding Bear Valley). Average altitude, 6,422 feet. Laximum altitude, 11,485 feet. Average slope, 42 percent. Length of maln

stream channel, 28 miles. Average slope of maln stream channel, 6.4 percent.

Gage-height record.- Water-stage recorder graph except 9 a.m. Mar. 2 to Mar. 23.

Discharge record.- Stage-discharge relation definea by current-meter measurements below 900 second-feet prior to Mar. 2 and below 400 second-feet after Mar. 23. Maximum discharge by slope-area methot. Diecharge for maximum 24 hours determined from rainfali-runoff relation, using the peoords from recoraing rain gages at Devils Canjon and Del Ros a a basis for alstribution of the storm rainfall; discharge for Mar. 2-22 obtained by comparison with adjecent streams.

Maxima.- I938: Discharge, 52,300 second-feet about 7 p.m. Mar. 2.

1896-1937: D1scharge, 29,100 second-feet Jan. 27, 1916, by slope-area method.

Remarks.- Records fair except those for Mar. 2-23, which are poor. Practically no release from Bear Valley Reservolr auring flood perlod. D1soharge 1s for natural flow of the stream and Inciudes flow in Southern Galifornia Bdison Co. 's oanal and Greenspot plpe line; no flow in canal and pipe line Fob. 28 to Apr. 30 .

Mean da1ly discharge, in second-feet, 1938

\begin{tabular}{|c|c|c|c|c|c|c|c|c|c|c|c|}
\hline Day & Feb. & Mar. & Apr. & Day & Feb. & Mar. & Apr. & Day & Feb. & Mar. & $\mathrm{Apr}$. \\
\hline $\begin{array}{r}1 \\
2 \\
3 \\
4 \\
5 \\
6 \\
7 \\
8 \\
9 \\
10\end{array}$ & $\begin{array}{r}72 \\
64 \\
90 \\
277 \\
105 \\
85 \\
64 \\
61 \\
78 \\
108\end{array}$ & $\begin{array}{r}979 \\
15,500 \\
8,080 \\
2,550 \\
1,550 \\
1,000 \\
800 \\
670 \\
600 \\
550\end{array}$ & $\begin{array}{l}248 \\
250 \\
255 \\
266 \\
271 \\
259 \\
250 \\
246 \\
250 \\
255\end{array}$ & $\begin{array}{l}11 \\
12 \\
13 \\
14 \\
15 \\
16 \\
17 \\
18 \\
19 \\
20\end{array}$ & $\begin{array}{r}119 \\
207 \\
126 \\
108 \\
86 \\
76 \\
75 \\
67 \\
100 \\
85\end{array}$ & $\begin{array}{r}500 \\
1,240 \\
1,970 \\
1,150 \\
760 \\
580 \\
480 \\
430 \\
390 \\
370\end{array}$ & $\begin{array}{l}264 \\
250 \\
280 \\
255 \\
250 \\
248 \\
257 \\
268 \\
330 \\
379\end{array}$ & $\begin{array}{l}21 \\
22 \\
23 \\
24 \\
25 \\
26 \\
27 \\
28 \\
29 \\
30 \\
31\end{array}$ & $\begin{array}{r}70 \\
71 \\
71 \\
62 \\
63 \\
63 \\
129 \\
611\end{array}$ & $\begin{array}{l}355 \\
345 \\
342 \\
340 \\
321 \\
304 \\
297 \\
285 \\
285 \\
285 \\
253\end{array}$ & $\begin{array}{l}431 \\
440 \\
424 \\
400 \\
379 \\
338 \\
352 \\
352 \\
355 \\
347\end{array}$ \\
\hline \multicolumn{9}{|c|}{ 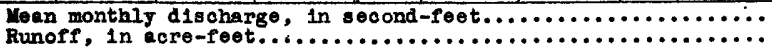 } & 6,330 & $\begin{array}{r}1,405 \\
86,400\end{array}$ & $\begin{array}{r}305 \\
18,150\end{array}$ \\
\hline
\end{tabular}


Gage helght, in feet, and discharge, in second-feet, at indicated time, 1938

\begin{tabular}{|c|c|c|c|c|c|c|}
\hline \multirow{2}{*}{ Hour } & Feot & Sec.ft. & Feet & Soc.ft. & Feot & Sec.ft \\
\hline & \multicolumn{2}{|c|}{ Fobruary 28} & \multicolumn{2}{|c|}{ March 1} & \multicolumn{2}{|c|}{ March 2} \\
\hline $\begin{array}{l}1 \\
2 \\
3 \\
4 \\
5 \\
6\end{array}$ & $\begin{array}{l}2.76 \\
2.90 \\
3.05 \\
3.10 \\
3.16 \\
3.40\end{array}$ & $\begin{array}{l}343 \\
385 \\
438 \\
455 \\
476 \\
530\end{array}$ & $\begin{array}{l}4.15 \\
5.60 \\
5.70 \\
5.75 \\
5.70 \\
5.50\end{array}$ & $\begin{array}{r}865 \\
1,650 \\
1,720 \\
1,760 \\
1,720 \\
1,580\end{array}$ & $\begin{array}{l}3.62 \\
3.60 \\
3.59 \\
3.58 \\
3.59 \\
3.65\end{array}$ & $\begin{array}{l}578 \\
570 \\
566 \\
562 \\
566 \\
590\end{array}$ \\
\hline $\begin{array}{r}7 \\
8 \\
9 \\
10 \\
11 \\
11\end{array}$ & $\begin{array}{l}3.70 \\
3.95 \\
4.00 \\
3.95 \\
3.95 \\
3.88\end{array}$ & $\begin{array}{l}690 \\
815 \\
840 \\
815 \\
815 \\
770\end{array}$ & $\begin{array}{l}5.00 \\
4.80 \\
4.70 \\
4.50 \\
4.36 \\
4.25\end{array}$ & $\begin{array}{r}1,280 \\
1,160 \\
1,100 \\
990 \\
920 \\
865\end{array}$ & $\begin{array}{l}3.92 \\
4.15 \\
4.45\end{array}$ & $\begin{array}{l}700 \\
815 \\
965\end{array}$ \\
\hline $\begin{array}{l}1 \\
2 \\
3 \\
4 \\
5 \\
6\end{array}$ & $\begin{array}{l}3.82 \\
3.72 \\
3.68 \\
3.65 \\
3.62 \\
3.58\end{array}$ & $\begin{array}{l}730 \\
674 \\
650 \\
630 \\
618 \\
602\end{array}$ & $\begin{array}{l}4.12 \\
4.00 \\
3.90 \\
3.85 \\
3.80 \\
3.78\end{array}$ & $\begin{array}{l}800 \\
740 \\
690 \\
670 \\
650 \\
642\end{array}$ & & \\
\hline $\begin{array}{r}7 \\
8 \\
9 \\
10 \\
11 \\
\mathbb{M} \\
\end{array}$ & $\begin{array}{l}3.56 \\
3.54 \\
3.52 \\
3.50 \\
3.50 \\
3.75\end{array}$ & $\begin{array}{l}594 \\
586 \\
578 \\
570 \\
570 \\
670 \\
\end{array}$ & $\begin{array}{l}3.76 \\
3.73 \\
3.71 \\
3.69 \\
3.66 \\
3.65 \\
\end{array}$ & $\begin{array}{l}634 \\
622 \\
614 \\
606 \\
594 \\
590 \\
\end{array}$ & & \\
\hline
\end{tabular}

Santa Ana River near Prado, Calif.

Location. - Water-stage recorder, lat. $33^{\circ} 52^{\prime} 05^{\prime \prime}$, long. $117^{\circ} 40^{\prime} 20^{\prime \prime}$, in Lomas de Santlago grant, at Riversiâ-Orange County line, in lower Santa Ana Canjon, 3 miles downstream from Rincon bridge, and 3 miles southwest of Prado, Riverside County. Altitude, about 400 feet.

Drainage area.- Area, 1,520 square miles (exaluding Bear Valley and San Jacinto River). Average altitude, 2,494 feot. Maximum altitude, 11,485 foet. Average slope, 28 percent. Length of main stream channel, 66.7 miles. A verage slope of maln stream channel, 3.1 percent.

Gage-holght record.- Water-stage recorder graph except 4 p.m. Mar. 2 to Mar. 29. Staff gage read 6 to io times daily Mar. 9-29.

D1scharge record.- Stage-discharge relation defined by current-meter measurements below 3,000 second-feet and extended above on bas is of mean depth-mean velocity relation. Kaximum dischargo obtained from studies of results of slope-area determinations, mean depth-mean velocity relation, and a cross section made Mar. 5, 1938, together with comparison with maximum dischargo of Santa Ana River at Santa Ana (seo pp. 182-183). D1scharge for some periods ostimated by comparison with Santa Ana River at Santa Ana.

Maxima. - 1938: Discharge, about 100,000 second-feet at 12:15 a.m. Mar. 3 (gage ho1ght, 17.32 feet).

1919-37: Discharge, about 18,000 second-feet Fob. 16, 1927.

Remarks. - Records falr, except for the perlod Mar. 2-20, which are poor. No diversions during flood period. Slight regulation at Bear Valley Reservo1r.

Mean dally discharge, in second-feet, 1938

\begin{tabular}{|r|c|r|c|c|c|c|c||c|c|c|c|}
\hline Day & Fob. & Mar. & Apr. & Day & Fob. & Mar. & Apr. & Day & Fob. & Kar. & Apr. \\
\hline 1 & 186 & 1,750 & 326 & 11 & 195 & 400 & 240 & 21 & 128 & 480 & 395 \\
2 & 146 & 12,300 & 290 & 12 & 324 & 3,020 & 272 & 22 & 125 & 430 & 456 \\
3 & 130 & 28,600 & 295 & 13 & 180 & 5,210 & 302 & 23 & 125 & 410 & 485 \\
4 & 170 & 4,480 & 290 & 14 & 149 & 3,500 & 290 & 24 & 125 & 410 & 522 \\
5 & 144 & 1,900 & 302 & 15 & 141 & 2,800 & 313 & 25 & 118 & 420 & 616 \\
6 & 110 & 1,280 & 326 & 16 & 132 & 1,100 & 302 & 26 & 118 & 410 & 529 \\
7 & 108 & 1,020 & 295 & 17 & 128 & 1,300 & 277 & 27 & 176 & 410 & 420 \\
8 & 106 & 780 & 235 & 18 & 128 & 1,300 & 272 & 28 & 345 & 400 & 400 \\
9 & 162 & 570 & 199 & 19 & 146 & 890 & 302 & 29 & & 380 & 390 \\
10 & 196 & 440 & 224 & 20 & 132 & 610 & 350 & 30 & & 470 & 413 \\
\end{tabular}


Gage height, in feet, and discharge, in second-feet, at indicated time, 1938

\begin{tabular}{|c|c|c|c|c|c|c|}
\hline \multirow{2}{*}{ Hour } & Feet & Sec.ft. & Fevt & Sec.ft. & Feet & Sec.ft. \\
\hline & \multicolumn{2}{|c|}{ Febmuary 28} & \multicolumn{2}{|c|}{ March 1} & \multicolumn{2}{|c|}{ March 2} \\
\hline $\begin{array}{l}1 \\
2 \\
3 \\
4 \\
5 \\
6\end{array}$ & & & $\begin{array}{l}3.60 \\
4.25 \\
3.82 \\
3.55 \\
3.65 \\
3.95\end{array}$ & $\begin{array}{r}870 \\
1,360 \\
1,030 \\
840 \\
905 \\
1,120\end{array}$ & $\begin{array}{l}3.20 \\
3.05 \\
3.00 \\
2.90 \\
3.10 \\
4.00\end{array}$ & $\begin{array}{r}630 \\
555 \\
530 \\
480 \\
580 \\
1,160\end{array}$ \\
\hline $\begin{array}{r}7 \\
8 \\
9 \\
10 \\
11 \\
N\end{array}$ & 2.30 & 247 & $\begin{array}{l}4.20 \\
4.65 \\
4.70 \\
4.65 \\
4.70 \\
4.75\end{array}$ & $\begin{array}{l}1,320 \\
1,620 \\
1,760 \\
1,620 \\
1,760 \\
1,810\end{array}$ & $\begin{array}{l}4.70 \\
5.25 \\
5.60 \\
6.20 \\
6.90 \\
8.00\end{array}$ & $\begin{array}{l}1,760 \\
2,350 \\
2,780 \\
3,600 \\
4,700 \\
6,850\end{array}$ \\
\hline $\begin{array}{l}1 \\
2 \\
3 \\
4 \\
5 \\
6\end{array}$ & 2.58 & 334 & $\begin{array}{l}5.00 \\
5.50 \\
6.00 \\
6.00 \\
6.20 \\
6.00\end{array}$ & $\begin{array}{l}2,070 \\
2,650 \\
3,300 \\
3,300 \\
3,600 \\
3,300\end{array}$ & $\begin{array}{r}9.20 \\
9.90 \\
10.20 \\
10.40\end{array}$ & $\begin{array}{r}9,800 \\
11,700 \\
12,800 \\
13,400\end{array}$ \\
\hline $\begin{array}{r}7 \\
8 \\
9 \\
10 \\
11 \\
M\end{array}$ & 3.25 & 648 & $\begin{array}{l}5.35 \\
4.65 \\
4.20 \\
3.90 \\
3.55 \\
3.35\end{array}$ & $\begin{array}{r}2,470 \\
1,720 \\
1,320 \\
1,090 \\
840 \\
720\end{array}$ & & \\
\hline
\end{tabular}

Santa Ana River at Santa Ana, Cal1f.

Locet1on. - Water-stage recorder and concrete we1r control, 1at. $33^{\circ} 45^{\prime} 00^{\prime \prime}, 10 n g .117^{\circ}$ $54^{\prime} 0^{\prime \prime}$, In Las Bolses land grant, on elghth of a mile kbove Fifth Street Bridge in Santa Ana, Orange County, and 2 miles below junction with Santiago Creex. Altitude, about 80 feet.

Gage-he1ght record. - Water-stage recorder graph except Mar. 3-6 and Apr. 18-30, when stiling well was filled with silt.

Discharge record. - Stage-discharge relation defined by current-meter measurements beIow 10,000 second-feet; extended to peak discharge determined by slope-aren method. Discharge 7 a.m. Mar. 3 to Mar. 6 based on comparison with Santiago Creek at Santa Ane and current-meter mepsurements.

Maxima.; 1938: Discharge, 46,300 second-feet kbout 3 a.m. Mar. 3 (gage helght, 10.2 fe日t).

1923-37: Discharge, about 25,000 second-feet Feb. 16, 1927.

Remarks.- Records falr, except those for Mar, 3-6, which are poor. No diversions during flood period. Some regulation at Bear Valiey and Santiago creek Reservolrs.

Mean dally discharge, in second-feet, 1938

\begin{tabular}{|c|c|c|c|c|c|c|c|c|c|c|c|}
\hline Day & Feb. & Mar. & Apr. & Dey & Feb. & Mar. & Apr. & Day & Feb. & Mar. & Apr. \\
\hline $\begin{array}{r}1 \\
2 \\
3 \\
4 \\
5 \\
6 \\
7 \\
8 \\
9 \\
10\end{array}$ & $\begin{array}{r}14 \\
0 \\
0 \\
0 \\
0 \\
0 \\
0 \\
0 \\
87 \\
6\end{array}$ & $\begin{array}{r}991 \\
8,590 \\
20,300 \\
5,070 \\
2,100 \\
1,500 \\
1,370 \\
920 \\
470 \\
300\end{array}$ & $\begin{array}{l}57 \\
52 \\
51 \\
52 \\
53 \\
54 \\
54 \\
53 \\
53 \\
68\end{array}$ & $\begin{array}{l}11 \\
12 \\
13 \\
14 \\
15 \\
16 \\
17 \\
18 \\
19 \\
20\end{array}$ & $\begin{array}{l}32 \\
82 \\
15 \\
1.7 \\
.3 \\
.2 \\
.1 \\
3.0 \\
30 \\
28\end{array}$ & $\begin{array}{r}250 \\
2,700 \\
5,710 \\
3,660 \\
2,770 \\
1,020 \\
1,200 \\
1,100 \\
700 \\
400\end{array}$ & $\begin{array}{l}71 \\
70 \\
68 \\
64 \\
60 \\
56 \\
52 \\
45 \\
41 \\
39\end{array}$ & $\begin{array}{l}21 \\
22 \\
23 \\
24 \\
25 \\
26 \\
27 \\
28 \\
29 \\
30 \\
31\end{array}$ & $\begin{array}{c}10 \\
0 \\
0 \\
0 \\
1.0 \\
.2 \\
23 \\
74\end{array}$ & $\begin{array}{l}350 \\
200 \\
180 \\
170 \\
170 \\
160 \\
150 \\
140 \\
110 \\
80 \\
60 \\
\end{array}$ & $\begin{array}{l}37 \\
36 \\
34 \\
33 \\
31 \\
30 \\
29 \\
28 \\
27 \\
25\end{array}$ \\
\hline \multicolumn{12}{|c|}{ 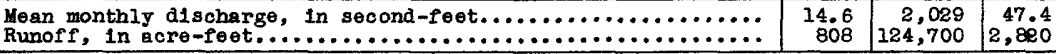 } \\
\hline
\end{tabular}


Gage helght, in feet, and discharge, in second-feet, at indieated time, 1938

\begin{tabular}{|c|c|c|c|c|c|c|c|c|}
\hline \multirow{2}{*}{ Hour } & Feet & sec.ft. & Feet & Sec.ft. & Feet & sec.ft. & Feet & Sec.ft. \\
\hline & \multicolumn{2}{|c|}{ February 28} & \multicolumn{2}{|c|}{ March 1} & \multicolumn{2}{|c|}{ March 2} & \multicolumn{2}{|c|}{ March 3} \\
\hline $\begin{array}{l}1 \\
2 \\
3 \\
4 \\
5 \\
6\end{array}$ & $\begin{array}{r}0.68 \\
.65 \\
.70 \\
.80 \\
.90 \\
1.05\end{array}$ & $\begin{array}{l}18 \\
16 \\
21 \\
33 \\
53 \\
90\end{array}$ & $\begin{array}{l}1.70 \\
2.10 \\
2.30 \\
2.30 \\
2.22 \\
2.15\end{array}$ & $\begin{array}{r}700 \\
900 \\
1,270 \\
1,270 \\
948 \\
800\end{array}$ & $\begin{array}{l}1.92 \\
1.78 \\
1.70 \\
1.65 \\
1.68 \\
2.00\end{array}$ & $\begin{array}{r}1,460 \\
1,090 \\
900 \\
800 \\
860 \\
1,700\end{array}$ & $\begin{array}{c}6.60 \\
8.0 \\
10.2 \\
9.0 \\
8.5 \\
8.0\end{array}$ & $\begin{array}{l}22,500 \\
30,400 \\
46,300 \\
36,600 \\
33,400 \\
30,400\end{array}$ \\
\hline $\begin{array}{r}7 \\
8 \\
9 \\
10 \\
11 \\
N\end{array}$ & $\begin{array}{r}1.30 \\
1.22 \\
1.22 \\
1.10 \\
1.02 \\
.94\end{array}$ & $\begin{array}{r}194 \\
158 \\
158 \\
109 \\
84 \\
64\end{array}$ & $\begin{array}{l}2.00 \\
1.90 \\
1.75 \\
1.65 \\
1.60 \\
1.65\end{array}$ & $\begin{array}{l}540 \\
420 \\
275 \\
206 \\
176 \\
206\end{array}$ & $\begin{array}{l}2.40 \\
2.70 \\
2.72 \\
2.86 \\
3.25 \\
3.40\end{array}$ & $\begin{array}{l}3,200 \\
4,350 \\
4,430 \\
4,950 \\
6,500 \\
7,100\end{array}$ & & \\
\hline $\begin{array}{l}1 \\
2 \\
3 \\
4 \\
5 \\
6\end{array}$ & $\begin{array}{l}.82 \\
.77 \\
.74 \\
.80 \\
.77 \\
.74\end{array}$ & $\begin{array}{r}46 \\
44 \\
43 \\
55 \\
48 \\
46\end{array}$ & $\begin{array}{l}1.77 \\
1.80 \\
1.80 \\
1.80 \\
1.78 \\
1.88\end{array}$ & $\begin{array}{l}388 \\
420 \\
540 \\
540 \\
668 \\
984\end{array}$ & $\begin{array}{l}3.60 \\
3.90 \\
4.40 \\
4.80 \\
5.00 \\
5.20\end{array}$ & $\begin{array}{r}7,900 \\
9,100 \\
11,400 \\
13,300 \\
14,300 \\
15,200\end{array}$ & & \\
\hline $\begin{array}{r}7 \\
8 \\
9 \\
10 \\
.11 \\
11\end{array}$ & $\begin{array}{r}.68 \\
.68 \\
.72 \\
.78 \\
1.00 \\
1.50\end{array}$ & $\begin{array}{r}36 \\
36 \\
46 \\
59 \\
95 \\
420\end{array}$ & $\begin{array}{l}2.10 \\
2.14 \\
2.20 \\
2.22 \\
2.20 \\
2.05\end{array}$ & $\begin{array}{l}1,750 \\
2,210 \\
2,450 \\
2,530 \\
2,450 \\
1,880\end{array}$ & $\begin{array}{l}5.30 \\
5.40 \\
5.60 \\
5.70 \\
6.00 \\
6.40\end{array}$ & $\begin{array}{l}15,700 \\
16,200 \\
17,200 \\
17,700 \\
19,200 \\
21,400\end{array}$ & & \\
\hline
\end{tabular}

M111 Creek near Craftonville, Calif.

Location. - Water-stage recorder and concrete control, lat. $34^{\circ} 05^{\prime} 15^{\prime \prime}$, 10 ng. $117^{\circ} \mathrm{C} 2^{\prime}$

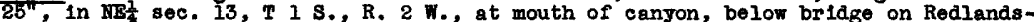
Bear Valley highway, and 5 miles northeast of Craftonvilie. Altitude, 2,950 feet.

Drainage area.- Area, 42.9 square miles. Average altitude, 6, 805 feet. Maximum altitude, 11,480 feet. Average slope, 41 percent. Length of main stream channel, 12.6 miles. Average slope of main stream channel, 11 percent.

Gage-helght record. - Record lost with the destruction of recorder shelter and control on Mar. 2.

Discharge record.- Maximum discharge obtained by slope-area method. Discharge for maximum 24-hour period determined from rainfall-runoff relation using the Del Rosa recording rain gage as a base for distribution of total storm precipitation. The remainder of the period was determined by comparison with records for Santa Ana Rfver near Mentone.

Maxima.- 1938: Discharge, 18,100 second-feet Mar. 2.

1919-37: Discharge, 4,500 second-feet (estimated) Feb. 16, 1927.

Remics.- Records poor. Discharge is for natural flow of the stream and includes flow in Mill Creek power canais Nos. 1, 2, and 3 ; no flow in canals Mar. 3 to Apr. 30.

Mean dally discharge, in second-feet, 1938

\begin{tabular}{|c|c|c|c|c|c|c|c|c|c|c|c|}
\hline Day & Feb. & Mar. & Apr. & Day & Feb. & Mar. & Apr. & Day & Feb. & Mar. & Apr. \\
\hline $\begin{array}{r}1 \\
2 \\
3 \\
4 \\
5 \\
6 \\
7 \\
8 \\
9 \\
10\end{array}$ & $\begin{array}{l}31 \\
31 \\
32 \\
45 \\
38 \\
34 \\
31 \\
30 \\
34 \\
36\end{array}$ & $\begin{array}{r}346 \\
6,300 \\
2,820 \\
1,080 \\
920 \\
690 \\
580 \\
580 \\
460 \\
350\end{array}$ & $\begin{array}{l}138 \\
138 \\
136 \\
136 \\
136 \\
137 \\
139 \\
140 \\
142 \\
146\end{array}$ & $\begin{array}{l}11 \\
12 \\
13 \\
14 \\
15 \\
16 \\
17 \\
18 \\
19 \\
20\end{array}$ & $\begin{array}{l}38 \\
46 \\
43 \\
37 \\
35 \\
32 \\
32 \\
31 \\
35 \\
32\end{array}$ & $\begin{array}{l}350 \\
360 \\
570 \\
350 \\
240 \\
200 \\
180 \\
170 \\
160 \\
155\end{array}$ & $\begin{array}{l}148 \\
150 \\
152 \\
156 \\
158 \\
158 \\
160 \\
160 \\
158 \\
155\end{array}$ & $\begin{array}{l}21 \\
22 \\
23 \\
24 \\
25 \\
26 \\
27 \\
28 \\
29 \\
30 \\
31\end{array}$ & $\begin{array}{r}31 \\
30 \\
31 \\
31 \\
30 \\
30 \\
36 \\
216\end{array}$ & $\begin{array}{l}152 \\
150 \\
148 \\
146 \\
145 \\
144 \\
142 \\
140 \\
140 \\
140 \\
140 \\
\end{array}$ & $\begin{array}{l}152 \\
150 \\
146 \\
144 \\
142 \\
140 \\
139 \\
136 \\
135 \\
135\end{array}$ \\
\hline \multicolumn{12}{|c|}{ 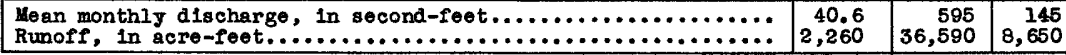 } \\
\hline
\end{tabular}


Plunge Creok near Bast Highlands, Calif.

Location. - Water-stage recorder, lat. $34^{\circ} 07^{\prime} 10^{n}$, long. $117^{\circ} 08^{\prime} 30^{m}$, in NRtNR sec. 1 , T. IS., R. 3 W., at mouth of oanyon at crossing at North Fork diteh siphon, 2 wilos northeast of kast Highlands. Altitude, about 1,625 foet.

Drainsgs area.- Area, 16.9 square miles. Avsrage elt1tude, 4,036 foet. Meximum alt1tuds, 6,350 foet. A verage slopo, 38 peroent. Longth of main stroem chennel, 7.5 m1108. Average slope of main stream channel, 12 percant.

Gage-helght record. - Water-stage recorder graph exoept Mar, 3-11.

Discharge record. - Stage-discharge relation definod by current-meter measurements beIow 300 ssoond-feet; extended to 2,900 second-feet on besis of mean depth-mean velocity relation. Maximim discharge determined by slope-area method ana checked by.rainfall-runoff study. Discharge for the maximum 24 hours determinod by rainfallminoff studies; the rainfall ras distributed on the basis of the Del Rosa recording rain gags rocord. Discharge lar. 1-1I based on gage holght, rainfall-munoff study, and comparative hydrographs.

Maxima;- 1938: D1scharge, 5,340 second-feet about 6 p.m. Kar. 2 (gage height, 7.0 (oot).

1919-37: Discharge, 1,420 second-foet Feb. 16, 1927 (gage helght, 3.8 feet).

Remarks.- Records good, except those for Mar.1-23, wh1ch are poor.

Mean daily discharge, in second-feet, 1938

\begin{tabular}{|c|c|c|c|c|c|c|c|c|c|c|c|}
\hline Day & Feb. & Mar. & Apr. & Day & Feb. & Mar. & Apr. & Day & Feb. & Mar. & Apr. \\
\hline $\begin{array}{r}1 \\
2 \\
3 \\
4 \\
5 \\
6 \\
7 \\
8 \\
9 \\
10\end{array}$ & $\begin{array}{c}22 \\
17 \\
35 \\
75 \\
27 \\
15 \\
11 \\
9.5 \\
14 \\
17\end{array}$ & $\begin{array}{r}219 \\
1,500 \\
1,130 \\
413 \\
240 \\
162 \\
110 \\
87 \\
74 \\
67\end{array}$ & $\begin{array}{l}32 \\
31 \\
28 \\
26 \\
27 \\
23 \\
20 \\
18 \\
17 \\
16\end{array}$ & $\begin{array}{l}11 \\
12 \\
13 \\
14 \\
15 \\
16 \\
17 \\
18 \\
19 \\
20\end{array}$ & $\begin{array}{l}36 \\
53 \\
31 \\
23 \\
20 \\
16 \\
14 \\
13 \\
26 \\
18\end{array}$ & $\begin{array}{r}62 \\
174 \\
189 \\
126 \\
100 \\
84 \\
73 \\
67 \\
61 \\
55\end{array}$ & $\begin{array}{l}13 \\
12 \\
32 \\
24 \\
20 \\
19 \\
16 \\
15 \\
14 \\
14\end{array}$ & $\begin{array}{l}21 \\
22 \\
23 \\
24 \\
25 \\
26 \\
27 \\
28 \\
29 \\
30 \\
31 \\
\end{array}$ & $\begin{array}{r}15 \\
14 \\
13 \\
12 \\
10 \\
10 \\
29 \\
167\end{array}$ & $\begin{array}{l}53 \\
50 \\
45 \\
46 \\
44 \\
42 \\
42 \\
40 \\
42 \\
38 \\
33 \\
\end{array}$ & $\begin{array}{l}12 \\
13 \\
11 \\
16 \\
32 \\
23 \\
19 \\
17 \\
23 \\
30\end{array}$ \\
\hline $\begin{array}{l}\text { Mes } \\
\text { Rur }\end{array}$ & 1 & & , & & & & & & $\begin{array}{r}27.2 \\
1,510 \\
\end{array}$ & $\begin{array}{r}176 \\
10,850\end{array}$ & $\begin{array}{r}20.4 \\
1,220\end{array}$ \\
\hline
\end{tabular}

Gage helght, in feet, and discharge, in second-feet, et indlceted time, 1938

\begin{tabular}{|c|c|c|c|c|c|c|c|c|c|c|c|c|}
\hline \multirow{2}{*}{ 岁 } & Feet & seo.ft. & Feet & Sec.ft. & Feet & Sec.ft. & Feet & sec.ft. & Feet & Sec.ft. & Feet & sec.ft. \\
\hline & \multicolumn{2}{|c|}{ February 27} & \multicolumn{2}{|c|}{ February 28} & \multicolumn{2}{|c|}{ March 1} & \multicolumn{2}{|c|}{ Yaroh 2} & \multicolumn{2}{|c|}{ March 3} & \multicolumn{2}{|c|}{ March 4} \\
\hline $\begin{array}{l}1 \\
2 \\
3 \\
4 \\
5 \\
6\end{array}$ & & & $\begin{array}{l}1.72 \\
1.72 \\
1.71 \\
1.69 \\
1.75 \\
1.98\end{array}$ & $\begin{array}{l}144 \\
144 \\
142 \\
138 \\
150 \\
193\end{array}$ & $\begin{array}{l}3.00 \\
3.40 \\
3.00 \\
2.66 \\
2.50 \\
2.35\end{array}$ & $\begin{array}{l}435 \\
555 \\
435 \\
345 \\
305 \\
271\end{array}$ & $\begin{array}{l}1.56 \\
1.62 \\
1.62 \\
1.65 \\
1.70 \\
2.00\end{array}$ & $\begin{array}{l}116 \\
126 \\
126 \\
128 \\
140 \\
197\end{array}$ & & & & \\
\hline $\begin{array}{r}7 \\
8 \\
9 \\
10 \\
11 \\
17\end{array}$ & 0.73 & 14 & $\begin{array}{l}2.07 \\
2.09 \\
2.06 \\
1.98 \\
1.92 \\
1.87\end{array}$ & $\begin{array}{l}211 \\
215 \\
209 \\
193 \\
182 \\
172\end{array}$ & $\begin{array}{l}2.20 \\
2.12 \\
2.02 \\
2.00 \\
1.96 \\
1.90\end{array}$ & $\begin{array}{l}238 \\
221 \\
201 \\
187 \\
189 \\
178\end{array}$ & $\begin{array}{l}2.30 \\
2.70 \\
2.84 \\
3.10 \\
3.60\end{array}$ & $\begin{array}{l}260 \\
355 \\
393 \\
465 \\
620\end{array}$ & & & & \\
\hline $\begin{array}{l}1 \\
2 \\
3 \\
4 \\
5 \\
6\end{array}$ & 1.00 & 35 & $\begin{array}{l}1.90 \\
1.94 \\
1.91 \\
1.86 \\
1.82 \\
1.77\end{array}$ & $\begin{array}{l}178 \\
186 \\
180 \\
170 \\
163 \\
153\end{array}$ & $\begin{array}{l}1.85 \\
1.80 \\
1.75 \\
1.73 \\
1.71 \\
1.70\end{array}$ & $\begin{array}{l}169 \\
159 \\
160 \\
146 \\
142 \\
140\end{array}$ & & & & & & \\
\hline $\begin{array}{r}7 \\
8 \\
9 \\
10 \\
11 \\
4\end{array}$ & 1.40 & 91 & $\begin{array}{l}1.72 \\
1.69 \\
1.67 \\
1.66 \\
1.80 \\
2.15\end{array}$ & $\begin{array}{l}144 \\
138 \\
135 \\
133 \\
159 \\
228\end{array}$ & $\begin{array}{l}1.67 \\
1.64 \\
1.62 \\
1.60 \\
1.58 \\
1.56\end{array}$ & $\begin{array}{l}135 \\
130 \\
126 \\
123 \\
119 \\
116\end{array}$ & & & & & & \\
\hline
\end{tabular}

Supplemental reoord.- Mar. I, 1:30 a.m., 605 sec.-ft. 
San TImotoo Orook noar Rodlando, Oal1f.

Locat1on. - Fator-stage rooorder and oonoreto oentrol, lat. $34^{\circ} 01^{\prime} 65^{n}$, long. $117^{\circ} 12^{\prime} 30^{\prime \prime}$, In 4 , ant soo. 4, T. 8 S., R. 3 m., 2 mllow outhoant of Rediands. Altitudo, about 1,260 root.

Dralnaqe sxea.- Aroa, 118 quaro m1lon. Avorago alt1tudo, 3,048 foot. Maximum alt1tria, 8,700 foot. Average ilopo, 36 peroont. Iongth of mein itream ohanne1, 22.0 milel. Avorage ilope of maln atroam ohannel, 6.4 poroont.

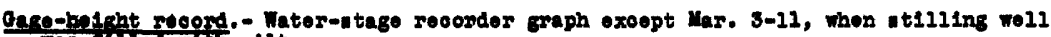

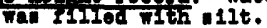

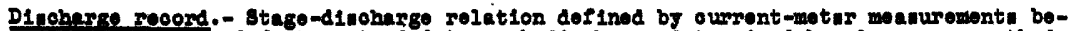
101 1,800 100ond-foet, extendod to poak dinobnerge dotermined. by lope-asea mothod. Dinohargo astimated kar. 3-11 by oomparinon with noarby itroims.

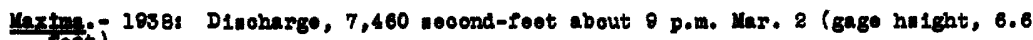

IOb6-578 DInohurge, 3,600 wooond-foot Fob. 6, I937 (gage ho1ght, B.5 fout).

Beraris..- Rocordn ralr.

Moun dalls d1woharge, in mooond-100t, 1938

\begin{tabular}{|c|c|c|c|c|c|c|c|c|c|c|c|}
\hline Dey & Bob. & Var. & Apr. & Day & Fob. & ras. & Apr. & Dar & Fob. & Mar. & Apr. \\
\hline $\begin{array}{r}7 \\
8 \\
3 \\
4 \\
8 \\
6 \\
7 \\
8 \\
0 \\
10\end{array}$ & $\begin{array}{l}1.2 \\
1.1 \\
.4 \\
2.4 \\
8.1 \\
1.7 \\
1.8 \\
1.0 \\
.8 \\
.8\end{array}$ & \begin{tabular}{|r|}
288 \\
1,880 \\
296 \\
38 \\
18 \\
7 \\
3.7 \\
2.0 \\
1.1 \\
.06
\end{tabular} & $\begin{array}{l}0.1 \\
.1 \\
.1 \\
.1 \\
.1 \\
.1 \\
.1 \\
.1 \\
.1 \\
.1\end{array}$ & $\begin{array}{l}11 \\
18 \\
13 \\
14 \\
18 \\
16 \\
17 \\
18 \\
18 \\
80\end{array}$ & $\begin{array}{l}28 \\
0^{-1} \\
0 \\
0 \\
0 \\
0 \\
0 \\
0 \\
0\end{array}$ & $\begin{array}{c}0.3 \\
61 \\
20 \\
8 \\
3 \\
2 \\
1.5 \\
1.0 \\
.8 \\
.6\end{array}$ & $\begin{array}{l}0.1 \\
0 \\
0 \\
0 \\
0 \\
0 \\
0 \\
0 \\
0 \\
0\end{array}$ & $\begin{array}{l}21 \\
82 \\
28 \\
84 \\
85 \\
88 \\
87 \\
88 \\
89 \\
30 \\
31\end{array}$ & $\begin{array}{l}0 \\
0 \\
0 \\
0 \\
0 \\
0 \\
0 \\
2.4\end{array}$ & $\begin{array}{r}0.8 \\
.3 \\
.8 \\
.8 \\
.2 \\
.1 \\
.1 \\
.1 \\
.1 \\
.1 \\
.1\end{array}$ & $\begin{array}{l}0 \\
0 \\
0 \\
0 \\
0 \\
0 \\
0 \\
0 \\
0 \\
0 \\
0\end{array}$ \\
\hline \multicolumn{9}{|c|}{ 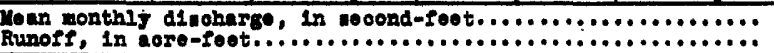 } & $\begin{array}{r}1.56 \\
86\end{array}$ & $\begin{array}{r}84.0 \\
8,160\end{array}$ & 0.04 \\
\hline
\end{tabular}

D1woharpe, in nooond-foot, at Indloatod timo, 1938

\begin{tabular}{|c|c|c|c|c|c|}
\hline Bour & Fob. 28 & Mar. 1 & Mar. 2 & Mar. 3 & Mas. 4 \\
\hline $\begin{array}{l}1 \\
2 \\
3 \\
4 \\
5 \\
6\end{array}$ & $\begin{array}{l}0 \\
0 \\
.5 \\
0 \\
0 \\
0\end{array}$ & $\begin{array}{r}24 \\
61 \\
1,900 \\
1,780 \\
775 \\
500\end{array}$ & $\begin{array}{l}70 \\
68 \\
68 \\
65 \\
68 \\
60\end{array}$ & $\begin{array}{r}1,300 \\
800 \\
680 \\
500 \\
400 \\
320\end{array}$ & $\begin{array}{l}61 \\
47 \\
48\end{array}$ \\
\hline $\begin{array}{r}7 \\
8 \\
9 \\
10 \\
11 \\
17\end{array}$ & $\begin{array}{l}1.3 \\
3.0 \\
1.8 \\
1.2 \\
1.0 \\
4.0\end{array}$ & $\begin{array}{l}350 \\
850 \\
828 \\
200 \\
180 \\
160\end{array}$ & $\begin{array}{r}60 \\
60 \\
60 \\
60 \\
100 \\
810\end{array}$ & $\begin{array}{l}860 \\
220 \\
190 \\
165 \\
180 \\
185\end{array}$ & $\begin{array}{l}38 \\
36 \\
33\end{array}$ \\
\hline $\begin{array}{l}1 \\
2 \\
3 \\
4 \\
5 \\
6\end{array}$ & $\begin{array}{l}8 \\
5.8 \\
3.8 \\
2.0 \\
1.0 \\
1.8\end{array}$ & $\begin{array}{r}140 \\
180 \\
110 \\
100 \\
98 \\
90\end{array}$ & $\begin{array}{l}1,900 \\
2,380 \\
2,480 \\
2,880 \\
3,600 \\
3,600\end{array}$ & $\begin{array}{r}120 \\
110 \\
100 \\
95 \\
86 \\
80\end{array}$ & $\begin{array}{l}30 \\
28 \\
26\end{array}$ \\
\hline $\begin{array}{c}7 \\
8 \\
9 \\
10 \\
11 \\
4\end{array}$ & $\begin{array}{l}1.7 \\
1.5 \\
1.5 \\
1.8 \\
2.8 \\
13\end{array}$ & $\begin{array}{l}85 \\
80 \\
75 \\
75 \\
70 \\
70\end{array}$ & $\begin{array}{l}4,400 \\
5,500 \\
7,460 \\
5,100 \\
3,200 \\
1,800\end{array}$ & $\begin{array}{l}76 \\
71 \\
66 \\
65 \\
60 \\
57\end{array}$ & $\begin{array}{l}24 \\
22 \\
21\end{array}$ \\
\hline
\end{tabular}


Tarm Creek near Colton, Cal1f.

Location. - Water-stage recorder, lat, $34^{\circ} 04^{\prime} 00^{\prime \prime}$, long. $117^{\circ} 18^{\prime} 30^{\prime \prime}$, In San Bernardino grant, at Colton Avenue Bridge, lit miles east of Colton, San Bernardino County. Altitude, about 970 feot.

Qage-he1ght record. - Water-stage recorder graph prior to 6 a.m. Mar. 2.

Discharge record. - Stage-discharge relation defined by current-meter messurements be1ow 1,250 second-feet. D1scharge 6 a.m. Har. 2 to Apr. 30 based on comparative hydragraph and five discharge measurements.

Uaxima.- 1938: D1scharge, 27,500 second-feet 7 p.m. Mar. 2.

1920-37: D1scharge, 2,780 second-feet Dec. 21, 1921 .

Remarks. - Records fa1r. Disoharge is for natural flow of stream and includes flow in leeks and Daley canal; flow in canal Fob. $1-28$ and Mar. 6 to Apr. 30 was 0.1 secondfoot or less and did not affect flood flow.

Yean daily discharge, in second-feet, 1938

\begin{tabular}{|c|c|c|c|c|c|c|c|c|c|c|c|}
\hline Day & Fob. & Mar. & Apr. & Day & Fob. & Mar. & Apr. & Day & Fob. & Mar. & Apr. \\
\hline $\begin{array}{r}1 \\
2 \\
3 \\
4 \\
5 \\
6 \\
7 \\
8 \\
9 \\
10\end{array}$ & $\begin{array}{r}98 \\
36 \\
77 \\
172 \\
36 \\
36 \\
36 \\
35 \\
55 \\
34\end{array}$ & $\begin{array}{r}666 \\
10,225 \\
5,715 \\
925 \\
490 \\
349 \\
277 \\
239 \\
214 \\
194\end{array}$ & $\begin{array}{l}101 \\
102 \\
103 \\
104 \\
105 \\
107 \\
108 \\
109 \\
110 \\
109\end{array}$ & $\begin{array}{l}11 \\
12 \\
13 \\
14 \\
15 \\
16 \\
17 \\
18 \\
19 \\
20\end{array}$ & $\begin{array}{r}112 \\
94 \\
61 \\
53 \\
47 \\
47 \\
45 \\
47 \\
53 \\
44\end{array}$ & $\begin{array}{r}185 \\
329 \\
413 \\
308 \\
220 \\
170 \\
138 \\
116 \\
101 \\
94\end{array}$ & $\begin{array}{r}108 \\
107 \\
106 \\
105 \\
104 \\
102 \\
101 \\
100 \\
99 \\
98\end{array}$ & $\begin{array}{l}21 \\
22 \\
23 \\
24 \\
25 \\
26 \\
27 \\
28 \\
29 \\
30 \\
31\end{array}$ & $\begin{array}{r}45 \\
45 \\
46 \\
45 \\
44 \\
45 \\
106 \\
448\end{array}$ & $\begin{array}{r}88 \\
86 \\
85 \\
87 \\
89 \\
92 \\
94 \\
95 \\
97 \\
99 \\
100\end{array}$ & $\begin{array}{l}97 \\
96 \\
95 \\
94 \\
93 \\
91 \\
90 \\
88 \\
87 \\
85\end{array}$ \\
\hline & & & & & & & & & $\begin{array}{r}72,9 \\
4,050 \\
\end{array}$ & $\begin{array}{r}722 \\
44,390 \\
\end{array}$ & $\begin{array}{r}100 \\
5,960 \\
\end{array}$ \\
\hline
\end{tabular}

Dlscharge, in second-feet, at indlested time, 1938

\begin{tabular}{|c|c|c|r|r|}
\hline Hour & Fob. 27 & Fob. 28 & Mar. 1 & Mar. 2 \\
\hline 1 & 49 & 184 & 652 & 225 \\
2 & 50 & 163 & 850 & 225 \\
3 & 50 & 157 & 1,140 & 225 \\
4 & 50 & 163 & 1,260 & 225 \\
5 & 50 & 224 & 1,360 & 253 \\
6 & 50 & 305 & 1,270 & 543 \\
7 & 50 & 370 & 1,130 & \\
8 & 55 & 488 & 975 & \\
9 & 70 & 498 & 853 & \\
10 & 120 & 407 & 782 & \\
11 & 160 & 416 & 702 & \\
1 & 240 & 524 & 637 & \\
1 & 150 & 750 & & \\
1 & 100 & 822 & 494 & \\
3 & 92 & 828 & 434 & \\
4 & 90 & 768 & 352 & \\
5 & 91 & 641 & 316 & \\
6 & 93 & 562 & 270 & \\
7 & 104 & 524 & 254 & \\
8 & 114 & 486 & 237 & \\
9 & 156 & 377 & 232 & \\
10 & 194 & 354 & 228 & \\
11 & 220 & 349 & 226 & \\
1 & 204 & 522 & 225 & \\
\hline
\end{tabular}


Strawberry Creok near Arrowhead Springs, Callf.

Location.- Water-stage recorder and broad-crested weir control, lat. $34^{\circ} 10^{\prime} 45^{n}$, long.

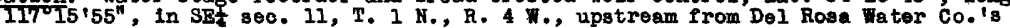
lower diversion dam, and half a mile south of Arrowhead Springs. Altitude, about 1,650 feot.

Drainage area.- Area, 8.6 square mlles. Average altitude, 3,520 feet. Maximum altitude, 6,150 feet. Average slope, 47 percent. Iength of main stream channel, about 4.8 miles. Average slope of main stream channel, 18 percent.

Gage-helght record. - Water-stage recorder graph except $12 \mathrm{~m}$. Mar. 2 to Mar. 15.

Discharge record.- Stage-discharge relation defined by current-meter measurements below 300 second-feet; extended above on the basis of mean depth-mean velocity relation and levele of March 1938 and confirmed by rainfall-runoff data. Maximum discherge estimated by consideration of slope-area studies, rainfall-runoff studies, and knowledge of loeal conditions. Records for maximum 24 hours derived by rainfallmunoff study, using Del Rosa recording rain gage as a base for alstribution of total storm precipitation.

Maxima.- 1938: Discharge, 3,360 second-feet 4 p.m. Mar. 2 (gage helght, 5.30 fe日t). 1919-37: Discharge, 492 second-feet Feb. 6, 1937 (gage helght, 4.15 foet).

Remarks.- Records good except those for $12 \mathrm{~m}$. Mar. 2 to Mar. 15, which are poor.

Mean dally discharge, in second-feet, 1938

\begin{tabular}{|c|c|c|c|c|c|c|c|c|c|c|c|}
\hline Day & Pob. & Mar. & Apr. & Day & Feb. & Mar. & Apr. & Day & Feb. & Mar. & Apr. \\
\hline $\begin{array}{r}2 \\
2 \\
3 \\
4 \\
5 \\
6 \\
7 \\
8 \\
9 \\
10\end{array}$ & $\begin{array}{c}12 \\
6.5 \\
26 \\
50 \\
19 \\
12 \\
9 \\
8 \\
14 \\
12\end{array}$ & $\begin{array}{r}106 \\
792 \\
414 \\
132 \\
76 \\
62 \\
55 \\
51 \\
39 \\
34\end{array}$ & $\begin{array}{l}22 \\
20 \\
20 \\
20 \\
21 \\
19 \\
18 \\
17 \\
17 \\
17\end{array}$ & $\begin{array}{l}11 \\
12 \\
13 \\
14 \\
15 \\
16 \\
17 \\
18 \\
19 \\
20\end{array}$ & $\begin{array}{c}13 \\
18 \\
14 \\
11 \\
10 \\
9 \\
8 \\
8 \\
11 \\
8.5\end{array}$ & $\begin{array}{r}34 \\
148 \\
206 \\
92 \\
63 \\
55 \\
49 \\
44 \\
42 \\
39\end{array}$ & $\begin{array}{l}17 \\
18 \\
22 \\
19 \\
17 \\
16 \\
16 \\
14 \\
14 \\
14\end{array}$ & $\begin{array}{l}21 \\
22 \\
23 \\
24 \\
25 \\
26 \\
27 \\
28 \\
29 \\
30 \\
31\end{array}$ & $\begin{array}{l}7.5 \\
7 \\
6.5 \\
6 \\
5.5 \\
5.5 \\
12 \\
63\end{array}$ & $\begin{array}{l}35 \\
34 \\
31 \\
29 \\
28 \\
26 \\
26 \\
26 \\
26 \\
24 \\
22\end{array}$ & $\begin{array}{l}14 \\
14 \\
13 \\
16 \\
22 \\
18 \\
16 \\
14 \\
21 \\
26\end{array}$ \\
\hline \multicolumn{9}{|c|}{ 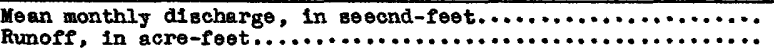 } & $\begin{array}{r}14.0 \\
778\end{array}$ & $\begin{array}{r}91.6 \\
5,630\end{array}$ & $\begin{array}{r}17.7 \\
2,060\end{array}$ \\
\hline
\end{tabular}

Gage height, in feet, and discharge, in second-feet, at indicated time, 1938

\begin{tabular}{|c|c|c|c|c|c|c|c|c|c|c|c|c|}
\hline \multirow{2}{*}{ 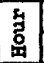 } & Poet & soc.et. & Feet & Sec.ft. & Feot & sec.et. & Feet & sec.ft. & Poet & sec.et. & Foet & sec.ft. \\
\hline & \multicolumn{2}{|c|}{ February 27} & \multicolumn{2}{|c|}{ February 28} & \multicolumn{2}{|c|}{ March 1} & \multicolumn{2}{|c|}{ March 2} & \multicolumn{2}{|c|}{ March 3} & \multicolumn{2}{|c|}{ March 4} \\
\hline $\begin{array}{l}1 \\
2 \\
3 \\
4 \\
5 \\
6\end{array}$ & 2.54 & 5.5 & $\begin{array}{l}2.25 \\
2.32 \\
2.28 \\
2.20 \\
2.20 \\
2.31\end{array}$ & $\begin{array}{l}40 \\
47 \\
42 \\
35 \\
35 \\
45\end{array}$ & $\begin{array}{l}3.50 \\
3.55 \\
3.28 \\
3.10 \\
3.02 \\
2.95\end{array}$ & $\begin{array}{l}275 \\
295 \\
223 \\
175 \\
157 \\
145\end{array}$ & $\begin{array}{l}2.27 \\
2.31 \\
2.33 \\
2.36 \\
2.52 \\
2.74\end{array}$ & $\begin{array}{r}42 \\
45 \\
48 \\
49 \\
69 \\
103\end{array}$ & & - & & \\
\hline $\begin{array}{r}7 \\
8 \\
9 \\
10 \\
11 \\
\mathbf{4}\end{array}$ & 1.57 & 6 & $\begin{array}{l}2.43 \\
2.44 \\
2.36 \\
2.36 \\
2.48 \\
2.62\end{array}$ & $\begin{array}{l}56 \\
57 \\
49 \\
49 \\
62 \\
81\end{array}$ & $\begin{array}{l}2.85 \\
2.79 \\
2.72 \\
2.66 \\
2.59 \\
2.56\end{array}$ & $\begin{array}{r}115 \\
111 \\
99 \\
89 \\
78 \\
74\end{array}$ & $\begin{array}{l}3.14 \\
3.35 \\
3.50 \\
3.75 \\
4.00 \\
4.10\end{array}$ & $\begin{array}{l}180 \\
250 \\
310 \\
425 \\
580 \\
740\end{array}$ & & & & \\
\hline $\begin{array}{l}1 \\
2 \\
3 \\
4 \\
5 \\
6\end{array}$ & 1.85 & 14 & $\begin{array}{l}2.62 \\
2.70 \\
2.74 \\
2.60 \\
2.54 \\
2.46\end{array}$ & $\begin{array}{l}81 \\
93 \\
98 \\
77 \\
68 \\
59\end{array}$ & $\begin{array}{l}2.54 \\
2.50 \\
2.48 \\
2.43 \\
2.41 \\
2.38\end{array}$ & $\begin{array}{l}71 \\
66 \\
63 \\
58 \\
55 \\
52\end{array}$ & & & & & & \\
\hline $\begin{array}{r}7 \\
8 \\
9 \\
10 \\
21 \\
4\end{array}$ & 1.85 & 14 & $\begin{array}{l}2.43 \\
2.40 \\
2.39 \\
2.44 \\
2.80 \\
3.14\end{array}$ & $\begin{array}{r}55 \\
52 \\
51 \\
54 \\
108 \\
181\end{array}$ & $\begin{array}{l}2.36 \\
2.34 \\
2.32 \\
2.29 \\
2.28 \\
2.27\end{array}$ & $\begin{array}{l}50 \\
48 \\
46 \\
43 \\
42 \\
41\end{array}$ & & & & & & \\
\hline
\end{tabular}


Waterman Oanjon Oroek near Arrowhead Springs, Callf.

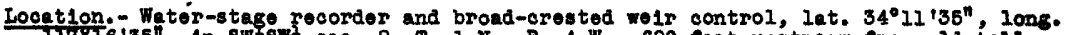

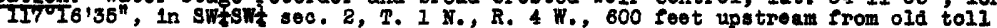
house, and I mile northwest of Arrowhead Springs. Alt1tude, about 2,125 reot.

Dreingge area.- Area, 4.65 square miles. Arerago alt1tude, 3,620 foot. Ylaximum alt1thide, 5,400 foet. A verage s lope, 50 percent. Longth of main stream ohennel, 4.5 miles. Arerage slope of maln stream channel, 14 percent.

Gage-ho1ght record.- Water-stago recorder graph, excopt 10 a.m. Kar. 2 to Mar. 15.

D1soharge reoord. - Stage-discharge relationshlp defined by current-meter measurements below 100 second-feet and extended to 600 second-foet on bas is of mean depth-mean velocity relation. Oonerete control was destroyed during afternoon of Mar. 2.

Vaximum discharge estimated by consideration of slopo-area studies, reinfail-runofs studies, and knowledgo of locel conditions. Discharge for the moximum 24-hour porlod dotermined from ralnfali-runoff study, using the Del Rosa, Etiwanda, and Dev1l Canyon recording raln gage rocords as a basis for distribution of total torm prec1p1tat1on. D1scharge for the rema1nder of the per10d, Mar. 2-15, determined from retention study and comparison with nearby stations.

Yaxima.- 1938:. D1scharge, 2,350 second-feet about 4 p.m. Har. 2.

1919-37: D1scharge, 390 sesond-foet Fob. 6, 1937 (gage holght, 4.10 foet).

Remarks.- Records good, except for the period Mar. 2-15, which are poor. Several small diversions for domestio supply above station.

Moan dally discherge, in sooond-feet, 1938

\begin{tabular}{|c|c|c|c|c|c|c|c|c|c|c|c|}
\hline Day & Fob. & Mar. & Apr. & Dey & Feb. & Mar. & Apr. & Day & Peb. & Mar. & Apr. \\
\hline $\begin{array}{r}1 \\
2 \\
3 \\
4 \\
5 \\
6 \\
7 \\
8 \\
9 \\
10\end{array}$ & $\begin{array}{c}12 \\
3.1 \\
26 \\
21 \\
7 \\
5.5 \\
4.8 \\
4.3 \\
2.9 \\
1.3\end{array}$ & $\begin{array}{r}55 \\
478 \\
156 \\
74 \\
51 \\
41 \\
35 \\
32 \\
30 \\
29\end{array}$ & $\begin{array}{l}12 \\
12 \\
11 \\
11 \\
12 \\
11 \\
11 \\
10 \\
10 \\
10\end{array}$ & $\begin{array}{l}11 \\
12 \\
13 \\
14 \\
16 \\
16 \\
17 \\
18 \\
19 \\
20\end{array}$ & $\begin{array}{r}8.7 \\
17.5 \\
8.5 \\
6.5 \\
5.5 \\
5.5 \\
4.8 \\
5.5 \\
6.5 \\
4.8\end{array}$ & $\begin{array}{r}29 \\
109 \\
111 \\
63 \\
48 \\
43 \\
42 \\
38 \\
35 \\
34\end{array}$ & $\begin{array}{l}9.5 \\
10 \\
12 \\
10 \\
9.5 \\
9 \\
9 \\
8.5 \\
8.5 \\
8.2\end{array}$ & $\begin{array}{l}21 \\
22 \\
23 \\
24 \\
25 \\
26 \\
27 \\
28 \\
29 \\
30 \\
31\end{array}$ & $\begin{array}{r}4.5 \\
4.3 \\
3.8 \\
3.8 \\
3.6 \\
3.4 \\
13 \\
38 \\
\end{array}$ & $\begin{array}{l}31 \\
29 \\
27 \\
25 \\
23 \\
20 \\
19 \\
17 \\
17 \\
15 \\
14\end{array}$ & $\begin{array}{c}7.5 \\
7 \\
7 \\
8.5 \\
12 \\
9 \\
8.5 \\
9 \\
10 \\
9\end{array}$ \\
\hline Fin & & & & & & & & & $\begin{array}{r}8.22 \\
456\end{array}$ & $\begin{array}{r}57.1 \\
3,510\end{array}$ & $\begin{array}{r}9.72 \\
579\end{array}$ \\
\hline
\end{tabular}

Gage ho1ght, in foet, and discharge, in socond-feet, at indioated time, 1938

\begin{tabular}{|c|c|c|c|c|c|c|c|c|c|c|c|c|}
\hline \multirow[t]{2}{*}{5} & Feot & Seo.ft. & Feet & seo.ft. & Feet & seo.ft. & Peet & sec.ft. & Peot & sec.ft. & Feet & sec.ft. \\
\hline & \multicolumn{2}{|c|}{ February 27 } & \multicolumn{2}{|c|}{ February 28} & \multicolumn{2}{|c|}{ Maroh 1} & \multicolumn{2}{|c|}{ March 2} & \multicolumn{2}{|c|}{ March 3} & \multicolumn{2}{|c|}{ March 4} \\
\hline $\begin{array}{l}1 \\
2 \\
3 \\
4 \\
5 \\
6\end{array}$ & $\begin{array}{l}2.78 \\
2.78 \\
2.79 \\
2.79 \\
2.79 \\
2.80\end{array}$ & $\begin{array}{l}3.4 \\
3.4 \\
3.6 \\
3.6 \\
3.6 \\
3.8\end{array}$ & $\begin{array}{l}3.15 \\
3.12 \\
3.09 \\
3.15 \\
3.23 \\
3.21\end{array}$ & $\begin{array}{l}33 \\
27 \\
21 \\
31 \\
42 \\
38\end{array}$ & $\begin{array}{l}3.65 \\
3.70 \\
3.47 \\
3.51 \\
3.52 \\
3.51\end{array}$ & $\begin{array}{r}120 \\
134 \\
60 \\
65 \\
65 \\
62\end{array}$ & $\begin{array}{l}3.37 \\
3.37 \\
3.39 \\
3.49 \\
3.50 \\
3.56\end{array}$ & $\begin{array}{r}29 \\
29 \\
34 \\
69 \\
86 \\
120\end{array}$ & & 1. & & . \\
\hline $\begin{array}{r}7 \\
8 \\
9 \\
10 \\
11 \\
15\end{array}$ & $\begin{array}{l}2.82 \\
2.83 \\
2.85 \\
2.90 \\
2.94 \\
2.94\end{array}$ & $\begin{array}{l}4.3 \\
4.5 \\
5 \\
6.5 \\
9 \\
9\end{array}$ & $\begin{array}{l}3.15 \\
3.13 \\
3.33 \\
3.34 \\
3.37 \\
3.36\end{array}$ & $\begin{array}{l}27 \\
24 \\
60 \\
62 \\
68 \\
65\end{array}$ & $\begin{array}{l}3.52 \\
3.50 \\
3.50 \\
3.50 \\
3.49 \\
3.48\end{array}$ & $\begin{array}{l}62 \\
57 \\
57 \\
57 \\
54 \\
51\end{array}$ & $\begin{array}{l}3.59 \\
3.63 \\
3.85\end{array}$ & $\begin{array}{l}154 \\
199 \\
308\end{array}$ & & & & \\
\hline $\begin{array}{l}1 \\
2 \\
3 \\
4 \\
5 \\
6\end{array}$ & $\begin{array}{l}2.99 \\
3.04 \\
3.01 \\
2.96 \\
2.93 \\
2.95\end{array}$ & $\begin{array}{l}13 \\
18 \\
15 \\
11 \\
8.5 \\
10\end{array}$ & $\begin{array}{l}3.35 \\
3.32 \\
3.20 \\
3.18 \\
3.16 \\
3.13\end{array}$ & $\begin{array}{l}62 \\
51 \\
25 \\
22 \\
20 \\
15\end{array}$ & $\begin{array}{l}3.47 \\
3.46 \\
3.45 \\
3.44 \\
3.43 \\
3.42\end{array}$ & $\begin{array}{l}49 \\
46 \\
44 \\
42 \\
40 \\
38\end{array}$ & & & & & & \\
\hline $\begin{array}{r}7 \\
8 \\
9 \\
10 \\
11\end{array}$ & $\begin{array}{l}2.98 \\
3.04 \\
3.15 \\
3.17 \\
3.14 \\
3.14\end{array}$ & $\begin{array}{l}12 \\
18 \\
33 \\
36 \\
31 \\
31\end{array}$ & $\begin{array}{l}3.16 \\
3.18 \\
3.26 \\
3.50 \\
3.50\end{array}$ & $\begin{array}{r}15 \\
17 \\
18 \\
.29 \\
88 \\
88\end{array}$ & $\begin{array}{l}3.40 \\
3.41 \\
3.43 \\
3.41 \\
3.40 \\
3.37\end{array}$ & $\begin{array}{l}35 \\
36 \\
40 \\
36 \\
35 \\
29\end{array}$ & & & & & & \\
\hline
\end{tabular}


City Creek near Highland, Cal1f.

Looation. - Water-stage recorder and conerete control, lat. $34^{\circ} 08^{\prime} 20^{\prime \prime}$, $10 n g .117^{\circ} 11^{\prime 2} 5^{\prime \prime}$,

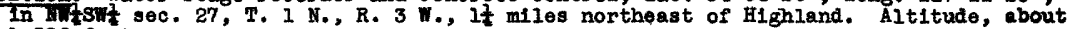
1,520 feet.

Dralnage area.- Axea, 19.8 square miles. Average alt1tuae, 3,830 feet, Maximum alt1tude, 6,350 feet. Average $310 \mathrm{pe}, 47$ percent. Iength of main stream channel, 7.6 mlles. Average slope of main stream channel, 12 percent.

Gage-he Ight record.- Water-stage recorder graph except $12 \mathrm{~m}$. Mar. 2 to Mar. 11.

Discharge recora.- Stage-discharge relation defined by current-meter measurements below, 250 second-feet; extended to peak disoharge determined by slope-area method. D1scharge for the maximu 24 hours determined from rainfall-runoff relation, using the Del Rosa recording rain gags record as a base for distribution of total storil prscipitation. Discharge Mar. 2-11 determined from retention study and comparison with adjecent stations.

Haxtma- 1938: Discharge, 6,900 second-feet 4 p.m. Mar. 2. 1919-37: Discharge, 2,360 second-feet Apr. $5,1926$.

Remarka.- Records good except those for Nar. 2-10, whlch are poor. D1scharge 1s for natural fiow of the strean and includes flow in City Oroek Fater Co.'s canal.

Mean daily discharge, in second-feet, 1988

\begin{tabular}{|c|c|c|c|c|c|c|c|c|c|c|c|}
\hline Day & Feb. & Mar. & Apr. & Day & Feb. & Mar. & Apr. & Day & Feb. & Mar. & Apr. \\
\hline $\begin{array}{r}1 \\
2 \\
3 \\
4 \\
5 \\
6 \\
7 \\
8 \\
9 \\
10\end{array}$ & $\begin{array}{l}23 \\
14 \\
33 \\
97 \\
45 \\
27 \\
20 \\
16 \\
25 \\
26\end{array}$ & $\begin{array}{r}255 \\
1,990 \\
738 \\
264 \\
208 \\
160 \\
144 \\
96 \\
80 \\
78\end{array}$ & $\begin{array}{l}43 \\
43 \\
42 \\
41 \\
42 \\
38 \\
36 \\
34 \\
33 \\
32\end{array}$ & $\begin{array}{l}11 \\
12 \\
13 \\
14 \\
15 \\
16 \\
17 \\
18 \\
19 \\
20\end{array}$ & $\begin{array}{l}36 \\
52 \\
35 \\
28 \\
24 \\
20 \\
16 \\
16 \\
22 \\
19\end{array}$ & $\begin{array}{r}74 \\
338 \\
491 \\
336 \\
240 \\
194 \\
155 \\
125 \\
108 \\
96\end{array}$ & $\begin{array}{l}32 \\
32 \\
44 \\
36 \\
35 \\
34 \\
32 \\
30 \\
28 \\
28\end{array}$ & $\begin{array}{l}21 \\
22 \\
23 \\
24 \\
25 \\
26 \\
27 \\
28 \\
29 \\
30 \\
31\end{array}$ & $\begin{array}{r}17 \\
15 \\
14 \\
13 \\
12 \\
12 \\
26 \\
170\end{array}$ & $\begin{array}{l}86 \\
78 \\
70 \\
64 \\
60 \\
55 \\
54 \\
54 \\
58 \\
51 \\
46\end{array}$ & $\begin{array}{l}27 \\
27 \\
26 \\
28 \\
40 \\
34 \\
31 \\
28 \\
32 \\
42\end{array}$ \\
\hline \multicolumn{9}{|c|}{ 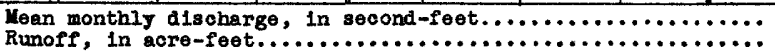 } & $\begin{array}{r}31.2 \\
1,730\end{array}$ & $\begin{array}{r}221 \\
13,580\end{array}$ & $\begin{array}{r}34,3 \\
2,040\end{array}$ \\
\hline
\end{tabular}

Discharge, in second-feet, at indicated time, 1938

\begin{tabular}{|c|c|c|c|c|}
\hline Hour & Peb. 27 & Feb. 28 & Mar. 1 & Nar. 2 \\
\hline $\begin{array}{l}1 \\
2 \\
3 \\
4 \\
5 \\
6\end{array}$ & 13 & $\begin{array}{l}103 \\
103 \\
104 \\
107 \\
107 \\
124\end{array}$ & $\begin{array}{l}397 \\
661 \\
529 \\
450 \\
357 \\
328\end{array}$ & $\begin{array}{l}122 \\
126 \\
128 \\
130 \\
147 \\
251\end{array}$ \\
\hline $\begin{array}{r}7 \\
8 \\
9 \\
10 \\
11 \\
1\end{array}$ & & $\begin{array}{l}166 \\
186 \\
188 \\
175 \\
177 \\
186\end{array}$ & $\begin{array}{l}299 \\
279 \\
261 \\
242 \\
224 \\
210\end{array}$ & $\begin{array}{r}322 \\
427 \\
600 \\
741 \\
1,100 \\
2,600\end{array}$ \\
\hline $\begin{array}{l}1 \\
2 \\
3 \\
4 \\
5 \\
6\end{array}$ & 30 & $\begin{array}{l}205 \\
230 \\
228 \\
224 \\
208 \\
196\end{array}$ & $\begin{array}{l}196 \\
182 \\
169 \\
160 \\
152 \\
148\end{array}$ & \\
\hline $\begin{array}{r}7 \\
8 \\
9 \\
10 \\
11 \\
4\end{array}$ & 55 & $\begin{array}{l}177 \\
168 \\
168 \\
157 \\
206 \\
290\end{array}$ & $\begin{array}{l}143 \\
136 \\
133 \\
125 \\
122 \\
118\end{array}$ & \\
\hline
\end{tabular}


Devil Canyon Creek near San Bernaraino, Calif.

Location.- Water-stage recorder and concrete control, lat. $34^{\circ} 12^{\prime} 05^{\prime \prime}, 10 n g .117^{\circ} 20^{\prime} 10^{\prime \prime}$, In fuscuplabe grant, 7.3 miles northrest of San Bernardino, San Bernardino County. Alt lude, about 1,800 feet.

Drainage area.- Area, 6.16 square miles, Average altitude, 3,560 feet. Haximum alt1tude, 5,350 feet. Average slope, 42 percent. Iength of maln stream channel, 4.5 miles. Average slope of main stream channel, 13 percent.

Gage-height record. - Water-stage recorder graph except 2 p.m. Mar. 2 to Mar. 7.

Discharge record. - Stage-discharge relation defined by current-meter measurements below 80 second-feet; extended logarithmically to 600 second-feet. Stream divided above station during storm period, only part of the flow passing gage (data in table includes both channe1s). Maximum discharge estimated by consideration of slope-area studies, rainfall-runoff studies, and kmowledge of local conditions. Discharge for the maximum 24 hours determined from rainfall-runoff study, using the recorde from the recoraing rain gages at Del Rosa, Etiwanda, and Devil Canyon to determine distribution of total storm rainfall.

Maxima.- 1938: Discharge, 3,320 second-feet about 4 p.m. Mar. 2. 1913-14, 1919-37: Discharge, 220 second-feet Apr. 7, 1926.

Remarks.- Records good except for the period Mar. 2-11, which are poor. C1ty of San Bermardino diverts water above gage. There was no diversion during period of flood Plov.

Mean daily discharge, in second-feet, 1938

\begin{tabular}{|c|c|c|c|c|c|c|c|c|c|c|c|}
\hline Day & Feb. & Mar. & Apr. & Day & Feb. & Mar. & Apr. & Day & Feb. & Mar. & Apr. \\
\hline $\begin{array}{r}1 \\
2 \\
3 \\
4 \\
5 \\
6 \\
7 \\
8 \\
9 \\
10\end{array}$ & $\begin{array}{r}0.1 \\
.1 \\
5.5 \\
15.0 \\
8.0 \\
5.5 \\
3.3 \\
1.3 \\
2.2 \\
2.4\end{array}$ & $\begin{array}{r}48 \\
495 \\
359 \\
137 \\
60 \\
46 \\
40 \\
36 \\
34 \\
32\end{array}$ & $\begin{array}{l}21 \\
21 \\
20 \\
19 \\
20 \\
19 \\
18 \\
16 \\
15 \\
15\end{array}$ & $\begin{array}{l}11 \\
12 \\
13 \\
14 \\
15 \\
16 \\
17 \\
18 \\
19 \\
20\end{array}$ & $\begin{array}{l}3.9 \\
7 \\
5.5 \\
4.0 \\
2.2 \\
1.5 \\
1.0 \\
1.3 \\
2.1 \\
1.8\end{array}$ & $\begin{array}{r}31 \\
143 \\
141 \\
70 \\
50 \\
48 \\
48 \\
44 \\
42 \\
39\end{array}$ & $\begin{array}{l}14 \\
15 \\
17 \\
1.5 \\
13 \\
13 \\
12 \\
11 \\
11 \\
11\end{array}$ & $\begin{array}{l}21 \\
22 \\
23 \\
24 \\
25 \\
26 \\
27 \\
28 \\
29 \\
30 \\
31 \\
\end{array}$ & $\begin{array}{r}1.6 \\
1.4 \\
1.0 \\
.8 \\
.7 \\
1.6 \\
32\end{array}$ & $\begin{array}{l}37 \\
36 \\
33 \\
32 \\
29 \\
28 \\
27 \\
25 \\
25 \\
23 \\
23 \\
\end{array}$ & $\begin{array}{l}11 \\
10 \\
12 \\
14 \\
21 \\
14 \\
14 \\
.13 \\
14 \\
16\end{array}$ \\
\hline \multicolumn{9}{|c|}{ 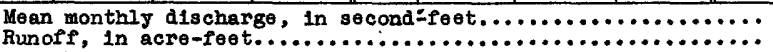 } & $\begin{array}{r}4.03 \\
224\end{array}$ & $\begin{array}{r}72,9 \\
4,480\end{array}$ & $\begin{array}{r}15.2 \\
902\end{array}$ \\
\hline
\end{tabular}

Gage height, in feet, and discharge, in second-feet, at indicated time, 1938

\begin{tabular}{|c|c|c|c|c|c|c|}
\hline \multirow{2}{*}{ Hour } & Feet & Sec.ft. & Feet & Sec.ft. & Feet & Sec.ft. \\
\hline & \multicolumn{2}{|c|}{ February 28} & \multicolumn{2}{|c|}{ March 1} & \multicolumn{2}{|c|}{ March 2} \\
\hline $\begin{array}{l}1 \\
2 \\
3 \\
4 \\
5 \\
6\end{array}$ & $\begin{array}{l}2.00 \\
2.04 \\
2.10 \\
2.23 \\
2.38 \\
2.46\end{array}$ & $\begin{array}{l}8.5 \\
9 \\
11 \\
14 \\
19 \\
22\end{array}$ & $\begin{array}{l}3.00 \\
3.07 \\
3.17 \\
3.12 \\
3.07 \\
3.02\end{array}$ & $\begin{array}{l}65 \\
77 \\
90 \\
80 \\
72 \\
67\end{array}$ & $\begin{array}{l}2.65 \\
2.65 \\
2.66 \\
2.67 \\
2.72 \\
2.85\end{array}$ & $\begin{array}{l}29 \\
29 \\
30 \\
31 \\
35 \\
46\end{array}$ \\
\hline $\begin{array}{r}7 \\
8 \\
9 \\
10 \\
\times 11 \\
\mathbf{N}\end{array}$ & $\begin{array}{l}2.51 \\
2.51 \\
2.52 \\
2.55 \\
2.66 \\
2.75\end{array}$ & $\begin{array}{l}24 \\
24 \\
25 \\
27 \\
35 \\
43\end{array}$ & $\begin{array}{l}2.99 \\
2.91 \\
2.90 \\
2.87 \\
2.82 \\
2.78\end{array}$ & $\begin{array}{l}63 \\
53 \\
52 \\
49 \\
44 \\
39\end{array}$ & $\begin{array}{l}3.02 \\
3.14 \\
3.18 \\
3.35 \\
3.37 \\
3.50\end{array}$ & $\begin{array}{r}67 \\
83 \\
90 \\
118 \\
130 \\
150\end{array}$ \\
\hline $\begin{array}{l}1 \\
2 \\
3 \\
4 \\
5 \\
6\end{array}$ & $\begin{array}{l}2.82 \\
2.82 \\
2.82 \\
2.78 \\
2.75 \\
2.73\end{array}$ & $\begin{array}{l}51 \\
51 \\
51 \\
45 \\
42 \\
40\end{array}$ & $\begin{array}{l}2.75 \\
2.74 \\
2.73 \\
2.71 \\
2.70 \\
2.68\end{array}$ & $\begin{array}{l}37 \\
36 \\
36 \\
34 \\
33 \\
32\end{array}$ & & $\begin{array}{r}250 \\
1,300\end{array}$ \\
\hline $\begin{array}{r}7 \\
8 \\
9 \\
10 \\
11 \\
11\end{array}$ & $\begin{array}{l}2.70 \\
2.68 \\
2.67 \\
2.71 \\
2.83 \\
2.92\end{array}$ & $\begin{array}{l}37 \\
35 \\
34 \\
36 \\
47 \\
57\end{array}$ & $\begin{array}{l}2.67 \\
2.66 \\
2.66 \\
2.65 \\
2.64 \\
2.63 .\end{array}$ & $\begin{array}{l}31 \\
30 \\
30 \\
29 \\
29 \\
28\end{array}$ & & \\
\hline
\end{tabular}


Lytle Creek near Fontana, Calif.

Location.- Two water-stage recorders, lat. $34^{\circ} 12^{\prime} 05^{\prime \prime}$, long. $117^{\circ} 26^{\prime} 50^{\prime \prime}$, in mit sec. 6 , T. IN., R. 5 W. (unsurvejed), a quarter of a milo bolow Lytlo Creok power plant or Southern Californla Edison Co., and $7 \frac{1}{2}$ miles north of Fontana. Alt1tude, about 2,200 feot.

Drainage area.- Area, 47.9 square miles. Average alt1tude, 6,038 foet. Uaximum alt1tude, 10,080 foot. Average slope, 45 percent. Iength of main stream channel, 15.7 square miles. Average slope of maln stream chennel, 9.4 percent.

Gage-helght record.- Oraphs from two water-stage recorders of little value due to inability to confine flow to channel near gages.

D1scharge record. - Maximum discherge determined by slope-erea measurement. Discharge for the maxtimum 24 hours determined from rainfall-minoff studies, using the records from the recording rain gages at Camp Baldy and Bt1wanda as a basis for distribution of the rainfal1. Rainfali-runoff relation based on the rocord of Fern Canyon Watershed Ho. 2. The remainder of the record determined by current-meter measurements and daliy estimates of flow made by the Fontana Union Water Co.

Laxima. - 1938: Discharge, 25,200 second-foet about 5 p.m. Mar. 2. 1918-21, 1922-37: Discharge, about 5,300 second-feet Feb. 16, 1927.

Remarics.- Records poor. Discharge 1s for naturgl flow of the stream and includes flow in Fontuna pipe line; no flow in pipe line Har. 3-9.

Mean da1ly discharge, in seciond-feet, 1938

\begin{tabular}{|c|c|c|c|c|c|c|c|c|c|c|c|}
\hline Day & Feb. & Har. & Apr. & Day & Fob. & yar. & Apr. & Day & Fob. & Mar. & Apr. \\
\hline $\begin{array}{r}1 \\
2 \\
3 \\
4 \\
5 \\
6 \\
6 \\
7 \\
8 \\
9 \\
10\end{array}$ & $\begin{array}{r}67 \\
41 \\
268 \\
268 \\
88 \\
60 \\
53 \\
50 \\
83 \\
72\end{array}$ & $\begin{array}{r}1,020 \\
8,960 \\
2,330 \\
1,080 \\
883 \\
692 \\
600 \\
550 \\
550 \\
502\end{array}$ & $\begin{array}{l}191 \\
191 \\
189 \\
187 \\
184 \\
180 \\
172 \\
173 \\
169 \\
168\end{array}$ & $\begin{array}{l}11 \\
12 \\
13 \\
14 \\
15 \\
16 \\
17 \\
18 \\
19 \\
20\end{array}$ & $\begin{array}{r}156 \\
130 \\
89 \\
85 \\
72 \\
67 \\
56 \\
58 \\
58 \\
60\end{array}$ & $\begin{array}{l}714 \\
944 \\
805 \\
436 \\
361 \\
289 \\
284 \\
254 \\
382 \\
301\end{array}$ & $\begin{array}{l}168 \\
166 \\
166 \\
164 \\
164 \\
160 \\
156 \\
153 \\
151 \\
152\end{array}$ & $\begin{array}{l}21 \\
22 \\
23 \\
24 \\
25 \\
26 \\
27 \\
28 \\
29 \\
30 \\
31\end{array}$ & $\begin{array}{r}56 \\
54 \\
54 \\
51 \\
49 \\
49 \\
156 \\
830\end{array}$ & $\begin{array}{l}274 \\
258 \\
257 \\
244 \\
232 \\
221 \\
211 \\
197 \\
186 \\
191 \\
193\end{array}$ & $\begin{array}{l}155 \\
158 \\
160 \\
161 \\
175 \\
177 \\
168 \\
174 \\
178 \\
184\end{array}$ \\
\hline \multicolumn{9}{|c|}{ 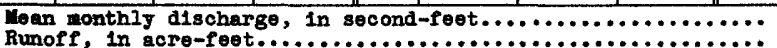 } & $\begin{array}{r}113 \\
6,300\end{array}$ & $\begin{array}{r}765 \\
48,280\end{array}$ & $10, \frac{170}{100}$ \\
\hline
\end{tabular}

Iytle Creok (east channel) at San Bernardino, Calif.

Locat1on. - Water-stage recorder, 1at. $34^{\circ} 05^{\prime} 50^{n}$, 1ong. $117^{\circ} 19^{\prime} 05^{n}$, In San Bermardino grant, near Atch1son, Topoka se Sante Fe Rallway bridge, a quarter of a milo upatreain from Mt. Vernon Street Bridge, at San Bernardino, San Bermardino County. Alt1tude, about 1,050 reot.

Gage-helght record.- Water-stage recorder graph prior to $3 \mathrm{a.m.} \mathrm{Mar.} 1$.

D1scharge record.- Stage-discharge relation defined by current-meter measurements be10w 1,500 second-feets.peak discharge determined by contracted-opening method. D1scharge 3 a.m. Mar. 1 to Mar. 4 obtained by comparison with Iytle Creek at Fontena, Cafon and Lone PIne Creeks near Keenbrook, and west channel of Iytle Creek at Colton, and Har. 4 to Apr. 30 by comparison with da1ly discharge of Iytle Creek at Foothill Boulevard (about $i$ mlle upstreand) as furnished by the Fontana water Co., and station on Warm Groek.

Kaxima. - 1938: D1scharge, 21,500 second-feet 7:3C p.m. Mar. 2. 1929-37: D1 scharge, 1,060 second-feet, Feb. 14, 1937.

Remarks.- Records poor.

$4547200-42-15$ 
Yean dally discharge, in second-feet, 1938

\begin{tabular}{|c|c|c|c|c|c|c|c|c|c|c|c|}
\hline Day & Feb. & Mar. & Apr. & Day & Feb. & Mar. & Apr. & Day & $\mathrm{Feb}$. & Mar. & Apr. \\
\hline $\begin{array}{r}1 \\
2 \\
3 \\
4 \\
5 \\
6 \\
7 \\
8 \\
9 \\
10\end{array}$ & $\begin{array}{r}18 \\
1.0 \\
91 \\
167 \\
4.3 \\
2.8 \\
.5 \\
.1 \\
.7 \\
2.2\end{array}$ & $\begin{array}{r}604 \\
7,640 \\
4,460 \\
625 \\
228 \\
160 \\
100 \\
68 \\
44 \\
27\end{array}$ & $\begin{array}{l}20 \\
19 \\
18 \\
17 \\
17 \\
17 \\
17 \\
17 \\
19 \\
20\end{array}$ & $\begin{array}{l}11 \\
12 \\
13 \\
14 \\
15 \\
16 \\
17 \\
18 \\
19 \\
20\end{array}$ & $\begin{array}{l}77 \\
41 \\
3.8 \\
0.3 \\
0 \\
0 \\
0 \\
0 \\
0\end{array}$ & $\begin{array}{r}32 \\
103 \\
119 \\
74 \\
54 \\
56 \\
43 \\
38 \\
27 \\
12\end{array}$ & $\begin{array}{l}23 \\
25 \\
27 \\
28 \\
29 \\
28 \\
27 \\
26 \\
27 \\
28\end{array}$ & $\begin{array}{l}21 \\
22 \\
23 \\
24 \\
25 \\
26 \\
27 \\
28 \\
29 \\
30 \\
31\end{array}$ & $\begin{array}{c}0 \\
0 \\
0 \\
0 \\
0 \\
0 \\
2.7 \\
255\end{array}$ & $\begin{array}{l}24 \\
20 \\
22 \\
27 \\
30 \\
33 \\
34 \\
33 \\
30 \\
26 \\
22 \\
\end{array}$ & $\begin{array}{l}28 \\
29 \\
28 \\
26 \\
25 \\
27 \\
32 \\
39 \\
47 \\
64\end{array}$ \\
\hline \multicolumn{9}{|c|}{ 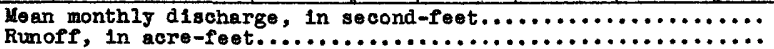 } & $\begin{array}{r}23.8 \\
1,320\end{array}$ & 29,378 & $\begin{array}{r}26.5 \\
1,580\end{array}$ \\
\hline
\end{tabular}

\begin{tabular}{|c|c|c|c|}
\hline Hour & February 87 & Febmuary 28 & March 1 \\
\hline $\begin{array}{l}1 \\
2 \\
3 \\
4 \\
5 \\
6\end{array}$ & $\begin{array}{l}0 \\
0 \\
0 \\
0 \\
0 \\
0\end{array}$ & $\begin{array}{l}50 \\
50 \\
50 \\
50 \\
50 \\
70\end{array}$ & $\begin{array}{r}110 \\
950 \\
1,350\end{array}$ \\
\hline $\begin{array}{r}7 \\
8 \\
9 \\
10 \\
11 \\
11\end{array}$ & $\begin{array}{l}0 \\
0 \\
0 \\
0 \\
0 \\
0\end{array}$ & $\begin{array}{l}140 \\
220 \\
200 \\
190 \\
350 \\
600\end{array}$ & - \\
\hline $\begin{array}{l}1 \\
2 \\
3 \\
4 \\
5 \\
6\end{array}$ & $\begin{array}{l}0 \\
0 \\
0 \\
0 \\
0 \\
0\end{array}$ & $\begin{array}{l}940 \\
860 \\
650 \\
450 \\
320 \\
210\end{array}$ & \\
\hline $\begin{array}{r}7 \\
8 \\
9 \\
10 \\
11 \\
y\end{array}$ & $\begin{array}{r}0 \\
0 \\
6 \\
18 \\
24 \\
36\end{array}$ & $\begin{array}{r}165 \\
130 \\
105 \\
95 \\
98 \\
100\end{array}$ & \\
\hline
\end{tabular}

Lytle Creek (west channel) at Colton, Cal1f.

Location. - Water-atage recorder, lat. $34^{\circ} 04^{\prime} 00^{\prime \prime}$, 1ong. $117^{\circ} 19^{\prime} 15^{\prime \prime}$, In San Bermardino grant, at F Street near Colton Avenue, at Coiton, San Bermardino County. Altitude, grout 980 feet.

Gage-height record.- Water-stage recorder graph except 2 p.m. Mar. 2 tc 4 p.m. Mar. 3, and 5 a.m. Har. 4 to Mar: 15 .

Discharge record.- Stage-discharge relation defined by current-meter measurementa beIow 480 second-feet; extended logarithmically to peak stage. Records for periods of missing gage helghts obtained from peak discharge compratation and by comparison with east channel of Lytle Creek.

Maxime.- 1938: Discharge, 7,900 second-feet 7 p.m. Mar. 2. 1929-37: Discharge, 20 second-feet Jan. 5, 1935.

Remarks.- Records fair except 2 p.m. Mar. 2 to 4 p.m. Mar. 3 , and 5 a.m. Kar. 4 to lar. 15, which are pcor. Flood flow not affected by diversions.

Mean daily discharge, in second-feet, 1938

\begin{tabular}{|c|c|c|c|c|c|c|c|c|c|c|c|}
\hline Day & Feb. & Mar. & Apr. & Day & $\mathrm{Feb}$. & Mar. & Apr. & Day & $\mathrm{Feb}$ & Mar. & Apr. \\
\hline $\begin{array}{r}1 \\
2 \\
3 \\
4 \\
5 \\
6 \\
7 \\
8 \\
9 \\
10\end{array}$ & $\begin{array}{l}0.6 \\
0 \\
.1 \\
0.1 \\
0 \\
0 \\
0 \\
0 \\
0.6\end{array}$ & $\begin{array}{c}6.2 \\
2,180 \\
817 \\
27 \\
1.6 \\
0 \\
0 \\
0 \\
0\end{array}$ & $\begin{array}{l}0 \\
0 \\
0 \\
0 \\
0 \\
0 \\
0 \\
0 \\
0 \\
0\end{array}$ & $\begin{array}{l}11 \\
12 \\
13 \\
14 \\
15 \\
16 \\
17 \\
18 \\
19 \\
20\end{array}$ & $\begin{array}{l}0.1 \\
0 \\
0 \\
0 \\
0 \\
0 \\
0 \\
0 \\
0 \\
0\end{array}$ & $\begin{array}{l}0 \\
0 \\
0 \\
0 \\
0 \\
0 \\
0 \\
0 \\
0 \\
0\end{array}$ & $\begin{array}{l}0 \\
0 \\
0 \\
0 \\
0 \\
0 \\
0 \\
0 \\
0 \\
0\end{array}$ & $\begin{array}{l}21 \\
22 \\
23 \\
24 \\
25 \\
26 \\
27 \\
28 \\
29 \\
30 \\
31\end{array}$ & $\begin{array}{l}0 \\
0 \\
0 \\
0 \\
0 \\
0 \\
.3 \\
.1\end{array}$ & $\begin{array}{l}0 \\
0 \\
0 \\
0 \\
0 \\
0 \\
0 \\
0 \\
0 \\
0 \\
0\end{array}$ & $\begin{array}{l}0 \\
0 \\
0 \\
0 \\
0 \\
0 \\
0 \\
0 \\
0 \\
0\end{array}$ \\
\hline \multicolumn{9}{|c|}{ 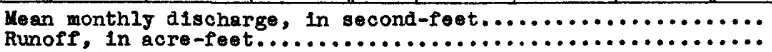 } & $\begin{array}{r}0.07 \\
3.8\end{array}$ & $\begin{array}{r}97.8 \\
6,010\end{array}$ & $\begin{array}{l}0 \\
0\end{array}$ \\
\hline
\end{tabular}


D1soharge, in second-foet, at indioeted time, 1938

\begin{tabular}{|c|c|c|c|c|}
\hline Hoir & Maroh 1 & Maroh 2 & Maroh 3 & March 4 \\
\hline $\begin{array}{l}1 \\
2 \\
3 \\
4 \\
5 \\
6\end{array}$ & $\begin{array}{l}9.5 \\
6.5 \\
1.4 \\
6.5 \\
29\end{array}$ & $\begin{array}{c}0.1 \\
.6 \\
4.7 \\
4.7 \\
9 \\
14\end{array}$ & & $\begin{array}{l}47 \\
36 \\
34 \\
49 \\
41\end{array}$ \\
\hline $\begin{array}{r}7 \\
8 \\
9 \\
10 \\
11 \\
11\end{array}$ & $\begin{array}{l}12 \\
7 \\
5 \\
4.2 \\
3.7 \\
2.4\end{array}$ & $\begin{array}{l}15 \\
10 \\
8.5 \\
10 \\
16 \\
12\end{array}$ & & \\
\hline $\begin{array}{l}1 \\
2 \\
3 \\
4 \\
5 \\
6\end{array}$ & $\begin{array}{r}1.7 \\
.9 \\
.3 \\
.3 \\
.2 \\
.2\end{array}$ & $\begin{array}{l}1,200 \\
2,250\end{array}$ & $\begin{array}{l}280 \\
216 \\
168\end{array}$ & \\
\hline $\begin{array}{r}7 \\
8 \\
9 \\
10 \\
11 \\
Y\end{array}$ & $\begin{array}{l}.1 \\
.1 \\
.1 \\
.1 \\
.1 \\
.1\end{array}$ & & $\begin{array}{r}172 \\
192 \\
170 \\
118 \\
88 \\
72\end{array}$ & \\
\hline
\end{tabular}

Cajon Creek near Keonbrook, Calif.

Loogt10n.- Two water-gtage rocorders, lat. $34^{\circ} 15150^{\prime \prime}$, long. $117^{\circ} 27^{\prime} 50^{\prime \prime}$, near north boundary of sec. 13, T. 2 N., R. 6 V., 300 feet and 1,800 feet above mouth of Ione Pline Creek, and about $i$ mile north of Keenbrook. Altitude, about 2,620 feet.

Drainage area.- Area, 40.9 equare m1lea. Average alt1tude, 3,960 leet. Maximum alt1tude, 6,917 feet. Average slope, 47 percent. Length of maln stream channe1, 13 mlles. Average s lope of main stream channe1, 6.1 percent.

Gage-helght record. - Water-stage recorder graph at lower station except 10 a.m. Mar. 2 to lar. II; at upper station except 2 p.m. Mar. 2 to Mar. 17.

D1scharge record. - Stage-discharge relation defined by current-meter measurements beIow 600000nd-feet; peak discharge determined by slope-area measurement. Dis-

oharge for the maximum 24 hourg determined largely from rainfal1-runoff relation, using the Dol Ross and Devil Canyon recording rain gage records as a basis for distribution of the storm rainfall. Disoharge lar. 2-15 besed on rainfali-runoff studies, partial gage-holght record, discharge mosaurements and comparison with nearby streams.

Maxima.- 1938: Discharge, 14,500 second-foet about 5 p.m. Mar. 2. 1919-37: Discharge, about 8,000 socond-feet Dec. 20, 1921 .

Remarks. - Records good exoept those for Mar. 2-15, which are poor. No diversion or regulation above gage.

Mean dally discharge, in seoond-feet, 1938

\begin{tabular}{|c|c|c|c|c|c|c|c|c|c|c|c|}
\hline Day & Feb. & Kar. & Apr. & $\overline{D a y}$ & Fob. & Kar. & Apr. & Day & Seb. & Mar. & Apr. \\
\hline \begin{tabular}{r|}
1 \\
2 \\
3 \\
4 \\
5 \\
6 \\
7 \\
8 \\
9 \\
10
\end{tabular} & $\begin{array}{r}52 \\
11 \\
83 \\
112 \\
29 \\
22 \\
18 \\
15 \\
73 \\
41\end{array}$ & $\begin{array}{r}580 \\
3,800 \\
2,050 \\
535 \\
231 \\
160 \\
130 \\
110 \\
90 \\
80\end{array}$ & $\begin{array}{l}26 \\
24 \\
26 \\
24 \\
22 \\
21 \\
21 \\
21 \\
21 \\
21\end{array}$ & $\begin{array}{l}11 \\
12 \\
13 \\
14 \\
16 \\
16 \\
17 \\
18 \\
19 \\
20\end{array}$ & $\begin{array}{r}222 \\
77 \\
34 \\
25 \\
21 \\
18 \\
17 \\
19 \\
23 \\
17\end{array}$ & $\begin{array}{r}75 \\
150 \\
80 \\
75 \\
64 \\
56 \\
48 \\
40 \\
43 \\
44\end{array}$ & $\begin{array}{l}22 \\
22 \\
20 \\
19 \\
17 \\
14 \\
13 \\
12 \\
12 \\
12\end{array}$ & $\begin{array}{l}21 \\
22 \\
23 \\
24 \\
25 \\
26 \\
27 \\
28 \\
29 \\
30 \\
31\end{array}$ & $\begin{array}{r}14 \\
13 \\
12 \\
11 \\
11 \\
10 \\
76 \\
533\end{array}$ & $\begin{array}{l}42 \\
34 \\
33 \\
30 \\
28 \\
26 \\
31 \\
29 \\
28 \\
27 \\
29\end{array}$ & $\begin{array}{l}11 \\
11 \\
11 \\
11 \\
14 \\
12 \\
11 \\
11 \\
13 \\
12\end{array}$ \\
\hline & & & & & & & & & $\begin{array}{r}61.0 \\
3,390\end{array}$ & $\begin{array}{r}283 \\
17,410\end{array}$ & $\begin{array}{r}16.9 \\
1,010\end{array}$ \\
\hline
\end{tabular}




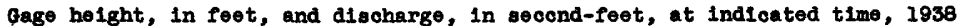

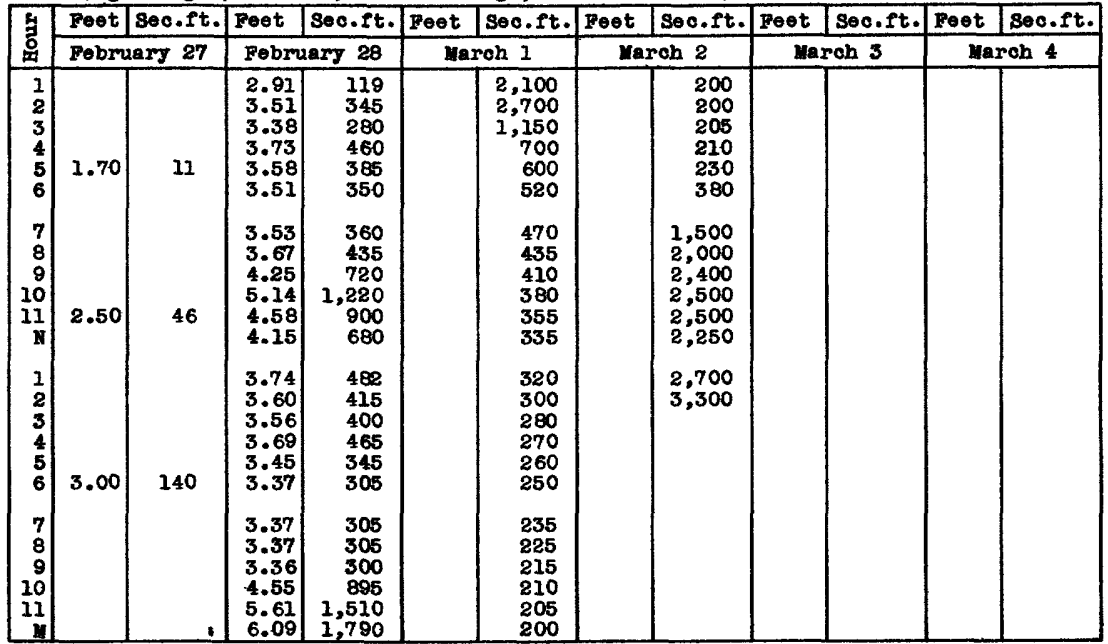

Lone Pine Creok near Keonbrook, Gallf.

Looation. - Water-stage recorder, lat. $34^{\circ} 15^{\prime} 55^{\prime \prime}$, long. $117^{\circ} 27^{\prime} 55^{\prime \prime}$, In s4d sec. 12, T. 2 1., T. 6 W., 50 feet upstreain from Atohison, Topolka Santa Pe Railway bridge, and 1 mile north of Koenbrook. Altitude, about 2,630 foet.

Drainage area.- Area, 15.3 square miles. Average altitude, 4,700 foet. Maximum altitưte, 8,500 feet. Average alope, 48 percent. Length of min atroam channel, 10.2

iniles. Average slope of main atream channel, il percent.

Gage-height record.- Record lost due to destruction of gage shelter and well.

Disoharge reoord. - Maximim discharge obtained by slope-area measurement. Disoharge for the maximum 24 hourg detervined from rainfall-runoff study; the average rainfail on the area was distributed on the basis of records from the D61 Rosa and Devil Canyon recording rain gages." Remainder of rocord determined from current-meter measurements and by comparieon with record for Cajon Creek near Koenbrook.

Hexim.- 1938: Disoharge, 6,180 second-foet Mar. 2.

1919-37: Discharge; 810 second-feet (estinated) Dec, 19, 1921.

Remarks.- Records poor. No diversion or regulation above atation.

Lean daily discharge, in second-fèt, 1938

\begin{tabular}{|c|c|c|c|c|c|c|c|c|c|c|c|}
\hline Day & Fob. & Nax: & Apr. & Day & Fob. & Mar. & Apr. & Day & Pob. & Mar. & Apr. \\
\hline $\begin{array}{r}1 \\
2 \\
3 \\
4 \\
5 \\
6 \\
7 \\
8 \\
9 \\
10\end{array}$ & $\begin{array}{r}18 \\
10^{.7} \\
9.5 \\
1.3 \\
1.1 \\
.9 \\
12^{.8} \\
8\end{array}$ & $\begin{array}{r}206 \\
1,480 \\
840 \\
190 \\
90 \\
46 \\
28 \\
18 \\
13 \\
9\end{array}$ & $\begin{array}{l}5.5 \\
5.5 \\
5.5 \\
6 \\
5.5 \\
5.5 \\
5.5 \\
5 \\
5 \\
5\end{array}$ & $\begin{array}{l}11 \\
12 \\
13 \\
14 \\
15 \\
16 \\
17 \\
18 \\
19 \\
20\end{array}$ & $\begin{array}{r}60 \\
12 \\
3.9 \\
1.8 \\
.9 \\
1.0 \\
1.2 \\
1.4 \\
1.7 \\
1.4\end{array}$ & $\begin{array}{c}7 \\
34 \\
8 \\
5 \\
4.7 \\
4.5 \\
4.2 \\
3.9 \\
3.8 \\
3.7\end{array}$ & $\begin{array}{l}5 \\
5 \\
5 \\
5 \\
4.9 \\
4.8 \\
4.8 \\
4.8 \\
4.7 \\
4.7\end{array}$ & $\begin{array}{l}21 \\
22 \\
23 \\
24 \\
25 \\
26 \\
27 \\
28 \\
29 \\
30 \\
31\end{array}$ & $\begin{array}{r}1.2 \\
1.1 \\
.9 \\
.9 \\
.8 \\
.7 \\
4.0 \\
67\end{array}$ & $\begin{array}{l}3.5 \\
3.4 \\
3.4 \\
3.3 \\
3.2 \\
3.2 \\
3.4 \\
3.7 \\
4.2 \\
4.7 \\
5.5 \\
\end{array}$ & $\begin{array}{l}4.7 \\
4.7 \\
4.8 \\
5 \\
5 \\
5 \\
5 \\
5 \\
5 \\
5.5\end{array}$ \\
\hline \multicolumn{9}{|c|}{ 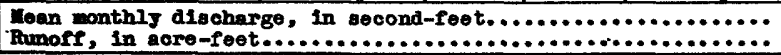 } & $\begin{array}{r}8.01 \\
445 \\
\end{array}$ & $\begin{array}{r}98,1 \\
6,030 \\
\end{array}$ & $\begin{array}{r}5.08 \\
302 \\
\end{array}$ \\
\hline
\end{tabular}


Day Creok near Et1wanda, Cal1f.

Location. - Water-atage recorder and broad-crested weir, 1at. $34^{\circ} 11^{\prime} 00^{\prime \prime}, 10 n g .1^{\circ} 7^{\circ} 32^{\prime}$ $25^{\circ}$, in SW sec. 8, T. I N., R. 6 . , a quarter of a mile dornstream from confluence of two main forks, and 6 miles north of Etiwanda. Altitudo, about 2,940 feet.

Drainage area.- Area, 4.8 square miles. Average altitude, 5,208 feet. Maximum altitude, 8,911 feet. Average slope, 52 percent. Length of main stream chamel, 4.0 miles. Slope of main stream channel, 28 percent.

Gage-he1ght record. - Record lost due to destruction of recoraing gage.

Discharge record. - Maximum discharge and discharge fer the maximum 24-hour period obtained by rainfall-munoff studies, using records from Camp Baldy and $5 t$ iwanda recording rain gages as basis for distributing the rainfall. Records Mar. 4-11 obtained by use of retention curves; other records oomputed from current-meter measurewents and by comparis on with nearby stations.

Maxima.- 1938: Diecharge, 4,200 second-feet Mar. 2

1929-37: Discharge, 192 second-feet Feb. il, 1936.

Remarks.- Records poor. No regulation or diversion above gage during flood period.

Kean dally discharge, in second-feet, 1938

\begin{tabular}{|r|c|c|c||c|c|c|c|c|c|c|c|}
\hline Day & Feb. & Mar. & Apr. & Day & Feb. & Mar. & Apr. & Day & Feb. & Mar. & Apr. \\
\hline 1 & 11 & 108 & 31 & 11 & 31 & 50 & 23 & 21 & 17 & 40 & 25 \\
2 & 7 & 1,055 & 31 & 12 & 33 & 130 & 22 & 22 & 14 & 39 & 23 \\
3 & 50 & 449 & 30 & 13 & 25 & 180 & 23 & 23 & 13 & 38 & 23 \\
4 & 49 & 121 & 29 & 14 & 20 & 78 & 24 & 24 & 12 & 37 & 23 \\
5 & 29 & 80 & 28 & 15 & 19 & 63 & 23 & 25 & 12 & 36 & 20 \\
6 & 19 & 60 & 27 & 16 & 19 & 55 & 18 & 26 & 12 & 36 & 20 \\
7 & 13 & 55 & 27 & 17 & 17 & $50 \ldots$ & 17 & 27 & 14 & 34 & 20 \\
8 & 11 & 52 & 26 & 18 & 17 & 46 & 18 & 28 & 107 & 33 & 20 \\
9 & 16 & 50 & 25 & 19 & 17 & 43 & 23 & 29 & & 33 & 20 \\
10 & 19 & 50 & 24 & 20 & 17 & 42 & 26 & 30 & & 32 & 22 \\
\hline
\end{tabular}

Cucamonga Creek near Upland, Calif.

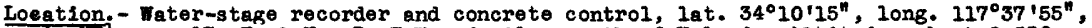
In lis sec. $17, T .1$ H., R. 7 . ., 6 miles north of Upland. Altitude, about 2,550 feet.

Drainage area.- Area, 10.1 square miles. Average altitude, 5,275 feet. Maximum altitude, 8,911 feet. Average slope, 54 percent. Length of in stream channel, 5 miles. Average slope of main atream channel, 24 percent.

Gage-helght record.- Vater-stage recorder graph except Mar. 2 to Apr. 15.

Discharge record.- Stage-discharge relation well defined below 100 eecond-feet prior to Harch 2. Concrete control destroyed and station buried in debris on March 2 . Haximum discharge estimated by consideration of slope-area studies, rainfeli-punoff studies, and knowledge of local conditions. Discharge for the meximum 24 hours determined from rainfall-munoff study, using the records from Camp Baldy and Etiwands recording rain gages as a bas for distributing the storn rainfall. Disoharge lar. 4-11 obtained bJ use of retention curves.

Maxima. - 1938: Discharge, 10,300 second-feet about 3 p.m. Mar. 2

1928-37: Discharge, 475 second-feet Feb. 11, 1936.

Remarks.- Records fair. No regulation or diversions above gage during flood period.

Mean deily discharge, in second-feet, 1938

\begin{tabular}{|c|c|c|c|c|c|c|c|c|c|c|c|}
\hline Day & Feb. & Mar. & Apr. & Day & Feb. & Mar. & Apr. & Day & Feb. & Mar. & Apr. \\
\hline $\begin{array}{r}1 \\
2 \\
3 \\
4 \\
5 \\
6 \\
7 \\
8 \\
9 \\
10\end{array}$ & $\begin{array}{r}14 \\
7 \\
60 \\
53 \\
32 \\
21 \\
14 \\
12 \\
22 \\
24\end{array}$ & $\begin{array}{r}223 \\
2,120 \\
719 \\
266 \\
149 \\
122 \\
109 \\
81 \\
68 \\
68\end{array}$ & $\begin{array}{l}36 \\
35 \\
34 \\
33 \\
32 \\
31 \\
30 \\
30 \\
29 \\
28\end{array}$ & $\begin{array}{l}11 \\
12 \\
13 \\
14 \\
15 \\
16 \\
17 \\
18 \\
19 \\
20\end{array}$ & $\begin{array}{l}43 \\
46 \\
33 \\
25 \\
20 \\
20 \\
18 \\
17 \\
18 \\
16\end{array}$ & $\begin{array}{r}68 \\
167 \\
242 \\
108 \\
80 \\
69 \\
66 \\
63 \\
60 \\
58\end{array}$ & $\begin{array}{l}28 \\
27 \\
28 \\
29 \\
30 \\
23 \\
19 \\
20 \\
29 \\
29\end{array}$ & $\begin{array}{l}21 \\
22 \\
23 \\
24 \\
25 \\
26 \\
27 \\
28 \\
29 \\
30 \\
31\end{array}$ & $\begin{array}{r}14 \\
13 \\
12 \\
12 \\
11 \\
11 \\
16 \\
222\end{array}$ & $\begin{array}{l}55 \\
53 \\
51 \\
49 \\
47 \\
45 \\
43 \\
42 \\
40 \\
39 \\
37\end{array}$ & $\begin{array}{l}29 \\
26 \\
24 \\
25 \\
21 \\
21 \\
21 \\
21 \\
22 \\
22\end{array}$ \\
\hline \multicolumn{9}{|c|}{ 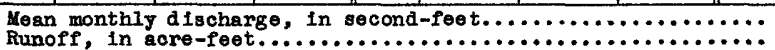 } & $\begin{array}{r}29.5 \\
1,640\end{array}$ & 10.720 & $1,61 . \frac{1}{0}$ \\
\hline
\end{tabular}


Gage he1ght, In feet, and d1scharge, In seoond-feet, at indicated t1me, 1938

\begin{tabular}{|c|c|c|c|c|c|c|}
\hline \multirow{2}{*}{ Hour } & Feet & sec.ft. & Feet & Sec.ft. & Feet & Seo.fto \\
\hline & \multicolumn{2}{|c|}{ Fobmuary 27} & \multicolumn{2}{|c|}{ Februery 28} & \multicolumn{2}{|c|}{ Maroh 1} \\
\hline $\begin{array}{l}1 \\
2 \\
3 \\
4 \\
5 \\
6\end{array}$ & $\begin{array}{l}1.76 \\
1.78 \\
1.80 \\
1.78 \\
1.78 \\
1.78\end{array}$ & $\begin{array}{l}11 \\
12 \\
12 \\
12 \\
12 \\
12\end{array}$ & $\begin{array}{l}2.82 \\
3.01 \\
3.21 \\
3.46 \\
3.64 \\
3.67\end{array}$ & $\begin{array}{r}37 \\
49 \\
67 \\
96 \\
123 \\
129\end{array}$ & $\begin{array}{l}4.87 \\
4.60 \\
4.65 \\
4.45 \\
4.45 \\
4.35\end{array}$ & $\begin{array}{l}520 \\
355 \\
418 \\
336 \\
336 \\
302\end{array}$ \\
\hline $\begin{array}{r}7 \\
8 \\
9 \\
10 \\
11 \\
11\end{array}$ & $\begin{array}{l}1.79 \\
1.82 \\
1.88 \\
1.83 \\
1.93 \\
1.92\end{array}$ & $\begin{array}{l}12 \\
13 \\
14 \\
16 \\
15 \\
16\end{array}$ & $\begin{array}{l}3.66 \\
3.86 \\
4.00 \\
4.10 \\
4.02 \\
4.43\end{array}$ & $\begin{array}{l}127 \\
167 \\
188 \\
225 \\
203 \\
328\end{array}$ & $\begin{array}{l}4.30 \\
4.15 \\
3.75 \\
3.65 \\
3.68 \\
3.90\end{array}$ & $\begin{array}{l}285 \\
240 \\
140 \\
125 \\
130 \\
175\end{array}$ \\
\hline $\begin{array}{l}1 \\
2 \\
3 \\
4 \\
5 \\
6\end{array}$ & $\begin{array}{l}1.92 \\
1.08 \\
2.01 \\
2.02 \\
2.02 \\
2.02\end{array}$ & $\begin{array}{l}16 \\
17 \\
18 \\
18 \\
18 \\
18\end{array}$ & $\begin{array}{l}4.25 \\
4.20 \\
4.00 \\
4.28 \\
3.85 \\
4.03\end{array}$ & $\begin{array}{l}270 \\
265 \\
188 \\
278 \\
186 \\
206\end{array}$ & $\begin{array}{l}4.08 \\
3.65 \\
3.65 \\
3.75 \\
3.75 \\
3.80\end{array}$ & $\begin{array}{l}220 \\
126 \\
125 \\
144 \\
144 \\
154\end{array}$ \\
\hline $\begin{array}{r}7 \\
8 \\
9 \\
10 \\
11 \\
11\end{array}$ & $\begin{array}{l}2.02 \\
2.05 \\
2.20 \\
2.21 \\
2.20 \\
2.18\end{array}$ & $\begin{array}{l}18 \\
18 \\
23 \\
23 \\
23 \\
22\end{array}$ & $\begin{array}{l}4.07 \\
4.06 \\
4.31 \\
4.68 \\
5.15 \\
5.15\end{array}$ & $\begin{array}{l}217 \\
214 \\
288 \\
431 \\
580 \\
680\end{array}$ & $\begin{array}{l}3.78 \\
3.78 \\
3.68 \\
3.65 \\
3.64 \\
3.72\end{array}$ & $\begin{array}{l}160 \\
150 \\
132 \\
125 \\
123 \\
138\end{array}$ \\
\hline
\end{tabular}

San Jacinto River near San Jacinto, 0ailf.

Location. - Staff-gage, lat. $33^{\circ} 44^{\prime} 05^{\prime \prime}$, long. $116^{\circ} 49^{\prime} 46^{\prime \prime}$, In SIt sec. 13, T. E S., R 1 H, at hlghway bridge, giles southoat of $\operatorname{san}$ Jacinto. Altitude, about 1,980 foet.

Drainage area.- Area, 140 quare miles. Average alt1tude, 5,250 feet. Maximum alt1tude, 10,800 leet. Average lope, 41 peroent. Iength of maln tream ohannel, 21 m1les. Average lope of ma in tream ohannel, 8.7 percent.

Qage-helght reoord.- Staff gage read dally; more frequently during flood perlod.

Discherge record. - Stage-divcharge relation falrly well defined by ourrent-moter moerurement below 200 second-fest; poak dlocharge 1o mo an of slope-area and con-

tracted opening determinatione. Discharge Feb. 28 to Mar. 4 baued on gage-holght, ourrent-moter moauremente, and entlmater of diecharge furniohod by kotropolitan Water Diotrict of Southern Cal1fornia.

Mextma.- 1938: D10 charge, 14,300 socond-foet about 12 p.m. Mar. 2.

1820-37, Discharge; 46,000 vecond-foet (estimated) Fob. i6, 1827.

Remerke.- Recorde poor. Some regulation during rlood at Lake Hemet; no diveralons.

Mean da1ly discherge, in weoond-feet, 1938

\begin{tabular}{|c|c|c|c|c|c|c|c|c|c|c|c|}
\hline$\overline{D a y}$ & Fob. & Mar. & Apr. & Day & Feb. & Mar. & Apr. & Day & $F \in b$, & Mar. & Apr. \\
\hline $\begin{array}{r}1 \\
2 \\
3 \\
4 \\
5 \\
6 \\
7 \\
8 \\
9 \\
10\end{array}$ & $\begin{array}{c}0.6 \\
16 \\
4.6 \\
45 \\
35 \\
28 \\
17 \\
13 \\
13 \\
37\end{array}$ & $\begin{array}{r}890 \\
3,170 \\
5,100 \\
1,600 \\
780 \\
622 \\
407 \\
368 \\
343 \\
298\end{array}$ & $\begin{array}{c}134 \\
128 \\
128 \\
128 \\
112 \\
115 \\
126 \\
103 \\
99 \\
99\end{array}$ & $\begin{array}{l}11 \\
12 \\
13 \\
14 \\
15 \\
16 \\
17 \\
18 \\
19 \\
20 .\end{array}$ & $\begin{array}{l}29 \\
74 \\
46 \\
30 \\
24 \\
24 \\
18 \\
23 \\
23 \\
21\end{array}$ & $\begin{array}{r}368 \\
2,070 \\
1,280 \\
940 \\
658 \\
550 \\
494 \\
444 \\
374 \\
368\end{array}$ & $\begin{array}{r}98 \\
98 \\
99 \\
111 \\
98 \\
100 \\
98 \\
84 \\
88 \\
88\end{array}$ & $\begin{array}{l}21 \\
22 \\
23 \\
24 \\
25 \\
26 \\
27 \\
28 \\
28 \\
30 \\
31\end{array}$ & $\begin{array}{r}17 \\
16 \\
16 \\
16 \\
18 \\
16 \\
22 \\
316\end{array}$ & $\begin{array}{l}356 \\
358 \\
174 \\
162 \\
163 \\
153 \\
170 \\
157 \\
157 \\
145 \\
140\end{array}$ & $\begin{array}{r}88 \\
86 \\
79 \\
76 \\
84 \\
188 \\
138 \\
110 \\
94 \\
108\end{array}$ \\
\hline & & & & & & & & & $\begin{array}{r}33.8 \\
1,880\end{array}$ & $\begin{array}{r}745 \\
46,670\end{array}$ & $\begin{array}{r}108 \\
6,260\end{array}$ \\
\hline
\end{tabular}


San Jacinto River at Railroad Cangon Reservoir, near Blsinore, Cal1f.

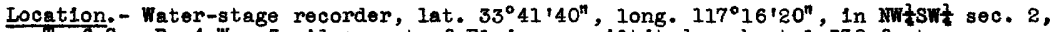
1. 6 S., R. 4 W., 3 miles east of Elsinore. Altitude, about 1,330 feet.

Draingge area.- 709 square miles.

Gage-he1ght record.- Water-stage recorder graph.

Inflow record. - Obtalned from stage-capacity curve and operation records.

Maximum. - 1938: Inflow, 3,150 second-feet about 8 p.m. Mar. 2 .

Remerks.- Records furnished by Corps of Bnglneers, U. S. Army.

Mean dally inflow, in second-feet, 1938

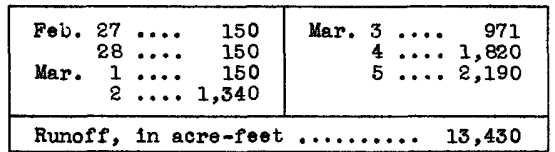

Inflow, in second-feet, at indicated time, 1938

\begin{tabular}{|c|c|c|c|c|c|}
\hline Bour & March 2 & March 3 & March 4 & March 5 & March 6 \\
\hline $\begin{array}{r}1 \\
2 \\
3 \\
4 \\
5 \\
6\end{array}$ & $\begin{array}{l}150 \\
200 \\
230 \\
260 \\
300 \\
400\end{array}$ & $\begin{array}{l}1,800 \\
1,650 \\
1,500 \\
1,400 \\
1,300 \\
1,200\end{array}$ & $\begin{array}{r}900 \\
1,000 \\
1,050 \\
1,140 \\
1,250 \\
1,350\end{array}$ & $\begin{array}{l}2,600 \\
2,500 \\
2,370\end{array}$ & $\begin{array}{l}1,850 \\
1,750 \\
1,700\end{array}$ \\
\hline $\begin{array}{r}7 \\
8 \\
9 \\
10 \\
11 \\
\mathbb{N}\end{array}$ & $\begin{array}{r}475 \\
575 \\
725 \\
900 \\
1,100 \\
1,160\end{array}$ & $\begin{array}{r}1,130 \\
1,100 \\
1,000 \\
900 \\
800 \\
750\end{array}$ & $\begin{array}{l}1,430 \\
1,530 \\
1,620 \\
1,690 \\
1,760 \\
1,850\end{array}$ & $\begin{array}{l}2,250 \\
2,150 \\
2,100\end{array}$ & $\begin{array}{l}1,620 \\
1,620\end{array}$ \\
\hline $\begin{array}{l}1 \\
2 \\
3 \\
4 \\
5 \\
6\end{array}$ & $\begin{array}{l}1,350 \\
1,500 \\
1,650 \\
1,900 \\
2,150 \\
2,500\end{array}$ & $\begin{array}{l}700 \\
660 \\
650 \\
630 \\
620 \\
625\end{array}$ & $\begin{array}{l}1,920 \\
2,000 \\
2,100 \\
2,170 \\
2,250 \\
2,300\end{array}$ & $\begin{array}{l}2,100 \\
2,050 \\
2,030\end{array}$ & \\
\hline $\begin{array}{r}7 \\
8 \\
9 \\
10 \\
11 \\
M\end{array}$ & $\begin{array}{l}2,900 \\
3,150 \\
2,980 \\
2,400 \\
2,100 \\
1,900\end{array}$ & $\begin{array}{l}640 \\
670 \\
700 \\
740 \\
780 \\
800\end{array}$ & $\begin{array}{l}2,400 \\
2,450 \\
2,500 \\
2,580 \\
2,630 \\
2,650\end{array}$ & $\begin{array}{l}2,000 \\
1,900 \\
1,880\end{array}$ & \\
\hline
\end{tabular}

San Jacinto River near Elsinore, Cal1f.

Locat1on.- Water-stage recorder, lat. $33^{\circ} 39^{\prime} 45^{\prime \prime}$, long. $117^{\circ} 17^{\prime} 45^{\prime \prime}$, near east 11 ne of sec. 9, T. 6 S., R. 4 W., 2 milee east of Elsinore, and $2 \frac{1}{2}$ miles upstream from Elsinore Lake (low-water stage). Altitude, about 1,270 feet.

Drainage area.- Area, 717 square miles. Average altitude, 2,710 feet. Maximum alt1tude, 10,805 feet. Average slope, 34 percent. Length of ma in stream channe1, 61.5 miles. Áverage slope of main stream channel, 1.6 percent.

Gage-helght record.- We r-atage recorder graph.

Discharge record. - Stage-d1scharge relation defined by current-meter measurements below 1,790 second-feet; extended logarlthmlcally to peak stage. Record verlfled by comparison with outflow from Rallroad Canyon Reservolr li miles upstream.

Haxima.- 1938: Discharge, 2,790 second-feet 2 to 4 a.m. Mar. 5 (gage helght, 8.41 feet). 1916.

Remarks.- Records good. Storage at Rallroad Canyon Reservoir and Lake Hemet; several divers Ions for irrigation. Seepage from Metropolitan Water District's San Jacinto tunnel was pumped Into San Jacinto River above gage. 
Mean dally discharge, in second-feet, 1938

\begin{tabular}{|c|c|c|c|c|c|c|c|c|c|c|c|}
\hline Day & Feb. & Mar. & Apr. & Day & Feb. & Nar. & Apr. & Day & Feb. & Mar. & Apr. \\
\hline $\begin{array}{r}1 \\
2 \\
3 \\
4 \\
5 \\
6 \\
7 \\
8 \\
9 \\
10\end{array}$ & $\begin{array}{l}52 \\
48 \\
47 \\
55 \\
51 \\
46 \\
46 \\
46 \\
53 \\
54\end{array}$ & $\begin{array}{r}180 \\
961 \\
1,400 \\
1,570 \\
2,470 \\
1,500 \\
964 \\
604 \\
594 \\
536\end{array}$ & $\begin{array}{r}126 \\
115 \\
103 \\
88 \\
79 \\
81 \\
78 \\
78 \\
74 \\
59\end{array}$ & $\begin{array}{l}11 \\
12 \\
13 \\
14 \\
15 \\
16 \\
17 \\
18 \\
19 \\
20\end{array}$ & $\begin{array}{l}\mathbf{5 9} \\
66 \\
59 \\
56 \\
54 \\
52 \\
51 \\
52 \\
58 \\
53\end{array}$ & $\begin{array}{l}476 \\
468 \\
399 \\
436 \\
675 \\
765 \\
684 \\
576 \\
496 \\
420\end{array}$ & $\begin{array}{l}49 \\
47 \\
45 \\
53 \\
68 \\
75 \\
, 70 \\
65 \\
58 \\
50\end{array}$ & $\begin{array}{l}21 \\
22 \\
23 \\
24 \\
25 \\
26 \\
27 \\
28 \\
29 \\
30 \\
31\end{array}$ & $\begin{array}{r}50 \\
51 \\
53 \\
56 \\
53 \\
52 \\
86 \\
100\end{array}$ & $\begin{array}{l}371 \\
324 \\
288 \\
252 \\
220 \\
194 \\
169 \\
149 \\
143 \\
141 \\
136\end{array}$ & $\begin{array}{r}49 \\
49 \\
45 \\
41 \\
42 \\
48 \\
81 \\
105 \\
99 \\
86\end{array}$ \\
\hline \multicolumn{9}{|c|}{ 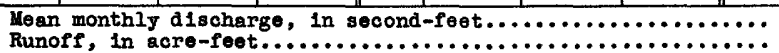 } & $\begin{array}{r}55.7 \\
3,090\end{array}$ & 36,820 & $\begin{array}{r}70.2 \\
4,180\end{array}$ \\
\hline
\end{tabular}

Gage helght, in feet, and discharge, in second-feet, at indicated time, 1938

\begin{tabular}{|c|c|c|c|c|c|c|c|c|}
\hline \multirow{2}{*}{ Hour } & Feet & sec.ft. & Feet & Sec.ft. & Feot & Sec.ft. & Feot & Sec.ft. \\
\hline & \multicolumn{2}{|c|}{ Karch 2} & \multicolumn{2}{|c|}{ March 3} & \multicolumn{2}{|c|}{ Karch 4} & \multicolumn{2}{|c|}{ March 5} \\
\hline$\frac{1}{2}$ & 4.20 & 169 & 7.92 & 2,270 & 5.78 & 730 & 8.41 & 2,790 \\
\hline 4 & 4.22 & 174 & 7.70 & 2,070 & 5.73 & 706 & 8.41 & 2,790 \\
\hline 6 & 4.30 & 192 & 7.44 & 1,840 & 5.72 & 702 & 8.37 & 2,750 \\
\hline $\begin{array}{l}7 \\
8\end{array}$ & 4.52 & 246 & 7.18 & 1,630 & 5.78 & 730 & 8.33 & 2,710 \\
\hline 10 & 4.70 & 297 & 6.92 & 1,430 & 5.96 & 830 & 8.26 & 2,610 \\
\hline $\mathbf{n}$ & 5.20 & 472 & 6.71 & 1,270 & 7.25 & 1,690 & 8.19 & 2,540 \\
\hline $\begin{array}{l}1 \\
2\end{array}$ & 5.70 & 689 & 6.50 & 1,140 & 7.52 & 1,920 & 8.11 & 2,430 \\
\hline 4 & 6.90 & 1,410 & 6.29 & 1,020 & 7.79 & 2,160 & 8.03 & 2,330 \\
\hline 6 & 7.42 & 1,820 & 6.14 & 930 & 8.03 & 2,390 & 7.93 & 2,220 \\
\hline $\begin{array}{l}7 \\
8\end{array}$ & 7.88 & 2,230 & 6.02 & 860 & 8.20 & 2,570 & 7.84 & 2,120 \\
\hline 10 & 8.19 & 2,550 & 5.92 & 806 & 8.32 & 2,700 & 7.75 & 2,020 \\
\hline M & 8.04 & 2,390 & 5.84 & 760 & 8.38 & 2,760 & 7.66 & $1,92,0$ \\
\hline
\end{tabular}

Elsinore Lake at Elsinore, Calif.

Location.- Staff gage, lat. $33^{\circ} 40^{\prime} 35^{\prime \prime}$, long. $117^{\circ} 21^{1} 30^{n}$, in La Laguna grant, on northeast shiore at Aloha Beach Club, Elsinore, Riverside County. Zero of gage is 1,200 feet above mean sea level.

Gage-helght record.- Staff-gage readings about once dally during flood perlods.

Remarks.- Elsinore Lake overflows only during and after years of heavy rainfall. Tomescal Creek is the high water outlet of lake. There was flow in creek during most of 1916 and the f1rst half of 1917. A hlstory of the lake $18 \mathrm{~g} 1$ ven in WaterSupply Papers 426, 429, and 441. Part of record furnlshed by D. R. Crane, Elsincre, callf.

Gage height, in feet, 1938

\begin{tabular}{|c|c|c|c|c|c|c|c|c|c|c|c|}
\hline Day & Feb. & Mar. & Apr. & Day & $\mathrm{Feb}$. & Mar. & Apr. & Day & Feb. & Mar. & Apr. \\
\hline $\begin{array}{r}1 \\
2 \\
3 \\
4 \\
5 \\
6 \\
7 \\
8 \\
9 \\
10\end{array}$ & $\begin{array}{l}50.0 \\
50.0\end{array}$ & $\begin{array}{l}51.0 \\
51.1 \\
52.4 \\
52.9 \\
53.7 \\
54.5 \\
55.0 \\
55.4 \\
55.6 \\
55.8\end{array}$ & $\begin{array}{l}58.5 \\
58.5 \\
58.6 \\
58.6 \\
58.6 \\
58.6 \\
58.6 \\
58.6 \\
58.6 \\
58.7\end{array}$ & $\begin{array}{l}11 \\
12 \\
13 \\
14 \\
15 \\
16 \\
17 \\
18 \\
19 \\
20\end{array}$ & 50.4 & $\begin{array}{l}56.0 \\
56.3 \\
56.4 \\
56.6 \\
56.7 \\
57.0 \\
57.2 \\
57.4 \\
57.6 \\
57.7\end{array}$ & $\begin{array}{l}58.7 \\
58.7 \\
58.7 \\
58.7 \\
58.7 \\
58.7 \\
58.7 \\
58.7 \\
58.8 \\
58.7\end{array}$ & $\begin{array}{l}21 \\
22 \\
23 \\
24 \\
25 \\
26 \\
27 \\
28 \\
29 \\
30 \\
31\end{array}$ & 50.5 & $\begin{array}{l}57.8 \\
57.9 \\
58.0 \\
58.1 \\
58.2 \\
58.3 \\
58.3 \\
58.3 \\
58.4 \\
58.4 \\
58.4\end{array}$ & $\begin{array}{l}58.8 \\
58.8 \\
58.8 \\
58.8 \\
58.8 \\
58.8 \\
58.8 \\
58.8 \\
58.8 \\
58.9\end{array}$ \\
\hline
\end{tabular}


Elsinore Lake, capacity trble*

\begin{tabular}{|c|c|c|c|c|c|}
\hline $\begin{array}{c}\text { Gage-height } \\
\text { (feet) }\end{array}$ & Acre-feet & $\begin{array}{c}\text { Gage-height } \\
\text { (feet) }\end{array}$ & Acre-feet & $\begin{array}{c}\text { Gege-height } \\
\text { (feet) }\end{array}$ & Acre-feet \\
\hline 45 & 60,000 & 50 & 82,000 & 55 & 107,500 \\
46 & 64,500 & 51 & 86,500 & 56 & 112,500 \\
47 & 68,500 & 52 & 92,000 & 57 & 118,000 \\
48 & 73,000 & 53 & 97,500 & 58 & 124,000 \\
49 & 77,500 & 54 & 102,500 & 59 & 130,000 \\
\hline
\end{tabular}

* From unpublished report by P. H. Albright, Blsinore Valley Water Supply.

Temescal Creek near Corona, Gallf.

Location. - Water-stage recorder, lat. $33^{\circ} 50^{\prime} 30^{\prime \prime}$, long. $117^{\circ} 30^{\prime} 45^{\prime \prime}$, in Bl Sobrante de San Jacinto grant, half a mlle upstream from Blue Dlamond Quarry, and 4 mfles southeast of Dorona, Riverside County. Altitude, 730 feet.

Dreinage area below Elsinore Lake.- Area, 157 square miles (924 square miles including San Jacinto River). Average a ltitude, 1,451 foet. Maximum altitude, 5,680 foet. Average slope, 33 percent. Length of main stream channel, 22 miles. Slope of main stream channel, 4.1 percent.

Gage-he1ght record. - Water-stage recorder graph except Mar. 2-14.

D1scherge record. - Stage-discharge relation poorly defined by current-meter measurements belon 100 second-fe日t; peak discharge determined by slope-area measurement. Discharge for maximum 24 hours determined from rainfall-runofe relation, using the S1lverado and Riverside recording rain gage records as a basis for distribution of the storm precipitation. D1scharge Mar. 4-14 determined from one current-meter measurement and by comparison with nearby stations.

Maxima. - 1938: D1scharge, 14,900 second-feet 7 p.m. Mar. 2.

1929-37: Discharge, 2,350 second-feet Peb. 6, 1937.

Remarks. - Records poor. Drainage area above Blsinore Lake d1d not contribute ony runoff dưring flood. Lee Lake dam falled in the late afternoon of Mar. 2 .

Mean dally discharge, in second-feet, 1938

\begin{tabular}{|r|l|r|r||r|r|r|r|r||c|c|c|c|}
\hline Day & Feb. & Mar. & Apr. & Day & Feb. & Mar. & Apr. & Day & Feb. & Mar. & Apr. \\
\hline 1 & 4.0 & 88 & 100 & 11 & 8 & 216 & 64 & 21 & 4.4 & 75 & 46 \\
2 & 9 & 3,460 & 88 & 12 & 8 & 152 & 55 & 22 & 4.4 & 96 & 46 \\
3 & 7 & 2,520 & 82 & 13 & 6 & 125 & 50 & 23 & 4.0 & 108 & 42 \\
4 & 7.5 & 432 & 84 & 14 & 7 & 120 & 46 & 24 & 4.0 & 104 & 42 \\
5 & 6 & 194 & 91 & 15 & 7 & 116 & 46 & 25 & 4.0 & 100 & 39 \\
6 & 5.5 & 136 & 87 & 16 & 6 & 112 & 46 & 26 & 4.8 & 118 & 33 \\
7 & 6 & 112 & 72 & 17 & 5 & 107 & 47 & 27 & 10 & 115 & 24 \\
8 & 7 & 106 & 68 & 18 & 5 & 123 & 48 & 28 & 11 & 129 & 22 \\
9 & 7.5 & 102 & 65 & 19 & 5.5 & 100 & 46 & 29 & & 130 & 28 \\
10 & 8 & 100 & 64 & 20 & 4.4 & 87 & 49 & 30 & & 122 & 25 \\
\end{tabular}

Chino Creok near Prado, Calif.

Location.- Water-atage recorder, lat. $33^{\circ} 53^{\prime} 40^{\prime \prime}$, long. $117^{\circ} 38^{\prime} 40^{\prime \prime}$, in Il Rincon grant, at Chino-Rincon road, $1 \mathrm{mlle}$ west of Prado, Riverside County. Altitude, 460 feet.

Drainsge ares.- About 176 square miles.

Gage-he1ght record. - Water-3tage recorder graph except 1 p.m. Mar. 2 to Mar. 21.

Discharge record.- Stage-discharge relation defined by currant-reter measurements below 300 seconâ-feet; extended logarithmically to peak discharge determined by slope-area measurement of the main chanmel $(5,000$ sec.-ft.) plus estimated discharge of secondary channels. Discharge Mar. 2 to Apr. $3 C$ computed by interpolating between discharge measurements and by comparison with adjacent streams.

Kexima.- 1938: Discharge, 5,200 second-feet about 6 p.m. Mar. 2 . i929-37: D1scharge, 1,440 second-fe日t Jan. 1, 1934 .

Remarks.- Records fair. Ho diversion or regulation during flood. 
Mean dally discharge, in second-feet, 1938

\begin{tabular}{|c|c|c|c|c|c|c|c|c|c|c|c|}
\hline Day & Feb. & Mar. & Apr. & Day & Feb. & Mar. & Apr. & Day & Fob. & Mar. & Apr. \\
\hline $\begin{array}{r}1 \\
2 \\
3 \\
4 \\
5 \\
6 \\
7 \\
8 \\
9 \\
10\end{array}$ & $\begin{array}{l}78 \\
32 \\
21 \\
50 \\
21 \\
13 \\
11 \\
9.5 \\
47 \\
67\end{array}$ & $\begin{array}{r}302 \\
1,580 \\
1,200 \\
371 \\
205 \\
155 \\
140 \\
132 \\
130 \\
128\end{array}$ & $\begin{array}{l}13 \\
11 \\
9 \\
8.5 \\
8.5 \\
9 \\
8 \\
6.5 \\
6 \\
6\end{array}$ & $\begin{array}{l}11 \\
12 \\
13 \\
14 \\
15 \\
16 \\
17 \\
18 \\
19 \\
20\end{array}$ & $\begin{array}{l}47 \\
96 \\
29 \\
20 \\
19 \\
17 \\
15 \\
14 \\
19 \\
16\end{array}$ & $\begin{array}{l}130 \\
190 \\
250 \\
190 \\
140 \\
100 \\
90 \\
86 \\
76 \\
66\end{array}$ & $\begin{array}{l}6.5 \\
7 \\
7.5 \\
8 \\
8 \\
8.5 \\
8.5 \\
8 \\
8 \\
8.5\end{array}$ & $\begin{array}{l}21 \\
22 \\
23 \\
24 \\
25 \\
26 \\
27 \\
28 \\
29 \\
30 \\
31\end{array}$ & $\begin{array}{l}15 \\
14 \\
14 \\
14 \\
14 \\
13 \\
42 \\
89\end{array}$ & $\begin{array}{l}60 \\
50 \\
44 \\
88 \\
35 \\
32 \\
28 \\
24 \\
21 \\
18 \\
15\end{array}$ & $\begin{array}{l}9.5 \\
11 \\
13 \\
14 \\
15 \\
14 \\
12 \\
10 \\
9 \\
10\end{array}$ \\
\hline \multicolumn{9}{|c|}{ 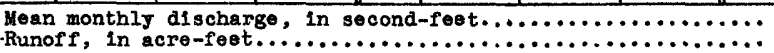 } & $\begin{array}{r}30.6 \\
1.700\end{array}$ & 12,050 & $\begin{array}{r}9.38 \\
558\end{array}$ \\
\hline
\end{tabular}

Gage helght, in feet, and discharge, in second-feet, at indlcated time, 1938

\begin{tabular}{|c|c|c|c|c|c|c|c|c|c|c|c|c|}
\hline \multirow{2}{*}{$\begin{array}{l}5 \\
0 \\
0\end{array}$} & Feet & sec.ft. & Feot & Sec.ft. & Feet & Sec.ft. & Feet & Sec.ft. & Feet & sec.ft. & Feet & Sec.ft. \\
\hline & \multicolumn{2}{|c|}{ Febmary 27} & \multicolumn{2}{|c|}{ Fobruary 28} & \multicolumn{2}{|c|}{ March 1} & \multicolumn{2}{|c|}{ March 2} & \multicolumn{2}{|c|}{ March 3} & \multicolumn{2}{|c|}{ March 4} \\
\hline $\begin{array}{l}1 \\
2 \\
3 \\
4 \\
5 \\
6\end{array}$ & 2.14 & 13 & $\begin{array}{l}3.93 \\
3.75 \\
3.56 \\
3.45 \\
3.35\end{array}$ & $\begin{array}{l}76 \\
68 \\
59 \\
54 \\
50\end{array}$ & $\begin{array}{l}4.60 \\
4.70 \\
5.00 \\
6.20 \\
7.30 \\
7.90\end{array}$ & $\begin{array}{l}116 \\
122 \\
142 \\
237 \\
345 \\
411\end{array}$ & $\begin{array}{l}5.48 \\
5.35 \\
5.25 \\
5.15 \\
5.25 \\
5.75\end{array}$ & $\begin{array}{l}178 \\
168 \\
160 \\
153 \\
160 \\
199\end{array}$ & & $\begin{array}{l}2,200 \\
1,850 \\
1,600\end{array}$ & & $\begin{array}{l}510 \\
480 \\
440\end{array}$ \\
\hline $\begin{array}{r}7 \\
8 \\
9 \\
10 \\
11 \\
1\end{array}$ & 2.27 & 15 & 3.25 & 46 & $\begin{array}{l}8.40 \\
8.62 \\
8.50 \\
8.25 \\
7.90 \\
7.60\end{array}$ & $\begin{array}{l}476 \\
503 \\
491 \\
456 \\
411 \\
379\end{array}$ & $\begin{array}{r}6.20 \\
7.30 \\
8.20 \\
9.30 \\
10.85 \\
11.00\end{array}$ & $\begin{array}{l}238 \\
346 \\
452 \\
593 \\
835 \\
858\end{array}$ & & $\begin{array}{l}1,300 \\
1,150 \\
1,000\end{array}$ & & $\begin{array}{l}410 \\
380 \\
350\end{array}$ \\
\hline $\begin{array}{l}1 \\
2 \\
3 \\
4 \\
5 \\
6\end{array}$ & $\begin{array}{l}2.45 \\
2.55 \\
2.65 \\
2.77 \\
2.95 \\
3.50\end{array}$ & $\begin{array}{l}20 \\
22 \\
25 \\
29 \\
35 \\
36\end{array}$ & $\begin{array}{l}3.55 \\
4.20 \\
4.65 \\
4.75 \\
4.85 \\
5.07\end{array}$ & $\begin{array}{r}59 \\
92 \\
119 \\
125 \\
132 \\
147\end{array}$ & $\begin{array}{l}7.40 \\
7.30 \\
7.20 \\
7.00 \\
6.77 \\
6.51\end{array}$ & $\begin{array}{l}357 \\
346 \\
335 \\
314 \\
291 \\
265\end{array}$ & 10.90 & $\begin{array}{r}900 \\
1,000 \\
1,200 \\
2,200 \\
3,500 \\
5,200\end{array}$ & & $\begin{array}{l}900 \\
810 \\
730\end{array}$ & & $\begin{array}{l}330 \\
310 \\
290\end{array}$ \\
\hline $\begin{array}{r}7 \\
8 \\
9 \\
10 \\
11 \\
M\end{array}$ & $\begin{array}{l}4.23 \\
4.77 \\
4.85 \\
4.70 \\
4.40 \\
4.13\end{array}$ & $\begin{array}{r}93 \\
126 \\
131 \\
121 \\
103 \\
87\end{array}$ & $\begin{array}{l}5.18 \\
5.20 \\
5.10 \\
4.90 \\
4.70 \\
4.50\end{array}$ & $\begin{array}{l}154 \\
156 \\
149 \\
135 \\
122 \\
110\end{array}$ & $\begin{array}{l}6.30 \\
6.15 \\
6.05 \\
5.90 \\
5.75 \\
5.60\end{array}$ & $\begin{array}{l}247 \\
233 \\
224 \\
212 \\
200 \\
188\end{array}$ & & $\begin{array}{l}4,400 \\
4,000 \\
3,600 \\
3,200 \\
2,900 \\
2,700\end{array}$ & & $\begin{array}{l}660 \\
600 \\
550\end{array}$ & & $\begin{array}{r}280 \\
265 \\
255\end{array}$ \\
\hline
\end{tabular}

San Antonio Creek near Claremont, Cal1f.

Location. - Water-stage recorder and concrete control, lat. $34^{\circ} 12^{\prime} 50^{\prime \prime}, 10 n g .117^{\circ} 40^{\prime} 00^{\prime \prime}$, In illifSEt sec. 36 , T. 2 H., R. 8 W., at highway bridge, half a mile above Southern California Edison Co.' S Sierra power plant, and 8 miles northeast of Claremont. Altitude, about 3,400 feet.

Drainage area.- Area, 16.9 square miles. Average alt itude, 6,580 feet. Maximm altitude, 10,080 feet. Average slope, 49 percent. Length of aln stream channel, 5.75 miles. Average slope of mein stream channel, 21 percent.

Gage-helght record.- Water-stage recorder graph except 10 a.m. Mar. 2 to Mar. 29.

Discharge record. - Stage-discharge relation defined by current-ineter measurements below I25 second-feet; extended logarithmically to 900 second-feet, 0oncrete control was destroyed on Mar. 2. The stream changed 1ts channel above gage, so that only a small part of the flood runoff passed the gage. Maximum discharge estimated by consideration of slope-area studies, rainfall-manoff studies, and knowledge of local conditions. Discharge for the maximum 24 hours determined from rainfall-rumoff study using Camp Baldy recording rain gage record as basis for distribution of total storm precipitation; for remalnder of the period of missing record determined from measurements made by the Los Angeles County Flood Control District at the mouth of the canyon, from retention studies, and by a comparison with other streams.

Maxima.- 1938: Discharge, 21,400 second-fe日t about 4 p.m. Mar. 2.

1917-37: Discharge, 1,020 second-fe日t Dec. 19, 1921.

Remarks.- Records good except for Mar. 2-29, which are poor. Discharge is for natural flow of the stream and includes flow in Southern California Fdison Co. 's canal; no flow in canal Mar. 2 to Apr. 30. 
Wean da11y disoherge, in seoond-reet, 1938

\begin{tabular}{|c|c|c|c|c|c|c|c|c|c|c|c|}
\hline Dey & Fob. & Yar. & Apr. & Day & Feb. & Yar. & Aps. & Day & $\overline{B a b}$. & Mar. & Ape. \\
\hline $\begin{array}{r}1 \\
2 \\
3 \\
4 \\
5 \\
6 \\
7 \\
8 \\
9 \\
10\end{array}$ & $\begin{array}{l}10 \\
16 \\
26 \\
30 \\
27 \\
23 \\
28 \\
20 \\
28 \\
26\end{array}$ & $\begin{array}{r}227 \\
4,110 \\
854 \\
436 \\
364 \\
272 \\
227 \\
227 \\
136 \\
136\end{array}$ & $\begin{array}{l}125 \\
125 \\
130 \\
140 \\
140 \\
140 \\
150 \\
150 \\
150 \\
140\end{array}$ & $\begin{array}{l}11 \\
18 \\
13 \\
14 \\
16 \\
16 \\
17 \\
18 \\
19 \\
20\end{array}$ & $\begin{array}{l}52 \\
35 \\
25 \\
24 \\
26 \\
26 \\
25 \\
24 \\
26 \\
25\end{array}$ & $\begin{array}{l}136 \\
274 \\
375 \\
208 \\
175 \\
168 \\
163 \\
158 \\
154 \\
163\end{array}$ & $\begin{array}{l}140 \\
130 \\
130 \\
125 \\
120 \\
115 \\
110 \\
105 \\
102 \\
98\end{array}$ & $\begin{array}{l}21 \\
28 \\
23 \\
24 \\
25 \\
26 \\
27 \\
28 \\
29 \\
30 \\
31\end{array}$ & $\begin{array}{r}25 \\
24 \\
24 \\
24 \\
24 \\
24 \\
26 \\
119\end{array}$ & $\begin{array}{l}151 \\
151 \\
150 \\
150 \\
148 \\
143 \\
140 \\
138 \\
135 \\
133 \\
130\end{array}$ & $\begin{array}{l}94 \\
98 \\
90 \\
67 \\
88 \\
82 \\
80 \\
78 \\
78 \\
75\end{array}$ \\
\hline \multicolumn{9}{|c|}{ 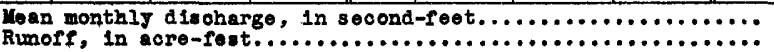 } & 1,620 & 21,060 & 6,780 \\
\hline
\end{tabular}

Discharge, in second-feet, at indioated time, 1938

\begin{tabular}{|c|c|c|c|}
\hline Hour & February 28 & Naroh 1 & Maroh 2 \\
\hline $\begin{array}{l}1 \\
2 \\
3 \\
4 \\
5 \\
6\end{array}$ & $\begin{array}{l}31 \\
36 \\
41 \\
47 \\
49 \\
53\end{array}$ & $\begin{array}{l}771 \\
581 \\
451 \\
356 \\
301 \\
266\end{array}$ & $\begin{array}{r}94 \\
95 \\
98 \\
102 \\
110 \\
120\end{array}$ \\
\hline $\begin{array}{r}7 \\
8 \\
9 \\
10 \\
11 \\
11\end{array}$ & $\begin{array}{l}56 \\
64 \\
70 \\
83 \\
83 \\
86\end{array}$ & $\begin{array}{l}231 \\
211 \\
193 \\
176 \\
166 \\
153\end{array}$ & $\begin{array}{l}140 \\
180 \\
350 \\
740\end{array}$ \\
\hline $\begin{array}{l}1 \\
2 \\
3 \\
4 \\
5 \\
6\end{array}$ & $\begin{array}{r}96 \\
109 \\
109 \\
128 \\
123 \\
118\end{array}$ & $\begin{array}{r}130 \\
112 \\
108 \\
104 \\
100 \\
98\end{array}$ & \\
\hline $\begin{array}{r}7 \\
8 \\
9 \\
10 \\
11 \\
11\end{array}$ & $\begin{array}{l}137 \\
119 \\
141 \\
196 \\
435 \\
921\end{array}$ & $\begin{array}{l}96 \\
95 \\
94 \\
93 \\
93 \\
93\end{array}$ & \\
\hline
\end{tabular}

Santiago Creek near Villa Park, Callf.

Loeation.- Water-gtage recorder, lat. $33^{\circ} 49^{\prime} 10^{\prime \prime}$, 3ong. $117^{\circ} 46^{\prime} 30^{\prime \prime}$, in Sw' $800.13, T$. 4 S., R. 9 W., five-eighths of a mile below diversion dam of serrano and oarpenter Irrigation Distriots, and $1-3 / 4$ miles northeast of Vilia Park, Altitude, about 420 feet.

Draingge area.- Area, 83.8 oquare miles. Average altitude, 1,954 reet. Inximun alt1tude, 5,680 fest. Average lope, 43 percent. Length of main otreain channe1, 16.5 miles. Slope of main stream obannel, 6.0 percent.

Gage-height record.- Water-stage recorder graph except Feb. 1-14, Iar. 4-6, and Apr. 21-30.

Discharge record.- Stage-discharge relation defined by current-meter meavurementa beIow 1,200 second-feet; extended above on basis of a loperarea measurement of the peak. Discharge for period of missing gage-helght record besed on diecharge measuremente and comparison with Santiago Oreek at Sente Ana.

Maxima.- 1938: Divcharge, 5,200 vecond-feet 7 p.m. Uar. 2 (gege holght, 6.27 feet). 1920-37: Discharte, 21,000 second-Peet Feb. 16,1927 (gage height, 8.4 feet), by slope-area method.

Remarke.- Reoords fair. Flow partly regulated by torage in santlago Reservolr. Irvine Company and Serreno and Oarpenter Irrigation Distriots divert abeve gege. 
Yean daily discharge, in second-feet, 1938

\begin{tabular}{|c|c|c|c|c|c|c|c|c|c|c|c|}
\hline Day & Feb. & Mar. & Apr. & Day & Feb. & Mar. & Apr. & Day & Peb. & Nar. & Apr. \\
\hline $\begin{array}{r}1 \\
8 \\
3 \\
4 \\
5 \\
6 \\
7 \\
8 \\
9 \\
10\end{array}$ & $\begin{array}{r}14 \\
5 \\
3.0 \\
2.0 \\
1.5 \\
1.2 \\
1.0 \\
.9 \\
.8 \\
.7\end{array}$ & $\begin{array}{r}176 \\
2,660 \\
3,190 \\
1,190 \\
420 \\
275 \\
264 \\
253 \\
219 \\
197\end{array}$ & $\begin{array}{l}68 \\
68 \\
62 \\
62 \\
62 \\
54 \\
44 \\
32 \\
36 \\
54\end{array}$ & $\begin{array}{l}11 \\
12 \\
13 \\
14 \\
15 \\
16 \\
17 \\
18 \\
19 \\
20\end{array}$ & $\begin{array}{l}0.7 \\
.8 \\
.8 \\
.8 \\
.8 \\
.8 \\
.6 \\
.7 \\
.9 \\
.8\end{array}$ & $\begin{array}{r}186 \\
583 \\
1,030 \\
646 \\
442 \\
322 \\
281 \\
253 \\
214 \\
197\end{array}$ & $\begin{array}{l}65 \\
68 \\
65 \\
62 \\
65 \\
46 \\
27 \\
17 \\
17 \\
17\end{array}$ & $\begin{array}{l}21 \\
22 \\
23 \\
24 \\
25 \\
26 \\
27 \\
28 \\
29 \\
30 \\
31\end{array}$ & $\begin{array}{r}0.7 \\
.6 \\
.6 \\
.5 \\
.3 \\
1.3 \\
13\end{array}$ & $\begin{array}{r}180 \\
160 \\
150 \\
117 \\
101 \\
70 \\
82 \\
79 \\
73 \\
70 \\
70\end{array}$ & $\begin{array}{r}10 \\
9 \\
8 \\
6 \\
15 \\
12 \\
10 \\
8 \\
8 \\
7\end{array}$ \\
\hline \multicolumn{9}{|c|}{ 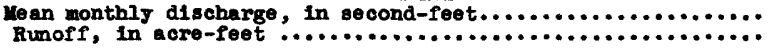 } & $\begin{array}{r}1.97 \\
109\end{array}$ & $\begin{array}{r}456 \\
28,070\end{array}$ & $\begin{array}{r}36.1 \\
2,150\end{array}$ \\
\hline
\end{tabular}

Gage helght, In feet, and diecharge, in second-feet, at indicated time, 1938

\begin{tabular}{|c|c|c|c|c|c|c|c|c|c|c|c|c|}
\hline \multirow{2}{*}{ 点 } & Feet & sec.ft. & Peet & soc.ft. & Feet & sec.ft. & Feet & sec.et. & Feet & Sec.ft. & Feet & soc.ft. \\
\hline & \multicolumn{2}{|c|}{ February 87} & \multicolumn{2}{|c|}{ February 28} & \multicolumn{2}{|c|}{ Nareh 1} & \multicolumn{2}{|c|}{ March 8} & \multicolumn{2}{|c|}{ March 3} & \multicolumn{2}{|c|}{ Varch 4} \\
\hline $\begin{array}{l}1 \\
2 \\
3 \\
4 \\
5 \\
6\end{array}$ & $\begin{array}{r}0.52 \\
.52 \\
.52 \\
.52 \\
.52 \\
.52\end{array}$ & $\begin{array}{r}0.3 \\
.3 \\
.3 \\
.3 \\
.3 \\
.3\end{array}$ & $\begin{array}{r}0.83 \\
.80 \\
.80 \\
.82 \\
.91 \\
.94\end{array}$ & $\begin{array}{l}4.4 \\
3.7 \\
3.7 \\
4.2 \\
7 \\
8\end{array}$ & $\begin{array}{l}2.70 \\
3.03 \\
3.05 \\
2.35 \\
1.96 \\
1.77\end{array}$ & $\begin{array}{l}590 \\
722 \\
730 \\
447 \\
309 \\
241\end{array}$ & $\begin{array}{r}0.85 \\
.90 \\
.94 \\
1.30 \\
2.00 \\
2.57\end{array}$ & $\begin{array}{r}96 \\
109 \\
139 \\
287 \\
750 \\
1,230\end{array}$ & $\begin{array}{l}5.63 \\
5.47 \\
5.33 \\
5.15 \\
5.04 \\
4.95\end{array}$ & $\begin{array}{l}4,480 \\
4,290 \\
4,130 \\
3,950 \\
3,840 \\
3,740\end{array}$ & & 1,640 \\
\hline $\begin{array}{r}7 \\
8 \\
9 \\
10 \\
11 \\
7\end{array}$ & $\begin{array}{l}.52 \\
.53 \\
.56 \\
.59 \\
.62 \\
.63\end{array}$ & $1^{\circ}$ & $\begin{array}{r}1.06 \\
1.01 \\
.96 \\
.90 \\
.89 \\
.87\end{array}$ & $\begin{array}{l}15 \\
12 \\
9 \\
6.5 \\
6.5 \\
5.5\end{array}$ & $\begin{array}{r}1.54 \\
1.40 \\
1.25 \\
1.12 \\
1.04 \\
.97\end{array}$ & $\begin{array}{r}158 \\
117 \\
82 \\
59 \\
51 \\
43\end{array}$ & $\begin{array}{l}2.77 \\
2.79 \\
2.95 \\
3.40 \\
3.93 \\
3.84\end{array}$ & $\begin{array}{l}1,410 \\
1,430 \\
1,590 \\
2,050 \\
2,650 \\
2,570\end{array}$ & $\begin{array}{l}4.87 \\
4.84 \\
4.77 \\
4.67 \\
4.56 \\
4.47\end{array}$ & $\begin{array}{l}3,660 \\
3,620 \\
3,550 \\
3,440 \\
3,320 \\
3,820\end{array}$ & & $\begin{array}{l}1,350 \\
1,120\end{array}$ \\
\hline $\begin{array}{l}1 \\
2 \\
3 \\
4 \\
5 \\
6\end{array}$ & $\begin{array}{l}.64 \\
.65 \\
.66 \\
.67 \\
.68 \\
.70\end{array}$ & $\begin{array}{l}1.1 \\
1.1 \\
1.3 \\
1.5 \\
1.6 \\
1.9\end{array}$ & $\begin{array}{l}.85 \\
.85 \\
.82 \\
.80 \\
.79 \\
.78\end{array}$ & $\begin{array}{l}4.9 \\
4.4 \\
4.2 \\
3.7 \\
3.5 \\
3.3\end{array}$ & $\begin{array}{l}.93 \\
.88 \\
.85 \\
.81 \\
.77\end{array}$ & $\begin{array}{l}41 \\
38 \\
38 \\
39 \\
40 \\
43\end{array}$ & $\begin{array}{l}4.03 \\
4.35 \\
4.80 \\
4.90 \\
4.90 \\
5.73\end{array}$ & $\begin{array}{l}2,750 \\
3,080 \\
3,580 \\
3,690 \\
3,690 \\
4,600\end{array}$ & $\begin{array}{l}4.37 \\
4.26 \\
4.09 \\
4.00 \\
3.91 \\
3.82\end{array}$ & $\begin{array}{l}3,110 \\
2,990 \\
2,810 \\
2,720 \\
2,630 \\
2,540\end{array}$ & & 930 \\
\hline $\begin{array}{r}7 \\
8 \\
9 \\
10 \\
11 \\
1\end{array}$ & $\begin{array}{l}.72 \\
.73 \\
.75 \\
.77 \\
.89 \\
.85\end{array}$ & $\begin{array}{l}2.2 \\
2.3 \\
2.6 \\
3.1 \\
6.5 \\
4.9\end{array}$ & $\begin{array}{r}.77 \\
.76 \\
.77 \\
.95 \\
1.85 \\
1.94\end{array}$ & $\begin{array}{r}3.1 \\
2.8 \\
3.1 \\
10 \\
41 \\
281\end{array}$ & $\begin{array}{l}.70 \\
.68 \\
.68 \\
.67 \\
.68\end{array}$ & $\begin{array}{l}44 \\
49 \\
58 \\
56 \\
58 \\
69\end{array}$ & $\begin{array}{l}6.27 \\
6.28 \\
6.27 \\
6.20 \\
6.18 \\
5.93\end{array}$ & $\begin{array}{l}5,200 \\
5,120 \\
5,800 \\
5,100 \\
5,080 \\
4,880\end{array}$ & $\begin{array}{l}3.71 \\
3.60 \\
3.54 \\
3.47 \\
3.40\end{array}$ & $\begin{array}{l}2,410 \\
2,280 \\
2,210 \\
2,130 \\
2,050 \\
2,000\end{array}$ & & 650 \\
\hline
\end{tabular}

Santiago Creek at Santa Ana, Galif.

Iocation. - Water-atage recorder, lat. $33^{\circ} 46^{\prime} 00^{\prime \prime}$, long. $117^{\circ} 52^{\prime} 45^{\prime \prime}$, in Santiago de Santa ina grant, at end of Baker Street, Santa Ana, Orange County. Áltitude, about 120 reet.

Gage-height record.- Water-stage recorder graph except for 10 il etages when water was beIow Intake, and var. 5-6, when intalce was clogged by allt.

Discherge record.- Stage-diecharge relation fairly well defined below 1, 000 second-feet by current-1eter measurements extended to peak etage on besie of mean depth-mean veloeity relation. shifting control corrections used throughout. Discharge for periods of miss the gage-helght record based on discharge neasurements and comparison with Santa Ana River at Santa Ana.

Laxima. - 1938; Discharge, 4,400 second-feet 8:30 p.m. Har, 2 (gage height, 7.40 feet). 1929-37/ Discharge, 3,270 second-feet Feb. 6, igs7 (gage helght, 6.90 feet).

Remaris.- Records fair. Regulation at Santiago Reservoir.

Lean daily discharge, in second-feet, 1958

\begin{tabular}{|c|c|c|c|c|c|c|c|c|c|c|c|}
\hline Day & Feb. & Mar. & Apr. & Day & Peb. & Mar. & Apr. & Day & Feb. & Mar. & Apr. \\
\hline $\begin{array}{r}1 \\
8 \\
3 \\
4 \\
5 \\
6 \\
7 \\
8 \\
9 \\
10\end{array}$ & $\begin{array}{c}38 \\
0 \\
0 \\
0 \\
0 \\
0 \\
0 \\
0 \\
68 \\
2.5\end{array}$ & $\begin{array}{r}340 \\
1,990 \\
8,320 \\
1,060 \\
650 \\
300 \\
151 \\
149 \\
116 \\
69\end{array}$ & $\begin{array}{l}17 \\
17 \\
17 \\
21 \\
25 \\
29 \\
28 \\
25 \\
29 \\
62\end{array}$ & $\begin{array}{l}11 \\
12 \\
13 \\
14 \\
15 \\
16 \\
17 \\
18 \\
19 \\
20\end{array}$ & $\begin{array}{c}53 \\
15 \\
.5 \\
0 \\
0 \\
0 \\
0 \\
10 \\
37 \\
30\end{array}$ & $\begin{array}{r}54 \\
254 \\
650 \\
500 \\
323 \\
208 \\
166 \\
136 \\
111 \\
91\end{array}$ & $\begin{array}{r}64 \\
68 \\
68 \\
68 \\
71 \\
67 \\
35 \\
12 \\
1.0 \\
.5\end{array}$ & $\begin{array}{l}21 \\
22 \\
23 \\
24 \\
25 \\
26 \\
27 \\
28 \\
29 \\
30 \\
31\end{array}$ & $\begin{array}{c}10 \\
1.0 \\
0 \\
0 \\
10 \\
5 \\
40 \\
80\end{array}$ & $\begin{array}{l}74 \\
71 \\
67 \\
61 \\
55 \\
47 \\
43 \\
39 \\
34 \\
28 \\
25\end{array}$ & $\begin{array}{l}0 \\
0 \\
0 \\
0 \\
7 \\
0 \\
0 \\
0 \\
0 \\
4.2\end{array}$ \\
\hline 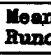 & nth & & & & & & & & $\begin{array}{r}14.1 \\
785\end{array}$ & $\begin{array}{r}329 \\
20,220\end{array}$ & $\begin{array}{r}24.5 \\
1,460\end{array}$ \\
\hline
\end{tabular}


Gage holght, in feet, and discharge, in second-feet, at indicated time, 1938

\begin{tabular}{|c|c|c|c|c|c|c|c|c|}
\hline \multirow{2}{*}{ Hour } & Poet & Sec.ft. & Foet & Sec.ft. & Fest & Sec.ft. & Feet & Sac.ft. \\
\hline & \multicolumn{2}{|c|}{ March 1} & \multicolumn{2}{|c|}{ March 2} & \multicolumn{2}{|c|}{ Harch 3} & \multicolumn{2}{|c|}{ Maroh 4} \\
\hline $\begin{array}{l}1 \\
2 \\
3 \\
4 \\
5 \\
6\end{array}$ & $\begin{array}{l}2.70 \\
4.30 \\
3.00 \\
2.65 \\
2.40 \\
2.00\end{array}$ & $\begin{array}{r}710 \\
1,460 \\
830 \\
690 \\
600 \\
465\end{array}$ & 3.10 & $\begin{array}{r}180 \\
210 \\
250 \\
350 \\
500 \\
1,050\end{array}$ & $\begin{array}{l}6.95 \\
6.85 \\
6.50 \\
6.20 \\
5.95 \\
5.70\end{array}$ & $\begin{array}{l}3,950 \\
3,850 \\
3,520 \\
3,270 \\
3,050 \\
2,890\end{array}$ & $\begin{array}{l}3.20 \\
3.15 \\
3.12 \\
3.07 \\
3.05 \\
3.00\end{array}$ & $\begin{array}{l}1,260 \\
1,230 \\
1,220 \\
1,190 \\
1,180 \\
1,160\end{array}$ \\
\hline $\begin{array}{r}7 \\
8 \\
9 \\
10 \\
11 \\
11\end{array}$ & & $\begin{array}{l}380 \\
300 \\
240 \\
190 \\
180 \\
170\end{array}$ & $\begin{array}{l}3.50 \\
3.55 \\
3.30 \\
3.25 \\
4.00 \\
4.20\end{array}$ & $\begin{array}{l}1,250 \\
1,280 \\
1,150 \\
1,120 \\
1,510 \\
1,630\end{array}$ & $\begin{array}{l}5.55 \\
5.40 \\
5.24 \\
5.10 \\
4.9 \\
4.75\end{array}$ & $\begin{array}{l}2,740 \\
2,620 \\
2,500 \\
2,410 \\
2,270 \\
2,170\end{array}$ & $\begin{array}{l}2.95 \\
2.90 \\
2.87 \\
2.85 \\
2.80 \\
2.77\end{array}$ & $\begin{array}{l}1,140 \\
1,120 \\
1,100 \\
1,090 \\
1,060 \\
1,060\end{array}$ \\
\hline $\begin{array}{l}1 \\
2 \\
3 \\
4 \\
5 \\
6\end{array}$ & & 150 & $\begin{array}{l}4.70 \\
5.00 \\
6.20 \\
6.40 \\
5.90 \\
5.60\end{array}$ & $\begin{array}{l}1,930 \\
2,130 \\
3,010 \\
3,180 \\
2,780 \\
2,620\end{array}$ & $\begin{array}{l}4.60 \\
4.40 \\
4.20 \\
4.05 \\
3.90 \\
3.77\end{array}$ & $\begin{array}{l}2,070 \\
1,940 \\
1,820 \\
1,700 \\
1,640 \\
1,560\end{array}$ & $\begin{array}{l}2.74 \\
2.70 \\
2.66 \\
2.63\end{array}$ & $\begin{array}{r}1,040 \\
1,020 \\
1,000 \\
990\end{array}$ \\
\hline $\begin{array}{r}7 \\
8 \\
9 \\
10 \\
11 \\
1 \\
\end{array}$ & & & $\begin{array}{l}6.00 \\
7.25 \\
7.30 \\
7.00 \\
7.05 \\
7.00\end{array}$ & $\begin{array}{l}3,010 \\
4,300 \\
4,300 \\
4,000 \\
4,050 \\
4,000\end{array}$ & $\begin{array}{l}3.66 \\
3.57 \\
3.50 \\
3.40 \\
3.33 \\
3.25\end{array}$ & $\begin{array}{l}1,500 \\
1,450 \\
1,410 \\
1,360 \\
1,320 \\
1,280\end{array}$ & 2.50 & 930 \\
\hline
\end{tabular}

Supplomental rocord. - Mar. 2, 8:30 p.m., 7.40 ft., 4,400 sec.-ft.

Irvine Ranch drainage canal near Tustin, Calle.

Location. - Water-stage recorder, lat. $33^{\circ} 40^{\circ} 30^{\prime \prime}$, long. $117^{\circ} 50^{\prime} 05^{\prime \prime}$, in San Joaquin grant at Lane Road Bridge, 5 milos south of Tustin, Orange County. Áltitude, about 26 feot.

Drainage area. 93 square miles.

Gage-holght record. - Water-stage recorder graph.

Dlacharge record. - Stage-diacharge ralation falrly well defined below 2,200 second-feet by current-meter measurements, extended to peak on basis of slope-area:determinations.

Maxima.- 1938: Discharge, 5,710 second-feot Mar. 2 (gage helght, 18.2 foet). 1930-37: Discharge, 5,020 second-feot Fob. 6, 1937.

Remarks. - Canal carrles aturm munoff from foothlils, orosses ranoh, and arains a small area between Tustin and the ocean. Records furnished by Orange County Flood Control District, through M. M. Thompsan, chlef ongineer.

\begin{tabular}{|r|c|c|c||c|c|c|c|c|c|c|c|c|}
\hline Day & Fob. & Mar. & Apr. & Day & Fob. & Mar. & Apr. & Day & Feb. & Mar. & Apr. \\
\hline 1 & 10 & 373 & 0.5 & 11 & 12 & 1.0 & 0.3 & 21 & 0.1 & 1.0 & 0.2 \\
2 & .1 & 3,200 & .4 & 12 & 5 & 146 & .3 & 22 & 0 & 1.0 & .2 \\
3 & 0 & 1,040 & .4 & 13 & .3 & 98 & .3 & 23 & 0 & 1.0 & .2 \\
4 & 7 & 12 & .4 & 14 & .3 & 6 & .3 & 24 & 0 & 1.0 & .2 \\
5 & 0 & 10 & .3 & 15 & .2 & 5 & .3 & 25 & 0 & 1.0 & .2 \\
6 & 0 & 7 & .3 & 16 & .2 & 4.0 & .3 & 26 & 0 & .9 & .2 \\
7 & 0 & 5 & .3 & 17 & .2 & 3.0 & .2 & 27 & 1.8 & .8 & .2 \\
8 & 0 & 4.0 & .3 & 18 & .2 & 2.0 & .2 & 28 & 30 & .7 & .2. \\
9 & 15 & 3.0 & .3 & 19 & .1 & 1.0 & .2 & 29 & & .6 & .2 \\
10 & 2.2 & 2.0 & .3 & 20 & .1 & 1.0 & .2 & 30 & & .5 & .2 \\
\end{tabular}


Oage: holght, in foet, and dwedinge, in woond-foet, at indicated time, 2938

\begin{tabular}{|c|c|c|c|c|c|c|c|c|c|c|c|c|}
\hline \multirow{2}{*}{8} & Foet & $800.1 t$. & Feot & $800 .{ }^{2}$ & Beot & Boc.ft. & Poet & seo.ft. & Peet & sec.tt. & Feet & Eeo.ft. \\
\hline & \multicolumn{2}{|c|}{ Fobruary 27} & \multicolumn{2}{|c|}{ Fobruary 28} & \multicolumn{2}{|c|}{ Maroh 1} & \multicolumn{2}{|c|}{ March 2} & \multicolumn{2}{|c|}{ Marah 3} & \multicolumn{2}{|c|}{ Maroh 4} \\
\hline $\begin{array}{l}1 \\
2 \\
3 \\
4 \\
5 \\
6\end{array}$ & & & & 7.0 & $\begin{array}{r}10.00 \\
11.70 \\
12.70 \\
11.95 \\
10.10 \\
8.30\end{array}$ & $\begin{array}{r}1,010 \\
1,800 \\
2,040 \\
1,700 \\
1,050 \\
590\end{array}$ & $\begin{array}{r}3.38 \\
5.25 \\
6.85 \\
7.50 \\
9.20 \\
11.70\end{array}$ & $\begin{array}{r}14 \\
93 \\
330 \\
430 \\
800 \\
1,610\end{array}$ & & $\begin{array}{l}3,880 \\
3,310 \\
2,670 \\
2,040 \\
1,420 \\
1,120\end{array}$ & & - \\
\hline $\begin{array}{r}7 \\
8 \\
8 \\
20 \\
21 \\
17\end{array}$ & $\begin{array}{l}3.0 \\
3.05\end{array}$ & & $\begin{array}{l}4.10 \\
4.35 \\
5.00 \\
4.45 \\
4.25 \\
4.10\end{array}$ & $\begin{array}{l}25 \\
33 \\
61 \\
38 \\
33 \\
28\end{array}$ & $\begin{array}{l}7.00 \\
6.20 \\
5.60 \\
5.00 \\
4.60 \\
4.25\end{array}$ & $\begin{array}{r}340 \\
210 \\
130 \\
71 \\
49 \\
37\end{array}$ & $\begin{array}{l}12.85 \\
13.30 \\
13.80 \\
14.65 \\
15.60 \\
15.30\end{array}$ & $\begin{array}{l}2,120 \\
2,340 \\
2,620 \\
3,120 \\
3,720 \\
3,520\end{array}$ & & $\begin{array}{r}1,030 \\
1,510 \\
1,570 \\
980 \\
590 \\
420\end{array}$ & & \\
\hline $\begin{array}{l}1 \\
2 \\
3 \\
4 \\
5 \\
6\end{array}$ & $\begin{array}{l}3.20 \\
3.87 \\
3.40\end{array}$ & & $\begin{array}{l}3.80 \\
3.78 \\
3.75 \\
3.80 \\
3.60 \\
3.55\end{array}$ & $\begin{array}{l}23 \\
20 \\
20 \\
17 \\
17\end{array}$ & $\begin{array}{l}4.00 \\
3.75 \\
3.80 \\
3.40\end{array}$ & $\begin{array}{l}29 \\
22 \\
17\end{array}$ & $\begin{array}{l}15.10 \\
15.45 \\
16.75 \\
17.40 \\
17.60 \\
17.80\end{array}$ & $\begin{array}{l}3,380 \\
3,620 \\
4,540 \\
5,030 \\
5,200 \\
5,480\end{array}$ & & $\begin{array}{r}300 \\
200 \\
240 \\
92 \\
68 \\
56\end{array}$ & & \\
\hline $\begin{array}{r}7 \\
8 \\
9 \\
20 \\
11 \\
17\end{array}$ & $\begin{array}{l}3.30 \\
3.25\end{array}$ & & $\begin{array}{l}3.45 \\
7.00\end{array}$ & 334 & .3 .00 & 12 & $\begin{array}{l}18.10 \\
18.20 \\
18.10\end{array}$ & $\begin{array}{l}5,620 \\
5,710 \\
5,680 \\
5,450 \\
5,110 \\
4,660\end{array}$ & & $\begin{array}{l}47 \\
39 \\
35 \\
31 \\
27 \\
25\end{array}$ & & \\
\hline
\end{tabular}

\section{Sen Gabriel River Basin}

Bast Fork of San Gabriel River near Camp Bonita, Oalif.

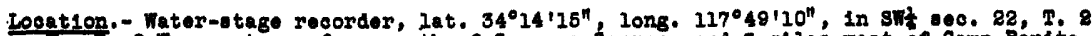
i., R. 9 \%., upetream from mouth of Sueanne Canjon, and 3 miles west of Oamp Bonita. Altitude, i,500 reot.

Drainage area. - Area, 01.4 square m1les. Average alt1tude, 5,830 root. Maximum alt1tudo, 10,0BO foet. Average lope, 54 percent. Inength of main channel, $17,8 \mathrm{ml} 100$. Average lope of main otream ohannel, 9.1 peroent.

Gage-he1ght reoord. - Vater-stage reoorder graph.

D1echarge record. - Stage-disoharge relation defined by ourrent-moter measuremente beloin 21,300 socond-reot and extended to peak otage on baels of moan depth-mean velocity reiation.

Lexign, 1936s Diooharge, 46,000 ceoond-feet Mar, 2 (gage holght, 10.1 feet). i932-37: D10charge; 8,500 eccond-reet Jan. 1, 1934.

Remarke. - Maximum discharge determined by the Geolog10al Survey; other recorde rurFiohod by Loe Angeles dounty Flood Oontrol D10trict, through H. B. Hedger, Ohlef onginoer.

Moan de1ly disoharge, in second-feet, 1938

\begin{tabular}{|c|c|c|c|c|c|c|c|c|c|c|c|}
\hline Dar & Peb. & Mas. & Apr. & Dar & Eob. & Mar. & Apr. & Des & Pob. & Mar. & Apr. \\
\hline $\begin{array}{r}7 \\
2 \\
3 \\
4 \\
8 \\
6 \\
7 \\
8 \\
9 \\
10\end{array}$ & $\begin{array}{r}79 \\
60 \\
188 \\
289 \\
186 \\
184 \\
110 \\
108 \\
183 \\
186\end{array}$ & $\begin{array}{r}1,980 \\
10,000 \\
5,100 \\
2,750 \\
2,000 \\
1,850 \\
1,260 \\
1,160 \\
900 \\
720\end{array}$ & $\begin{array}{l}350 \\
346 \\
340 \\
340 \\
350 \\
340 \\
330 \\
320 \\
320 \\
318\end{array}$ & $\begin{array}{l}11 \\
12 \\
13 \\
14 \\
16 \\
16 \\
17 \\
18 \\
19 \\
20\end{array}$ & $\begin{array}{l}364 \\
350 \\
972 \\
228 \\
197 \\
172 \\
160 \\
164 \\
160 \\
142\end{array}$ & $\begin{array}{r}620 \\
2,100 \\
1,860 \\
1,400 \\
1,200 \\
1,050 \\
930 \\
850 \\
740 \\
680\end{array}$ & $\begin{array}{l}310 \\
305 \\
295 \\
290 \\
280 \\
270 \\
265 \\
265 \\
280 \\
300\end{array}$ & $\begin{array}{l}21 \\
22 \\
23 \\
24 \\
25 \\
26 \\
27 \\
28 \\
28 \\
30 \\
31\end{array}$ & $\begin{array}{r}137 \\
137 \\
187 \\
132 \\
137 \\
132 \\
168 \\
1,870\end{array}$ & $\begin{array}{l}640 \\
600 \\
570 \\
540 \\
680 \\
490 \\
460 \\
400 \\
400 \\
370 \\
350\end{array}$ & $\begin{array}{l}295 \\
285 \\
280 \\
268 \\
271 \\
264 \\
836 \\
238 \\
838 \\
238\end{array}$ \\
\hline & & & & & & & & & $\begin{array}{r}212 \\
11,760 \\
\end{array}$ & $\begin{array}{r}1,450 \\
87,850\end{array}$ & $17^{\prime}, 494$ \\
\hline
\end{tabular}


Discharge, in second-feet, at indicated time, 1938

\begin{tabular}{|c|c|c|c|}
\hline Hour & February 27 & February 28 & March 1 \\
\hline $\begin{array}{r}1 \\
2 \\
3 \\
4 \\
5 \\
6 \\
7 \\
8 \\
9 \\
10 \\
11 \\
13 \\
1 \\
2 \\
3 \\
4 \\
5 \\
6 \\
7 \\
8 \\
9 \\
10 \\
11 \\
14 \\
\end{array}$ & $\begin{array}{l}130 \\
132 \\
134 \\
137 \\
142 \\
163 \\
168 \\
174 \\
180 \\
198 \\
236 \\
242 \\
264 \\
264\end{array}$ & $\begin{array}{r}284 \\
396 \\
850 \\
1,180 \\
1 \\
1,430 \\
1,310 \\
2,120 \\
1,550 \\
1,520 \\
1,670 \\
1,650 \\
1,940 \\
1,760 \\
1,850 \\
2,400 \\
1,480\end{array}$ & $\begin{array}{l}4,400 \\
4,630 \\
4,340 \\
3,150 \\
2,040 \\
1,510 \\
2,000 \\
2,510 \\
2,180 \\
1,260 \\
1,270 \\
1,300 \\
1,400 \\
1,380 \\
1,650\end{array}$ \\
\hline
\end{tabular}

San Gabriel River at Foothill Boulevard, near Azusa, Calif.

Location. - Water-stage recorder, lat. $34^{\circ} 08^{\prime} 15^{\prime \prime}$, long. $117^{\circ} 56^{\prime} 35^{\prime \prime}$, in Azusa (Duarte) land grant, at highway bridge at Foothill Boulevard, 2 miles west of Azusa, Los Angeles county. Alt tude, a bout 580 feet.

Drainage area.- Area, 230 square mlles. Average altitude, 4,330 feet. Maximum altitude, 10,080 feet. Average slope, 43 percent. Length of main stream channel, 30 miles. Average siope of main stream channel, 5.9 percent.

Gage-height record.- Water-stage recorder graph prior to 1:30 a.m. Mar. 3 .

Discharge record. - Stage-discharge relation defined by current-meter measurements below 35,500 second-feet; extended to maximum discharge determined by slope-area method.

Maxime; 1938: Discharge, 61,800 second-feet 7:35 p.m. Mar. 2 (gage-he1ght, 20.5

1932-37: Discharge, 10,000 second-feet Jan. 19, 1933.

Remarks.-Records fair. Flow partially regulated by San Gabriel River flood-control dams Nos. 1 and 2 and Morris Dam. Water diverted near mouth of San Gabriel Canyon for irrigation and spreading. Base data for Feb. 27 to Mar. 3 and entire record Feb. 1-26, Apr. 20, 27, and 29, furmished by Los Angeles County Flood Control District, through H. E. Hedger, chlef engineer.

Mean daily discharge, in second-feet, 1938

\begin{tabular}{|c|c|c|c|c|c|c|c|c|c|c|c|}
\hline Day & Feb. & Mar. & Apr. & Day & Feb. & Mar. & Apr. & Day & Feb. & Mar. & Apr. \\
\hline $\begin{array}{r}1 \\
2 \\
3 \\
4 \\
5 \\
6 \\
7 \\
8 \\
9 \\
10\end{array}$ & $\begin{array}{r}27 \\
12 \\
154 \\
118 \\
58 \\
37 \\
30 \\
26 \\
42 \\
50\end{array}$ & $\begin{array}{r}913 \\
11,600\end{array}$ & & $\begin{array}{l}11 \\
12 \\
13 \\
14 \\
15 \\
16 \\
17 \\
18 \\
19 \\
20\end{array}$ & $\begin{array}{r}114 \\
124 \\
98 \\
76 \\
72 \\
68 \\
62 \\
45 \\
35 \\
31\end{array}$ & & $* 410$ & $\begin{array}{l}21 \\
22 \\
23 \\
24 \\
25 \\
26 \\
27 \\
28 \\
29 \\
30 \\
31\end{array}$ & $\begin{array}{r}29 \\
28 \\
27 \\
79 \\
254 \\
217 \\
160 \\
527\end{array}$ & & $\begin{array}{l}* 23 \\
* 27\end{array}$ \\
\hline $\begin{array}{l}\text { Mean } \\
\text { Runo }\end{array}$ & $\begin{array}{c}n+h] \\
\text { In }\end{array}$ & 80 & & & & & & & $\begin{array}{r}92,9 \\
5,160\end{array}$ & & \\
\hline
\end{tabular}

* Discharge measurement. 
Gage holght, in feot, and discharge, in second-feot, at indicated time, 1938

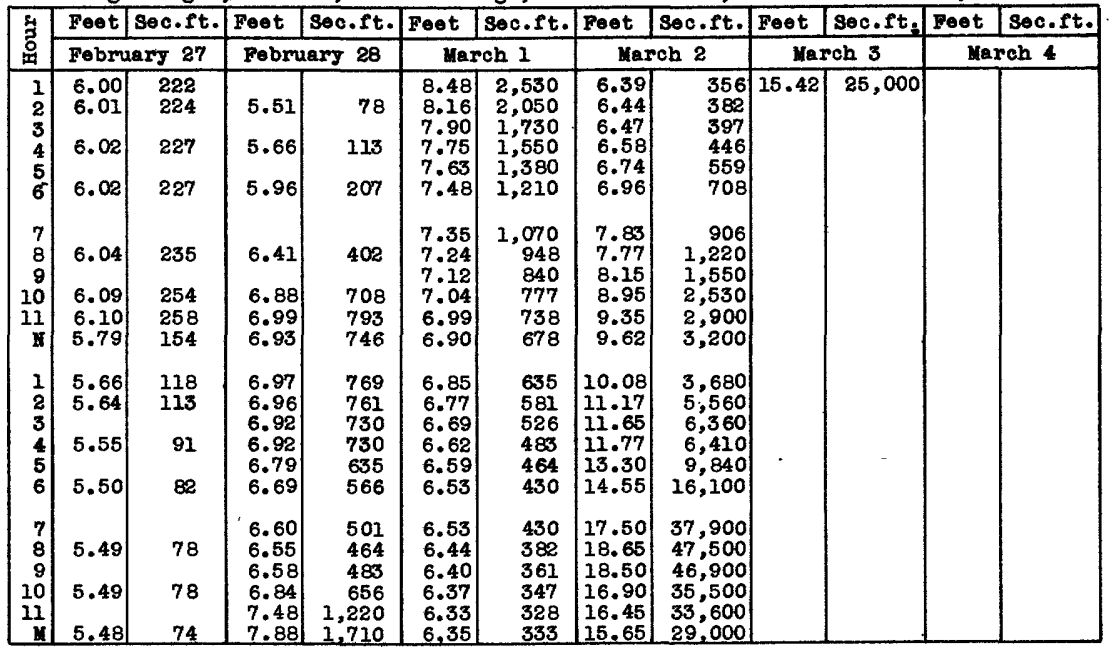

Supplemental record.- Mar. 2, 7:35 p.m., 20.5 ft., 61,800 sec. $-\mathrm{ft}$.

San Gabriel River at Plco, Calif.

Location. - Water-stage recorder, lat. $34^{\circ} 00^{\prime} 20^{n}$, long. $118^{\circ} 04^{\prime} 05^{n}$, in Paso de Bartolo grant, at Beverly Boulevard Bridge, half a mile east of Plco, Los Angeles County. Altitude, about 180 foot.

Drainage area. - Area indeterminate due to a natural split near Arrow Highway, which d1vides the San Gsbriel RIver into two branches; the west branch, known as the R10 Hondo, flows into the Los Angeles River; the east branch retalns the name San Gabriel. R1ver.

Gege-he1ght record. - Water-stage recorder graph except Mar. 4-6.

D1scharge record.- Stage-dlscharge relation defined by current-meter measurements below 12,700 second-feet; extended logarithmically to peak discharge on basis of slopearea measurement of the peak. Discharge for period of missing gage-he1ght record obtained from measurements and comparable hydrographs of ad jacent streams.

Maxima.- 1938: D1scharge, 22,700 second-feet 10:30 p.m. Mar. 2. 1928-37: Discharge, 22,000 second-feet Jan. 1, 1934.

Remarks.- Records falr. Flow partially regulated by San Gabriel River flood-control dams Nos. 1 and 2, Morr1s Dam, B1g Dalton Croek, San Dimas Oreek, Puddingstone, Ifve Oak Creok, and Thompson Oreek flood-control dams, and Pudaingstone diversion dam. The C1ty of Pasadena diverts water from the San Gabriel R1ver at Morris Dam. There are also several diversions for Irrigation. Records furnished by Los Angeles County Flood Control District, through H. B. Hedger, chief eng ineer.

Mean da1ly discharge, in second-feet, 1938

\begin{tabular}{|c|c|c|c|c|c|c|c|c|c|c|c|}
\hline Day & Feb. & Mar. & Apr. & Day & Feb. & Mar. & Apr. & Day & Feb. & Lar. & Apr. \\
\hline $\begin{array}{r}1 \\
2 \\
3 \\
4 \\
5 \\
6 \\
7 \\
8 \\
9 \\
10\end{array}$ & $\begin{array}{r}304 \\
55 \\
60 \\
88 \\
60 \\
55 \\
52 \\
47 \\
326 \\
95\end{array}$ & $\begin{array}{r}2,040 \\
11,400 \\
6,080 \\
3,190 \\
1,420 \\
1,030 \\
916 \\
1,270 \\
2,030 \\
1,050\end{array}$ & $\begin{array}{r}12 \\
167 \\
254 \\
221 \\
384 \\
503 \\
469 \\
242 \\
278 \\
314\end{array}$ & $\begin{array}{l}11 \\
12 \\
13 \\
14 \\
15 \\
16 \\
17 \\
18 \\
19 \\
20\end{array}$ & $\begin{array}{r}321 \\
196 \\
106 \\
69 \\
66 \\
77 \\
69 \\
66 \\
63 \\
49\end{array}$ & $\begin{array}{r}615 \\
1,020 \\
759 \\
704 \\
597 \\
386 \\
203 \\
254 \\
278 \\
221\end{array}$ & $\begin{array}{r}254 \\
150 \\
100 \\
54 \\
35 \\
61 \\
78 \\
78 \\
65 \\
39\end{array}$ & $\begin{array}{l}21 \\
22 \\
23 \\
24 \\
25 \\
26 \\
27 \\
28 \\
29 \\
30 \\
31\end{array}$ & $\begin{array}{r}47 \\
38 \\
38 \\
42 \\
106 \\
95 \\
216 \\
840\end{array}$ & $\begin{array}{r}121 \\
115 \\
176 \\
314 \\
384 \\
384 \\
134 \\
78 \\
121 \\
140 \\
70\end{array}$ & $\begin{array}{l}48 \\
65 \\
61 \\
61 \\
69 \\
69 \\
52 \\
52 \\
61 \\
56\end{array}$ \\
\hline \multicolumn{9}{|c|}{ 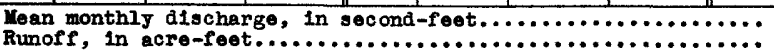 } & 7,230 & $\begin{array}{r}1,210 \\
74,380\end{array}$ & 8,680 \\
\hline
\end{tabular}


Gage height, in foet, and discherge, in second-foet, at indicated time, 1938

\begin{tabular}{|c|c|c|c|c|c|c|c|c|c|c|c|}
\hline \multirow{2}{*}{ 㟔 } & \begin{tabular}{l|l|} 
Foet & Sec.ft \\
\end{tabular} & Feot & sec.ft. & Feet & sec.ft. & Foet & Sec.ft. & Feet & Soc.ft. & Feet & sec.ft. \\
\hline & Pobruary 27 & \multicolumn{2}{|c|}{ Fobruary 28} & \multicolumn{2}{|c|}{ March 1} & \multicolumn{2}{|c|}{ March 2} & \multicolumn{2}{|c|}{ March 3} & \multicolumn{2}{|c|}{ Maroh 4} \\
\hline $\begin{array}{l}1 \\
2 \\
3 \\
4 \\
5 \\
6\end{array}$ & & $\begin{array}{l}5.87 \\
5.87 \\
5.87\end{array}$ & $\begin{array}{l}95 \\
95 \\
95\end{array}$ & $\begin{array}{l}9.00 \\
9.30 \\
9.60 \\
9.20 \\
8.75 \\
8.20\end{array}$ & $\begin{array}{l}5,600 \\
6,500 \\
7,900 \\
6,400 \\
4,700 \\
3,100\end{array}$ & $\begin{array}{l}5.76 \\
5.78 \\
6.10 \\
6.70 \\
7.57 \\
8.60\end{array}$ & $\begin{array}{r}400 \\
400 \\
800 \\
2,000 \\
4,200 \\
7,400\end{array}$ & & $\begin{array}{l}8,000 \\
5,800 \\
5,100 \\
4,500 \\
3,900 \\
3,500\end{array}$ & & \\
\hline $\begin{array}{r}7 \\
8 \\
9 \\
10 \\
11 \\
Y\end{array}$ & & $\begin{array}{l}6.00 \\
6.40 \\
6.57 \\
6.69 \\
6.57 \\
7.43\end{array}$ & $\begin{array}{r}151 \\
459 \\
670 \\
845 \\
670 \\
2,550\end{array}$ & $\begin{array}{l}7.85 \\
7.25 \\
6.90 \\
6.65 \\
6.45 \\
6.30\end{array}$ & $\begin{array}{r}2,500 \\
1,600 \\
1,100 \\
900 \\
700 \\
650\end{array}$ & $\begin{array}{r}9.45 \\
9.90 \\
10.12 \\
10.85 \\
10.52 \\
10.05\end{array}$ & $\begin{array}{r}9,800 \\
9,800 \\
13,400 \\
14,800 \\
11,800 \\
10,700\end{array}$ & & & & \\
\hline $\begin{array}{l}1 \\
2 \\
3 \\
4 \\
5 \\
6\end{array}$ & & $\begin{array}{l}7.25 \\
6.95 \\
6.77 \\
6.64 \\
6.53 \\
6.52\end{array}$ & $\begin{array}{r}2,180 \\
1,550 \\
1,220 \\
1,020 \\
848 \\
836\end{array}$ & $\begin{array}{l}6.12 \\
5.98\end{array}$ & 500 & $\begin{array}{r}10,20 \\
10.08 \\
9.90 \\
10.55 \\
12.45 \\
11.35\end{array}$ & $\begin{array}{l}11,400 \\
10,000 \\
11,400 \\
16,400 \\
19,200 \\
17,400\end{array}$ & & & & \\
\hline $\begin{array}{r}7 \\
8 \\
9 \\
10 \\
11 \\
4\end{array}$ & & $\begin{array}{l}6.40 \\
6.41 \\
6.47 \\
6.47 \\
6.65 \\
7.45\end{array}$ & $\begin{array}{r}692 \\
704 \\
776 \\
776 \\
1,020 \\
2,620\end{array}$ & $\begin{array}{r}5.70 \\
5.75\end{array}$ & 400 & $\begin{array}{l}11.00 \\
11.25 \\
11.02\end{array}$ & $\begin{array}{l}16,400 \\
18,300 \\
20,300 \\
22,000 \\
20,000 \\
12,600\end{array}$ & & & & \\
\hline
\end{tabular}

San Gabriel River at Spring Street, near Ios Alamitos, Calif.

- Location.- Fater-stage recorder, lat, $33^{\circ} 48^{\prime} 40^{n}$, long. $118^{\circ} 05^{\prime} 30^{\prime \prime}$, in Los Alamitos grant, at Spring Street Bridge, 1 is miles west of Los Alamitos, Orange Oounty. Altitude, about 22 feet.

Drainage area. - Area indeterminate due to a natural split near Arrow Blghway, which divides the San Gabriel R1ver Into two branches; the west branch known as the Rio Hondo, Plows into the Los Angeles River; the east branch retains the name of San Gabriel River.

Gage-helght record. - Water-stage recorder graph.

D1scharge record.- Stage-discharge relation poorly defined; shifting-control corrections used throughout period. Discharge Mar. 2, 3, 6, 8, and 11 determined by comparison with other stations. Maximum discharge determined by slope-area computation.

Max1ma. - 1938: Discharge, 27,300 second-feet Mar. 2 .

1928-37: Discharge, 15,000 second-feet Jan. 1, 1934.

Remarks.- See San Gabriel River at Pico for regulations and diversions. Naximum diecharge determined by the Geological Survey; other records furnished by Los Angeles County Flood Control District, through B. B. Hedger, chlef engineer.

Hean dally discharge, in second-feet, 1938

\begin{tabular}{|r|c|r|r||r|r|r|r||c|c|c|c|}
\hline Day & Feb. & Mar. & Apr. & Day & Feb. & Mar. & Apr. & Day & Feb. & Mar. & Apr. \\
\hline 1 & 56 & 1,870 & 5.5 & 11 & 89 & 248 & 87 & 21 & 0 & 103 & 0 \\
2 & .8 & 14,500 & 0 & 12 & 207 & 690 & 36 & 22 & 0 & 42 & 0 \\
3 & 0 & 9,250 & 5.5 & 13 & 6 & 416 & 0 & 23 & 0 & 0 & 0 \\
4 & 3.0 & 6,190 & 35 & 14 & 0 & 440 & 0 & 24 & 0 & 34 & 0 \\
5 & 0 & 1,950 & 95 & 15 & 0 & 312 & 0 & 25 & 0 & 54 & 0 \\
6 & 0 & 1,070 & 210 & 16 & 0 & 202 & 0 & 26 & 0 & 34 & 0 \\
7 & 0 & 802 & 248 & 17 & 0 & 142 & 0 & 27 & 46 & 27 & 0 \\
8 & 0 & 743 & 270 & 18 & 0 & 112 & 0 & 28 & 362 & 28 & 0 \\
9 & 156 & 1,660 & 166 & 19 & 0 & 109 & 0 & 29 & & 38 & 0 \\
10 & 75 & 911 & 188 & 20 & 0 & 112 & 0 & 30 & & 37 & 0 \\
\hline
\end{tabular}

$4547200-42-16$ 
West Fork of San Gabriel River at Camp Rincon, Callf.

Iocation. - Nater-stage recorder, lat. $34^{\circ} 14^{\prime} 30^{n}$, long. $117^{\circ} 51^{\prime} 50^{n}$, near center of sec. $19,1.2$ N., R. 9 i., a quartar of a mile above Camp Rincon, and half a mile downstream from North Fork of San Gabrial River. Altitude, 1,600 feot.

Drainage area.- Araa, 102 square miles. Averaga altitude, 4,260 feot. Maximum alt1tuda, 8,240 feet. Average slope, 50 percent. Length of main atream channel, 14.4 miles. Averaga slope of main stream channal, 8.7 percent.

Gage-helght record. - Nater-stage recordar graph axcept Mar. 2 to Apr. 4.

Discharge record.- Stage-diacharge ralation fairly well defined. Discharge obtalned by comparison with adjacent stations Mar. 2 to Apr. 4.

Maxima.- 1938: D1scharge, 34,000 second-faat Mar. 2 (astimated).

1927-37: Diacharge; 5,320 a econd-feet Jan. 1, 1934

Remarks.- Fl ow partiy regulated by flood-control dam upstream. Baae data and peak d1scharge for March and entire record for February and April furnished by Los Angeles County Flood Control District, through H. F. Hedger, chief engineer.

Mean da1ly discharge, in second-feet, 1938

\begin{tabular}{|c|c|c|c|c|c|c|c|c|c|c|c|}
\hline Day & Feb. & Mar. & Apr. & Day & $F \oplus b$. & Har. & Apr. & Day & Feb. & Mar. & Apr. \\
\hline $\begin{array}{r}1 \\
2 \\
3 \\
4 \\
5 \\
6 \\
7 \\
8 \\
9 \\
10\end{array}$ & $\begin{array}{r}136 \\
69 \\
322 \\
448 \\
231 \\
151 \\
117 \\
102 \\
166 \\
181\end{array}$ & $\begin{array}{r}1,900 \\
17,000 \\
8,900 \\
3,650 \\
1,800 \\
1,650 \\
1,650 \\
1,350 \\
1,950 \\
980\end{array}$ & $\begin{array}{l}330 \\
330 \\
320 \\
315 \\
320 \\
302 \\
276 \\
241 \\
212 \\
180\end{array}$ & $\begin{array}{l}11 \\
12 \\
13 \\
14 \\
15 \\
16 \\
17 \\
18 \\
19 \\
20\end{array}$ & $\begin{array}{l}598 \\
441 \\
280 \\
218 \\
179 \\
156 \\
139 \\
139 \\
151 \\
126\end{array}$ & $\begin{array}{r}480 \\
1,200 \\
1,000 \\
935 \\
960 \\
690 \\
670 \\
690 \\
720 \\
660\end{array}$ & $\begin{array}{l}177 \\
186 \\
186 \\
182 \\
164 \\
164 \\
158 \\
156 \\
152 \\
150\end{array}$ & $\begin{array}{l}21 \\
22 \\
23 \\
24 \\
25 \\
26 \\
27 \\
28 \\
29 \\
30 \\
31\end{array}$ & $\begin{array}{r}115 \\
111 \\
108 \\
106 \\
102 \\
100 \\
197 \\
1,310\end{array}$ & $\begin{array}{l}660 \\
750 \\
670 \\
670 \\
650 \\
620 \\
515 \\
490 \\
490 \\
435 \\
385\end{array}$ & $\begin{array}{l}144 \\
140 \\
135 \\
146 \\
150 \\
142 \\
132 \\
122 \\
130 \\
124\end{array}$ \\
\hline \multicolumn{9}{|c|}{$\begin{array}{l}\text { Mean monthly discharge, in second-feat. } \ldots \ldots \ldots \ldots \ldots \ldots \ldots \ldots \ldots \ldots \ldots \\
\text { Runoff, in acre-feet. } \ldots \ldots \ldots \ldots \ldots \ldots \ldots \ldots \ldots\end{array}$} & $\begin{array}{r}232 \\
12,890\end{array}$ & $\begin{array}{r}1,776 \\
109,200\end{array}$ & $\begin{array}{r}196 \\
11,640\end{array}$ \\
\hline
\end{tabular}

Rogerg Creok near Azusa, Calif.

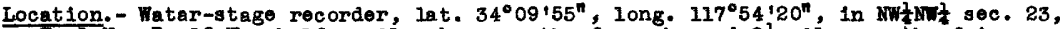
T. I K., R. 10 W., half mile above mouth of creok, and $2 \frac{1}{8}$ miles north of $A z u s a$. Aititude, a bout 800 foet.

Drainage area.- Araa, 6.4 square miles. Average alt1tude, 2,500 feet. Maximum alt1tude, 4,54i feet. Avaraga slope, 50 percent. Length of min stream channel, 5.0 miles. Average slope of main stream channel, 14 percent.

Gage-hajght record.- Watar-stage recorder graph except 7 a.m. Mar. 2 to Mar. 15.

Diacharge record. - Stage-discharge relation defined by current-mater moaurementa below 319 second-feet; axtended logarithmically to peak diacharge. Discharge for parlod of missing gege-helght record determined from rainfall records, comparieon with nearby streams, and one discharge measurement.

Maxima- 1938: Discharge, about 2,070 second-fakt 4:30 p.m. Mar. 2.

1917-37: Discharge, a bout 2,600 second-feet Apr. 7, 1926.

Ramarks.- Racorda good except thosa for period of missing gage-helght racord, which are poor. Diversions abova gage at times.

Mean daliy discharge, in second-feet, 1938

\begin{tabular}{|c|c|c|c|c|c|c|c|c|c|c|c|}
\hline Day & Feb. & Mar. & Apr. & Day & Fob. & Mar. & Apr. & Day & Feb. & Mar. & Apr. \\
\hline $\begin{array}{r}1 \\
2 \\
3 \\
4 \\
5 \\
6 \\
7 \\
8 \\
9 \\
10\end{array}$ & $\begin{array}{c}11 \\
5.5 \\
131 \\
50 \\
20 \\
11 \\
9.5 \\
9 \\
18 \\
16\end{array}$ & $\begin{array}{r}233 \\
738 \\
202 \\
108 \\
86 \\
62 \\
45 \\
41 \\
41 \\
38\end{array}$ & $\begin{array}{l}15 \\
15 \\
15 \\
15 \\
15 \\
15 \\
15 \\
14 \\
14 \\
13\end{array}$ & $\begin{array}{l}11 \\
12 \\
13 \\
14 \\
15 \\
16 \\
17 \\
18 \\
19 \\
20\end{array}$ & $\begin{array}{l}54 \\
42 \\
23 \\
19 \\
17 \\
9.5 \\
8 \\
8 \\
8.5 \\
7\end{array}$ & $\begin{array}{l}28 \\
87 \\
85 \\
75 \\
69 \\
62 \\
51 \\
44 \\
37 \\
34\end{array}$ & $\begin{array}{l}13 \\
14 \\
14 \\
12 \\
11 \\
11 \\
10 \\
9.5 \\
9.5 \\
9.5\end{array}$ & $\begin{array}{l}21 \\
22 \\
23 \\
24 \\
25 \\
26 \\
27 \\
28 \\
29 \\
30 \\
31\end{array}$ & $\begin{array}{c}6 \\
6 \\
5.5 \\
4.9 \\
4.9 \\
4.7 \\
8.5 \\
212\end{array}$ & $\begin{array}{l}30 \\
28 \\
25 \\
24 \\
23 \\
22 \\
19 \\
19 \\
18 \\
17 \\
16\end{array}$ & $\begin{array}{l}9.5 \\
9.5 \\
9 \\
9.5 \\
10 \\
9.5 \\
9 \\
8.5 \\
9 \\
9\end{array}$ \\
\hline $\mathrm{Yes}$ & in & & & & & & & & $\begin{array}{r}26.1 \\
1,450 \\
\end{array}$ & $\begin{array}{r}77.6 \\
4.770 \\
\end{array}$ & $\begin{array}{r}11.7 \\
698 \\
\end{array}$ \\
\hline
\end{tabular}


aage holght, in foot, and disoharge, in socond-foet, at Indioated timo, 1988

\begin{tabular}{|c|c|c|c|c|c|c|}
\hline \multirow{2}{*}{ Eour } & Feet & See.5t. & Peet & 8eo.It. & Foet & Soo.ft. \\
\hline & \multicolumn{2}{|c|}{ Fobruary 28} & \multicolumn{2}{|c|}{ Laroh 1} & \multicolumn{2}{|c|}{ Maroh 2} \\
\hline $\begin{array}{l}1 \\
2 \\
3 \\
4 \\
5 \\
6\end{array}$ & $\begin{array}{l}2.91 \\
5.02 \\
3.23 \\
5.82 \\
3.80 \\
3.96\end{array}$ & $\begin{array}{r}24 \\
20 \\
35 \\
62 \\
91 \\
213\end{array}$ & $\begin{array}{l}6.98 \\
6.87 \\
6.34 \\
5.97 \\
5.97 \\
8.87\end{array}$ & $\begin{array}{l}512 \\
461 \\
301 \\
336 \\
336 \\
802\end{array}$ & $\begin{array}{l}4.36 \\
4.46 \\
4.80 \\
4.86 \\
4.85 \\
5.28\end{array}$ & $\begin{array}{l}135 \\
146 \\
160 \\
168 \\
190 \\
236\end{array}$ \\
\hline $\begin{array}{r}7 \\
8 \\
9 \\
10 \\
12 \\
7\end{array}$ & $\begin{array}{l}4.08 \\
4.93 \\
4.89 \\
5.10 \\
5.10 \\
5.87\end{array}$ & $\begin{array}{l}188 \\
242 \\
837 \\
289 \\
269 \\
299\end{array}$ & $\begin{array}{l}5.82 \\
5.88 \\
5.21 \\
5.08 \\
4.94 \\
4.88\end{array}$ & $\begin{array}{l}272 \\
264 \\
235 \\
218 \\
201 \\
180\end{array}$ & 5.76 & 304 \\
\hline $\begin{array}{l}1 \\
2 \\
3 \\
4 \\
5 \\
6\end{array}$ & $\begin{array}{l}5.28 \\
5.28 \\
5.12 \\
4.98 \\
4.88 \\
4.78\end{array}$ & $\begin{array}{l}314 \\
518 \\
300 \\
270 \\
854 \\
286\end{array}$ & $\begin{array}{l}4.74 \\
4.70 \\
4.88 \\
4.49 \\
4.48 \\
4.38\end{array}$ & $\begin{array}{l}177 \\
178 \\
189 . \\
149 \\
148 \\
137\end{array}$ & & \\
\hline $\begin{array}{r}7 \\
8 \\
9 \\
10 \\
21 \\
21\end{array}$ & $\begin{array}{l}4.78 \\
4.75 \\
4.85 \\
5.82 \\
6.47 \\
6.90\end{array}$ & $\begin{array}{l}827 \\
220 \\
228 \\
308 \\
448 \\
801\end{array}$ & $\begin{array}{l}4.36 \\
4.35 \\
4.38 \\
4.32 \\
4.38 \\
4.80\end{array}$ & $\begin{array}{l}135 \\
131 \\
130 \\
130 \\
130 \\
127\end{array}$ & & \\
\hline
\end{tabular}

F1ah Oreok noar Duarte, Oal1f.

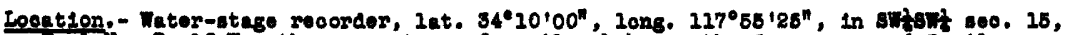

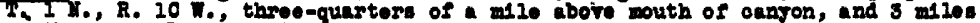
northeast of Drarte. Altlude, about 1,000 Fót:

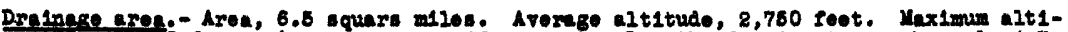

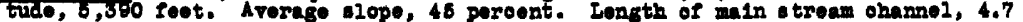
mf 10. Average lope of maln atream obannol, 17.6 percent.

Qege-pelent reoond. - Reporder not in operation during flood. Dally staff-gage readIngt Obtalnod after Mur. 15.

D1soharge reoord.- D1soharge prior to Kar. 15 ot1mated rrom ourront-motor measure-

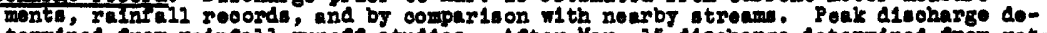
tormined from reinfall-munoff atudies. After var. 15 disoharge doterained from ratIng curve defined by ourrent-meter measurements.

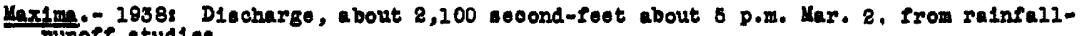
Furots tudies.

1916-371 Disoharge, ebout 2,180 secona-feet Apr. 4, 1925.

Remarka.- Rocosda poor. Ho regulation or Alveralons.

Uean da11y a1soharge, in seoond-feet, 1938

\begin{tabular}{|c|c|c|c|c|c|c|c|c|c|c|c|}
\hline Day & Fob. & Mar. & Apr. & $\overline{\text { Day }}$ & Feb. & Mar. & Apr. & Day & Fob. & Mar. & Apr. \\
\hline \begin{tabular}{r|}
1 \\
2 \\
3 \\
4 \\
5 \\
6 \\
7 \\
8 \\
9 \\
90
\end{tabular} & $\begin{array}{r}19 \\
8 \\
140 \\
89 \\
83 \\
12 \\
11 \\
10 \\
20 \\
18\end{array}$ & $\begin{array}{c}267 \\
752 \\
303 \\
138 \\
96 \\
70 \\
61 \\
58 \\
44 \\
35\end{array}$ & $\begin{array}{l}24 \\
28 \\
23 \\
20 \\
20 \\
20 \\
20 \\
19 \\
19 \\
19 \\
18\end{array}$ & $\begin{array}{l}11 \\
18 \\
13 \\
14 \\
18 \\
16 \\
17 \\
18 \\
18 \\
20\end{array}$ & $\begin{array}{l}56 \\
46 \\
25 \\
21 \\
19 \\
18 \\
8.6 \\
9 \\
9.6 \\
7.6\end{array}$ & $\begin{array}{l}33 \\
90 \\
80 \\
68 \\
68 \\
58 \\
49 \\
46 \\
46 \\
41\end{array}$ & $\begin{array}{l}16 \\
17 \\
16 \\
16 \\
18 \\
13 \\
12 \\
11 \\
11 \\
11\end{array}$ & $\begin{array}{l}21 \\
22 \\
23 \\
24 \\
25 \\
26 \\
87 \\
28 \\
89 \\
30 \\
31\end{array}$ & $\begin{array}{c}6.5 \\
6.5 \\
8 \\
5.5 \\
8.5 \\
5 \\
8 \\
190\end{array}$ & $\begin{array}{l}40 \\
37 \\
34 \\
35 \\
30 \\
30 \\
28 \\
88 \\
87 \\
28 \\
86\end{array}$ & $\begin{array}{l}11 \\
11 \\
11 \\
12 \\
13 \\
11 \\
10 \\
10 \\
11 \\
11\end{array}$ \\
\hline \multicolumn{9}{|c|}{ 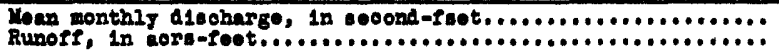 } & $\begin{array}{r}27.4 \\
1,580\end{array}$ & $\begin{array}{r}87.8 \\
5,300\end{array}$ & $\begin{array}{r}18.8 \\
008 \\
\end{array}$ \\
\hline
\end{tabular}


Sawplt Creek near Monrovia, Cailf.

Ioeation.- Water-stage recorder and broad-crested we1r control, 1at. $34^{\circ} 10^{\prime} 20^{*}$, $10 \mathrm{ng}$.

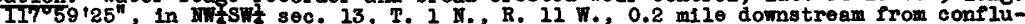
ence of two main branches, and 2 miles north of Monrovla. Alt1tude, about 1,100 leet.

Drainage area.- Area, 5.7 square miles. Average alt1tude, 3,050 feet. Maximum alt1tude, 5,390 feet. Average slope, 50 percent. Length of main stream channel, 3.6 miles. Average slope of main stream channe 2, 14 percent.

Gage-he1ght record. - Water-stage recorder graph Feb. 2-21 and Apr. 12, 16-23, and $25-30$.

D1scharge record. - Stage-discharge relation well defined within the range of ua crete control and station destroyed Har. 2. Discharge for Feb. 12 to Apr. 11, Apr. 13-15, and 24 determined from hydrograph based on records at flood-control dam and five discharge measurements.

Yaxima.- 1938: D1scharge, about 2,800 second-feet Har. 2, computed on basis of inflow to flood-control reservolr above gage.

1916-37: Discharge, about 2,000 second-feet Apr. 7, 2926, estimated from flow of Rogers Creek.

Remarks.- Records falr. Flow partly regulated by operation of flood-control dam about 0.3 mile upstream.

Msan da1ly discharge, in second-feet, 1938

\begin{tabular}{|c|c|c|c|c|c|c|c|c|c|c|c|}
\hline Day & Feb. & Mar. & Apr. & Day & Feb. & Mar. & Apr. & Day & Feb. & Mar. & Apr. \\
\hline $\begin{array}{r}1 \\
2 \\
3 \\
4 \\
5 \\
6 \\
7 \\
8 \\
9 \\
10\end{array}$ & $\begin{array}{c}1.1 \\
.5 \\
21 \\
29 \\
9.5 \\
8 \\
5 \\
8 \\
10 \\
1.5\end{array}$ & $\begin{array}{r}124 \\
450 \\
235 \\
108 \\
92 \\
91 \\
76 \\
37 \\
25 \\
24\end{array}$ & $\begin{array}{l}13 \\
13 \\
13 \\
13 \\
12 \\
12 \\
12 \\
12 \\
11 \\
21\end{array}$ & $\begin{array}{l}21 \\
12 \\
23 \\
24 \\
25 \\
16 \\
17 \\
18 \\
29 \\
20\end{array}$ & $\begin{array}{l}14 \\
12 \\
12 \\
10.5 \\
7 \\
5 \\
4.5 \\
3.5 \\
2.8 \\
1.4\end{array}$ & $\begin{array}{l}22 \\
41 \\
34 \\
30 \\
26 \\
24 \\
22 \\
20 \\
19 \\
18\end{array}$ & $\begin{array}{r}10 \\
10 \\
10 \\
10 \\
10 \\
9 \\
9 \\
9 \\
9 \\
9\end{array}$ & $\begin{array}{l}21 \\
22 \\
23 \\
24 \\
25 \\
26 \\
27 \\
28 \\
29 \\
30 \\
31\end{array}$ & $\begin{array}{r}1.0 \\
.9 \\
.8 \\
.8 \\
.7 \\
20 \\
89\end{array}$ & $\begin{array}{l}17 \\
16 \\
18 \\
14 \\
13 \\
13 \\
13 \\
13 \\
14 \\
14 \\
13\end{array}$ & $\begin{array}{l}9 \\
8.5 \\
8 \\
7.5 \\
7 \\
6 \\
6 \\
6 \\
6 \\
7\end{array}$ \\
\hline \multicolumn{9}{|c|}{ 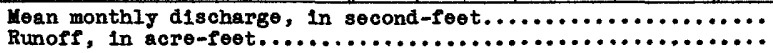 } & $\begin{array}{r}8.82 \\
490\end{array}$ & $\begin{array}{r}53.7 \\
3,300 \\
\end{array}$ & $\begin{array}{r}9.60 \\
571 \\
\end{array}$ \\
\hline
\end{tabular}

San Dimas Creek near San Dimas, Calif.

Location.- Water-stage recorder and broad-orested weir control, lat. $34^{\circ} 08^{\prime} 45^{\prime \prime}$, $10 \mathrm{ng}$.

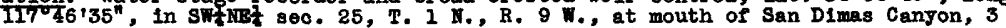
miles northeast of San Dimas. Aititude, about $i, 245$ fert.

Drainage area.- Area, 28.3 square miles. Average altitude, 3,130 feet. Maximum alt1tude, 5,558 feөt. Average slope, 42 percent. Iength of main stream ohannel, 7.5

miles. Average slope of main stream channel, 10 percent.

Gege-he1ght reoord.- Water-stage recorder graph except Feb. 18-27 and Mar. $20-27$.

Discharge record.- Stage-dischargs relation well defined below 250 seccnd-feet prior to Har. 2 ; on Mar. 2 control was partiy destroyed. D1schargs Feb. 18-27, Mar. 2-9 and 20-27, determined from outflow from flood-control reservolr.

Maxima. - 2938: Discharge, 4,250 seoond-feet 6 p.m. Mar. 2.

1914, 1916-37: Discharge, 2,950 second-feet February 1914.

Remarks.- Records good. Flow almost completely regulated by flood-control reservolr 0.7 mile upstream.

\begin{tabular}{|c|c|c|c|c|c|c|c|c|c|c|c|}
\hline Day & Feb. & Mar. & Apr. & Day & $\mathrm{Feb}$. & Mar. & Apr. & Day & Feb. & Mar. & Apr. \\
\hline $\begin{array}{r}1 \\
2 \\
3 \\
4 \\
5 \\
6 \\
7 \\
8 \\
9 \\
10\end{array}$ & $\begin{array}{r}1.6 \\
.5 \\
1.7 \\
2.0 \\
1.2 \\
.9 \\
.7 \\
.6 \\
1.7 \\
1.3\end{array}$ & $\begin{array}{r}281 \\
1,340 \\
750 \\
340 \\
210 \\
110 \\
96 \\
65 \\
62 \\
42\end{array}$ & $\begin{array}{l}27 \\
26 \\
25 \\
25 \\
25 \\
25 \\
25 \\
25 \\
25 \\
25\end{array}$ & $\begin{array}{l}11 \\
12 \\
13 \\
14 \\
15 \\
16 \\
17 \\
18 \\
19 \\
20\end{array}$ & $\begin{array}{l}2.8 \\
8 \\
15 \\
15 \\
15 \\
12 \\
12 \\
11 \\
10 \\
8\end{array}$ & $\begin{array}{r}36 \\
88 \\
115 \\
127 \\
117 \\
93 \\
90 \\
47 \\
93 \\
70\end{array}$ & $\begin{array}{l}24 \\
24 \\
9.5 \\
2.1 \\
2.1 \\
2.2 \\
2.2 \\
2.2 \\
2.4 \\
2.4\end{array}$ & $\begin{array}{l}21 \\
22 \\
23 \\
24 \\
25 \\
26 \\
27 \\
28 \\
29 \\
30 \\
31 \\
\end{array}$ & $\begin{array}{r}6 \\
5 \\
4 \\
2 \\
1 \\
2 \\
15 \\
87\end{array}$ & $\begin{array}{l}\mathbf{5 7} \\
\mathbf{5 7} \\
\mathbf{5 3} \\
\mathbf{4 9} \\
\mathbf{4 8} \\
\mathbf{4 6} \\
\mathbf{3 9} \\
\mathbf{3 3} \\
\mathbf{3 3} \\
\mathbf{3 3} \\
\mathbf{3 2} \\
\end{array}$ & $\begin{array}{l}2.4 \\
2.3 \\
2.4 \\
2.8 \\
2.7 \\
1.8 \\
4.0 \\
10 \\
14 \\
14\end{array}$ \\
\hline \multicolumn{9}{|c|}{ 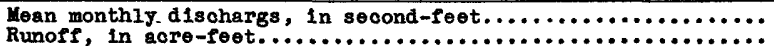 } & $\begin{array}{r}8.68 \\
482\end{array}$ & $\begin{array}{r}150 \\
9,230\end{array}$ & $\begin{array}{l}12.8 \\
75.9\end{array}$ \\
\hline
\end{tabular}


Dalton Crook noar Glendora, Calif.

Looation. - Water-stage recorder and concrete control, lat. $34^{\circ} \mathrm{C} 9^{\prime} 20^{\prime \prime}$, long. $117^{\circ} 49^{\prime} 50^{n}$, in center of sec. 21, T. I H., R. Q W., at Glendora Irrigation Co.'s dam, a quarter of a mlle above mouth, and 2 miles northeast of Glendora. Altitude, about 1,150 foot.

Drainage area.- Area, 7.5 square miles. Average altitude, 2,440 leet. Maximum alt1tude, 3,686 feet. Average slope, 42 percent. Iength of main stream chamel, 5.0 miles. Average slope of maln stream channel, 10 percent.

Gage-helght record. - Water-stage recorder graph except Feb. 25-27 and Mar. 24-28.

Discharge record.- Stage-discharge relation well defined by current-meter measurements belo 80 second-5eet. Discherge Feb. 25-27 Interpolated. Discherge 1ar 2-7 determined by release from flood-control reservolr and computed runoff for area between flood-control dam and gaging station. Diacharge Mar. 24-28 taken from comparative hydrograph based on four discherge measurements, release from flood-control reservolr, and estimated inflow betweon dam and gage.

Haxima. - 1938: D1scharge, about 850 second-foet 5 p.m. Mar. 2 (gage helght, 4.90 feot). 1914, 1919-37: D1scharge, 1,070 second-feet Februars 1914.

Remarks.- Records good except those for Har. 2-7, which are fair. Flood-control dam located about $1 \mathrm{mile}$ upstream.

Wean dally discharge, in seeond-foet, 1958

\begin{tabular}{|c|c|c|c|c|c|c|c|c|c|c|c|}
\hline Day & Feb. & Mar. & Apr. & Day & Feb. & Mar. & Apr. & $\mathrm{Day}$ & Feb. & Mar. & Apr. \\
\hline $\begin{array}{r}1 \\
2 \\
3 \\
4 \\
5 \\
6 \\
7 \\
8 \\
9 \\
10\end{array}$ & $\begin{array}{r}0.9 \\
.3 \\
1.8 \\
1.3 \\
.9 \\
.6 \\
.4 \\
.3 \\
1.0 \\
.8\end{array}$ & $\begin{array}{r}28 \\
266 \\
219 \\
193 \\
128 \\
118 \\
76 \\
70 \\
54 \\
50\end{array}$ & $\begin{array}{l}9 \\
8 \\
8 \\
8 \\
8 \\
7.5 \\
7.5 \\
7 \\
5 \\
3.1\end{array}$ & $\begin{array}{l}11 \\
12 \\
13 \\
14 \\
16 \\
16 \\
17 \\
18 \\
19 \\
80\end{array}$ & $\begin{array}{r}2.8 \\
3.0 \\
2.4 \\
2.2 \\
1.7 \\
1.0 \\
.6 \\
.5 \\
.8\end{array}$ & $\begin{array}{l}30 \\
37 \\
28 \\
28 \\
30 \\
30 \\
40 \\
33 \\
27 \\
23\end{array}$ & $\begin{array}{l}3.0 \\
2.7 \\
2.3 \\
1.8 \\
1.6 \\
1.6 \\
1.4 \\
1.3 \\
1.4 \\
1.4\end{array}$ & $\begin{array}{l}21 \\
22 \\
23 \\
24 \\
25 \\
26 \\
27 \\
28 \\
89 \\
30 \\
31\end{array}$ & $\begin{array}{r}0.3 \\
.3 \\
.3 \\
.2 \\
.2 \\
.1 \\
14^{.1}\end{array}$ & $\begin{array}{l}23 \\
22 \\
16 \\
12 \\
10 \\
10 \\
13 \\
16 \\
12 \\
11 \\
10\end{array}$ & $\begin{array}{l}1.4 \\
1.4 \\
1.6 \\
2.6 \\
2.6 \\
2.1 \\
1.4 \\
1.5 \\
1.6\end{array}$ \\
\hline \multicolumn{12}{|c|}{ 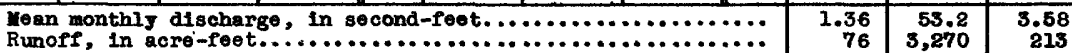 } \\
\hline
\end{tabular}

Gage height, In feet, and discharge, in second-feet, at Indieated time, 1958

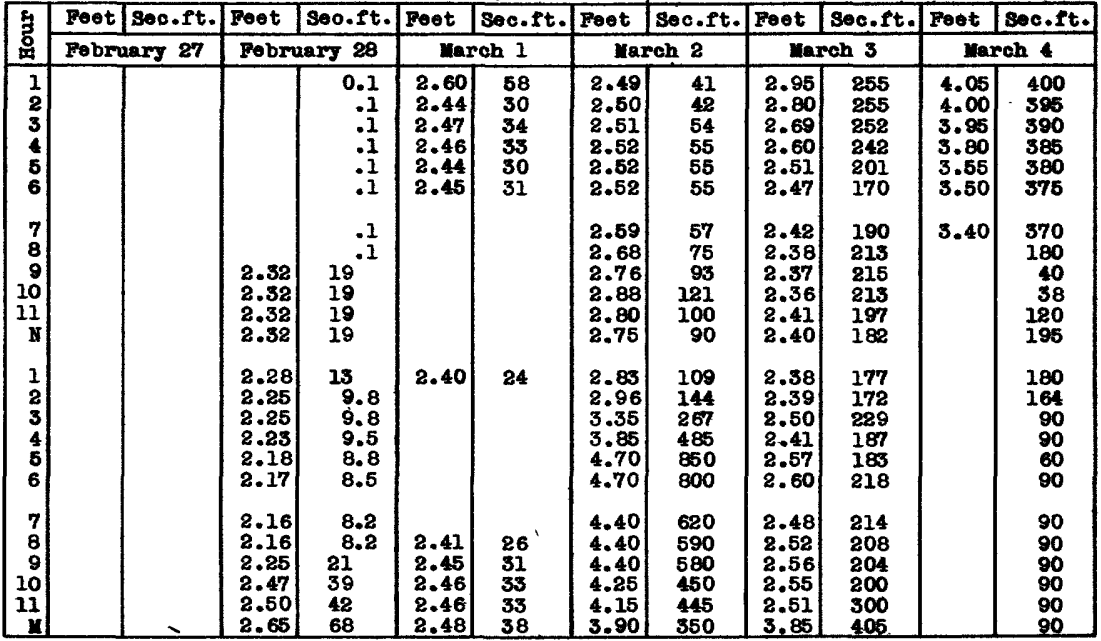


Ilttle Dalton Creok near Glendora, Cal1f.

Location.- Water-stage recorder, lat. $34^{\circ} 09130^{n}$, long. $117^{\circ} 50^{\prime} 15^{n}$, in SFt sec. $20, T$. 1 . R. 9 W., 500 feet above mouth of Littio Dalton Canyon, and 2 miles northerst of Giendora. 'Altitude, about 1,110 feet.

Drainage area. - 3.3 square miles.

Gage-helght record.- Water-stage recorder graph except 7 p.m. Mar. 2 to Mar. 12.

Discharge record.- Stage discharge relation defined by ourrent-meter messurements below I5I second-reet; extended logarithmicaliy to peak discharge as determined by a slopearea measurement. Discherge for perlods of missing record estimated from discharge measurements and records of Inflow to flood-control reservolr on Dalton Creek.

Mexima.- 1938: D1scharge, 960 second-feet 4:55 p.m. Mar. 2 (gage height, 5.9 feet). 1914, 1929-37: Discharge, 1,020 second-feet Februery 1914 .

Remarks. - Records falr. Small diversion above station. Record furnished by Los ingoles County Flood Control Distriet through B. E. Hedger, ohlef englneer.

Mean dally discharge, in second-feet, 1938

\begin{tabular}{|c|c|c|c|c|c|c|c||c|c|c|c|}
\hline Day & Feb. & Mar. & Apr. & Day & Feb. & Mar. & Apr. & Day & Feb. & Mar. & Apr. \\
\hline 1 & 2.0 & 81 & 6.5 & 11 & 6.0 & 12 & 4.3 & 21 & 1.5 & 13 & 3.3 \\
2 & .8 & 381 & 6 & 12 & 5.5 & 31 & 4.3 & 22 & 1.3 & 12 & 2.6 \\
3 & 9.5 & 105 & 6 & 13 & 4.2 & 30 & 4.3 & 23 & 1.2 & 12 & 2.6 \\
4 & 5.5 & 66 & 6 & 14 & 3.2 & 22 & 4.1 & 24 & 1.1 & 10 & 3.3 \\
5 & 3.0 & 30 & 5.5 & 15 & 2.6 & 20 & 3.8 & 26 & 1.0 & 9.5 & 4.3 \\
6 & 2.1 & 23 & 5 & 16 & 2.4 & 19 & 3.8 & 26 & 1.0 & 9 & 3.8 \\
7 & 1.8 & 18 & 4.8 & 17 & 1.8 & 16 & 3.3 & 27 & 3.7 & 8.5 & 3.3 \\
8 & 1.5 & 18 & 4.8 & 18 & 2.1 & 15 & 3.1 & 28 & 45 & 8.5 & 3.1 \\
9 & 3.3 & 17 & 4.5 & 19 & 2.6 & 15 & 3.1 & 29 & & 8 & 2.8 \\
10 & 1.6 & 12 & 4.5 & 20 & 1.8 & 14 & 3.6 & 30 & & 7.5 & 3.6 \\
\hline
\end{tabular}

Gage height, In feet, and discharge, in second-feet, at indicated time, 1838

\begin{tabular}{|c|c|c|c|c|c|c|c|c|c|c|c|c|}
\hline \multirow{2}{*}{ 跂 } & Feot & sec.ft. & Feot & seo.ft. & Feet & sec.ft. & Foet & sec.ft. & Foet & sec.ft. & Feet & sec.et. \\
\hline & \multicolumn{2}{|c|}{ February 27} & \multicolumn{2}{|c|}{ Pobruary 28} & \multicolumn{2}{|c|}{ March I } & \multicolumn{2}{|c|}{ March 2} & \multicolumn{2}{|c|}{ March 3} & \multicolumn{2}{|c|}{ March 4} \\
\hline $\begin{array}{l}1 \\
2 \\
3 \\
4 \\
5 \\
6\end{array}$ & & & $\begin{array}{r}0.72 \\
.81\end{array}$ & $\begin{array}{r}8.3 \\
17.8\end{array}$ & $\begin{array}{l}3.08 \\
2.45 \\
2.23 \\
2.05\end{array}$ & $\begin{array}{r}346 \\
214 \\
169 \\
132 \\
90\end{array}$ & $\begin{array}{l}1.42 \\
1.53 \\
1.95\end{array}$ & $\begin{array}{r}48 \\
68 \\
132\end{array}$ & & & & \\
\hline \begin{tabular}{r|}
7 \\
8 \\
9 \\
10 \\
11 \\
11
\end{tabular} & $\begin{array}{r}0.48 \\
.50 \\
.65\end{array}$ & $\begin{array}{l}1.4 \\
1.6 \\
6.0\end{array}$ & $\begin{array}{l}.95 \\
2.03 \\
1.30 \\
1.31 \\
1.25 \\
1.15\end{array}$ & $\begin{array}{c}19.7 \\
148 \\
46 \\
47 \\
42 \\
33\end{array}$ & 1.60 & $\begin{array}{r}63 \\
52 \\
49 \\
\cdot 44\end{array}$ & $\begin{array}{l}2.50 \\
3.24 \\
3.36 \\
3.36\end{array}$ & $\begin{array}{l}234 \\
387 \\
412 \\
412\end{array}$ & & & & \\
\hline $\begin{array}{l}1 \\
2 \\
3\end{array}$ & .57 & 3.7 & 1.36 & 52.0 & & 39 & $\begin{array}{l}4.04 \\
4.05\end{array}$ & $\begin{array}{l}559 \\
561\end{array}$ & & & & \\
\hline $\begin{array}{l}4 \\
5\end{array}$ & & & & 34 & 1.47 & 35 & 4.70 & 700 & & & & \\
\hline 6 & & & & 23 & & & 5.40 & 851 & & . & & \\
\hline $\begin{array}{l}7 \\
8 \\
8\end{array}$ & & & $\begin{array}{r}.86 \\
.86 \\
1.12\end{array}$ & $\begin{array}{l}19 \\
19 \\
30\end{array}$ & & 24 & 5.00 & 765 & & & & \\
\hline 10 & .53 & 2.8 & 1.65 & 87 & & 18 & & & & & & \\
\hline 11 & .55 & 3.2 & $\begin{array}{l}2.05 \\
2.36\end{array}$ & $\begin{array}{l}149 \\
205\end{array}$ & $\begin{array}{l}1.20 \\
1.26\end{array}$ & $\begin{array}{l}15 \\
19\end{array}$ & & & & & & \\
\hline
\end{tabular}

Supplementel record.- Mar. 2, 4:55 p.m., 5.9 ft., 960 sec.-ft. 
Nalnut Creek near Baldwin Park, Cal1f.

Location.- Fater-atage recorder, lat. $34^{\circ} 03^{\prime} 55^{\prime \prime}$, long. $117^{\circ} 59^{\prime} 00^{\prime \prime}$, in Ia Puente grant, at Covina Boulevard, near Baldwin Park, Los Angeles County. Ait 1tude, about 335 feet.

Drainage area.- 99 square miles.

Gage-height recora.- Nater-atage recorder graph except 2 a.m. Xar. 3 to $12 \mathrm{~m}$. Mar. 7. tage heights estimated from occasional staff-gage readings for period of inssing record.

Discharge record. - Stage-discharge relation defined by 13 current-meter measurements, made during period Feb. 27 to $\mathrm{Mar}, 3$, up to 4,200 second-feet and extended above.

Maxima:- 1938: D1scharge, 4,280 second-feet 5 p.m. Mar. 2 (gage height, 5.50 feet). 1928-37: Discharge, 8,060 second-feet Jan. $1,1934$.

Remarks. - Recorda good. Flow partially regulated by Dalton, San Dimas, Pudaingstone diversion, Pudaingstone, IIve Oak, and Thompson Creok Dams. Some water diverted for Irrigation at Puddingstone Dam. Records furnished by Los Angeles Oounty Flood Control District, through H. B. Hedger, chief engineer.

Mean dally discharge, in second-feet, 1938

\begin{tabular}{|c|c|c|c|c|c|c|c|c|c|c|c|}
\hline Day & $\mathrm{Peb}$ & Mar. & Apr. & Day & Feb. & Mar. & Apr. & Daf & Feb. & Mar. & Apr. \\
\hline $\begin{array}{r}1 \\
2 \\
3 \\
4 \\
5 \\
6 \\
7 \\
8 \\
9 \\
10\end{array}$ & $\begin{array}{c}93 \\
0 \\
5.5 \\
1.2 \\
0 \\
0 \\
0 \\
0 \\
70 \\
0\end{array}$ & $\begin{array}{r}443 \\
2,580 \\
517 \\
253 \\
117 \\
123 \\
45 \\
124 \\
110 \\
89\end{array}$ & $\begin{array}{l}0.4 \\
0.4 \\
.2 \\
.2 \\
0.2 \\
.2 \\
.8 \\
.8 \\
.2 \\
.2\end{array}$ & $\begin{array}{l}11 \\
12 \\
13 \\
14 \\
15 \\
16 \\
17 \\
18 \\
19 \\
20\end{array}$ & $\begin{array}{r}85 \\
35 \\
0 \\
0 \\
0 \\
0 \\
0 \\
0 \\
0 \\
0\end{array}$ & $\begin{array}{r}36 \\
94 \\
35 \\
99 \\
89 \\
103 \\
89 \\
45 \\
64 \\
86\end{array}$ & $\begin{array}{l}0 \\
2.8 \\
2.8 \\
2.4 \\
2.4 \\
1.2 \\
2.8 \\
1.1 \\
.6 \\
1.1\end{array}$ & $\begin{array}{l}21 \\
22 \\
23 \\
24 \\
25 \\
26 \\
27 \\
28 \\
29 \\
30 \\
31\end{array}$ & $\begin{array}{r}0 \\
0 \\
0 \\
0 \\
0 \\
0 \\
20 \\
406\end{array}$ & $\begin{array}{r}69 \\
64 \\
86 \\
64 \\
33 \\
20 \\
17 \\
3.6 \\
.8 \\
.4 \\
.4\end{array}$ & $\begin{array}{l}1.4 \\
2.8 \\
2.8 \\
0 \\
0.6 \\
0.1 \\
0 \\
0.9\end{array}$ \\
\hline $\begin{array}{l}\text { Mea } \\
\text { Run }\end{array}$ & , in & - & in & & & & & & $\begin{array}{r}25.6 \\
1,420\end{array}$ & $\begin{array}{r}177 \\
10,900\end{array}$ & $\begin{array}{r}0.96 \\
57\end{array}$ \\
\hline
\end{tabular}

Gage height, in feet, and discharge, in seconamfeet, at inaicated time, 1938

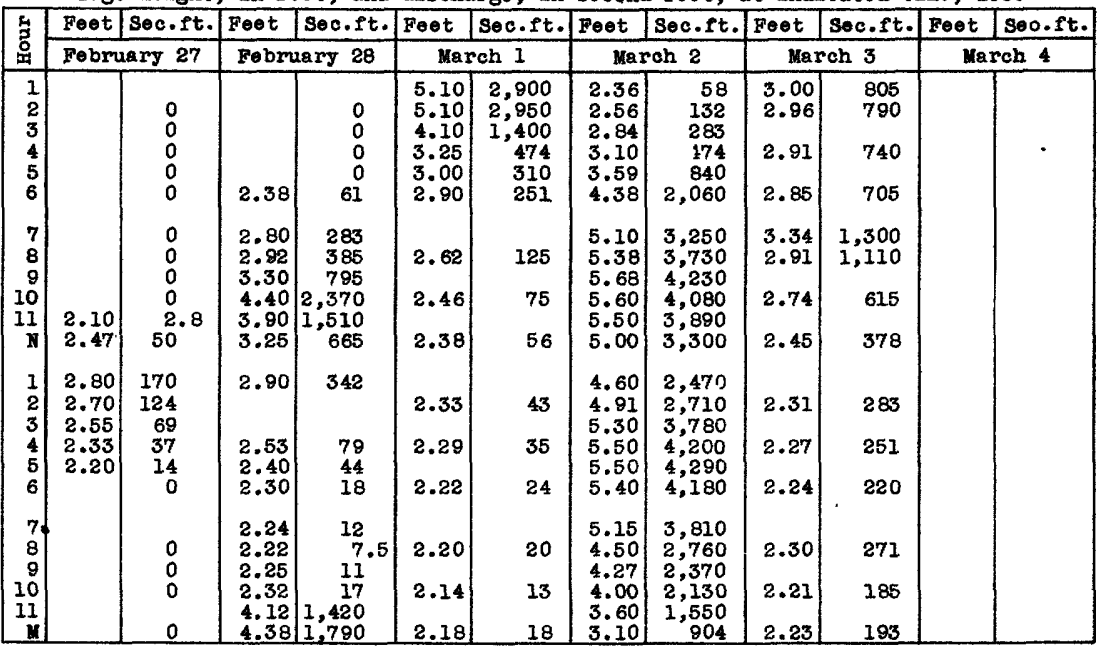


San Jose Creek near Whittier, Calif.

Locetion.- Water-stage recoxder, lat. $34^{\circ} 01^{\prime} 25^{\prime \prime}$, 1ong. $118^{\circ} 02^{\prime} 05^{\prime \prime}$, in Paso de Bartolo grant, at Workman-Mill road bridge, and about $3 \mathrm{miles}$ north of Whittiex, Ios Angeles county. Altitude, about 230 feet.

Drainage area.- 85.2 square miles.

Gage-he1ght record. - Tater-stage recordex greph except Apr11 21-29.

Discharge record.- Stage-discharge relation defined by current-meter measurements below 7,500 second-feet; extended logarithmically to peak discharge. Discharge for period of missing gage-helght record based on current-meter measurements.

Maxima.- 1938: Discharge, 9,350 second-feet 4:40 p.m. Mar. 2 (gage he1ght, 10.3 feet).. 1929-37: Discharge, 13,100 second-feet Jan. 1,1934 .

Remarks.- Flow partially regulated by Thompson Creek Dam. Several small diversions. Records furnished by Los Angeles County Flood Control District, through H. E. Hedger, chief engineex.

Nean dally discharge, in second-feet, 1938

\begin{tabular}{|c|c|c|c|c|c|c|c|c|c|c|c|}
\hline Day & Feb. & Max. & Apr. & Dey & Feb. & Mar. & Apr. & Day & Feb. & Max. & Apr. \\
\hline $\begin{array}{r}1 \\
2 \\
3 \\
4 \\
5 \\
6 \\
7 \\
8 \\
9 \\
10\end{array}$ & $\begin{array}{r}156 \\
6.0 \\
4.4 \\
31 \\
2.4 \\
.8 \\
.8 \\
.8 \\
193 \\
26\end{array}$ & \begin{tabular}{|r|}
768 \\
4,380 \\
505 \\
115 \\
32 \\
9 \\
3.9 \\
3.9 \\
4.4 \\
3.9
\end{tabular} & $\begin{array}{l}1.3 \\
1.3 \\
1.6 \\
1.3 \\
1.4 \\
1.3 \\
1.4 \\
2.3 \\
1.6 \\
1.1\end{array}$ & $\begin{array}{l}11 \\
12 \\
13 \\
14 \\
15 \\
16 \\
17 \\
18 \\
19 \\
20\end{array}$ & $\begin{array}{r}165 \\
81 \\
14.0 \\
2.1 \\
1.0 \\
.8 \\
.7 \\
.7 \\
.8 \\
.8\end{array}$ & $\begin{array}{c}5.4 \\
342 \\
55 \\
23 \\
7 \\
4.4 \\
2.3 \\
1.6 \\
1.6 \\
1.6\end{array}$ & $\begin{array}{l}1.6 \\
2.3 \\
2.3 \\
1.8 \\
1.8 \\
2.3 \\
1.3 \\
1.1 \\
1.6 \\
1.8\end{array}$ & $\begin{array}{l}21 \\
22 \\
23 \\
24 \\
25 \\
26 \\
27 \\
28 \\
29 \\
30 \\
31\end{array}$ & $\begin{array}{r}0.9 \\
.9 \\
.9 \\
2.0 \\
2.7 \\
1.3 \\
86 \\
340\end{array}$ & $\begin{array}{l}1.6 \\
1.6 \\
1.6 \\
1.6 \\
1.6 \\
1.6 \\
1.8 \\
1.4 \\
1.6 \\
1.3 \\
1.3\end{array}$ & $\begin{array}{l}1.8 \\
1.9 \\
1.9 \\
2.1 \\
2.1 \\
2.2 \\
2.2 \\
2.2 \\
2.3 \\
2.3\end{array}$ \\
\hline \multicolumn{9}{|c|}{ 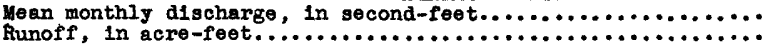 } & $\begin{array}{r}40.1 \\
2,230\end{array}$ & $\begin{array}{r}203 \\
12,470 \\
\end{array}$ & $\begin{array}{r}1.78 \\
106\end{array}$ \\
\hline
\end{tabular}

Gage helght, in feet, and discharge, in second-feet, at indicated time, 1938

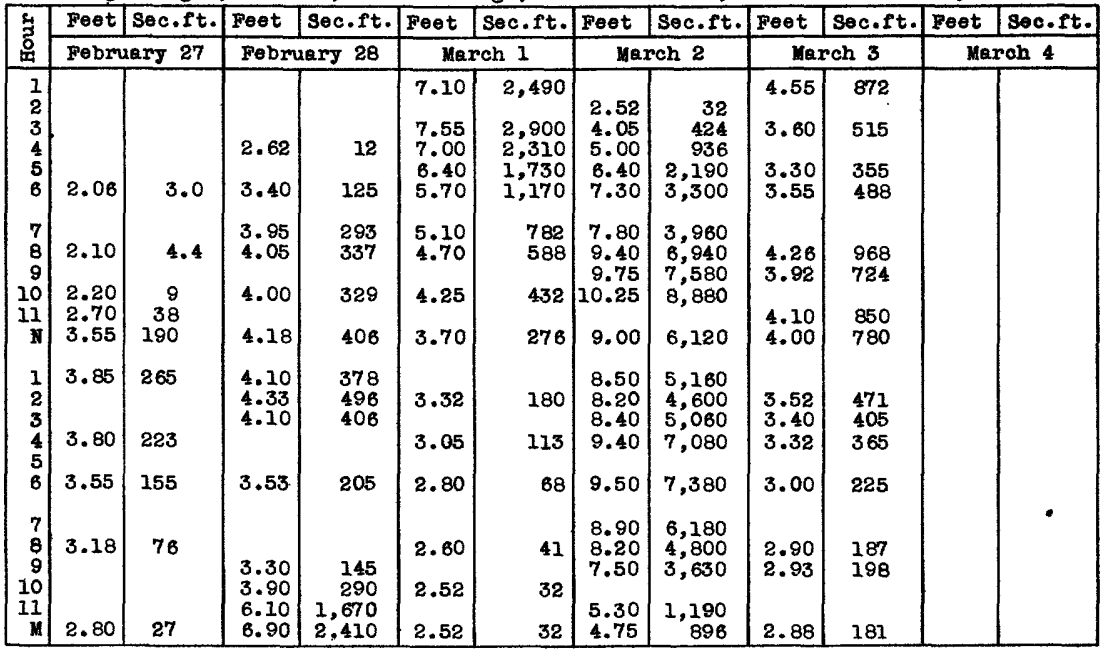

Supplemental record.- Mar. 2, 4240 p.m., 10.3 ft., 9,350 sec.-ft. 
Cojote Crook nesr Artes1a, Callf.

Locat1on. - Water-stage recorder, lat. $33^{\circ} 50^{\prime} 45^{n}$, long. $118^{\circ} 03^{\prime} 30^{n}$, in Coyote grant, on Iine between Orange and Los Angeles Counties, just above Pacific Blectric Ra1lway bridge $2 \frac{1}{2}$ miles southeast of Artesia. Altitude, about 35 feet.

Drainage area. - 110 square miles.

Gage-helght record. - Water-s tage recorder graph.

D1scharge record.- Stage-discharge relation defined by current-meter measurements be$10 \pi 3,580$ gecond-feet; oxtended logerlthmically to peak s tage.

Max1ma.- 1938: Discharge, 4,000 second-feet 8 p.m. Mar. 2 (gage height, 12.15 feet). 1929-37: Discharge, 4,190 second-feet Feb. 6, 1937 (gage height, 1C.i feet).

Remarks.- Records good. No regulation or diversions. Records furnished by Los Angeles county Flood Control Dletrlet, through H. E. Hedger, chief ongineer, and by Corps of Brigineers, U. S. Army.

Mean dally discharge, in second-feet, 1938

\begin{tabular}{|c|c|c|c|c|c|c|c|c|c|c|c|}
\hline Day & Fob. & Mar. & Apr. & Day & Feb. & Mar. & Apr. & Day & Feb. & Mar. & Apr. \\
\hline $\begin{array}{r}1 \\
2 \\
3 \\
4 \\
5 \\
6 \\
7 \\
8 \\
9 \\
10\end{array}$ & $\begin{array}{c}7.5 \\
22 \\
4.0 \\
4.4 \\
7.5 \\
6.5 \\
2.2 \\
1.2 \\
131 \\
149\end{array}$ & $\begin{array}{r}886 \\
2,540 \\
2,840 \\
228 \\
69 \\
38 \\
23 \\
18 \\
14 \\
13\end{array}$ & $\begin{array}{l}2.2 \\
2.0 \\
2.2 \\
2.0 \\
2.0 \\
2.2 \\
2.4 \\
1.9 \\
1.4 \\
1.3\end{array}$ & $\begin{array}{l}11 \\
12 \\
13 \\
14 \\
15 \\
16 \\
17 \\
18 \\
19 \\
20\end{array}$ & $\begin{array}{l}37 \\
94 \\
32 \\
11 \\
4.4 \\
2.4 \\
1.8 \\
1.2 \\
1.4 \\
1.9\end{array}$ & $\begin{array}{c}8.5 \\
156 \\
80 \\
32 \\
13 \\
6 \\
6 \\
4.4 \\
4.4 \\
4.2\end{array}$ & $\begin{array}{r}1.3 \\
1.2 \\
1.0 \\
.8 \\
.8 \\
.7 \\
.6 \\
.6 \\
.5 \\
.5\end{array}$ & $\begin{array}{l}21 \\
22 \\
23 \\
24 \\
25 \\
26 \\
27 \\
28 \\
29 \\
30 \\
31\end{array}$ & $\begin{array}{r}1.8 \\
1.3 \\
1.0 \\
.8 \\
.7 \\
.6 \\
109\end{array}$ & $\begin{array}{l}4.2 \\
3.2 \\
4.0 \\
4.2 \\
4.2 \\
4.2 \\
3.2 \\
3.0 \\
3.2 \\
3.4 \\
2.7\end{array}$ & $\begin{array}{r}0.5 \\
.5 \\
.5 \\
.7 \\
1.3 \\
1.6 \\
1.0 \\
.8 \\
.8 \\
1.4\end{array}$ \\
\hline \multicolumn{9}{|c|}{$\begin{array}{l}\text { Mean monthly discharge, in second-feet } \ldots \ldots \ldots \ldots \ldots \ldots \ldots \ldots \ldots \ldots \ldots \ldots \\
\text { Runoff, in acre-feet. } \ldots \ldots \ldots \ldots \ldots \ldots \ldots \ldots \ldots \ldots \ldots \ldots\end{array}$} & $\begin{array}{r}23.1 \\
1,280 \\
\end{array}$ & $\begin{array}{r}227 \\
13,930 \\
\end{array}$ & 1.22 \\
\hline
\end{tabular}

Gage height, in feet, and discharge, in second-feet, at indicated time, 1938

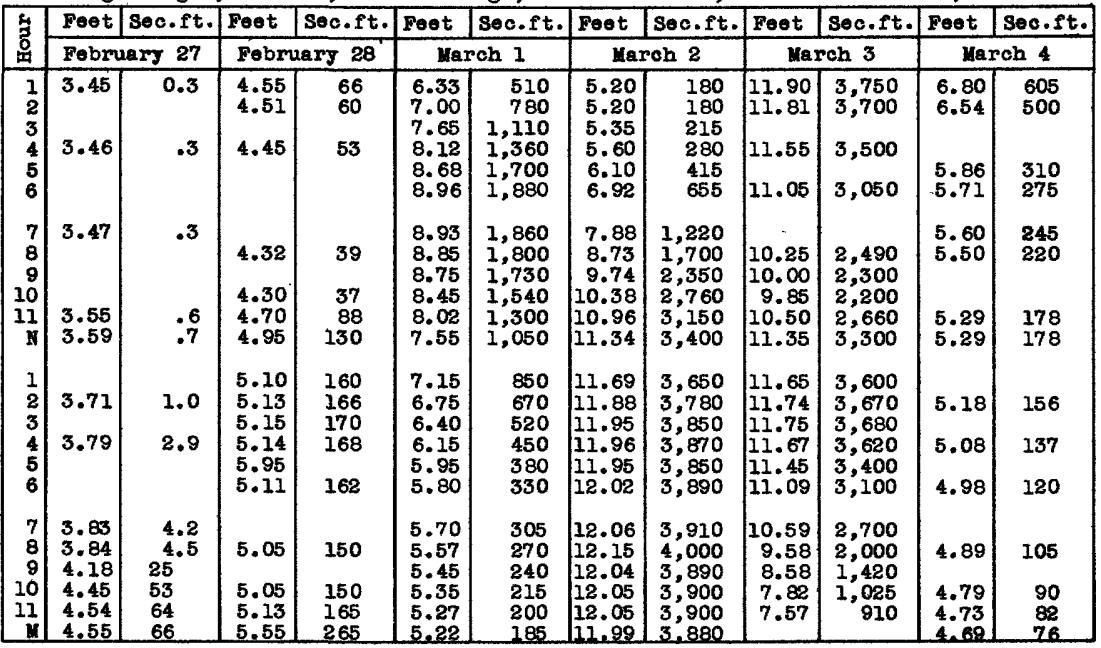


Brea Creek at Fullerton, Calif.

Location. - Water-stage recorder, lat. $33^{\circ} 52^{\prime} 25^{\prime \prime}$, long. $117^{\circ} 55^{\prime} 40^{\prime \prime}$, in San Juan Cajon de Santa Ana grant, at Ford Averue Bridge, at Fullerton, Orange County. Altitude, about 250 feet.

Drainage area.- Area, 26.4 square miles. Average altitude, 800 feet. Maximum alt1tude, 1,476 feet. Average slope, 21 percent. Iength of main stream channel, 16 miles. Average slope of main stream channel, 1.5 percent.

Gage-height record.- Water-stage recorder graph.

Discharge record.- Stage-discharge relation fairly well defined by measurements below 250 second-feet and extended above on basis of slope-area determinations.

Maxima. - 1938: Discharge, 1,970 second-feet Mar. 2 (gage he1ght, 9.55 feet). 1930-37: Discharge, 1,600 second-feet Oct. 17, 1934 (gage he1ght, $7.60 \mathrm{feet}$ ).

Remarks.- Records furnished by Orange County Flood Control District, through M. N. thompson, chier engineer.

Mean dally discharge, in second-feet, 1938

\begin{tabular}{|c|c|c|c|c|c|c|c|c|c|c|c|}
\hline Day & Feb. & Mar. & Apr. & Day & Feb. & Mar. & Apr. & Day & Feb. & Mar. & Apr. \\
\hline $\begin{array}{r}1 \\
2 \\
3 \\
4 \\
5 \\
6 \\
7 \\
8 \\
9 \\
10\end{array}$ & $\begin{array}{l}8.5 \\
0 \\
.6 \\
0 \\
0 \\
0 \\
0 \\
0 \\
59 \\
12\end{array}$ & $\begin{array}{r}157 \\
944 \\
22 \\
1.8 \\
.9 \\
.5 \\
.2 \\
.1 \\
.1 \\
.1\end{array}$ & $\begin{array}{l}0 \\
0 \\
0 \\
0 \\
0 \\
0 \\
0 \\
0 \\
0 \\
0\end{array}$ & $\begin{array}{l}11 \\
12 \\
13 \\
14 \\
15 \\
16 \\
17 \\
18 \\
19 \\
20\end{array}$ & $\begin{array}{l}8 \\
8.5 \\
.4 \\
0 \\
0 \\
0 \\
0 \\
0 \\
0.1\end{array}$ & $\begin{array}{l}0.1 \\
2.4 \\
.4 \\
.2 \\
0 \\
0 \\
0 \\
0 \\
0 \\
0\end{array}$ & $\begin{array}{l}0 \\
0 \\
0 \\
0 \\
0 \\
0 \\
0 \\
0 \\
0 \\
0\end{array}$ & $\begin{array}{l}21 \\
22 \\
23 \\
24 \\
25 \\
26 \\
27 \\
28 \\
29 \\
30 \\
31\end{array}$ & $\begin{array}{l}0 \\
0 \\
0 \\
0 \\
0 \\
0 \\
6.5 \\
50\end{array}$ & $\begin{array}{l}0 \\
0 \\
0 \\
0 \\
0 \\
0 \\
0 \\
0 \\
0 \\
0 \\
0\end{array}$ & $\begin{array}{l}0 \\
0 \\
0 \\
0 \\
0 \\
0 \\
0 \\
0 \\
0 \\
0\end{array}$ \\
\hline \multicolumn{9}{|c|}{ 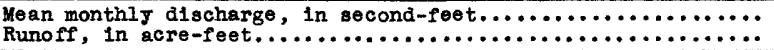 } & $\begin{array}{r}5.51 \\
306\end{array}$ & $\begin{array}{r}36.4 \\
2,240\end{array}$ & $\begin{array}{l}0 \\
0\end{array}$ \\
\hline
\end{tabular}

Gage height, in feet, and discharge, in second-feet, at indicated time, 1938

\begin{tabular}{|c|c|c|c|c|c|c|c|c|c|c|c|c|}
\hline \multirow{2}{*}{ 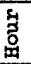 } & Peet & sec.ft. & Feet & sec.ft. & Feet & Sec.ft. & Feet & sec.ft. & Feet & Sec.ft. & Feet & sec.ft. \\
\hline & \multicolumn{2}{|c|}{ February 27} & \multicolumn{2}{|c|}{ February 28} & \multicolumn{2}{|c|}{ March 1} & \multicolumn{2}{|c|}{ March 2} & \multicolumn{2}{|c|}{ March 3} & \multicolumn{2}{|c|}{ March 4} \\
\hline $\begin{array}{l}1 \\
2 \\
3 \\
4 \\
5 \\
6\end{array}$ & & $\begin{array}{l}0 \\
0 \\
0 \\
0 \\
0 \\
0\end{array}$ & $\begin{array}{r}1.00 \\
.96 \\
.90 \\
.86 \\
1.05 \\
1.86\end{array}$ & $\begin{array}{l}3.9 \\
3.4 \\
2.8 \\
2.4 \\
5 \\
50\end{array}$ & $\begin{array}{l}4.65 \\
4.26 \\
3.60 \\
3.11 \\
3.32 \\
3.06\end{array}$ & $\begin{array}{l}580 \\
490 \\
330 \\
240 \\
280 \\
230\end{array}$ & $\begin{array}{l}1.90 \\
2.12 \\
2.20 \\
3.00 \\
5.00 \\
6.93\end{array}$ & $\begin{array}{r}62 \\
90 \\
102 \\
280 \\
870 \\
1,450\end{array}$ & $\begin{array}{r}1.90 \\
1.50 \\
1.20 \\
1.00 \\
.82 \\
1.60\end{array}$ & $\begin{array}{r}62 \\
35 \\
22 \\
14 \\
9 \\
40\end{array}$ & & \\
\hline $\begin{array}{r}7 \\
8 \\
9 \\
10 \\
11 \\
N\end{array}$ & $\begin{array}{r}0.90 \\
.83 \\
1.40 \\
1.24 \\
1.27\end{array}$ & $\begin{array}{c}0 \\
1.8 \\
1.2 \\
14 \\
7.5 \\
8.5\end{array}$ & $\begin{array}{l}1.90 \\
1.90 \\
1.83 \\
1.82 \\
1.82 \\
1.83\end{array}$ & $\begin{array}{l}54 \\
54 \\
49 \\
49 \\
50 \\
50\end{array}$ & $\begin{array}{l}3.12 \\
2.80 \\
2.50 \\
2.18 \\
2.12 \\
2.03\end{array}$ & $\begin{array}{r}240 \\
180 \\
140 \\
95 \\
89 \\
79\end{array}$ & $\begin{array}{l}6.60 \\
5.80 \\
6.38 \\
6.79 \\
6.30 \\
5.78\end{array}$ & $\begin{array}{l}1,350 \\
1,100 \\
1,280 \\
1,410 \\
1,260 \\
1,100\end{array}$ & $\begin{array}{r}1.92 \\
1.50 \\
1.17 \\
.97 \\
.93 \\
1.00\end{array}$ & $\begin{array}{l}64 \\
35 \\
21 \\
13 \\
12 \\
14\end{array}$ & & \\
\hline $\begin{array}{l}1 \\
2 \\
3 \\
4 \\
5 \\
6\end{array}$ & $\begin{array}{l}1.33 \\
1.40 \\
1.50 \\
1.48 \\
1.40 \\
1.34\end{array}$ & $\begin{array}{l}11 \\
15 \\
20 \\
20 \\
16 \\
13\end{array}$ & $\begin{array}{l}1.77 \\
1.73 \\
1.72 \\
1.69 \\
1.62 \\
1.60\end{array}$ & $\begin{array}{l}47 \\
44 \\
44 \\
42 \\
38 \\
36\end{array}$ & $\begin{array}{l}1.95 \\
1.85 \\
1.78 \\
1.72 \\
1.65 \\
1.59\end{array}$ & $\begin{array}{l}74 \\
67 \\
64 \\
61 \\
57 \\
55\end{array}$ & $\begin{array}{l}6.40 \\
9.00 \\
8.70 \\
6.80 \\
8.50 \\
7.32\end{array}$ & $\begin{array}{l}1,280 \\
1,910 \\
1,860 \\
1,420 \\
1,830 \\
1,560\end{array}$ & $\begin{array}{l}.91 \\
.80 \\
.77 \\
.73 \\
.70 \\
.68\end{array}$ & $\begin{array}{l}11 \\
8.5 \\
8 \\
7 \\
6.5 \\
6\end{array}$ & & \\
\hline $\begin{array}{r}7 \\
8 \\
9 \\
10 \\
11 \\
4\end{array}$ & $\begin{array}{l}1.28 \\
1.22 \\
1.14 \\
1.09 \\
1.00 \\
1.00\end{array}$ & $\begin{array}{l}10 \\
8.5 \\
6 \\
5 \\
3.6 \\
3.7 \\
\end{array}$ & $\begin{array}{l}1.56 \\
1.60 \\
2.05 \\
2.40 \\
2.55 \\
3.10\end{array}$ & $\begin{array}{r}34 \\
37 \\
74 \\
113 \\
136 \\
230\end{array}$ & $\begin{array}{l}1.53 \\
1.47 \\
1.42 \\
1.39 \\
1.42 \\
1.75\end{array}$ & $\begin{array}{l}52 \\
50 \\
49 \\
49 \\
53 \\
88\end{array}$ & $\begin{array}{l}5.90 \\
4.50 \\
4.10 \\
3.60 \\
3.00 \\
2.38\end{array}$ & $\begin{array}{r}1,140 \\
720 \\
600 \\
450 \\
280 \\
140\end{array}$ & $\begin{array}{l}.65 \\
.62 \\
.60 \\
.58 \\
.56 \\
.53\end{array}$ & $\begin{array}{l}5.5 \\
5 \\
4.6 \\
4.3 \\
4.0 \\
3.5\end{array}$ & & \\
\hline
\end{tabular}


Carbon Creok at Olinda, Cal1s.

Loontion. - Water-atage recorder, lat. $35^{\circ} 53120^{n}$, long. $117^{\circ} 80^{\prime} 40^{\prime \prime}$, in $\operatorname{san}$ Juan Oa jon do Sante Ana grant, at Golden Avenue Bridge, li miles outh of O1lnda, Orange Oounty. Altitudo, 362 soot.

Drelrgze erea,- Area, 80.C square mileg. Average alt1tude, 1,010 feet. Yaximum alt1tula, 1,760 feot. Average slope, 30 percent. Iongth of maln a trean ohannol, 9.5 m11es. Average slope of min stream ahamel, 1.8 peresnt.

Gege-helent reoord. - Water-stage recorder graph to lar. 1 ; no record thereafter. Peale stago srom illoodmarks.

D1soherge record. - Stage-disohargo relation defined by ourrent-moter moasurewents bolow 410 coond-Ioet, and extended to peak stage on basis of slops-area deterifinations.

Iexima - $1838:$ Disoharge, 1,760 second-feot Mar. 8 (gage holght, 4.0 feet). 1930-37: D1soharge; 728 second-feet Jane $i, 1084$.

Remarke. - Record furnishod by Orange dounty Flood Control Distriot, throuch M. H. Iromps on, ohlep eng ineer.

Koan de11y discharge, in second-seet, 1088

\begin{tabular}{|c|c|c|c|c|c|c|c|c|c|c|c|}
\hline Day & Fob. & Mar. & ADr. & $\overline{D a y}$ & Pob. & Iar. & Apr. & Dar & Fob. & nar. & Apr. \\
\hline $\begin{array}{r}1 \\
2 \\
5 \\
4 \\
8 \\
8 \\
7 \\
8 \\
9 \\
10\end{array}$ & $\begin{array}{l}2.8 \\
0 \\
.8 \\
1.0 \\
0 \\
0 \\
0 \\
0 \\
7 \\
3.8\end{array}$ & 46 & & $\begin{array}{l}11 \\
18 \\
13 \\
14 \\
18 \\
16 \\
17 \\
18 \\
10 \\
20\end{array}$ & $\begin{array}{l}8 \\
3.6 \\
0 \\
0 \\
0 \\
0 \\
0 \\
0 \\
0 \\
0\end{array}$ & & & $\begin{array}{l}81 \\
88 \\
88 \\
84 \\
88 \\
80 \\
87 \\
80 \\
89 \\
30 \\
31\end{array}$ & $\begin{array}{c}0 \\
0 \\
0 \\
0 \\
0 \\
0 \\
2.5 \\
18\end{array}$ & & \\
\hline 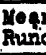 & & & & & & & & & $\begin{array}{r}1.68 \\
86\end{array}$ & & \\
\hline
\end{tabular}

Gage bo1ght, in feot, and disoharge, in wecond-feet, at indicated timo, 1038

\begin{tabular}{|c|c|c|c|c|c|c|c|c|c|c|c|c|}
\hline \multirow{2}{*}{ है } & Pest & $800 . \mathrm{ft}$ & Peet & Beo.et. & Det & soo.st. & Peet & sec.et. & Feet & seo,ft. & Feet & sec.ft. \\
\hline & Bobrv & any 87 & \multicolumn{2}{|c|}{ Pobruary 88} & \multicolumn{2}{|c|}{ Marah 1} & \multicolumn{2}{|c|}{ March 2} & \multicolumn{2}{|c|}{ Maroh 3} & \multicolumn{2}{|c|}{ Laroh 4} \\
\hline $\begin{array}{l}1 \\
8 \\
3 \\
4 \\
3 \\
6\end{array}$ & & & $\begin{array}{r}-0.85 \\
.10 \\
.03 \\
.08\end{array}$ & $\begin{array}{l}0 \\
0 \\
0 \\
6.5 \\
4.8 \\
8.8\end{array}$ & $\begin{array}{r}1.40 \\
2.60 \\
1.00 \\
.68 \\
.80 \\
.48\end{array}$ & $\begin{array}{r}164 \\
465 \\
90 \\
83 \\
32 \\
26\end{array}$ & & & & & & \\
\hline $\begin{array}{r}7 \\
8 \\
9 \\
10 \\
11 \\
1\end{array}$ & $\left|\begin{array}{r}-0.28 \\
.08 \\
-.10 \\
-.20\end{array}\right|$ & & $\begin{array}{l}.80 \\
.30 \\
.30 \\
.80 \\
.18 \\
.18\end{array}$ & $\begin{array}{l}10 \\
18 \\
16 \\
14 \\
8.8 \\
9.8\end{array}$ & $\begin{array}{l}.30 \\
.34 \\
.31 \\
.80 \\
.26 \\
.23\end{array}$ & $\begin{array}{l}22 \\
18 \\
16 \\
16 \\
13 \\
18\end{array}$ & & & & & & \\
\hline $\begin{array}{l}1 \\
2 \\
3 \\
4 \\
5 \\
6\end{array}$ & $\begin{array}{l}.80 \\
.20 \\
.18 \\
.05 \\
.06 \\
.08\end{array}$ & & $\begin{array}{l}.80 \\
.28 \\
.82 \\
.14 \\
.08 \\
.08\end{array}$ & $\begin{array}{r}10 \\
13 \\
11 \\
8 \\
6 \\
6\end{array}$ & $\begin{array}{l}.21 \\
.80 \\
.18 \\
.18 \\
.16 \\
.18\end{array}$ & $\begin{array}{l}11 \\
10 \\
9.5 \\
9.5 \\
8.5 \\
8.5\end{array}$ & & & & & & \\
\hline $\begin{array}{r}7 \\
8 \\
8 \\
10 \\
11 \\
11\end{array}$ & $\begin{array}{l}=.01 \\
=.09 \\
=.17 \\
=.18 \\
=.81 \\
=.80\end{array}$ & & $\begin{array}{r}0 \\
.10 \\
.38 \\
.38 \\
.70 \\
1.10\end{array}$ & $\begin{array}{r}3.7 \\
6.5 \\
22 \\
28 \\
68 \\
114\end{array}$ & $\begin{array}{l}.13 \\
.18 \\
.11 \\
.11 \\
.12 \\
.26\end{array}$ & $\begin{array}{c}7.8 \\
7 \\
7 \\
7 \\
7 \\
15\end{array}$ & & & & & & \\
\hline
\end{tabular}




\section{Los Angeles River Basin}

Los Angeles River near Van Nuys, Calif.

Location. - Water-stage recorder, lat. $34^{\circ} 09^{\prime} 30^{\prime \prime}$, long. $118^{\circ} 26^{\prime} 55^{\prime \prime}$, in Bx Mission San Fernando grant, on Van kuys Boulevard Bridge, about 2 miles south of Van huys, Los Angeles county. Zero of gage is 635.75 feet above mean sea level.

Drainage area.- 157 square miles.

Gage-he1ght record. - Water-stage rscorder graph Feb. 1 to $2: 30$ p.m. Mar. 2 ; peak stage observed on stafe gage.

Discharge record. - Stage-discharge relation defined by $81 x$ discharge measurements made reb. 27 to Lar. 2 .

Haxima.- 1938: Discharge, 11,600 second-fe日t 5 p.m. Mar. 2

1928-37: Discharge, 7,380 second-fe日t, Jan. 1,1934 .

Remarks.- Flow slightly regulated by Chatsworth Reservoir, Upper and Lower San Fernando Reservoirs, Twin Lakes Dams, dam on tributary of Iimekín Creek, and Encino Reservoir. Peak discharge determined by the Geologlcal Survey, other records furnished by Ios Angeles County Flood Control District, through H. I. Hedger, chief engineer. Mean dally discharge, in second-feet, 1938

\begin{tabular}{|c|c|c|c|c|c|c|c|c|c|c|c|}
\hline Day & Feb. & Mar. & Apr. & Day & Fab. & Mar. & Apr. & Day & Feb. & Mar. & Apr. \\
\hline $\begin{array}{r}1 \\
2 \\
3 \\
4 \\
5 \\
6 \\
7 \\
8 \\
9 \\
10\end{array}$ & $\begin{array}{c}211 \\
10 \\
58 \\
50 \\
8.5 \\
6.5 \\
6.5 \\
7 \\
73 \\
20\end{array}$ & $\begin{array}{r}974 \\
5,870\end{array}$ & & $\begin{array}{l}11 \\
12 \\
13 \\
14 \\
15 \\
16 \\
17 \\
18 \\
19 \\
20\end{array}$ & $\begin{array}{c}477 \\
45 \\
11 \\
9 \\
8 \\
8 \\
7.5 \\
8 \\
10 \\
7.5\end{array}$ & 、 & & $\begin{array}{l}21 \\
22 \\
23 \\
24 \\
25 \\
26 \\
27 \\
28 \\
29 \\
30 \\
31\end{array}$ & $\begin{array}{c}7 \\
7 \\
6.5 \\
6.5 \\
6 \\
6 \\
45 \\
509\end{array}$ & & $\begin{array}{l}11 \\
11\end{array}$ \\
\hline Mea & anthl & Ischa & In & & 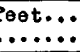 & & & $\ldots$ & $\begin{array}{r}58.4 \\
3,240\end{array}$ & & \\
\hline
\end{tabular}

Qsge height, in feet, snd discharge, in second-feet, at indicsted time, 1938

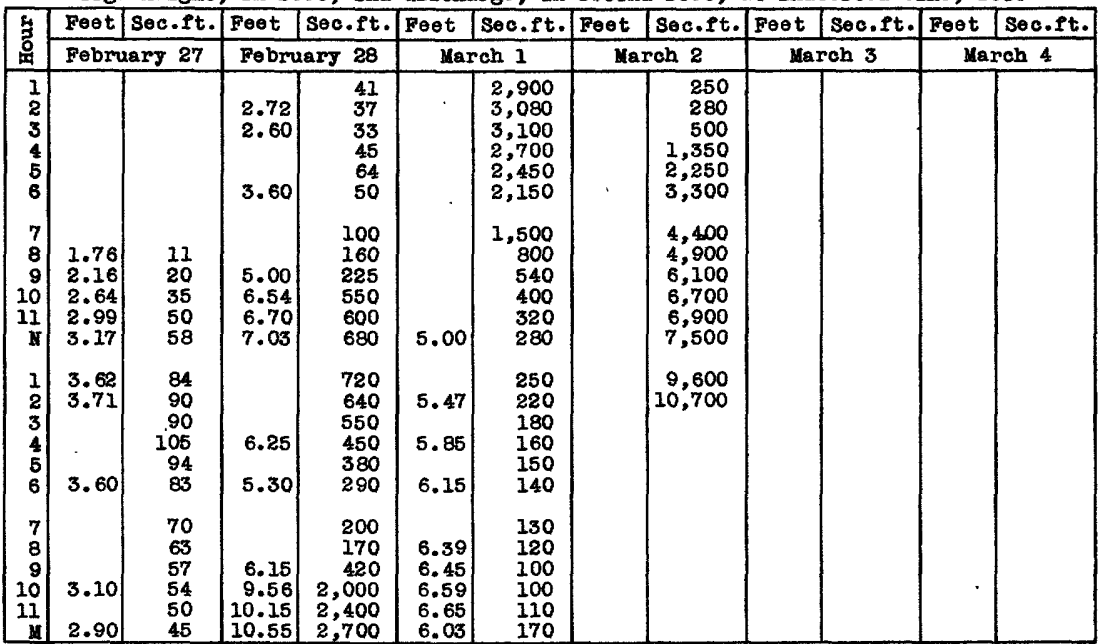


Ios Angeles River near Universal City, Galif.

Locat1on. - Water-atage recorder, lat. $34^{\circ} 08^{\prime} 35^{\prime \prime}$, long. $118^{\circ} 22^{\prime} 10^{\prime \prime}$, in Ex Mission San Fermando grant, at Vineland Átemue Bridge, near Universal City, Los Angeles County. Altitude, about 550 feet.

Drainage erea.- After leaving 1ta canjon, Tujunga Creek splits into two normally dry washes, one of which, kmown as Tujunga Wash, enters Ios Angeles River upstream from this station and the other downstream. The drainage area of Los Angeles River, exclusive of Tujunga Creek Basin above the split (157 square miles), is 245 square niles.

Gage-helght record.- Water-stage rocorder graph 7 a.m. Feb. 27 to 8250 a.m. Mar. 2.

Discharge record. - Stage-discharge relation defined by four current-meter and two float measurements, madeduring period Peb. 27 to Mar. 2, and a slope-area measurement of the peak.

Yaxima.- 1938: Discharge, 37,700 second-feet Nar. 2 .

1930-36: Discharge, 9,140 second-feet Jan. $1,1934$.

Remarks,-Records poor. Flow pertially regulated by Tujunga Dam, Pacolma Dam, Haines Debris Basin, Twin Iakes Dams, Chatsworth Reservo1r, Upper and Iower Ban Fernando Reservolrs, Enolno Reservolr, and a dam on a tributary of Limekiln Creek. Records furnished by Ios Angeles County Flood Control District, through H. B. Hedger, chlef engineer, and by Corps of Engineers, U. S. Army.

Hean dally discharge, in second-feet, 1938

\begin{tabular}{|lr|}
\hline February 27 & 48 \\
February 28 & 589 \\
Jreh 1 & 1,240 \\
\hline
\end{tabular}

Gage helght, in reet, and discharge, in second-feet, at indicated time, 1938

\begin{tabular}{|c|c|c|c|c|c|c|c|c|c|c|c|c|}
\hline \multirow{2}{*}{ 崬 } & Feet & sec.rt. & Feet & Sec.ft. & Feet & Sec.ft. & Feet & secoft. & Peet & sec.ft. & Feet & Sec-ft. \\
\hline & \multicolumn{2}{|c|}{ February 27} & \multicolumn{2}{|c|}{ Fobruary 28} & \multicolumn{2}{|c|}{ March 1} & \multicolumn{2}{|c|}{ March 2} & \multicolumn{2}{|c|}{ Maroh 3} & \multicolumn{2}{|c|}{ Maroh 4} \\
\hline $\begin{array}{l}1 \\
8 \\
5 \\
4 \\
5 \\
6\end{array}$ & 1 & 0 & $\begin{array}{l}8.66 \\
8.65\end{array}$ & $\begin{array}{r}82 \\
28 \\
19 \\
35 \\
79 \\
105\end{array}$ & $\begin{array}{c}8.18 \\
8.06 \\
7.95 \\
7.48\end{array}$ & $\begin{array}{l}3,700 \\
3,400 \\
3,300 \\
3,100 \\
2,650 \\
2,400\end{array}$ & $\begin{array}{l}4.26 \\
4.35 \\
4.70 \\
5.30 \\
7.95\end{array}$ & $\begin{array}{r}240 \\
260 \\
370 \\
620 \\
1,770 \\
3,050\end{array}$ & & $\cdot$ & & \\
\hline $\begin{array}{r}7 \\
8 \\
9 \\
10 \\
11 \\
11\end{array}$ & $\begin{array}{l}1.35 \\
1.40 \\
1.60 \\
2.15 \\
2.60 \\
2.90\end{array}$ & $\begin{array}{l}0 \\
.1 \\
6 \\
20 \\
37\end{array}$ & $\begin{array}{l}4.55 \\
4.82 \\
5.07 \\
5.46 \\
5.76\end{array}$ & $\begin{array}{l}280 \\
320 \\
410 \\
520 \\
700 \\
860\end{array}$ & $\begin{array}{l}6.60 \\
6.05 \\
5.55 \\
5.80 \\
4.97\end{array}$ & $\begin{array}{r}2,030 \\
1,500 \\
1,070 \\
750 \\
580 \\
480\end{array}$ & $\begin{array}{l}8.65 \\
9.26\end{array}$ & $\begin{array}{l}4,100 \\
5,400\end{array}$ & & & & \\
\hline $\begin{array}{l}1 \\
8 \\
3 \\
4 \\
5 \\
6\end{array}$ & $\begin{array}{l}3.35 \\
3.45\end{array}$ & $\begin{array}{r}85 \\
94 \\
110 \\
149 \\
124 \\
110\end{array}$ & $\begin{array}{l}5.45 \\
4.86\end{array}$ & $\begin{array}{l}855 \\
925 \\
855 \\
700 \\
545 \\
420\end{array}$ & 4.86 & $\begin{array}{l}425 \\
360 \\
280 \\
845 \\
\\
840\end{array}$ & & & & & & \\
\hline $\begin{array}{r}7 \\
8 \\
9 \\
10 \\
11 \\
1\end{array}$ & $\begin{array}{l}3.24 \\
2.94 \\
2.82 \\
2.85 \\
2.75\end{array}$ & $\begin{array}{l}95 \\
70 \\
40 \\
38 \\
33 \\
89\end{array}$ & $\begin{array}{l}4.62 \\
4.44 \\
4.85 \\
7.50 \\
8.20\end{array}$ & $\begin{array}{r}350 \\
300 \\
480 \\
1,180 \\
8,500 \\
3,450\end{array}$ & $\begin{array}{r}4.86 \\
4.86 \\
4.86\end{array}$ & $\begin{array}{l}240 \\
240 \\
240\end{array}$ & & & & & & \\
\hline
\end{tabular}

Supplemental record.- Float measurements lar. 8, 9:30 a.m., 13,400 sec.-ft.; 3845 p.m., 86,300 sec. $-\mathrm{ft}$. 
Los Angeles River at Los Angeles, Callf.

Iocation. - Water-stage reoorder, lat. $34^{\circ} 04^{\prime} 50^{\prime \prime}$, Iong. $118^{\circ} 13^{\prime} 35^{\prime \prime}$, In San Rafael grant, at Figueroa Stroet Bridge (formeriy Dayton Áveme), Los Angeleb, Los Angeles County, 0.1 mile upstream from Arroyo seco. Altitude, 298 feet.

\section{Dreinaze area. - 510 square miles.}

Gage-helght record.- Water-stage recorder graph for most of period.

Discharge record.- Computed on basis of ourrent-meter measurements and area and reloofty tudies; 140 current-meter messurements, ranging up to 22,000 second-feet, were made during period $\mathrm{Peb}$. I to Apr. $3 \mathrm{C}$, of whloh 44 were made during pertod Fob. 27 to Mar. 4. Peak discherge determined by lope-area method.

Mexima. - 1238: Dischargo, 67,000 second-foet 10 p.m. Mar. 2. 1929-37: Discharge, 22,000 second-feet Jan. 1, 1934.

Remark.- Records fair. Regulation by Twin Lakez Dans, Ohatsworth Reservolr, Upper and Lower San Bernando Reservolrs, Bnoino Reservolr; Pacolm Dam, and Tujunga Dam. Records except peak discharge furniahed by Los Angeles County Fiood Control Distriet, through H. I. Hedger, chief engineer.

Mean dally discharge, in seoond-feet, 1938

\begin{tabular}{|c|c|c|c|c|c|c|c|c|c|c|c|}
\hline Dey & Dob. & Mar. & Apr. & Day & Feb. & Mar. & Apr. & Day & Feb. & Mar. & Apr. \\
\hline $\begin{array}{r}1 \\
2 \\
3 \\
4 \\
5 \\
6 \\
7 \\
8 \\
9 \\
10\end{array}$ & $\begin{array}{r}598 \\
111 \\
260 \\
242 \\
92 \\
61 \\
45 \\
43 \\
271 \\
110\end{array}$ & $\begin{array}{r}2,300 \\
27,900 \\
8,210 \\
1,790 \\
930 \\
650 \\
600 \\
900 \\
800 \\
770\end{array}$ & $\begin{array}{l}66 \\
65 \\
64 \\
62 \\
61 \\
60 \\
68 \\
57 \\
56 \\
55\end{array}$ & $\begin{array}{l}11 \\
12 \\
13 \\
14 \\
16 \\
16 \\
17 \\
18 \\
19 \\
20\end{array}$ & $\begin{array}{r}766 \\
188 \\
84 \\
65 \\
56 \\
48 \\
44 \\
45 \\
95 \\
55\end{array}$ & $\begin{array}{r}800 \\
1,320 \\
1,180 \\
800 \\
540 \\
360 \\
340 \\
370 \\
350 \\
360\end{array}$ & $\begin{array}{l}54 \\
53 \\
62 \\
51 \\
49 \\
47 \\
45 \\
48 \\
41 \\
39\end{array}$ & $\begin{array}{l}21 \\
22 \\
23 \\
24 \\
25 \\
26 \\
27 \\
28 \\
29 \\
30 \\
31\end{array}$ & $\begin{array}{r}42 \\
41 \\
40 \\
43 \\
38 \\
40 \\
252 \\
1,390\end{array}$ & $\begin{array}{l}340 \\
340 \\
330 \\
330 \\
180 \\
80 \\
80 \\
74 \\
68 \\
68 \\
67\end{array}$ & $\begin{array}{l}38 \\
37 \\
37 \\
37 \\
37 \\
37 \\
37 \\
36 \\
36 \\
50\end{array}$ \\
\hline Int & it & & & & & & & & $\begin{array}{c}184 \\
10,240\end{array}$ & $\begin{array}{r}1,720 \\
105,600\end{array}$ & $\begin{array}{r}48.7 \\
2,890\end{array}$ \\
\hline
\end{tabular}

Discharge, in seoond-feet, at indicated time, 1938

\begin{tabular}{|c|c|c|c|c|c|c|}
\hline Hour & Fob. 27 & Fob. 28 & Mar. 1 & Nar. 2 & Mar. 3 & Har. 4 \\
\hline $\begin{array}{l}1 \\
2 \\
3 \\
4 \\
5 \\
6\end{array}$ & $\begin{array}{l}0 \\
0 \\
0 \\
0 \\
0 \\
0\end{array}$ & $\begin{array}{r}116 \\
280 \\
330 \\
580 \\
1,000 \\
1,800\end{array}$ & $\begin{array}{l}8,780 \\
5,800 \\
4,400 \\
4,700 \\
4,390 \\
4,210\end{array}$ & $\begin{array}{l}1,240 \\
1,100 \\
2,000 \\
3,500 \\
6,200 \\
8,100\end{array}$ & $\begin{array}{r}29,000 \\
23,000 \\
18,000 \\
14,000 \\
11,200 \\
8,800\end{array}$ & $\begin{array}{l}2,700 \\
2,540 \\
2,600 \\
2,500 \\
2,320 \\
2,130\end{array}$ \\
\hline $\begin{array}{r}7 \\
8 \\
9 \\
10 \\
11 \\
1\end{array}$ & $\begin{array}{r}0 \\
0 \\
340 \\
510 \\
621 \\
724\end{array}$ & $\begin{array}{l}2,500 \\
1,380 \\
1,680 \\
1,880 \\
1,440 \\
1,160\end{array}$ & $\begin{array}{l}3,220 \\
2,680 \\
2,230 \\
1,690 \\
1,210 \\
1,010\end{array}$ & $\begin{array}{l}10,900 \\
15,600 \\
19,100 \\
25,000 \\
17,700 \\
14,900\end{array}$ & $\begin{array}{l}7,000 \\
5,460 \\
5,670 \\
5,120 \\
4,820 \\
4,640\end{array}$ & $\begin{array}{l}1,860 \\
1,750 \\
1,810 \\
1,800 \\
1,810 \\
1,650\end{array}$ \\
\hline $\begin{array}{l}1 \\
2 \\
3 \\
4 \\
5 \\
6\end{array}$ & $\begin{array}{l}673 \\
648 \\
400 \\
312 \\
265 \\
220\end{array}$ & $\begin{array}{r}1,100 \\
1,120 \\
1,170 \\
1,090 \\
900 \\
860\end{array}$ & $\begin{array}{l}880 \\
790 \\
690 \\
630 \\
590 \\
540\end{array}$ & $\begin{array}{l}22,800 \\
30,600 \\
36,000 \\
44,800 \\
46,300\end{array}$ & $\begin{array}{l}4,640 \\
4,700 \\
4,640 \\
4,150 \\
3,800 \\
3,400\end{array}$ & $\begin{array}{l}1,560 \\
1,450 \\
1,430 \\
1,550 \\
1,420 \\
1,330\end{array}$ \\
\hline $\begin{array}{r}7 \\
8 \\
9 \\
10 \\
11 \\
4 \\
\end{array}$ & $\begin{array}{l}190 \\
170 \\
150 \\
140 \\
130 \\
120\end{array}$ & $\begin{array}{r}800 \\
680 \\
1,070 \\
3,120 \\
4,030 \\
6,600\end{array}$ & $\begin{array}{l}490 \\
495 \\
490 \\
510 \\
550 \\
890\end{array}$ & $\begin{array}{l}67,000 \\
36,000\end{array}$ & $\begin{array}{l}3,310 \\
3,310 \\
3,130 \\
2,860 \\
2,860 \\
2,790\end{array}$ & $\begin{array}{l}1,380 \\
1,370 \\
1,400 \\
1,360 \\
1,300 \\
1,230\end{array}$ \\
\hline
\end{tabular}


Los Angeles River near Domey, Calif.

Locetion. - Water-atage recorder, lat. $33^{\circ} 56^{\prime} 45^{\prime \prime}$, 1ong. $118^{\circ} 10^{\prime} 25^{n}$, In San Antonio grent, at Stewart and Gray Road Bridge, half a milo above Junction with Rio Fondo, and $2 \frac{1}{\mathrm{~s}} \mathrm{mles}$ west of Domey, Los Angeles County. Staff gages for slope readings 500 reot upstream and downstream. After Mar. 4, gage moved 0.4 of a mile upstream to Firestone Boule vard Bridge. Altitude, about 100 foet at now site.

Drainage area.- 614 square miles.

Gage-holght record.- Vater-stage recorder graph Feb, 1-16 and Apr. 11-30. Frequent stalt gage readings during s torm perlod $\mathrm{Peb}, 28$ to Mar. 4. After 9 p.m. Mar. 2 , roadings taken at upstream slope gago.

D1scharge record.- Stage discharge relation at Stewart and Gray Road defined by currentmotor mesurements belor 19,700 seoond-feet; extended to perk dischargo on bas 1s of slope-ares determinations. Discharge Mar. 3-4 based on Manning formula and a cross sectien taken after flood. Occaslonal measurements during periods Fob. 15-27 and Mar. 5 to Apr. 1l. Stage d lacharge relation at Firestone Boulevard defined by ourrent-meter measurements below 988 second-feet.

Mexima.- 1938: D1scharge, 79,700 second-feet 10 p.m. Mar. 2.

1928-37: D1scharge, 29,400 second-foet Jen. 1, 1934.

Remarka.- Records fair. Regulation at Pacolma Croek flood-control dam, Tujunga Crook I100d-control dam, and Devils Gate dam on Arroyo Seco. Records except peak discharge furnishod by Los Angeles County Flood Control D1striet, through H. B. Hedger, chlef engineer.

Mean dally discharge, in second-feet, 1938

\begin{tabular}{|c|c|c|c|c|c|c|c|c|c|c|c|}
\hline Day & Fөb. & Mar. & Apr. & Day & Feb. & Mar. & Apr. & Das & Fob. & Mar. & Apr. \\
\hline $\begin{array}{r}1 \\
2 \\
3 \\
4 \\
5 \\
6 \\
7 \\
8 \\
9 \\
10\end{array}$ & $\begin{array}{r}1,580 \\
200 \\
525 \\
418 \\
130 \\
80 \\
72 \\
71 \\
512 \\
140\end{array}$ & $\begin{array}{r}4,910 \\
40,000 \\
15,500 \\
2,500 \\
1,600 \\
1,300 \\
1,000 \\
1,500 \\
1,200 \\
1,050\end{array}$ & $\begin{array}{l}82 \\
75 \\
70 \\
64 \\
59 \\
54 \\
50 \\
44 \\
39 \\
34\end{array}$ & $\begin{array}{l}11 \\
12 \\
13 \\
14 \\
15 \\
16 \\
17 \\
18 \\
19 \\
20\end{array}$ & $\begin{array}{r}1,300 \\
830 \\
235 \\
180 \\
115 \\
68 \\
58 \\
60 \\
110 \\
80\end{array}$ & $\begin{array}{r}1,000 \\
1,500 \\
1,100 \\
840 \\
680 \\
560 \\
520 \\
560 \\
550 \\
540\end{array}$ & $\begin{array}{l}30 \\
27 \\
26 \\
38 \\
29 \\
31 \\
27 \\
26 \\
22 \\
34\end{array}$ & $\begin{array}{l}21 \\
22 \\
23 \\
24 \\
25 \\
26 \\
27 \\
28 \\
29 \\
30 \\
31\end{array}$ & $\begin{array}{r}55 \\
42 \\
39 \\
37 \\
39 \\
41 \\
400 \\
2,750\end{array}$ & $\begin{array}{r}530 \\
520 \\
510 \\
500 \\
250 \\
130 \\
125 \\
120 \\
108 \\
96 \\
88\end{array}$ & $\begin{array}{l}29 \\
22 \\
21 \\
26 \\
39 \\
52 \\
52 \\
42 \\
42 \\
81\end{array}$ \\
\hline $\begin{array}{l}\text { Mea } \\
\text { Ru }\end{array}$ & in & & & & & & & & $\begin{array}{r}363 \\
20,170\end{array}$ & $\begin{array}{r}2,625 \\
161,400\end{array}$ & $\begin{array}{r}42.3 \\
2,520\end{array}$ \\
\hline
\end{tabular}

Discharge, in second-feet, at indicated tims, 1938

\begin{tabular}{|c|c|c|c|}
\hline Hour & February 28 & Sarch 1 & Narch 2 \\
\hline $\begin{array}{l}1 \\
2 \\
3 \\
4 \\
5 \\
6\end{array}$ & $\begin{array}{r}320 \\
540 \\
670 \\
800 \\
1,400 \\
2,600\end{array}$ & $\begin{array}{r}16,700 \\
18,500 \\
12,000 \\
8,300 \\
7,100 \\
6,300\end{array}$ & $\begin{array}{r}2,400 \\
2,900 \\
2,800 \\
4,500 \\
10,300 \\
16,300\end{array}$ \\
\hline $\begin{array}{r}7 \\
8 \\
9 \\
10 \\
11 \\
11\end{array}$ & $\begin{array}{l}3,700 \\
5,400 \\
7,300 \\
5,100 \\
4,000 \\
2,900\end{array}$ & $\begin{array}{l}7,100 \\
6,000 \\
4,600 \\
3,700 \\
3,700 \\
2,750\end{array}$ & $\begin{array}{l}20,700 \\
25,000 \\
35,000 \\
37,000 \\
38,000 \\
36,500\end{array}$ \\
\hline $\begin{array}{l}1 \\
2 \\
3 \\
4 \\
5 \\
6\end{array}$ & $\begin{array}{l}2,200 \\
1,530 \\
1,300 \\
1,330 \\
1,330 \\
1,200\end{array}$ & $\begin{array}{l}2,200 \\
1,750 \\
1,720 \\
1,600 \\
1,450 \\
1,400\end{array}$ & $\begin{array}{l}35,000 \\
40,000\end{array}$ \\
\hline $\begin{array}{r}7 \\
8 \\
9 \\
10 \\
11 \\
\mathbf{u}\end{array}$ & $\begin{array}{r}1,040 \\
940 \\
2,900 \\
5,000 \\
7,300 \\
10,000\end{array}$ & $\begin{array}{l}1,320 \\
1,230 \\
1,160 \\
1,080 \\
1,000 \\
1,300\end{array}$ & · \\
\hline
\end{tabular}


Los Angeles River at Long Beach, Calif.

Iocation. - Water-stage recorder, lat. $33^{\circ} 47^{\prime} 25^{\prime \prime}$, Iong. $118^{\circ} 12^{\prime} 20^{\prime \prime}$, In Los Cerritos grant, at State Street Bridge tiong Beach, 'Los Angeles County.

Gage-height record.- Water-stege recorder graph.

Discharge record.- Stage-discharge relation poorly defined; shifting-control method used throughout period. Discharge Mar. 2-11 determined by comparison with edjacent stetions.

Maxima.- 1938: Discharge, 99,000 second-feet Mar. 2.

1928-37: Discharge, 20,500 second-feet Feb. 14, 1937.

Remarks. - See Los Angeles River at Los Angeles for regulation. Record furnished by Los Angeles County Flood Control District, through H. B. Hedger, chief engineer.

Mean daily discharge, in second-feet, 1938

\begin{tabular}{|c|c|c|c|c|c|c|c|c|c|c|c|}
\hline Day & Feb. & Mar. & Apr. & Day & Feb. & Nar. & Apr. & Day & Feb. & Mar. & Apr. \\
\hline $\begin{array}{r}1 \\
2 \\
3 \\
4 \\
5 \\
6 \\
7 \\
8 \\
9 \\
10\end{array}$ & $\begin{array}{r}1,960 \\
262 \\
690 \\
815 \\
233 \\
60 \\
32 \\
24 \\
1,370 \\
450\end{array}$ & $\begin{array}{r}7,090 \\
50,000 \\
30,000 \\
8,000 \\
5,400 \\
3,900 \\
3,800 \\
4,400 \\
4,800 \\
4,100\end{array}$ & $\begin{array}{r}831 \\
1,400 \\
1,500 \\
1,650 \\
2,290 \\
2,460 \\
2,580 \\
2,690 \\
2,600 \\
2,060\end{array}$ & $\begin{array}{l}11 \\
12 \\
13 \\
14 \\
15 \\
16 \\
17 \\
18 \\
19 \\
20\end{array}$ & $\begin{array}{r}1,720 \\
873 \\
160 \\
107 \\
75 \\
63 \\
58 \\
58 \\
81 \\
50\end{array}$ & $\begin{array}{l}2,300 \\
2,630 \\
2,880 \\
2,370 \\
2,150 \\
1,900 \\
1,420 \\
1,420 \\
1,630 \\
1,130\end{array}$ & $\begin{array}{r}1,730 \\
1,360 \\
784 \\
310 \\
266 \\
288 \\
255 \\
233 \\
222 \\
200\end{array}$ & $\begin{array}{l}21 \\
22 \\
23 \\
24 \\
25 \\
26 \\
27 \\
28 \\
29 \\
30 \\
31\end{array}$ & $\begin{array}{r}45 \\
41 \\
37 \\
32 \\
22 \\
26 \\
1,140 \\
3,250\end{array}$ & $\begin{array}{r}1,060 \\
986 \\
914 \\
1,140 \\
1,190 \\
1,210 \\
1,130 \\
1,130 \\
1,080 \\
1,050 \\
980\end{array}$ & $\begin{array}{l}190 \\
352 \\
450 \\
510 \\
465 \\
422 \\
366 \\
324 \\
288 \\
244\end{array}$ \\
\hline \multicolumn{9}{|c|}{ 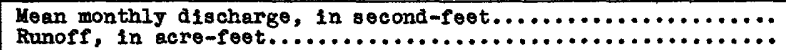 } & 27,280 & $\begin{array}{r}4,942 \\
303,800\end{array}$ & 58,160 \\
\hline
\end{tabular}

Browns Creek at Chatsworth, Calif.

Locetion. - Water-stage recorder, lat. $34^{\circ} 15^{\prime} 25^{n}$, Iong. $118^{\circ} 35^{\prime} 4^{n}$, in Bx Mission San Fermando grant, at Devonshire Street Bridge at Chatsworth, Las Angeles County. Altituae, bout 1,100 feet.

Drainage erea.- 14.3 square miles.

Gage-he1ght record. - Water-stage recorder graph except Mar. 4-11.

Discharge record. - Stage discharge relation defined by current-meter meesurements be10 296 second-feet; extended by computations based on cross section at gage and mean depth-mean velocity reletion. Diecharge for period of missing gage-height

record determined by discharge measurements and comparis on with adjecent stetions.

Yeximum.- 1938: D1scherge, 870 second-feet 10 a.m. Mar. 2 (gage height, 2.83 feet).

Remarks.- Records poor. FIow sIightly rogulated by Twin Lakes Dams. No divergions. Records, except for the period Mar. 1-3, furnished by Los Angeles County Flood Control District through H. E. Hedger, chlef engineer.

Mean delly discharge, in second-feet, 1938

\begin{tabular}{|c|c|c|c|c|c|c|c|c|c|c|c|}
\hline Day & Feb. & Mar. & Apr. & Day & Feb. & Mar. & Apr. & Day & Feb. & Har. & Apr. \\
\hline $\begin{array}{r}1 \\
2 \\
3 \\
4 \\
5 \\
6 \\
7 \\
8 \\
9 \\
10\end{array}$ & $\begin{array}{l}0 \\
0 \\
0 \\
0 \\
0 \\
0 \\
0 \\
0 \\
0 \\
0\end{array}$ & $\begin{array}{r}53 \\
512 \\
198 \\
70 \\
18 \\
8 \\
3.0 \\
1.7 \\
1.1 \\
.8\end{array}$ & $\begin{array}{l}1.2 \\
1.1 \\
1.0 \\
.9 \\
.8 \\
.7 \\
.6 \\
.6 \\
0 \\
0\end{array}$ & $\begin{array}{l}11 \\
12 \\
13 \\
14 \\
15 \\
16 \\
17 \\
18 \\
19 \\
20\end{array}$ & $\begin{array}{l}17 \\
0 \\
0 \\
0 \\
0 \\
0 \\
0 \\
0 \\
0 \\
0\end{array}$ & $\begin{array}{l}1.7 \\
26 \\
7 \\
8 \\
7 \\
6 \\
3.0 \\
2.5 \\
2.4 \\
2.3\end{array}$ & $\begin{array}{l}0 \\
0 \\
0 \\
0 \\
0 \\
0 \\
0 \\
0 \\
0 \\
0\end{array}$ & $\begin{array}{l}21 \\
22 \\
23 \\
24 \\
25 \\
26 \\
27 \\
28 \\
29 \\
30 \\
31\end{array}$ & $\begin{array}{l}0 \\
0 \\
0 \\
0 \\
0 \\
0 \\
0 \\
9\end{array}$ & $\begin{array}{l}2.2 \\
2.1 \\
2.0 \\
1.9 \\
1.8 \\
1.8 \\
1.7 \\
1.6 \\
1.5 \\
1.4 \\
1.3\end{array}$ & $\begin{array}{l}0 \\
0 \\
0 \\
0 \\
0 \\
0 \\
0 \\
0 \\
0 \\
.4\end{array}$ \\
\hline \multicolumn{9}{|c|}{ 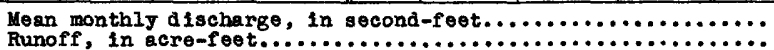 } & $\begin{array}{r}0.96 \\
53\end{array}$ & $\begin{array}{r}30.7 \\
1,890\end{array}$ & 0.24 \\
\hline
\end{tabular}


Gage height, in feet, and discharge, in second-feet, at indicated time, 1938

\begin{tabular}{|c|c|c|c|c|c|c|c|c|c|c|c|c|}
\hline \multirow{2}{*}{ 总 } & Feet & Sec.ft. & Feet & sec.ft. & Feet & Sec.ft. & Feet & Sec.ft. & Feot & Sec.ft. & Feot & Bec.ft. \\
\hline & \multicolumn{2}{|c|}{ February 27} & \multicolumn{2}{|c|}{ February 28} & \multicolumn{2}{|c|}{ March 1} & \multicolumn{2}{|c|}{ March 2} & \multicolumn{2}{|c|}{ March 3} & \multicolumn{2}{|c|}{ Iarch 4} \\
\hline $\begin{array}{r}1 \\
2 \\
3 \\
4 \\
5 \\
6 \\
7 \\
7 \\
8 \\
9 \\
10 \\
11 \\
1 \\
1 \\
1 \\
3 \\
4 \\
4 \\
5 \\
6 \\
7 \\
8 \\
9 \\
10 \\
11 \\
4\end{array}$ & 0.03 & $\begin{array}{l}0 \\
1.4\end{array}$ & 0.06 & $\begin{array}{l}3.0 \\
9 \\
5.8\end{array}$ & $\begin{array}{l}0.29 \\
.28 \\
.23 \\
.21\end{array}$ & $\begin{array}{l}53 \\
49 \\
38 \\
30 \\
27\end{array}$ & $\begin{array}{r}0.48 \\
.82 \\
2.62 \\
2.83 \\
2.45 \\
2.81 \\
2.17 \\
2.44 \\
1.95 \\
1.60 \\
1.30 \\
1.08\end{array}$ & $\begin{array}{r}77 \\
170 \\
794 \\
\\
870 \\
719 \\
859 \\
817 \\
712 \\
529 \\
402 \\
296 \\
225\end{array}$ & $\begin{array}{r}0.97 \\
.93 \\
1.04 \\
1.04 \\
.92 \\
.87\end{array}$ & $\begin{array}{l}212 \\
212 \\
176 \\
161\end{array}$ & & $\cdot$ \\
\hline
\end{tabular}

Pacoima Creek near San Fernando, Calif.

Location. - Water-stage recorder, lat. $34^{\circ} 20^{\prime} 02^{\prime \prime}$, 1ong. $118^{\circ} 23^{\prime} 55^{\prime \prime}$, in SEt NEt $8 \theta c .24$, T. 3 N., R. 15 w., 600 feet upstream from mouth of canjon, $0.2^{\prime}$ mile below Pacolma flood-control dam, and 4 miles northeast of San Fernando.

Drainage area.- 27.9 square miles .

Remarks.- For records of discharge see Pacolma Creek at flood-control reservoir near San Fernando, outflow records.

Pacolma Creek at Mission Acres, Calif.

Location. - Water-stage recorder, lat. $34^{\circ} 13^{\prime} 40^{\prime \prime}$, 1ong. $118^{\circ} 27^{\prime} 30^{\prime \prime}$, in Ex Miss 1on San Fernando grant, at Parthenia street Bridge in Mission Acres, Los Angeles County, 3 m1les northwest of Van Nuys. Altitude, about 820 feet.

Dreinege area.- 50.6 square miles.

Gage-he1ght record. - Water-stage recorder graph Feb. 1 to 3:40 a.m. Mar. 3; occas 1onal staf-gage readings thereafter.

Discharge record. - Stage-discharge relation defined by current-meter measurements beIow 1,270 second-feet; extended logarithmically to peak stege. After $3: 40$ a.m. Mar. 3 discharge estimated from outflow at Pacolma Dam and Beveral current-meter measurements .

Maxime-- 1938: Discharge, 2,270 second-feet 3:40 a.m. Mar. 3 .

i 92977 : Discharge, 477 second-fe日t Feb. 8, 1932.

Remarks.- Records poor. Flow partially regulated by Pacolma Dam. Small diversions near mouth of canjon. Records, except peak discherge, furnished by IOs Angeles County Flood Oontrol District, through H. E. Hedger, chief engineer.

Mean delly discharge, in second-feet, 1938

\begin{tabular}{|c|c|c|c|c|c|c|c|c|c|c|c|}
\hline Day & $F \in b$. & Mar. & Apr. & Day & Feb. & Mar. & Apr. & Day & Feb. & Mar. & Apr. \\
\hline $\begin{array}{r}1 \\
2 \\
3 \\
4 \\
5 \\
6 \\
7 \\
8 \\
9 \\
10\end{array}$ & $\begin{array}{l}6.5 \\
0 \\
5.5 \\
.44 \\
0 \\
0 \\
0 \\
0 \\
1.3 \\
0\end{array}$ & $\begin{array}{r}34 \\
470\end{array}$ & & $\begin{array}{l}11 \\
12 \\
13 \\
14 \\
15 \\
16 \\
17 \\
18 \\
19 \\
20\end{array}$ & $\begin{array}{l}12 \\
0 \\
0 \\
3.7 \\
1.5 \\
0 \\
0 \\
0 \\
0 \\
0\end{array}$ & & & $\begin{array}{l}21 \\
22 \\
23 \\
24 \\
25 \\
26 \\
27 \\
28 \\
29 \\
30 \\
31\end{array}$ & $\begin{array}{c}0 \\
0 \\
0 \\
0 \\
0 \\
0 \\
26^{.6}\end{array}$ & , & \\
\hline \multicolumn{9}{|c|}{ 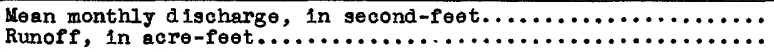 } & $\begin{array}{r}2.05 \\
114\end{array}$ & & \\
\hline
\end{tabular}


Oage he1ght, in foet, and disoharge, in oocond-reet, at indicated tine, 1936

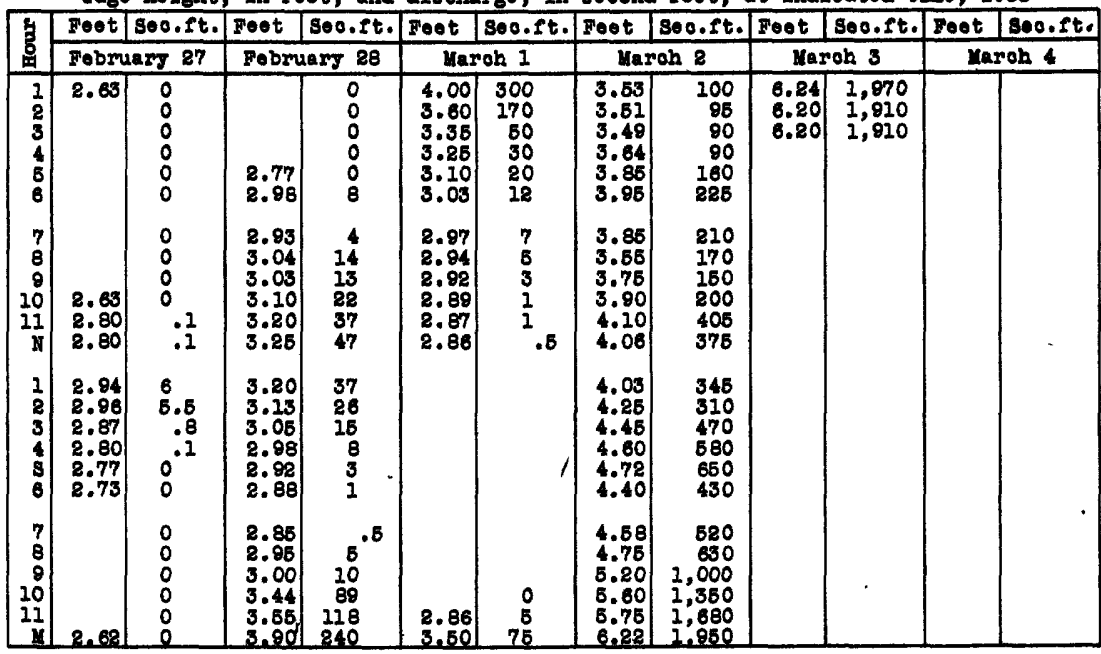

Supplemental record. - Mar. 3, 3840 a.m., 2,270 000.-ft.

Tujunga Oreak near Colby Ranoh, Oal1s.

Iopat10n. - Water-stage recorder, lat. $34^{\circ} 18^{\prime} 10^{n}$, 10ng. $118^{\circ} 09^{\prime 35^{n}}$, 28 Ieet upatream Irom oroesing of Hdison Road, 300 feet downetream from Iuoas oreek, 3 t miles west of Oolby Ranob, Los Angeles County, and 4 milea upotream from thufunga flood-0ontrol dam No. I. Altitude, 2,450 reet.

Dralngge areg.- Area, 66.9 equare m1les. Average altitude, 4,510 feet. unximun elt1-

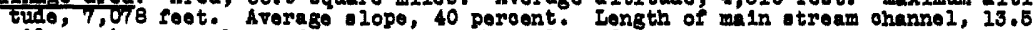
m1les. Average lope of main atream ohannel, 6.6 peroent.

Qage-helght reoord. - Water-atage rooorder graph oxoopt for Fob. 14-21 and Xar. 1-28.

D1eoharge reoord.- Stage-diocharge relation falrig woll dorinod. D1ooharge Fob. 14-z1 and Lar. 1-28 determined by ocmparioon with adjacont otations.

Pexima. - 18388 D1eoharge not determinod; ooourred Mar. 2. 1230-37, D1soharge, 3,910 cooond-Foot Fob. 8, 1232.

Remarke, - No regulation or diversion. Record furniohed by Los Angeles County Flood control Dietriot, through H. B. Hedger, ohler ong inoor.

Yean da11g disobarge, in seoond-feet, 2938

\begin{tabular}{|c|c|c|c|c|c|c|c|c|c|c|c|}
\hline Dey & Fob. & $\operatorname{Max}$. & Apr. & Dey & Fob. & Mas. & Apr. & Das & Dob. & Mar. & Apr. \\
\hline $\begin{array}{r}1 \\
8 \\
3 \\
4 \\
5 \\
6 \\
7 \\
8 \\
9 \\
10\end{array}$ & $\begin{array}{r}48 \\
18 \\
87 \\
110 \\
44 \\
28 \\
28 \\
20 \\
44 \\
74\end{array}$ & $\begin{array}{r}870 \\
8,200 \\
1,600 \\
690 \\
450 \\
330 \\
255 \\
205 \\
175 \\
150\end{array}$ & $\begin{array}{l}86 \\
82 \\
80 \\
77 \\
72 \\
68 \\
68 \\
65 \\
65 \\
65\end{array}$ & $\begin{array}{l}11 \\
18 \\
15 \\
14 \\
15 \\
16 \\
17 \\
18 \\
19 \\
20\end{array}$ & $\begin{array}{l}517 \\
208 \\
89 \\
67 \\
59 \\
50 \\
43 \\
37 \\
32 \\
30\end{array}$ & $\begin{array}{l}180 \\
580 \\
390 \\
285 \\
240 \\
210 \\
195 \\
180 \\
185 \\
150\end{array}$ & $\begin{array}{l}63 \\
65 \\
65 \\
60 \\
56 \\
52 \\
51 \\
49 \\
47 \\
46\end{array}$ & $\begin{array}{l}21 \\
22 \\
23 \\
24 \\
25 \\
28 \\
27 \\
28 \\
29 \\
30 \\
31\end{array}$ & $\begin{array}{r}26 \\
24 \\
28 \\
20 \\
20 \\
19 \\
87 \\
480\end{array}$ & $\begin{array}{r}140 \\
130 \\
125 \\
180 \\
118 \\
110 \\
108 \\
108 \\
98 \\
88 \\
84\end{array}$ & $\begin{array}{l}44 \\
48 \\
48 \\
43 \\
44 \\
48 \\
41 \\
40 \\
42 \\
41\end{array}$ \\
\hline \multicolumn{9}{|c|}{ 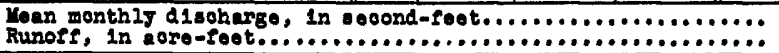 } & $\begin{array}{r}80.1 \\
4,480\end{array}$ & 33.060 & $\begin{array}{r}56.8 \\
3.370\end{array}$ \\
\hline
\end{tabular}


Tugunga Creok near sunland, Calif.

Location. - Water-stage recorder, lat, $34^{\circ} 17^{\prime} 55^{\prime \prime}$, long. $118^{\circ} 16^{\prime} 10^{\prime \prime}$, near center of eec. $32, T .3 \mathrm{~K} ., R_{.} 13 \mathrm{~W}$. (unsurvejed), a quarter of a mile downstream from a partly constructed and abandoned dam, 2 miles upstream from mouth of oanyon, and 4 miles northeast of Bunland. Altitude, 1,600 feet.

Drainage area.- Area, 106 square miles. Average altitude, 4,220 feet. Maximim altitude, 7,078 feet. Average slope, 40 percent. Length of main stream channel, 21.5 m1les. Average slope of main stream channel, 3.9 percent.

Qage-helght record.- Water-stage recorder graph.

D1scherge reoord. - Stage-discherge relation fairly well defined below 1,7c0 second-feet. Discherge Feb. 15, 16, Mar. 2, 3, 8-12, 14-30, and Apr. 8-20, obtained by comparison with record at flood-control dam.

Maxima.- 1938: Discharge 50,000 second-feet Mar. 2.

ig16-37: Discharge, 8,500 second-feet Dec. i9, 1921.

Remarks.- Flow pertly regulated by flood-control reservoir 5.4 miles upstream. Record Iurnished by Los Angeles County Flood Control District, through H. B. Hedger, chler engineer.

Moan dally discharge, in second-feet, 1938

\begin{tabular}{|r|r|r|r||r|c|c|c|c||c|c|c|c|}
\hline Day & Feb. & Mar. & Apr. & Day & Feb. & Mar. & Apr. & Day & Feb. & Kar. & Apr. \\
\hline 1 & 38 & 763 & 159 & 11 & 206 & 280 & 105 & 21 & 68 & 300 & 27 \\
2 & 22 & 13,000 & 157 & 12 & 147 & 600 & 105 & 22 & 66 & 260 & 26 \\
3 & 98 & 3,700 & 154 & 13 & 96 & 643 & 105 & 23 & 67 & 230 & 24 \\
4 & 79 & 1,280 & 150 & 14 & 86 & 475 & 105 & 24 & 68 & 240 & 28 \\
5 & 62 & 841 & 168 & 15 & 94 & 440 & 90 & 25 & 64 & 130 & 30 \\
6 & 50 & 910 & 190 & 16 & 48 & 520 & 49 & 26 & 62 & 230 & 24 \\
7 & 46 & 1,220 & 186 & 17 & 62 & 510 & 48 & 27 & 50 & 230 & 22 \\
8 & 42 & 886 & 140 & 18 & 59 & 380 & 38 & 28 & 206 & 200 & 21 \\
9 & 38 & 692 & 105 & 19 & 46 & 340 & 31 & 29 & & 160 & 23 \\
10 & 24 & 445 & 105 & 20 & 69 & 300 & 27 & 30 & & 160 & 40 \\
\hline
\end{tabular}

Tujunga Creek near San Fernando, Cal1f.

Location.- Water-9tage recorder, lat. $34^{\circ} 15^{\prime} 50^{\prime \prime}$, long. $118^{\circ} 22^{\prime} 50^{\prime \prime}$, in Ex Mission San Fernando grant, at stonehurst Avenue Bridge, about 3 miles s outheast of San Fernando, LOS Angeles County. Alti tude, a bout 995 feet.

Drainege area. - 148 square miles.

Gage-height record. - Water-stage recorder graph.

Discharge record.- Stage-discharge relation defined by current-meter measurements below 1,360 second-feet; extended to peak discharge on basis of a slope-area measurement of the peak.

Maxima.- 1938: D1scharge, 51,000 second-feet about 6 p.m. Mar. 2 . 1931-36: Dlecharge, 3,750 eecond-feet (estimated) Jan. 1, 1934.

Remarks.- Records poor. Flow partlally regulated by Tujunga Dam and by Haines Debris Basin. Reoords furnished by Los Angeles County Flood Control District, through H. E. Hedger, chlef engineer, and by Corps of Engineers, U. S. Arny.

Mean daily discharge, in second-reet, 1938

\begin{tabular}{|c|c|c|c|c|c|c|c|c|c|c|c|}
\hline Day & Feb. & Mar. & Apr. & Day & Fob. & Mar. & Apr. & Day & Feb. & Mar. & Apr. \\
\hline $\begin{array}{r}1 \\
2 \\
3 \\
4 \\
5 \\
6 \\
7 \\
8 \\
9 \\
10\end{array}$ & $\begin{array}{r}41 \\
24 \\
129 \\
108 \\
69 \\
49 \\
44 \\
47 \\
47 \\
36\end{array}$ & 1,120 & - & $\begin{array}{l}11 \\
12 \\
13 \\
14 \\
15 \\
16 \\
17 \\
18 \\
19 \\
20\end{array}$ & $\begin{array}{r}234 \\
178 \\
132 \\
110 \\
81 \\
38 \\
61 \\
61 \\
47 \\
61\end{array}$ & & & $\begin{array}{l}21 \\
22 \\
23 \\
24 \\
25 \\
26 \\
27 \\
28 \\
29 \\
30 \\
31\end{array}$ & $\begin{array}{r}61 \\
61 \\
61 \\
66 \\
59 \\
56 \\
56 \\
211\end{array}$ & & \\
\hline $\begin{array}{l}\text { Sean } \\
\text { Runo }\end{array}$ & & & & & & & & & $\begin{array}{r}79.6 \\
4,420\end{array}$ & & \\
\hline
\end{tabular}


Gage height, in feet, and discharge, in second-feet, at indicated time, 1938

\begin{tabular}{|c|c|c|c|c|c|c|c|c|c|c|c|}
\hline$\xi$ & \begin{tabular}{l|l} 
Feet & Sec.ft. \\
\end{tabular} & Feet & Sec.ft. & Feet & Sec.ft. & Feet & Sec.ft. & Feet & Sec.ft. & Feet & Sec.ft. \\
\hline 商 & February 27 & Febr & darg 28 & Ma: & $\operatorname{coh} 1$ & Ma) & $\mathrm{ch} 2$ & & $\operatorname{coh} 3$ & $\mathrm{Ha}$ & $\operatorname{ch~} 4$ \\
\hline $\begin{array}{l}1 \\
2 \\
3 \\
4 \\
5 \\
6\end{array}$ & & $\begin{array}{l}2.95 \\
2.87 \\
2.88 \\
2.84 \\
2.94 \\
2.94\end{array}$ & $\begin{array}{l}27 \\
20 \\
18 \\
19 \\
26 \\
26\end{array}$ & $\begin{array}{l}5.20 \\
5.20 \\
4.40 \\
3.95 \\
3.77 \\
3.62\end{array}$ & $\begin{array}{r}4,000 \\
4,000 \\
2,000 \\
1,350 \\
1,100 \\
960\end{array}$ & $\begin{array}{l}3.68 \\
3.70 \\
3.72 \\
3.78 \\
3.88 \\
3.96\end{array}$ & $\begin{array}{r}980 \\
1,000 \\
1,050 \\
1,120 \\
1,220 \\
1,360\end{array}$ & & & & \\
\hline $\begin{array}{r}7 \\
8 \\
9 \\
10 \\
11 \\
\text { N }\end{array}$ & & $\begin{array}{l}3.07 \\
3.20 \\
3.33 \\
3.38 \\
3.48 \\
3.55\end{array}$ & $\begin{array}{r}39 \\
56 \\
88 \\
100 \\
140 \\
160\end{array}$ & $\begin{array}{l}3.56 \\
3.50 \\
3.44 \\
3.35 \\
3.35 \\
3.38\end{array}$ & $\begin{array}{l}860 \\
820 \\
780 \\
700 \\
700 \\
720\end{array}$ & $\begin{array}{l}3.95 \\
4.25 \\
4.62 \\
5.00 \\
5.50 \\
5.65\end{array}$ & $\begin{array}{l}1,350 \\
1,750 \\
2,600 \\
3,400 \\
4,900 \\
5,600\end{array}$ & & & & \\
\hline $\begin{array}{l}1 \\
2 \\
3 \\
4 \\
5 \\
6\end{array}$ & & $\begin{array}{l}3.70 \\
3.78 \\
3.78 \\
3.72 \\
3.63 \\
3.55\end{array}$ & $\begin{array}{l}260 \\
300 \\
300 \\
265 \\
220 \\
160\end{array}$ & $\begin{array}{l}3.29 \\
3.26 \\
3.26 \\
3.28 \\
3.30 \\
3.31\end{array}$ & $\begin{array}{l}640 \\
600 \\
600 \\
620 \\
660 \\
670\end{array}$ & $\begin{array}{l}5.65 \\
8.20 \\
9.10 \\
9.15 \\
8.50\end{array}$ & $\begin{array}{r}5,600 \\
20,400 \\
27,500 \\
27,700 \\
22,500\end{array}$ & & & & \\
\hline $\begin{array}{r}7 \\
8 \\
9 \\
10 \\
\end{array}$ & & $\begin{array}{l}3.60 \\
3.80 \\
3.82 \\
3.88 \\
4.30 \\
4.47\end{array}$ & $\begin{array}{r}200 \\
310 \\
320 \\
390 \\
1,000 \\
1,500\end{array}$ & $\begin{array}{r}3.31 \\
3.32 \\
3.33 \\
.3 .45 \\
3.68 \\
3.61\end{array}$ & $\begin{array}{l}670 \\
680 \\
690 \\
780 \\
980 \\
920\end{array}$ & & & & & & \\
\hline
\end{tabular}

Little Tujunga Creek near San Fernando, Calif.

Location.- Water-stage recorder, lat. $34^{\circ} 16^{1} 30^{n}$, long. $118^{\circ} 22^{1} 20^{n}$, In Tujunga grant, at Foothill Boulevard Bridge, 4 miles east of 8 an Fernando, Los Angeles County. Alt tude, 1,250 feet.

Dralnage area.- Area, 21.0 square miles. Average altitude, 2,500 feet. Waximum altitude, 5,215 feet. Average slope, 38 percent. Length of main stream channel, 8.0 miles. Average slope of main s tream channel, 9.3 percent.

Gage-he1ght record.- Water-stage recorder graph except Mar. 2 to Apr. 12.

Discharge record. - Stage-discharge relation fairly well defined. Discherge, Mar. 2 to Apr. 12, computed by comparison with adjacent stations; discharge, Apr. 12-30, obtained by interpolation between discharge measurements.

Maxima.- 1938: Discharge, 8,500 second-feet Mar. 2 .

1928-37: Discharge, 1,360 second-feet Jan. 1, 1934.

Remarks.- Records furnished by Los Angeles County Flood Control District, through H. $B$. Hedger, chlef engineer.

Mean daily discharge, in second-feet, 1938

\begin{tabular}{|r|c|r|r||r|r|r|r||l|l|l|l|}
\hline Day & Feb. & Mar. & Apr. & Day & Feb. & Mar. & Apr. & Day & Feb. & Mar. & Apr. \\
\hline 1 & 7 & 209 & 8.5 & 11 & 71 & 41 & 6.5 & 21 & 1.4 & 34 & 5.5 \\
2 & 1.5 & 1,300 & 8 & 12 & 28 & 95 & 6.5 & 22 & 1.3 & 29 & 5.5 \\
3 & 36 & 750 & 7.5 & 13 & 15 & 139 & 6.5 & 23 & 1.1 & 25 & 5.5 \\
4 & 22 & 275 & 7 & 14 & 9 & 108 & 6 & 24 & 1.0 & 22 & 5.5 \\
5 & 5.5 & 123 & 6.5 & 15 & 7.5 & 84 & 6 & 25 & 1.0 & 19 & 5.5 \\
6 & 3.2 & 93 & 6.5 & 16 & 6 & 71 & 6 & 26 & .8 & 16 & 5 \\
7 & 1.3 & 76 & 6.5 & 17 & 4.1 & 61 & 6 & 27 & 4.4 & 14 & 5 \\
8 & 1.1 & 62 & 6.5 & 18 & 4.6 & 52 & 6 & 28 & 64 & 12 & 5 \\
9 & 3.5 & 51 & 6.5 & 19 & 3.8 & 45 & 6 & 29 & & 11 & 4.6 \\
10 & 4.3 & 45 & 6.5 & 20 & 1.7 & 39 & 5.5 & 30 & & 9.5 & 4.2 \\
\end{tabular}


Halnes Creek near Tujunga, Cal1f.

Locat1on.- Water-stage recorder and concrete control, lat. $34^{\circ} 15^{\prime} 50^{\prime \prime}$, long. $118^{\circ} 16^{\prime} 15^{\prime \prime}$, In $1 \frac{1}{2} 1 w^{\frac{1}{4}}$ sec. 17, T. 2 N., R. $13 W_{.}, 800$ feet above mouth of canyon, and $1 \frac{1}{8}$ m1les northeast of Tujunga. Altitude, about 2,200 feet.

Drainage area.- Area, 1.2 squaro miles. Average alt1tude, 3,322 feet. Maximum altitude, 5,000 foet. Average slope, 71 percent. Length of main stream channel, 2.0 miles. Average slope of ma in stream channel, 26 percent.

Gage-he1ght record. - Water-stage recorder graph except Mar. 5, 6, and Apr. 3-7.

D1scharge record. - Stage-discharge relation defined by current-meter measurements below 3.2 second-feet; extended to peak a tage by weir formula. Discharge Mar. 5, 6 , Apr. 3-7, estimated.

Maxime.- 1938: D1scharge, 265 second-feet 1:30 p.m. Mar. 2 (gage helght, 4.60 foet). 1917-34, 1935-37: Stage, 11.0 feet Jan. 1, 1934 (discharge not determined).

Remarks.- Records falr. No diversion or regulation during flood period.

Mean da1ly discharge, in second-feet, 1938 *

\begin{tabular}{|c|c|c|c|c|c|c|c||c|c|c|c|}
\hline Day & Feb. & Mar. & Apr. & Day & Feb. & Mar. & Apr. & Day & Feb. & Mar. & Apr. \\
\hline 1 & 0.3 & 8 & 0.9 & 11 & 4.0 & 1.6 & 0.8 & 21 & 0 & 1.6 & 0.6 \\
2 & 0 & 70 & 1.0 & 12 & .8 & 2.8 & 1.0 & 22 & 0 & 1.5 & .6 \\
3 & 1.4 & 30 & 1.0 & 13 & .2 & 3.3 & 1.0 & 23 & 0 & 1.4 & .6 \\
4 & .1 & 12 & .9 & 14 & 0 & 2.4 & .8 & 24 & 0 & 1.2 & .6 \\
5 & 0 & 6.5 & .9 & 15 & 0 & 2.2 & .6 & 25 & 0 & 1.2 & .7 \\
6 & 0 & 4.3 & .9 & 16 & 0 & 2.0 & .6 & 26 & 0 & 1.1 & .7 \\
7 & 0 & 3.4 & .9 & 17 & 0 & 1.9 & .6 & 27 & .1 & 1.0 & .7 \\
8 & 0 & 3.1 & .8 & 18 & 0 & 1.8 & .6 & 28 & 5.5 & 1.0 & .7 \\
9 & .3 & 2.1 & .8 & 19 & 0 & 1.7 & .6 & 29 & & 1.0 & .7 \\
10 & .4 & 1.6 & .8 & 20 & 0 & 1.6 & .6 & 30 & & 1.0 & .7 \\
\hline
\end{tabular}

Gage helght, in feet, and discharge, in second-feet, at indicated time, 1938

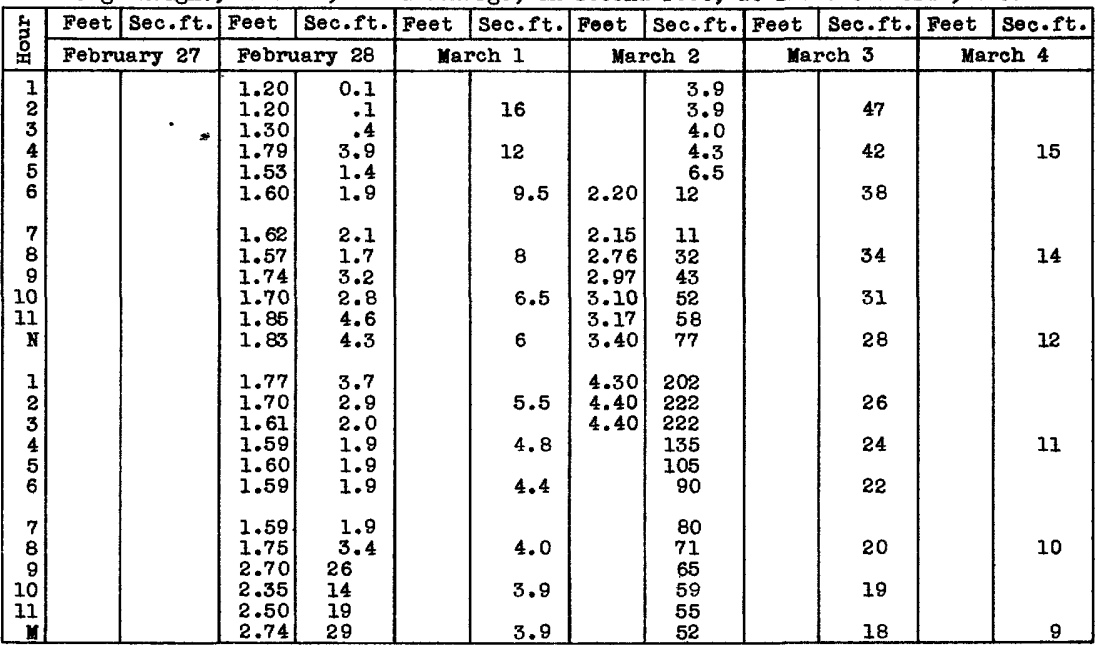

Supplemental records.- Fob. 28, 11:30 p.m., 3.18 feet, 54 sec.-ft.; Mar. 2, 11:15 a.m., 3.85 feet, 130 sec.-ft.; $1: 30$ p.m., 4.60 feet, 265 sec. $-f t$. 
Looat1on.- Water-stage recorder, lat. $34^{\circ} 11^{\prime} 05^{\prime \prime}$, long. $118^{\circ} 13^{\prime} 35^{\prime \prime}$, in San Rafael grant, at Del Valle Avenue, Qlendale, Los Angeles County. Alt1tude, ábout 915 feet.

Drainage area. - 19.1 square mlles.

Qage-helght reoord. - Water-stage recorder graph.

D1soharge record. - Stage-discharge relation determined by computat1ons of discharge by Laming equation; the ohannel is conorete lined and of uniform oros seotion.

Maxima. - 1938 : Discharge, 3,500 second-feet 2812 p.m. Mar. 2 (gege helght, 2.10 feet). 1935-37: Discharge, 1,020 second-feet Jan. 5, 1835.

Remarks.-Records good. Basic data fumlshed by Los Angeles County Plood Control Distriot, through.H. $\mathrm{E}$. Hedger, ohlef engineer, and by Corps of Engineers, J. 8 . Army.

Mean dally disoharge, in second-feet, 1938

\begin{tabular}{|cc|}
\hline Pebruary 27 & 0.8 \\
February 28 & 50 \\
March 1 & 40 \\
Maroh 2 & 662 \\
\hline Runoff, in acre-feet & 1,490 \\
\hline
\end{tabular}

Gage helght, in feet, and discharge, in seoond-feet, at indioated time, 1938

\begin{tabular}{|c|c|c|c|c|c|c|c|c|c|c|c|c|}
\hline \multirow{2}{*}{ 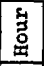 } & Feet & sec.ft. & Feet & sec.ft. & Feet & sec.ft. & Feot & seo.ft. & Feet & Seo.ft. & Peot & seo.ft. \\
\hline & \multicolumn{2}{|c|}{ February 27} & \multicolumn{2}{|c|}{ Fobruary 28} & \multicolumn{2}{|c|}{ Maroh 1} & \multicolumn{2}{|c|}{ March 2} & \multicolumn{2}{|c|}{ Maroh 3} & \multicolumn{2}{|c|}{ Maroh 4} \\
\hline $\begin{array}{l}1 \\
2 \\
3 \\
4 \\
5 \\
6\end{array}$ & $\begin{array}{l}0 \\
0 \\
0 \\
0 \\
.04 \\
.04\end{array}$ & $\begin{array}{l}0 \\
0 \\
0 \\
0 \\
.1 \\
.1\end{array}$ & $\begin{array}{r}0.08 \\
.09 \\
.07 \\
.22 \\
.17 \\
.21\end{array}$ & $\begin{array}{r}0.5 \\
.7 \\
.4 \\
8 \\
4.5 \\
7.5\end{array}$ & $\begin{array}{r}0.73 \\
.62 \\
.53 \\
.47 \\
.41 \\
.38\end{array}$ & $\begin{array}{r}207 \\
134 \\
90 \\
65 \\
45 \\
37\end{array}$ & $\begin{array}{l}0.36 \\
.28 \\
.31 \\
.58 \\
.67 \\
.68\end{array}$ & $\begin{array}{r}32 \\
16 \\
21 \\
113 \\
165 \\
171\end{array}$ & & , & & \\
\hline $\begin{array}{r}7 \\
8 \\
9 \\
10 \\
11 \\
1\end{array}$ & $\begin{array}{l}.06 \\
.12 \\
.15 \\
.16 \\
.16 \\
.16\end{array}$ & $\begin{array}{l}.3 \\
1.7 \\
3.0 \\
3.6 \\
3.6 \\
3.6\end{array}$ & $\begin{array}{l}.31 \\
.28 \\
.32 \\
.38 \\
.38 \\
.36\end{array}$ & $\begin{array}{l}21 \\
16 \\
24 \\
37 \\
37 \\
32\end{array}$ & $\begin{array}{l}.33 \\
.30 \\
.27 \\
.25 \\
.22 \\
.19\end{array}$ & $\begin{array}{l}25 \\
19 \\
15 \\
12 \\
8 \\
5.5\end{array}$ & $\begin{array}{r}.78 \\
.84 \\
1.22 \\
1.53 \\
1.07 \\
1.32\end{array}$ & $\begin{array}{r}247 \\
301 \\
895 \\
1,590 \\
590 \\
1,100\end{array}$ & & . & & \\
\hline $\begin{array}{l}1 \\
2 \\
3 \\
4 \\
5 \\
6\end{array}$ & $\begin{array}{l}.13 \\
.06 \\
.06 \\
.09 \\
.05 \\
.06\end{array}$ & $\begin{array}{r}2.0 \\
.3 \\
.3 \\
.7 \\
.2 \\
.3\end{array}$ & $\begin{array}{l}.36 \\
.32 \\
.28 \\
.22 \\
.20 \\
.18\end{array}$ & $\begin{array}{l}32 \\
24 \\
16 \\
8 \\
6.5 \\
5\end{array}$ & $\begin{array}{l}.18 \\
.17 \\
.16 \\
.15 \\
.15 \\
.15\end{array}$ & $\begin{array}{l}5 \\
4.5 \\
3.6 \\
3.0 \\
3.0 \\
3.0\end{array}$ & $\begin{array}{r}1.52 \\
1.90 \\
1.88 \\
1.40 \\
1.18 \\
.97\end{array}$ & $\begin{array}{r}1,570 \\
2,720 \\
2,660 \\
1,280 \\
810 \\
446\end{array}$ & & & & \\
\hline $\begin{array}{r}7 \\
8 \\
9 \\
10 \\
11 \\
4\end{array}$ & $\begin{array}{l}.04 \\
.03 \\
.03 \\
.05 \\
.03 \\
.03\end{array}$ & $\begin{array}{l}0.1 \\
0 \\
0 \\
0\end{array}$ & $\begin{array}{r}.18 \\
.22 \\
.46 \\
.87 \\
.78 \\
1.01\end{array}$ & $\begin{array}{r}5 \\
8 \\
62 \\
330 \\
247 \\
500\end{array}$ & $\begin{array}{l}.15 \\
.16 \\
.16 \\
.16 \\
.18 \\
.27\end{array}$ & $\begin{array}{l}3.0 \\
3.6 \\
3.6 \\
3.6 \\
5 \\
15\end{array}$ & $\begin{array}{l}.85 \\
.82 \\
.73 \\
.68\end{array}$ & $\begin{array}{l}310 \\
280 \\
205 \\
170 \\
140 \\
120\end{array}$ & & & & \\
\hline
\end{tabular}

Supplemental recora.- Mar. 2, $2: 12$ p.m., $2.10 \mathrm{ft} ., 3,500$ sec. $-\mathrm{ft}$. 
Verdugo Creek at Glendale, Cal1f.

Iocat1on.- Water-8tage recorder, 1at. $34^{\circ} 09^{\prime} 25^{\prime \prime}$, Iong. $118^{\circ} 16^{\prime} 25^{\prime \prime}$, in San Rafael grant, at betelie Ave., Glendale, Los Angeles County, 0.4 mile above junction with Ios Angeles River. 'Altitude, about 465 feet.

Drainage area.- 22.4 Bquare m110日.

Gage-helght record.- Water-8tage recorder graph except 4 p.m. Mar. 2 to Har. 9. Staff ge'ge readingr Ler. 3 and 8 .

Discharge record.- Stage-discharge relation defined by current-meter measurements Feb. $1-26$ and Har. 14 to Apr. 30 . Diecharge Feb. 27 to Mar. 2 and Mar. 8-13 computed by Manning 's formula, laing coefflcients based on current-meter measurements of 110 second-feet and less. Discharge estimated Mar, 3-7.

Haxima:- 1938: Discharge, 4,500 second-feot $2825 \mathrm{p}, \mathrm{m}$. Mar. 2 (gage he1ght, 2.9 foet 1835-37: Diecharge, 1,100 second-feet Mar. 30, 1936.

Remarks.- Records good except those estimated, whlch are poor. Channel 18 concrete Iined and of uniform $8 \theta 0$ tion and 8 lope. Basic data for Feb. 27 to Mar. 13 and ont1re record for Fob. $1-26$ and Mar. 14 to Apr. 30 furnished by Los Angeles County Flood Control District, through $\mathrm{H}$. F. Hedger, chier engineer.

Mean daily discharge, in second-foet, 1938

\begin{tabular}{|c|c|c|c|c|c|c|c|c|c|c|c|}
\hline Day & Fob. & Mar. & Apr. & Day & Fob. & Mar. & Apr. & Day & Feb. & Mar. & Apr. \\
\hline $\begin{array}{r}1 \\
2 \\
3 \\
4 \\
5 \\
6 \\
7 \\
8 \\
9 \\
10\end{array}$ & $\begin{array}{c}33 \\
3.5 \\
18 \\
31 \\
8.5 \\
3.8 \\
2.5 \\
1.6 \\
23 \\
6.5\end{array}$ & \begin{tabular}{|c|}
121 \\
1,250 \\
95 \\
43 \\
25 \\
18 \\
14 \\
16 \\
8 \\
9.5
\end{tabular} & $\begin{array}{l}0.4 \\
.4 \\
.4 \\
.4 \\
.4 \\
.4 \\
.4 \\
.4 \\
.4 \\
.4\end{array}$ & $\begin{array}{l}11 \\
1.2 \\
13 \\
14 \\
1.5 \\
16 \\
17 \\
18 \\
10 \\
20\end{array}$ & $\begin{array}{r}59 \\
13 \\
3.8 \\
1.5 \\
.5 \\
1.2 \\
.5 \\
.5 \\
.6 \\
.4\end{array}$ & $\begin{array}{l}17 \\
38 \\
27 \\
11 \\
9.5 \\
9.5 \\
13 \\
8.5 \\
6.5 \\
4.5\end{array}$ & $\begin{array}{r}0.4 \\
.4 \\
.5 \\
1.5 \\
.8 \\
.8 \\
.6 \\
.4 \\
.4 \\
.5\end{array}$ & $\begin{array}{l}21 \\
22 \\
23 \\
24 \\
25 \\
26 \\
27 \\
28 \\
29 \\
30 \\
31\end{array}$ & $\begin{array}{r}0.4 \\
.4 \\
.3 \\
.3 \\
.2 \\
1.3 \\
140\end{array}$ & $\begin{array}{l}2.0 \\
1.2 \\
1.2 \\
.6 \\
.5 \\
.6 \\
.6 \\
.8 \\
.6 \\
.6 \\
.4\end{array}$ & $\begin{array}{r}0.8 \\
1.2 \\
1.2 \\
.8 \\
.8 \\
1.2 \\
.8 \\
.6 \\
.5 \\
.8\end{array}$ \\
\hline \multicolumn{9}{|c|}{ 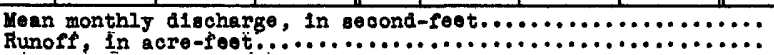 } & $\begin{array}{r}13.1 \\
727\end{array}$ & $\begin{array}{r}56.6 \\
3.480\end{array}$ & 0.65 \\
\hline
\end{tabular}

Gage height, in feet, and discharge, in eecond-feet, at indicated time, 1938

\begin{tabular}{|c|c|c|c|c|c|c|c|c|c|c|c|c|}
\hline \multirow{2}{*}{$\begin{array}{l}0 \\
8 \\
5\end{array}$} & Feet & sec.ft. & Feet & sec.et. & Fe日t & seo.ft. & Peet & sec.ft. & Feet & sec.ft. & Feot & Seo.ft. \\
\hline & \multicolumn{2}{|c|}{ Pebruary 27} & \multicolumn{2}{|c|}{ February 28} & \multicolumn{2}{|c|}{ Maroh 1} & \multicolumn{2}{|c|}{ March 2} & \multicolumn{2}{|c|}{ March 3} & \multicolumn{2}{|c|}{ March 4} \\
\hline $\begin{array}{l}1 \\
2 \\
3 \\
4 \\
5 \\
0\end{array}$ & $\begin{array}{l}0.06 \\
.06 \\
.15 \\
.20 \\
.19 \\
.15\end{array}$ & $\begin{array}{l}0.2 \\
.2 \\
2.3 \\
5 \\
4.2 \\
2.3\end{array}$ & $\begin{array}{l}0.18 \\
.33 \\
.32 \\
.40 \\
.39 \\
.40\end{array}$ & $\begin{array}{l}3.7 \\
19 \\
18 \\
32 \\
30 \\
32\end{array}$ & $\begin{array}{r}1.19 \\
.97 \\
.85 \\
.75 \\
.65 \\
.58\end{array}$ & $\begin{array}{r}650 \\
385 \\
243 \\
171 \\
117 \\
86\end{array}$ & $\begin{array}{r}0.53 \\
.52 \\
.54 \\
.87 \\
1.06 \\
1.12\end{array}$ & $\begin{array}{r}68 \\
64 \\
71 \\
258 \\
480 \\
515\end{array}$ & & & & \\
\hline $\begin{array}{r}7 \\
8 \\
9 \\
10 \\
11 \\
\mathbf{N}\end{array}$ & $\begin{array}{l}.15 \\
.20 \\
.35 \\
.40 \\
.46 \\
.44\end{array}$ & $\begin{array}{l}2.3 \\
5 \\
23 \\
32 \\
46 \\
41\end{array}$ & $\begin{array}{l}.71 \\
.58 \\
.65 \\
.61 \\
.62 \\
.61\end{array}$ & $\begin{array}{r}149 \\
86 \\
117 \\
98 \\
103 \\
98\end{array}$ & $\begin{array}{l}.52 \\
.49 \\
.47 \\
.45 \\
.44 \\
.42\end{array}$ & $\begin{array}{l}64 \\
55 \\
49 \\
44 \\
41 \\
36\end{array}$ & $\begin{array}{l}1.36 \\
1.44 \\
1.76 \\
2.37 \\
1.71 \\
1.82\end{array}$ & $\begin{array}{r}920 \\
1,080 \\
1,740 \\
3,180 \\
1,640 \\
1,860\end{array}$ & & & & \\
\hline $\begin{array}{l}1 \\
2 \\
3 \\
4 \\
5 \\
6\end{array}$ & $\begin{array}{l}.38 \\
.30 \\
.30 \\
.30 \\
.31 \\
.30\end{array}$ & $\begin{array}{l}28 \\
15 \\
15 \\
15 \\
16 \\
15\end{array}$ & $\begin{array}{l}.57 \\
.53 \\
.47 \\
.39 \\
.35 \\
.34\end{array}$ & $\begin{array}{l}82 \\
68 \\
49 \\
30 \\
23 \\
21\end{array}$ & $\begin{array}{l}.41 \\
.41 \\
.41 \\
.41 \\
.40 \\
.40\end{array}$ & $\begin{array}{l}34 \\
34 \\
34 \\
34 \\
32 \\
32\end{array}$ & $\begin{array}{l}2.14 \\
2.50 \\
2.51 \\
2.05\end{array}$ & $\begin{array}{l}2,600 \\
3,500 \\
3,520 \\
2,410\end{array}$ & & & & \\
\hline $\begin{array}{r}7 \\
8 \\
9 \\
10 \\
11 \\
4\end{array}$ & $\begin{array}{l}.29 \\
.20 \\
.17 \\
.16 \\
.16 \\
.18\end{array}$ & $\begin{array}{l}14 \\
5 \\
3 \\
2.5 \\
2.5 \\
3.7\end{array}$ & $\begin{array}{r}.33 \\
.35 \\
.65 \\
1.35 \\
1.19 \\
1.48\end{array}$ & $\begin{array}{r}19 \\
23 \\
117 \\
900 \\
650 \\
1,160\end{array}$ & $\begin{array}{l}.40 \\
.40 \\
.40 \\
.40 \\
.41 \\
.52\end{array}$ & $\begin{array}{l}32 \\
32 \\
32 \\
32 \\
34 \\
64\end{array}$ & & & & & & \\
\hline
\end{tabular}

Supplemental record.- Mar. 2, 2.25 p.m., 2.9 ft., 4,500 sec.-ft. 
Arroyo Seco near Pasadena, Calif.

Iocation.- Water-stage recorder and concrete control, 1at. $34^{\circ} 13^{\prime} 20^{\prime \prime}, 10 \mathrm{~g} .118^{\circ} 10^{\prime}$ $40^{\prime \prime}$, near north line of sec. 3I, T. 2 N., R. 12 W., lis miles above mouth of Millard Canyon, and $5 \frac{1}{k}$ miles northwest of Pasadena. Altitude, about 1,400 feet.

Drainage area.- Area, 16.4 square miles. Average altitude, 3,692 feet. Maximum alt1tude, 6,152 feet. Average slope, 46 percent. Length of main stream channel, 6.6 miles. Average slope of main stream channel, 14 percent.

Gage-height record.- Water-stage recorder graph except I0 a.m. Mar. 2 to Mar. 28.

Discharge record. - Stage-discharge relation falrly well defined by current-meter measurements belo 300 second-feet; peak discharge obtained by slope-area method.

Discharge for the maximum 24 hours obtained from rainfall-runoff studies, and for

the remainder of the period Mar. $2-28$ by comparison with the inflow into Devils

Gate flood-control reservolr, 1 miles downstream.

Maxima.- 1938: Discharge, 8,620 second-feet about 4 p.m. Mar. 2 .

1910-37: Discharge, about 5,630 second-feet Feb. 20, 1914.

Remarks.- Records falr. No diversions or regulation above gage.

Mean daily discharge, in second-feet, 1938

\begin{tabular}{|c|c|c|c|c|c|c|c|c|c|c|c|}
\hline Day & Feb. & Mar. & Apr. & Day & Feb. & Mar. & Apr. & Day & Feb. & Mar. & Apr. \\
\hline $\begin{array}{r}1 \\
2 \\
3 \\
4 \\
5 \\
6 \\
7 \\
8 \\
9 \\
10\end{array}$ & $\begin{array}{c}21 \\
8.5 \\
164 \\
118 \\
49 \\
28 \\
19 \\
15 \\
37 \\
42\end{array}$ & $\begin{array}{r}565 \\
2,700 \\
808 \\
261 \\
208 \\
178 \\
158 \\
143 \\
125 \\
112\end{array}$ & $\begin{array}{l}50 \\
46 \\
42 \\
40 \\
38 \\
36 \\
35 \\
32 \\
31 \\
30\end{array}$ & $\begin{array}{l}11 \\
12 \\
13 \\
14 \\
15 \\
16 \\
17 \\
18 \\
19 \\
20\end{array}$ & $\begin{array}{l}232 \\
81 \\
50 \\
38 \\
34 \\
21 \\
17 \\
16 \\
16 \\
14\end{array}$ & $\begin{array}{r}108 \\
158 \\
152 \\
147 \\
135 \\
125 \\
117 \\
109 \\
102 \\
94\end{array}$ & $\begin{array}{l}30 \\
29 \\
26 \\
26 \\
25 \\
25 \\
25 \\
25 \\
25 \\
24\end{array}$ & $\begin{array}{l}21 \\
22 \\
23 \\
24 \\
25 \\
26 \\
27 \\
28 \\
29 \\
30 \\
31\end{array}$ & $\begin{array}{c}12 \\
11 \\
10 \\
10 \\
9.5 \\
9.5 \\
20 \\
351\end{array}$ & $\begin{array}{l}87 \\
80 \\
73 \\
72 \\
71 \\
70 \\
69 \\
68 \\
64 \\
58 \\
54 \\
\end{array}$ & $\begin{array}{l}24 \\
24 \\
24 \\
24 \\
24 \\
23 \\
23 \\
23 \\
25 \\
25\end{array}$ \\
\hline \multicolumn{9}{|c|}{ 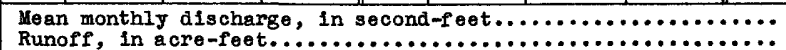 } & $\begin{array}{r}51.9 \\
2,880\end{array}$ & $\begin{array}{r}235 \\
14,420\end{array}$ & $\begin{array}{r}29.3 \\
1,740\end{array}$ \\
\hline
\end{tabular}

Discharge, in second-feet, at indicated time, 1938

\begin{tabular}{|c|c|c|c|c|}
\hline Hour & Feb. 27 & Feb. 28 & Mar. 1 & Har. 2 \\
\hline $\begin{array}{l}1 \\
2 \\
3 \\
4 \\
5 \\
6\end{array}$ & $\begin{array}{r}9 \\
9 \\
9 \\
9 \\
10 \\
10\end{array}$ & $\begin{array}{l}30 \\
30 \\
30 \\
30 \\
62 \\
74\end{array}$ & $\begin{array}{r}2,000 \\
1,400 \\
1,080 \\
940 \\
820 \\
740\end{array}$ & $\begin{array}{l}225 \\
220 \\
200 \\
280 \\
390 \\
510\end{array}$ \\
\hline $\begin{array}{r}7 \\
8 \\
9 \\
10 \\
11 \\
N\end{array}$ & $\begin{array}{l}10 \\
11 \\
14 \\
19 \\
24 \\
26\end{array}$ & $\begin{array}{l}155 \\
160 \\
200 \\
255 \\
340 \\
450\end{array}$ & $\begin{array}{l}680 \\
620 \\
554 \\
490 \\
440 \\
390\end{array}$ & $\begin{array}{r}770 \\
940 \\
1,050 \\
1,500\end{array}$ \\
\hline $\begin{array}{l}1 \\
2 \\
3 \\
4 \\
5 \\
6\end{array}$ & $\begin{array}{l}27 \\
30 \\
32 \\
32 \\
33 \\
37\end{array}$ & $\begin{array}{l}480 \\
450 \\
410 \\
390 \\
340 \\
320\end{array}$ & $\begin{array}{l}335 \\
300 \\
255 \\
230 \\
215 \\
195\end{array}$ & \\
\hline $\begin{array}{r}7 \\
8 \\
9 \\
10 \\
11 \\
11\end{array}$ & $\begin{array}{l}36 \\
35 \\
33 \\
32 \\
31 \\
30\end{array}$ & $\begin{array}{r}240 \\
200 \\
250 \\
600 \\
1,230 \\
1,850\end{array}$ & $\begin{array}{l}178 \\
170 \\
165 \\
162 \\
165 \\
210\end{array}$ & . \\
\hline
\end{tabular}


Santa An1ta Creek near Sierra Madre, Calif.

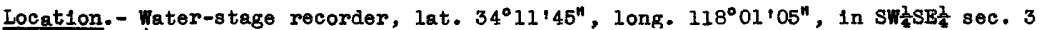
(revisea), T. I W., R. Il W., at head of Hermits Falls, 4 miles northeast of Sierra Madre. Aititude, about 1,400 feet.

Drainage area.- Area, 10.5 square miles. Average altitude, 3,560 feet. Maximum alt1tude, 5,886 feet. Average slope, 47 percent. Length of main stream chamnel, 4.0 miles. Average slope of main atream channel, 20 percent.

Gage-he1ght record.- Water-gtage recorder graph Mar. 18 to Apr. 30. Station was destroyed Var. 2 1ncluding the gage-height record for the perlod Feb. 1 to Mar. 2; station reestablished Mar. 18.

Discharge record.- Discharge Feb. I to Mar. 17 determined by comparison with inflow into flood-control reservoir about half a mile below station. Stage-discharge relation falrly well deflned Mar. I8 to Apr. 30 .

Maxima.- 1938: Discharge, about 5,200 second-feet Mar. 2 .

1916-37: Discharge, a bout 3,300 second-feet Apr. 7, 1926.

Remarks.- Records fair. No regulation or diversion above station.

Mean da1ly discharge, in second-feet, 1938

\begin{tabular}{|c|c|c|c|c|c|c|c|c|c|c|c|}
\hline Day & Feb. & Mar. & Apr. & Day & Feb. & Max. & Apr. & Day & Feb. & Mar. & Apr. \\
\hline \begin{tabular}{r|}
1 \\
2 \\
3 \\
4 \\
5 \\
6 \\
7 \\
8 \\
9 \\
10
\end{tabular} & $\begin{array}{r}17 \\
8 \\
43 \\
63 \\
31 \\
19 \\
14 \\
12 \\
21 \\
24\end{array}$ & $\begin{array}{r}433 \\
1,580 \\
511 \\
230 \\
162 \\
130 \\
107 \\
90 \\
79 \\
72\end{array}$ & $\begin{array}{l}36 \\
34 \\
33 \\
33 \\
33 \\
30 \\
28 \\
26 \\
25 \\
25\end{array}$ & $\begin{array}{l}11 \\
12 \\
13 \\
14 \\
15 \\
16 \\
17 \\
18 \\
19 \\
20\end{array}$ & $\begin{array}{l}60 \\
60 \\
42 \\
28 \\
25 \\
19 \\
17 \\
16 \\
14 \\
12\end{array}$ & $\begin{array}{r}68 \\
138 \\
122 \\
105 \\
94 \\
88 \\
84 \\
82 \\
79 \\
73\end{array}$ & $\begin{array}{l}25 \\
27 \\
27 \\
25 \\
24 \\
23 \\
22 \\
21 \\
20 \\
22\end{array}$ & $\begin{array}{l}21 \\
22 \\
23 \\
24 \\
25 \\
26 \\
27 \\
28 \\
29 \\
30 \\
31\end{array}$ & $\begin{array}{c}10 \\
9 \\
8.5 \\
8 \\
7.5 \\
7 \\
17 \\
334\end{array}$ & $\begin{array}{l}67 \\
62 \\
57 \\
54 \\
52 \\
48 \\
45 \\
44 \\
42 \\
40 \\
37 \\
\end{array}$ & $\begin{array}{l}22 \\
21 \\
20 \\
24 \\
22 \\
20 \\
19 \\
19 \\
20 \\
22\end{array}$ \\
\hline $\begin{array}{l}\text { Moan } \\
\text { Runo }\end{array}$ & ath & $1 \mathrm{sch}$ & in & & & & & & $\begin{array}{r}33.4 \\
1,860\end{array}$ & $\begin{array}{r}157 \\
9,670\end{array}$ & $\begin{array}{r}24.9 \\
1,480\end{array}$ \\
\hline
\end{tabular}

Little Santa Anita Creek near Sierra Madre, Calif.

Locat1on:- Water-stage recorder, lat. $34^{\circ} 11^{\prime} 15^{\prime \prime}$, Iong. $118^{\circ} 02^{\prime} 35^{\prime \prime}$, near center of Nw/ sec. 9, T. ㄱ. N., R. II W., 2 miles northeast of Sierre Madre. AItitude, about 2,200 feet.

Drainage area.- Area, 1.9 square miles. Average alt1tude, 3,430 feet. Maximum alt1tude, 5,433 feet. Average slope, 50 percent. Length of main atream channe1, 2.0

miles. Average slope of main stream channel, 31 percent.

Gage-he1ght record. - Water-stage recorder graph except Mar. 1-18.

Discharge record.- Stage-discharge relation fairly well defined below 60 second-feet.

Discharge tar. I-18 obtalned from records of inflow into flood-control reservolr

about 1 mile below station.

Maxima.- 1938: Discharge, 536 second-feet Mar. 2.

i916-37: Stage, 11.75 feet Apr. 7, 1926 (discharge not determined).

Remarks.- Records fair. No regulation or diversion above gage.

Mean da1ly discharge, in second-feet, 1938

\begin{tabular}{|c|c|c|c|c|c|c|c|c|c|c|c|}
\hline Day & Feb. & Mar. & Apr. & Day & Feb. & Mar. & Apr. & Day & Feb. & Mar. & Apr. \\
\hline $\begin{array}{r}1 \\
2 \\
3 \\
4 \\
5 \\
6 \\
7 \\
8 \\
9 \\
10\end{array}$ & $\begin{array}{l}1.7 \\
1.1 \\
5.5 \\
8 \\
3.6 \\
2.6 \\
2.0 \\
1.6 \\
2.2 \\
2.6\end{array}$ & $\begin{array}{r}57 \\
182 \\
100 \\
41 \\
33 \\
26 \\
23 \\
14 \\
16 \\
14\end{array}$ & $\begin{array}{l}10 \\
10 \\
10 \\
10 \\
10 \\
7.5 \\
6 \\
6 \\
6 \\
6\end{array}$ & $\begin{array}{l}11 \\
12 \\
13 \\
14 \\
15 \\
16 \\
17 \\
18 \\
19 \\
20\end{array}$ & $\begin{array}{l}5.5 \\
6.5 \\
7.5 \\
4.0 \\
3.3 \\
3.1 \\
2.7 \\
2.5 \\
2.4 \\
2.0\end{array}$ & $\begin{array}{l}16 \\
32 \\
30 \\
16 \\
25 \\
18 \\
16 \\
14 \\
13 \\
12\end{array}$ & $\begin{array}{l}6 \\
6 \\
6 \\
5.5 \\
4.9 \\
4.7 \\
4.2 \\
3.9 \\
3.6 \\
3.4\end{array}$ & $\begin{array}{l}21 \\
22 \\
23 \\
24 \\
25 \\
26 \\
27 \\
28 \\
29 \\
30 \\
31\end{array}$ & $\begin{array}{r}1.6 \\
1.5 \\
1.4 \\
1.3 \\
1.3 \\
1.2 \\
2.0 \\
42\end{array}$ & $\begin{array}{l}12 \\
12 \\
11 \\
10 \\
10 \\
10 \\
10 \\
10 \\
10 \\
10 \\
10\end{array}$ & $\begin{array}{l}3.3 \\
3.3 \\
3.0 \\
3.0 \\
2.7 \\
2.7 \\
2.6 \\
2.6 \\
2.6 \\
2.6\end{array}$ \\
\hline \multicolumn{9}{|c|}{ 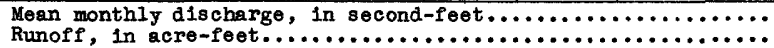 } & $\begin{array}{r}4.38 \\
243\end{array}$ & $\begin{array}{r}26.2 \\
1,610\end{array}$ & $\begin{array}{r}5.27 \\
314\end{array}$ \\
\hline
\end{tabular}


Baton Orook noar Pasadene, Oalif.

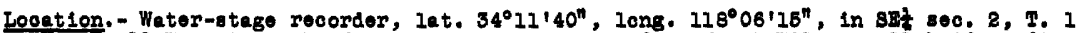
.., K. 12 W., at mouth of canyon, just upstroam from Mount W1lson toll bridge site, and 4 mllos northeast of Pasedena. Altitude, 1,230 foet.

Dreinaze area.- Area, 6.5 square milos. Average eltitude, 3,830 foot, Maximum alt1tude, 6,156 foot. A verage slope, 61 percent. Iongth of main atream ohannel, 4.0 ml108. Avorage slope of main tream ohannel, 23 percent.

aese-he1ght rocord. - Water-atage rocorder graph oxcept Fob. 16 to Kar. $29 ;$ staff gago road twioo dali, Mar. 21-29. Station destrojed Mar. 2 with logs of gage-holght rocord for Fob. 16 to Kar. 2; tation roostablishod Mar. 21.

Discharge record.- Stage-discharge reletion fairly well defined below 100 g00ond-foet. Disohargo yob. 16 to Kar. 29 obtained by comparis on with the inflow into floodcontrol reservolr e bout 2 miles down troam.

Maxima.- 1938: Discharge, 2,400 socond-100t 5 p.m. Mar. 2 .

1818:378 Disoharge, ebout 1,360 second-foet Apr. $7,1926$.

Remarke.- Records good except those for Fob. 16 to Mar. 29 , which aro poor. Diversions above gage by oity of Pasadona not inoludad in dally and monthly rocarda.

Mean daliy discharge, in second-feet, 1838

\begin{tabular}{|c|c|c|c|c|c|c|c|c|c|c|c|}
\hline Day & Fob. & Mar. & Apr. & Day & Feb. & Mar. & Apr. & Dey & Fob. & Mar. & Apr. \\
\hline $\begin{array}{r}1 \\
2 \\
3 \\
4 \\
5 \\
6 \\
7 \\
8 \\
8 \\
10\end{array}$ & $\begin{array}{l}9 \\
1.9 \\
36 \\
46 \\
20 \\
4.6 \\
0 \\
0 \\
8 \\
6.5\end{array}$ & $\begin{array}{r}142 \\
836 \\
268 \\
111 \\
82 \\
70 \\
63 \\
58 \\
50 \\
37\end{array}$ & $\begin{array}{c}18 \\
13 \\
8 \\
11 \\
12 \\
11 \\
11 \\
10 \\
8 \\
8.5\end{array}$ & $\begin{array}{l}11 \\
12 \\
13 \\
14 \\
15 \\
16 \\
17 \\
18 \\
18 \\
20\end{array}$ & $\begin{array}{c}45 \\
40 \\
20 \\
11 \\
7.5 \\
76 \\
12 \\
5 \\
5 \\
3\end{array}$ & $\begin{array}{l}43 \\
66 \\
66 \\
60 \\
51 \\
45 \\
40 \\
35 \\
38 \\
35\end{array}$ & $\begin{array}{c}9 \\
14 \\
12 \\
10 \\
8.5 \\
8 \\
7 \\
6 \\
6 \\
7.8\end{array}$ & $\begin{array}{l}21 \\
28 \\
23 \\
24 \\
25 \\
26 \\
27 \\
28 \\
28 \\
30 \\
31\end{array}$ & $\begin{array}{r}2 \\
2 \\
2 \\
2 \\
2 \\
20 \\
20 \\
144\end{array}$ & $\begin{array}{l}32 \\
28 \\
26 \\
24 \\
23 \\
24 \\
24 \\
23 \\
22 \\
18 \\
17\end{array}$ & $\begin{array}{l}8 \\
7 \\
6 \\
5.5 \\
5 \\
4.8 \\
4.8 \\
4.5 \\
3.8 \\
4.8\end{array}$ \\
\hline \multicolumn{12}{|c|}{ 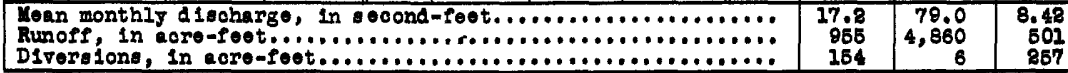 } \\
\hline
\end{tabular}


Eaton Creek near El Monte, Calif.

Location.- Water-stage recorder, lat. $34^{\circ} 05^{\prime} 16^{\prime \prime}$, Iong. $118^{\circ} 03^{\prime} 56^{\prime \prime}$, In san Franc1squ1to grant, at Sunset Avenue Bridge, neax El Monte, Los Angsies County. Alt1tude, about $8 B$ feot.

Drainage area.- 18.4 squars miles.

Qage-he1ght record,- Water-etage rscorder graph and staff-gage readings.

Discherge record.- Defined by current-meter measurements below 400 second-feet; extended to peak discharge determined by slope-area method.

Lexima.- 1938: Discharge, bout 2,300 second-feet 6 p.m. Mar. 2 (gage he1ght, 9.0 fe日t).

1930-37: Discharge, 2,180 second-feet Jan. 1, 1934.

Remarks.- Record poor. Partial regulation at Baton Creok flood-control dam. The Pasadena Water Department diverts water fust abovs Baton Wash dobris dem. Records except for the period Feb. 28 to Har. 2 furnished by Los Angeles County Flood Oontrol District, through H. E. Hedger, chief engineer.

Mean da11y discharge, in second-feet, 1938

\begin{tabular}{|c|c|c|c|c|c|c|c|c|c|c|c|}
\hline Day & Feb. & Mar. & Apr. & Day & Feb. & Mar. & Apr. & Day & $\mathrm{Feb}$. & Mar. & Apr. \\
\hline $\begin{array}{r}1 \\
2 \\
3 \\
4 \\
5 \\
6 \\
7 \\
8 \\
9 \\
10\end{array}$ & $\begin{array}{c}38 \\
0 \\
29 \\
4.5 \\
0 \\
0 \\
0 \\
0 \\
30 \\
0\end{array}$ & $\begin{array}{r}200 \\
770 \\
251 \\
138 \\
92 \\
100 \\
62 \\
23 \\
34 \\
44\end{array}$ & & $\begin{array}{l}11 \\
12 \\
13 \\
14 \\
15 \\
16 \\
17 \\
18 \\
19 \\
20\end{array}$ & $\begin{array}{l}18 \\
3.5 \\
0 \\
0 \\
0 \\
0 \\
0 \\
1.1 \\
2.6 \\
0\end{array}$ & $\begin{array}{c}0 \\
13 \\
14 \\
0 \\
7.5 \\
16 \\
8 \\
9.5 \\
11 \\
5\end{array}$ & & $\begin{array}{l}21 \\
22 \\
23 \\
24 \\
25 \\
26 \\
27 \\
28 \\
29 \\
30 \\
31\end{array}$ & $\begin{array}{r}0 \\
0 \\
0 \\
0 \\
0 \\
0 \\
18 \\
107\end{array}$ & $\begin{array}{l}0 \\
0 \\
0 \\
0 \\
0 \\
0 \\
0 \\
0 \\
6 \\
.8\end{array}$ & 1 \\
\hline \multicolumn{9}{|c|}{ 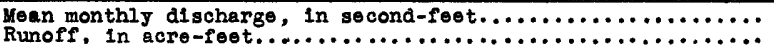 } & $\begin{array}{r}8.99 \\
499\end{array}$ & $\begin{array}{r}58.2 \\
3,580\end{array}$ & $\begin{array}{l}0 \\
0\end{array}$ \\
\hline
\end{tabular}

Gage height, in feet, and discharge, in second-feet, at indicated time, 1938

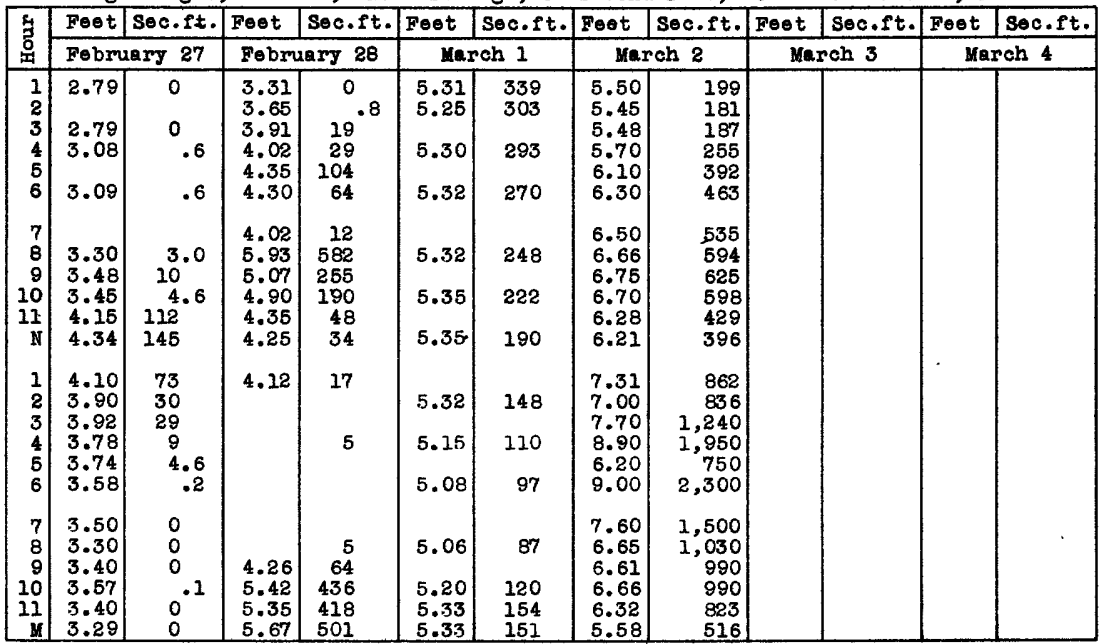


Rub10 Canyon Wash at Rosemead, Cal1f.

Location.- Water-stage recorder, lat. $34^{\circ} 04^{\prime} 30^{\prime \prime}$, long. $118^{\circ} 04^{\prime} 35^{\prime \prime}$, in mith sec. 19 , T. IS., R. Il W., at Glendon Wáy in Rosemead. 'Altitude, about 290 feet.

Dralnage area.- 13.4 square miles.

Gage-he1ght record.- Water-stage recorder graph.

D1scharge record.- Stage-discharge relation defined by current-meter measurements below 1,470 second-feet; extended logar1thmically to peak stage.

Maxima.- 1938: Discharge, 2,700 second-feet 9 a.m. Mar. 2 (gage he1ght, 2.55 feet). 1930-37: Discharge, 2,070 second-feet Dec. 31, 1933.

Remarks. - Records good. Channel is concrete lined and of uniform section. Base data Feb. 27 to Mar. 3 and ent1re record Feb. 1-26 and Lar. 27 to Apr. 30 furn1shed by Los Angeles County Flood Control District, through H. B. Hedger, chief engineer.

Mean daliy discharge, in second-feet, 1938

\begin{tabular}{|c|c|c|c|c|c|c|c|c|c|c|c|}
\hline Day & Feb. & Mar. & Apr. & Day & Feb. & Mar. & Apr. & Day & Feb. & Mar. & Apr. \\
\hline $\begin{array}{r}1 \\
2 \\
3 \\
4 \\
5 \\
6 \\
7 \\
8 \\
9 \\
10\end{array}$ & $\begin{array}{c}107 \\
7.5 \\
54 \\
21 \\
.1 \\
0.1 \\
0.9 \\
2.9 \\
61 \\
2.5\end{array}$ & $\begin{array}{c}101 \\
815 \\
59 \\
10 \\
6.5 \\
2.5 \\
6.5 \\
14 \\
2.1 \\
1.2\end{array}$ & $\begin{array}{r}0.2 \\
.2 \\
.2 \\
.2 \\
.4 \\
.4 \\
.2 \\
.1 \\
.2 \\
.2\end{array}$ & $\begin{array}{l}11 \\
12 \\
13 \\
14 \\
15 \\
16 \\
17 \\
18 \\
19 \\
20\end{array}$ & $\begin{array}{c}69 \\
9 \\
.4 \\
1.6 \\
0.1 \\
0 \\
0 \\
4.1 \\
10 \\
0\end{array}$ & $\begin{array}{c}9 \\
58 \\
18 \\
4.8 \\
4.1 \\
3.3 \\
2.5 \\
2.1 \\
2.1 \\
1.6\end{array}$ & $\begin{array}{l}0.2 \\
1.1 \\
4.8 \\
1.6 \\
.2 \\
.1 \\
.1 \\
0 \\
0 \\
0\end{array}$ & $\begin{array}{l}21 \\
22 \\
23 \\
24 \\
25 \\
26 \\
27 \\
28 \\
29 \\
30 \\
31\end{array}$ & $\begin{array}{r}0.8 \\
.4 \\
.2 \\
0 \\
0 \\
0 \\
86 \\
322\end{array}$ & $\begin{array}{r}1.2 \\
.8 \\
.8 \\
.4 \\
.4 \\
.4 \\
.4 \\
.4 \\
.4 \\
.2 \\
.2\end{array}$ & $\begin{array}{l}0 \\
0 \\
0 \\
2.3 \\
4.1 \\
0.2 \\
0 \\
0 \\
0 \\
.4\end{array}$ \\
\hline $\begin{array}{l}\text { Hea } \\
\text { Run }\end{array}$ & $\begin{array}{l}\text { onthl } \\
\text { in }\end{array}$ & scha & $: 1$ & - & eet. & ${ }^{*}$ & $\cdot$ & $\because \cdots$ & $\begin{array}{r}27.1 \\
1.510\end{array}$ & $\begin{array}{r}36.4 \\
2,240\end{array}$ & $\begin{array}{r}.58 \\
35\end{array}$ \\
\hline
\end{tabular}

Gage helght, in feet, and discharge, in second-feet, at indicated time, 1938

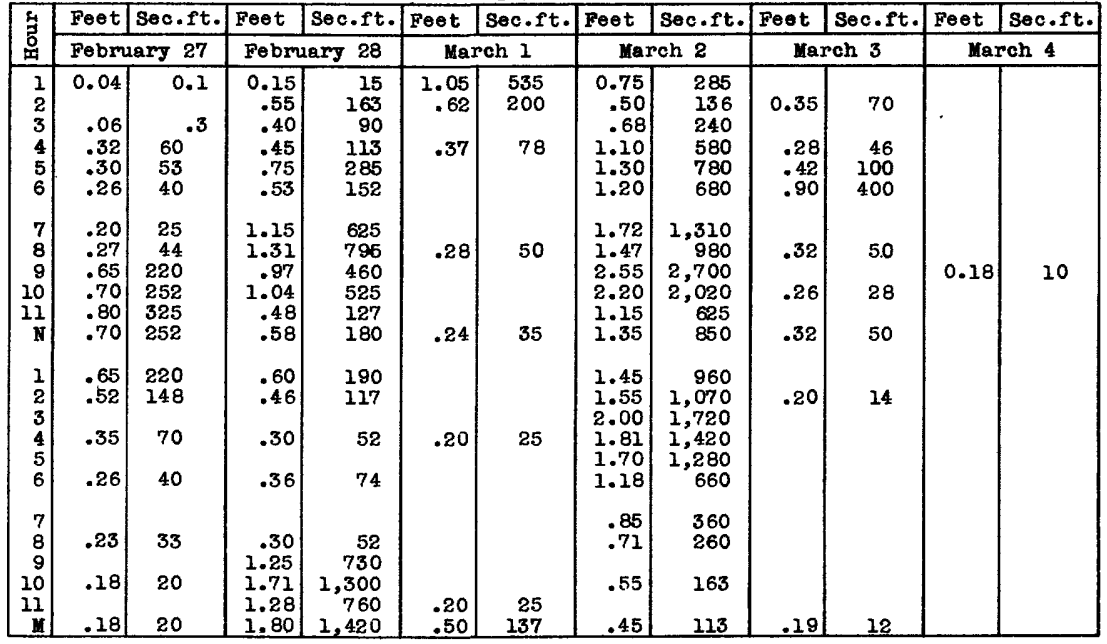


Alhambra Wash at Alhambra, Cal1f.

Location. - Water-atage recorder, lat. $34^{\circ} 03^{\prime} 20^{\prime \prime}$, long. $118^{\circ} 05^{\prime} 10^{\prime \prime}$, in Potrero Grande grant, about 250 feet above Short Street in Álhambra, Los Angeles County. Zero of gage is 243.8 feet above mean sea level.

Drainage erea.- 14.5 square miles.

Gage-helght record.- Water-stage recorder graph.

Discharge record.- Stage-discharge relation defined by current-meter measurements below 456 second-feet; extended to peak discharge on basis of a slope-area measurement of the peak. Discharge Feb. 27 to Mar. 2, computed by Manning 's formula and five

current-meter measurements made during the period.

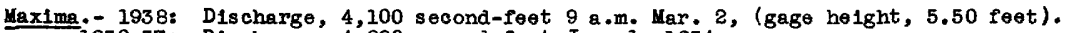
1930-37: Discharge, 4,890 second-feet Jan. i, 1934.

Remarks.- Records falr. No regulation or diversion. Channel is concrete lined and of uniform section and slope. Records furnished by Los Angeles County Flood Control District, through H. E. Hedger, chief engineer, and by Corps of Engineers, U. S. A rmy. Discharge Feb. 27 to Mar. 2 computed by Corps of Engineers, U. S. Army.

Mean dally discharge, in second-feet, 1938

\begin{tabular}{|c|c|c|c|c|c|c|c|c|c|c|c|}
\hline Day & $F \oplus b$. & Mar. & Apr. & Day & $F \oplus b$. & Mar. & Apr. & Day & Feb. & Mar. & Apr. \\
\hline $\begin{array}{r}1 \\
2 \\
3 \\
4 \\
5 \\
6 \\
7 \\
8 \\
9 \\
10\end{array}$ & $\begin{array}{c}177 \\
8^{174} \\
11 \\
0 \\
0 \\
0 \\
0 \\
76 \\
12\end{array}$ & $\begin{array}{c}101 \\
803 \\
30 \\
0^{.2} \\
2^{2.1} \\
7 \\
0 \\
0\end{array}$ & $\begin{array}{l}0 \\
0 \\
0 \\
0 \\
0 \\
0 \\
0 \\
0 \\
0 \\
0\end{array}$ & $\begin{array}{l}11 \\
12 \\
13 \\
14 \\
15 \\
16 \\
17 \\
18 \\
19 \\
20\end{array}$ & $\begin{array}{c}113 \\
7 \\
0 \\
0 \\
0 \\
0 \\
0 \\
4.4 \\
0.8\end{array}$ & $\begin{array}{l}3.9 \\
75 \\
33 \\
7 \\
1.6 \\
.4 \\
0.4 \\
0 \\
0\end{array}$ & $\begin{array}{l}0 \\
0 \\
0 \\
0 \\
0 \\
0 \\
0 \\
0 \\
0 \\
0\end{array}$ & $\begin{array}{l}21 \\
22 \\
23 \\
24 \\
25 \\
26 \\
27 \\
28 \\
29 \\
30 \\
31\end{array}$ & $\begin{array}{r}0 \\
0 \\
0 \\
0 \\
0 \\
0 \\
178 \\
562\end{array}$ & $\begin{array}{l}0 \\
0 \\
0 \\
0 \\
0 \\
0 \\
0 \\
0 \\
0 \\
0 \\
0\end{array}$ & $\begin{array}{l}0 \\
0 \\
0 \\
0 \\
0 \\
0 \\
0 \\
0 \\
0 \\
0\end{array}$ \\
\hline \multicolumn{9}{|c|}{ 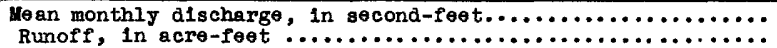 } & $\begin{array}{l}43.8 \\
2,430\end{array}$ & $\begin{array}{r}34,4 \\
2,110\end{array}$ & $\begin{array}{l}0 \\
0\end{array}$ \\
\hline
\end{tabular}

Gage helght, in feet, and discharge, in second-feet, at indicated time, 1938

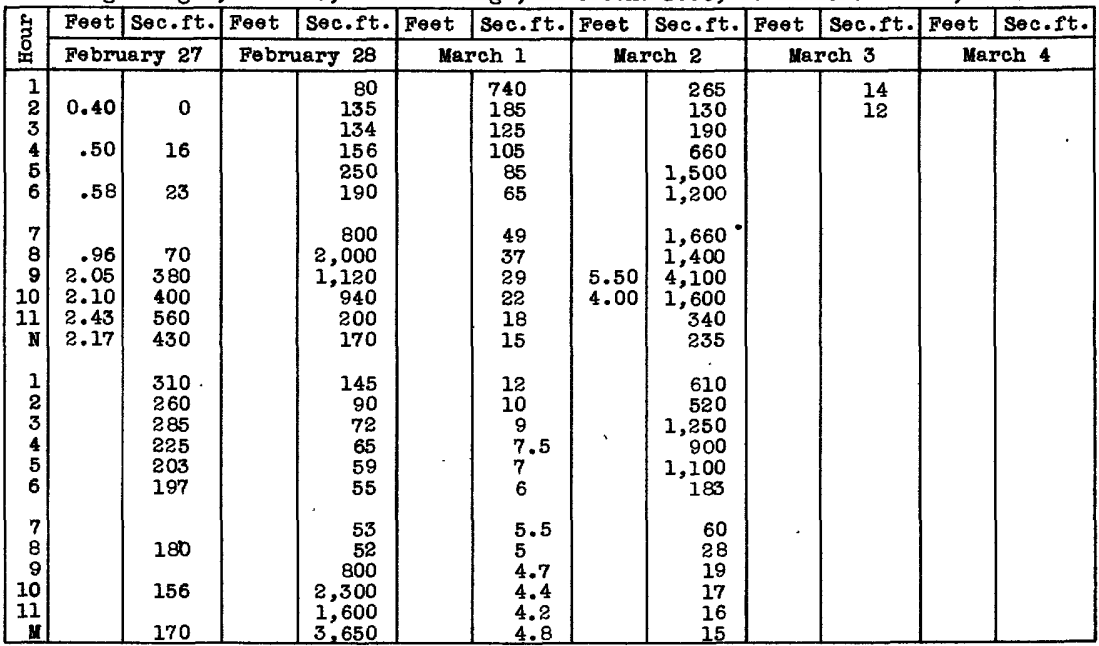


R10 Hondo near El Monte, Cal1f.

Location. - Water-s tage recorder, lat. $34^{\circ} 05^{\prime} 35^{\prime \prime}$, long. $118^{\circ} 01^{\prime} 55^{\prime \prime}$, in San Franelequito grant, at Lower Azusa road bridgo, it mlle日 north of $\mathrm{gl}$ Monto, Los Angelos County. Altitude, bout 285 foet.

Drainage area. - A natural aplit noar Arrow Highway divides the San Gabriel River Into two branohes. Tho west branch is know as the R10 Hondo. Tho San Gabriel River dralnage area above the split is 230 square miles; the Rio Hondo drainage area from the oplit to Lower Azusa road is 54.9 square miles.

Gege-helght record. - Water-stage recorder graph.

D1soharge record.- Stage-discharge relation defined by current-meter measurements below 3,860 second-feet; extended logarlthmioally to peak discharge on basis of alopearea measurement of the peak.

Maximg.- 1938: D1scharge, 31,800 second-feet 9 p.m. Mar. 2 (gage he1ght, 7.56 feet). 1932-36: Ditcharge; 5,860 sooond-foet Jan. 1 , 1834 .

Remarice.- Recorde poor. Regulation by Santa Anita, San Gabrlel No. 1, San Gabriel No. 2, and Morrlo Dams. The City of Pasadena diverts water from the San Gabrlel River and the City of Monrovia diverts water from Monrovia Creek; there are several diversions for Irrigation. Records furntshed by Los Angeles County Flood Control Distriot, through H. E. Hedger, ohief engineer, and by Corps of Engineers, U. S. Army.

Mean daily discharge, in second-feet, 1838

\begin{tabular}{|c|c|c|c|c|c|c|c|c|c|c|c|}
\hline Day & Fob. & Mar. & Apr. & Day & Fab. & Mar. & Apr. & Day & Feb. & Mar. & Apr. \\
\hline $\begin{array}{r}1 \\
2 \\
3 \\
4 \\
5 \\
6 \\
7 \\
8 \\
8 \\
10\end{array}$ & $\begin{array}{r}64 \\
0 \\
76 \\
62 \\
32 \\
13 \\
10 \\
11 \\
36 \\
11\end{array}$ & $\begin{array}{r}1,270 \\
7,700 \\
11,000 \\
2,960 \\
2,800 \\
2,730 \\
4,280 \\
3,660 \\
4,510 \\
2,200\end{array}$ & $\begin{array}{r}331 \\
837 \\
531 \\
735 \\
909 \\
1,780 \\
1,800 \\
1,780 \\
1,780 \\
1,520\end{array}$ & $\begin{array}{l}11 \\
12 \\
13 \\
14 \\
15 \\
16 \\
17 \\
18 \\
19 \\
20\end{array}$ & $\begin{array}{r}51 \\
68 \\
48 \\
35 \\
25 \\
24 \\
13 \\
10 \\
7 \\
0\end{array}$ & $\begin{array}{r}1,230 \\
1,550 \\
1,990 \\
1,720 \\
1,390 \\
1,050 \\
780 \\
1,200 \\
1,460 \\
1,120\end{array}$ & $\begin{array}{r}1,120 \\
305 \\
225 \\
222 \\
229 \\
245 \\
284 \\
260 \\
210 \\
199\end{array}$ & $\begin{array}{l}21 \\
22 \\
23 \\
24 \\
25 \\
26 \\
27 \\
28 \\
28 \\
30 \\
31\end{array}$ & $\begin{array}{c}0 \\
0 \\
1.4 \\
9 \\
83 \\
81 \\
79 \\
415\end{array}$ & $\begin{array}{r}596 \\
290 \\
773 \\
1,010 \\
986 \\
986 \\
996 \\
986 \\
986 \\
986 \\
674\end{array}$ & \begin{tabular}{c}
225 \\
241 \\
237 \\
222 \\
117 \\
\multicolumn{1}{c}{$0^{.2}$} \\
0 \\
0 \\
0
\end{tabular} \\
\hline \multicolumn{9}{|c|}{ 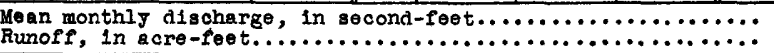 } & $\begin{array}{r}46.2 \\
2,610\end{array}$ & $\begin{array}{r}2,12 \\
130,60\end{array}$ & $\begin{array}{r}544 \\
32,400\end{array}$ \\
\hline
\end{tabular}

Gage helght, in feet, and discharge, In second-feet, at indicated time, 1838

\begin{tabular}{|c|c|c|c|c|c|c|c|c|c|c|c|c|}
\hline \multirow{2}{*}{ 点 } & Feet & sec.ft. & Feet & Sec.ft. & Feet & Sec.ft. & Feet & seo.ft. & Feet & $800.1 \mathrm{ft}$ & Feet & Seo.ft. \\
\hline & \multicolumn{2}{|c|}{ Fobruary 27} & \multicolumn{2}{|c|}{ February 28} & \multicolumn{2}{|c|}{ March 1} & \multicolumn{2}{|c|}{ March 2} & \multicolumn{2}{|c|}{ March 3} & \multicolumn{2}{|c|}{ Maroh 4} \\
\hline $\begin{array}{l}7 \\
2 \\
3 \\
4 \\
5 \\
6\end{array}$ & $\begin{array}{l}2.51 \\
2.52\end{array}$ & $\begin{array}{l}85 \\
88\end{array}$ & $\begin{array}{l}2.17 \\
2.28\end{array}$ & $\begin{array}{c}33 \\
50 \\
.\end{array}$ & $\begin{array}{l}4.21 \\
4.45 \\
4.15 \\
4.00 \\
3.82 \\
3.83\end{array}$ & $\begin{array}{l}3,300 \\
3,800 \\
2,700 \\
2,200 \\
1,750 \\
1,700\end{array}$ & $\begin{array}{l}3.28 \\
3.30 \\
3.49 \\
3.67 \\
3.92\end{array}$ & $\begin{array}{r}520 \\
526 \\
730 \\
1,000 \\
1,450 \\
1,800\end{array}$ & $\begin{array}{l}6.04 \\
5.44 \\
5.36 \\
5.20 \\
5.94\end{array}$ & $\begin{array}{r}14,400 \\
9,200 \\
8,500 \\
7,400 \\
13,500\end{array}$ & $\begin{array}{l}4.57 \\
4.21 \\
4.10\end{array}$ & $\begin{array}{l}4,950 \\
3,440 \\
3,110\end{array}$ \\
\hline $\begin{array}{r}7 \\
8 \\
9 \\
10 \\
11 \\
11\end{array}$ & $\begin{array}{l}2.54 \\
2.65 \\
2.73\end{array}$ & $\begin{array}{r}96 \\
130 \\
170\end{array}$ & $\begin{array}{l}2.70 \\
3.16 \\
3.45 \\
3.21 \\
3.10 \\
3.07\end{array}$ & $\begin{array}{l}190 \\
440 \\
900 \\
610 \\
500 \\
460\end{array}$ & $\begin{array}{c}3.81 \\
3.67 \\
3.56 \\
3.47\end{array}$ & $\begin{array}{r}1,600 \\
1,300 \\
1,050 \\
860\end{array}$ & $\begin{array}{l}4.06 \\
4.12 \\
4.25 \\
4.20 \\
4.30\end{array}$ & $\begin{array}{l}1,800 \\
1,900 \\
2,300 \\
2,100 \\
2,400 \\
3,100\end{array}$ & $\begin{array}{l}6.13 \\
6.06 \\
5.84 \\
5.88 \\
5.76\end{array}$ & $\begin{array}{l}15,500 \\
15,000 \\
13,000 \\
13,500 \\
12,000 \\
11,600\end{array}$ & 3.89 & 2,23 \\
\hline $\begin{array}{l}1 \\
2 \\
3 \\
4 \\
5 \\
6\end{array}$ & $\begin{array}{l}2.53 \\
2.28 \\
2.17\end{array}$ & $\begin{array}{l}98 \\
43 \\
30\end{array}$ & $\begin{array}{l}3.05 \\
3.07 \\
2.98\end{array}$ & $\begin{array}{l}440 \\
460 \\
380\end{array}$ & $\begin{array}{l}3.43 \\
3.42 \\
3.35\end{array}$ & $\begin{array}{l}800 \\
740 \\
640\end{array}$ & $\begin{array}{l}4.72 \\
4.96 \\
5.07 \\
5.38 \\
6.03 \\
6.14\end{array}$ & $\begin{array}{r}4,000 \\
5,300 \\
6,000 \\
8,100 \\
13,600 \\
15,000\end{array}$ & $\begin{array}{l}5.83 \\
5.70 \\
5.75 \\
5.84 \\
5.71 \\
5.42\end{array}$ & $\begin{array}{r}13,000 \\
12,000 \\
12,400 \\
13,000 \\
12,000 \\
9,600\end{array}$ & 3.49 & 1,780 \\
\hline $\begin{array}{r}7 \\
8 \\
9 \\
10 \\
11 \\
M \\
\end{array}$ & $\begin{array}{l}2.16 \\
2.16 \\
2.15\end{array}$ & $\begin{array}{l}28 \\
30 \\
28 \\
\end{array}$ & $\begin{array}{l}2.95 \\
3.00 \\
3.40 \\
3.60 \\
3.60 \\
\end{array}$ & $\begin{array}{r}360 \\
400 \\
860 \\
1,200 \\
1,200\end{array}$ & $\begin{array}{c}3.33 \\
3.23 \\
3.38\end{array}$ & $\begin{array}{l}600 \\
480 \\
630 \\
\end{array}$ & $\begin{array}{l}6.02 \\
5.90 \\
7.56 \\
6.84 \\
6.71 \\
6.41\end{array}$ & $\begin{array}{l}14,000 \\
13,000 \\
31,800 \\
24,000 \\
21,500 \\
18,000\end{array}$ & $\begin{array}{l}5.21 \\
4.87 \\
5.02 \\
5.12 \\
5.13 \\
5.03\end{array}$ & $\begin{array}{l}8,000 \\
5,800 \\
6,800 \\
7,500 \\
7,600 \\
6,800\end{array}$ & 3.48 & 1,980 \\
\hline
\end{tabular}


R10 Eondo near Montebel10, Cal1f.

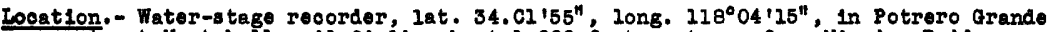
grant, at Montebel10 o11 Pleid, about 1,000 foet upstream from MIasion Bridge, and 2 milos northesst of Montebeilo, Los Angeles County. Altitude, about 200 foet.

Drainage area.- A natural split near Arrow Elghway divides the San Gabriel River into two branohos; the west brenoh $1 \mathrm{~s}$ known as the k10 Bondo. The San Gabrlel River drathage area above the aplit is 230 square miles; the klo Bondo drainage area from the split to this station 18113 quere mlies.

Gage-he1ght reoord. - Water-stage reoorder graph sxoept Feb. 1, Mar. 3-8, 8-11, 17, 2123, 26-29, Apr. 12-15, 28-30.

Dsecharge record. - Stage-discharge relation defined by current-meter measurements belon 8,800 socond-feot; extended to peale disoharge determined by slope-area method on besis of other disoharge information. Discharge estimated for dags of missing recond.

Maxima.- 1938: D1scharge; 28,000 second-feet 11 p.m. Nar. 2 (gage helght, 12.83 foet). 1928-37:. Dlscharge, 11,800 second-feet Jan. 1, 1934.

Remarks.- Reoorde falr. Flow partialiy regulated by Slerra Madre, Santa Anlta, Sawplt, San Gabrlol Ho. 2, and Morris Dams. The C1ty of Pasadena diverts water from Eaton Creok and $f$ rom the $\operatorname{San}$ Gabrlel RIver; the 01ty of Monrovia diverts water from Monrovla Creok; several diversions for 1rrigation. Records furnished by Los Angeles Dounty Flood Control District, through $B$. $\mathbb{B}$. Bedger, chlef onglneor.

Mean delly disoharge, in seoond-foet, 1938

\begin{tabular}{|c|r|r|r||r|r|r|r||r|r|r|r|}
\hline Day & Feb. & Mar. & Apr. & Day & Fob. & Mar. & Apr. & Dag & Fob. & Mar. & Apr. \\
\hline 1 & 560 & 1,290 & 556 & 11 & 364 & 1,400 & 1,300 & 21 & 25 & 578 & 332 \\
2 & 72 & 9,500 & 1,120 & 12 & 230 & 1,820 & 325 & 22 & 32 & 255 & 300 \\
3 & 335 & 12,600 & 968 & 13 & 163 & 1,860 & 250 & 23 & 34 & 610 & 280 \\
4 & 187 & $2,96 C$ & 1,320 & 14 & 125 & 1,770 & 250 & 24 & 38 & 1,010 & 249 \\
5 & 66 & 2,600 & 2,040 & 15 & 80 & 1,790 & 269 & 25 & 96 & 980 & 122 \\
6 & 54 & 2,800 & 2,240 & 16 & 63 & 1,490 & 259 & 26 & 110 & 1,000 & 30 \\
7 & 62 & 2,900 & 1,960 & 17 & 49 & 915 & 232 & 27 & 419 & 1,000 & 26 \\
8 & 76 & 3,490 & 1,760 & 18 & 46 & 1,100 & 172 & 28 & 1,520 & 1,000 & 28 \\
9 & 345 & 3,640 & 1,720 & 19 & 60 & 1,080 & 207 & 29 & & 1,060 & 31 \\
10 & 65 & 2,200 & 2,010 & 20 & 11 & 854 & 241 & 30 & & 1,280 & 34 \\
\hline
\end{tabular}

Gage holght, in feet, and disoharge, in seoond-feet, at indioated time, 1938

\begin{tabular}{|c|c|c|c|c|c|c|c|c|c|c|c|c|}
\hline \multirow{2}{*}{ 绾 } & Feot & soo.ft. & Foet & seo.ft. & Feet & Seo.ft. & Feet & seo.ft. & Feot & seo.ft. & Foet & seo.ft. \\
\hline & \multicolumn{2}{|c|}{ February 27} & \multicolumn{2}{|c|}{ Fobmuary 28} & \multicolumn{2}{|c|}{ Maroh 1} & \multicolumn{2}{|c|}{ Maroh 2} & \multicolumn{2}{|c|}{ March 3} & \multicolumn{2}{|c|}{ March 4} \\
\hline $\begin{array}{l}1 \\
2 \\
3 \\
4 \\
5 \\
6\end{array}$ & $\begin{array}{c}4.89 \\
4.93 \\
5.04\end{array}$ & $\begin{array}{l}132 \\
150 \\
211\end{array}$ & $\begin{array}{l}4.81 \\
5.35 \\
5.20 \\
6.06 \\
5.65\end{array}$ & $\begin{array}{r}200 \\
200 \\
600 \\
500 \\
1,100 \\
800\end{array}$ & $\begin{array}{l}8.42 \\
7.55 \\
7.37 \\
6.85 \\
6.58 \\
6.34\end{array}$ & $\begin{array}{l}5,000 \\
3,600 \\
2,500 \\
2,000 \\
1,700 \\
1,400\end{array}$ & $\begin{array}{l}6.15 \\
5.86 \\
5.85 \\
6.85 \\
7.90 \\
8.45\end{array}$ & $\begin{array}{l}1,300 \\
1,200 \\
1,000 \\
2,500 \\
4,700 \\
6,300\end{array}$ & & $\begin{array}{l}22,000 \\
20,000 \\
18,000 \\
16,000 \\
14,000 \\
11,000\end{array}$ & & \\
\hline $\begin{array}{r}7 \\
8 \\
9 \\
10 \\
11 \\
N\end{array}$ & $\begin{array}{l}5.05 \\
5.49 \\
5.96 \\
6.10 \\
6.19\end{array}$ & $\begin{array}{r}222 \\
532 \\
1,020 \\
1,190 \\
1,320\end{array}$ & $\begin{array}{l}5.55 \\
7.75 \\
7.57 \\
7.59 \\
6.45 \\
6.15\end{array}$ & $\begin{array}{r}700 \\
4,000 \\
3,600 \\
3,800 \\
2,000 \\
1,400\end{array}$ & $\begin{array}{l}6.12 \\
6.00 \\
5.78 \\
5.60\end{array}$ & $\begin{array}{r}874 \\
759\end{array}$ & $\begin{array}{r}8.80 \\
9.29 \\
10.13 \\
9.57 \\
8.82 \\
8.07\end{array}$ & $\begin{array}{r}6,700 \\
7,300 \\
10,000 \\
8,500 \\
6,000 \\
4,300\end{array}$ & & $\begin{array}{r}9,000 \\
7,800 \\
12,000 \\
16,000 \\
15,000 \\
14,000\end{array}$ & & \\
\hline $\begin{array}{l}1 \\
2 \\
3 \\
4 \\
5 \\
6\end{array}$ & $\begin{array}{l}5.92 \\
5.66 \\
5.38 \\
5.20 \\
4.80 \\
4.78\end{array}$ & $\begin{array}{r}1,020 \\
820 \\
630 \\
540 \\
306 \\
274\end{array}$ & $\begin{array}{l}\mathbf{5 . 6 8} \\
5.77 \\
5.50 \\
5.35 \\
5.33 \\
\mathbf{5 . 1 5}\end{array}$ & $\begin{array}{r}1,200 \\
1,300 \\
1,000 \\
800 \\
700 \\
600\end{array}$ & $\begin{array}{l}5.48 \\
5.30 \\
5.28\end{array}$ & $\begin{array}{l}682 \\
580 \\
520\end{array}$ & $\begin{array}{r}8.82 \\
8.91 \\
9.94 \\
10.15 \\
10.77 \\
10.65\end{array}$ & $\begin{array}{r}7,000 \\
7,700 \\
12,000 \\
13,000 \\
15,000 \\
16,000\end{array}$ & & $\begin{array}{l}14,000 \\
13,000 \\
13,000 \\
12,000 \\
12,000 \\
11,000\end{array}$ & & \\
\hline 10 & $\begin{array}{l}4.50 \\
4.52 \\
4.76 \\
\end{array}$ & $\begin{array}{l}152 \\
157 \\
234 \\
\end{array}$ & $\begin{array}{l}5.05 \\
5.50 \\
7.45 \\
7.95 \\
8.19 \\
\end{array}$ & $\begin{array}{r}500 \\
500 \\
1,400 \\
3,600 \\
4,100 \\
4,400 \\
\end{array}$ & $\begin{array}{l}5.14 \\
5.10 \\
5.16 \\
\end{array}$ & $\begin{array}{r}503 \\
492 \\
538 \\
\end{array}$ & $\begin{array}{r}10.30 \\
10.02 \\
9.97 \\
11.62 \\
12.83\end{array}$ & $\begin{array}{l}13,000 \\
12,000 \\
12,000 \\
18,000 \\
28,000 \\
24,000\end{array}$ & & $\begin{array}{l}9,800 \\
8,700 \\
7,600\end{array}$ & & \\
\hline
\end{tabular}


R1o Hondo near Downey, Cal1f.

Location. - Water-stage recorder, lat. $33^{\circ} 56^{\prime} 40^{n}$, long. $118^{\circ} 09^{\prime} 50^{n}$, in San Antonio grant, at Stewart and Gray Road Bridge, half a mfle upstream from confluence with Los Angeles River, and $1 \frac{1}{c}$ miles west of Downey, Los Angeles County. Altitude, about 95 foet.

Drainage area.- 140 square miles not including San Gabriel River above the split which forms R10 Hondo (see Rio Hondo near el Konte).

Gage-height record. - Water-stage recorder graph except 7 p.m. Mar. 2 to 9 a.m. Mar. 3. Maximum stage read on s taff gage.

Discharge record.- Stage-discharge relation defined by current-meter measurements below 9,420 second-feet; extended logarithmicaliy to peak discharge as determined by slope-area measurement. Discharge for the period of missing gage-helght record determined by comperison with adjacent stations.

Maxima.- 1938: Discharge, 24,400 second-feet 1 a.m. Mar. 3 (gage he1ght, 13.5 feet). 1914, 1928-37: Discharge, 20,900 second-feet February 1914.

Remarks.- Records poor. Regulation at San Gabriel No. 1, San Gabriel No. 2, Morris, Sawpit, and Santa Anita Dams, and Eaton Creek Debris Dam. The City of Pasadena diverts water from the San Gabriel River, the City of Monrovia diverts water from Monrovia Creek; several diversions for Irrigation. Records furntshed by Los Angeles County Flood Control District, through H. E. Hedger, chlef engineer.

Mean dally discharge, in second-feet, 1938

\begin{tabular}{|c|c|c|c|c|c|c|c|c|c|c|c|}
\hline Day & Feb. & Mar. & Apr. & Day & Feb. & Mar. & Apr. & Day & Feb. & Mar. & Apr. \\
\hline $\begin{array}{r}1 \\
2 \\
3 \\
4 \\
5 \\
6 \\
7 \\
8 \\
9 \\
10\end{array}$ & $\begin{array}{c}415 \\
0 \\
205 \\
159 \\
9 \\
1.1 \\
0 \\
0 \\
272 \\
40\end{array}$ & $\begin{array}{r}1,870 \\
11,100 \\
12,700 \\
3,690 \\
2,690 \\
2,500 \\
2,520 \\
2,780 \\
3,520 \\
2,570\end{array}$ & $\begin{array}{r}444 \\
908 \\
800 \\
1,110 \\
1,750 \\
1,930 \\
1,700 \\
1,400 \\
1,340 \\
1,220\end{array}$ & $\begin{array}{l}11 \\
12 \\
13 \\
14 \\
15 \\
16 \\
17 \\
18 \\
19 \\
20\end{array}$ & $\begin{array}{r}222 \\
111 \\
9 \\
0 \\
0 \\
0 \\
0 \\
0 \\
0 \\
0\end{array}$ & $\begin{array}{r}1,210 \\
1,630 \\
1,850 \\
2,050 \\
1,820 \\
1,380 \\
788 \\
997 \\
968 \\
707\end{array}$ & $\begin{array}{l}799 \\
291 \\
172 \\
128 \\
136 \\
141 \\
156 \\
166 \\
161 \\
161\end{array}$ & $\begin{array}{l}21 \\
22 \\
23 \\
24 \\
25 \\
26 \\
27 \\
28 \\
29 \\
30 \\
31 \\
\end{array}$ & \begin{tabular}{|c|}
0 \\
0 \\
0 \\
0 \\
2.0 \\
3.3 \\
279 \\
1,360
\end{tabular} & $\begin{array}{l}544 \\
158 \\
456 \\
824 \\
800 \\
752 \\
728 \\
800 \\
788 \\
812 \\
831 \\
\end{array}$ & $\begin{array}{r}172 \\
205 \\
223 \\
235 \\
14 \\
0 \\
0 \\
0 \\
0 \\
-\quad .1\end{array}$ \\
\hline \multicolumn{9}{|c|}{ 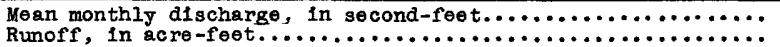 } & $\begin{array}{r}110 \\
6,120\end{array}$ & $\begin{array}{r}2,156 \\
132,600\end{array}$ & $\begin{array}{r}525 \\
31,260\end{array}$ \\
\hline
\end{tabular}

Gage height, In feet, and discharge, in second-feet, at indicated time, 1938

\begin{tabular}{|c|c|c|c|c|c|c|c|c|c|c|c|c|}
\hline \multirow{2}{*}{ 急 } & Feet & Sec.ft. & Feet & sec.ft. & Feet & sec.ft. & Feet & Sec.ft. & Feet & Sec.ft. & Feet & Sec.ft. \\
\hline & \multicolumn{2}{|c|}{ February 27} & \multicolumn{2}{|c|}{ Febmuary 28} & \multicolumn{2}{|c|}{ March 1} & \multicolumn{2}{|c|}{ March 2} & \multicolumn{2}{|c|}{ March 3} & \multicolumn{2}{|c|}{ March 4} \\
\hline $\begin{array}{l}1 \\
2 \\
3 \\
4 \\
5 \\
6\end{array}$ & & & $\begin{array}{l}5.96 \\
5.95 \\
6.05\end{array}$ & $\begin{array}{l}115 \\
113 \\
138\end{array}$ & $\begin{array}{r}10.05 \\
9.55 \\
8.77 \\
8.03 \\
7.65 \\
7.37\end{array}$ & $\begin{array}{l}8,600 \\
7,100 \\
5,000 \\
3,400 \\
2,750 \\
2,300\end{array}$ & $\begin{array}{l}6.52 \\
6.87 \\
7.48 \\
7.39 \\
8.20 \\
9.80\end{array}$ & $\begin{array}{r}820 \\
1,100 \\
1,800 \\
1,650 \\
2,800 \\
6,300\end{array}$ & 13.50 & 24,400 & 1- & \\
\hline $\begin{array}{r}7 \\
8 \\
9 \\
10 \\
11 \\
\mathbf{N}\end{array}$ & $\begin{array}{l}6.20 \\
7.05\end{array}$ & $\begin{array}{l}225 \\
695\end{array}$ & $\begin{array}{l}6.30 \\
7.21 \\
7.80 \\
9.40 \\
8.90 \\
8.08\end{array}$ & $\begin{array}{r}210 \\
700 \\
1,280 \\
4,900 \\
3,800 \\
2,500\end{array}$ & $\begin{array}{l}7.13 \\
7.01 \\
6.91 \\
6.80 \\
6.70 \\
6.64\end{array}$ & $\begin{array}{l}2,000 \\
1,850 \\
1,750 \\
1,500 \\
1,310 \\
1,180\end{array}$ & $\begin{array}{l}10.35 \\
10.60 \\
10.85 \\
11.03 \\
10.98 \\
10.35\end{array}$ & $\begin{array}{r}7,900 \\
8,600 \\
9,400 \\
11,000 \\
11,600 \\
10,200\end{array}$ & 9.10 & 9,700 & & \\
\hline $\begin{array}{l}1 \\
2 \\
3 \\
4 \\
5 \\
6\end{array}$ & $\begin{array}{l}7.59 \\
7.66 \\
7.45 \\
7.13 \\
6.88 \\
6.65\end{array}$ & $\begin{array}{r}1,180 \\
1,220 \\
970 \\
675 \\
560 \\
365\end{array}$ & $\begin{array}{l}7.25 \\
6.97 \\
6.75 \\
6.60 \\
6.54 \\
6.46\end{array}$ & $\begin{array}{r}1,480 \\
1,260 \\
1,120 \\
1,020 \\
1,000 \\
950\end{array}$ & $\begin{array}{l}6.57 \\
6.53 \\
6.56 \\
6.54 \\
6.41\end{array}$ & $\begin{array}{r}1,090 \\
1,030 \\
1,040 \\
1,010 \\
870\end{array}$ & $\begin{array}{r}9.45 \\
10.22 \\
10.40 \\
11.15 \\
11.60 \\
12.05\end{array}$ & $\begin{array}{r}7,900 \\
10,500 \\
11,000 \\
14,000 \\
15,700 \\
17,700\end{array}$ & & & & \\
\hline $\begin{array}{r}7 \\
8 \\
9 \\
10 \\
11 \\
\mathbf{M}\end{array}$ & $\begin{array}{l}6.48 \\
6.30 \\
6.25 \\
6.16 \\
6.05\end{array}$ & $\begin{array}{l}290 \\
215 \\
200 \\
173 \\
140\end{array}$ & $\begin{array}{l}6.46 \\
6.41 \\
6.49 \\
6.89 \\
8.76 \\
9.40\end{array}$ & $\begin{array}{r}960 \\
940 \\
1,020 \\
1,480 \\
4,800 \\
6,500\end{array}$ & $\begin{array}{l}6.36 \\
6.33 \\
6.39\end{array}$ & $\begin{array}{l}790 \\
730 \\
730\end{array}$ & 12.40 & 19,000 & & & & \\
\hline
\end{tabular}


Ballona Creek Basin

Centinela Creek near Culver clty, Calif.

Location. - Water-stage recorder, lat. $33^{\circ} 58^{\prime} 35^{\prime \prime}$, long., $118^{\circ} 23^{\prime} 05^{\prime \prime}$, In Agua fe de la Centinela grant, 25 feet south of Centinela Boulevard, between Centinela Boulevard and Pacific Blectric R. R., and $2 t$ miles south of Culver City, Los Angeles County. Altitude, about 47 feot.

Drainage area.- 5.17 square miles.

Gage-helght record.- Water-stage recorder graph Feb. 27 to 5 p.m. Mar. 2; staff gage reading a $12 \mathrm{~m}$. Mar. 4.

D1scharge record.- Stage-discharge relation defined by current-meter measurements below 580 second-feet; extended logarithmically to peak discharge determined by slopearea mothod.

Maxima.- 1938: Discharge, 1,900 second-feet 5 a.m. Mar. 2 (gage helght, 7.83 feet). 1932-37: Discharge, 1,590. second-feet Mar. 2, 1935.

Remarks.- Records fair. No regulation or diversions. Records furnished by Los Angeles County Flood Control District, through H. E. Hedger, chler engineer, and by Corps of Bngineers, J. S. Army.

Mean dally discharge, in second-feet, 1938

\begin{tabular}{|ll|}
\hline \begin{tabular}{ll} 
February 28 & 267 \\
March 1 & 117 \\
\hline
\end{tabular} \\
\hline
\end{tabular}

Gage height, in feet, and discharge, in second-feet, at indicated time, 1938

\begin{tabular}{|c|c|c|c|c|c|c|c|c|c|c|c|c|}
\hline \multirow{2}{*}{$\begin{array}{l} \\
0 \\
0 \\
0\end{array}$} & Feet & sec.ft. & Feot & sec.ft. & Feet & sec.ft. & Feet & sec.ft. & Feet & sec.ft. & Feet & Sec.ft. \\
\hline & \multicolumn{2}{|c|}{ Februaxy 27} & \multicolumn{2}{|c|}{ February 2S } & \multicolumn{2}{|c|}{ March I } & \multicolumn{2}{|c|}{ March 2} & \multicolumn{2}{|c|}{ March 3} & \multicolumn{2}{|c|}{$\operatorname{March}, 4$} \\
\hline $\begin{array}{l}1 \\
2 \\
3 \\
4 \\
5 \\
6\end{array}$ & & & $\begin{array}{l}2.00 \\
2.00 \\
2.00 \\
2.00 \\
2.50 \\
4.50\end{array}$ & $\begin{array}{r}1 \\
1 \\
1 \\
1 \\
13 \\
480\end{array}$ & $\begin{array}{l}4.50 \\
2.76 \\
2.76 \\
2.76\end{array}$ & $\begin{array}{r}480 \\
41 \\
41 \\
41\end{array}$ & $\begin{array}{l}3.75 \\
6.50 \\
7.58 \\
7.52 \\
7.83 \\
7.30\end{array}$ & $\begin{array}{r}250 \\
1,260 \\
1,780 \\
1,730 \\
1,900 \\
1,640\end{array}$ & & & & \\
\hline \begin{tabular}{r|}
7 \\
8 \\
9 \\
10 \\
11 \\
1
\end{tabular} & 3.23 & 125 & $\begin{array}{l}5.74 \\
5.74 \\
4.25 \\
2.75 \\
2.68 \\
2.61\end{array}$ & $\begin{array}{r}950 \\
950 \\
395 \\
41 \\
34 \\
21\end{array}$ & $\begin{array}{l}2.76 \\
2.75 \\
2.75\end{array}$ & $\begin{array}{l}41 \\
40 \\
40\end{array}$ & $\begin{array}{l}7.15 \\
7.00 \\
6.00 \\
3.90 \\
4.57 \\
4.00\end{array}$ & $\begin{array}{r}1,570 \\
1,500 \\
1,060 \\
290 \\
505 \\
320\end{array}$ & & & 2.92 & 0 \\
\hline $\begin{array}{l}1 \\
2 \\
3 \\
4 \\
5 \\
6\end{array}$ & $\begin{array}{l}3.25 \\
2.86 \\
2.70 \\
2.55 \\
2.40 \\
2.27\end{array}$ & $\begin{array}{r}128 \\
55 \\
34 \\
16 \\
8 \\
6\end{array}$ & $\begin{array}{l}2.60 \\
2.59 \\
2.58 \\
2.57 \\
2.57 \\
2.56\end{array}$ & $\begin{array}{l}20 \\
19 \\
18 \\
18 \\
18 \\
17\end{array}$ & $\begin{array}{l}2.75 \\
2.75 \\
2.74\end{array}$ & $\begin{array}{l}40 \\
40 \\
39\end{array}$ & $\begin{array}{l}4.50 \\
4.75 \\
5.70 \\
4.75 \\
3.43\end{array}$ & $\begin{array}{l}480 \\
565 \\
935 \\
565 \\
163\end{array}$ & - & & & \\
\hline $\begin{array}{r}7 \\
8 \\
9 \\
10 \\
11 \\
y\end{array}$ & $\begin{array}{l}2.18 \\
2.09 \\
2.00 \\
2.00 \\
2.00 \\
2.00\end{array}$ & $\begin{array}{l}3 \\
2 \\
1 \\
1 \\
1 \\
1\end{array}$ & $\begin{array}{l}2.55 \\
3.50 \\
5.10 \\
5.00 \\
6.55 \\
6.17\end{array}$ & $\begin{array}{r}16 \\
185 \\
695 \\
660 \\
1,285 \\
1,120\end{array}$ & $\begin{array}{r}2.74 \\
2.74 \\
4.00 \\
4.70 \\
3.60\end{array}$ & $\begin{array}{r}39 \\
39 \\
320 \\
550 \\
210\end{array}$ & & & & & & \\
\hline
\end{tabular}

$4547200-42-18$ 
Ballona Oreek near Oulver O1ty, Oalie.

Locetion. - Water-stage recorder, lat. $33^{\circ} 59^{\prime} 50^{\prime \prime}$, long. $118^{\circ} 24^{\prime} 10^{\prime \prime}$, in Ia Ballona grant, at Sawtelie Boulevard Bridge, abut lz miles south of Oulver Oity, Los Angeles

County. Alt1tude, about. 40 foet.

Dreinage area.- 112 square miles.

Gege-he1ght reoord. - Water-stage reoorder graph.

Discharge record. - Stage-disoharge relation defined by current-meter measurements be10x 18,000 8eo ond-feet; extended logar 1 thmioal1y to peak stage.

Maxima.- 1938 : Disoharge, 19,000 seoond-feet 9:40 a.m. Mar. \& (gage he1ght, 15.25 Ieet).

1988-37: D18charge, 11,300 second-feet Jan. 1, 1934.

Remarias.- Records fair. Reoords furnished by Los Angeles Oounty F1ood Oontrol District, through B. F. Hedzer, chief engineer, except those for period Fob. 27 to

Har. 5, whioh were fumished by Corps of Ingineers, J. S. Arry.

Mean da1ly discharge, in second-feet, 1938

\begin{tabular}{|c|c|c|c|c|c|c|c|c|c|c|c|}
\hline Day & Fob. & Mar. & Apr. & Day & Feb. & Har. & Apr. & Day & Fob. & Mar. & Apr. \\
\hline $\begin{array}{r}1 \\
2 \\
3 \\
4 \\
5 \\
6 \\
7 \\
8 \\
9 \\
10\end{array}$ & $\begin{array}{r}2,050 \\
134 \\
375 \\
864 \\
17 \\
17 \\
17 \\
92 \\
698 \\
88\end{array}$ & $\begin{array}{r}1,210 \\
7,410 \\
280 \\
100 \\
69 \\
59 \\
55 \\
84 \\
59 \\
59\end{array}$ & $\begin{array}{l}9.5 \\
10 \\
12 \\
12 \\
12 \\
11 \\
12 \\
12 \\
12 \\
13\end{array}$ & $\begin{array}{l}11 \\
18 \\
13 \\
14 \\
15 \\
16 \\
17 \\
18 \\
18 \\
20\end{array}$ & $\begin{array}{r}991 \\
119 \\
18 \\
65 \\
17 \\
17 \\
17 \\
85 \\
114 \\
17\end{array}$ & $\begin{array}{r}368 \\
605 \\
251 \\
108 \\
103 \\
89 \\
86 \\
72 \\
68 \\
56\end{array}$ & $\begin{array}{l}14 \\
13 \\
14 \\
15 \\
15 \\
16 \\
16 \\
16 \\
17 \\
17\end{array}$ & $\begin{array}{l}21 \\
22 \\
23 \\
24 \\
25 \\
28 \\
27 \\
28 \\
89 \\
30 \\
31\end{array}$ & $\begin{array}{r}17 \\
17 \\
17 \\
17 \\
17 \\
17 \\
948 \\
2,710\end{array}$ & $\begin{array}{r}46 \\
36 \\
27 \\
22 \\
20 \\
19 \\
18 \\
16 \\
18 \\
9 \\
9\end{array}$ & $\begin{array}{r}18 \\
18 \\
17 \\
30 \\
180 \\
18 \\
16 \\
16 \\
16 \\
85\end{array}$ \\
\hline \multicolumn{9}{|c|}{ 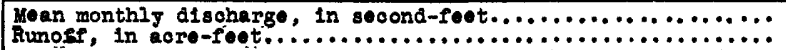 } & $\begin{array}{r}320 \\
17,800\end{array}$ & 22,650 & $\begin{array}{r}22.6 \\
1,350\end{array}$ \\
\hline
\end{tabular}

Gage height, In feet, and discharge, in second-feet, at indioated time, 1838

\begin{tabular}{|c|c|c|c|c|c|c|c|c|c|c|c|c|}
\hline \multirow{2}{*}{ 월 } & Feet & sec.ft. & Feet & Seo.ft. & Feet & seo.f.t. & Deet & sec.ft. & Feet & seo.et. & Feet & sec.ft. \\
\hline & \multicolumn{2}{|c|}{ February 27} & \multicolumn{2}{|c|}{ February 28} & \multicolumn{2}{|c|}{ March 1} & \multicolumn{2}{|c|}{ Maroh 8} & \multicolumn{2}{|c|}{ Naroh 3} & \multicolumn{2}{|c|}{ March 4} \\
\hline $\begin{array}{l}1 \\
2 \\
3 \\
4 \\
5 \\
6\end{array}$ & $\begin{array}{l}2.61 \\
2.60 \\
2.60 \\
2.91 \\
4.35 \\
3.96\end{array}$ & $\begin{array}{l}170 \\
168 \\
168 \\
230 \\
683 \\
526\end{array}$ & $\begin{array}{l}3.00 \\
4.05 \\
3.95 \\
3.90 \\
6.35 \\
5.47\end{array}$ & $\begin{array}{r}250 \\
558 \\
523 \\
505 \\
1,900 \\
1,260\end{array}$ & $\begin{array}{r}10.30 \\
7.55 \\
6.15 \\
5.08 \\
4.50 \\
4.20\end{array}$ & $\begin{array}{r}6,820 \\
2,970 \\
1,740 \\
1,030 \\
750 \\
616\end{array}$ & $\begin{array}{r}8.35 \\
7.30 \\
7.33 \\
11.05 \\
14.00 \\
12.70\end{array}$ & $\begin{array}{r}3,890 \\
2,730 \\
2,770 \\
8,240 \\
15,300 \\
11,800\end{array}$ & & & & \\
\hline $\begin{array}{r}7 \\
8 \\
9 \\
10 \\
11 \\
1\end{array}$ & $\begin{array}{l}3.52 \\
3.48 \\
6.50 \\
7.40 \\
7.40 \\
7.20\end{array}$ & $\begin{array}{r}386 \\
374 \\
2,020 \\
2,820 \\
2,820 \\
2,630\end{array}$ & $\begin{array}{r}10.80 \\
11.25 \\
10.10 \\
7.75 \\
6.50 \\
5.70\end{array}$ & $\begin{array}{r}7,770 \\
8,640 \\
6,470 \\
3,190 \\
2,020 \\
1,410\end{array}$ & $\begin{array}{l}3.99 \\
3.80 \\
3.60 \\
3.47 \\
3.38\end{array}$ & $\begin{array}{l}537 \\
500 \\
470 \\
410 \\
371 \\
344\end{array}$ & $\begin{array}{r}14.30 \\
14.58 \\
14.70 \\
14.85 \\
12.00 \\
9.80\end{array}$ & $\begin{array}{r}16,200 \\
17,000 \\
17,300 \\
17,700 \\
10,200 \\
5,990\end{array}$ & & & & \\
\hline $\begin{array}{l}1 \\
2 \\
3 \\
4 \\
5 \\
6\end{array}$ & $\begin{array}{l}6.60 \\
6.10 \\
5.40 \\
5.07 \\
4.95 \\
4.45\end{array}$ & $\begin{array}{r}2,100 \\
1,700 \\
1,220 \\
1,020 \\
965 \\
728\end{array}$ & $\begin{array}{l}4.97 \\
5.00 \\
4.17 \\
3.73 \\
3.59 \\
3.46\end{array}$ & $\begin{array}{r}975 \\
990 \\
603 \\
449 \\
407 \\
368\end{array}$ & $\begin{array}{l}3.31 \\
3.30 \\
3.29 \\
3.28 \\
3.26 \\
3.26\end{array}$ & $\begin{array}{l}323 \\
320 \\
318 \\
315 \\
310 \\
310\end{array}$ & $\begin{array}{c}10.60 \\
10.25 \\
10.2 \\
8.52 \\
10.95 \\
8.89\end{array}$ & $\begin{array}{l}7,390 \\
6,730 \\
6,640 \\
4,100 \\
8,050 \\
4,580\end{array}$ & & & & \\
\hline $\begin{array}{r}7 \\
8 \\
9 \\
10 \\
11 \\
4 \\
\end{array}$ & $\begin{array}{l}4.07 \\
3.58 \\
3.24 \\
3.12 \\
3.05 \\
3.01\end{array}$ & $\begin{array}{l}565 \\
404 \\
305 \\
277 \\
261 \\
252\end{array}$ & $\begin{array}{r}3.37 \\
3.51 \\
7.40 \\
12.10 \\
9.50 \\
13.70\end{array}$ & $\begin{array}{r}341 \\
383 \\
2,820 \\
, 10,400 \\
5,510 \\
14,500\end{array}$ & $\begin{array}{l}3.26 \\
3.26 \\
3.26 \\
7.95\end{array}$ & $\begin{array}{r}310 \\
310 \\
310 \\
3.420\end{array}$ & $\begin{array}{l}7.48 \\
6.52 \\
5.92 \\
5.55 \\
5.20\end{array}$ & $\begin{array}{l}2,900 \\
2,030 \\
1,560 \\
1,310 \\
1,100\end{array}$ & & & & \\
\hline
\end{tabular}

Supplemental record.- Mar. 2, 9:40 a.m., 15.25 ft., 19,000 se0.-ft. 
Benediot Canjon torm drain at Culver O1ty, Calle.

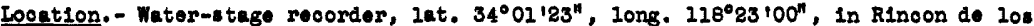

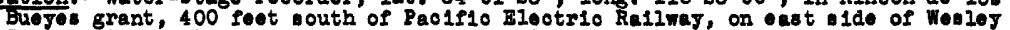
street, CuIver City, Ios Angeles County. Alt1tude, bout os feet.

Drainage areg.- 6.98 -quare m1108.

Gage-height record. - Water-atage reoorder graph.

D1scharge reoord.- D18charge through 90-inoh conorete pipe, oomputed by Kutter's rommin.

Maxtma, - 19388 D1echarge, 648 seoond-feet $7: 10$ a.m. Mar. 2 (gage he1ght, 7.0 foet). 1935-37: D1soharge, 248 vecond-feet Feb. 12, 1936.

Remarke. - Record good. No regulation or d1vers10ns. Recorde furmlshed by Ios Angeles County Flood Control District, through H. B. Hedger, chlef onglneer, and by Corpa of Inglneere, D. 8. Army.

Mean de11y d1echarge, in seoond-feet, 1838

\begin{tabular}{|lc|}
\hline February 28 & 128 \\
Maroh 1 & 65 \\
Maroh 2 & 308 \\
Maroh 3 & 91 \\
\hline Runoff, In acre-feet & 1,170 \\
\hline
\end{tabular}

Gage height, In feet, and discharge, in seoond-feet, at Indioated time, 1938

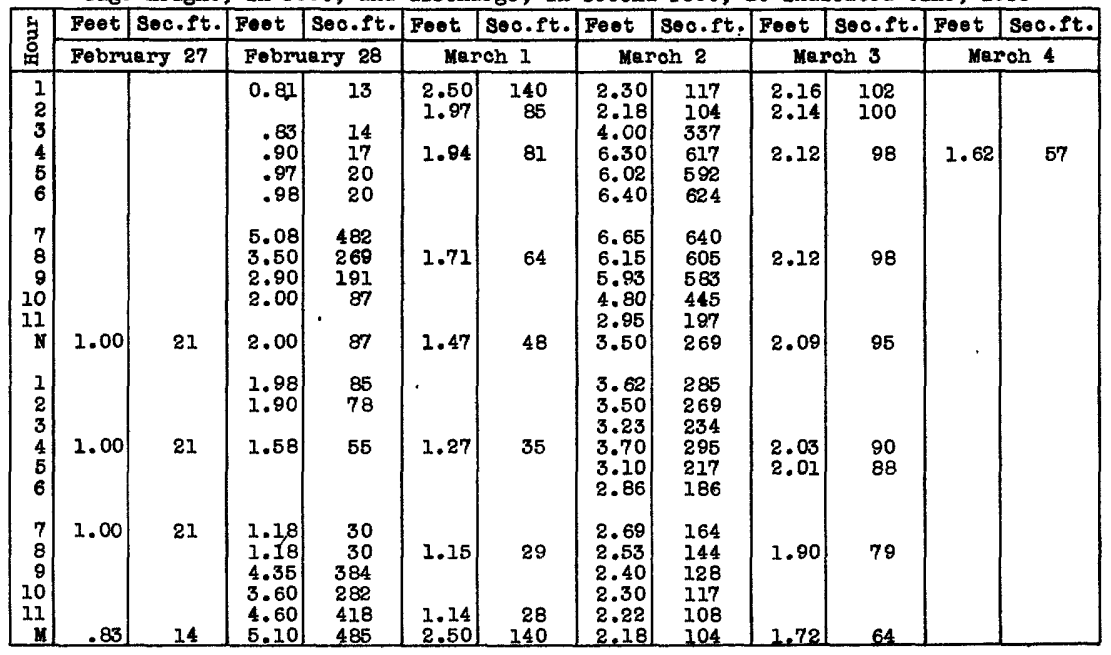

Supplementel record.- Mar. 2, 7:10 a.m., 7.0 ft., 648 sec.-ft. 
Topanga Crook Bas in

Topanga Creek near Topanga Beach, Cal1f.

Location. - Water-stage recorder, lat. $34^{\circ} 03^{\prime} 50^{n}$, long. $118^{\circ} 35^{\prime} 10^{n}$, in Boca de Santa Ionica grant, at highway briage, 2 miles north of Topanga Beach, Ios Angeles County. Altitude, about 270 peet.

Drainage area.- Area, 17.9 square miles. Average altitude, 1,355 feet. Maximum alt1tude, 2,477 feet. Average slope, 40 percent. Length of the main'stream channel, 6.4 miles. Average slope of the min stream channel, 5.2 percent.

Gage-height record. - Water-stage recorder graph.

Discharge record.- Stage-discharge relation defined by current-meter measurements be$10 \mathrm{w}$ 6,100 second-feet; extended above to s lope-area determination of peak discharge.

Maxima,- 1938: Discharge, 7,960 second-feet 9:40 a.m. Mar. 2 (grge he1ght, 11.20 feet). 1930-37: Discharge, 4,510 second-feet Dec. 31, 1933.

Remarks:- Records, except those for Feb. 27 to Mer. 4, furnished by Los Angeles County Flood Control District, through H. E. Hedger, chief engineer.

Mean da1ly discharge, in second-feet, 1938

\begin{tabular}{|c|c|c|c|c|c|c|c|c|c|c|c|}
\hline Day & Feb. & Mar. & Apr. & Day & Feb. & Mar. & Apr. & Day & Feb. & Mar. & Apr. \\
\hline $\begin{array}{r}1 \\
2 \\
3 \\
4 \\
5 \\
6 \\
7 \\
8 \\
9 \\
10\end{array}$ & $\begin{array}{c}213 \\
3.1 \\
135 \\
86 \\
48 \\
31 \\
18 \\
9 \\
49 \\
16\end{array}$ & $\begin{array}{r}501 \\
2,670 \\
269 \\
101 \\
63 \\
46 \\
36 \\
36 \\
33 \\
33\end{array}$ & $\begin{array}{l}6 \\
6 \\
5.5 \\
5.5 \\
5.5 \\
4.8 \\
5 \\
5 \\
5.5 \\
5.5\end{array}$ & $\begin{array}{l}11 \\
12 \\
13 \\
14 \\
15 \\
16 \\
17 \\
18 \\
19 \\
20\end{array}$ & $\begin{array}{c}239 \\
147 \\
63 \\
28 \\
16 \\
7 \\
5 \\
4.8 \\
5 \\
4.1\end{array}$ & $\begin{array}{r}49 \\
213 \\
209 \\
81 \\
53 \\
34 \\
21 \\
21 \\
22 \\
22\end{array}$ & $\begin{array}{l}6 \\
6 \\
6 \\
4.8 \\
4.8 \\
4.3 \\
3.4 \\
3.4 \\
3.4 \\
3.4\end{array}$ & $\begin{array}{l}21 \\
22 \\
23 \\
24 \\
25 \\
26 \\
27 \\
28 \\
29 \\
30 \\
31\end{array}$ & $\begin{array}{r}4.1 \\
3.9 \\
3.6 \\
3.3 \\
1.9 \\
1.9 \\
12 \\
643\end{array}$ & $\begin{array}{l}23 \\
23 \\
23 \\
21 \\
16 \\
13 \\
11 \\
10 \\
9 \\
7.5 \\
5.5\end{array}$ & $\begin{array}{l}3.4 \\
3.2 \\
2.6 \\
2.9 \\
3.2 \\
2.9 \\
3.4 \\
4.3 \\
4.6 \\
5.5\end{array}$ \\
\hline \multicolumn{9}{|c|}{ 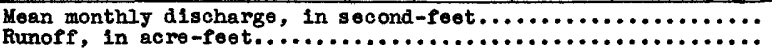 } & $\begin{array}{r}64.3 \\
3,570\end{array}$ & $\begin{array}{r}151 \\
9,270\end{array}$ & $\begin{array}{r}4.53 \\
270\end{array}$ \\
\hline
\end{tabular}

Discharge, in second-feet, at indicated time, 1938

\begin{tabular}{|c|c|c|c|c|c|c|}
\hline Hour & Fob. 27 & Feb. 28 & Nar. 1 & Mar. 2 & Mar. 3 & Mar. 4 \\
\hline $\begin{array}{l}1 \\
2 \\
3 \\
4 \\
5 \\
6\end{array}$ & $\begin{array}{l}8.5 \\
8.5 \\
8.4 \\
8.3 \\
8.4 \\
8.5\end{array}$ & $\begin{array}{r}28 \\
29 \\
35 \\
55 \\
113 \\
136\end{array}$ & $\begin{array}{r}2,090 \\
1,750 \\
1,720 \\
977 \\
732 \\
514\end{array}$ & $\begin{array}{r}134 \\
206 \\
259 \\
679 \\
1,700 \\
2,410\end{array}$ & $\begin{array}{l}464 \\
414 \\
376 \\
345 \\
334 \\
339\end{array}$ & $\begin{array}{l}148 \\
143 \\
137 \\
131 \\
128 \\
121\end{array}$ \\
\hline $\begin{array}{r}7 \\
8 \\
9 \\
10 \\
11 \\
\text { N }\end{array}$ & $\begin{array}{l}8.4 \\
8.3 \\
8.5 \\
13 \\
10 \\
14\end{array}$ & $\begin{array}{r}302 \\
449 \\
935 \\
1,730 \\
1,140 \\
793\end{array}$ & $\begin{array}{l}400 \\
325 \\
275 \\
233 \\
202 \\
169\end{array}$ & $\begin{array}{l}4,370 \\
4,160 \\
5,580 \\
7,020 \\
4,950 \\
4,525\end{array}$ & $\begin{array}{l}355 \\
307 \\
294 \\
278 \\
252 \\
240\end{array}$ & $\begin{array}{r}117 \\
112 \\
108 \\
104 \\
100 \\
96\end{array}$ \\
\hline $\begin{array}{l}1 \\
2 \\
3 \\
4 \\
5 \\
6\end{array}$ & $\begin{array}{l}18 \\
21 \\
23 \\
37 \\
41 \\
39\end{array}$ & $\begin{array}{l}546 \\
370 \\
278 \\
219 \\
185 \\
173\end{array}$ & $\begin{array}{l}149 \\
134 \\
123 \\
114 \\
109 \\
103\end{array}$ & $\begin{array}{l}6,490 \\
4,840 \\
4,490 \\
3,590 \\
2,420 \\
1,680\end{array}$ & $\begin{array}{l}238 \\
224 \\
217 \\
208 \\
202 \\
189\end{array}$ & $\begin{array}{l}92 \\
89 \\
85 \\
81 \\
78 \\
78\end{array}$ \\
\hline $\begin{array}{r}7 \\
8 \\
9 \\
10 \\
11 \\
M\end{array}$ & $\begin{array}{l}35 \\
33 \\
29 \\
29 \\
28 \\
26\end{array}$ & $\begin{array}{r}200 \\
355 \\
1,120 \\
2,940 \\
1,890 \\
2,810\end{array}$ & $\begin{array}{r}100 \\
95 \\
89 \\
89 \\
87 \\
94\end{array}$ & $\begin{array}{r}1,280 \\
1,020 \\
815 \\
669 \\
593 \\
526\end{array}$ & $\begin{array}{l}181 \\
175 \\
167 \\
162 \\
158 \\
155\end{array}$ & $\begin{array}{l}77 \\
75 \\
73 \\
71 \\
70 \\
70\end{array}$ \\
\hline
\end{tabular}

Supplemental record.- Mar. 2, 9:40 a.m., 11.20 ft., 7,960 sec. $-\mathrm{ft}$. 


\section{Malibu Creek Basin}

Malibu Creok at Crater Camp, near Calabasas, Calif.

Locat10n. - Water-stage recorder, lat. $34^{\circ} 04^{\prime} 30^{\prime \prime}$, 1ong. $118^{\circ} 42^{\prime} 10^{\prime \prime}$, in sift sec. 18, T. I S., R. 17 W., a quarter of a mile below Crater Camp, and 6 miles southwest of Calabasas. Altitude, about 460 feot.

Drainage area.- Area, 103 square miles. Average altitude, 1,226 feet. Maximum alt1tude, 3,05C feet. Average slope, 38 percent. Length of main stream channel, 17.5 miles. Average slope of the main stream channel, 6.9 percent.

Aage-he1ght record.- Water-stage recorder graph except Mar. 3-8.

Discharge record.- Stage-diacharge relation defined by current-meter measurements below 1,200 second-feet and extended above to estimated maximum discharge. Record Mar. 38 obtalned by comparison 1 th adjacent stations and one current-meter measurement

Maxima.- 1938: D1scharge, 10,000 second-feet 1 p.m. Mar. 2 (gage he1ght, 15.9 feet). 1931-37: D1scharge, 9,650 second-feet Jan. 1, 1934 (gage he1ght, 13.73 feet).

Remarks. - Records furn1shed by Los Angeles County Flood Control D1strict, through H. F. Hedger, chlef englneer.

Mean dally discharge, in second-feet, 1938

\begin{tabular}{|c|c|c|c|c|c|c|c|c|c|c|c|}
\hline Day & Feb. & Mar. & Apr. & Day & Feb. & Mar. & Apr. & Day & Feb. & Mar. & Apr. \\
\hline $\begin{array}{r}1 \\
2 \\
3 \\
4 \\
5 \\
6 \\
7 \\
8 \\
9 \\
10\end{array}$ & $\begin{array}{r}146 \\
38 \\
100 \\
86 \\
32 \\
26 \\
25 \\
25 \\
37 \\
28\end{array}$ & $\begin{array}{r}1,960 \\
5,090 \\
1,600 \\
609 \\
330 \\
250 \\
190 \\
150 \\
123 \\
98\end{array}$ & $\begin{array}{l}27 \\
25 \\
26 \\
24 \\
24 \\
22 \\
21 \\
19 \\
18 \\
20\end{array}$ & $\begin{array}{l}11 \\
12 \\
13 \\
14 \\
15 \\
16 \\
17 \\
18 \\
19 \\
20\end{array}$ & $\begin{array}{r}300 \\
185 \\
61 \\
42 \\
39 \\
29 \\
23 \\
22 \\
30 \\
13\end{array}$ & $\begin{array}{r}103 \\
835 \\
809 \\
509 \\
299 \\
190 \\
135 \\
109 \\
96 \\
87\end{array}$ & $\begin{array}{l}19 \\
19 \\
19 \\
18 \\
18 \\
18 \\
16 \\
15 \\
13 \\
13\end{array}$ & $\begin{array}{l}21 \\
22 \\
23 \\
24 \\
25 \\
26 \\
27 \\
28 \\
29 \\
30 \\
31\end{array}$ & $\begin{array}{r}11 \\
12 \\
12 \\
12 \\
12 \\
12 \\
19 \\
612\end{array}$ & $\begin{array}{l}77 \\
66 \\
60 \\
56 \\
56 \\
55 \\
53 \\
55 \\
57 \\
56 \\
41\end{array}$ & $\begin{array}{l}14 \\
14 \\
14 \\
13 \\
13 \\
13 \\
12 \\
12 \\
12 \\
11\end{array}$ \\
\hline \multicolumn{9}{|c|}{ 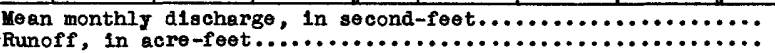 } & $\begin{array}{r}71.0 \\
3,950\end{array}$ & $\begin{array}{r}458 \\
28,170\end{array}$ & $\begin{array}{r}17.4 \\
1,040\end{array}$ \\
\hline
\end{tabular}

Gage helght, in feet, and discherge, in second-feet, at Indicated time, 1938

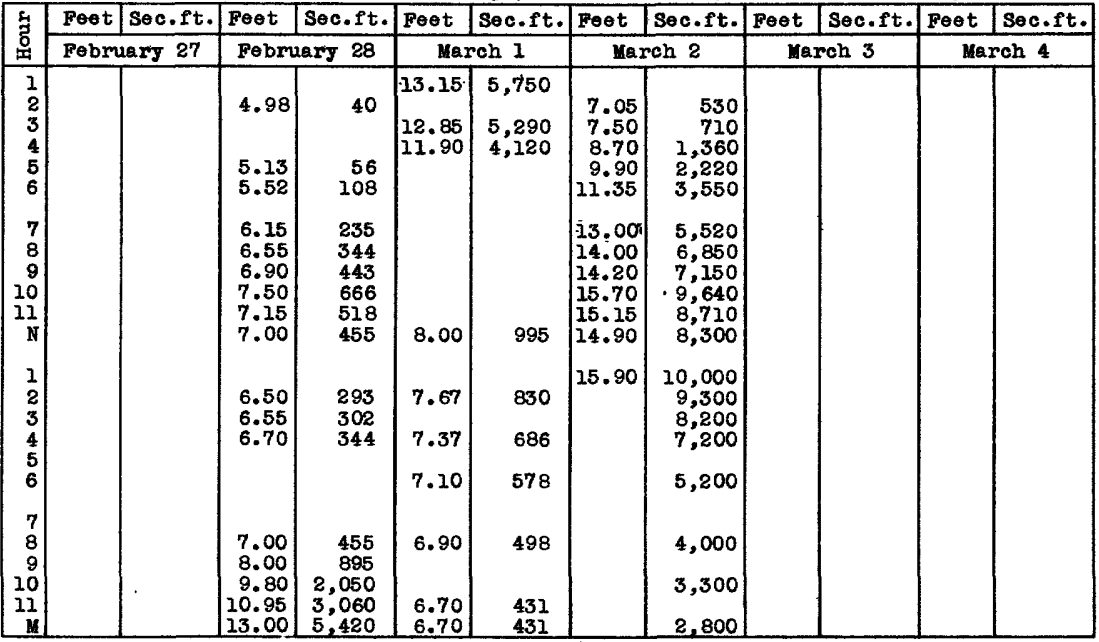




\section{Santa Clare River Basin}

\section{Santa Clara River noar Saugus, Calif.}

Location. - Water-8 tage recorder, lat. $34^{\circ} 25^{\prime} 42^{\prime \prime}$, long. $118^{\circ} 35^{\prime} 23^{\prime \prime}$, in San Franc1sco grant, on pler of old hlghway bridge, 3 milee west of Saugus, Los Angelos County.

Drainage area.- 355 square m1les.

Qege-he1ght record. - Water-stage recorder graph.

D1scharge record.- Stage-discharge relation poorly defined. Shifting-control corrections used throughout period. Discherge determined by comperison with adjacent stations Feb. 6-9, 13-26, Mar. 5-9, 17, 18. D1scharge Mar. 22 to Apr. 30 determined by Interpolation between discharge mossurements.

Maxima.- 1938: D1scharge, 24,000 second-feet (eatimated) 7:30 p.m. Mar. 2 . 1929-37: D1scharge, 3,870 second-fe日t Jan. 1, 1834 .

Remarks.- Slight regulation at reserrolrs in Bouquet and Dry Cenrons. Records furnishod by Los Angeles County Flood Control District, through H. F. Hedger, chlef engineer.

Mean da1ly discharge, in second-feet, 1938

\begin{tabular}{|r|c|c|c||c|c|c|c||c|c|c|c|}
\hline Daj & Feb. & Mar. & Apr. & Day & Fob. & Mar. & Apr. & Day & Fob. & Mar. & Apr. \\
\hline 1 & 38 & 349 & 51 & 11 & 175 & 80 & 11 & 21 & 0.9 & 89 & 9.5 \\
2 & .4 & 6,370 & 44 & 12 & 20 & 275 & 10 & 22 & .8 & 88 & 9.5 \\
3 & 58 & 2,310 & 37 & 13 & 1.5 & 513 & 10 & 23 & .7 & 87 & 9 \\
4 & 11 & 394 & 30 & 14 & 1.4 & 306 & 10 & 24 & .6 & 86 & 9 \\
5 & 5 & 102 & 24 & 15 & 1.3 & 99 & 10 & 25 & .5 & 85 & 9 \\
6 & 4.4 & 65 & 16 & 16 & 1.2 & 67 & 10 & 26 & .5 & 84 & 9 \\
7 & 4.4 & 84 & 11 & 17 & 1.1 & 43 & 10 & 27 & 2.0 & 84 & 9 \\
8 & 4.4 & 46 & 11 & 18 & 1.0 & 49 & 10 & 28 & 113 & 78 & 8.5 \\
9 & 4.4 & 46 & 11 & 19 & 1.1 & 68 & 10 & 29 & & 71 & 8.5 \\
10 & 4.4 & 67 & 11 & 20 & 1.0 & 84 & 9.5 & 30 & & 64 & 8.5 \\
\end{tabular}

Discharge, in second-feet, at indicated time, 1938

\begin{tabular}{|c|r|r|}
\hline Eour & March 2 & Karch 3 \\
\hline 1 & 100 & 4,700 \\
2 & 150 & 3,900 \\
3 & 300 & 3,500 \\
4 & 450 & 3,000 \\
5 & 450 & 2,800 \\
6 & 900 & 2,600 \\
7 & 1,350 & 2,650 \\
8 & 1,600 & 2,650 \\
9 & 1,650 & 2,500 \\
10 & 1,800 & 2,400 \\
11 & 2,200 & 2,300 \\
N & 2,700 & 2,200 \\
& & \\
1 & 3,100 & 2,100 \\
2 & 5,200 & 2,000 \\
3 & 5,900 & 2,000 \\
4 & 11,300 & 1,950 \\
5 & 16,000 & 1,550 \\
6 & 21,500 & 1,400 \\
7 & 23,400 & 1,300 \\
8 & 20,000 & 1,200 \\
9 & 12,500 & 1,150 \\
10 & 10,000 & 1,050 \\
11 & 7,000 & 1,000 \\
M & 5,500 & 1,000 \\
\hline
\end{tabular}

Supplemental records.- Mar, 2, 7.830 p.m., 24,000 sec.-ft. 
P1ru Creek near P1m, Cal1f.

Loot1 ion. - Water-stage recorder, lat. $34^{\circ} 25^{1} 30^{\prime \prime}$, long. $118^{\circ} 45^{\prime} 45^{\prime \prime}$, In outhern part of yomocal grant, ebout 1-3/4 miles northeast of P1ru, Vonture County, and 2 miles uptream Irom junotion with Santa Clare RIver. Altitude, about 780 feet.

Drainage erea.- Area, 432 square miles. Average ltitude, 4,200 seet. Maximum alt1tude, 8,800 feet. Average slope, 35.5 percent. Iongth of mein stream ohannel, 54 m110s. Average lope of main atream channel, 2.8 percent.

Gege-he1 ght record.- Nater-stage reoorder graph except $12 \mathrm{~m}$. Kar. 2 to $12 \mathrm{m.}$ Mar. 4.

Disoharge reoord.- Stage-d1soharge relation defined by ourrent-meter moasurements belor 8,110 seoond-foet ; extended logar fthmionlly to peak disoharge determined by alopearea mothod. Shifting-control correotions applied during period of reoord. Dis oharge from $12 \mathrm{~m}$. Mar. 2 to $12 \mathrm{~m}$. Mar. 4 and lar. 13-21 dotermined from comparative hrdrograph based on reoorde of Santa Pavle Creek near Sante Paula and Coyote Oreek noar venture.

Kaxima. - 19388 D1soharge, 35,600 second-feot 1:30 p.m. Mas. 2. 1911-13, 1927-37; Discharge, 15,800 vecond-feet Feb. 9, 1932.

Remarks.- Records falr. Flood flow not affectod by diveralons. Station maintained in cooperation with Venture County Water survey.

Mean dally discharge, in second-feet, 1938

\begin{tabular}{|c|c|c|c|c|c|c|c|c|c|c|c|}
\hline Day & Feb. & Mar. & Apr. & Day & Feb. & Mar. & Apr. & Day & Pob. & Mar. & Apr. \\
\hline $\begin{array}{r}1 \\
8 \\
3 \\
4 \\
5 \\
6 \\
7 \\
8 \\
8 \\
10\end{array}$ & $\begin{array}{r}657 \\
159 \\
655 \\
440 \\
192 \\
95 \\
45 \\
37 \\
199 \\
294\end{array}$ & $\begin{array}{r}5,510 \\
13,140 \\
4,980 \\
2,110 \\
1,560 \\
1,150 \\
774 \\
742 \\
678 \\
618\end{array}$ & $\begin{array}{l}232 \\
221 \\
221 \\
221 \\
224 \\
224 \\
217 \\
215 \\
210 \\
202\end{array}$ & $\begin{array}{l}11 \\
12 \\
13 \\
14 \\
15 \\
16 \\
17 \\
18 \\
19 \\
20\end{array}$ & $\begin{array}{r}2,610 \\
681 \\
418 \\
300 \\
245 \\
208 \\
188 \\
188 \\
233 \\
181\end{array}$ & $\begin{array}{r}855 \\
1,530 \\
819 \\
750 \\
700 \\
620 \\
550 \\
580 \\
490 \\
450\end{array}$ & $\begin{array}{l}192 \\
195 \\
198 \\
201 \\
194 \\
165 \\
150 \\
138 \\
133 \\
126\end{array}$ & $\begin{array}{l}21 \\
22 \\
23 \\
24 \\
85 \\
26 \\
27 \\
28 \\
29 \\
30 \\
31\end{array}$ & $\begin{array}{r}160 \\
150 \\
130 \\
127 \\
120 \\
111 \\
114 \\
1,610\end{array}$ & $\begin{array}{l}400 \\
395 \\
405 \\
400 \\
375 \\
347 \\
317 \\
288 \\
273 \\
256 \\
242\end{array}$ & $\begin{array}{l}125 \\
124 \\
124 \\
118 \\
127 \\
126 \\
118 \\
120 \\
120 \\
128\end{array}$ \\
\hline \multicolumn{9}{|c|}{ 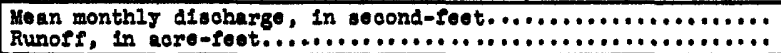 } & $\begin{array}{r}376 \\
20.910\end{array}$ & $\begin{array}{r}1,356 \\
83,350\end{array}$ & 10,180 \\
\hline
\end{tabular}

Gage holght, in feot, and discharge, in second-feot, at indicated time, 1958

\begin{tabular}{|c|c|c|c|c|c|c|c|c|c|c|c|}
\hline \multirow{2}{*}{ 量 } & Feet Beo. It. & Feet & sec.ft. & Feet & seo.ft. & Feet & Boo.ft. & Foet & seceft. & Feet & Beo.ft. \\
\hline & Fobruary 27 & \multicolumn{2}{|c|}{ Februaxy 28} & \multicolumn{2}{|c|}{ Maroh 1} & \multicolumn{2}{|c|}{ Maroh 2} & \multicolumn{2}{|c|}{ Maroh 8} & \multicolumn{2}{|c|}{ Maroh 4} \\
\hline $\begin{array}{l}1 \\
8 \\
3 \\
4 \\
5 \\
6\end{array}$ & & $\begin{array}{l}3.00 \\
3.13 \\
3.46\end{array}$ & $\begin{array}{l}134 \\
177 \\
337\end{array}$ & $\begin{array}{l}6.70 \\
6.46 \\
6.25 \\
6.00 \\
6.15 \\
6.80\end{array}$ & $\begin{array}{l}6,700 \\
6,380 \\
5,960 \\
5,680 \\
5,960 \\
7,450\end{array}$ & $\begin{array}{l}4.57 \\
4.76 \\
5.10 \\
5.35 \\
5.87 \\
6.70\end{array}$ & $\begin{array}{l}4,440 \\
4,790 \\
5,460 \\
6,380 \\
7,690 \\
9,900\end{array}$ & & $\begin{array}{l}7,000 \\
6,600 \\
7,500\end{array}$ & & 2,300 \\
\hline $\begin{array}{r}7 \\
8 \\
9 \\
10 \\
11 \\
\mathbb{1 1}\end{array}$ & & $\begin{array}{l}3.55 \\
3.65 \\
3.93 \\
3.82 \\
3.92 \\
4.03\end{array}$ & $\begin{array}{r}424 \\
605 \\
1,100 \\
964 \\
1,220 \\
1,620\end{array}$ & $\begin{array}{l}7.15 \\
6.70 \\
6.25 \\
5.94 \\
5.65 \\
5.45\end{array}$ & $\begin{array}{l}8,480 \\
7,650 \\
6,600 \\
6,140 \\
5,560 \\
5,150\end{array}$ & $\begin{array}{r}7.30 \\
7.60 \\
7.80 \\
8.10 \\
9.15 \\
11.70\end{array}$ & $\begin{array}{l}11,600 \\
12,000 \\
12,800 \\
13,800 \\
17,800 \\
25,900\end{array}$ & & $\begin{array}{l}6,800 \\
5,800 \\
5,400\end{array}$ & & 8,130 \\
\hline $\begin{array}{l}7 \\
8 \\
8 \\
1 \\
5 \\
6\end{array}$ & & $\begin{array}{l}4.08 \\
3.96 \\
3.98 \\
3.97 \\
3.93 \\
3.92\end{array}$ & $\begin{array}{l}1,610 \\
1,520 \\
1,550 \\
1,540 \\
1,490 \\
1,480\end{array}$ & $\begin{array}{l}5.25 \\
5.10 \\
4.90 \\
4.85 \\
4.75 \\
4.65\end{array}$ & $\begin{array}{l}4,980 \\
4,860 \\
4,680 \\
4,680 \\
4,590 \\
4,400\end{array}$ & 14.00 & $\begin{array}{l}34,600 \\
30,000 \\
28,000 \\
18,000 \\
14,000 \\
18,000\end{array}$ & & $\begin{array}{l}4,300 \\
3,600 \\
3,500\end{array}$ & & 2,010 \\
\hline $\begin{array}{r}7 \\
8 \\
9 \\
10 \\
11 \\
\end{array}$ & & $\begin{array}{l}4.08 \\
4.19 \\
4.53 \\
5.20 \\
6.80 \\
7.10 \\
\end{array}$ & $\begin{array}{l}1,610 \\
1,850 \\
2,340 \\
3,600 \\
5,460 \\
7,650 \\
\end{array}$ & $\begin{array}{l}4.56 \\
4.51 \\
4.48 \\
4.44 \\
4.45 \\
4.48\end{array}$ & $\begin{array}{l}4,320 \\
4,240 \\
4,310 \\
4,230 \\
4,250 \\
4,310 \\
\end{array}$ & & $\begin{array}{r}11,000 \\
9,800 \\
9,800 \\
8,800 \\
9,000 \\
7,500\end{array}$ & & $\begin{array}{l}3,300 \\
3,000 \\
2,700\end{array}$ & & \\
\hline
\end{tabular}

Supplementel record.- Har. 2,1830 p.m., 35,600 sec. -ft. 
Sespe Creok near F1llmore, Calif.

Location. - Water-stage recorder, lat. $34^{\circ} 26^{\prime} 55^{\prime \prime}$, long. $118^{\circ} 55^{\prime} 35^{\prime \prime}$, in NEt $800,12, T$. 4 H., R. 20 W., $0.1 \mathrm{mile}$ downstream from junction w1th I1ttle Sespe Creok, and $3 \frac{1}{8}$ mlles north of Filimore. Alt1tude, about 590 foet.

Dralnage area.- Area, 254 square mlles. Average alt1tude, 4,040 feot. Maximum alt1tude, 7,480 feet. Average slope, 37 percent. Length of main stream channel, 44 miles. Average slope main stream channel, 2.9 percent.

Gage-he1ght record. - Water-stage rocorder graph Feb. 1-11; no rocerd Feb. 12 to Apr. 30.

D1scharge record.- Stage-d1scharge relation defined by current-meter asurements below 2,160 second-feet; extended logarlthmlcally to peak discharge determined by slopearea method. Shifting-control corrections a pplied for period of record. Discharge for Fob. 12 to Apr. 30 determined from comparative hydrograph based on Santa Paula Oreek record, nine current-meter measurements, and slcpe-area determination of peak dis charge.

Max1ma. - 1938: D1scharge, 56,000 second-feet about 2 p.m. Mar. 2 . 1927-37: Diacharge, about 34,000 second-feet Dec. 31, 1933.

Remarks, - Records +falr except those for Feb. 1-11, whlch are good. No diversions above gage. Station maintained in cooperation with Ventura County Water Survey.

Mean dafly discherge, in second-feet, 1938

\begin{tabular}{|r|r|r|r||r|r|r|r|r||r|r|r|r|}
\hline Day & Fob. & Har. & Apr. & Day & Fob. & Mar. & Apr. & Day & Fob. & Mar. & Apr. \\
\hline 1 & 2,560 & 3,800 & 370 & 11 & 10,680 & 1,810 & 244 & 21 & 430 & 775 & 177 \\
2 & 301 & 14,840 & 350 & 12 & 2,160 & 4,410 & 230 & 22 & 384 & 700 & 177 \\
3 & 4,320 & 7,090 & 340 & 13 & 970 & 3,000 & 220 & 23 & 344 & 640 & 177 \\
4 & 1,620 & 4,110 & 340 & 14 & 758 & 2,100 & 210 & 24 & 290 & 586 & 177 \\
5 & 672 & 2,920 & 339 & 15 & 660 & 1,600 & 200 & 25 & 261 & 550 & 180 \\
6 & 396 & 2,350 & 328 & 16 & 600 & 1,290 & 193 & 26 & 257 & 520 & 176 \\
7 & 354 & 2,120 & 314 & 17 & 580 & 1,110 & 185 & 27 & 262 & 490 & 174 \\
8 & 343 & 2,080 & 295 & 18 & 566 & 960 & 180 & 28 & 1,570 & 460 & 172 \\
9 & 956 & 1,840 & 277 & 19 & 572 & 880 & 177 & 29 & & 435 & 171 \\
20 & 1,400 & 1,600 & 260 & 20 & 480 & 820 & 177 & 30 & & 418 & 170 \\
\end{tabular}


Sante Paula Greek near Santa Paula, Callf.

Location. - Water-stage recorder, lat. $34^{\circ} 23^{\prime} 40^{n}$, long. $119^{\circ} 04^{\prime} 35^{\prime \prime}$, near east boundary of $\mathrm{kx}$ Mission San Buenaventura grant, 50 feet upstream from Santa Paula Water Works diversion dam, and about 3 miles north of Santa Paula, Ventura County. Altitude, about 650 feet.

Drainage area.- Area, 39.8 square miles. Average altitude, 3,220 feet. Maximum altitude, 6,704 feet. Average slope, 44 percent. Iength of ma in stream chamel, 10 miles. Average slope of main stream channel, 11 percent.

Gage-helght record.- Tater-stage recorder graph except 6 p.m. Mar. 2 to 6 p.m. Mar. 4, and $1 \mathrm{p.m.} \mathrm{Mar.} 12$ to $1 \mathrm{p.m}$. Mar. 14. Gage heights interpolated $6 \mathrm{p.m.} \mathrm{to} 12 \mathrm{p.m}$. Mar. 2 and 1 p.m. Mar. 12 to 1 p.m. Mar. 14.

Discharge record.- Stage-discharge relation defined by current-meter measurements below 575 second-feet; extended logarithmically to peak discharge determined by slope-ares method. Shifting-control corrections applied to gage-height record. Discharge Mar. 3 and 4 determined from comparative hydrograph.

Maxima.- 1938: Discharge, 13,500 second-feet 2 p.m. Mar. 2 (gage height, 10.56 feet). 1927-37: Discharge, about 10,000 second-feet Dec. $31,1933$.

Remarks. - Records good except those for Mar. 2-4 and 12-14, which are fair. Flood flow not affected by diversion above gage. Station maintained in cooperation with Ventura County Water Survey.

Mean dally discharge, in second-feet, 1938

\begin{tabular}{|c|c|c|c|c|c|c|c|c|c|c|c|}
\hline Day & Peb. & Mar. & Apr. & Day & $\mathrm{Feb}$. & Mar. & Apr. & Day & $\mathrm{P} \in \mathrm{b}$. & Mar. & Apr. \\
\hline $\begin{array}{r}1 \\
2 \\
3 \\
4 \\
5 \\
6 \\
7 \\
8 \\
9 \\
10\end{array}$ & $\begin{array}{r}287 \\
48 \\
819 \\
312 \\
120 \\
90 \\
81 \\
79 \\
144 \\
166\end{array}$ & $\begin{array}{r}683 \\
3,040 \\
1,100 \\
639 \\
434 \\
339 \\
299 \\
299 \\
269 \\
247\end{array}$ & $\begin{array}{l}90 \\
81 \\
79 \\
79 \\
77 \\
72 \\
68 \\
66 \\
61 \\
61\end{array}$ & $\begin{array}{l}11 \\
12 \\
13 \\
14 \\
15 \\
16 \\
17 \\
18 \\
19 \\
20\end{array}$ & $\begin{array}{r}771 \\
384 \\
201 \\
155 \\
108 \\
95 \\
95 \\
90 \\
98 \\
88\end{array}$ & $\begin{array}{l}299 \\
754 \\
565 \\
400 \\
326 \\
280 \\
247 \\
225 \\
211 \\
201\end{array}$ & $\begin{array}{l}59 \\
58 \\
53 \\
48 \\
45 \\
44 \\
43 \\
42 \\
42 \\
45\end{array}$ & $\begin{array}{l}21 \\
22 \\
23 \\
24 \\
25 \\
26 \\
27 \\
28 \\
29 \\
30 \\
31 \\
\end{array}$ & $\begin{array}{r}85 \\
81 \\
77 \\
66 \\
62 \\
61 \\
66 \\
410\end{array}$ & $\begin{array}{l}196 \\
174 \\
151 \\
140 \\
133 \\
124 \\
117 \\
111 \\
108 \\
101 \\
95 \\
\end{array}$ & $\begin{array}{l}44 \\
44 \\
43 \\
45 \\
51 \\
44 \\
42 \\
39 \\
43 \\
44\end{array}$ \\
\hline tear & in & & 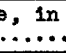 & & & & & & $\begin{array}{r}184 \\
10,190 \\
\end{array}$ & $\begin{array}{r}397 \\
24,410 \\
\end{array}$ & $\begin{array}{r}55.1 \\
3,280 \\
\end{array}$ \\
\hline
\end{tabular}

Gage height, in feet, and discharge, in second-feet, at indicated time, 1938

\begin{tabular}{|c|c|c|c|c|c|c|c|c|c|c|c|c|}
\hline \multirow{2}{*}{ 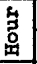 } & Feet & sec.ft. & Peet & Sec.ft. & Feet & sec.ft. & Feet & sec, ft. & Feet & Sec.ft. & Feet & Sec.ft. \\
\hline & \multicolumn{2}{|c|}{ Pebruary 27} & \multicolumn{2}{|c|}{ February 28} & \multicolumn{2}{|c|}{ March 1} & \multicolumn{2}{|c|}{ March 2} & \multicolumn{2}{|c|}{ March 3} & \multicolumn{2}{|c|}{ March 4} \\
\hline $\begin{array}{l}1 \\
2 \\
3 \\
4 \\
5 \\
6\end{array}$ & & & $\begin{array}{l}2.58 \\
2.58 \\
2.59 \\
2.63 \\
2.75 \\
2.84\end{array}$ & $\begin{array}{r}68 \\
68 \\
70 \\
79 \\
111 \\
140\end{array}$ & $\begin{array}{l}4.50 \\
4.22 \\
4.05 \\
3.95 \\
3.85 \\
3.75\end{array}$ & $\begin{array}{r}1,540 \\
1,230 \\
1,060 \\
960 \\
865 \\
778\end{array}$ & $\begin{array}{l}3.60 \\
3.75 \\
3.95 \\
4.45 \\
4.90 \\
4.93\end{array}$ & $\begin{array}{r}650 \\
778 \\
960 \\
1,480 \\
2,000 \\
2,040\end{array}$ & & $\begin{array}{l}1,060 \\
1,030 \\
1,350 \\
1,550 \\
1,780 \\
1,500\end{array}$ & & 750 \\
\hline $\begin{array}{r}7 \\
8 \\
9 \\
10 \\
11 \\
\mathbf{4}\end{array}$ & & & $\begin{array}{l}2.95 \\
3.12 \\
3.07 \\
3.07 \\
3.10 \\
3.15\end{array}$ & $\begin{array}{l}182 \\
275 \\
247 \\
247 \\
264 \\
299\end{array}$ & $\begin{array}{l}3.70 \\
3.65 \\
3.60 \\
3.55 \\
3.50 \\
3.46\end{array}$ & $\begin{array}{l}735 \\
692 \\
650 \\
610 \\
570 \\
538\end{array}$ & $\begin{array}{l}4.80 \\
4.72 \\
4.80 \\
5.50 \\
7.12 \\
7.25\end{array}$ & $\begin{array}{l}1,880 \\
1,780 \\
1,880 \\
2,810 \\
5,540 \\
5,800\end{array}$ & & $\begin{array}{l}1,160 \\
1,120 \\
1,180 \\
1,210 \\
1,150 \\
1,090\end{array}$ & & 660 \\
\hline $\begin{array}{l}1 \\
2 \\
3 \\
4 \\
5 \\
6\end{array}$ & & & $\begin{array}{l}3.15 \\
3.23 \\
3.20 \\
3.15 \\
3.12 \\
3.10\end{array}$ & $\begin{array}{l}299 \\
352 \\
332 \\
299 \\
280 \\
275\end{array}$ & $\begin{array}{l}3.42 \\
3.40 \\
3.38 \\
3.35 \\
3.33 \\
3.31\end{array}$ & $\begin{array}{l}506 \\
490 \\
478 \\
455 \\
441 \\
427\end{array}$ & $\begin{array}{r}8.55 \\
10.56 \\
7.95 \\
6.00 \\
5.00 \\
4.35\end{array}$ & $\begin{array}{r}8,450 \\
13,500 \\
7,800 \\
4,050 \\
2,530 \\
1,700\end{array}$ & & $\begin{array}{r}1,060 \\
1,030 \\
1,000 \\
970 \\
950 \\
920\end{array}$ & & 600 \\
\hline $\begin{array}{r}7 \\
8 \\
9 \\
10 \\
11 \\
4\end{array}$ & & & $\begin{array}{l}3.10 \\
3.25 \\
3.72 \\
4.48 \\
4.90 \\
4.98\end{array}$ & $\begin{array}{r}275 \\
372 \\
735 \\
1,507 \\
1,990 \\
2,104\end{array}$ & $\begin{array}{l}3.30 \\
3.28 \\
3.26 \\
3.28 \\
3.32 \\
3.40\end{array}$ & $\begin{array}{l}420 \\
406 \\
393 \\
406 \\
434 \\
490\end{array}$ & $\begin{array}{l}4.17 \\
4.05 \\
3.97 \\
3.88 \\
3.82 \\
3.77\end{array}$ & $\begin{array}{l}1,510 \\
1,380 \\
1,290 \\
1,190 \\
1,130 \\
1,080\end{array}$ & & $\begin{array}{l}900 \\
880 \\
860 \\
840 \\
820 \\
800\end{array}$ & & 545 \\
\hline
\end{tabular}


Ventura R17or Besin

Mat111 Ja Orook at Mat111ja, Oal1s.

Location. - Water-stage rooorder, lat. $34^{\circ} 29^{\prime} 05^{n}$, long. $118^{\circ} 18^{\prime} 30^{\prime \prime}$, in NEt s00. $29, \mathrm{~T}$. 5 H., R. 23 W., half a mlle northwest of Matilifa. Alt1tude, about 970 foet.

Drainege area.- Area, 55 square m1les. Average elt1tude, 3,630 soot. Maximim alt1tude, 6,025 foet. Average slope, 47 peroent. Length of main stream channel, 13.7 m1les. A perage lope of maln stroam channel, 6.8 percent.

Gage-helght reoord.- Water-8tage recorder graph excopt $10 \mathrm{a.m.} \mathrm{Mar.} 2$ to Mar. 4 and Mer. 28 to sopt. 30 .

D18oharge reocrd. - 8tage-d1scharge relation defsned by current-meter measurements belor 800 second-Ieet; extended to B lope-area determination of peak dischargo. Discharge 10 a.m. Mar. 2 to Mar. 4 and Mar. 28 to Bept. 30 based on 43 discherge measurement: and comparativo hydrograph.

Max1me.- 1838: D1scharge, 15,800 socond-feet 12830 p.m. Mar. 2. 1927-37: Discharge, 7,000 second-feet Dec. 31, 1833 (gage he1ght, 7.20 foet).

Remarks.- Records good, except those for Mar. 2-4, and Mar. 28 to Apr. 30, which exe Ialr. No diverolons above gage. Station mainteined in cooperation with Ventura County Water Burvey.

Moan deily discharge, in second-seet, 1938

\begin{tabular}{|c|c|c|c|c|c|c|c|c|c|c|c|}
\hline Day & Fob. & Mer. & Apr. & Dey & Feb. & Lar. & Apr. & Day & Fob. & Mar. & Apr. \\
\hline $\begin{array}{r}1 \\
2 \\
3 \\
4 \\
5 \\
6 \\
7 \\
8 \\
9 \\
10\end{array}$ & $\begin{array}{r}427 \\
66 \\
516 \\
268 \\
155 \\
111 . \\
80 \\
81 \\
235 \\
318\end{array}$ & $\begin{array}{r}888 \\
4,850 \\
2,120 \\
1,280 \\
1,270 \\
816 \\
765 \\
786 \\
694 \\
632\end{array}$ & $\begin{array}{l}170 \\
160 \\
150 \\
147 \\
143 \\
137 \\
129 \\
128 \\
116 \\
112\end{array}$ & $\begin{array}{l}11 \\
12 \\
13 \\
14 \\
15 \\
16 \\
17 \\
18 \\
18 \\
20\end{array}$ & $\begin{array}{r}1,870 \\
766 \\
408 \\
338 \\
268 \\
217 \\
191 \\
188 \\
175 \\
157\end{array}$ & $\begin{array}{r}792 \\
1,860 \\
1,350 \\
816 \\
544 \\
480 \\
445 \\
412 \\
377 \\
356\end{array}$ & $\begin{array}{r}109 \\
105 \\
96 \\
89 \\
83 \\
79 \\
76 \\
76 \\
76 \\
82\end{array}$ & $\begin{array}{l}21 \\
28 \\
23 \\
24 \\
85 \\
26 \\
27 \\
28 \\
28 \\
30 \\
31\end{array}$ & $\begin{array}{l}143 \\
134 \\
123 \\
115 \\
113 \\
113 \\
111 \\
410\end{array}$ & $\begin{array}{l}332 \\
313 \\
284 \\
276 \\
262 \\
245 \\
232 \\
220 \\
205 \\
182 \\
180\end{array}$ & $\begin{array}{l}82 \\
82 \\
88 \\
88 \\
95 \\
86 \\
86 \\
83 \\
83 \\
79\end{array}$ \\
\hline & & & & & & & & & $\begin{array}{r}293 \\
16,260\end{array}$ & $\begin{array}{r}784 \\
48,170\end{array}$ & $\begin{array}{r}105 \\
6,150\end{array}$ \\
\hline
\end{tabular}

Gage holght, In soet, and discharge, in second-seot, at indioated time, 1938

\begin{tabular}{|c|c|c|c|c|c|c|c|c|c|c|c|c|}
\hline \multirow{2}{*}{ है } & Foet & sec.ft. & Foot & 800.ft. & Foet & sec.ft. & Feet & sec.ft. & Feet & sec.st. & Feet & sec.ft. \\
\hline & \multicolumn{2}{|c|}{ Fobruary 27} & \multicolumn{2}{|c|}{ Febmuary 28} & \multicolumn{2}{|c|}{ March 1} & \multicolumn{2}{|c|}{ Merch 2} & \multicolumn{2}{|c|}{ March 3} & \multicolumn{2}{|c|}{ Merch 4} \\
\hline $\begin{array}{l}1 \\
2 \\
3 \\
4 \\
5 \\
6\end{array}$ & & & $\begin{array}{l}2.94 \\
2.94 \\
2.95 \\
3.00 \\
3.06 \\
3.14\end{array}$ & $\begin{array}{l}109 \\
109 \\
111 \\
123 \\
136 \\
157\end{array}$ & $\begin{array}{l}5.60 \\
5.20 \\
4.95 \\
4.80 \\
4.55 \\
4.40\end{array}$ & $\begin{array}{r}1,840 \\
1,500 \\
1,270 \\
1,140 \\
948 \\
840\end{array}$ & $\begin{array}{l}4.35 \\
4.70 \\
5.07 \\
5.35 \\
6.30 \\
6.75\end{array}$ & $\begin{array}{r}808 \\
1,070 \\
1,410 \\
1,700 \\
2,810 \\
3,640\end{array}$ & & $\begin{array}{l}2,450 \\
2,350 \\
2,700 \\
3,600 \\
3,800 \\
2,800\end{array}$ & & 1,420 \\
\hline $\begin{array}{r}7 \\
.8 \\
9 \\
10 \\
11 \\
11\end{array}$ & & & $\begin{array}{l}3.19 \\
3.22 \\
3.23 \\
3.24 \\
3.24 \\
3.24\end{array}$ & $\begin{array}{l}170 \\
180 \\
183 \\
188 \\
188 \\
188\end{array}$ & $\begin{array}{l}4.35 \\
4.35 \\
4.40 \\
4.43 \\
4.43 \\
4.46\end{array}$ & $\begin{array}{l}808 \\
808 \\
856 \\
880 \\
888 \\
810\end{array}$ & $\begin{array}{l}6.40 \\
6.65 \\
6.50\end{array}$ & $\begin{array}{r}2,920 \\
3,380 \\
3,070 \\
7,000 \\
13,000 \\
15,000\end{array}$ & & $\begin{array}{l}2,400 \\
2,200 \\
2,120 \\
2,050 \\
2,000 \\
1,940\end{array}$ & & 1,310 \\
\hline $\begin{array}{l}1 \\
2 \\
3 \\
4 \\
5 \\
6\end{array}$ & & & $\begin{array}{l}3.23 \\
3.22 \\
3.21 \\
3.20 \\
3.20 \\
3.18\end{array}$ & $\begin{array}{l}188 \\
185 \\
183 \\
183 \\
183 \\
183\end{array}$ & $\begin{array}{l}4.30 \\
4.15 \\
4.10 \\
4.07 \\
4.02 \\
3.98\end{array}$ & $\begin{array}{l}793 \\
694 \\
670 \\
654 \\
626 \\
597\end{array}$ & & $\begin{array}{r}14,000 \\
8,500 \\
6,500 \\
5,500 \\
4,600 \\
4,100\end{array}$ & & $\begin{array}{l}1,880 \\
1,830 \\
1,780 \\
1,740 \\
1,700 \\
1,670\end{array}$ & & 1,230 \\
\hline $\begin{array}{r}7 \\
8 \\
9 \\
10 \\
11 \\
4\end{array}$ & & & $\begin{array}{l}3.25 \\
3.50 \\
4.15 \\
5.50 \\
6.30 \\
6.20 \\
\end{array}$ & $\begin{array}{r}202 \\
287 \\
597 \\
1,760 \\
2,700 \\
2,570 \\
\end{array}$ & $\begin{array}{l}3.92 \\
3.89 \\
3.93 \\
3.95 \\
4.05 \\
4.20\end{array}$ & $\begin{array}{l}561 \\
538 \\
561 \\
573 \\
626 \\
712 \\
\end{array}$ & & $\begin{array}{l}3,700 \\
3,400 \\
3,100 \\
2,800 \\
2,700 \\
2,550 \\
\end{array}$ & & $\begin{array}{l}1,630 \\
1,600 \\
1,570 \\
1,540 \\
1,510 \\
1,490\end{array}$ & & 1,180 \\
\hline
\end{tabular}

Supplemental record.- Mar. 2, $12: 30$ p.m., 15,900 sec.-ft. 
Ventura River noar Ventura, Calif.

Iocat10n.- Water-itage recorder, let. $34^{\circ} 20^{\prime} 55^{\prime \prime}$, Iong. $118^{\circ} 18^{\prime} 20^{n}$, in southeast corner or santi Ans grant, at h1ghway bridgo et entranse to Foster Momorial Park, auarter of m110 downstream from Ventura diversion dam and Ooyote Oreek, and 5 mIles north of Venture, Venture County. Altstude, bout 210 feet.

Draingze area.- Aroa, 187 square miles. Avorage altitude, 2,310 foot. Haximum alt1tude, 6,025 foet. Averege lope, 48 percent. Length of main tream channel, 23.7 m1les. Averege slope of ma in etream channel, 5 percent.

Gege-helght reoord. - Water-g tage recorder graph exoept 1 p.m. Mar. 2 to 9:30 a.m. Mar. 3. Polk gago holght observod.

Discharge reoord. - Stege-discherge relation defined by ourrent-meter measurements belor 6,600 second-feet; extended to alope-area deternination of peak d1scharge. Dlacharge 1 p.m. Mer. 2 to 9830 .m. Mar. 3 based on hrdrograph.

Max1ma.- 1938: D1soharge, 39,200 socond-foot 2:15 p.m. Uar. 2 (gage helght 19.2 foet). 1811-13, 1829-37: D socharge, 23,000 second-foet Dec. 31, 1933 (gage he1ght, 14.6 foet).

Remarkg.- Rocords good. Flood flow not affooted by diversions. Station malntained in cooperation with Venture County Water Survey.

Moan da1ly disgharge, in sooond-foot, 1938

\begin{tabular}{|c|c|c|c|c|c|c|c|c|c|c|c|}
\hline Dey & Feb. & Tar. & Aps. & Day & Fób. & Mar. & Apr. & Day & F॰b. & Mer. & Apr. \\
\hline $\begin{array}{r}1 \\
2 \\
3 \\
4 \\
5 \\
8 \\
7 \\
8 \\
9 \\
10\end{array}$ & $\begin{array}{r}1,700 \\
174 \\
1,540 \\
812 \\
406 \\
264 \\
206 \\
177 \\
421 \\
494\end{array}$ & $\begin{array}{r}2,890 \\
17,950 \\
6,970 \\
2,770 \\
1,660 \\
1,300 \\
1,100 \\
1,310 \\
1,020 \\
882\end{array}$ & $\begin{array}{l}304 \\
288 \\
267 \\
257 \\
257 \\
247 \\
237 \\
228 \\
228 \\
218\end{array}$ & $\begin{array}{l}11 \\
12 \\
13 \\
14 \\
15 \\
18 \\
17 \\
18 \\
18 \\
20\end{array}$ & $\begin{array}{r}6,410 \\
1,890 \\
832 \\
730 \\
524 \\
407 \\
341 \\
301 \\
295 \\
255\end{array}$ & $\begin{array}{r}1,180 \\
5,840 \\
3,060 \\
1,880 \\
1,480 \\
1,170 \\
1,010 \\
848 \\
746 \\
652\end{array}$ & $\begin{array}{l}218 \\
219 \\
219 \\
206 \\
197 \\
188 \\
180 \\
172 \\
161 \\
165\end{array}$ & $\begin{array}{l}21 \\
22 \\
23 \\
24 \\
25 \\
26 \\
27 \\
28 \\
29 \\
30 \\
31\end{array}$ & $\begin{array}{r}231 \\
206 \\
201 \\
197 \\
185 \\
182 \\
182 \\
1,060\end{array}$ & $\begin{array}{l}592 \\
541 \\
510 \\
478 \\
449 \\
421 \\
400 \\
381 \\
363 \\
344 \\
321\end{array}$ & $\begin{array}{l}161 \\
153 \\
149 \\
161 \\
184 \\
165 \\
148 \\
148 \\
146 \\
153\end{array}$ \\
\hline \multicolumn{9}{|c|}{ 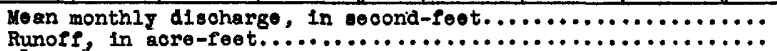 } & $\begin{array}{r}737 \\
40,810\end{array}$ & $\begin{array}{r}1,853 \\
120,100\end{array}$ & $\begin{array}{r}201 \\
11,960\end{array}$ \\
\hline
\end{tabular}

Gage helght, in foet, and disoharge, in second-feet, at indicated time, 1938

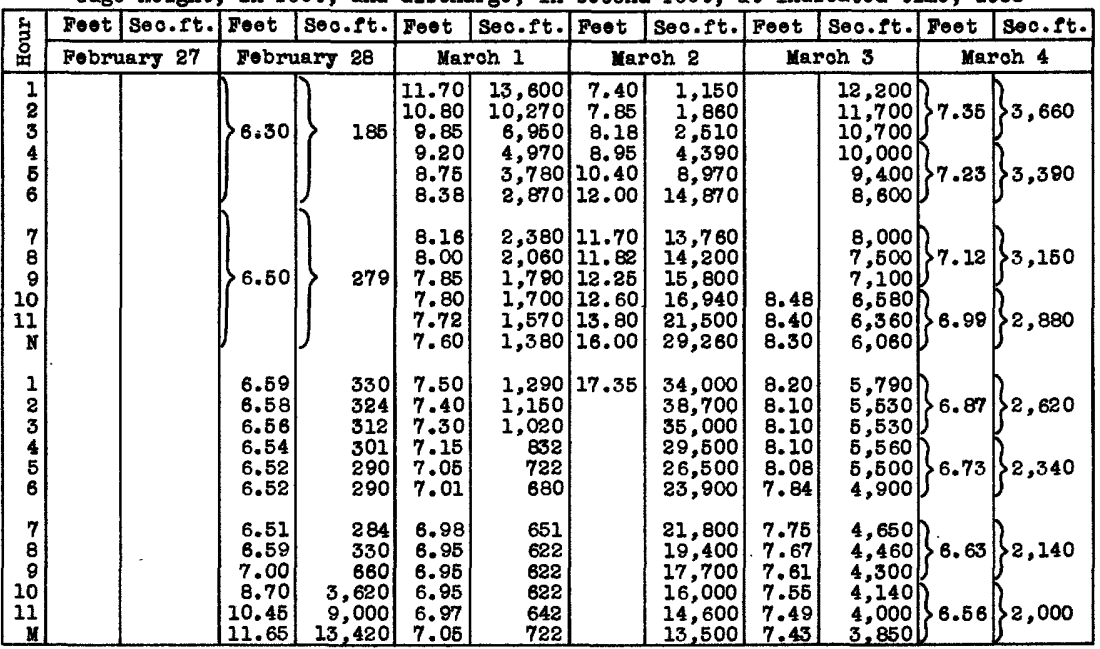

Supplemental record.- Mar. 2, 2:15 p.m., $19.2 \mathrm{ft.,} 39,200$ sec.-ft. 
Coyote Creek near Ventura, Calif.

Location. - Water-stage recorder, lat. $34^{\circ} 21^{\prime} 20^{n}$, long. $119^{\circ} 18^{\prime} 50^{n}$, near southeast corner of Santa Ana grant, 200 feet downstream from highway bridge, half a mile upstream from junction with Ventura River, and $5 \frac{1}{8}$ miles northwest of Ventura, Ventura County. Altitude, about 250 feet.

Drainage area.- Area, 41.1 square miles. Average altitude, 1,590 feet. Maximum altitude, 4,870 feet. Average slope, 34 percent. Length of main stream channe1, 11.0 miles. Average slope of main stream channel, 7.9 percent.

Gage-height record.- Water-stage recorder graph.

Discharge record.- Stage-discharge relation defined by current-meter measurements below 525 second-feet; extended to slope-area determination of peak discharge.

Maxima.- 1938: Discharge, 11,500 second-feet 1:30 p.m. Mar. 2 (gage helght, 12.00 feet). 1927-32, 2933-37: Discharge, 5,600 second-feet Feb. 14, 1937.

Remarks.- Records good. Ho diversions above gage. Station maintained in cooperation ith Ventura County Water Survey.

Mean dally discharge, in second-feet, 1938

\begin{tabular}{|c|c|c|c|c|c|c|c|c|c|c|c|}
\hline Day & Feb. & Mar. & Apr. & Day & Feb. & Mar. & Apr. & Day & Feb. & Mar. & Apr. \\
\hline $\begin{array}{r}1 \\
2 \\
3 \\
4 \\
5 \\
6 \\
7 \\
8 \\
9 \\
10\end{array}$ & $\begin{array}{r}465 \\
54 \\
303 \\
254 \\
100 \\
51 \\
36 \\
28 \\
96 \\
91\end{array}$ & $\begin{array}{r}524 \\
3,470 \\
800 \\
350 \\
247 \\
162 \\
157 \\
142 \\
120 \\
106\end{array}$ & $\begin{array}{l}34 \\
32 \\
32 \\
28 \\
27 \\
27 \\
27 \\
27 \\
26 \\
25\end{array}$ & $\begin{array}{l}11 \\
12 \\
13 \\
14 \\
15 \\
16 \\
17 \\
18 \\
19 \\
20\end{array}$ & $\begin{array}{r}663 \\
271 \\
131 \\
104 \\
71 \\
61 \\
50 \\
44 \\
51 \\
37\end{array}$ & $\begin{array}{r}173 \\
516 \\
303 \\
190 \\
145 \\
122 \\
102 \\
91 \\
80 \\
72\end{array}$ & $\begin{array}{l}25 \\
25 \\
24 \\
24 \\
23 \\
22 \\
21 \\
20 \\
19 \\
18\end{array}$ & $\begin{array}{l}21 \\
22 \\
23 \\
24 \\
25 \\
26 \\
27 \\
28 \\
29 \\
30 \\
31\end{array}$ & $\begin{array}{r}31 \\
30 \\
26 \\
25 \\
21 \\
20 \\
20 \\
328\end{array}$ & $\begin{array}{l}66 \\
62 \\
61 \\
59 \\
58 \\
51 \\
48 \\
46 \\
42 \\
38 \\
36\end{array}$ & $\begin{array}{l}18 \\
18 \\
16 \\
16 \\
15 \\
15 \\
14 \\
14 \\
14 \\
16\end{array}$ \\
\hline \multicolumn{9}{|c|}{ 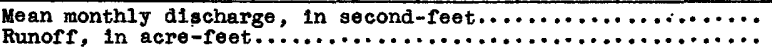 } & 6,870 & $\begin{array}{r}272 \\
16,740\end{array}$ & 1,310 \\
\hline
\end{tabular}

Gage height, in feet, and discharge, in second-feet, at indicated time, 1938

\begin{tabular}{|c|c|c|c|c|c|c|c|c|c|c|c|c|}
\hline \multirow{2}{*}{$\begin{array}{l}\text { 岁 } \\
\text { 总 }\end{array}$} & Feet & Sec.ft. & Feet & Sec.ft. & Feet & Sec.ft. & Feet & sec.ft. & Feet & Sec.ft. & Feet & Sec.rt. \\
\hline & \multicolumn{2}{|c|}{ February 27} & \multicolumn{2}{|c|}{ February 28} & \multicolumn{2}{|c|}{ March 1} & \multicolumn{2}{|c|}{ March 2} & \multicolumn{2}{|c|}{ March 3} & \multicolumn{2}{|c|}{ March 4} \\
\hline $\begin{array}{l}1 \\
2 \\
3 \\
4 \\
5 \\
6\end{array}$ & & & $\begin{array}{l}1.95 \\
1.95 \\
2.00 \\
2.04 \\
2.07 \\
2.10\end{array}$ & $\begin{array}{l}19 \\
19 \\
22 \\
28 \\
32 \\
36\end{array}$ & $\begin{array}{l}5.20 \\
4.60 \\
4.31 \\
4.13 \\
4.00 \\
3.75\end{array}$ & $\begin{array}{r}1,550 \\
1,080 \\
896 \\
788 \\
713 \\
578\end{array}$ & $\begin{array}{l}3.56 \\
3.60 \\
3.69 \\
3.86 \\
6.91 \\
8.20\end{array}$ & $\begin{array}{r}486 \\
504 \\
548 \\
636 \\
3,240 \\
4,950\end{array}$ & $\begin{array}{l}4.31 \\
4.25 \\
4.31 \\
4.91 \\
5.11 \\
4.57\end{array}$ & $\begin{array}{r}896 \\
860 \\
896 \\
1,320 \\
1,470 \\
1,050\end{array}$ & & \\
\hline $\begin{array}{r}7 \\
8 \\
9 \\
10 \\
11 \\
N\end{array}$ & & & $\begin{array}{l}2.19 \\
2.30 \\
2.30 \\
2.40 \\
2.61 \\
2.61\end{array}$ & $\begin{array}{r}47 \\
62 \\
64 \\
79 \\
118 \\
118\end{array}$ & $\begin{array}{l}3.62 \\
3.51 \\
3.48 \\
3.39 \\
3.31 \\
3.27\end{array}$ & $\begin{array}{l}514 \\
463 \\
449 \\
409 \\
373 \\
357\end{array}$ & $\begin{array}{r}8.01 \\
7.34 \\
6.89 \\
7.61 \\
9.51 \\
10.32\end{array}$ & $\begin{array}{l}4,680 \\
3,790 \\
3,220 \\
4,140 \\
6,940 \\
8,310\end{array}$ & $\begin{array}{l}4.39 \\
4.38 \\
4.43 \\
4.34 \\
4.21 \\
4.11\end{array}$ & $\begin{array}{l}938 \\
926 \\
956 \\
902 \\
818 \\
759\end{array}$ & & \\
\hline $\begin{array}{l}1 \\
2 \\
3 \\
4 \\
5 \\
6\end{array}$ & & & $\begin{array}{l}2.56 \\
2.48 \\
2.41 \\
2.39 \\
2.37 \\
2.34\end{array}$ & $\begin{array}{l}99 \\
93 \\
80 \\
79 \\
76 \\
71\end{array}$ & $\begin{array}{l}3.22 \\
3.19 \\
3.13 \\
3.10 \\
3.08 \\
3.04\end{array}$ & $\begin{array}{l}338 \\
326 \\
303 \\
291 \\
284 \\
268\end{array}$ & $\begin{array}{r}11.15 \\
11.79 \\
8.71 \\
7.01 \\
6.11 \\
5.66\end{array}$ & $\begin{array}{r}9,840 \\
11,000 \\
5,650 \\
3,360 \\
2,380 \\
1,950\end{array}$ & $\begin{array}{l}4.01 \\
3.97 \\
3.96 \\
3.89 \\
3.94 \\
3.95\end{array}$ & $\begin{array}{l}702 \\
674 \\
669 \\
625 \\
652 \\
658\end{array}$ & & $\cdot$ \\
\hline $\begin{array}{r}7 \\
8 \\
9 \\
10 \\
11 \\
\mathbf{M} \\
\end{array}$ & & & $\begin{array}{l}2.40 \\
2.70 \\
3.29 \\
6.10 \\
6.49 \\
6.00\end{array}$ & $\begin{array}{r}82 \\
145 \\
338 \\
2,320 \\
2,720 \\
2,270 \\
\end{array}$ & $\begin{array}{l}3.00 \\
2.99 \\
2.97 \\
2.98 \\
3.02 \\
3.41\end{array}$ & $\begin{array}{l}257 \\
254 \\
247 \\
250 \\
264 \\
418 \\
\end{array}$ & $\begin{array}{l}5.26 \\
4.98 \\
4.79 \\
4.64 \\
4.51 \\
4.38\end{array}$ & $\begin{array}{r}1,600 \\
1,370 \\
1,220 \\
1,110 \\
1,020 \\
938 \\
\end{array}$ & $\begin{array}{l}3.89 \\
3.88 \\
3.69 \\
3.65 \\
3.61 \\
3.58\end{array}$ & $\begin{array}{l}620 \\
614 \\
514 \\
495 \\
476 \\
458 \\
\end{array}$ & & \\
\hline
\end{tabular}

Supplemental record.- Mar. $2,1: 30$ p.m., 12.00 ft., 11,500 sec.-ft. 


\section{Santa Ynoz River Basin}

Santa Ynez River at Juncal Reservoir, near Montecito, Calif.

Location. - Water-stage recorder, lat. $34^{\circ} 29^{\prime} 30^{\prime \prime}$, long. $119^{\circ} 30^{\prime} 50^{n}$, in sec. $28, T .5$ K., R. 25 W., at Juncal Reservolr dam, 8.5 miles northeast of fontecito. Altitude of stream bed, about 1,950 feet.

Draingge area.- Area, 16.0 square miles. Average a Ititude, 3,550 feet. Maximum alt1tude, 6,025 feet. Average slope, 64 percent. Length of main stream channel, 5.2 miles. Average slope of main stream channel, 15 percent.

Gage-he1ght record. - Water-stage recorder graph.

Discharge record.- Discharge computed from reservoir gage-helght record, rainfall, evaporation, release, and overfiow.

Maximum.- 1938: Mean hourly discharge, 4,160 second-feet Mar. 2 .

Remarks. - No diversion during flood period. Record furmished by Montecito County Water District.

\begin{tabular}{|c|c|c|c|c|c|c|c|c|c|c|c|}
\hline Day & Feb. & Mar. & Apr. & Day & $\mathrm{Feb}$, & Mar. & ADr. & Dar & $\mathrm{Feb}$ & Mar & Anr \\
\hline $\begin{array}{r}1 \\
2 \\
3 \\
4 \\
5 \\
6 \\
7 \\
8 \\
9 \\
10\end{array}$ & $\begin{array}{r}162 \\
85 \\
194 \\
85 \\
30 \\
17 \\
11 \\
10 \\
29 \\
57\end{array}$ & $\begin{array}{r}272 \\
1,240 \\
467 \\
197 \\
116 \\
89 \\
119 \\
185 \\
125 \\
87\end{array}$ & $\begin{array}{l}32 \\
32 \\
32 \\
32 \\
31 \\
32 \\
31 \\
31 \\
31 \\
31\end{array}$ & $\begin{array}{l}11 \\
12 \\
13 \\
14 \\
15 \\
16 \\
17 \\
18 \\
19 \\
20\end{array}$ & $\begin{array}{l}585 \\
147 \\
71 \\
32 \\
26 \\
26 \\
13 \\
13 \\
15 \\
17\end{array}$ & $\begin{array}{r}78 \\
509 \\
293 \\
159 \\
124 \\
97 \\
75 \\
61 \\
47 \\
42\end{array}$ & $\begin{array}{l}29 \\
29 \\
28 \\
29 \\
28 \\
27 \\
27 \\
27 \\
27 \\
15\end{array}$ & $\begin{array}{l}21 \\
22 \\
23 \\
24 \\
25 \\
26 \\
27 \\
28 \\
29 \\
30 \\
31\end{array}$ & $\begin{array}{l}15 \\
13 \\
14 \\
12 \\
10 \\
10 \\
10 \\
15\end{array}$ & $\begin{array}{l}38 \\
47 \\
49 \\
41 \\
38 \\
37 \\
34 \\
35 \\
32 \\
30 \\
25\end{array}$ & $\begin{array}{l}15 \\
13 \\
16 \\
22 \\
25 \\
23 \\
22 \\
19 \\
19 \\
21\end{array}$ \\
\hline \multicolumn{9}{|c|}{ 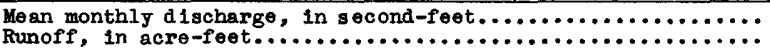 } & $\begin{array}{r}61.6 \\
3,420\end{array}$ & 9,500 & $\begin{array}{r}25.9 \\
1,540\end{array}$ \\
\hline
\end{tabular}

Discharge, in second-feet, at indicated time, 1938

\begin{tabular}{|c|c|c|c|c|}
\hline Hour & March 1 & March 2 & March 3 & March \\
\hline $\begin{array}{l}1 \\
2 \\
3 \\
4 \\
5 \\
6\end{array}$ & $\begin{array}{l}943 \\
625 \\
519 \\
419 \\
343 \\
290\end{array}$ & $\begin{array}{l}135 \\
163 \\
195 \\
260 \\
563 \\
891\end{array}$ & $\begin{array}{l}547 \\
519 \\
510 \\
547 \\
595 \\
557\end{array}$ & $\begin{array}{l}233 \\
233 \\
226 \\
206 \\
200 \\
195\end{array}$ \\
\hline $\begin{array}{r}7 \\
8 \\
9 \\
10 \\
11 \\
N\end{array}$ & $\begin{array}{l}252 \\
208 \\
188 \\
170 \\
157 \\
153\end{array}$ & $\begin{array}{l}1,050 \\
1,030 \\
1,010 \\
1,140 \\
1,720 \\
2,550\end{array}$ & $\begin{array}{l}547 \\
527 \\
481 \\
437 \\
384 \\
375\end{array}$ & $\begin{array}{l}195 \\
195 \\
195 \\
189 \\
189 \\
189\end{array}$ \\
\hline $\begin{array}{l}1 \\
2 \\
3 \\
4 \\
5 \\
6\end{array}$ & $\begin{array}{l}140 \\
129 \\
129 \\
124 \\
115 \\
110\end{array}$ & $\begin{array}{l}3,740 \\
4,160 \\
3,450 \\
2,440 \\
1,790 \\
1,460\end{array}$ & $\begin{array}{l}375 \\
375 \\
367 \\
343 \\
320 \\
297\end{array}$ & $\begin{array}{l}189 \\
189 \\
182 \\
182 \\
176 \\
163\end{array}$ \\
\hline $\begin{array}{r}7 \\
8 \\
9 \\
10 \\
11 \\
11\end{array}$ & $\begin{array}{l}105 \\
105 \\
110 \\
110 \\
115 \\
124\end{array}$ & $\begin{array}{r}1,200 \\
1,010 \\
868 \\
752 \\
667 \\
605\end{array}$ & $\begin{array}{l}290 \\
275 \\
260 \\
253 \\
246 \\
233\end{array}$ & $\begin{array}{l}157 \\
152 \\
146 \\
140 \\
135 \\
135\end{array}$ \\
\hline
\end{tabular}


Santa Inez River near Santa Barbara, Cal1f.

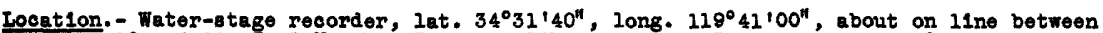
sec8. 10 and 11, T. 5 H., R. 27 W., at Gibraítar Dam, 7 miles north of Santa Barbara. At ltude, about 1,200 fét.

Draingge area, - Area, 219 square miles. Average alt1tude, 3,400 feet. Maximum alt1tude, 6,828 feet. A verage slope, 42 percent. Length of main otream chennel, 17.5 miles. Average slope of maln stroam channe1, 6 percent.

Gage-he1ght record. - Water-8tage rocorder graph.

Disoharge record. - Inflow computed from fluctuation in reservolr level.

Maximum. - 1838: Discharge (mean from 1:45 p.m. to 2 p.m.), 38,200 second-feet Mar. 2 .

Remarks. - Records represent flow into reservior at olbralter Dam and are good. Regalation at Juncal Reservolr except during flood perlods. Records furnished by olty of Santa Barbara.

Mean de11y discharge, in second-feet, 1938*

\begin{tabular}{|c|c|c|c|c|c|c|c|c|c|c|c|}
\hline Day & $\mathrm{Feb}$. & Mar. & Apr. & Day & Feb. & Mar. & Apr. & Day & Feb. & Mar. & Apr. \\
\hline $\begin{array}{r}1 \\
2 \\
3 \\
4 \\
5 \\
6 \\
7 \\
8 \\
9 \\
10\end{array}$ & $\begin{array}{l}782 \\
122 \\
775 \\
604 \\
310 \\
191 \\
150 \\
128 \\
180 \\
408\end{array}$ & $\begin{array}{r}1,390 \\
1,130 \\
5,440 \\
2,020 \\
1,130 \\
794 \\
618 \\
1,000 \\
870 \\
698\end{array}$ & $\begin{array}{l}222 \\
216 \\
210 \\
203 \\
201 \\
198 \\
188 \\
180 \\
174 \\
168\end{array}$ & $\begin{array}{l}11 \\
12 \\
13 \\
14 \\
15 \\
16 \\
17 \\
18 \\
19 \\
20\end{array}$ & $\begin{array}{r}4,400 \\
1,630 \\
552 \\
406 \\
351 \\
276 \\
247 \\
244 \\
252 \\
207\end{array}$ & $\begin{array}{r}713 \\
4,480 \\
2,620 \\
1,560 \\
1,100 \\
835 \\
669 \\
562 \\
481 \\
450\end{array}$ & $\begin{array}{l}162 \\
164 \\
161 \\
156 \\
155 \\
155 \\
151 \\
145 \\
144 \\
139\end{array}$ & $\begin{array}{l}21 \\
22 \\
23 \\
24 \\
25 \\
26 \\
27 \\
28 \\
29 \\
30 \\
31\end{array}$ & $\begin{array}{l}197 \\
212 \\
220 \\
165 \\
150 \\
131 \\
126 \\
175\end{array}$ & $\begin{array}{l}405 \\
340 \\
334 \\
328 \\
312 \\
300 \\
280 \\
267 \\
259 \\
248 \\
231\end{array}$ & $\begin{array}{l}130 \\
122 \\
120 \\
124 \\
152 \\
139 \\
130 \\
122 \\
124 \\
126\end{array}$ \\
\hline \multicolumn{9}{|c|}{ 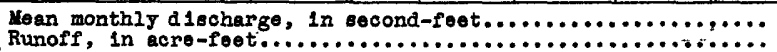 } & 26,986 & $\begin{array}{r}1,286 \\
79,090\end{array}$ & 9,480 \\
\hline
\end{tabular}

* Discharges are for 24-hour period ending $6 \mathrm{p} . \mathrm{m}$, of day Indicated.

* Mean discharge for calendar day Kar. 2, 11,300 second-feet.

Discharge, in second-feet, at indlogted time, 1938

\begin{tabular}{|c|c|c|c|}
\hline Hour & March 1 & March 2 & Harch 3 \\
\hline $\begin{array}{l}1 \\
2 \\
3 \\
4 \\
5 \\
6\end{array}$ & & $\begin{array}{r}781 \\
895 \\
1,890 \\
1,870 \\
2,850 \\
4,600\end{array}$ & $\begin{array}{l}5,600 \\
5,320 \\
6,100 \\
4,930 \\
4,800 \\
4,660\end{array}$ \\
\hline $\begin{array}{r}7 \\
8 \\
9 \\
10 \\
11 \\
11\end{array}$ & & $\begin{array}{r}6,170 \\
6,940 \\
7,390 \\
8,790 \\
12,800 \\
21,300\end{array}$ & $\begin{array}{l}4,490 \\
4,280 \\
4,120 \\
4,020 \\
3,880 \\
3,740\end{array}$ \\
\hline $\begin{array}{l}1 \\
2 \\
3 \\
4 \\
5 \\
6\end{array}$ & 489 & $\begin{array}{l}31,800 \\
35,200 \\
29,500 \\
22,200 \\
16,900 \\
13,800\end{array}$ & $\begin{array}{l}3,590 \\
3,460 \\
3,350 \\
3,230 \\
3,120 \\
2,990\end{array}$ \\
\hline $\begin{array}{r}7 \\
8 \\
9 \\
10 \\
11 \\
M\end{array}$ & $\begin{array}{l}467 \\
481 \\
526 \\
582 \\
641 \\
718\end{array}$ & $\begin{array}{r}11,700 \\
19,300 \\
8,340 \\
7,370 \\
6,630 \\
6,020\end{array}$ & \\
\hline
\end{tabular}

8upplemental record.- Mar. 2, mean from 1:45 to 2 p.m., 38,200 second-feet. 
Santa Ynez R170r below Olbraltar Dam, near Santa Barbara, Oallf.

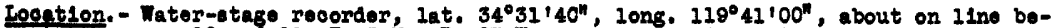
twon soos. 10 and 11, T. 5 K., R. 27 ., domntroam from albraltar Dam, 7 mf 108 north of Senta Barbara. Altitudo, about 1,200 feot.

Drelnage nrea. - Area, 819 square miles. Average alt1tude, 3,400 foot. Maximum alt1trido, 6,8:8 soot, Average slope, 42 percent. Iongth of ma in otroam chennel, 17,5 milos. Average slopo of main struam channol, 6 percent.

Gage-holght reoord.- Nater-8tage rocorder graph.

Disoharge record. - D1soharge computed from rating of gato valve Fob, 18-17 and Mar. 1-6, and rating or splilway, Fob. 8 to Apr. 30 .

Yextmum. - 1938 8 Disobarge, 35,600 second-feot 2816 p.m. Mar. 2.

Remariss. - Flow rogulated by storage in reservolr and diversion to o ity of Santa Barbara; during flood perlod City of Santa Barbara d 1 verted 149 acre-foet. Mo diversfons from Glbraitar Roservolr Fob. 3-14 and Mar. 1 to Apr. 3. Records rurniohod by Oity of Santa Barbara.

Moan daily discharge, in socond-feet, $1938^{*}$

\begin{tabular}{|c|c|c|c|c|c|c|c|c|c|c|c|}
\hline Day & F॰b. & Mar. & Apr. & Day & Fob. & Har. & Apr. & Day & Fob. & Mar. & Apr. \\
\hline \begin{tabular}{r|}
1 \\
2 \\
3 \\
4 \\
5 \\
6 \\
7 \\
8 \\
9 \\
10
\end{tabular} & $\begin{array}{r}0 \\
0 \\
623 \\
669 \\
365 \\
206 \\
168 \\
188 \\
166 \\
396\end{array}$ & $\begin{array}{r}1,360 \\
48,630 \\
5,950 \\
2,150 \\
1,170 \\
821 \\
658 \\
981 \\
888 \\
714\end{array}$ & $\begin{array}{l}222 \\
816 \\
809 \\
203 \\
200 \\
197 \\
188 \\
179 \\
173 \\
167\end{array}$ & $\begin{array}{l}11 \\
12 \\
13 \\
14 \\
18 \\
16 \\
17 \\
18 \\
18 \\
80\end{array}$ & $\begin{array}{r}4,130 \\
1,900 \\
595 \\
415 \\
369 \\
286 \\
849 \\
236 \\
264 \\
810\end{array}$ & $\begin{array}{r}696 \\
4,380 \\
2,730 \\
1,610 \\
1,130 \\
853 \\
683 \\
670 \\
497 \\
454\end{array}$ & $\begin{array}{l}161 \\
161 \\
161 \\
165 \\
168 \\
158 \\
149 \\
143 \\
140 \\
137\end{array}$ & $\begin{array}{l}81 \\
22 \\
23 \\
24 \\
25 \\
26 \\
27 \\
28 \\
29 \\
30 \\
31\end{array}$ & $\begin{array}{l}195 \\
201 \\
282 \\
170 \\
149 \\
131 \\
123 \\
156\end{array}$ & $\begin{array}{l}410 \\
346 \\
333 \\
330 \\
316 \\
301 \\
283 \\
268 \\
259 \\
849 \\
234\end{array}$ & $\begin{array}{l}129 \\
120 \\
117 \\
121 \\
153 \\
137 \\
129 \\
120 \\
128 \\
129\end{array}$ \\
\hline \multicolumn{9}{|c|}{ 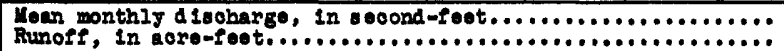 } & 25,854 & $\begin{array}{r}1,890 \\
79,430\end{array}$ & $\begin{array}{r}158 \\
9,400\end{array}$ \\
\hline
\end{tabular}

- Discharges are for 24-hour perlod ending $6 \mathrm{p.m.}$ of day indloated.

* Hean discharge for oslendar day Mar. 2, 10,900 second-feet.

Dlsoharge, In socond-foot, at Indioated timo, 1938

\begin{tabular}{|c|c|c|c|}
\hline Bour & Maroh 1 & March 2 & Maroh 3 \\
\hline $\begin{array}{l}1 \\
8 \\
3 \\
4 \\
5 \\
6\end{array}$ & & $\begin{array}{r}646 \\
695 \\
795 \\
1,100 \\
1,550 \\
8,470\end{array}$ & $\begin{array}{l}6,110 \\
5,690 \\
5,380 \\
5,170 \\
4,980 \\
4,840\end{array}$ \\
\hline $\begin{array}{r}7 \\
8 \\
9 \\
10 \\
11 \\
17\end{array}$ & & $\begin{array}{r}4,160 \\
5,930 \\
6,740 \\
7,270 \\
9,150 \\
14,000\end{array}$ & $\begin{array}{l}4,690 \\
4,850 \\
4,880 \\
4,180 \\
4,060 \\
3,910\end{array}$ \\
\hline $\begin{array}{l}1 \\
8 \\
8 \\
4 \\
5 \\
6\end{array}$ & 560 & $\begin{array}{l}24,800 \\
34,800 \\
35,400 \\
25,100 \\
80,200 \\
16,500\end{array}$ & $\begin{array}{l}8,780 \\
3,630 \\
3,610 \\
3,400 \\
8,280 \\
3,170\end{array}$ \\
\hline $\begin{array}{r}7 \\
8 \\
8 \\
10 \\
11 \\
11\end{array}$ & $\begin{array}{l}587 \\
506 \\
508 \\
516 \\
550 \\
586 \\
\end{array}$ & $\begin{array}{r}13,800 \\
11,800 \\
9,680 \\
8,890 \\
7,400 \\
6,680 \\
\end{array}$ & \\
\hline
\end{tabular}

Supplomental rooord. - Mar. 2, 2816 p.m., 38,600 cooona-foet. 
Location. - Water-stage recorder, lat. $34^{\circ} 35^{\prime} 20^{n}$, long. $120^{\circ} 01^{\prime} 25^{\prime \prime}$, in Canada de los Pinos grant, at San Marcos Road Bridge, 4 miles scutheast of Senta Ynez, Santa Barbara County. Altitude, about 500 feet.

Drainage area.- Area, 435 square miles. Average altitude, 2,890 feet. Maximum altitude, 6,828 feet. Average slope, 34 percent. Iength of main stream channel, 41.5 miles. Average slope of main stream channel, 3 percent.

Gage-he1ght record.- Water-stage recorder graph.

Discharge record.- Stage-discharge relaticn defined by current-meter measurements beIow 3,360 second-feet and by flost megsurements up to 34,100 second-feet; extended logarithmically to peak s tage.

Maxima.- 1938: Discharge, 43,700 second-feet 6 p.m. Mar. 2 (gage height, 17.9 feet). 1929-31, 1932-37: Discharge, 7,990 second-feet Feb. 6, 1937 (gage height, 10.67 feet).

Remarks.- Records good. The cities of Santa Barbara and Montecito divert water above gage. Flood flows affected by regulation at Gibralter and Juncal Reservolrs.

Mean daily discharge, in second-feet, 1938

\begin{tabular}{|c|c|c|c|c|c|c|c|c|c|c|c|}
\hline Day & Feb. & Mar. & Apr. & Day & Feb. & Mar. & Apr. & Day & Feb. & Mar. & Apr. \\
\hline $\begin{array}{r}1 \\
2 \\
3 \\
4 \\
5 \\
6 \\
7 \\
8 \\
9 \\
10\end{array}$ & $\begin{array}{r}796 \\
172 \\
1,490 \\
2,040 \\
974 \\
441 \\
278 \\
197 \\
265 \\
728\end{array}$ & $\begin{array}{r}2,800 \\
17,910 \\
9,140 \\
4,210 \\
3,250 \\
2,470 \\
2,940 \\
3,280 \\
2,520 \\
2,180\end{array}$ & $\begin{array}{l}535 \\
509 \\
488 \\
463 \\
473 \\
428 \\
400 \\
380 \\
366 \\
356\end{array}$ & $\begin{array}{l}11 \\
12 \\
13 \\
14 \\
15 \\
16 \\
17 \\
18 \\
19 \\
20\end{array}$ & $\begin{array}{r}9,700 \\
3,400 \\
1,660 \\
1,250 \\
1,010 \\
766 \\
591 \\
491 \\
496 \\
478\end{array}$ & $\begin{array}{l}2,130 \\
9,180 \\
6,120 \\
4,120 \\
3,190 \\
2,380 \\
1,920 \\
1,600 \\
1,390 \\
1,260\end{array}$ & $\begin{array}{l}343 \\
343 \\
348 \\
325 \\
309 \\
289 \\
281 \\
266 \\
254 \\
242\end{array}$ & $\begin{array}{l}21 \\
22 \\
23 \\
24 \\
25 \\
26 \\
27 \\
28 \\
29 \\
30 \\
31 \\
\end{array}$ & $\begin{array}{l}386 \\
356 \\
331 \\
335 \\
294 \\
269 \\
254 \\
421\end{array}$ & $\begin{array}{r}1,170 \\
1,030 \\
958 \\
894 \\
810 \\
750 \\
715 \\
679 \\
644 \\
600 \\
572 \\
\end{array}$ & $\begin{array}{l}224 \\
214 \\
203 \\
217 \\
277 \\
250 \\
200 \\
183 \\
193 \\
193\end{array}$ \\
\hline \multicolumn{9}{|c|}{ 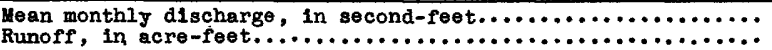 } & $\begin{array}{r}1,067 \\
59,240 \\
\end{array}$ & $\begin{array}{r}2,994 \\
184,100 \\
\end{array}$ & $\begin{array}{r}318 \\
18,950 \\
\end{array}$ \\
\hline
\end{tabular}

Gage helght, in feet, and discharge, In second-feet, at indicated time, 1938

\begin{tabular}{|c|c|c|c|c|c|c|c|c|c|c|c|c|}
\hline \multirow{2}{*}{ 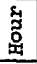 } & Feet & sec.ft. & Feet & Sec.ft. & Feet & sec.ft. & Feet & sec.ft. & Feet & sec.ft. & Feet & Sec.ft. \\
\hline & \multicolumn{2}{|c|}{ February 27} & \multicolumn{2}{|c|}{ Febmary 28} & \multicolumn{2}{|c|}{ March 1} & \multicolumn{2}{|c|}{ March 2} & \multicolumn{2}{|c|}{ March 3} & \multicolumn{2}{|c|}{ March 4} \\
\hline $\begin{array}{l}1 \\
2 \\
3 \\
4 \\
5 \\
6\end{array}$ & & & $\begin{array}{l}4.79 \\
4.80 \\
4.82 \\
4.83 \\
4.84 \\
4.84\end{array}$ & $\begin{array}{l}248 \\
254 \\
263 \\
266 \\
269 \\
272\end{array}$ & $\begin{array}{l}8.02 \\
8.20 \\
8.66 \\
8.34 \\
8.04 \\
7.82\end{array}$ & $\begin{array}{l}2,908 \\
3,180 \\
3,960 \\
3,430 \\
2,980 \\
2,690\end{array}$ & $\begin{array}{l}6.85 \\
6.88 \\
6.95 \\
7.12 \\
7.40 \\
7.71\end{array}$ & $\begin{array}{l}1,520 \\
1,560 \\
1,610 \\
1,780 \\
2,060 \\
2,410\end{array}$ & $\begin{array}{l}11.69 \\
11.40 \\
11.44 \\
11.70 \\
11.34 \\
11.12\end{array}$ & $\begin{array}{l}13,600 \\
12,400 \\
12,600 \\
13,600 \\
12,200 \\
11,400\end{array}$ & $\begin{array}{l}8.94 \\
8.84 \\
8.80 \\
8.74 \\
8.69 \\
8.64\end{array}$ & $\begin{array}{l}5,130 \\
4,940 \\
4,840 \\
4,260 \\
4,620 \\
4,540\end{array}$ \\
\hline $\begin{array}{r}7 \\
8 \\
9 \\
10 \\
11 \\
\mathrm{~N}\end{array}$ & & & $\begin{array}{l}4.84 \\
4.84 \\
4.85 \\
4.86 \\
4.87 \\
4.88\end{array}$ & $\begin{array}{l}272 \\
275 \\
278 \\
285 \\
288 \\
294\end{array}$ & $\begin{array}{l}8.04 \\
8.74 \\
9.14 \\
8.98 \\
8.59 \\
8.32\end{array}$ & $\begin{array}{l}3,010 \\
4,110 \\
4,840 \\
4,540 \\
3,860 \\
3,430\end{array}$ & $\begin{array}{r}8.24 \\
8.89 \\
9.79 \\
10.61 \\
12.50 \\
14.20\end{array}$ & $\begin{array}{r}3,120 \\
4,150 \\
5,900 \\
7,840 \\
16,840 \\
24,400\end{array}$ & $\begin{array}{l}10.74 \\
10.54 \\
10.54 \\
10.56 \\
10.40 \\
10.30\end{array}$ & $\begin{array}{r}10,100 \\
9,420 \\
9,420 \\
9,480 \\
9,000 \\
8,700\end{array}$ & $\begin{array}{l}8.54 \\
8.54 \\
8.50 \\
8.52 \\
8.50 \\
.8 .42\end{array}$ & $\begin{array}{l}4,330 \\
4,350 \\
4,260 \\
4,330 \\
4,280 \\
4,140\end{array}$ \\
\hline $\begin{array}{l}1 \\
2 \\
3 \\
4 \\
5 \\
6\end{array}$ & & & $\begin{array}{l}4.89 \\
4.89 \\
4.89 \\
4.91 \\
4.94 \\
4.97\end{array}$ & $\begin{array}{l}298 \\
301 \\
301 \\
311 \\
324 \\
338\end{array}$ & $\begin{array}{l}7.99 \\
7.82 \\
7.64 \\
7.46 \\
7.34 \\
7.20\end{array}$ & $\begin{array}{l}2,910 \\
2,660 \\
2,420 \\
2,200 \\
2,060 \\
1,900\end{array}$ & $\begin{array}{l}15.80 \\
16.35 \\
16.00 \\
15.75 \\
16.70 \\
17.90\end{array}$ & $\begin{array}{l}32,100 \\
34,900 \\
33,100 \\
31,800 \\
36,900 \\
43,700\end{array}$ & $\begin{array}{r}10.19 \\
10.14 \\
10.04 \\
9.94 \\
9.80 \\
9.60\end{array}$ & $\begin{array}{l}8,370 \\
8,220 \\
7,920 \\
7,620 \\
7,250 \\
6,750\end{array}$ & $\begin{array}{l}8.34 \\
8.34 \\
8.30 \\
8.30 \\
8.30 \\
8.25\end{array}$ & $\begin{array}{l}3,980 \\
4,000 \\
3,920 \\
3,940 \\
3,940 \\
3,870\end{array}$ \\
\hline $\begin{array}{r}7 \\
8 \\
9 \\
10 \\
11 \\
\end{array}$ & & & $\begin{array}{l}5.02 \\
5.12 \\
5.30 \\
5.87 \\
6.90 \\
7.69\end{array}$ & $\begin{array}{r}360 \\
402 \\
482 \\
785 \\
1,590 \\
2,460 \\
\end{array}$ & $\begin{array}{l}7.09 \\
7.01 \\
6.90 \\
6.87 \\
6.85\end{array}$ & $\begin{array}{l}1,790 \\
1,700 \\
1,590 \\
1,550 \\
1,540 \\
1,510\end{array}$ & $\begin{array}{l}17.20 \\
15.60 \\
14.65 \\
13.60 \\
12.65 \\
12.10\end{array}$ & $\begin{array}{l}39,700 \\
31,000 \\
26,400 \\
21,400 \\
17,400 \\
15,200\end{array}$ & $\begin{array}{l}9.56 \\
9.49 \\
9.36 \\
9.30 \\
9.20 \\
9.05\end{array}$ & $\begin{array}{l}6,650 \\
6,480 \\
6,150 \\
6,000 \\
5,750 \\
5,380\end{array}$ & $\begin{array}{l}8.24 \\
8.22 \\
8.18 \\
8.15 \\
8.14 \\
8.74\end{array}$ & $\begin{array}{l}3,850 \\
3,830 \\
3,750 \\
3,720 \\
3,700 \\
3,720\end{array}$ \\
\hline
\end{tabular}


Santa Ynez River near Lompoc, Callf.

Location.- Water-stage recorder, lat. $34^{\circ} 38^{\prime} 30^{\prime \prime}$, long. $120^{\circ} 25^{\prime} 50^{\prime \prime}$, near boundary of Ia Kission Vieja de la Purisima grant, at highway bridge, $1^{\frac{1}{8}}$ milee east of Lompoc, Santa Barbara County. Alt tude, about 100 feet.

Drainage area.- Area, 790 square miles. Average altitude, 1,884 feet. Uaximum altitude, 6,828 feet. Average s lope, 28 percent. Iength of ma in stream ohannel, 71 miles. Average $\$$ lope of main stream channel, 1.7 percent.

Gage-height reoord. - Water-stage recorder graph except 9 p.m. Mar. 2 to $4: 30$ a.m. Mar. 3.

Discharge record. - Stage-discharge relation defined by current-meter measurements beIow 5,050 second-feet and by float messurements below 37,600 second-feet; extended logarithmically to peak stage. Shifting-control corrections used throughout the period. Discharge estimated 9 p.m. Mar. 2 to $4: 30$ a.m. Mar. 3 .

Maxime.- 1938: Discharge, 50,100 second-feet 4 a.m. Mar. 3 (gage height, 28.10 feet). 1906-18, 1925-37; Discharge, 41,800 second-feet Jan. 25, 1914 (gage helght, 13.0 feet, former site and datum).

Remarks.- Records good. The citles of Santa Barbara and Montecito divert water above gage. Flood flows affected by regulation at Gibralter and Juncal Reservolrs.

Mean dally discharge, In second-feet, 1938

\begin{tabular}{|c|c|c|c|c|c|c|c|c|c|c|c|}
\hline Day & Feb. & Mar. & Apr. & Day & Feb. & Mar. & Apr. & Day & Feb. & Mar. & Apr. \\
\hline $\begin{array}{r}1 \\
2 \\
3 \\
4 \\
5 \\
6 \\
7 \\
8 \\
9 \\
10\end{array}$ & $\begin{array}{r}643 \\
746 \\
992 \\
2,190 \\
1,940 \\
1,100 \\
628 \\
441 \\
522 \\
738\end{array}$ & $\begin{array}{r}2,590 \\
8,350 \\
24,760 \\
7,050 \\
4,160 \\
3,180 \\
2,670 \\
2,960 \\
3,050 \\
2,980\end{array}$ & $\begin{array}{l}726 \\
679 \\
646 \\
620 \\
601 \\
588 \\
562 \\
531 \\
506 \\
493\end{array}$ & $\begin{array}{l}11 \\
12 \\
13 \\
14 \\
15 \\
16 \\
17 \\
18 \\
19 \\
20\end{array}$ & $\begin{array}{r}6,330 \\
15,010 \\
3,520 \\
2,220 \\
1,540 \\
914 \\
1,040 \\
865 \\
791 \\
766\end{array}$ & $\begin{array}{l}2,970 \\
4,890 \\
6,620 \\
4,740 \\
3,830 \\
3,430 \\
2,420 \\
2,210 \\
2,010 \\
1,970\end{array}$ & $\begin{array}{l}475 \\
444 \\
434 \\
428 \\
422 \\
417 \\
400 \\
379 \\
358 \\
342\end{array}$ & $\begin{array}{l}21 \\
22 \\
23 \\
24 \\
25 \\
26 \\
27 \\
28 \\
29 \\
30 \\
31\end{array}$ & $\begin{array}{l}694 \\
623 \\
542 \\
485 \\
458 \\
388 \\
355 \\
463\end{array}$ & $\begin{array}{r}1,680 \\
1,460 \\
1,190 \\
1,150 \\
1,090 \\
1,020 \\
955 \\
895 \\
858 \\
812 \\
774\end{array}$ & $\begin{array}{l}337 \\
318 \\
288 \\
293 \\
313 \\
322 \\
303 \\
288 \\
279 \\
298\end{array}$ \\
\hline \multicolumn{9}{|c|}{ 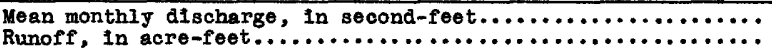 } & $\begin{array}{r}1,676 \\
93,110\end{array}$ & $\begin{array}{r}3,501 \\
215,300\end{array}$ & $\begin{array}{r}436 \\
25,960\end{array}$ \\
\hline
\end{tabular}

Gage height, in feet, and discharge, in second-feet, at indicated time, 1938

\begin{tabular}{|c|c|c|c|c|c|c|c|c|c|c|c|c|}
\hline \multirow{2}{*}{ 骂 } & Feet & Sec.ft. & Feet & Sec.ft. & Feet & Sec.ft. & Feet & sec.ft. & Feet & sec.st. & Feet & Sec.ft. \\
\hline & Febm & 1ary 27 & \multicolumn{2}{|c|}{ February 28} & \multicolumn{2}{|c|}{ March 1} & \multicolumn{2}{|c|}{ March 2} & \multicolumn{2}{|c|}{ March 3} & \multicolumn{2}{|c|}{ March 4} \\
\hline $\begin{array}{l}1 \\
2 \\
3 \\
4 \\
5 \\
6\end{array}$ & & & $\begin{array}{l}12.10 \\
12.09 \\
12.09 \\
12.09 \\
12.09 \\
12.09\end{array}$ & $\begin{array}{l}339 \\
334 \\
334 \\
334 \\
334 \\
329\end{array}$ & $\begin{array}{l}15.44 \\
15.00 \\
14.58 \\
14.20 \\
13.88 \\
13.70\end{array}$ & $\begin{array}{l}2,580 \\
2,120 \\
1,720 \\
1,420 \\
1,180 \\
1,040\end{array}$ & $\begin{array}{l}17.32 \\
17.56 \\
17.50 \\
17.33 \\
16.97 \\
16.91\end{array}$ & $\begin{array}{l}4,830 \\
5,230 \\
5,180 \\
5,000 \\
4,520 \\
4,510\end{array}$ & $\begin{array}{l}28.10 \\
27.43 \\
26.35\end{array}$ & $\begin{array}{l}32,000 \\
39,000 \\
46,700 \\
50,100 \\
45,600 \\
39,000\end{array}$ & $\begin{array}{l}20.05 \\
19.85 \\
19.60 \\
19.40 \\
19.20\end{array}$ & $\begin{array}{r}11,300 \\
10,600 \\
9,900 \\
9,350 \\
8,600\end{array}$ \\
\hline $\begin{array}{r}7 \\
8 \\
9 \\
10 \\
11 \\
\mathbf{1}\end{array}$ & & & $\begin{array}{l}12.08 \\
12.08 \\
12.08 \\
12.08 \\
12.08 \\
12.08\end{array}$ & $\begin{array}{l}324 \\
324 \\
324 \\
324 \\
324 \\
324\end{array}$ & $\begin{array}{l}14.00 \\
14.65 \\
15.14 \\
15.33 \\
15.30 \\
15.22\end{array}$ & $\begin{array}{l}1,220 \\
1,680 \\
2,090 \\
2,250 \\
2,200 \\
2,090\end{array}$ & $\begin{array}{l}16.97 \\
16.95 \\
17.10 \\
17.17 \\
17.39 \\
17.81\end{array}$ & $\begin{array}{l}4,680 \\
4,720 \\
5,030 \\
5,210 \\
5,600 \\
6,380\end{array}$ & $\begin{array}{l}25.25 \\
24.05 \\
23.50 \\
23.10 \\
22.85 \\
22.73\end{array}$ & $\begin{array}{l}32,900 \\
27,000 \\
24,500 \\
22,700 \\
21,600 \\
20,900\end{array}$ & 18.20 & 6,720 \\
\hline $\begin{array}{l}1 \\
2 \\
3 \\
4 \\
5 \\
6\end{array}$ & & & $\begin{array}{l}12.08 \\
12.08 \\
12.08 \\
12.08 \\
12.08 \\
12.09\end{array}$ & $\begin{array}{l}324 \\
324 \\
310 \\
300 \\
290 \\
285\end{array}$ & $\begin{array}{l}15.45 \\
15.75 \\
16.00 \\
16.25 \\
16.43 \\
16.42\end{array}$ & $\begin{array}{l}2,300 \\
2,570 \\
2,820 \\
3,120 \\
3,360 \\
3,400\end{array}$ & $\begin{array}{l}18.10 \\
18.58 \\
18.83 \\
18.67 \\
18.35 \\
18.47\end{array}$ & $\begin{array}{l}6,980 \\
7,910 \\
8,500 \\
8,180 \\
7,620 \\
7,880\end{array}$ & $\begin{array}{l}22.63 \\
22.35 \\
22.25 \\
22.00 \\
21.55 \\
21.17\end{array}$ & $\begin{array}{l}20,500 \\
19,400 \\
19,000 \\
18,000 \\
16,300 \\
15,000\end{array}$ & 17.13 & 5,560 \\
\hline $\begin{array}{r}7 \\
8 \\
9 \\
10 \\
11 \\
11\end{array}$ & & & $\begin{array}{l}12.15 \\
12.17 \\
12.35 \\
13.70 \\
14.50 \\
15.44\end{array}$ & $\begin{array}{r}295 \\
280 \\
355 \\
1,180 \\
1,740 \\
2,610\end{array}$ & $\begin{array}{l}16.37 \\
16.51 \\
16.76 \\
16.92 \\
17.15 \\
17.21\end{array}$ & $\begin{array}{l}3,360 \\
3,570 \\
3,920 \\
4,150 \\
4,500 \\
4,620\end{array}$ & $\begin{array}{l}18.63 \\
19.12 \\
20.00\end{array}$ & $\begin{array}{r}8,360 \\
9,490 \\
11,700 \\
18,700 \\
23,000 \\
28,000\end{array}$ & $\begin{array}{l}20.82 \\
20.70 \\
20.55 \\
20.38 \\
20.18 \\
20.00\end{array}$ & $\begin{array}{l}13,800 \\
13,400 \\
13,000 \\
12,400 \\
11,800 \\
11,300\end{array}$ & 16.80 & 5,270 \\
\hline
\end{tabular}

$4547200-42-19$ 


\section{Santa Maria River Basin}

Cuyama River near Santa Maria, Callf.

Location. - Water-stage recorder, lat. $35^{\circ} 00^{\prime} 50^{\prime \prime}$, long. $120^{\circ} 16^{\prime} 45^{\prime \prime}$, In Suey grant, at highiay brldgo, 3 miles upstroam from Alamos $C$ rook, and 10 miles northerst of sants Mar1a, Santa Barbara County. Alt 1tude, a bout 610 foet.

Dra1nage area.- Area, 902 square m1los. Maximum alt1tude, 7,488 foet. Length of main Btroam channo 1,76 m1les.

Gage-ho1ght record. - Water-stage recorder graph excopt $6 \mathrm{a} . \mathrm{m}$. Fob. 6 to Fob. 12 and 9 a.m. tar. 3 to iar. 9. Maximum gage holght determined from hlgh-water marks in gage we13.

D1soharge reoord. - Stage-disoharge relation dof1ned by ourrent-meter measurements be10T 4,370 Becond-feet; extended logar1 thmioally to peak d1 sohargo determined by lopo-area mothod. Shifting-control corrections usod through the perlod of record. D1soharge Fob, 6-12 and Mar. 3-9 based on comparablo hydrograph of Euane River noar Santa Mar1a.

Maxima.- 1988 8 Disoharge, 17,300 seoond-foet 9 a.m. Mar. 3 (gage holght, 16.6 fogt'). 1929-37, D180harg०, 5,220 socond-foet Pob. 6, 1937 (gago ho1ght, 7.60 foot).

Remerya.- Records falr oxoept those for Fob. 6-12 and Mar, 3-9, which are poor. Ho aiversion above station.

Moan da11y discharge, in second-peot, $193 \mathrm{~S}$

\begin{tabular}{|c|c|c|c|c|c|c|c|c|c|c|c|}
\hline Day & seb. & Mar. & Apr. & Day & $F \bullet b$. & Mar. & Apr. & Day & $F \in b$. & Mar. & Apr. \\
\hline $\begin{array}{r}1 \\
2 \\
3 \\
4 \\
5 \\
6 \\
7 \\
8 \\
9 \\
10\end{array}$ & $\begin{array}{r}73 \\
48 \\
326 \\
157 \\
134 \\
115 \\
100 \\
94 \\
200 \\
428\end{array}$ & $\begin{array}{r}124 \\
325 \\
5,350 \\
2,180 \\
800 \\
400 \\
290 \\
378 \\
290 \\
280\end{array}$ & $\begin{array}{l}71 \\
74 \\
72 \\
69 \\
76 \\
68 \\
64 \\
60 \\
58 \\
66\end{array}$ & $\begin{array}{l}11 \\
12 \\
13 \\
14 \\
16 \\
16 \\
17 \\
18 \\
18 \\
20\end{array}$ & $\begin{array}{r}2,920 \\
992 \\
326 \\
246 \\
144 \\
104 \\
87 \\
76 \\
87 \\
78\end{array}$ & $\begin{array}{r}280 \\
968 \\
1,240 \\
690 \\
616 \\
400 \\
332 \\
290 \\
256 \\
232\end{array}$ & $\begin{array}{l}66 \\
58 \\
68 \\
68 \\
58 \\
85 \\
61 \\
49 \\
47 \\
44\end{array}$ & $\begin{array}{l}21 \\
22 \\
23 \\
24 \\
26 \\
26 \\
27 \\
28 \\
29 \\
30 \\
31\end{array}$ & $\begin{array}{l}74 \\
71 \\
68 \\
68 \\
66 \\
64 \\
64 \\
72\end{array}$ & $\begin{array}{r}204 \\
183 \\
169 \\
146 \\
189 \\
119 \\
104 \\
96 \\
90 \\
78 \\
78\end{array}$ & $\begin{array}{l}43 \\
39 \\
38 \\
51 \\
68 \\
64 \\
51 \\
46 \\
58 \\
67\end{array}$ \\
\hline \multicolumn{9}{|c|}{ 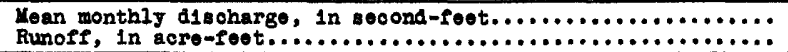 } & $\begin{array}{r}260 \\
14,440\end{array}$ & $\begin{array}{r}548 \\
33,320\end{array}$ & $\begin{array}{r}57,6 \\
3,430\end{array}$ \\
\hline
\end{tabular}

Buasna River noar Santa Mar1a, Calse.

Loont1on. - Vater-stage recorder, lat. $35^{\circ} 0 l^{\prime} 20^{\prime \prime}$, long. $120^{\circ} 18^{\prime} 20^{\prime}$, In Buez grant, half a

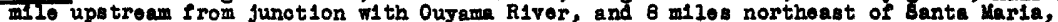
Santa Barbara CountJ. Alt1tude, about 600 foot.

Dre1mage areg.- Arog, 119 squiro m1los. Avorago alt1tudo, 1,371 foot. Maximum alt1tudo, 3,165 foot. Avorge slopo, 31 percent. Iongth of ma1n stroam obannol, 88 miler. Average slope of main tream channel, 22 percont.

Gage-belght reoord.- Wator-stage rocorder graph.

D1 scharse record. - Stage-d1soharge relation defined by current-meter meacuroments bo10W 1,080 B00ond-foet; extended to peak d1schargo determined by lopo-ares mothod. Shifting-control oorreotions used throughout the perlod.

Maxima.; Karoh 1938: D10charge, 1,190 second-foet 5 p.m. Mar. 2 (gage he1ght, 4.47 I00t).

1929-Fobruary 18382 D180hargo, 11,400 socond-foet 10:20 g.m. Fob. 11, 1938 (gage holght, 11.26 foot).

Remarke. - Records falr. Ho diverions above station. 
Mean dally discharge, in second-feet, 1938

\begin{tabular}{|c|c|c|c|c|c|c|c|c|c|c|c|}
\hline Day & Peb. & Mar. & Apr. & Day & Feb. & Mar. & Apr. & Day & Fob. & Mar. & Apr. \\
\hline $\begin{array}{r}1 \\
2 \\
3 \\
4 \\
5 \\
6 \\
7 \\
8 \\
9 \\
10\end{array}$ & $\begin{array}{l}304 \\
143 \\
597 \\
427 \\
243 \\
163 \\
127 \\
105 \\
193 \\
340\end{array}$ & $\begin{array}{l}271 \\
546 \\
910 \\
615 \\
427 \\
334 \\
377 \\
490 \\
334 \\
264\end{array}$ & $\begin{array}{l}59 \\
56 \\
52 \\
48 \\
51 \\
47 \\
44 \\
43 \\
42 \\
42\end{array}$ & $\begin{array}{l}11 \\
12 \\
13 \\
14 \\
15 \\
16 \\
17 \\
18 \\
19 \\
20\end{array}$ & $\begin{array}{r}4,630 \\
986 \\
493 \\
552 \\
395 \\
306 \\
240 \\
218 \\
252 \\
173\end{array}$ & $\begin{array}{r}261 \\
929 \\
1,156 \\
640 \\
467 \\
363 \\
295 \\
243 \\
212 \\
190\end{array}$ & $\begin{array}{r}-40 \\
39 \\
39 \\
36 \\
36 \\
35 \\
33 \\
32 \\
30 \\
30\end{array}$ & $\begin{array}{l}21 \\
22 \\
23 \\
24 \\
25 \\
26 \\
27 \\
28 \\
29 \\
30 \\
31\end{array}$ & $\begin{array}{r}147 \\
123 \\
101 \\
87 \\
74 \\
64 \\
59 \\
78\end{array}$ & $\begin{array}{r}169 \\
157 \\
139 \\
135 \\
121 \\
111 \\
97 \\
89 \\
85 \\
72 \\
64\end{array}$ & $\begin{array}{l}28 \\
26 \\
25 \\
28 \\
40 \\
35 \\
30 \\
30 \\
35 \\
35\end{array}$ \\
\hline \multicolumn{9}{|c|}{ 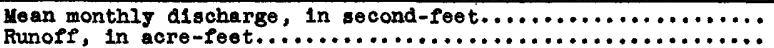 } & $\begin{array}{r}415 \\
23,050\end{array}$ & $\begin{array}{r}341 \\
20,950\end{array}$ & $\begin{array}{r}38.2 \\
2,270\end{array}$ \\
\hline
\end{tabular}

Gage height, in feet, and discharge, in second-feet, at indicated time, 1938

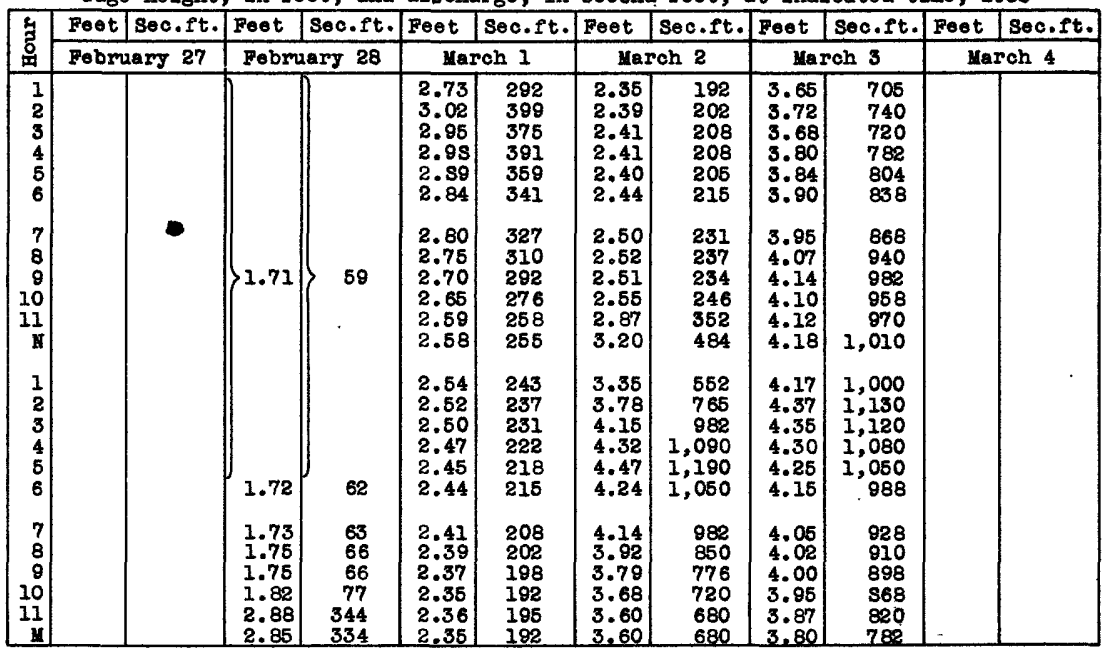

\section{Salton Sea Basin}

Palm Canyon Greek near Palm Springs, Cal1f.

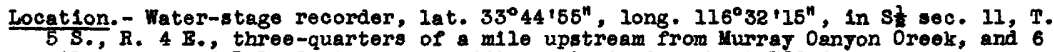
miles south of Palm Springs post office. Alt1tude, about 700 feet.

Drainage area.- 94 square miles.

Gage-he1ght record.- Water-stage recorder graph except Kar. 15-30.

Discharge record. - Stage-discharge relation falrly well defined below 30 second-feet; extended above by mean velocity-mean depth relation. Diacharge Mar. 15-30 obtalned by comparison with Mill Creek near Craftonvilie and Sante Ane River near Mentone.

Maxima.- 1938: D1scharge, 2,380 second-feet 10 p.m. Mar. 2 (gage he1ght, 4.43 feet). 1930-37: Discharge, 3,850 second-feet Feb. 6, 1937 (gage he1ght, 5.60 feet).

Remarks.- Records good. No regulation or diversion above gage. 
Mean dally discharge, in second-fest, 1938

\begin{tabular}{|c|c|c|c|c|c|c|c|c|c|c|c|}
\hline Day & Feb. & Mar. & Apr. & Day & Feb. & Mar. & Apr. & Day & Feb. & Mar. & Apr. \\
\hline $\begin{array}{r}1 \\
2 \\
3 \\
4 \\
5 \\
6 \\
7 \\
8 \\
9 \\
10\end{array}$ & $\begin{array}{c}12 \\
6.5 \\
3.9 \\
11 \\
11 \\
5.5 \\
4.2 \\
3.6 \\
4.5 \\
5.5\end{array}$ & $\begin{array}{r}106 \\
731 \\
813 \\
224 \\
77 \\
50 \\
41 \\
38 \\
31 \\
26\end{array}$ & $\begin{array}{l}15 \\
15 \\
15 \\
15 \\
14 \\
13 \\
13 \\
12 \\
12 \\
12\end{array}$ & $\begin{array}{l}11 \\
12 \\
13 \\
14 \\
15 \\
15 \\
17 \\
18 \\
19 \\
20\end{array}$ & $\begin{array}{c}5.5 \\
10 \\
6 \\
4.8 \\
4.5 \\
4.5 \\
3.9 \\
3.3 \\
4.2 \\
3.6\end{array}$ & $\begin{array}{r}16 \\
125 \\
82 \\
34 \\
26 \\
22 \\
20 \\
19 \\
18 \\
17\end{array}$ & $\begin{array}{l}12 \\
12 \\
14 \\
15 \\
13 \\
13 \\
12 \\
11 \\
11 \\
10\end{array}$ & $\begin{array}{l}21 \\
22 \\
23 \\
24 \\
25 \\
26 \\
27 \\
28 \\
29 \\
30 \\
31\end{array}$ & $\begin{array}{r}3.1 \\
2.8 \\
2.6 \\
2.6 \\
2.4 \\
2.4 \\
9 \\
22\end{array}$ & $\begin{array}{l}16 \\
16 \\
16 \\
16 \\
16 \\
16 \\
16 \\
16 \\
16 \\
16 \\
16\end{array}$ & $\begin{array}{l}10 \\
10 \\
9.5 \\
9.5 \\
13 \\
13 \\
10 \\
9.5 \\
9.5 \\
9.5\end{array}$ \\
\hline \multicolumn{9}{|c|}{ 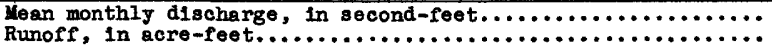 } & $\begin{array}{r}5.89 \\
327\end{array}$ & $\begin{array}{r}86.8 \\
5,340\end{array}$ & $\begin{array}{r}12.1 \\
719\end{array}$ \\
\hline
\end{tabular}

Discharge, in second-feet, at indicated tims, 1938

\begin{tabular}{|c|c|c|c|}
\hline Hour & March 1 & March 2 & March 3 \\
\hline 1 & 19 & 56 & 1,120 \\
2 & 21 & 55 & 1,000 \\
3 & 30 & 54 & 936 \\
4 & 100 & 54 & 825 \\
5 & 248 & 56 & 754 \\
6 & 238 & 64 & 721 \\
7 & 202 & 70 & 862 \\
8 & 183 & 86 & 1,050 \\
9 & 175 & 150 & 1,080 \\
10 & 148 & 366 & 1,260 \\
11 & 130 & 491 & 1,110 \\
N & 123 & 533 & 1,000 \\
1 & 112 & 658 & 862 \\
2 & 100 & 616 & 804 \\
3 & 95 & 501 & 754 \\
4 & 89 & 599 & 664 \\
5 & 84 & 741 & 628 \\
6 & 77 & 1,012 & 570 \\
7 & & 1,900 & 544 \\
8 & 72 & 2,200 & 528 \\
9 & 69 & 2,210 & 522 \\
10 & 66 & 2,380 & 496 \\
11 & 62 & 1,840 & 470 \\
M & 59 & 1,460 & 440 \\
\hline & 58 & & \\
\hline
\end{tabular}

\section{Mojave River Bas In}

Deep Creek near Hesperla, Calif.

Location. - Water-atage recorder, lat. $34^{\circ} 20^{\prime} 30^{\prime \prime}$, long. $117^{\circ} 13^{\prime} 40^{m}$, In SEt sec. 18, T. 3 K., R. 3 T., haif a mile upatream from confluence with Weat Fork of Mojeve River, and 8 miles southeast of Hesperla. Altitude, about 3,050 feet.

Dralnage area.- Arsa, 137 square miles. Average altitude, 5,850 feet. Maximum altitude, 8,500 feet. Average slope, 38 percent. Iength of main stream channel, 21.6 mlles. Average slope of main atream chennel, 4.5 percent.

Qage-helght record. - No record.

Discharge record.- Discharge Feb. I to Apr. 20 computed on basis of discharge measurements and studies of $f(0)$ at other stations in the Mojave River Basin: April 21-30 by standerd methods at temporary station a quarter of a mile downstream. Naximum discharge determined by slope-area method.

Maxima.- 1938: Discharge, 46,600 second-feet Mar. 2.

1929-37: Discharge, 7,900 second-feet Feb. 9, 1932.

Remarks.- Records poor. Storage in Lake Arrowhead. Ho diversion during flood period. 
Mean daily discharge, in second-feet, 1938

\begin{tabular}{|c|c|c|c|c|c|c|c|c|c|c|c|}
\hline Day & Feb. & Mar. & Apr. & Day & Feb. & Mar. & Apr. & Day & Feb. & Mar. & Apr. \\
\hline $\begin{array}{r}1 \\
2 \\
3 \\
4 \\
5 \\
6 \\
7 \\
8 \\
9 \\
10\end{array}$ & $\begin{array}{r}100 \\
340 \\
530 \\
1,230 \\
370 \\
160 \\
60 \\
40 \\
250 \\
265\end{array}$ & $\begin{array}{r}2,850 \\
12,000 \\
5,700 \\
1,900 \\
1,200 \\
880 \\
740 \\
600 \\
550 \\
530\end{array}$ & $\begin{array}{l}240 \\
240 \\
260 \\
290 \\
305 \\
290 \\
250 \\
235 \\
240 \\
235\end{array}$ & $\begin{array}{l}11 \\
12 \\
13 \\
14 \\
15 \\
16 \\
17 \\
18 \\
19 \\
20\end{array}$ & $\begin{array}{r}730 \\
560 \\
295 \\
190 \\
160 \\
98 \\
52 \\
38 \\
155 \\
74\end{array}$ & $\begin{array}{r}550 \\
2,200 \\
2,700 \\
2,100 \\
1,600 \\
1,550 \\
1,375 \\
1,210 \\
960 \\
850\end{array}$ & $\begin{array}{l}225 \\
215 \\
240 \\
235 \\
240 \\
380 \\
360 \\
300 \\
280 \\
260\end{array}$ & $\begin{array}{l}21 \\
22 \\
23 \\
24 \\
25 \\
26 \\
27 \\
28 \\
29 \\
30 \\
31\end{array}$ & \begin{tabular}{|r|}
50 \\
43 \\
41 \\
36 \\
30 \\
30 \\
490 \\
3,100 \\
\end{tabular} & $\begin{array}{l}760 \\
620 \\
510 \\
430 \\
420 \\
420 \\
380 \\
350 \\
350 \\
300 \\
300\end{array}$ & $\begin{array}{l}235 \\
218 \\
190 \\
178 \\
250 \\
278 \\
191 \\
155 \\
159 \\
167\end{array}$ \\
\hline \multicolumn{9}{|c|}{ 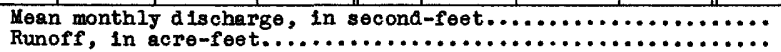 } & 18,880 & $\begin{array}{r}1,512 \\
93,000\end{array}$ & $\begin{array}{r}245 \\
14,560\end{array}$ \\
\hline
\end{tabular}

Mojave River at lower narrows, near Victorv1110, Calif.

Location.- Water-stage recorder, lat. $34^{\circ} 34^{\prime} 25^{n}$, long. $117^{\circ} 19^{\prime} 10^{n}$, in switset sec. 29 , T. 6 N., R. 4 W., 500 feet upstream from bridge on U. S. Highway No. 66, and 3 miles northwest of Victorville. Altitude, sbout 2,640 feet.

Drainage area. - 530 square miles.

Gage-helght record. - Water-stage recorder graph except 7:30 p.m. Mar. 2 to Mar. 29.

Discharge record.- Stage-discharge relation defined by current-meter measurements beIov 10,300 second-feet; peak discharge determined by slope-area method. Discharge Mar. 2-29 determined from discharge measurements and studies of flow at other stations in the Mojave River Besin.

Maxtma:- 1938: Discharge, 70,600 second-fe日t 7:30 p.m. Mar. 2 (gage helght, 16.70 feet).

1930-37: Discharge, 12,500 second-feet Feb. 9, 1932.

Remarks.- Records fair except those for Mar. 2-29, which a re poor. Storage at Lake Arrowhead and Lake aregory. No diversions during flood.

Mean dally d ischarge, in second-feet, 1938

\begin{tabular}{|r|c|r|r||r|r|r|r||r|r|r|r|}
\hline Day & Feb. & Mar. & Apr. & Day & Feb. & Mar. & Apr. & Day & Feb. & Mar. & Apr. \\
\hline 1 & 47 & 4,350 & 330 & 11 & 54 & 780 & 297 & 21 & 77 & 1,050 & 245 \\
2 & 48 & 16,000 & 330 & 12 & 288 & 3,000 & 285 & 22 & 64 & 890 & 221 \\
3 & 50 & 10,000 & 365 & 13 & 166 & 4,200 & 330 & 23 & 60 & 760 & 187 \\
4 & 48 & 3,000 & 403 & 14 & 120 & 3,400 & 319 & 24 & 61 & 620 & 176 \\
5 & 50 & 1,800 & 440 & 15 & 102 & 2,400 & 323 & 25 & 57 & 580 & 226 \\
6 & 48 & 1,300 & 416 & 16 & 90 & 2,200 & 405 & 26 & 55 & 530 & 360 \\
7 & 49 & 1,100 & 342 & 17 & 75 & 2,050 & 376 & 27 & 65 & 485 & 303 \\
8 & 52 & 960 & 319 & 18 & 64 & 1,850 & 303 & 28 & 2,660 & 430 & 215 \\
9 & 50 & 850 & 330 & 19 & 73 & 1,400 & 271 & 29 & & 410 & 159 \\
10 & 53 & 800 & 309 & 20 & 107 & 1,200 & 271 & 30 & & 354 & 209 \\
\end{tabular}


Mojeve River at Barstow, Calif.

Iocation, - Nater-8tage recorder, lat. $34^{\circ} 54^{\prime} 25^{\prime \prime}$, long. $117^{\circ} 01^{\prime 2} 0^{\prime \prime}$, in Swhst, sec. 31 , I. 10 N., R. 1 W., on J. S. Highway No. 91 at Barstow. Altitude, about 2,090 feet.

Gage-he1ght record.- Nater-8tage recorder graph.

Discharge record.- Stage-discharge relation defined by current-meter measurements below 5,200 second-feet; extended to peak discharge determined by slope-area method. Discharge Mar, 4-11, 20-31 determined from discharge measurements and studies of $110 \mathrm{w}$ at other stations in the Mojave River Basin. Discharge for Apr. 1-30 determined by interpolation between measurements.

Maxima.- 1938: Discharge, 64,300 second-feet 2 a.m. Mar. 3 (gage he1ght, 8.60 feet). 1930-37: Discherge, 8,300 second-feet Feb. 9, 1932.

Remarks.- Records fair. Storage at Lake Arrowhead and Lake Gregory. No divers ions during flood.

Mean daily disoharge, in seoond-feet, 1938

\begin{tabular}{|c|c|c|c|c|c|c|c|c|c|c|c|}
\hline Day & Feb. & Mar. & Apr. & Day & Feb. & Mar. & Apr. & Day & Feb. & Mar. & Apr. \\
\hline $\begin{array}{r}1 \\
2 \\
3 \\
4 \\
5 \\
6 \\
7 \\
8 \\
9 \\
10\end{array}$ & $\begin{array}{l}0 \\
0 \\
0 \\
0 \\
0 \\
0 \\
0 \\
0 \\
0 \\
0\end{array}$ & $\begin{array}{r}3,070 \\
3,720 \\
28,100 \\
3,200 \\
1,850 \\
1,250 \\
1,050 \\
920 \\
820 \\
770\end{array}$ & $\begin{array}{l}290 \\
285 \\
280 \\
275 \\
270 \\
265 \\
260 \\
255 \\
250 \\
245\end{array}$ & $\begin{array}{l}11 \\
12 \\
13 \\
14 \\
15 \\
16 \\
17 \\
18 \\
19 \\
20\end{array}$ & $\begin{array}{l}0 \\
0 \\
0 \\
0 \\
0 \\
0 \\
0 \\
0 \\
0 \\
0\end{array}$ & $\begin{array}{r}730 \\
1,600 \\
4,000 \\
3,500 \\
2,240 \\
2,060 \\
1,890 \\
1,920 \\
1,350 \\
1,140\end{array}$ & $\begin{array}{l}240 \\
236 \\
232 \\
228 \\
224 \\
220 \\
216 \\
212 \\
208 \\
204\end{array}$ & $\begin{array}{l}21 \\
22 \\
23 \\
24 \\
25 \\
26 \\
27 \\
28 \\
29 \\
30 \\
31\end{array}$ & $\begin{array}{l}0 \\
0 \\
0 \\
0 \\
0 \\
0 \\
0 \\
0\end{array}$ & $\begin{array}{l}900 \\
757 \\
680 \\
580 \\
520 \\
460 \\
410 \\
360 \\
340 \\
320 \\
300\end{array}$ & $\begin{array}{l}200 \\
197 \\
198 \\
199 \\
200 \\
201 \\
202 \\
203 \\
204 \\
205\end{array}$ \\
\hline \multicolumn{9}{|c|}{ 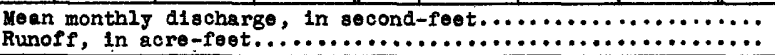 } & $\begin{array}{l}0 \\
0\end{array}$ & $\begin{array}{r}1,962 \\
120,600\end{array}$ & $\begin{array}{r}230 \\
13,690\end{array}$ \\
\hline
\end{tabular}

Gage height, In feet, and discharge, in second-feet, et indicated time, 1938

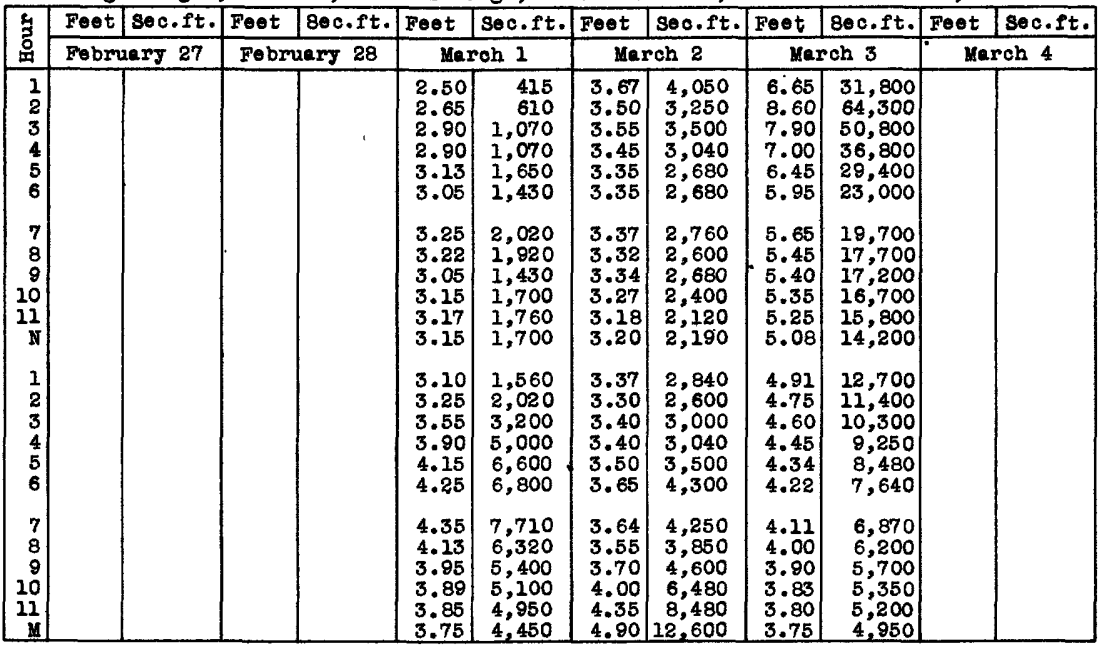


West Fork of Mojave River near Hosperla, Oal1f.

Loogtson.- Water-stage reoorder, lat. $34^{\circ} 20^{\prime} 20^{n}$, long. $117^{\circ} 14^{\prime} 35^{n}$, in SEs sec. 13, T. SH., R. 4 W., at hlghway bridge, haif a mile upstream from confluence with Deep Oreek, and 7 miles southeset of Hsaperia. Altitude, about 3,050 fset.

Drainige area. - Area, 74.8 square m1108. Average altstuds, 4,270 foot. Maximum alt1tude, 6,150 foet. Length of main stroam channel, 12.5 msles. Average slope of main strsam ohannel, 3.7 percent.

Gage-hesght record. - Water-8tage recorder graph except 11 a.m. War. 2 to Mar. 14.

DIscharge record. - Stags-discharge relation derined by current-moter measurements below 2,060 socond-foet; oxtended logarl thmicaliy to maximum discharge dotermined by

siope-area mothod. Disoharge for perlod of missing gage-helght record doterminod

from discharge measurements and studies of flow at other stations in the Mojeve River Besin.

Maxime. - 1938: . D1scharge, 26,100 second-feet Mar. 2.

1930-37: D1scharge, 6,000 second-feet Fob. 8, 1932.

Remarks. - Records good except those for Mar. 2-14, whlch are poor. Storage at Lake Gregory.

Mean da1ly discharge, in second-feet, 1938

\begin{tabular}{|c|c|c|c||c|c|c|c||c|r|r|r|}
\hline Day & Feb. & Mar. & Apr. & Day & Feb. & Mar. & Apr. & Day & Feb. & Mar. & Apr. \\
\hline 1 & 67 & 2,090 & 178 & 11 & 375 & 330 & 108 & 21 & 64 & 388 & 74 \\
2 & 197 & 7,000 & 164 & 12 & 298 & 1,300 & 102 & 22 & 58 & 324 & 74 \\
3 & 287 & 3,300 & 161 & 13 & 174 & 1,600 & 150 & 23 & 56 & 275 & 70 \\
4 & 603 & 1,100 & 158 & 14 & 122 & 1,200 & 114 & 24 & 50 & 235 & 67 \\
5 & 212 & 700 & 164 & 15 & 103 & 844 & 108 & 25 & 43 & 232 & 171 \\
6 & 108 & 520 & 147 & 16 & 89 & 740 & 102 & 26 & 43 & 232 & 128 \\
7 & 71 & 410 & 128 & 17 & 67 & 660 & 98 & 27 & 264 & 214 & 102 \\
6 & 54 & 380 & 125 & 18 & 52 & 560 & 91 & 28 & 1,410 & 201 & 88 \\
9 & 151 & 330 & 114 & 19 & 106 & 480 & 78 & 29 & & 201 & 90 \\
10 & 160 & 310 & 111 & 20 & 79 & 429 & 88 & 30 & & 187 & 174 \\
\hline
\end{tabular}

Gage helght, in feet, and discharge, in second-feet, at indlcated time, 1838

\begin{tabular}{|c|c|c|c|c|c|c|c|c|c|c|c|c|}
\hline \multirow{2}{*}{$\begin{array}{l}5 \\
\text { 至 }\end{array}$} & Feot & soo.ft. & Feet & sec.ft. & Foet & Sec.ft. & Feet & Sec.ft. & Feet & sec.ft. & Feet & sec.ft. \\
\hline & \multicolumn{2}{|c|}{ February 27} & \multicolumn{2}{|c|}{ Fobmuary 28} & \multicolumn{2}{|c|}{ March 1} & \multicolumn{2}{|c|}{ March 2} & \multicolumn{2}{|c|}{ March 3} & \multicolumn{2}{|c|}{ March 4} \\
\hline \begin{tabular}{l|}
1 \\
2 \\
3 \\
4 \\
5 \\
6
\end{tabular} & 0.87 & 43 & $\begin{array}{l}3.20 \\
3.10 \\
3.15 \\
3.22 \\
3.40 \\
3.65\end{array}$ & $\begin{array}{l}690 \\
646 \\
666 \\
710 \\
800 \\
930\end{array}$ & $\begin{array}{l}6.00 \\
7.75 \\
8.70 \\
9.33 \\
8.20 \\
6.75\end{array}$ & $\begin{array}{l}2,480 \\
3,780 \\
4,540 \\
5,050 \\
4,130 \\
3,020\end{array}$ & $\begin{array}{l}3.43 \\
3.38 \\
3.39 \\
3.42 \\
3.58 \\
3.66\end{array}$ & $\begin{array}{r}940 \\
920 \\
925 \\
940 \\
1,020 \\
1,070\end{array}$ & & & & \\
\hline $\begin{array}{r}7 \\
8 \\
9 \\
10 \\
11 \\
1\end{array}$ & $\begin{array}{r}.87 \\
.95 \\
1.03 \\
1.12\end{array}$ & $\begin{array}{l}43 \\
52 \\
62 \\
74\end{array}$ & $\begin{array}{l}3.86 \\
4.15 \\
4.40 \\
4.50 \\
4.60 \\
5.10\end{array}$ & $\begin{array}{l}1,040 \\
1,210 \\
1,370 \\
1,430 \\
1,500 \\
1,870\end{array}$ & $\begin{array}{l}6.00 \\
5.70 \\
5.43 \\
5.15 \\
4.85 \\
4.67\end{array}$ & $\begin{array}{l}2,480 \\
2,290 \\
2,120 \\
1,940 \\
1,750 \\
1,650\end{array}$ & $\begin{array}{l}3.95 \\
5.00 \\
6.25 \\
7.10 \\
7.70\end{array}$ & $\begin{array}{l}1,220 \\
1,840 \\
2,660 \\
3,220 \\
3,710\end{array}$ & & - & & \\
\hline $\begin{array}{l}1 \\
2 \\
3 \\
4 \\
5 \\
6\end{array}$ & $\begin{array}{l}1.28 \\
1.57 \\
1.85 \\
2.20 \\
2.65 \\
3.00\end{array}$ & $\begin{array}{r}98 \\
148 \\
208 \\
298 \\
446 \\
582\end{array}$ & $\begin{array}{l}5.60 \\
5.94 \\
5.70 \\
5.52 \\
5.18 \\
4.83\end{array}$ & $\begin{array}{l}2,220 \\
2,440 \\
2,290 \\
2,170 \\
1,950 \\
1,740\end{array}$ & $\begin{array}{l}4.47 \\
4.38 \\
4.25 \\
4.15 \\
4.05 \\
3.95\end{array}$ & $\begin{array}{l}1,530 \\
1,470 \\
1,400 \\
1,340 \\
1,280 \\
1,220\end{array}$ & & & & & & \\
\hline $\begin{array}{r}7 \\
8 \\
9 \\
10 \\
11 \\
\forall\end{array}$ & $\begin{array}{l}3.27 \\
3.30 \\
3.32 \\
3.35 \\
3.30 \\
3.25\end{array}$ & $\begin{array}{l}695 \\
715 \\
730 \\
745 \\
725 \\
705\end{array}$ & $\begin{array}{l}4.60 \\
4.35 \\
4.16 \\
4.05 \\
4.10 \\
4.50\end{array}$ & $\begin{array}{l}1,600 \\
1,460 \\
1,340 \\
1,280 \\
1,310 \\
1,550\end{array}$ & $\begin{array}{l}3.85 \\
3.75 \\
3.65 \\
3.60 \\
3.55 \\
3.50\end{array}$ & $\begin{array}{l}1,170 \\
1,120 \\
1,070 \\
1,040 \\
1,010 \\
990\end{array}$ & & & & & & \\
\hline
\end{tabular}


In table 23, "Summary of flood discharges," are assembled the results of the determinations of maximum flood discharges at stream-gaging stations and other places on streams in southern California. For convenience, several gaging stations that are plotted on the isohyetal maps are included, although the maximum discharge at those stations was not determined. The table gives the following information: (1) Map reference number, to aid in locating the place where the discharge was determined, as plotted on plates 11, 12, and 13; (2) name of stream and place where discharge was determined; (3) latitude and longitude of place of measurement; (4) approximate altitude of place of measurement as determined from topographic maps; (5) drainage area, in square miles, tributary to the stream at the place where discharge was determined; (6) period of recordwhich conforms to period of operation of the station-given only for ex1sting stream-gaging stations; (7) date and magnitude of the maximum discharge recorded prior to the March 1938 floods, that is, during the period of record given in the preceding column; (8) day and hour, if known, and rate in second-feet, total and per square mile, of the maximum discharge in March 1938; (9) a brief notation as to the method used in determining the maximum discharge.

Water-Supply Papers 391 (pp. 89-91) and 447 (pp. 544-550) give, among other data, tables of estimated flood discharge of streams in the vicinity of Los Angeles, Calif., during the storms of January and February 1914. At that time, under the direction of J. B. Lippincott, of the board of engineers for flood control, Los Angeles County, sections were selected and data collected for computing flood flow. The summary of flood discharges as given in this report (table 23) is primarily concerned with records made since continuous and systematic stream-gaging was inaugurated, and therefore the estimates of flood discharge from the storms of January and February 1914 were not generally considered in the preparation of the table. A summary of information concerning floods prior to the general period of record is given in the section of this report on "History of floods in southern Califormia". (See pp. 385-394.)

The methods emplojed are described in detail in the section of this report entitled "Determination of flood discharge". (See pp. 168-182.) The basic data used in determining the discharges are filed in the Los Angeles office of the Geological Survey, where they may be examined. 


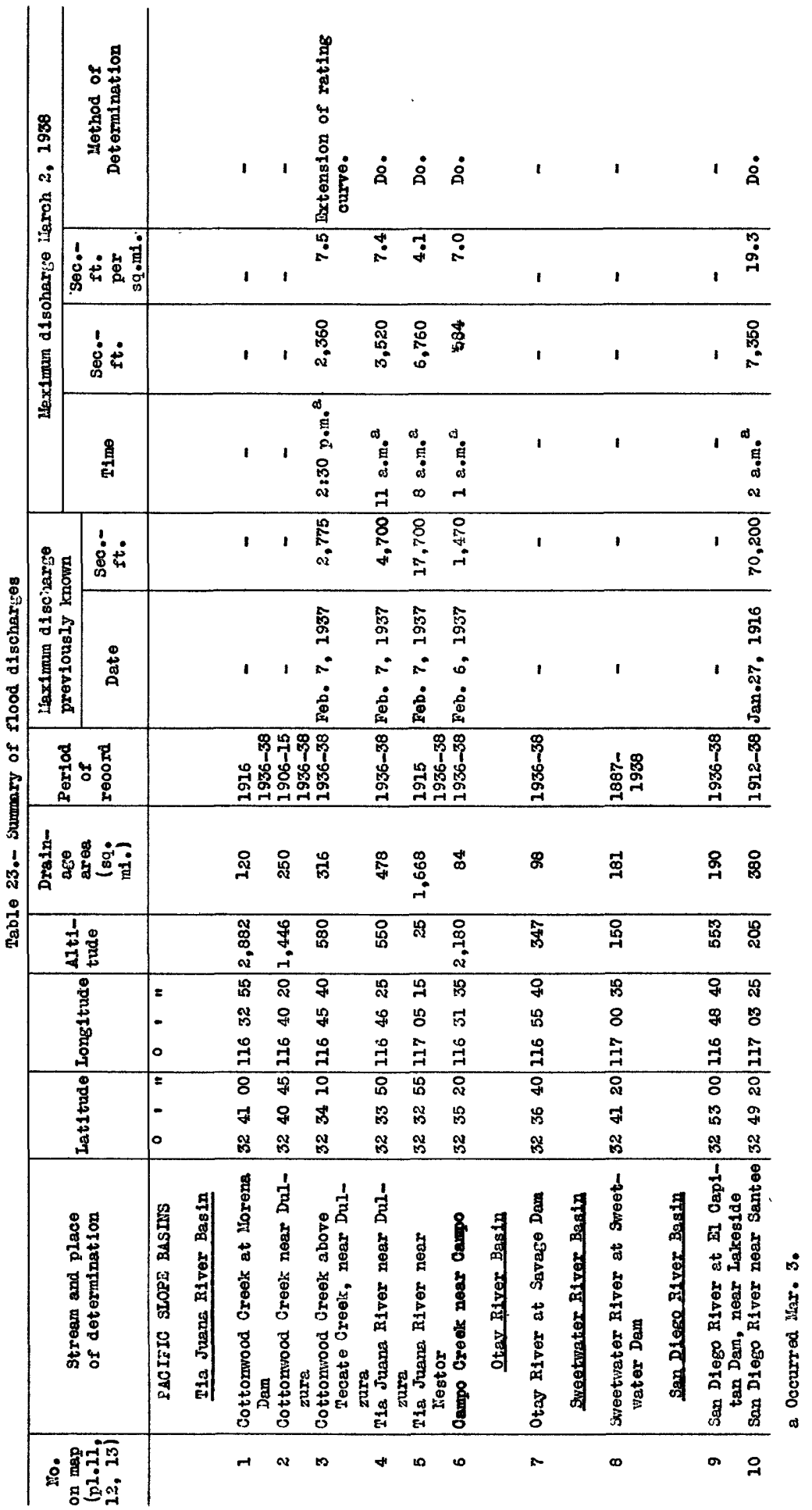




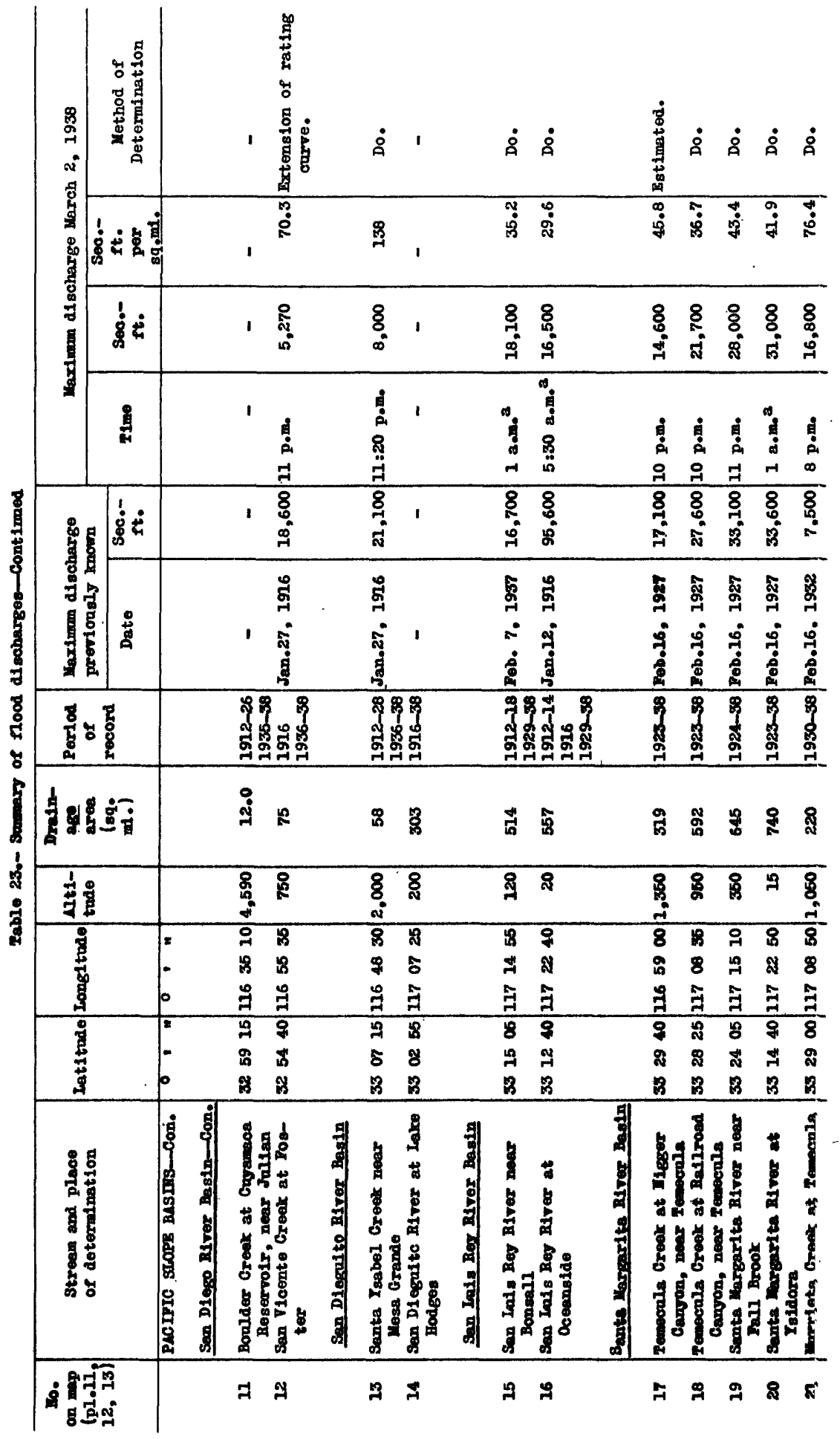




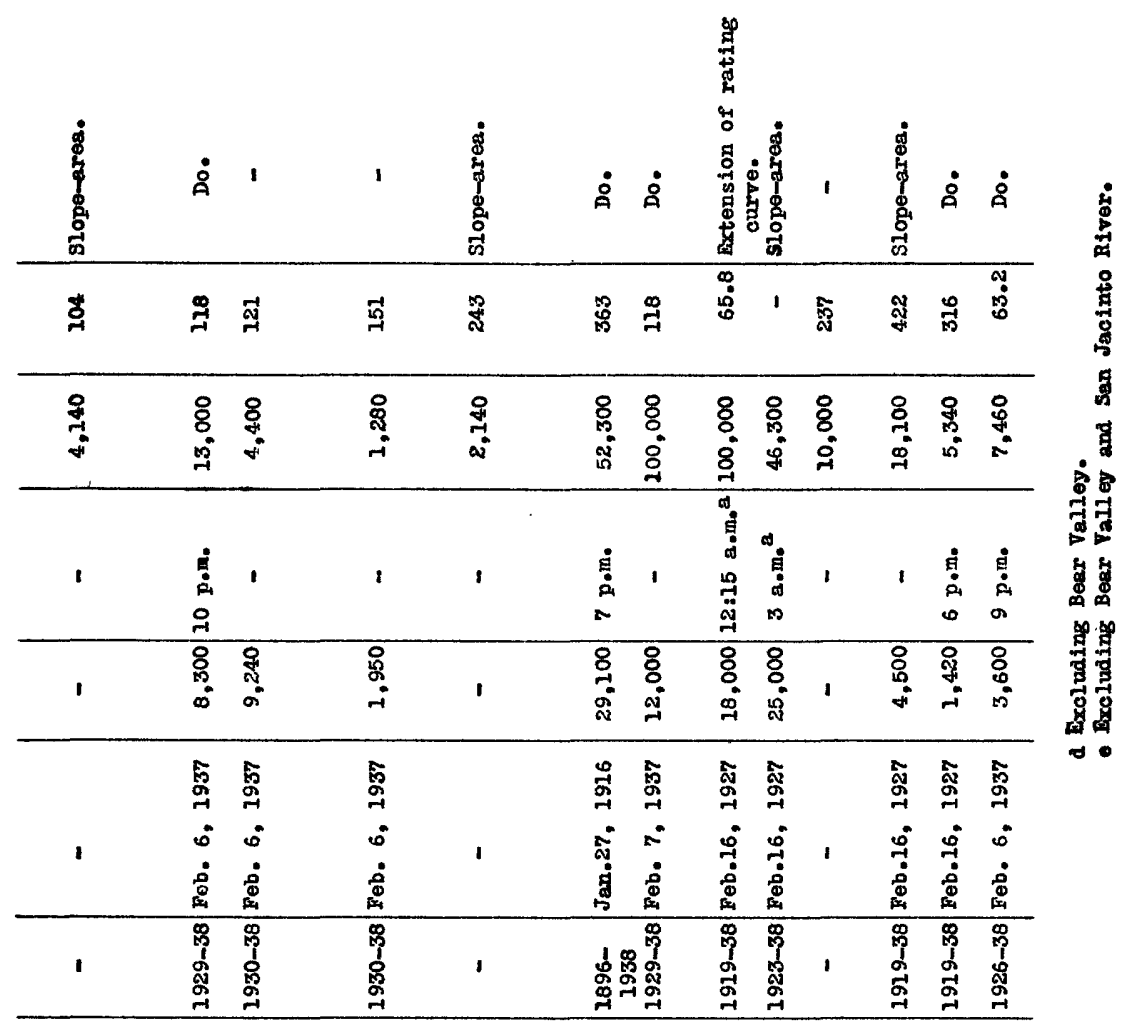

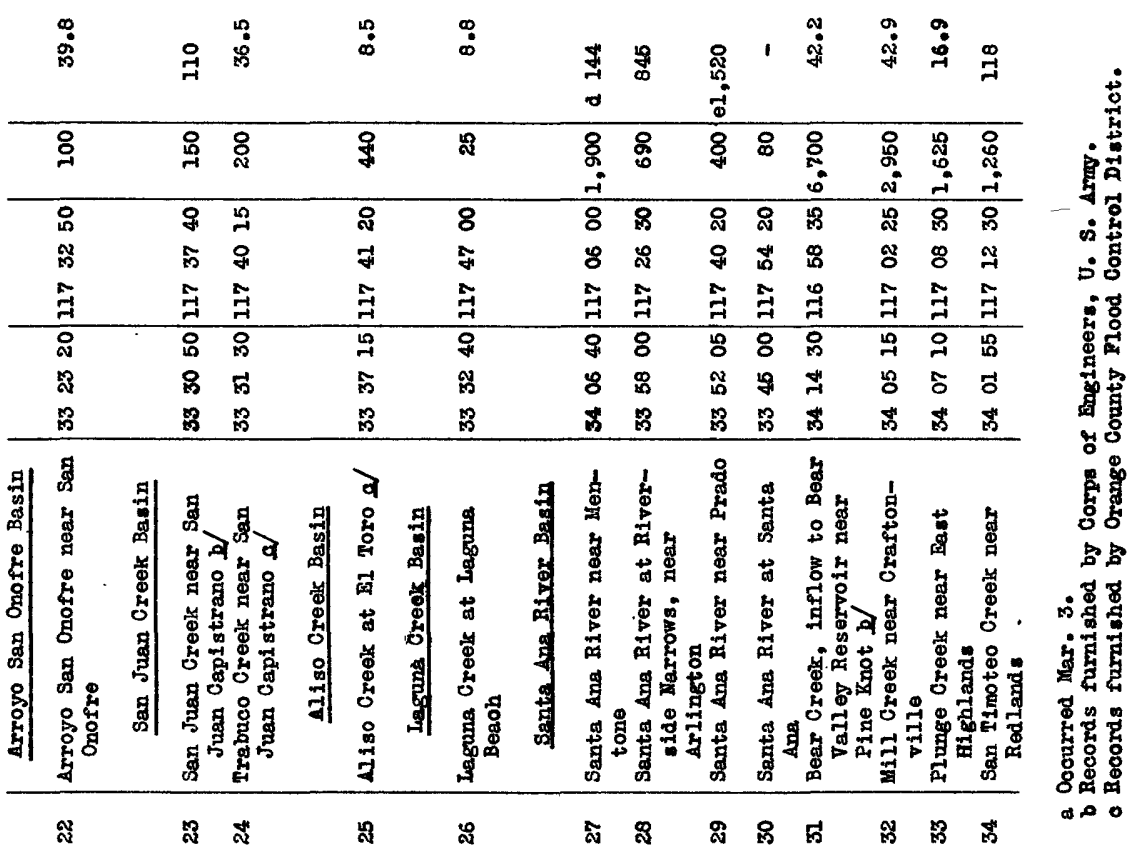




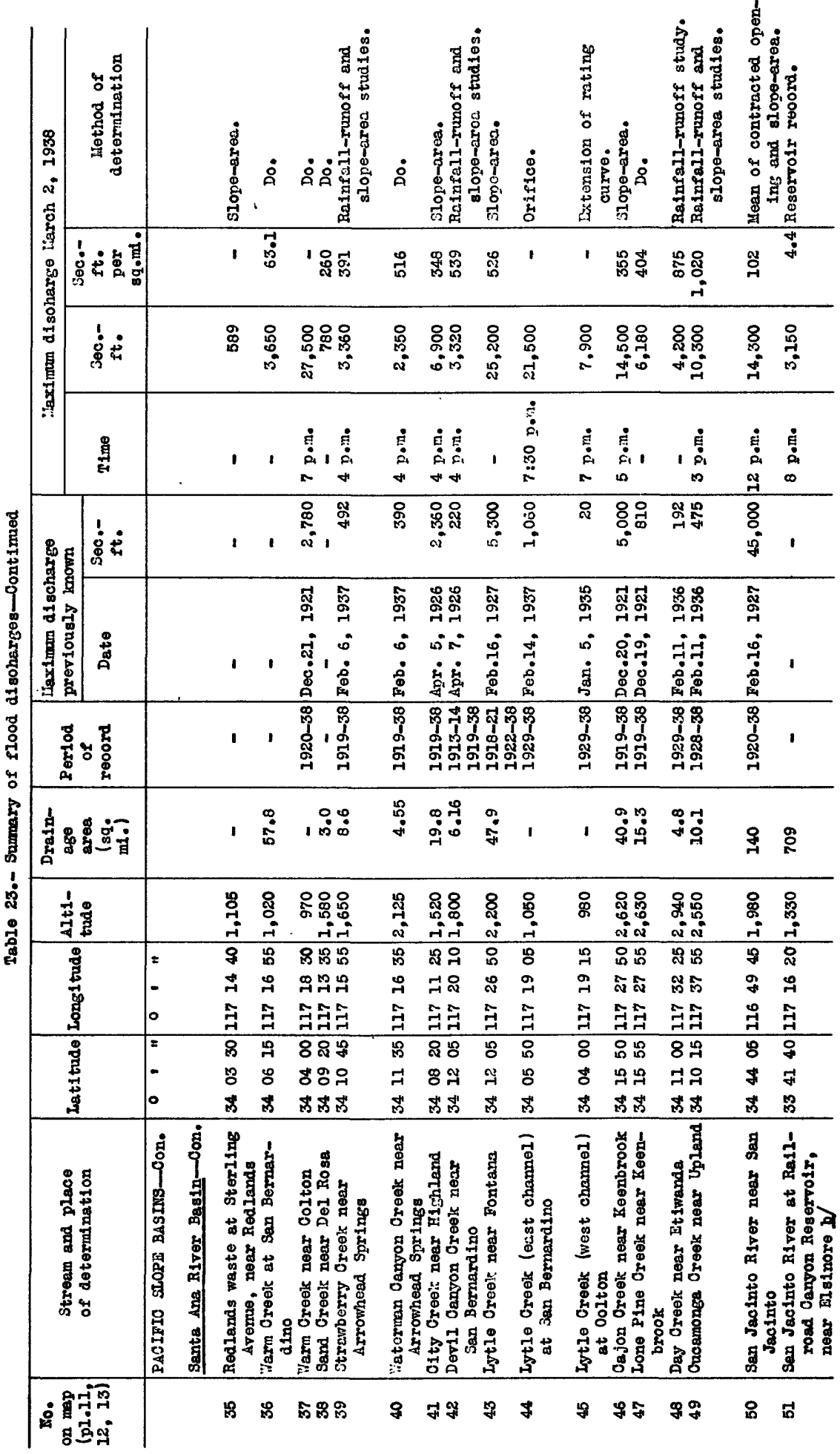



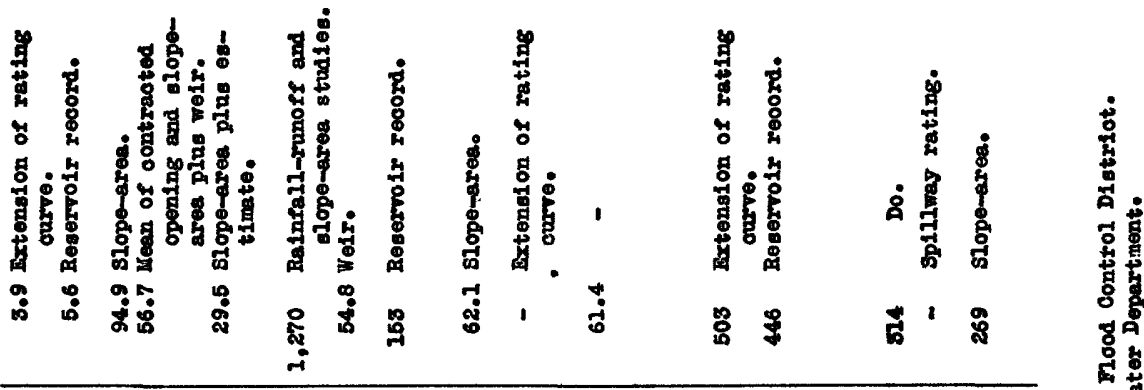

\begin{tabular}{|c|c|c|}
\hline 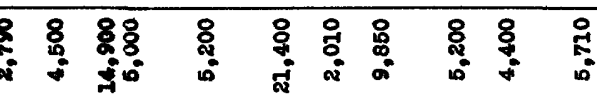 & $\begin{array}{l}88 \\
80 \\
10\end{array}$ & 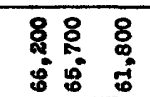 \\
\hline 这 & & $\begin{array}{l}1 \\
\end{array}$ \\
\hline
\end{tabular}

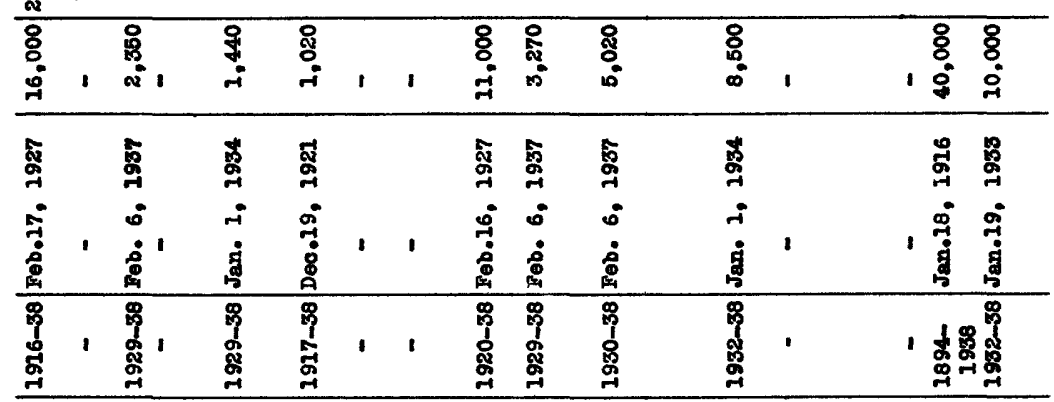

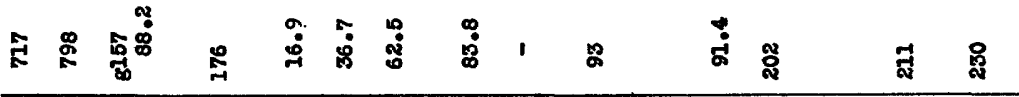

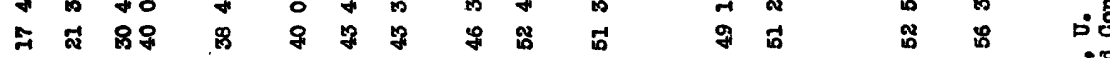

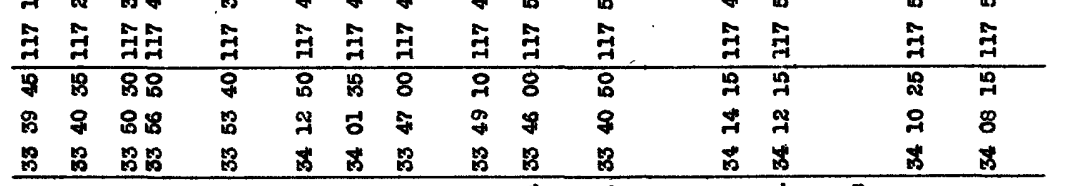

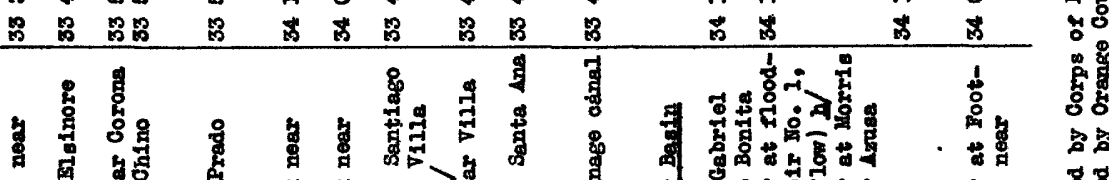

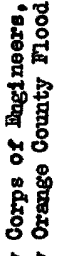

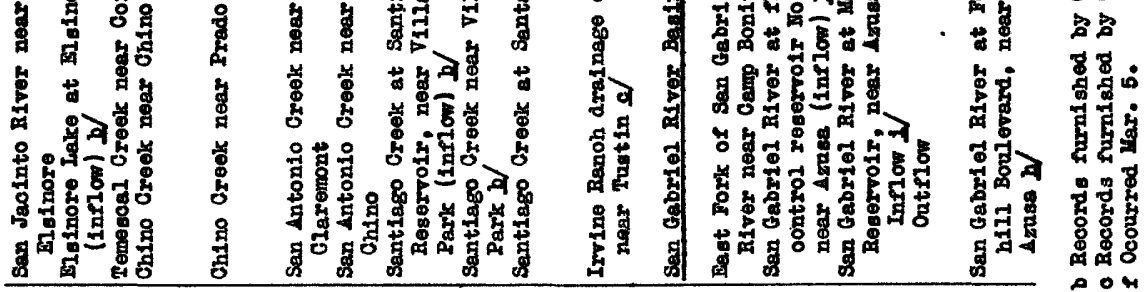
㽞 


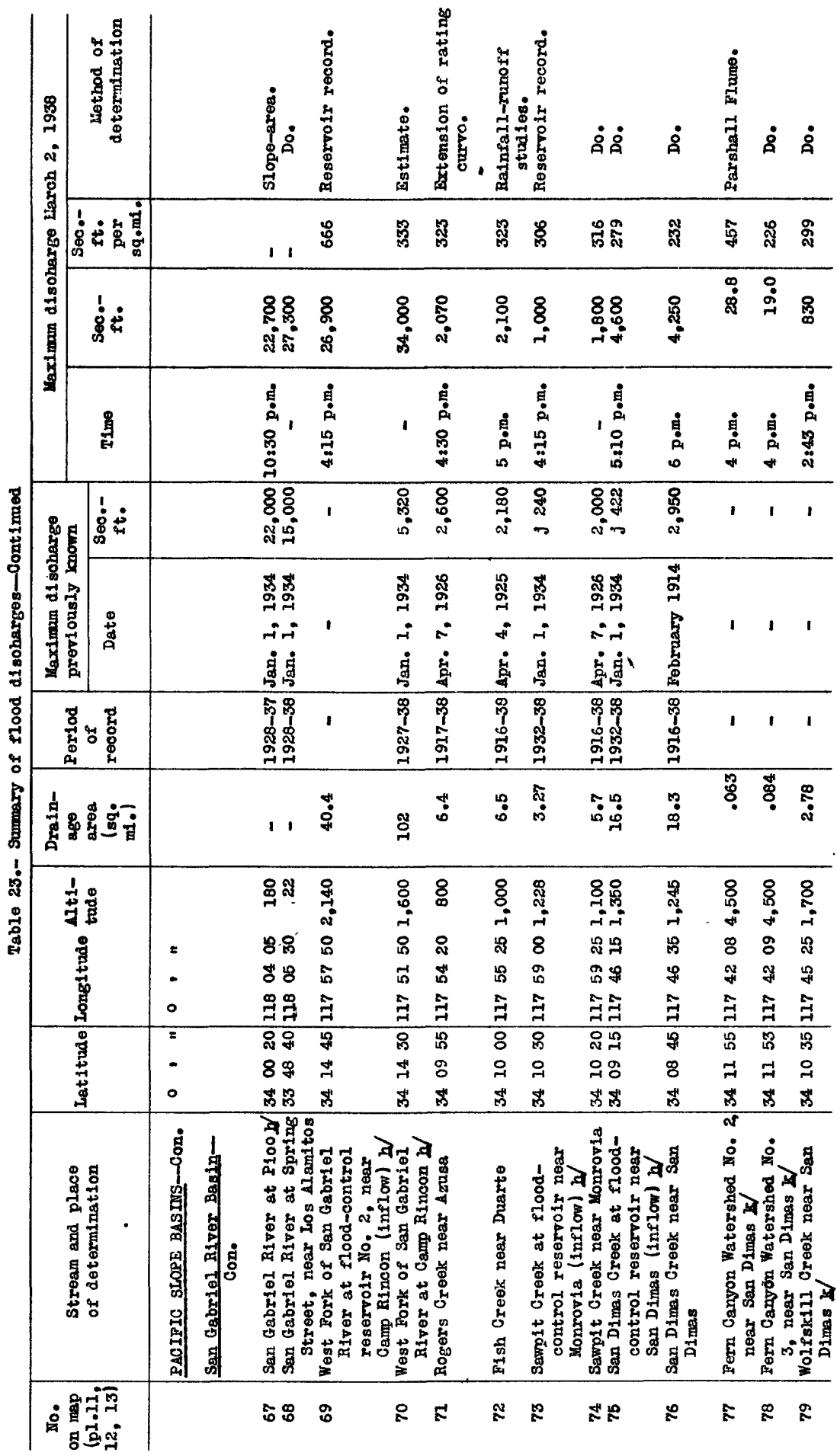




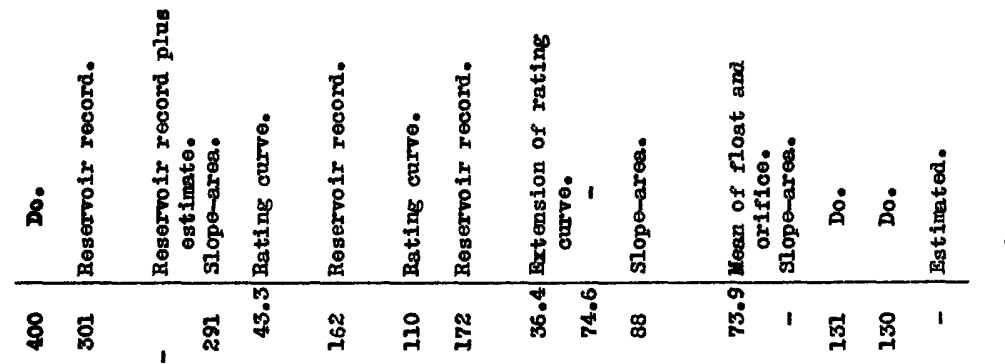

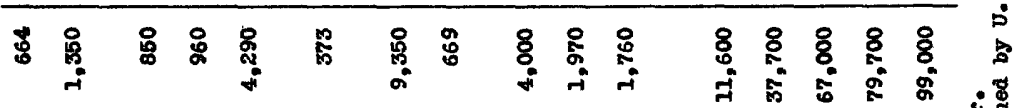

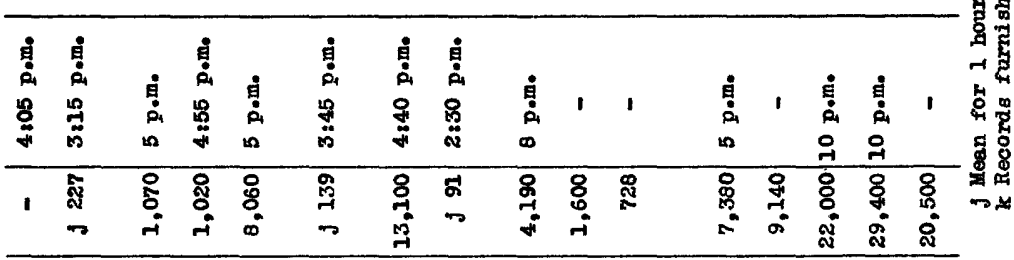

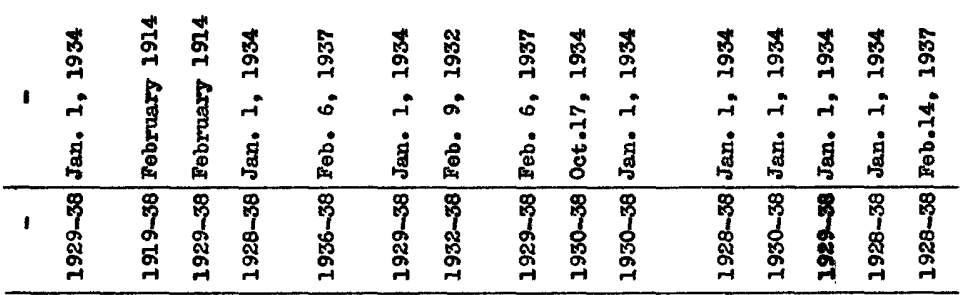

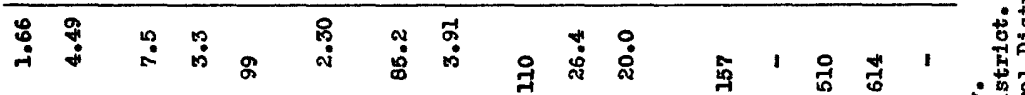
4 它

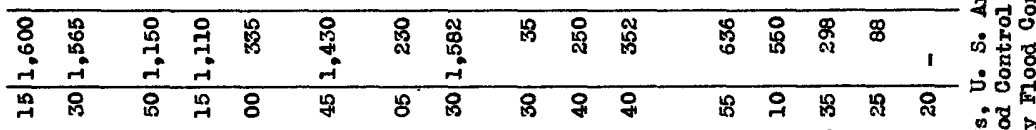
\% 年

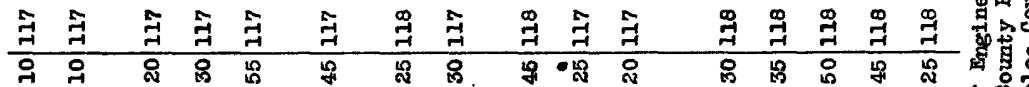

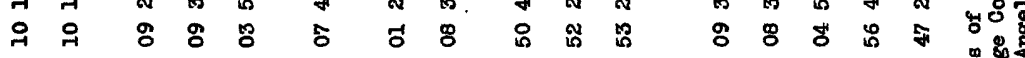

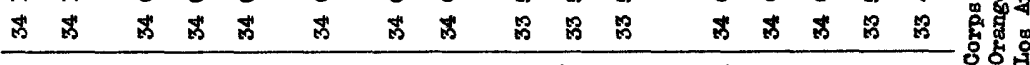

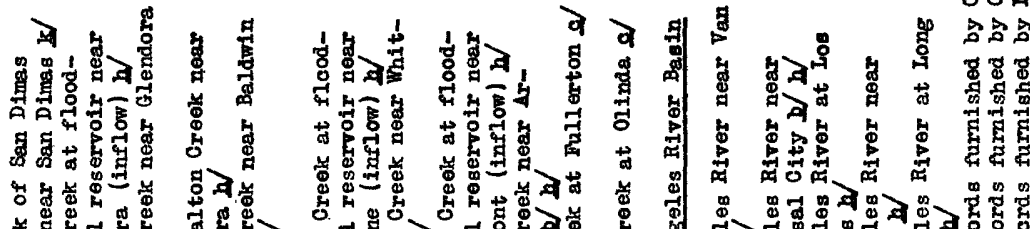

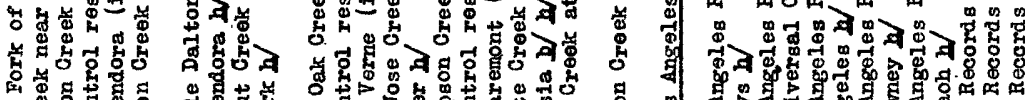

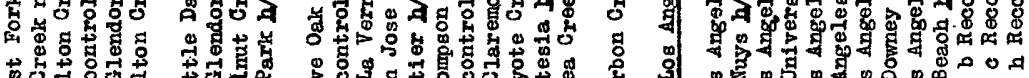

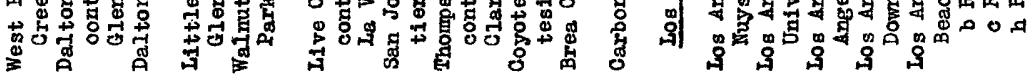

ஓ 


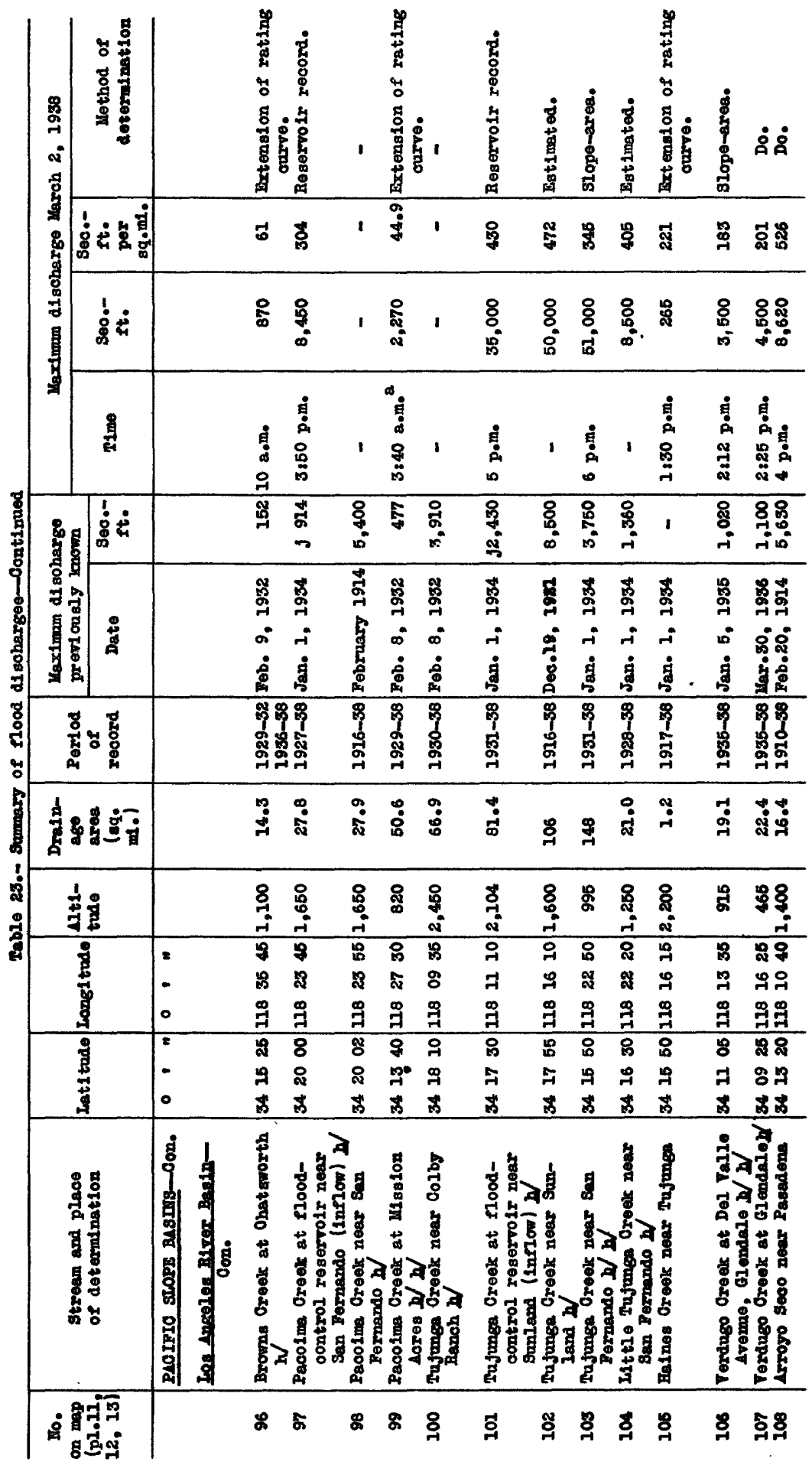




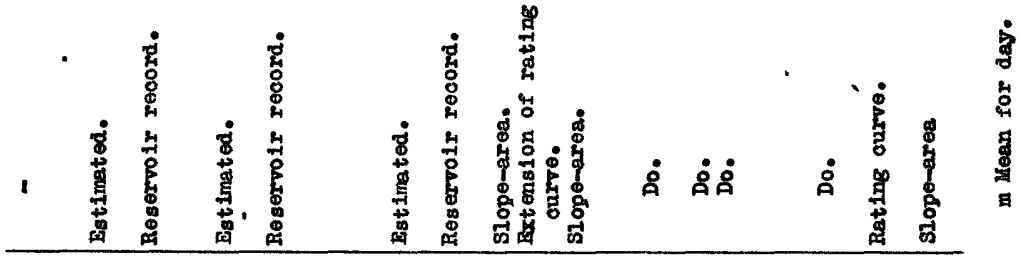

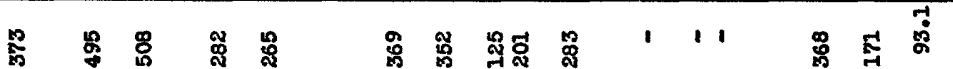

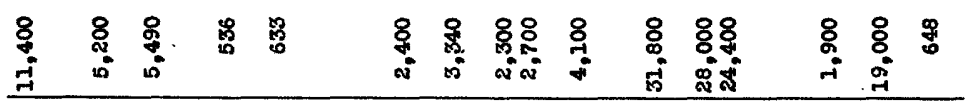

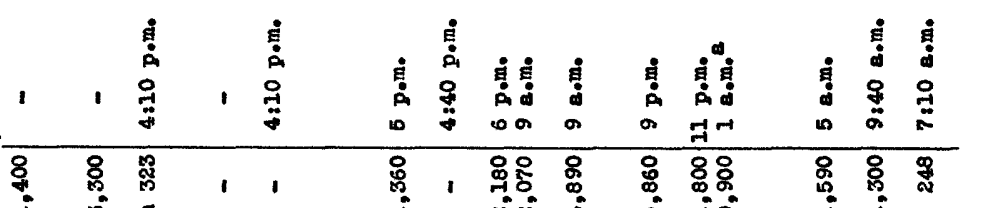
产 焉

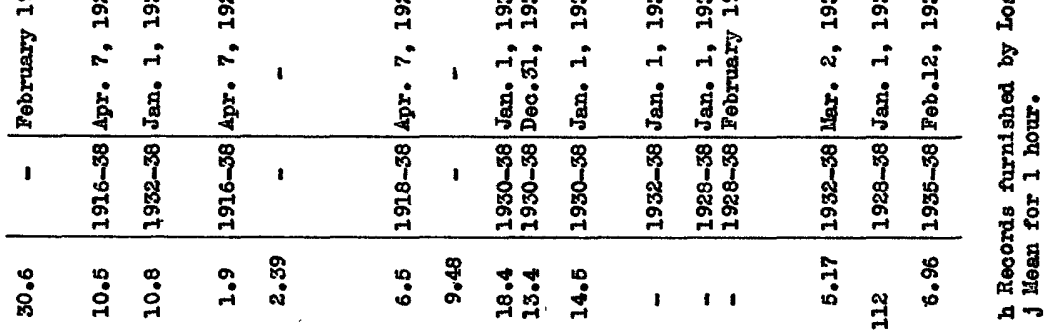

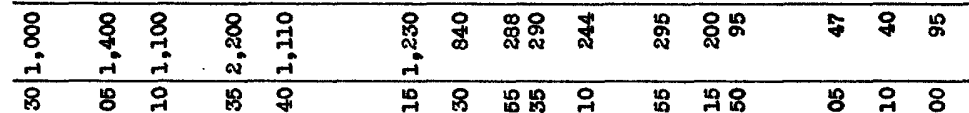

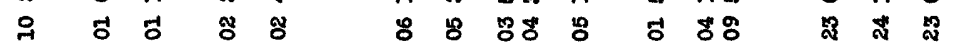
弯

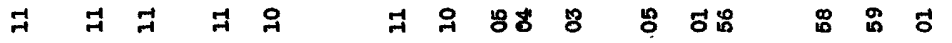

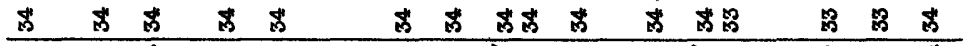

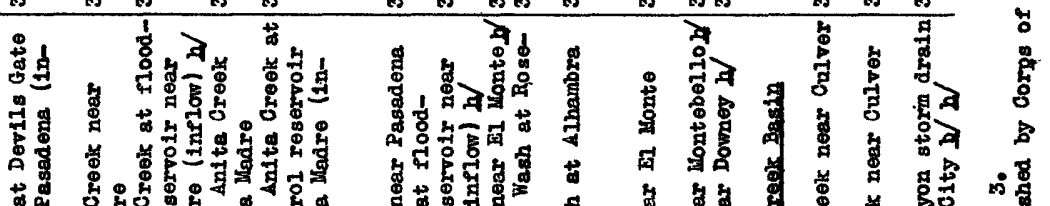
के

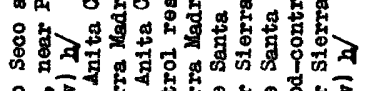

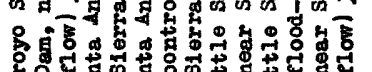

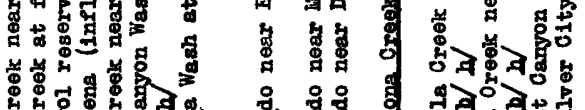

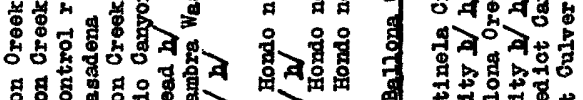

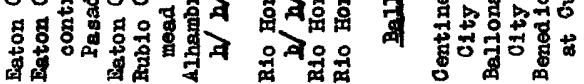

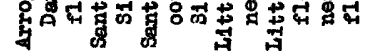
(1)

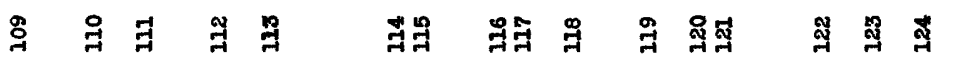




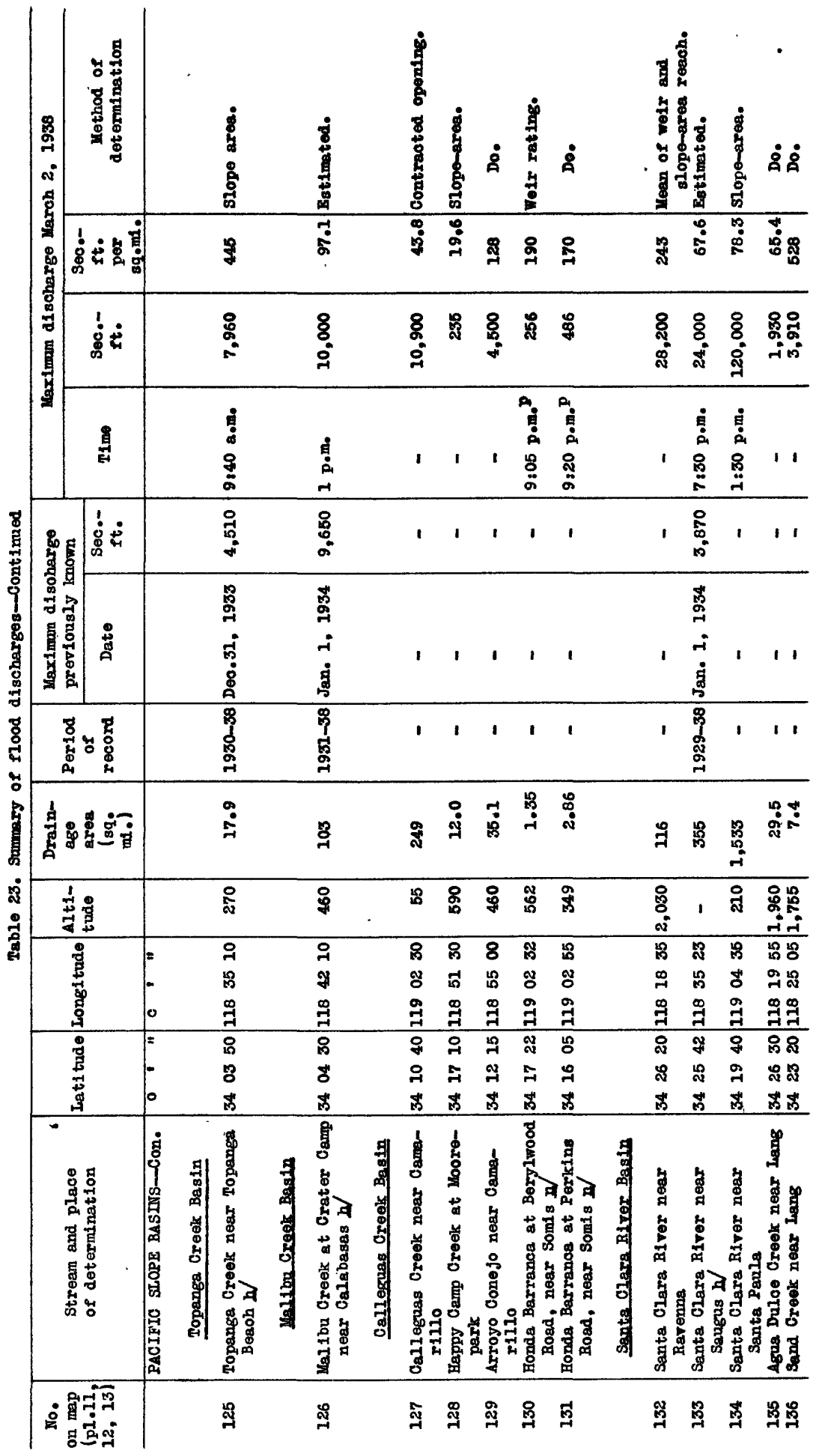




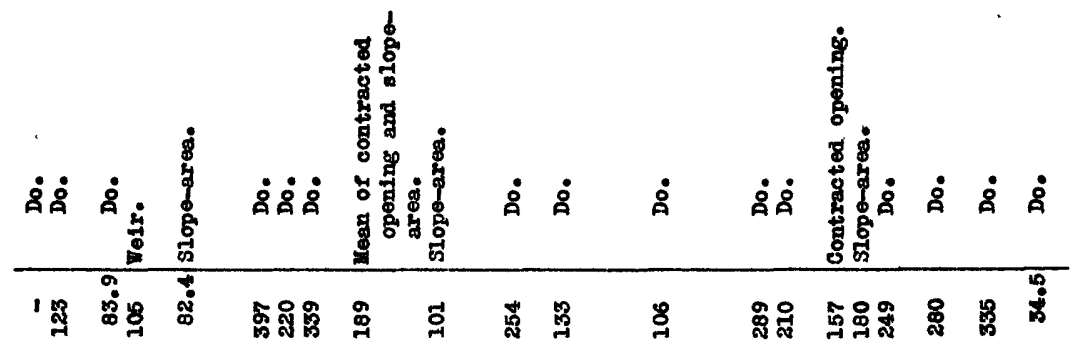

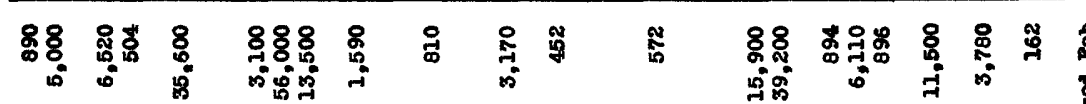

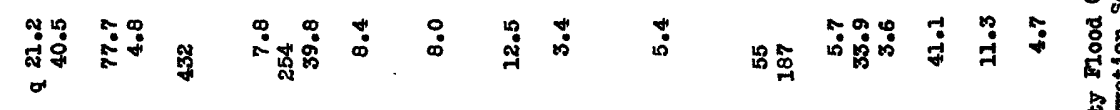

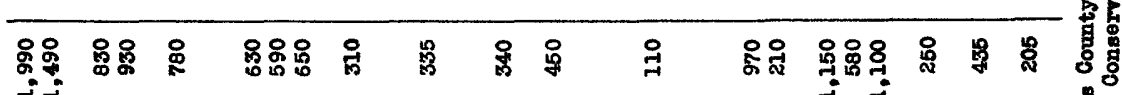

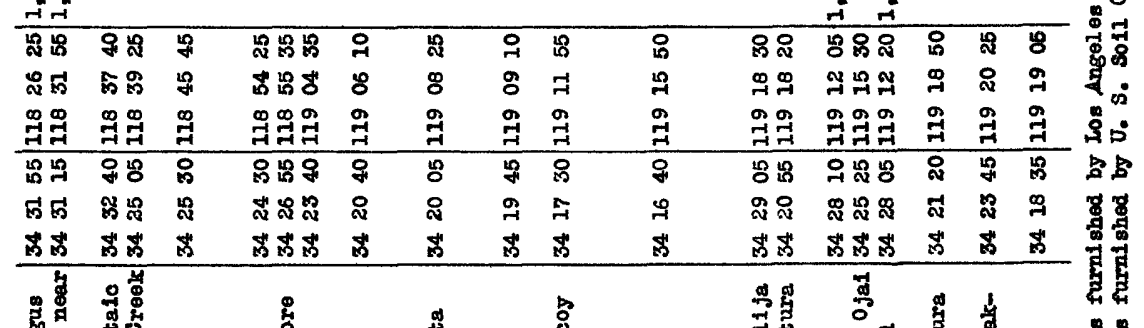

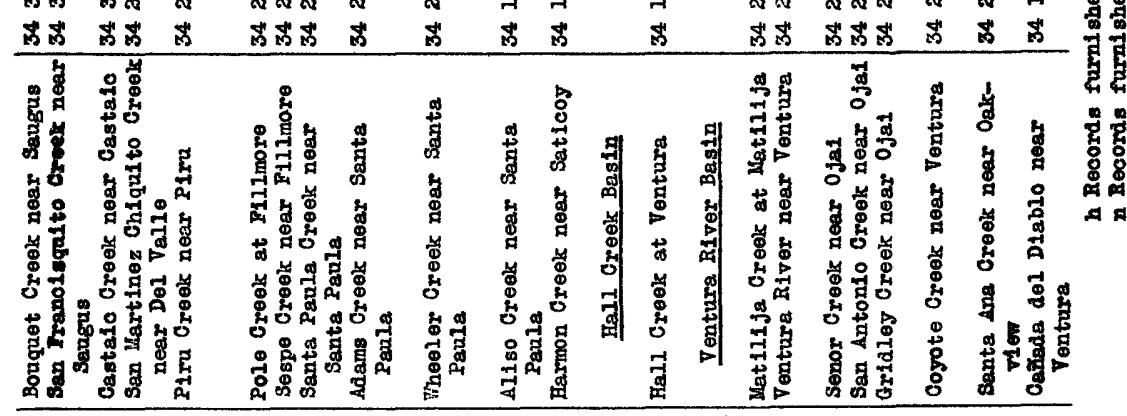

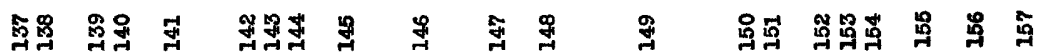




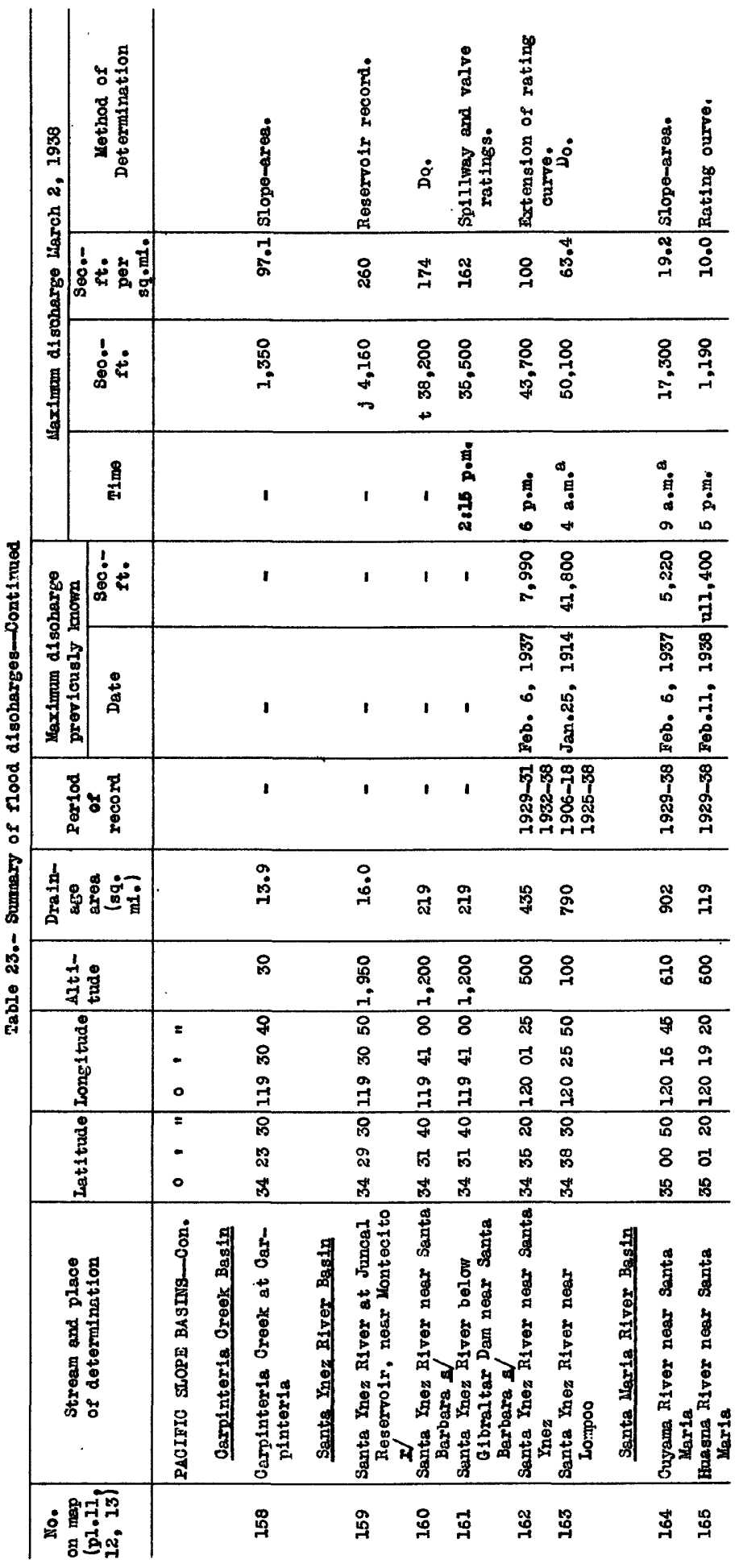




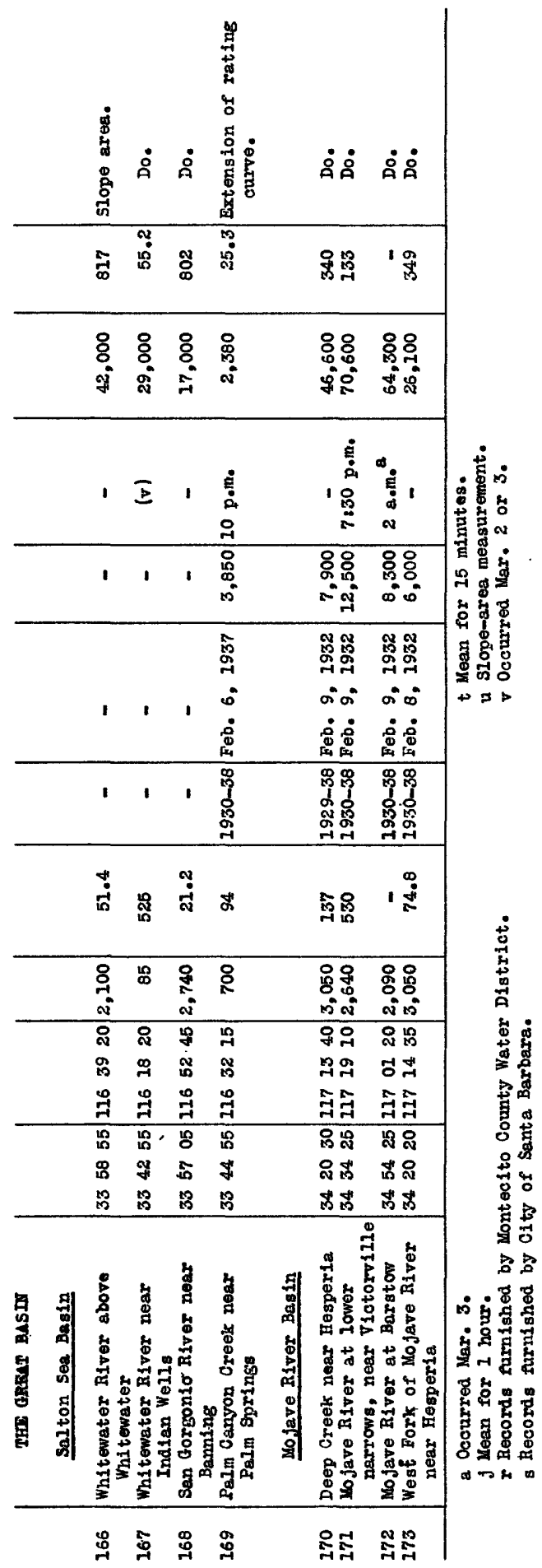




\section{PHYSIOGRAPHIC AND GEOLOQIC FEATURES AFFECTING FLOOD RUNOFF}

By Harold V. Peterson

\section{Introduction}

In a region in which the structures are as diverse and the topographic and geologic contrasts as sharp as they are in southern California it is evident that any attempt to discuss the relation between flood runoff and the physiographic and geologic features on an areal basis, must be a generalization. Stream flow resulting from rain falling on a arainage basin represents the combined influence of many factors, chief among which are distribution, rate, and amount of rainfall, antecedent rain, climate, shape of the drainage area, condition of the channel, and the ability of the surface mantle and underlying bedrock to absorb and to retain the rain. Geology has an important bearing on the last-named factor, because the type and texture of the surface mantle and the character and structure of the underlying bedrock are the result of geologic agencles and processes. So1l conditions often determine the type and extent of vegetal covering, which has an important influence on the rainfall-runoff relation, although the climate of the region has a far-reaching influence on this relation as well as on stream development.

As rain falls on the surface of the ground, it is disposed of in three ways: it may be evaporated, it may be absorbed by the soll mantle, or it may run off as surface flow. Of the rain that becomes runoff from any particular area, a part may evaporate from the open water surface and the remainder may flow by devious routes to the ultimate arainage channel or be partly or entirely absorbed into avallable subsurface storage along the route. The storage capacity of the surface mantle and the underlying formations and the rate at which absorption w111 take place are factors that affect the rainfall-runoff relation. Both are largely determined by the geologic characteristics of the drainage basin. Runoff studies of many southern California streams indicate that geologic features exert considerable influence on both ordinary and flood flows, and no doubt most of the other streams of the region also, are affected by the geology of their drainage basins, although the relationship is not so obvious nor so eas 1ly recognized.

The southern California region consists of a series of mountain ranges representing great upfaulted or upfolded blocks of the earth's crust. The intervening valleys occupy depressed segments filled with 
debris eroded from the surrounding mountains. Surface features affecting runoff throughout this region range from steep outcrops of practically impervious unweathered rock to almost level surfaces underlaln by thick deposits of highly permeable alluvium. In areas of the first type practically all the rain that falls will become runoff, but in areas of the second type the permeable material will absorb all the rain and prevent runoff. Between these extremes are gradations, which vary according to the topography, character of the surface mantle, and condition of the subsurface bedrock.

Physlographic features of the region are youthful in character. The steep mountain ranges rise abruptly from the relatively flat valleys. Streams within the mountain area occupg deep V-shaped canyons separated by sharp narrow ridges. The canyon walls are, as a rule, extremely steep, their slope in many places being only slightly less than the angle of repose of the materials covering them. Mechenical weathering is the dominant factor in rock disintegration and is instrumental in maintaining the steep and rugged form of the mountain area. This prooess is constantly acting to supply loose material susceptible of being transported by stream action.

Erosion within this mountain area and deposition on the floors of the adjacent valleys are proceoding at an active rate. The great alluvial cones found at the mouths of practically all the canyons are products of these processes. Owing to the seasonal or cyollc character of the storm common to the region, erosion and deposition do not occur at regular intervals; Instead, dry periods, during which many of the stream beds are boing aggraded, altemate with flood periods, when great quantities of water transport the already disintegrated and loosened debris from the mountains to the valleys. This sharp contrast in both quantity of flow and debris load causes unstable channel conditions, especially over the cone area, with the result that streams often overflow and shift their positions during heavy storms. The recurrence of this shifting of the streame with its accompanying deposition of debris over a long period of years has, of course, been responsible for the natural development of the oones.

The floors of the alluvium-filled vallegs represent the flood plains. They consist of wide expanse of nearly flat lands that merge into the gently sloping cones near the valley margin. The streams, as a rule, are not established in permanent courses but occupy shallow channels cut in the soft, unconsolidated valley fill. In times of flood they overflow 
and spread, often forming new chennels, with the partial or complete abandonment of the old. Wide shifting is kmown to have occurred on the Santa Ana, Los Angeles, and San Gabriel Rivers within historic times, and a repetition of this action is possible on these and other streams during any future major flood. Efforts to confine the streams are successful only to the limit of capacity of levees or other flood-control structures, and once their capacity is exceeded, as it was in many localities during the flood of March 1938, the flow is essentially uncontrolled.

Owing to the torrential nature of the storms common to the region, coupled with the unstable character of the stream beds and the low relief of the valley floors, floods in southerm callformia are a greater potential source of damage than in areas where the stream channels and flood plains are well defined and.easily distinguished.

In the following discussion the purpose has been to outline briefly the main geologic features of the region and to point out what appear to be some of the relations between these features and the runoff from rainfall. Some of the relations seem well-defined and easily recognized, but others are indistinct or obscured by other factors. Neither the time nor the opportunity was avaliable to make detailed fleld investigations on any phase of the subject, and as a consequence only general aspects of the relations are herein considered. Information covering the general geology of the region has been obtained largely from publications of the Geological Survey of the Department of the Interior, the Division of Water Resources of the State of California, and the University of California. Other reports and studies relating to the subject have been made available by the Los Angeles County Flood Control District. For convenlence in discussion, the region has been divided into districts, each of which is characterized by similarity of topographic and geologic features.

\section{San Diego area 18}

That part of western San Diego County extending from the crest of the main mountain range west to the Pacific Ocean and from the international boundary north to the Orange County line is naturally divided into two physlographic and topographic units. To the east lie the mountain highlands, and to the west the narrow coastal belt characterized by dissected terrace formations that terminate at the ocean in a ine of sea cliffs.

18 See also Ellis, A. J., and Lee, C. H., Geology and ground water of the restern part of San Diego County, Calif.: U. S. Geol. Survey Water Supply Paper 446, 1919. 


\section{Mountain highlands 19}

The mountains of the San Diego area belong to the Peninsular Range, which extends southeast from the Santa Ana Range through the peninsula of Lower California. In the higher, eastern part the general altitude of the range is more than 3,000 feet above sea level; in the western part it is 500 to 1,500 feet above sea level. The highest peaks rise to altitudes of about 6,000 feot. The topography, as a rulo, is that of a mature, eroded surface. Slopes are gentle to moderately steep, and the main streams have cut to or near a graded surface.

Within the mountain highlands are several broad valleys, the most notable being Cajon, Santa Marla, Green, Escond1do, Poway, San Jose, and San Felipe Valleys. In general, each is partly or completely surrounded by hills or mountalns, and their floors are level or gently rolling, occasionally broken by low hills or knobs of granite. Their isolated position and the remoteness of most of them from any large river channel indicate that these valleys were formerly a part of an old peneplained surface.

Some faulting has occurred within the mountain highlands. Two faults have been mapped by Ell1s, 20 both apparently southeast extensions of the Elsinore fault systems. One of these strikes southeast from the town of Temecula. The upper part of the San Luis Rey River, above the Pala Indian Reservation, follows the line of this fault. About 8 miles to the northeast a rather prominent northeastward-facing scarp extending from Pauba Valley to San Jose Valley marks the line of a second fault parallel to the first. The upper part of Temecula creek flows a long the course of this fault for a distance of about 15 miles. Farther west several lines of topographic expression indicate the presence of faults, but definite evidence of their existence is not ava1lable.

The broken character of the crystalline complex, which allowed pronounced weathering and deep disintegration, is undoubtedly due to strains in the earth's crust that were contemporary with faulting, but the sharp stmactural breaks between mountains and valleys and the gravel-filled troughs common to the San Bernardino and San Gabrlel Ranges are not found In the San Diego area. Certain surface and subsurface conditions created

19 See also Sauer, Carl, Land forms in the Peninsular Range of Cal1formia as developed about Warner's Hot Springs and Mesa Grande: Calif. Univ., Pub. In Geography, vol. 3, no. 4, pp. 199-290, 1929; Miller, W. J., Geomorphology of the southern Peninsular Range of California: Geol. Soc. Am. Bull., vol. 46, pp. 1535-62, October 1935. 20 Ellis, A. J., and Lee, C. H., op. c1t., pl. 3. 
by faulting no doubt have some effect on the flood munof, but definfte. ovidence of a relationship between the structural features and munof is lacking.

\section{Coastal belt}

A narrow belt underiain by sedimentary rocks of kocene age and younger follows the coast line and extends eastward a distance of 5 to 20 miles. As previously noted, it cons1sts largely of level or gently rollIng broad-topped terraces dissected by streams. North of the Otay River these terraces slope toward the sea from an altitude of 500 foot along the eastern margin of the belt to 300 feet near the coast. south of the river they slope in the opposite direction and range in altitude from 550 foet at the coast to 500 foet 4 miles inland. Structural deformation appears to be responsible for this contrast.

The terraces, locally called mesas, are capped by a shallow reddishbrown soll containing mixtures of partly woathered sand, gravel, and clay. The underlying formations consist of well-consolldated marine sediments, Including sandstones, shales, and conglomerates. For the most part both the surface and subsurface deposits are rather t1ght and impervious, a condition that permits only a small amount of absorption and consequently a small jield of water from underground sources.

\section{River valleys}

The Tla Juana, Sweetwater, San Dlego, San Dlegulto, and San Lu1s Rey R1vers all head near the summit of the Peninsular Range and flow southwest or west to the ocean. The Otay RIver occuples a small basin that heads near Lyong Poak, whlch 1s several m1les west of the main divide. The Santa Margarita River has cut beyond the divide and drains a large area between the Santa Ana and San Jacinto Ranges.

As with other features of the San Dlego area, the regimen of the streams has been controlled to a large extent by osolllation of the land. within the mountain highlands the streams have cut deop gorges w1th steop side slopes, but the valleys are generally wide and their floors are graded, indicating that at one time the land surface was considerably h1gher then at present. Subsequent sinking and fllling on the valley floors resulted in the present conditions. The fill consists of alluvial deposits of sand, gravel, and clay, in which sand predominates. Within the coastal belt a similar condition prevalls. The sides of the valley are steop, although comparatively low. The floors are wlde and flat. 
Near the coast most of the streams are partly drowned, giving rise to marshy tidelands of considerable extent.

The valley fill ranges in thlckness from a thin layer to more than 100 feet. In some reaches of the canyons, especially in the higher mountain areas, the stream beds are narrow and the bedrock crops out at the surface. Commonly, however, the valleys are wider both above and below the canyons and the bodrock is burled under alluvial f111. Differential movement in the earth's crust appears to be responsible for this cond1tion. The few avallable well logs that show the total thickess of alluvium in the valley $1111 \mathrm{~s}$ indicate that the deposits range in depth from 60 to 150 feet in the Tia Juana, Sweetwater, and San Diego River Basins, to more than 200 feet in the San Luis Rey and Santa Margarita River Basins. Much of this $f 111$ is favorable to the storage of water, and a large part of the agricultural development in San Diego County has taken place on these valley lands, from the subsurface reservolrs of which water for irrigation is pumped.

\section{Effect of geology on flood munoff}

The greater part of the San Diego area is characterlzed by geologic features favorable to the absorption of rainfall. The widespread distribution in the mountain highlands of deeply weathered and disintegrated granite, with its granular and porous soll covering, is especially favorable to this action. The moderate slopes and $11 \mathrm{mited}$ extent of outcrops of the bare rock 11kewise facilitate absorption. The quantity of rainfall that such on area will absorb is not accurately known, nor can the rate of. absorption be definitely stated. It is known, however, that storms of moderate intensity and duration produce littie runoff unless they have been preceded by substantial amounts of rain.

Large supplies of ground water have been developed in the granite residium in a number of the upland valleys and flatter slopes. In the vicinity of Fallbrook, for example, a group of wells sunk into the disintegrated granite yields eufficient water to support a considerable acreago of citrus groves. Similar conditions exist in a number of other 10cal1ties, indicating that a large part of the mountain area has surface and subsurface characteristics favorable to the absorption of rainfall.

Conditions over the coastal belt generally are less favorable to rainfall absorption. Except in areas covered by recent alluvial deposits, the solls are shallow, in most areas not exceeding 12 to 18 inches in depth. They tend toward the compact clayey type and are underlain by a 
tight, partly or completely cemented subso1l, which merges into imperviaus marine sediments below. Such surfaces are likely to yleld large runoff from rains, because their retentive capacity is limited to a part of the pore space of the upper few inches.

The river valleys, with their extensive alluvial deposits, have a large capacity for subsurface storage and hence should contribute materially to the reduction of runoff peaks. However, two factors tend to prevent this reduction. The first is the factor of time: peak flows last only a few hours as a rule and the amount of percolation that can take place during such a short period is necessarily relatively small. The second is the factor of available storage space: in many of the valleys no attempt is made to utilize the subsurface water supplies, so that in some places the ground water stands within a foot of the surface during most of the jear, and in many areas where pumping is practiced the water table is lowered only a few feet during the irrigation season. The net result is a relatively small reduction of flood flows. This condition is probably responsible, in part, for the high stream flows that result from rains that have been preceded by heavy storms. The small available storage is filled by the runoff from the earlier storms, leaving no capacity for retention of subsequent flows. The effect of antecedent precipitation is carried over from one season to the next and may extend over two seasons, as is indicated by the following table prepared by Charles H. Lee on the basis of his investigations in this area.21 (See table 24.)

Table 24.- Average depth of precipitation, in inches, required to produce runoff on certain streams in the San Diego area

\begin{tabular}{|c|c|c|c|}
\hline Stream & $\begin{array}{l}\text { First year } \\
\text { after year } \\
\text { with less } \\
\text { than } 90 \\
\text { percent of } \\
\text { average } \\
\text { rainfall or } \\
\text { second year } \\
\text { after one } \\
\text { with } 70 \\
\text { percent or } \\
\text { less }\end{array}$ & $\begin{array}{l}\text { Following an } \\
\text { average year } \\
\text { (90 to } 110 \\
\text { percent of } \\
\text { average } \\
\text { rainfail) }\end{array}$ & $\begin{array}{l}\text { Following a } \\
\text { year with } \\
\text { more than } \\
\text { llo percent } \\
\text { of average } \\
\text { ralnfall, } \\
\text { except } \\
\text { second year } \\
\text { after one } \\
\text { with } 70 \\
\text { percent or } \\
\text { less }\end{array}$ \\
\hline $\begin{array}{l}\text { San Luis Rey River near Pala } \\
\text { Santa Ysabel Creek near Ramona } \\
\text { San Diego River at diverting dam } \\
\text { Sweetwater River at Sweetwater dam }\end{array}$ & $\begin{array}{r}6.7 \\
7.6 \\
8.5 \\
11.3\end{array}$ & $\begin{array}{l}4.3 \\
4.9 \\
5.3 \\
7.8\end{array}$ & $\begin{array}{l}1.4 \\
3.7 \\
3.1 \\
5.7\end{array}$ \\
\hline
\end{tabular}

21 Ellis, A. J., and Lee, C. H., Geology and ground waters of the westerm part of San Diego County, Calif.: U. S. Geol. Survey WaterSupply Paper 446, p. 97, 1919. 
It is not clear whether the water retalned from wet years is distributed over an entire drainage basin or is confined to its alluvial valleys, but it is probable that the whole basin is affected.

In general, geologic conditions are such that runoff from the Tia Juana, Otay, Sweetwater, San Diego, San D1eguito, San Luls Rey, and Santa Margarita Rivers will be affected approximately as follows: in the part of the area underlain by the granite, light and moderately heavy rains occurring early in the raing season will be largely absorbed by the surface mantle, and any water that reaches the streams will probably percolate into the alluvial fill of the river beds. On the mesa lands of the cosstal belt, runoff is likely to follow all except the 11ghtest storms. Heavy and Intense late-season storms, especially if preceded by other heavy rains, are likely to produce flood flows from the mountain areas, and such flows will probably not be reduced by absorption in the river channels. Heavy precipitation in the preceding year will cause greater runoff from all parts of the area and from storms of all types.

\section{San Bernardino mountain area 22}

The San Bernardino Mountains include the eastern part of the great uplifted mountain block that extends from the Salton Sea depression northwest to the Santa Clara River Valley. The San Andreas fault cuts diagonelly through the block near its central part, forming Cajon Pass, which has been adopted as the dividing line between the San Bernardino Range on the east and the San Gabriel Range on the west.

The San Bernardino Range is bounded on the south by that part of the San Andreas fault which extends southesstward from Cajon Creek through Potato Canyon and San Gorgonio Pass. The remarkably straight southrest front of the mountains is the dissected scarp of this fracture. San Gorgonio Pass is considered to be a graben assoclated with this same faulting, the depressed block forming the divide between the San Bernardino and San Jacinto Ranges. On the north side of the San Bernardino Range, less spectacular but nevertheless distinct faut lines and escarpments mark the boundary between the mountains and the Mojave Desert. Thus the mountain mass consists of a great block of the earth's crust pushed up several thousand feet between two lines of faulting.

22 See also Vaughan, F. F. Geology of San Bernardino Mountains north of San Gorgonio Pass: Caiffornia Univ., Dept. Geol. Sc1., Bu11., vol. 13, no. 9, pp. 319-411, 1922. Eckis, Roilin, Geology and ground water storage capacity of valley f1ll: California Dept. Public Works, Water Resources DIv., Bul1. 45, 1934; Noble, L. F., Excursion to the San Andreas fault and Cajon Pass: $16 \mathrm{th}$ Internat. Geol. Cong. Guidebook 15, pp. 10-21, 1932 . 
The structural features assoclated with the foundation of the range have caused two distinct and contrasting types of topography--ste日p north and south boundarles and a comparatively subdued plateau area that forms the interior of the range. This plateau area, which includes Bear Valley and the part of the Santa Ana drainage basin above Seven Oaks, is 10 to $15 \mathrm{miles}$ wide, and about $30 \mathrm{miles}$ long. The subdued topography, gentle slopes, and extensive open valleys indicate that it may formerly have been a partly peneplained area uplifted with the mountain range. North of this plateau the surface slopes sharply to the level of the desert, and south of it to the upper Santa Ana River Valley. Here the streams and washes have cut deep gorges into the frontal scarps, resulting in the characteristic rugged terrain common to the mountain boundaries.

\section{Geology of the San Bernardino Range}

Information concerning the distribution of rock types in the San Bernardino Range is incomplete, but the general extent of the various formations and their effect on the rainfall-runoff relation may be outlined. The most widespread formation found in the mountain area consists of a coarse crystalline granite locally intruded by dikes of siliceous porphyry. One large mass of this granite extends from Clenaga Seca and Rattlesnake Creoks eastward to the limits of the range; another mass extends south and west from B1g Meadows and Santa Ana Canjon to the faulted sections along the southern boundary; and a third mass extends west from Bear Lake, which is north of the crest of the range, to Cajon Pass. In certain areas along the stream beds the granite is buried under fan depos1ts of unconsolidated alluvium. One such area parallels the Santa Ana River between BIg Meadows and Seven Oaks; and others occur in Bear Valley, along Holcomb Creek, and in Millard, Hathaway, and Protroro Canjons. These deposits represent remnants of a formation that formerly extended across the canjon floors when the range was at a considerably lower altitude. Subsequent uplift and rejuvenated stream action have caused partial erosion, leaving the remainder of these deposits as benchlike features flanking the stream beds.

Assoclated with the granite are widespread masses and belts of contorted schists and gnelsses of variable character. The largest mass extends south from the San Bernardino and San Gorgonio Mountains to San Gorgonio Pass and the mouth of Whitewater River. Another mass follows the crest of the range west from Bear Creok Gorge to Cajon Pass and occupies practically all the area botween the San Andreas and Cleghorn 
faults. The metamorphic rocks are intruded in many places by granite, with the follation commonly extending through the granite.

Sedimentary rocks including limestone and quartzites of carbonfferous age or older, make up much of the range on the desert side east of Arrastre and Rattlesnake Creeks. These rocks are folded and faulted, and in many places are intimately associated with the adjacent sohistose and granitic formations. One belt of quartzite extends north from cienaga Seca and Sugarloaf Mountain across Broom Flat and Baldwin Lake. Holcomb Valley, northwest of Baldwin Lake, is surrounded by exposures of limestone and quartzite, with younger alluvium occupying its central parts.

Deposits of shale, sandstone, and conglomerate of Tertiary Age or younger occupy narrow belts and wedges along the San Andreas fault below Cajon Pass and San Gorgonio Pass. East of Devil Canyon the fault block between the San Andress and Mission Creok faults consists largely of a coarse-grained arkose sandstone and conglomerate. The upper reaches of the Cajon Creok Basin, including the area north of the San Andreas fault, are underlain by coarse-grained sandstone interbedded with small deposits of shale and Impure limestone. This same formation appears between Cleghorn Mountain and Horsethlef Canjon and the flanks of the mountains along the Mojave Desert. The position of these isolated sedimentary blocks is due to fault movements, and the beds as a rule are highly distorted and displaced. Dips up to $70^{\circ}$ are not uncommon, and some of the strata are overturned.

Wuch of the major faulting in the San Bernardino Range is confined to the south-border areas and is a part of or closely associated with the San Andreas fault system. The Mission Creek fault is one of this group. Its scarp lies a few miles to the north of and parallel with the San Andreas fault, and it extends from Devil Canjon through Mill Creek and the headwaters of the Whitewater River into Mission Creek. The spectacular box canjon at the head of Mill Creek appears to have resulted from atream erosion along this break. There is evidence that saveral other faults, whose strikes range from east to northeast, branch off from the M1ssion Creek fault. Most of these appear to be 1nactive and their effect on the topography has been largely obliterated by erosion. On the north side of the range several fault are known to strike northwest from the mountain area into the desert, but their influence on runoff is probably not s1gnifioant. 
Between Waterman Canyon and Ione Pine Canyon several lines of faultIng that strike east and southeast branch off from the San Andreas system One line cuts through Waterman Canyon and is the source of the hot springs known as Arrowhead Hot Springs, while another branches off at Cable Canyon and cuts through Devil Canyon about $1 \frac{1}{2}$ miles from 1ts mouth. The complex structure found in the lower part of Lone Pine Canyon and reaching through Cleghorn Canjon into the West Fork of Mojave River has resulted from movement along the San Andreas and Cleghorn faults.

In addition to the major faulting in the San Bernardino mountain area, practically the whole of the mountain surface is broken by inmumerable closely spaced intersecting fractures and joints. Although this faulting action was probably not so severe as that affecting the San Gabriel Range, nevertheless it is of major importance in its effect on munoff. Each break offers opportunity for the absorption and retention of rainfall. It likewise is conducive to weathering and the creation of a residual covering, because fractured and jointed surfaces are subject to deep disintegration and rock decay due both to the weakened condition of the bedrock and to the accessibility of percolating waters.

\section{Effect of the geology on the runoff}

Several geologic features of the San Bermardino Mountains have notable effect on the runoff. There are, for example, considerable differences In the types of soll mantle covering the different formations. Generally, the metamorphic rocks are more resistant to weathering than the granites, and in the main they form steeper slopes and are covered with a shallower, more altered, and less pervious mentle, which in turn supports a lighter growth of vegetation. Bedrock fractures, although prominent, do not exhibit the deep disintegration found in the granites, and as a consequence there is less opportunity for percolation of rainfall. Runoff from such areas is, therefore, likely to be at a maximum. The unconsolidated fan deposits found in places along the rivers and in some of the upland valleys are, on the other hand, exceptionally well situated to absorb large quantities of rain, and runoff from these areas should be at a minimum. The granite areas, with their deep mantle of granular so11 and heavy vegetal covering, and the areas of older sedimentary rocks, blanketed with extensive accumulations of talus, will show runoff characteristics between these extremes. 
Faulting has an influence on manoff for a muber of reasons. The recently formed fault scarps are generally very steep, their soll mantle 1s shallow, and rock outcrops are common. These factors, unfavorable to rainfall absorption, are somewhat offset by the deep gravel deposits generally found in the beds of the streams that have eroded their channels along fault lines. Some water probably percolates to considerable depth along fault lines, but the amount may be too small to have any noticeable effect on direct runoff.

Streams draining the San Bernardino Range may be divided into two classes--those that drain the interior parts of the range and those that drain only the steep frontal area surrounding the range. The Santa Ana and Mojave Rivers belong to the first class; while many smaller streams and washes, each of wich debouches independently upon the desert or valley floor, make up the second class. Mill, Cajon, and Lone Pine Creeks have drainage basins that have been materialiy affected by faulting, and they $\mathbf{1 1 1}$ be discussed separately.

\section{Mojave River}

The basin of the Mojeve River Includes all the northrest part of the San Bernardino mountain area and extends from the divide west of Bear Lake to Cleghorn Mountain. Although this arainage area is extensive, most of the flow from it originates in a belt a fer miles wide fust north of the divide. The rainfall diminishes rapidy from this belt to the northern edge of the plateau, where desert conditions are prevalent.

In the northerm part of the basin the streams have cut deop gorges, but these are separated by wide stretches of flat or gently rolling tablelands. The country rock is predominantly a massive coarse-grained granite, closely jointed and fractured. It weathers to a coarse porous mantle that is only slightly altered. Vegetation is scattered and sparse. Generally, conditions are those characteristic of a non-wafer-producing area. The shallow side washes and the lack of erosion channels indicate a scanty munoff, and it is unlikely that this area produces runoff in any considerable amount except during the most intense storms.

In the upper part of this arainage basin, near the crest, there is a marked change in both topography and surfece covering. The canyons change to open valleys with subdued divides and gentle slopes, and in many pleces they widen to form broad flats in which the ground-water table is near the surface and marshy conditions preva1l. The country rock which includes both schist and granite, is generally buried under a thick soll 
mantle. Observations in dam excavations and road cuts show that the mantle may be from 5 to 30 feet deep in the valley areas and somewhat less on the side slopes. In section it ranges from a fine loam at the surface through a well-decomposed somewhat clayey subsoll to the disintegrated bedrock below. The area is well forested with pines, except on the higher ridges, where a heavy growth of chaparral thrives.

The prevaling type of so11 mantle has a large capacity for absorbIng water, but apparently the infiltration takes place slowly. Drainage I1kewise is slow, as Inalcated by the prevalence of seeps and springs in road cuts and in favorable spots along the slopes, many of which flow until late in the summer. Marshlands remain saturated throughout the year. The amount of flood runoff from such areas 1s largely controlled by the Intensity of the rainfall and the amount of antecedent rain. So long as the rain falls at a rate that does not exceed the rate at which infiltration $\mathbf{w l l}$ take place and ground storage remains avallable, there 1s $11 k e 1 \mathrm{~J}$ to be little surface runoff, but as the rainfell increases and the ground storage becomes filled only a part of the water is retained and munoff takes place. Again, if the antecedent rainfall has been sufficient to partly or completely f1ll all the ground storage, runoff can be expected from any storm. This was substantialiy the condition during the storm of March 1938. Most of the heavy precipitation of the 1936-37 season had been in the form of snow, and mach of the water had been retained as ground water, as indicated by the prevalence of springs, seeps, and marshlands throughout the area during the late summer of 1837. Raing prior to the storm of March 1838 may have been sufficlent to practically f1ll the remaining storage space, and therefore the high intensities of the March storms were especially effective in producing the disastrous floods.

\section{Santa Ana River}

The drainage basin of the Santa Ana River above Mentone may be roughly divided into an upper and a lower part, distinguished malnly by their topographic differences. The lower third of the basin, which includes the area between the valley floor and Fllirea Flat and Bear Valleg, has a rugged terrain characterized by the deep $v$-gorges of the river and Its larger tributaries, exceptionally steep to precipitous slopes, narrow ridges, and an almost total absence of flat or moderately sloping lands. The country rock cons1sts of schist and gne1ss intruded by coarse granite, both types being intensely fractured and jointed. The thetamorphic rocks 
break down to a compact, well-altered, somewhat clayey type of soil, which, on the south slopes is usually shellow and capable of supporting only a light growth of vegetation, but on the north slopes is somewhat deeper and carries heavier vegetation. Such areas produce practically no talus accumulation and only a small amount of rock litter. The granites, on the other hand, weather to a coarse granular soll of considerable depth, on which rock litter is prevalent and vegetation as a mie is heary.

The upper two-thirds of the basin, Including most of the Santa Ana River Valley above Fllirea Flat and all of Bear Valley, is in the high plateau region already described. With the exception of a few steep slopes leading from the higher mountains and ridges, the topography is mature and characterized by subdued ridges and broad open valleys.

Both Bear Valley and the lower part of the Santa Ana River Valley above Fillrea Flat are underlain by fan deposits. In Bear Valley a considerable part of these deposits is submerged under the Bear Valley Reservolr and hence has no visible effect on the rainfall-runoff relation. Along the south bank of the Santa Ana, however, they occupy a bench ranging in width from a few hundred feet to more than a mile and extending from Big Meadows to Filirea Flat. Smaller fan deposits elso occur along the north side of the river at the mouth of clenaga Seca Creek, betwern Holcomb Creek and Convers Flat, and at Fllirea Flat. These deposits consist largely of unconsolidated coarse gravel, boulders, and sand, and their permeability is shown by the result of spreading operations being carried on by the Bear Valley Irrigation Co. at Barton Flat. This compeny has succeeded in increasing the summer flow in some of the tributaries of the Santa Ana River by spreading several thousand acre-feet of the spring runoff from South Fork.

The country rock along the mountain slopes above the bench south of the river consists of schist and gneiss intruded by granite. At lower altitudes the surface mantie is a deep, much disintegrated, but somewhat rocky soll. These lower areas are covered with trees. On the hifher reaches near the summits of San Bernardino Peak and San Gorgonio Hountain and the ridge connecting the two, vegetation is sparse and the slopes are mantled with a disintegrated but unaltered rock litter. North of the river sedimentary rocks, consisting of quartzite with minor amounts of limestone, crop out along the steep south flanks of Sugarloaf Mountain and the ridges leading from the mountains to the east. The quartzite. 
breaks down into blocky fragments, occasionally forming talus slopes of angular rock particles almost devold of fine material. West of Sugarloaf Mountain the country rock is granlte, and the slopes are covered with a shallow, porous mantle formed by the disintegration of this rock. All of the area except the quartzite talus slopes support a moderately heavy growth of brush.

In any generallzation regarding the effect of geology on the flood runoff from the Santa Ana River Basin the relative influence of the two parts of the basin mast be considered. Because argillaceous types of shallow soll cover most of the steep slopes of the lower part, percolation is restricted and ground storage is limited. This part of the drainage basin is, therefore, likely to show some munoff after any except light or moderate rains occurring early in the raing season. If antecedent rains have been sufficient to fill the larger part of the pore space in the soil, its retentive capacity is $11 \mathrm{mited}$ and the runoff is $11 k e 1 \mathrm{y}$ to be substantially increased. Under these conditions, rains of high intensity and long duration are practically certain to furnish runoff of flood proportions. These were the prevaliling conditions during the storm of March 1938, and it is probable that a large part of the Santa Ane River flood that followed that storm originated on these slopes.

- With extensive subsurface storage avallable in the upper reaches of the drainage basin, only the larger storms are likely to produce runoff in any great amount. The belt of alluvial deposits that parallels the Santa Ana River on the south between Seven Oaks and Big Meadows shows evidence of only a small amount of munoff from the storm of March 1938. The opposite or north side of the river yielded considerable munoff from the same storm. No extensive deposits of porous material are found along the south slopes of Sugarloaf Mountain. The light vegetation and the comparative1y tight quartzite bedrock of those slopes appear to favor copious runoff. The flood waters that damaged Seven oaks and other resorts along the river appear to have originated on these quartzite slopes. Convers Flat, Fillrea Flat, and Big Meadows are alluvial-fill areas providing considerable ground-water storage, and no doubt they acted to reduce the runoff to some extent. The porous granite slopes west of Sugarloaf Mountain would have a similar effect.

The records indicate that the direct runoff resulting from the storm of Harch 1938 was proportionately less along the Santa Ana River than along any of the neighboring streams. The subsequent summer runof was likerise less and the retention correspondingly greater. The fact that 
the Santa Ana River is the only stream in the immediate vicinity that has belts of alluvium along its upstream drainage area appears to explain the condition adequately. These areas absorb water readily and apparently yield it slowly, the carry-over perhaps extending from one season to another or even longer.

In considering the munoff from an entire drainage basin, such as the Santa Ana River above Mentone, the geologic factors may effect a balance between those areas that will yield almost complete direct munoff from rainfall and those that 111 absorb most of the rainfall and yield it mainly from subsurface sources. Where geologic formations produce tight, nonabsorbent surfaces, such as rock outcrops or impervious clay solls, the flood peaks will be at a maximum and the aralnage will be rapid, but where geologic formations produce surfaces that favor rapid infiltration, the flood peaks w11l be reduced and the draining process will extend pver a longer period. The parts of the Santa Ana River Basin possessing characteristics favorable for retention of water have a tendency to shom a smaller flood peak and higher subsequent munoff then other parts of the basin.

\section{M111 Creek}

Although M111 Creek drains a part of the frontal area, it shows a rainfall-munof relation differing materially from that of the other streams. Both the flood munoff and the summer munof from its bas in are consistently higher than from neighboring basins. Geologic features appear to be, In a large measure, responsible for this difference.

Mill Creek is a typical structural stream, and from its headpaters, just south of San Gorgonio Mountain, to a point about 2 milles northeast of the Crafton H1.11s it has excavated its canjon along the line of the Mission Creek fault. About 2 miles above 1ts mouth the stream turns abruptly to the southwest, leaves the fault line, and cuts through the narrow block separating the Mission Creek and San Andreas faults, entering the Santa Ana Valley about 2 miles east of Mentone.

For about five-sixths of Its length the creek is flanked on the north by a steep, precipitous fault scarp that is only slightly eroded and dissected by a few steep, narrow gorges. Most of the tributaries entering from this side pass over waterfalls or steep cascades or flow down steep, shallow rock-floored washes. The slopes on the opposite side of the stream are considerably less precipitous in the lower parts of the basin but become steeper upstream, especially above High Creek, where they form the south wall of the box canyon. 
The bedrock north of the canjon consists of granites, schists, and gneisses. The granites appear to predominate in the lower part of the. canyon, and the metamorphic rocks in the upper reaches. The rocks of both types are only slightly disintegrated, rock exposures are comon, and the mantle, if present, is shallow and rocky. Vegetation is sparse.

The high, steep ridge on the south that separates the basins of Mill and Potato Creoks, is mado up largely of granite, although parts of it are underlain by a hard, coarse-grained, well-consolidated sandstone, designated by Vaughan 23 as the Potato sandstone. A wedge-shaped mass of the same material occurs north of the creek below the point where the canyon leaves the fault line near Morton Peak.' Both the sandstone and granite have weathered on the moderate slopes, to fairly deep, granular mantles, which support a heavy growh of vegetation. The upstream steoper slopes are barren.

Mill Creek Canyon is floored by coarse gravel and boulder alluvium. The deposits range in width from 200 feet above Fall Creok to a quarter of a mile or more in certain sections near the mouth of the canjon. The thiokness of this alluvial fill is not known, but it is probably 100 fet or more. The many seeps and springs that originate in the fill are ovidence of its pervious character and account for the comparatively large summer flow.

The rainfall-runoff relation in Mill Creok Basin may bo interpreted from its geologic features. Within the northern part of the basin, where steep slopes, shallow soll mantles that support only sparse vegetation, and large exposures of rock, are characteristic, all storms are likely to furnish rapid runoff. Features such as these-are especially prominent

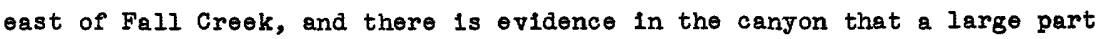
of the floodwaters of March 1938 originated in washes draining from this area. In the box canyon at the head of the basin, both sides furnished heavy runoff. In contrast, the southern part of the basin, with its dense vegetation and porous mantlo, would probably have little or no runoff from any but heavy storms. It is possible that the storm of March 1938 produced some runoff from this area but from surface appearances most of the rain that foll here was absorbed. 
The sumer slow in Mill Creek seems to come largely from the alluviel 111 in the channel. A few streams on the north, 1noluding Fall, Mountain Home, and Town Creeks, have the1r sources on the high slopes of the San Bernardino Mountains and are perenn1al, but their contributions account for only part of the flow. All the streams draining from the south ordinarlig dry up during the sumer. The opportunity for infiltration presented by the flood waters flowling over several mlles of the porous 111 in M1ll creek ohannel favors a large absorption during storm periods with subsequent draining out during spring and summer.

The frontal streams

The frontal area of the San Bernardino Mountains may be deffned as the steep belt surrounding the range and forming the transition between the hlgh and comparatively level interlor plateau and the lowlands of the Santa Ane Valley and the Hojave Desert. It extends from San Gorgonio Pass on the southeast around through Cajon Pass on the west to Burns Canyon on the northeast.

of the streams that drain this frontal are only those debovohing Into the upper Santa Ana River Basin have rlood problems that are of oconomlo 1mportance. The north frontal washes draining the belt between Oajon Pass and Burns Canjon are subject to per1odic and at times Intense and spectacular sloods. Thej debouch, however, onto a sparsels settled or uninhabited desert area, and their geologio features are not here disoussed.

From Cajon Pass to the Santa Ana River the south front of the San Bernardino Range represents the dissected scarps of the San Androas and assoclate fault systems. A number of streams have established drainage basins on the mountain front, each of these empties independently onto the valley floor. For the most part, they are tjploal consequent streams that have carved the1r pattern on the uplifted mountain block. In general, the area 1s characterized by steep slopes and narrow ridges, rugged In places, with bedrook outcrops, deep main gorges with shallower, steeper tributary gorges arranged in dendrit1c patterns. A medium to heavy growth of chaparral and brush covers the area except on rooks patches and on some of the steopest slopes, where the soll mantle is too shallow and unstable to support plant growth.

The bedrook of the frontal area consists mainly of sohlsts and gnelsses Intruded by dikes and large bodies of massive coarsely crystal11ne granite. The location and extent of the Intruded granite rocks are 
not accurately known, but outcrops have been observed in the lower parts of Devil and Waterman Canyons and in the upper reaches of Strawberry Creek. It is probable that a detalled examination would disclose these rocks in other parts of the area. Minor exposures of sedimentary rocks are also found in the area, distributed along the north side of the San Andreas fault. From Cable Canjon a narrow belt of nonmarine sandstone and conglomerate, half a mile to a mile wide, extends west along the fault line into cajon Canyon. Another belt of rocks of the same type extends east from Dev1l Canyon and forms the block that separates the San Andreas and Mission Creek faults. Both the sedimentary and crystalline rocks are closely fractured, although the sedimentary deposits, being rather poorly consolidated, show little surface evidence of this weakmess. The surface mantle ranges in character from a deep granular, much disintegrated and altered soll in the more level areas, which are underlain by the granite formations, to the shallow clayey types of soll found on the steeper slopes, which are underlain by the metamorphic rocks. Occasional small outcrops of bare rock are found on the steepest slopes. Accumulations of talus are rare, and the stream beds are, for the most part, swept clean of debris.

The frontal area is similar in many respects to the lower part of the Santa Ana River Basin and the rainfal1-runoff relationship of the two areas is comparable. Iight rains are retained, but as ground storage is limited, owing to the shallowness of the soll covering and the lack of talus deposits, intense storms cause rapid runoff and flashy floods with sharp peaks, followed by rapid recession as the storm ends. The summer flow is small and is limited to the larger streams. The smaller streams are dry except during short perlods following substantial rains.

\section{Cajon Creek}

The headwaters of cajon creok and most of its drainage basin are in a wide valley lying between the San Andreas fault and the sumit of the San Bernardino Range and known locally as the Cajon Ampitheatre. Faulted and tilted sedimentary rocks, predominantly sandstones of Miocene and Pllocene age, underlie most of the basin, and thick gravel deposits of Pleistocene age overlie the sandstones along the rim to the north, forming a serles of infacing bluffs. Nearly half of the basin is blanketed under deposits of alluvial gravel. These follow the main stream channel and extend in a fingerlike pattern over the lower depressions of the basin. 
Southeast of this basin, in the vicinity of cloghom Mountain and between Cleghorn Canyon and the San Andreas fault, the country rock consists of granite, schist, and gneiss. These resistant rocks form steep slopes, many of them showing rock exposures, and the remainder only partly covered by a thin veneer of sandy and rocky material. Below the point where Cajon Creek crosses the San Andreas fault at the mouth of Lone Pine Canyon, the stream is flanked on the south by a low ridge of schist and granite and on the north by a belt, a mile or so wide, of low ridges and mounds lying between the channel and the fault. These ridges and mounds are capped by gravel deposits that are apparently remnants of old terraces. Below Cable Canyon, the canyon widens as the wash enters the upper reaches of the broad, flat alluvial cone.

The influence of geology on the rainfali-runoff relation in Cajon Creek Basin appears to be rostricted to the ability of the porous alluvial gravels to absorb the runoff from the surrounding impervious formations. The exposed sedimentary rocks in the upper basin appear as steeply tilted blocks, the inclined strata, In many instances forming steep dip slopes that are almost devold of any covering. In localities where the slopes are less steep the mantle covering usually consists of a very thin layer of porous sand. The underlying bedrock, though well jointed, may be considered as too impermeable to have any appreciable effect on runaff. The grantte and metamorphic formations found in cleghorm Canyon and in the steep northeast escarpment of the San Andreas fault which parallels the lower part of Cajon Canyon are also characterized by a thin soll mantle, sparse vegetation, and considerable areas of bare rock slopes. These areas can be expected to yield a high runoff from any but light rains. The many local floods which are common in this vicinity originate on these slopes, and, although they are'flashy and spectacular at particular points, their effect is not widespread, because their waters are usually absorbed by the porous gravels of the lower stream bed. Under ordinary storm conditions it appears that all runoff would be absorbed by the gravels and that only during long intense rains would flood conditions prevall over the entire stream.

Lone PIne Creek

Lone Pine Creek is unusual among the streams of the Immediate mountain area in that it not only shows very little munoff from individual storms but it also requires a longer period in draining out. Gealogical conditions appear to be responsible for this behavior. The stream follows 
the Iine of the San Andreas fault for 1t entire longth, and 1t drainage bas in has the long narrow outline typical of struotural basins. Although this basin is 10 miles in length, its maximum width does not exoeed 2 m1les. In common with other areas along the fault in this vioinity, opposite sides of the valley show dissimila rock formations. The ridge on the south is composed of Pelone schist, the formation which is found also on the north side of Iytle creok and is described in connection with that stream. The relatively gentle slopes of this ridge are mantiod with a fino-textured micacoous soli that oarries a small amount of rock litter but no talus accumulations. Although the surface is covered by a moderate growth of brush, there is evidence of considerable erosion, and many of the shallow side washes are floored on bedrock.

Rocks found in the ridge along the opposite north flank of the canyon Include both sedimentary and grenitio types. For several miles upstream from the mouth of the canyon at Cajon Creek, the ridge is made up of sedimentary rock broken into a series of complex fault blocks. A wellconsolidated arkosic sandstone is the predominating type. North of these faulted structures, the ridge continues as a narrow wedge of only slightly weathered granite. Both the sedimentary and granitic slopes have charaoteristios unfavorable to the retention of rainfall. The soll mantlo is thin and scattered, being interrupted by many small areas of exposed rock. Vegetation is sparse and consists malniy of scattered clumps of stunted brush.

The floor of the valley is covered with deposits of alluvial f1ll throughout most of its length. On the south thsse deposits extend well up on the slopes, but on the north there is a sharp line of demarcation between the comparatively level floor of the valley and the steop sandatone and granite slopes. A cross ssction of the fill as exposed in a recently eroded wash near the mouth of the canyon shows it to be composed of unconsolidated gravel and sand, with many thin beds of micacoous silt. The depth of the 1111 at this point is about 60 feet, but 1nasmuch as the exposure occurs near the ridge several hundred feet south of the fault Iine and at a point of rising ground water, this depth cannot be considered as representative of the entire valley. Recent action along the San Andreas fault has affected the fill struoture. The sag pond near the mouth of the canyon, knom locally as Lost Lake, and several low ridges and scarps farther up the valley appear to be the results of movement along the fault line. 
The comparatively low flood runoff from stiorms falling on the Lono P1ne Cpreok Basin may be attributed to the absorptive capac1ty of the gravels in the valley floor, but the reason for the slow araining out is not entirely cloar. One possible explanation is that recent fault action may have formed series of isolated pervious pockets in the fill slow drainage from these pockets would furnish a stable flow to the stfoam for long perlods. It is possible also that water may escapo along the fault and appear at the surface at points outside the drainago area. some observers belleve that water passes through the complex fault lines at the mouth of the canyon, moving from the Lost Lake area Into Cajon Creok at a point above the Cajon Creek measuring station. Again it may be that the presence of lenses and bods of the fine micaceous silts in the fill has. some effoct on runoff. These saturated beds would yleld water at slow rates for long periods. There is no visible evidence to support any of these assumptions, and it may be that the stream's behavior is due to a combination of all of them.

\section{Upper Santa Ana Valley}

The southwest front of the San Bernardino Range is flanked by the oastorn part of the uppor Santa Ana Valley. Structurally this part of tho valley is a burled fault block depressed between the San Andreas and San Jacinto faults. On the northeast side the boundary is sharply defined by the straight-line contact between the valley alluvium and the crystaline rock of the mountains. The San Jacinto fault has a less distinct, though definite, surface expression in the low escarpment that marks the south benk of Lytle Creek. It is well known that this escarpment, known locally as the Bunker H1Il Dike, acts as a barrier to underground flow.

The valley floor is a broad alluvial plain whose slopes convergo toward the intersection of Warm Creek and the Santa Ana River near Colton. At the front of the mountains the streams have bullt up a serles of. alluvial cones that extends eastward from Lytle Creek to the Santa Ana River. From Dovil Canyon to the Santa Ana River the cones coalesce to form a continuous upland bench broken at the valley margins by the interconal dopressions. Near their apexes the slope of the cones is from 150 to 250 feet to the mile, but it gradually decreases unt1l at the lower end of the valley floor it is 25 to 50 feet to the mile. The upper parts of the cones have been dissected and the parent streams occupy well-defined channels. Lower down these channels are broader and divide to form a network of gravel-strewn washes, ary except during periods of high stream flow. 
The valley fill consists of unconsolidated sands, clays and gravels In many places more than 1,000 feet deep. There is little or no evidence of sorting or classification of materials except along the stream courses. There the deposits reflect the changing gradients from the relatively steep alluvial cones to the flat valley lands, with coarse materials predominating in the area near the mountains and fine silts and clays predominating in the adjoining low lands. This sorting is by no means regular, however, for accumulations of coarser debris in the valley fill occur throughout its extent. In the vicinity of San Bernardino several of these more pervious beds are capped with partly impervious clay blankets near the surface, and this clay covering together with the barrier formed by the San Jacinto fault, is responsible for the well-know San Bernardino artesian basin.

The Santa Ana Valley is an important source of underground water in southern California. The capacity of the basin to absorb additional water no doubt exceeds the runoff from any single storm or series of storms likely to occur, but the opportunity for absorption is limited by the rate at which percolation can take place during the usually short periods of high runoff. Ordinarily the water of streams entering the valley is entirely absorbed within a few miles beyond the mountains. Because of their low flow the streams seldom spread to any great extent, and the absorption takes place along a single channel area. As the runoff increases the streams tend to spread and occupy additional channels and washes, thus increasing the area over which absorption can take place. During the larger floods the streams, unless artificlally restricted, spread and occupy a large part of the cone area; percolation thereby is naturally increased and peak flows are proportionately decreased. The cones at the mouths of the Santa Ana River, City Creek, and Plunge Creek are especlally well situated to absorb additional water by this process. The relatively short perlod of the flood flows--seldom more than a few days--limits the amount of flood water actually percolating underground.

\section{San Gabriel mountain area}

The San Gabrlel Mountains and the valleys flanking them on the south form two distinct physlographic and geologic units. The mountain area characterlzed by high, sharp crests, deep canyons, steep and preclpitous slopes, and sharply dissected boundaries, is in direct contrast to the broad, gently sloping valieys beyond. The economic value of the tillable lands in these vallejs is due to this contrast, for without the mountains 
to induce precipitation from the molsture-laden air masses moving eastward from the Pacific ocean and thus develop streams, the fertile valley lands would be nothing more than a desert. At the same time this contrast is responsible for a flood hazard that increases in gravity as the population increases and the lands become more valuable.

\section{San Gabriel Mountains}

The San Gabriel Mountains represent the western part of the great uplifted block of which the San Bernardino Range is the eastern part. In extent the San Gabriel Mountains reach from Cajon Pass on the east to San Fernando Pass and the Santa Clara River on the west and northwest, and from the Mojave Desert on the north to the Santa Ana, San Gabriel, and San Fernando Valleys on the south and southwest. Within this ared the mountain tops range in altitude from 3,000 feet in the foothills south of the Santa Clara River to more than 10,000 feet at San Antonio Peak, near the northeast edge of the range. The main crest of the range follows an Irregular line nearer to the north boundary than the south boundary, but there is a secondary, parallel crest of slightly lower altitude extending along the south edge of the mountains and forming the ridge between the East and West Forks of San Gabriel River and the valley.

A narron belt of more subdued topography occurs just south of the main divide, extending from Charlton Flats on Tujunga Creek to Pine Flat on the North Fork of San Gabriel River. This belt ranges in altitude from 3,000 to 6,000 feet and is considered to be the uplifted segment of an ancient land surface. Älthough its topographic features are similar to those found in the plateau region of the San Bernardino Mountains, the total area of the San Gabriel belt is much less extensive. It is probably sufficiently large, however, to exert an influence on flood runoff, as its topography, 'geology, and vegetation are all favorable to the rapid absorption of rainfall.

The main streams draining the San Gabriel Range have cut deep Vgorges, and most of these are at or near grade. There is evidence of some aggrading in the stream channels, particularly in the lower reaches of the San Cabriel, Tujunga, and Arroyo Seco Canyons. The channels of most of the other streams are swept clean. Tributaries to the main rivers and creeks are numerous, with the result that the mountain topography is a complex network of steep canyons and sharp narrow ridges. 
Geology of the mountain area 24

Igneous and metamorphic rocks are the predominant types of rock formations found within the San Gabriel Mountalns. Alluvial deposits flank the range on the south at the elevation of the stream valieys and on the north at the level of the desert, and a few scattered remnants of old alluvial stream terraces are found in belts and patches along some of the stream channels. A small area of sedimentary rocks consisting largely of sandstone and conglomerate of Tertiary age crops out in the uplifted fault block south of the San Gabriel fault between Tujunga Canjon and Pacoime Wash. With these exceptions no unmetamorphosed deposits of sedimentary origin are found within the mountain area.

The metamorphic rocks have the greatest areal extent. They consist mainly of a complex mass of granitic and dioritic schists and gnelsses with occasional lenses of quartzite and altered limestones, some of which are more than a hundred feet in width. This aggregation has been intruded by large masses of crystaline granitic rocks, and the whole mass has in turn been cut by sharply defined dikes of aplite and diabase. In typical sections of gneiss the bands vary from a few inches to a foot in width, the 11ghter bands being composed of quartz and feldspar, the darker ones usualiy including biotite and hornblend. Much of the schist has a ribbon11ke structure. Parting along the follation planes in both the gneisses and the schists is not common in the unweathered rock, but as weathering increases the formations show a tendency to part along these planes and thus give rise to talus and litter composed of flot spalis.

The 1gneous rocks consist chiefly of granites, granodiorites, and diorites, but there is a large area of anorthosite in the northwestern part of the range, extending from the upper Tujunga and Pacoima Basins to Soledad Canyon. The igneous rocks show considerable variation in texture and structure, which is, in turn, reflected in the type of mantle they produce. The granite at the head of Eaton, Rub10, and M1liard canyonsils coarsely crystaline and disintegrates to a deep, loose, granular soll.

24 See also Eckis, Rollin, Geology and ground-water storage capac1ty of valiey f1ll: Callfomia Dept. Publ1c Works, Div. Water Resources Div., Bul1. 45, 1934; H111, M. L., Structure of the San Gabriel Mountains north of Los Angeles, California: Cal1fornia Univ., Dept. Geol. Sc1., Bul1., 19, no. 6, pp. 137-170, 1930; Noble, I. F., The San Andreas rift and some other active faults in the desert region of southeastern callfornia: Carnegie Inst. Washington Year Book, No. 25, pp. 415-428, 1926; Milier, W. J., Geology of the western San Gabriel Mountains of Cailfornia: California Univ., Pub. In Math. and Phys. Sc1., vol. 1, no. 1, pp. 1-114, 1934 . 
The granodiorites and anorthosites in the western part of the range have a similar texture but produce only shallow mantles of granular partioles containing considerable rook litter. On the other hand, the dionites, which outorop at the head of the North Fork of San Gabriel River in the vioinity of Mount Iolip and Mount San Anton10, are fine-grainod and dense and weather lowly to blookg iragments only slightiy disintegrated.

Deta1ls conoerning the distribution of the various rook formation,, so far as they are known, w1ll be discussed in connection with the desoriptions of the individual drainage besins.

\section{Faulting}

The northeast boundary of the San Gabriel Range follows the trend of the well-known San Andreas rift. Toward the east the fault line louts ' through the flank of the range and is reflected in the long, narrow depressions of Lone Pine Canyon and Swarthout Valley. West of Swarthout Valley the boundary of the range swings several miles south of the fault, formins a broad reentrant between Rook Creok and the mouth of Leonls Valloy. Boginning at the latter loodity the faultagain outs the rlank of the range; pasing northweatward through the narrow Loonis Valley and Pine Canjon sections. On the south of the range, the irregular but sharp boundary between the valiey and mountain areas is formed by the slerra Madre fault systems. In adaition to these major faults, the interior part of the range is cut into a series of blooks by other faults whose displacoments vary from a fow foet to hundreds of feot.

Many of the larger faults in the interior part of the range lare reflected in the topography, because the weakened fracture planes are espocially susceptible to stream erosion. The great canyon of the Bast and West Forks of San Gabriel River has been eroded along the line of the San Gabriel rault. The courses or Pacolma and Tufunga Creeks have been determined in part by fault I1nes. Iytle Creek follows the line of the San Jacinto fault. Several of the frontal streams along the south flank of the range, notably Sawplt, Dalton, and San Dimas Croeks, have had their courses determined in part by arcuate faults branching off from the slerra Madre system. La Cañada and Tujunga Valleys occupy a narrow fault graben that separates the Verdugo and San Rafael Hills from the main San Gabriel Rango. 
Minor faults and fractures are so numerous that it is inadvisable, in a report such as the present one, to attempt to discuss their Iocation or movement. Their combined effect has been to convert the entire range into a weakened and frectured mass. Miller writes: 25

St1ll another feature should be mentloned here, namely, the high degree of minor fracturing of the whole San Gabriel region of 1,200 square miles. The writer has never seen another area of comparable size so remarkably fractured. * * It would seem that, during the uplift of the vast San Gabriel block of crystalline rocks, the whole mass was subjected to stresses which, in part at least, found rellef in the development of countless fractures running in all directions.

\section{Effect of geology on flood runoff}

The San Gabriel Mountains possess two contrasting characteristics that affect the runoff from rainfall. The first is the very steep topography, which would ordinarily be expected to produce a large volume and high rate of runoff; the second is the exceptionally porous nature of the greater part of the mountain surface, which acts as an absorbent and tends to reduce direct runoff. The actual runoff from both ordinary and floodproducing storms represents a balance between these influences. Another unusual condition 1s the apparent lack of direct surface runoff in certain areas, even under the most intense rains, although the total discharge from such areas is substantial and the elapsed time between the peak rainfall on the drainage basin and the peak discharge in the river channel at the gaging station is practically the same as would normally be expected In areas where direct surface runoff takes place. The explanation of this seeming contradiction is probably to be found in certain geologic and phys $10 \mathrm{graph1c}$ characteristics. Except where rock surfaces are exposed, the mountain area is for the most part covered with a mantle of disintegrated but only partly altered rock particles, which ranges in depth from a few Inches to several feet. On steep slopes this material shows a tendency to creep, with the result that the mass is continually being disturbed, the fine particles are buried and the coarse particles remain near the surface. Plants establish their root systems in the finer subsurface layers and deposit their litter at the surface, where 1t mingles with the coarser rock fragments. When it rains this surface functions as an absorbent, and water enters the mantle. Some of this water percolates to a considerable depth, and the remainder moves about through the myriad of

25 Miller, W. J., Geomorphology of the southwestern San Gabriel Mountains of California: Cal1f. Univ., Dept. Geology, Bull. 17, No. 6. pp. $203-4,1928$. 
pore openings in the mixture of coarse fragments and litter and eventually escapes into the many gullies and canyons leading down the slopes During intense storms, such as those prevalent in March 1938, it is fikely that most of the rain water remains under ground for only a short period and reappears almost immediately as stream flow, yet there is relktively little evidence of erosion except in the channels of streams that drain a large area, where a concentration of water is to be expected. In many of the drainage basins, the general surface shows little effect of erosion and the vegetal litter and soll particles are undisturbed, jet the floors of the stream channels are cut to bedrock.

Streams draining the San Gabriel Mountains may be divided into tro general groups--those that drain the steep frontal slopes, and those that have cut through the frontal scarp and drain the interior of the range. Streams of the first group include Deer, Cucamonga, San Antonio, San Dimas, Dalton, Rogers, Fish, Sampit, Santa Anita, Eaton, and Millard Crepks and Arroyo Seco and many smaller streams. Although the courses of many of these streams show the influence of faulting, they are for the most part typical consequent streams developed on the frontal scarp of the range and are characterized by steep slopes, many tributaries arranged in a dendritic pattern, and fanshaped watersheds. Tujunga and Pacoima Creeks and San Gabriel River belong in the second group. These are characterized by larger watersheds of 1rregular outline, main stream beds of low gradient, following fault linos over a large part of their courses, and tributaries of high gradient that generally enter the parent stream at right angles.

\section{San Gabriel River}

Although the courses of the East and West Forks of San Gabriel River follow the line of a recognized fault, it is doubtful if this structural feature has any signiflcant influence on flood runoff. The canyan does not appear to have any of the characteristics of a fault graben, and although the zone of fracturing seems to be falriy wide, the surface features of the zone are not materlally different from those of the areas flanking 1t. Type of country rock, depth and stage of disintegration and overlying vegetal cover appear to be the controlling factors in the relation of runoff to rainfall.

A belt of rocks consisting mainly of diorite gnefss intruded by masses of granodiorite and diorite extends along the East and West Forks of San Gabriel River from the head of Con Oanjon to the head of the West 
Fork. North of this belt, which inoludes the larger part of the drainage basins of Devil Canyon, Bear Creok, and North Fork of San Gabriel River and the part of the East Fork of San Gabriel River above Laurel Guloh, the country rock is predominantis granodiorite. A small outorop of mioa sonist is found in the vioinity of Vincent Guloh and Prairie Fork at the hoad of East Fork of San Gabriel River, and the Crystal Lake region is buried under material that in some ways resembles morainal deposits. West of Chileno Canyon and south of the West Fork of San Gabriel River, the underlying bedrook is predominantig diorite and granodiorite; these are part of the same intrusive mass that appears throughout the sawpit, Santa Anita, and Baton Creek Basins.

Several features that influenoe the rainfall-runoff relation and the behavior of flood flows over certain areas appear to be traceable to the type of the underlying bedrook. One noticeable example is the sharp contrast between the north- and south-faoing slopes along the West Fork of San Gabriel River. On the north-faoing slopes south of the river the granodiorite and diorite are weathered to deep granular solls that contain considerable humus and support a heavy growth of ohaparral and conifers. Wuch of this area lies in a zone of heavier than ordinary rainfall, as noted in the section on $100 a$ r rainfall characteristios, p. 49. There are practicaliy no exposures of bedrock, and the stream channels are, for the most part, onis silghtly eroded, indioating that even during the storm of March 1938 the runoff was not excessive. In contrast to this condition the south-froing slopes north of the river, underlain by gnelsses and schists, show unmistakable ovidence of heavy runoff. Here the planes of sohistosity stand almost vertioal and the rock breaks down to small, angular, flat fragments only slightly disintegrated or decomposed. The soil covering is thin and finely granular. Steeper slopes show many areas devold of soll covering, and the whole region is out into a network of shallow washes and gullies, each of which 1s almost invariably eroded to bodrook.

A similar contrast, though perhaps not so distinct, is appareńt alang the north-faoling slopes south of the East Fork of San Gabriel River. The resistant metamorph10 formations found north of the West Fork of San Gabriel River continue eastward Into the valley of East Fork. The bedrook of the prominent, bold slopes along both the north and south flanks of the river is of this type. There slopes are bare or thinly mantled with so11, the vegetation is sparse, and the washes are rook-floored, all of 
which features inalcate a heavy runoff. About 2 miles south of the river the bedrock changes to a deeply disintegrated and well-altered schist. The schist is exposed in the upper part of Horse Canyon and in road cuts along the divide between San Dimas and Dalton Creeks and the San Gabriel River. Rounded ridges, moderate slopes, deep soil, and a heavy growth of brush characterize the area, all of them evidences of a comparatively light munof.

The granodiorite that underlies most of the northern part of the San Gabriel River Basin is commonly of porphyritic texture and consists of white or light-colored plagloclese feldspar and quertz through which crystals of blotite and hornblende are scattered. The blotite and harnblende constitute only 1 to 5 percent of the total volume. Although the granodiorite is somewhat disintegrated by weathering, it shows little evidence of decomposition, and the thin rocky to sandy mantle contains very little clay. The lower slopes are covered by coarse talus deposits of medium depth. Vegetation is sparse on the steeper slopes but is somewhat thicker over flatter areas. At the head of Devil Canyon and on the slopels of Mount Waterman, Mount IsI1p, and other observed places, the gullies and washes have all been eroded to bedrock. In most areas the larger tributary channels have been similarly eroded. The high runoff and cancentration of flow in the West Fork of San Gabriel River during the starm of March 1938 appear to have resulted from the rain that fell on the thinly protected slopes of the Bear Creek, Devil Canyon, and Trail Fork Basins. Precipitation during the same storm seems to have been sufficient to saturate the talus accumulations as well as the soil mantle. Storms that do not provide enough water to saturate the soll will ordinarily give little runoff.

The formation at the head of the East Fork of San Gabriel River appears to be a part of the Pelona schist described by Noble, 26 which occurs between the San Andreas and Iytle Creek faults. The formation radily disintegrates to particles of dustlike fineness and produces a deep mantle of flne, compact soll, whlch would probably show high runoff from any but light rains. Farther south, in the basins of Iron Fork and South Fork of Iron Fork, the rock is decidediy more resistant, and weathering has produced great quantities of only slightly disintegrated rock slabsd These basins, for the most part, are devold of soll or vegetation, and their surface would furnish direct runoff from any storm.

26 Noble, L. F., Excursion to the San Andreas fault and Cajon Pass: 16th Internat. Geol. Cong. Guidebook 15, p. 12, 1932. 
The floor of the valley of North Fork of San Gabriel River is covered with a heterogenous mixture of angular rock fragments, soil, and litter that has the appearance of a glacial deposit. Crystal Lake occupies a lower depression in the characteristically humocky surface of the area. The head of the canjon is a cirquelike amphitheatre considerably modified by erosion. Slopes surrounding this amphitheatre are steep to precipitous; many of them showing rock outcrops, and the remainder are mantled by shallow coverings of coarse rock fragments and talus. Several springs of substantial flow occur along the south margin of the alluvium above Sycamore Flat, indicating that these deposits are highly pervious. It is probable that in moderate storms much of the runoff from the surrounding area is absorbed, but when rainfall is intense the steep slopes of the amphitheatre must jield almost complete runoff, and from appearances only a part of this flow infiltrates to the subsurface storage. The wide, deeply eroded wash that crosses the area indicates a high runoff from the storm of Harch 1938, and it is probable that any storm of similar intensity lasting more than a few hours, will yield a proportionately high runoff.

\section{Tujunga and Pacolma Creeks}

The basins of Tujunga and Pacoima Creeks have for the most part, neither the altitude nor the sharp, rugged topography characteristic of the San Gabriel Range farther east. Underlying rock formations are in pert responsible for the difference. A belt composed of gneiss and schist occupies the south front of the range, forming an areal link between the granite and diorite that occur in the vicinity of Echo Mountain and Mount Wilson and the sedimentary rocks that crop out in the head of Placerita Canyon. This belt covers the lower part of Pacoima Wash and the part of Tujunga Creek Basin south of an Irregular line runing from the mouth of Trail Canyon to Mount Josephine. The gneiss and schist have been Intruded by several wide dikes and other irregular masses of diorite and granodiorite, but in both its topographic form and the character of its mantle this belt resembles the belt of similar rocks north of the San Gabriel River. Sharp, craggy ridges, steep slopes, narrow V-shaped canyons, a thin, rock-11ttered mantle, and many rock outcrops are characteristio features.

On the north side of this metamorphic belt the country rock is of a distinctly different character. Pacoima Creok Basin is underlain by diorite and anorthosite. The bedrock in the upper reaches of Tujunga Creek 
Basin consists of granodiorite, diorite, and anorthosite. These rocks are less resistant than the metamorphic types and have weathered to subdued ridges and somewhat open stream valleys. The moderately deep mantle covoring them consiats of rather finely disintegrated particles that grade almost imperceptibly into the intensely fractured parent rock. On the higher slopes this covering is perceptibly thimer, and there are occasional outcrops of rock, but on the more level surfaces and in the valley areas it increases in thickness, forming heavy accumblations of rather compact, well-altered clay soils. Vegetation 1s aparse on the steeper slopes but moderately heavy on so1l-covered reaches. Both the surface and subsurface characteristics of this formation are favorable to the absorption of rainfall in small amounts, and for this reason light to moderate atorms of short duration seldom produce increased stream flow. However, in intense storms, especially those of long duration, the comparatively shallow mantle along the slopes would probably reach saturation and, In that event, with deductions limited to the water that might percolate to the cracks and fissures of the bedrock, the runoff would be high. The impervious nature of the clay-filled valley solls would also contribute to high runoff from intense storms. These features probably account for the flashy floods shown by the streams under heavy precipitation.

\section{The frontal streams}

The frontal streams of the San Gabriel Range are similar to those of the San Bernardino Range in that they drain only the steop mountain fronts, in contrast to the larger streams that cut into and drain the interior of the range. The San Gabriel frontal streams include Cucamonga, San Antonio, San Dimas, Dalton, Rogers, Fish, Sawpit, Santa Anita, Eaton, and M11lard Creeks and Arroyo Seco and many other small streams. Their basins as a rule are fan-shaped, the stream and 1 ts tributaries commonly forming a dendritic pattern. Considerable variation both in type of bedrock and in nature of soll and vegetal covering is found in the frontal area, which In turn accounts for a noticeable difference in muoff characteristics.

The bedrock in the frontal area between Iytle Creok and the San $\mathrm{C}_{a-}$ briel River is almost exclusively metamorphic, the predominating type being a. diorite gneiss in wich the gneissic bands are almost vertical and trend In an east-west direction, parallel vith the range. The bands in general range in width from a fraction of an inch to 3 or 4 inches, but a few 
widen to approximately a foot. In many areas the bands are very narrow and have a ribbonlike appearance. The formation has been considerably shattered and broken.

The main stream channels have cut directly across the trend of this banding, forming deep cenyons with steep walls, and this steepness, combinded with the structural arrangement of the bedrock, which exposes only the edges of the bends, has prevented the formation of a deep mantle. Such soil as does exist consists largely of a thin blanket of disintegrated bedrock with a smaII amount of clay and considerable rock l1tter. Many of the slopes are almost devold of soll, but are covered by shallow talus accumulations of small, flat rock fragments. Side washes and gullies are generally floored with bedrock. The vegetation is moderately heavy to sparse.

The areas drained by Etiwanda Creek, Day Canyon, Deer Canyon, a large part of Cucamonga Canyon and the lower part of San Antonio Creek Canyon all show these features. The runoff from such areas depends to a large extent on the characteristics of the storm that produced it. Both the so1l mantie and the talus slopes are highly absorbent, but their retention capacity is low. They act, however, in carrying the water to the cracks and flssures of the underlying bedrock. In intense stoms the rainfail is 11kely to exceed the retention capacity of both the mantle and the bedrock, and then the excess mast appear as runoff, the amount of which w1II be in direct proportion to the amount of rainfall. Th1s water accumalates In the many side washes and gullies, where the increased flow has removed all debris and cleaned the channels to bedrock. Drainage from the watersheds is rapid, and all these streams, with the exception of San Antonio Creek, show only small amounts of summer munoff.

San Antonio Creek Basin differs from the basins of the adjacent streams to the east in that it has a substantial summer runoff. Naturaliy, mach of the ordinary summer flow can be attributed to the snowfall along the higher slopes of San Antonio Peak, but certain geologic conditions also favor this runoff. North of an east-west line crosing the saddle at the head of Cattle Canyon, which is about the line of the eastward extension of the San Gabriel fault, the bedrock changes from the metamorphic types already described to slightly altered granodiorite and dorite. Jointing in these formations is more widely spaced, and the rock breaks down to coarse angular, blocky fragments. Rock exposures are common along the higher slopes, but the lower slopes are blanketed with heavy accumelations of talus. The upper reaches of the tributary stream channels are 
eroded to bedrock but the lower parts, extending for some distance above the confluence with the main stream are floored with coarse angular alluจ1um.

In the vicinity of the old Toll House and extending upstream to about the mouth of Icohouse Canyon the bed of San Antonio Creek consists of gravel deposits. These range from about 200 feet to nearly a quarter of a mile in width. Above Icohouse Canyon the gravels are confined to a narrow belt along the stream channel. The thickness of these deposits is not known, but in one area above Icehouse Canyon more than 100 feet is exposed.

With storm precipitation in the form of rain, as in March 1938, these gravels act as an equalizing influence, absorbing a part of the alrect runoff from the surrounding region, to be released later. The extensive talus deposits along the slopes and in the lower reaches of the side streams would act in the same way. It is evident that with storms of high Intensity aimilar to that of March 1938 only a part of the runoff is retained, but with moderate rains 1 appears that only minor amounts of munoff would escape. The remarkably consistent sumer flow of San Antonjo Creok indicates a long period of carry-over, extending from one season to another or perhaps longer.

The bedrock throughout the greater part of the San Dimas and DaIton Creek Basins is a soft, well-disintegrated, and partly decomposed darkcolored schist with occesional areas of unaltered granite. The formation weathers to rounded ridges, broad canyons, and moderately steep slopes. The surface mantlo is a granular and porous soll containing many partly disintegrated grains of quartz and feldspar and a small amount of rock 11tter. It verios in thickness from 1 or 2 feet on the slopes to 20 feet or more on the rounded ridges and in the flat areas. Observations in road cuts and other places where the subsurface material is exposed show that the so1l mantle merges almost imperceptibly into the shattered, welldisintegrated, underlying bedrock. There are practically no accumula tions of talus, vers few exposures of rock, and most of the area included in the two basins is covered with a moderate to heavy growth of chaparral.

The amount of runoff from rain falling on this type of watershed dopends in a large measure on the rate of rainfall and on the amount of antecedent precipitation. Although the degree of poroaity of the mantio 1s high, its rate of absorption 1s necessar1ly 11mited by the small pore openings and does not approach that prevaliling in the coarse rocky solls of other drainage areas. Drainage likewlse takes place slowly. Ilght to 
moderate rains will seldom yield an appreciable amount of munoff unless they have been preceded during the season by an average or greater than average amount of precipitation. The floods of 1914, 1916, and 1938 show an aggravated stage of these unfavorable conditions, an intense storm of several hours duration occurring in a season of unusualig high antecedent rainfal1. Under these circumstances, the porous but relatively impermeable subsoll and disintegrated bedrock were probably near saturation before the storm occurred, and absorption during the rain was limited to the available pore space in the top few inches of the mantle. Although there, was coplous stream flow during the storm of March 1938, only a small part of it appeared as direct surface flow, as show by the observations of the United States Forest Service on plots in the San Dimas Experimental Forest.

The various drainage basins between the San Gabriel and Arrojo Seco Canyons are underlain mostly by diorite and granodiorite. Occasional narrow belts of schist and gneiss are found closely associated with and paralleling the fault lines, but with these exceptions the rock 1s uneltered and retains most of 1ts original crystalline texture. In most of the basins the rock is intensely fractured and disintegrated to a depth of several feet. Partial decomposition at the surface has produced a granular sol1 mantle carrying large quantities of rock particles. Some of the steepest slopes in the mountain region are found in this area, jet there are few exposures of rock, and the slopes are generally covered with a medium to dense growth of vegetation. Iarge accumulations of talus are lacking. The main stream chapnels are generally floored with bedrock in the upper reaches but are covered with alluvium near the valley. In Arroyo Seco Canyon these deposits form the aquifers for a number of springs and seeps. Runoff characteristics in this area are similar to those in the San Dimas and Dalton Creek Basins, with the amount of runoff depending to a large extent on the rate and duration of the rainfall and the amount of antecedent rainfall.

The effects of geologic conditions on the rainfall-runoff relationship are evident in the contrast between this area of granitic rocks and the area underlain by metamorphic rocks fust north of the San Gabriel River. These two areas recelve comparable amounts of rainfall, both have southward-facing slopes, and the topography is similar, yet owing to the difference in the bedrock there is a notable difference in the type and thickness of the so1l mantle, in the density of vegetation, and consequently in the runoff characteristics. On the other hand, a comparison 
of the same San Gabriel area with the Cucamonga Creek and Iower San Antonio Canyon drainage basins, showa a marked similarity in topography and surface features and a very close relationship in munoff characteristics. These two areas are underlain by metamorphic rocks of the same general type.

In addition to the absorption that takes place over the mountain area of the frontal drainage basins flood peaks are further reduced by percolation in the extensive cone structures developed at the mouth of each stream. Two conditions, however, mitigate against the full influence of percolation as a factor--first, the short duration of flood flow, and second, artificial restriction of the stream channels. Any unfavorable alteration of these conditions such as speeding up the flow by diverting the stream to Iined condults or further increase in the practice of channel confinement will, by reducing percolation in the coarse gravels of the cone, tend to increase the amount of flood water reaching the valley floor.

\section{Lytle Creek}

The characteristics of flood runoff in this basin appear to be materially influenced by both the stmuctural formation of the canyon and the type of underlying bedrock. The lower part of the main stream and the Middle Fork follow the line of the San Jacinto fault, and the upper reach of the stream above Glen Ranch follows the line of the Loma Linda fault. It is probable that the canjon above Glen Ranch occuples a depressed fault slice, although the evidence of a double line of faulting is hidden under alluvial f1l1.

The movement along these faults has been of sufficient magnitude to cause unlike formations to appear on opposite sides of the canyon throughout practically 1ts entire length. The long narrow ridge to the northeast which forms the divide between Iytle Creek Basin and Cajon and Ione Plne Creek drainage basins is composed of Pelona schist, a formation which Noble 27 describes as being "composed chiefly of bluish-gray quartzsericite-albite schist but includes beds of chlorite schist, actinolite schist, greenstone, quartzite, and limestone". The rock disintegrates readily, forming a mantle of fine, compact soll that supports a heavy growth of brush. Slopes throughout this area are moderately steep, with rounded ridges and shallow washes. There are practically no extensive exposures of rock and talus accumilations are almost unknown, but many of 
tho washes show bedrock floors. The upturned edges of the sohists may be observed in these channels. Southwest of the fault the country rook 1s largely diorite gnolss and granodiorite. Metamorphio types are exposed from the mouth of the oanyon to a point above alen Ranch, but northwest of this point the predominating rook is a fine-gralned orystalline granodiorite, the same formation as that found in the headwaters of San Antonto Creok. In fact, conditions along the west side of the Ijtlo Crook Basin are simflar in many ways to those in the upper San Antonio Crook Basin. Heavy acoumulations of angular talus cover the lower slopes whoreas the hlgher slopes wow many areas of thin mantlo or bare rook.

The opposite sides of the canyon show alstinct dissimlarity in conditions of munoff from rainfall. Although the slopes underlain by the Pelona sohlst are well covered, it is apparent that they would jleld a hlgh proportionate munoff from intense storme. The very fine micacoous 1011s have a high porosity and high retention capacity but an excoptionally low permeability. Percolation to the subsurface storage would, therefore, be extremely slow, and the surface munoff would be inereased. On the other hand, the coarse talus acoumulations common to the opposito s1des of the canjon favor absorption of rainfall in large quantities. The topographio expresilon of this dissimilar behavior is olearls ohow in the I1l above alen Ranch. Throughout thls part of the canjon, where the wldth of the alluvium ranges from about 500 feot to more than a quarter of a mile, the northeast margin of the f1ll is 20 to 30 foot higher than the southwest margin. Moreover the stream channel, whloh would normalls be expected to oocups the lowest depression, follows a course near the top of the f1ll close to the schlst slopes. The lower depression shows little ovidenco of stroam flow, but the higher watercourse is a wide, mach-eroded wash. Thls condition apparently results from high runoff and-movement of debris from the sohlstose slopes, In contrast to low runoff and slight orosion from the opposite s1de. Th1s behavior would probably provall only In storms of high intensity. Under light to moderate rains, 1t would appear that the heavy vegetal covering on the sohlstose slopes would dolay surface flow long enough to allow peroolation of the water to underground storage.

In addition to high diroot runoff from hoavy storms, Iytlo Creok also furnishes a substantial summer flow. Part of this flow no doubt oomes from water retalnod in the extensive talus deposits of the southern slopes, but it is likely that a larger part has its source in the extensive porous gravel deposits of the canyon. These deposits extend through almost the 
entire length of the stream and range in width from a fow hundred feet to nearly half a mile. All of the tributaries empty on the deposit, and many of them have built small alluvial cones flanking the main stream. The probable fault-slice structure of the canyon above Glen Ranch appears to be especially well sulted for storage, as shown by the substantial amounts of water that rise to the surface between Glen Ranch and the mouth of Middle Fork of Iytle Creek, where the main stream cuts through a block separating two lines of faulting. Contributions from the Middle and South Forks of Iytle Creok are mainly from talus deposits, as these streams have only small amounts of channel gravel. It is $11 \mathrm{ke}$. part of the early summer flow originates in melting snow on the northeast slopes of the San Antonio Peak riage, percolating through the gravel fill of the canyon.

The vallegs 28

San Fernando and San Gabriel Vallejs, and the west half of the San -Bernardino Valley flank the San Gabriel Range on the south, and each of the streams draining from the mountain area south of the crest passes through one of them in its course to the Pacific Ocean. In topography and structure the valleys are similar--each represents a depressed segment of the earth's cmast buried under deposits of alluvial fill, and each has a comparatively flat surface that slopes gently away from the base of the mountains. From the standpoint of their influence on the flood flow of streams, the important feature of the valley structures is the great depth and porous character of the alluvial cones, which occur at the mouths of all canjons and washes, and of the widespread valley fill. The cones extend in an almost continuous coalescing belt from the mouth of Arroyo Seco Canyon to the mouth of Cajon Canyon. Naturally the height of the individual cones and the distance they extend into the valley floor vary with the size and drainage area of the parent streams, but their internal structure is similar. Coarse gravel and boulders predominate near the apexes of the cones, fine silts and clays are the chief constituents at their outer edges, and material of intermediate texture occuples their central zones. This distribution, however, is by no means regular, for,

28 See also Mendenhall, W. C., Groundwater and irrigation enterprises in the foothill belt, southern Califormia: U. S. Geol. Survey Water-Supply Paper 719, 1908; Eckis, Rollin, Geology and ground-water storage capacity of vailey fill: Callfornla Dept. Public Works, Water Resources Div., Bull. $45,1934$. 
as the cones are the result of deposition by streams in all stages of flow, there has been constant shifting of deposition zones, with the result that many types and textures of debris are indiscriminately distributed throughout the mass of the cone. This heterogeneous collection of debris is, as a mie, very porous, and 1ts effect on flood flow is limited by the rate at which water can percolate during the comparatively short duration of the flood. Confining the stream to a single channel or otherwise restricting its lateral spread over the cone naturaliy reduces the absorption area, and infiltration is correspondingly reduced. DistributIng the water over the ground, on the other hand, augments percolation.

The coastal plain 29

An intermediate belt of $10 \mathrm{w}$ hlls and mounteins, including the Santa Monica Mountains, the Repetto Hills, the Puente H11ls, and the northwestern part of the Santa Ana Mountains, separates the San Fernando, San Gabriel, and San Bernardino Vallejs from the coastal plain. The Los Angeles, San Gabriel, and Santa Ana Rivers enter this plain through gaps cut through the mountalnous belt, and each has deposited a low, broad alluvial cone at the southerm base of the hills. From the base of the cones the surface slopes gradusily to the Pacific Ocean as a flood plain broken only by occasional hills and low mesas and the Isolated San Pedro Hills, which rise abruptly at the coast line in the southwestern part. The flood plain is about $50 \mathrm{miles}$ long, extending from Sante Monica to Newport Beach, and 12 miles wide, spreading from the ocean to the hills.

Oscillations in sea level, together with some differential land movement, have left the surface somewhat uneven. Some areas are well above the level of present deposition and are being actively eroded, whereas others are below that level and are being aggraded. The higher surfaces coincide with segments of the earth's crust that have been uplifted by faulting or folding, and the lower surfaces occupy the troughs between the uplifted blocks. The Beverly-Newport uplift, extending southeast from Beverly Hills to Newport Beach, and the Santa Fe Springs-Coyote Hills up1ift, extending from the subdued Santa Fe Springs dome to the mach higher Coyote Hills, several miles ferther east, are the most prominent of these areas of uplift. Between them are broad troughs filled with several hundred feet of Plelstocene and Recent alluvium. Where the streams have cut

29 See also Eckis, Rollin, Geology and ground-water storage of valley fill: California Dept. Pubilc Works, Water Resources Div.; Buil. 45, 1934. 
through the uplifted segments, they occupy deep, weil entrenched channels, but across the troughs, in which the underlying formation is largely alluvial fill, the channels are usually unstable and shallow, most of them less than 10 feet in depth. There is considerable evidence to show that the streams have, in the past, meandered over a large part of the trough area. It is known, for example, that within historical time the Los Angeles River has reached the ocean through Ballona Creok. During the samo period the San Gabriel River has shifted several times between its present channel, the bed of the Rio Hondo, and the former courge of the Los Angeles River. The Santa Ana River, also, has occupied several channels witin an area extending from Anaheim Creok to Newport Bay. Thus the lower part of the coastal basin is in reality a flood plain fed by the flows of these.rivers, and in times of high flood discharge it is not unusual to find free intermingling of their waters in a sheot spreading across the lowlands between Anaheim, Nigger Slough, and the ocean.

Before the arrival of man and the restrictions he imposed on the streams by building various bridges, dikes, and other confining structures it is probable that none of the streams had stable channels but that they meandered over the wide flat plains, as in fact, they do today in places where natural and artificial channels are inadequate to carry the runoff.

In the coastal plain conditions favoring the infiltration of water to underground storage are less favorable than in the upper Santa Ana, San Gabriel, and San Fernando Valleys. The material making up the alluvium represents the outwash from small streams draining the predominantly sedimentary formations of the surrounding hills plus contributions of fine debris from the three lo ger rivers. As a consequence, the channel bottoms are covered with fine, poorly assorted gravel and sand that includes a substantial proportion of clay and silt. Wuch of the surrounding area is covered with a surface mantle of heary clay soll. Under these conditions it is apparent that the capacity for absorbing flood water is IImted and that the greater part of the water mast escape through some channel to the ocean. 
Santa Clara, Ventura, Santa Ynez, and Santa Maria drainage basins 30.

The basins of the Santa Clara, Ventura, Santa Ynez, and Santa Maria Rivers differ materially in geologic structures and rock types from those of the streams that drain from the San Gabriel Range. Basin characteristics, including shape, slope, and topography, as well as mantle covering and vegetation reflect this difference. The influence that such factors exert on flood munoff from rainfall is likewise shown to some extent.

The country rock of the grea is predominantly sedimentary. Small outcrops of lgneous and metamorphic rocks are found along the west edge of the San Gabriel Range in the headwaters of the Santa Clara River, and again along the foothills of the San Rafael Range, reaching from zaca Creek, southwest of Lookout Mountain, to the nelghborhood of Little Pine Mountain, but these are comparatively unimportant in their effect on flood runoff. The exposures of extrusive andesite and basalt found in the Senta Susana Mountains and the western part of the Santa Monica Mountains are too limited in areal extent to have any appreciable effect on the rainfallmunoff relation in the area. Of the sedimentary rocks, sandstone, conglomerate, and shale predominate, and impure limestone and siliceous shale occur in smaller amounts. For the most part, the shales weather to rounded hills with gentle slopes, and the harder sandstones, conglomerates, and siliceous shales appear as steep ridges and cliffs. The mantle cover, ing is largely residual and varies in thickness and texture with the slope of the terrain and the type of the underlying formations. Sandstones and conglomerates weather to coarse porous mantles, shallow on the steep slopes and thicker on the gentler ones, but shale forms an almost impervious, compact covering on any slopes. The veget growth reflects, to a great extent, the underlying formation. The sandy solls support a heavy cover of chaparral, especially on the protected northern slopes of the mountains, but the shaly solls are usually entirely barren or have only a sparse growth of short grass or stunted sage. Between these extremes are variations of all types of cover and vegetation, depending on the proportion of sand or shale in the soil, the direction of slope, and the availability of water.

30 See also Eldridge, G. H., and Arnold, Ralph, The Santa Clara Valley, Puente Hills, and Los Angeles oll districts, southern California: U. S. Geol. Survey Bull. 309, 1907; Arnold, Ralph, Geology and o1l resources of the Summerland district, Santa Barbara County, Calif.: U. $\mathbf{S}$. Geol. Survey Bull. 321, 1907; Arnold, Ralph, and Anderson, Robert, Geology and oil resources of the Santa Maria oll district, Santa Barbara County, Calif.: U. S. Geol. Survey Bull. 322, 1907; and Kow, W. S. W., Ios Angeles to Santa Barbara: 16th Internat. Geol. Cong., Guidobook 15, pp. 48-68, 1932. 
The structural features of the area under discussion differ considerably from thcse of the San Gabrlel Range. Uplift in the San Gabrlel Range occurred along fault lines, and the mossive Igneous and metamorphic rooks jlelded by fracture rather than folding, the fractures being distributed through the mess of the mountain block in the form of innumerable joints, faults, and f1ssures. The sedimentary country rock of the area northwest of the San Gabrlel Range ylelded to mountain-bullding forces mainly by folding, with faulting of secondary importance. As a result of this action a series of east-west trending folds have been developed, these folds determining the outline of the characteristic parallel mountain ranges and their intervening vallegs. In this pattem the vallegs generally occupy the synclinal troughs, and the mountains represent the anticlinal crests. The pattern has been modiffed somewhat by differential erosion and, in some small areas, by faulting, but in general the drainage follows the structural outline. For the most part, the main streams flow westward, following the synolinal troughs, and their tributarles enter at right angles, having out deep, narrow canyons across the upfolded mountainous areas.

\section{Relation of rainfall and runoff to geology}

Whatever effect geologic formations have on runoff from rainfall depends on the amount of water that can be absorbed by the surface mantle and the pervious parts of the underljing bedrock and the rate at which the absorption proceeds. The topography and the thickness, texture, and composition of the mantle are the prinolpal factors affecting this relationshlp, and all are the result of geologic processes.

The sedimentary rocks of this area have yielded to deformation by folding. The process required a long perlod of time, during which geologic forces were constently being applied and readjustments in the rock structure were continualis taking place. Both the nature of the change and the plastic oharacter of the deeply buried sediments were unfavorable to the fracturing and associated disintegration such as took place in the San Gabriel Range. Consequently, the country rock of the area is characteristically impervious, and percolation of rainfall is therefore limited to the porous areas of the surface mantle and to the alluvial 1111 of the river valleys. In areas covered by a deep sandy mantle little runoff from rainfall is to be expected. The heavy growth of vegetation usualis found on such areas stimulates both the percolation and the retention of 
water. On the other hand, rock outcrops favor complete runoff from rainfall, and shaly areas, with their impervious clay mantle and sparse vegetation, are almost as effective in preventing the percolation of rainfall. Coarse valley fill favors percolation from both rainfall and stream flow, but with finer textured fills percolation is less effective. The proportionate acreage of the different types of rock and surface cover found in the various areas will determine the characteristies of the rainfall-runoff relationship.

\section{Santa Clara River}

The Santa Clara River occuples a somewhat modified structural depression that extends westward from the west flank of the San Gabriel Range to the Pacific Ocean, a distance of $75 \mathrm{miles}$. Its headwaters are on the western edge of the San Gabriel Mountains, but most of 1ts drainage area lies north of the stream, between Antelope Valley and Pine Mountains. All of its larger tributaries, including Castaic, Piru, Sespe, and Santa Paula Creeks, named from east to rest, drain from the north and flow in a general southerly direction through tortuous courses that have been determined in part by rock stmucture and in part by erosion. Their canjons are deep, many of them cliff-bound, and almost their entire drainage basins are steep and magged. The mountainous parts of their basins are generally covered with a fairly thick growth of chaparral, but in the foothills vegetation is sparse.

Both the Santa Clara Valley and the lower parts of its tributary valleys are floored with alluvium. These deposits range in width from half a mile to 3 miles in the main valley, and from a fer hundred feot to half a mile in the valleys of the side streams. The texture and porosity of the fill vary according to the locality; deposits in the upper part of the main stream channel consist principally of clean coarse gravel and sand derived from the igneous and conglomerate formations common to the headwater area, whereas those in the lower part of the main channel and in the tributary streams include sand, elay, and silt oroded from the surrounding fine-textured sedimentary rocks. The depth of the flll ranges from a few inches along the edge to more than a hundred feet in the central areas. At a few places in the channel the deposits are intermupted by exposures of bedrock. One such exposure occurs in the main stream about $3 \mathrm{miles}$ east of PIma Creek and forms the area of rising ground water reaching upstream to the mouth of Castalc Creek. Another occurs between Filmore and Santa Paula, forming a similar but smaller area of rising ground water. 
It is evident that only in certain reaches of the stream channel, that is, above the mouth of Cestaic Creek and between the bedrock exposures east of Piru and the mouth of Sespe Creok, are conditions favorable for an appreciable reduction of flood flow by percolation to the alluvial 111. The fineness of the alluvium in the lower section of the valley and in valleys of the tributaries makes it unfevorable to rapid percolation, and, naturally, areas of rising water would have no capacity for additional storage.

The bedrock of the Santa Clara Basin is sedimentary throughout, except on the northwest slopes of the San Gabriel Mountains. In this locality it is igneous, predominantly anorthosite intruded by many dikes of dark-colored diabase, and conditions are similar to those in the upper Tujunga and Pacolma drainage basins. The surface features are favorable for the absorption of rain only in small amounts, and when precipitation is heavy copious runoff is to be expected. The rock-floored gullies and severely eroded washes are ovidences of the large runoff from the storm of March 1938.

In the pert of the basin that Iies above PIma Creek the country rock consists of thick deposits of conglomerate and sandstone with lenses of variegated shale and fine-grained sandstone. These beds heve been throm Into monoclines and more complex folds. Over much of the area east of Castaic Creek they crop out as a series of more or less parallel hogback ridges, with massive resistant conglomerate or sandstone strata occupying the crests. Dip slopes are commonly entirely barren. Between Castaic and Piru Creeks the beds have been thrown into a number of eastward-trending folds that appear to have had some influence on the topography. Broad ridges alternate with open stream valleys, and the slopes are moderate.

The rocks weather slowly to form a shallow soll mantle that is sandy and filled with rock litter where it overlies sandstone or conglomerate, but dense and Impervious where it is derived from shale. Brush is generally sparse, but much of the area is covered with a fair growth of grass and desert annuals.

The bedrock of the area is somewhat pervious, a few wells derive small flows of water from some of the more porous strata, and it is possible that some rainfall percolates to considerable depth. The amount appears to be relatively small, however, and it is belleved that absorption is limited mainly to areas covered with a porous soil mantle. 
PIru Oreek

The formations underlying the P1mu Oreek Basin are somewhat finer in texture than those immediately to the east. They consist mainly of shale, 811100048 shale, and fine-grained sandstone with occasional beds of conglomerate. Structural deformation has cast the beds into a great number of more or 1088 parallel folds; some of these are broad with the beds 1nolined at a low angle, and others are squeezed, so that the beds orten stand nearly vert1cal. Plmu Creek has out across the prevaling trend of these folds, and 1ts tributarles drain through canjons that are more or $108 \mathrm{~s}$ parallel to them.

Slopes along the lower $15 \mathrm{mlles}$ of the oreok and 1 ts tributarles are exceptionaliy steep. A shallow sandy and rooky soll mantle, in most places not more than a few 1nohes thiok, is the prevaling type of oover and a considerable part of the area shows bare rook slopes. Many of these appear to be due to small landslides that were generated during the storm of March 1938. Nearly all the steeper washes are eroded to bedrook. Sollcovered spots support a moderate growth of brush and grass. The finetextured, rather thin-bedded rocks show little tendenoy to break into spalls or large fragments, and consequently there are no talus deposits.

- The topography in the upper reaches of the drainage basins is more mature; the canyons open to valleys, the slopes are moderate, and there are few areas of exposed rock. The soll mantle, although somewhat thicker than on the steeper slopes, is relatively shallow, but the vegetal coverIng 18 moderate to heavy. With the exception of rook exposures, surface features appear to be favorable to the absorption of moderate amounts of rainfall. However, as absorption in this basin is definitely 11 imited to the surface mantie because of the impervious character of the bedrock, heavy rains of long duration are likely to furnish a proportionately higher runoff, than in the San Gabriel Range, but probably not so high as in the neighboring Sespe Creok Besin.

\section{Sespe Creok}

Features that favor the absorption and retention of rainfall are probably less prevalent in the Sespe Creek Basin that in any similar area in the Santa Clara drainage basin. The type of bedrook, the charaoter and small extent of the surface mantle, and the structural arrangement of the mountain mass appear to be responsible for this condition. The bedrook throughout the larger part of this oreek basin is dark brown or 
chocolate-colored and is composed of fine-grained sandstone interbedded with shale and, in a few places, deposits of conglomerate. These beds are easily recognized and are known to geologists as the Sespe formation. In the lower part of the drainage basin, between Sespe and Ilttle sespe Creoks, the beds are mainly thin and shaly, but in the higher parts of the basin they become thick and masive. In both localities, however, the rocks are dense and compact, and appear to be practically impervious to water. Folding and faulting have caused many varlations in structure, and in most areas the beds dip at high angles. Dip slopes are prevalent, and many of them have been eroded unt1l they are entirely berren of soll or vegetation. In the plateau section, between Sespe and Little Sespe Creeks, 1t is estimated that as much as 25 percent of the area has been denuded of soll; in other sections the percentage is much less.

The Sespe formation weathers to a shallow flne-textured soll mantle, somewhat sandy in most areas but tight and clay-filled in localitles underlain by shaly deposits. The bedrock shows practically no weathering or disintegration below the so1l mantle, and extensive talus deposits are lacking. Vegetation on soll-covered areas is, as a rule, heavy. The Sespe Creek channel is filled with alluvium below the function of Sespe and Ilttle Sespe. Creeks, but above that point both streams and most of their tributaries contain only shallow fllls of debris or have been eroded to bedrock.

The unusually high percentage of bare rock slopes in the Sespe Creek Basin is naturally reflected in the runoff characteristics of the stream, since these slopes furnish complete and direct runoff from storms of practicaliy any type or intensity. Much of the remaining soll-covered area has characteristics that are unfavorable to extensive absorption. The soll covering when dry, appears to be open and porous, but close examination reveals that most of it contains enough clay to render it nearly Impervious when wet. This condition prevalis on the plateau between Sespe and Ilttlo Sespe Creeks and was noted also on the north slopes of the Topatopa Mountaing. Once this materlal has been thoroughly soaked at the surface by antecedent rains, its capacity for absorbing additional quantities of water is greatly reduced. Even in areas where the soll covering is highly porous, the retention of rainfall is limited by the shallowness of the mantle and the compact, impervious nature of the bedrock. 
Other parts of Santa Clara drainage basin

Conditions over the remalnder of the Santa Clare drainage basin, which includes the north-facing slopes of the Santa Susana Mountains, Oak Ridge, South Mountain, and the south-facing slopes of Sulphur Mountain, are somewhat more favorable to retention of rainfall, and comparable rains are therefore not likely to show as high a proportion of munoff as on Sespe Creek. The bedrock over the area is of the same type and character as that in the PIru and Sespe Creek Basins, but the structural features are not so sharply defined and the slopes are less steep. Barren dip slopes are lacking, and although there are a few patches of exposed rock, they are fewer than in the P1ru Creek Basin. The soll mantle, although relatively shallow, is generally sandy and porous. Brush is, as a rule, sparse, especially on north-facling slopes, but the surface is well protected durIng the winter by a heavy growth of native grass. The absorptive capacity of the cover appears to be sufficient to enable it to retain practically all the rainfall from light or moderate storms, and the only runoff likely to appear under these conditions is from the small areas of exposed bedrock. Intense and protracted rains, on the other hand, are likely to furnish heavy runoff.

\section{Ventura River}

The Ventura River and its chief tributaries, Coyote Creok and Katilija Creok, drain an area that includes the 0 jal Valley and the mountainous region to the north, west, and east. ojai Valloy is an alluvium-filled structural depression, resulting partiy from fault action and modified by erosion, measuring about 12 miles from east to west and 2 miles from north to south. Streams from the mountains have built up steep alluvial cones along the north margin of the valley, and from the bese of these cones the valley floor slopes gradualiy toward the southwest. Both the cones and the valley fill are important as sources of ground water, and their capaclty for storage of additional water is usually sufficient to insure the absorption of the ordinary runoff of streams and washes draining across them.

A comparatively low but rugged range of hills lles between the 0ja1 Valiey and the Pacific Ocean. This range is a part of the complex, upfolded mass that extends west from Piru Creok to the ocean, and 1ts sed1mentary bedrock is of the same age and approximately the same character as that found in the Piru and Sespe Creek Basins. Ventura River has cut 
directly across this range, forming a wide but steep-sided canyon. Cojote Creek, entering from the west, and Canada Larga, from the east, are the largest tributaries. The lower parts of the basins of these streams, together with the Ventura Valley, are graded and fliled with alluvium. They provide considerabie underground storage.

The largest and most Important part of the Ventura River Basin, from the standpoint of water production, lies to the northwest of the Ojal Valley. It is drained by Matili ja Creek and its tributaries. This area Includes the northward-facing slopes at the western end of the Santa Ynez Mountains and the southward-facing slopes of Ortega Hills.

The country rock in the area is of sedimentary origin and consists of Interstratifled shale, sandstone, and conglomerate, all so well consolldated that it may be considered to be impervious. The beds have been folded and tilted, though probably less than in the lower reaches of Piru and Sespe Creeks, and over much of the area they appear as a succession of sharp ridges and canjons, with masive resistant strata forming the ridge crests and softer beds flooring the valleys. Slopes are generally steep but as a rule are not preclpitous except in occasional stretches along some of the steeper, narrower canyons.

The soll mantle is residual and varies in texture and composition according to the nature of the parent rock. Sandy types seem to prevall. The covering is rarely more than 1 or 2 feet thick, but it is widespread and there are only occasional small areas of exposed bedrock. Vegetation is usualiy heavy on the north-facing slopes but scattered and sparse in other places. Clay solls overlying shale beds can generally be distingulshed by thelr sparse vegetal covering.

As impervious bedrock underlies the greater part of the Ventura River Basin, the absorption of rainfall will be restricted to the soll mantle of the mountain section and the alluvial fill of the stream and valley areas. As the capacity of the soll to hold water is necessarily small because of Its shallowness, It is evident that only light or moderate rainfall w1ll be absorbed. Storms of greater intensity and longer duration must eventually jield the greater part of their precipitation as runoff, and this will appear in the valley and in the alluvium-filled sections of the streans. The most favorable locality for absorption of either ordinary or flood runoff is along the Ventura River where it crosses the ofal Valley, but storage in this area is limited by the high ground-water level that usualig prevalls in the lower parts of the valley. 
Measurements made by the State Division of Water Resources 31 indicate that considerable rainfall may percolate to ground storage during earlyseason storms, but that the amount dwindles rapidis as later storms tend to flll the avallable storage. Storage in the alluvial belts found along the Ventura River between Ojal Valley and the ocean and the lower parts of the basins of the larger tributaries, notably Coyote, San Antonio, and Santa Ane Creoks and Canada Larga, is limited by the relatively small extent of the deposits.

The limited storage avaliable in both the mountain and valley areas would indicate a relatively high flood runoff for the Ventura River.

\section{Santa Ynez River}

The Santa Ynez River occuples a structural trough that trends slightly north of east and follows the north flank of the Santa Ynez Mountains. Besides the north slope of these mountains its drainage basin Includes the southweat alope of the eastern part of the San Rafael Mountains, the south slope of the Purisime Hills, and the wide valley areas around Santa Inez and north of Lompoc. Toward the south the slopes rise steeply in a series of steps to the crest. of the Santa Ynez Mountains, but toward the north they rise gradually from the terraces and and hills west of the Santa Ynez Valley, becoming steeper, with pronounced ridges and sharp canjons, as they merge with the south slopes of the San Rafael Range.

In its upper reaches the river flows through a well-defined canjon, and has a gradient of about 125 feet to the m1le. Throughout most of this atretch there is a little fill on the canyon floor and that only in thin deposits. Beginning at a point east of Santa Ynez and extending to the coast, the gradient drops to 25 feet to the mile and the valley opens to the north over wide terraces. In this stretch deposits of recent elluvium underlie the river channel.

The north-facing slopes of the Santa Ynez Range carry a fairly heavy growth of vegetation, but north of the river the covering is IImited to a sparse growth of brush and grass, with occasional patches of more luxuriant vegetation in shaded and protected places.

The rock of the Santa Ynez Range consists of marine sediments, Including sandstone, shele, and conglomerate, with small areas of limestone. Sandstone occurs in the foothills, shele in the intermediate belt, and sandstone and conglomerate at the creat of the range. Both the sandatone 1834,

31 Conkling, Harold, Ventura Oounty Invest1gation: Bull. 46, p. 62, 
and the shale are well consolidated, so that the absorption of rainfall would take place only in the soll mantle.

Terrace deposits of considerable extent ooour in the Santa $Y_{n e z} V_{a l-}$ ley at 1ts cosstal end, west and north of Lompoo, and throughout Its middle and upper reaches. The doposits consist of a veneer of and and gravel, usually not more than 25 feet th10k, normally soft and porous but In pleces compacted and cemented. Where they are soft and porous, the deposits are capable of absorbing the rainfall from all but the most intense storms. These deposits and the underlying formations probably also not as reservolrs for water percolating from the river, though of oourse this percolation oan take plece only in the imediate vioinity of the stream.

The bedrook in the Purisima H11ls and in small areas in the footh111s of the San Rafael Range Inoludes thlok deposits of diatomecous shale. This shale also underlies much of the terrace deposits of the river valley. Where the hale has not been altered by olose folding or alliceous comentation 1t has a porous texture favorable to absorption of water. Althouch definite Information 1. laoking, it appears that in a large part of the Purisima Hills area and a smaller part of the San Rafael diatriot the exposed bedrook 1s of this oharaoter. Areas underiain by g11101f1ed and folded atrata are likely to j1eld complete runoff from ang but the 11 ght. est atorms.

The remalning areas. In the San Rafael part of the Santa Ynez River Basin are underlain by sedimentary rooks ranging in texture from coarse sands and conglomerates of fluvial origin to rell-oompaoted fine sands and shale of marine or1gin. The harder beds prevall over the higher part of the area, and the softer beds ocoupg the foothill area near the river. A narrow belt of old orystaline rook half a mile to a mile in width and several miles in length strikes southwest from the head of IIsque Oreek.

Owing to the diversified tjpes of bedrook and mantlo found within the Santa Ynez RIver Basin anj relation between flood runoff and geologio ooriditions is inderinite. Although the exposures of impervious bedrook are limited in their areal extent, medium rains are likely to provide almost complete runoff from these areas and looal floods are to be expected. Exoept in the more Intense and w1despread torms, suoh runoff ehould bo largely absorbed by the more porous sands and the valley f1ll. 


\section{Santa Maria River}

The Santa Marla Valley is a broad trlangular area bounded on the southwest by the Casmalla and Solomon Hills and on the northeast by the San Rafael Mountains. The. Cuyama and Sisquoc Rivers with their tributarles drain the San Rafael Mountains and debouch on the valley floor to form the wash of the Sante Maria River. The Santa Marla flows only in times of flood. Streams from the Solomon Hills also debouch on the valley floor but seldom, if ever, reach the river. The runoff from Casmalia Hills is largely Intercepted by Guadalupe Lake.

The valley is a wide flood plain, the interior part of which is floored with thick deposits of Recent alluvium. Along the edges of the valley are terraces that fringe the San Rafael Mountains on the northeast and rise gradually to the Casmalla and Solomon Hilis on the southwest. The alluvial fill consists mainly of coarse gravel and sand, with some finer sediments near the ocean, all capped by fine-textured soll. The $f 111$ is favorable to water percolation and atorage and is the source of irrigation water used in the extensive agricultural development of the valley. The terrace deposits are similar to those found along the Santa Ynez River, and percolation in them is limited to the more porous areas. The principal source of supply for the Santa Maria River is the runoff from the southwest slopes of the San Rafael Mountains. The bedrock on these slopes includes a belt of thin-bedded siliceous shale paralleling the valley along the foothills and, contiguous to it on the northwest, older sedimentary rocks, mainly sandstone, conglomerate, and shale. The beds are considerably folded, and in many places their thinly mantled edges appear at the surface. A wide belt which follows the crest of the range, is made up of a thick series of alternating thin-bedded darkcolored shale and more massive sandetone. These beds are not so strongly folded as the younger formations, but in places they are steoply upturned.

In general, flood flows in the Santa Marla River originate on the southwest slopes of the San Rafael Mountains. The higher mountains have the greatest rainfall, and the thin-bedded shales and the hard, massive sandstone found in this area are least favorable to water absorption; the shallow soll mantle, especialiy where it overlies shale, is itself largely impervious, and the underlying rock is almost completely so. Softer parts of the siliceous shale favor absorption on low to moderate slopes, and these areas probably do not contribute runoff except in severe storms. 
RAINFALL, RUNOFF, AND RETENTION

The amount of runoff that reached stream channels during the flood of March 1938 and the degree to which this runoff was concentrated with respect to time were governed mainly by the rates of rainfall, absorption, and retention during the storm period and by the amount of water in the ground at the beginning of the storm period. The antecedent rainfali, to the extent that it was fliling the natural surface and subsurface storage at the beginning of the storm, had an important effect on the runoff and on the maximum rates of discharge, as continuing rains not accompanied by a corresponaing draining out of the infiltrated rater may result in a condition approaching saturation. Any decrease in the ablilty of the ground to absorb the storm rainfall, especially during periods of heavy precipitation, would tend to increase the flood runoff.

Practically all the major floods of record in southern California have been preceded by about 8 inches of antecedent rainfall within a period of a few weeks, and the floods of March 1938 were no exception.32 In February 1938, as shown in table 8 on page 82, there had been coplous rainfa1l, in excess of normal, throughout southern California, and as a result a part of the absorptive capacity of the drainage basins was ut1$11 z e d$ when the storm period began.

\section{Antecedent precipitation and retention}

The retention in arainage area is the difference between the rainfall and the runoff (Including water losses), accumulated from an appropriately selected time of beginning. It may serve as an index of the state of the ground-storage capacity in the area immediately before a storm and hence of the effect of antecedent precipitation on direct runoff from the storm. To 1llustrate this point a study of retention has boen made In Strawberry and Santa Anita Creek Basins for the rainy seascas of 1925-26, 1926-27, and 1937-38. Strawberry Creek, with arainage area of 8.6 square miles above the gaging station, is in the San Bernardino Mountains, and Santa Anita Creek,with a drainage area of 10.5 square miles, is in the San Gabriel Mountains.

In this study it is necessary to make a distinction between gross retention and net retention. The gross retention in a drainage area is the difference between the rainfall and the runoff from a given time, as

32 Lynch, H. B., The history of floods in Callfornia; Am. Geophys. Union Trans., 1939. 
for example, the beginning of the raing season, and exceeds in amount the actual water in ground storage in the drainage area by the amount removed by evapo-transpiration or other depletion. For Strawberry and Santa Anita Creek Basins, the Influence of ground-water flow to or from the drainage area at the point of measurement is assumed to be negligible. Moreover, the phreatic divide is assumed to conform to the divide of surface drainage.

In southern California the short mild winters with considerable molsture are more favorable to the growth of vegetation than the long hot, dry summers. Consequently, in this area, unlike mountain areas in many other States, there 18 considerable winter evaporation and transpiration. The average annual evapo-transpiration is estimated to range from 15 to 18 inches in the valley areas to 20 to 25 inches in the highest mountain areas. The type of vegetation and hence the transpiration would seem to bear a relation to the avallablo water supply.33

Although there has been some investigation of evapo-transpiration losses, the data are not sufficlent for satisfactory ovaluation of net retention. For thls reason and because of the possible influence of ground-water flow to or from the basin, any determination of net retention mast be subject to some speculation.

As a rough approximation for use in the present 1llustration, it is assumed that, in the drainage areas considered, the rate of transplration is I Inch per month in winter, and, on the basis that transpiration is largoly a function of solar radiation, that this rate increases to 2 inches per month in summer. The rate of $108 \mathrm{~s}$ will be modified somewhat by the amount of water avallable at the surface, and therefore after a heavy storm it may exceod the estimated monthly rate. In this study the evaporation loss following a heavy stom has boen assumed to be 0.5 inch for each stom per1od. If the rainfall for the ontire storm period was $10 \mathrm{ss}$ than $0.5 \mathrm{inch}$, the entire amount is assumed to have boen lost. The avorage rainfall for Strawberry Croek Basin is based on the rocords at Squirrel Inn (altitude, 5,300 feet), which is within the arainage basin, and at San Bernardino (alt1tude, 1,048 feet), which is juet south of the mouth of the canyon of Strawberry Creek. The average rainfall for Santa Anita Creek Basin is based on the records at Mount Wilson (altitude, 5,850 feet) and at Santa Anita Canyon ranger station (altitude, 1,950 feet), both of which are within the arainage basin.

33 Sonderegger, A. L., Water supply from rainfall on valley floor, Am. Soc. C1v. Eng. Trans., vol. 94 p. 1263, 1930. 
Table 25 shows for Strawberry and Santa Antta Creek Basins the rainral1, the runoff, and the net retention, estimated as explained above, for periods preceding the for storms and during the major storm for the raing seasons of 1925-26, 1926-27, 1937-38. The rainfall for the major storm period as given in this table is the actual rainfall minus the estimated amount of water lost by evaporation during the storm. The flood runoff for these three years was the highest in about 20 years of streamflow records at these two gaging stations. The estimated net retention in each basin, although subject to insccurecy, as previously explained, is an Index of the soll molsture in that basin before and after the major storm periods.

Jable 25.- Net retention in Strawberry and Santa Anita Creek Basins for the rainy seasons 1925-26, 1926-27, and 1937-38

\begin{tabular}{|c|c|c|c|c|c|c|c|c|c|c|}
\hline \multirow[b]{2}{*}{$\begin{array}{l}\text { Basin, stream, } \\
\text { and season }\end{array}$} & \multicolumn{5}{|c|}{ Period preceding major storm } & \multicolumn{4}{|c|}{ Najor storm period } & \multirow{2}{*}{$\begin{array}{c}\text { Potal } \\
\text { net } \\
\text { reten- } \\
\text { tion }\end{array}$} \\
\hline & Date & $\begin{array}{l}\text { Rain- } \\
\text { fall }\end{array}$ & $\begin{array}{l}\text { Erapo- } \\
\text { loes } \\
\text { (a) }\end{array}$ & Run- & $\left\{\begin{array}{c}\text { Net } \\
\text { reten- } \\
\text { tion }\end{array}\right.$ & Date & $\begin{array}{c}\text { Rain- } \\
\text { fall } \\
(b)\end{array}$ & $\begin{array}{l}\text { Run- } \\
\text { off }\end{array}$ & \begin{tabular}{|c|} 
Net \\
reten- \\
tion
\end{tabular} & \\
\hline \multicolumn{11}{|l|}{$\begin{array}{l}\text { Santa Ane River } \\
\text { Strawberry Creek } \\
\text { near Arrowhead } \\
\text { Springs }\end{array}$} \\
\hline $\begin{array}{l}1925-26 \\
1926-27 \\
1937-38\end{array}$ & $\begin{array}{l}\text { Peo. } 1 \text { to } \\
\text { Har. } 30 \\
\text { Yov. 21 to } \\
\text { Feb. } 10 \\
\text { Deo. } 11 \text { to } \\
\text { Feb. } 25\end{array}$ & $\begin{array}{l}14.68 \\
11.09 \\
20.65\end{array}$ & $\begin{array}{l}7.73 \\
6.08 \\
6.71\end{array}$ & $\begin{array}{r}1.28 \\
.93 \\
2.10\end{array}$ & $\begin{array}{r}5.67 \\
4.08 \\
11.84\end{array}$ & $\begin{array}{l}\text { Apr. } 1 \text { to } \\
10 \\
\text { Feb. } 11 \text { to } \\
20 \\
\text { Feb. } 25 \text { to } \\
\text { Mar. } 3\end{array}$ & $\begin{array}{l}18.76 \\
15.62 \\
16.39\end{array}$ & $\begin{array}{l}1.97 \\
2.83 \\
5.24\end{array} \mid$ & $\begin{array}{l}16.79 \\
12.79 \\
11.15\end{array}$ & $\begin{array}{l}22.46 \\
26.87 \\
22.99\end{array}$ \\
\hline $\begin{array}{l}\text { Ios Anzeles Bize } \\
\text { Senta Anita Cree } \\
\text { near Sierra } \\
\text { Lisdre }\end{array}$ & & & & & & & & & & \\
\hline $\begin{array}{l}1925-26 \\
1926-27\end{array}$ & $\begin{array}{l}\text { Dec. } 1 \text { to } \\
\text { Har. } 30 \\
\text { Dec. } 25 \text { to }\end{array}$ & $\begin{array}{l}14.48 \\
13.07\end{array}$ & $\begin{array}{l}8.27 \\
6.06\end{array}$ & $\begin{array}{r}1.07 \\
.90\end{array}$ & 5.14 & $\begin{array}{l}\text { Apr. } 1 \text { to } \\
15 \\
\text { Feb. } 11 \text { to }\end{array}$ & $\begin{array}{l}16.23 \\
16.62\end{array} \mid$ & $\begin{array}{l}3.90 \\
4.60\end{array}$ & 12.33 & 17.47 \\
\hline $1937-38$ & $\begin{array}{l}\text { Feb } 10 \\
\text { Dec. } 5 \text { to } \\
\text { Feb. } 26\end{array}$ & 24.68 & 7.30 & 2.70 & 14.68 & $\begin{array}{l}\text { Mar. } 5 \\
\text { Feb. } 27 \text { to } \\
\text { Har. } 3\end{array}$ & 24.60 & 11.11 & 13.49 & 28.17 \\
\hline
\end{tabular}

a Estimateà evaporation from rainfall and transpiration.

$b$ Adjusted for estinated evaporation losses during the period.

The retention and therefore the minoff reeulting from a given storm depend fundamentally on the following factors:

(1) The retention in the basin from antecedent rainfall, which serves to occupy some of the avallable storage and thus to decrease the absorptive capacity of the soil.

(2) The amount and rate of storm rainfall. 
The data in table 25 indicate that in Strawberry Creek Basin the retention preceding each of the three storm periods listed was of about the same magnitude as in Santa Anita Creek Basin. In Strawberry Creok Basin, the rainfall during the 1926 and 1927 storm periods averaged about the same as for 1938, but the retention in this basin prior to the March 1938 storm was 11.84 inches, as compared with 5.67 and 4.08 inches during the earlier seasons, and as a result the proportion of the storm rainfall appearing as runoff was greater than for the two earlier storms.

In Santa Anita Creek Basin the retention preceding the storms of 1926 and 1927 was 5.14 and 6.11 inches, respectively. The rainfall during each of these two storm periods was about the same and averaged about 16.4 inches; the runoff averaged 4.3 inches, adding an incroment to the basin retention that averaged 12.2 inches, giving a total average retention of 17.8 inches. However, the net retention in the basin preceding the storm of March 1938 was 14.68 inches, which was about 9 inches more than the average for the earlier years. As a result of 24.6 inches of storm rainfall there was a runoff of 11.11 inches, adding an increment to the basin retention of 13.49 inches, or an average of only 1.3 inches more than resulted from the materially 11ghter storms of 1926 and 1927. The munoff that resulted from the storm of March 1938 was correspondingly greater than that from the 1926 and 1927 storms, and seemingly for two reasons--greater antecedent retention ani greater storm rainfall.

Figures 37 and 38 show the precipitation, munoff, gross retention, and net retention, estimated as indicated above, for Strawberry and Santa Anita Creek Basins, for the raing seasons of 1926-27. and 1937-38.

In the "New Year's storm of 1934," at Hoegee's camp, in the headwaters of Santa Anita Creek, 19.20 inches of rain fell in 2 days, but the maximum discharge at the gaging station was only 54 second-feet per square mile. This low runoff is explained by the relatively light antecedent precipitation of only 13.59 inches during the preceding 3 months, moh of which had already been lost by transpiration and evaporation, thus affording a relatively large retention capacity.

One of the outstanding floods of the perlod of record in southern California occurred in Jamary 1916.34 Analysis of that flood on the San Dlego River near Lakeside indicates the outstanding influence that retention within the basin has on subsequent flood manoff. Prior to Jamaary

34 MoGlashan, H. D., and Ebert, F. C., Southern California floods of Jamary 1916: Geol. Survey Water-Supply Paper 426, 1918. 


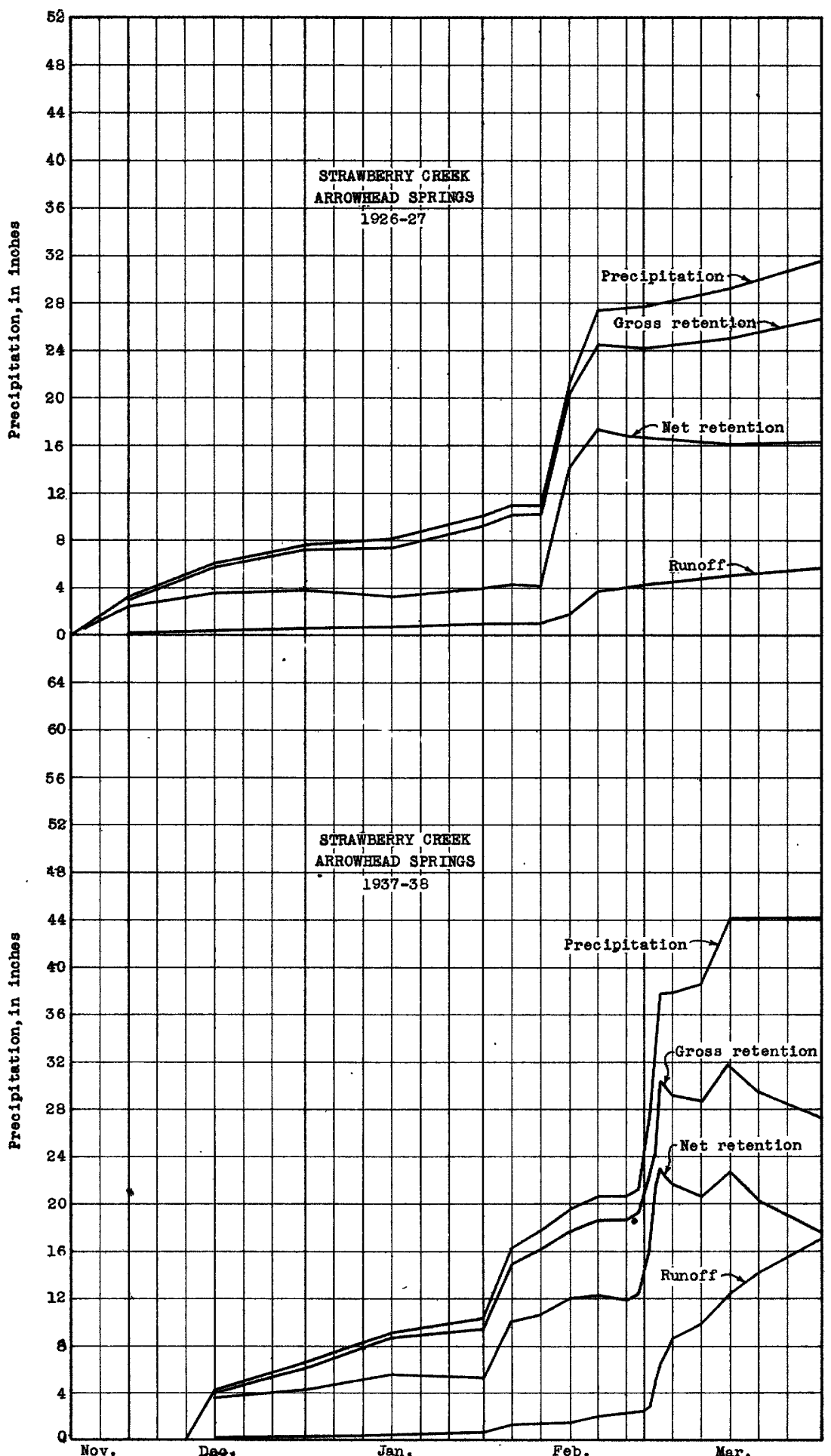

Figure 37.- Precipitation, runoff, and gross and net retention on Strawberry Creek during rainy sessons of 1926-27 and 1937-38. 


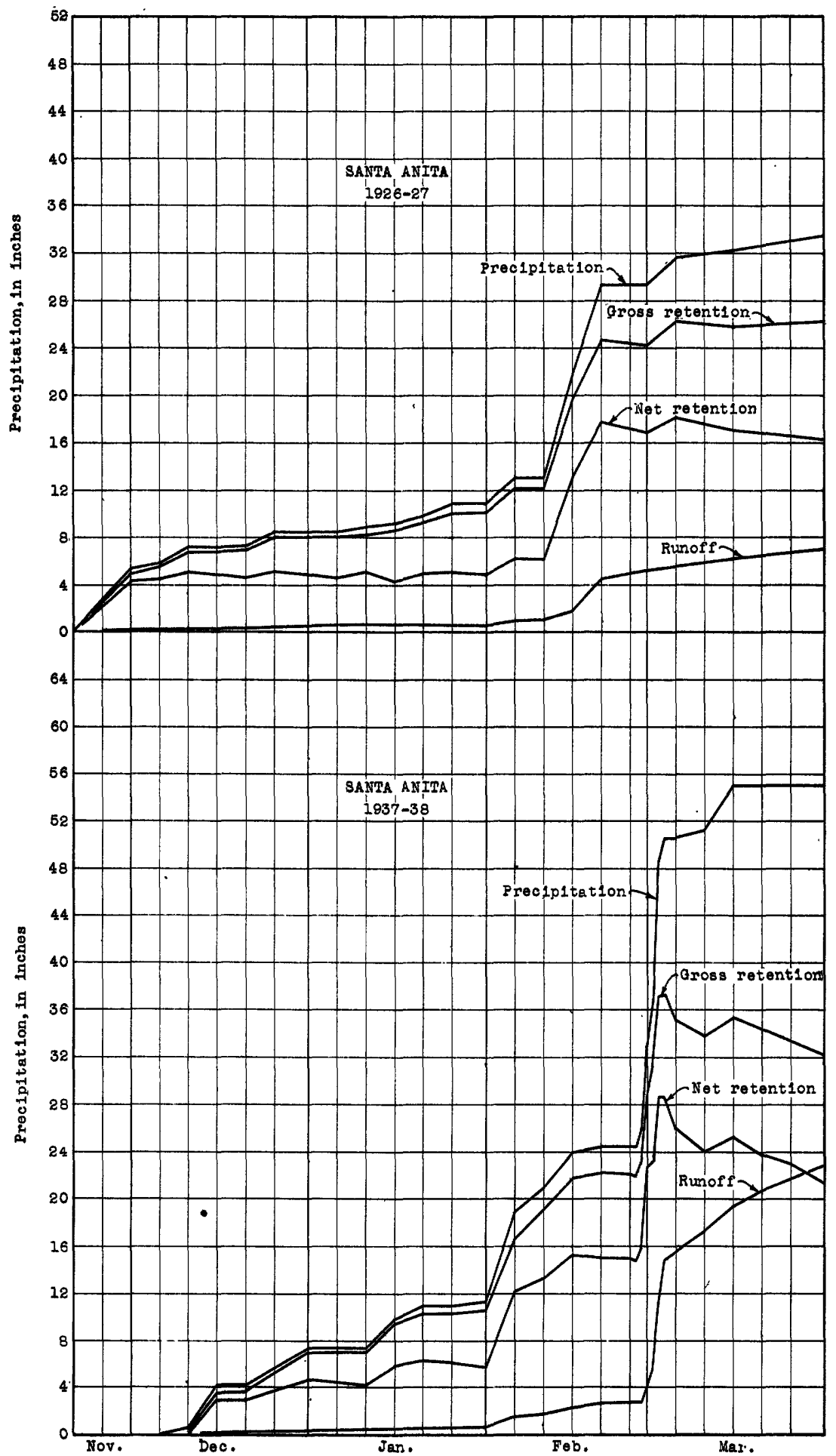

F1gure 38. - Prec1pitation, munoff, and gross and net retention on Santa An1ta Creek during rainy seasons of 1926-27 and 1937-38. 
13, 1916, the total preclpitation for the season had amounted to an average of 10.02 inches on the San Dlego River drainage basin, fully half of which is estimated to have evaporated or transpired by January 13. Between January 13 and 21 the prec1pitation was 16.33 inches, of which about 4.94 Inches ran off, leaving a gross retention from the beginning of the storm of 11.39 inches on January 23. Durlng a 7-day storm period beginning January 23, 11.27 inohes of rain fell, which was about 70 percent of the rainfall in the first storm. The runoff from this socond storm was about 100 percent, Indicating that the limlt of storage capacity in the ground had been practlcally reached durlng the flrst storm.

The rather large absorptive capac1ty shown for Strawberry and Santa Anite Creok Basins seoms to be typical of mach of the San Gabriel and San Bernardino mountaln areas. Additional ovidence of absorptive capacity can be seen by an inspection of the runoff records subsequent to the storm of March 1938. The subsequent period ut1lized for this study extended to September 30, 1938. The detalled records of stream flow subsequent to March 10 are given in Goolog1cal Survey Water-Supply Paper 861, covering stream-flow measurements in southern California for the year ending september 30, 1938. The runoff data for the period following Maroh 10 as used or cited in this disoussion were obtained from that source.

The runoff of San Antonio Crejek Basin near Claremont during the perlod March 4 to September 30, 1938, was 32.1 inches. During this period the rainfall was only about 7.5 inches, of whioh it 1s estimated about 1.5 Inches evaporated. The loss by transpiration during the period is estimated at about 11.5 inches. On the basis of these records and est1mates, and assuming also that the influence of ground-water flow to or from the basin at the point of measurement was negligible, there must have been a net retention of about 37.6 inches $(32.1+1.5+11.5-7.5)$ In San Antonio Creok Basin above the river-measurement station near Claremont on March 4. A calculation based on the same rainfall, evaporation, and transpiration, Indicates that Mill Creok Basin had about 28.2 inches in retention on March 4, and Lytle Creok Basin about 30.6 inches. If this analysis is oorreot, these three stream basins showed the ability to retain at least 28 to 37 inches of water in subsurface storage. If the estimates of evaporation following rains ( 1.5 lnohes) and of transpiration from the chaparral cover (11.5 inches) during the perlod March 4 to September 30, 1938, are too small, the estimate of retention must be Inoreased accordingly. In order to supply the observed stream flow during 
the period March 4 to September 30 and the estimated evapo-transpiration requirements for the same period, it is assumed that there must have been in storage prior to the March storm at lesst 20.9 inches (37.6 inches, total retention, minus 16.7 inches, retention from Narch storm) in San Antonio Creek Basin, 10.5 inches in Mill Creek Basin, and 13.5 inches in Lytie Creek Basin. To each of these amounts should be added the groundwater storage st111 retained on September 30, 1938, to obtain the actual net retention in each basin prior to the March 1938 storm.

Variations in the geologic structure of the besins of San Antonio, M111, and Lytle Creeks undoubtediy affect the absorption, storage, and flow of ground water, and perhaps the position of the phreatic divide in relation to the surface divide. Consequently, the results for these three streams may indicate a retention that is higher than would be generally representative of streams in the region.

For example, the drainage area of Lone Pine Creek is contiguous to that of Iytle Creek, referred to above. The retention for the storm period ending March 3, 1938, was 16.83 inches for the former and 17.09 inches for the latter. (See table 26) However, the runoff March 4 to September 30, following the storm, was only 3.1 inches for Lone Pine Creek as compared with 25.1 inches for Iytle Creek. The difference of 22 inches in munof indicates dissimflar geologic formations or other conditions that affect the rate at which ground-water storage is released to the streams in these basins.

\section{San Diego, San Dieguito, San Luls ReJ, and Santa Margarita River Basins}

As show on the isohyetal map, the storm of March 1938 was relatively 11ght in San Diego County and flood conditions were not severe. Table 26 lists for 10 stream-measurement stations in San Diego County the average rainfall for the period February 27 to March 3 ; the runoff in inches and acre-feet for the perlods February 27 to March 3 and March 4 to 10 and also for the maximum 24-hour period; and the difference between rainfall and runoff at midnight on March 3 and March 10. The Indicated retention relates only to the Warch flood period and does not include retention from precipitation prior to February 27. The periods used in table 26, as previousiy indicated in the text, show characteristic phases of the March flood. 
A. Iimited amount of regulation during the flood period was effected by Cuyamace and Fl Capitan Reservolrs on San Dlego Rlver and Lake Henshaw on San Luis Rey River. The records for all other streams in San Diego County 118 ted in table 26 represent conditions of natural flow.

The average rainfall by basins for the storm period of larch 1938 in San Diego County ranged from 8.41 to 15.10 inches, which is considerably less than was recorded during the storm of 1916.35 The rainfall on San Diogo River Basin upstream from the gaging station near Santee was 21.4 Inches during the storm perlod of January 1916, as compared with only 9.53 Inches during the storm of March 1938. Ilkewise the rain on San Iuis Rey River Basin upstream from Oceanside was 22.6 inches during the $1916 \mathrm{storm}$ and only 11.20 inches during the March 1938 storm.

The runoff for the different basins ranged from 0.59 to 2.91 inches during the period when 1t was heaviest, Pebruary 27 to March 3 . The least munoff was in San Diego River near Santee, possibly a result in part of regulation at El Capitan Reservolr, where 12,560 acre-feet, or 0.62 inch depth on the dralnage area above Santee, was placed in storage during the March flood perlod. The flood runoff of San Dlego River Basin for the March storm has been exceeded four times during the perlod of record, 1912-38, as follows: January 26-30, 1916 (at 01d Town Station), 5.16 Inches; February 14-18, 1927, 3.54 Inches; December 26-28, 1921, 2.00 Inches; and Apr11 6-9, 1926, 0.96 Inch.

Table 26 shows that the runoff during the maximum 24-hour period represented from 58 to 75 percent of the total storm runoff of February 27 to March 3, 1938, with the San Diego River Basin near Santeo showing the greatest concentration and Temecula Creek Basin at Ra1lroad Canyon, near Temecula, the least. High concentration of flood runoff with respect to time seems to be typical of semiarid areas. The peak discharge rate of Santa Ysabel Creek near Mesa Grande was 2.8 times that of the maximum 24-hour period, which is the highest ratio in the entire group. The lowest ratio between rate of peak discharge and maximum 24-hours was 1.4 on San Luis Rey River at Oceanside; this may have been due in part to regulation by Lake Henshaw.

35 Mcalashan, H. D., and Ebert, F. C. Southern California floods of January 1916, U. S. Geol. Survey Water-Supply Paper 426, 1918. 
Tablo 26.- Rainfall, runoff, and retention for seleoted s treams

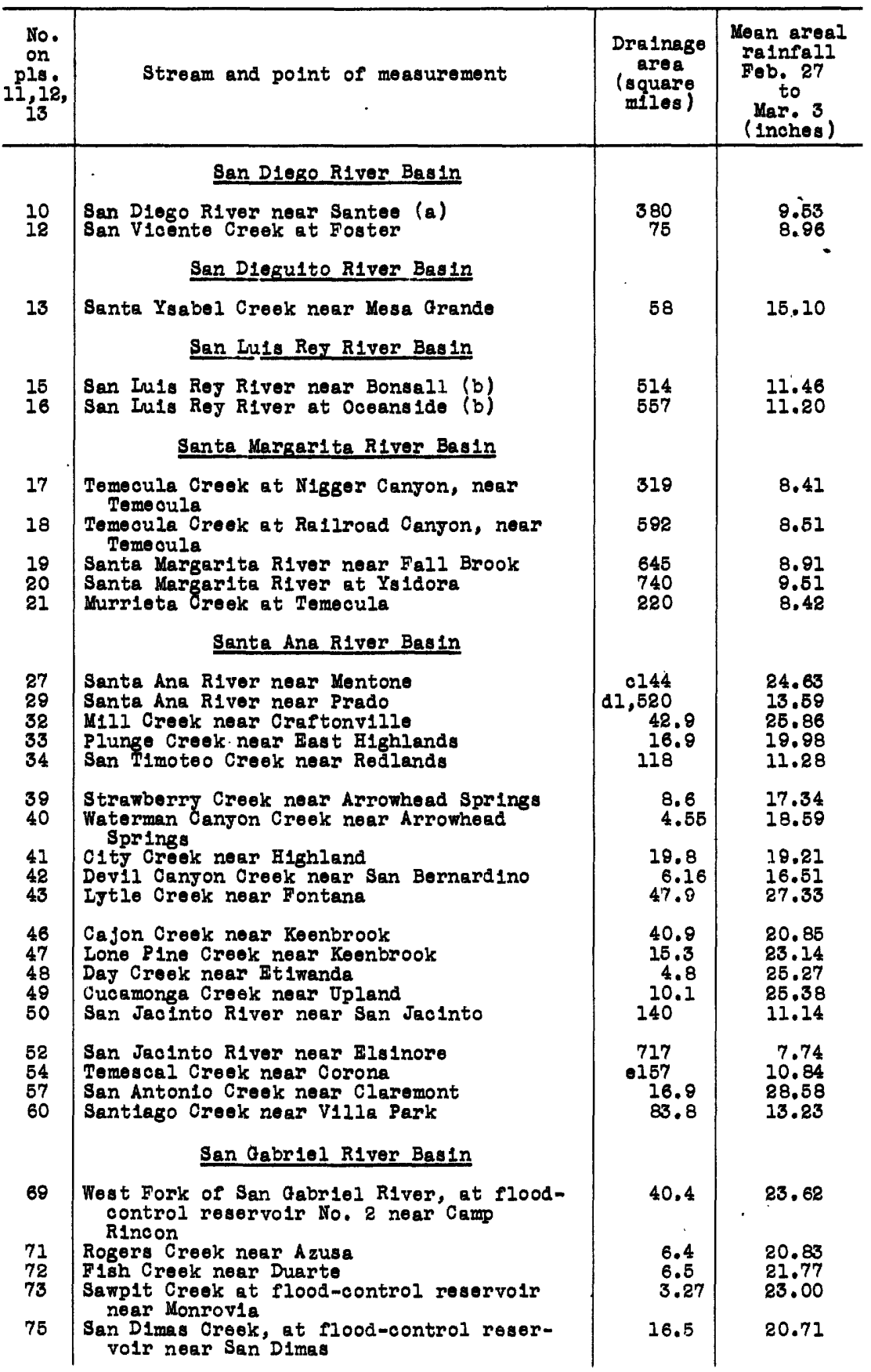

* Maximum da11j mean.

a Runoff not corrected for regulation by Cuyuma and El Capltan Reservolr.

b Runoff not corrected for regulation by Lake Henshaw. 
for the period February 27 to March 10, 1938 , in southern Callfornie

\begin{tabular}{|c|c|c|c|c|c|c|c|}
\hline \multicolumn{6}{|c|}{ Runoff } & \multirow{2}{*}{\multicolumn{2}{|c|}{$\begin{array}{c}\text { Retention } \\
\text { (1nches) }\end{array}$}} \\
\hline & Inche & & & 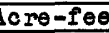 & & & \\
\hline $\begin{array}{c}\text { Feb. } 27 \\
\text { to } \\
\text { Mar. } 3\end{array}$ & Mar: & $\begin{array}{c}\text { Maximum } \\
24 \\
\text { hours }\end{array}$ & $\begin{array}{c}\text { Feb. } 27 \\
\text { to } \\
\text { Mar. } 3\end{array}$ & ${ }_{4-10}^{\text {Mar. }}$ & $\begin{array}{c}\text { Maximum } \\
24 \\
\text { hours }\end{array}$ & $\begin{array}{l}12 \text { p.m. } \\
\text { Mar. } 3\end{array}$ & $\begin{array}{l}12 \mathrm{p} \cdot \mathrm{m} . \\
\text { Mar. } 10\end{array}$ \\
\hline $\begin{array}{l}0.59 \\
1.88\end{array}$ & $\begin{array}{r}0.23 \\
.77\end{array}$ & $\begin{array}{l}0.44 \\
1.25\end{array}$ & $\begin{array}{r}11,890 \\
7,520\end{array}$ & $\begin{array}{l}4,650 \\
3,060\end{array}$ & $\begin{array}{l}8,830 \\
5,020\end{array}$ & $\begin{array}{l}8.04 \\
7.08\end{array}$ & $\begin{array}{l}8.71 \\
6.31\end{array}$ \\
\hline 2.91 & 1.26 & 1.77 & 9,030 & 3,890 & 5,490 & 12.19 & 10.93 \\
\hline $\begin{array}{l}1.21 \\
1.10\end{array}$ & $\begin{array}{l}.44 \\
.51\end{array}$ & $\begin{array}{l}.79 \\
.77\end{array}$ & $\begin{array}{l}33,220 \\
32,850\end{array}$ & $\begin{array}{l}12,130 \\
15,260\end{array}$ & $\begin{array}{l}21,560 \\
22,910\end{array}$ & $\begin{array}{l}10.25 \\
10.10\end{array}$ & $\begin{array}{l}9.81 \\
9.58\end{array}$ \\
\hline .92 & .26 & .68 & 15,600 & 4,490 & 11,620 & 7.49 & 7.23 \\
\hline 1.43 & .25 & .83 & 45,020 & 7,890 & 26,320 & 7.08 & 6.83 \\
\hline $\begin{array}{l}1.56 \\
1.81 \\
2.14\end{array}$ & $\begin{array}{l}.89 \\
.43 \\
.14\end{array}$ & $\begin{array}{r}.86 \\
1.03 \\
1.48\end{array}$ & $\begin{array}{l}53,610 \\
71,300 \\
25,110\end{array}$ & $\begin{array}{r}30,550 \\
17,020 \\
1,590\end{array}$ & $\begin{array}{l}33,220 \\
40,780 \\
17,320\end{array}$ & $\begin{array}{l}7.35 \\
7.70 \\
6.28\end{array}$ & $\begin{array}{l}6.46 \\
7.27 \\
6.14\end{array}$ \\
\hline $\begin{array}{r}6.56 \\
1.06 \\
8.17 \\
6.70 \\
.77\end{array}$ & $\begin{array}{r}2.01 \\
.26 \\
3.94 \\
2.54 \\
.02\end{array}$ & $\begin{array}{r}5.41 \\
.82 \\
6.67 \\
4.74 \\
.65\end{array}$ & $\begin{array}{r}50,020 \\
85,630 \\
19,180 \\
6,040 \\
4,850\end{array}$ & $\begin{array}{r}14,310 \\
20,770 \\
9,240 \\
2,280 \\
128\end{array}$ & $\begin{array}{r}41,300 \\
66,510 \\
15,650 \\
4,270 \\
4,120\end{array}$ & $\begin{array}{l}18.07 \\
12.53 \\
17.69 \\
13.88 \\
10.51\end{array}$ & $\begin{array}{l}16.06 \\
12.27 \\
13.75 \\
10.74 \\
10.49\end{array}$ \\
\hline $\begin{array}{l}5.89 \\
6.05\end{array}$ & $\begin{array}{l}1.94 \\
2.39\end{array}$ & $\begin{array}{l}4.15 \\
4.45\end{array}$ & $\begin{array}{l}2,750 \\
1,470\end{array}$ & $\begin{array}{l}891 \\
578\end{array}$ & $\begin{array}{l}1,900 \\
1,080\end{array}$ & $\begin{array}{l}11 \cdot 35 \\
12.54\end{array}$ & $\begin{array}{r}9.41 \\
10.15\end{array}$ \\
\hline $\begin{array}{r}5.97 \\
5.65 \\
10.24\end{array}$ & $\begin{array}{l}1.93 \\
2.33 \\
3.76\end{array}$ & $\begin{array}{l}4.31 \\
4.17 \\
7.69\end{array}$ & $\begin{array}{r}6,280 \\
1,850 \\
26,190\end{array}$ & $\begin{array}{r}2,040 \\
762 \\
9,600\end{array}$ & $\begin{array}{r}4,540 \\
1,370 \\
19,660\end{array}$ & $\begin{array}{l}13.24 \\
10.86 \\
17.09\end{array}$ & $\begin{array}{r}11.31 \\
8.53 \\
13.33\end{array}$ \\
\hline $\begin{array}{r}6.40 \\
6.31 \\
13.43 \\
12.14 \\
2.51\end{array}$ & $\begin{array}{l}1.21 \\
.96 \\
3.63 \\
3.18 \\
1.26\end{array}$ & $\begin{array}{r}4.26 \\
4.59 \\
9.51 \\
8.91 \\
71.36\end{array}$ & $\begin{array}{r}13,860 \\
5,150 \\
3,440 \\
6,550 \\
18,700\end{array}$ & $\begin{array}{r}2,650 \\
781 \\
928 \\
1,710 \\
9,360\end{array}$ & $\begin{array}{r}9,300 \\
3,750 \\
2,430 \\
4,790 \\
* 10,120\end{array}$ & $\begin{array}{r}14.45 \\
16.85 \\
11.84 \\
13.26 \\
8.63\end{array}$ & $\begin{array}{r}13.24 \\
15.87 \\
8.21 \\
10.08 \\
7.37\end{array}$ \\
\hline $\begin{array}{r}.14 \\
1.07 \\
11.84 \\
2.68\end{array}$ & $\begin{array}{r}.43 \\
.21 \\
3.96 \\
1.25\end{array}$ & $\begin{array}{r}.14 \\
.88 \\
10.14 \\
1.80\end{array}$ & $\begin{array}{r}5,410 \\
12,080 \\
10,680 \\
11,980\end{array}$ & $\begin{array}{r}16,340 \\
2,340 \\
3,570 \\
5,590\end{array}$ & $\begin{array}{r}5,180 \\
10,000 \\
9,140 \\
8,050\end{array}$ & $\begin{array}{r}7.60 \\
9.77 \\
16.74 \\
10.55\end{array}$ & $\begin{array}{r}7.17 \\
9.56 \\
12.78 \\
9.30\end{array}$ \\
\hline 12.06 & 3.01 & 8.26 & 25,860 & 6,470 & 17,790 & 11.56 & 8.55 \\
\hline $\begin{array}{l}8.10 \\
8.70 \\
7.20\end{array}$ & $\begin{array}{l}2.45 \\
2.82 \\
2.25\end{array}$ & $\begin{array}{l}4.42 \\
4.80 \\
4.57\end{array}$ & $\begin{array}{l}2,760 \\
3,020 \\
1,250\end{array}$ & $\begin{array}{l}835 \\
978 \\
391\end{array}$ & $\begin{array}{r}1,510 \\
1,660 \\
798\end{array}$ & $\begin{array}{l}12.73 \\
13.07 \\
15.80\end{array}$ & $\begin{array}{l}10.28 \\
10.25 \\
13.55\end{array}$ \\
\hline 5.69 & 1.55 & 3.84 & 5,010 & 1,370 & 3,469 & 15.02 & 13.47 \\
\hline
\end{tabular}

c Excluding Bear Valley.

d Excluding Bear Valley and San Jacinto River.

- Area below Blsinore Lake. 
Table 26.- Rainfall, muoff, and retention for selected streams in southern

\begin{tabular}{|c|c|c|c|}
\hline $\begin{array}{c}\text { No. } \\
\text { on } \\
\text { plis. } \\
11,12 \\
13\end{array}$ & Stream and point of measurement & $\begin{array}{l}\text { Drainage } \\
\text { area } \\
\text { (aquare } \\
\text { miles) }\end{array}$ & $\begin{array}{l}\text { Mean areal } \\
\text { ralnfali } \\
\text { Feb. } 27 \\
\text { to } \\
\text { Mar. } 3 \\
\text { (1nches) }\end{array}$ \\
\hline & San Gabriel River Basin--Continued & & \\
\hline $\begin{array}{l}81 \\
83 \\
85\end{array}$ & $\begin{array}{l}\text { Dalton Creek at flood-control reservolr } \\
\text { near Glendora } \\
\text { Little Dalton Creek near Glendora } \\
\text { Live Oak Creek at flood-control reser- }\end{array}$ & $\begin{array}{l}4.49 \\
3.3 \\
2.30\end{array}$ & $\begin{array}{l}17.85 \\
19.87 \\
17.41\end{array}$ \\
\hline $\begin{array}{l}86 \\
87\end{array}$ & $\begin{array}{l}\text { San Jose Creek near Whittier } \\
\text { Thompa on Creek at flood-control reser- } \\
\text { rolr near Claremont }\end{array}$ & $\begin{array}{l}85.2 \\
3.91\end{array}$ & $\begin{array}{l}10.74 \\
17.41\end{array}$ \\
\hline 89 & $\begin{array}{l}\text { Brea Creek at Fullerton } \\
\text { Los Angeles River Basin }\end{array}$ & 26.4 & 11.22 \\
\hline 97 & $\begin{array}{l}\text { Pacolma Creek at flood-control reservolr } \\
\text { near San Fernando }\end{array}$ & 27.8 & 18.90 \\
\hline 101 & $\begin{array}{l}\text { Tujunga Creok at flood-control reservolr } \\
\text { near Sunland }\end{array}$ & 81.4 & 18.10 \\
\hline $\begin{array}{l}105 \\
108 \\
111\end{array}$ & $\begin{array}{l}\text { Haines Creek near Tujunga } \\
\text { Arroyo Seco near Pesadena } \\
\text { Santa Anita Creek at flood control reser- } \\
\text { rolr near Slerra Uadre }\end{array}$ & $\begin{array}{l}1.2 \\
16.4 \\
10.8\end{array}$ & $\begin{array}{l}19 \cdot 38 \\
21 \cdot 86 \\
25.76\end{array}$ \\
\hline 113 & $\begin{array}{l}\text { Iltte Santa Anita Creok at flood-control } \\
\text { reservolr near Slerra Madro }\end{array}$ & 2.39 & 22.74 \\
\hline 115 & $\begin{array}{l}\text { Eaton Creok at flood-control reservolr } \\
\text { near Pasadena }\end{array}$ & 9.48 & 22.39 \\
\hline & Santa Clara River Basin & & \\
\hline $\begin{array}{l}141 \\
143 \\
144\end{array}$ & $\begin{array}{l}\text { Plmu Creek near Plru } \\
\text { Sespe Creek near Fillmore } \\
\text { Santa Paule Creek near Santa Paula } \\
\text { Ventura River Basin }\end{array}$ & $\begin{array}{l}432 \\
254 \\
39.8\end{array}$ & $\begin{array}{l}12.36 \\
14.96 \\
16.89\end{array}$ \\
\hline $\begin{array}{l}150 \\
151 \\
155\end{array}$ & $\begin{array}{l}\text { Matilija Croek at Matilija } \\
\text { Ventura River near Ventura } \\
\text { Coyote Creek noar Ventura } \\
\text { Santa Ynoz River Basin }\end{array}$ & $\begin{array}{r}56 \\
187 \\
41.1\end{array}$ & $\begin{array}{l}15 \cdot 44 \\
13 \cdot 32 \\
12 \cdot 78\end{array}$ \\
\hline 159 & $\begin{array}{l}\text { Santa Ynez River at Juncal Reservoir near } \\
\text { Montecito }\end{array}$ & 16.0 & 14.69 \\
\hline 161 & $\begin{array}{l}\text { Santa Ynez River bolow Gibraltar Dem, near } \\
\text { Santa Barbara }\end{array}$ & 219 & 12.62 \\
\hline $\begin{array}{l}162 \\
163\end{array}$ & $\begin{array}{c}\text { Santa Ynez River near Santa Ynez } \\
\text { Santa Ynez River near Lompoc } \\
\text { Santa Maria River Bas in }\end{array}$ & $\begin{array}{l}435 \\
790\end{array}$ & $\begin{array}{l}12.12 \\
10.08\end{array}$ \\
\hline $\begin{array}{l}164 \\
165\end{array}$ & $\begin{array}{l}\text { Cuyama River near Santa Maria } \\
\text { Huasna River near Santa Marla }\end{array}$ & $\begin{array}{l}902 \\
119\end{array}$ & $\begin{array}{l}5.31 \\
4.16 \\
\end{array}$ \\
\hline
\end{tabular}

* Maximum daily mean. 
the per1od February 27 to Harch 10, 1938, for California--Continued

\begin{tabular}{|c|c|c|c|c|c|c|c|}
\hline \multicolumn{6}{|c|}{ Runoff } & \multirow{2}{*}{\multicolumn{2}{|c|}{$\begin{array}{r}\text { Retention } \\
\text { (Inches) }\end{array}$}} \\
\hline & Inches & $>$ & & $e r \theta-f e \theta t$ & & & \\
\hline $\begin{array}{c}\mathrm{Feb}_{\text {to }} 27 \\
\text { to } \\
\text { far. } 3\end{array}$ & $\operatorname{Mar}_{4-10}$ & \begin{tabular}{|c|} 
Maximum \\
24 \\
hours
\end{tabular} & $\begin{array}{c}\text { Feb. } 27 \\
\text { to } \\
\text { Mar. } 3\end{array}$ & Mar. & $\begin{array}{c}\text { Maximum } \\
24 \\
\text { hours }\end{array}$ & $\begin{array}{l}12 \text { p.m. } \\
\text { Mar. } 3\end{array}$ & $\begin{array}{l}12 \text { p.m. } \\
\text { Mar. } 10\end{array}$ \\
\hline 5.93 & 2.08 & 3.83 & 1,420 & 496 & 917 & 11.92 & 9.84 \\
\hline $\begin{array}{l}6.11 \\
3.73\end{array}$ & $\begin{array}{r}1.94 \\
.69\end{array}$ & $\begin{array}{r}13.14 \\
2.50\end{array}$ & $\begin{array}{r}1,010 \\
457\end{array}$ & $\begin{array}{r}321 \\
85\end{array}$ & $\begin{array}{r}* 520 \\
307\end{array}$ & $\begin{array}{l}13.76 \\
13.68\end{array}$ & $\begin{array}{l}11.82 \\
12.99\end{array}$ \\
\hline $\begin{array}{l}2.62 \\
3.95\end{array}$ & $\begin{array}{l}.08 \\
.89\end{array}$ & $\begin{array}{r}* 1.93 \\
2.75\end{array}$ & $\begin{array}{r}11,940 \\
845\end{array}$ & $\begin{array}{l}341 \\
186\end{array}$ & $\begin{array}{r}* 8,790 \\
573\end{array}$ & $\begin{array}{r}8.12 \\
13.46\end{array}$ & $\begin{array}{r}8.04 \\
12.57\end{array}$ \\
\hline 1.66 & .01 & $\$ 1.33$ & 2,340 & 734 & $* 1,870$ & 9.56 & 9.55 \\
\hline 5.98 & 2.60 & 4.17 & 8,820 & 3,850 & 6,177 & 12.92 & 10.32 \\
\hline 7.21 & 1.36 & 5.73 & 31,290 & 5,820 & 24,865 & 10.89 & 9.54 \\
\hline $\begin{array}{r}3.52 \\
10.10 \\
11.06\end{array}$ & $\begin{array}{l}1.02 \\
2.69 \\
3.52\end{array}$ & $\begin{array}{l}2.48 \\
6.83 \\
6.84\end{array}$ & $\begin{array}{r}225 \\
8,820 \\
6,380\end{array}$ & $\begin{array}{r}65 \\
2,350 \\
2,020\end{array}$ & $\begin{array}{r}159 \\
5,970 \\
3,941\end{array}$ & $\begin{array}{l}15.86 \\
11.76 \\
14.70\end{array}$ & $\begin{array}{r}14.84 \\
9.07 \\
11.18\end{array}$ \\
\hline 6.94 & 2.97 & 3.87 & 888 & 377 & 493 & 15.80 & 12.83 \\
\hline 5.90 & 1.80 & 3.79 & 2,970 & 916 & 1,918 & 16.49 & 14.69 \\
\hline $\begin{array}{l}2.18 \\
4.02 \\
4.95\end{array}$ & $\begin{array}{r}.66 \\
2.48 \\
2.36\end{array}$ & $\begin{array}{l}1.16 \\
2.30 \\
2.89\end{array}$ & $\begin{array}{l}50,250 \\
54,670 \\
10,510\end{array}$ & $\begin{array}{r}15,140 \\
33,760 \\
5,010\end{array}$ & $\begin{array}{r}26,710 \\
31,220 \\
6,140\end{array}$ & $\begin{array}{l}10.17 \\
10.94 \\
10.94\end{array}$ & $\begin{array}{l}9.51 \\
8.46 \\
8.58\end{array}$ \\
\hline $\begin{array}{l}5.66 \\
5.78 \\
4.65\end{array}$ & $\begin{array}{l}4.22 \\
2.00 \\
1.16\end{array}$ & $\begin{array}{l}3.49 \\
3.90 \\
3.16\end{array}$ & $\begin{array}{l}16,620 \\
57,620 \\
10,200\end{array}$ & $\begin{array}{r}12,380 \\
19,920 \\
2,550\end{array}$ & $\begin{array}{r}10,240 \\
38,900 \\
6,930\end{array}$ & $\begin{array}{l}9.78 \\
7.54 \\
8.13\end{array}$ & $\begin{array}{l}5.56 \\
5.54 \\
6.97\end{array}$ \\
\hline 4.67 & 2.14 & 3.32 & - 3,980 & 1,820 & 2,830 & 10.02 & 7.88 \\
\hline 2.76 & 1.21 & 2.07 & 32,250 & 14,130 & 24,160 & 9.86 & 8.65 \\
\hline $\begin{array}{l}2.61 \\
1.71\end{array}$ & $\begin{array}{l}1.78 \\
1.23\end{array}$ & $\begin{array}{l}1.83 \\
1.22\end{array}$ & $\begin{array}{l}60,550 \\
72,040\end{array}$ & $\begin{array}{l}41,360 \\
51,670\end{array}$ & $\begin{array}{r}42,880 \\
.51,190\end{array}$ & $\begin{array}{l}9.51 \\
8.37\end{array}$ & $\begin{array}{l}7.73 \\
7.14\end{array}$ \\
\hline $\begin{array}{l}.24 \\
.58\end{array}$ & .19 & $\begin{array}{l}.22 \\
.28\end{array}$ & $\begin{array}{r}11,800 \\
3,700\end{array}$ & $\begin{array}{l}8,920 \\
5,640\end{array}$ & $\begin{array}{r}10,600 \\
1,800\end{array}$ & $\begin{array}{l}5.07 \\
3.58\end{array}$ & $\begin{array}{l}4.88 \\
2.69\end{array}$ \\
\hline
\end{tabular}


The basin in which the natural storage drains out most rapidiy is that of Santa Ysabol Creok. During the period March 4 to 10 the runoff in this basin was 1.26 inches and during the longer poriod March 4 to Soptomber 30 1t was 5.90 inches, an amount which with the transpiration $1088 \theta$ during the same per1od, must have exhausted the retention of 12.19 Inches left by the March storm. In the San Vincente Crook Basin, the munoff was 0.77 Inch for the period March 4-10 and 2.13 inches for the perlod March 4 to September 30, 1938; in th1s bas1n, also, It appears that the retention of 7.08 inches from the March storm was hardly onough to satisfy the demands of transpiration and the runoff during the period. Murrieta Creek Basin had the least runoff--0.14 inch March 4-10 and 0.50 inch March 4 to September 30. As in the other stream basins already discussed, it is doubtful if the retention of 6.28 inches on March 3 could have been more than oqual to the losses by transpiration and the runoff during the period of March 4 to september 30. It seems very probablo, therefore, that antecedent precipitation had an important but indeterminate effect on the munoff during this period.

\section{Santa Ana, San Gabriol, and Los Angelos River Basins}

The rainfall during the storm of March 1938 was heavter in the Santa Ana, San Gabriel, and Los Angeles River Basins than in any other parts of southern California. The amount of rainfall for these basins, together with the runoff for two characteristic phases of the storm period, February 27 to March 3 and March 4 to 10, and the differences between rainfall and runoff are shown in table 26.

The average storm rainfall on the drainage areas listed ranged from 7.74 inches on the valley floor to 28.58 inches at high altitudes in the mountains. In most of the area it was the heaviest rainfall experienced in many years.

The discharge for the maximum 24-hour period was apparently governed by the rates of rainfall, the rates of infiltration, and the amount of water already in groundwater storage. During the maximum 24-hour period, the records from the flood-control reservoirs in Los Angeles County showed an average runoff of 4.55 inches, which represents about 66 percent of the entire flow during the 5-day period February 27 to March 3 . of these reservoir stations, that of the West Fork of San Gabriel River recorded the greatest runoff for the maximum 24-hour period, a total of 8.26 inches, which was 68 percent of the flood runoff to and including March 3. 
The runoff from the dralnage area above the I1ve Oak Creek Reservolr was the least for the group--2.50 inches during the maximum 24-hour perlod, or 67 percent of the munoff for the part of the flood period ending March 3 , a percentage almost Identical with that of the West Fork of the San Gabriel R1ver. The discharge for the maximum 24-hour period on Tujunge Creok accounted for 80 percent of the flood munoff for the same perlod and was the greatest concentration of inflow reported for any of the reservolrs. Ilttio Santa Anita Creok showed the least concentration of munoff, that for the maximum 24-hour period belng equal to 56 percent of that for the 5-day storm period Fobmary 27 to March 3 . On the whole, most of the munoff records complled from the other reservolrs were much more consistent, the maximum 24-hour munoff generalis falling within 4 percent of the average, indicating unfformity of storm characteristics and other conditions in these particular areas.

In that part of the San Gabriel and San Bernardino Mountains drained by the Santa Ane River, the computed runoff for the maximum 24-hour perlod was 75 percent of the runoff for the 5-day storm period, a percentage conslderably higher then that indicated by the reservolr records. This greater concentration of munoff may have resulted from the heavier rainfall and the generaliy steeper slopes, especially in that part of the san Gabriel Mounta1ns tributary to the Santa Ana River.

The three main streams in the Santo Ane R1ver Basin draining the San Gabriel Mountains are San Antonio, Cucamonga, and Day Creeks, of these streams, San Antonio Creek had the greatest runoff during the maximum 24hour period as well as the greatest cancentration. During the maximum 24-hour perlod the runoff amounted to 86 percent of that for the 5-day period. Day Creek had a concentration during the maximum 24 hours of 71 percent, and Cucamonga Creek 73 percent.

The dralnage areas of Iytle, Cajon, and Lone Pine Creeke have many characterlstics in common, Including slope and altitude, and all three streams drain both the San Gabriel and San Bernardino Mountains. The runoff of Iytle Creek during the maximum 24-hour period was 74 percent of the munoff during the 5-day storm period Febmary 27 to March 3; that of Cajon Creek was 67 percent; and Lone PIne Creek, 73 percent. These rat1os are in fairly olose agreement with the average of the Santa Ana River stations In the San Gabriel and San Bernardino Mountains. 
The southern slopes of the San Bernardino Mountains are drained by the Santa Ana River and by Mil1, Plunge, City, Strawberry, Waterman CanJon, and Devil Canyon Creeks. The discharge of Santa Ana River and of Mill Creek for the maximum 24 hours indicated a munof equal to 82 percent of the storm runoff for the period February 28 to Karch 3, which represents the greatest concentration of runoff in this group. Strawberry Creek showed the least concentration--69 percent. The average concentration of runoff for these streams is greater than that shown by the floodcontrol reservoir records in the basins of San Gabriel and Los Angeles Rivers.

The San Jacinto River Basin above San Jacinto passesses many characteristics similar to those of the other tributaries of Santa Ana River. However, owing to a lighter rainfall in this area, there was no exceptionally high runoff during the storm of March 1938. The maximum daily runoff was 1.36 inches, or 54 percent of that for the 5-day storm period. The peak discharge during the March storm was 14,300 second-feet on March 2, as compared with 14,000 second-feet on February 6, 1937. A peak discharge of 30,000 second-feet near San Jacinto was recorded in January 1916, and one of 45,000 second-feet on Febmary 16, 1927, the latter caused, in part, by the failure of a dike at Hemet Reservoir.

The longest record of munof in the San Gabriel River Basin is that for the station on the main stem of the river near Azusa, which was estab11shed in 1894. The determination of the natural runoff at this station is now difficult owing to the regulation at the two flood-control reservoirs and at Morris Dam. The maximum discharge of the San Gabriel River at the Azusa station might have been as high as 100,000 second-feet during the flood of March 1938 had there been no regulation. The maximum previously recorded was 40,000 second-feet on January 18, 1916.

All the streams in the Los Angeles River Basin listed in table 26 drain the San Gabriel Mountains. The discharge for the maximum 24-hour period for stations on these streams shows a greater variation in degree of concentration than in any of the other areas in southern california. The two extremes for this basin, as already mentioned, are Tujunga and Little Santa Anita Creeks.

The storm munoff March 4 to 10 represents a draining out of ground water, wich in its characteristics of amount and rate. of movement would seem to be closely related to the characteristics of the geologic formations with respect to their capacity for storing water. The draining out 
was also dependent on the retention from the March storm and from antecedent precipitation, which mag have included some ground-water storage remining from the abnormally high rainfall during the season of 1936-37. During the period March 4 to 10, the average runoff, as based on the flood-control reservolr records, was 2.16 inches. Santa Anita Creok had the largest runoff--3.51 inches--and Iive Oak Creek the least--0.69 inch.

For the Santa Ana River near Mentone the runoff during the draining out period, March 4 to 10, was 2.01 inches. For the adjacent besin of M111 Creok during the same perlod the runoff was 3.94 inches, or nearly twice as great. For the per1od March 4 to September 30, 1938, the runoff for the Santa Ana River near Mentone was 13.27 inches, as compared with 22.61 inches for M111 creek. During the same period, the runoff from Lone Pine Creek was only 3.1 inches. As shown by table 26, the retention in these three besins was nearly the same at midnight March 3.

Strawbery Creek, which is typical of the short mountain tributaries in the San Bernardino Mountains, had a runoff of 1.94 inches for the 7day period March 4 to 10 and for the longer period March 4 to September $30,13.7$ inches.

In that part of the San Gabriel River Basin draining the San Gabriol Mountains, the West Fork of San Gabriel River showed the most rapid release of ground water, with 3.01 inches of munoff between March 4 and 10 . San Dimas Creek had the least runoff with 1.55 inches for the same period.

Because of storage losses and diversion during the summer, it was not practicable to compare the runoff from the basins of the controlled streams for the longer period Warch 4 to September 30. Fish Creek, in the San Gabrlel River Basin, which is not regulated, had a runoff during this period of 14.1 inches, which is comparable to the runoff for Straw-, berry Creek, in the Santa Ana River Basin.

In the Los Angeles River Basin-during the period March 4 to 10 Santa Antta Creek had the largest runoff, 3.51 inches, and Haines Creek had the least, 1.02 inches. The average runoff for the entire group in this basin as shown in table 26 was 2.28 inches. During the period March 4 to september 30, 1938, the runoff of Arroyo Seco was 11.8 inches, and that of Santa Anfta Creek was 14.3 inches. 


\section{Santa Clare and Ventura River Basins}

There was no regulation or diversion on any of the streams in the Senta Olare and Ventura River Basins for the perlod February 27 to March 10. The average runof at the stations on these streams was 4.54 1nohes for the perlod Fobruary 27 to Maroh 3, whioh is somewhat less than the average for the streams in the Santa Ana, San Gabriel, and Los Angeles River Basins, probably on account of the 21ghter ralnfall.

In the Santa Clare River Basin, the rat10 of the runoff for the maximum 24-hour perlod to the runoff for the perlod February 27 to March 3 was 53 percent on P1ru Orook, 57 percent on Sespe Orook, and 58 peroent on Santa Paula Orook.

In the ventura River Basin, the rat10 of runoff for the maximum 24 - hours to that for the perlod Fobruary 27 to Maroh 3 was 62 percent on Mat111ja Crook, 67 percent on Venture River, and 68 percent on coyote Oroek.

During the draining-out perlod Maroh 4 to 10, the average munoff from these s1X streams in the Sante Olara and Venture River Basins was 2.15 Inches, whioh approximates olosely the average of the reoords from the flood-control reservolrs in Los Angeles County. P1ru Oreok had the loast runoff, 0.66 inch during the perlod karch 4 to 10 and 2.5 inches during the per1od Maroh 4 to September 30. Sespe Oreek, an adjacent stream, had a. munoff of 2.48 Inches during the per1od March 4 to 10 and 7.8 inohes March 4 to Soptember 30 .

\section{Sente Ynez and Sente Marle R1ver Besins}

The Santa Ynez and Santa Mar1a River Bas1ns are situated on the extreme northern edge of the area affected by the storm of Karoh 1838. In the main, the charaoteristios of the rainfall, runofs, and retention in these two basins were very similar to those in the Santa clara and Ventura River Basins. The heavy rainfall in Fobruary had inereased the ground storage in about the same proportion as in some of the basins to the south.

The average runoff from stroams in these basins (seo table 26) for the maximum 24-hour perlod was 1.48 1nohes, wh10h is 71 percent of the total average runoff for the perlod Fobmary 27 to Maroh 3 . Santa Ynez River at Junoel Reservolr had the highest maximum 24-hour runoff in these. basins, 3.32 1nohes. This rate 1s only slightly less than that for San 
Dimas, Baton, Dalton, and I1ttle Santa Anita Creeks. Although those latter stations had a rainfall for the maximum 24-hour period of 9.5 to 11.3 1nches, 1t is doubtful if the rainfall on the Santa Inez River Basin above Juncal Reservo1r exceeded 8.0 inches for the same per1od. The other stat1ons on the Santa Ynez R1ver, and those on the Santa Marla River show considerably less runoff, owing principally to a much 11ghter rainfall.

The munoff of Santa Ynez River at Juncal Reservolr during the period March 4 to 10 was 2.14 inches and for the period March 4 to september 30 , 11.22 1nches.

Tho-rainfall in the Santa Maria River Basin was comparatively light and the flood was not especially severe.

\section{ARTIFICIAI STORAGE}

The detention and retardation of flood flows by storage reduces the peak stages of streams at critical times and, to the limit of the ava1lable storage capacity, is an effective method of reducing flood damage. In the mountalnous headrater areas of the Santa Ana, San Cabriel, and Los Angeles River Basins are a number of reservolrs used e1ther for water supply or flood control purposes.

The influence of reservolrs on the flood of March 1938 is shown by the data presented in table 27, many of which are taken from the report by Burke.36 The table gives the total storage capac1ty and the storage available at the beginning of the maximum 24-hour period of munoff, the runoff for the 3 days prior to the maximum 24-hour period, the runoff for the maximum 24-hour period, the peak inflow, the peak outflow, the inter: val between time of peak inflow and peak outflow, and other pertinent data.

The difficulty of operating the reservolrs for material reduction of flood peaks such as occurred on March 2, 1938, can be seen by comparing the capac1ty with the volume of inflow. The total capacity, at spillway level, of the reservolrs operated primarily for flood control in San Qabriel and Los Angeles River Basins was 105,000 acre-feet; the runoff during the 3 days prior to the maximum 24-hour period was about 41,400 acre-feet, and the runoff during the maximum 24-hour period was about 146,000 acre-fert.

36 Burke, H. F., Flood of Mar. 2, 1938, Los Angeles County Flood Control District, May 20, 1938. (Mimeographéd) 


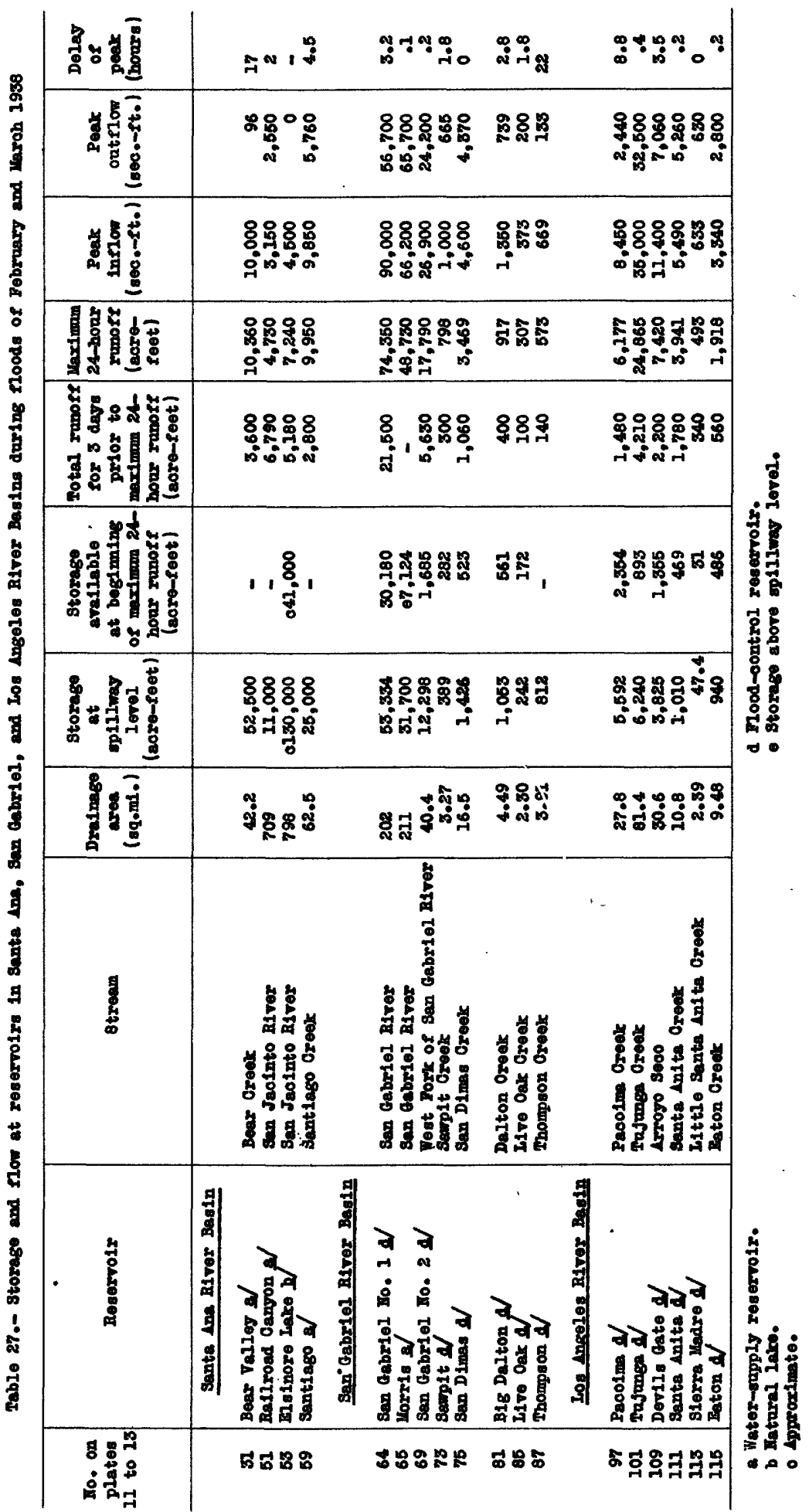


The runoff incident to the first phase of the stcrm utilized about half of the total available capacity, and in consequence only about a third of the runoff during the 24 hours of maximum discharge on Narch 2 could be retained. Although on some of the streams the effect on the maximum peak discharge may have been relatively small, the general effect was to reduce the total amount of water that accumulated in overflow areas below the reservoirs, and all the reservoirs acted as debris basins, effectively trapping large accumulations of silt that might otherwise have been deposited outside the mountain areas.

At several flood-control dams the silt and debris accumulated to an altitude above that of some of the outlets, which interfered with the regulating of the gates to achieve maximum flood-control efficiency.

Several reservolrs on smaller streams in the Santa Ana River Basin contributed substantially to the lowering of the flood peaks on those streams but had little effect on the peak in the Santa Ana River. Els1nore Lake, fed by the San Jacinto River, did not overflow. Overflow from this lake would pass down Temescal Creek to the Santa Ana River.

The San Gabriel River was effectively controlled by storage in San Gabrlel River Flood-control Reservolr No. 1, the peak discharge being reduced from 90,000 to 56,700 second-feet. No water was released from Puddingstone Reservolr, storage in which reduced the peak discharge on Walnut Creek. This reservoir also stored water diverted from San Dimas Creek, thereby reducing the flow in the lower reaches of that stream. other smaller flood-control reservolrs in the basin had little effect on the flood in the San Gabriel River.

In the Los Angeles River Basin there are a number of reservoirs, whose combined capacity, at spillway level, is about 54,000 acre-feet, of wich about 18,000 acre-feet is operated solely for flood control. The effect of these reservolrs on the flood peak in the Los Angeles River was not material.

Although reservolrs on the smaller streams did not appreciably reduce the peak discharge of the larger rivers, they did retain large amounts of silt and debris and thus materially reduced the damage to the highly developed areas adjoining the streams at lower altitudes. 


\section{DEBRIS}

\section{Interception of debris by reservolrs and debris basins}

As already noted, the streams that have their sources on the southern slopes of the San Gabriel and San Bernardino Mountains flow Into the San Fernando, San Gabriel, Chino, and upper Santa Ana Vallejs. As disclosed by well drilling in the central part of San Gabriel Valley, all these valleys are, in the main, underlain by alluvial deposits 1,000 to 2,000 feet deep. At the heads of the valleys are a ser1es of debris cones, some of enormous s1ze, all bearing witness to large debris movements in the past.

In earlier years the debris-laden streams meandered during flood perlods more or less at w1ll over the debris cones and valley floor, as explained in the subsequent chapter on history of floods. As the valley floor was populated, it became more and more necessary to confine the meandering streams to fixed channels. Unt1l recent jears the accommodation of the accumulating debris was not a serlous problem, as the debris cones still afforded areas for further deposition.

As settlement has increased, however, the tillable lands along the cones have been utilized for orchards, homes, and in some places for considerable urban development. Because of this use of the lands the control of the movement of debris on the cones has become an important economic problem. Streams that formerly meandered and dropped much of their bed load and silt content over the cones in a normal, natural manner now have to be confined within fixed channels in order to protect man's improvements. The practice of confining these streams to direct routes across the debris cones has necessarily resulted in the transportation to the valleys below of much of the debris that formerly was deposited on the cones. (See pl. 9.) In an attempt to alleviate this aituation, at least in part, debris or catchment basins have been excavated in the stream channels near the apexes of some of the cones. The capacity of these basins ranges from about 12.5 acre-feet to 98 acre-feet.

One of the debris basins, located on the Dunsmuir Creek debris cone, is 1llustrated on plate 19, which shows the inlet structure and the outlet structure with the overflow weir. Between these two structures 1s the excavated basin in the cone, which has debris storage capacity of about 80 acre-feet. The steepness of the cone is clearly shown in plate $19, \mathrm{~A}$, and a part of the urban development in the valley below can be seen in plate $19, \mathrm{~B}$. 
To maintain the effectiveness of these basins it is necessary to remove the accumilated debris after each heavy storm and to transport $1 t$ elsewhere. With the continued extension of improvements in the area, the disposal of the material trapped in the debris basins has become a serious problem. Plate 20 shows the Dunsmulr Creok debris basin 1 modiately following the March flood and before 1ts re-excavation had started.

The flood-control reservolrs that have been constructed for the storage and temporary retention of flood runoff also act as debris basins. Table 28 gives pertinent data, furnished by the Los Angeles County Flood Control District and the United States So11 Conservation Serv10e, relating to deposits of debris in the debris basins and flood-control reservolrs. For the flood-control reservolrs, the column "Debr1s deposited" Indicates the total volume of debrls per square m1le removed from each drainage area; but for the debris basins, because their capacity for catchment may have been exceeded, the volume shown in this column may not be the total volume of debris so removed. The volume of debris 11 sted in the.table includes not only the material actualiy trapped in the basin or reservolr, but also the depo'sits in the channel just upstream from the debris basin. The runoff in acre-feet per square mile for the maximum 24-hour perlod is given if avallable.

The loss in capacity of the reservolrs as shown in table 29 is for the perlod between the last previous survey and the flood of March 1938. It is belleved thet practically all of the movement of debris causing this loss in capac1ty occurred during the storm perlod of March 1938 and that most of the debris was deposited within the maximum 24-hour period of flood runoff.

The data in table 28, ehown graphically in figure 39, Indicate a relation between the average slope of a drainage basin and the acoumulation of debris. The slope of these dreinage basins is extraordinarily steop, the average increase in altitude within a horizontal alstance of 100 feet ranging from 36 to 76 feet. Detalled field studies made in connection with the flood on New Year's Day, 1934, In the La Cañada area 37 Indicate that the debris moved out of the canyon area during severe storns represents the gradual accumalation in channels during a period of jears of low runoff rather than the direct movement from the area as a whole during a single flood period.

37 Troxell, Harold C., and Peterson, John Q. Flood in La Cañada. Val1ey, Cal1f., January 1. 1934: U. S. Geol. Survey Water-Supply Paper $796-\mathrm{C}$, p. 93 , i 937 . 
Table 28.- Deposition of debris in reservoirs and debris basins of the Los Angeles County Flood Control District

\begin{tabular}{|c|c|c|c|c|}
\hline Reservolr or basin & $\begin{array}{c}\text { Drainage } \\
\text { area } \\
(\mathrm{sq} \cdot \mathrm{mt})\end{array}$ & $\begin{array}{l}\text { Average } \\
\text { slope of } \\
\text { arainage } \\
\text { area } \\
\text { (percent) }\end{array}$ & $\begin{array}{l}\text { Debris } \\
\text { deposited } \\
\text { (acre- } \\
\text { feet per } \\
\text { sq.mi.) }\end{array}$ & $\begin{array}{l}\text { Maximum } \\
24 \text {-hour } \\
\text { inflow } \\
\text { (acre- } \\
\text { feet per } \\
\text { sq. mi.) }\end{array}$ \\
\hline \multicolumn{5}{|l|}{ Flood-control reservolrs } \\
\hline $\begin{array}{l}\text { Big Dalton } \\
\text { Devils Gate (Arroyo Seco) } \\
\text { Eaton Creek } \\
\text { Live Oak Creek } \\
\text { Pacolma Creek }\end{array}$ & $\begin{array}{r}4.5 \\
30.6 \\
9.5 \\
2.3 \\
27.8\end{array}$ & $\begin{array}{l}40 \\
5 \overline{8} \\
36 \\
41\end{array}$ & $\begin{array}{r}19.6 \\
41.5 \\
50.9 \\
6.5 \\
21.4\end{array}$ & $\begin{array}{l}204 \\
242 \\
203 \\
134 \\
225\end{array}$ \\
\hline $\begin{array}{l}\text { San Dimas Creek } \\
\text { San Gabriel River No. I } \\
\text { San Gabriel River No. } 2 \text { (West Fork) } \\
\text { Santa Anlta Creek } \\
\text { Sierra Madre (IIttle Santa Anita } \\
\text { Creek) }\end{array}$ & $\begin{array}{l}16.5 \\
202 \\
40.4 \\
10.8 \\
2.4\end{array}$ & $\begin{array}{l}42 \\
49 \\
47 \\
47 \\
50\end{array}$ & $\begin{array}{l}17.3 \\
38.3 \\
39.2 \\
40.1 \\
16.7\end{array}$ & $\begin{array}{l}212 \\
368 \\
442 \\
360 \\
207\end{array}$ \\
\hline $\begin{array}{l}\text { Sawp1t Creek } \\
\text { Thompson Creek } \\
\text { Tujunga Creek }\end{array}$ & $\begin{array}{r}3.3 \\
3.9 \\
81.4\end{array}$ & $\begin{array}{l}50 \\
36 \\
44\end{array}$ & $\begin{array}{r}24.5 \\
7.7 \\
19.7\end{array}$ & $\begin{array}{l}242 \\
147 \\
306\end{array}$ \\
\hline \multicolumn{5}{|l|}{ Debris basins } \\
\hline $\begin{array}{l}\text { Brand } \\
\text { Dunsmuir } \\
\text { Eagle-Goss } \\
\text { Falr Oaks } \\
\text { Fern }\end{array}$ & $\begin{array}{r}1.03 \\
.84 \\
.61 \\
.21 \\
.30\end{array}$ & $\begin{array}{l}62 \\
67 \\
65 \\
61 \\
62\end{array}$ & $\begin{array}{r}8.1 \\
43.4 \\
41.5 \\
35.3 \\
42.8\end{array}$ & 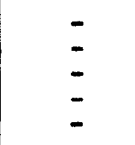 \\
\hline $\begin{array}{l}\text { Haines } \\
\text { Hall-Beckley } \\
\text { Hay } \\
\text { Las Flores } \\
\text { Lincoln }\end{array}$ & $\begin{array}{r}1.53 \\
.84 \\
.20 \\
.42 \\
.50\end{array}$ & $\begin{array}{l}71 \\
74 \\
56 \\
65 \\
51\end{array}$ & $\begin{array}{l}21.1 \\
68.8 \\
39.0 \\
55.2 \\
21.1\end{array}$ & $\begin{array}{l}- \\
- \\
-\end{array}$ \\
\hline $\begin{array}{l}\text { Hichols } \\
\text { Plckens } \\
\text { Shields } \\
\text { Snover } \\
\text { West Ravine }\end{array}$ & $\begin{array}{r}.94 \\
1.84 \\
.27 \\
.23 \\
.25\end{array}$ & $\begin{array}{l}54 \\
69 \\
76 \\
49 \\
69 \\
\end{array}$ & $\begin{array}{l}11.8 \\
50.8 \\
74.4 \\
45.2 \\
73.1 \\
\end{array}$ & $\begin{array}{l}- \\
\overline{-} \\
\end{array}$ \\
\hline
\end{tabular}

Runoff that might be sufficient to transport debris on slopes as steep as these canyon walls may lack the force to move it along slopes as flat as those found in the canyon floors. Thus material washed off the mountainsides may accumulate in stream beds until such time as the area is visited by a major flood, when most of the accumulation may be moved out on the debris cone or valley floor. This is, in a measure, indicated by the observations at the reservolrs. For example, San Dimas Creek floodcontrol reservolr, bullt in September 1922, has lost to date 22.8 percent of its cepacity, of which it is estimated 18.1 percent was lost during the flood of March 1938. For the 15-year perlod prior to this flood the capacity of the reservolr had been reduced by only about 4.7 percent, although during that time there were at least three severe storms. It seems 


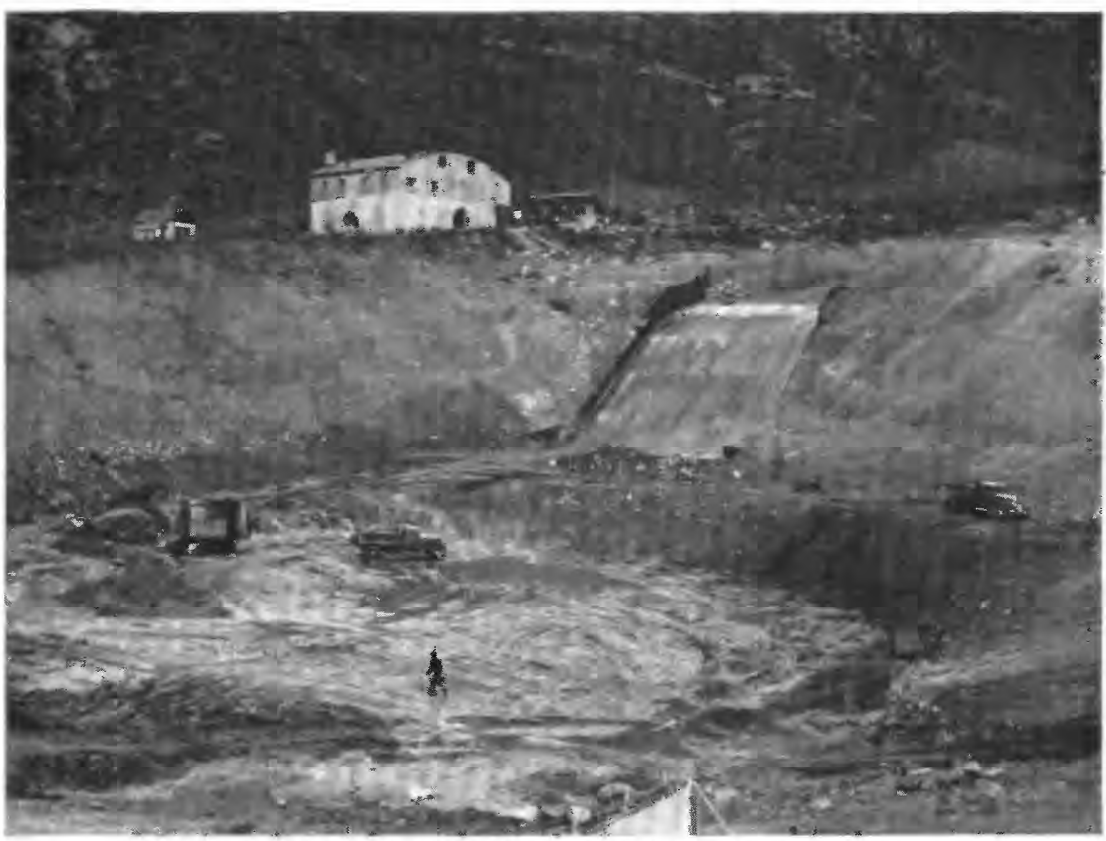

A. EXCAVATED BASIN AND INFLOW STRUCTCRE.

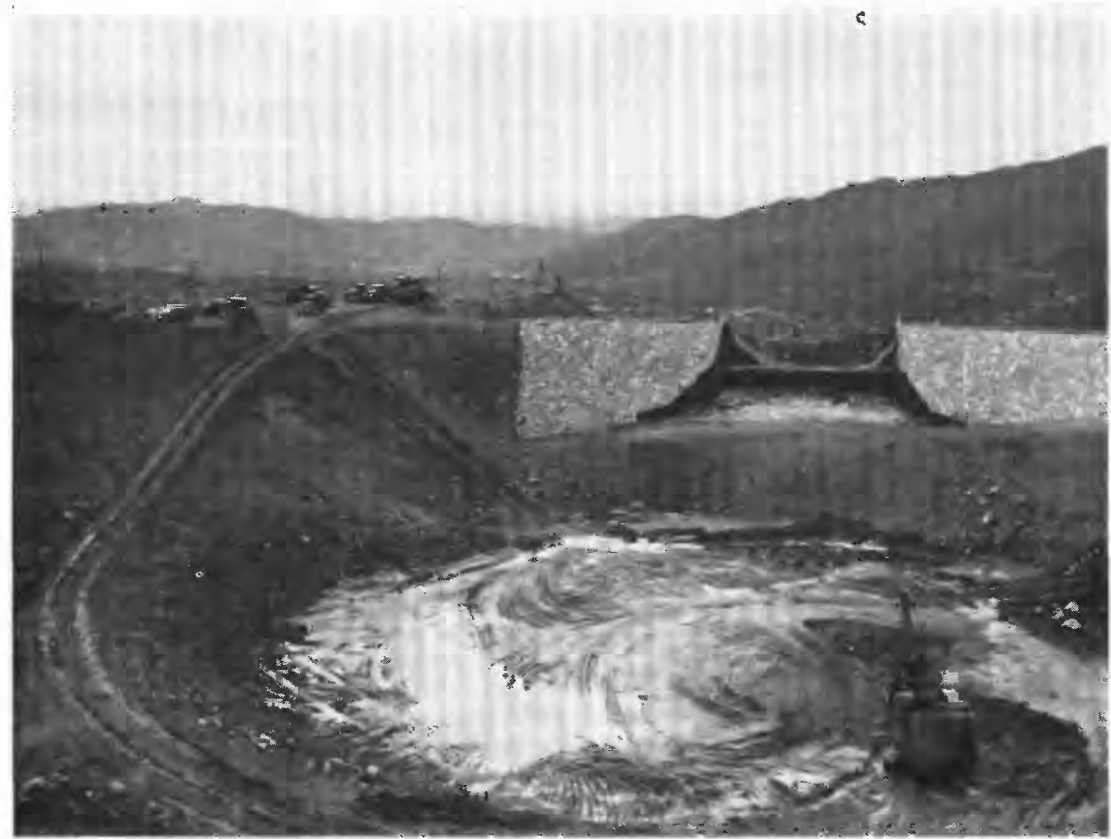

B. OUTFLOW STRUCTURE.

DUNSMUIR DEBRIS BASIN AFTER EXCAVATION OF DEBRIS.

Courtesy of Corps of Engineers, U. S. Army. 


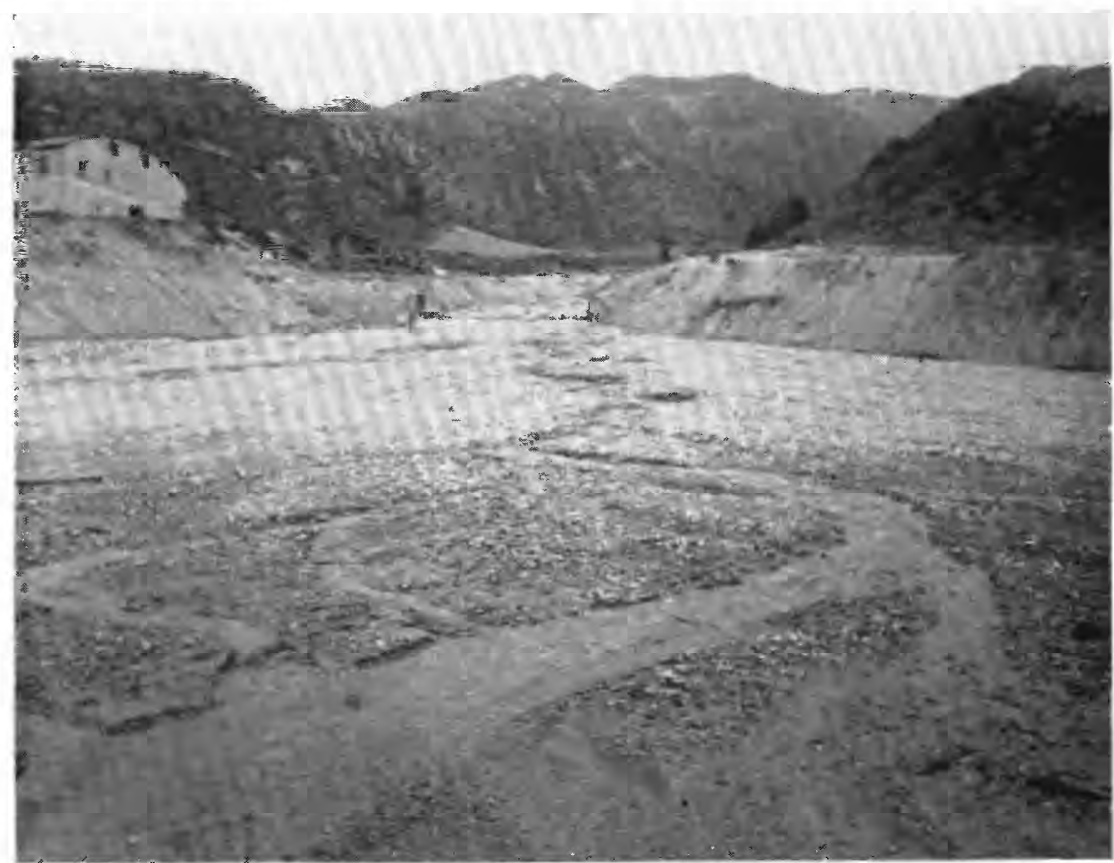

A. VIEW UPSTREAM SHOWING DEBRIS DEPOSITS UP TO ALTITUDE OF INFLOW STRUCTURES.

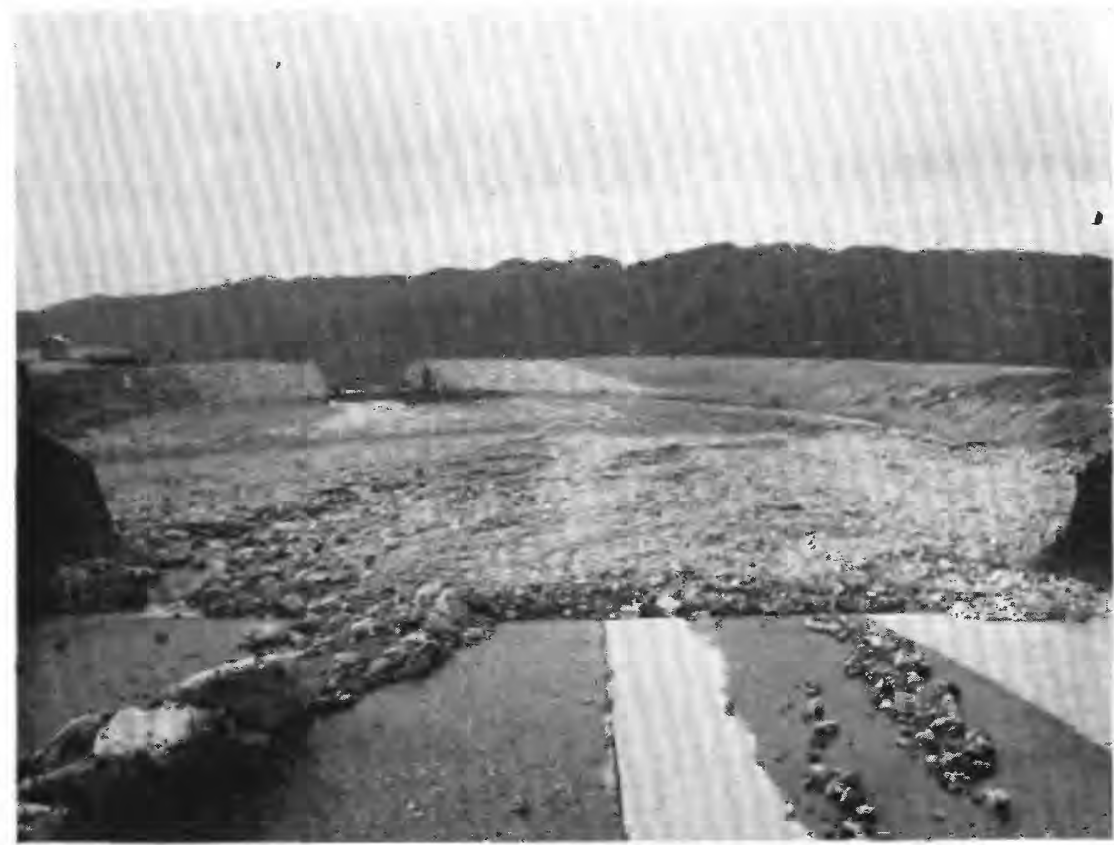

B. VIEW DOWNSTREAM.

DUNSMUIR DEBRIS BASIN FULL, OF DEBRIS, MARCH 1], 1938.

Courtesy of Corps of Engineers, U. S. Army. 
Table 29.- Loss in capacity of reservoirs of the Los Angeles County Flood Control District

\begin{tabular}{|c|c|c|c|c|c|c|c|c|}
\hline \multirow{2}{*}{ Reserroir or basin } & \multirow{2}{*}{$\begin{array}{c}\text { Alti- } \\
\text { tude } \\
\text { of } \\
\text { spill- } \\
\text { way* }\end{array}$} & \multirow{2}{*}{$\begin{array}{l}\text { Date } \\
\text { storage } \\
\text { began }\end{array}$} & \multirow{2}{*}{$\begin{array}{c}\text { Original } \\
\text { oapacity } \\
\text { aere- } \\
\text { feet }\end{array}$} & \multirow{2}{*}{$\begin{array}{l}\text { Date of } \\
\text { last } \\
\text { survey } \\
\text { prior to } \\
\text { lar.1938 }\end{array}$} & \multicolumn{2}{|c|}{$\begin{array}{l}\text { Capacity below } \\
\text { gpl11way level }\end{array}$} & \multicolumn{2}{|c|}{$\begin{array}{l}\text { Ioss in } \\
\text { capacity }\end{array}$} \\
\hline & & & & & $\mid \begin{array}{l}\text { Prior to } \\
\text { Mar.1938 }\end{array}$ & Apr. 1938 & $\begin{array}{l}\text { Aore- } \\
\text { foet }\end{array}$ & $P$ \\
\hline $\begin{array}{l}\text { Big Dalton } \\
\text { Derils Gate (Arroyo } \\
\text { Seco) }\end{array}$ & $\mid \begin{array}{l}1,706 \\
1,054\end{array}$ & $\begin{array}{l}\text { Ang. } 1929 \\
\text { June } 1920\end{array}$ & $\begin{array}{l}1,060 \\
4,563\end{array}$ & $\begin{array}{l}\text { Det.1.1937 } \\
\text { Dec.1936 }\end{array}$ & $\begin{array}{l}1,040 \\
3,825\end{array}$ & $\begin{array}{r}+974 \\
2,967\end{array}$ & $\begin{array}{r}66 \\
848\end{array}$ & $\begin{array}{r}6.2 \\
18.6\end{array}$ \\
\hline $\begin{array}{l}\text { Beton Creek } \\
\text { Ifre Oak Creek } \\
\text { Iacolms Creek }\end{array}$ & $\begin{array}{r}888 \\
1,497 \\
1,950\end{array}$ & $\begin{array}{l}\text { Feb. } 1937 \\
\text { Fov. } 1922 \\
\text { Feb. } 1929\end{array}$ & $\begin{array}{r}940 \\
250 \\
6,060\end{array}$ & $\begin{array}{l}\text { Kov.1937 } \\
\text { Kar.1936 } \\
\operatorname{Jan} .1936\end{array}$ & $\begin{array}{r}940 \\
242 \\
5,592\end{array}$ & $\begin{array}{r}698 \\
229 \\
5,004\end{array}$ & $\begin{array}{r}242 \\
13 \\
588\end{array}$ & $\begin{array}{r}25.7 \\
5.2 \\
9.7\end{array}$ \\
\hline $\begin{array}{l}\text { Sen Dimas Creele } \\
\text { Sen Gabriel River } \\
\text { No. } 1\end{array}$ & $\begin{array}{l}1,462 \\
1,453\end{array}$ & $\begin{array}{l}\text { Sept.1922 } \\
\text { Rov. 193? }\end{array}$ & $\begin{array}{r}1,496 \\
53,334\end{array}$ & $\begin{array}{l}\text { Oot.1937 } \\
\operatorname{Jan} \cdot 1938\end{array}$ & $\begin{array}{r}1,399 \\
53,384\end{array}$ & $\begin{array}{l}+1,188 \\
47,191\end{array}$ & 6,211 & $\begin{array}{l}14.1 \\
11.5\end{array}$ \\
\hline $\begin{array}{l}\text { San Gabriel River } \\
\text { To. } 2 \text { (West Fork) }\end{array}$ & 2,385 & Apr. 1935 & 12,320 & Oct.1935 & 12,298 & 10,787 & 1,511 & 12.3 \\
\hline $\begin{array}{l}\text { Santa Anita Creek } \\
\text { Sampit Creok }\end{array}$ & $\begin{array}{l}1,316 \\
1,360\end{array}$ & $\begin{array}{l}\text { Mar. } 1927 \\
\text { June } 1927\end{array}$ & $\begin{array}{r}1,043 \\
431\end{array}$ & $\begin{array}{l}\text { Feb.1936 } \\
\text { Oct.1935 }\end{array}$ & $\begin{array}{r}1,010 \\
389\end{array}$ & $\begin{array}{l}683 \\
320\end{array}$ & $\begin{array}{r}327 \\
69\end{array}$ & $\begin{array}{l}31.4 \\
16.0\end{array}$ \\
\hline $\begin{array}{l}\text { Sierra Nadre } \\
\text { (Little Santa } \\
\text { Anita Creak) }\end{array}$ & 1,172 & Feb. 1928 & 49.5 & Mar.1932 & 47.4 & 8.5 & 38.9 & 78.6 \\
\hline $\begin{array}{l}\text { Thompson Creak } \\
\text { Trujunga Creek }\end{array}$ & $\begin{array}{l}1,640 \\
2,290\end{array}$ & $\begin{array}{l}\text { Mar. } 1928 \\
\text { July } 1931\end{array}$ & $\begin{array}{r}812 \\
6,240\end{array}$ & $\begin{array}{l}\Delta p r .1932 \\
\text { Feb.1929 }\end{array}$ & $\begin{array}{r}812 \\
6,240\end{array}$ & $\begin{array}{r}786 \\
4,734\end{array}$ & $\begin{array}{r}26 \\
1,506\end{array}$ & $\begin{array}{r}3.2 \\
24.1\end{array}$ \\
\hline Total & & & $88,608.5$ & & $87,168.4$ & $75,579.5$ & $11,588.9$ & 13.1 \\
\hline
\end{tabular}

Iotee- Date furmished by U. S. Soll Conservation Service.

* Altitude in feet abore mean sea level.

** Percentage of original capacity lost since last survey.

+ October 1938 .

probable that the debris moved into the reservolr by the storm of March 1938 represents a part of the debris removed from the canyon walls and accumulated in the stream bed during a relatively long preceding period.

Santa Anita Creek flood-control reservoir has had a reduction of 34.5 percent in capacity since its construction in March 1927, of which about 31.4 percent occurred during the storm of March 1938. Thus during the 11 years preceding March 1938 the reduction was only 3.1 percent.

A large part of the mountain drainage area in the vicinity of La Cañada was burned over in the fall of 1933, and the fire was followed by the severe storm that caused the New Year's flood of 1934. Large amounts of debris that had accumulated during the preceding years of below-normal precipitation were transported out of the canyons during that flood. The basins in which flood-control reservoirs had been constructed were not within the area in which the 1934 flood was heaviest, and the movement of debris down the stream channels was not nearly so great as in the La Cañada area. Following the New Year's flood of 1934, many debris basins were constructed, most of them in the La Cañada area. This may explain the indication of figure 39 that for the storm of March 1938, in drainage basins of equal slope, the debris movement into the reservoirs, in acrefeet per square mile of drainage area, was considerably greater than the movement into the debris basins. 

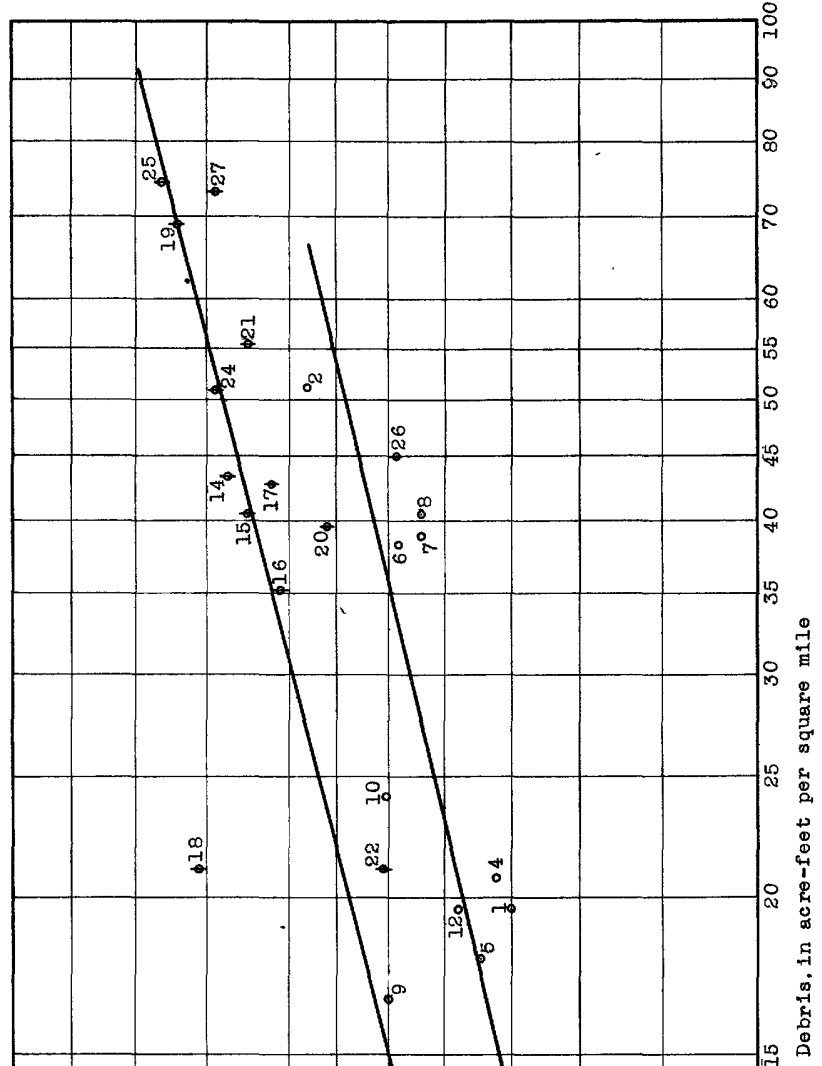

要

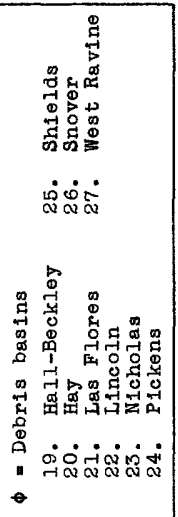

(1)

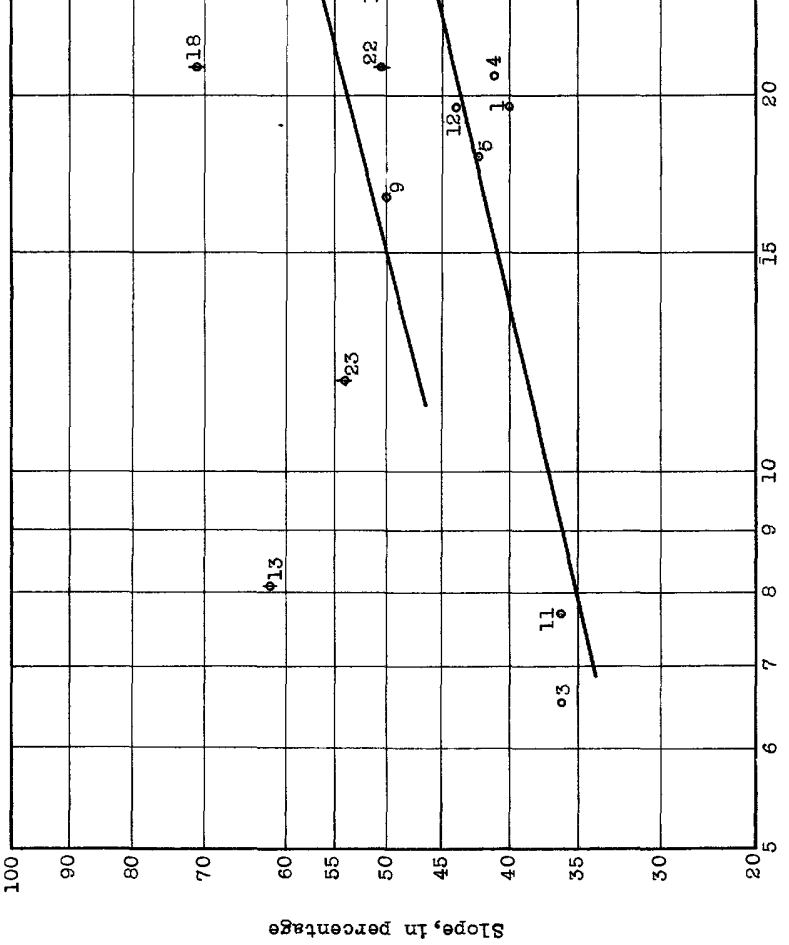

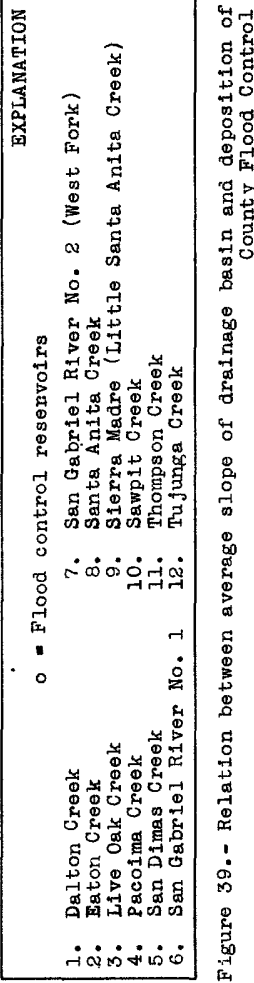




\section{Movement of debris in stream channels on the vailey floor}

The movement of debris in stream channels is not confined to the mountain areas but extends downstream to the flatter slopes, where the debris accumulates in the channels on the valley floor unt1l such time as the area is visited by a major storm. Figure 40 shows the change in altitude of the point of zero flow for the channel of the Santa Ana River near Prado during the period 1919-38. This change is an indicator of streambed scour and f111. The maximum discharge and the date of 1 ts occurrence are given for each flood that caused noticeable scour in the channel. The storm of February 1927 lowered the point of zero flow 3.5 feet and appears to have caused the greatest stream-bed scour during the period of record. Except for the scouring and flling, the channel characteristics at the gaging station have remained fairly constant throughout the period of record.

It will be noted that following each storm period there is a noticeable deposition in the stream channel, amounting in some years to almost 0.5 foot. During the comparatively ary period from July 1927 to December 1931 the channel appears to have been bullt up about 1.3 feet.

About three months before the storm of March 1938, in connection with . a study of certain hydraulic features of the Prado flood-control dam, the Corps of Engineers, United States Army, surveyed a serles of 22 cross sections in the channel of the Santa Ane River over a stretch of $4 \frac{1}{4} \mathrm{miles}$ below the site of the proposed dam. Immediately following the storm the sites of these sections were relocated and new cross sections were determined. The changes in the sections with respect to scour or fill are show in table 30 .

During February 1937 the Orange County Flood Control District surveyed a series of cross sections under each of the highway briages across the Santa Ana River in Orange County. Immediately after the flood of March 1938 these sections were redetermined. The indicated scour at each section is likewise shown in table 30 .

Only 2 of the 30 sections included in table 30 showed a fill in the channel during the flood period. At the other sections the scour was as much as 6.79 feet at the State Highway bridge at the mouth of the river. The average depth of scour for the entire group was about 1.47 feet over the entire width of the channel. 


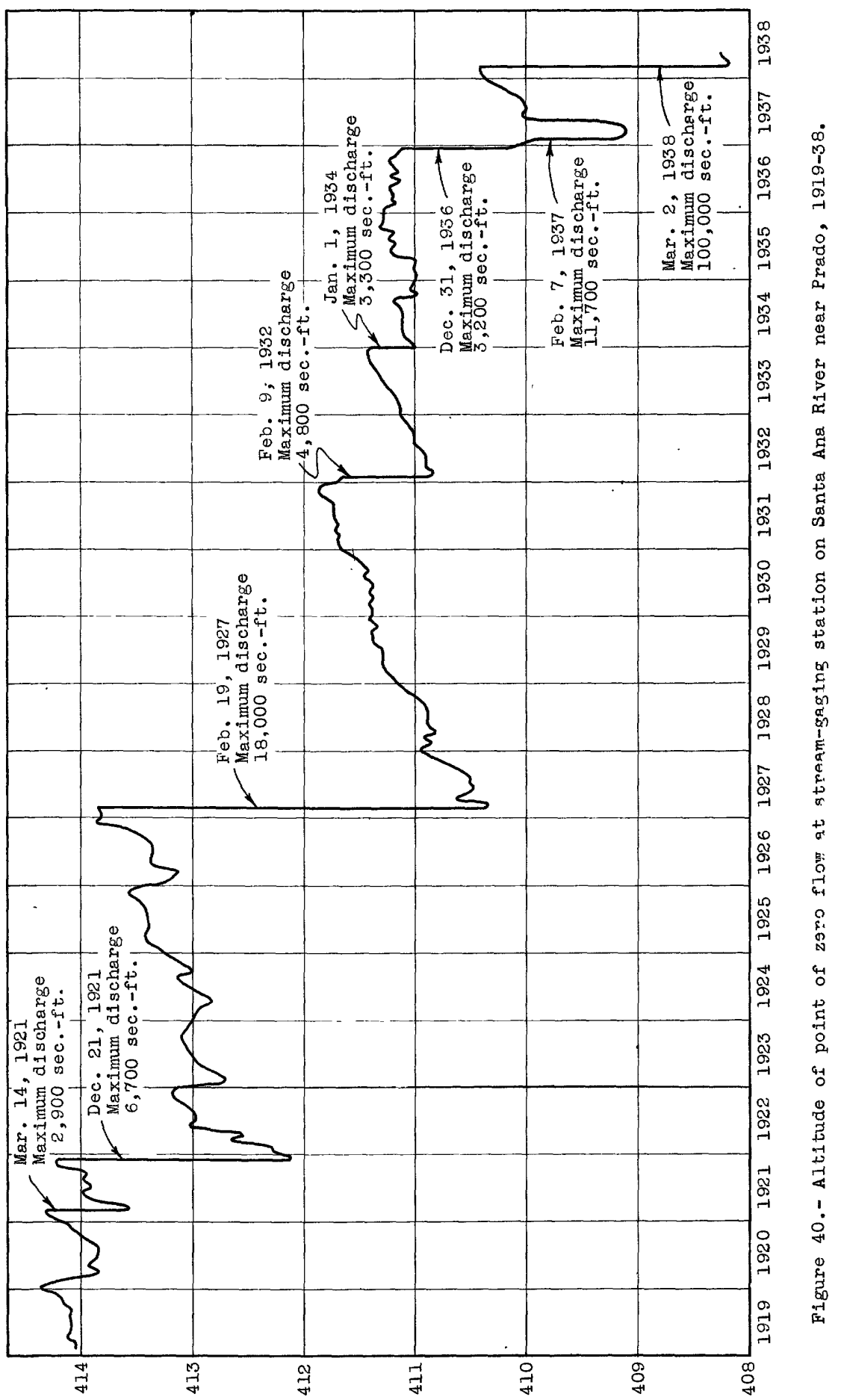


Table 30.- Scour and fill in channel of the Santa Ana River due to flood of March 1938

\begin{tabular}{|c|c|c|c|c|c|}
\hline \multirow{2}{*}{$\begin{array}{l}\text { Distance } \\
\text { above coast } \\
\text { highway } \\
\text { (miles) }\end{array}$} & \multirow{2}{*}{$\begin{array}{l}\text { Width } \\
\text { (feet) }\end{array}$} & \multicolumn{2}{|c|}{ Change in cross section } & \multicolumn{2}{|c|}{ Change in mean depth } \\
\hline & & $\begin{array}{c}\text { Scour } \\
\text { (square feet) }\end{array}$ & $\begin{array}{c}\text { F111 } \\
\text { (square feet) }\end{array}$ & $\begin{array}{l}\text { Scour } \\
\text { (feet) }\end{array}$ & $\begin{array}{l}\text { Fill } \\
\text { (feet) }\end{array}$ \\
\hline $\begin{array}{l}31.59 \\
31.50 \\
31.46 \\
31.43 \\
31.41\end{array}$ & $\begin{array}{l}2,000 \\
1,850 \\
1,900 \\
2,000 \\
1,400\end{array}$ & $\begin{array}{l}- \\
100 \\
1,220 \\
1,830 \\
1,430\end{array}$ & $\begin{array}{c}1,190 \\
= \\
= \\
=\end{array}$ & $\begin{array}{r}- \\
0.05 \\
.64 \\
.92 \\
1.02\end{array}$ & $\begin{array}{l}0.60 \\
= \\
= \\
=\end{array}$ \\
\hline $\begin{array}{l}31.37 \\
31.34 \\
31.30 \\
31.21 \\
30.99\end{array}$ & $\begin{array}{l}1,450 \\
1,250 \\
1,350 \\
1,300 \\
1,300\end{array}$ & $\begin{array}{r}1,820 \\
1,630 \\
1,250 \\
590 \\
2,570\end{array}$ & $\begin{array}{l}- \\
\overline{-} \\
-\end{array}$ & $\begin{array}{r}1.26 \\
1.30 \\
.93 \\
.45 \\
1.98\end{array}$ & $\begin{array}{l}- \\
- \\
-\end{array}$ \\
\hline $\begin{array}{l}30.80 \\
30.61 \\
30.41 \\
30.19 \\
29.91\end{array}$ & $\begin{array}{l}1,600 \\
2,000 \\
1,750 \\
1,250 \\
1,800\end{array}$ & $\begin{array}{r}500 \\
780 \\
2,560 \\
4,000 \\
3,860\end{array}$ & $\begin{array}{l}- \\
- \\
- \\
-\end{array}$ & $\begin{array}{r}.31 \\
.39 \\
1.46 \\
3.20 \\
2.14\end{array}$ & $\begin{array}{l}- \\
- \\
- \\
-\end{array}$ \\
\hline $\begin{array}{l}29.68 \\
29.38 \\
29.07 \\
28.84 \quad \text { (a) } \\
28.40\end{array}$ & $\begin{array}{r}1,750 \\
3,250 \\
1,700 \\
750 \\
2,000\end{array}$ & $\begin{array}{c}4,350 \\
2,110 \\
2,250 \\
1,330 \\
=\end{array}$ & $\begin{array}{c}- \\
- \\
\overline{7,340}\end{array}$ & $\begin{array}{r}2.49 \\
.65 \\
1.32 \\
1.77 \\
-\end{array}$ & $\begin{array}{l}\overline{-} \\
\bar{z} \\
2.67\end{array}$ \\
\hline $\begin{array}{l}27.93 \\
27.36 \\
20.43 \text { (b) } \\
17.51 \text { (c) } \\
17.06 \text { (d) }\end{array}$ & $\begin{array}{c}1,500 \\
1,800 \\
1,000 \\
- \\
942\end{array}$ & $\begin{array}{r}2,010 \\
8,500 \\
1,150 \\
274 \\
1,726\end{array}$ & $\begin{array}{l}- \\
\overline{-} \\
\overline{-}\end{array}$ & $\begin{array}{l}1.34 \\
4.72 \\
1.15 \\
- \\
1.83\end{array}$ & $\begin{array}{l}- \\
- \\
-\end{array}$ \\
\hline $\begin{array}{rr}15.45 & (\mathrm{e}) \\
13.70 & (\mathrm{f}) \\
8.83 & (\mathrm{~g}) \\
5.28 & (\mathrm{~h}) \\
0 & \text { (i) }\end{array}$ & $\begin{array}{r}1,000 \\
1,277 \\
346 \\
291 \\
520\end{array}$ & $\begin{array}{r}1,824 \\
594 \\
166 \\
611 \\
3,531\end{array}$ & $\begin{array}{l}- \\
- \\
- \\
-\end{array}$ & $\begin{array}{r}1.82 \\
.47 \\
.48 \\
2.10 \\
6.79\end{array}$ & $\begin{array}{l}- \\
- \\
- \\
-\end{array}$ \\
\hline
\end{tabular}

a River-measurement station near Prado.

b Yorba Bridge.

c Jefferson Street Bridge.

d A.T.\& S.F.Ry.Bridge.

e Anaheim-01ive Bridge.

$f$ Southern Pacific Co. Bridge.

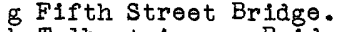

h Talbert Avenue Bridge.

1 Coast Highway Bridge.

There is a wide variation in the degree to which the sand deposits in the channel will scour during periods of flood runoff. Many bridge piers were undermined and many buried pipe lines across the stream channels were wrecked during the flood of March 1938.

Not all sections of the stream channels across the valley floor were subject to appreciable scour. In order to obtain additional data on scour, the Corps of Engineers, United States Army, on October 1, 1936 , placed three series of boulders in the deep sandy channel of the Los Angeles River between Firestone Boulevard and Stewart and Grey Road. 
The first series consisted of five boulders weighing about 400 pounds each, which were placed in line across the channel and burled a short distance below the surface. Two of these boulders have shown no change in position except for a slight settlement; one moved downstream about 40 feet during the relatively moderate storm of February 1937, but there was no movement in March 1938; another disappeared in February 1937; and only one appears to have moved during the flood of March, 1938.

Two hundred feet farther downstream was the second series, a row of six rocks each weighing 200 pounds and similarly placed. One of these was lost and two moved between 300 and 400 feet in February 1937; three disappeared and two showed no change in location during the flood of March 1938.

The third serles of six rocks, each weighing 100 pounds, was placed 200 feet downstream from the second serles. All the boulders in this serles disappeared during the storm of February 1937.

The results of this experiment seem to indicate that the storm of February 1937 had a greater scouring effect at this particular location than the considerably more severe storm of March 1938.

Much debris set in motion by the runoff from the storm of March 1938 was deposited in the ocean at the mouths of the streams. The report by the Corps of Engineers 38 indicates that at the mouth of the Los Angeles River a deposit of $8,120,000$ cublc yards of debris formed above an altitude of 30 feet bélow sea level and within a strip 3,000 feet wide, bounded by the breakwater on the west and extending approximately $1 \mathrm{mile}$ seaward. If the estimated quantity of f1ll beyond the limit of surveys is Included, the total deposit was approximately 11,000,000 cublc yards. This represents the volume of debris deposited during a period of more than 10 years, as the last previous survey was made in 1927, by the c1ty of Long Beach. Durlng that perlod tidal currents undoubtedly had an effect on the deposition of debris. From a study of aerial photographs and a few soundings made between 1927 and 1938, it is estimated that 60 percent of the total, or $6,600,000$ cubic jards of debris, was deposited by the flood of March 1938 in the immediate area. Further uncertainty as to the total volume carrled by the river is caused by the possibility that some part of the debris may have been deposited outside this immediate area.

38 Corps of Englneers, U. S. Army, Report on engineering aspects, flood of March 1938, p. 37, 1938. 
Similar data collected at the mouth of the San Gabriel River show a deposit of 273,000 cubic yards of debris above an altitude of 15 feet below sea level within a strip 1,600 feet wide extenaing directly seaward for 2,200 feet from the mouth of the river. The volume at the mouth of the San Gabriel River has undoubtedly been more accurately determined than that at the mouth of the Los Angeles River. It is based upon a survey made in september 1937. The absence of a breakwater here reduced the probability of excessive tidal effects, although tidal deposits and scour remain undetermined factors. As before, no estimate can be made of the volume of debris deposited outside the area surveyed.

\section{FLOOD DAMAGE}

Southerm California sustained damages amounting to $\$ 78,602,000$ from the storm of February 27 to March 4, 1938, according to the report compiled by the Corps of Engineers, United States Army, Los Angeles district; of this total, $\$ 27,4 i 3,000$ has been estimated as indirect damage and $\$ 51,189,000$ as direct damage. A large number of homes and business houses were either completely destroyed or badly damaged. Railroads 39 and highways suffered losses estimated at many millions of dollars, owing to. destruction of bridges and roadbeds. Other utilities, such as the telegraph, telephone, gas, and water systems, were severely crippled. For a time some localities were cut off from all lines of communication and others were served only by alrplane or radio.

The Inundated area in Los Angeles County included about 108,000 acres; In the Santa Ana River Basin in San Bernardino, Riverside, and Orange Counties, 182,300 acres; and in Ventura and Santa Clara River Basins, 6,200 acres. Plates 2l-26, from photographs made March 3 show flood damage along the Santa Ana River.

Maps of southern California showing areas damaged by the flood of March 1938 have been prepared by the Corps of Engineers, United States Army; by the United States Department of Agriculture; and by the Los Angeles County Flood Control District (Ios Angeles County only).

39 Kirkbride, W. H., A rallroad fights floods: Civil Engineering, vol. 9, No. 12, pp: 7il-7í, December 1939. 


\section{Loss of I1fe}

The total number of deaths caused by flood in southern California in 1938 was reported as 87 , of which 77 occurred in the Los Angeles and Sente Ana River Basins. Three months after the flood, 9 other persons were still reported as missing. The number of lives lost in each river basin is given in table 31 .

Table 31.- Loss of life in southern Callfornia due to storm of February 27 to March 3, 1938

$$
\text { Drainage basin Lives }
$$

San Juan and Aliso Creek and other small basins in

Orange County.................... 2

Santa Ana River, including Coyote Creek . . . . . . . 34

San Gabriel River, excluding Coyote Creek . . . . . . . 1

Los Angeles River, excluding Rio Hondo .. . . . . . . 43

Rlo Hondo... . . . . . . . . . . . . . . . 2

Ballona and Topanga Creek and other coastal areas in.

Los Angeles County ................. 3

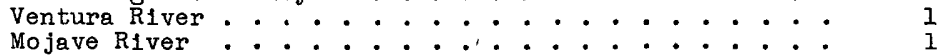

87

\section{Flood-damage survey}

Irmediately after the storm the Corps of Engineers, United States Army began a thorough field investigation to determine the extent of the damage. Every property owner or renter in the affected areas that could be located was interviewed, and an 1 temized estimate of flood damage was made. Reports on the damage were obtalned also from State, county, and c1ty officlals, from public and private ut1lity agencles, and from private corporations.

In making this flood-damage survey the Corps of Engineers endeavored to determine, with reasonable accuracy, the indirect damage as well as the direct physical damage caused by the storm. According to the report, 40 direct and indirect damage are defined as follows:

a. Direct damage--Physical damage to property, measured by the cost of repair or replacement in kind, including cost of clean-up.

40 Wyman, Theodore, Jr., Report on flood damage in Los Angeles Engineers D1str1ct from storm of February 27--March 3, 1938. 
b. Indirect damege--Indirect losses resulting from the direct flood damage :

(1) Loss of wages by employed persons.

(2) Loss of income from interruption of or delay in bus1ness, other than (4) or (5), below.

(3) Depreciation of property values in areas damaged.

(4) Loss of business by transportation companies due to damaged bridges and roadways.

(5) Loss to utilities of income of nonrecoverable types, owing to intermuption of services.

As it was not practicable to obtain a complete evaluation of the indirect damage in all areas, ratios of indirect to direct damage were determined from the fairly complete data avallable for the Los Angeles and San Gabriel River Basins, Including San Antonio Creek, and applied to other areas for which only data on direct damage were available.

Flood damages, segregated in table 32 for various types of property, are based on compilations made by the Los Angeles District, Corps of Engineers, United States Army. Table 32 does not in the main, except for public utilities, include any of the losses that occurred in the mountain areas, most of which are within the boundaries of the Angeles, Padre, San Bernardino, and Cleveland National Forests. The losses due to the flood, in certain of these areas, though large, have not been completely determined. Among the items for which figures are available are $\$ 89,500$ for Government-owned recreational facilities and $18 \mathrm{miles}$ of Govemment-owned telephone lines valued at about $\$ 12,550$, both within the Angeles National Forest. The flood damage in San Antonio Canyon, also in the Angeles $\mathrm{Na}-$ tional Forest, as determined by a survey conducted by the Corps of Engineers, amounted to $\$ 328,700$, the largest item of which was the destruction of 219 privately-owned cabins on Government leases. The supervisor of the Angeles National Forest reported the loss, in all, of 520 privately-owned cabins on Govermment leases. In most of the mountain canyons, however, the losses were considerably less than in San Antonio Canyon. 


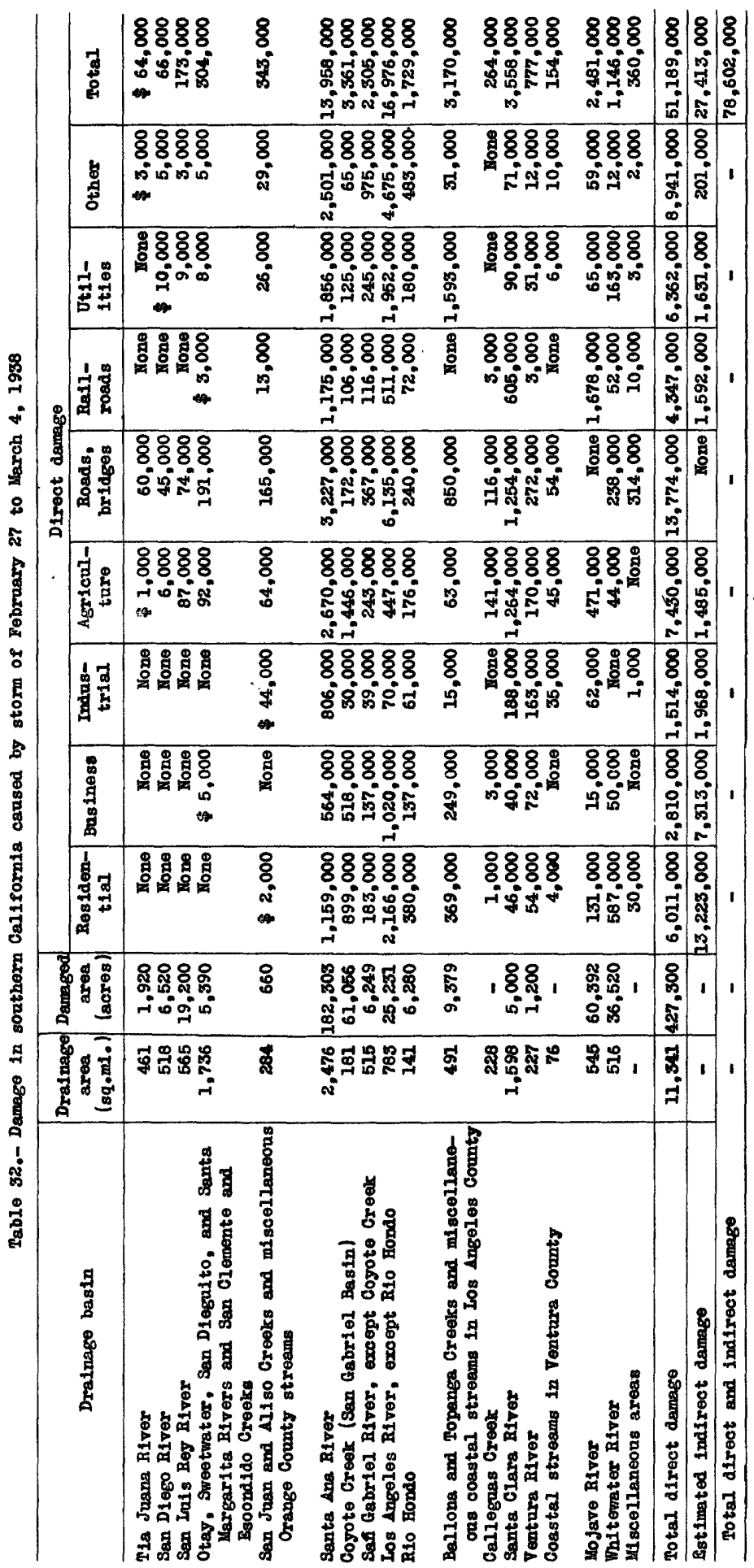


HISTORY OF FLOODS IN SOUTHERN CALIFORNIA

Since January 7, 1770, when Father Juan Crespi first observed the evidence of a great flood along the Los Angeles River, the flood runoff from the San Gabrlel and San Bernardino Mountains and other parts of the Coast Ranges has periodically submerged, damaged, and washed away crops, homes, missions, bridges, highways, railroads, and other structures located by man for his convenience in, on, or over flood channels and flood plains that had boen establishod by Nature for the passage of flood waters from the mountains to the sea. Only through discriminating consideration of these past floods can plans be formulated for protection from and control of future floods, which will inevitably occur. That damaging floods occurred in the past is unquestionable; little is known, however, of their relative magnitude. All were sufflciently severe to cause damage and temporary inconvenience, and some caused loss of lifo. Residents of southern California should real1ze that, in spite of the application of our most advanced technical and scientific knowledge of flood-control structures and practices, those who continue to occupy natural flood channols, flood plains, and dobris cones are defying inexorablo laws of nature.

Records of floods in southern California are found in a wide variety of publications and in mission records, speclal reports, transcripts of court hearings, and statements of old-time residents of the country. The earliest references to floods are found in the diaries of the Spanish Mission Fathers. These early records indicate that there were floods in $1770-71,1771-72,1775-76$, and 1779-80.

Testimony in the case of Daner1 v. The Southern California Rallway Co., In the Superior Court of Los Angeles County in 1897, and statiements obtained in 1915 from a large number of old residents and complled by Reagan 41 were to the effect that there were floods on the Los Angeles and $\operatorname{San} G a b r 101$ R1vers in 1811, 1815, 1822, 1825, 1832, 1842, 1851-52, $1859-60,1862,1867,1884,1886,1889,1891$, and 1914 .

Transcripts of public hearings concerning flood control held during 1938 by the United States district ongineer, War Department, recorded floods on the Ventura River in 1832, 1862, 1867, 1875, 1884, 1905, 1907, 1911, 1914, 1926, 1927, 1934, and 1937, and on the Santa Ana River in $1862,1867,1884,1886 ; 1889,1890,1906,1908,1909,1914,1916,1918$, 1921 , and 1926.

41 Rergan, J.W., Flood history of Los Angeles County, complled from all avaliable sources, 1914-15 (unpublished report). 
Official weather observations in southern California were begun at San Diego in 1851, at Santa Barbara in 1867, and at Los Angeles in 1878: Except for the floods recorded in their diaries by the Mission Fathers and a few isolated recorded statements by others, floods pior to 1851 mentioned in the various reports are described by the various witnesses from their personal recollections or from what others had told them about earlier floods. It is quite posslble that there may have been other floods that were not recalled and also that the relative magnitude of the floods as given may be open to question.

The first known estimates of flood discharge actually made at the time of a flood were two separate and independent estimates made on the Los Angeles River for the flood of 1889, one by William Mulholland and the other by H. Hawgood. 42

Actual current-meter measurements of major peak discharges have never been made, but since about 1894, continuous stream-flow records have been collected for the major streams in southern California, and peak discharges have been computed or estimated by various methods. These records are avaliable in the annual reports on surface-water supply and special reports published by the Geological Survey.

1770

Father Juan Crespi 43 gives us the first record of a flood in Cal1fornia. The following is quoted from his diary:

Jan. 17, 1770, * * we saw the Poiciuncula [Los Angeles] River. We crossed the plain in a southeasterly direction, arrived at the river, and forded it, observing on its sand, rubbish, fallen trees, and pools on elther side, for a few days previously there had been a great flood which had caused it to leave its bed.

The San Gabriel Mission was built about a mile from the banks of the San Gabriel River channel and about 3 miles directly south of El Monte. The fatiners planted and cultivated the low bottom lands around the mission, buc the crops of the first year were overflowed and destroyed in 1772. In 1776, owing to the recurrence of heavy flood flows, the mission was moved to 1 ts present location in San Gabriel, some 6 miles back from! the river.

42 Reports of the Board of Flood Control Englneers to the Board of Supervisors, Los Angeles County, Cal1f., p. 144, 1915.

43 Crespi, Father Juan, in Bolton, H. E., New California, vol. 2 , p. 256, 1926 . 
The flood of 1780 is reported in the following note, taken from the annual report dated December 31,1780 , at San Diego, by Fathers Lasuen and Serra: 44

A few days ago we had a heavy rainfall which filled the river bed and the lowlands where the wheat and barley had been planted ***. The indlans are now working hard to remedy the trouble for the present and to prevent similar disasters in the future.

Little is recorded of the flood of 1811 .

\section{5}

In 1815 there was a flood that changed the course of the Los Angeles River within what is now the city limits of Los Angeles from the east to the west side of the valley. The river turned and ran down where Alameda Street now 1s, broke westward near Fourth and Fifth Streets and jolned Ballona Creek, thence emptying into Santa Monica-Bay.

1821

A flood on the San Diego River in 1821 is reported by Smythe.45

By the year 1821, the little patches of cultivated land had mult1plied at the base of Presidio Hill and even spread up and across Mission Valley. Don Blas Agullar, who was born in San Diego in 1811 , recalled 15 such rancherlas, as they were called, which were occupled prior to the great flood of that jear. At two places in the valley there were vineyards. Most of the rancherlas were washed away or greatly damaged by the flood, which occurred in September or October and in a single night filled the valley and changed the course of the river.

\section{2}

In 1822 another great flood on the Los Angeles River covered all the lowlands and reached a greater helght than was ever known before. 46

Probably the greatest of the earlier recorded floods was that of 1824-25, of which Warner 47 writes:

In 1825 the rivers of this country were so swollen that their beds, their banks, and the adjoining lands were greatif changed. At the date of the settiement of Los Angeles City a large portion of the country from the central part of the city to the tidewater of the sea, through and over which the Los Angeles River now finds its way to the ocean, was largely covered with a forest, interspersed with tracts of marsh. From that time until 1825 it was seldom, if in any year, that the river discharged, even during the rainy season, its waters into the sea. Instead of having a riverway to the sea the waters spread over the country, filling the depressions in the surface and forming lakes, ponds, and marshes.

44 Engelhardt, Father Zephyrin, Mission San Diego, p. 109, 1920. 45 Smythe, W. E., History of San Diego, p. 99, 1907.

46 McGlashan, H. D., and Ebert, F. C., Southern Californla floods of January 1916: U. S. Geol. Survey Water-Supply Paper 426, p. 36, 1918. 47 Warner, J. J., Hayes, Benjamin, and. Widney, J. P., Historical sketch of Los Angeles County, p. 17, 1876. 
The river water, if any, that reached the ocean, drained off from the land at so many places, and in such small volume, thet no channel existed until the flood of 1825 , which by cutting a riverway to tidewater drained the marshland and caused the forests to disappear.

There are numerous references that testify to the fact that the Los Angeles River made the big change in its course during the flood of 182425. It was commonly understood and talked of in early days by old Mexican. settlers that, until the flood of 1825, the Los Angeles River flowed out through the southwest part of the c1ty into Ballona Creek and thence into Santa Monica Bay. But in 1825 the floods were probably the greatest for many years, causing the river to break eastward into San Pedro Bay. '

Pio Pico, former Governor of Alta California under the Mexican regime, and others have afflrmed that up to 1825 the Los Angeles River flowed along the high bank fust east of kain street down to about seventeenth street, thence southwesterly into cienega creek, which in turn flowed into Ballona Creek.

J. R. Ramirez, who furnished the following account, was born in Los Angeles in 1839. His great-great-grandfather settled there in 1786, and this pioneer's descerdants have lived on the old home place ever since. Mr. Ramirez says: 48

In those early days up to 1825 the river flowed along San Fernando street, which is just below North Broadway at this point. In 1825, the floods were the greatest in the past 100 years. This flood filled the whole Los Angeles River Valley from the bank between North Broadway and San Fernando Streets to the southern Pacific yards on the other side of the river. This flood changed the course of the Los Angeles River eastward from its old bank, along Main street, to somewhere near Alamada street. Its entire course was changed to the south side of the city. The river flowed out through Ballona Creek before this flood, but after this time it passed near the foot of the hills at Dominguez and into San Pedro Bay.

The flood of 1825 changed the course of the Santa Ana River also. Previous to that year the Santa Ana entered the ocean several miles to the northwest of its present channel.

1832

According to Warner, 49 the flood of 1832 produced considerable change in the contour of the country south of the city. He says:

The flood of 1832 so changed the drainage in the neighborhood of Compton and the northeastern portion of the San Pedro Ranch that a number of lakes and ponds, covering a large area of the latter ranch, lying north and westerly from Wilmington, which to that date had been permanent, became dry a few years thereafter.

The drainage of these ponds and lakes completed the destruction of the forests that Warner says covered a large part of the county south and 
west of the city. These forests were in all probability willow thickets or copse, the same as grew until quite recently on the low grounds near the mouth of the Santa Ana River and in the swampy lands along the San Gabriel River.

In Venturs County, the flood of 1832 destroyed the chapel of San Miguel. This is the first authentic record of flood damage along the lower reaches of the Ventura River. Father Engelhardt states:50

Fr. Uria remarks, In his report (1832), that the chapel of San Miguel, the pride of Father Senan, could not be saved, the floods having destroyed it entirely.

1849 to 1860

Floods of various magnitude occurred in 1849-50, 1851-52, 1859-60.

$1861-62$

The flood of 1861-62, has been called the "great flood" and the "Noachian deluge of California floods." During the flood period in 1862 the entire valley area from Los Angeles to the ocean, both toward San Pedro and toward Ballona, was a great lake. The Los Angeles River in the city of Los Angeles extended from Alameda Street to the bluff on the Pico Heights side. A little below Vermon, about where Vernon Avenue now is, the river split, and part of it went through Los Cienagas grant into Ballona Creok.

During the same flood the San Gabrlel River overflowed its banks, broke from 1ts course east of El Monte, and started a new channel to the west of El Monte, taking about the same course as later taken in 1867 to form what was then known as New River.

In San Bernardino, Riverside, and Orange Counties, the Santa Ana River became a raging torrent during the flood of 1862. The prosperous colonies along the banks of the river were completely inundated, and vineyards, orchards, and grain fields were transformed into a barren waste.

Flood waters of 1862 on the Ventura River are reported to have spread from the bluff on the Taylor Ranch to the hills east of Ventura Avenue, thus attaining a width of about 4,000 feet. Many houses were submerged and several destroyed. Parts of the old Mission waterworks, which brought water to Ventura, were destroyed. 1930.

50 Enge Ihardt, Father Zephyrin, San Buenaventura Mission, p. 68, 
In describing this flood, Guinn 51 states:

The great flood of 1861-62 was the Noachian deluge of California floods. The season's rainfall footed up to nearly 50 inches. The valley of the Sacramento was a vast inland sea. * * * In our country, on account of the smaller area of the valleys, there was but little loss of property. The rivers spread over the low lands, but stock found safety from the flood on the hills. The Santa Ana for a time rivaled the "Father of Waters" in magnitude. In the town of Anaheim, 4 miles from the river, the water ran 4 feet deep and spread in an unbroken sheet to the Coyote Hilis, 3 miles beyond. The Arroyo Seco, swollen to a mighty river, brought down from the mountains and canjons great rafts of driftwood which were scattered over the plains below the city and furnished fuel for the poor people of the city for several years. It began raining on December 24, 1861, and continued for thirty days with but two slight interruptions. The "Star" published the following local: "A Phenomenon--on Tuesday last the sun made its appearance. The phenomenon lasted several minutes and was witnessed by a great number of persons."

An interesting account of this flood is given by Mrs. Crafts, 52 who says:

The fall of 1861 was sunny, dry, and warm unt1l Christmas, which proved to be a ralny day. All through the holidays there continued what we would call a nice, pleasant rain, as it often rains in this section for days at a time. This much-needed molsture and wetting of the parched earth lasted until the 18th of January, 1862, when there was a downpour for 24 hours or longer. AIl the flat, from the Santa Ana River to Pine's hotel was under water, a perfect sea of water, Inundating the valley for miles up and down the rlver, and Lytle Creek came rushing down D Street, across Third, finding an outlet through an open space into Warm Creek. Many families fled in the night to higher ground, losing everything they had stored away for the winter. * * * The constant rain on the adobe houses turned them to mud, and, of course, they fell to pleces.

Heavy rains extended into San Diego County, according to a report by Davis, 53 in which he states:

In the winter of 1861-62 unusually heavy rains fell in San Diego County, being 30 inches, the average fall for that section of the state being 9 inchies.

$1867-68$

The flood of 1867-68 caused the San Gabriel River to break out of its channel and form New River. It broke out a little above the old Temple place and continued south, instead of making the turn toward the point of hills just below Old Mission settlement, and all the water of the San Gabriel went into the New River. Several thousand acres of land were washed away. Willows had grown up so thlck and large down by the old Mission settlement that when the flood came it simply knocked the trees down and covered them up with sand and boulders, forming a barrier that changed the channel and caused the flow to pass by the Pico Rancho and on to Los Alamitos Bay.

51 Guinn, J. M., A history of California, p. 428, 1915.

52 Crafts, Mrs. E. P. R., Ploneer days in San Bernardino Valley, p. 71,1906 .

53 Davis, W. H., Seventy-five jears in California, p. 335, 1929. 
A short time before the founding of Downey, a small settlement named Galatin had been started nearby, but the flood of 1868 caused the otherwise dry rivers to change their courses and Galatin was washed away. 54 Concerning the Santa Ana River, Brown and Boyd, 55 report as follows:

There was no further year of heavy rainfall unt1l that of 1867-68, when the winter proved rainy, but, while the precipitation was continuous, it was not as heavy as in 1862, and as a result less damage resulted.

The Ventura River overflowed its banks, and according to Hobson, 56 the whole of that portion of the town west of the point of the schoolhouse hill (tnis location being a little north and east of the present junction of Main Street and Ventura Avenue) was submerged.

1884

No serious floods occurred from 1868 to 1884 , a period of 16 years. Then southern California experienced a series of wet years, with floods in $1885,1886,1889$, and 1891 .

The flood of 1884 ranks among the major floods--in fact, there were two floods in 1884. The first came the latter part of February; it did little damage, but a great quantity of water fell, apparently utilizing much of the absorptive capacity of the ground. A second flood came within 6 or 8 days after the first and did a great deal of damage. AII the bridges across the Los Angeles River were washed out except one, the old Downey Avenue Bridge. Many houses were washed away, and several people dromed. The water broke out of the river banks and flowed toward Alameda Street, which was lower than the river bed; came up on First Street halfway between Los Angeles and Main Streets, and also flooded the lower part of the city. At Maple and Twenty-fourtr Streets the water was between 3 and 4 feet deep. The flood waters flowed westward along the then Washington Road and Jefferson Street and thence southwest into $\mathrm{Ba}$ lone Creek through which it reached Ballona Bay. All of the Cienega country became a great lake, and the country back of Venice a veritable sea.

San Fernando Valley was flooded from Chatsworth to Glendale. The Southern Pacific railroad was washed out in many places.

54 Newmark, Harris, Sixty years in southern California (1853-1913), p. 362,1926 .

55 Brown, John, Jr., and Boyd, James, History of San Bernardino and Riverside Counties, vol. i, p. 145, 1922.

56 Hobson, W. D., unpubilshed manuscript, 1876. 
The Santa Ana River cut a new channel to the sea. Beginning at a point below where Santiago Creek enters the Santa Ana, the river cut through the fertile lands east of the old channel and discharged into the ocean about 3 miles southeast of its former outlet. According to Brown and Boyd 57

The year of 1884 proved to be the great flood year of later times, and 37.50 inches were reported during the season for San Bernardino, while over 40 inches were registered at Los Angeles and more at other points.

In San Diego County the flood of 1884 was very severe. Smythe 58 says:

Thus far all appeared to be going well, but there was more trouble in store for San D1ego and 1ts raliroad hopes. In February 1884 a serles of violent storms descended and literally destroyed the section of the railroad through Temecula Canyon.

The season of 1883-84 was the wettest winter on record in San Diego County, w1th 27.59 inches of rainfall at San Diego and 50.51 inches at Escondido.

In Ventura County, 30 inches of rain fell in 27 days, of which 6 inches fell in 34 hours, causing the venture River to rise higher than it had been since the flood of 1867. It was estimated that 65 inches fell during February and March in the San Antonio valley, whose drainage is tributary to the Ventura River, causing the worst flood known in the valley. Casitas Bridge, crossing Ventura River at Foster Park, was washod out.

1886

Heavy rainfall with streams reaching flood stages and causing considerable damage was recorded in 1886-87. According to Newmark 59

The great flood of 1886 reached its first serlous state on January 19th. All of Los Angeles between Wilmingtion Street and the hills on the east side was inundated; levees were carried off as if they were so mich loose sand and stubble, and for two or three weeks railway communication with the outside world was impossible.

1889

The flood of 1889 was comparable in magnitude to that of 1884 . It came on Christmas Day, and the greatest damage occurred on that day. The Evening Express, Los Angeles, in 1ts issue of December 26, 1889, says:

Much damage has been done in and about the city. Streets cut up, conduits filled with sand, bridges carried away, railroad tracks covered with dirt, and much other damage done.

57 Brown, John, Jr., and Boyd, James, op. c1t., vol. 1, p. 145.

58 Smythe, W. E., op. c1t., p. 405.

59 Newmark, Harris, op. cit., p. 551. 
The approaches to the Kuhrts Street bridge were washed away. About half the Santa Fe bridge over the Los Angeles River swept away and dashed against the Buena Viste bridge, weakening the piers and badiy damaging it. The S. P. [Southern Pacific] pile bridge, below Kuhrts Street Bridge, almost entirely carried away. The 7 th and 9 th street bridges so badiy damaged as to render them unserviceablo.

The storm was general all over the southern section of the State. No trains on the Santa Fe or S. P. have either arrived or departed except one to Santa Monica. Levees in numerous places have been washed away.

The country between this city and the sea is in many places covered by water, and many ranchers will lose heavily. The oxtensive vegetable gardeners south of the city will lose heavily.

At Compton the water is very high. The citizens resorted to boats and made their way about tow in that way. The country in that vicinity is flooded, and it will be weoks before the surface will.be dry.

Hon. H. T. Gage says that the now San Gabriol, tho old San Gabriel, and the Los Angeles Rivers have formed one body neai Downey and are sweeping toward the ocean, carrying everything with them. On the Laguna Ranch a lake $5 \mathrm{miles}$ in width has formed, and the water nearly to the top of the hay stacks. The Los Angeles River, 2 miles below the city has swerved from its channel and is running down the Downey Road.

\section{1}

In 1891, floods were recorded on the Santa Ana River and some of 1 ts tributaries, and on the San Luis Rey River above Bonsa.11.60

\section{9}

The following extract from the RIverside Daily Press gives an account of a flood on the Santa Ana River in 1909:

December 31, 1909.- Santa Ana RIver was at its highest flood stage in 20 years. Portions of the Crestmore Electric Railroad line bridge, between Riverside and Crestmore, washed out. Chinese truck gardens, roads, crops, trees, fences, and farm buildings in the path of the flood were washed out. Head works of the power company's canal at the Salt Lake Ra1lroad bridge washed out. * * Barn on the Voorhes Ranch, above the Salt Lake Raliroad bridge, was washed away. Flood waters destroyed Evens Lake and dam below the bridge on the west side of the river. Ranch homes threatened. Many large cottonwood trees were uprooted and carried downstream.

Between Menifee and Ethanac, flood waters rushing of the San Jacinto Mountains into San Jacinto River and Bautista Canyon Ereek washed out the Santa Fe Railroad. Heavy runoff through Cajon Pass caused landslides on Santa Fe Rallroad along a stretch of 13 miles between Sumit and Devore stations. All train schedules abandoned. At Etiwanda, the Santa Fe Railroad washed out. Same road badly damaged at East Highland by mush of flood water off the San Bernardino Mountains. Railroad service between San Bermardino and Los Angeles abandoned.

With reference to this same storm, the San Bernardino Sun for Januery

1, 1810, says:

At 2 o'clock this morning, storm and flood conditions are quite serious. Rain was falling in torrents. Unless relief comes by daybreak, damages will run into thousands of dollars. Engine and three cosches of work train turned over $1 \mathrm{mile}$ north of Devore.

The Santa Fe Railroad roundhouse at San Eernardino surrounded by floodwater and that city has suffered more severely from floodwater than at any time in its history.

Approaches to Colton Avenue Bridge, Pacific Electric bridge, washed out. Colton entirely cut off from outside world. Iytlo Creok out of its channel and threatening Salt Lake Railroad shops at Colton. * * * 
The Southern Pacific "newspaper special train" from Los Angeles plunged into the Santa Ana River while crosing the bridge near Colton. Floodwaters had undermined piling of the bridge. Two coaches toppled over on the 30-inch pipe line of the RIverside-Highland Water Co., breaking 1t. Two sections of the plpe ine toppled into the river when the cement plers crumbled under the force of the raging stream.

Traffic on the Santa Fe Rallroad through the Santa Ana River canyon was abandoned because of landslides.

At San Jacinto, floodwaters out of the San Jacinto River and Bautiste Creek canyon ripped bad breaks in the river levee near San Jacinto and the lowlands between Gilman Hot Springs and Rallroad Canjon Dam of the Temescal Water Co. near Elsinore were badly flooded. Four inches of rain fell at Lake Hemet, raising the water in the lake to 112 feet.

Because of the washout of the approach to the West Riverside Bridge on the west side of the Santa Ana River, entrance to Riverside was by way of the Salt Lake Rallroad bridge and by way of Colton. Two spans of the Crestmore trolley line bridge were washed out.

\section{1}

In March 1911 there was a serlous flood on the Ventura River. January and February of that year were ralny months. Although no excessive downfall occurred, the soll was wet and conditions were ripe for a rapid runoff following the heavy rain of March 9. According to the Ventura Free Press for March 9, 1911,

The Ventura River perhaps was never higher. It is overflowing 1ts banks from Casitas to the sea. The Casitas Bridge, 17 feet above the water at normal flow, is under water; the Avenue is awash; the western part of Ventura is under water; at 3:15 the big steel bridge [Southern Pacific Railroad] broke and 50 feet of the structure went down and out into the ocean.

Floods of January and February 1914 were very destructive in southern California and were responsible for increased interest in stream-flow records. Water-Supply Papers 391 and 447 contain estimates of peak discharges avallable for this period.

1916

The flood of 1916 is covered by Geological Survey Water-Supply Paper 426, Southern California floods of January, 1916, by H. D. McGlashan and F. C. Ebert.

\section{6 to 1938}

No mejor floods occurred in the southern California region between 1916 and 1938, except the flood of January 1, 1934, which did considerable damage in Ia Cañada Valley area. This flood is described in Water-Supply Paper 796-C, Flood in La Cañada Valley, Californla, January 1, 1934, by Harold $C$. Troxell and John Q. Peterson. Minor floods, causing damage in certain areas, occurred during 1918, 1921-22, 1926, 1927, 1931, 1932, 1934,1936 , and 1937 . 


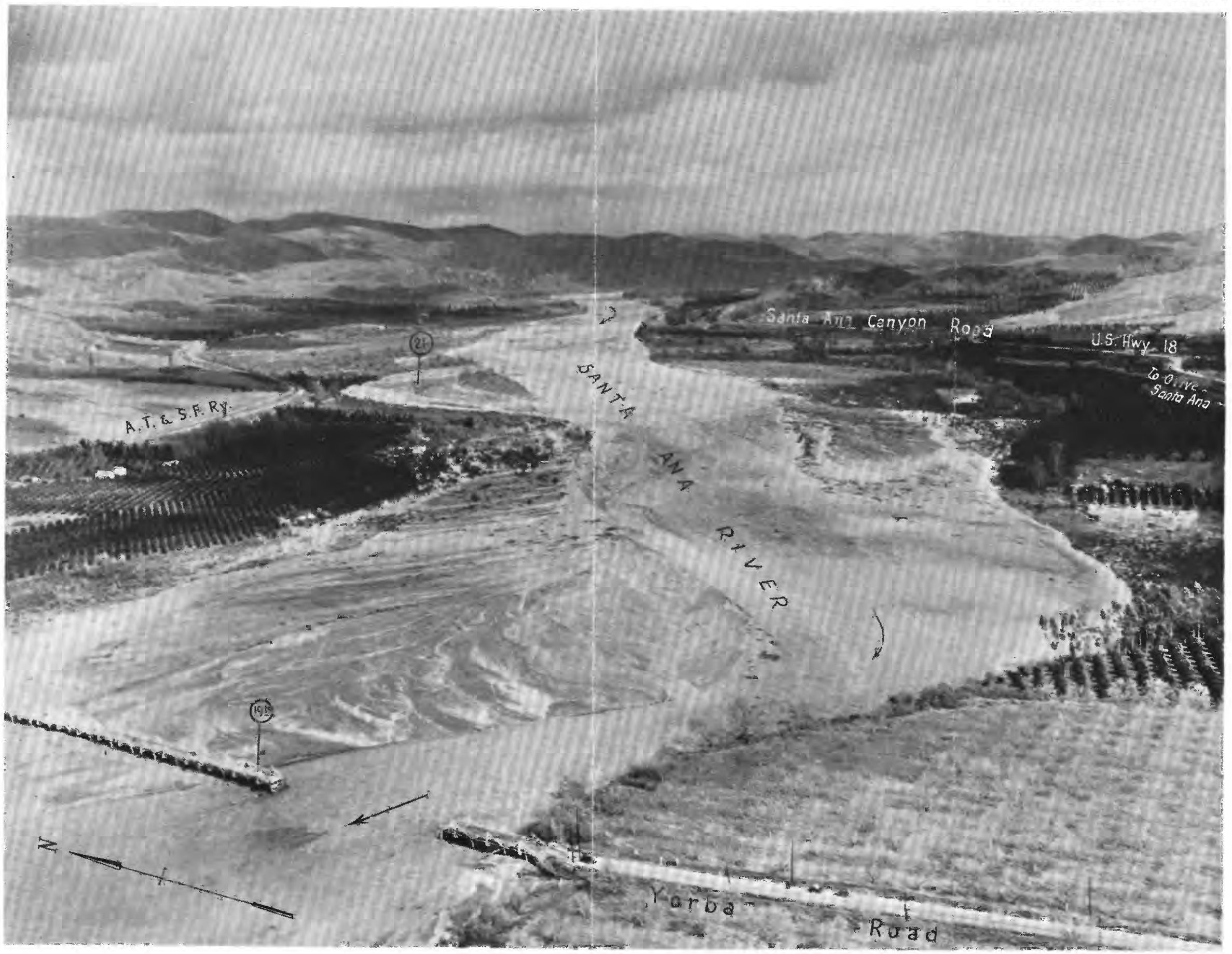

SANTA ANA RIVER BELOW LOWER SANTA ANA CANYON, MARCH 3, 1938.

Courtesy of Fairshild Aerial Surveys, Inc. 



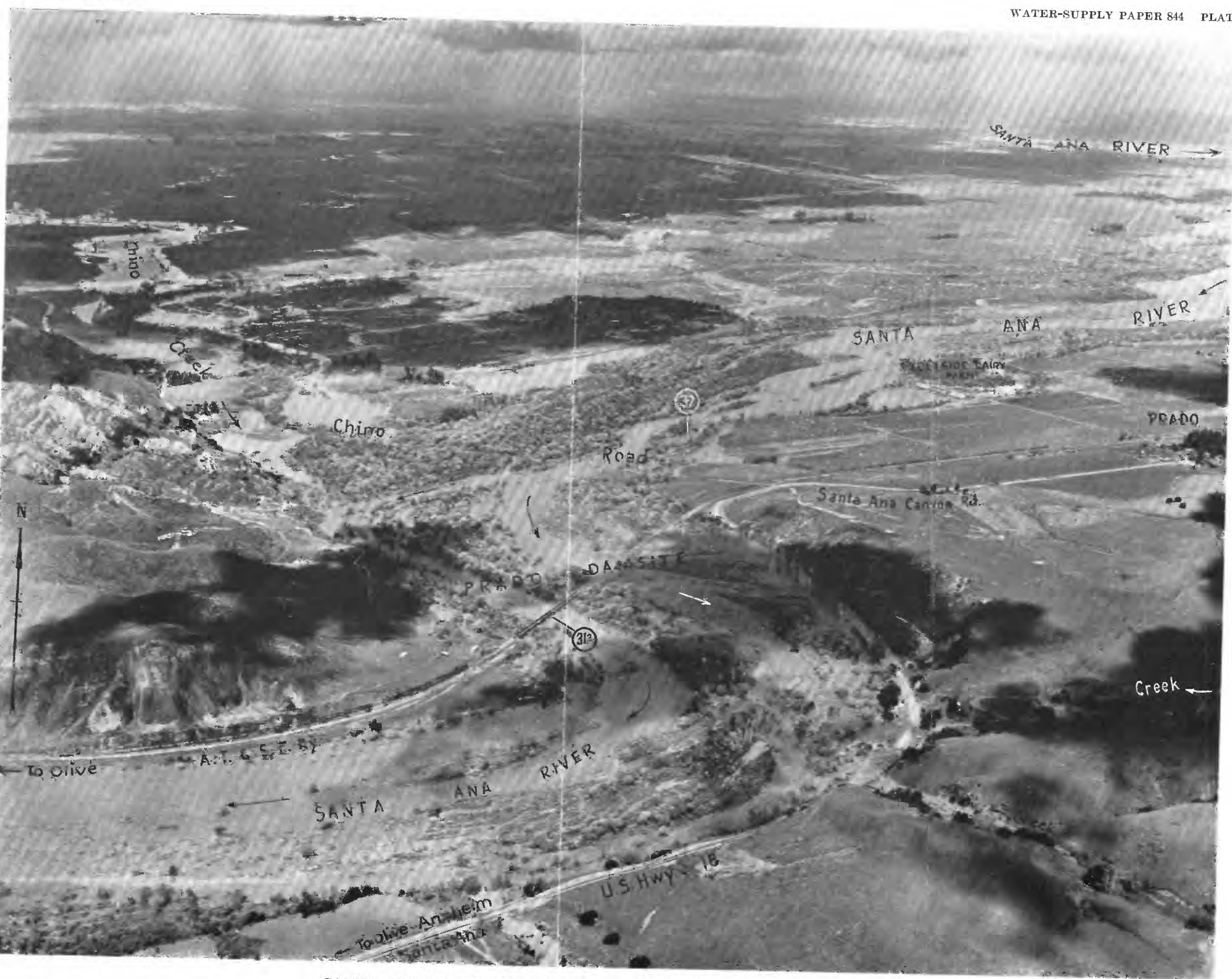

SANTA ANA RIVER AT MOLTH OF CHINO CREEK, MARCH 3, 1938.

Courtesy of Fairchild Aerial Surveys, Inc. 



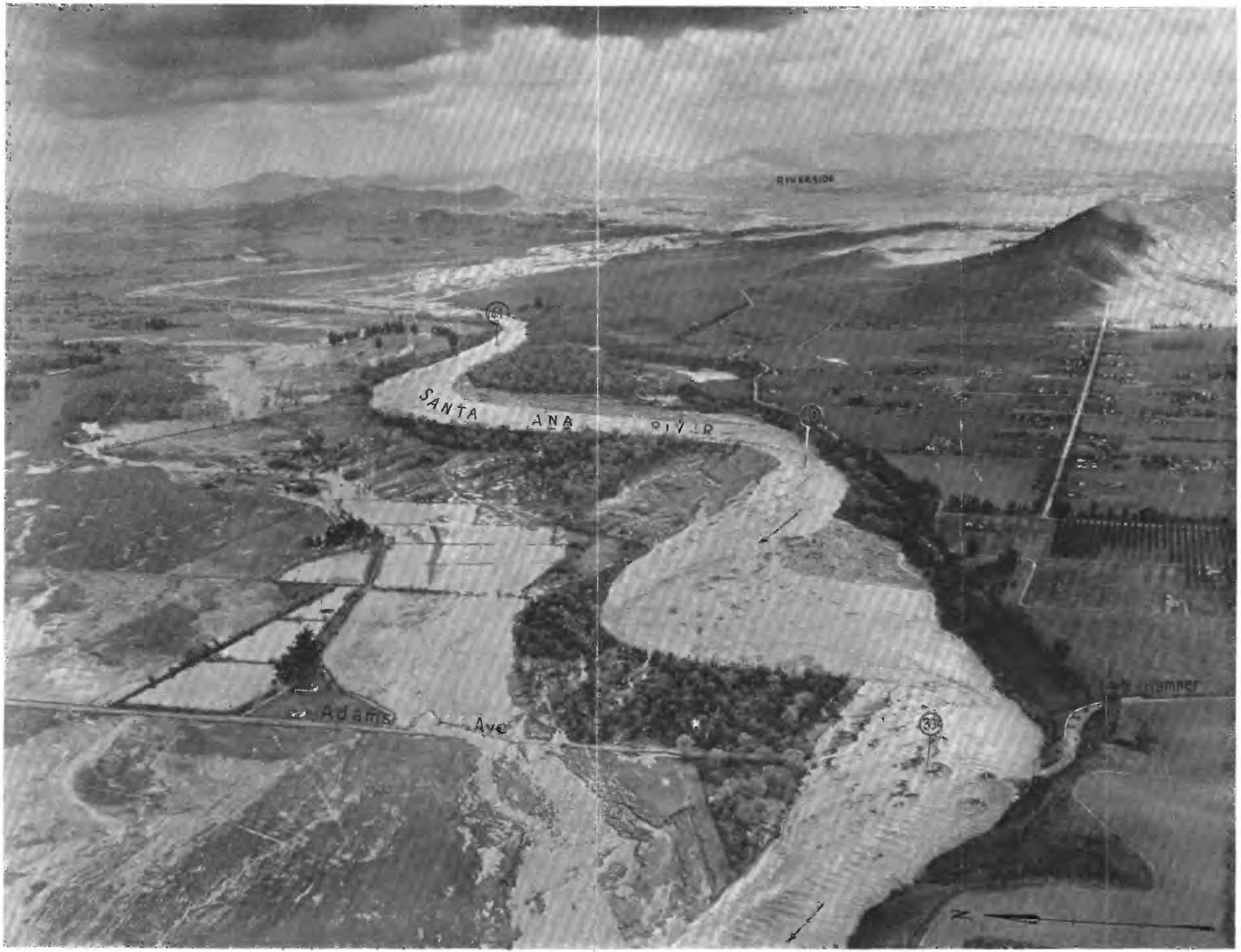

SANTA ANA RIVER NEAR NORCO, MARCH 3, 1938. Courtesy of Fairchild Aerial Surveys, Inc. 



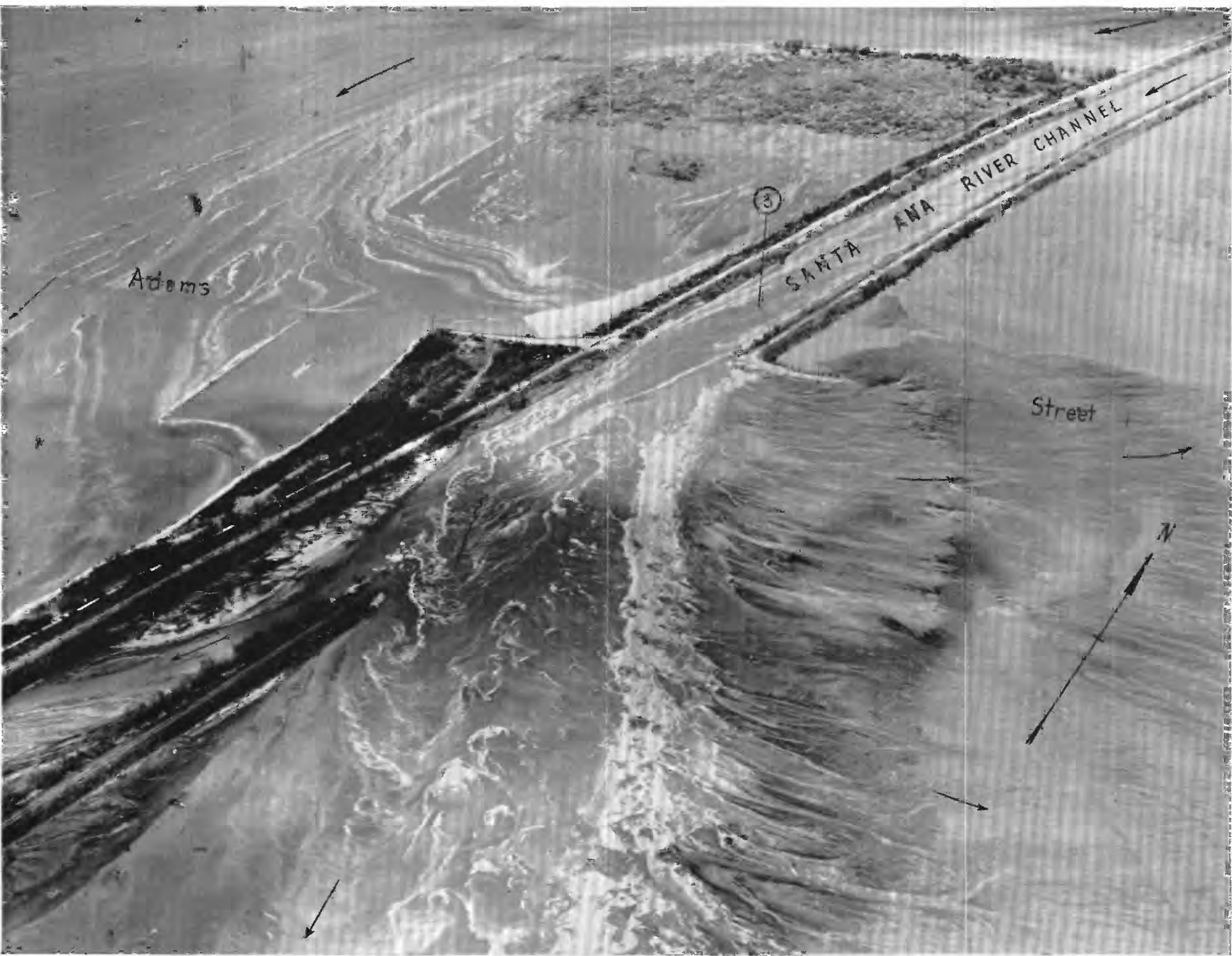

BREAK IN LEVEE OF SANTA ANA RIVER, MARCH 3, 1938.

Note development of debris cone, lower right, which formed bank of new channel. Courtesy of Fairchild Aerial Surveys, Ine. 


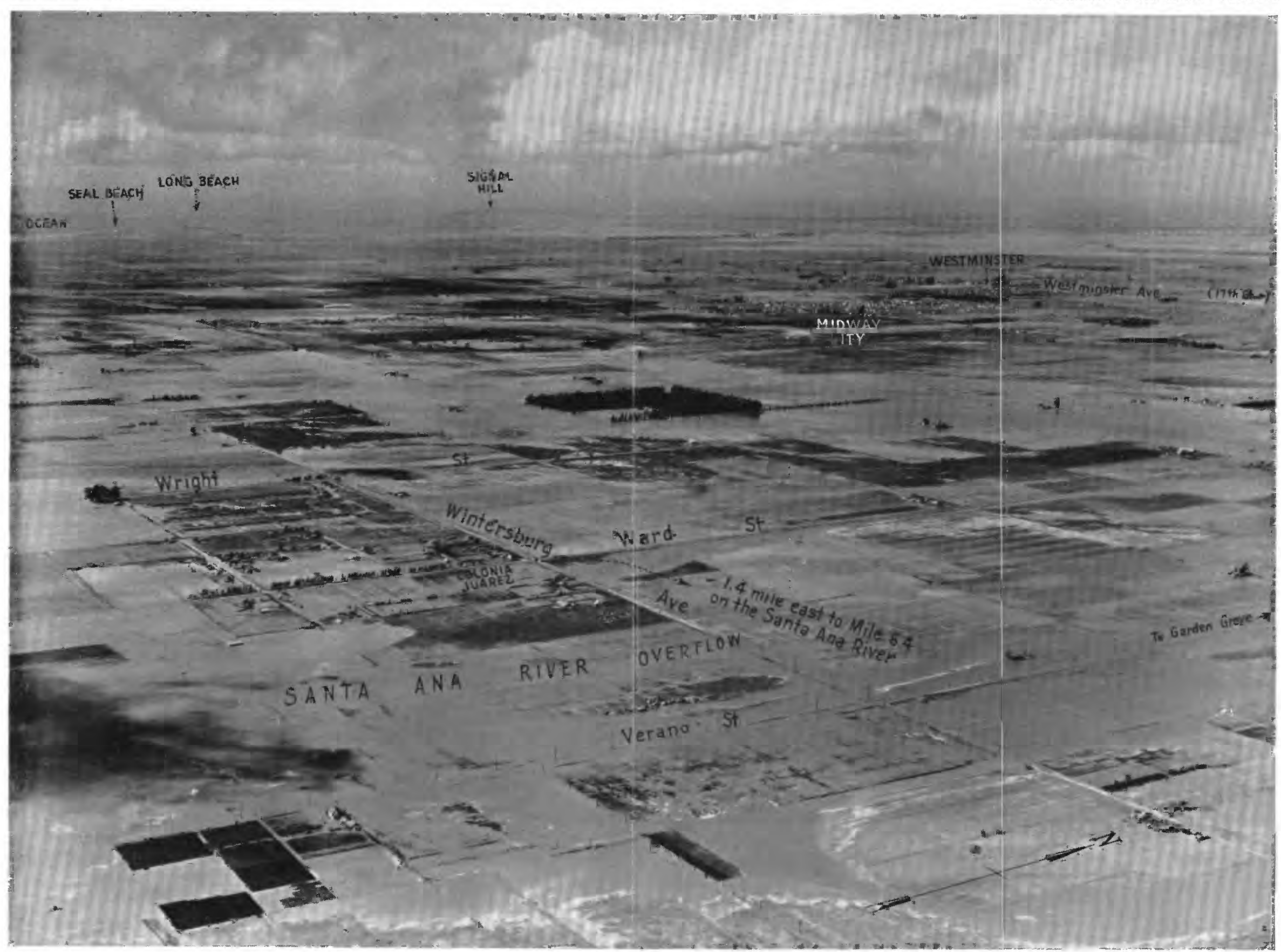

OVERFLOW AREA ALONG SANTA ANA RIVER, MARCH 3, 1938. Courtesy of Fairchild Aerial Surveys, Inc. 


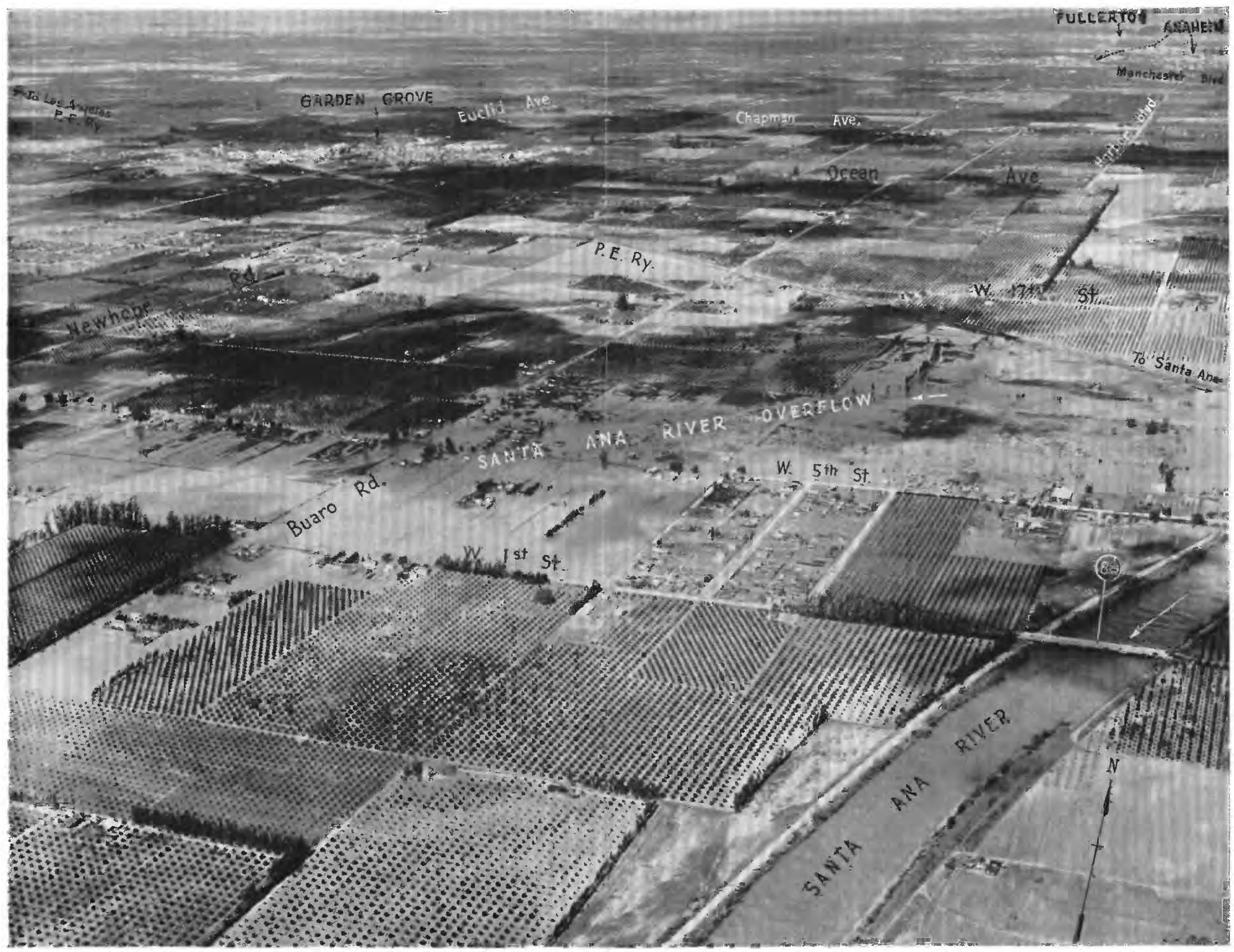

FLOODED CITRUS AND WALNUT GROVES ALONG SANTA ANA RIVER, MARCH 3, 1938. 
Acknowledgments ..........

Administration and personnel. .

Alhambra Wash, at Alhambra. . .

Al1so Creek, at EI Toro...203-204

Al1so Creok Basin, discharge in . 203-204,289

rainfall in....... 18-19

Alluvial cones, character of. . . 337-338

origin of . inition in relition

Altitude, precipitation in relation

Arrowhead Springs, Strawberry Creok near cinjoi Crék near.: :

Waterman Canyon Creek near. in....................

Arroyo Seco, near Pasadena. . :

Artesia, Coyote Creok near. . . .

Azusa, Rogers Creek near. . . . 232-233

San Gabriel River

near. . . 106-109,229-230,pl. 17,A B

Baldwin Park, Walnut Creek near. . Ballona Creek, near Culver C1ty.

Ballona Creok Basin, dischargo

floods in, previous: : : : : $263-265,295$

Barstow, Mojave River at. - . . : 284

"Basin," meaning of, in parts of report.......... 137

Benedict Canyon storm drain at Culver City $\cdot \cdot \cdot \cdot \cdot \cdot \cdot 265$

Bonsal1, San Lu1s Rey River near. 197-198 Boulder Creek, at Cuyamaca Reservoir, near Julian......

Brea Creek, at Fullerton. . . .

Brown, John, Jr., and Boyd, James, quoted. . .........391,392

Browns Creek at Chatsworth.: : : 246-247

\section{C}

Cajon Creek, near Keenbrook . . . 217-218 Cajon Creek Basin, phys lographic and geologic features of. . 318-319

Calabasas, Mallbu Creek near. .

Calleguas Croek Basin, discharge

In. . . . . * $129-133,296$

rainfail in: : : $36-37,72-73,77,80$ Camp Bonita, East Fork of San

Gabriel River near. - 174-176,228-229

West Fork of San Gabriel River

near. ........ 109-111,232

Campo Creek, near Campo . . . . 190-191

Carbon Creok, at Olinda . .

Carpinteria Creek Basin, discharge

In $\dot{c}$ rék, near culver city.

Centinela Creek, near Culver City.

Chat sworth, Browns Creek at . . . 246-247

Chino Creek, at mouth...:. : pl. 22

near Prado. . . . . . . . . 223-224

Chino Valloy, rainfali in. $28,43,70,77,80$

C1ty Creek, near Highland . . 176-180,213

Claremont, San Antonio Creek

near. . . . . 224-225, pl. 18, A

Thompson Creek near..... 120-121

Coastal plains, physiographic and geologic features of . $4338-339$

rainfali in.... . $31-35,44,71-72,77,80$

Colby Ranch, Tujunga Creek near. . 248

Colton, debris deposits in....

Iytle Creok at......: : 216-217

Warm Creok near : . . : :

Cones, aliuvial, origin and character of...... 301,337-338

debris, origin and control of . . 372

Contracted openings, flow through,

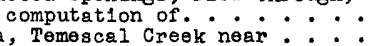

Corona, Temescal Creek near.

Creek, near Dulzura . . . 187-188

near Dulzura.: : *: : : : : : 186-187

Coyote Creek (San Gabriei River Bas in), near Artesia. $\dot{0}$.

Coyote Creok' (Ventura River Basin), near Ventura. . . . . . Craftonv1lie, M1ll Creek near. . Crafts, Mrs. E.P.R., quoted . : :
Cucamong a Creek, near Upland -210-220,pl. 17,

Culver CIty, Ballona Creek near. . Benedict Canyon storm drain at. - 265 Centinela Creek near. ...... 263 Cuyama R1ver, near Santa Maria. . . 280

\section{D}

Daingerfleld, I. H., quoted. Dalton Creek, at flood-control reservoir near Glendora... 117 - 118

near Glendorg 235

phys lographic and geologic

features of ........ 333-334

Davis, W. H., quoted. . . . . . 390

Day Creek, near Et1wanda..... 219

Debris, deposition of, in reser-

volrs and debris basins . . 372-376

effect of, on discharge : : : 100-101 movement of .....: i69,377-381 views showing........pls. 3, 6 ,

Debris basins, deposition $10, \mathrm{~B}, 16, \mathrm{~A}, 20, \mathrm{~A}$

views of......... . . is. $9,19,20$ Debris cones, artificial channels across. . . . ... 13, pl. 9

orituin and control of.

Deep Creek, near Hesper1a .282-283, pl. 18,B

Del Rosa, maximum precipitation

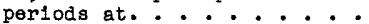

Devil Canyon, maximum precipitation perlods at..........

Dev1l Canyon Creek, near San Bernardino. - - * -

Discharge, into reservoirs, graphs showlng .......... 104

peaks of, computation of i3i-136,168-174 in relation to artificial storage......... 369-371 in relation to retention. . - 181 summary of.......... 286-299 records of. . . . . . 98-130,183-285 basic, definition of. . . . 98-99 consistency of. . : .:.:182-183 cont inuous, computation of. . 174-176 effect of debris on . . . 100-101 in relation to besin characterlstics and rainfall. . . 136-167 methods of obtalning. .98-i03, 168-184 See also Runoff; Stage and dis-

charge; names of streams and gaging stations.

Downey, Los Angeles River near. . . 245

R10 Hondo near. . . . . . . .

Drainage basins, area above floodcontrol dams. . . . 136-139

debris in, accumulation and movement of .......... 372-381 in flooded region, map showing.

phys fographic and geologic festures of. 139-140,149-167,300-350

See also RaInfall; Runoff; names of basins.

Duarte, Fish Creek near . . . . . 233

Dulzura, Cottonwood Creek near.: 186-188

Tia Juana R1ver near. . . . . 188-189

Dunsmuir debris basin, $372-373$ features cf. . . pls. 9, A, 19, 20

Eagle-Goss debris basin, outlet

channel from. - . . . pl. 9, B

East H1ghlands, Plunge Creok near. 208

rainfall in : íjod-controi reser-

volrnear Pasadena. . . . 127-128

near El Monte. : 257

near Pasadena . . . . . . . . 256

El Monte, Eaton Creek near. . . . 257

R1o Hondo near. . . . . . : 260

El Toro, Aliso Creok at....203-204

Elsinore, San Jacinto R1ver near. 221-222

Elsinore Lake at Elsinore. : $222-223$

Enge Ihardt, Father Zephyrin, quoted. 387,389

tIwanda, Day Creek near..... 219

East Et 1wanda Creek near. . . .pl. 16,A

Eapo-transpiration, effect of, on $351-352$

Evening Express, of Los Angeies, quoted........ 392-393 
Fa11 Brook, Santa Margarita R1ver

Fern Centon plots, rainfili and runoff on ........ 166-167

Fern Canyon Watershed $\mathrm{Nc}_{\mathrm{c}} \mathrm{2}$, near San Dimas.........

Fern Canyon Watershed No: 3 , near San D1mas . . . . . . . 114-115

Fern Canyon watersheds, description of........... . . . . pl. 15

rainfall and munoff on. 152,154-155, 167

F111, in stream channels. See Scour and fill.

F111more, Sespe Creek near. . . .

Flah Creek, near Duarte......

Flood of Harch 1938 , damage caused

by. . $13-14,381-384$, pls. $1-8,10,21-26$

general features of ...... 4-14

region affected by, maps showing. 5,8

Flood-control dams, rainfall, munoff, and retention at?.. 142-145

Floods, in southerm Callfornia, history of. . *.. 385-394

previous, in Baliona Creik Basin: 389-39C

In La Cañada Valley . . . . . . 394

In Los Angeles River Bas1n. . 386-393

in San Diego River Basin. . . 387,392

in $\operatorname{San}$ Gabriel River

Basin..... 386,389-390,393

In San Luls Rey River Basin . 3.93

in Santa Ana River Bas In. . . 388-394

in Ventura River Basin. $389^{\circ}-390,392,394$

Flood-control reservolrs, debris

In. . . . 100-101,372-376

discharge into. . : 103-104,131-136

effectiveness of. . . . 369-371

on Dalton Creek near Glendora : 117-118

on Eaton Creek near Pasadena. : 127-128

on Ilttie Santa Anita Creek near

Sierra Madre. . . . . 126-129

on Live 0ak Creek near La Verne. 118-119

on Pacolma Creek near San Fermando. 121-122

on San Dimas Creek near San Dimas. 112-113

on San Gabriel River near Azusa. 106-108

on San Gabriel River, West Fork

of, near Camp Rincon. . . 109-111

on Sàta Anita Creek near Sierra

uadre........ 124-125

on Santiago Creek near vilia

Park. . . ...... 105-106

on Sawpit Creek near Monrovia . 111-112

on Thompson Creek near Claremont. 120-121

on Tujunga Creek near Sunland . 123-124

Flood plains, character of . 301-3c2, 338-339

Fontana, Lytí Creik near . . . . 215

Foster, San Vicente Creek at. . . . 195

Fullertion, Brea Creok at. . : :

\section{G}

Gage heights, records of. . . . 183-285

Gaging stations. See Stream-gaging stations.

Geologic features of the area. . 300-350

alendale, Verdugo Creek at. . : 252-253

Glendore, Dalton Creek near: - i17-118,235

Ilt le Dalton Creek near. . . . 236

Great Basin, discharge in... . 299

rainfall in....... * 41

"Great" flood, accounts of: : : $389-390$

Gulnn, J. K., quoted....... 390

\section{$\mathrm{H}$}

Haines Creek near Tujunga . . . Hall Creek Basin, discharge in.

Hesperia, Deep Creek near. 282-283,pl. $18, \mathrm{~B}$

Wegt Fork of Mojave River near . 285

Highland, C1ty Creek near. . 176-180,213

Honda Barranca, at Berylwood Road,

near Somis. . . . . .

129

at Perkins Road, near Somis: : 129-130

Huasna River, near 8anto Mar1a. . 280-281

Huntington Beach, flooded area near. pl. 2

Hydrologic conditions in the area. 15-97

Infiltration, relation of, to retention and runoff. . . 166-167
Irvine Ranch drainage canal near

Tustin. . . 227-228 Is ohyetal maps, of 'San Dimas

Experimental Forest. . . . 57 cf southern California. . . pls. 11-13,

(In pooket), 14 preparation of. . . . . . . 46-49

$$
J \text {. }
$$

Jullan, Boulder Creek near. ...

Jurupa Basin, rainfall

In. . . . . 29,44,70-71,77,80

Keenbrook, Cajon Creek near . . 217-218

Ione P1ne Creek near. . .171,218,pl. 16, B L

La Cañada Valley, flood 1n. . . . 375,394 Lag, between rainfall and runoff. - 162-166 Lagune Creok Basin, discharge 1n. 289 Lake Hodges, San Diegulto River at. 197 Lakeside, San Diego River near. . 192-193 La Verne, Live Oak Creek near . : 118-119 Life, loss of, by flood..... 38 Ittie Dalton Creok, near ilendora. 236 Little Santa Anita Creek, near Sierre Madre... . *. 126-127,255 Little Tujunga Creek, near San Fermando. . . ...... 250

IIve Oak Creek, at flood-control reservolr near La Verre. . 118-118

Live Oak Creek Basin, rainfali, runof, and retention

in. ?...? $143,145,151-153$

Location of region affected by

flood........... . 4,5

Lompoc, Santa Inez River near. . : 278 Lone Píne Creek, near Keenbrook . . . . 171,218,pl. 16,B

Lone Pine Creek Basin, physiographic and geologic features of. . 319-321 rainfall, rumoff, and retention in. 358 Long Beach, Ios Angeles River at. • 246 Los Alamitos, San Gabriel River

near. ..........23I Los Angeles, Los Angeles River at .244, pl. 7 Los Angeles River, at Long Beach. 246 at Los Angeles........ 244, pl. 7 channel of, scour and f111 in . 379-38c near Downey ........... . 245 near Universal city. . . . . . 243 near Van Nuys.:.:.:. : 242 Los Angeles Rlver Basin, area above flood-contrcl dams in... 137-139 discharge in. . . . . 121-128,242-262 peaks of. . . 132-135,293-295 floods in, previous: : * : 386-393 physiographic and geologic $140,322-327$, features of . . 330-335,337-338 rainfall in . . is-35,42-44,55,63-72, $75-77,79-81,82-83,150$
$156-157,362-363,364-367$ reservoirs in, storage and

flow at............370-371 retention in.: : 181,362-363,364-367 runofe in . . 134,147,150,156-157, $164,362-363,364-367$

Lytle Creek, at Colton. . . 216-217, pl. 3 at San Bernardino . . . . . 215-216 near Fontena. . . . . 215 Lytle Creek Basin, physiographic and bologic features of. . 335-337 rainfall, munoff, and retention

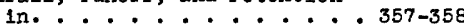

M

Mal1bu Creek, at Crater Camp, near Calabasas 267

Malibu Creok Basin, discharge in. 267-296 rainfall in? : 36-37, 45,72-73,77,80 Manning formula, use of, in computing peak dischárge... . 169-170 Matilija Creek, at Matil1 ja... . 272 Mentone, Santa Ana River near . 204-205 Mesa Grande, Santa Yaabel Creek near. 196 
Meteorologic conditions in the area. Page

Mill Creok, near Craftonvillo.

Mili Creok Basin, phys lographic and geologio features of. . . rainfall, runoff, and retention

Misolon Acros, Pacoima croik at: $357-358$

Mission Fethers, records by, on

floods. . . 386,387,389

Mojave RIver, at Barstow. : . . 284 at lower narrows, near victor-

villo *. : . . . . . 283

West Fork of, near Hesperia... 285

Mojavo River Basin, dischargo $282-285,298$

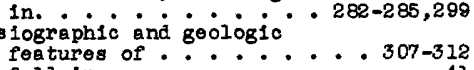
rainfall in : íriniar: ili-112,234

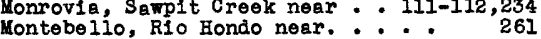

Montec1to, Santa Ynez River near. - 275

Norena Dam, Cottonwood Creok at - 186

Morr 1s Reservolr, records at. *: : 108-108

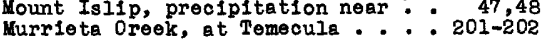

\section{$\mathrm{N}$}

Nestor, Tia Juana River near. . . 189-190 Newmark, Harris, quoted : : : : : pl. 392 Norco, Santa Ana Rive

Oceanside, San Luls Rey River at. 198-199 Olinda, Cárbon Creek at...... 241 Orange, Santiago Creok near . . . pl. 5 Otay River, at Savage Dam : Otay River Basin, discharge in. : 191,287 physlographic and goologic

features of . . . . 304-307 rainfall in. . . … $16,42,63,75$

\section{$\mathrm{P}$}

Pacific slcpe besins, discharge In, peaks of. : : : $287-298$ Pecolma Creek, at flood-control reservolr near San Fermando - 121-122

at Miss ion Acres. . . . . . 247-248

near San Fermando ........

Pacolma Creok Basin, physiographio and geologie features of. . 330-331

Pala, San Luls Rey River near: : 306
-282

Palm Canyon Creok, near Palm Springs 281-282

Pasadena, Arroyo Soco near. : : i27-128,256

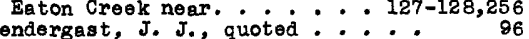

personnel: : : quoted: : :

Physlographic and geologic features of the eren 300-350

P1co, San Gabriel River at. . . 230-231

Piru Creok, noar Piru. . . . .

Piru Creok Basin, physiographic and geologic fentures of. ....

Plunge Oreok, near East Hlghlands.

rado, Chino Creok near . - : . 223-224

Santa Ana River noar.: 205-206,377,378

Precipitation. See Rainfail; Snow.

Precipitation stations. Soe Rain gages.

\section{$\mathrm{R}$}

Rain gages, distribution and

grouping of .. 46-50,61-62,82-90

precipitation at. . . . 16-45,61-90

Rainfali, altitude in rolation

to.......... 47-60,82-93

entecedent to storm of Feb $27=$

Mar. 4, 1938. 95-96,353-358

chronologic distribution of,

Fob. 27-Mar. 4, 1938. . . 58-61

da11y. . . . . $16-46,63-74$

hourly: : : $60,63-90,150-162, \mathrm{pl} 1.14$

Infiltration of :. . . . . . 166-167

intensity of. . . . . . . . 58-95

isohyotal maps showing. $46-49,54$, $57-58, \mathrm{pls}$. $11-13$ (in pocket), 14 local characteristica of. meximum if coilection of . $58-62,79-95,162-165$
ReInfal1--Continued.

retention of. . . . 166-167,351-369 runoff in relation

to. . . 136-167, 176-179,35i-369

Ramirez, J. R., quoted. . . ... 388

Ramona, Santa Ysabel Creek near : $\quad 306$

Rating curves, computation of peak discharge from. ..... 172-174

Redlands, San Timotoo Creok near. - 200

Reservolis, records at. ? - 105-113,117-128,194,221,275 See also Fiood-control reservolrs; storage.

Retention, definition of. .... 351

factors affecting... . . 353-358 relation of, to ovapotranspiration......351-352

to infiltration..... 166-167

to peak discharge...... 181

to rainfall . . . 140-149,351-369

to munoff . : : : 140-149,351-369

Rio Hondo, near Downey. . . . . 262

near El Monte........... 260

near Montebel10......... . 261

Riverside Dally Press, quoted.: 393

Rogers Oreek, near Azusa. : . : 232-233

Rosemead, Rubio Canyon Wash at: : 258

Rubio Canyon Wash at Rosemead. . 258

Runoff characteristics of. : 140-149

lag between precipitation and: 162-166 physlographic and geologio features affecting. 136-140,300-350 records of collection of. . . . 14 relation of, to artificial

atorage...". . . . 369-371

to Infiltration: : : 166-167

to rainfal1... 140-166,176-179, $306,351-360$

to retention. . . 140-149,351-368 $\mathrm{S}$

Salton Ser Basin, discharge in .279,281-282 phys lographic and geologio

features of . . .... 307-311

San Antonio Creok, near

Claremont . . . 224-225, pl. 18, A

San Antonlo Creok Basin,

phys fographic and goclogic

features of . . . 332-333

reinfall, runoff, and retention

in. . . 357-358

San Bernariino, Devil Canyon Creok

near. . . . . . . . . 214

Lotle Creok at.:- : $: 215-216$

San Bernardino mountain area, physiographic and goolog 10

features of ....... 307-321

rainfall in : : $23,43,67,76,79,84$

San Bernardino Sun, quoted. : 393-394

San Bernardino valioy, physiographic and geologic features of. . 337-338

San Diego area, physiographic and

geologic fertures of. . 302-307

San Diego RIver, at El Capitan Dam.

near Lakeálde. . . . . . 192-193

near Santeo.:. : : : 193-194

San Dlego River Basin, aischarge

In.

floods in, previous: : * 387-392

physlographic and goclogic features of. 304-307

rainfall, runoff, ind rotention

in. 16-17, 358-364

San Dieguito River, it Lake Hodges. 197

San Dieguito River Basin, discherge in. . . . . . 196-197,288

phys lographic and goologio

fentures of . 304-307

rainfall, runoff, and retention

in. : $\cdot 16-17,358-359,360-361,364$

San Dimas, Ferm Canyon watersheds

near. . . . . 114-115

San Dimas Creok near. . . . 112-113,234

West Fork of San Dimas Crook

noar. ........... 116

Wolfsk1I Creok noar. . . . 115-116

San Dimas Creok, at flood-control

reservolr near San Dimas. . 112-113 
San Dimas Creek--Contimued.

near San Dlmas. -

San Dimas Creek Basin, als charge in 131,116 physlographic and geologic

features of . . . . . 333-334

rainfali and runoff in.

154

San Dimas Experimental Forest.

infiltration experiments in . 166-167

rainfali in ........ 54-58

San Fermando, Littio Tujunga Creek

near. ......... 250

Pacoims Creek near. * : : i2i-122,247

Tujunga Creek near. ..... 249-250

San Fernando Valley, physiographic

and geologic features of. . 337-338

ralnfal1 in..... $23,25,43,67,76,80$

view of............. pl. 8

San Gabriel mountain area.

physiographic and geologic

features of ....... 322-338

raln gages in : : : : : : : : $322-338$

rainfali in . . . . . 19-23,42-43,

San Gabriel River, at flood-control

reservolr No. 1, near Azusa. 106-108

at Foothill Boulevard, near

A zusa . . . . . . 229-230

at Morris Reservoir, near Azusa . 108 109

at $\mathrm{P} 100 . . . . . . .230-231$

at Spring Street, near Los

Alamitos. . . . . . . . 231

channel. of, scour and fili in : $\quad 380$

East Fork of, near Camp

Bontta. . . . 174-176,228-229

profile of............ . . 10

gaging station on :-pl. 17,

Fest Fork of, at Camp Rinoon.

flood-control reservolr No.2,

rainfall and runoff on: : : : 142,153

San Gabriel River Basin, area ibove flood-control dams in . 137-139

discharge in...... 106-121,228-241

peaks of. . . . . 132-135,291-293

floods in, previous .. . 386,389-390,393 physiographic and geologic

features of . - 140,327-330,337-338

rainfall in . . 19-35,42-44,55,63-72,

$75-77,79-81,82-83,142,150$

$153,156-162,360-363,364-367$

reservoirs in, storage and

flow at. 369-371

retention in. . . i isi, 360-363,364-367

runoff in : $: 134,142,146,150,153$,

San Gabriel Valley, physiographic

and geologic features of. . 337-338

rainfali in... 25-27,43,68-69,76-80

San Jacinto, San Jacinto River near. 220

San Jacinto River, at Railroad

Canyon Reservolr, near

Elsinore.

221

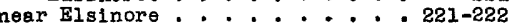

near San Jacinto........ 220

San Jacinto Rivor Basin, rainfali

In. . . 29-30

San Jose Creek, near Whittier: :

San Juan Capistrano, San Juan Crook

near. . ........... 202

Trabuco Creek near.: : : : 202-203

San Juan Capistrano Creok Basin, rainfall in...... 18-19

San Juan Creok, near San Juan

Capistrano. : . . . .

In. . . . . 202-203,289

San Luis Obispo Creok Basin,

rainfali in ...39-40,45,74,77,80

San Luis Rey River, at Dceans1de. 188-199

at $\mathrm{Pa} 1 \mathrm{a} . .$. . . . . . . 306

near Bonsail: : : : : : : : $197-198$

San Iuis Rey River Basin, discharge

in. . . . . 197-199,288

floods in, previous: 393

physlographic and goologic

features of :- *303-307

rainfal1, runoff, and retention

in.? 18,56,358-364

San Rafael Hilis, rainfall.

San Timoteo Creok, near Rediands. $\quad 209$
San Vicente Oreek, at Foster. . Page

Santa Ana, Santa Ana River at: : 206-207 Santlago Creek at ....... 226-227

Santa Ana R1 ver, at Senta Ana . 206-207 channel of, scour and fill in: 377-379 near Mentóne. . . . . . . . 204-205 near Prado.: : : $205-206,377,378$ proflle of. - . - * $\cdot \dot{i} 9$ views of........pls. 1,2,4,21-26

Sante Ana River Basin, discharge

1n. . - . 105-106,204-228,377-378 discharge in, peaks

of..? . . 132,134,135,289-291

floods in, previous . 388-394 physlographic and geologic $140,307-311$ features of ? rainfall in.? i9-35,42-44,56,63-72, $75-77,79-81,82-83,150$,
$360-361,364-367$

reservolrs in, storage and flow retention in.: : : i i $1,360-36 i, 364-367$ runoff in . 134,150,180,360,361,364-367

Santa Anita Creek, at flood-control reservolr near slerra Madre 124-125

near Slerra Madre....... . 124-125 Santa Antta Crook Basin, rainfili, runoff, and retention in. - 351-357

Santa Barbara, Santa Ynez River near. .......... . 276-277

Santa Clara River, near Saugus: : 276

Santa Clara River Basin, dischargo

in. . . . 268-271,296-297

physlographic and geologic features of . . . 322-327, 340-346 rainfall, runoff, and retention 1n. : $362-363,368$

Santa Margarite River, at Ysidora. 201

near Fall Brook . . . . . . 200-201

Santa Margarita River Basin, . 199-202,288 discharge in. .
physiographic and geologic . " 199-202,288

rainfall, munoff, and retention

in. : * : . 18,358-359,360-361

Santa Maria, Cuyama River near. . : 280

Huasna River near....:. 280-281

Santa Maria River Basin, dischargo

In. . . . . . . 280-281, 298

physlographic and geologic features of ? $340-342,350$ rainfall, runoff, and retention in. 39-40,362-363,368-369

Santa Paula Croek, noar Santa Paula. 27l

Santa Ynez, Santa Ynez River near. 278

Santa Ynez River, at Juncal Reservoir, near Montecito. . .

below Gibraltar Dam, near Santa Barbara.........

near Santa Barbara. . . . . . 276

near Santa Ynez......... 278

Sante Ynoz River Basin, discharge

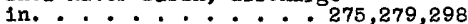

physiographic and geologic features of . . . 340-342,348-349

Santa Ynez River Basin, rainfell, runoff, and reteintion

in. 39-40,362-363,368-369

Santa Ysabel Creek, near Mesa

Grande........... 196

near Ramona . . . . . . . 306

Sante日, San Diego River near. . 193-194

Santiago Creok, at Santo Ana. . 226-227

at Santiagc Reservolr, near

villa Park. ........ 105-106

flooded lands along : : : : : : pl. 5

near Orange . . . . - . -

near Villa Park . . . . 225-226,pl, 5

Santiago Creok Basin, rainfall

In. . * : $30,44,70,77,80$

Santiago Reservoir, recoras at. ? 105-106 Saugus, Santa Clara River near. . 268

Savage Dam, Otay River at..... 191

Sawplt Creok, at flood-control reservoir near Monrovia. . 111-112

near Monrovia........ . . 234 scour and fill, in siream channels. . 174-176,377-380

Sespe Creok, near Fililmore. . . . 270

Sespo Creok Basin, physiographic and geologic features of. . 344-345 
Sierra Madre, Iittle Santa Anita

Creek near. .... 126-127,255

Santa Anita Creek near. : : 124-125,255

Slope-area method, computation of peak discharge by ..... 169-171

typical reach used in application

of. . . . . . . . . . pl. 16,8

Smythe, W. E., quoted ...... 387,392

Snowfali, records of. . . . : : 96-97

Somis, Honda Barranca near. . . 129-130

Stage and discharge, records of . 183-285

relation between. ...... 172-175

Storage, artificial, effect of, on fiood discharge .... . 369-371

Storm of Feb. 27-Mar. $4,193 \dot{8}$,

damage caused by. . . . . 382-384

general features of . . $\cdot$. $\cdot$ - 4-14

movement of . . . . 58-59,93-95, pl. 14

rainfall and retention preceding . 351-358

region affected by, maps showing. $5,7,8$

Strawberry Creek, near Arrowhead

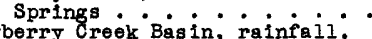

Stramberry Creek Basin, rainfali, runoff, and retention in. . 351-357

Stream-gaging stations, stage and discharge at, records of. . 183-285 views of, after reconstruction. p1s. 17,18 Sweetwater River, at Sweetwater

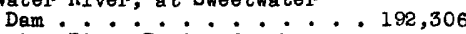
Sweetwater River Basin, discharge

in. . . . . 192,287 phys iographic and geologic

features of . . . . . 304-307

rainfall in ........ 16,42,63,75

Sunland, Tujunga Creek near. 123-124,249 $\mathrm{T}$

Temecula, Murrieta Creek at. . . 201-202 Temecula Creek near ..... . 199-200

Temecula Creek, at Nigger Canyon,

near Temecula.....? 199-200

at Railroad Canyon, near

Temecula.:. * *.... 200

Temecula Creek Basin, rainfail in:

Temescal Creek near Corona. . . .

Temescal Creek Basin, rainfall in

Thomps on Creek, at flood-control reservolr near Claremont. . 120-121

Tia Juana River, near Dulzura . : 188-189 near Nestor......... 189-190

Tia Juana River Basin, discharge

in. $: . * . . . * 186-191,287$

physlographic and geologic

features of . ..... 304-307

rainfall in........16,42,63,75

Topanga Creek, near Topanga Beach
Topenga Creek Besin, discherge in 266,206

rainfall in... 36-37,72-73,77, 80 Trabuco Creek, near San Juan Capistrano. ....... 202-203 Transpiration. See Evapo-

transpiration.

Tujunga, Haines Creek near. $\dot{\text { Tuja }}$. reservolr near Sunland. . . 123-124

near Colby Ranch. . . . . . . 248

near San Fernando...... 249-250

near Sunland. . . . . . . 249

Tujunga Creek Basin, physiographic and geologic features

of. . 137-140,330-331

Tustin, Irvine Ranch drainage canal near. . $\mathrm{U}_{\mathrm{U}} \cdot{ }^{*} \cdot . \cdot 227-228$

Universal City, Los Angeles River

near. 243

Upland, Cucamonga creek near $219-220$, pl.17,

\section{$\mathrm{V}$}

Van Nuys, Los Angeles River near. - 242

Venturs, Coyote Creek near. . . . 274

Ventura River near...... 273

Ventura County, rainfail in: : : 54,55

Ventura Free Press, quoted. . . . 384

Ventura River, near ventura . . . $\quad 273$

Ventura River Basin, discharge

1n.......... 272-274,297

rloods in, previous: : 389-390,392,394 physiographic and geologic

features of .?. 340-342,346-348

rainfall, runoff, and retention

in. . 37-39, 45,73-74,77,362-363,368

Verdugo Creek, at Glendale. . . 252-253 Verdugo Mountains, rainfali

in................... $43,68,76,80$

Victorville, Mojave River near. . 283

Villa Park, Santiago Creek near. . . . 105-106,225-226,pl. 5

\section{W}

Walnut Creek, near Baldwin Park . . 237

Warm Creek, near Colton .... . 210

Warner, J. J., quoted.:.: : : 387-388 Waterman Canjon Creek, near Arrow-

head Springs. . . . . . 212

We1rs, flow over, computation of. , 171-172

Whittier, San Jòse Creek near. . . 238

Wolfskili Creek, near San Dimas: : $115-116$ $\mathrm{Y}$

Ysidora, Santa Margarita River at. 201 

The use of the subjoined mailing label to return this report will be official business, and no postage stamps will be required

\section{UNITED STATES \\ DEPARTMENT OF THE INTERIOR}

GEOLOGICAL, SURVEY

OFFICIAL BUSINESS

This label can be used only for returning official publications. The address must not be changed.
PENALTY FOR PRIVATE USE TO AVOID PAYMENT OF POSTAGE, \$300

\section{GEOLOGICAL SURVEY,}

